\title{
Enantioselective Fluorescent Sensors for Chiral Alpha-Hydroxycarboxylic Acids, Amino Acid derivatives and Diamines
}

\author{
Shanshan Yu \\ Dezhou, China
}

B. S., Wuhan University, China, 2008

A Dissertation presented to the Graduate Faculty of the University of Virginia in Candidacy for the Degree of

Doctor of Philosophy

Department of Chemistry

University of Virginia

Aug, 2013 Degree will be Conferred 


\begin{abstract}
A series of enantioselective fluorescent sensors based on chiral 1,1'-bi-2-naphathol (BINOL) was designed and developed for the recognition of chiral $\alpha$-hydroxycarboxylic acids, amino acid derivatives and diamines.

A new concept with the use of a pseudoenantiomeric fluorescent sensor pair for enantioselective fluorescent sensing was introduced. A pseudoenantiomeric fluorescent sensor pair is composed of two fluorescent sensors that emit at different wavelengths and have the opposite enantioselectivity. When a 1:1 mixture of BINOL-based and $\mathrm{H}_{8} \mathrm{BINOL}$-based sensors are used to interact with mandelic acid, the sum and difference of the fluorescence intensity at the two different emission signals of the two sensors allow the determination of both the concentration and the enantiomeric composition of a mandelic acid sample by a single fluorescence measurement.

The fluorescent properties of a series of $\mathrm{H}_{8} \mathrm{BINOL}-\mathrm{amine}$ compounds were investigated. It is revealed that the intramolecular hydrogen bonds of these compounds can shift the emission of their $\mathrm{H}_{8}$ BINOL unit to a much longer wavelength. Binding with the acid suppressed its intramolecular hydrogen bonding and restores the short wavelength emission of the $\mathrm{H}_{8} \mathrm{BINOL}$ unit, giving high sensitivity and good enantioselectivity.
\end{abstract}

A BINOL-amino alcohol enantiomeric pair is discovered to be able to conduct both enantioselective and diastereoselective fluorescent discrimination of the four stereoisomers of threonine derivatives. This is the first example that one enantiomeric sensor pair can be used to recognize four stereoisomers of chiral substrates containing 
two chiral centers.

A BINOL-trifluoroacetyl compound is discovered to exhibit high sensitivity at one emission wavelength and high enantioselectivity at another when treated with a chiral diamine. By using this fluorescent sensor it was demonstrated for the first time that both the concentration and enantiomeric composition of a chiral substrate can be determined simultaneously with one fluorescent measurement.

These discoveries represent important progress in the field of enantioselective fluorescent sensors and have fundamental significance for the potential application of fluorescence in chiral catalyst screening. 


\section{Acknowledgements}

I would like to thank Dr. Lin Pu for providing me the opportunity to pursue my graduate studies. My work on this project would not have been possible without his innovative ideas, critical thinking and careful guidance. He is more than just academic advisor for me. I benefit a lot from conversations with him when I encountered tough problems in my life. My gratitude also goes to my committee: Dr. Harman, Dr. Gunnoe and Dr. Chruma. I have learned much from them and appreciate their encouragement. I would also like to say thank you to Dr. Demas. For many times I interfered with him but he is always patient with my questions. I especially appreciate Dr. Li's willingness to serve as the outside member of my committee. I also appreciate Dr. Michal Sabat's help with crystals. I would also like to thank Dr. Guoqing Zhang for his help in my career.

I would like to thank my families for their enduring love and support through every step of my graduate school. Their understanding and support make me who I am. My gratitude also goes to Mark Turlington and Albert DeBerardinis. I am grateful for their training of me and their patient helpfulness with all of my questions, especially with my candidacy exam. Tiandong Liu, Lihong Zhou and Yang Yue helped me immensely with adjusting to the life in US and starting my work in the lab. I am also thankful for the many other great labmates that I have had the privilege of working with: Li Xiao, Yuhao Du, Wei Chen, Betrand Tran, Junliang Liu, Man Xu, Jun Ying, Chao Wang, Andrea Mulas, Winston Plunkett, Michael Kim, Abbey Pfister and Elaine Wu. Thank you guys for the invaluable friendship. 


\section{Table of Contents}

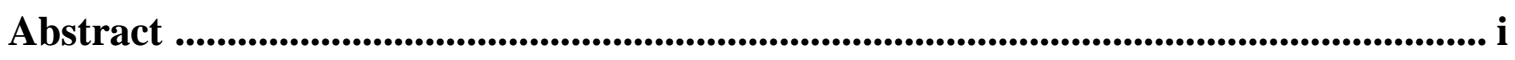

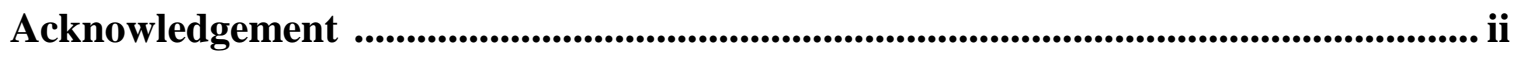

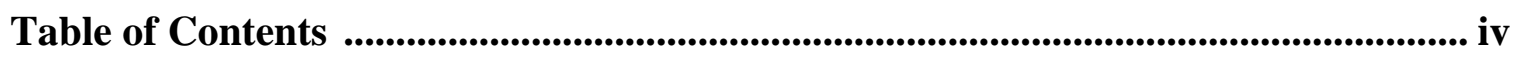

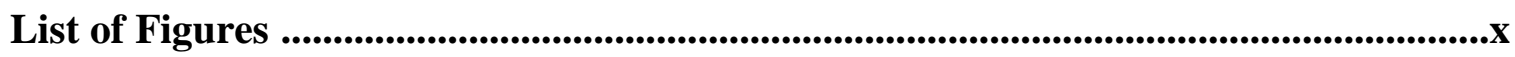

List of Schemes

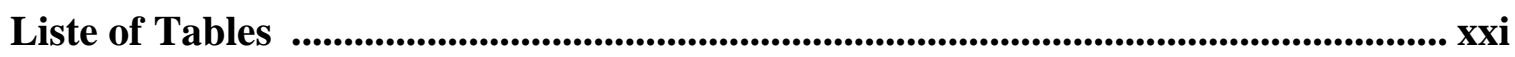

Chapter 1. Enantioselective Fluorescent Sensors ..........................................................1

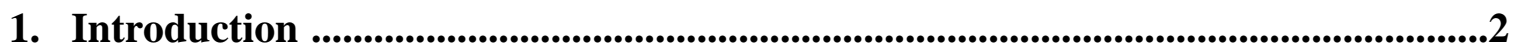

2. High-throughput Screening Techniques for $e e$ Determination ...............................3

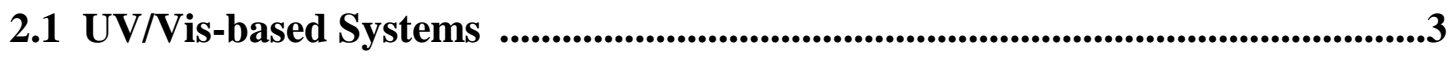

2.2 IR-thermographic Assays ..............................................................................................4

2.3 Electron Spray Mass Spectrometry ....................................................................5

2.4 Capillary Electrophoresis .........................................................................................7

2.5 Circular Dichroism ...............................................................................................................8

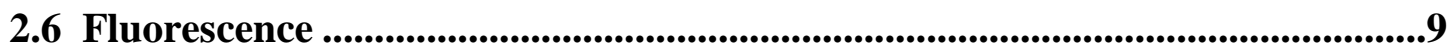

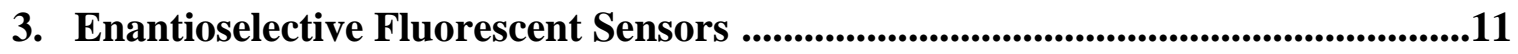

3.1 Hydrogen Bonding .....................................................................................................12

3.2 Metal Complexation ..................................................................................23

3.3 Reversible Covalent Interactions .............................................................28

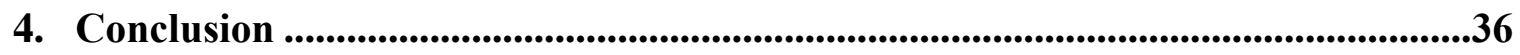


Chapter 2. Pseudoenantiomeric Fluorescent Sensors in a Chiral Assay .....................43

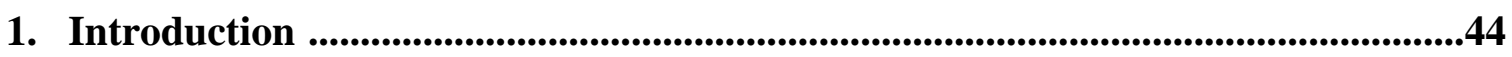

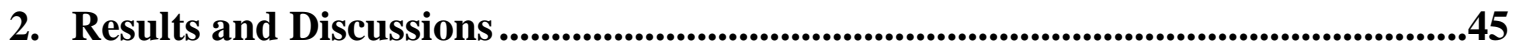

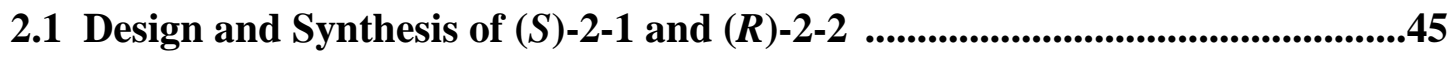

2.2 Enantioselective Fluorescent Recognition of Chiral Acids .............................47

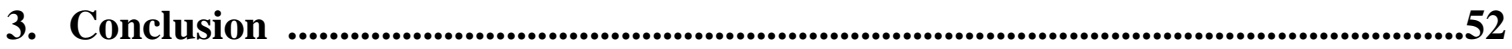

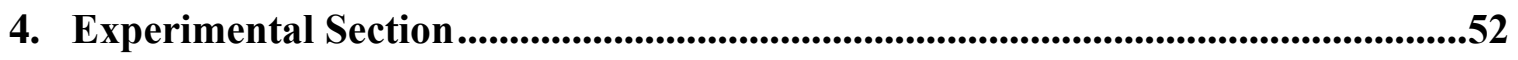

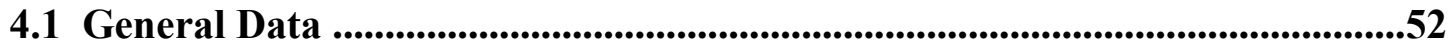

4.2 Preparation and Characterization of Compounds ........................................53

4.3 Preparation of Samples for Fluorescence Measurement …............................59

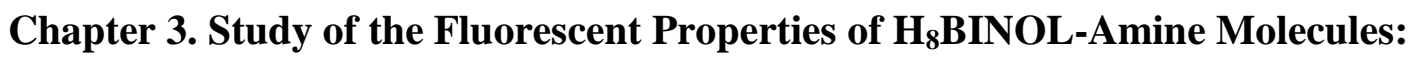

A New Window for the Enantioselective Fluorescent Recognition ...........63

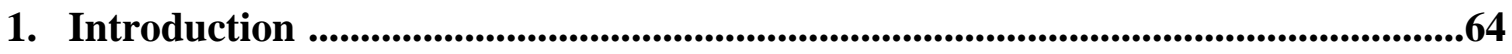

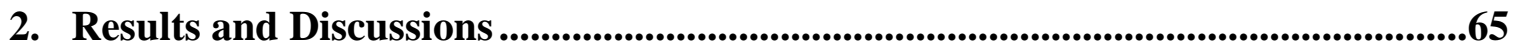

2.1 Synthesis of the $\mathrm{H}_{8}$ BINOL-Amine Molecules and Study of Their Fluorescent Properties

2.2 Study of the Interaction of $(S)-3-5$ with Mandelic Acid ...................................74

2.3 Interaction of the $\mathrm{H}_{8} \mathrm{BINOL-Amine} \mathrm{Compounds}(S)-3-6-(S)-3-10$ with

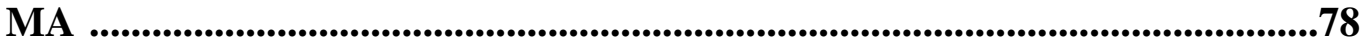

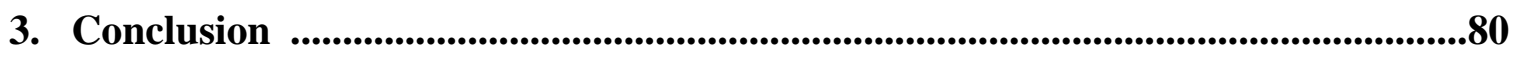

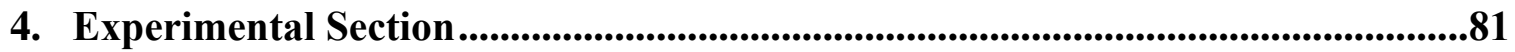

4.1 Synthesis and Characterization of Compounds .................................................81 
Chapter 4. One Enantiomeric Fluorescent Sensor Pair to Discriminate Four Stereoisomers of Threonines ....................................................................88

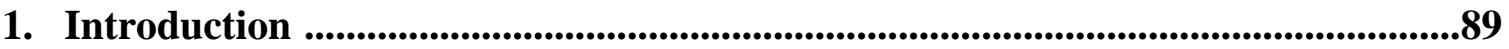

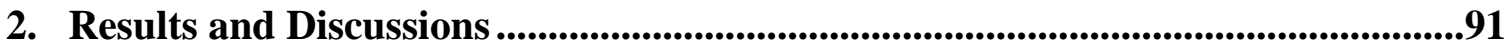

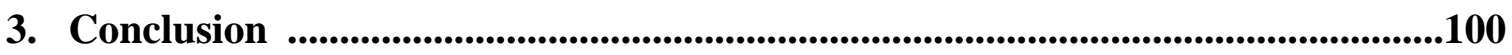

4. Experimental Section ..................................................................................................100

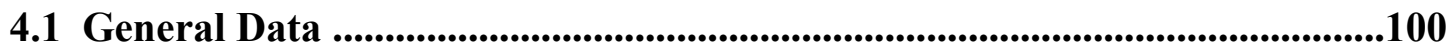

4.2 Synthesis and Characterization of Compounds ..........................................101

4.3 Preparation of Samples for Fluorescence Measurement .................................102

Chapter 5. Simultaneous Determination of Both the Enantiomeric Composition and Concentration of a Chiral Substrate with One Fluorescent Sensor ....................106

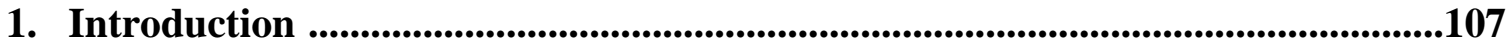

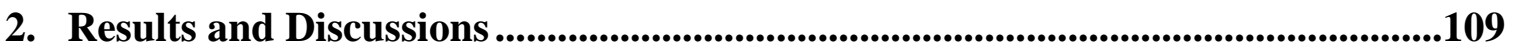

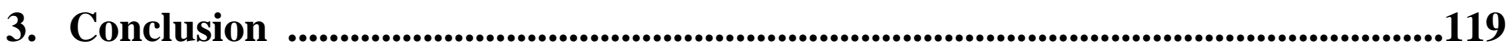

4. Experimental Section ................................................................................119

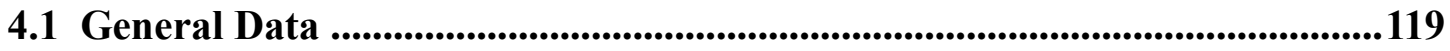

4.2 Synthesis and Characterization of Compounds ................................................120

4.3 Preparation of Samples for Fluorescence Measurement .................................123

4.4 Preparation of Samples for ${ }^{19}$ F NMR Titration ..............................................123 
Chapter 6.

1. Introduction.

2. Results and Discussions

2.1 Synthesis of Characterization of the BINOL-deketone Compounds

2.2 UV Spectra of Compounds (S)-6-3, (S)-6-4, (R)-6-8, (S)-6-10, (S)-6-12 and

(S)-6-16

2.3 Fluorescence Spectra of Compounds (S)-6-3, (S)-6-4, (R)-6-8, (S)-6-10, (S)-6-12 and (S)-6-16

2.4 Interaction of (S)-6-4 with Amines 138

A. Fluorescent Study

B. UV Study

C. NMR Study

D. X-Ray Structures .155

2.5 Interaction of the other analogs of $(S)-6-4$ with amines .159

2.6 Interaction of (S)-6-4 with Tetrabutylammonium Salts of Amino Acids .....165

2.7 Interaction of (S)-6-4 with Chiral Amino Alcohols . .167

3. Conclusion .169

4. Experimental Section . .170

4.1 General Data . .170

4.2 Preparation of Samples for Fluorescence Measurement . .171

4.2 Synthesis and Characterization of Compounds .171 


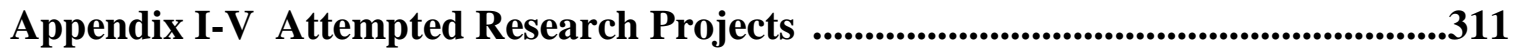




\section{List of Figures}

Figure 1-1. Fluorescence spectra of $(S, S)-\mathbf{1 - 1 8}\left(9.5 \times 10^{-5} \mathrm{M}\right.$ in benzene/2\% DME) with and without MA $\left(5 \times 10^{-3} \mathrm{M}\right)$.

Figure 1-2. Fluorescence spectra of $(R, R)-\mathbf{1 - 1 8},(R, R)-\mathbf{1 - 2 0},(R, R)-\mathbf{1 - 2 1}\left(3.1 \times 10^{-6}\right.$ M, benzene/0.1\% DME) with and without $(\mathrm{R})-\mathrm{MA}\left(1 \times 10^{-3} \mathrm{M}\right)$.

Figure 1-3. Fluorescence spectra of $(S)$-1-22 $\left(1 \times 10^{-4} \mathrm{M}\right.$, benzene/2\% DME) with and without MA $\left(2 \times 10^{-2} \mathrm{M}\right)$.

Figure 1-4. Fluorescence spectra of $(S)-1-23\left(1 \times 10^{-5} \mathrm{M}\right.$, benzene/0.05\% DME) with and without MA $\left(5 \times 10^{-4} \mathrm{M}\right)$.

Figure 1-5. Photographs of $(S)-\mathbf{1 - 2 4}\left(5 \times 10^{-4} \mathrm{M}\right.$, benzene/0.4\% DME) with $(R)$ - and $(S)$-MA $\left(4 \times 10^{-3} \mathrm{M}\right)$.

Figure 1-6. Fluorescence spectra of $(S)-\mathbf{1 - 2 4}\left(5 \times 10^{-4} \mathrm{M}\right.$, benzene/0.4\% DME) with and without MA $\left(4 \times 10^{-3} \mathrm{M}\right)$.

Figure 1-7. Fluorescent enantioselectivity of $(S)$-1-25 toward various chiral $\alpha$ hydroxycarboxylic acids.

Figure 1-8. Fluorescence spectra of $(S)-\mathbf{1 - 2 5}\left(2 \times 10^{-4} \mathrm{M}\right.$, benzene/0.4\% DME) with and without phenyllactic acid $\left(5 \times 10^{-3} \mathrm{M}\right)$.

Figure 1-9. Calculated structure of the proposed 1:1 complex of $(S)-\mathbf{1 - 2 5}+(\mathrm{R})$ phenyllatic acid.

Figure 1-10. Stern-Volmer plots showing enantioselective fluorescence quenching of ()-1-45 in the presence of 1-36 and 1-39.

Figure 1-11. Structural elements considered in the design of enantioselective 
cyclodextrins.

Figure 1-12. Fluorescence spectra of 1-51 $\left(5.0 \times 10^{-7} \mathrm{M}\right)$ in $\mathrm{CH}_{2} \mathrm{Cl}_{2} /$ n-hexane $(2: 3)$ in the presence of $(\mathrm{R})$ - and $(\mathrm{S})$-phenylglycinol $\left(5.0 \times 10^{-4} \mathrm{M}\right)$.

Figure 1-13. a) Fluorescence spectra of $1-52+\mathrm{Cu}(\mathrm{II})\left(1 \times 10^{-5} \mathrm{M}+2 \times 10^{-5} \mathrm{M}\right)$ with $\mathrm{D}$ - or L-Phe $\left(1 \times 10^{-4} \mathrm{M}\right)$. (b) Plots of $\mathrm{I} / \mathrm{I}_{0}$ vs Phe concentration during titration.

Figure 1-14. Generic design of fluorescent PET sensors with the boronic acid recognition unit.

Figure 1-15. The effect of saccharide complexation and $\mathrm{pH}$ changes on the fluorescence of boronic acid.

Fluorescence intensity-pH profiles for titrations of $(R)$ - and $(S)$-1-58

Figure 1-16. $\left(5.0 \times 10^{-6} \mathrm{M}\right.$ in $0.05 \mathrm{M} \mathrm{NaCl}$ solution $(52.1 \%$ methanol in water) $)$ with Dor L-tartaric acid (0.02 M).

Fluorescence intensity-pH profiles for titrations of (a) 1-60, (b) $(R, R)-\mathbf{1 -}$

Figure 1-17. $61\left(3.0 \times 10^{-6} \mathrm{M}\right.$ in $0.05 \mathrm{M} \mathrm{NaCl}$ solution (52.1\% methanol in water)) with D- or L-tartaric acid (0.05 M).

Figure 1-18. (a) Structure of $(R, R)-1-60$. (b) Structure of $(R, R)-1-60$ complex with Ltartaric acid.

Fluorescence intensity-pH profiles of $(S, S)-\mathbf{1 - 6 2}\left(3.0 \times 10^{-6} \mathrm{M}\right.$ in $0.05 \mathrm{M}$

Figure 1-19. $\mathrm{NaCl}$ solution (52.1\% methanol in water)) with $\mathrm{D}$ - or L-tartaric acid (0.05 M).

Figure 1-20. Fluorescence intensity-pH profiles of $(S, S)-\mathbf{1 - 6 3}\left(5.0 \times 10^{-7} \mathrm{M}\right.$ in methanol/water 3:1) with (a) D- or L-tartaric acid (b) D- or L-mandelic 
$\operatorname{acid}(0.01 \mathrm{M})$.

Figure 2-1. (a) Fluorescence spectra of $(S)-\mathbf{2 - 1}\left(1.0 \times 10^{-4} \mathrm{M}, \mathrm{CH}_{2} \mathrm{Cl}_{2}\right)$ with/without MA (4.0x $\left.10^{-3} \mathrm{M}\right)$. (b) Three independent measurements for the fluorescence enhancement of $(S)-2-1\left(1.0 \times 10^{-4} \mathrm{M}, \mathrm{CH}_{2} \mathrm{Cl}_{2}\right)$ at $\lambda_{1}=374 \mathrm{~nm}$ with varying MA concentration. $\left(\lambda_{\mathrm{exc}}=290 \mathrm{~nm}\right.$, slit $\left.=4.0 / 4.0 \mathrm{~nm}\right)$.

Figure 2-2. (a) Fluorescence spectra of $(R)-2-2\left(1.0 \times 10^{-4} \mathrm{M}, \mathrm{CH}_{2} \mathrm{Cl}_{2}\right)$ with/without $(R)$ - and $(S)$-MA $\left(4.0 \times 10^{-3} \mathrm{M}\right)$. (b) Three independent measurements for the fluorescence enhancement of $(R)-\mathbf{2 - 2}\left(1.0 \times 10^{-4} \mathrm{M}, \mathrm{CH}_{2} \mathrm{Cl}_{2}\right)$ at $\lambda_{2}=330$ $\mathrm{nm}$ with varying MA concentration. $\left(\lambda_{\mathrm{exc}}=290 \mathrm{~nm}\right.$, slit $\left.=4.0 / 4.0 \mathrm{~nm}\right)$.

Figure 2-3. (a) Plot of $\left(\mathrm{I}_{1} / \mathrm{I}_{10}-\mathrm{I}_{2} / \mathrm{I}_{20}\right)$ versus $[(R)-\mathrm{MA}] \%$ at varying MA concentrations (mM). (b) Plot of $\left(\mathrm{I}_{1} / \mathrm{I}_{10}+\mathrm{I}_{2} / \mathrm{I}_{20}\right)$ versus MA concentration at varying $[(R)$ MA $] \% .\left(\lambda_{\text {exc }}=290 \mathrm{~nm}\right.$, slit $\left.=4.0 / 4.0 \mathrm{~nm}\right)$.

Figure 2-4. (a) $3 \mathrm{D}$ and $2 \mathrm{D}$ plots of $\left(\mathrm{I}_{1} / \mathrm{I}_{10}-\mathrm{I}_{2} / \mathrm{I}_{20}\right)$ and $\left(\mathrm{I}_{1} / \mathrm{I}_{10}+\mathrm{I}_{2} / \mathrm{I}_{20}\right)$ with the MA concentration (mM). (b) $3 \mathrm{D}$ and $2 \mathrm{D}$ plots of $\left(\mathrm{I}_{1} / \mathrm{I}_{10}-\mathrm{I}_{2} / \mathrm{I}_{20}\right)$ and $\left(\mathrm{I}_{1} / \mathrm{I}_{10}+\right.$ $\left.\mathrm{I}_{2} / \mathrm{I}_{20}\right)$ with $[(R)-\mathrm{MA}] \%$.

Figure 3-1. UV spectra of sensor (S)-3-5 in methylene chloride at various concentrations.

Figure 3-2. Fluorescence spectra of $(S)-3-5$ in $\mathrm{CH}_{2} \mathrm{Cl}_{2}$ at various concentrations $\left(\lambda_{\mathrm{exc}}=\right.$ $290 \mathrm{~nm}$, slit $=3.0 / 3.0 \mathrm{~nm})$.

Figure 3-3. Fluorescence spectrum of $(S)-\mathrm{H}_{8} \mathrm{BINOL}\left(2.0 \times 10^{-4} \mathrm{M}\right.$ in $\left.\mathrm{CH}_{2} \mathrm{Cl}_{2}\right)\left(\lambda_{\text {exc }}=\right.$ $288 \mathrm{~nm}$. slit $=4.0 / 4.0 \mathrm{~nm})$.

Figure 3-4. (a) UV and (b) fluorescence spectra of sensor (S)-3-5 in methanol at 
various concentrations $\left(\lambda_{\mathrm{exc}}=291 \mathrm{~nm}\right.$, slit $\left.=4.0 / 4.0 \mathrm{~nm}\right)$.

Figure 3-5. (a) UV and (b) fluorescence spectra of sensor $(S)-3-5$ in $\mathrm{CH}_{3} \mathrm{CN}$ at various concentrations $\left(\lambda_{\mathrm{exc}}=291 \mathrm{~nm}\right.$, slit $\left.=4.0 / 4.0 \mathrm{~nm}\right)$.

Figure 3-6. Fluorescence spectra of compounds $(S)-3-6-(S)-3-10\left[2.0 \times 10^{-4} \mathrm{M}\right.$ in $\mathrm{CH}_{2} \mathrm{Cl}_{2} . \quad$ exc $=292 \mathrm{~nm}$ for $(S)-3-6-(S)-3-9$ and $291 \mathrm{~nm}$ for $(S)-3-10$. Slit: 5.0/5.0 $\mathrm{nm}$ for $(S)-3-6,(S)-3-7$ and $(S)-3-9$ and 4.0/4.0 $\mathrm{nm}$ for $(S)-3-8$ and $(S)-3-10]$.

Figure 3-7. Fluorescence spectra of (a) $(S)-3-6$ (b) $(S)$-3-7 (c) $(S)$-3-8 (d) $(S)-3-9$ (e) (S)-3-10 in $\mathrm{CH}_{2} \mathrm{Cl}_{2}$ at various concentrations $\left(\lambda_{\text {exc }}=290 \mathrm{~nm}\right.$, slit $=5.0 / 5.0$ $\mathrm{nm})$.

Figure 3-8. (a) Fluorescence spectra of $(S)-3-5\left(1.0 \times 10^{-4} \mathrm{M}, \mathrm{CH}_{2} \mathrm{Cl}_{2}\right)$ with/without $(R)$ and $(S)$-MA $\left(4.0 \times 10^{-3} \mathrm{M}\right)$. (b) Three independent measurements of Fluorescence enhancement of $(S)-3-5\left(1.0 \times 10^{-4} \mathrm{M}, \mathrm{CH}_{2} \mathrm{Cl}_{2}\right)$ at $\lambda_{\mathrm{em}}=330$ $\mathrm{nm}$ with varying acid concentration. $\left(\lambda_{\mathrm{exc}}=290 \mathrm{~nm}\right.$, slit $\left.=3.0 / 3.0 \mathrm{~nm}\right)$.

Figure 3-9. Fluorescence spectra of $(S)-\mathrm{H}_{8} \mathrm{BINOL}\left(2.0 \times 10^{-4} \mathrm{M}\right.$ in $\left.\mathrm{CH}_{2} \mathrm{Cl}_{2}\right)$ in the presence of $(R)$ - and $(S)$-mandelic acid $\left(4.0 \times 10^{-3} \mathrm{M}\right)\left[\lambda_{\mathrm{exc}}=288 \mathrm{~nm}\right.$. Slit $=$ $4.0 / 4.0 \mathrm{~nm}]$.

Figure 3-10. Job plots of $(S)$-3-5 with $(R)$-MA obtained by using the ${ }^{1} \mathrm{H}$ NMR signal change $\left(\Delta \delta=\delta_{0}-\delta\right)$ of (a) the $\mathrm{H}_{\alpha}$ of $(R)-\mathrm{MA}\{\mathrm{X}=[$ acid $] /([$ acid $]+[$ sensor $]\}$, and (b) the $\mathrm{H}_{\mathrm{b}}$ of $(\mathrm{S})-3-5\{\mathrm{X}=[$ sensor $] /([$ sensor $]+[$ acid $])\}$.

Figure 3-11. Fluorescence spectra of $(S)-3-6-(\mathrm{S})-3-10\left(2.0 \times 10^{-4} \mathrm{M}\right.$ in $\left.\mathrm{CH}_{2} \mathrm{Cl}_{2}\right)$ in the presence of $(R)$ - and $(S)$-MA $\left(4.0 \times 10^{-3} \mathrm{M}\right)\left[\lambda_{\text {exc }}=292 \mathrm{~nm}\right.$ for $(S)-3-6-$ 
(S)-3-9 and $291 \mathrm{~nm}$ for $(S)$-3-10. Slit: 5.0/5.0 $\mathrm{nm}$ for $(S)-3-6,(S)-3-7$ and (S)-3-9 and 4.0/4.0 nm for $(S)-3-8$ and $(S)-3-10]$.

Figure 4-1. Stereoisomers of Threonines and the N-Cbz-Threonines.

Figure 4-2. Fluorescence spectra of (a) $(S)-4-1\left(5.0 \times 10^{-4} \mathrm{M}\right)$ and $(\mathrm{b})(R)-\mathbf{4 - 1}\left(5.0 \times 10^{-4}\right.$ M) with four stereoisomers of $\alpha-\mathrm{N}-\mathrm{Cbz}$-amino acid $\left(1.0 \times 10^{-3} \mathrm{M}\right)$. (Solvent: toluene $/ 2 \%$ DME. $\lambda_{\mathrm{exc}}=341 \mathrm{~nm}, 3.0 / 3.0 \mathrm{~nm}$ )

Figure 4-3. Fluorescence enhancements of (a) $(S)$-4-1 and (b) $(R)-4-1$ both at $5.0 \times 10^{-}$ ${ }^{4} \mathrm{M}$ with varying acid concentrations. ( $\mathrm{I}_{1}$, fluorescence intensity at $370 \mathrm{~nm}$ with the acid, and $\mathrm{I}_{10}$ without the acid. $\lambda_{\mathrm{exc}}=341 \mathrm{~nm}$, slit $=3.0 / 3.0 \mathrm{~nm}$ ).

Figure 4-4. Interactions of $(S)$-4-1 (toluene/2\% DME, $5.0 \times 10^{-4} \mathrm{M}$ ) with LAT at varying concentrations (from left to right: 0, 1E-4, 2E-4, 5E-4, 1E-3, 2E-3, 3E-3, 4E-3, 5E-3 M).

Figure 4-5. Plots of $\mathrm{I}_{2} / \mathrm{I}_{1}$ for (a) $(S)$-4-1 and (b) (R)-4-1 versus the concentration of the stereoisomeric threonines. ( $\mathrm{I}_{1}$ : fluorescence intensity at $370 \mathrm{~nm}$. $\mathrm{I}_{2}$ : fluorescence intensity at $445 \mathrm{~nm}$. Solvent: toluene $/ 2 \%$ DME. $\lambda_{\mathrm{exc}}=341$ $\mathrm{nm}$, slit $=3.0 / 3.0 \mathrm{~nm})$

Figure 4-6. (a) Plots of $I_{1} / I_{0}$ of $(S)$-4-1 versus the concentration of the optically pure LAT and the percent of LAT in the mixture of the four stereoisomers. (b) Plots of $\mathrm{I}_{1} / \mathrm{I}_{0}$ of $(R)-4-1$ versus the concentration of the optically pure DAT and the percent of DAT in the mixture of the four stereoisomers (total acid concentration in the mixture: $0.5 \mathrm{mM}$. Solvent: toluene $/ 2 \%$ DME. $\lambda_{\mathrm{exc}}=$ $341 \mathrm{~nm}$, slit $=3.0 / 3.0 \mathrm{~nm})$ 
Figure 4-7. (a) Plots of $I_{2} / I_{1}$ of $(S)-4-1$ versus the concentration of the optically pure DTH as well as the percent of DTH in the mixture of four stereoisomers. (a) Plots of $\mathrm{I}_{2} / \mathrm{I}_{1}$ of $(R)-4-1$ versus the concentration of the optically pure LTH as well as the percent of LTH in the mixture of four stereoisomers. (Total acid concentration in the mixture: $0.5 \mathrm{mM}$. Solvent: toluene/2\% DME. $\lambda_{\mathrm{exc}}=341 \mathrm{~nm}$, slit $\left.=3.0 / 3.0 \mathrm{~nm}\right)$

Figure 5-1. UV/Vis absorption spectra (a) and fluorescence spectra (b) of $(S)-5-3$ (1.0 $\left.\times 10^{-5} \mathrm{M}\right)$ with/without $(R, R)-$ and $(S, S)-5-4\left(5.0 \times 10^{-3} \mathrm{M}\right)($ Solvent: $\mathrm{CH}_{2} \mathrm{Cl}_{2} \cdot \lambda_{\text {exc }}=343 \mathrm{~nm}$, slit $=2 / 2 \mathrm{~nm}$.).

Figure 5-2. Plots of $I_{1}(a), I_{2}(b), I_{1} / I_{2}(c)$ for $(S)-5-3\left(1.0 \times 10^{-5} \mathrm{M}\right)$ in the presence of varying concentrations of $(R, R)$ - and $(S, S)-5-4$. (Fluorescence intensity $\mathrm{I}_{1}$ at $\quad{ }_{1}=370 \mathrm{~nm}$ and $\mathrm{I}_{2}$ at $\quad 2=438 \mathrm{~nm}$. Solvent: $\mathrm{CH}_{2} \mathrm{Cl}_{2} \cdot \lambda_{\mathrm{exc}}=343 \mathrm{~nm}$, slit $=2 / 2 \mathrm{~nm}$.)

Figure 5-3. Plots of $\mathrm{I}_{1} / \mathrm{I}_{2}$ vs $(S, S)-\mathbf{5 - 4} \%$ at various diamine concentrations $(\mathrm{mM})$ (Solvent: $\mathrm{CH}_{2} \mathrm{Cl}_{2} \cdot \lambda_{\mathrm{exc}}=343 \mathrm{~nm}$, slit $=2 / 2 \mathrm{~nm}$.).

Figure 5-4. Plot of $\mathrm{I}_{1}, \mathrm{I}_{1} / \mathrm{I}_{2}$ vs the total concentration of 5-4 with various enantiomeric composition.

Figure 5-5. Time dependent fluorescence spectra of $(S)-3-3$ solution $\left(1 \times 10^{-5} \mathrm{M}\right)$ with (a) $(S, S)-5-4\left(5 \times 10^{-3} \mathrm{M}\right)$ and (b) $(R, R)-5-4\left(5 \times 10^{-3} \mathrm{M}\right)$. (Solvent: $\mathrm{CH}_{2} \mathrm{Cl}_{2}$. $\left.\lambda_{\mathrm{exc}}=343 \mathrm{~nm}, \mathrm{slit}=2 / 2 \mathrm{~nm}.\right)$

Figure 6-1. $\quad$ UV spectra of (a) $(S)-6-3,($ b) $(R)-6-8,($ c) $(S)-6-10,(S)-6-12$ and (d) $(S)-6-$ $16\left(1.0 \times 10^{-5} \mathrm{M}\right)$ in comparison with that of $(S)-6-4$ in $\mathrm{CH}_{2} \mathrm{Cl}_{2}$. 
Figure 6-2. Fluorescence spectra of $(S)-6-3,(S)-6-4,(R)-6-8,(S)-6-10,(S)-6-12$ and $(S)-6-16\left(1.0 \times 10^{-5} \mathrm{M}\right)$ in $\mathrm{CH}_{2} \mathrm{Cl}_{2}$. $\left(\lambda_{\text {exc }}=343 \mathrm{~nm}\right.$ for $(S)-6-3,(S)-6-4,(S)$ 6-10, $(S)-6-12$ and $(S)-\mathbf{6 - 1 6}, \lambda_{\mathrm{exc}}=290 \mathrm{~nm}$ for $(R)-\mathbf{6 - 8}$, slit $\left.=2.0 / 2.0 \mathrm{~nm}\right)$.

Figure 6-3. Fluorescence spectra of (a) $(S)-6-4$, (b) $(S)-6-10$ and (c) $(S)-6-12\left(1.0 \times 10^{-5}\right.$ M) in various solvents. $\left(\lambda_{\mathrm{exc}}=343 \mathrm{~nm}\right.$, slit $\left.=2.0 / 2.0 \mathrm{~nm}\right)$.

Figure 6-4. Structures of various amines.

Figure 6-5. Fluorescence spectra of $(S)-6-4\left(1.0 \times 10^{-5} \mathrm{M}\right)$ in the presence of (a) various achiral amines N1-N14, (b) three isomers of N15 and (c) two enantiomers of N16 $\left(1.0 \times 10^{-3} \mathrm{M}\right) .\left(\lambda_{\mathrm{exc}}=343 \mathrm{~nm}\right.$, slit $\left.=2.0 / 2.0 \mathrm{~nm}\right)$.

Figure 6-6. Plots of $I_{1} / I_{2}$ for $(S)-6-4\left(1.0 \times 10^{-5} \mathrm{M}\right)$ in the presence of varying concentrations of $(R, R)-(S, R)$ - and $(S, S)$-N15. (Fluorescence intensity $\mathrm{I}_{1}$ at $\lambda_{1}=370 \mathrm{~nm}$ and $\mathrm{I}_{2}$ at $\lambda_{2}=438 \mathrm{~nm}$. Solvent: $\mathrm{CH}_{2} \mathrm{Cl}_{2} \cdot \lambda_{\mathrm{exc}}=343 \mathrm{~nm}$, slit $=2 / 2 \mathrm{~nm}$.

Figure 6-7. Plots of (a) $I_{1}$, (b) $I_{2}$ and (c) $I_{1} / I_{2}$ for $(S)-6-4\left(1.0 \times 10^{-5} \mathrm{M}\right)$ in the presence of varying concentrations of (R)- and (S)-N16. (Fluorescence intensity $\mathrm{I}_{1}$ at $\lambda_{1}=370 \mathrm{~nm}$ and $\mathrm{I}_{2}$ at $\lambda_{2}=438 \mathrm{~nm}$. Solvent: $\mathrm{CH}_{2} \mathrm{Cl}_{2} \cdot \lambda_{\mathrm{exc}}=343 \mathrm{~nm}$, slit $=2 / 2 \mathrm{~nm}$.

Figure 6-8. Excitation spectra of $(S)-6-4\left(1.0 \times 10^{-5} \mathrm{M}, \mathrm{CH}_{2} \mathrm{Cl}_{2}\right)$ in the presence of (a) $(R, R)$ - or $(\mathrm{b})(S, S)-\mathbf{N 1 5}\left(4.0 \times 10^{-3} \mathrm{M}\right) .(\mathrm{slit}=2.0 / 2.0 \mathrm{~nm})$.

Figure 6-9. Job plot of $(S)-6-4\left(1.0 \times 10^{-5} \mathrm{M}\right)$ with (a) N9 and (b) N12. (total concentration $=0.1 \mathrm{mM}, \lambda_{\mathrm{exc}}=343 \mathrm{~nm}$, slit $\left.=2.0 / 2.0 \mathrm{~nm}\right)$.

Figure 6-10. Fluorescence spectra of $(S)-6-4\left(1.0 \times 10^{-5} \mathrm{M}\right.$ in $\left.\mathrm{CH}_{2} \mathrm{Cl}_{2}\right)$ in the presence of 
propylamine $\mathbf{N 5}$ at various concentrations $(0-0.05 \mathrm{M}) .\left(\lambda_{\mathrm{exc}}=343 \mathrm{~nm}\right.$, slit $=2.0 / 2.0 \mathrm{~nm})$.

Figure 6-11. UV spectra of $(S)-6-4\left(1.0 \times 10^{-5} \mathrm{M}\right)$, amines $\left(1.0 \times 10^{-3} \mathrm{M}\right)$ and their mixtures. (The black line represents UV absorption of $(S)-\mathbf{6 - 4}$, the blue line represents amine and the red line represents the mixture.)

Figure 6-12. UV-Vis absorption spectra of $(S)-6-4\left(1.0 \times 10^{-5} \mathrm{M}\right.$ in $\left.\mathrm{CH}_{2} \mathrm{Cl}_{2}\right)$ in the presence of propylamine $\mathbf{N 5}$ at various concentrations $(0-0.05 \mathrm{M})$.

Figure 6-13. X-ray structures of the complexes of (S)-6-4 with (a) N9, (b) N10 and (c) $(S, S)-\mathbf{N 1 5}$

Figure 6-14. Fluorescence spectrum of $(S, R, R)-\mathbf{6 - 2 5}\left(1 \times 10^{-5} \mathrm{M}\right.$ in $\left.\mathrm{CH}_{2} \mathrm{Cl}_{2}\right) \cdot\left(\lambda_{\mathrm{exc}}=343\right.$ $\mathrm{nm}$, slit $=2.0 / 2.0 \mathrm{~nm})$

Figure 6-15. Fluorescence spectra of $(R)-6-8$ in the presence of N1, N2, N5, N9 (a), and $(R, R)$ - or $(S, S)$-N15 (b) (amine: $\left.1.0 \times 10^{-3} \mathrm{M}\right) \cdot\left(\lambda_{\mathrm{exc}}=286 \mathrm{~nm}\right.$, slit $=$ 2.0/2.0 nm). UV spectra of $(R)-6-8$ in the presence of $(R, R)$ - and $(S, S)$ N15 $\left(5 \times 10^{-3} \mathrm{M}\right)$ (c). (sensor: $1.0 \times 10^{-5} \mathrm{M}$ in $\left.\mathrm{CH}_{2} \mathrm{Cl}_{2}\right)$

Figure 6-16. Fluorescence spectra of $(S)-6-10$ in the presence of N1, N2, N5, N9 (a), and $(R, R)$ - or $(S, S)-\mathbf{N 1 5}(\mathrm{b}) .\left(\lambda_{\mathrm{exc}}=343 \mathrm{~nm}\right.$, slit $\left.=2.0 / 2.0 \mathrm{~nm}\right) . \mathrm{UV}$ spectra of $(S)-6-10$ in the presence of $(R, R)$ - and $(S, S)$-N15 (c). (sensor: $1.0 \times 10^{-5} \mathrm{M}$ in $\mathrm{CH}_{2} \mathrm{Cl}_{2}$. amine: $\left.5.0 \times 10^{-3} \mathrm{M}\right)$

Figure 6-17. Fluorescence spectra of $(S)-6-12\left(1.0 \times 10^{-5} \mathrm{M}^{\text {in }} \mathrm{CH}_{2} \mathrm{Cl}_{2}\right)$ in the presence of N1, N2, N5, N9, $(R, R)-$ or $(S, S)-\mathbf{N 1 5}\left(5.0 \times 10^{-3} \mathrm{M}\right) .\left(\lambda_{\mathrm{exc}}=343 \mathrm{~nm}\right.$, slit $=3.0 / 3.0 \mathrm{~nm})$. 
Figure 6-18. Fluorescence spectra of $(S)-6-16$ in the presence of N1, N2, N5, N9, N12 (a), and $(R, R)$ - or $(S, S)-\mathbf{N 1 5}(\mathrm{b}) .\left(\lambda_{\mathrm{exc}}=343 \mathrm{~nm}\right.$, slit $=1.0 / 1.0 \mathrm{~nm}$ for N1, N2, N5, N9 and N12, slit $=2.0 / 2.0 \mathrm{~nm}$ for $(R, R)$ - or $(S, S)-\mathbf{N 1 5})$. UV spectra of $(S)-6-16$ in the presence of $(R, R)$ - and $(S, S)$-N15 (c). (sensor: $1.0 \times 10^{-5} \mathrm{M}$ in $\mathrm{CH}_{2} \mathrm{Cl}_{2}$. amine: $\left.5.0 \times 10^{-3} \mathrm{M}\right)$

Figure 6-19. (a) Fluorescence spectra of $(S)-6-3$ in the presence of $(R, R)$ - and $(S, S)$ N15. $\left(\lambda_{\text {exc }}=343 \mathrm{~nm}\right.$, slit $\left.=2.0 / 2.0 \mathrm{~nm}\right)$. (b) UV spectra of $(S)-\mathbf{6 - 3}$ in the presence of $(R, R)$ - and $(S, S)$-N15. (sensor: $1.0 \times 10^{-5} \mathrm{M}$ in $\mathrm{CH}_{2} \mathrm{Cl}_{2}$. amine: $\left.5.0 \times 10^{-3} \mathrm{M}\right)$

Figure 6-20. Fluorescence spectra of $(S)-6-3$ (a) and $(S)-6-16$ (b) in the presence of $(R$, $R$ )- and $(S, \mathrm{~S})-\mathrm{N} 15$ (sensor: $1.0 \times 10^{-5} \mathrm{M}$ in $\mathrm{CH}_{2} \mathrm{Cl}_{2}$, amine: $5.0 \times 10^{-3} \mathrm{M}$ ) $\left(\lambda_{\mathrm{exc}}=290 \mathrm{~nm}\right.$, slit $\left.=2.0 / 2.0 \mathrm{~nm}\right)$.

Figure 6-21. Fluorescence spectra of $(S)-6-4\left(1.0 \times 10^{-5} \mathrm{M}\right)$ in the presence of the tetrabutylammonium salts of D- and L-amino acids $\left(2 \cdot 0 \times 10^{-4} \mathrm{M}\right) .\left(\lambda_{\mathrm{exc}}=\right.$ $343 \mathrm{~nm}$, slit $=2.0 / 2.0 \mathrm{~nm})$

Figure 6-22. Structures of tested chiral amino alcohols.

Figure 6-23. Fluorescence spectra of $(S)-6-4\left(1.0 \times 10^{-5} \mathrm{M}\right.$, in $\left.\mathrm{CH}_{2} \mathrm{Cl}_{2}\right)$ in the presence of chiral amino alcohols $\left(2.0 \times 10^{-4} \mathrm{M}\right)$ and the maximum emission change with increasing concentrations. $\left(\lambda_{\mathrm{exc}}=343 \mathrm{~nm}\right.$, slit $\left.=2.0 / 2.0 \mathrm{~nm}\right)$. 


\section{List of Schemes}

Scheme 1-1. Kinetic resolution of ester catalysed by mutant lipases.

Scheme 1-2. Lipase-catalyzed enantioselective synthesis of $(R)$-1-7 from rac-1-6.

Scheme 1-3. a) Asymmetric transformation of a mixture of pseudo-enantiomers involving cleavage of the functional groups FG and labeled functional groups FG*. b) Asymmetric transformation of a mixture of pseudoenantiomers involving either cleavage or bond formation at the functional group FG. c) Asymmetric transformation of a pseudo-meso substrate involving cleavage of the functional groups FG and labeled functional groups $\mathrm{FG}^{*}$. d) Asymmetrictransformation of a pseudo-p rochiral substrate involving cleavage of the functional groups FG and labeled functionalgroups $\mathrm{FG}^{*}$.

Scheme 1-4. Mass-spectrometric ee determination in the kinetic resolution of chiral alcohols.

Scheme 1-5. Derivatization of chiral amines for the determination of $e e$.

Scheme 1-6. Asymmetric activation of diol-zinc catalysts by nitrogen ligands.

Scheme 1-7. Enantioselective catalytic acylation of trans-(+/-)-1-15.

Scheme 2-1. Synthesis of BINOL-amino alcohol (S)-2-1.

Scheme 2-2. Synthesis of $\mathrm{H}_{8} \mathrm{BINOL-amino} \mathrm{alcohol}(R)$-2-2

Scheme 3-1. Synthesis of the $\mathrm{H}_{8}$ BINOL-Amino Alcohol (S)-3-5.

Scheme 3-2. Synthesis of the $\mathrm{H}_{8} \mathrm{BINOL}-\mathrm{amine}$ compounds $(S)-3-6-(S)-3-10$.

Scheme 5-1. Preparation of Compounds $(S)$ - and $(R)-5-3$. 
Scheme 5-2. A proposed mechanism for the reaction of $(S)-5-3$ with the chiral diamine.

Scheme 6-1. Synthesis of the BINOL-diketone (S)-6-4.

Scheme 6-2. Synthesis of the $\mathrm{H}_{8} \mathrm{BINOL-diketone}(R)-6-8$.

Scheme 6-3. Synthesis of the BINOL-dialdehyde $(S)-6-10$.

Scheme 6-4. Synthesis of the BINOL-diketone $(S)-6-12$.

Scheme 6-5. Synthesis of the BINOL-diketone $(S)-6-16$.

Scheme 6-6. Proposed mechanism for the fluorescent responses of $(S)-6-4$.

Scheme 6-7. Proposed binding mechanisms.

Scheme 6-8. A proposed mechanism for the reaction of $(S)-6-4$ with ethylene diamine. 


\section{List of Tables}

Table 1-1. Enantioselectivity $\Delta \mathrm{F}_{\mathrm{D}} / \Delta \mathrm{F}_{\mathrm{L}}$ of $\mathrm{Cu} \mathbf{- 1 - 4 6}$ towards amino acids.

Table 1-2. Stability constants and fluorescence enhancements for saccharides with $(R)$ 1-58.

Table 5-1. Determination of (S,S)-5-4\% and concentration of test samples.

Table 6-1. Maximum absorption peaks and extinction coffecient of $(S)-\mathbf{6 - 3},(S)-\mathbf{6 - 4}$, $(R)-6-8,(S)-6-10,(S)-6-12$ and $(S)-6-16\left(1.0 \times 10^{-5} \mathrm{M}^{\text {in }} \mathrm{CH}_{2} \mathrm{Cl}_{2}\right)$.

Table 6-2. The ratio of $\mathrm{I}_{\mathrm{D}} / \mathrm{I}_{\mathrm{L}}$ of tested amino acid salts. ( $\mathrm{I}_{\mathrm{D}}$ : the fluorescence intensity maximum of $(S)-6-4$ when treated with D-amino acid salts. $\mathrm{I}_{\mathrm{L}}$ : the fluorescence intensity at maximum of $(S)-\mathbf{6 - 4}$ when treated with L-amino acid salts.) 
Chapter 1

\section{Enantioselective Fluorescent}

Sensors 


\section{Introduction}

Enantioselectivity is one of the essential research focuses for sensor science, ${ }^{1-3}$ since the enantiomerically pure compounds have fundamental importance in pharmaceutical industry, ${ }^{4}$ agrochemical area, ${ }^{5}$ and food analysis. ${ }^{6}$ Biological activity of drugs is tightly correlated with stereochemistry due to the inherent chiral environment in biological systems. Therefore, fast and easily performed method for acquiring enantiomerically pure compounds is in demand.

Asymmetric catalysis has been intensively investigated in the past two decades ${ }^{7}$ because it can provide very convenient synthetic routes to chiral compounds, avoiding the labor-intensive and time-consuming separation of enantiomers. However, thousands of experiments need to be conducted in order to develop an efficient catalyst and a suitable reaction condition, which still requires tremendous investment in time and labor. The development of parallel synthesis and combinatorial chemistry provides fast, reliable methods for screening the properties of enormous number of chiral catalysts. ${ }^{8,} 9$ However, the traditional analytical techniques, such as gas chromatography (GC) or high-performance liquid chromatography (HPLC), are not fast enough to determine the enantiomeric excess $(e e)$ of products generated from combinatorial catalyst screening due to the serial and time-consuming nature of the chromatographic techniques. Therefore, high-throughput screening (HTS) techniques have become essentially important for the application of rapid chiral assay. 


\section{High-throughput screening techniques for $e e$ determination}

Researchers have been exploring in this area and some achievements have been made. ${ }^{2,3,8,10}$ A number of techniques are being developed for the high-thoughput ee screening of chiral compounds, such as UV/Vis, ${ }^{11}{ }^{12}$ time resolved IR-thermographic method, ${ }^{13,} 14$ electron spray mass spectrometry, capillary electrophoresis, circular dichroism and fluorescence.

\subsection{UV/Vis-based systems}

In 1997, Manfred T. Reetz, et al. established a rather crude UV/Vis-based screening system for the lipase-catalyzed kinetic resolution of chiral p-nitrophenol esters (Scheme 1-1). ${ }^{12}$ The $\mathrm{R}$ and $\mathrm{S}$ esters were tested separated pairwise on a 96-well micro titer plate using a simple UV/Vis-based plate reader. The process of reactions was monitored by measuring the absorption of the $p$-nitrophenolate anion at $410 \mathrm{~nm}$ as a function of time.

Scheme 1-1. Kinetic resolution of ester catalysed by mutant lipases.

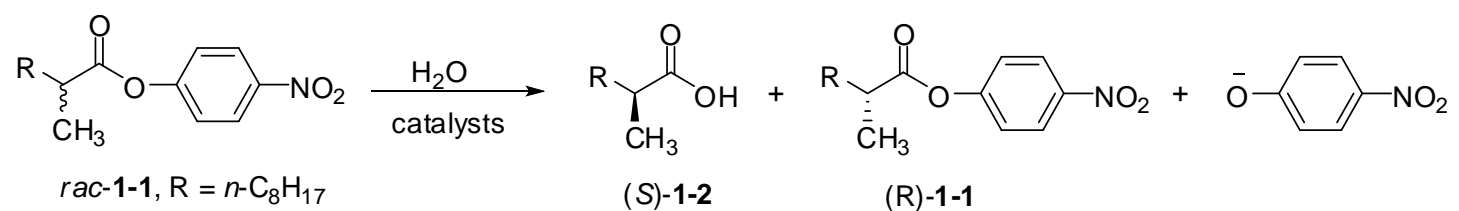

Another typical example is the use of enantioselective indicator displacement assays (eIDAs) by Eric V. Anslyn for the determination of $e e$ of $\alpha$-amino acids in 2008. ${ }^{11}$ Chrome azurol S (1-5) was used as the indicator. Coordination of the indicator the 
$\mathrm{Cu}(\mathrm{II})$ metal center of the $\mathrm{Cu}(\mathrm{II})$-diamine complexes $\left([\mathrm{Cu}(\mathbf{1 - 3})]^{2+}\right.$ and $\left.[\mathrm{Cu}(\mathbf{1 - 4})]^{2+}\right)$ produced an absorbance band at $602 \mathrm{~nm}$, while the free indicator showed absorption at $429 \mathrm{~nm}$. When amino acids were added to the complex solution, the indicator was replaced and the absorption at 429 was increased differently for amino acids enantiomers. Then they applied this system to 96-well plate and demonstrated its ability to be used in an HT format.

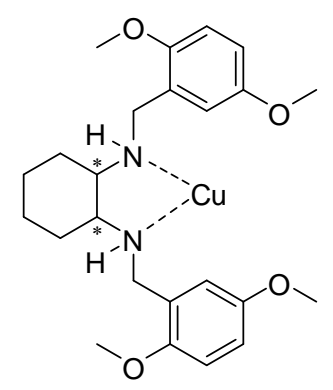

$[\mathrm{Cu}(1-3)]^{2+}$

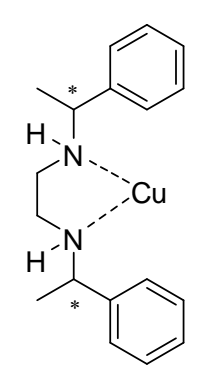

$[\mathrm{Cu}(1-4)]^{2+}$

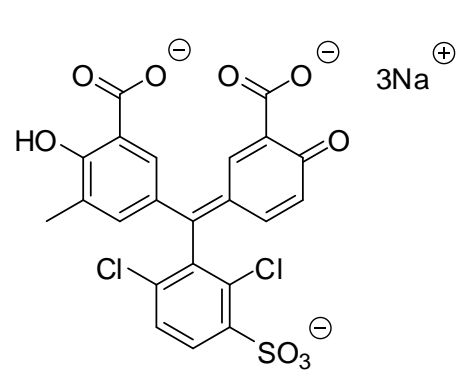

$1-5$

\subsection{IR-thermographic assays}

In 1998, Reetz et al. used an infrared camera to monitor the temperature change of the lipase-catalysed acylation of 1-phenylethanol (Scheme 1-2) on a special microtiter plate. $^{14}$ The results showed good spatial resolution and clearly identified the "hottest" reaction. They then applied this method to transition metal-catalyzed enantioselective ring-opening hydrolysis of epoxides and successfully demonstrated that time-resolved IR-thermographic method was feasible in the screening of enantioselectivity in catalytic reactions.

In 2002, Mahmoudian et al. tested the same lipase-catalysed acylation (Scheme 1-2) 
on a 96-well plate and plotted a calibration curve to quantify ee from the temperature change. ${ }^{13}$ Then they studied the screening of 96 racemic alcohols for acylation on a 96-well plate but only crude readout could be obtained, which suggested that with its limitations IRT can provide a crude but practical intitial method when screening large numbers of catalysts and/or substrates.

Scheme 1-2. Lipase-catalyzed enantioselective synthesis of $(R)-1-7$ from $r a c-1-6$.

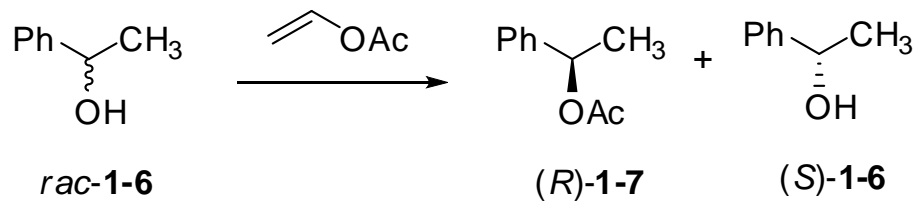

\subsection{Electron spray mass spectrometry}

Since the $\mathrm{R}$ and $\mathrm{S}$ forms of a chiral compound show identical mass spectra, the basic principle to apply MS to the enantioselective catalysis is to create mass differences. In 1999, Reetz et al. utilized isotopically labeled substrates in the form of pseudo-enantiomers or pseudo-prochiral compounds (Scheme 1-3). ${ }^{15}$ The kinetic resolution of racemates and the asymmetric transformation of prochiral substrates with enantiotopic groups could be monitored by detecting the relative amounts of reactants and/or products by ESI-MS.

Scheme 1-3. a) Asymmetric transformation of a mixture of pseudo-enantiomers involving cleavage of the functional groups FG and labeled functional groups FG*. b) 
Asymmetric transformation of a mixture of pseudo-enantiomers involving either cleavage or bond formation at the functional group FG. c) Asymmetric transformation of a pseudo-meso substrate involving cleavage of the functional groups FG and labeled functional groups $\mathrm{FG}^{*}$. d) Asymmetric transformation of a pseudo-prochiral substrate involving cleavage of the functional groups FG and labeled functionalgroups FG*.

a)

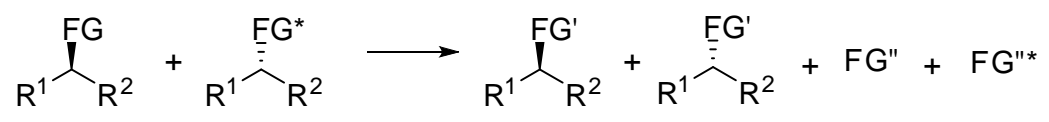

b)

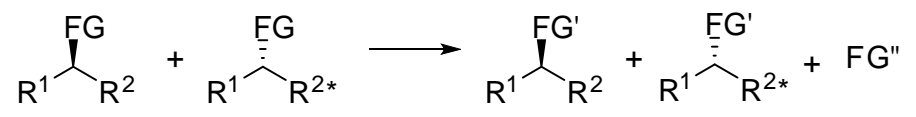

c)

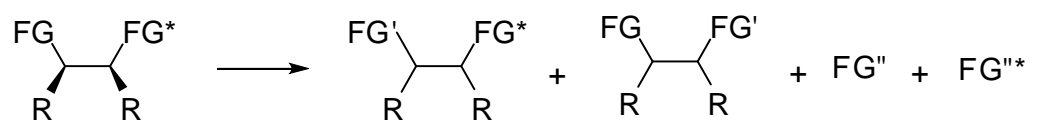

d)

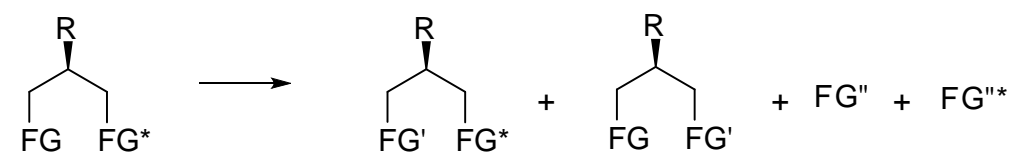

In the same year, Finn et al. employed a different approach, by using pseudo-enantiomeric "mass-tagged" chiral acylating agents 1-8 and 1-9 to determine ee values of chiral alcohols or amines (Sheme 1-4). ${ }^{16}$ Nine secondary alcohols and five primary and secondary amines were tested with this method and the measured value fell within $10 \%$ ee of the true value.

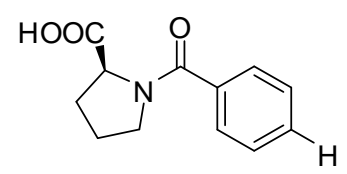

1-8

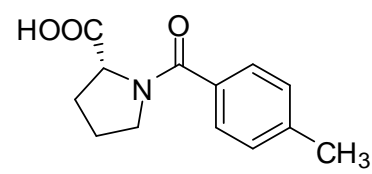

1-9 
Scheme 1-4. Mass-spectrometric ee determination in the kinetic resolution of chiral alcohols.

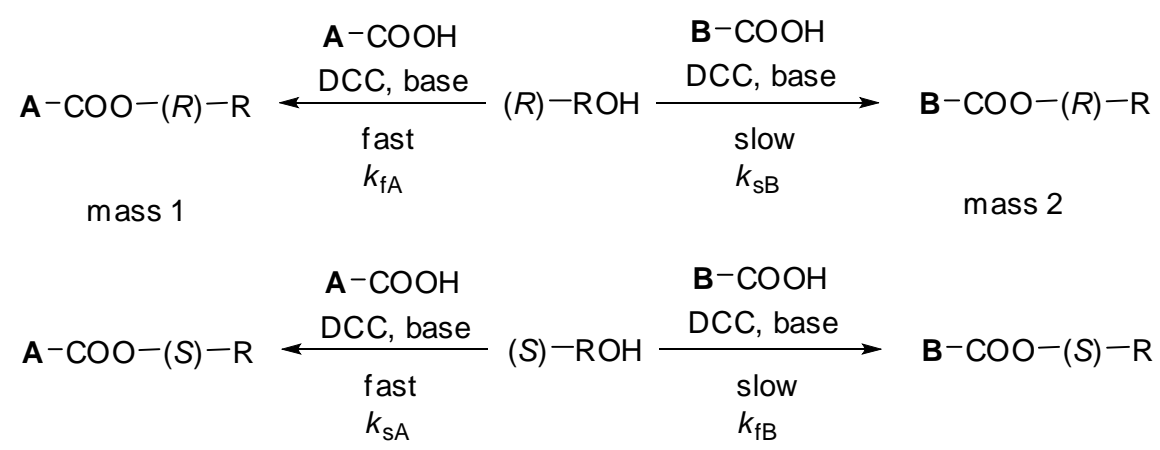

\subsection{Capillary electrophoresis}

The high-throughput ee-screening system based on capillary electrophoresis was developed by Reetz et al. by using the MegaBase system consisting of 6 bundles of 16 capillaries which spatially address standard 96-well microtiter plates. ${ }^{17}$ Chiral amines 1-10 were derivatized with fluorescein isothiocyanate 1-11 for the parallel optical detection (Scheme 1-5). Soluble cyclodextrins derivatives were employed as chiral selectors to enable chiral separation. It was demonstrated that at least 7000 ee determinations could be performed per day, proving the ability of capillary electrophoresis in high-throughput manner.

Scheme 1-5. Derivatization of chiral amines for the determination of $e e$. 


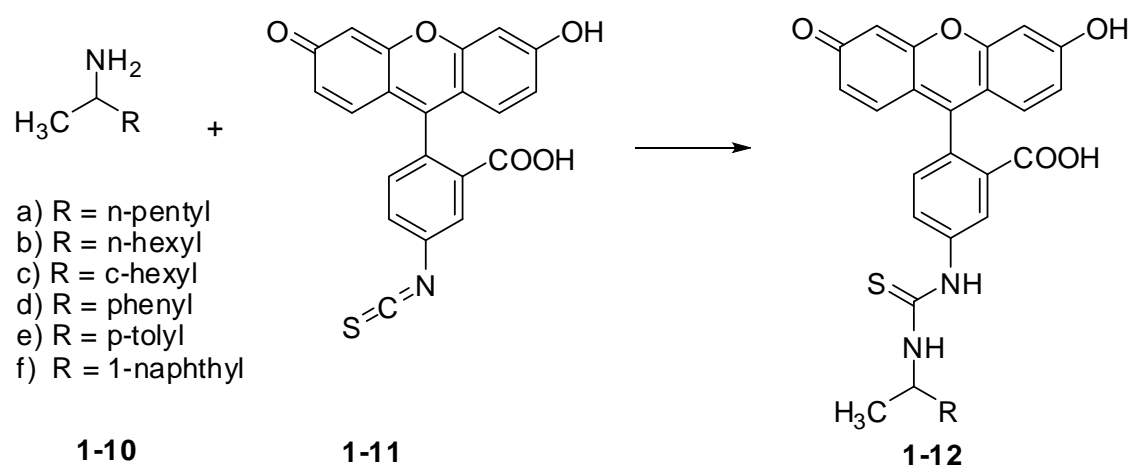

\subsection{Circular dichroism (CD)}

In 1999, Koichi Mikami et al. developed an HPLC-CD-based system ${ }^{18}$, which allows the simultaneous detection of the CD signal $\Delta \varepsilon$, the absorption $\varepsilon$, and their ratio $g=\Delta \varepsilon / \varepsilon$. The dissymmetry factor $\mathrm{g}$ is independent of concentration and is linearly associated to the $e e$ value. They applied this technique to the parallel asymmetric addition of diethylzinc to aldehydes in the presence of combinations of chiral diol ligands and nitrogen activators (Scheme 1-6).

Scheme 1-6. Asymmetric activation of diol-zinc catalysts by nitrogen ligands.

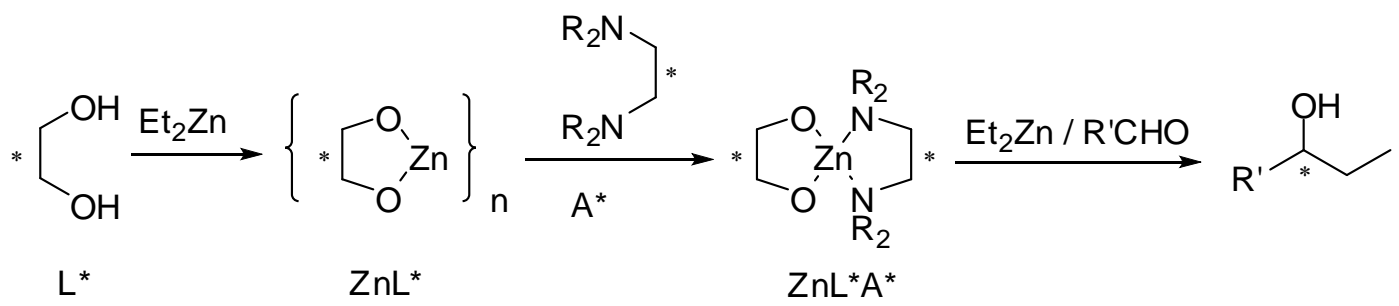

Anslyn et al. explored the use of the CD active metal-to-ligand charge transfer (MLCT) transitions in ee determination ${ }^{19}$. Metal complex [Cu(I)(BINAP)(MeCN) ${ }_{2} \mathrm{PF}_{6}$ (1-13) was used to interact with enantiomers of four chiral diamines and it was 
demonstrated that fingerprinting chemical identity, chirality and concentration could be achieved. Automation of a 96-well plate CD spectrophotometer made this system compatible with HTS.

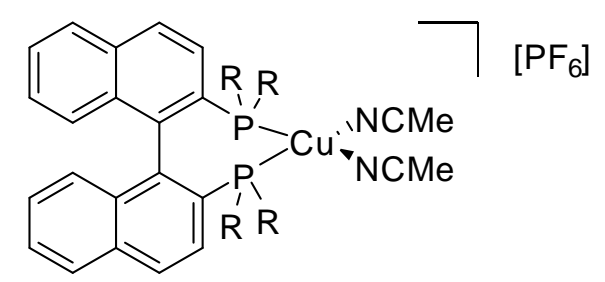

1-13

\subsection{Fluorescence}

In 1999, Scott J. Miller et al. employed a fluorescent acetic acid sensor (1-14) to examine the catalyst activity for the enantioselective acylation of trans-1,2acetamidocyclohexanol 1-15 (Scheme 1-7). ${ }^{20}$ They found that the most selective catalysts typically afforded the fastest reactions. The $\mathrm{pH}$-sensitive fluorophore could monitor the evolution of acetic acid by turning on the fluorescence.<smiles>COC(=O)C1CCN(Cc2c3ccccc3cc3ccccc23)CC1</smiles>

1-14

Scheme 1-7. Enantioselective catalytic acylation of trans-(+/-)-1-15.

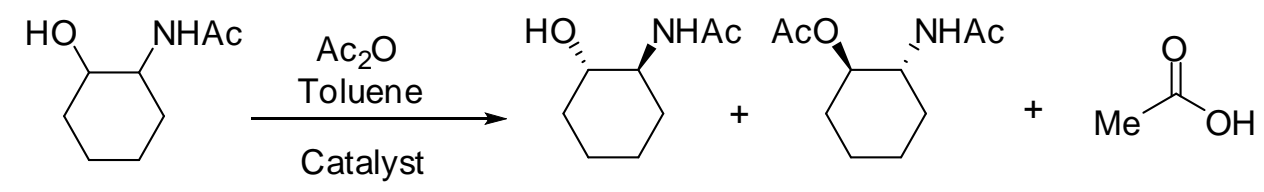
trans-(+/-)-1-15 
In 2001, Mattew D. Shair et al. adapted the reaction mircoarray method for the high-throughput determination of ee. ${ }^{21}$ Amino acids were attached to a glass surface and pseudoenantiomeric fluorescent probes 1-16 and 1-17 were coupled with amino acids. Parallel kinetic resolution during the amide coupling reaction converted the ee information of the sample into a ratio of fluorophores. With this technique approximately 75,000 samples can be arrayed onto a $25 \mathrm{~mm} \times 75 \mathrm{~mm}$ glass slide and analysed in a comparable amount of time.

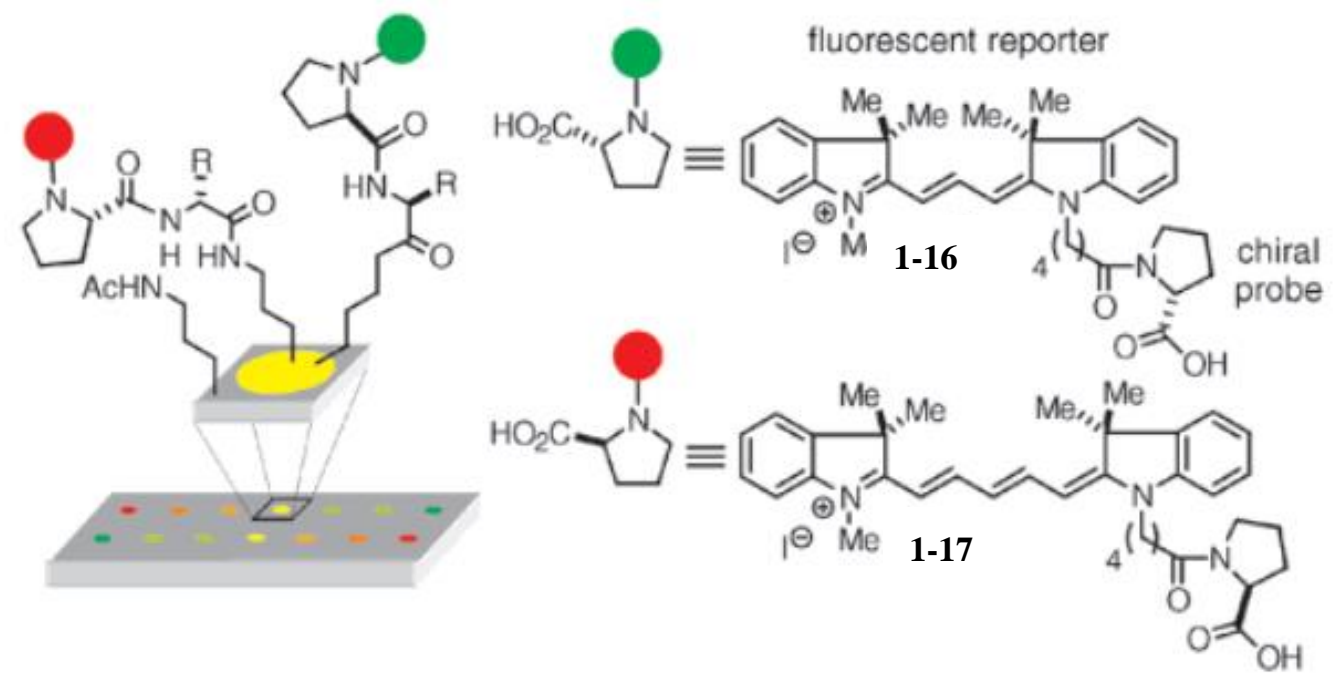

Among all the analytical methods mentioned above, the use of fluorescence has many incomparable advantages. Fluorescence spectrometers are of low cost and widely available. It has very high sensitivity, which is favorable for the small loading amount in high-throughput screening. It provides many different detection modes, such as intensity, wavelength and lifetime. Also, its ability to be used in real-time analysis is another attractive feature. 


\section{Enantioselective Fluorescent sensors}

The two examples of use of fluorescence mentioned above are not straightforward for ee determination of chiral products, one through detection of the achiral product and the other through derivatization of the chiral compounds. In recent years, researchers have been exploring the direct enantioselective fluorescent sensors for the ee determination and a number of highly enantioselective fluorescent sensors for the recognition of chiral molecules such as carboxylic acids, amines, alcohols, amino alcohols and amino acid derivatives have been reported.

The three key components for the successful design of enantioselective fluorescent sensors are an appropriate fluorophore, binding sites and sensing mechanisms. ${ }^{22} \mathrm{~A}$ fluorophore is the site for both photonic excitation and emission, which allows the detection of fluorescence. Binding sites are responsible for guest complexation and decomplexation and in enantioselective recognition must be selective for one enantiomer over the other. Common fluorescence recognition mechanisms are based on many structural features of molecules including $\pi-\pi^{*}$ and $n-\pi^{*}$ transitions, structural rigidity, noncovalent interactions (e.g., hydrogen bonds, $\pi-\pi$ interactions, and hydrophilic and hydrophobic interactions), intra- or intermolecular energy transfer, and photoinduced electron transfers (PET). When a chiral fluorescent sensor interacts with enantiomers of chiral substrates, the chirality-matched enantiomer will form stronger interactions with the sensor than the chirality-mismatched enantiomer. This difference in interactions 
leads to the different structural changes in fluorophore. As a result, different fluorescent responses can be observed. Some examples of the previously reported enantioslective fluorescent sensors are described below.

\subsection{Hydrogen bonding}

Our group has developed a series of 1,1'-bi-2-naphthol (BINOL) based enantioselective fluorescent sensors for the recognition of chiral $\alpha$-hydroxycarboxylic acids. $^{2}$ BINOL is a very commonly used framework for the construction of enantioselective fluorescent sensors due to its fluorescent properties of naphthyl rings, hydrogen bonding ability of the hydroxyl group and chiral recognition ability caused by the hindered rotation of the two naphthyl rings.

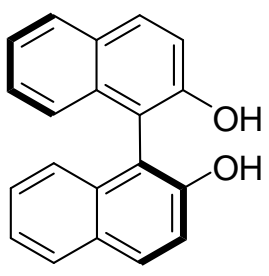

(S)-BINOL<smiles>Oc1ccc2ccccc2c1-c1c(O)ccc2ccccc12</smiles>

$(R)$-BINOL

The bisBINOL compound 1-18 was designed for the recognition of chiral mandelic acid (MA). ${ }^{23}$ Structure 1-19 was a proposed complex between $(S, S)-\mathbf{1 - 1 8}$ and (S)-MA and features a binding site of three specific hydrogen bonds, which forms a more rigidified structure and enhances the fluorescence. Due to different binding constants of the sensor with two enantiomers of MA, different fluorescence enhancement was 
achieved. Figure 1-1 gives the fluorescence response of $(S, S)-\mathbf{1 - 1 8}$ in the presence of $(R)$ - or $(S)$-MA in benzene in which $2 \%$ of dimethoxyethane (DME) was added to improve the solubility of MA. $(S)$-MA $\left(5 \times 10^{-3} \mathrm{M}\right)$ increases the fluorescence of $(S)$-1-18 $\left(9.5 \times 10^{-5} \mathrm{M}\right)$ more than $(\mathrm{R})-\mathrm{MA}$ with $\mathrm{IS} / \mathrm{IO}=2.87$ and the enantiomeric fluorescence enhancement ratio ef was $2.49\left[\mathrm{ef}=\left(\mathrm{I}_{\mathrm{S}}-\mathrm{I}_{0}\right) /\left(\mathrm{I}_{\mathrm{R}}-\mathrm{I}_{0}\right)\right]$.

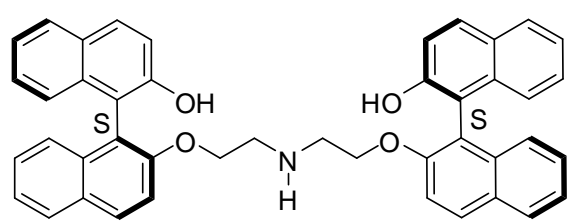

$(S, S)-1-18$

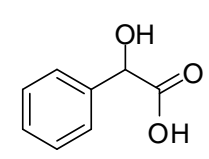

mandelic acid (MA)

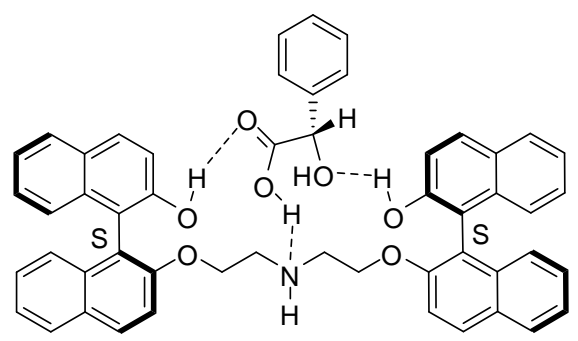

$1-19$

Figure 1-1. Fluorescence spectra of $(S, S)$-1-18 $\left(9.5 \times 10^{-5} \mathrm{M}\right.$ in benzene/2\% DME) with and without MA $\left(5 \times 10^{-3} \mathrm{M}\right)$.

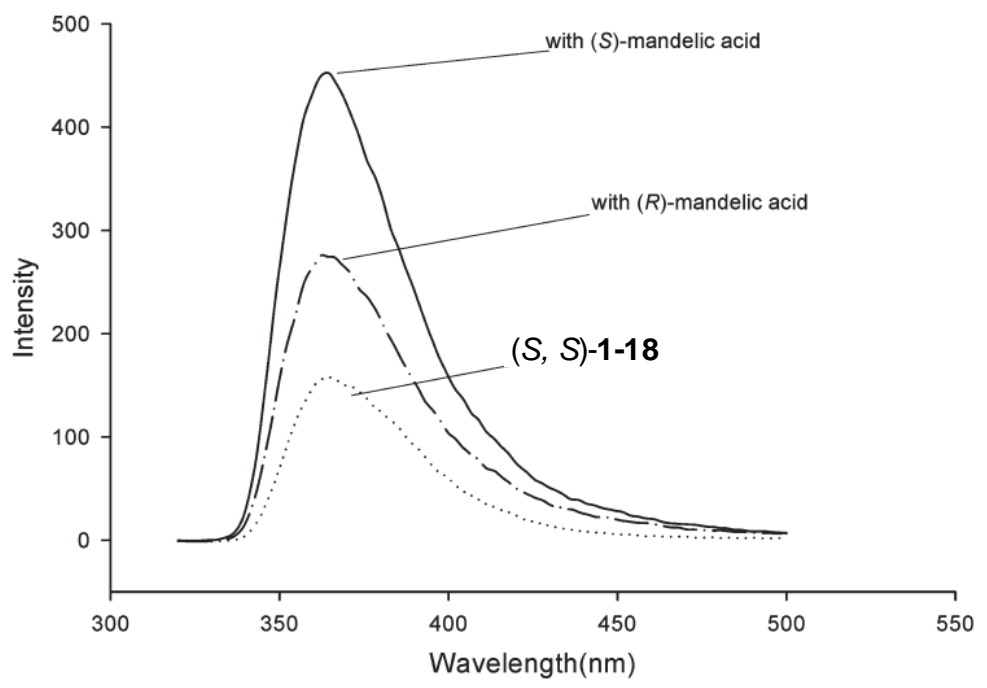

Compounds $(R, R)-\mathbf{1 - 2 0}$ and $(R, R)-\mathbf{1 - 2 1}$, the dendritic derivatives of $(R, R)-\mathbf{1 - 1 8}$ with phenylene dendrons, were also synthesized. ${ }^{24}$ By introducing dendritic branches to the 
chiral receptor unit, the light-harvesting effect of the dendritic structure amplified the fluorescence signal of the receptors and greatly increased their sensitivity (Figure 1-2). The enantioselectivity of $(R, R)-\mathbf{1 - 2 0}$ was similar to that of the core $(R, R)-\mathbf{1 - 1 8}$ with an ef value of 2.05 and $(R, R)$-1-21 was a little lower with an ef of 1.49.

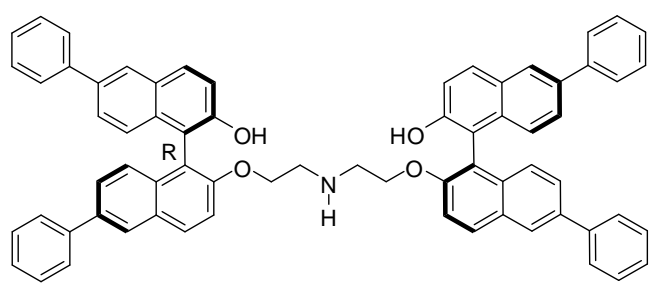

$(R, R)-1-20$

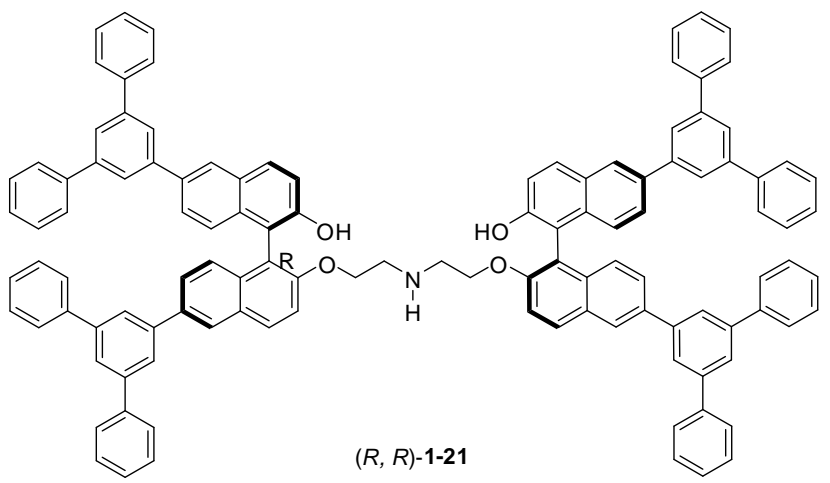

Figure 1-2. Fluorescence spectra of $(R, R)-\mathbf{1 - 1 8},(R, R)-\mathbf{1 - 2 0},(R, R)-\mathbf{1 - 2 1}\left(3.1 \times 10^{-6} \mathrm{M}\right.$, benzene/0.1\% DME) with and without $(\mathrm{R})-\mathrm{MA}\left(1 \times 10^{-3} \mathrm{M}\right)$.

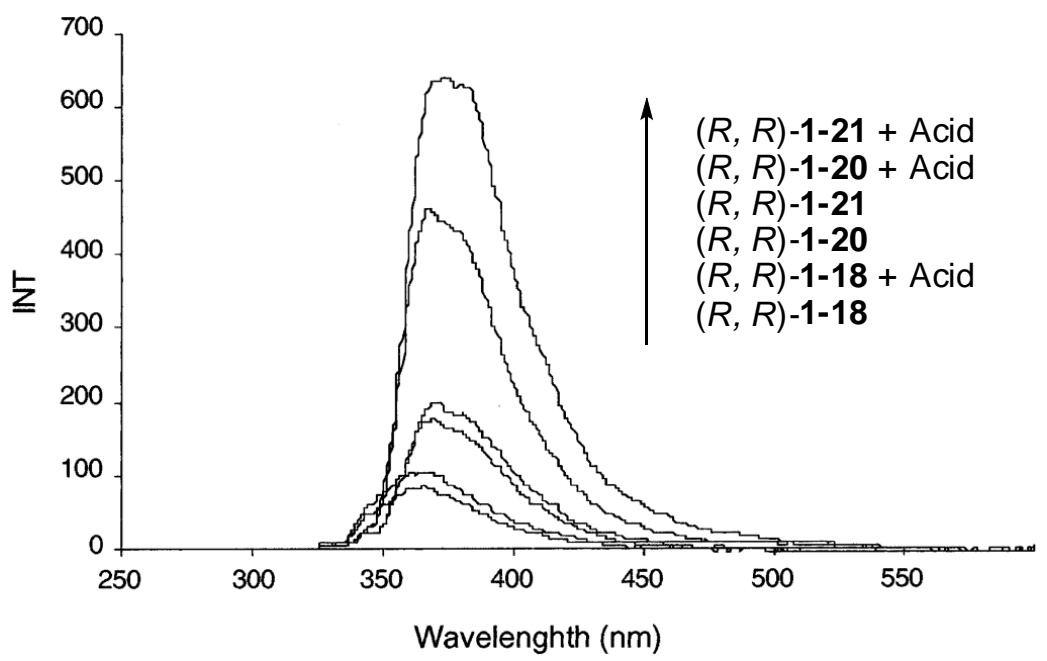

In order to improve the enantioselectivity, two bisBINOL-based macrocycles $(S)-\mathbf{1 - 2 2}$ and $(S)-\mathbf{1 - 2 3}$ were prepared. $^{25,26}(S)-\mathbf{1 - 2 2}$ show dual emissions at $365 \mathrm{~nm}$ and $424 \mathrm{~nm}$, which were assigned to the monomer emission and excimer emission, respectively. As 
shown in Figure 1-3, $(S)$-MA increased the fluorescence at $424 \mathrm{~nm}$ to 2.9 times of its original value and very high enantioselectivity with ef greater than 12 was obtained. The job plot indicated the formation of a 1:4 complex between the macrocycle and the acid. It is proposed that the interaction of the macrocycle nitrogen and the acidic proton of the substrate interferes with the intramolecular hydrogen bonding of the sensor and leads to the enhanced fluorescence.

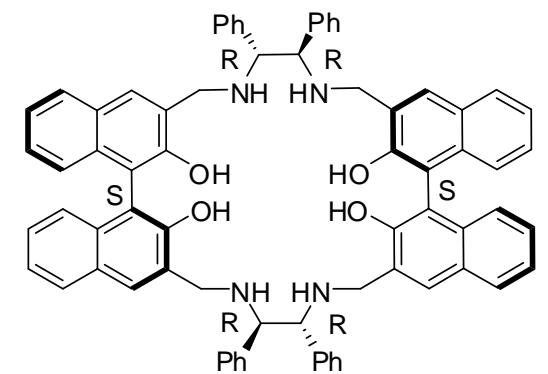

$(S)-1-22$

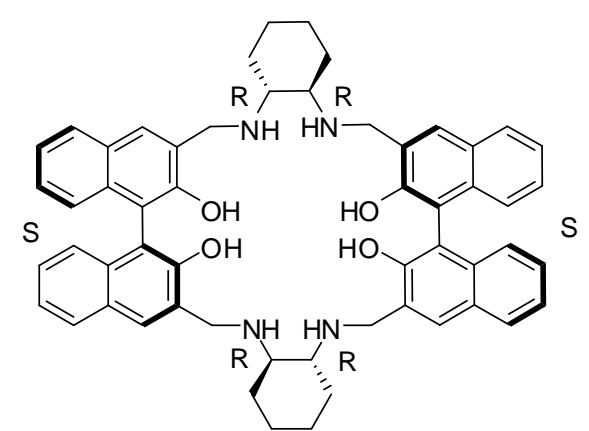

(S) $-1-23$

Figure 1-3. Fluorescence spectra of $(S)-\mathbf{1 - 2 2}\left(1 \times 10^{-4} \mathrm{M}\right.$, benzene/2\% DME $)$ with and without MA $\left(2 \times 10^{-2} \mathrm{M}\right)$.

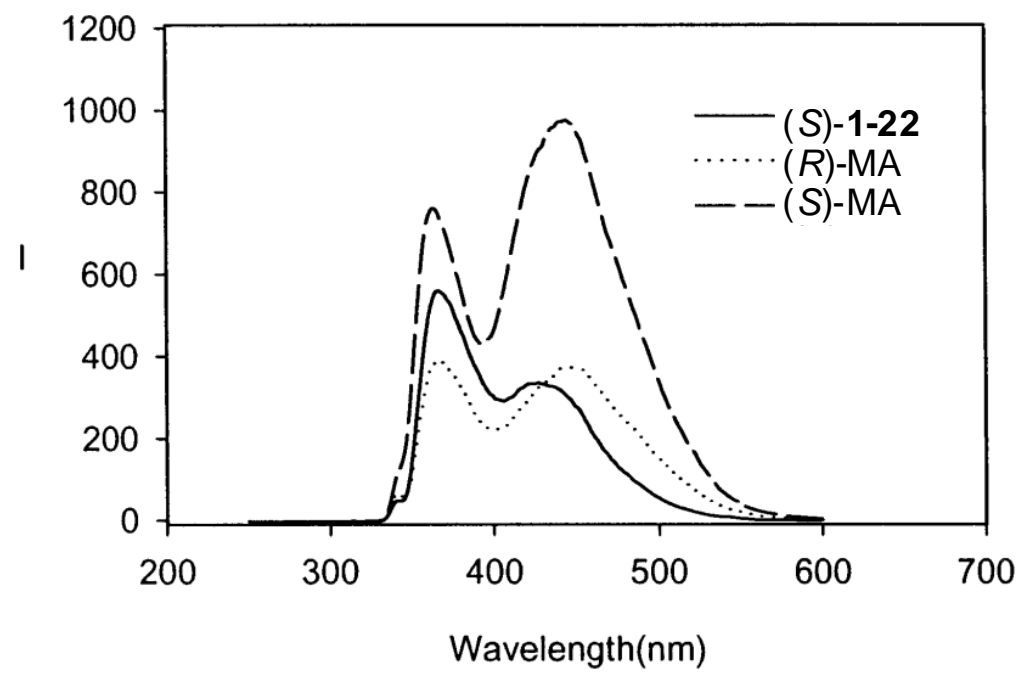


Its analogue $(S)-\mathbf{1 - 2 3}$, prepared from $(1 R, 2 R)$-diaminocyclohexane, showed very different fluorescent properties. $^{26}$ The addition of $(S)$-MA increased its monomeric emission by over 20 folds while (R)-MA caused very little change (Figure 1-4). Therefore, it exhibited extraordinary high enantioselectivity for MA with ef $=46 . \quad$ A two-stage recognition process was proposed to account for the significant fluorescent enhancement: $(S)-\mathbf{1 - 2 3}$ formed a structurally rigid 1:1 complex with MA through the host-guest inclusion inside the chiral cavity; additional MA located outside the cavity interacted with the complex through multiple hydrogen bonds to further increase the fluorescence.

Figure 1-4. Fluorescence spectra of $(S)-1-23\left(1 \times 10^{-5} \mathrm{M}\right.$, benzene/0.05\% DME) with and without MA $\left(5 \times 10^{-4} \mathrm{M}\right)$.

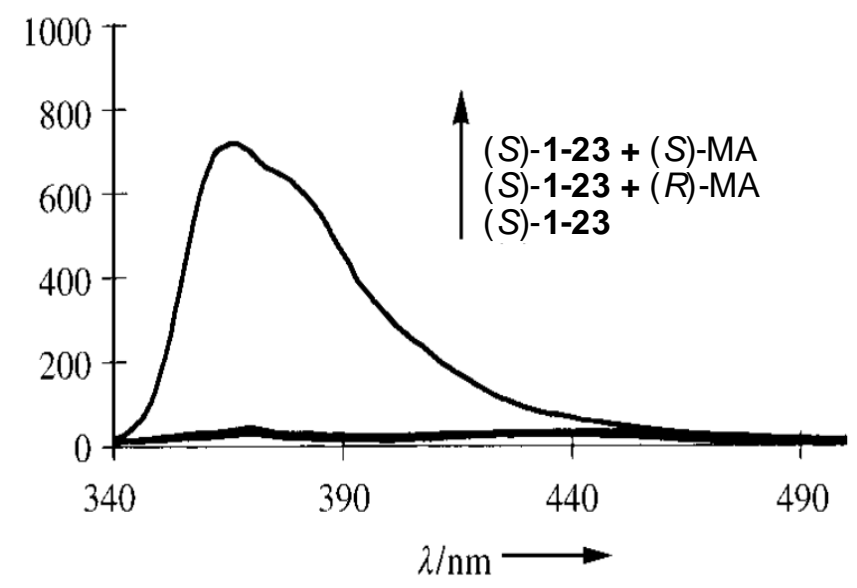

The acyclic BINOL-based amino alcohol (S)-1-24 exhibited very special enantioselective precipitation with MA. ${ }^{27}$ The clear solution of (S)-1-24 immediately becomes a white suspension with addition of $(S)$-MA $(>=3 \mathrm{mM})$ while remains clear 
with $(R)$-MA (3 mM $-8 \mathrm{mM}$ ) (Figure 1-5). Moreover, the observed enantioselective precipitation was associated with a dramatic (over 950-fold) solid-state fluorescence enhancement and ef was as high as 485 (Figure 1-6).

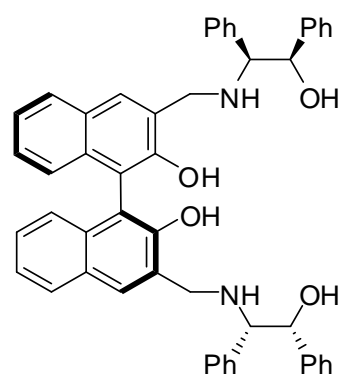

(S)-1-24

Figure 1-5. Photographs of $(S)-\mathbf{1 - 2 4}\left(5 \times 10^{-4} \mathrm{M}\right.$, benzene/0.4\% DME) with $(R)$ - and $(S)$-MA $\left(4 \times 10^{-3} \mathrm{M}\right)$.

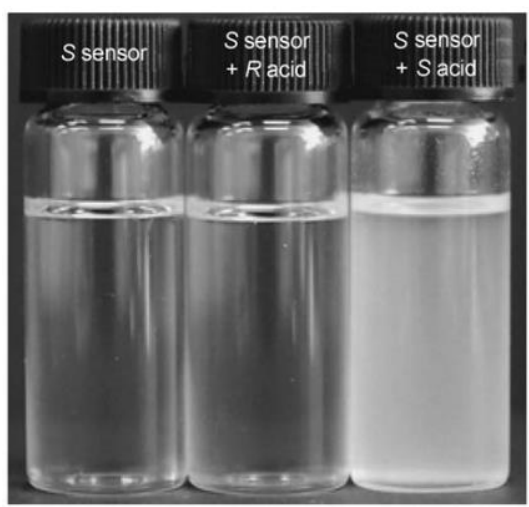

Figure 1-6. Fluorescence spectra of $(S)-\mathbf{1 - 2 4}\left(5 \times 10^{-4} \mathrm{M}\right.$, benzene/0.4\% DME) with and without MA $\left(4 \times 10^{-3} \mathrm{M}\right)$. 


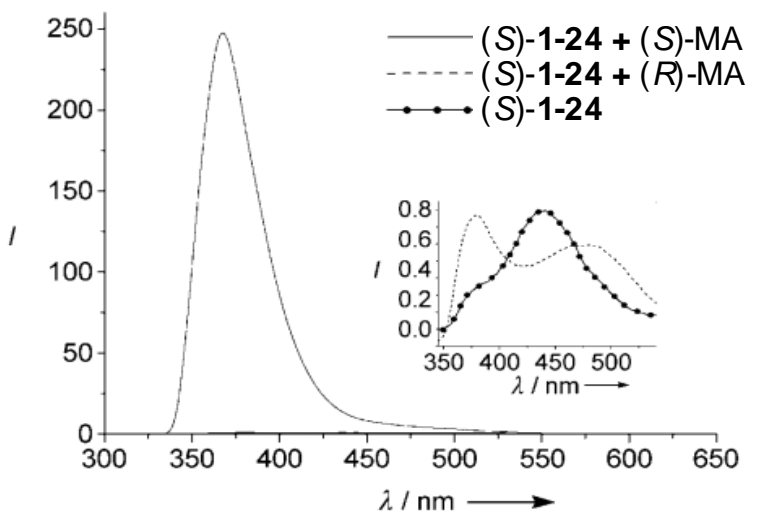

The compound $(S) \mathbf{- 1 - 2 5}$, which contains one more phenyl group than $(S) \mathbf{- 1 - 2 4}$, shows general enantioselectivity for structurally diverse $\alpha$-hydroxyl carboxylic acids, including aromatic, aliphatic and tertiary $\alpha$-hydroxyl carboxylic acids (Figure 1-7). ${ }^{28}$ When (S)-1-25 was treated with a chiral acid, one enantiomer of the acid greatly enhanced its monomer emission while the other quenched it (Figure 1-8). The $I_{R} / I_{S}$ ratio was used to represent the enantioselectivity. The ${ }^{1} \mathrm{H}$ NMR study indicated the formation of $1: 1$ sensor/acid complex. The proposed structure of the complex is shown in Figure 1-9.

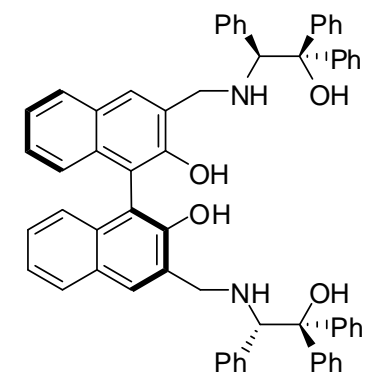

(S)-1-25

Figure 1-7. Fluorescent enantioselectivity of (S)-1-25 toward various chiral $\alpha$-hydroxycarboxylic acids. 

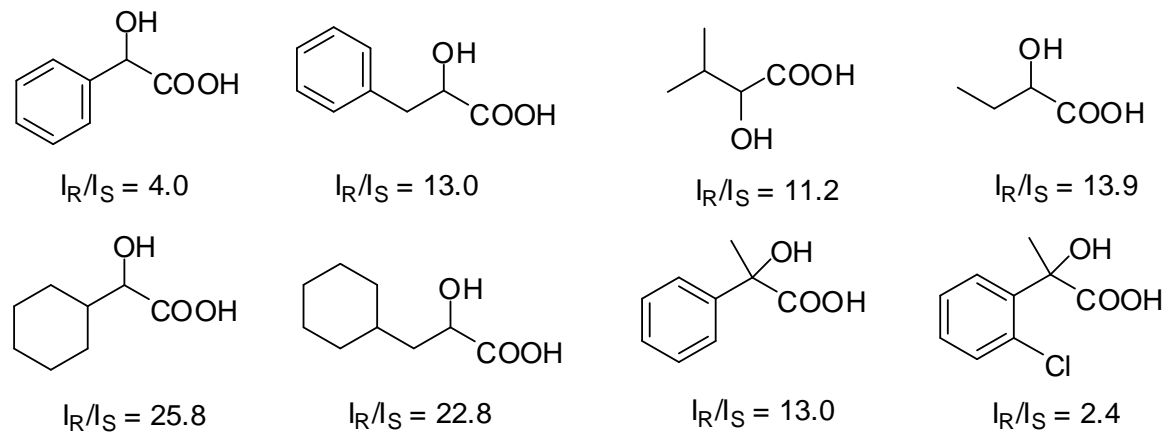

Figure 1-8. Fluorescence spectra of $(S)-\mathbf{1 - 2 5}\left(2 \times 10^{-4} \mathrm{M}\right.$, benzene/0.4\% DME) with and without phenyllactic acid $\left(5 \times 10^{-3} \mathrm{M}\right)$.

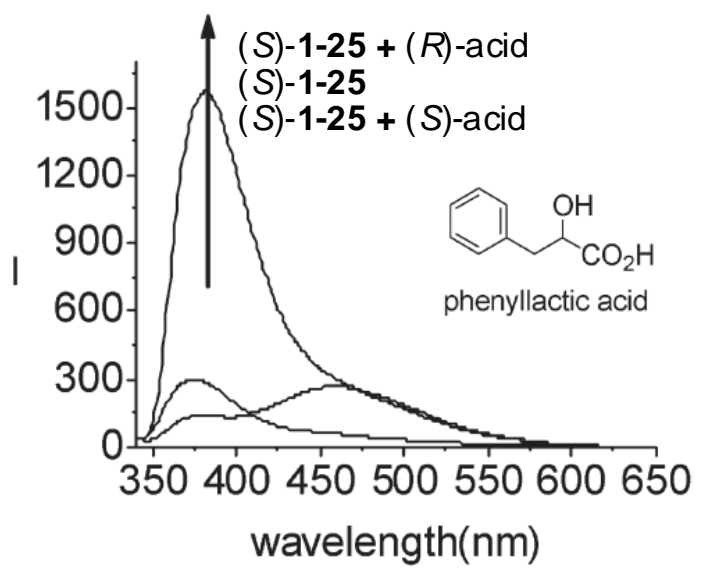

Figure 1-9. Calculated structure of the proposed $1: 1$ complex of $(S)-\mathbf{1 - 2 5}+$ (R)-phenyllatic acid.
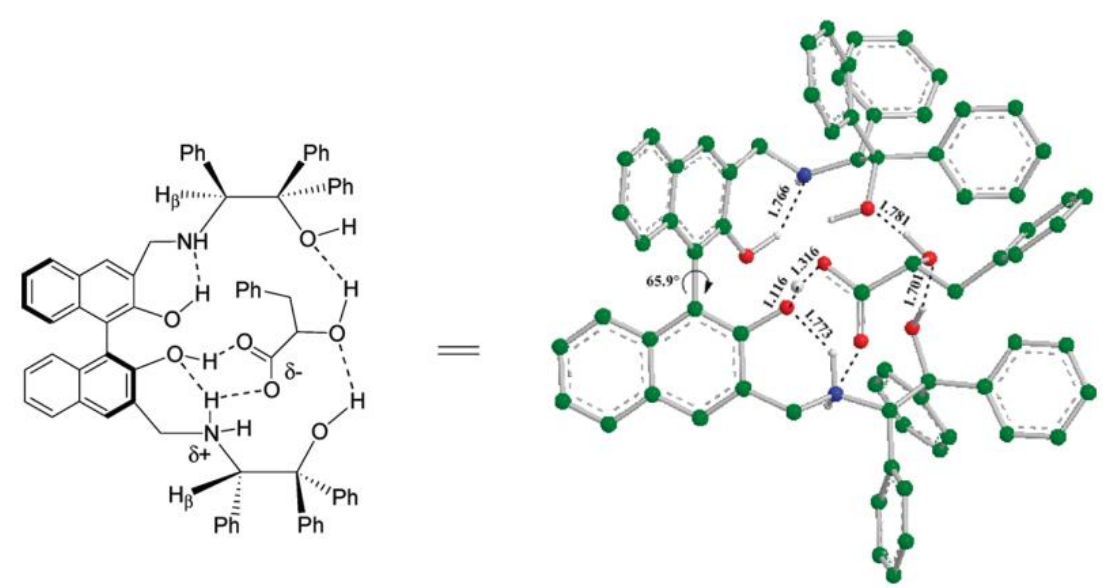
Crown ether is a commonly used binding unit in enantioselective recognition of organic ammonium salts due to the formation of tripod-like hydrogen binding complex 1-26. Huszthy et al. designed acridino-crown ligand 1-27 for the enantioselective recognition of organic ammonium perchlorates $\mathbf{1 - 2 8}$ and $\mathbf{1 - 2 9} .{ }^{29}$ Besides the tripod-like hydrogen bonding, the extended aromatic ring system of the ligand can form strong $\pi-\pi$ interaction with the substrates. When $(R)$ - or $(S)$-1-28 were added to a acetonitrile solution of 1-27, the fluorescence intensity of naphthalene $\left(\lambda_{\mathrm{em}}=330 \mathrm{~nm}\right)$ was almost completely quenched while the fluorescence of the acridine moiety $\left(\lambda_{\mathrm{em}}=440 \mathrm{~nm}\right)$ underwent only partial quenching. The association constants exhibited big differences with $4.4 \times 10^{5} \mathrm{M}^{-1}$ for $(R)-\mathbf{1 - 2 8}$ and $2.3 \times 10^{6} \mathrm{M}^{-1}$ for $(S)-\mathbf{1 - 2 8}$. This ligand also showed enantioselectivity for chiral ammonium 1-29 with association constant at $3.4 \times 10^{5} \mathrm{M}^{-1}$ for $(R)$-1-29 and $1.7 \times 10^{6} \mathrm{M}^{-1}$ for $(S)-\mathbf{1 - 2 9}$.
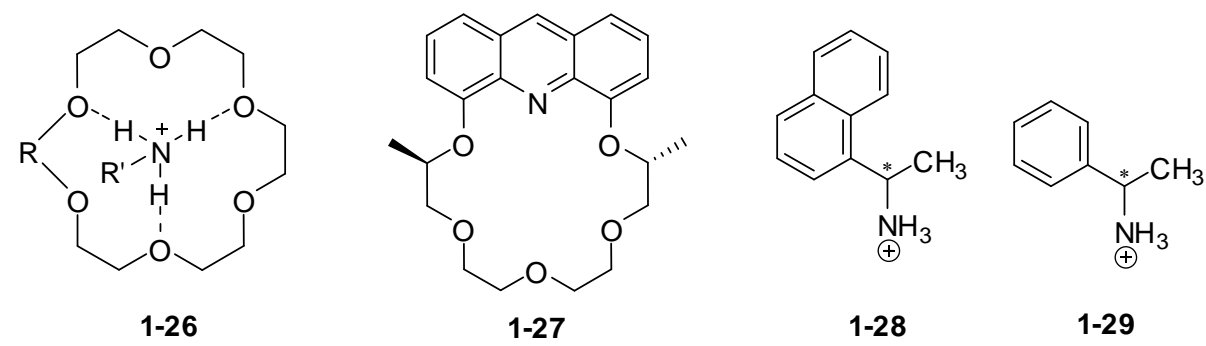

Karnik et al. developed a furo-fused BINOL based chiral crown 1-30 as an enantioselective chiral sensor for phenylethylamine 1-31 and ethyl ester of valine 1-32. ${ }^{30}$ Fluorescence enhancement was observed when 1-31 in chloroform was treated with perchlorate salts of 1-31 and 1-32. The fluorescence was increased to 1.25 times with 
$(R)-\mathbf{1 - 3 1}\left(1.64 \times 10^{-6} \mathrm{M}\right)$ but only 1.08 times with $(S)-\mathbf{1 - 3 1}$, which gave ef value 2.97 . It also exhibited enantioselective fluorescence enhancement for $(S) \mathbf{- 1 - 3 2}$ and ef was 2.55. A linear relationship was observed for the concentration of 1-31 versus fluorescent intensity ratio, which indicated $\mathbf{1 - 3 0}$ is useful for the ee determination of the amine 1-31.

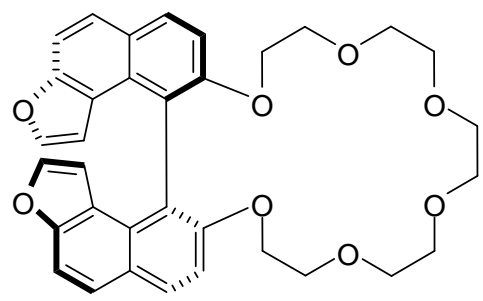

$1-30$

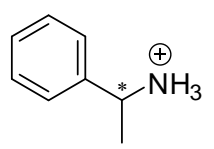

1-31

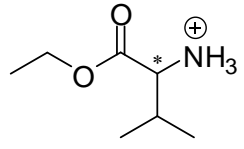

1-32

Wolf and coworkers have developed a series of $\mathrm{C}_{2}$-symmetric 1,8-diacrydylnaphthalene fluorescent sensors for a range of chiral hydrogen bond donor analytes. Compound 1-33 was found to undergo enantioselective quenching $\left(\lambda_{\mathrm{em}}=550\right.$ $\mathrm{nm})$ in acetonitrile with a variety of chiral carboxylic acids 1-34 - 1-41. ${ }^{31}$ Among all these acids, camphanic acid 1-41 exhibited highest enantioselectivity with $\alpha\left(\mathrm{K}^{\mathrm{R}}{ }_{\mathrm{SV}} / \mathrm{K}^{\mathrm{S}}{ }_{\mathrm{SV}}\right.$ or $\mathrm{K}_{\mathrm{SV}}^{\mathrm{S}} / \mathrm{K}_{\mathrm{SV}}^{\mathrm{R}}$ ) up to 4.5. Based on Stern-Volmer equation, compound 1-33 forms 1:1 complex with acids 1-34 - 1-37 and 1:2 complexes with acids 1-38 - 1-41. It was proposed that the enantioselective fluorescence quenching was due to static quenching through nonradiative releaxation of the diastereomeric acid-base adducts. 


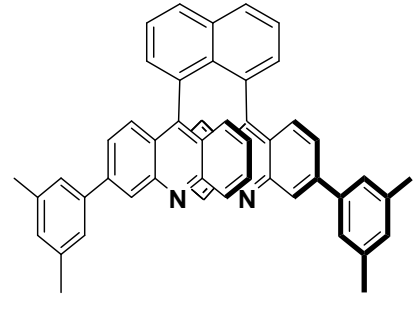

1-33
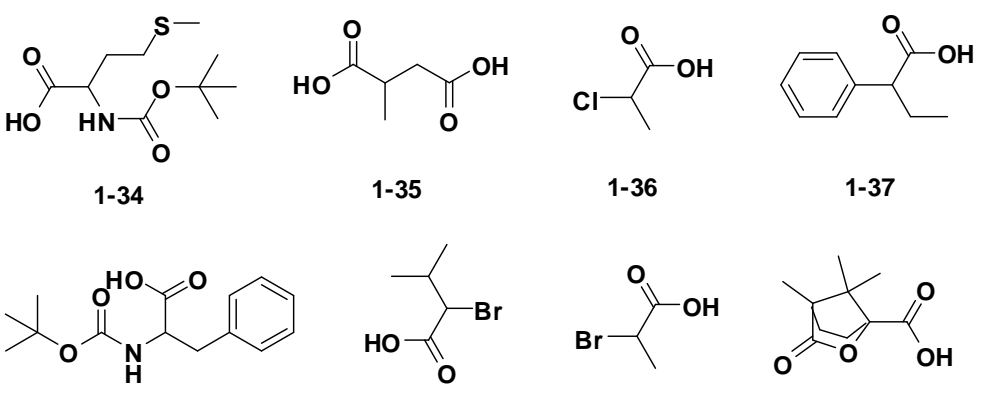

1-39

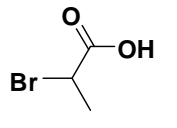

$1-40$

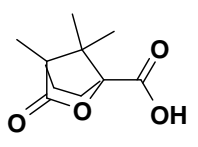

$1-41$

N,N'-dioxide 1-42 was also prepared and the twisting of the acrydyl rings can vary to accommodate analytes of varying size. ${ }^{32}$ Enantioselective quenching can be observed when treated with chiral bidentate analytes $\mathbf{1 - 4 3}$ and 1-44. The enantioselectivity factor $\alpha$ were 1.63 and 1.50 , respectively.
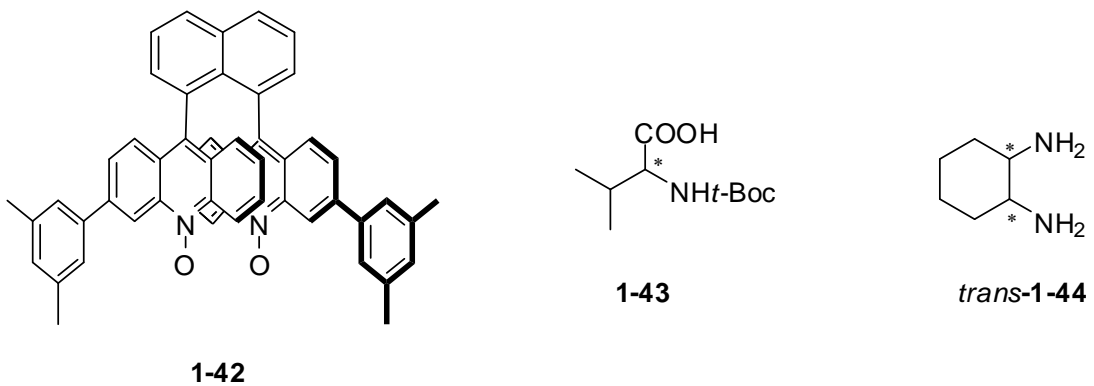

Similarly, compound $\mathbf{1 - 4 5}$ can enantioselectively recognize various carboxylic acids and amino acids. ${ }^{33}$ Taking 1-36 and 1-39 as examples, the fluorescence of 1-45 was dramatically quenched when treated with $(S)$-enantiomer while only a little quenching could be observed with $(R)$-enantiomer (Figure 1-10). It was proved that the total concentration and ee of a chiral substrate 1-36 could be determined by using the racemic and enantiopure form of $\mathbf{1 - 4 5}$. Six samples were tested and the results were within $+/-2 \%$ of the actual concentration and $+/-3 \%$ of the actual enantiopurity. 


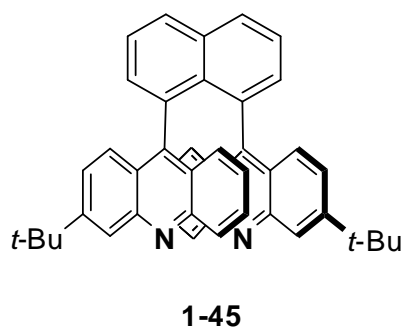

Figure 1-10. Stern-Volmer plots showing enantioselective fluorescence quenching of (-)-1-45 in the presence of 1-36 and 1-39.
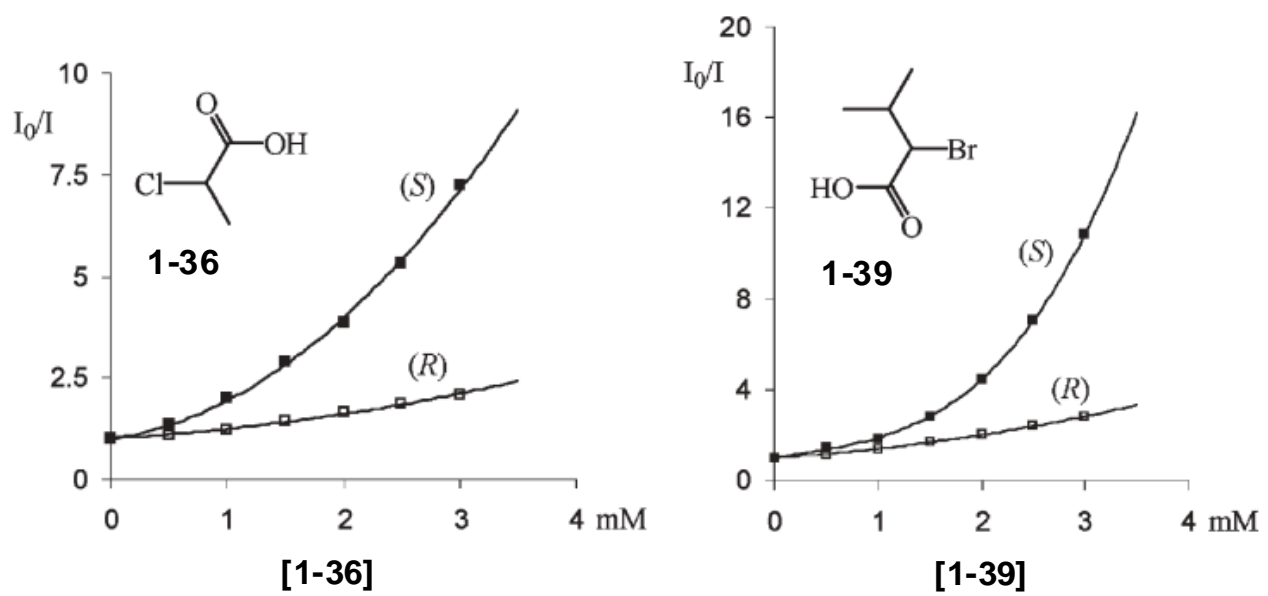

\section{2 metal complexation}

Transition metals, especially $\mathrm{Cu}$ (II), usually work as an efficient fluorescence quencher due to the formation of a metal-ligand charge transfer state. Treating a fluorophore with transition metals may turn off the fluorescence and produce a low background. If addition of guests to this complex can recover the fluorescence, a turn on fluorescent sensor with high sensitivity would be enabled.

Roberto Corradini and coworkers developed a series of monofunctionalized $\beta$-cyclodextrins bearing a copper (II) binding side arm and a dansyl group 
(CD-NH-AA-CH2CH2NH-DNS) for the enantioselective sensing of unmodified $\alpha$-amino acids. As shown in Figure 1-11, $\beta$-cyclodextrins was chosen as platform since it has the right size to include dansyl in its cavity to form rigid preorganized structure. The side arms and dansyl group provide an amino, an amide, and a sulfonamide group as binding sites for $\mathrm{Cu}(\mathrm{II})$. Chiral amino acid provides an additional chiral center to enhance enantioselectivity.

Figure 1-11. Structural elements considered in the design of enantioselective cyclodextrins.

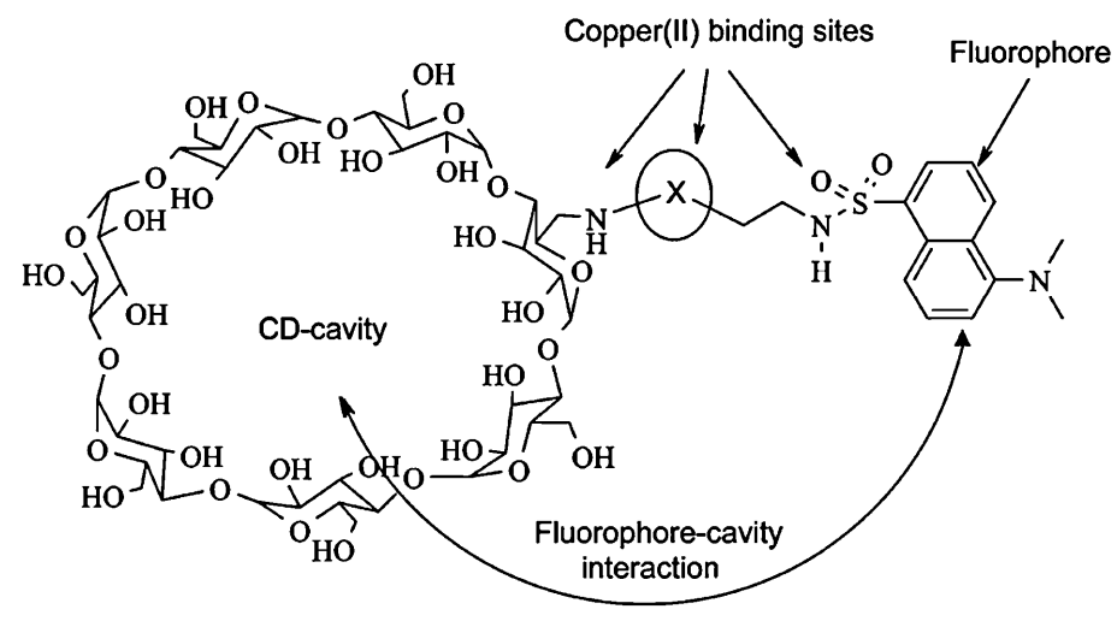

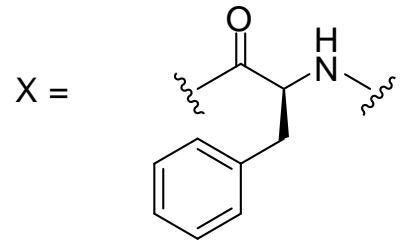

$1-46$<smiles>CCNC(C(=O)CC)c1ccccc1</smiles>

1-47<smiles>CCC(=O)C1CCCN1CC</smiles>

$1-48$<smiles>C=CNC(C(=O)CC)C1CCCCC1</smiles>

1-49

Compound 1-46 can form 1:1 complex with $\mathrm{Cu}(\mathrm{II})$ showing quenched fluorescence. ${ }^{34}$ When equimolar amount of D- or L-amino acids were added to this complex solution (6 
$\left.\times 10^{-5} \mathrm{M}\right)$ in $0.1 \mathrm{M}$ tetraborate buffer $(\mathrm{pH}=7.3)$, the fluorescence can be turned back on with enantioselectivity. This $\mathrm{Cu}$ complex showed good enantioselectivity for most of tested amino acids expect Ala, His and Asp (Table 1-1). ${ }^{35}$ The mechanism of sensing can be attributed to the competition between the formation of the ternary $\mathrm{Cu}(\mathrm{II}) /$ ligand/amino acid complex which is nonfluorescent and that of the binary $\mathrm{Cu}(\mathrm{II}) /$ amino acid complex which is fluorescent because of displacement of the dansyl group.

Table 1-1. Enantioselectivity $\Delta \mathrm{F}_{\mathrm{D}} / \Delta \mathrm{F}_{\mathrm{L}}$ of $\mathrm{Cu} \mathbf{- 1 - 4 6}$ towards amino acids.

\begin{tabular}{|c|c|c|c|c|c|c|c|c|c|c|c|c|}
\hline & Pro & Ala & Val & Leu & His & Asp & Lys & Ser & Phe & Phgly & Trp & Tyr \\
\hline$\Delta \mathrm{F}_{\mathrm{D}} / \Delta \mathrm{F}_{\mathrm{L}}$ & 3.93 & 1.04 & 0.34 & 0.31 & 0.94 & 0.89 & 0.79 & 0.68 & 0.19 & 0.43 & 0.62 & 0.44 \\
\hline
\end{tabular}

Another three cyclodextrins 1-47 - 1-49 were prepared and enantioselective fluorescence quenching was performed by adding $\mathrm{Cu}(\mathrm{II})$ complex of amino acid to a

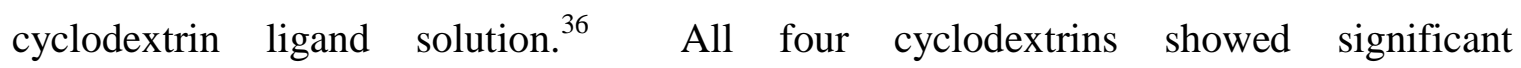
enantioselectivity for both proline and valine. Calibration curves were obtained by using the fluorescence microplate reader to measure the interaction of cyclodextrins 1-46 - 1-48 with valine samples of different enantiomeric excess. Six samples were analyzed with the calibration curve and it shows an average error of $6 \%$, which proved its accuracy and ability to be used for high-throughput screening. ${ }^{37}$

A BINOL-terpyridine-Cu(II) complex 1-51 was prepared by our group and its $\mathrm{CHCl}_{3}$ suspension at $3.75 \%(\mathrm{w} / \mathrm{v}, \mathrm{g} / \mathrm{mL})$ can form gel upon sonication. ${ }^{38}$ The gel collapsed 
when a $\mathrm{CHCl}_{3}$ solution $(0.1 \mathrm{~mL})$ of $(\mathrm{S})$-phenylglycinol $(0.1$ equiv) was added while remained stable with $(R)$-phenylglycinol upon sonication. Complexation with $\mathrm{Cu}$ quenched the fluorescence of 1-50. When the enantiomers of phenylglycinol were added to the solution of $\mathbf{1 - 5 1}\left(5.0 \times 10^{-7} \mathrm{M}\right.$ in $2: 3 \mathrm{CH}_{2} \mathrm{Cl}_{2} / \mathrm{n}$-hexane), it showed enantioselective fluorescence enhancement (Figure 1-12). This enantioselectivity can be attributed to the enantioselective displacement of the $\mathrm{Cu}$ (II) from 1-51 by the amino alcohol.
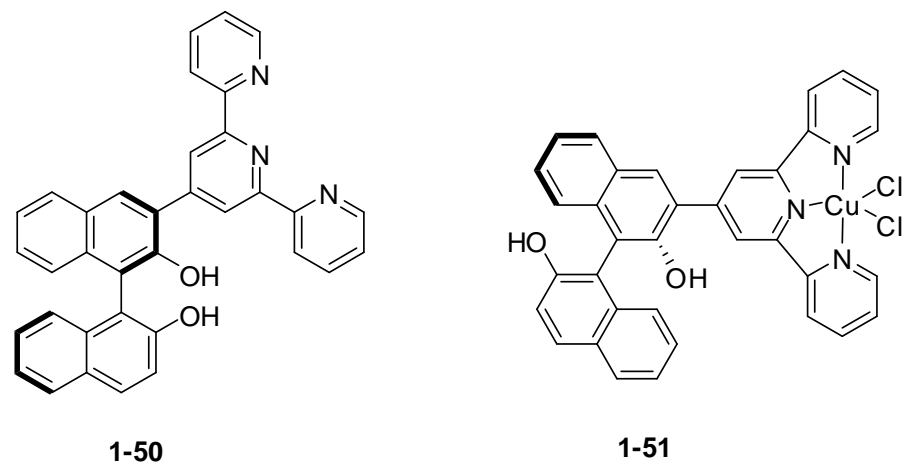

Figure 1-12. Fluorescence spectra of 1-51 $\left(5.0 \times 10^{-7} \mathrm{M}\right)$ in $\mathrm{CH}_{2} \mathrm{Cl}_{2} / \mathrm{n}$-hexane $(2: 3)$ in the presence of (R)- and (S)-phenylglycinol $\left(5.0 \times 10^{-4} \mathrm{M}\right)$.

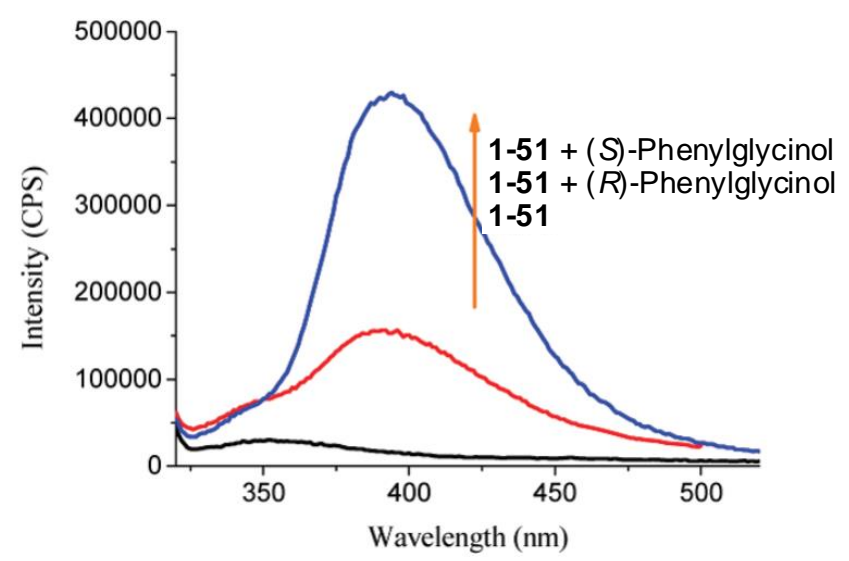


Zhu et al. synthesized a chiral perazamacrocyclic fluorescent sensor 1-52. ${ }^{39}$ Among the 14 tested metals, only $\mathrm{Cu}(\mathrm{II})$ could efficiently quench its fluorescence by forming a 1:1 complex. With excess amount of $\mathrm{Cu}(\mathrm{II})$, which is $2 \mathrm{eq}$, this ligand solution $\left(1 \times 10^{-5}\right.$ M, in 1:1 methanol/water) exhibited remarkable enantioselective fluorescence enhancement toward unmodified amino acids 1-53-1-57, in which 1-57 Phe showed the highest fluorescence enhancement with $\mathrm{I}_{\mathrm{D}} / \mathrm{I}_{0}=14.9$ and highest enantioselectivity with $\Delta \mathrm{I} / \mathrm{I}_{0}=4.9$ (Figure 1-13).

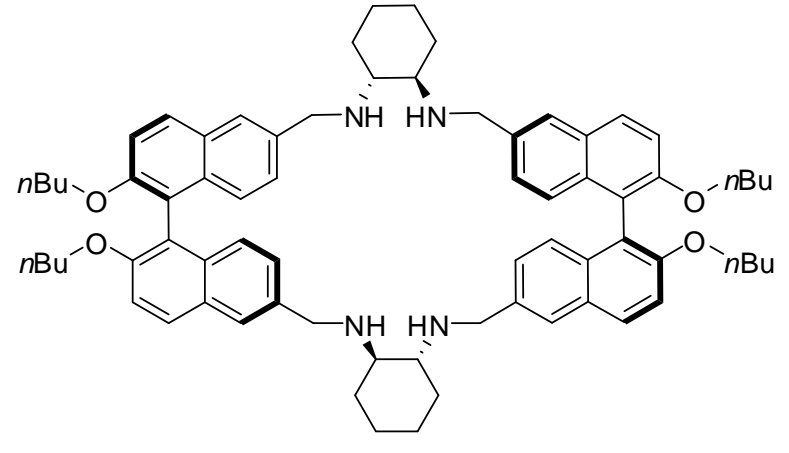

$1-52$

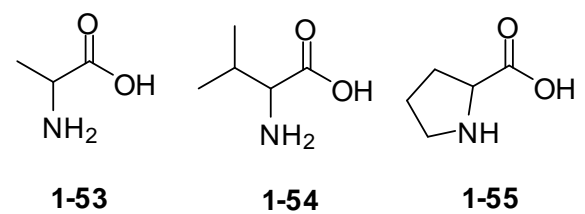<smiles>NC(Cc1ccccc1)C(=O)O</smiles>

$1-56$<smiles>NC(C(=O)O)c1ccccc1</smiles>

$1-57$

Figure 1-13. (a) Fluorescence spectra of $1-52+\mathrm{Cu}(\mathrm{II})\left(1 \times 10^{-5} \mathrm{M}+2 \times 10^{-5} \mathrm{M}\right)$ with $\mathrm{D}$ - or L-Phe $\left(1 \times 10^{-4} \mathrm{M}\right)$. (b) Plots of $\mathrm{I} / \mathrm{I}_{0}$ vs Phe concentration during titration.
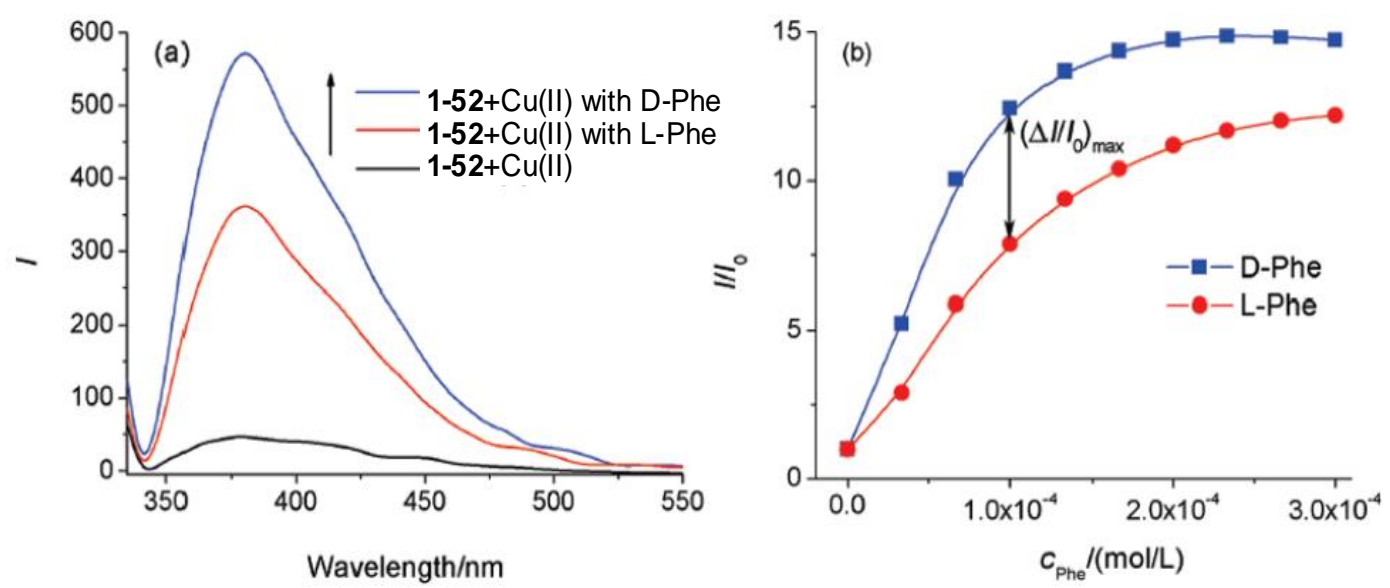


\subsection{Reversible covalent interactions}

The use of hydrogen bonding interactions has its limitations in protic solvents. Thus the fluorescent boronic acid based sensors have been extensively explored because they can covalently interact with diols in aqueous environment. ${ }^{40}$

Figure 1-14. Generic design of fluorescent PET sensors with the boronic acid recognition unit.<smiles>CC(C)OCCOCN(C)Cc1ccccc1B(O)O</smiles>

Figure 1-15. The effect of saccharide complexation and $\mathrm{pH}$ changes on the fluorescence of boronic acid.

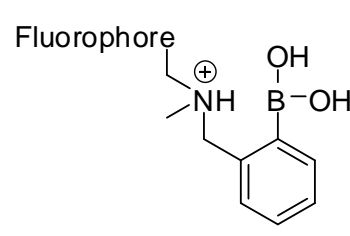

fluorescent

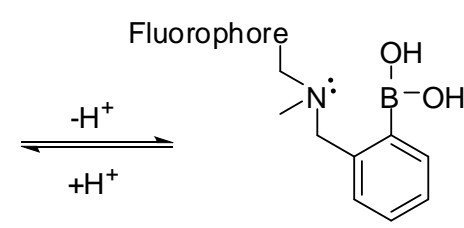

non-fluorescent
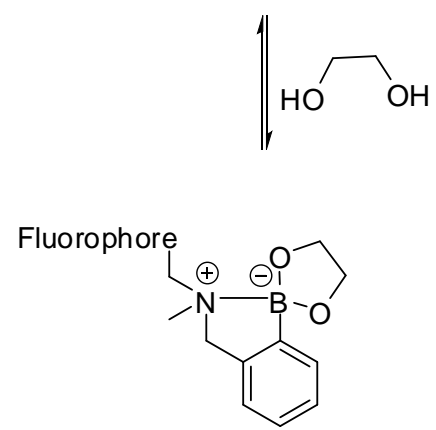

fluorescent

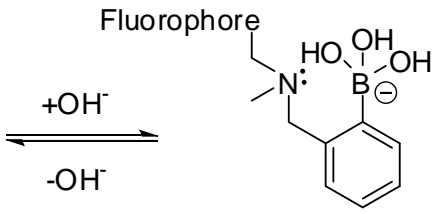

non-fluorescent

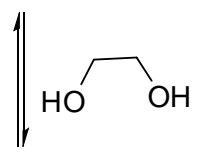

Fluorophore

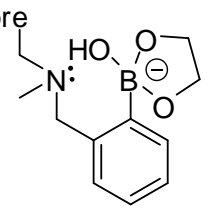

non-fluorescent

The design of these sensors follows fluorophore-amine base-boronic acid structure 
(Figure 1-14). As shown in Figure 1-15, the amine base-boronic acid interaction lowers the $\mathrm{pKa}$ of the boronic acid and allows binding to occur at neutral $\mathrm{pH}$. Also the lone pair of amine is available for photoinduced electron transfer (PET) to quench the fluorescence. Complexation with diols augments the N-B interaction and in turn distrupts PET, turning on the fluorescence. Seiji Shinkai, Tony D. James and Jianzhang Zhao have developed a series of enantioselective boronic acid sensors.

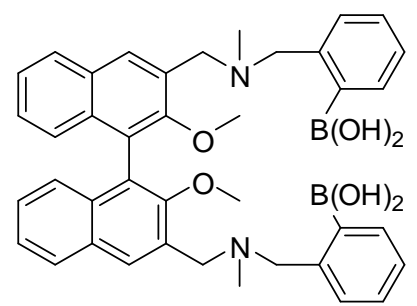

(R)-1-58 and (S)-1-58

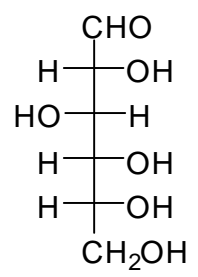

D-Glucose

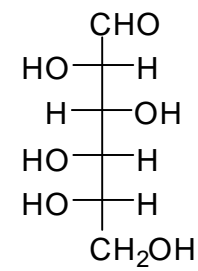

L-Glucose
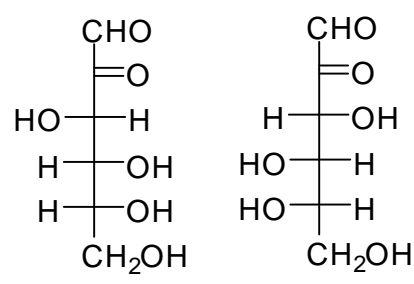

D-Fructose

L-Fructose

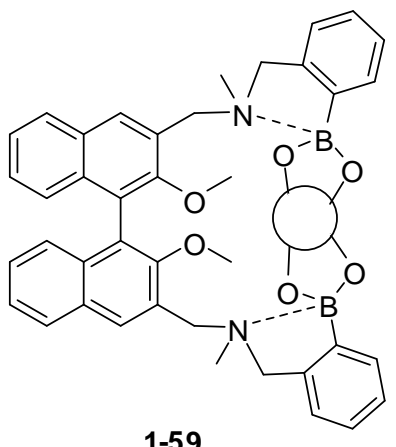

$1-59$

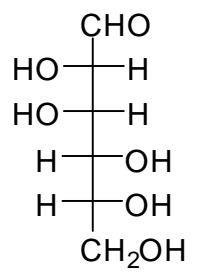

\begin{tabular}{c|c}
\multicolumn{2}{c}{$\mathrm{CHO}$} \\
$\mathrm{H}-\mathrm{OH}$ \\
$\mathrm{H}-\mathrm{OH}$ \\
$\mathrm{HO}$ & $\mathrm{H}$ \\
$\mathrm{HO}$ & $\mathrm{H}$ \\
\multicolumn{2}{c}{$\mathrm{CH}_{2} \mathrm{OH}$}
\end{tabular}

D-Mannose

L-Mannose

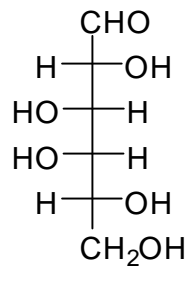

D-Galactose

The BINOL-bisboronic acid compound 1-58 was first designed for the chiral discrimination of $\mathrm{D}$ - and L-monosaccharides at $\mathrm{pH} 7.77$ (phosphate 33.3\% (w/w) 
methanol buffer). ${ }^{41}$ It formed 1:1 cyclic boronate ester 1-59 with guests and the fluorescence was enhanced due to suppressed PET. With (R)-1-58 the complex stabilities of D-fructose, D-glucose, D-mannose and L-galactose are greater than those of L-fructose, L-glucose, L-mannose and D-galactose, respectively (Table 1-2). The stronger binding corresponds to the stronger fluorescence.

Table 1-2. Stability constants and fluorescence enhancements for saccharides with (R)-1-58.

\begin{tabular}{|c|c|c|c|}
\hline Saccharide & D $\log \mathrm{K}$ & $\mathrm{L} \log \mathrm{K}$ & D/L fluorescence ratio \\
\hline Fructose & 4.0 & 3.5 & 1.47 \\
\hline Glucose & 3.3 & 3.1 & 1.93 \\
\hline Galactose & 3.1 & 3.3 & 0.82 \\
\hline Mannose & $<2.4$ & - & - \\
\hline
\end{tabular}

The same compound was then investigated for the interaction with a range of sugar acids over a range of $\mathrm{pH}$ values. ${ }^{42}$ As shown in Figure 1-16, the recognition of D- and L-tartaric acid is strongly $\mathrm{pH}$ dependent. At pH 5.6 the fluorescence of $(R)-\mathbf{1 - 5 8}$ was enhanced by D-tartaric acid but diminished by L-tartaric acid. At pH 8.3 both D- and L-tartaric acid could enhance the fluorescence with much stronger fluorescence for D-tartaric acid. Gluconic acid showed similar responses.<smiles>O=C(O)C(O)C(O)C(=O)O</smiles><smiles>O=C(O)C(O)C(O)C(=O)O</smiles>

D-Tartaric acid

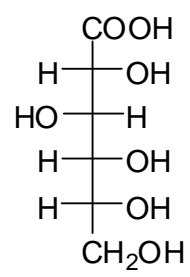

D-Gluconic acid L-Gluconic acid

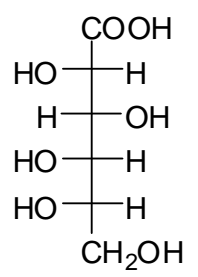

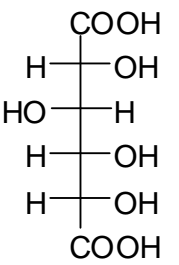

\begin{tabular}{c|cc}
\multicolumn{2}{c|}{$\mathrm{COOH}$} \\
$\mathrm{HO}$ & $\mathrm{H}$ \\
$\mathrm{H}$ & $\mathrm{OH}$ \\
$\mathrm{HO}$ & $\mathrm{H}$ & $\mathrm{H}$ \\
$\mathrm{COOH}$
\end{tabular}

D-Glucaric acid

L-Glucaric acid 
Figure 1-16. Fluorescence intensity-pH profiles for titrations of $(R)$ - and $(S)$-1-58 $\left(5.0 \times 10^{-6} \mathrm{M}\right.$ in $0.05 \mathrm{M} \mathrm{NaCl}$ solution $(52.1 \%$ methanol in water)) with D- or L-tartaric $\operatorname{acid}(0.02 \mathrm{M})$.

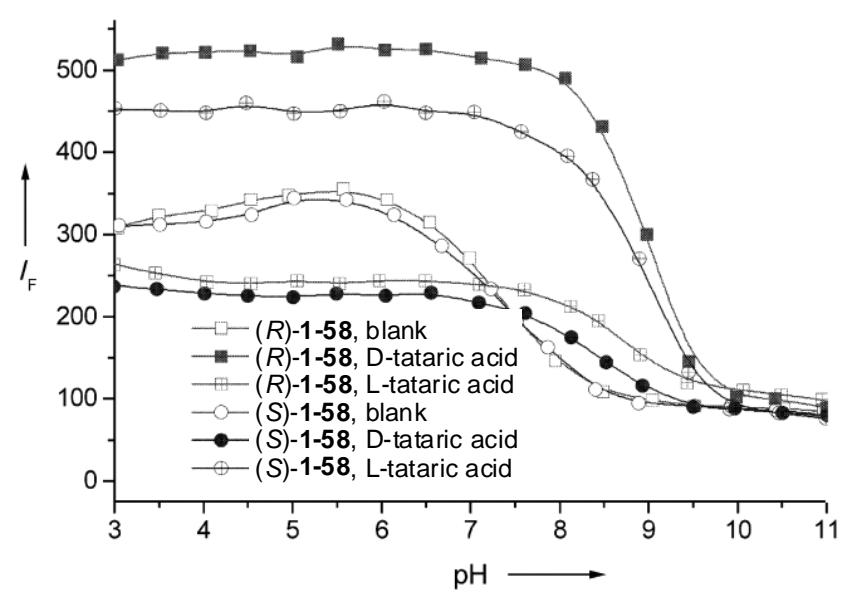

In order to make the chiral center in close proximity to the receptor's binding site, anthracene-bisboronic acid 1-60 was designed. ${ }^{43}$ It exhibited significantly improved sensitivity and enantioselectivity toward sugar acids, such as tartaric acid, glucaric acid and gluconic acid. At $\mathrm{pH}$ 8.3, titration of $(S, S)$-1-60 with L-tartaric acid caused a significant fluorescence enhancement $\left(\mathrm{I} / \mathrm{I}_{0}=8.24\right)$, whereas only a small change $\left(\mathrm{I} / \mathrm{I}_{0}=\right.$ 1.5) was observed with D-tartaric acid (Figure 1-17a). Calibration curve was generated and the ee of tartaric acid can be determined. Single-crystal X-ray data confirmed the 1:1 binding complex (Figure 1-18). It also exhibited significant enantioselectivity towards sugar alcohols. ${ }^{44}$ 


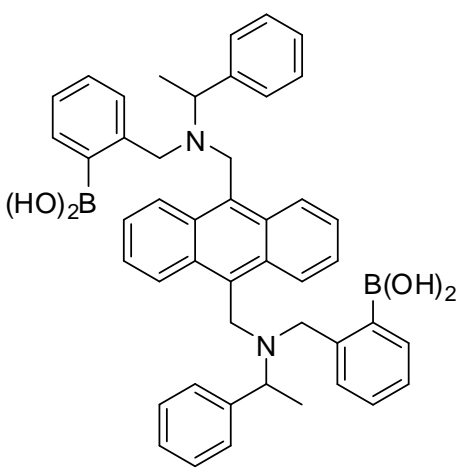

$(R, R)-1-60$ and $(S, S)-1-60$

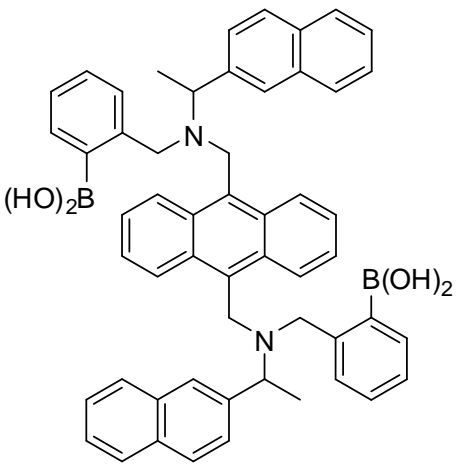

$(R, R)-1-61$ and $(S, S)-1-61$

Figure 1-17. Fluorescence intensity-pH profiles for titrations of (a) 1-60, (b) $(R, R)-\mathbf{1 - 6 1}$ $\left(3.0 \times 10^{-6} \mathrm{M}\right.$ in $0.05 \mathrm{M} \mathrm{NaCl}$ solution (52.1\% methanol in water)) with D- or L-tartaric acid $(0.05 \mathrm{M})$.
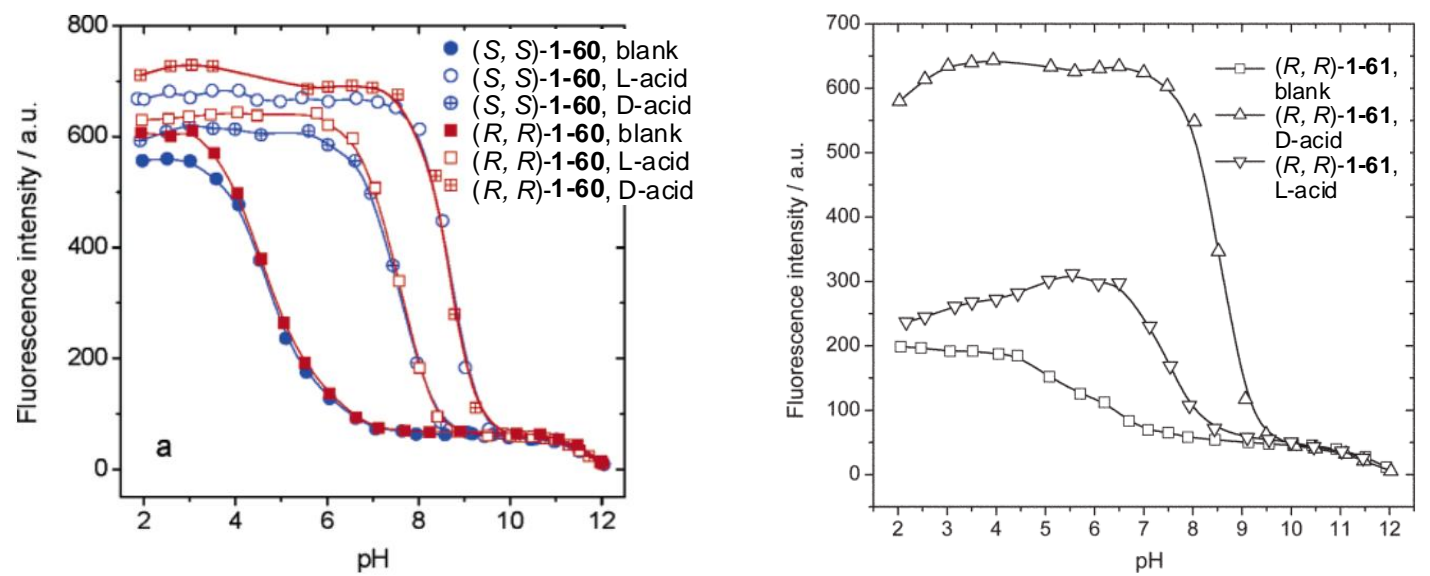

Figure 1-18. (a) Structure of $(R, R)$-1-60. (b) Structure of $(R, R)-\mathbf{1 - 6 0}$ complex with L-tartaric acid.

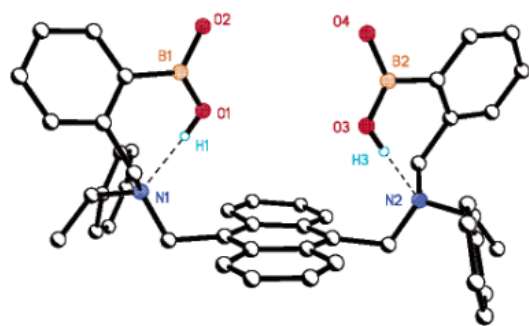

(a)

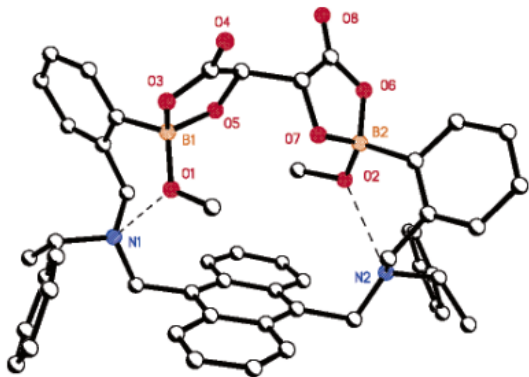

(b) 
By introducing naphthalene a dual fluorophore boronic acid receptor 1-61 was prepared. $^{45}$ It displayed reduced fluorescence response compared to 1-60 due to close contact of the naphthalene and anthracene fluorophores. Addition of D-tartaric acid to $(R, R)$-1-61 caused a large increase in fluorescence as a result of the formation of a rigid complex. Addition of L-tartaric acid only produced small changes in fluorescence.

The normal PET sensors, as described above, give stronger fluorescence at acidic conditions due to suppressed PET. The fluorophore acts as acceptor in PET process. However, the carbazole-bisboronic acid 1-62 displayed a reverse fluorescence intensity-pH relationship with diminished emission at acidic $\mathrm{pH}$ but enhanced emission at basic $\mathrm{pH}$ (Figure 1-19). ${ }^{46}$ It is proposed that the reduced fluorescence is due to electron transfer from the cabazole moiety to the protonated amine and boronic acid moiety, which is called d-PET (fluorophore as donor). This theory was supported by DFT/TDDFT calculations. 1-62 exhibited fluorescence enhancement /diminishment selectivity toward the enantiomers of tartaric acid at neutral $\mathrm{pH}$.

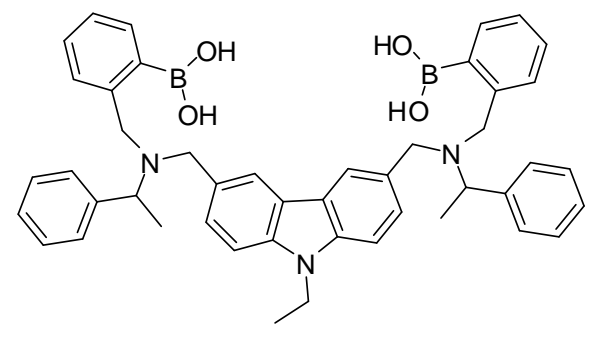

$(R, R)-1-62$ and $(S, S)-1-62$

Figure 1-19. Fluorescence intensity-pH profiles of $(S, S)-\mathbf{1 - 6 2}\left(3.0 \times 10^{-6} \mathrm{M}\right.$ in $0.05 \mathrm{M}$ $\mathrm{NaCl}$ solution $(52.1 \%$ methanol in water)) with D- or L-tartaric acid (0.05 M). 


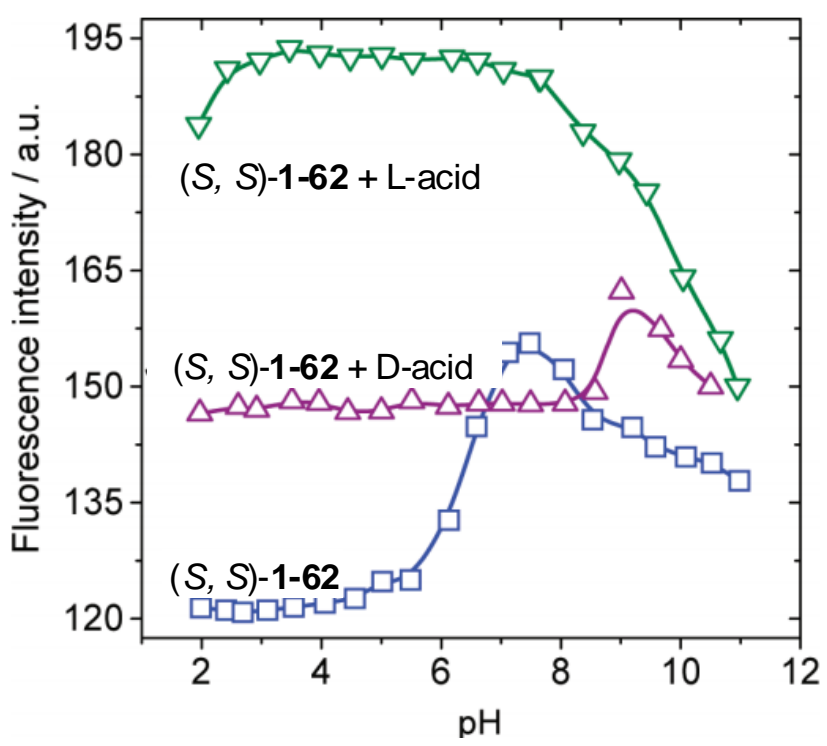

In order to extend the $\pi$-conjugation of the carbazole and to enhance the electron-donating ability of the fluorophore, thiophene was employed to acquire carbazole-thiophene-boronic acid sensor 1-63. ${ }^{47}$ Its emission maxima located at $413 \mathrm{~nm}$, which is red shifted by $38 \mathrm{~nm}$ compare to sensor 1-62. The d-PET contrast ratio is 3, which is 10-fold greater than 1-62. Enantioselective recognition toward tartaric acid and mandelic acid was observed (Figure 1-20).

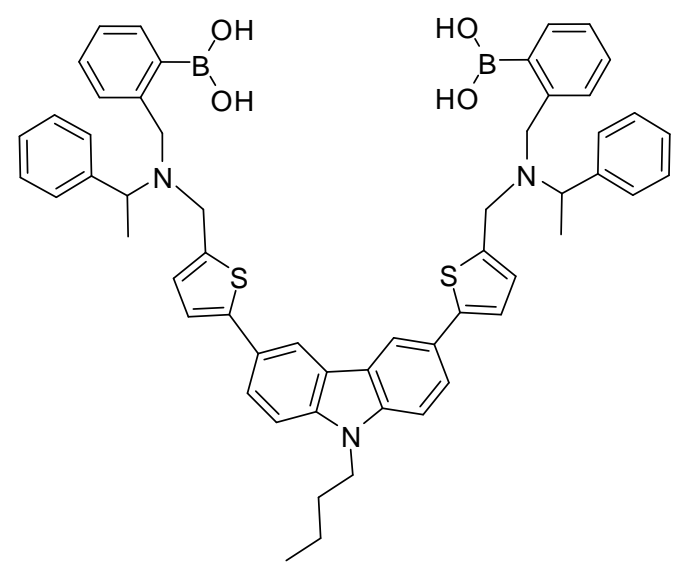

$(R, R)-1-63$ and $(S, S)-1-63$ 
Figure 1-20. Fluorescence intensity-pH profiles of $(S, S)-\mathbf{1 - 6 3}\left(5.0 \times 10^{-7} \mathrm{M}\right.$ in methanol/water 3:1) with (a) D- or L-tartaric acid (b) D- or L-mandelic acid (0.01 M).
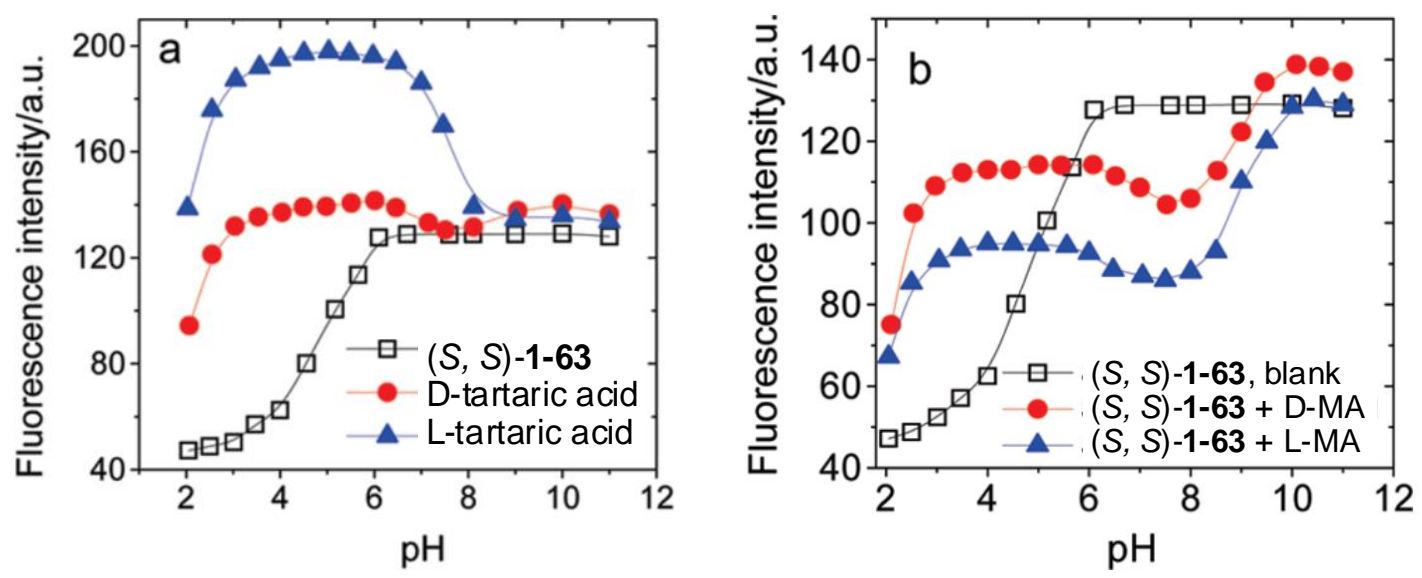

For all the boronic acid sensors mentioned above, the fluorophore, scaffold and chirogenic centers are integrated, limiting the design of boronic acid sensors. To solve this problem, modular chiral sensor 1-64 was deigned, in which the fluorophore (phenothiazine) and the chirogenic centers were modules and readily assembles onto a scaffold. $^{48}$ With this structural motif, any fluorophores that can be ethynylated are suitable for assembling a chiral sensor. The sensor 1-64 has emission maxima at 488 $\mathrm{nm}$ and the contrast ratio was about 6.0. It showed the fluorescence enhancement at acidic $\mathrm{pH}$ and diminishment at basic $\mathrm{pH}$ toward tartaric acids. 


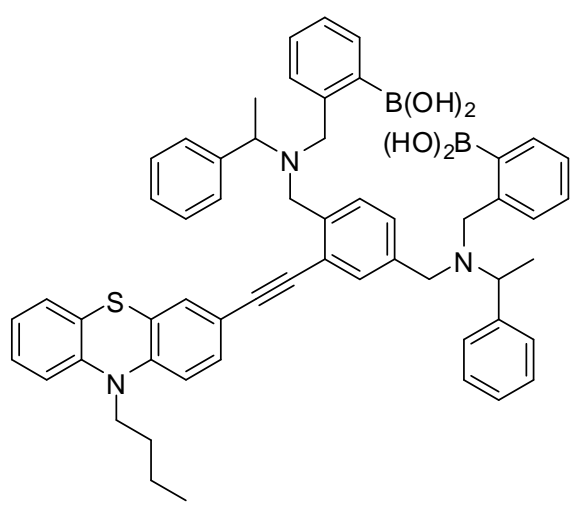

$(R, R)-1-64$ and $(S, S)-1-64$

\section{Conclusion}

Although a large number of enantioselective fluorescent sensors for the recognition of chiral compounds have been developed, it is still a challenge to use them in the screening of chiral catalysts for asymmetric synthesis. Most of the fluorescent sensors with high enantioselectivity can be used to determine ee of substrates only at a certain concentration, which means a separate method for determining the total concentration is needed. This drawback significantly limits the application of fluorescent sensors in HTS of chiral catalysts.

Our lab has developed a series of BINOL-based chiral fluorescent sensors with very high enantioselectivity. The subject of this thesis is to further develop those sensors and explore methods for simultaneous determination of concentration and ee of chiral substrates, which will be a significant step toward the application of fluorescent sensor in the high-throughput screening of asymmetric catalysts. 


\section{References}

1. $\mathrm{Pu}, \mathrm{L} .$, Chemical Reviews 2004, 104 (3), 1687-1716.

2. $\mathrm{Pu}, \mathrm{L} .$, Accounts of Chemical Research 2012, 45 (2), 150-163.

3. Leung, D.; Kang, S. O.; Anslyn, E. V., Chemical Society Reviews 2012, 41 (1), 448-479.

4. Nunez, M. C.; Garcia-Rubino, M. E.; Conejo-Garcia, A.; Cruz-Lopez, O.; Kimatrai, M.; Gallo, M. A.; Espinosa, A.; Campos, J. M., Current Medicinal Chemistry 2009, 16 (16), 2064-2074; Izake, E. L., Journal of Pharmaceutical Sciences 2007, 96 (7), 1659-1676.

5. Natarajan, R.; Basak, S. C., Current Topics in Medicinal Chemistry 2011, 11 (7), 771-787.

6. Simo, C.; Barbas, C.; Cifuentes, A., Electrophoresis 2003, 24 (15), 2431-2441; Herrero, M.; Simo, C.; Garcia-Canas, V.; Fanali, S.; Cifuentes, A., Electrophoresis 2010, 31 (13), 2106-2114.

7. Lin, G.-Q.; Li, Y.-M.; Chan, A. S. C., Principles and Applications of Asymmetric Synthesis. John Wiley \& Sons: 2001; Christmann, M.; Bräse, S., Asymmetric Synthesis: The Essentials. 2007.

8. Reetz, M. T., Angewandte Chemie-International Edition 2001, 40 (2), 284-310.

9. Maier, W. F.; Stowe, K.; Sieg, S., Angewandte Chemie-International Edition 2007, 46 (32), 6016-6067; Jandeleit, B.; Schaefer, D. J.; Powers, T. S.; Turner, H. W.; Weinberg, 
W. H., Angewandte Chemie-International Edition 1999, 38 (17), 2495-2532.

10. Finn, M. G., Chirality 2002, 14 (7), 534-540; Tsukamoto, M.; Kagan, H. B., Advanced Synthesis \& Catalysis 2002, 344 (5), 453-463; Traverse, J. F.; Snapper, M. L., Drug Discovery Today 2002, 7 (19), 1002-1012.

11. Leung, D.; Folmer-Andersen, J. F.; Lynch, V. M.; Anslyn, E. V., Journal of the American Chemical Society 2008, 130 (37), 12318-12327; Leung, D.; Anslyn, E. V., Journal of the American Chemical Society 2008, 130 (37), 12328-12333.

12. Tielmann, P.; Boese, M.; Luft, M.; Reetz, M. T., Chemistry-a European Journal 2003, 9 (16), 3882-3887; Reetz, M. T.; Jaeger, K. E., Chemistry-a European Journal 2000, 6 (3), 407-412; Reetz, M. T., Pure and Applied Chemistry 2000, 72 (9), 1615-1622; Reetz, M. T.; Zonta, A.; Schimossek, K.; Liebeton, K.; Jaeger, K. E., Angewandte Chemie-International Edition 1997, 36 (24), 2830-2832.

13. Millot, N.; Borman, P.; Anson, M. S.; Campbell, I. B.; Macdonald, S. J. F.; Mahmoudian, M., Organic Process Research \& Development 2002, 6 (4), 463-470.

14. Reetz, M. T.; Becker, M. H.; Kuhling, K. M.; Holzwarth, A., Angewandte Chemie-International Edition 1998, 37 (19), 2647-2650.

15. Reetz, M. T.; Becker, M. H.; Klein, H. W.; Stockigt, D., Angewandte Chemie-International Edition 1999, 38 (12), 1758-1761.

16. Guo, J. H.; Wu, J. Y.; Siuzdak, G.; Finn, M. G., Angewandte Chemie-International Edition 1999, 38 (12), 1755-1758. 
17. Reetz, M. T.; Kuhling, K. M.; Deege, A.; Hinrichs, H.; Belder, D., Angewandte Chemie-International Edition 2000, 39 (21), 3891-+.

18. Ding, K. L.; Ishii, A.; Mikami, K., Angewandte Chemie-International Edition 1999, $38(4), 497-501$.

19. Nieto, S.; Lynch, V. M.; Anslyn, E. V.; Kim, H.; Chin, J., Journal of the American Chemical Society 2008, 130 (29), 9232-+.

20. Copeland, G. T.; Miller, S. J., Journal of the American Chemical Society 1999, 121 (17), 4306-4307.

21. Korbel, G. A.; Lalic, G.; Shair, M. D., Journal of the American Chemical Society 2001, $123(2), 361-362$.

22. de Silva, A. P.; Gunaratne, H. Q. N.; Gunnlaugsson, T.; Huxley, A. J. M.; McCoy, C. P.; Rademacher, J. T.; Rice, T. E., Chemical Reviews 1997, 97 (5), 1515-1566.

23. Lin, J.; Hu, Q. S.; Xu, M. H.; Pu, L., Journal of the American Chemical Society 2002 , 124 (10), 2088-2089.

24. Xu, M. H.; Lin, J.; Hu, Q. S.; Pu, L., Journal of the American Chemical Society 2002, 124 (47), 14239-14246.

25. Lin, J.; Zhang, H. C.; Pu, L., Organic Letters 2002, 4 (19), 3297-3300.

26. Li, Z. B.; Lin, J.; Pu, L., Angewandte Chemie-International Edition 2005, 44 (11), 1690-1693.

27.Liu, H. L.; Hou, X. L.; Pu, L., Angewandte Chemie-International Edition 2009, 48 (2), 
$382-385$.

28. Liu, H. L.; Peng, Q.; Wu, Y. D.; Chen, D.; Hou, X. L.; Sabat, M.; Pu, L., Angewandte Chemie-International Edition 2010, 49 (3), 602-606.

29. Prodi, L.; Bolletta, F.; Montalti, M.; Zaccheroni, N.; Huszthy, P.; Samu, E.; Vermes, B., New Journal of Chemistry 2000, 24 (10), 781-785.

30. Upadhyay, S. P.; Pissurlenkar, R. R. S.; Coutinho, E. C.; Karnik, A. V., Journal of Organic Chemistry 2007, 72 (15), 5709-5714.

31. Mei, X. F.; Wolf, C., Journal of the American Chemical Society 2004, 126 (45), $14736-14737$.

32. Mei, X. F.; Wolf, C., Chemical Communications 2004, (18), 2078-2079.

33. Wolf, C.; Liu, S. L.; Reinhardt, B. C., Chemical Communications 2006, (40), $4242-4244$.

34. Pagliari, S.; Corradini, R.; Galaverna, G.; Sforza, S.; Dossena, A.; Marchelli, R., Tetrahedron Letters 2000, 41 (19), 3691-3695.

35.Pagliari, S.; Corradini, R.; Galaverna, G.; Sforza, S.; Dossena, A.; Montalti, M.; Prodi, L.; Zaccheroni, N.; Marchelli, R., Chemistry-a European Journal 2004, 10 (11), 2749-2758.

36. Corradini, R.; Paganuzzi, C.; Marchelli, R.; Pagliari, S.; Sforza, S.; Dossena, A.; Galaverna, G.; Duchateau, A., Chirality 2003, 15, S30-S39.

37. Corradini, R.; Paganuzzi, C.; Marchelli, R.; Pagliari, S.; Sforza, S.; Dossena, A.; 
Galaverna, G.; Duchateau, A., Journal of Materials Chemistry 2005, 15 (27-28), 2741-2746.

38. Chen, X.; Huang, Z.; Chen, S. Y.; Li, K.; Yu, X. Q.; Pu, L., Journal of the American Chemical Society 2010, 132 (21), 7297-+.

39. Yang, X.; Liu, X. C.; Shen, K.; Zhu, C. J.; Cheng, Y. X., Organic Letters 2011, 13 (13), 3510-3513.

40. James, T. D.; Shinkai, S., Host-Guest Chemistry: Mimetic Approaches to Study Carbohydrate Recognition 2002, 218, 159-200; James, T. D., Creative Chemical Sensor Systems 2007, 277, 107-152.

41. James, T. D.; Sandanayake, K.; Shinkai, S., Nature 1995, 374 (6520), 345-347.

42. Zhao, J. Z.; Fyles, T. M.; James, T. D., Angewandte Chemie-International Edition 2004, 43 (26), 3461-3464.

43. Zhao, J. Z.; Davidson, M. G.; Mahon, M. F.; Kociok-Kohn, G.; James, T. D., Journal of the American Chemical Society 2004, 126 (49), 16179-16186.

44. Zhao, J. Z.; James, T. D., Journal of Materials Chemistry 2005, 15 (27-28), 2896-2901.

45. Zhao, J. Z.; James, T. D., Chemical Communications 2005, (14), 1889-1891.

46. Han, F.; Chi, L. N.; Liang, X. F.; Ji, S. M.; Liu, S. S.; Zhou, F. K.; Wu, Y. B.; Han, K. L.; Zhao, J. Z.; James, T. D., Journal of Organic Chemistry 2009, 74 (3), 1333-1336.

47. Wu, Y. B.; Guo, H. M.; James, T. D.; Zhao, J. Z., Journal of Organic Chemistry 2011, 
76 (14), 5685-5695.

48. Wu, Y. B.; Guo, H. M.; Zhang, X.; James, T. D.; Zhao, J. Z., Chemistry-a European Journal 2011, 17 (27), 7632-7644. 


\section{Chapter 2}

\section{Pseudoenantiomeric Fluorescent Sensors in a Chiral Assay}




\section{Introduction}

In recent years, there have been growing interests in developing enantioselective fluorescent sensors because of their potential application as a rapid analytical tool in chiral assay. $^{1,2}$ A number of highly enantioselective fluorescent sensors have been reported for the recognition of chiral molecules such as carboxylic acids, amines, alcohols, amino alcohols and amino acid derivatives. ${ }^{1-3}$ These sensors can be used to determine the enantiomeric composition of a chiral substrate at a given concentration. Because the fluorescence of a chiral sensor is strongly influenced by both the concentration and the enantiomeric composition of the substrate, these two parameters need to be determined separately. ${ }^{1 \mathrm{~m}}$ It would be highly advantageous if both the concentration and the enantiomeric composition of the substrate could be determined simultaneously by one fluorescence measurement. This should greatly simplify the analysis of the reaction products generated from high throughput screening experiments.

We propose to develop a novel strategy to simultaneously measure both the concentration and the enantiomeric composition of a chiral substrate by using pseudo-enantiomeric fluorescent sensor pairs. An enantiomeric fluorescent sensor pair is a racemic mixture which cannot be used for the desired chiral recognition. Whereas, in a pseudo-enantiomeric fluorescent sensor pair, the two sensors, e.g., $(S)$-A and $(R)-\mathrm{B}$, will have opposite enantioselectivity and emit at two distinctively different wavelengths, e.g., $\lambda_{\mathrm{A}}$ and $\lambda_{\mathrm{B}}$. When a mixture of the pseudo-enantiomeric sensor pair $(S)$-A and $(R)-\mathrm{B}$ 
is treated with an enantiomeric mixture of a chiral substrate, we assume that one enantiomer of the substrate should enhance the fluorescence of $(S)$-A at $\lambda_{\mathrm{A}}$ giving a fluorescence intensity $I_{A}$, and the other enantiomer of the substrate should enhance the fluorescence of $(R)$ - $\mathrm{B}$ at $\lambda_{\mathrm{B}}$ giving a fluorescence intensity $\mathrm{I}_{\mathrm{B}}$. It is proposed that the fluorescence intensity difference $\mathrm{I}_{\mathrm{A}}-\mathrm{I}_{\mathrm{B}}$ could be used to determine the concentration difference of the two enantiomers of the substrate and the sum of the fluorescence intensity $\mathrm{I}_{\mathrm{A}}+\mathrm{I}_{\mathrm{B}}$ could be used to determine the total concentration of the two enantiomers. That is, both the enantiomeric composition of the substrate and its concentration could be determined by one fluorescence measurement with the use of the pseudo-enantiomeric sensor pair.

\section{Results and Discussion}

\subsection{Design and Synthesis of $(S)-2-1$ and $(R)-2-2$}

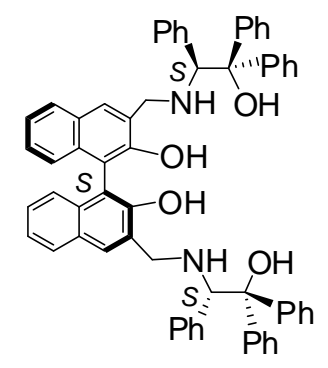

$(S)-2-1$

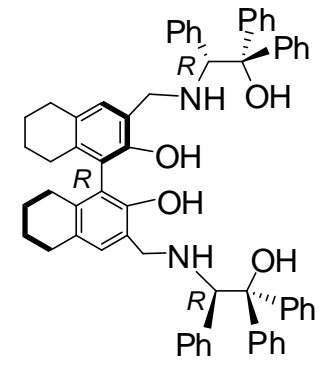

$(R)-2-2$

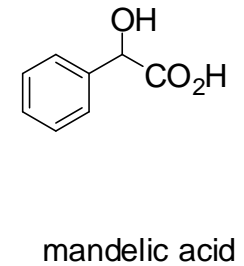

(MA)

We have conceived the use of the 1,1'-binaphthol (BINOL)-amino alcohol (S)-2-1 and its analog (R)-2-2 as a pseudo-enantiomeric sensor pair. These two compounds have the opposite chiral configuration at both the axially chiral biaryl centers and the 
amino alcohol units. They are expected to exhibit emission at different wavelengths because of the much reduced conjugation of $(R)$-2-2 versus $(S)-\mathbf{2 - 1}$.

Scheme 2-1. Synthesis of BINOL-amino alcohol (S)-2-1.

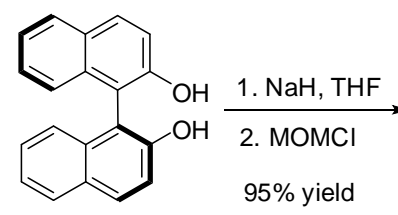

(S)-BINOL

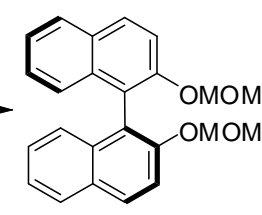

(S)-2-3

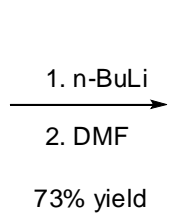

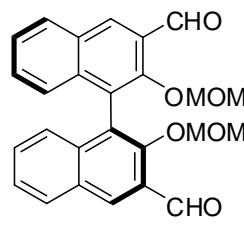

(S) -2-4

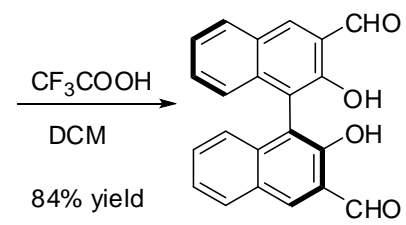

(S)-2-5

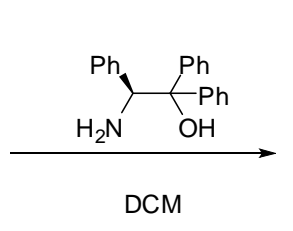

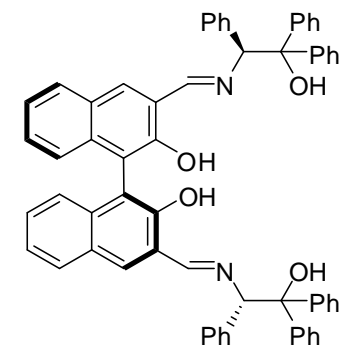

(S) $-2-6$

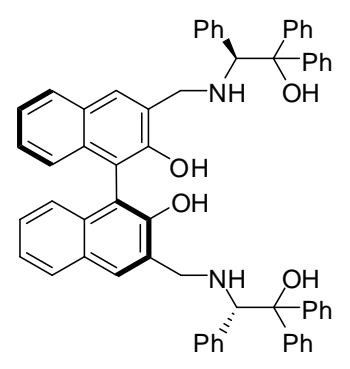

(S)-2-1

Scheme 2-2. Synthesis of $\mathrm{H}_{8} \mathrm{BINOL}$-amino alcohol $(R)-2-2$.

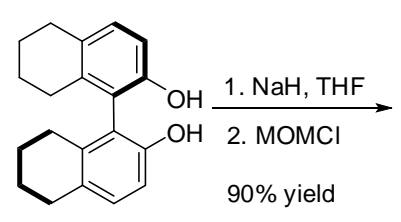

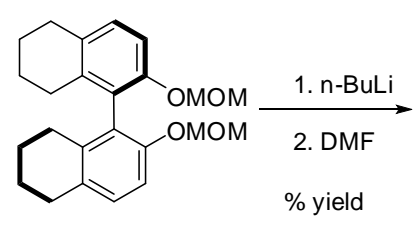

$(R)-2-7$

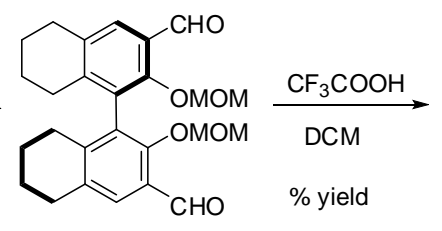

$(R)-2-8$

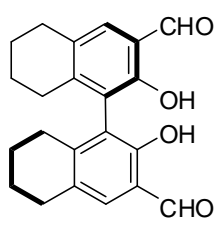

$(R)-2-9$

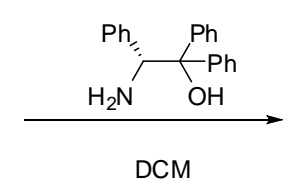

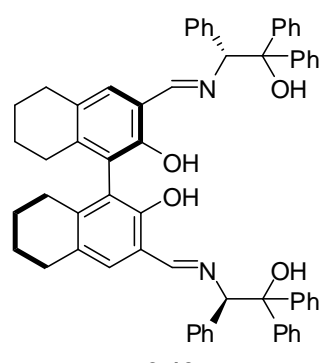

(R)-2-10

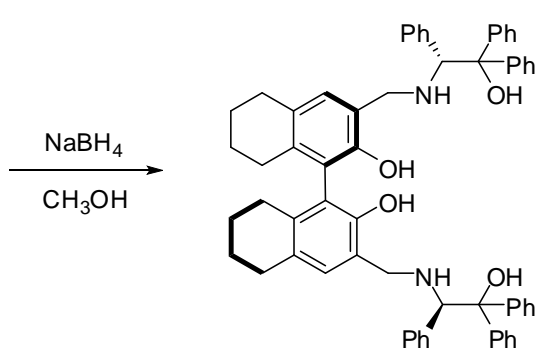

$(R)-2-2$

Compound $(S)$-2-1 was readily prepared according to Scheme 2-1. Reaction of (S)-1,1'-bi-2-naphthol [(S)-BINOL] with $\mathrm{NaH}$ followed by treatment with $\mathrm{MOMCl}$ gave 
the protected BINOL $(S)-\mathbf{2 - 3}$ in 95\% yield. Ortho-lithiationg followed by addition of DMF and hydrolysis gave BINOL dialdehyde $(S)-\mathbf{2 - 5}$. Condensation of $(S)-\mathbf{2 - 3}$ with the amino alcohol followed by reduction produced the BINOL-amino alcohol (S)-2-1. The $\mathrm{H}_{8} \mathrm{BINOL}$ amino alcohol $(R)-\mathbf{2 - 2}$ was obtained following similar procedures starting with partially hydrogenated BINOL, $(R)-\mathrm{H}_{8} \mathrm{BINOL}$ (Scheme 2-2).

\subsection{Enantioselective Fluorescent Recognition of Chiral Acids.}

$(S)$-2-1 was reported as a generally enantioselective fluorescent sensor for $\alpha$-hydroxycarboxylic acids in benzene solution. ${ }^{3}$ Because of the reduced conjugation of $(R)$-2-2 versus $(S)-\mathbf{2 - 1}$, benzene interferes with the fluorescence spectrum of $(R)-\mathbf{2 - 2}$ and is not a suitable solvent for this pseudo-enantiomeric pair. We have thus examined the fluorescent response of (S)-2-1 toward mandelic acid (MA) in $\mathrm{CH}_{2} \mathrm{Cl}_{2}$. Even though $\mathrm{CH}_{2} \mathrm{Cl}_{2}$ is a much more polar solvent, highly enantioselective fluorescent responses are still observed. As shown in Figure 2-1a, $(R)$-MA greatly enhances the fluorescence of $(S)$-2-1 at $\lambda_{1}=374 \mathrm{~nm}$ but $(S)$-MA only causes a very small fluorescence enhancement. It is found that $I_{R} / I_{0}=11.4$ and ef [enantioselective fluorescence enhancement ratio $=$ $\left.\left(\mathrm{I}_{R}-\mathrm{I}_{0}\right) /\left(\mathrm{I}_{\mathrm{S}}-\mathrm{I}_{0}\right)\right]=26.0 . \quad$ Figure 2-1b gives the fluorescence responses of $(S)-\mathbf{2 - 1}$ at various concentrations of $(R)$ - and $(S)$-MA.

Figure 2-1. (a) Fluorescence spectra of $(S)-2-1\left(1.0 \times 10^{-4} \mathrm{M}, \mathrm{CH}_{2} \mathrm{Cl}_{2}\right)$ with/without MA $\left(4.0 \times 10^{-3} \mathrm{M}\right)$. (b) Three independent measurements for the fluorescence enhancement of 
(S)-2-1 $\left(1.0 \times 10^{-4} \mathrm{M}, \mathrm{CH}_{2} \mathrm{Cl}_{2}\right)$ at $\lambda_{1}=374 \mathrm{~nm}$ with varying MA concentration. $\left(\lambda_{\text {exc }}=\right.$ $290 \mathrm{~nm}$, slit $=4.0 / 4.0 \mathrm{~nm})$.
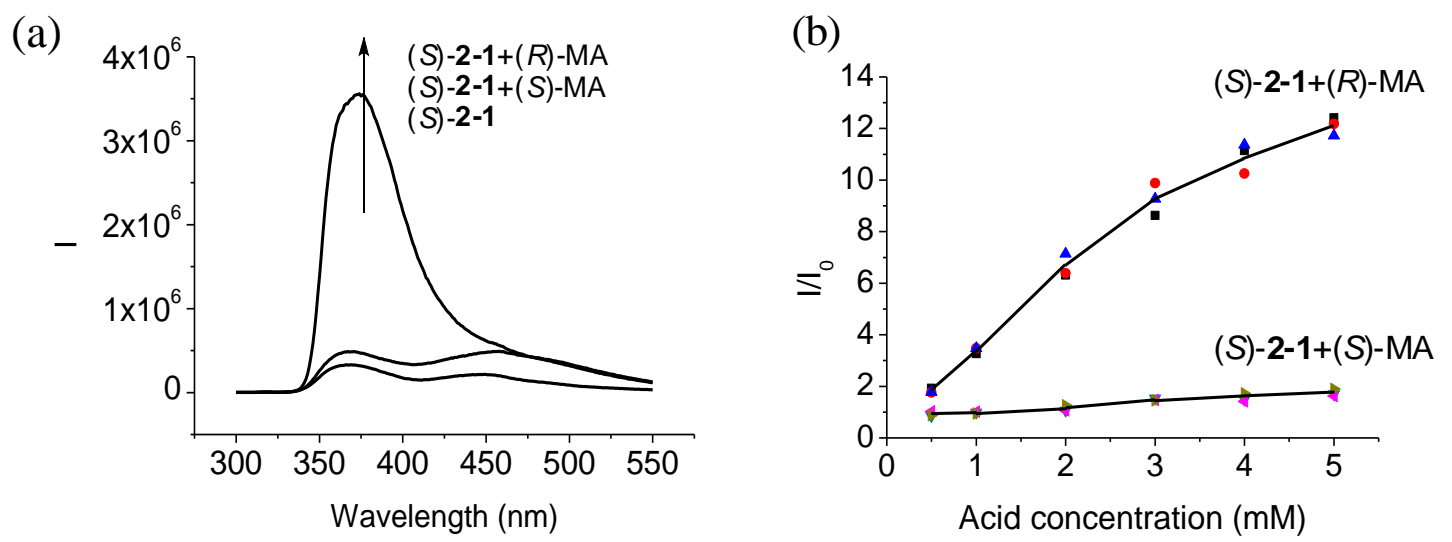

Figure 2-2. (a) Fluorescence spectra of $(R)-2-2\left(1.0 \times 10^{-4} \mathrm{M}, \mathrm{CH}_{2} \mathrm{Cl}_{2}\right)$ with/without $(R)$ and $(S)$-MA $\left(4.0 \times 10^{-3} \mathrm{M}\right)$. (b) Three independent measurements for the fluorescence enhancement of $(R)-2-2\left(1.0 \times 10^{-4} \mathrm{M}, \mathrm{CH}_{2} \mathrm{Cl}_{2}\right)$ at $\lambda_{2}=330 \mathrm{~nm}$ with varying $\mathrm{MA}$ concentration. $\left(\lambda_{\mathrm{exc}}=290 \mathrm{~nm}\right.$, slit $\left.=4.0 / 4.0 \mathrm{~nm}\right)$.
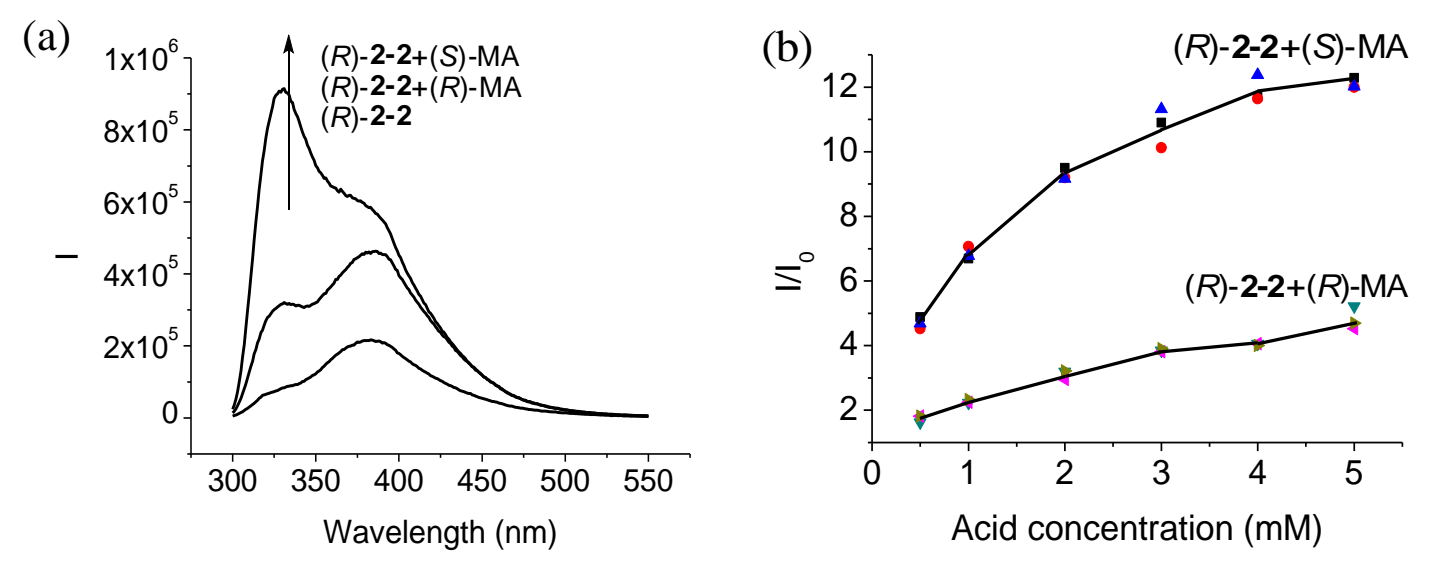

We studied the fluorescent response of $(R)-2-2$ toward $(R)$ - and $(S)-\mathrm{MA}$ in $\mathrm{CH}_{2} \mathrm{Cl}_{2}$. As show in Figure 2-2a, $(S)$-MA greatly enhances the fluorescence of $(R)-\mathbf{2 - 2}$ at $\lambda_{2}=330$ nm but $(R)$-MA causes a much smaller fluorescence enhancement. It is found that $\mathrm{I}_{\mathrm{S}} / \mathrm{I}_{0}$ 
$=11.7$ and ef $=3.6$. Figure $2-2 \mathrm{~b}$ gives the fluorescence responses of $(R)-\mathbf{2 - 2}$ at various concentrations of $(R)$ - and $(S)$-MA.

The distinctively different fluorescent response wavelengths between $(S)-\mathbf{2 - 1}$ and $(R)-2-2$ and their good and opposite enantioselectivity in the recognition of MA have encouraged us to study the use of this pseudo-enantiomeric sensor pair to interact with MA. A 1:1 mixture of $(S)-\mathbf{2 - 1}$ and $(R)-\mathbf{2 - 2}$ in $\mathrm{CH}_{2} \mathrm{Cl}_{2}$ is prepared in which each sensor's concentration is $1.0 \times 10^{-4} \mathrm{M}$. This sensor pair solution is treated with MA of varying enantiomeric composition as well as total concentration. The fluorescence intensity at $\lambda_{1}=374 \mathrm{~nm}$ is labeled as $\mathrm{I}_{10}$ without MA and $\mathrm{I}_{1}$ with $\mathrm{MA}$; and the fluorescence intensity at $\lambda_{2}=330 \mathrm{~nm}$ is labeled as $\mathrm{I}_{20}$ without MA and $\mathrm{I}_{2}$ with MA.

Figure 2-3a plots the fluorescence intensity difference at $\lambda_{1}$ and $\lambda_{2},\left(I_{1} / I_{10}-I_{2} / I_{20}\right)$, versus the enantiomeric purity of MA, $[(R)-\mathrm{MA}] \%$, with the total acid concentration varying from $2.0 \times 10^{-4} \mathrm{M}$ to $5.0 \times 10^{-3} \mathrm{M}$. It shows that when pure $(R)$-MA, [(R)-MA]\% $=1$, is used, $\mathrm{I}_{1} / \mathrm{I}_{10}>\mathrm{I}_{2} / \mathrm{I}_{20}$ and when pure $(S)$-MA, $[(R)-\mathrm{MA}] \%=0$, is used, $\mathrm{I}_{1} / \mathrm{I}_{10}<\mathrm{I}_{2} / \mathrm{I}_{20}$. As $[(R)$-MA $] \%$ increases, $\left(I_{1} / I_{10}-I_{2} / I_{20}\right)$ changes from the negative region to the positive region. At the higher acid concentrations, there are greater fluorescence intensity differences, and as the acid concentration decreases, the fluorescence intensity difference decreases. Figure $2-3 b$ plots the sum of the fluorescence intensity at $\lambda_{1}$ and $\lambda_{2}$, $\left(\mathrm{I}_{1} / \mathrm{I}_{10}+\mathrm{I}_{2} / \mathrm{I}_{20}\right)$, versus the total acid concentration at varying $[(R)-\mathrm{MA}] \%$. It shows that as the acid concentration increases, $\left(\mathrm{I}_{1} / \mathrm{I}_{10}+\mathrm{I}_{2} / \mathrm{I}_{20}\right)$ increases. 
Figure 2-3. (a) Plot of $\left(\mathrm{I}_{1} / \mathrm{I}_{10}-\mathrm{I}_{2} / \mathrm{I}_{20}\right)$ versus $[(R)-\mathrm{MA}] \%$ at varying MA concentrations $(\mathrm{mM})$. (b) Plot of $\left(\mathrm{I}_{1} / \mathrm{I}_{10}+\mathrm{I}_{2} / \mathrm{I}_{20}\right)$ versus MA concentration at varying $[(R)-\mathrm{MA}] \%$. $\left(\lambda_{\mathrm{exc}}=\right.$ $290 \mathrm{~nm}$, slit $=4.0 / 4.0 \mathrm{~nm})$.
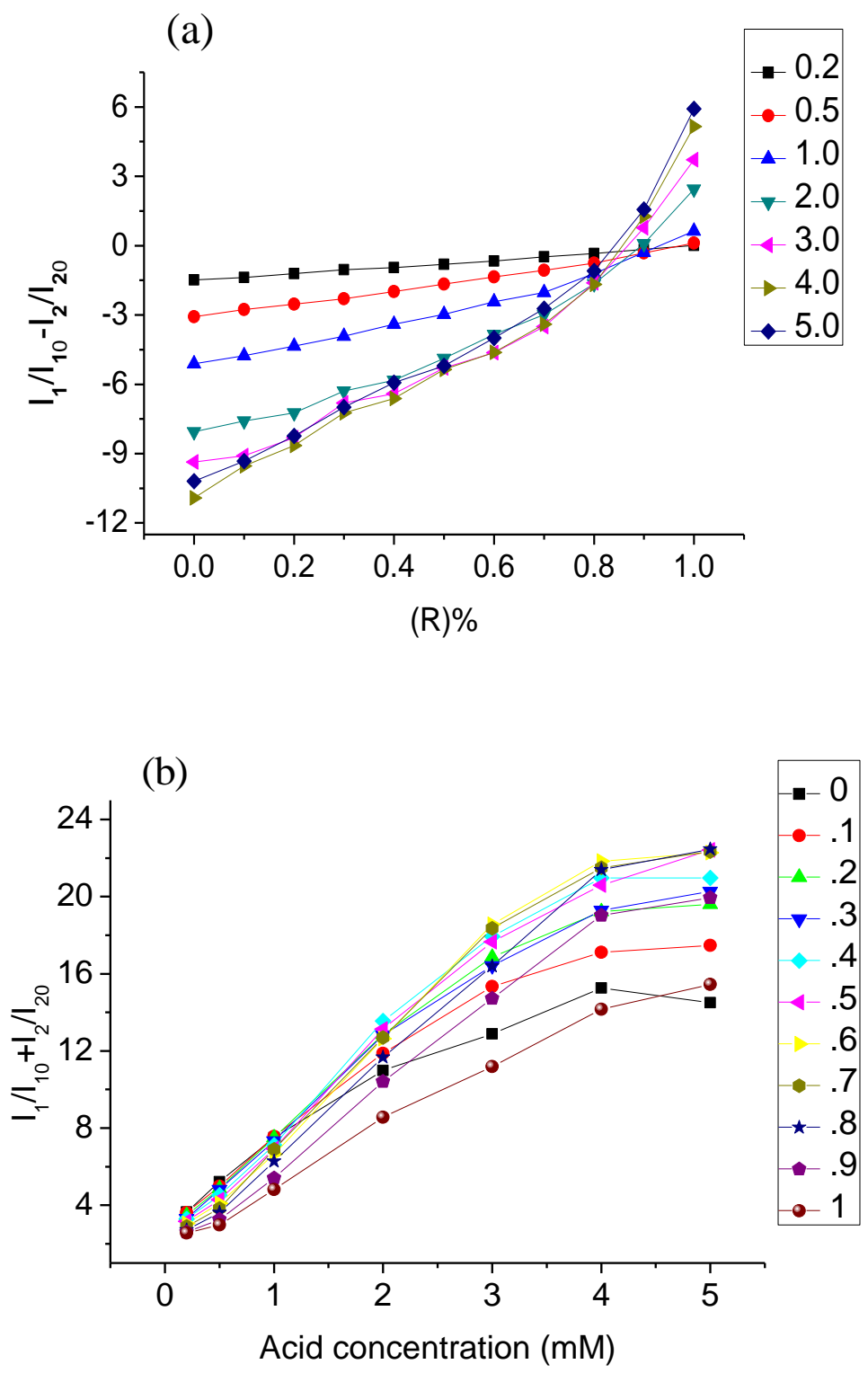

On the basis of Figure 2-3a,b, we have plotted $\left(\mathrm{I}_{1} / \mathrm{I}_{10}-\mathrm{I}_{2} / \mathrm{I}_{20}\right)$ and $\left(\mathrm{I}_{1} / \mathrm{I}_{10}+\mathrm{I}_{2} / \mathrm{I}_{20}\right)$ against the MA concentration and $[(R)-\mathrm{MA}] \%$ respectively in Figure 2-4. In Figure 2-4a, both 
$3 \mathrm{D}$ and $2 \mathrm{D}$ graphs are used to show the relation of $\left(\mathrm{I}_{1} / \mathrm{I}_{10}-\mathrm{I}_{2} / \mathrm{I}_{20}\right)$ and $\left(\mathrm{I}_{1} / \mathrm{I}_{10}+\mathrm{I}_{2} / \mathrm{I}_{20}\right)$ with the MA concentration and the data are color-coded according to the MA concentration. These graphs show that in the concentration range of $0.5 \mathrm{mM}$ to $4 \mathrm{mM}$, using $\left(\mathrm{I}_{\mathbf{1}} / \mathrm{I}_{10}-\mathrm{I}_{\mathbf{2}} / \mathrm{I}_{\mathbf{2} 0}\right)$ and $\left(I_{1} / I_{10}+I_{2} / I_{20}\right)$ can determine the total concentration of MA. The points start to overlap outside this concentration range. In Figure $2-4 b$, both $3 D$ and $2 \mathrm{D}$ graphs are used to show the relation of $\left(\mathrm{I}_{1} / \mathrm{I}_{10}-\mathrm{I}_{2} / \mathrm{I}_{20}\right)$ and $\left(\mathrm{I}_{1} / \mathrm{I}_{10}+\mathrm{I}_{2} / \mathrm{I}_{20}\right)$ with $[(R)-\mathrm{MA}] \%$, and the data are color-coded according to $[(R)-\mathrm{MA}] \%$. When $\left(\mathrm{I}_{1} / \mathrm{I}_{10}+\mathrm{I}_{2} / \mathrm{I}_{20}\right)>5$, that is, the concentration of MA > $0.5 \mathrm{mM}$ according to Figure 2-4a, the enantiomeric purity can be determined by using $\left(\mathrm{I}_{1} / \mathrm{I}_{10}-\mathrm{I}_{2} / \mathrm{I}_{20}\right)$ and $\left(\mathrm{I}_{1} / \mathrm{I}_{10}+\mathrm{I}_{2} / \mathrm{I}_{20}\right)$. Therefore, Figures $2-4 \mathrm{a}, \mathrm{b}$ allow the direct determination of both the concentration and the enantiomeric concentration of MA by one fluorescence intensity measurement of the sensor-substrate sample.

Figure 2-4. (a) $3 \mathrm{D}$ and $2 \mathrm{D}$ plots of $\left(\mathrm{I}_{1} / \mathrm{I}_{10}-\mathrm{I}_{2} / \mathrm{I}_{20}\right)$ and $\left(\mathrm{I}_{1} / \mathrm{I}_{10}+\mathrm{I}_{2} / \mathrm{I}_{20}\right)$ with the MA concentration $(\mathrm{mM})$. (b) $3 \mathrm{D}$ and $2 \mathrm{D}$ plots of $\left(\mathrm{I}_{1} / \mathrm{I}_{10}-\mathrm{I}_{2} / \mathrm{I}_{20}\right)$ and $\left(\mathrm{I}_{1} / \mathrm{I}_{10}+\mathrm{I}_{2} / \mathrm{I}_{20}\right)$ with $[(R)-\mathrm{MA}] \%$.

(a)

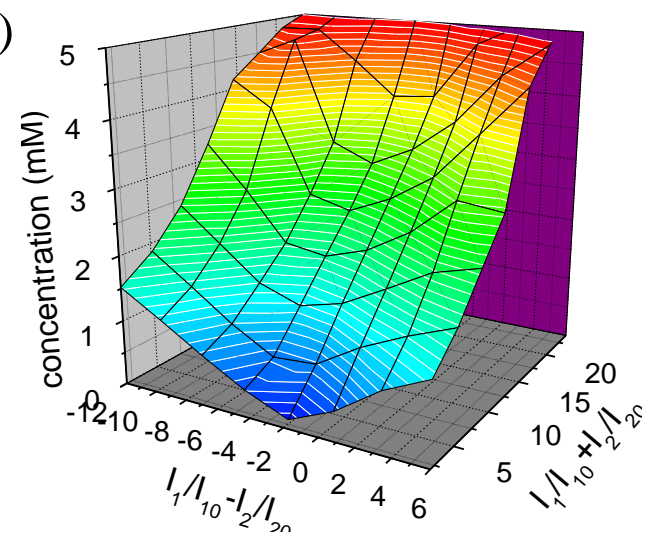

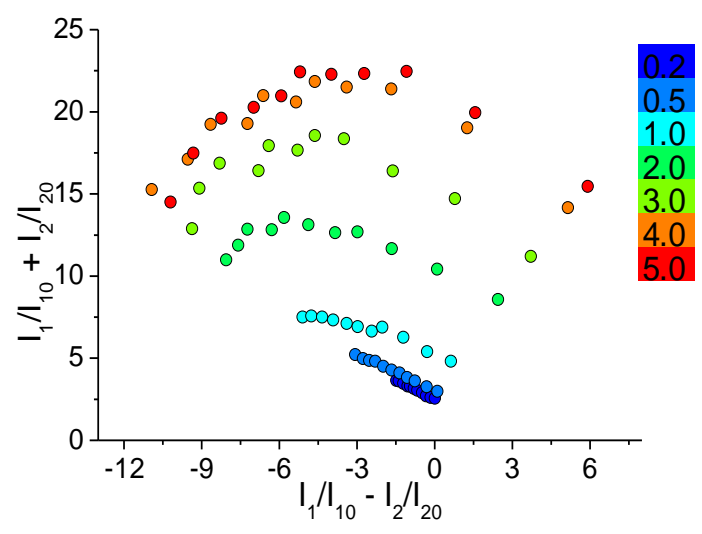


(b)
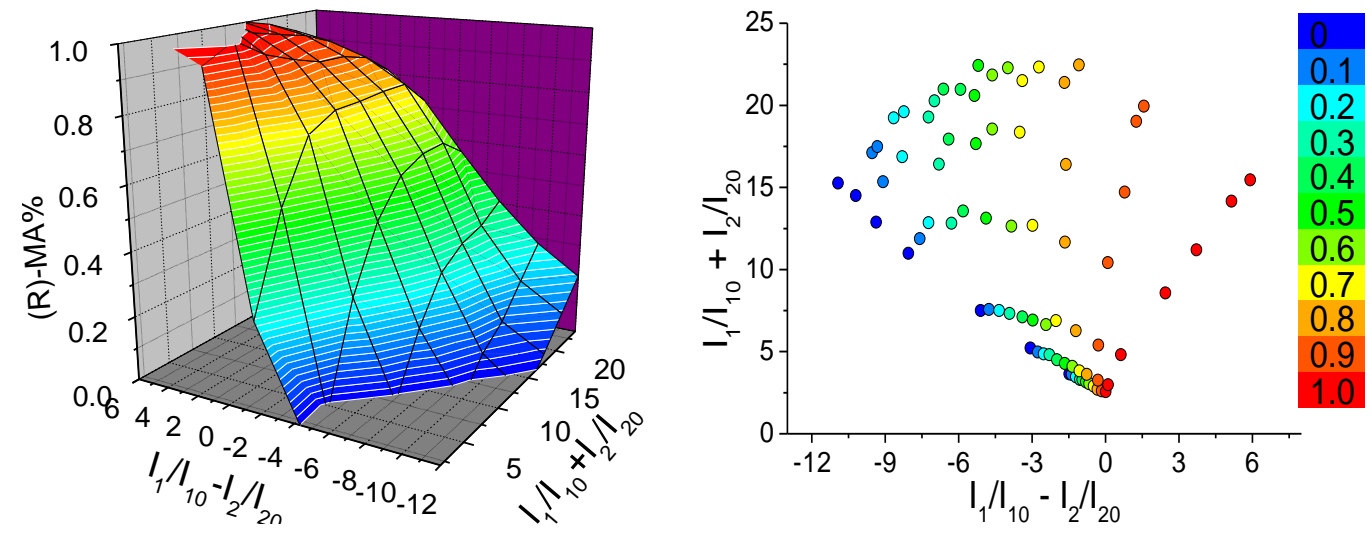

\section{Conclusion}

In conclusion, we have demonstrated that the pseudo-enantiomeric molecular pair $(S)-2-1$ and $(R)-2-2$ are highly enantioselective fluorescent sensors toward MA with distinctively different emission response wavelengths. Each molecule in this sensor pair recognizes the opposite enantiomer of MA. When a 1:1 mixture of $(S)-\mathbf{2 - 1}$ and $(R)-\mathbf{2 - 2}$ is used to interact with the chiral acid, in one fluorescence measurement, both the sum and the difference of the fluorescence intensity at the two emission wavelengths can be obtained to directly determine both the concentration and the enantiomeric composition of the chiral substrate. This new strategy is potentially useful for the analysis of the chiral substrate generated from the high throughput catalyst or reaction screening experiments which are expected to produce a great number of samples with varying concentrations and enantiomeric compositions.

\section{Experimental Section}

\subsection{General Data}


All reactions were carried out under nitrogen unless otherwise noted. All chemicals were purchased from Sigma Aldrich Chemical Co. or Alfa Aesar. THF was distilled over sodium and benzophenone under nitrogen atmosphere. Methylene chloride and diethyl ether were dried by passing through activated alumina columns under nitrogen. Solvents were stored over $4 \AA$ molecular sieves.

Melting points were uncorrected and obtained on a Mel-Temp II capillary melting point apparatus. Optical rotations were measured on a Jasco P-2000 digital polarimeter. NMR spectra were recorded on a Varian-300 MHz or Bruker-600 MHz spectrometer. High resolution mass spectra were obtained from the University of Illinois at Urbana-Champaign (UIUC) Mass Spectrometry Facility. UV-Vis spectra were recorded on a Hewlett-Packard 8452A diode-array. Steady-state fluorescence emission spectra were recorded on a Horiba Fluorolog-3 Model FL3-22 spectrofluorometer (double-grating excitation and double-grating emission monochromators).

\subsection{Preparation and Characterization of Compounds}

\section{Preparation and Characterization of (S)-2,2'-bis(methoxymethoxy)-1,1'-}

binaphthyl, (S)-2-3. Under nitrogen, (S)-1,1'-binaphthyl-2,2'-diol, (S)-BINOL (17.5 mmol, $5.0 \mathrm{~g})$ was dissolved in THF $(200 \mathrm{~mL})$. The solution was cooled to $0{ }^{\circ} \mathrm{C}$, and $\mathrm{NaH}(43.75 \mathrm{mmol}, 60 \%$ in mineral oil, $1.75 \mathrm{~g})$ was added in small portions. The reaction mixture was stirred for $15 \mathrm{~min}$ and then chloromethyl methyl ether $(43.75 \mathrm{mmol}$, 
$3.3 \mathrm{~mL}$ ) was added slowly. The reaction mixture was allowed to warm to room temperature and stirred for $1 \mathrm{~h}$. Water was added slowly to quench the reaction. The organic layer was separated, and the aqueous layer was extracted with ethyl acetate (three $30 \mathrm{~mL}$ aliquots). The combined organic extracts were washed with brine, and dried over $\mathrm{Na}_{2} \mathrm{SO}_{4}$. After evaporation of the solvent, the residue was purified by column chromatography on silica gel eluted with hexane/ethyl acetate (15/1) to afford compound (S)-2-3 as a white solid in 95\% yield (16.6 mmol, 6.22g). ${ }^{1} \mathrm{H}$ NMR $\left(300 \mathrm{MHz}, \mathrm{CDCl}_{3}\right)$ $\delta 3.15(\mathrm{~s}, 6 \mathrm{H}), 4.98(\mathrm{~d}, \mathrm{~J}=6.9 \mathrm{~Hz}, 2 \mathrm{H}), 5.09(\mathrm{~d}, \mathrm{~J}=6.6 \mathrm{~Hz}, 2 \mathrm{H}), 7.14-7.25(\mathrm{~m}, 4 \mathrm{H})$, 7.32-7.37 (m, 2H), $7.58(\mathrm{~d}, \mathrm{~J}=9.3 \mathrm{~Hz}, 2 \mathrm{H}), 7.88(\mathrm{~d}, \mathrm{~J}=8.1 \mathrm{~Hz}, 2 \mathrm{H}), 7.96(\mathrm{~d}, \mathrm{~J}=9.0 \mathrm{~Hz}$, $2 \mathrm{H})$.

Preparation and Characterization of (S)-2,2'-bis(methoxymethoxy)-1,1'binaphthyl-3,3'-dicarbaldehyde, (S)-2-4. Under nitrogen, 2,2'-bismethoxymethyl-1,1'-binaphthyl, $(S)$-2-3 (3.0 mmol, $1.12 \mathrm{~g})$ was dissolved in ether (33 $\mathrm{mL}$ ). The solution was cooled to $0{ }^{\circ} \mathrm{C}$, and $\mathrm{n}$-BuLi (10.5 mmol, $2.5 \mathrm{M}$ in hexane, 4.2 $\mathrm{mL}$ ) was added dropwise. The reaction mixture was stirred for $3 \mathrm{~h}$ at room temperature and cooled to $0{ }^{\circ} \mathrm{C}$, and then anhydrous DMF (12.0 mmol, $\left.1.3 \mathrm{~mL}\right)$ was added slowly. The reaction mixture was allowed to warm to room temperature and stirred for $2 \mathrm{~h}$ to afford a cream-like mixture. A saturated aqueous $\mathrm{NH}_{4} \mathrm{Cl}$ solution was added to quench the reaction. The organic layer was separated, and the aqueous layer was extracted with ethyl acetate (three $20 \mathrm{~mL}$ aliquots). The combined organic extracts were washed with 
brine, and dried over $\mathrm{Na}_{2} \mathrm{SO}_{4}$. After evaporation of the solvent, the residue was purified by column chromatography on silica gel eluted with hexane/ethyl acetate (4/1) to afford compound $(S)-\mathbf{2 - 4}$ as a yellow oil in $73 \%$ yield $(2.2 \mathrm{mmol}, 943 \mathrm{mg}) . \quad{ }^{1} \mathrm{H}$ NMR $(300$ $\left.\mathrm{MHz}, \mathrm{CDCl}_{3}\right) \delta 2.88(\mathrm{~s}, 6 \mathrm{H}), 4.76(\mathrm{~d}, \mathrm{~J}=1.2 \mathrm{~Hz}, 2 \mathrm{H}), 4.78(\mathrm{~d}, \mathrm{~J}=1.5 \mathrm{~Hz}, 2 \mathrm{H}), 7.27(\mathrm{~d}, \mathrm{~J}=$ $8.4 \mathrm{~Hz}, 2 \mathrm{H}), 7.42(\mathrm{t}, \mathrm{J}=6.9 \mathrm{~Hz}, 2 \mathrm{H}), 7.53(\mathrm{~d}, \mathrm{~J}=6.9 \mathrm{~Hz}, 2 \mathrm{H}), 8.10(\mathrm{~d}, \mathrm{~J}=8.1 \mathrm{~Hz}, 2 \mathrm{H})$, $8.67(\mathrm{~s}, 2 \mathrm{H}), \quad 10.59(\mathrm{~s}, 2 \mathrm{H})$.

\section{Preparation and Characterization of (S)-2,2'-dihydroxy-1,1'-binaphthyl-3,3'-}

dicarbaldehyde, (S)-2-5. After compound $(S)-2-4(1.1 \mathrm{mmol}, 471 \mathrm{mg})$ was dissolved in a minimum amount of $\mathrm{CH}_{2} \mathrm{Cl}_{2}$, trifluroacetic acid $(1.0 \mathrm{~mL})$ was added slowly, and the mixture was stirred at room temperature for $1 \mathrm{~h}$. A saturated aqueous $\mathrm{Na}_{2} \mathrm{CO}_{3}$ solution was added to quench the reaction. The organic layer was separated, and the aqueous layer was extracted with $\mathrm{CH}_{2} \mathrm{Cl}_{2}(3 \times 20 \mathrm{~mL})$. The combined organic extracts were washed with brine, and dried over $\mathrm{Na}_{2} \mathrm{SO}_{4}$. After evaporation of the solvent, the residue was purified by recrystallization with $\mathrm{CH}_{2} \mathrm{Cl}_{2}$ /ethanol to afford compound $(S)-\mathbf{2 - 5}$ as a yellow solid in $84 \%$ yield $(0.92 \mathrm{mmol}, 316 \mathrm{mg}) .{ }^{1} \mathrm{H}$ NMR $\left(300 \mathrm{MHz}, \mathrm{CDCl}_{3}\right) \delta$ 7.19-7.22 (m, 2H), 7.38-7.43 (m, 4H), 7.98-8.02 (m, 2H), 8.35 (s, 2H), $10.19(\mathrm{~s}, 2 \mathrm{H}), 10.59(\mathrm{~s}, 2 \mathrm{H})$.

Preparation and Characterization of (S)-3,3'-bis(((S)-2-hydroxy-1,2,2triphenylethylamino)methyl)-1,1'-binaphthyl-2,2'-diol, (S)-2-1. Under nitrogen, to a $50 \mathrm{~mL}$ flask were added (S)-2-5 (224 mg, $0.65 \mathrm{mmol}), \quad(\mathrm{S})-2$-amino-1,1,2triphenylethanol $(378 \mathrm{mg}, 1.30 \mathrm{mmol})$ and methylene chloride $(30 \mathrm{~mL})$. The mixture 
was heated at reflux for overnight, and monitored by $1 \mathrm{H}$ NMR spectroscopy. When the reaction was complete, the solution was cooled to room temperature and dried over $\mathrm{Na}_{2} \mathrm{SO}_{4}$. After filtration and evaporation, the resulting yellow imine product was dissolved in anhydrous methanol (25 mL), and NaBH4 (99 mg, $2.60 \mathrm{mmol}$ ) was added to the mixture at $0{ }^{\circ} \mathrm{C}$. The reaction temperature was maintained at $0{ }^{\circ} \mathrm{C}$ until the solution became colorless and transparent. It was then allowed to proceed at room temperature for additional $30 \mathrm{~min}$. Methanol was removed, and the residue was dissolved in ethyl acetate $(50 \mathrm{~mL})$ and washed with water $(15 \mathrm{~mL})$. The aqueous layer after separation was extracted with ethyl acetate $(3 \times 30 \mathrm{~mL})$. The combined ethyl acetate layer was washed with brine $(15 \mathrm{~mL})$ and dried over anhydrous $\mathrm{Na}_{2} \mathrm{SO}_{4}$. After evaporation of the solvent, the residue was purified by flash column chromatography on silica gel eluted with ethyl acetate/petroleum ether (1/3) to afford (S)-2-1 as a white solid in 78\% yield.

${ }^{1} \mathrm{H}$ NMR (300 MHz, $\mathrm{CDCl}_{3}$ ) $\delta 3.62($ br s, $2 \mathrm{H}), 3.78(\mathrm{~d}, \mathrm{~J}=13.8 \mathrm{~Hz}, 2 \mathrm{H}), 4.16(\mathrm{~d}, \mathrm{~J}=13.8$ $\mathrm{Hz}, 2 \mathrm{H}), 4,61(\mathrm{~s}, 2 \mathrm{H}), 6.90-7.38(\mathrm{~m}, 34 \mathrm{H}), 7.48$ (d, J = $7.2 \mathrm{~Hz}, 4 \mathrm{H}), 7.77$ (d, J = 6.6 Hz, $2 \mathrm{H})$.

Preparation and Characterization of (R)-2,2'-bis(methoxymethoxy)5,5',6,6',7,7',8,8'-octahydro-1,1'-binaphthyl, (R)-2-7. Under nitrogen, (R)5,5',6,6',7,7',8,8'-octahydro-1,1'-binaphthyl-2,2'-diol ( $\left.\mathrm{H}_{8} \mathrm{BINOL}\right),(25 \mathrm{mmol}, 7.4 \mathrm{~g}$ ) was dissolved in THF (300 mL). The solution was cooled to $0{ }^{\circ} \mathrm{C}$, and $\mathrm{NaH}(62.8 \mathrm{mmol}, 60 \%$ in mineral oil, $2.52 \mathrm{~g}$ ) was added in small portions. The reaction mixture was stirred for 
$30 \mathrm{~min}$ and then chloromethyl methyl ether $(62.8 \mathrm{mmol}, 4.8 \mathrm{~mL})$ was added slowly. The reaction mixture was allowed to warm to room temperature and stirred for overnight. Water was added slowly to quench the reaction. The organic layer was separated, and the aqueous layer was extracted with ethyl acetate (three $30 \mathrm{~mL}$ aliquots). The combined organic extracts were washed with brine, and dried over $\mathrm{Na}_{2} \mathrm{SO}_{4}$. After evaporation of the solvent, the residue was purified by column chromatography on silica gel eluted with hexane/ethyl acetate (15/1) to afford compound $(R)-2-7$ as a white solid in 90\% yield (22.5 mmol, 8.6 g). ${ }^{1} \mathrm{H}$ NMR (300 $\left.\mathrm{MHz}, \mathrm{CDCl}_{3}\right) \delta 1.65-1.76(\mathrm{~m}, 8 \mathrm{H})$, 2.07-2.17 (m, 2H), 2.27-2.37 (m, 2H), $2.78(\mathrm{t}, \mathrm{J}=6.0 \mathrm{~Hz}, 4 \mathrm{H}), 3.30(\mathrm{~s}, 6 \mathrm{H}), 4.97(\mathrm{~d}, \mathrm{~J}=$ $6.6 \mathrm{~Hz}, 2 \mathrm{H}), 5.03(\mathrm{~d}, \mathrm{~J}=6.6 \mathrm{~Hz}, 2 \mathrm{H}), 6.99(\mathrm{~d}, \mathrm{~J}=8.4 \mathrm{~Hz}, 2 \mathrm{H}), 7.05(\mathrm{~d}, \mathrm{~J}=8.4 \mathrm{~Hz}, 2 \mathrm{H})$.

Preparation and Characterization of $(\mathbf{R})-2-8$. Under nitrogen, $(R)-2-7 \quad(15.5$ mmol, $5.9 \mathrm{~g})$ was dissolved in ether $(180 \mathrm{~mL})$. The solution was cooled to $0{ }^{\circ} \mathrm{C}$, and n-BuLi (62.5 mmol, 2.5 M in hexane, $25.0 \mathrm{~mL}$ ) was added dropwise. The reaction mixture was stirred for $4 \mathrm{~h}$ at room temperature and cooled to $0{ }^{0} \mathrm{C}$, and then anhydrous DMF (70.0 mmol, $5.4 \mathrm{~mL}$ ) was added slowly. The reaction mixture was allowed to warm to room temperature and stirred for $2 \mathrm{~h}$ to afford a cream-like mixture. A saturated aqueous $\mathrm{NH}_{4} \mathrm{Cl}$ solution was added to quench the reaction. The organic layer was separated, and the aqueous layer was extracted with ethyl acetate (three $40 \mathrm{~mL}$ aliquots). The combined organic extracts were washed with brine, and dried over $\mathrm{Na}_{2} \mathrm{SO}_{4}$. After evaporation of the solvent, the residue was purified by column 
chromatography on silica gel eluted with hexane/ethyl acetate (4/1) to afford compound $(S)-\mathbf{2 - 8}$ as a light yellow oil in 58\% yield. ${ }^{1} \mathrm{H}$ NMR $\left(300 \mathrm{MHz}, \mathrm{CDCl}_{3}\right) \delta$ 1.68-1.76 (m, 8H), 2.16-2.26 (m, 2H), 2.42-2.52 (m, 2H), $2.83(\mathrm{t}, \mathrm{J}=6.0 \mathrm{~Hz}, 4 \mathrm{H}), 2.98(\mathrm{~s}, 6 \mathrm{H}), 4.78(\mathrm{~d}$, $\mathrm{J}=6.6 \mathrm{~Hz}, 2 \mathrm{H}), 4.83(\mathrm{~d}, \mathrm{~J}=6.6 \mathrm{~Hz}, 2 \mathrm{H}), 7.62(\mathrm{~s}, 2 \mathrm{H}), 10.22(\mathrm{~s}, 2 \mathrm{H})$.

Preparation and Characterization of (R)-2-9. After compound (S)-2-8 (6.02 mmol, $2.64 \mathrm{~g}$ ) was dissolved in a minimum amount of $\mathrm{CH}_{2} \mathrm{Cl}_{2}$, trifluroacetic acid (10 mmol, $1.4 \mathrm{~mL}$ ) was added slowly, and the mixture was stirred at room temperature for overnight. A saturated aqueous $\mathrm{Na}_{2} \mathrm{CO}_{3}$ solution was added to quench the reaction. The organic layer was separated, and the aqueous layer was extracted with $\mathrm{CH}_{2} \mathrm{Cl}_{2}(3 \mathrm{x}$ $20 \mathrm{~mL}$ ). The combined organic extracts were washed with brine, and dried over $\mathrm{Na}_{2} \mathrm{SO}_{4}$. After evaporation of the solvent, the residue was purified by recrystallization with $\mathrm{CH}_{2} \mathrm{Cl}_{2} /$ ethanol to afford compound $(S)-2-9$ as a light yellow solid in $86 \%$ yield. ${ }^{1} \mathrm{H}$ NMR $\left(300 \mathrm{MHz}, \mathrm{CDCl}_{3}\right) \delta$ 1.64-1.82 (m, 8H), 2.16-2.26 (m, 2H), 2.44-2.55 (m, 2H), $2.83(\mathrm{t}, \mathrm{J}=6.0 \mathrm{~Hz}, 4 \mathrm{H}), 7.32(\mathrm{~s}, 2 \mathrm{H}), 9.84(\mathrm{~s}, 2 \mathrm{H}), 10.93(\mathrm{~s}, 2 \mathrm{H})$.

Preparation and Characterization of (R)-3,3'-bis(((R)-2-hydroxy-1,2,2triphenylethylamino)methyl)-5,5',6,6',7,7',8,8'-octahydro-1,1'-binaphthyl-2,2'-diol,

(R)-2-2. (R)-3,3'-DiformylH ${ }_{8}$ BINOL, (R)-2-9 (245 mg, 0.70 mmol) was dissolved in $\mathrm{CH}_{2} \mathrm{Cl}_{2}(22 \mathrm{~mL})$ in the presence of $4 \AA$ molecular sieves and combined with (R)-2-amino-1,1,2-triphenylenthanol $(607 \mathrm{mg}, 2.10 \mathrm{mmol})$. The reaction mixture was heated at reflux for $3 \mathrm{~d}$, and monitored by using ${ }^{1} \mathrm{H}$ NMR spectroscopy. When the 
reaction was complete, the solution was cooled to room temperature and dried over anhydrous $\mathrm{Na}_{2} \mathrm{SO}_{4}$. After filtration, the filtrate was concentrated under vacuum. The residue was passed through a silica gel column eluted with hexanes/ethyl acetate (8/1) to give the corresponding Schiff base. The Schiff base was dissolved in methanol $(28 \mathrm{~mL})$ and cooled down to $0{ }^{\circ} \mathrm{C}$. $\mathrm{NaBH}_{4}(106 \mathrm{mg}, 2.80 \mathrm{mmol})$ was added in small portions. The reaction temperature was maintained at $0{ }^{\circ} \mathrm{C}$ until the solution became colorless and transparent. Then, it was allowed to proceed at room temperature for additional $30 \mathrm{~min}$. Methanol was removed by roto-evaporation, and the residue was dissolved in ethyl acetate $(50 \mathrm{~mL})$ and washed with water $(15 \mathrm{~mL})$. The aqueous layer after separation was extracted with ethyl acetate $(3 \times 30 \mathrm{~mL})$. The combined organic layer was washed with brine $(15 \mathrm{~mL})$ and dried over anhydrous $\mathrm{Na}_{2} \mathrm{SO}_{4}$. After evaporation of the solvent, the residue was purified by flash column chromatography on silica gel eluted with hexanes/ethyl acetate (3/1) to afford (R)-2-2 as a white solid in $73 \%$ yield. ${ }^{1} \mathrm{H}$ NMR $\left(300 \mathrm{MHz}, \mathrm{CDCl}_{3}\right) \delta 1.72(\mathrm{~m}, 8 \mathrm{H}), 2.17(\mathrm{~m}, 2 \mathrm{H}), 2.34(\mathrm{~m}, 2 \mathrm{H}), 2.66(\mathrm{~m}, 4 \mathrm{H}), 3.59(\mathrm{~d}, \mathrm{~J}=$ $13.5 \mathrm{~Hz}, 2 \mathrm{H}), 3.77(\mathrm{~d}, \mathrm{~J}=13.5 \mathrm{~Hz}, 2 \mathrm{H}), 4.63(\mathrm{~s}, 2 \mathrm{H}), 6.58(\mathrm{~s}, 2 \mathrm{H}), 7.02-7.31(\mathrm{~m}, 28 \mathrm{H})$, $7.59(\mathrm{~d}, \mathrm{~J}=7.2 \mathrm{~Hz}, 2 \mathrm{H}) . \quad{ }^{13} \mathrm{C} \mathrm{NMR}\left(75 \mathrm{MHz}, \mathrm{CDCl}_{3}\right) \delta 23.5,23.6,27.5,29.5,49.4$, 69.0, $80.5,121.8,122.0,126.4,126.7,126.9,127.6,127.7,127.8,127.9,128.7,128.8,130.1$, 130.4, 136.4, 137.4, 144.5, 145.4, 151.4. HRMS Calcd for $\mathrm{C}_{62} \mathrm{H}_{61} \mathrm{~N}_{2} \mathrm{O}_{4}\left(\mathrm{MH}^{+}\right)$: 897.4631. Found: 897.4623. m.p. $124-125{ }^{0} \mathrm{C} . \quad[\alpha]_{\mathrm{D}}=119.3\left(\mathrm{c}=0.470, \mathrm{CHCl}_{3}\right)$. 


\subsection{Preparation of Samples for Fluorescence Measurement.}

Sensors were purified by column chromatography and then stored in a refrigerator. The enantiomers of mandelic acid were purchased from Aldrich and recrystallized from methanol. They were then passed through a short column of silica gel (eluted with diethyl ether) and dried under vacuum. All of the solvents were either HPLC or spectroscopic grade. The stock solutions of the sensors were freshly prepared for each measurement. A $0.01 \mathrm{M}$ stock solution of mandelic acid in methylene chloride was freshly prepared. For the fluorescence enhancement study, a sensor solution was mixed with the mandelic acid solution at room temperature in a $5 \mathrm{~mL}$ volumetric flask and diluted to the desired concentration. The resulting solution was allowed to stand at room temperature for 2 - $3 \mathrm{~h}$ before the fluorescence measurement. 


\section{References}

1. Selected references of enantioselective fluorescent sensors: (a) James, T. D.; Sadanayake, K. R. A. S.; Shinkai, S. Nature 1995, 374, 345-347. (b) Pugh, V.; Hu, Q. -S.; Pu, L. Angew. Chem. Int. Ed. 2000, 39, 3638-3641. (c) Reetz, M. T.; Sostmann, S. Tetrahedron 2001, 57, 2515-2520. (d) Korbel, G. A.; Lalic, G.; Shair, M. D. J. Am. Chem. Soc. 2001, 123, 361-362. (e) Jarvo, E. R.; Evans, C. A.; Copeland, G. T.; Miller, S. J. J. Org. Chem. 2001, 66, 5522-5527. (f) Wong, W.-L.; Huang, K.-H.; Teng, P.-F.; Lee, C.-S.; Kwong, H.-L. Chem. Commun. 2004, 384-385. (g) Zhao, J.-Z.; Fyles, T. M.; James, T. D. Angew. Chem., Int. Ed. 2004, 43, 3461-3464. (h) Pagliari, S.; Corradini, R.; Galaverna, G.; Sforza, S.; Dossena, A.; Montalti, M.; Prodi, L.; Zaccheroni, N.; Marchelli, R. Chem. Eur. J. 2004, 10, 2749-2758. (i) Matsushita, H.; Yamamoto, N.; Meijler, M. M.; Wirsching, P.; Lerner, R.A.; Matsushita, M.; Janda, K. D. Mol. Biosyst. 2005, 1. 303-306. (j) Zhu, L.; Anslyn, E. V. J. Am. Chem. Soc. 2004, 126, 3676-3677. (k) Mei, X. F. ; Wolf, C. J. Am. Chem. Soc. 2004, 126, 14736-14737. (1) Li, Z.-B.; Lin, J.; Pu, L. Angew. Chem., Int. Ed. 2005, 44, 1690-1693. (m) Wolf, C.; Liu, S.; Reinhardt, B. C. Chem. Commun. 2006, $4242-4244$.

2. A review on enantioselective fluorescent sensing: Pu, L. Chem. Rev. 2004, 104, 1687-1716.

3. Liu, H. L.; Peng, Q.; Wu,Y. D.; Chen, D.; Hou, X. L.; Sabat, M.; Pu, L. Angew. Chem., 
Int. Ed. 2010, 49, 602-606. 
Chapter 3

Study of the Fluorescent Properties of $\mathrm{H}_{8}$ BINOL-Amine Molecules: A New Window for the

Enantioselective Fluorescent

Recogniton 


\section{Introduction}

Fluorescent chemical sensors have been applied to the detection of metal cations, $\mathrm{pH}$, anions, proteins and DNAs. ${ }^{1-3}$ Enantioselective fluorescent sensors are also receiving increasing attentions because they can provide real time analysis for the enantiomeric composition of chiral compounds and enhance the sensitivity in the detection of chiral substrates. ${ }^{4,5}$ Among the enantioselective fluorescent sensors developed, those based on the chiral structure of 1,1'-bi-2-naphthol (BINOL) have been actively investigated and a few highly enantioselective fluorescent sensors have been discovered for the recognition of $\alpha$-hydroxycarboxylic acids, amines, amino alcohols and amino acids. ${ }^{6} \mathrm{H}_{8} \mathrm{BINOL}$ is a partially hydrogenated derivative of BINOL. The increased steric bulkiness of $\mathrm{H}_{8} \mathrm{BINOL}$ because of the $\mathrm{sp}^{3}$ carbons and the increased electron density on the aromatic rings make this compound both sterically and electronically quite different from BINOL. The use of $\mathrm{H}_{8} \mathrm{BINOL}$ and its derivatives in asymmetric catalysis has been actively pursued and a number of efficient catalysts have been developed. ${ }^{7}$ However, no study on using $\mathrm{H}_{8} \mathrm{BINOL}$ to build enantioselective fluorescent sensor was reported before. In our laboratory, we have synthesized a series of $\mathrm{H}_{8} \mathrm{BINOL}$-amine compounds and have explored their application in the fluorescent recognition of mandelic acid (MA), a representative of $\alpha$-hydroxycarboxylic acids. This investigation demonstrates that although the $\mathrm{H}_{8} \mathrm{BINOL}-$ based compounds do not have the extended conjugation as those

derived from BINOL, they still exhibit very interesting fluorescent properties and have 
opened a new window in the fluorescence spectrum for the enantioselective fluorescent recognition. Herein, these results are reported. ${ }^{8}$
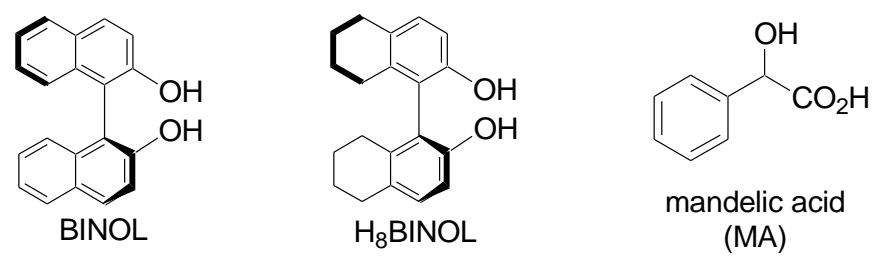

\section{Results and Discussion}

\subsection{Synthesis of the $\mathrm{H}_{8}$ BINOL-Amine Molecules and Study of Their Fluorescent}

\section{Properties}

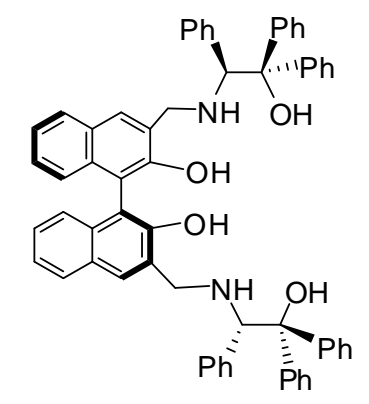

(S)-3-1

Recently, we reported that the BINOL-amino alcohol molecule (S)-3-1 is a highly enantioselective fluorescent sensor for the structurally diverse $\alpha$-hydroxycarboxylic acids. ${ }^{9}$ In order to explore the application of $\mathrm{H}_{8} \mathrm{BINOL}$ in fluorescent recognition, we have prepared a $\mathrm{H}_{8} \mathrm{BINOL}$ analog of $(S)$-3-1. As shown in Scheme 3-1, protection of (S)- $\mathrm{H}_{8} \mathrm{BINOL}$ with methoxymethyl (MOM) group gave (S)-3-2. Ortho-lithiation followed by addition of DMF and hydrolysis gave the $\mathrm{H}_{8} \mathrm{BINOL}$ dialdehyde $(S)-\mathbf{3 - 3}{ }^{10}$ Condensation of $(S)$-3-3 with the amino alcohol $(S)$-3-4 followed by reduction produced 
the $\mathrm{H}_{8}$ BINOL-amino alcohol $(S)-3-5$ in $71 \%$ yield. ${ }^{11} \quad$ The specific optical rotation of this compound is $[\alpha]_{\mathrm{D}}=-119.9\left(\mathrm{c}=0.865, \mathrm{CHCl}_{3}\right)$, greater than that of $(S)-\mathbf{3 - 1}\left\{[\alpha]_{\mathrm{D}}=-93.7\right.$ $\left.\left(\mathrm{c}=0.80, \mathrm{CH}_{2} \mathrm{Cl}_{2}\right)\right\}$. The ${ }^{1} \mathrm{H}$ NMR spectrum of $(S)-\mathbf{3 - 5}$ in $\mathrm{CDCl}_{3}$ shows two doublets at $\delta 3.59(\mathrm{~d}, \mathrm{~J}=13.5 \mathrm{~Hz}, 2 \mathrm{H})$ and $3.77(\mathrm{~d}, \mathrm{~J}=13.5 \mathrm{~Hz}, 2 \mathrm{H})$ for the two diastereotopic protons $\left(\mathrm{H}_{\mathrm{a}}\right)$ of the 3,3'-methylene substituents which are significantly more upfield than those observed for $(S)-\mathbf{3 - 1}$ at $\delta 3.78(\mathrm{~d}, J=13.8 \mathrm{~Hz}, 2 \mathrm{H})$ and $4.16(\mathrm{~d}, J=13.8 \mathrm{~Hz}, 2 \mathrm{H})$. This is consistent with the more electron rich aromatic rings of $(S)-3-5$.

Scheme 3-1. Synthesis of the $\mathrm{H}_{8} \mathrm{BINOL-Amino} \mathrm{Alcohol} \mathrm{(S)-3-5.}$

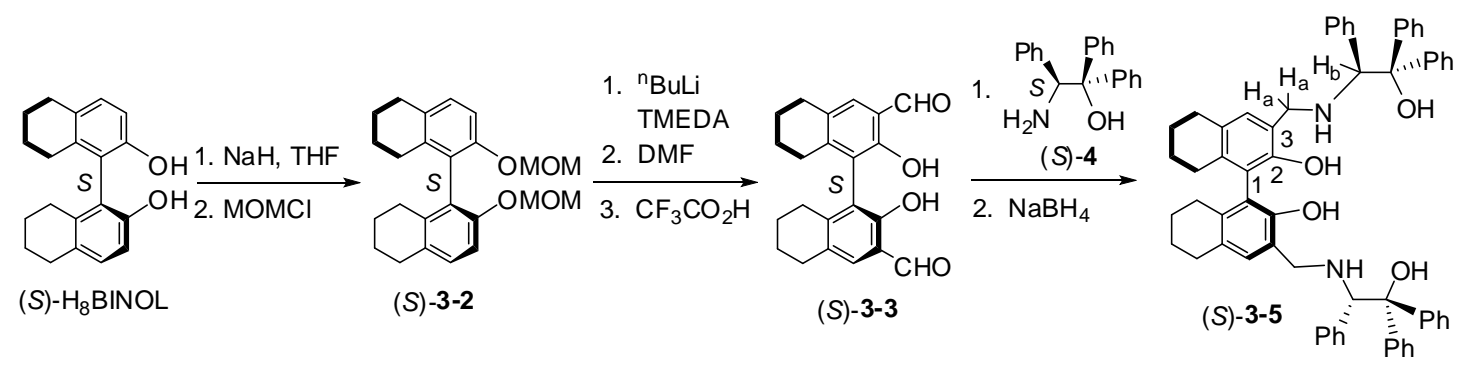

As shown in Figure 3-1, the UV spectrum of $(S)-3-5$ in $\mathrm{CH}_{2} \mathrm{Cl}_{2}$ gives absorptions at $\lambda_{\max }(\varepsilon)=232\left(2.6 \times 10^{4}\right)$ and $290\left(7.4 \times 10^{3}\right) \mathrm{nm}$, without the long wavelength absorption of $(S)-3-1$ at $\lambda_{\max }=334 \mathrm{~nm}$ due to the reduced conjugation system in $(S)-3-5 . \quad$ The peak position and shape of the absorption signals of (S)-3-5 do not change while the concentration changes. The fluorescence spectra of $(S)-3-5$ in $\mathrm{CH}_{2} \mathrm{Cl}_{2}$ at various concentrations are shown in Figure 3-2. Compound (S)-3-5 gives a major emission at $\lambda_{\text {emi }}$ $=390 \mathrm{~nm}$ whose shape and position also do not change with concentration. The major 
emission wavelength of $(S)-\mathbf{3 - 5}$ is much longer than that observed for $(S)-\mathrm{H}_{8} \mathrm{BINOL}$ at $\lambda_{\mathrm{emi}}=323 \mathrm{~nm}$ as shown in Figure 3-3. Compound $(S)$-3-5 only gives a very weak short wavelength emission signal at $\lambda_{\mathrm{emi}}=318 \mathrm{~nm}$. The concentration independence of both the absorption and emission of $(S)$-3-5 indicates that the long wavelength fluorescence signal of $(S)-3-5$ is not due to the formation of either excimer or ground state aggregate. The fluorescence spectrum of $(S)$-3-5 is also very different from that of $(S)$-3-1 which exhibits dual emission signals at $\lambda_{\text {emi }}=372$ and $448 \mathrm{~nm}$ in $\mathrm{CH}_{2} \mathrm{Cl}_{2}{ }^{9} \quad$ The fluorescence quantum yield of $(S)-\mathbf{3 - 5}$ is estimated to be $0.36 \%$ by using 2 -aminopyridine as the standard. $^{12}$

Figure 3-1. UV spectra of sensor $(S)$-3-5 in methylene chloride at various concentrations.

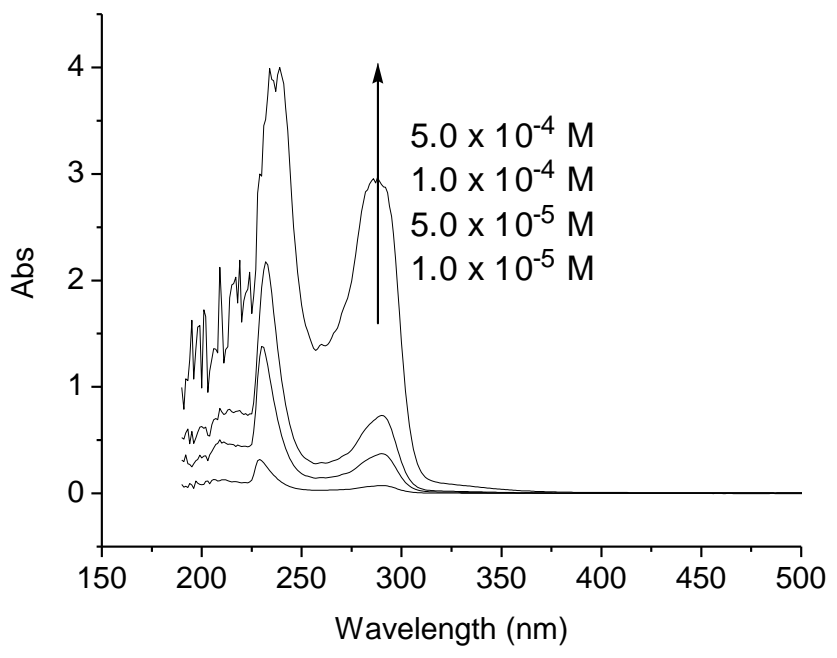

Figure 3-2. Fluorescence spectra of $(S)-3-5$ in $\mathrm{CH}_{2} \mathrm{Cl}_{2}$ at various concentrations $\left(\lambda_{\text {exc }}=\right.$ $290 \mathrm{~nm}$, slit $=3.0 / 3.0 \mathrm{~nm})$. 


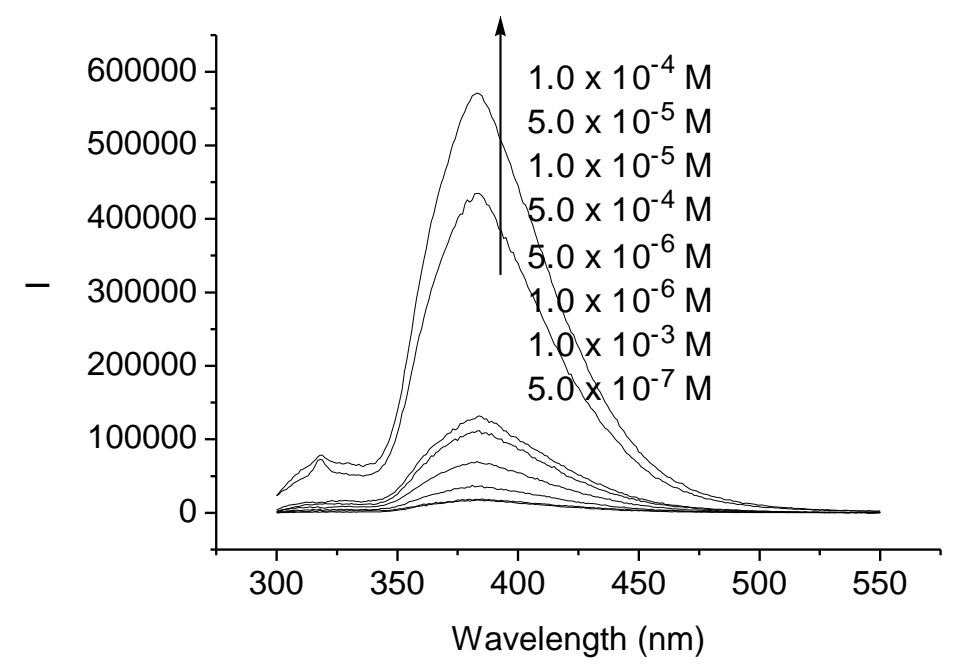

Figure 3-3. Fluorescence spectrum of $(S)-\mathrm{H}_{8} \mathrm{BINOL}\left(2.0 \times 10^{-4} \mathrm{M}\right.$ in $\left.\mathrm{CH}_{2} \mathrm{Cl}_{2}\right)\left(\lambda_{\mathrm{exc}}=288\right.$ nm. slit $=4.0 / 4.0 \mathrm{~nm})$.

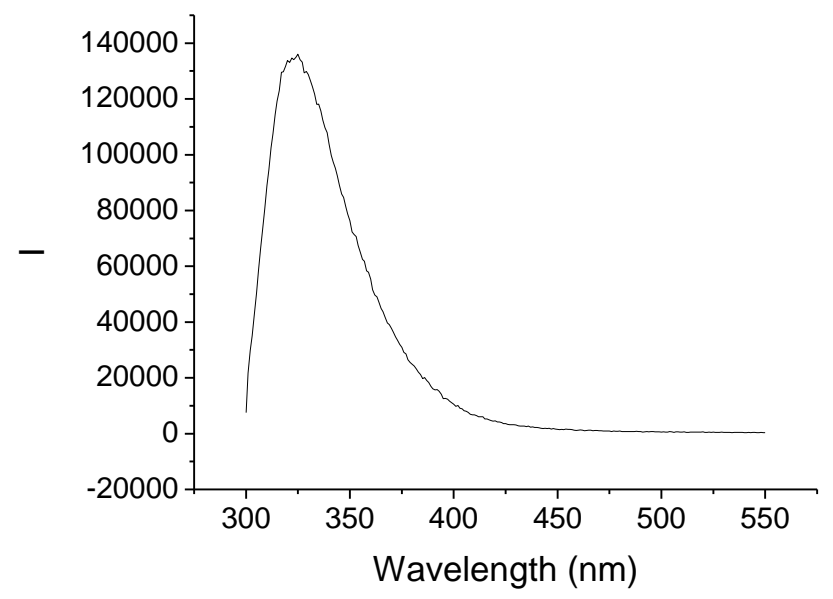

We have studied the solvent effect on the fluorescence property of $(S)-3-5$. Changing the solvent from $\mathrm{CH}_{2} \mathrm{Cl}_{2}$ to $\mathrm{CH}_{3} \mathrm{OH}$ caused little change on the absorption as well as the fluorescence spectra of (S)-3-5 at various concentrations (Figure 3-4). This indicates that the intermolecular hydrogen bonding interaction of (S)-3-5 has little 
influence on its electronic properties. Similarly, as shown in Figure 3-5, the use of acetonitrile as the solvent also maintains the absorption and fluorescence properties of (S)-3-5.

Figure 3-4. (a) UV and (b) fluorescence spectra of sensor (S)-3-5 in methanol at various concentrations $\left(\lambda_{\mathrm{exc}}=291 \mathrm{~nm}, \mathrm{slit}=4.0 / 4.0 \mathrm{~nm}\right)$.
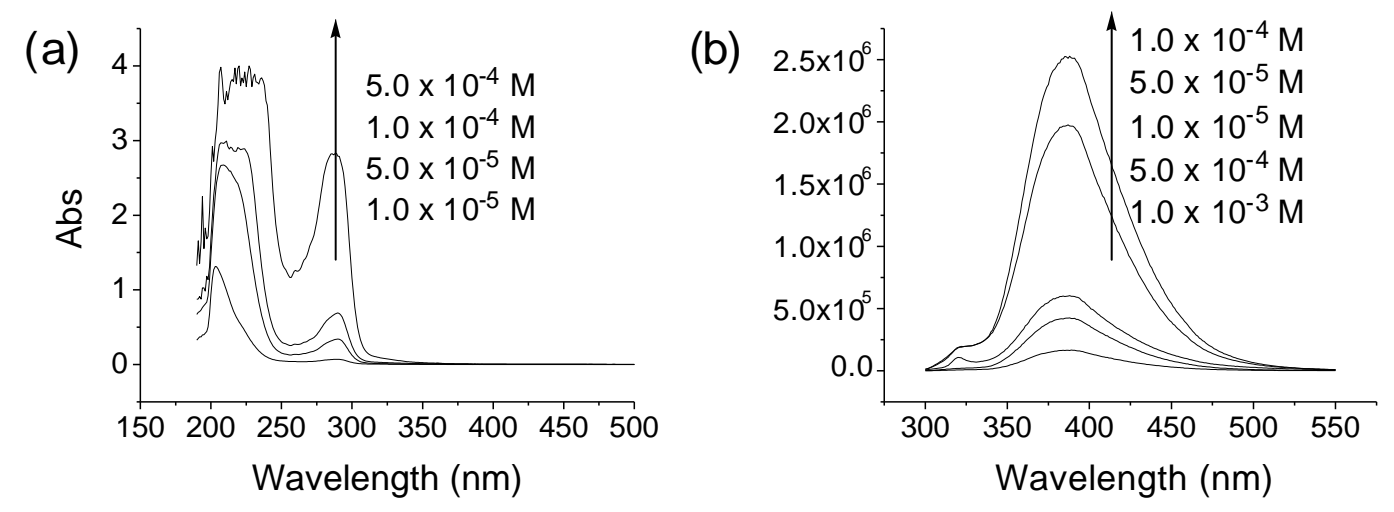

Figure 3-5. (a) UV and (b) fluorescence spectra of sensor (S)-3-5 in $\mathrm{CH}_{3} \mathrm{CN}$ at various concentrations $\left(\lambda_{\mathrm{exc}}=291 \mathrm{~nm}\right.$, slit $\left.=4.0 / 4.0 \mathrm{~nm}\right)$.
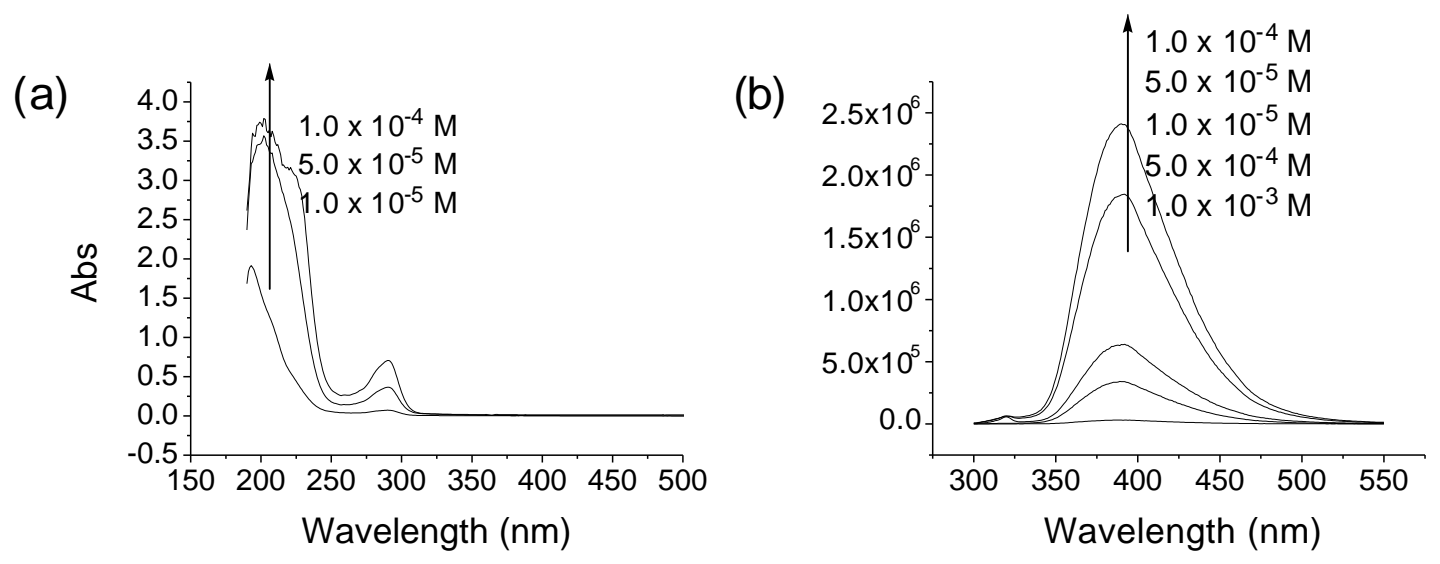

In order to gain further understanding on the fluorescent properties of $(S)-3-5$, we 
have prepared the $\mathrm{H}_{8} \mathrm{BINOL}$-amine molecules $(S)$-3-6 $-(S)$-3-10 by using our recently developed one-step reaction of $\mathrm{H}_{8} \mathrm{BINOL}$ with an in situ generated aminomethanol (Scheme 3-2). ${ }^{13}$

Scheme 3-2. Synthesis of the $\mathrm{H}_{8} \mathrm{BINOL}-$ amine compounds $(S)-3-6-(S)-3-10$.

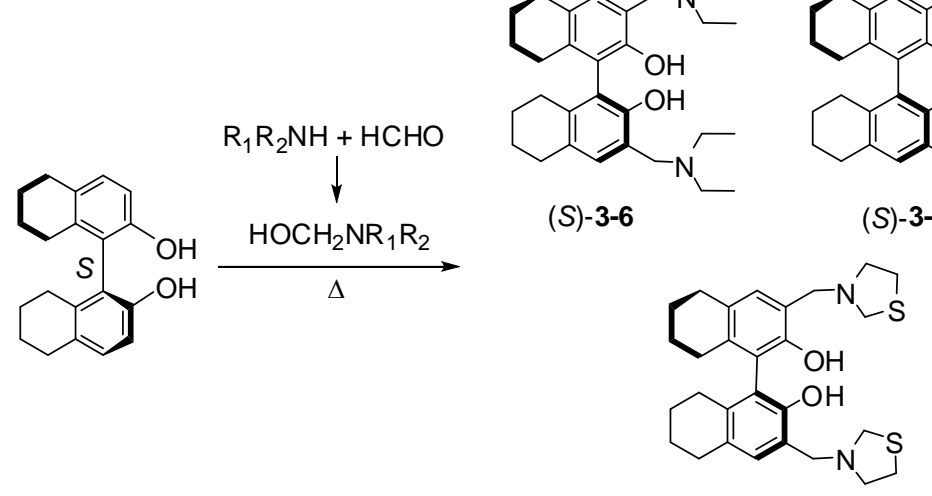

(S)-3-9

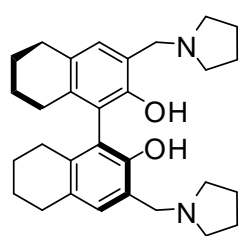

(S)-3-7

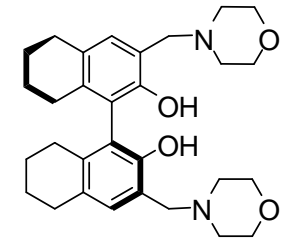

(S)-3-8

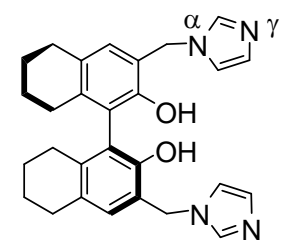

(S)-3-10

These $\mathrm{H}_{8} \mathrm{BINOL-amine}$ compounds such as $(S)-3-7$ and $(S)-3-8$ have exhibited excellent enantioselectivity in the asymmetric arylzinc addition to aldehydes. ${ }^{13}$ The fluorescence spectra of these compounds in $\mathrm{CH}_{2} \mathrm{Cl}_{2}\left(2.0 \times 10^{-4} \mathrm{M}\right)$ are obtained. As shown in Figure 3-6, compounds $(S)-3-6,(S)-3-7$ and $(S)$-3-8 show a major emission peak at about $380 \mathrm{~nm}$, similar to $(S)-\mathbf{3 - 5}$. However, compound (S)-3-9 gives dual emissions as $\lambda_{\mathrm{emi}}=329$ and $370(\mathrm{sh}) \mathrm{nm}$, and compound (S)-3-10 gives only a short wavelength emission at $\lambda_{\mathrm{emi}}=324 \mathrm{~nm}$. The fluorescence signal of $(S)-3-10$ is very similar to that of $(S)-\mathrm{H}_{8} \mathrm{BINOL}$. No change in peak position and shape is observed when their concentration is varied (Figure 3-7). 
Figure 3-6. Fluorescence spectra of compounds $(S)-3-6-(S)-3-10\left[2.0 \times 10^{-4} \mathrm{M}\right.$ in $\mathrm{CH}_{2} \mathrm{Cl}_{2} . \lambda_{\text {exc }}=292 \mathrm{~nm}$ for $(S)-\mathbf{3 - 6}-(S)-\mathbf{3 - 9}$ and $291 \mathrm{~nm}$ for $(S)-3-10$. Slit: $5.0 / 5.0 \mathrm{~nm}$ for $(S)-3-6,(S)-3-7$ and $(S)-3-9$ and 4.0/4.0 $\mathrm{nm}$ for $(S)$-3-8 and $(S)-3-10]$.

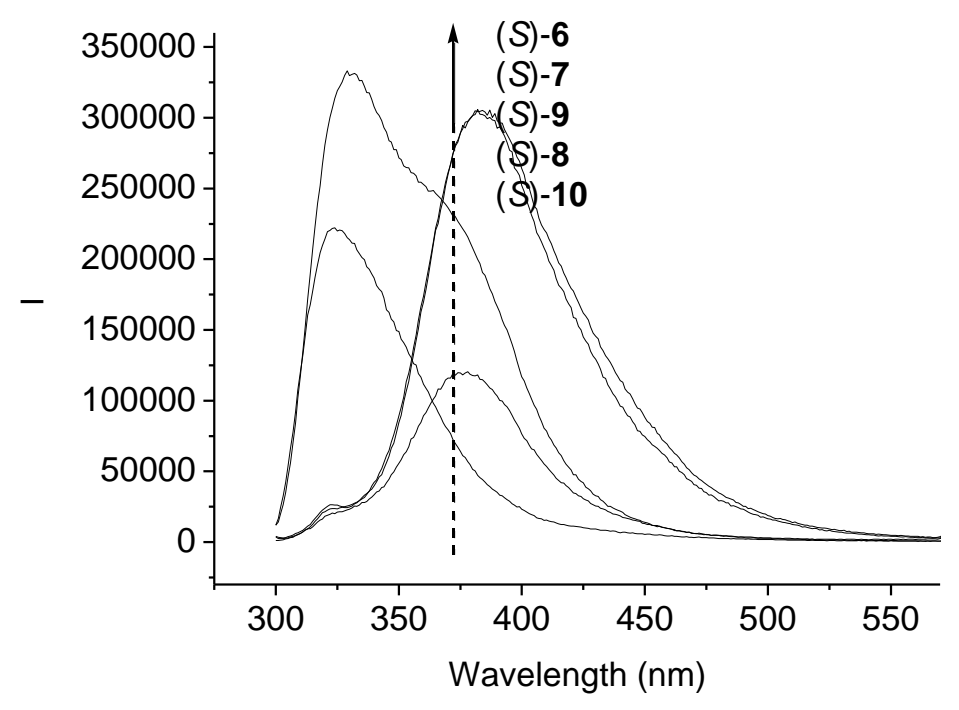

Figure 3-7. Fluorescence spectra of (a) (S)-3-6 (b) (S)-3-7 (c) (S)-3-8 (d) (S)-3-9 (e) $(S)-3-10$ in $\mathrm{CH}_{2} \mathrm{Cl}_{2}$ at various concentrations $\left(\lambda_{\text {exc }}=290 \mathrm{~nm}\right.$, slit $\left.=5.0 / 5.0 \mathrm{~nm}\right)$.
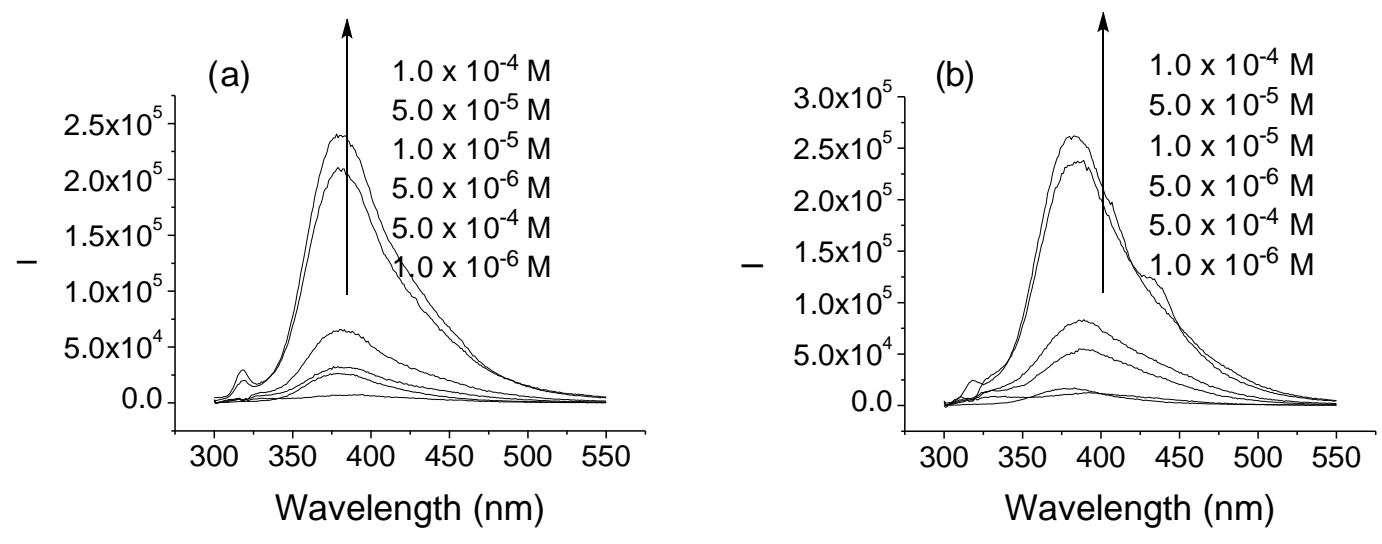

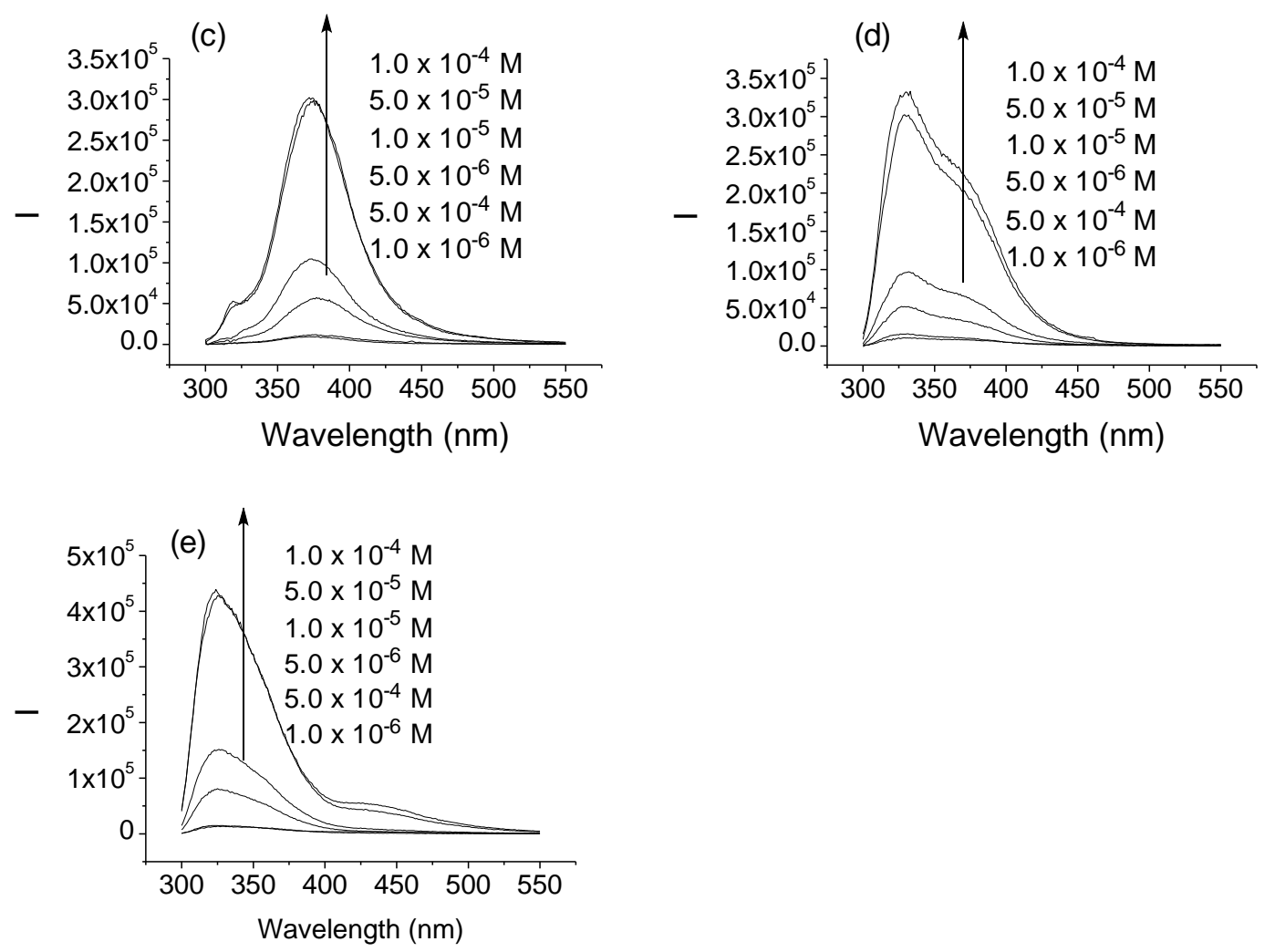

In the ${ }^{1} \mathrm{H}$ NMR spectra of compounds $(S)-\mathbf{3 - 6},(S)-\mathbf{3 - 7}$ and $(S)-\mathbf{3 - 8}$, their $\mathrm{H}_{8} \mathrm{BINOL}$ hydroxyl proton signals appeared at $\delta 11.13,11.91$, and 10.38 , respectively. ${ }^{13}$ These greatly downfield-shifted ${ }^{1} \mathrm{H}$ NMR signals indicate strong intramolecular hydrogen bonds between the hydroxyl protons and the basic nitrogen atoms. The chemical shift of the hydroxyl proton signal of $(S)$-3-9 $(\delta 9.52)$ is about 2 ppm less downfield than that of (S)-3-7. This may imply a weaker hydrogen bond in (S)-3-9 probably because of the reduced basicity of the nitrogen atom adjacent to the sulfur atom. The $\mathrm{H}_{8} \mathrm{BINOL}$ hydroxyl proton signal of $(S)$-3-10 is observed at $\delta 3.48$, greatly upfield-shifted in comparison with those of $(S)$-3-6 - (S)-3-9. This is attributed to the non-basic $\alpha$ nitrogen atoms of the imidazole rings of $(S)-\mathbf{3 - 1 0}$ which cannot form intramolecular hydrogen 
bond with the $\mathrm{H}_{8} \mathrm{BINOL}$ hydroxyl protons at all. The more basic $\gamma$ nitrogen atom in each of the imidazole rings of $(S)-\mathbf{3 - 1 0}$ is not sterically feasible to form an intramolecular hydrogen bond.

On the basis of the above analysis, we propose that the difference between the fluorescence spectra of the $\mathrm{H}_{8} \mathrm{BINOL}-\mathrm{amine}$ compounds $(S)-\mathbf{3 - 6}$ - $(S)-\mathbf{3 - 1 0}$ could arise from the different capability of their nitrogen atoms to form intramolecular hydrogen bond. Compound (S)-3-10 cannot form an intramolecular hydrogen bond and thus exhibits only the emission of its $\mathrm{H}_{8} \mathrm{BINOL}$ unit. Compounds $(S)-3-6,(S)-3-7$ and (S)-3-8 have strong intramolecular hydrogen bonds and thus show emission of either the intramolecularly hydrogen bonded complex or its subsequent excited state proton transfer complex. $^{14}$ Compound (S)-3-9 forms a weaker intramolecular hydrogen bond and it shows the emissions contributed by both the $\mathrm{H}_{8} \mathrm{BINOL}$ unit and the intramolecularly hydrogen bonded complex.

The study of compounds $(S)-3-6-(S)-3-10$ has revealed the nature of the emission of the $\mathrm{H}_{8} \mathrm{BINOL}$-amino alcohol $(S)-\mathbf{3 - 5}$ as a contribution from its intramolecularly hydrogen bonded complex. It explains why the fluorescence spectrum of $(S)$-3-5 is very different from that of $\mathrm{H}_{8} \mathrm{BINOL}$. Unlike that observed in the ${ }^{1} \mathrm{H}$ NMR spectra of compounds (S)-3-6 - $(S)-3-10$, the $\mathrm{H}_{8}$ BINOL hydroxyl proton signals of $(S)$-5 are invisible probably because of fast exchange in solution. 


\subsection{Study of the Interaction of $(S)-3-5$ with Mandelic Acid}

We have investigated the interaction of $(S)-3-5$ with the enantiomers of MA. As shown in Figure 3-8a, when $(S)-3-5\left(1.0 \times 10^{-4}\right.$ in $\left.\mathrm{CH}_{2} \mathrm{Cl}_{2}\right)$ was treated with $(R)$-MA (4.0 x $\left.10^{-3}\right)$, a large enhancement at the short wavelength emission $\left(\lambda_{\mathrm{emi}}=330 \mathrm{~nm}\right)$ was observed with $\mathrm{I}_{\mathrm{R}} / \mathrm{I}_{0}=11.7$. When $(S)$-MA was used under the same conditions, the enhancement at the short wavelength emission was much smaller. Thus, a good enantioselective fluorescent response was observed with ef $=3.5$ [ef: enantioselective fluorescence enhancement ratio $\left.=\left(\mathrm{I}_{\mathrm{R}}-\mathrm{I}_{0}\right) /\left(\mathrm{I}_{\mathrm{S}}-\mathrm{I}_{0}\right)\right]$. The fluorescence enhancement at the long wavelength emission of (S)-3-5 is much smaller and also with little enantioselectivity. Figure 3-8b displays the results of three independent measurements for the fluorescence enhancement of $(S)-3-5$ at the short wavelength emission while the concentration of the acid is varied. We have prepared $(R)-\mathbf{3 - 5}$, the enantiomer of $(S)-\mathbf{3 - 5}$, from $(R)-\mathrm{H}_{8} \mathrm{BINOL}$ and $(R)-3-4$, and studied its interaction with $(R)-$ and $(S)$-MA. It is found that $(S)$-MA causes much greater fluorescence enhancement for $(R)-3-5$ at the short wavelength emission than $(R)$-MA. This confirms the enantioselective nature of the observed different fluorescence enhancements for the sensor in the presence of the two enantiomers of the acid.

Figure 3-8. (a) Fluorescence spectra of $(S)-3-5\left(1.0 \times 10^{-4} \mathrm{M}, \mathrm{CH}_{2} \mathrm{Cl}_{2}\right)$ with/without $(R)$ and (S)-MA $\left(4.0 \times 10^{-3} \mathrm{M}\right)$. (b) Three independent measurements of Fluorescence 
enhancement of $(S)-3-5\left(1.0 \times 10^{-4} \mathrm{M}, \mathrm{CH}_{2} \mathrm{Cl}_{2}\right)$ at $\lambda_{\mathrm{em}}=330 \mathrm{~nm}$ with varying acid concentration. $\quad\left(\lambda_{\mathrm{exc}}=290 \mathrm{~nm}\right.$, slit $\left.=3.0 / 3.0 \mathrm{~nm}\right)$.

(a)

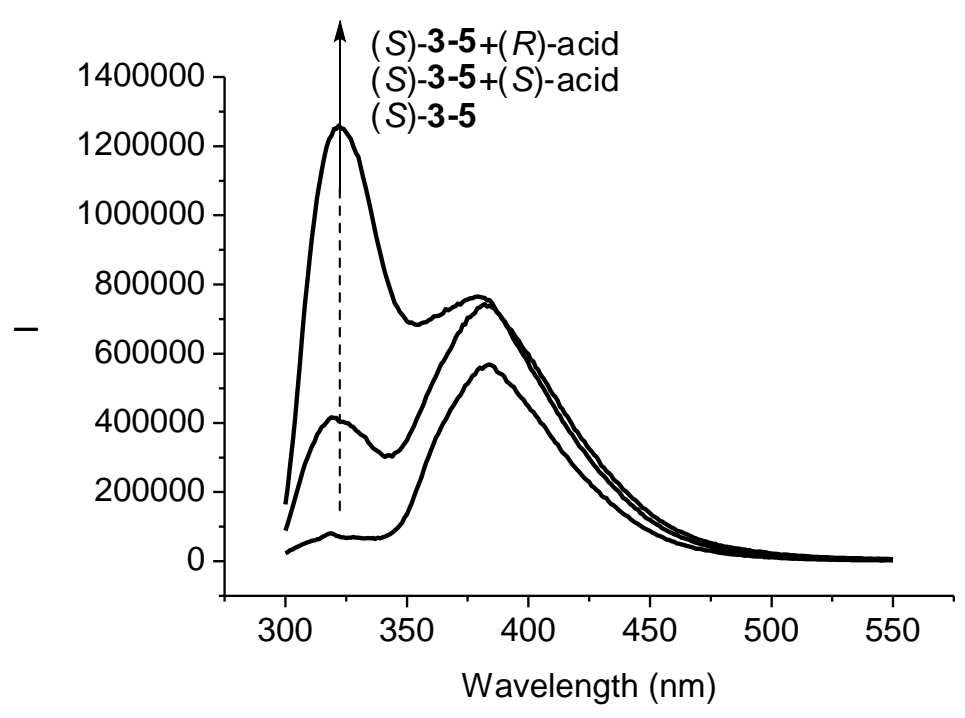

(b)

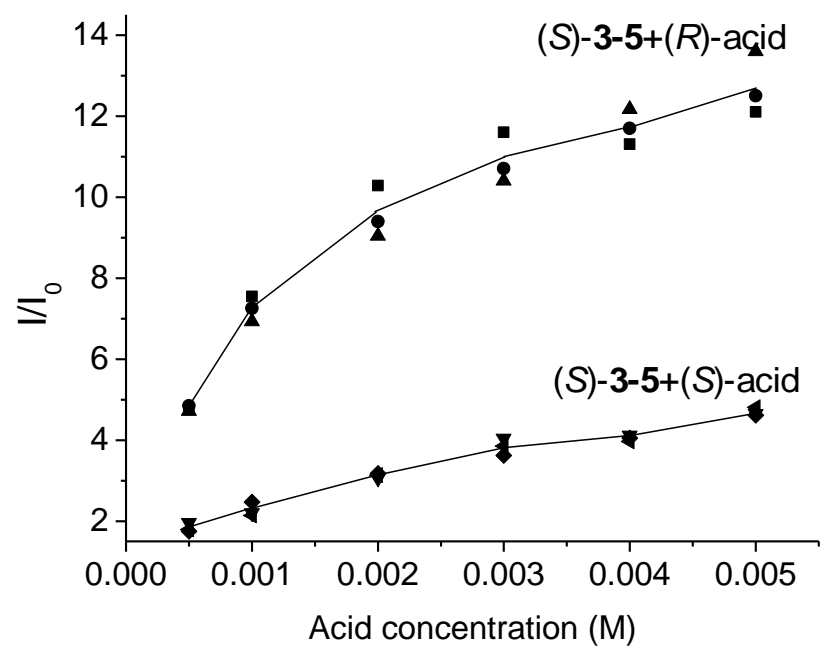

The greatly enhanced short wavelength emission of $(S)-3-5$ in the presence of $(R)$-MA demonstrates that protonation of the nitrogen atoms of $(S)-\mathbf{3 - 5}$ by the acidic proton of (R)-MA should have suppressed the intramolecular hydrogen bonding between the 
$\mathrm{H}_{8} \mathrm{BINOL}$ hydroxyl protons of $(S)-\mathbf{3 - 5}$ and its amine nitrogens and restored the emission of the $\mathrm{H}_{8} \mathrm{BINOL}$ unit. The overall fluorescence enhancement should be generated from the formation of the structurally more rigid intermolecular complex between $(S)-\mathbf{3 - 5}$ and (R)-MA.

When $(S)-\mathrm{H}_{8} \mathrm{BINOL}$ is treated with $(R)$ - or $(S)$-MA, there is almost no fluorescence enhancement and enantioselectivity (Figure 3-9). Thus, the intermolecular hydrogen bonding between the amino alcohol units of $(S)-\mathbf{3 - 5}$ and $(R)$-MA is important for the observed enantioselective fluorescent enhancement.

Figure 3-9. Fluorescence spectra of $(S)-\mathrm{H}_{8} \mathrm{BINOL}\left(2.0 \times 10^{-4} \mathrm{M}\right.$ in $\left.\mathrm{CH}_{2} \mathrm{Cl}_{2}\right)$ in the presence of $(R)$ - and $(S)$-mandelic acid $\left(4.0 \times 10^{-3} \mathrm{M}\right)\left[\lambda_{\mathrm{exc}}=288 \mathrm{~nm} . \quad\right.$ Slit $\left.=4.0 / 4.0 \mathrm{~nm}\right]$.

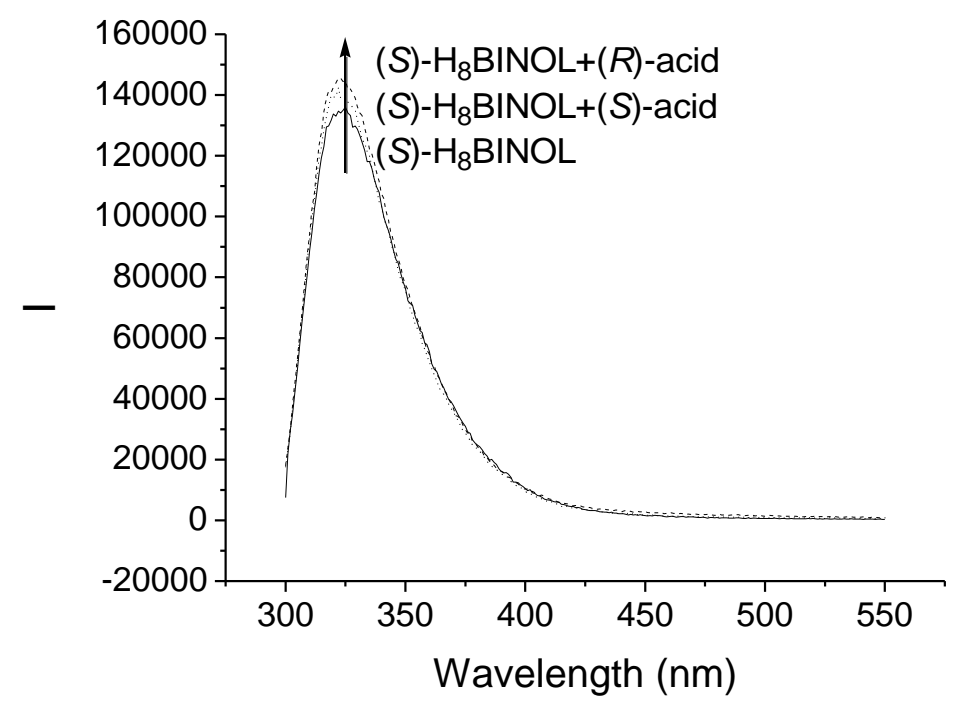

A ${ }^{1} \mathrm{H}$ NMR spectroscopic investigation on the interaction of $(S)-3-5$ with $(R)$-MA in $\mathrm{CDCl}_{3}$ was conducted while the total concentration was maintained at $6.0 \times 10^{-3} \mathrm{M}$. 
Addition of (S)-3-5 to the solution of (R)-MA caused the $\alpha$ proton signal of (R)-MA to undergo upfield shift from $\delta 5.26$ to $\delta 4.20$ as the ratio of $(S)-3-5$ relative $(R)$-MA reached 2:3. This large upfield shift indicates that the complexation between $(S)-3-5$ and (R)-MA might have placed the $\alpha$ proton of the acid in the electronically shielded region of the sensor. Further increasing the ratio of (S)-3-5 versus (R)-MA to 5:1 shifted the $\alpha$ proton signal of (R)-MA downfield to $\delta 4.53$. Thus, the structure of the complex probably changes as the amount of (S)-3-5 versus (R)-MA further increases. In the ${ }^{1} \mathrm{H}$ NMR spectrum, the two doublet signals of the diastereotopic protons $\mathrm{H}_{\mathrm{a}}$ of $(S)-\mathbf{3 - 5}$ began to move toward each other with the addition of $(R)$-MA and then merged into a singlet at $\delta 3.74$ when the ratio of $(R)$-MA relative to $(S)$-3-5 reached 0.43:1 (= 1:2.3). However, when the amount of $(R)$-MA was further increased, the singlet split back into two doublets, which were then moving away from each other with increasing $(R)$-MA. When the ratio of $(R)$-MA relative to $(S)$-3-5 reached 9:1, the $\mathrm{H}_{\mathrm{a}}$ signals of $(S)$-3-5 were observed at $\delta 4.24(\mathrm{~d}, \mathrm{~J}=12.3 \mathrm{~Hz})$ and $3.63(\mathrm{~d}, \mathrm{~J}=12.0 \mathrm{~Hz})$. We propose the following hypothesis to explain the above observed changes in the NMR signals of $(S)-3-5$. The intramolecular hydrogen bonds between the amine nitrogens and the core $\mathrm{H}_{8} \mathrm{BINOL}$ hydroxyl protons in $(S)-3-5$ should generate a rigid cyclic structure giving the two well-resolved diastereotopic proton signals for $\mathrm{H}_{\mathrm{a}}$. Addition of acid should protonate the amine group and allow free rotation of the 3,3'-substituents of $(S)-\mathbf{3 - 5}$, resulting in the merged signal of the $\mathrm{H}_{\mathrm{a}}$ protons. As the amount of $(R)$-MA increases, a structurally 
rigid intermolecular complex between $(S)$-3-5 and $(R)$-MA should be produced to give the well separated signals for the $\mathrm{H}_{\mathrm{a}}$ protons.

On the basis of the above NMR study, the Job plots for the interaction of $(S)-3-5$ with (R)-MA are produced. ${ }^{15}$ Figure $3-10 \mathrm{a}$ is obtained by monitoring the change of the $\alpha$ proton signal of $(R)-\mathrm{MA}$, and Figure $3-10 \mathrm{~b}$ by monitoring the change of the $\mathrm{H}_{\mathrm{b}}$ signal of $(S)-3-5$. Both plots indicate that $(S)-3-5$ and $(R)$-MA form a 1:2 complex in the ground state. Because the fluorescence response of $(S)-3-5$ toward $(R)-\mathrm{MA}$ at a constant total concentration of $1.0 \times 10^{-4} \mathrm{M}$ is very small, the Job plot could not be used to determine the excited state binding stoichiometry of $(S)-3-5$ with $(R)$-MA.

Figure 3-10. Job plots of $(S)$-3-5 with $(R)$-MA obtained by using the ${ }^{1} \mathrm{H}$ NMR signal change $\left(\Delta \delta=\delta_{0}-\delta\right)$ of (a) the $\mathrm{H}_{\alpha}$ of $(R)$-MA $\left\{\mathrm{X}=[\right.$ acid $] /([$ acid $]+[$ sensor $]\}$, and (b) the $\mathrm{H}_{\mathrm{b}}$ of $(\mathrm{S})-3-5\{\mathrm{X}=[$ sensor $] /([$ sensor $]+[$ acid $])\}$.
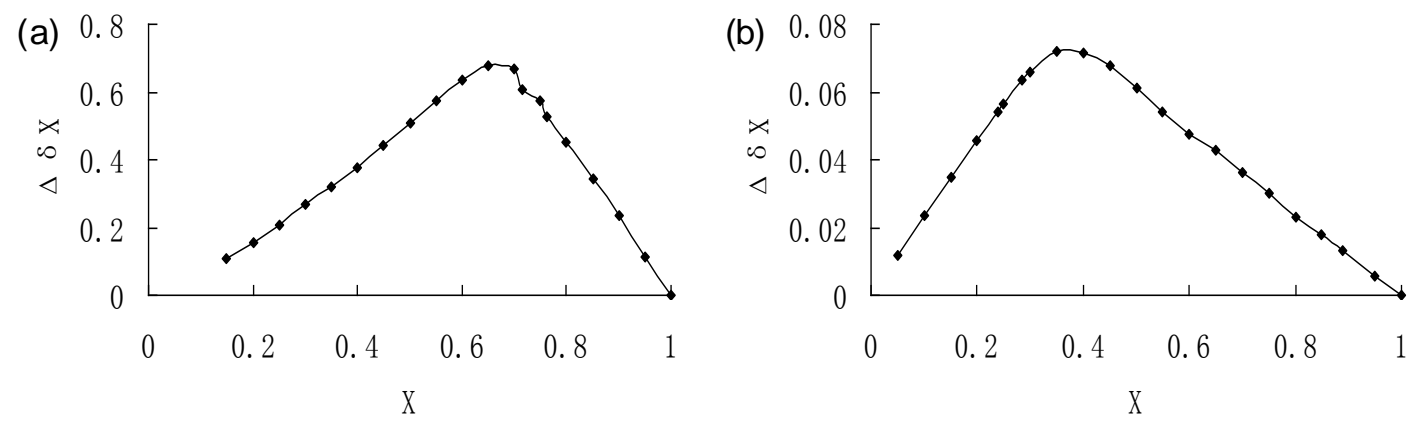

\subsection{Interaction of the $\mathrm{H}_{8} \mathrm{BINOL-Amine} \mathrm{Compounds}(S)-3-6$ - $(S)-3-10$ with MA}

The fluorescent responses of the $\mathrm{H}_{8} \mathrm{BINOL}-$ amine compounds $(S)-\mathbf{3 - 6}-(S)-\mathbf{3 - 1 0}$ in 
the presence of $(R)$ - and $(S)$-MA are studied. As shown in Figure 3-11, when compounds (S)-3-6, $(S)$-3-7 and $(S)$-3-8 were treated with MA, large fluorescence enhancements at the short wavelength were observed similar to that observed for $(S)-\mathbf{3 - 5}$. This is consistent with the suppressed intramolecular hydrogen bonding interaction of these compounds when their amine nitrogens are interacting with the carboxylic acid proton of the acid. However, almost no enantioselectivity was observed. When compound (S)-3-9 was treated with MA, little fluorescence enhancement was observed. This indicates that the intermolecular interaction of $(S)$-3-9 with MA should be much weaker than that of compounds $(S)-\mathbf{3 - 5}-(S)-\mathbf{3 - 8}$ because of the weaker basicity of the nitrogens in (S)-3-9. Although the $\alpha$ nitrogen atoms of $(S)-3-10$ are not basic at all, there is significant fluorescence enhancement in the presence of $(R)-\mathrm{MA}$ with $\mathrm{I}_{\mathrm{R}} / \mathrm{I}_{0}=3.2$. It is proposed that the much more basic $\gamma$ nitrogens of $(S)-\mathbf{3 - 1 0}$ should have participated in the complexation with $(R)$-MA to form a structurally more rigid fluorophore, leading to the observed fluorescence enhancement. When $(S)-\mathbf{3 - 1 0}$ was treated with the enantiomeric acid (S)-MA, the fluorescence enhancement was smaller, giving a good enantioselectivity of ef $=2.1$. This indicates that $(S)$-3-10 is a promising candidate for the enantioselective fluorescent recognition of MA.

Figure 3-11. Fluorescence spectra of (S)-3-6 - (S)-3-10 $\left(2.0 \times 10^{-4} \mathrm{M}\right.$ in $\left.\mathrm{CH}_{2} \mathrm{Cl}_{2}\right)$ in the presence of $(R)$ - and $(S)-\mathrm{MA}\left(4.0 \times 10^{-3} \mathrm{M}\right)\left[\lambda_{\mathrm{exc}}=292 \mathrm{~nm}\right.$ for $(S)-3-6-(S)-3-9$ and 291 
$\mathrm{nm}$ for $(S)$-3-10. Slit: 5.0/5.0 $\mathrm{nm}$ for $(S)-3-6,(S)-3-7$ and $(S)-3-9$ and 4.0/4.0 $\mathrm{nm}$ for (S)-3-8 and (S)-3-10].
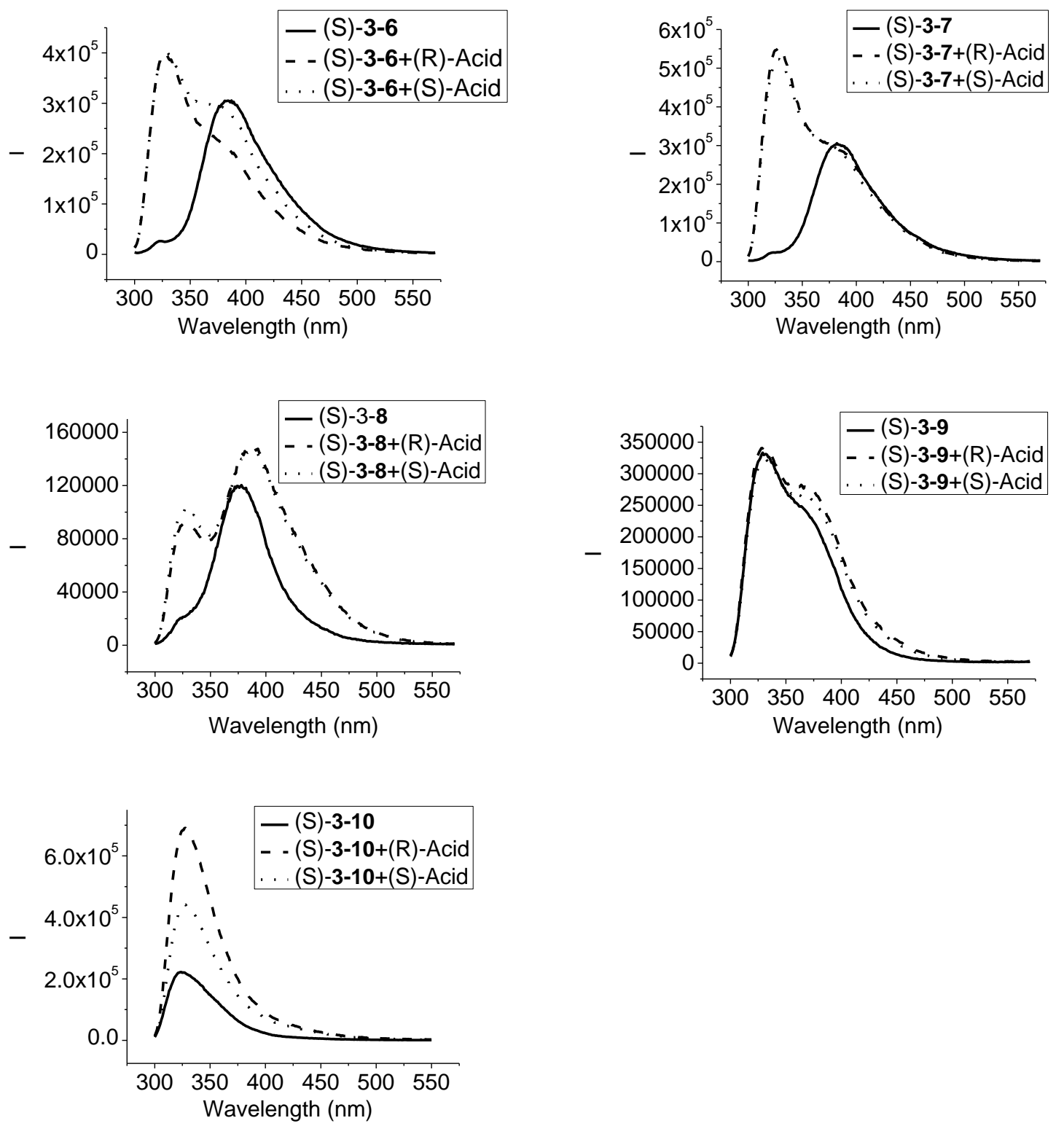

\section{Conclusion}

We have investigated the fluorescent properties of a series of $\mathrm{H}_{8}$ BINOL-amine 
compounds. This study reveals that the intramolecular hydrogen bonds of these compounds can shift the emission of their $\mathrm{H}_{8} \mathrm{BINOL}$ unit to a much longer wavelength. In spite of the much shorter conjugation in the $\mathrm{H}_{8} \mathrm{BINOL-based} \mathrm{fluorophore} \mathrm{of}(S)$-3-5 than that in the BINOL-based compound $(S)$-3-1, $(S)$-3-5 has exhibited very efficient fluorescent response toward MA. Binding of $(S)-\mathbf{3 - 5}$ with the acid suppresses its intramolecular hydrogen bonding and restores the short wavelength emission of the $\mathrm{H}_{8}$ BINOL unite, giving high sensitivity and good enantioselectivity. Thus, with appropriate design of the structure and functional groups, the $\mathrm{H}_{8} \mathrm{BINOL}$-based molecules are promising as a new class of enantioselective fluorescent sensors. In comparison with their BINOL analogs, the $\mathrm{H}_{8} \mathrm{BINOL}$-based compounds have opened a new window in the shorter emission wavelength to observe the enantioselective fluorescent recognition. $^{8}$

\section{Experimental Section}

\subsection{Synthesis and Characterization of Compounds}

Synthesis and Characterization of Sensor (S)-3-5. (1) (S)-3,3'-Diformyl$\mathrm{H}_{8} \mathrm{BINOL}$, $(S)-3-3(245 \mathrm{mg}, 0.70 \mathrm{mmol})$, was dissolved in $\mathrm{CH}_{2} \mathrm{Cl}_{2}(22 \mathrm{~mL})$ in the presence of $4 \AA$ molecular sieves and combined with (S)-2-amino-1,1,2triphenylenthanol, $(S)-3-4(607 \mathrm{mg}, 2.10 \mathrm{mmol})$. The reaction mixture was heated at reflux for $30 \mathrm{~h}$, and monitored by using ${ }^{1} \mathrm{H}$ NMR spectroscopy. When the reaction was 
complete, the reaction mixture was cooled to room temperature and filtered. The filtrate was concentrated under vacuum and passed through a silica gel column eluted with $\mathrm{CH}_{2} \mathrm{Cl}_{2}$ to give the corresponding Schiff base. (2) The Schiff base was dissolved in methanol $(28 \mathrm{~mL})$ and cooled down to $0{ }^{\circ} \mathrm{C}$. $\mathrm{NaBH}_{4}(106 \mathrm{mg}, 2.80 \mathrm{mmol})$ was added in small portions. The reaction temperature was maintained at $0{ }^{\circ} \mathrm{C}$ until the solution became colorless and transparent. Then, it was allowed to proceed at room temperature for additional $30 \mathrm{~min}$. Methanol was removed, and the residue was dissolved in ethyl acetate $(50 \mathrm{~mL})$ and washed with water $(15 \mathrm{~mL})$. The aqueous layer after separation was extracted with ethyl acetate $(3 \times 30 \mathrm{~mL})$. The combined organic layer was washed with brine $(15 \mathrm{~mL})$ and dried over anhydrous $\mathrm{Na}_{2} \mathrm{SO}_{4}$. After evaporation of the solvent, the residue was purified by flash column chromatography on silica gel eluted with hexanes/ethyl acetate (3/1) to afford $(S)-\mathbf{3 - 5}$ as a white solid in $71 \%$ yield. ${ }^{1} \mathrm{H}$ NMR $\left(300 \mathrm{MHz}, \mathrm{CDCl}_{3}\right) \delta 1.72(\mathrm{~m}, 8 \mathrm{H}), 2.17(\mathrm{~m}, 2 \mathrm{H}), 2.34(\mathrm{~m}, 2 \mathrm{H}), 2.66(\mathrm{~m}, 4 \mathrm{H}), 3.59(\mathrm{~d}, \mathrm{~J}=$ $13.5 \mathrm{~Hz}, 2 \mathrm{H}), 3.77(\mathrm{~d}, \mathrm{~J}=13.5 \mathrm{~Hz}, 2 \mathrm{H}), 4.63(\mathrm{~s}, 2 \mathrm{H}), 6.58(\mathrm{~s}, 2 \mathrm{H}), 7.02-7.31(\mathrm{~m}, 28 \mathrm{H})$, $7.59(\mathrm{~d}, \mathrm{~J}=7.2 \mathrm{~Hz}, 2 \mathrm{H}) . \quad{ }^{13} \mathrm{C} \mathrm{NMR}\left(75 \mathrm{MHz}, \mathrm{CDCl}_{3}\right) \delta 23.5,23.6,27.5,29.5,49.4,69.0$, $80.5,121.8,122.0,126.4,126.7,126.9,127.6,127.7,127.8,127.9,128.7,128.8,130.1$, 130.4, 136.4, 137.4, 144.5, 145.4, 151.4. HRMS Calcd for $\mathrm{C}_{62} \mathrm{H}_{61} \mathrm{~N}_{2} \mathrm{O}_{4}\left(\mathrm{MH}^{+}\right)$: 897.4631. Found: 897.4653. m.p. $124-125{ }^{0} \mathrm{C} . \quad[\alpha]_{\mathrm{D}}=-119.9\left(\mathrm{c}=0.865, \mathrm{CHCl}_{3}\right)$. The enantiomer (R)-3-5 was obtained in the same way by using (R)-3,3'-DiformylH ${ }_{8}$ BINOL and $(R)$-2-amino-1,1,2-triphenylenthanol. $[\alpha]_{\mathrm{D}}=119.3(\mathrm{c}=$ 
$\left.0.470, \mathrm{CHCl}_{3}\right)$.

\subsection{Preparation of Samples for Fluorescence Measurement}

Sensors were purified by column chromatography and then stored in a refrigerator. The commercially obtained enantiomers of MA were recrystallized from methanol. They were then passed through a short column of silica gel (eluted with diethyl ether) and dried under vacuum. All of the solvents were either HPLC or spectroscopic grade. The stock solutions of the sensors were freshly prepared for each measurement. A 0.01 M stock solution of MA in methylene chloride was freshly prepared. For the fluorescence enhancement study, a sensor solution was mixed with the MA solution at room temperature in a $5 \mathrm{~mL}$ volumetric flask and diluted to the desired concentration. The resulting solution was allowed to stand at room temperature for 2 - $3 \mathrm{~h}$ before the fluorescence measurement. 


\section{References}

1. (a) Fluorescent Chemosensors for Ion and Molecular Recognition; Czarnik, A. W., Ed.; ACS Symposium Series 538; American Chemical Society: Washington, DC, 1993. (b) de Silva, A. P.; Gunaratne, H. Q. N.; Gunnlaugsson, T.; Huxley, A. J. M.; McCoy, C. P.; Rademacher, J. T.; Rice, T. E. Chem. Rev. 1997, 97, 1515-1566. (c) Fabbrizzi, L.; Poggi, A. Chem. Soc. Rev. 1995, 24, 197. (d) Fluorescent and Luminescent Probes, 2nd ed.; Mason, W. T., Ed.; Academic: San Diego, 1999.

2. (a) Jelinek, R.; Kolusheva, S. Chem. Rev. 2004, 104, 5987-6015. (b) Johnson, K. S.; Needoba, J. A.; Riser, S. C.; Showers, W. J. Chem. Rev. 2007, 107, 623-640. (c) Basabe-Desmonts, L.; Reinhoudt, D. N.; Crego-Calama, M. Chem. Soc. Rev., 2007, 36, 993-1017. (d) McDonagh, C.; Burke, C. S.; MacCraith, B. D. Chem. Rev. 2008, 108, 400-422. (e) Borisov, S. M.; Wolfbeis, O. S. Chem. Rev. 2008, 108, 423-461. (f) Nolan, E. M.; Lippard, S. J. Acc. Chem. Res. 2009, 42, 193-203.

3. (a) Thompson, R. B. Fluorescence Sensors and Biosensors, CRC: U.S. 2005. (b) James, T. D.; Phillips, M. D.; Shinkai, S. Boronic Acids in Saccharide Recognition; RSC: UK, 2006.

4. A review: $\mathrm{Pu}$, L. Chem. Rev. 2004, 104, 1687-1716.

5. Selected references: (a) James, T. D.; Sandanayake, K. R. A. S.; Shinkai, S. Nature 1995, 374, 345-347. (b) Klein, G.; Reymond, J.-L. Helv. Chim. Acta 1999, 82, 400-407. (c) Pugh, V.; Hu, Q. -S.; Pu, L. Angew. Chem. Int. Ed. 2000, 39, 3638-3641. 
(d) Reetz, M. T.; Sostmann, S. Tetrahedron 2001, 57, 2515-2520. (e) Korbel, G. A.; Lalic, G.; Shair, M. D. J. Am. Chem. Soc. 2001, 123, 361-362. (f) Jarvo, E. R.; Evans, C. A.; Copeland, G. T.; Miller, S. J. J. Org. Chem. 2001, 66, 5522-5527. (g) Wong, W.-L.; Huang, K.-H.; Teng, P.-F.; Lee, C.-S.; Kwong, H.-L. Chem. Commun. 2004, 384-385. (h) Zhao, J.-Z.; Fyles, T. M.; James, T. D. Angew. Chem., Int. Ed. 2004, 43, 3461-3464. (i) Pagliari, S.; Corradini, R.; Galaverna, G.; Sforza, S.; Dossena, A.; Montalti, M.; Prodi, L.; Zaccheroni, N.; Marchelli, R. Chem. Eur. J. 2004, 10, 2749-2758. (j) Matsushita, H.; Yamamoto, N.; Meijler, M. M.; Wirsching, P.; Lerner, R.A.; Matsushita, M.; Janda, K. D. Mol. Biosyst. 2005, 1. 303-306. (k). Zhu, L.; Anslyn, E. V. J. Am. Chem. Soc. 2004, 126, 3676-3677. (1) Mei, X. F.; Wolf, C. J. Am. Chem. Soc. 2004, 126, 14736-14737.

6. (a) Lin, J.; Hu, Q.-S.; Xu, M. H.; Pu, L. J. Am. Chem. Soc. 2002, 124, 2088-2089.

(b) Xu, M.-H.; Lin, J.; Hu, Q.-S.; Pu, L. J. Am. Chem. Soc .2002, 124, 14239-14246.

(c) Li, Z.-B.; Lin, J.; Pu, L. Angew. Chem., Int. Ed. 2005, 44, 1690-1693. (d) Lin, J.; Rajaram, A. R.; Pu, L. Tetrahedron 2004, 60, 11277-11281. (e) He, X.; Cui, X.; Li, M.; Lin, L.; Liu, X.; Feng, X. Tetrahedron Lett. 2009, 50, 5853-5856. (f) Liu, H. -L.; Hou, X. -L.; Pu, L. Angew. Chem. Int. Ed. 2009, 48, 382 -385. (g) Chen, X.; Huang, Z.; Chen, S. -Y.; Li, K.; Yu, X. -Q.; Pu, L. J. Am. Chem. Soc. 2010, 132, 7297-7299.

(h) Liu, H. -L.; Hou, X. -L; Pu, L. Org. Lett. 2010, 12, 4172-4175. 
7. (a) A review: Au-Yeung, T.L.-L.; Chan, S.-S.; Chan. A. S. C. Adv. Synth. Catal. 2003, 345, 537-555. (b) Matsunaga, S.; Kinoshita, T.; Okada, S.; Harada, S.; Shibasaki, M. J. Am. Chem. Soc. 2004, 126, 7559-7570. (c) Kumaraswamy, G.; Jena, N.; Sastry, M. N. V.; Padmaja, M.; Markondaiah, B. Adv. Synth. Catal. 2005, 347, 867 -871. (d) Huang, H.; Liu, X.; Chen, H.; Zheng, Z. Tetrahedron: Asymmetry 2005, 16, 693-697. (e) Kim, J. G.; Camp, E. H.; Walsh, P. J. Org. Lett. 2006, 8, 4413-4416. (f) Wu, K.-H.; Gau, H.-M. J. Am. Chem. Soc. 2006, 128, 14808-14809. (g) Muramatsu, Y.; Harada, T. Chem. Eur. J. 2008, 14, 10560 - 10563. (h) Jiang, J.; Yu, J.; Sun, X.-X.; Rao, Q.-Q.; Gong, L.-Z. Angew. Chem. Int. Ed. 2008, 47, 2458 2462. (i) Zhou, S.; Wu, K.-H.; Chen, C.-A.; Gau, H.-M. J. Org. Chem. 2009, 74, $3500-3505$.

8. A preliminary study on $(R)-\mathbf{3 - 5}$, the enantiomer of $(S)-\mathbf{3 - 5}$, was recently communicated: Yu, S.; Pu, L. J. Am. Chem. Soc. 2010, 132, 17698-17700.

9. Liu, H. -L.; Peng, Q.; Wu, Y. -D.; Chen, D.; Hou, X. -L.; Sabat, M.; Pu, L. Angew. Chem. Int. Ed. 2010, 49, 602-606.

10. DeBerardinis, A. M.; Turlington, M.; Ko, J; Sole, L; Lin, Pu. J. Org. Chem. 2010, $75,2836-2850$.

11. Zhang, H. -C.; Huang, W. -S.; Pu, L. J. Org. Chem. 2001, 66, 481-487.

12. (a) Demas, J. N.; Crosby, J. J. Phys. Chem. 1971, 75, 991-1024 (see pages 1007-1011).

(b) Hu, Q-S; Pugh, V.; Sabat, M.; Pu, L. J. Org. Chem. 1999, 64, 7528-7536. 
13. DeBerardinis, A. M.; Turlington, M.; Ko, J.; Sole, L.; Pu, L. J. Org. Chem. 2010, $75,2836-2850$.

14. (a) Iwanek, W.; Mattay, J. J, Photochem. Photobiol. A: Chem., 1992, 67, 209-226. (b) Ofran, M.; Feitelson, J. Chem. Phys. Lett. 1973, 19, 427-431. (c) Solntsev, K. M.; Bartolo, E.-A.; Pan, G.; Muller, G.; Bommireddy, S.; Huppert, D.; Tolbert, L. M. Israel J. Chem. 2009, 49, 227-233. (d) Flegel, M.; Lukeman, M.; Wan, P. Can. J. Chem. 2008, 86, 161-169. (e) Xu, Y.; McCarroll, M. E. J. Photochem. Photobiol. A. Chem. 2006, 178, 50-56.

15. Blanda, M. T.; Horner, J. H.; Newcomb, M. J. Org. Chem. 1989, 54, 4626-4636. 


\section{Chapter 4}

One Enantiomeric Fluorescent Sensor Pair to Discriminate Four Stereoisomers of Threonines 


\section{Introduction}

The development of enantioselective fluorescent sensors for the recognition of chiral organic molecules has received increasing attention in the past decade because these sensors can potentially provide a real time technique of high sensitivity for high throughput chiral assay. ${ }^{1,2}$ Highly enantioselective fluorescent sensors for chiral amines, amino alcohols, $\alpha$-hydroxycarboxylic acids and amino acids have been obtained. Most of the substrates studied contain only one chiral center except in cases such as the sugar molecules investigated by Shinkai and others. ${ }^{3-5}$ No study on the development of a molecular sensor for the fluorescent discrimination of all the four stereoisomers of a compound with two chiral centers, such as threonines and its derivatives (Figure 4-1), was reported before. ${ }^{4,5}$ There is significant challenge to carry out the fluorescent recognition of these stereoisomers since it requires a fluorescent sensor to be both enantioselective and diastereoselective.

Figure 4-1. Stereoisomers of Threonines and the N-Cbz-Threonines.<smiles>CC(O)C(N)C(=O)O</smiles>

L-threonine<smiles>C[C@H](O)[C@H](NC(=O)OCc1ccccc1)C(=O)O</smiles>

$$
\text { LTH }
$$

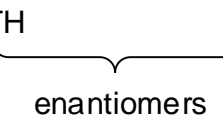

D-threonine DTH<smiles>CC(O)C(N)C(=O)O</smiles><smiles>CC(O)C(N)C(=O)O</smiles><smiles>CC(O)C(N)C(=O)O</smiles>

D-allo-threonine L-allo-threonine<smiles>CC(O)[C@H](NC(=O)OCc1ccccc1)C(=O)N[C@@H](C(=O)O)C(C)O</smiles>

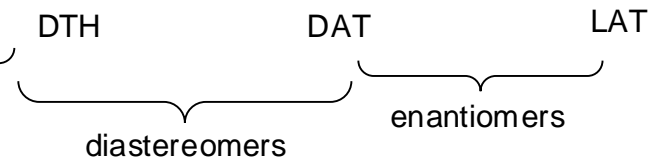


Threonines represent a class of $\beta$-hydroxy- $\alpha$-amino acids that are found to be structural units of many biologically significant natural products such as kaitocephalin, sphingofungins, clithioneine and myriocin. Among the four stereoisomers of threonines, L-threonine is an essential amino acid for humans. Recently, we reported that the 1,1'-bi-2-naphthol (BINOL)-amino alcohol $(S)-4-1$ is an enantioselective fluorescent sensor for the N-Cbz-serine, a molecule analogous to threonine but without the $\beta$-chiral center. $^{6,7}$ We have thus studied the interaction of $(S)-\mathbf{4 - 1}$ and its enantiomer $(R)-\mathbf{4 - 1}$ with the stereoisomeric N-Cbz-threonines LTH, DTH, LAT and DAT (Figure 4-1). This enantiomeric fluorescent sensor pair is found to exhibit different responses at two emission wavelengths toward the threonine derivatives, leading to both enantioselective and diastereoselective recognition of the threonines. In addition, an enantioselective precipitation is also observed which allows visual discrimination. Herein, these results are reported.

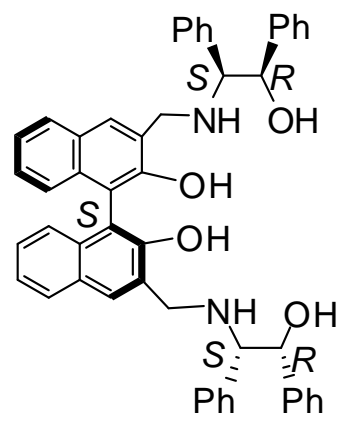

(S)-4-1

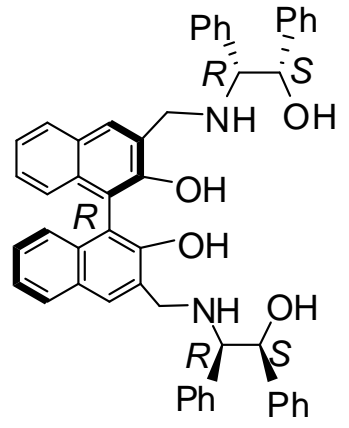

(R)-4-1 


\section{Results and Discussion}

In toluene solutions [2\% 1,2-dimethoxyethane (DME) was added to improve the solubility of the threonines], $(S)-\mathbf{4 - 1}$ was treated with the threonines. Figure 4-2a gives the fluorescence spectra of $(S)$-4-1 with or without the four stereoisomeric N-protected amino acids. It shows that (S)-4-1 gives dual emissions with $\lambda_{1}=370 \mathrm{~nm}$ and $\lambda_{2}=431$ $\mathrm{nm}$. When treated with the four stereoisomers of threonines, the fluorescence responses of (S)-4-1 at the two emission wavelengths are different. LAT causes large fluorescence enhancement at both $\lambda_{1}$ and $\lambda_{2}$. DTH enhances $\lambda_{2}$ greatly but little at $\lambda_{1}$. Both DAT and LTH show smaller enhancement at $\lambda_{1}$ and $\lambda_{2}$, but DAT enhances $\lambda_{2}$ more than LTH.

Figure 4-2. Fluorescence spectra of (a) $(S)-4-1\left(5.0 \times 10^{-4} \mathrm{M}\right)$ and $(b)(R)-4-1\left(5.0 \times 10^{-4} \mathrm{M}\right)$ with four stereoisomers of $\alpha$-N-Cbz-amino acid $\left(1.0 \times 10^{-3} \mathrm{M}\right)$. (Solvent: toluene/2\% DME. $\left.\lambda_{\text {exc }}=341 \mathrm{~nm}, 3.0 / 3.0 \mathrm{~nm}\right)$

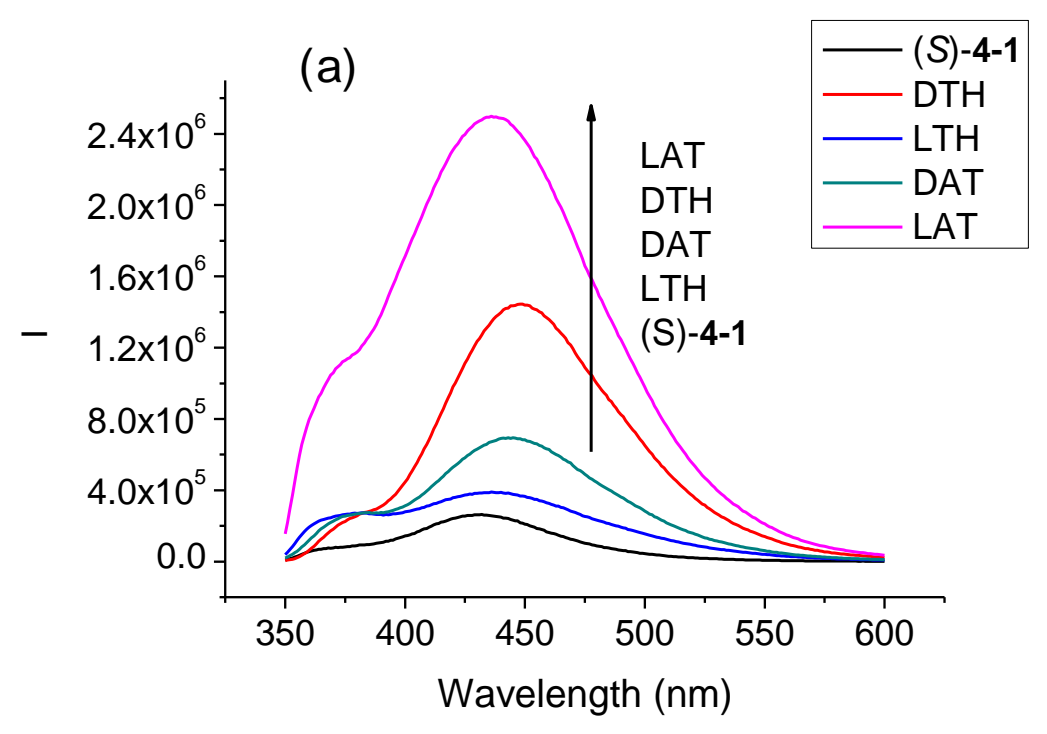




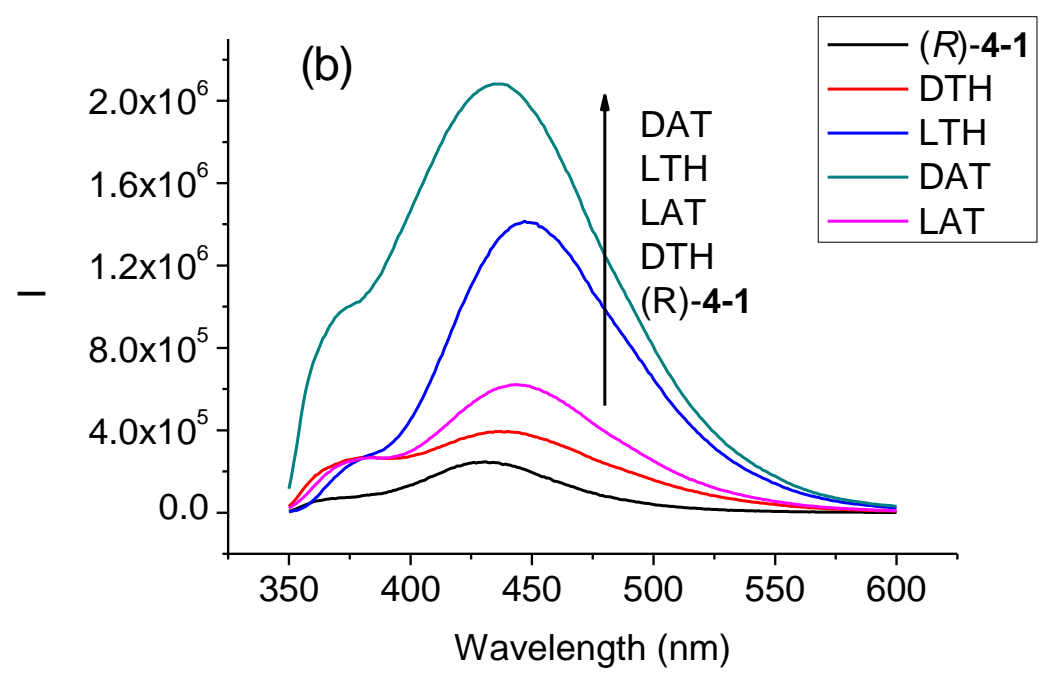

Figure 4-2b gives the fluorescence spectra of $(R)-4-1$ when treated with the four stereoisomers of the N-protected threonines. The fluorescence responses of Figure 4-2a and Figure 4-2b have a mirror image relationship for the enantiomeric amino acids. As shown in Figure 4-2b, DAT causes large fluorescence enhancement at both $\lambda_{1}$ and $\lambda_{2}$ and LTH enhances $\lambda_{2}$ greatly but not at $\lambda_{1}$. Both LAT and DTH show smaller enhancement at $\lambda_{1}$ and $\lambda_{2}$, but LAT enhances $\lambda_{2}$ more than DTH.

Figure 4-3a plots the fluorescence enhancement of $(S)-\mathbf{4 - 1}$ at $\lambda_{1}\left(\mathrm{I}_{1} / \mathrm{I}_{10}\right)$ in the presence of the four stereoisomers of threonines at various concentrations. It shows that in the acid concentration $\leq 2.0 \times 10^{-3} \mathrm{M}$, LAT enhances the fluorescence of $(S)$-4-1 much greater than the other three stereoisomers with $\mathrm{I}_{1} / \mathrm{I}_{10}$ up to 14.3 . Thus, the emission of (S)-4-1 at $\lambda_{1}$ can be used to detect LAT. When the concentration of acid was greater than $2.5 \times 10^{-3} \mathrm{M}$, all the four stereoisomers cause similar fluorescence enhancement of 
(S)-4-1. Figure 4-3b shows that the emission of $(R)-4-1$ at $\lambda_{1}$ can be used to sense DAT among the four stereoisomers in the acid concentration $\leq 2.0 \times 10^{-3} \mathrm{M}$.

Figure 4-3. Fluorescence enhancements of (a) $(S)-4-1$ and (b) $(R)-4-1$ both at $5.0 \times 10^{-4}$ M with varying acid concentrations. $\left(I_{1}\right.$, fluorescence intensity at $370 \mathrm{~nm}$ with the acid, and $\mathrm{I}_{10}$ without the acid. $\lambda_{\mathrm{exc}}=341 \mathrm{~nm}$, slit $=3.0 / 3.0 \mathrm{~nm}$ ).
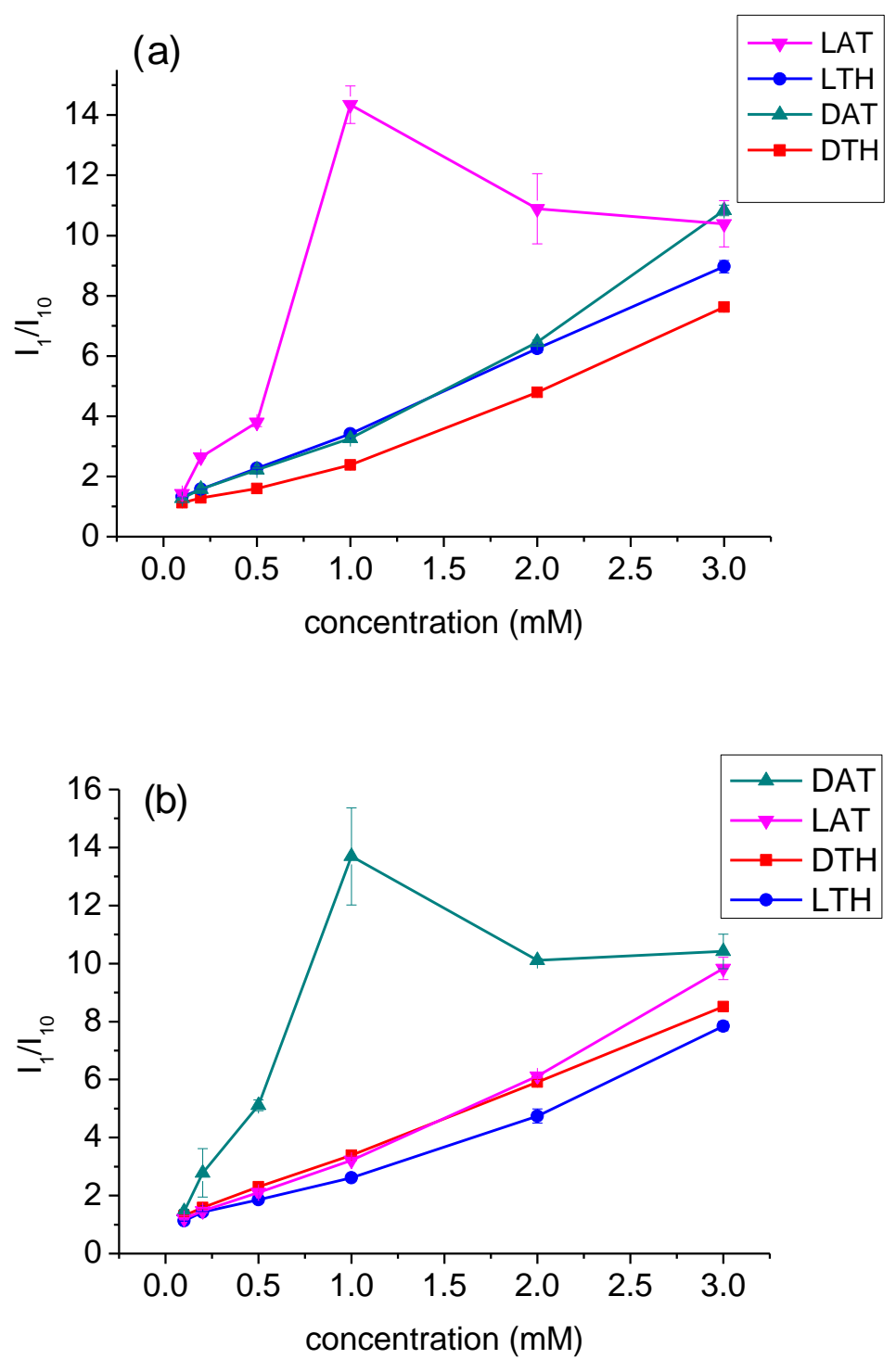
Figure 4-4. Interactions of $(S)$-4-1 (toluene/2\% DME, $5.0 \times 10^{-4} \mathrm{M}$ ) with LAT at varying concentrations (from left to right: $0,1 \mathrm{E}-4,2 \mathrm{E}-4,5 \mathrm{E}-4,1 \mathrm{E}-3,2 \mathrm{E}-3,3 \mathrm{E}-3,4 \mathrm{E}-3,5 \mathrm{E}-3 \mathrm{M}$ ).

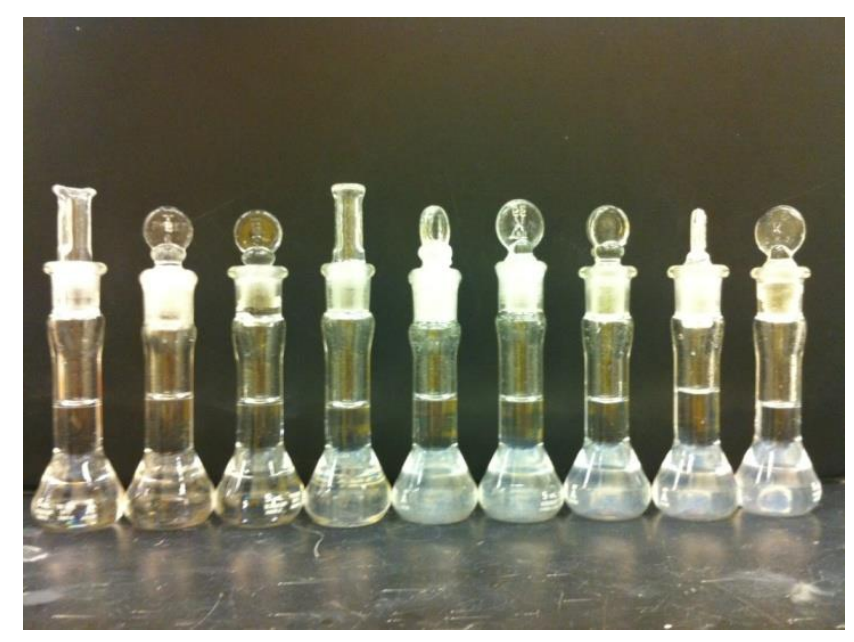

In addition, an enantioselective precipitation was also observed for the interaction of the sensor with LAT and DAT. When LAT $\left(\geq 5.0 \times 10^{-4} \mathrm{M}\right)$ was added to a solution of (S)-4-1 in toluene $(2 \% \mathrm{DME})\left(5.0 \times 10^{-4} \mathrm{M}\right)$, a white precipitate was produced immediately (Figure 4-4). As the concentration of LAT increased, more precipitates were generated. Under the same conditions, when DAT was added to a solution of (S)-4-1 in the concentration range of $0-3 \times 10^{-3} \mathrm{M}$, the solution remained clear. When the enantiomer $(R)$-4-1 was used, precipitation with DAT was observed but not with LAT. Thus, LAT and DAT can be visually detected by using $(S)$-4-1 and $(R)-4-1$.

Figure 4-5. Plots of $\mathrm{I}_{2} / \mathrm{I}_{1}$ for (a) $(S)-4-1$ and (b) (R)-4-1 versus the concentration of the stereoisomeric threonines. $\left(\mathrm{I}_{1}\right.$ : fluorescence intensity at $370 \mathrm{~nm} . \mathrm{I}_{2}$ : fluorescence intensity at $445 \mathrm{~nm}$. Solvent: toluene $/ 2 \%$ DME. $\lambda_{\text {exc }}=341 \mathrm{~nm}$, slit $=3.0 / 3.0 \mathrm{~nm}$ ) 

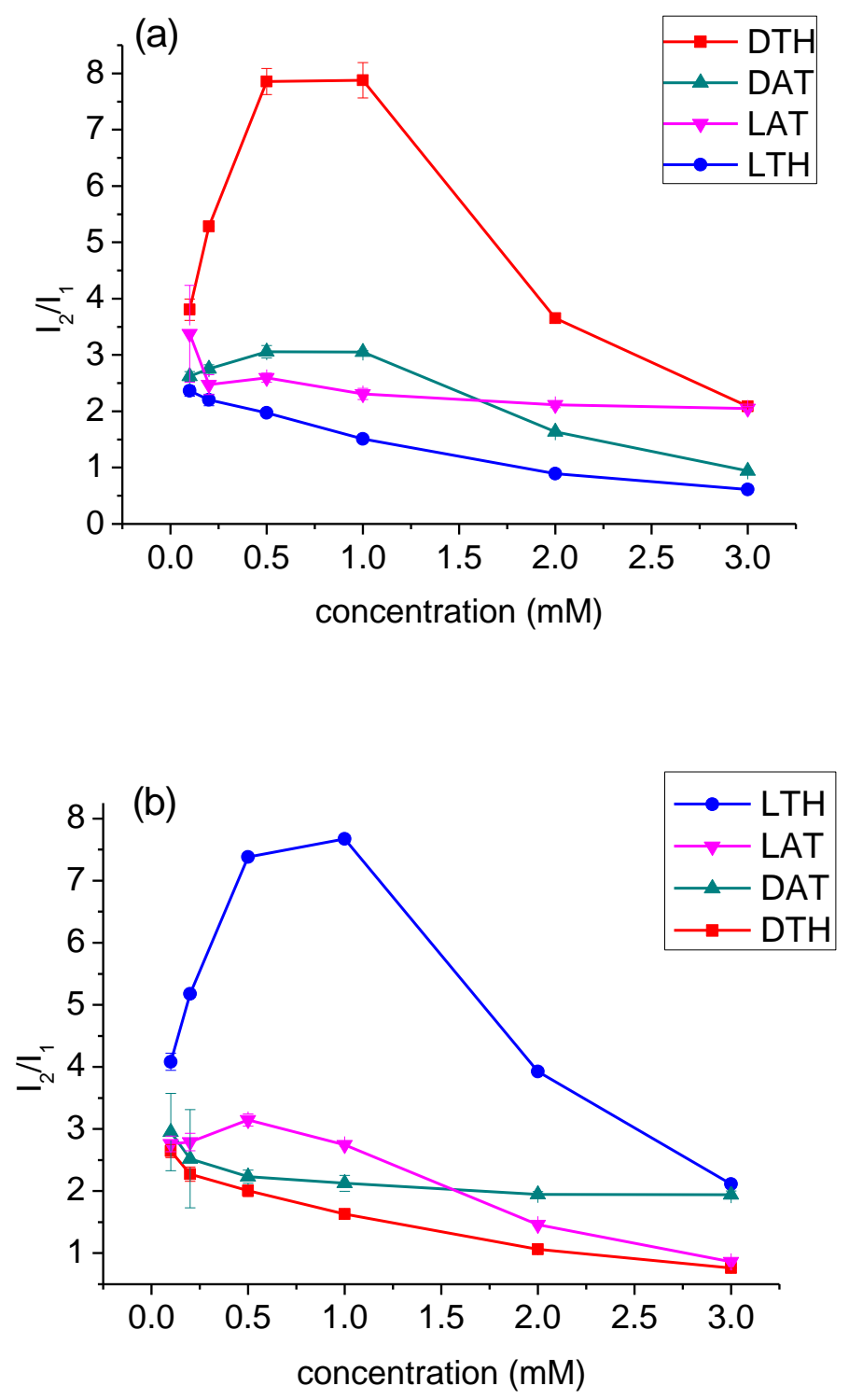

Because the two emission wavelengths of the sensor exhibit different responses toward the stereoisomers of threonines, we have plotted the ratio of the fluorescence intensity of $(S)$-4-1 at $\lambda_{2}$ and $\lambda_{1}\left(\mathrm{I}_{2} / \mathrm{I}_{1}\right)$ against the concentrations of the acids. As shown in Figure 4-5a, when the acid concentration $\leq 2.0 \times 10^{-3} \mathrm{M}$, DTH increases the $\mathrm{I}_{2} / \mathrm{I}_{1}$ ratio much greater than the other three stereoisomers with $I_{2} / I_{1}$ up to 7.8. Thus, the $I_{2} / I_{1}$ ratio 
of $(S)-4-1$ can be used to sense DTH. When the concentration of acid is greater than 2.5 x $10^{-3} \mathrm{M}$, the $\mathrm{I}_{2} / \mathrm{I}_{1}$ ratio of $(S)-4-1$ in the presence of all the four stereoisomers becomes close. Figure 4-5b shows that the fluorescence intensity ratio $\mathrm{I}_{2} / \mathrm{I}_{1}$ of $(R)-\mathbf{4 - 1}$ can be used to detect LTH out of the four stereoisomers when the acid concentration $\leq 2.0 \times 10^{-3}$ M.

We have studied the fluorescence responses of $(S)-4-1$ and $(R)-4-1$ toward samples containing a mixture of the threonine stereoisomers. The samples were prepared by gradually increasing the percentage of one stereoisomer in the mixture of three other stereoisomers whose proportion was maintained at 1:1:1. The top curve of Figure 4-6a shows the fluorescence enhancement of $(S)-4-1$ at $\lambda_{1}\left(\mathrm{I}_{1} / \mathrm{I}_{10}\right)$ versus the increasing percentage of LAT in the mixture. The bottom curve of Figure 4-6a shows the fluorescence enhancement of $(S)-4-1$ at $\lambda_{1}\left(\mathrm{I}_{1} / \mathrm{I}_{10}\right)$ when treated with the optically pure LAT at concentrations corresponding to those in the mixture. Thus, at the same amount of LAT, the mixture causes a greater fluorescence enhancement than the optically pure LAT because of the influence of the other three stereoisomers. Figure 4-6a indicates that $(S)$-4-1 can be used to estimate the relative concentration of LAT in the mixture of the four stereoisomers assuming that changing the ratio of the other three stereoisomers should have small effect on the overall fluorescence enhancement. This is supported by Figure 4-3a which shows much smaller effects of the other three stereoisomers on the fluorescence of $(S)-4-1$ than LAT. Figure 4-6b plots the fluorescence enhancement of 
(R)-4-1 at $\lambda_{1}\left(\mathrm{I}_{1} / \mathrm{I}_{10}\right)$ against the increasing percentage of DAT in the mixture as well as the optically pure DAT. Similar to the interaction of $(S)-4-1$ with LAT, $(R)-\mathbf{4 - 1}$ can be used to estimate the relative concentration of DAT in the mixture of the four stereoisomers. The differences between Figure 4-6a and 4-6b are attributed to the experimental errors in measuring the fluorescence of the mixtures. Therefore, these plots only provide estimates for the relative concentration of LAT and DAT.

Figure 4-6. (a) Plots of $I_{1} / I_{0}$ of $(S)-4-1$ versus the concentration of the optically pure LAT and the percent of LAT in the mixture of the four stereoisomers. (b) Plots of $\mathrm{I}_{1} / \mathrm{I}_{0}$ of (R)-4-1 versus the concentration of the optically pure DAT and the percent of DAT in the mixture of the four stereoisomers (total acid concentration in the mixture: $0.5 \mathrm{mM}$. Solvent: toluene $/ 2 \%$ DME. $\lambda_{\mathrm{exc}}=341 \mathrm{~nm}$, slit $=3.0 / 3.0 \mathrm{~nm}$ )

(a)

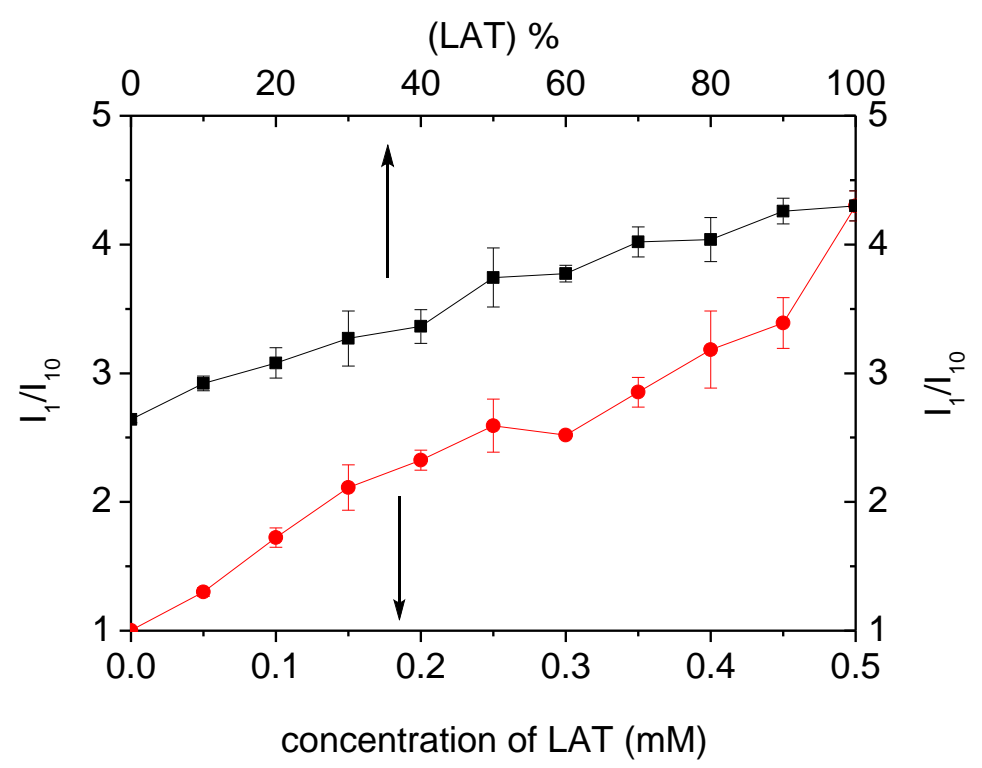




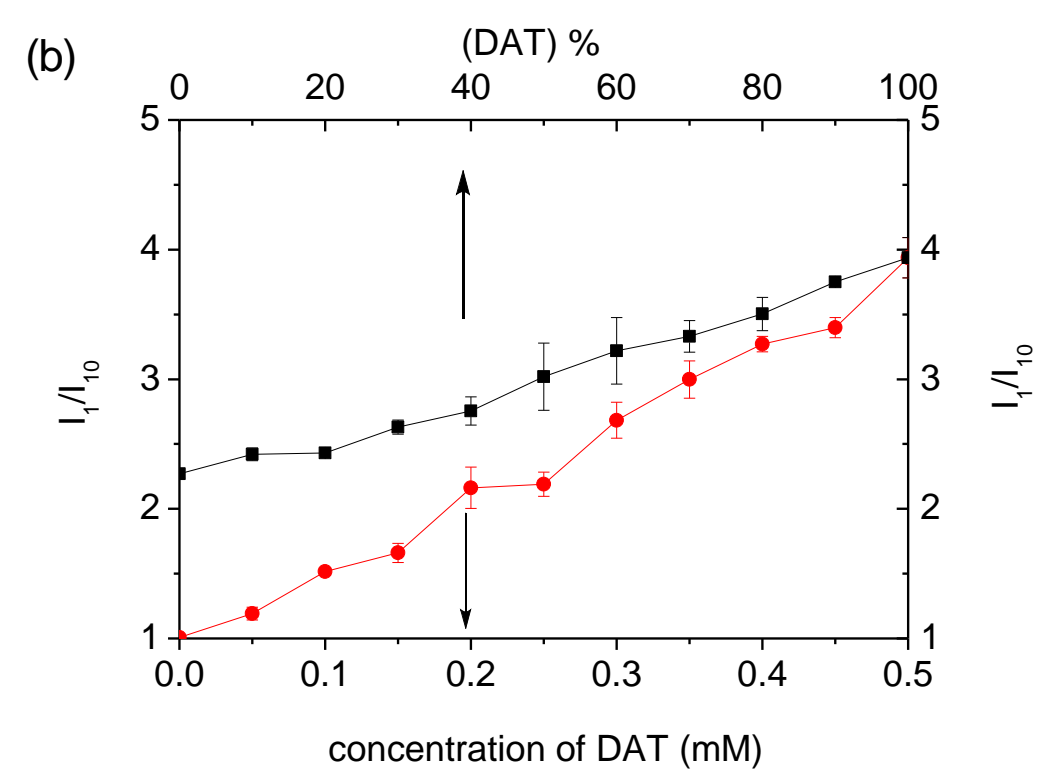

The fluorescence intensity ratio $\left(\mathrm{I}_{2} / \mathrm{I}_{1}\right)$ of $(S)$-4-1 versus the increasing percentage of DTH in the mixture of the stereoisomers is given by the bottom curve of Figure 4-7a. The top curve of Figure 4-7a gives the fluorescence intensity ratio $\left(\mathrm{I}_{2} / \mathrm{I}_{1}\right)$ of $(S)-4-1$ when treated with the optically pure DTH at concentrations corresponding to those in the mixture. At the same amount of DTH, the optically pure DTH generates a greater $\mathrm{I}_{2} / \mathrm{I}_{1}$ than the mixture due to the effect of the other stereoisomers. Assuming that the effect of the ratio among the other three stereoisomers in the mixture on $\mathrm{I}_{2} / \mathrm{I}_{1}$ is small as indicated by Figure 4-5a, Figure 4-7a would allow the relative concentration of DTH in the mixture of the four stereoisomers to be estimated. Similarly, from Figure 4-7b, $(R)-\mathbf{4 - 1}$ can be used to estimate the relative concentration of DTH in the mixture of the four stereoisomers. 
Figure 4-7. (a) Plots of $I_{2} / I_{1}$ of $(S)-4-1$ versus the concentration of the optically pure DTH as well as the percent of DTH in the mixture of four stereoisomers. (a) Plots of $I_{2} / I_{1}$ of (R)-4-1 versus the concentration of the optically pure LTH as well as the percent of LTH in the mixture of four stereoisomers. (Total acid concentration in the mixture: $0.5 \mathrm{mM}$. Solvent: toluene $/ 2 \%$ DME. $\left.\lambda_{\mathrm{exc}}=341 \mathrm{~nm}, \mathrm{slit}=3.0 / 3.0 \mathrm{~nm}\right)$

(a)

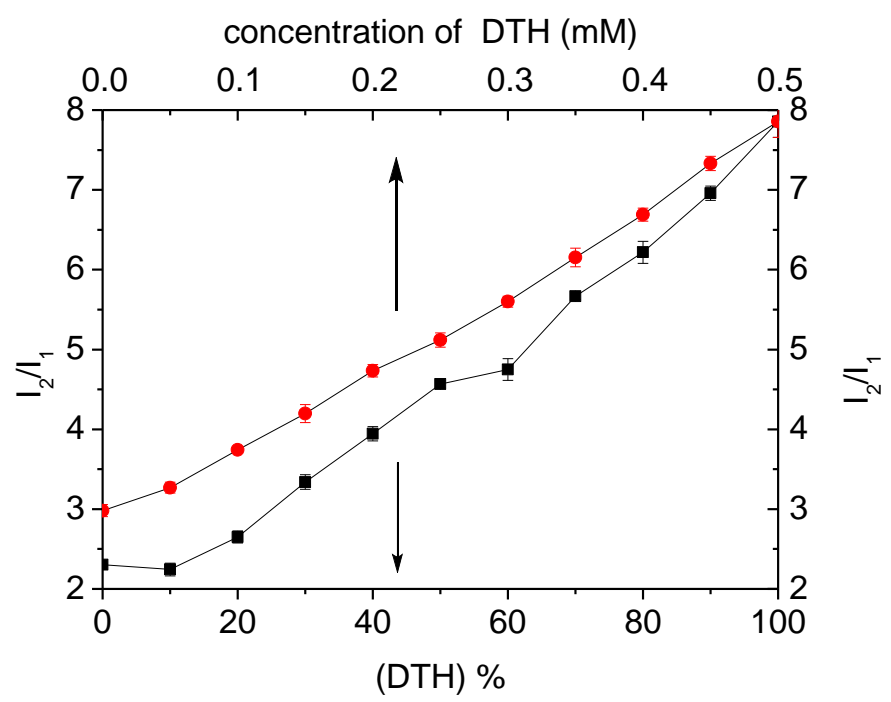

(b)

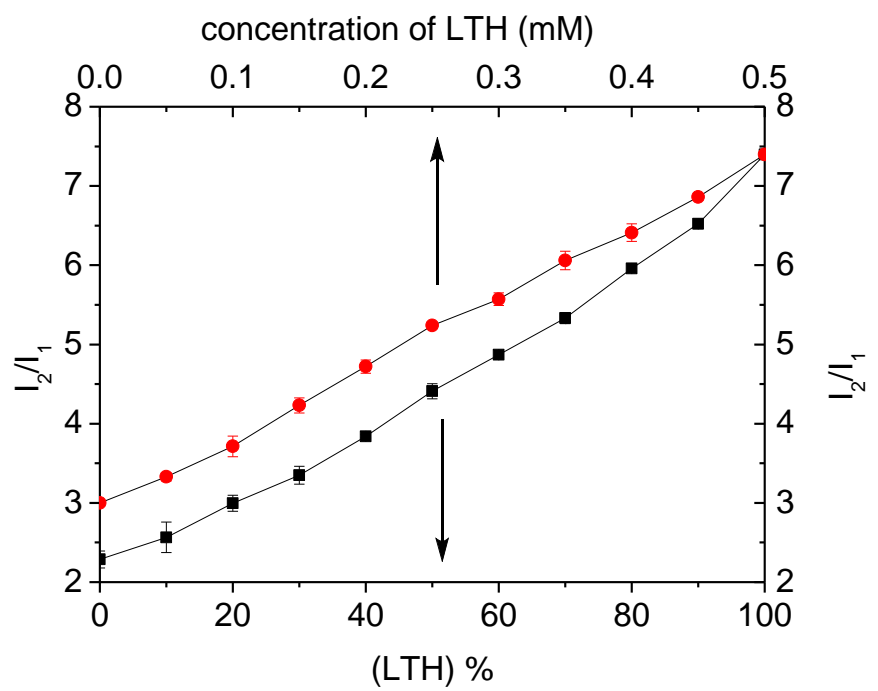




\section{Conclusion}

In summary, the chiral BINOL-amino alcohol enantiomeric pair $(S)-4-1$ and $(R)-4-1$ is found to be an efficient enantioselective and diastereoselective fluorescent sensor pair for the detection of all the four stereoisomers of the threonine derivatives. This work represents the first example that the four stereoisomers of a compound with two chiral centers can be discriminated by using a molecular fluorescent sensor. The enantioselective precipitation of the sensor by the amino acids also makes it possible for visual discrimination. In this study, we have utilized a sensor that exhibits different responses toward the stereoisomeric substrates at two emission wavelengths to expand its capability for both enantioselective and diastereoselective fluorescent sensing. Further development of this strategy has potential to significantly facilitate the discovery of stereoselective fluorescent sensors for chiral organic compounds.

\section{Experimental section}

\subsection{General Data}

All reactions were carried out under nitrogen unless otherwise noted. All chemicals were purchased from Sigma Aldrich Chemical Co. or Alfa Aesar. THF was distilled over sodium and benzophenone under nitrogen atmosphere. Methylene chloride and diethyl ether were dried by passing through activated alumina columns under nitrogen. Solvents were stored over $4 \AA$ A molecular sieves. 
NMR spectra were recorded on a Varian-300 MHz spectrometer. High resolution mass spectra were obtained from the University of Illinois at Urbana-Champaign (UIUC) Mass Spectrometry Facility. Steady-state fluorescence emission spectra were recorded on a Horiba FluoroMax-4 spectrofluorometer.

\subsection{Synthesis and Characterization of Compounds.}

Synthesis and Characterization of Sensor (S)-4-1 and (R)-4-1. Our previously reported procedure was applied: $(S)$-3,3'-DiformylBINOL (224 mg, $0.65 \mathrm{mmol}$ ) was dissolved in $\mathrm{CH}_{2} \mathrm{Cl}_{2}(20 \mathrm{~mL})$ in the presence of $4 \AA$ molecular sieves and combined with $(1 R, 2 S)$-2-amino-1,2-diphenylenthanol $(279 \mathrm{mg}, 1.30 \mathrm{mmol})$. The reaction mixture was heated at reflux for overnight, and monitored by using ${ }^{1} \mathrm{H}$ NMR spectroscopy. When the reaction was complete, the solution was cooled to room temperature. After filtration and evaporation, the resulting yellow condensation compound was dissolved in methanol $(25 \mathrm{~mL})$ and cooled down to $0{ }^{\circ} \mathrm{C}$. $\mathrm{NaBH}_{4}(99 \mathrm{mg}, 2.60 \mathrm{mmol})$ was added in small portions. The reaction temperature was maintained at $0{ }^{\circ} \mathrm{C}$ until the solution became colorless and transparent. Then, it was allowed to proceed at room temperature for additional $30 \mathrm{~min}$. Methanol was removed by roto-evaporation, and the residue was dissolved in ethyl acetate $(50 \mathrm{~mL})$ and washed with water $(15 \mathrm{~mL})$. The aqueous layer after separation was extracted with ethyl acetate $(3 \times 30 \mathrm{~mL})$. The combined organic layer was washed with brine $(15 \mathrm{~mL})$ and dried over anhydrous $\mathrm{Na}_{2} \mathrm{SO}_{4}$. After 
evaporation of the solvent, the residue was purified by flash column chromatography on silica gel eluted with hexanes/ethyl acetate (3/1) to afford $(S)-\mathbf{4 - 1}$ as a white solid in $85 \%$ yield. $\quad{ }^{1} \mathrm{H}$ NMR $\left(300 \mathrm{MHz}, \mathrm{CDCl}_{3}\right) \delta 3.86(\mathrm{~d}, \mathrm{~J}=13.5 \mathrm{~Hz}, 2 \mathrm{H}), 3.92(\mathrm{~d}, \mathrm{~J}=4.8 \mathrm{~Hz}, 2 \mathrm{H})$, $4.16(\mathrm{~d}, \mathrm{~J}=13.8 \mathrm{~Hz}, 2 \mathrm{H}), 4.98(\mathrm{~d}, \mathrm{~J}=5.1 \mathrm{~Hz}, 2 \mathrm{H}), 6.96-6.99(\mathrm{~m}, 4 \mathrm{H}), 7.06-7.31(\mathrm{~m}$, 22H), $7.48(\mathrm{~s}, 2 \mathrm{H}), 7.73(\mathrm{~d}, \mathrm{~J}=6.3 \mathrm{~Hz}, 2 \mathrm{H})$. The enantiomer $(R)-\mathbf{4 - 1}$ was obtained in the same way by using $(R)-3,3^{\prime}$-DiformylBINOL and (1S, 2R)-2-amino-1,2-diphenylenthanol.

Synthesis and Characterization of DAT and LAT. D-allo-threonine (101.4 mg, $0.85 \mathrm{mmol})$ was dissolved in a mixture of saturated $\mathrm{NaHCO}_{3}(10 \mathrm{~mL})$ and THF $(10 \mathrm{~mL})$, and the whole mixture was cooled to $0{ }^{0} \mathrm{C}$. To this solution, benzyl chloroformate (146 $\mu \mathrm{L}, 1.02 \mathrm{mmol}$ ) was added at $0{ }^{0} \mathrm{C}$ and the resulting mixture was stirred for $1 \mathrm{~h}$ at $0{ }^{0} \mathrm{C}$. After quenching the reaction with $3 \mathrm{M}$ aqueous $\mathrm{HCl}$ at $0{ }^{0} \mathrm{C}$, the organic layer was washed with $3 \mathrm{M}$ aqueous $\mathrm{HCl}$ and brine and dried over anhydrous sodium sulfate. After evaporation of the solvent, the residue was purified by flash column chromatography on silica gel eluted with methylene chloride/methanol (10/1) to afford DAT as a white solid in $75 \%$ yield. ${ }^{1} \mathrm{H}$ NMR $\left(300 \mathrm{MHz}, \mathrm{CDCl}_{3}\right) \delta 1.25(\mathrm{~d}, \mathrm{~J}=6.3 \mathrm{~Hz}, 3 \mathrm{H}), 4.14(\mathrm{br}, 1 \mathrm{H})$, 4.37 (br, 1H), 5.09 (s, 2H), $5.94(\mathrm{~d}, \mathrm{~J}=7.5 \mathrm{~Hz}, 1 \mathrm{H}), 6.06(\mathrm{br}, 2 \mathrm{H}), 7.32(\mathrm{br}, 5 \mathrm{H}) .{ }^{13} \mathrm{C}$ NMR (75 MHz, $\left.\mathrm{CDCl}_{3}\right) \delta 19.2,59.5,67.8,69.3,128.4,128.6,128.8,136.0,157.0,173.5$. HRMS Calcd for $\mathrm{C}_{12} \mathrm{H}_{15} \mathrm{NO}_{5}\left(\mathrm{MH}^{+}\right)$: 276.0848. Found: 276.0846. The enantiomer LAT was obtained in the same way by using L-allo-threonine. 


\subsection{Preparation of Samples for Fluorescence Measurement}

Sensors were purified by column chromatography and then stored in a refrigerator. N-benzyloxycabonyl-L-threonine and N-benzyloxycabonyl-D-threonine were purchased from Alfa Aesar. N-benzyloxycabonyl-D-allothreonine and N-benzyloxycabonyl-Lallothreonine were purified by column chromatography and recrystallized with ethyl acetate/hexanes. All of the solvents were either HPLC or spectroscopic grade. The $0.0025 \mathrm{M}$ stock solutions of the sensors in toluene were freshly prepared for each measurement. A $0.025 \mathrm{M}$ stock solution of $\alpha$-N-Cbz-amino acids was freshly prepared using toluene containing 10\% (v) DME. DME was added to improve the solubility of the substrates. For the fluorescence enhancement study, a sensor solution was mixed with $\alpha-\mathrm{N}-\mathrm{Cbz}$-amino acid solution at room temperature in a $5 \mathrm{~mL}$ volumetric flask and diluted to the desired concentration. The resulting solution was allowed to stand at room temperature for $2 \mathrm{~h}$ before the fluorescence measurement. 


\section{References}

1. A review: Pu, L. Chem. Rev. 2004, 104, 1687-1716.

2. Selected references: (a) James, T. D.; Sandanayake, K. R. A. S.; Shinkai, S. Nature 1995, 374, 345-347. (b) Pugh, V.; Hu, Q. -S.; Pu, L. Angew. Chem. Int. Ed. 2000, 39, 3638-3641. (c) Reetz, M. T.; Sostmann, S. Tetrahedron 2001, 57, 2515-2520. (d) Korbel, G. A.; Lalic, G.; Shair, M. D. J. Am. Chem. Soc. 2001, 123, 361-362. (e) Jarvo, E. R.; Evans, C. A.; Copeland, G. T.; Miller, S. J. J. Org. Chem. 2001, 66, 5522-5527. (f) Beer, G.; Rurack, K.; Daub, J. J. Chem. Soc., Chem. Commun. 2001, 1138-1139. (g) McCarroll, M. E.; Haddadian, F.; Warner, I. M. J. Am. Chem. Soc. 2001, 123; 3173-3174. (h) Wong, W.-L.; Huang, K.-H.; Teng, P.-F.; Lee, C.-S.; Kwong, H.-L. Chem. Commun. 2004, 384-385. (i) Zhao, J.-Z.; Fyles, T. M.; James, T. D. Angew. Chem. Int. Ed. 2004, 43, 3461-3464. (h) Pagliari, S.; Corradini, R.; Galaverna, G.; Sforza, S.; Dossena, A.; Montalti, M.; Prodi, L.; Zaccheroni, N.; Marchelli, R. Chem. Eur. J. 2004, 10, 2749-2758. (i) Zhu, L.; Anslyn, E. V. J. Am. Chem. Soc. 2004, 126, 3676-3677. (j) Mei, X. F.; Wolf, C. J. Am. Chem. Soc. 2004, 126, 14736-14737. (k) Matsushita, H.; Yamamoto, N.; Meijler, M. M.; Wirsching, P.; Lerner, R.A.; Matsushita, M.; Janda, K. D. Mol. Biosyst. 2005, 1, 303-306. (1) Dai, Z. H.; Xu, X. D.; Canary, J. W. Chirality 2005, 17, S227-S233. (m) Yu, S.; Pu, L. J. Am. Chem. Soc. 2010, 132, 17698-17700.

3. James,T. D.; Phillips, M. D.; Shinkai, S. Boronic Acids in Saccharide Recognition; 
RSC: UK, 2006.

4. Fluorescent discrimination of the two enantiomers of amino alcohols, diamines or diacids with two chiral centers was reported: (a) Grady, T.; Harris, S. J.; Smyth, M. R.; Diamond, D.; Hailey, P. Anal. Chem. 1996, 68, 3775-3782. (b) Zhao, J. Z.; James, T. D. Chem. Comm. 2005, 1889-1891. (c) Wang, Q.; Chen, X.; Tao, L.; Wang, L.; Xiao, D.; Yu, X. -Q.; Pu, L. J. Org. Chem. 2007, 72, 97-101. (d) Zheng, Y. -S.; Hu, Y. -J. J. Org. Chem. 2009, 74, 5660-5663.

5. A fluorescent sensor was reported to discriminate L- and D-threonines: Wang, H.; Chan, W. -H.; Lee, A. W. M. Org. Biomol. Chem. 2008, 6, 929-934.

6. Liu, H. L.; Hou, X. L; Pu, L. Org. Lett. 2010, 12, 4172-4175.

7. For an account on the enantioselective fluorescent sensors developed in our laboratory, see: Pu, L. Acc. Chem. Res. 2012, 45, 150-163. 


\section{Chapter 5}

Simultaneous Determination of Both the Enantiomeric

Composition and Concentration of

a Chiral Substrate with One

Fluorescent Sensor 


\section{Introduction}

The potential application of enantioselective fluorescent sensors in rapid chiral assay has attracted significant research activity in this area. In recent years, a number of highly enantioselective fluorescent sensors have been developed for the recognition of chiral substrates such as carboxylic acids, amino acids, amines and amino alcohols. ${ }^{1,2}$ These sensors can be used to determine the enantiomeric composition of a chiral substrate at a given concentration. Thus, an independent method to determine the concentration of the substrate is generally required. That is, two separate measurements are needed in order to determine both the concentration and the enantiomeric composition of a sample. ${ }^{3}$

In 2007, Anslyn reported the use of two UV absorption sensors (one chiral and one achiral with distinctively different absorptions) placed separately in a dual-chamber quartz cuvette to determine both the enantiomeric composition and concentration in one absorption measurement. ${ }^{4}$ In 2010, we reported the use of a pseudoenantiomeric sensor pair in a fluorescent chiral assay. ${ }^{5}$ A pseudoenantiomeric sensor pair contains a mixture of two sensors that have emissions at two different wavelengths $\left(\lambda_{1}\right.$ and $\left.\lambda_{2}\right)$ with the opposite fluorescent responses to the two enantiomers of a chiral molecule. When this pseudoenantiomeric sensor pair is applied to a chiral assay, we have demonstrated that using the fluorescent intensity difference $\left(\mathrm{I}_{1}-\mathrm{I}_{2}\right)\left(\mathrm{I}_{1}\right.$ : fluorescence intensity at $\lambda_{1}$. $\mathrm{I}_{2}$ : fluorescence intensity at $\lambda_{2}$ ) can determine the enantiomeric composition of the substrate 
and using the fluorescence intensity sum $\left(\mathrm{I}_{1}+\mathrm{I}_{2}\right)$ can determine the concentration. That is, one fluorescent measurement could give both data with the use of the sensor mixture.

The above study leads us to propose another fluorescent method to determine both the concentration and enantiomeric composition of a chiral molecule: If a dual emission fluorescent sensor could exhibit a highly concentration dependent emission at $\lambda_{1}$ and a highly enantioselective emission at $\lambda_{2}$, it might be possible to use the fluorescent responses of this sensor at the two emission wavelengths to determine both the concentration and the enantiomeric composition of a chiral molecule. Herein, we wish to report our discovery of the first example of such a system to simultaneously determine both the enantiomeric composition and the concentration of a chiral diamine with one fluorescent sensor.

\section{Results and Discussions}

We synthesized the 1,1'-bi-2-naphthol-based trifluoromethyl ketone molecule $(S)-5-3$ and its enantiomer $(R)-\mathbf{5 - 3}$ as a potential fluorescent sensor for chiral amines according to Scheme 5-1. When (S)-2,2'-bis(methoxymethoxy)-1,1'-binaphthyl, (S)-5-1, is deprotonated by $n$-BuLi followed by addition of ethyl trifluoroacetate, (S)-5-2 is obtained as a yellow oil in $62 \% . \quad \mathrm{CF}_{3} \mathrm{COOH}$ is then added to remove $\mathrm{MOM}$ groups to generate the desired compound (S)-5-3 as an orange solid in $84 \%$ yield. Its specific optical rotation was $[\alpha]_{\mathrm{D}}=-167.5\left(\mathrm{c}=0.355, \mathrm{CHCl}_{3}\right) . \quad$ The ${ }^{19} \mathrm{~F}$ NMR spectrum of $(S)-5-3$ in 
$\mathrm{CDCl}_{3}$ shows a singlet at $\delta-70.06$. The enantiomer (R)-5-3 is also prepared by using the enantiomer of the starting material.

Scheme 5-1. Preparation of Compounds $(S)$ - and $(R)-5-3$.

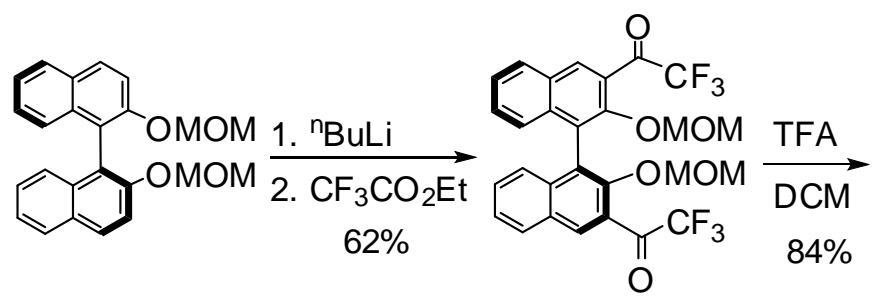

(S) -5-1

(S) -5-2

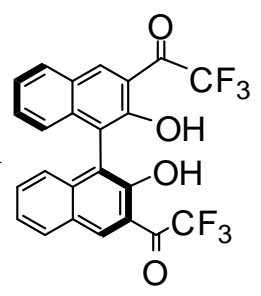

(S)-5-3

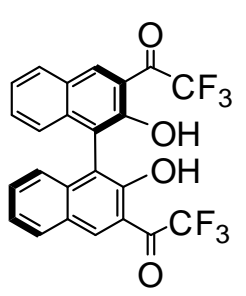

(R)-5-3

The use of trifluoromethyl ketone-based molecular sensors have been studied previously. In 1974, Herman reported the use of a trifluoromethyl aryl ketone for the selective electrochemical detection of carbonates. ${ }^{6}$ It was later established that this selectivity is due to the nucleophilic addition of carbonates to the highly electrophilic trifluoromethyl ketone. $^{7}$ In 1991, Simon reported the use of the trifluoromethyl aryl ketone-based membranes as optical sensors for humidity and ethanol. ${ }^{8}$ In these studies, the nucleophilic addition of water or ethanol to the trifluoromethyl carbonyl group disrupts the extended conjugation, leading to hypsochromic shifts of the absorption band. Further development of the trifluoromethyl ketone-based absorption and fluorescence sensors have been achieved in recent years for the recognition of many nucleophilic species such as alcohols, amines, and various anions. ${ }^{9} \quad$ In 2010, Anh also reported that a binaphthyl-based chiral trifluoromeththyl ketone could be used to distinguish the enantiomers of amino acids by using NMR spectroscopic methods. ${ }^{10}$ In spite of these 
studies, however, no report has appeared on using the trifluoromethyl ketone-based molecules for enantioselective fluorescent recognition.

We studied the optical properties of $(S)-5-3$. The UV spectrum of $(S)-5-3$ in methylene chloride displays absorptions at $\lambda_{\max }(\varepsilon)=228\left(4.5 \times 10^{4}\right), 263\left(5.2 \times 10^{4}\right), 319$ $\left(3.0 \times 10^{4}\right)$ and $432\left(4.8 \times 10^{3}\right) \mathrm{nm}\left(\right.$ Figure 5-1a). When $(S)-5-3\left(1.0 \times 10^{-5} \mathrm{M}\right.$ in $\left.\mathrm{CH}_{2} \mathrm{Cl}_{2}\right)$ was treated with a chiral diamine trans-1,2-diaminocyclohexane $(R, R)$ - or $(S, S)-5-4(5.0 \mathrm{x}$ $10^{-3} \mathrm{M}$ ), there were large absorption decreases at $\lambda_{\max }=263,319$ and $432 \mathrm{~nm}$, a large increase at $\lambda_{\max }=231 \mathrm{~nm}$ and a new absorption at $\lambda_{\max }=345 \mathrm{~nm}$, but no enantioselectivity was observed (Figure 5-1a).

Figure 5-1. UV/Vis absorption spectra (a) and fluorescence spectra (b) of $(S)-5-3(1.0 \times$ $\left.10^{-5} \mathrm{M}\right)$ with/without $(R, R)$ - and $(S, S)-5-4\left(5.0 \times 10^{-3} \mathrm{M}\right)\left(\right.$ Solvent: $\mathrm{CH}_{2} \mathrm{Cl}_{2} \cdot \lambda_{\text {exc }}=343 \mathrm{~nm}$, slit $=2 / 2 \mathrm{~nm}$.).
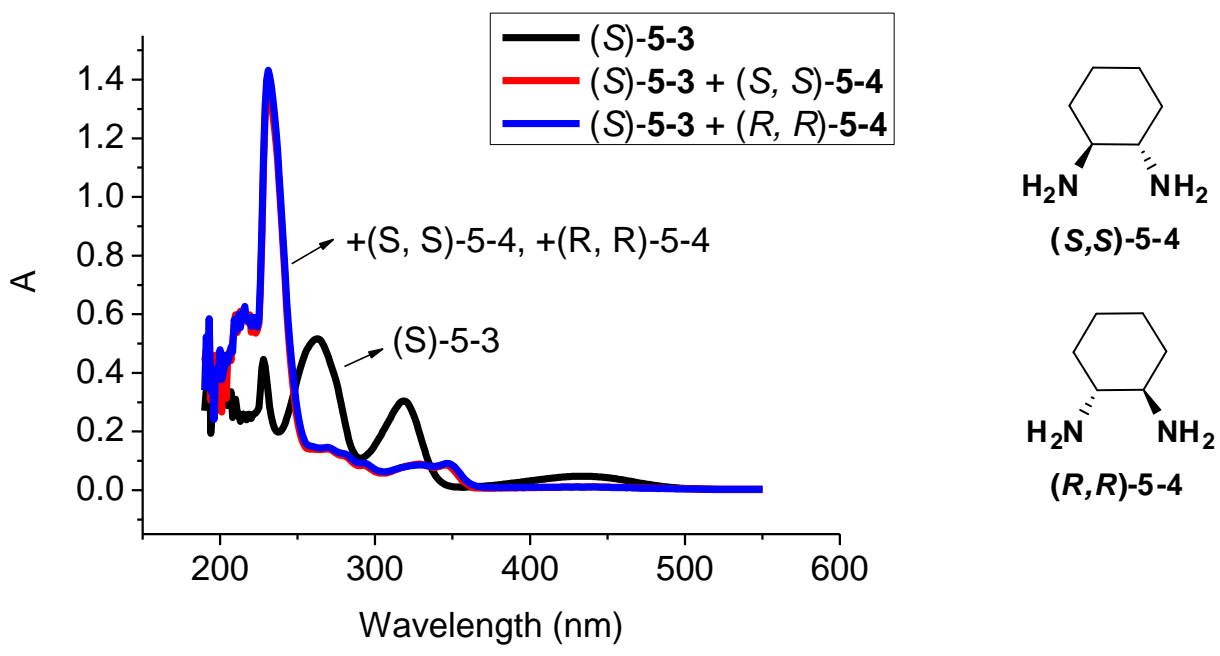

$(R, R)-5-4$ 


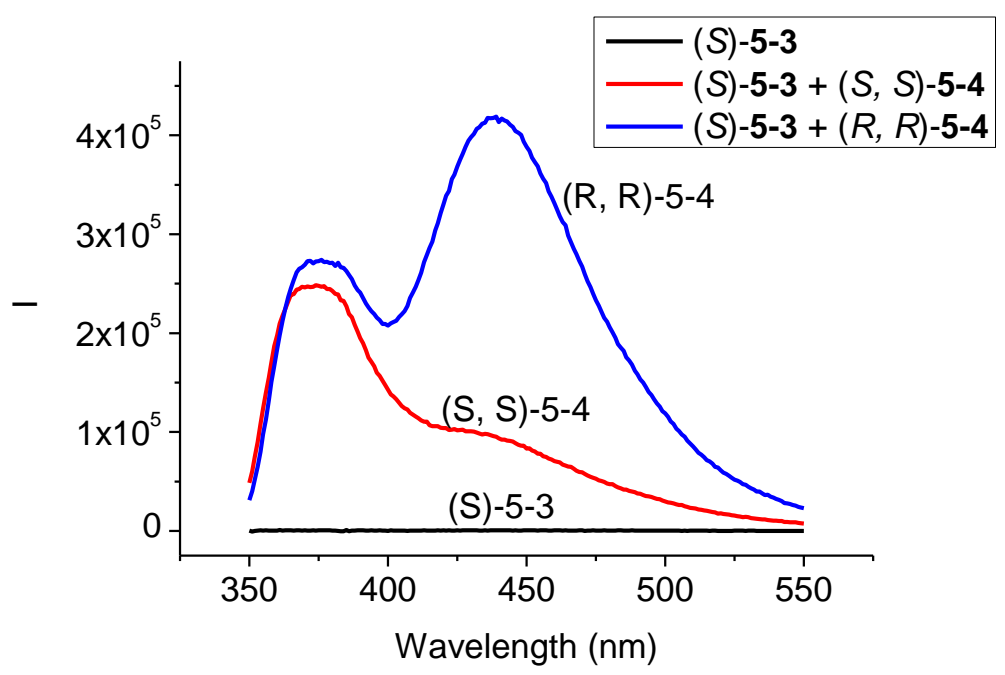

Unlike many 1,1'-binaphthyl molecules, $(S)-\mathbf{5 - 3}$ was found to be nonemissive at all in solution (Figure 5-1b). When its solution $\left(1.0 \times 10^{-5} \mathrm{M}\right.$ in $\left.\mathrm{CH}_{2} \mathrm{Cl}_{2}\right)$ was treated with $(R, R)-5-4\left(5.0 \times 10^{-3} \mathrm{M}\right)$, a dramatic fluorescent enhancement was observed with dual emissions at $370\left(\lambda_{1}\right)$ and $438\left(\lambda_{2}\right) \mathrm{nm}$ (Figure 5-1b). When $(S)$-5-3 was treated with $(S, S)-5-4$, a similar large fluorescence enhancement at $\lambda_{1}$ was also observed, but the fluorescence enhancement at $\lambda_{2}$ was much smaller. Thus, $(S)-5-3$ exhibits high sensitivity toward the chiral diamine at $\lambda_{1}$ and high enantioselectivity at $\lambda_{2}$. This molecule represents a rare example of an enantioselective fluorescent enhancement sensor for a chiral diamine. ${ }^{11}$ We also studied the fluorescence response of $(R)-\mathbf{5 - 3}$, the enantiomer of $(S)-\mathbf{5 - 3}$, toward the chiral diamine. The expected mirror image responses were observed which confirmed the observed chiral discrimination.

Figure 5-2. Plots of $I_{1}(a), I_{2}(b), I_{1} / I_{2}(c)$ for $(S)-5-3\left(1.0 \times 10^{-5} M\right)$ in the presence of 
varying concentrations of $(R, R)$ - and $(S, S)-5-4$. (Fluorescence intensity $\mathrm{I}_{1}$ at $\lambda_{1}=370 \mathrm{~nm}$ and $\mathrm{I}_{2}$ at $\lambda_{2}=438 \mathrm{~nm}$. Solvent: $\mathrm{CH}_{2} \mathrm{Cl}_{2} . \lambda_{\text {exc }}=343 \mathrm{~nm}$, slit $=2 / 2 \mathrm{~nm}$.)
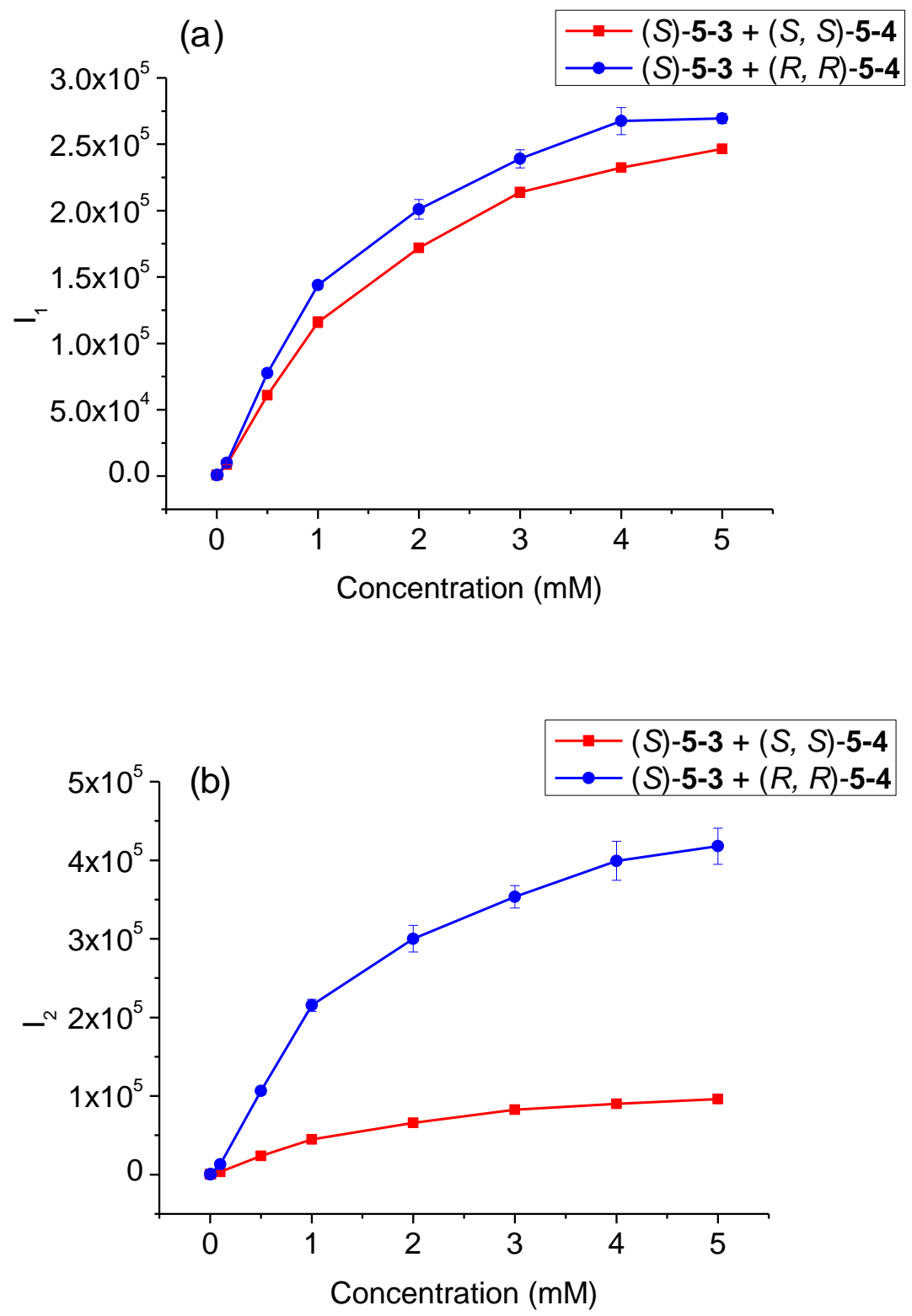


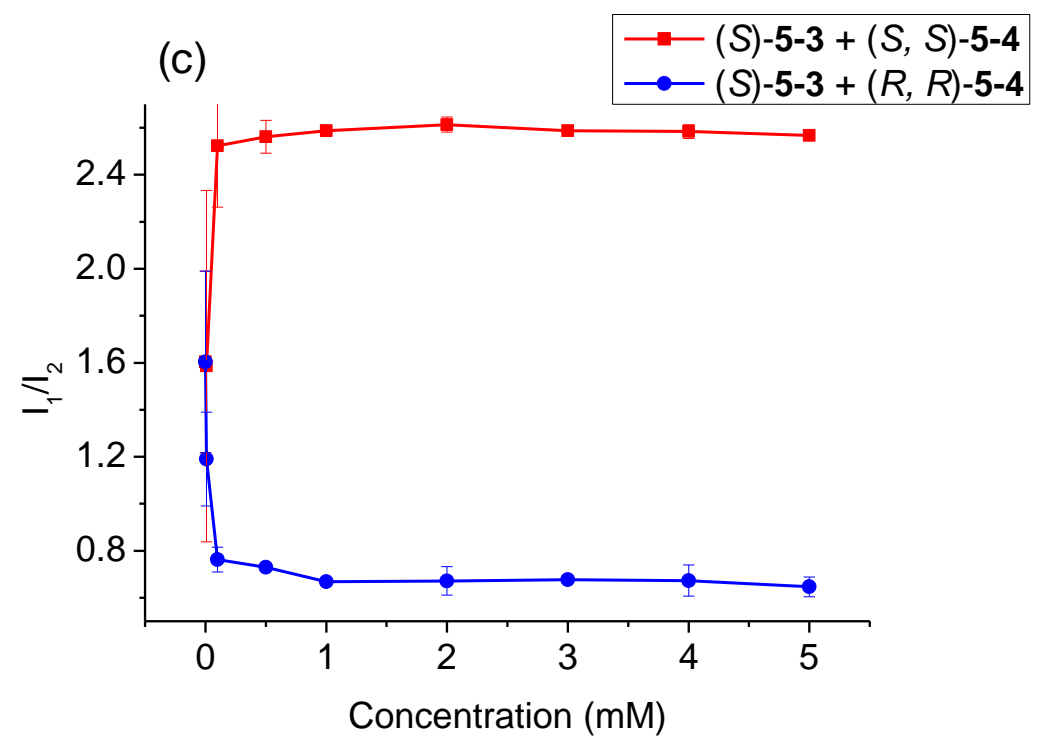

We have studied the effects of the concentration of the chiral diamine on the fluorescence responses of $(S)-\mathbf{5 - 3}$ at $\lambda_{1}$ and $\lambda_{2}$. Figure 5-2a plots the fluorescence intensity $\left(\mathrm{I}_{1}\right)$ of $(S)-\mathbf{5 - 3}$ at $\lambda_{1}$ versus the increasing concentration of $(R, R)$ - and $(S, S)-\mathbf{5 - 4}$. It shows that $I_{1}$ is strongly dependent on the concentration of the dimine but not significantly on its chiral configuration. Figure 5-2b plots the fluorescence intensity $\left(\mathrm{I}_{2}\right)$ of $(S)$-5-3 at $\lambda_{2}$ versus the increasing concentration of $(R, R)-$ and $(S, S)-5-4$ which shows high enantioselectivity. We further found that the fluorescence intensity ratio $I_{1} / I_{2}$ is independent of the concentration of the chiral diamine in the range of $5.0 \times 10^{-4} \mathrm{M}$ to 5.0 $\times 10^{-3} \mathrm{M}$ but is only dependent of the chiral configuration of the substrate. As shown in Figure 5-2c, the $\mathrm{I}_{1} / \mathrm{I}_{2}$ ratio for $(S, S)-5-4$ remains to be constant at 2.60 and that for $(R, R)-5-4$ at 0.67 .

We have plotted $\mathrm{I}_{1} / \mathrm{I}_{2}$ of $(S)-\mathbf{5 - 3}\left(1.0 \times 10^{-5} \mathrm{M}\right.$ in $\left.\mathrm{CH}_{2} \mathrm{Cl}_{2}\right)$ versus $(S, S)-\mathbf{5 - 4} \%$ for the 
chiral diamine samples with varying enantiomeric composition and concentration $(5.0 \mathrm{x}$ $10^{-4} \mathrm{M}-5.0 \times 10^{-3} \mathrm{M}$ ) in Figure 5-3. This plot demonstrates that the enantiomeric purity of the chiral diamine can be determined by measuring the fluorescence responses of $(S)-5-3$ at $\lambda_{1}$ and $\lambda_{2}$ without the need to know the concentration of the sample.

Figure 5-3. Plots of $\mathrm{I}_{1} / \mathrm{I}_{2}$ vs $(S, S)-\mathbf{5 - 4} \%$ at various diamine concentrations $(\mathrm{mM})$ (Solvent: $\mathrm{CH}_{2} \mathrm{Cl}_{2} \cdot \lambda_{\text {exc }}=343 \mathrm{~nm}$, slit $=2 / 2 \mathrm{~nm}$.).

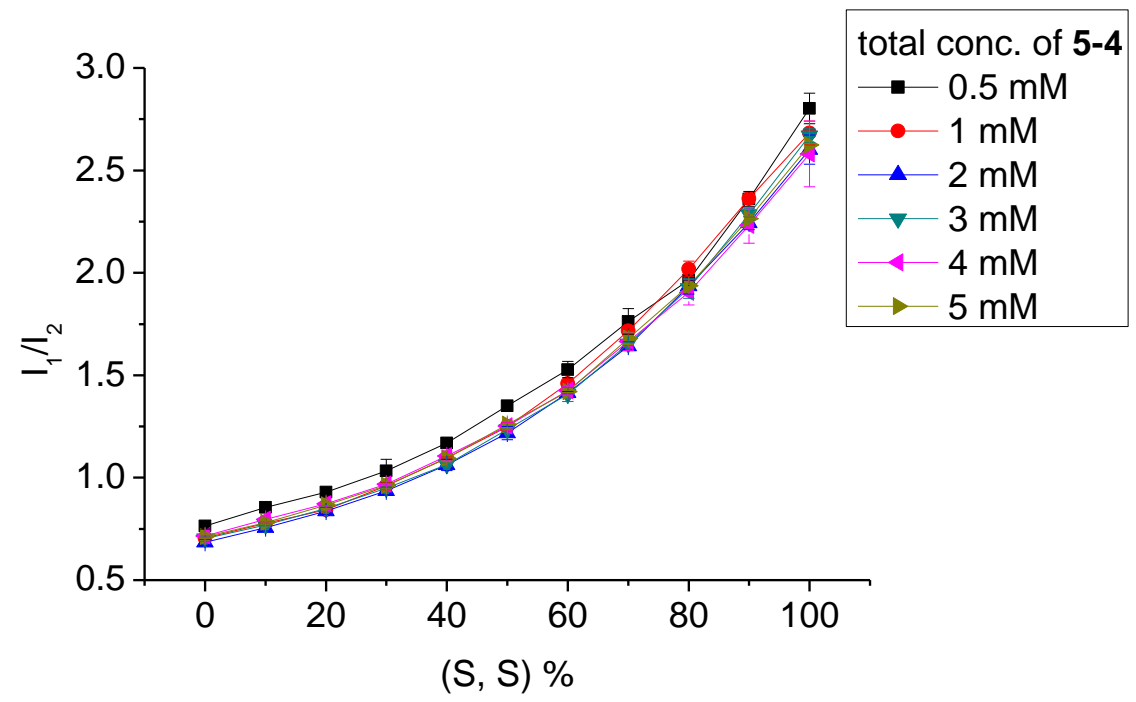

As described above, $\mathrm{I}_{1}$ is strongly influenced by both $(S, S)$ - and $(R, R)-5-4$ (Figure 5-2a) and $I_{1} / I_{2}$ is only dependent on the enantiomeric composition (Figure 5-3). Figure 5-2a also shows that the chiral configuration of the diamine has a small effect on $\mathrm{I}_{1}$. In order to more accurately determine the concentration of the substrate, we have plotted $\mathrm{I}_{1}$ and $I_{1} / I_{2}$ of $(S)$-5-3 against the diamine concentration of the samples containing varying 
compositions of $(S, S)$ - and $(R, R)-5-4$ in Figure 5-4. This plot takes into consideration the effects of the chiral configuration of the diamine. It demonstrates that the concentration of a chiral diamine sample can be determined by measuring the fluorescence responses $\mathrm{I}_{1}$ and $\mathrm{I}_{2}$ of the sensor $(S)-\mathbf{5 - 3}$.

Figure 5-4. Plot of $\mathrm{I}_{1}, \mathrm{I}_{1} / \mathrm{I}_{2}$ vs the total concentration of 5-4 with various enantiomeric composition.

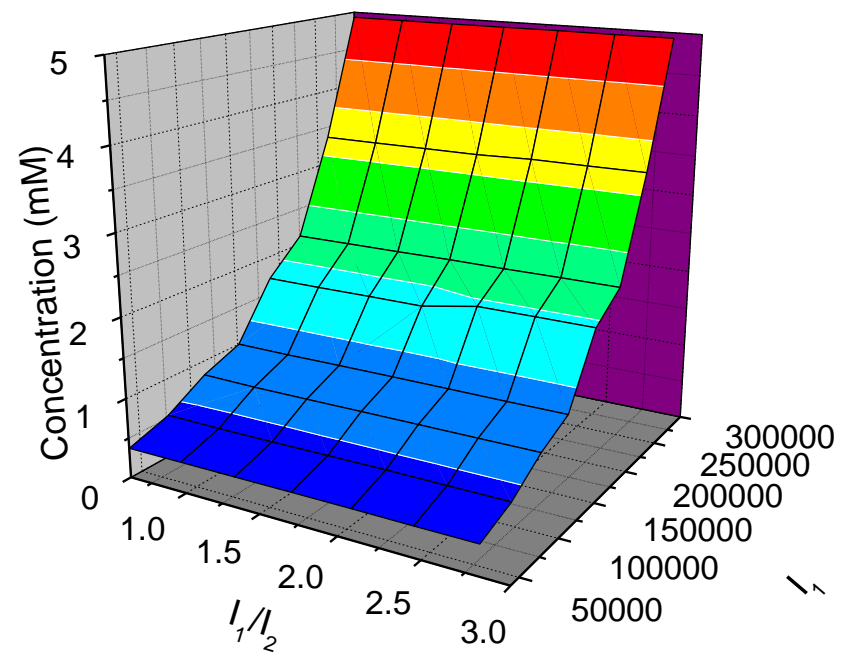

In the above experiments, when a given sample of the chiral diamine is treated with the fluorescent sensor $(S)-5-3$, one fluorescence measurement will give the fluorescence intensity $I_{1}$ and $I_{2}$. By using $I_{1} / I_{2}$, the enantiomeric composition of the sample can be determined from Figure 5-3. By using $I_{1}$ and $I_{1} / I_{2}$, the total concentration of the two enantiomers of the diamine can be determined from Figure 5-4. Therefore, both the 
concentration and the enantiomeric composition of a chiral molecule can be simultaneously determined by one fluorescence measurement with the use of only one fluorescent sensor.

We have applied Figure 5-3 and 5-4 to ananlyze the ee and concentration of five test samples of the chiral dimaine 5-4. As the results summarized in Table 5-1, the values of (S, S)-5-4\% and the sample concentration from the fluorescent measurements had average errors of $10.6 \%$ and $8 \%$, respectively.

Table 5-1. Determination of $(\mathrm{S}, \mathrm{S})-\mathbf{5 - 4} \%$ and concentration of test samples.

\begin{tabular}{|l|c|c|c|c|c|}
\hline sample & 1 & 2 & 3 & 4 & 5 \\
\hline measured (S, S) \% & 99 & 80 & 55 & 26 & 96 \\
\hline actual (S, S) \% & 95 & 75 & 50 & 20 & 95 \\
\hline error & $4 \%$ & $7 \%$ & $10 \%$ & $30 \%$ & $1 \%$ \\
\hline measured concentration (mM) & 0.6 & 0.9 & 1.6 & 2.9 & 3.4 \\
\hline actual concentration (mM) & 0.6 & 1 & 1.8 & 2.5 & 3.5 \\
\hline error & $0 \%$ & $10 \%$ & $11 \%$ & $16 \%$ & $3 \%$ \\
\hline
\end{tabular}

In order to gain further understanding on the interaction of $(S)-5-3$ with the chiral diamine, we have conducted a ${ }^{19} \mathrm{~F}$ NMR titration for the interaction of $(S)-5-3$ with $(S, S)$-5-4. To an NMR tube containing $(S)-5-3(0.4 \mathrm{~mL}, 5 \mathrm{mM})$ in $\mathrm{CDCl}_{3},(S, S)-5-4$ was gradually added. After each addition the solution was mixed well before its ${ }^{19} \mathrm{~F}$ NMR spectrum was taken. The ${ }^{19} \mathrm{~F}$ NMR spectrum of $(S)-5-3$ gave a singlet at $\delta-70.06$. With the addition of $(S, S)-\mathbf{5 - 4}$, two new peaks at $\delta-69.94$ and -83.83 started to appear with the same integration, while the signal of $(S)-5-3$ at $\delta-70.06$ was decreasing and then 
completely disappeared with the addition of 4.7 equiv of the diamine. This indicates the formation of the 1:1 adduct homosemiaminal 5-5 at this stage (Scheme 5-2). ${ }^{9 c, 12 a}$ After that, the signal at $\delta-69.94$ started to decrease while the signal at $\delta-83.83$ was increasing until all the peaks were converted to the peak at $\delta-83.83$ with the addition of 27 equiv of the diamine. This indicates the formation of the $2: 1$ adduct disemiaminal 5-6. ${ }^{9 c, 12 a}$ Further addition of the diamine didn't change the ${ }^{19} \mathrm{~F}$ NMR spectra during the $2 \mathrm{~h}$ period. In the subsequent several days, slow appearance of new peaks at $\delta-72.09$ and -80.64 was observed, which were then slowly converted to the peak at $\delta-80.64$. The signal at $\delta$ -72.09 is attributed to the formation of $5-7^{12 b}$ and that at $\delta-80.64$ is attributed to the formation of aminal 5-8. ${ }^{12 \mathrm{c}}$ Compound 5-8 was also prepared from the reaction of $(S)$-5-3 with $(S, S)-5-4$ in the presence of molecular sieves at room temperature in $2 \mathrm{~d}$. ${ }^{19}$ F NMR titration of $(S)$-5-3 with $(R, R)-5-4$ exhibited similar responses.

The above NMR study has revealed that the addition of the chiral diamine to $(S)-5-3$ led to a fast formation of the amine-ketone adducts 5-5 and 5-6, but the formation of the condensation product 5-7 and the subsequent cycloaddition product 5-8 was slow. Therefore, the observed large fluorescence enhancement of $(S)-5-3$ in the presence of the chiral diamine can be attributed to the formation of 5-5 and 5-6. We have examined the fluorescence spectra of the $(S)-\mathbf{5 - 3}$ solution $\left(1 \times 10^{-5} \mathrm{M}\right)$ with $(S, S)-\mathbf{5 - 4}$ or $(R, R)-\mathbf{5 - 4}$ $\left(5 \times 10^{-3} \mathrm{M}\right)$ over five hours after mixing, and we found no significant change in both the shape and intensity (Figure 5-5). 
Scheme 5-2. A proposed mechanism for the reaction of $(S)-5-3$ with the chiral diamine.

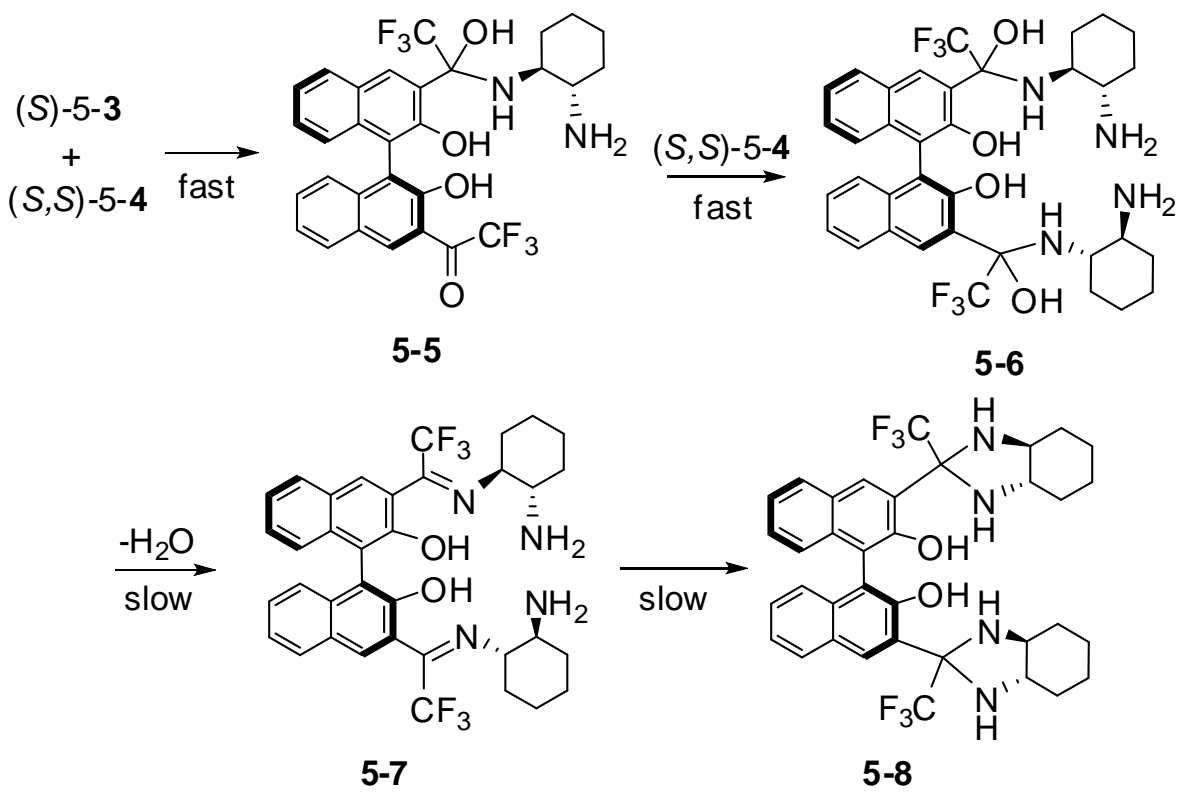

Figure 5-5. Time dependent fluorescence spectra of $(S)-3-3$ solution $\left(1 \times 10^{-5} \mathrm{M}\right)$ with (a) $(S, S)-5-4\left(5 \times 10^{-3} \mathrm{M}\right)$ and (b) $(R, R)-5-4\left(5 \times 10^{-3} \mathrm{M}\right)$. (Solvent: $\mathrm{CH}_{2} \mathrm{Cl}_{2} \cdot \lambda_{\text {exc }}=343 \mathrm{~nm}$, slit $=2 / 2 \mathrm{~nm}$.)

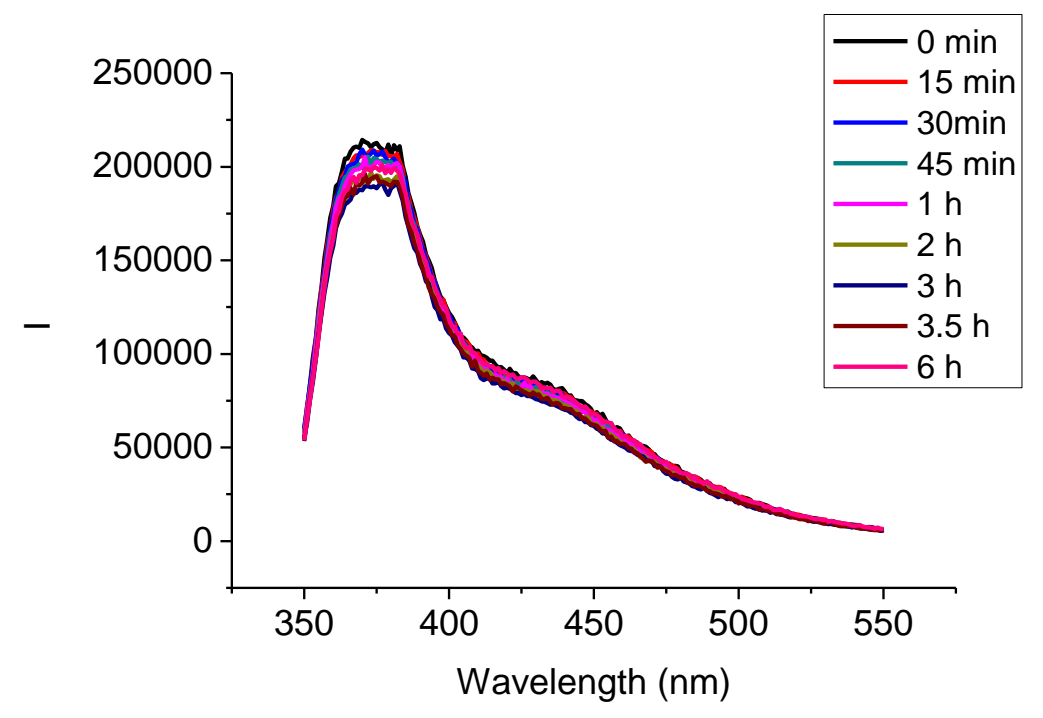




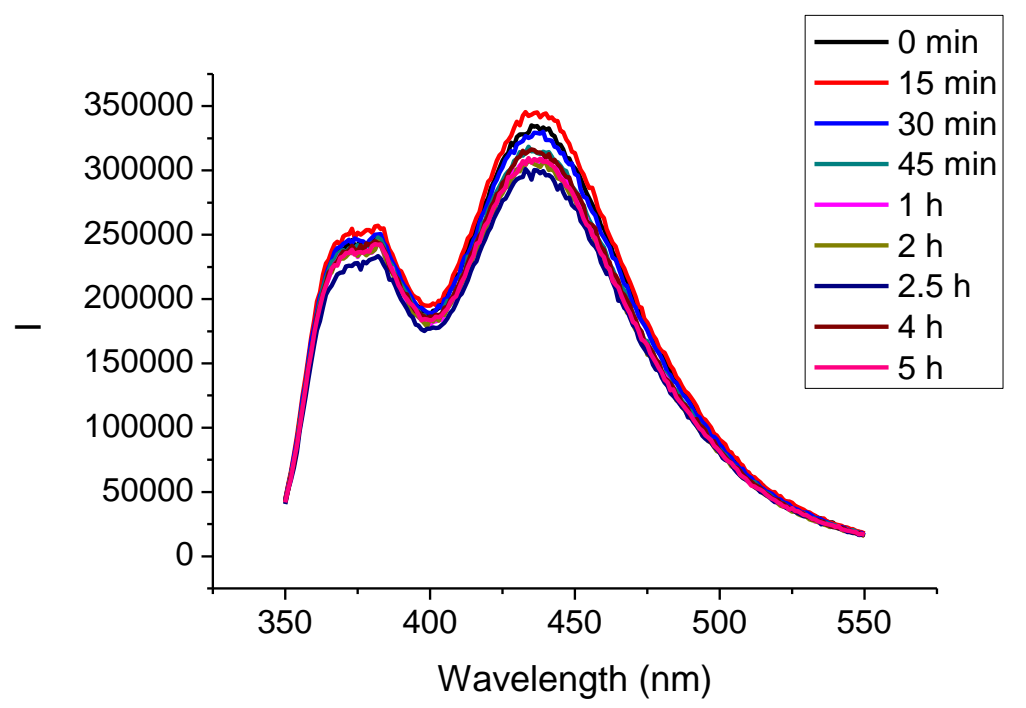

\section{Conclusion}

In summary, we have discovered a fluorescent sensor that exhibits very different fluorescence responses at two emission wavelengths toward a chiral diamine, one with high sensitivity and one with high enantioselectivity. On the basis of this difference in fluorescence response, it has been demonstrated for the first time that both the concentration and enantiomeric composition of a chiral substrate can be determined simultaneously by one fluorescence measurement with the use of only one fluorescent sensor. This system should significantly simplify the application of the enantioselective fluorescent sensor.

\section{Experimental section}

\subsection{General Data}


All reactions were carried out under nitrogen unless otherwise noted. All chemicals were purchased from Sigma Aldrich Chemical Co. or Alfa Aesar. THF was distilled over sodium and benzophenone under nitrogen atmosphere. Methylene chloride and diethyl ether were dried by passing through activated alumina columns under nitrogen. Solvents were stored over $4 \AA$ molecular sieves.

Melting points were uncorrected and obtained on a Mel-Temp II capillary melting point apparatus. Optical rotations were measured on a Jasco P-2000 digital polarimeter. NMR spectra were recorded on a Varian-300 MHz or Bruker-600 MHz spectrometer. Chemical shifts for ${ }^{1} \mathrm{H}$ NMR spectra were reported in parts per million relative to a singlet at $7.26 \mathrm{ppm}$ for deuterated chloroform. Chemical shifts for ${ }^{13} \mathrm{C}$ NMR were reported relative to the centerline of a triplet at $77.16 \mathrm{ppm}$ for deuterated chloroform. The ${ }^{19} \mathrm{~F}$ NMR spectra were reported in units of part per million (ppm) relative to trifluoroacetic acid $(\delta-76.55 \mathrm{ppm})$ as an external reference. High resolution mass spectra were obtained from the University of Illinois at Urbana-Champaign (UIUC) Mass Spectrometry Facility. UV-Vis spectra were recorded on a Hewlett-Packard 8452A diode-array spectrophotometer. Steady-state fluorescence emission spectra were recorded on a Horiba FluoroMax-4 spectrofluorometer.

\subsection{Synthesis and Characterization of Compounds}

Synthesis and Characterization of (S)-5-2. Under nitrogen, $(S)-5-1$ (3.0 mmol, 1.12 
g) was dissolved in ether $(36 \mathrm{~mL})$. The solution was cooled to $0{ }^{\circ} \mathrm{C}$, and $\mathrm{n}-\mathrm{BuLi}(12.0$ mmol, 2.5 $\mathrm{M}$ in hexane, $4.8 \mathrm{~mL}$ ) was added dropwise. The reaction mixture was stirred for $2 \mathrm{~h}$ at room temperature and cooled to $0{ }^{0} \mathrm{C}$, and then ethyl trifluoroacetate $(13.5$ mmol, $1.6 \mathrm{~mL}$ ) was added slowly. The reaction mixture was allowed to warm to room temperature and stirred for $1 \mathrm{~h}$ to afford a cream-like mixture. A saturated aqueous $\mathrm{NH}_{4} \mathrm{Cl}$ solution was added to quench the reaction. The organic layer was separated, and the aqueous layer was extracted with ethyl acetate (three $20 \mathrm{~mL}$ aliquots). The combined organic extracts were washed with brine, and dried over $\mathrm{Na}_{2} \mathrm{SO}_{4}$. After evaporation of the solvent, the residue was purified by column chromatography on silica gel eluted with hexane/methylene chloride (1/3) to afford compound $(S)-5-2$ as a yellow oil in $62 \%$ yield. ${ }^{1} \mathrm{H}$ NMR $\left(300 \mathrm{MHz}, \mathrm{CDCl}_{3}\right) \delta 2.77(\mathrm{~s}, 6 \mathrm{H}), 4.73(\mathrm{~d}, \mathrm{~J}=6.3 \mathrm{~Hz}, 2 \mathrm{H})$, $4.77(\mathrm{~d}, \mathrm{~J}=6.3 \mathrm{~Hz}, 2 \mathrm{H}), 7.25(\mathrm{~d}, \mathrm{~J}=8.7 \mathrm{~Hz}, 2 \mathrm{H}), 7.44-7.50(\mathrm{~m}, 2 \mathrm{H}), 7.53-7.58(\mathrm{~m}, 2 \mathrm{H})$, $8.05(\mathrm{~d}, \mathrm{~J}=8.1 \mathrm{~Hz}, 2 \mathrm{H}), 8.43(\mathrm{~s}, 2 \mathrm{H}) .{ }^{19} \mathrm{~F}$ NMR $\left(300 \mathrm{MHz}, \mathrm{CDCl}_{3}\right) \delta-73.62 .{ }^{13} \mathrm{C}$ NMR $\left(75 \mathrm{MHz}, \mathrm{CDCl}_{3}\right) \delta 56.6,100.7,116.5(\mathrm{q}, \mathrm{J}=290 \mathrm{~Hz}), 126.4,126.8,126.9,127.0$, 129.6, 129.8, 130.1, 132.7, 136.2, 151.6, $182.4(\mathrm{q}, \mathrm{J}=35.6 \mathrm{~Hz})$. HRMS Calcd for $\mathrm{C}_{28} \mathrm{H}_{20} \mathrm{O}_{6} \mathrm{~F}_{6} \mathrm{Na}\left(\mathrm{MNa}^{+}\right): 589.1062$, Found: 589.1053. $[\alpha]_{\mathrm{D}}=-18.056\left(\mathrm{c}=0.590, \mathrm{CHCl}_{3}\right)$.

Synthesis and Characterization of (S)-5-3. After compound (S)-5-2 (134.2 mg, 0.25 mmol) was dissolved in a minimum amount of $\mathrm{CH}_{2} \mathrm{Cl}_{2}$, trifluroacetic acid $(1.0 \mathrm{~mL})$ was added slowly, and the mixture was stirred at room temperature for $10 \mathrm{~min}$. A saturated aqueous $\mathrm{NaHCO}_{3}$ solution was added to quench the reaction. The organic layer was 
separated, and the aqueous layer was extracted with $\mathrm{CH}_{2} \mathrm{Cl}_{2}(3 \times 20 \mathrm{~mL})$. The combined organic extracts were washed with brine, and dried over $\mathrm{Na}_{2} \mathrm{SO}_{4}$. After evaporation of the solvent, the residue was purified by column chromatography on silica gel eluted with hexane/methylene chloride (2/1) to afford compound (S)-5-3 as an orange solid in $84 \%$ yield. ${ }^{1} \mathrm{H}$ NMR (300 MHz, $\left.\mathrm{CDCl}_{3}\right) \delta 7.16(\mathrm{~d}, \mathrm{~J}=7.5 \mathrm{~Hz}, 2 \mathrm{H}), 7.41-7.51$ $(\mathrm{m}, 4 \mathrm{H}), 8.02(\mathrm{~d}, \mathrm{~J}=7.5 \mathrm{~Hz}, 2 \mathrm{H}), 8.70(\mathrm{~s}, 2 \mathrm{H}), 10.51(\mathrm{~s}, 2 \mathrm{H}) . \quad{ }^{19} \mathrm{~F}$ NMR $(282 \mathrm{MHz}$, $\left.\mathrm{CDCl}_{3}\right) \delta-70.06 .{ }^{13} \mathrm{C} \mathrm{NMR}\left(150 \mathrm{MHz}, \mathrm{CDCl}_{3}\right) \delta 115.2,116.7(\mathrm{q}, \mathrm{J}=289.5 \mathrm{~Hz}), 117.8$, $124.7,125.3,127.2,131.2,132.2,136.1(\mathrm{q}, \mathrm{J}=3.75 \mathrm{~Hz}), 138.5,155.0,185.1(\mathrm{q}, \mathrm{J}=36.0$ Hz). HRMS Calcd for $\mathrm{C}_{24} \mathrm{H}_{13} \mathrm{O}_{4} \mathrm{~F}_{6}\left(\mathrm{MH}^{+}\right)$: 479.0718, Found: 479.0719. m.p. $231{ }^{0} \mathrm{C}$. $[\alpha]_{\mathrm{D}}=-167.50\left(\mathrm{c}=0.355, \mathrm{CHCl}_{3}\right)$.

Synthesis and Characterization of $(\boldsymbol{S}, \boldsymbol{S}, \boldsymbol{S})$-5-8. Under nitrogen, (S)-5-3 (0.1 mmol, $47.8 \mathrm{mg})$ was dissolved in $\mathrm{CH}_{2} \mathrm{Cl}_{2}(3 \mathrm{~mL}) .(S, S)-5-4(2.0 \mathrm{mmol}, 228.4 \mathrm{mg})$ and 4 Á molecular sieves were added. The reaction mixture was stirred for 2 days at room temperature. After filtration, the solvent was evaporated and the residue was purified by column chromatography on neutral aluminum oxide eluted with methylene chloride to afford compound $(S, S, S)-\mathbf{5 - 8}$ as a white solid in $65 \%$ yield. ${ }^{1} \mathrm{H}$ NMR $\left(300 \mathrm{MHz}, \mathrm{CDCl}_{3}\right)$ $\delta$ 1.20-1.34 (m, 8H), 1.84-1.87 (m, 4H), 2.06-2.09 (m, 2H), 2.25-2.29 (m, 2H), 2.47-2.58 (m, 8H), 7.27-7.29 (m, 6H), 7.78-7.82 (m, 2H), $8.03(\mathrm{~s}, 2 \mathrm{H}), 13.57(\mathrm{~s}, 2 \mathrm{H}) .{ }^{19} \mathrm{~F}$ NMR $\left(282 \mathrm{MHz}, \mathrm{CDCl}_{3}\right) \delta-80.63 .{ }^{13} \mathrm{C} \mathrm{NMR}\left(150 \mathrm{MHz}, \mathrm{CDCl}_{3}\right) \delta 24.8,24.9,28.8,29.5,64.7$, 65.4, $84.3(\mathrm{q}, \mathrm{J}=28.8 \mathrm{~Hz}), 117.7,122.0,123.4,125.0,125.7(\mathrm{q}, \mathrm{J}=283.5 \mathrm{~Hz}), 127.5$, 
127.6, 128.5, 129.4, 134.5, 152.8. HRMS Calcd for $\mathrm{C}_{36} \mathrm{H}_{37} \mathrm{~N}_{4} \mathrm{O}_{2} \mathrm{~F}_{6}\left(\mathrm{MH}^{+}\right)$: 671.2821, Found: 671.2831. m.p. $194{ }^{0} \mathrm{C} . \quad[\alpha]_{\mathrm{D}}=-217.0\left(\mathrm{c}=0.52, \mathrm{CHCl}_{3}\right)$.

Synthesis and Characterization of $(S, R, R)-5-8 . \quad(S, R, R)-5-8$ was prepared in a way similar to the preparation of $(S, S, S)-\mathbf{5 - 8}$ by starting with $(S)-\mathbf{5 - 3}$ and $(R, R)-\mathbf{5 - 4} . \quad{ }^{1} \mathrm{H}$ NMR (300 MHz, $\left.\mathrm{CDCl}_{3}\right) \delta 1.24-1.33(\mathrm{~m}, 8 \mathrm{H}), 1.84(\mathrm{~d}, \mathrm{~J}=8.1 \mathrm{~Hz}, 4 \mathrm{H}), 2.8(\mathrm{~d}, \mathrm{~J}=13.5$ $\mathrm{Hz}, 2 \mathrm{H}), 2.25(\mathrm{~d}, \mathrm{~J}=10.8 \mathrm{~Hz}, 2 \mathrm{H}), 2.37(\mathrm{~m}, 2 \mathrm{H}), 2.54-2.66(\mathrm{~m}, 6 \mathrm{H}), 7.07$ (d, J = 8.1 Hz, 2H), 7.16-7.28 (m, 4H), $7.81(\mathrm{~d}, \mathrm{~J}=7.8 \mathrm{~Hz}, 2 \mathrm{H}), 8.05(\mathrm{~s}, 2 \mathrm{H}), 13.24(\mathrm{~s}, 2 \mathrm{H}) .{ }^{19} \mathrm{~F} \mathrm{NMR}$ $\left(282 \mathrm{MHz}, \mathrm{CDCl}_{3}\right) \delta-80.29 .{ }^{13} \mathrm{C} \mathrm{NMR}\left(150 \mathrm{MHz}, \mathrm{CDCl}_{3}\right) \delta 24.76,24.82,29.0,29.7$, 64.8, 65.2, $84.0(\mathrm{q}, \mathrm{J}=29.9 \mathrm{~Hz}), 117.7,122.7,123.2,124.8,125.6(\mathrm{q}, \mathrm{J}=279.6 \mathrm{~Hz})$, 127.3, 127.6, 128.6, 129.2, 134.5, 153.6. HRMS Calcd for $\mathrm{C}_{36} \mathrm{H}_{37} \mathrm{~N}_{4} \mathrm{O}_{2} \mathrm{~F}_{6}\left(\mathrm{MH}^{+}\right)$: 671.2821, Found: 671.2816. m.p. $236{ }^{0} \mathrm{C} . \quad[\alpha]_{\mathrm{D}}=-36.3\left(\mathrm{c}=1.66, \mathrm{CHCl}_{3}\right)$.

\subsection{Preparation of Samples for Fluorescence Measurement.}

Sensors were purified by column chromatography followed by recrystallization and then stored in a refrigerator. The enantiomers of trans-1,2-diaminocyclohexane were purchased from Aldrich and redistilled. All of the solvents were either HPLC or spectroscopic grade. The stock solutions of the sensors were freshly prepared for each measurement. A 0.01 M stock solution of trans-1,2-diaminocyclohexane in methylene chloride was freshly prepared. For the fluorescence enhancement study, a sensor solution was mixed with the amine solution at room temperature in a $5 \mathrm{~mL}$ volumetric 
flask and diluted to the desired concentration. The resulting solution was allowed to stand at room temperature for $0.5 \mathrm{~h}$ before the fluorescence measurement and all the fluorescence spectra were taken within 2 hours.

\subsection{Preparation of Samples for ${ }^{19}$ F NMR Titration}

The $\mathrm{CDCl}_{3}$ solution of $(S)-\mathbf{5 - 3}(0.4 \mathrm{~mL}, 5.0 \mathrm{mM})$ was prepared in an NMR tube. The $\mathrm{CDCl}_{3}$ solution of $(S, S)$ or $(R, R)-\mathbf{5 - 4}(1 \mathrm{~mL}, 0.5 \mathrm{M})$ was prepared in a small vial as the stock solution. The NMR spectrum of the $(S)-5-3$ solution was first recorded and the stock solution of the diamine was added to the NMR tube in the following fashion: 1 x 2 $\mu \mathrm{L}, 1 \times 3 \mu \mathrm{L}, 3 \times 5 \mu \mathrm{L}, 8 \times 10 \mu \mathrm{L}$, and then $50 \mu \mathrm{Ls}$. After each addition the solution was mixed well and the NMR spectra were recorded. (In order to avoid the sensor being diluted with the addition of the diamine during the titration, a small amount of (S)-5-3 (5.0 $\mathrm{mM})$ was pre-added to the diamine stock solution. This way, the total concentration of the sensor was maintained at $5.0 \mathrm{mM}$ throughout the titration.) 


\section{References}

1. Selected references of enantioselective fluorescent sensors: (a) James, T. D.; Sandanayake, K. R. A. S.; Shinkai, S. Nature 1995, 374, 345-347. (b) Pugh, V.; Hu, Q. -S.; Pu, L. Angew. Chem. Int. Ed. 2000, 39, 3638-3641. (c) Reetz, M. T.; Sostmann, S. Tetrahedron 2001, 57, 2515-2520. (d) Korbel, G. A.; Lalic, G.; Shair, M. D. J. Am. Chem. Soc. 2001, 123, 361-362. (e) Jarvo, E. R.; Evans, C. A.; Copeland, G. T.; Miller, S. J. J. Org. Chem. 2001, 66, 5522-5527. (f) Wong, W.-L.; Huang, K.-H.; Teng, P.-F.; Lee, C.-S.; Kwong, H.-L. Chem. Commun. 2004, 384-385. (g) Zhao, J.-Z.; Fyles, T. M.; James, T. D. Angew. Chem., Int. Ed. 2004, 43, 3461-3464. (h) Pagliari, S.; Corradini, R.; Galaverna, G.; Sforza, S.; Dossena, A.; Montalti, M.; Prodi, L.; Zaccheroni, N.; Marchelli, R. Chem. Eur. J. 2004, 10, 2749-2758. (i) Matsushita, H.; Yamamoto, N.; Meijler, M. M.; Wirsching, P.; Lerner, R.A.; Matsushita, M.; Janda, K. D. Mol. Biosyst. 2005, 1. 303-306. (j) Zhu, L.; Anslyn, E. V. J. Am. Chem. Soc. 2004, 126, 3676-3677. (k) Mei, X. F. ; Wolf, C. J. Am. Chem. Soc. 2004, 126, 14736-14737. (1) Li, Z.-B.; Lin, J.; Pu, L. Angew. Chem., Int. Ed. 2005, 44, 1690-1693.

2. For reviews on enantioselective fluorescent recognition: (a) Pu, L. Chem. Rev. 2004, 104, 1687-1716. (b) Pu, L. Acc. Chem. Res. 2012, 45, 150-163. (c) A recent review on chiral optical sensors: Leung, D.; Kang, S. O.; Anslyn, E. V. Chem. Soc. Rev. 2012, $41,448-479$. 
3. (a) Mei, X. F.;Wolf, C. J. Am. Chem. Soc. 2006, 128, 13326-13327. (b) Wolf, C.; Liu, S.; Reinhardt, B. C. Chem. Commun. 2006, 4242-4244.

4. Zhu, L.; Shabbir, S. H.; Anslyn, E. V. Chem. Eur. J. 2007, 13, 99-104.

5. Yu, S. S.; Pu, L. J. Am. Chem. Soc. 2010, 132, 17698-17700.

6. Herman, H. B.; Rechnitz, G. A. Science 1974, 184, 1074-1075.

7. Meyerhoff, M. E.; Pretsch, E.; Welti, D. H.; Simon, W. Anal. Chem. 1987, 59, 144150.

8. (a) Wang, K.; Seiler, K.; Haug, J. -P.; Lehmann, B.; Hartman, S. W. K.; Simon, W. Anal. Chem. 1991, 63, 970-974. (b) Seiler, K.; Wang, K.; Kuratli, M.; Simon, W. Anal. Chim. Act. 1991, 244, 151-160.

9. (a) Mohr, G. J.; Tirelli, N.; Lohse, C.; Spichiger-Keller, U. E. Adv. Mater. 1998, 10, 1353-1357. (b) Mertz, E.; Zimmerman, S. C. J. Am. Chem. Soc. 2003, 125, 3424-3425. (c) Sasaki, S. -i.; Kotegawa, Y.; Tamiaki, H. Tetrahedron Lett. 2006, 47, 4849-4852. (d) Ryu, D.; Park, E.; Kim, D. -S.; Yan, S.; Lee, J. Y.; Chang, B. -Y.; Ahn, K. H. J. Am. Chem. Soc. 2008, 130, 2394-2395.

10. Sambasivan, S.; Kim, D. -s.; Ahn, K. H. Chem. Commun. 2010, 46, 541-543.

11. Tumambac, G. E.; Wolf, C. Org. Lett. 2005, 7, 4045-4048.

12. (a) Mertz, E.; Beil, J. B.; Zimmerman, S. C. Org. Lett. 2003, 5, 3127-3130. (b) Tamborski, C.; Prabhu, U. D. G.; Eapen, K. C. J. Fluor. Chem. 1985, 28, 139-150. (c) Prakash, G. K. S.; Mathew, T.; Panja, C.; Vaghoo, H.; Venkataraman, K.; Olah, G. A. 
Org. Lett. 2007, 9, 179-182. 


\section{Chapter 6}

\section{Study of BINOL-Dicarbonyl}

Compounds in Fluorescent

Recognition 


\section{Introduction}

Enantioselective fluorescent sensors have been extensively investigated due to their potential application in high-throughput screening of chiral catalyst. In recent years, a number of highly enantioselective fluorescent sensors have been developed for the recognition of chiral substrates such as carboxylic acids, amino acids, amines and amino alcohols. $^{1,2}$ Our group has developed a series of BINOL-based fluorescent sensors ${ }^{3}$ because BINOL can provide a good fluorophore, axial chirality and binding sites. Most of our sensors are based on hydrogen bonding mechanism, which significantly limits their application in highly polar solvents. The use of trifluoromethyl ketone-based molecular sensors has been studied previously and they could covalently interact with various nucleophiles, such as carbonates, alcohols and amines. ${ }^{4}$ Recently we reported the first example of using the trifluoromethyl ketone-based molecule for enantioselective fluorescent recognition. ${ }^{5}$ As described in Chapter 5, the BINOL-trifluoromethyl ketone compound (S)-5-3 is nonfluorescent in $\mathrm{CH}_{2} \mathrm{Cl}_{2}$ and shows dual emissions when treated with both enantiomers of trans-1,2-diaminocyclohexane. It exhibits high sensitivity at one emission wavelength and high enantioselectivity at another. By using this fluorescent sensor, both the concentration and enantiomeric composition of trans-1,2diaminocyclohexane can be determined simultaneously with one fluorescence measurement which points a new direction for the development of enantioselective fluorescent sensors. In order to gain more understanding on this type of sensors, we have synthesized a series of BINOL-based molecules containing 3,3'- or 6,6'-carbonyl groups and have explored their fluorescent responses towards a variety of amines, 
diamines, amino carboxylates and amino alcohols. Herein, these results are reported.

\section{Results and Discussion}

\subsection{Synthesis and Characterization of the BINOL-diketone compounds.}

As described in Chapter 5, we synthesized (S)-6-4 according to Scheme 6-1. ${ }^{5}$ The ${ }^{1} \mathrm{H}$ NMR spectrum of $(S)-6-4$ in $\mathrm{CDCl}_{3}$ shows a singlet at $\delta 10.51$ for the hydroxyl group, which indicates strong intramolecular hydrogen bonding.

Scheme 6-1. Synthesis of the BINOL-diketone $(S)-6-4$.<smiles>Oc1ccc2ccccc2c1-c1c(O)ccc2ccccc12</smiles>

(S)-6-1

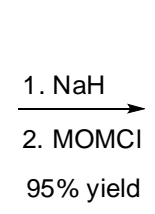<smiles>COc1ccc2ccccc2c1-c1c(OC)ccc2ccccc12</smiles>

(S)-6-2

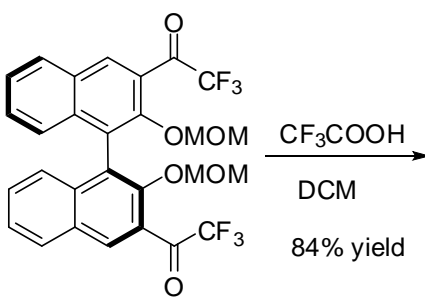

(S)-6-3

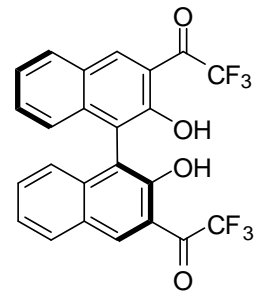

(S)-6-4

An $\mathrm{H}_{8} \mathrm{BINOL}$ analogue $(R)-6-8$ was prepared by following a similar procedure starting with $(R)-\mathrm{H}_{8} \mathrm{BINOL}$ (Scheme 6-2). Its specific optical rotation was $[\alpha]_{\mathrm{D}}=-38.5(\mathrm{c}$ $\left.=0.36, \mathrm{CHCl}_{3}\right)$. The ${ }^{1} \mathrm{H}$ NMR spectrum of $(R)-8$ in $\mathrm{CDCl}_{3}$ shows a singlet at $\delta 11.14$ for the hydroxyl group.

Scheme 6-2. Synthesis of the $\mathrm{H}_{8} \mathrm{BINOL-diketone}(R)-\mathbf{6 - 8}$.

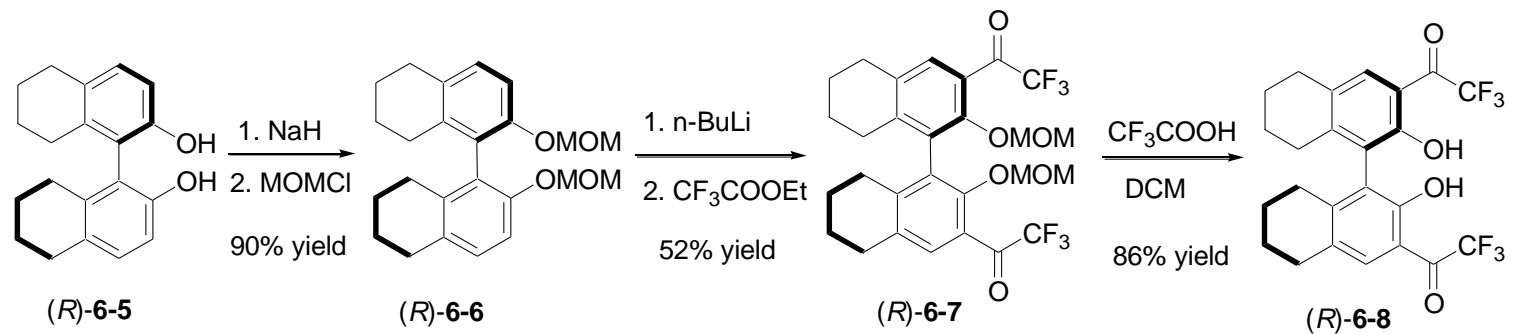


Compounds $(S)-\mathbf{6 - 1 0}$ and $(S)-6-12$ were prepared as the analogues of $(S)-\mathbf{6 - 4}$ with carbonyl groups of different electronic properties. Following procedures similar to the synthesis of (S)-6-4, we used DMF to prepare (S)-6-10 (Scheme 6-3) ${ }^{6}$ and ethyl difluoroacetate to prepare (S)-6-12 (Scheme 6-4). The specific optical rotation of (S)-612 was $[\alpha]_{D}=31.9\left(\mathrm{c}=0.19, \mathrm{CHCl}_{3}\right)$. The ${ }^{1} \mathrm{H}$ NMR spectrum in $\mathrm{CDCl}_{3}$ shows a singlet at $\delta 10.59$ for the hydroxyl group of $(S)-\mathbf{6 - 1 0}$ and at $\delta 10.75$ for $(S)-\mathbf{6 - 1 2}$.

Scheme 6-3. Synthesis of the BINOL-dialdehyde (S)-6-10.

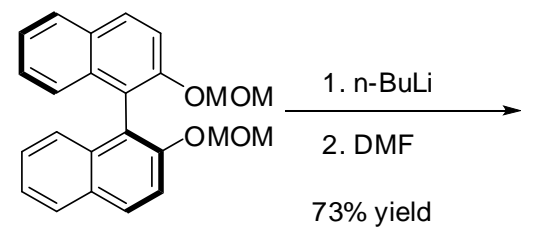

(S)-6-2

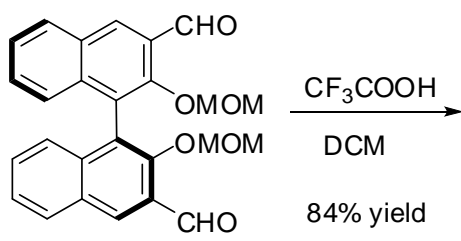

(S)-6-9

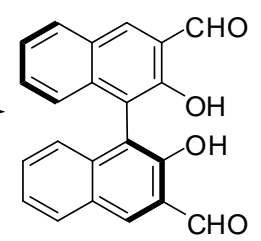

(S)-6-10

Scheme 6-4. Synthesis of the BINOL-diketone $(S)-6-12$.

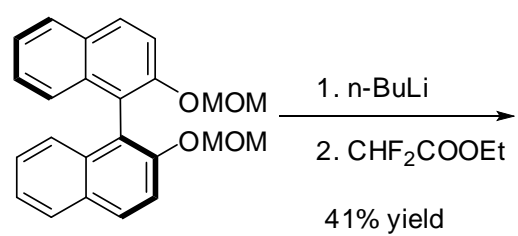

(S)-6-2

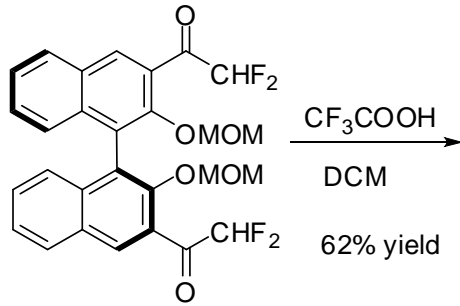

(S)-6-11

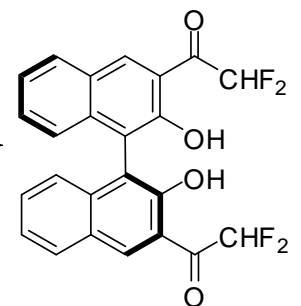

(S)-6-12

We also introduced trifluoroacetyl group to the 6,6'-postions of BINOL to make compound (S)-6-16. As shown in Scheme 6-5, $(S)-6-1$ was treated with bromine to give 6,6'-brominated BINOL in $87 \%$ yield. $^{7}$ After protected with the MOM group, ${ }^{7}$ the resulting compound was reacted with $n$-BuLi and ethyl trifluoroacetate to produce $(S)$-615 in $87 \%$ yield. $^{8}$ Then TFA was added to remove MOM to generate (S)-6-16 in 89\% yield. Its specific optical rotation was $[\alpha]_{\mathrm{D}}=333.6\left(\mathrm{c}=0.16, \mathrm{CHCl}_{3}\right)$. The ${ }^{1} \mathrm{H}$ NMR 
spectrum of $(S)$-16 in $\mathrm{CDCl}_{3}$ shows a singlet at $\delta 5.33$ for the hydroxyl group which is much more upfiled than those of the 3,3'-dicarbonylBINOLs because of the absence of the intramolecular $\mathrm{OH}^{\cdots} \mathrm{O}=\mathrm{C}$ hydrogen bond.

Scheme 6-5. Synthesis of the BINOL-diketone $(S)-6-16$.

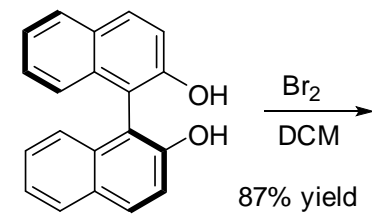

(S)-6-1

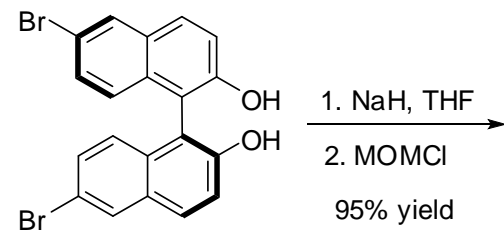

(S)-6-13

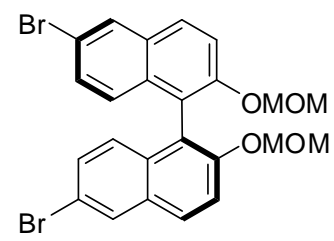

(S) $-6-14$

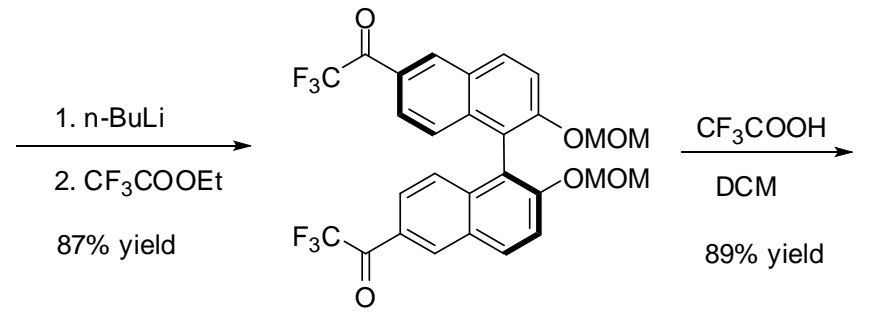

(S) -6-15

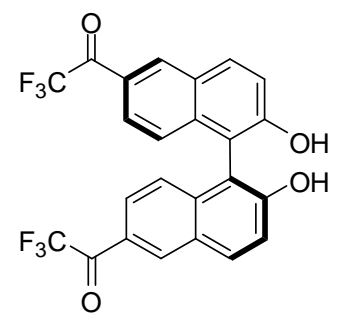

(S) -6-16

2.2 UV Spectra of Compounds $(S)-6-3,(S)-6-4,(R)-6-8,(S)-6-10,(S)-6-12$ and $(S)-6-$

16.

Figure 6-1 gives the UV spectra of $(S)-\mathbf{6 - 3},(S)-\mathbf{6 - 4},(R)-\mathbf{6 - 8},(S)-\mathbf{6 - 1 0},(S)-\mathbf{6 - 1 2}$ and (S)-6-16 in $\mathrm{CH}_{2} \mathrm{Cl}_{2}$ at $1.0 \times 10^{-5} \mathrm{M}$ and Table 6-1 summarizes their maximum absorption peaks and their extinction coefficients. As shown in Figure 6-1a, $(S)-6-4$ displays absorptions at $\lambda_{\max }=228,263,319$ and $432 \mathrm{~nm}$. Compared to $(S)-\mathbf{6 - 4}$, the long wavelength absorption is missing in the MOM protected compound (S)-6-3, which indicates that the intramolecular hydrogen bonding is responsible for the absorption at $432 \mathrm{~nm} . \quad \mathrm{H}_{8} \mathrm{BINOL}$ analogue $(R)-\mathbf{6 - 8}$ exhibits blue shifted absorptions due to its less conjugated structure but with patterns similar to those of $(S)-6-4$ (Figure 6-1b). The 
similar absorption patterns can also be observed in compounds (S)-6-10 and $(S)-\mathbf{6 - 1 2}$. (S)-6-16, in which two trifluoroacetyl groups are attached to the 6,6'-positions, gives very differently structured absorptions with the long wavelength absorption observed in $(S)$-64 missing. This observation is consistent with our proposed hydrogen bonding origin of the absorption at $432 \mathrm{~nm}$ in $(S)-6-4$.

Figure 6-1. UV spectra of (a) (S)-6-3, (b) $(R)-6-8$, (c) $(S)-6-10,(S)-6-12$ and (d) $(S)-6-16$ $\left(1.0 \times 10^{-5} \mathrm{M}\right)$ in comparison with that of $(S)-6-4$ in $\mathrm{CH}_{2} \mathrm{Cl}_{2}$.
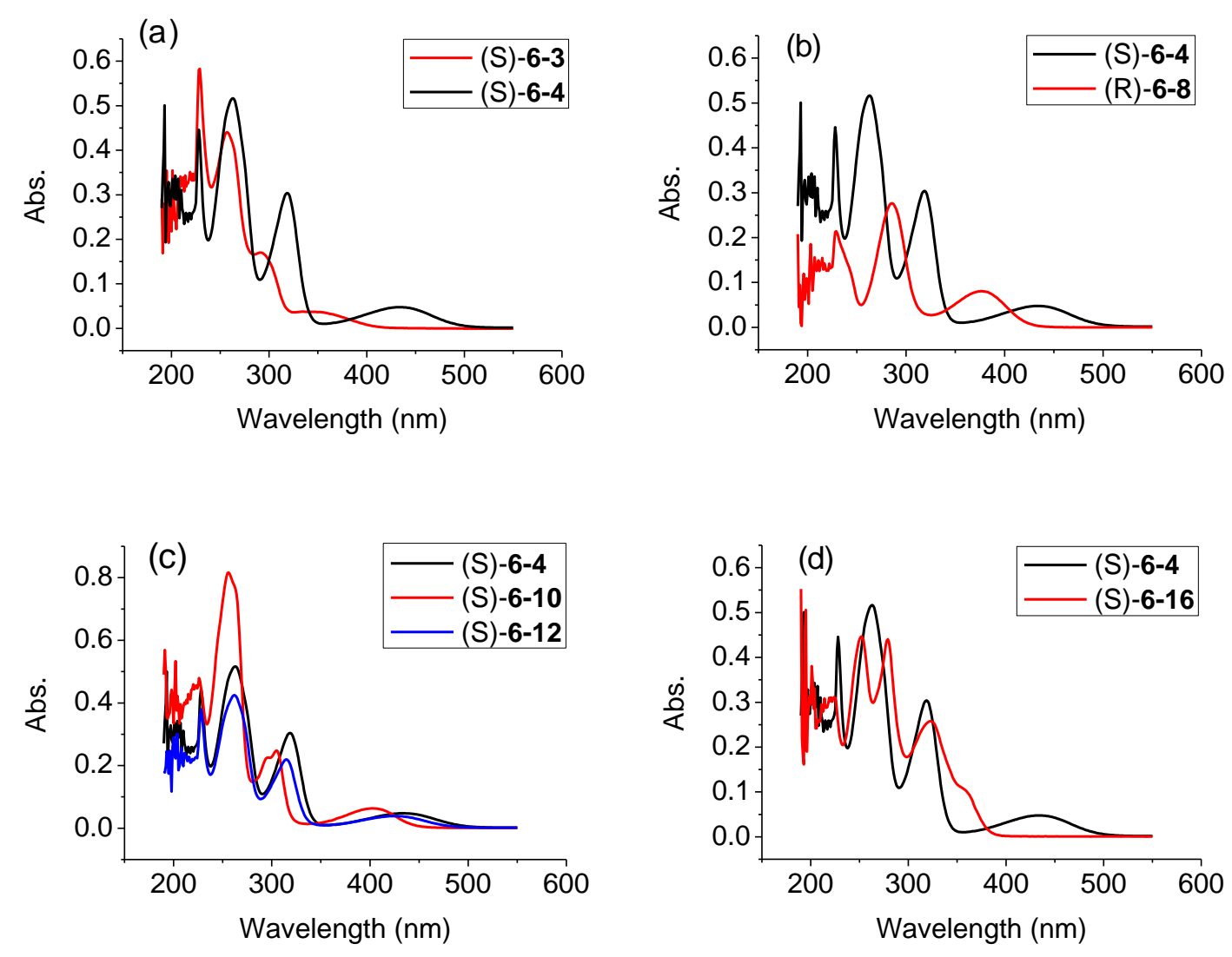
Table 6-1. Maximum absorption peaks and extinction coffecient of (S)-6-3, $(S)-\mathbf{6 - 4},(R)$ 6-8, (S)-6-10, $(S)$-6-12 and $(S)-6-16\left(1.0 \times 10^{-5} \mathrm{M}^{\text {in }} \mathrm{CH}_{2} \mathrm{Cl}_{2}\right)$.

\begin{tabular}{|c|c|}
\hline & $\lambda_{\max }(\varepsilon)$ \\
\hline$(S)-6-3$ & $229\left(5.8 \times 10^{4}\right), 257\left(4.4 \times 10^{4}\right), 291\left(1.7 \times 10^{4}\right), 357\left(3.5 \times 10^{3}\right) \mathrm{nm}$. \\
\hline$(S)-6-4$ & $228\left(4.5 \times 10^{4}\right), 263\left(5.2 \times 10^{4}\right), 319\left(3.0 \times 10^{4}\right), 432\left(4.8 \times 10^{3}\right) \mathrm{nm}$ \\
\hline$(R)-6-8$ & $229\left(2.1 \times 10^{4}\right), 285\left(2.8 \times 10^{4}\right), 377\left(8.1 \times 10^{3}\right) \mathrm{nm}$ \\
\hline$(S)-6-10$ & $255\left(8.1 \times 10^{4}\right), 305\left(2.5 \times 10^{4}\right), 402\left(6.3 \times 10^{3}\right) \mathrm{nm}$ \\
\hline$(S)-6-12$ & $228\left(3.8 \times 10^{4}\right), 262\left(4.2 \times 10^{4}\right), 315\left(2.2 \times 10^{4}\right), 425\left(3.9 \times 10^{3}\right) \mathrm{nm}$ \\
\hline$(S)-6-16$ & $252\left(4.5 \times 10^{4}\right), 279\left(4.4 \times 10^{4}\right), 323\left(2.6 \times 10^{4}\right), 359\left(1.0 \times 10^{4}\right) \mathrm{nm}$ \\
\hline
\end{tabular}

2.3 Fluorescence Spectra of Compounds $(S)-6-3,(S)-6-4,(R)-6-8,(S)-6-10,(S)-6-12$ and $(S)-6-16$.

The fluorescence spectrum of $(S)$-6-4 is compared with those of $(S)-\mathbf{6 - 3},(R)-\mathbf{6 - 8},(S)$ 6-10, $(S)-6-12$ and $(S)-6-16$ in $\mathrm{CH}_{2} \mathrm{Cl}_{2}$ at $1.0 \times 10^{-5} \mathrm{M}$. As shown by Figure 6-2, similar to (S)-6-4, compounds $(R)-6-8, \quad(S)-6-10$ and $(S)-6-12$ containing the intramolecular hydrogen bonds are found to be nonemissive. The MOM protected compound (S)-6-3, however, shows a very strong emission at $495 \mathrm{~nm}$. (S)-6-16 gives an even stronger emission at $444 \mathrm{~nm}$. The emissions of $(S)-\mathbf{6 - 3}$ and $(S)$-6-16 could be attributed to their internal charge transfer states.

Figure 6-2. Fluorescence spectra of $(S)$-6-3, $(S)-6-4,(R)-6-8,(S)-6-10,(S)-6-12$ and $(S)$ 6-16 $\left(1.0 \times 10^{-5} \mathrm{M}\right)$ in $\mathrm{CH}_{2} \mathrm{Cl}_{2}$. $\left(\lambda_{\text {exc }}=343 \mathrm{~nm}\right.$ for $(S)-\mathbf{6 - 3},(S)-\mathbf{6 - 4},(S)-\mathbf{6 - 1 0},(S)-\mathbf{6 - 1 2}$ and $(S)-\mathbf{6 - 1 6}, \lambda_{\mathrm{exc}}=290 \mathrm{~nm}$ for $(R)-\mathbf{6 - 8}$, slit $\left.=2.0 / 2.0 \mathrm{~nm}\right)$. 


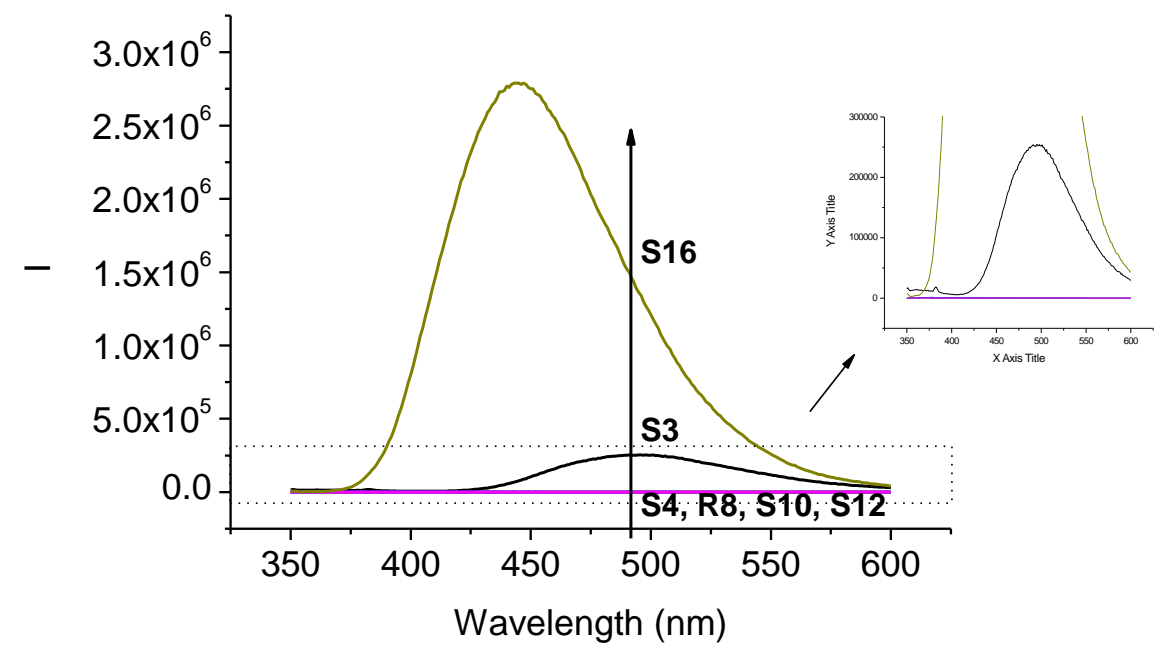

We have studied the solvent effect on the fluorescence properties of these compounds. As shown in Figure 6-3a, (S)-6-4 showed very little emission in $\mathrm{CH}_{2} \mathrm{Cl}_{2}, \mathrm{CHCl}_{3}$ and $\mathrm{CH}_{3} \mathrm{CN}$, moderate emission in THF, DMSO and very strong emission in $\mathrm{MeOH}$. Changing the solvent from $\mathrm{CH}_{3} \mathrm{CN}$ to $\mathrm{CH}_{3} \mathrm{CN} / \mathrm{H}_{2} \mathrm{O}$ (1:1) and from THF to THF/ $\mathrm{H}_{2} \mathrm{O}$ (1:1) caused significant fluorescence enhancement. However, changing the solvent from $\mathrm{CH}_{2} \mathrm{Cl}_{2}$ to $\mathrm{CH}_{2} \mathrm{Cl}_{2} / \mathrm{H}_{2} \mathrm{O}$ (1:1) and from $\mathrm{CHCl}_{3}$ to $\mathrm{CHCl}_{3} / \mathrm{H}_{2} \mathrm{O}$ (1:1) barely affect the fluorescent emission. Moreover, the colors of the solutions change accordingly with the fluorescence. When there is no emission or very weak fluorescence, the solution is light yellow, and when it shows strong emission, the solution becomes colorless.

Compound $(S)-\mathbf{6 - 1 2}$ shows similar solvent effect but the fluorescence is much weaker than $(S)-6-4$ (Figure 6-3c). (S)-6-10 shows no fluorescence or very weak fluorescence in all of the tested solvents (Figure 6-3b).

Figure 6-3. Fluorescence spectra of (a) $(S)-6-4$, (b) $(S)-\mathbf{6 - 1 0}$ and (c) $(S)-6-12\left(1.0 \times 10^{-5}\right.$ M) in various solvents. $\left(\lambda_{\mathrm{exc}}=343 \mathrm{~nm}\right.$, slit $\left.=2.0 / 2.0 \mathrm{~nm}\right)$. 

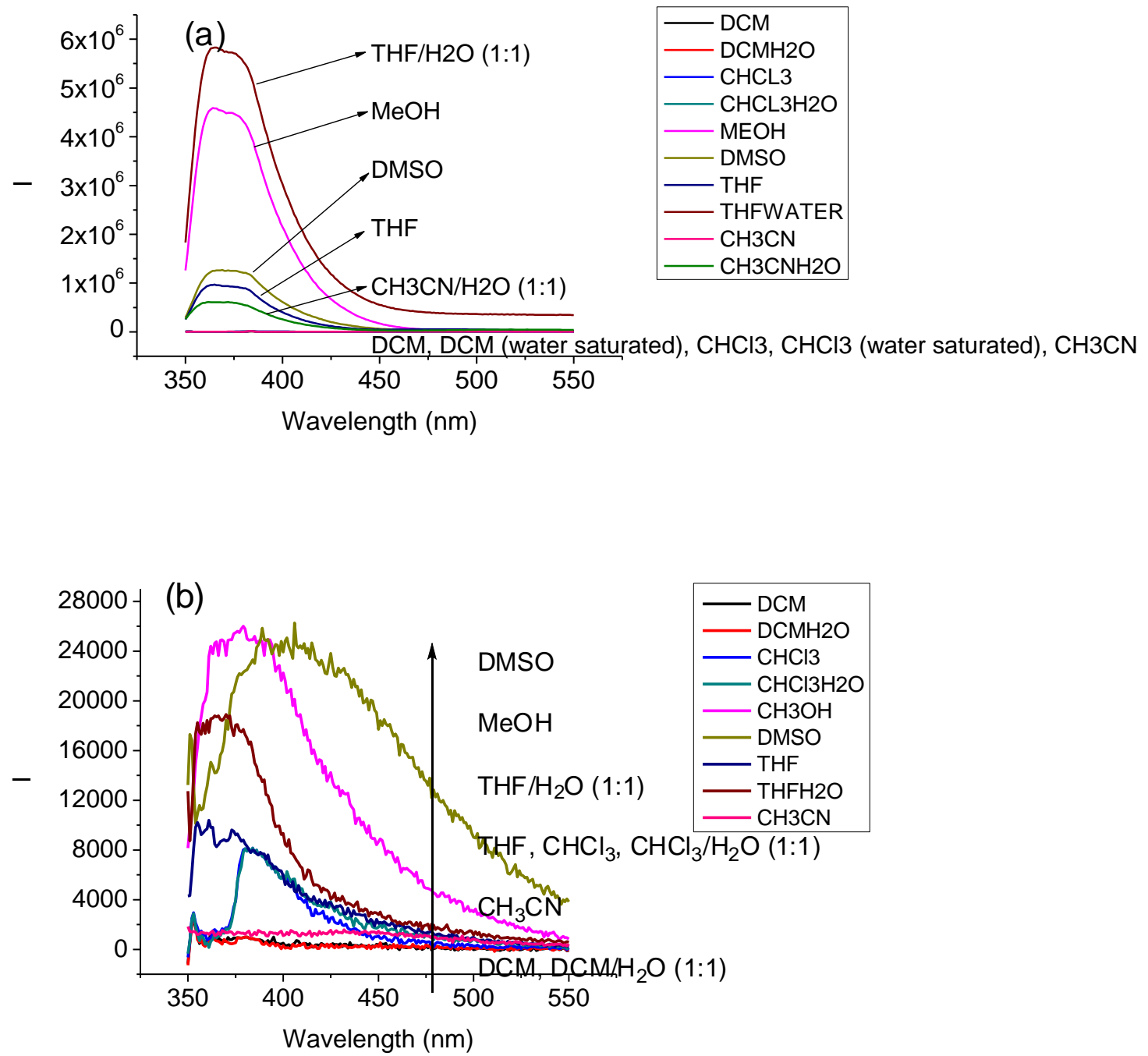

(c)

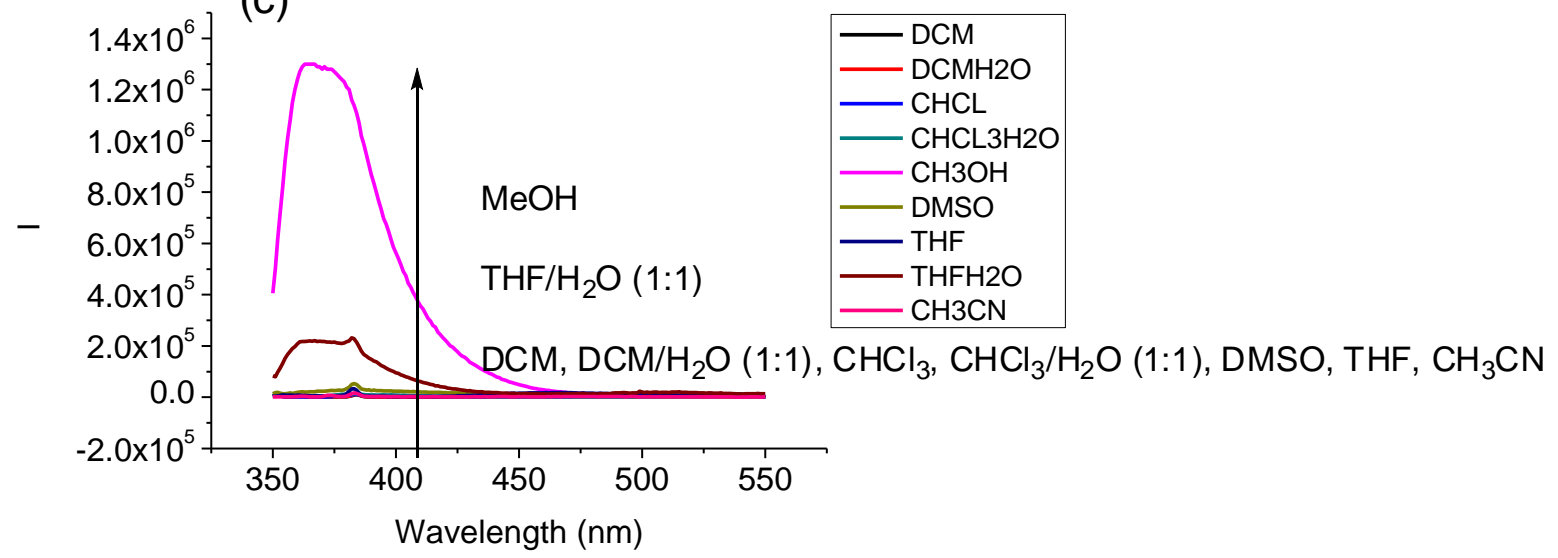


On the basis of the above observations, we propose that the quenched fluorescence of compound (S)-6-4 might be due to the excited state intramolecular proton transfer process. ${ }^{9}$ Absorption of photons frequently changes the electron distribution within a fluorophore. ${ }^{10}$ Aromatic alcohols or protonated amines become more acidic in the excited state than in the ground state because the electrons on the hydroxyl group or protonated amine are shifted into the aromatic ring in the excited state. ${ }^{11}$ Aromatic carboxylic acids and esters usually become more basic in the excited state because electron acceptors have vacant $\pi$ orbitals into which electrons can be transferred. In the case of 2-naphthol the $\mathrm{pK}_{\mathrm{A}}$ decreased from 9.2 in the ground state to 2.0 in the excited state. $^{12}$ In acid solution the emission is from naphthol with an emission maximum at 357 nm. In basic solution the emission is from the naphtholate anion with an emission maximum at $409 \mathrm{~nm}$. At intermediate $\mathrm{pH}$ values emission from both species is observed in water. ${ }^{13}$ It has been proposed that water cluster serves as proton acceptor which explains why naphtholate emission disappears in nonaquous solvents. ${ }^{14}$ When a molecule contains both the proton donor and acceptor, excited state proton transfer is facile to happen within the molecule since the transferring proton is very close to the acceptor. For example, Methyl 2-hydroxy-3-naphtholat (MNA), which contains both the proton donor and acceptor, is known to undergo intramolecular excited state proton transfer reaction. ${ }^{15}$ The transferring proton, which is already hydrogen bonded with ester group, is very easy to move to its adjacent oxygen atom in the excited state. This theory can also apply to compounds $(S)-\mathbf{6 - 4},(R)-\mathbf{6 - 8},(S)-6-10$ and $(S)-6-12$, in which the intramolecular hydrogen bond is formed. When methanol or water is added to the $(S)-\mathbf{6 - 4}$ 
solutions, nucleophilic attack happens, which changes the structure of the original intramolecular hydrogen bond and thus changes its fluorescent property.

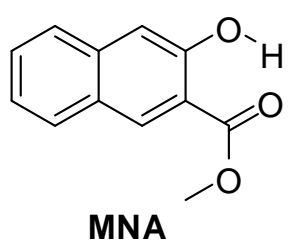

The following scheme shows a proposed mechanism for the fluorescence of $(S)-\mathbf{6}-\mathbf{4}$. In nonaqueous solution, the excited state proton transfer of (S)-6-4 upon irradiation could generate a nonemissive species $(S)-6-17$. In the presence of water or alcohol, $(S)$-6-18 could be generated. The excited state proton transfer of $(S)-\mathbf{6 - 4}$ upon irradiation could generate the naphthalate $(S)-6-19$ that could undergo both radiative and nonradiative decay.

Scheme 6-6. Proposed mechanism for the fluorescent responses of (S)-6-4.

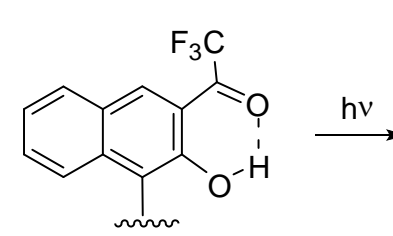

(S)-6-4

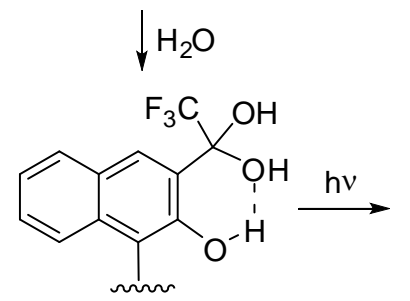

(S) $-6-18$

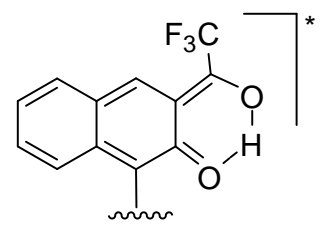

(S)-6-17 non-emissive

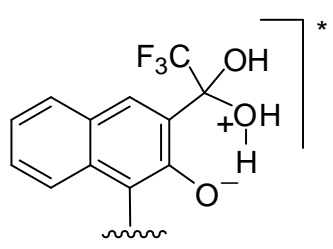

(S)-6-19 emissive 


\subsection{Interaction of (S)-6-4 with Amines.}

In Chapter 5, we described the interaction of $(S)-6-4$ with the two enantiomers of trans-cyclohexanediamine. We have further studied the interaction of $(S)-6-4$ with many other amines and diamines as listed in Figure 6-4.

Figure 6-4. Structures of various amines.

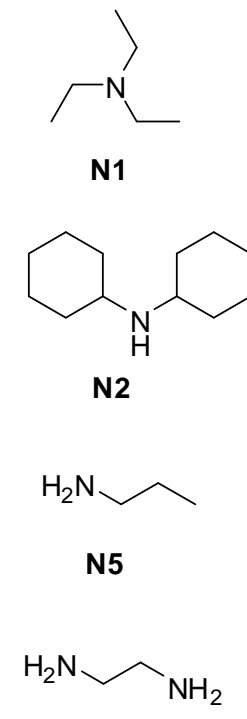

N9

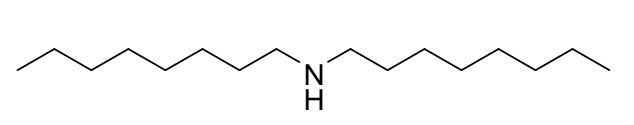

N3

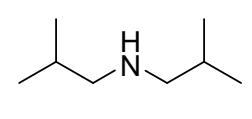

N4<smiles>Nc1ccccc1N</smiles><smiles>NCc1ccc(CN)cc1</smiles>

N13 N14<smiles>NC1CCCCC1N</smiles><smiles>NC1CCCCC1N</smiles><smiles>NC1CCCCC1N</smiles>

(1R, 2R)-N15
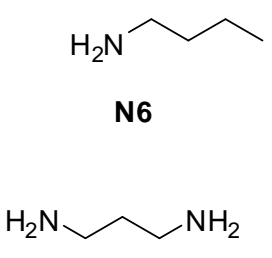

N10

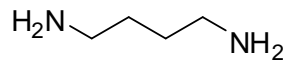

N11

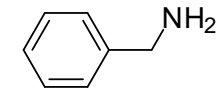

N8

N12

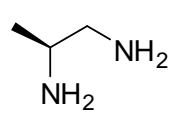

(S)-N16

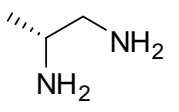

(R)-N16

\section{A. Fluorescence Study.}

When $(S)-6-4\left(1.0 \times 10^{-5} \mathrm{M}\right.$ in $\left.\mathrm{CH}_{2} \mathrm{Cl}_{2}\right)$ was treated with various monoamines N1-N8 $\left(1.0 \times 10^{-3} \mathrm{M}\right)$, including tertiary, secondary and primary monoamines, very little change 
in the fluorescence was observed (Figure 6-5a). When linear aliphatic diamines (N9-N12) were used under the same conditions, the fluorescence changed dramatically. Both 1,2diaminoethane N9 and 1,5-diaminopentane N12 showed significant fluorescence enhancement and gave dual emission peaks with $\lambda_{1}=370 \mathrm{~nm}$ and $\lambda_{2}=438 \mathrm{~nm}$ for $\mathbf{N 9}$ and $\lambda_{1}=384 \mathrm{~nm}$ and $\lambda_{2}=438 \mathrm{~nm}$ for N12. Both diamines enhanced the short wavelength emission more than long wavelength emission. In contrast, 1,3diaminopropane N10 and 1,4-diaminobutane N11 displayed much smaller fluorescence enhancement which is approximately only 3\% of N9. The aromatic diamine N13 and the benzylic diamine N14 didn't turn on the fluorescence at all.

We have compared the fluorescence responses of $(S)-6-4$ toward the transcyclohexane-1,2-diamine $[(R, R)-\mathrm{N} 15,(S, S)$-N15] described in Chapter 5 with those toward the meso-cis-cyclohexane-1,2-diamine $[(S, R)-\mathrm{N15}]$. As shown in Figure 6-5b, these three stereoisomers showed very interesting fluorescence enhancement. All three isomers turned on the fluorescence of $(S)-6-4$ and showed dual emission at $\lambda_{1}=370$ and $\lambda_{2}=438 \mathrm{~nm}$ but the fluorescence responses at the two emission wavelengths are different. $(1 R, 2 R)$-N15 causes large fluorescence enhancement at both $\lambda_{1}$ and $\lambda_{2}$, in which one at $\lambda_{1}$ is smaller than $\lambda_{2}$. (1S, 2S)-N15 enhances $\lambda_{1}$ greatly but much smaller at $\lambda_{2}$. The meso isomer $(1 S, 2 R)$-N15 enhances $\lambda_{1}$ greatly and a little smaller at $\lambda_{2}$. Similarly, both enantiomers of chiral 1,2-diaminopropane N16 cause significant fluorescence enhancement at both $\lambda_{1}$ and $\lambda_{2}$, in which $(R)$-N16 enhances more at $\lambda_{2}$ than $(S)$-N16 (Figure 6-5c) does.

Figure 6-5. Fluorescence spectra of $(S)-6-4\left(1.0 \times 10^{-5} \mathrm{M}\right)$ in the presence of (a) various 
achiral amines N1-N14, (b) three isomers of N15 and (c) two enantiomers of N16 $\left(1.0 \times 10^{-3} \mathrm{M}\right) .\left(\lambda_{\mathrm{exc}}=343 \mathrm{~nm}\right.$, slit $\left.=2.0 / 2.0 \mathrm{~nm}\right)$.

(a)
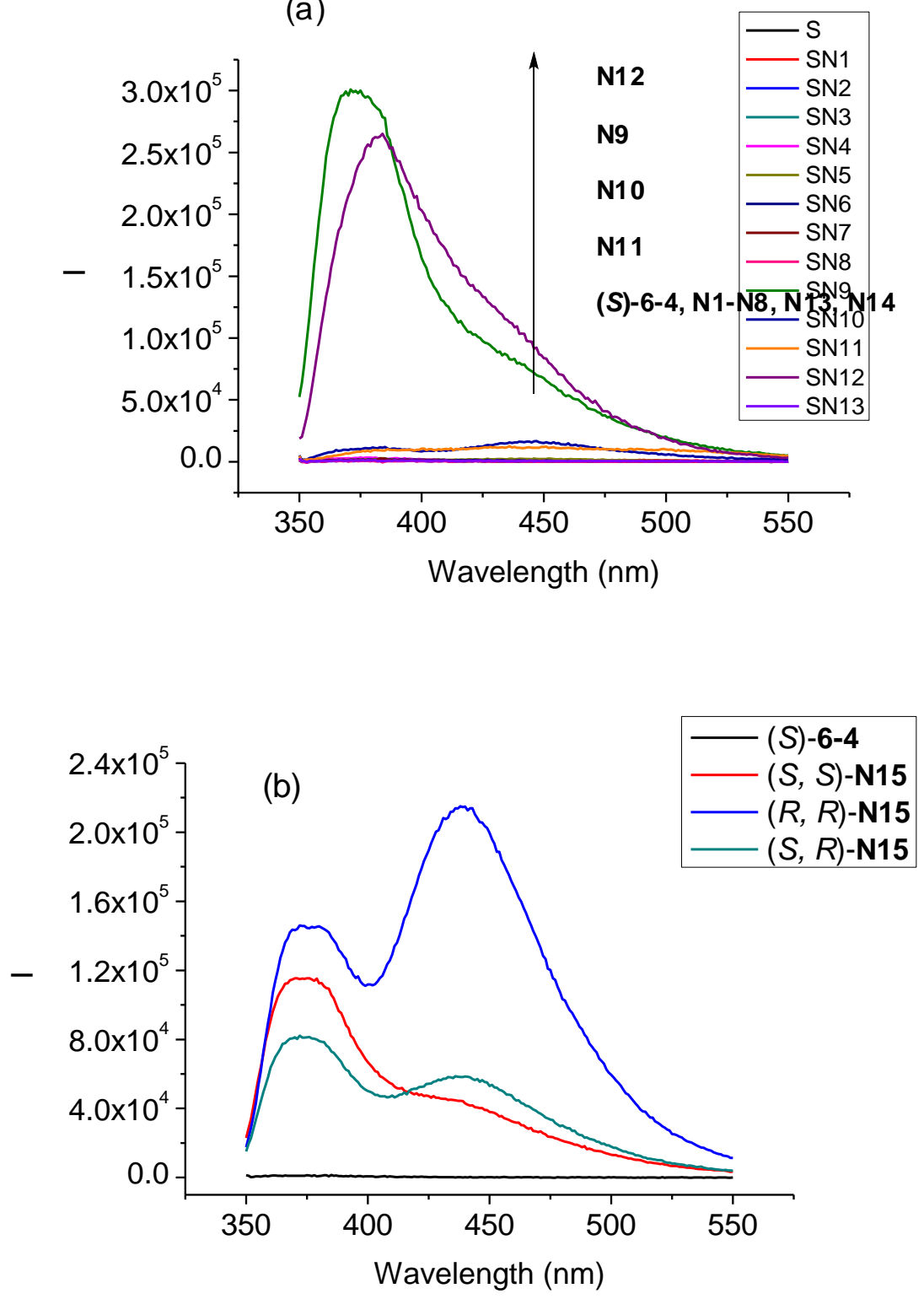


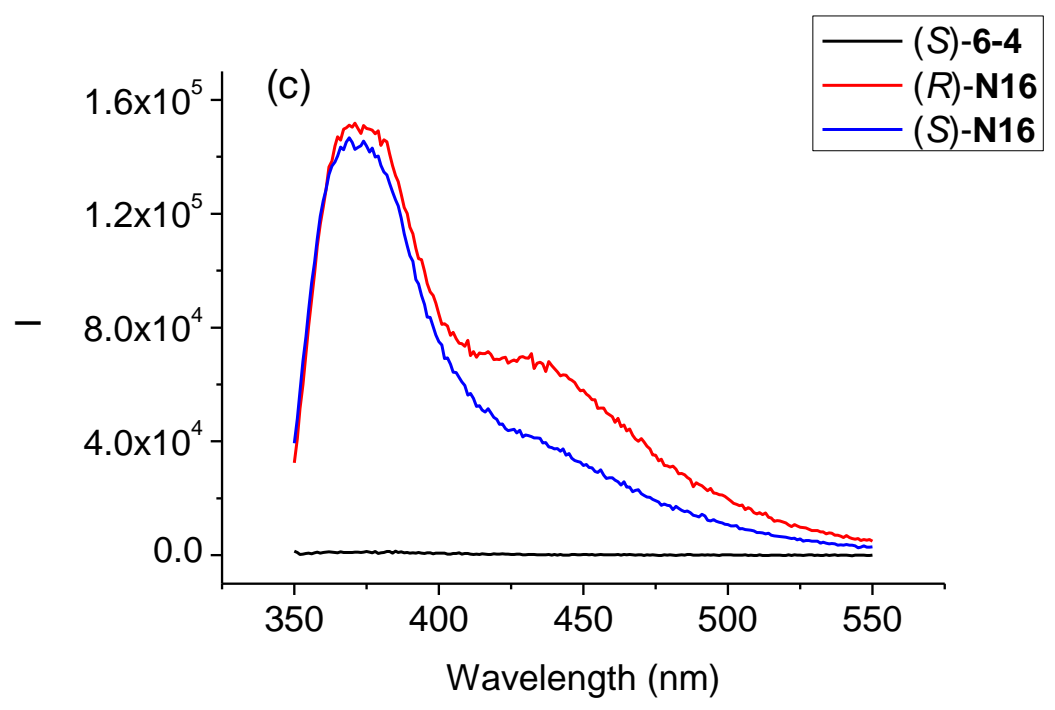

In Chapter 5, we described that the ratio of the fluorescence intensity of $(S)-6-4$ at $\mathrm{I}_{1}$ and $I_{2}\left(I_{1} / I_{2}\right)$ in the presence of trans-cyclohexanediamine only depends on the configuration of the enantiomer and is independent of the concentration. We also studied the effect of the concentration of meso-cis-cyclohexanediamine on $I_{1} / I_{2}$. As shown in Figure 6-6, similar to that observed for the enantiomers of trans-cyclohexanedimine, the $\mathrm{I}_{1} / \mathrm{I}_{2}$ remains constant (1.4) while the concentration of meso-cis-cyclohexanedimine varies. Therefore, using $I_{1} / I_{2}$ we can distinguish all the three stereoisomers of cyclohexanediamine.

Figure 6-6. Plots of $I_{1} / I_{2}$ for $(S)-6-4\left(1.0 \times 10^{-5} \mathrm{M}\right)$ in the presence of varying concentrations of $(R, R)-(S, R)$ - and $(S, S)$-N15. (Fluorescence intensity $\mathrm{I}_{1}$ at $\lambda_{1}=370 \mathrm{~nm}$ and $\mathrm{I}_{2}$ at $\lambda_{2}=438 \mathrm{~nm}$. Solvent: $\mathrm{CH}_{2} \mathrm{Cl}_{2} \cdot \lambda_{\mathrm{exc}}=343 \mathrm{~nm}$, slit $=2 / 2 \mathrm{~nm}$.) 


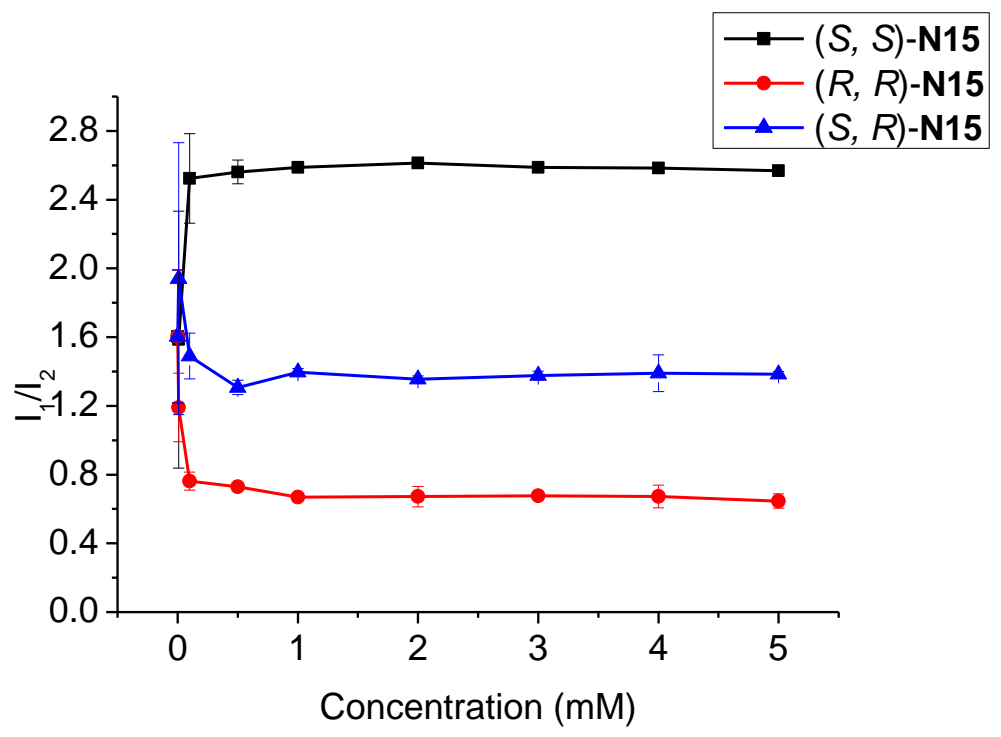

Similar study was also conducted on chiral diamine N16 in the concentration range of $5.0 \times 10^{-4} \mathrm{M}$ to $5.0 \times 10^{-3} \mathrm{M}$. As expected, $(S)-\mathbf{6 - 4}$ showed similar fluorescent responses when treated with N16. Figure 6-7a plots the fluorescent intensity of $(S)$-6-4 at $\lambda_{1}$ versus increasing concentration of $(R)$ - and $(S)$-N16. It shows that $\mathrm{I}_{1}$ is strongly dependent on the concentration of the diamine but not significantly on its chiral configuration. Figure $2 \mathrm{~b}$ plots the fluorescent intensity at $\lambda_{2}$ versus increasing concentration of $(R)$ - and $(S)$ N16 which shows significant enantioselectivity. We also found the $I_{1} / I_{2}$ ratio is nearly independent of the concentration and remains at 3.3-3.7 for $(S)$-N16 and 2.3-2.1 for $(R)$ N16 in the concentration range of $5.0 \times 10^{-4} \mathrm{M}$ to $5.0 \times 10^{-3} \mathrm{M}$.

Figure 6-7. Plots of (a) $I_{1}$, (b) $I_{2}$ and (c) $I_{1} / I_{2}$ for $(S)-6-4\left(1.0 \times 10^{-5} M\right)$ in the presence of varying concentrations of (R)- and (S)-N16. (Fluorescence intensity $I_{1}$ at $\lambda_{1}=370 \mathrm{~nm}$ and $\mathrm{I}_{2}$ at $\lambda_{2}=438 \mathrm{~nm}$. Solvent: $\mathrm{CH}_{2} \mathrm{Cl}_{2} \cdot \lambda_{\text {exc }}=343 \mathrm{~nm}$, slit $=2 / 2 \mathrm{~nm}$.) 

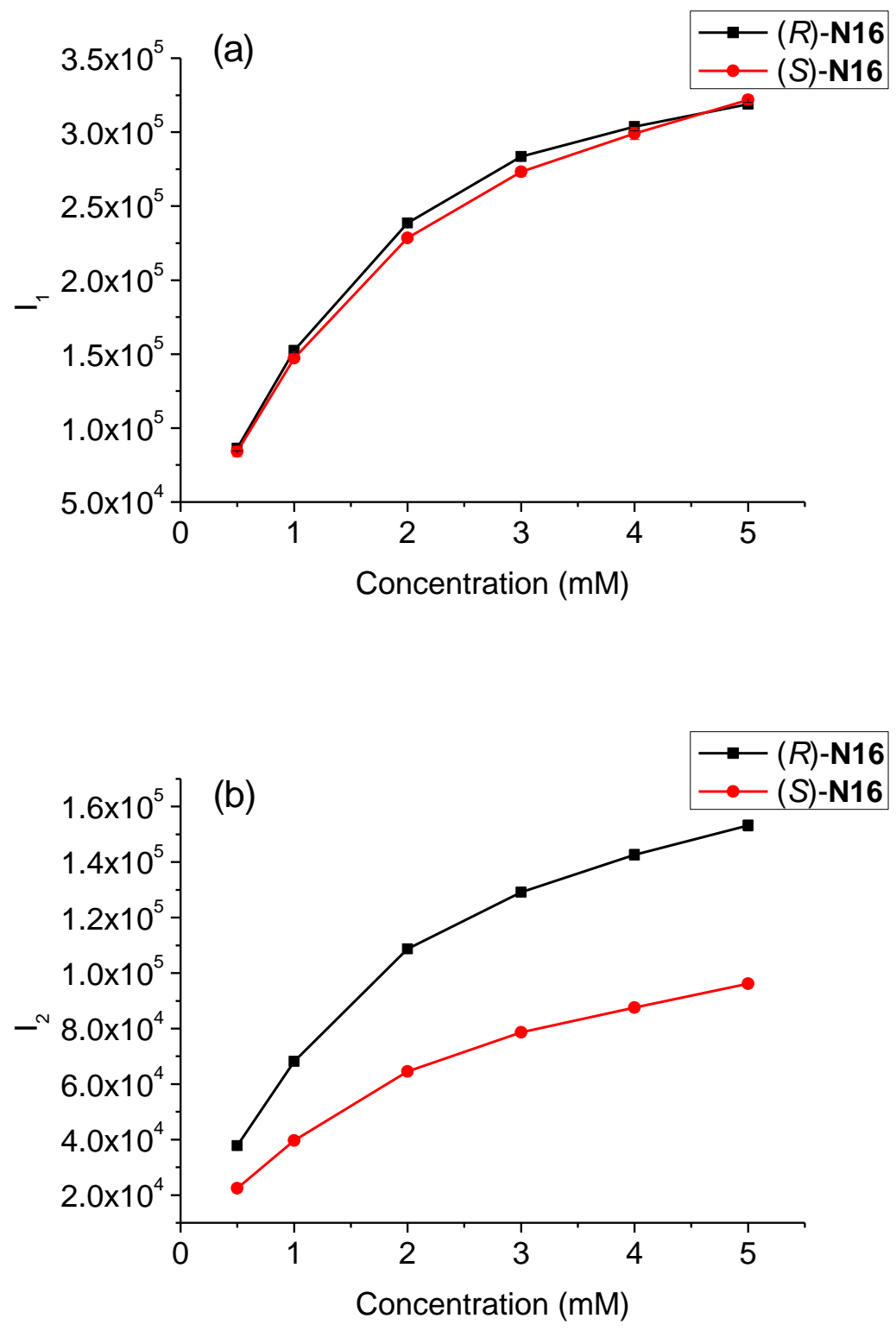


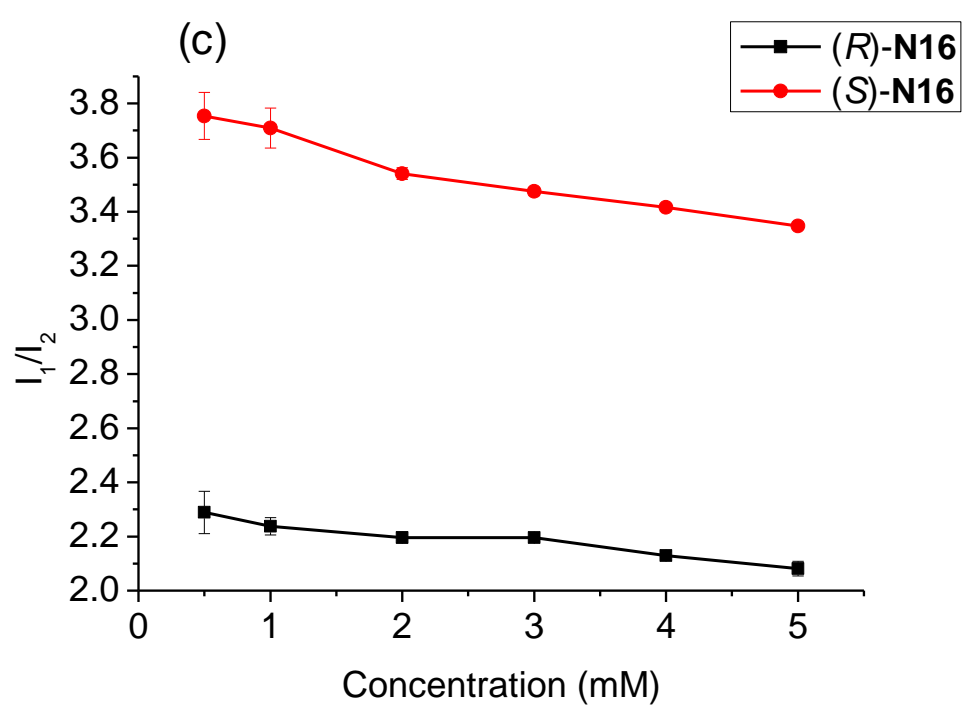

To better understand the dual emissions of $(S)-6-4$ when treated with chiral diamines N15 or N16, the excitation spectra were recorded for $(S)-6-4\left(1 \times 10^{-5} \mathrm{M}\right)$ in the presence of $(R, R)$ - and $(S, S)$-N15 $\left(4 \times 10^{-3} \mathrm{M}\right)$. As shown in Figure 6-8, both samples give the same excitation spectra with emissions at 370 or $438 \mathrm{~nm}$, which means both emissions at 370 and $438 \mathrm{~nm}$ are from the same ground state species. Lifetimes were also measured and it is $1 \mathrm{~ns}$ for emission at 370 and $1.7 \mathrm{~ns}$ for emission at 438. The difference in lifetime indicates that the two emission peaks are from two different emitting states. Therefore, after $(S)-6-4$ interacts with diamine, the resulting complex experiences excited state reactions and forms two different emitting species.

Figure 6-8. Excitation spectra of $(S)-6-4\left(1.0 \times 10^{-5} \mathrm{M}, \mathrm{CH}_{2} \mathrm{Cl}_{2}\right)$ in the presence of (a) $(R$, $R)$ - or (b) $(S, S)-\mathbf{N 1 5}\left(4.0 \times 10^{-3} \mathrm{M}\right) .($ slit $=2.0 / 2.0 \mathrm{~nm})$. 

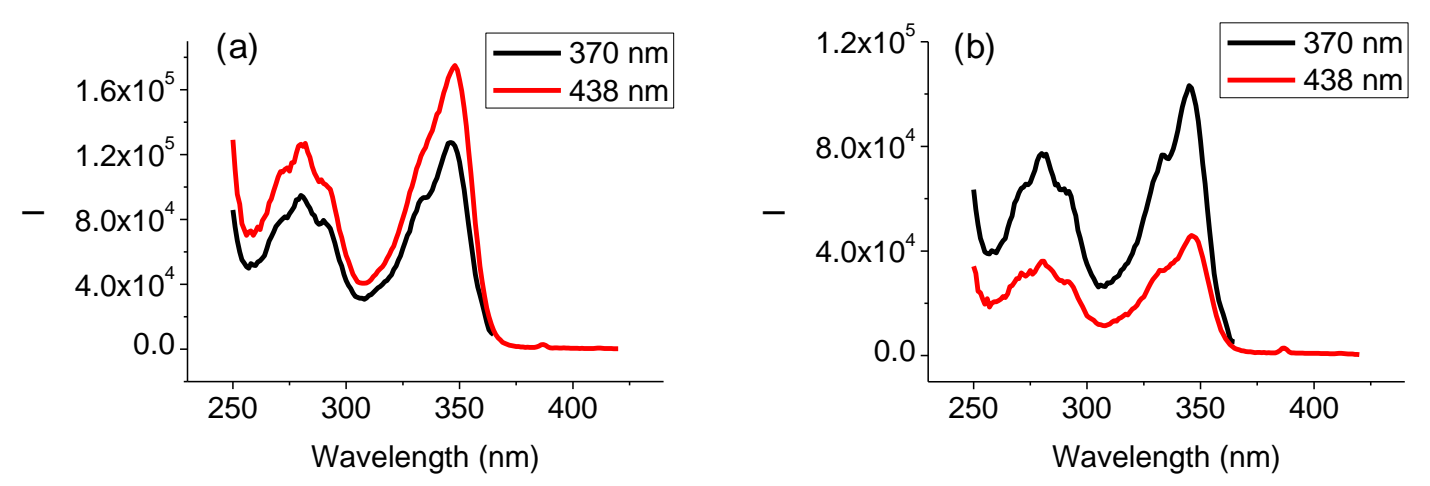

As shown in Figure 6-9, Job plots have been obtained for the interaction of $(S)-6-4$ with $\mathbf{N 9}$ and $\mathbf{N 1 2}$ at a total concentration of $0.1 \mathrm{mM}$. For $\mathbf{N 9}$ the fluorescence emission reached maximum at 30\% of $(S)-\mathbf{6 - 4}$, which indicates a 1:2 [(S)-6-4:N9] binding. However, for N12, the maximum fluorescence was shown at 50\% of $(S)-\mathbf{6 - 4}$ and the fluorescence emission stayed high in the range of $30 \%-50 \%$ of $(S)-\mathbf{6 - 4}$, which indicates more complicated binding mechanism. Both 1:1 and 1:2 [(S)-6-4:N12] bindings could exist in this system.

Figure 6-9. Job plot of $(S)-6-4\left(1.0 \times 10^{-5} \mathrm{M}\right)$ with (a) N9 and (b) N12. (total concentration $=0.1 \mathrm{mM}, \lambda_{\mathrm{exc}}=343 \mathrm{~nm}$, slit $\left.=2.0 / 2.0 \mathrm{~nm}\right)$.
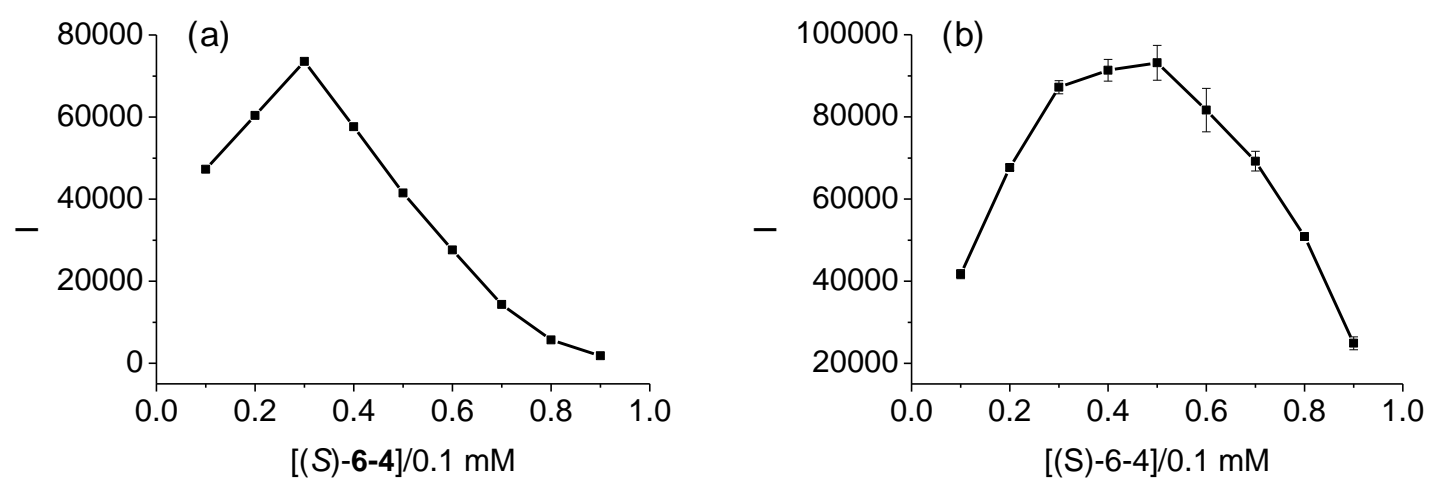
To better understand different fluorescence responses of $(S)-6-4$ with monoamines and diamines, we recorded the fluorescence spectra of $(S)-6-4\left(1.0 \times 10^{-5} \mathrm{M}\right.$ in $\left.\mathrm{CH}_{2} \mathrm{Cl}_{2}\right)$ with propylamine (N5) at higher concentrations. As is shown in Figure 6-10, N5 could not turn on the fluorescence of $(S)-6-4$ at $0.001 \mathrm{M}$. However, as the concentration of N5 increased, (S)-6-4 started to show dual emissions at $\lambda=380$ and $436 \mathrm{~nm}$ but the fluorescence intensities were still much lower than that with aliphatic diamines.

Figure 6-10. Fluorescence spectra of $(S)-6-4\left(1.0 \times 10^{-5} \mathrm{M}\right.$ in $\left.\mathrm{CH}_{2} \mathrm{Cl}_{2}\right)$ in the presence of propylamine $\mathbf{N 5}$ at various concentrations $(0-0.05 \mathrm{M}) .\left(\lambda_{\mathrm{exc}}=343 \mathrm{~nm}\right.$, slit $\left.=2.0 / 2.0 \mathrm{~nm}\right)$.

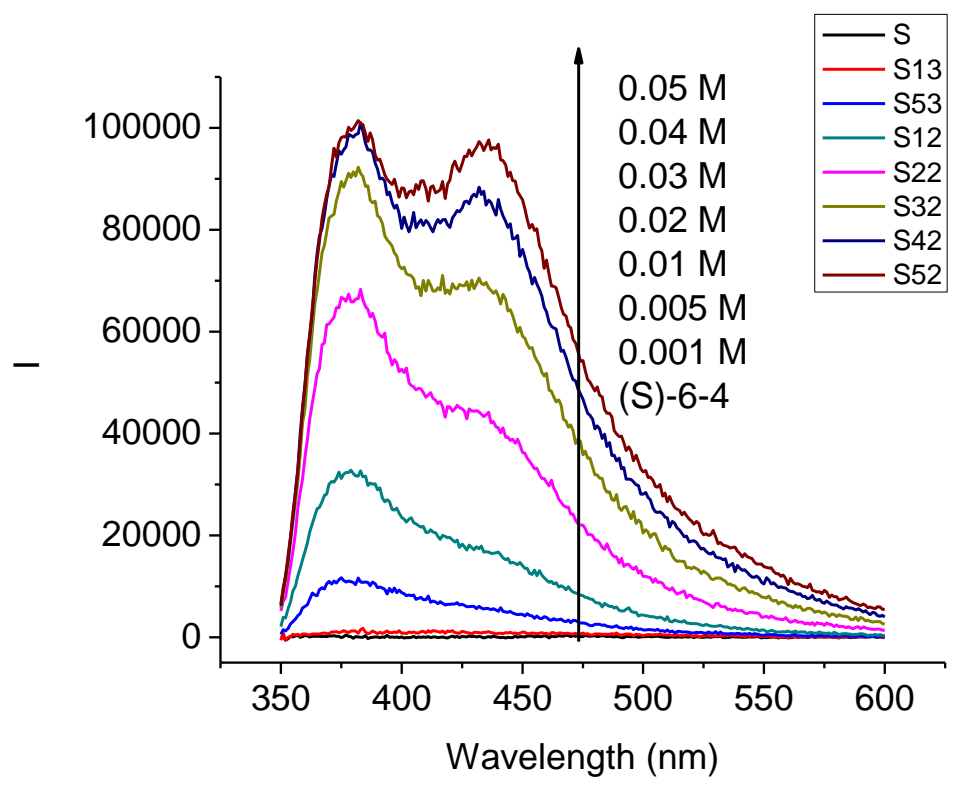

\section{B. UV Study.}

We have measured the UV-Vis absorption spectra of $(S)-6-4\left(1.0 \times 10^{-5} \mathrm{M}\right)$, amines $\left(1.0 \times 10^{-3} \mathrm{M}\right)$ and their mixtures. As shown in Figure 6-11, for N1-N8 and N13-N14, the UV spectra of the mixtures are simple addition of those of $(S)-6-4$ and amines, and the 
amines don't change the absorption of $(S)-6-4$ at all. The aliphatic diamines N9-N12 and the three stereoisomers of $\mathbf{N 1 5}$ caused similar changes to the UV absorption of $(S)-\mathbf{6 - 4}$, including a significant decrease in the absorbance intensity at $\lambda 263,319$ and $432 \mathrm{~nm}$, an increase at $231 \mathrm{~nm}$ and the appearance of a new absorption peak at $345 \mathrm{~nm}$. This indicates significant structural changes of $(S)-\mathbf{6 - 4}$ when interacted with the diamines.

Figure 6-11. UV spectra of $(S)-6-4\left(1.0 \times 10^{-5} \mathrm{M}\right)$, amines $\left(1.0 \times 10^{-3} \mathrm{M}\right)$ and their mixtures. (The black line represents UV absorption of $(S)-\mathbf{6 - 4}$, the blue line represents amine and the red line represents the mixture.)
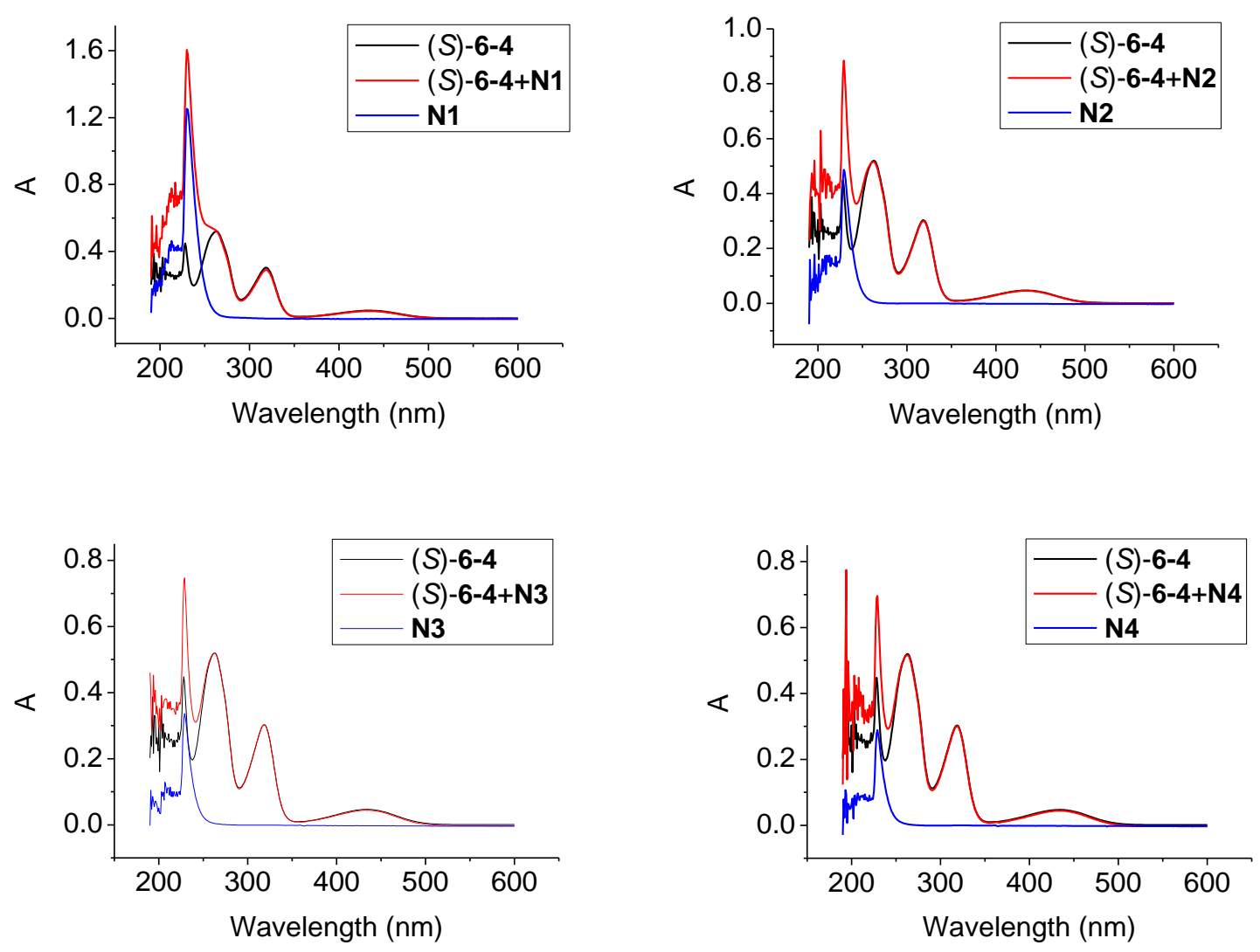

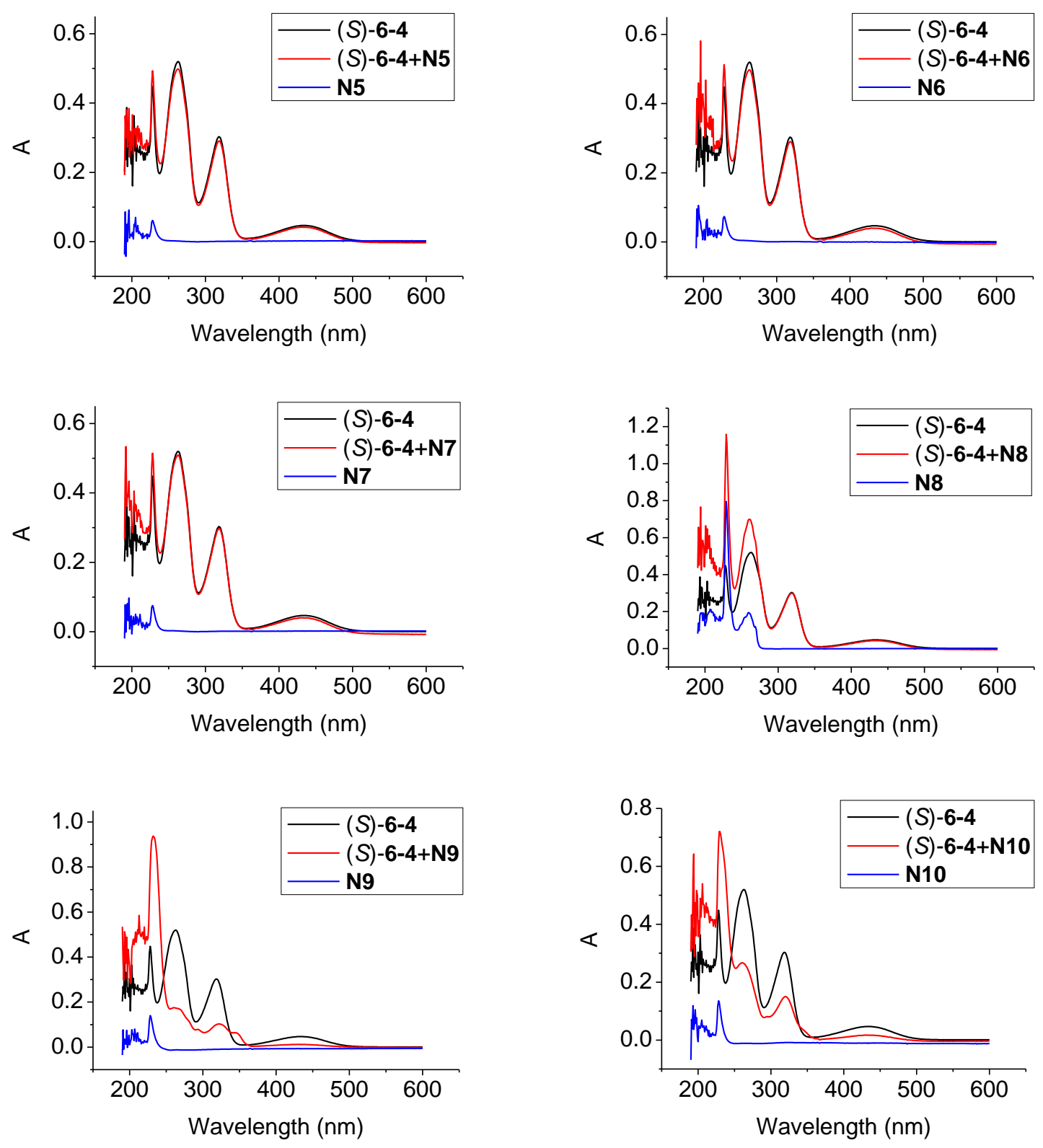

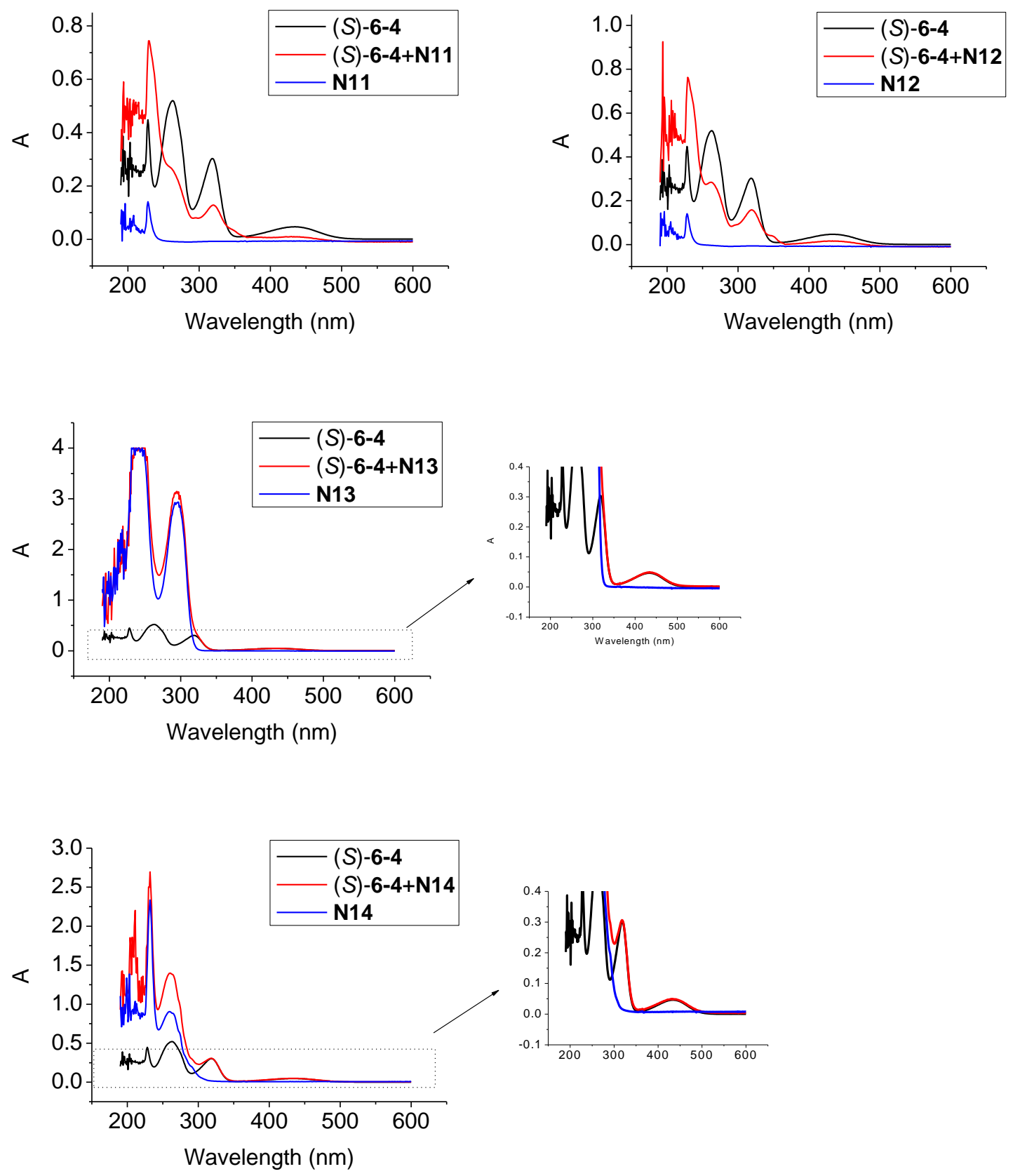

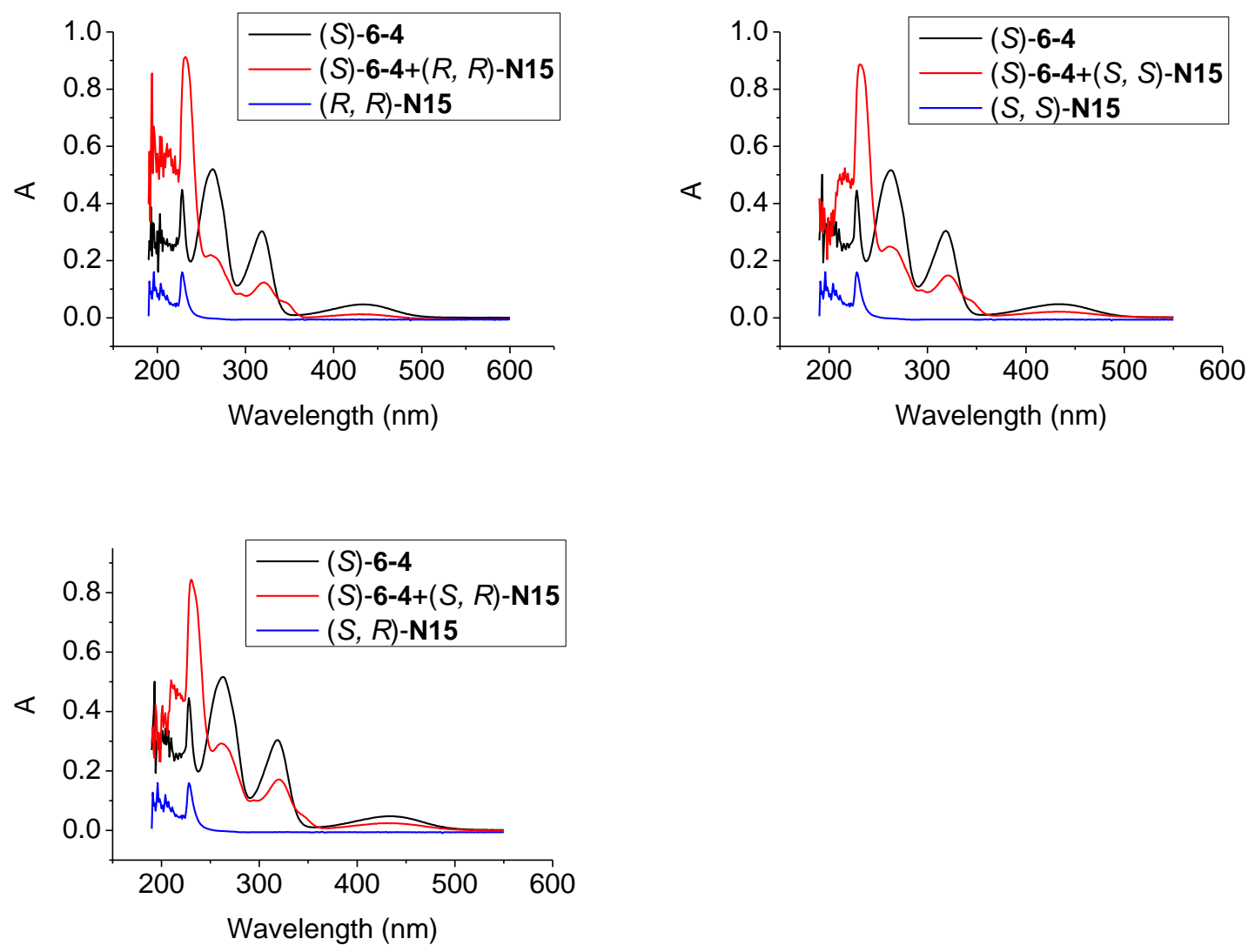

We further examined the UV absorption of $(S)-6-4\left(1.0 \times 10^{-5} \mathrm{M}\right.$ in $\left.\mathrm{CH}_{2} \mathrm{Cl}_{2}\right)$ with propylamine $\mathbf{N 5}$ at higher concentrations. As is shown in Figure 6-12, at concentrations higher than $0.005 \mathrm{M}$, N5 started to cause similar changes to the UV absorption of (S)-6-4 as the aliphatic diamines. This observation, along with fluorescence study, suggests that monoamines and diamines could cause similar structural changes to $(S)-6-4$ but much higher concentration is needed for the use of the monoamines.

Figure 6-12. UV-Vis absorption spectra of $(S)-6-4\left(1.0 \times 10^{-5} \mathrm{M}\right.$ in $\left.\mathrm{CH}_{2} \mathrm{Cl}_{2}\right)$ in the presence of propylamine $\mathbf{N 5}$ at various concentrations $(0-0.05 \mathrm{M})$. 


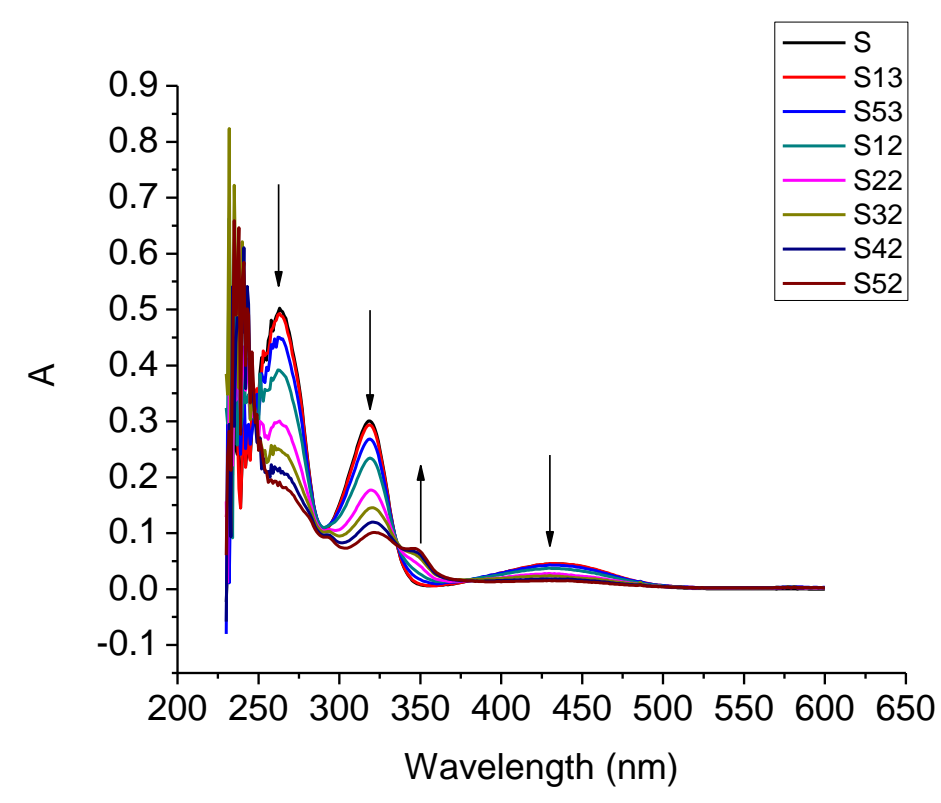

\section{NMR Study.}

In Chapter 5, we described the study of the ${ }^{19} \mathrm{~F}$ NMR spectra of $(S)-6-4$ in the presence of trans-cyclohexanediamine. We have further conducted a ${ }^{19} \mathrm{~F}$ NMR titration of $(S)-6-4$ with N9, N12 and the meso isomer of $\mathbf{N 1 5}$ in $\mathrm{CDCl}_{3}$. The $\mathrm{CDCl}_{3}$ solution of (S)-6-4 (0.4 mL, $5.0 \mathrm{mM})$ was prepared in an NMR tube. The $\mathrm{CDCl}_{3}$ solution of diamine $(1 \mathrm{~mL}, 0.5 \mathrm{M})$ was prepared in a small vial as the stock solution. The NMR spectrum of the $(S)-6-4$ was first recorded and the stock solution of the diamine was gradually added to the NMR tube. After each addition the solution was mixed well and the NMR spectra were recorded. In order to avoid the sensor being diluted with the addition of the diamine during the titration, a small amount of $(S)-6-4(5.0 \mathrm{mM})$ was pre-added to the diamine solution.

$(S)-6-4$ showed a singlet ${ }^{19} \mathrm{~F}$ signal at $\delta$-70.06. With addition of 0.2 eq $\mathbf{N 9}$, two peaks at $\delta-69.98$ and -83.64 appeared with the same integration. These two new peaks kept 
increasing while the signal at $\delta-70.06$ decreased until addition of 0.8 eq N9. These two peaks were assigned to the monohemiaminal product $(S)-\mathbf{6 - 2 0}$. After that a new peak at $\delta$ -83.53 started to show up and all other signals decreased until addition of 7.1 eq $\mathbf{N 9}$ when all other signals converted to the peak at $\delta-83.53$, which is assigned to the dihemiaminal product $(S)$-6-21. Further addition of amine generated new peaks at around $\delta-72.58$ and -80.20, which were assigned to imine $(S)-6-22$ and aminal $(S)-6-23$ respectively. The signal at $\delta-72.58$ remained at a low intensity all the time and the signal at around $\delta$ 80.20 increased while signal at $\delta-83.53$ decreased. This indicates that once the hemiaminal is converted to imine, the imine is converted to the aminal quickly. After 108 eq of amine was added over $2 \mathrm{~h}$, there was still an intense peak at $\delta-83.44$. After overnight, the hemiaminal signal was significantly reduced while the aminal signal was increased, which indicated that the conversion from the hemiaminal $(S)-\mathbf{6 - 2 1}$ to the aminal $(S)-6-23$ was slowly proceeding.

The NMR titration of $(S)-6-4$ with N12 showed more complicated chemical shift changes. With addition of 0.19 eq N12, a new peak at $\delta-83.81$ appeared and the signal of (S)-6-4 became a little broader, which became even broader and shifted upfield with more amine addition. With addition of 0.94 eq N12, two new peaks at $\delta-85.80$ and -86.65 appeared. When amine addition increased to $5.3 \mathrm{eq}$, the broad peak completely disappeared and only three peaks at $\delta-83.80,-85.81$ and -86.41 left. With even more amine added, the NMR spectrum started to become complicated.

The NMR titration of $(S)-\mathbf{6 - 4}$ with the three stereo isomers of N15 exhibited responses similar to those by $\mathbf{N 9}$ but with much slower reaction. When $(S)-\mathbf{6 - 4}$ was 
titrated with cyclohexanediamine, only hemiaminal signals could be observed in the first several hours and the signals of imines and aminals started to show up after overnight.

Scheme 6-7. Proposed binding mechanisms.<smiles>CC(C)(C)C(=O)c1cc2ccccc2c(-c2c(O)c(C(C)(O)NCCN)cc3ccccc23)c1Oc1c(O)c(C(=O)C(F)(F)F)cc2ccccc12</smiles>

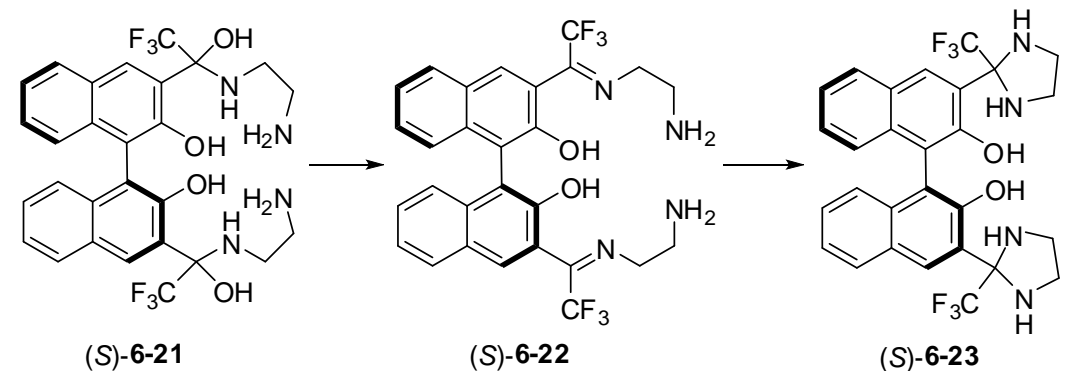

$(S)-6-21$

(S)-6-22

(S)-6-23

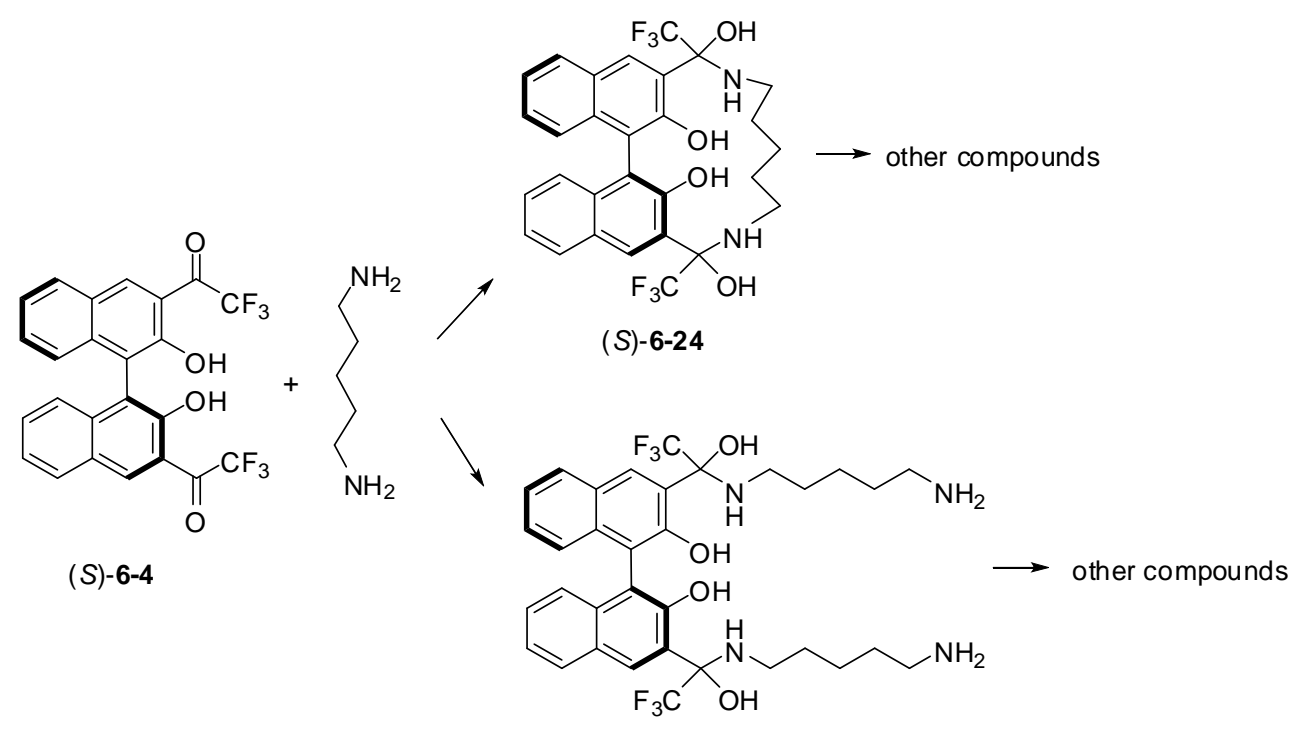

(S)-6-25 
On the basis of the above observations, we propose the following binding mechanisms. The reaction of ethylenediamine (N9) with $(S)-6-4$ is similar to that described for the reaction with trans-cyclohexanediamine in Chapter 5. As shown in Scheme 6-7, the 1,2-diamine favors 1:2 (ligand:diamine) binding and undergoes amine addition, water elimination and addition to imine to form an aminal of five-member ring. In contrast, the 1,5-diamine could form both $1: 1$ and 1:2 binding as indicated by its Job plot. One possible structure for the 1:1 adduct between 1,5-diaminopentane (N12) could be that generated from the addition to both of the $\mathrm{COCF}_{3}$ group. Formation of oligomers is also possible.

The ${ }^{19} \mathrm{~F}$ NMR titration of $(S)-\mathbf{6 - 1 6}$ with $(S, S)$-N15 in $\mathrm{CDCl}_{3}$ was also conducted. Similar to $(S)-6-4$, the fluorine signals were converting to a peak at -83.84 with addition of $(\mathrm{S}, \mathrm{S})-\mathrm{N} 15$, which indicated that addition of amino group to carbonyl also happened for $(S)-6-16$.

We also conducted a ${ }^{19} \mathrm{~F}$ NMR study for the reaction of $(S)-6-4$ with propylamine. For the NMR study, a much higher concentration of $(S)-6-4(5 \mathrm{mM})$ in $\mathrm{CDCl}_{3}$ than that used in the fluorescence and UV experiments $\left(10^{-5} \mathrm{M}\right)$ was needed for the signal detection. However, at this high concentration, when $(S)-6-4$ was treated with propylamine, the NMR spectrum showed the disappearance of the signal of $(S)-6-4$ with formation of a very weak and broad signal. Sometimes formation of a yellowish precipitate could be observed. This indicates that the solubility of the addition product of (S)-6-4 with propylamine is probably lower than the detection limit of the NMR experiments for characterization. In order to characterize the product for the reaction of 
(S)-4 with propylamine, we switched the solvent to DMSO- $d_{6}$ for the ${ }^{19} \mathrm{~F}$ NMR titration of (S)-4 with propylamine. In DMSO- $d_{6}$, the ${ }^{19}$ F NMR spectrum of $(S)-6-4$ showed multiple signals probably due to the reaction of the nucleophilic solvent and water molecules with the trifluoroacetyl groups of (S)-6-4. Addition of 20 equiv propylamine converted all the signals to a singlet at $\delta-81.51$. The product was isolated and further characterized by ${ }^{1} \mathrm{H} /{ }^{13} \mathrm{C}$ NMR and mass spectroscopes as a hemiaminal compound (S)-626.

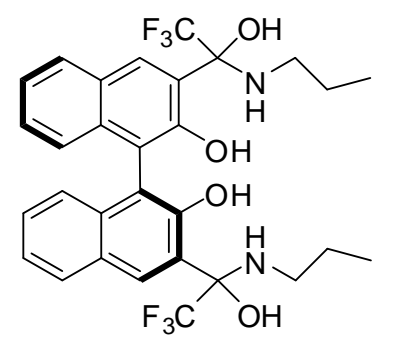

(S)-6-26

\section{X-Ray Structures.}

The crystals of the aminal product isolated from the reaction of $(S)-\mathbf{4}$ with $(S, S)$ N15 were obtained by slow evaporation of its chloroform solution and were used for Xray analysis. Crystals of the final addition products for the reactions of $(S)-\mathbf{4}$ with ethylenediamine $\mathbf{N 9}$ and propylenediamine $\mathbf{N 1 0}$ were obtained by mixing the sensor with the diamines in 1:10 ratio in chloroform followed by slow evaporation. The X-ray analyses of these crystals have confirmed their bis(cyclic aminal) structures (Figure 6-13). In these structures, intramolecular hydrogen bonding between the central hydroxyl groups and the nitrogen atoms of the aminal rings are observed. 
Figure 6-13. X-ray structures of the complexes of (S)-6-4 with (a) N9, (b) N10 and (c) (S, S)-N15.
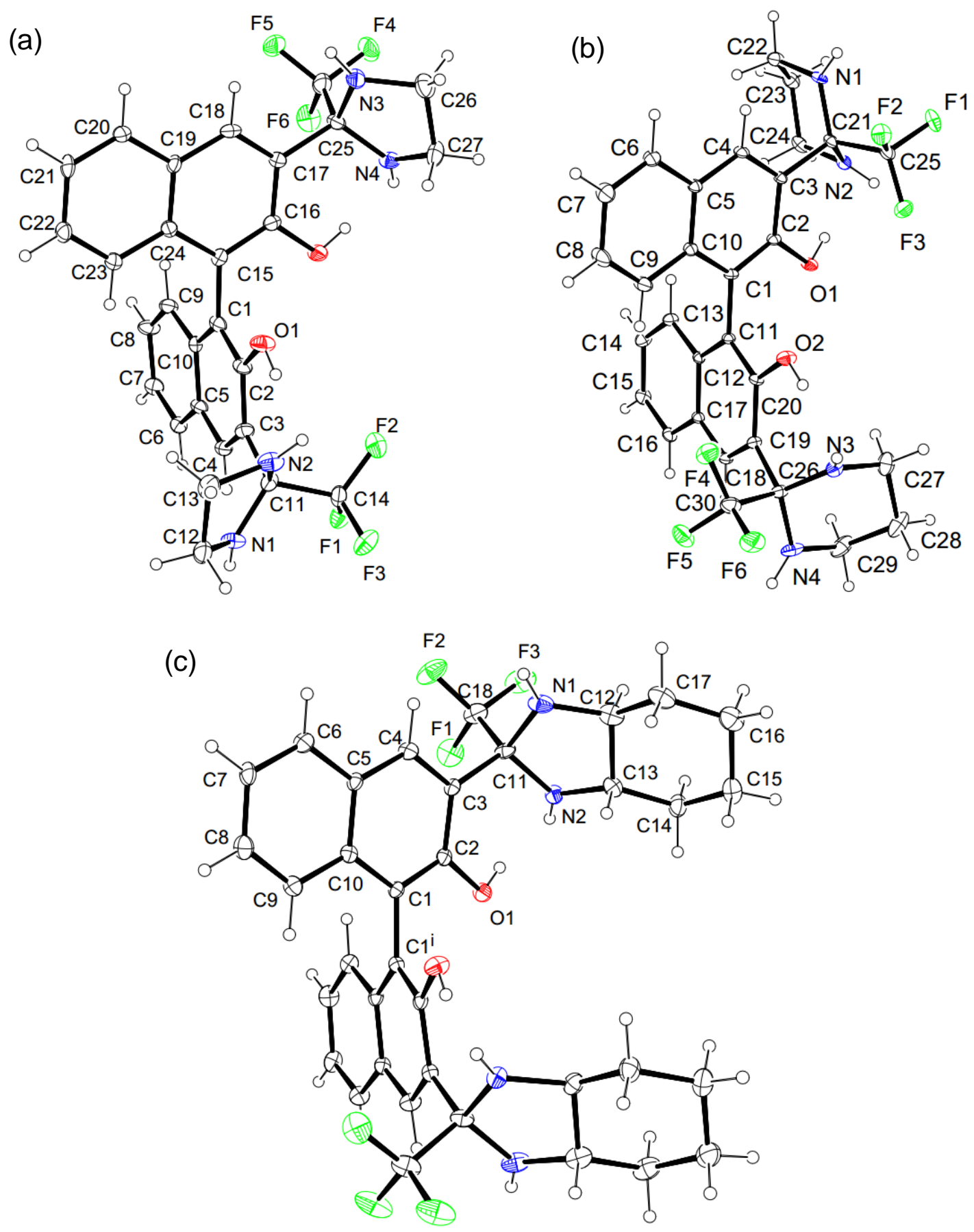
On the basis of the above observations for the interaction of $(S)-6-4$ with various amines, we found that the reactions of $(S)-6-4$ with the aliphatic primary diamines are much more facile than those with the primary monoamines, which lead to much greater fluorescence enhancement at lower concentration. The fluorescence responses of the sensor toward the 1,2-diamine and 1,5-diamine are much greater than those of the 1,3and 1,4-diamines. As shown in Scheme 6-8, it is proposed that the intermolecular hydrogen bond as shown by $(S)-\mathbf{6 - 2 7}$ could facilitate the reaction with the 1,2-diamines. In the resulting compound such as $(S)-\mathbf{6 - 2 8}$, the original hydrogen bond of $\mathrm{O}-\mathrm{H}^{\cdots} \mathrm{O}=\mathrm{C}$ has been disrupted which could contribute to the fluorescence enhancement. That is, although the $\mathrm{O}-\mathrm{H}^{\cdots} \mathrm{O}=\mathrm{C}$ hydrogen bonds of the sensor completely quench its fluorescence, the new $\mathrm{O}-\mathrm{H}^{\cdots} \mathrm{N}$ hydrogen bonds do not. In addition, the much greater fluorescence enhancement of the sensor caused by the 1,2-diamine than by propylamine could be further attributed to the formation of more rigid structures of $(S)-\mathbf{6 - 2 8}$ and $(S)-\mathbf{6 - 2 9}$ that contain multiple new hydrogen bonds of $\mathrm{O}-\mathrm{H}^{\cdots} \mathrm{N}$. The interaction of the second amine group of ethylenediamine with the two central hydroxyl groups of the sensor could also explain the observed highly enantioselective fluorescent response in the presence of the chiral 1,2-diamines. When the 1,3- and 1,4-diamines are used, there may be greater ring strains for the hydrogen bonding interactions similar to those shown by $(S)-\mathbf{6 - 2 7}-(S)-6-$ 28. Therefore, their fluorescence enhancements are much smaller. When the 1,5-diamine is used, the large fluorescence enhancement could be attributed to the formation of a compound like $(S)-\mathbf{6 - 2 4}$ where both carbonyl groups of the sensor could react with the amine to generate a rigid macrocyclic structure. 
Scheme 6-8. A proposed mechanism for the reaction of $(S)-6-4$ with ethylene diamine.

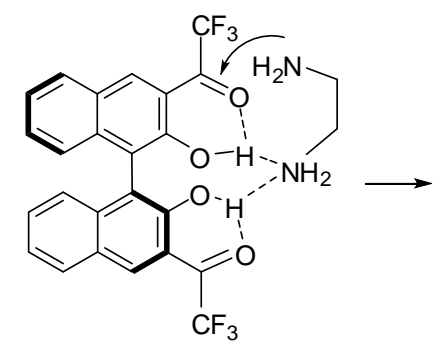

(S)-6-27

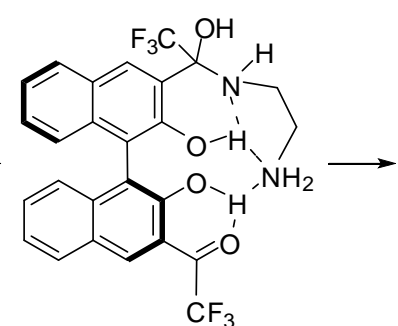

(S) $-6-28$

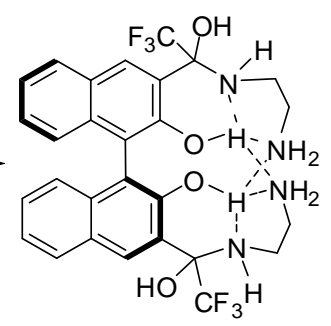

(S)-6-29

We have also isolated the final reaction product between $(S)-6-4$ and $(R, R)-\mathbf{N 1 5}$, compound $(S, R, R)$-6-30. Figure 6-14 gives the fluorescent spectrum of this compound. It gives a maximum emission at $383 \mathrm{~nm}$ and the fluorescent intensity is much higher than those intermediates.

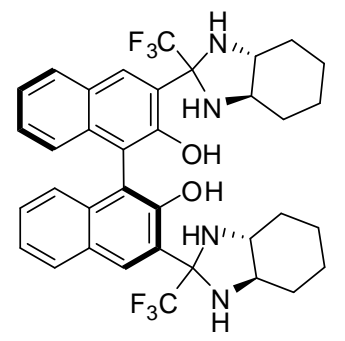

$(S, R, R)-6-30$

Figure 6-14. Fluorescence spectrum of $(S, R, R)-6-30\left(1 \times 10^{-5} \mathrm{M}\right.$ in $\left.\mathrm{CH}_{2} \mathrm{Cl}_{2}\right) \cdot\left(\lambda_{\text {exc }}=343\right.$ $\mathrm{nm}$, slit $=2.0 / 2.0 \mathrm{~nm})$

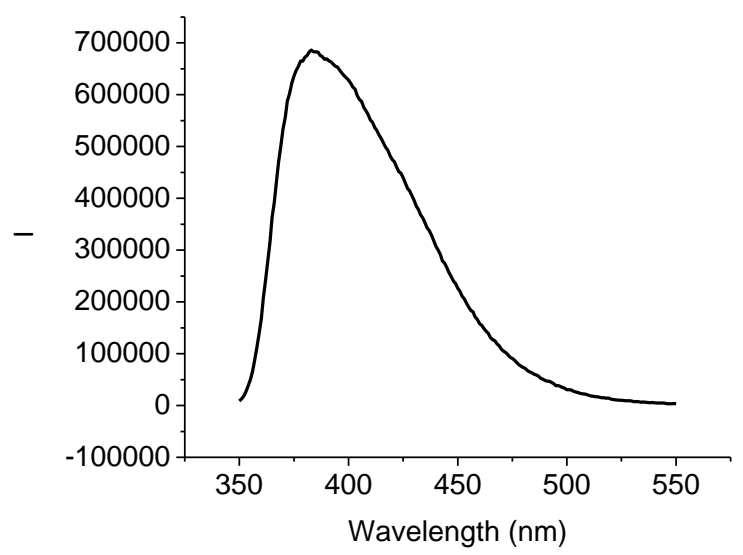




\subsection{Interaction of the other analogs of $(S)-6-4$ with amines}

The interactions of compounds $(R)-6-8,(S)-6-10,(S)-6-12$ and $(S)-6-16\left(1 \times 10^{-5} \mathrm{M}\right)$ with various amines in $\mathrm{CH}_{2} \mathrm{Cl}_{2}$ were also studied. The amines $\mathbf{N 1}, \mathbf{N 2}, \mathbf{N 5}, \mathbf{N 9},(R, R)-$ or $(S, S)$-N15 were selected as typical examples of tertiary, secondary and primary amines, achiral and chiral diamines. As shown in Figure 6-15a, b, monoamines N1, N2 and N5 caused very little change to the fluorescence of the $\mathrm{H}_{8} \mathrm{BINOL}$ analogue $(R)-\mathbf{6 - 8}$. The diamines turned on the fluorescence of $(R)-6-8$ with dual emissions. A low enantioselective fluorescence response was observed in the presence of $(R, R)$ - and $(S, S)$ N15 (Figure 6-15b). Thus, this compound could be used to sense the diamines but the sensitivity and enantioselectivity are much lower than those of $(S)-6-4$. The UV spectrum of $(R)-6-8$ in the presence of 500 equiv of the chiral diamine RR- and SS-N15 showed little change (Figure 6-15c).

Figure 6-15. Fluorescence spectra of $(R)-6-8$ in the presence of N1, N2, N5, N9 (a), and $(R, R)$ - or $(S, S)-\mathrm{N} 15$ (b) (amine: $\left.1.0 \times 10^{-3} \mathrm{M}\right) .\left(\lambda_{\mathrm{exc}}=286 \mathrm{~nm}\right.$, slit $\left.=2.0 / 2.0 \mathrm{~nm}\right) . \mathrm{UV}$ spectra of $(R)-\mathbf{6 - 8}$ in the presence of $(R, R)$ - and $(S, S)-\mathbf{N 1 5}\left(5 \times 10^{-3} \mathrm{M}\right)(\mathrm{c})$. (sensor: $1.0 \times 10^{-5} \mathrm{M}$ in $\mathrm{CH}_{2} \mathrm{Cl}_{2}$ )
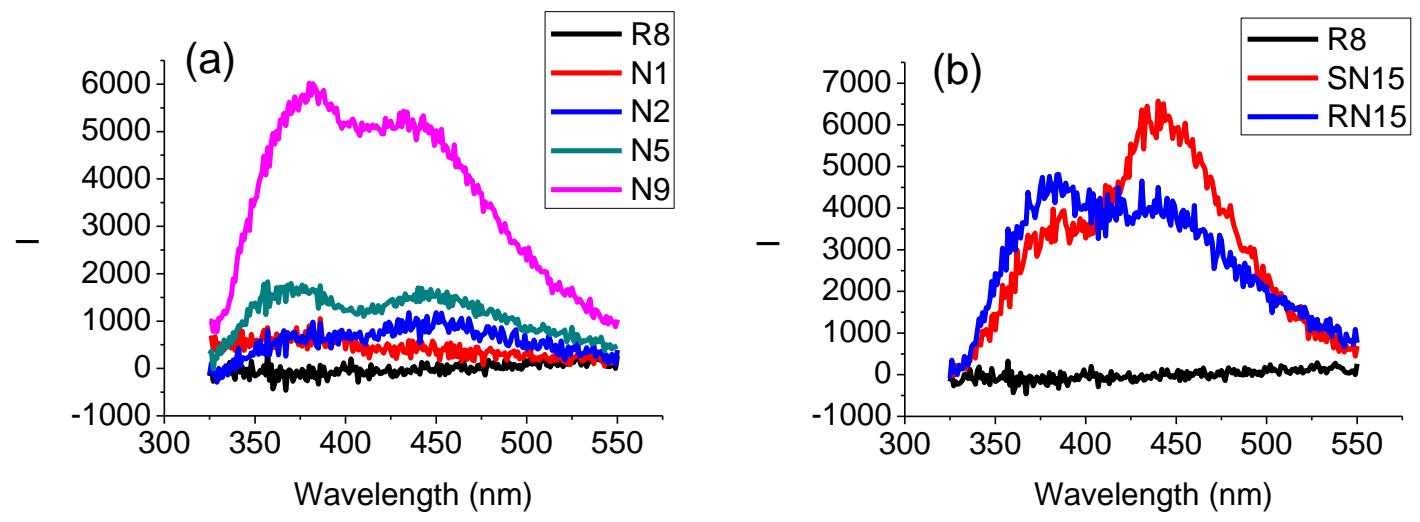


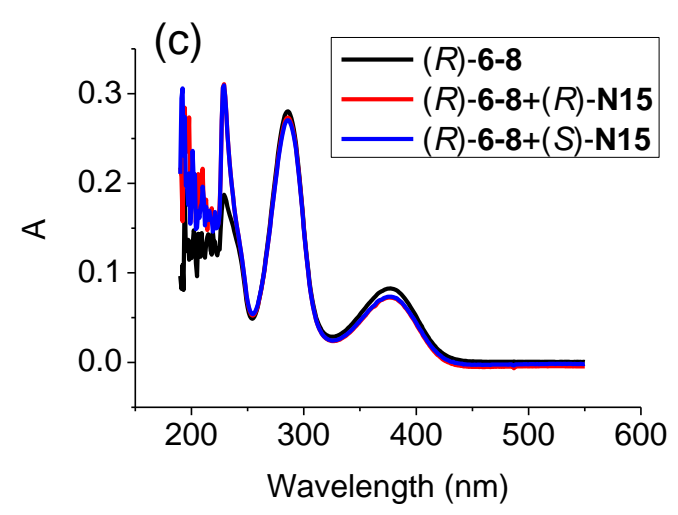

(S)-6-10, the BINOL-dialdehyde analogue, showed different fluorescence responses when treated with various amines. All of the tested monoamines and achiral diamines didn't turn on its fluorescence (Figure 6-16a). The chiral diamine N15 caused enantioselective fluorescence enhancement with $I_{R} / I_{S}=2.6$ at $\lambda=395 \mathrm{~nm}$ (Figure 6-16b). Figure 6-16c gives the UV absorption responses of $(S)-6-10$ when treated with $(R, R)$ - or $(S, S)$-N15. It shows that there were absorption increases at $\lambda=228,256,265,293$ and $304 \mathrm{~nm}$, an decrease at $\lambda=405 \mathrm{~nm}$, an new absorption at $\lambda=382 \mathrm{~nm}$.

Figure 6-16. Fluorescence spectra of $(S)-6-10$ in the presence of N1, N2, N5, N9 (a), and $(R, R)$ - or $(S, S)-\mathbf{N 1 5}(\mathrm{b}) .\left(\lambda_{\mathrm{exc}}=343 \mathrm{~nm}, \mathrm{slit}=2.0 / 2.0 \mathrm{~nm}\right)$. UV spectra of $(S)-\mathbf{6 - 1 0}$ in the presence of $(R, R)$ - and $(S, S)$-N15 (c). (sensor: $1.0 \times 10^{-5} \mathrm{M}$ in $\mathrm{CH}_{2} \mathrm{Cl}_{2}$. amine: $5.0 \times 10^{-3}$ M) 

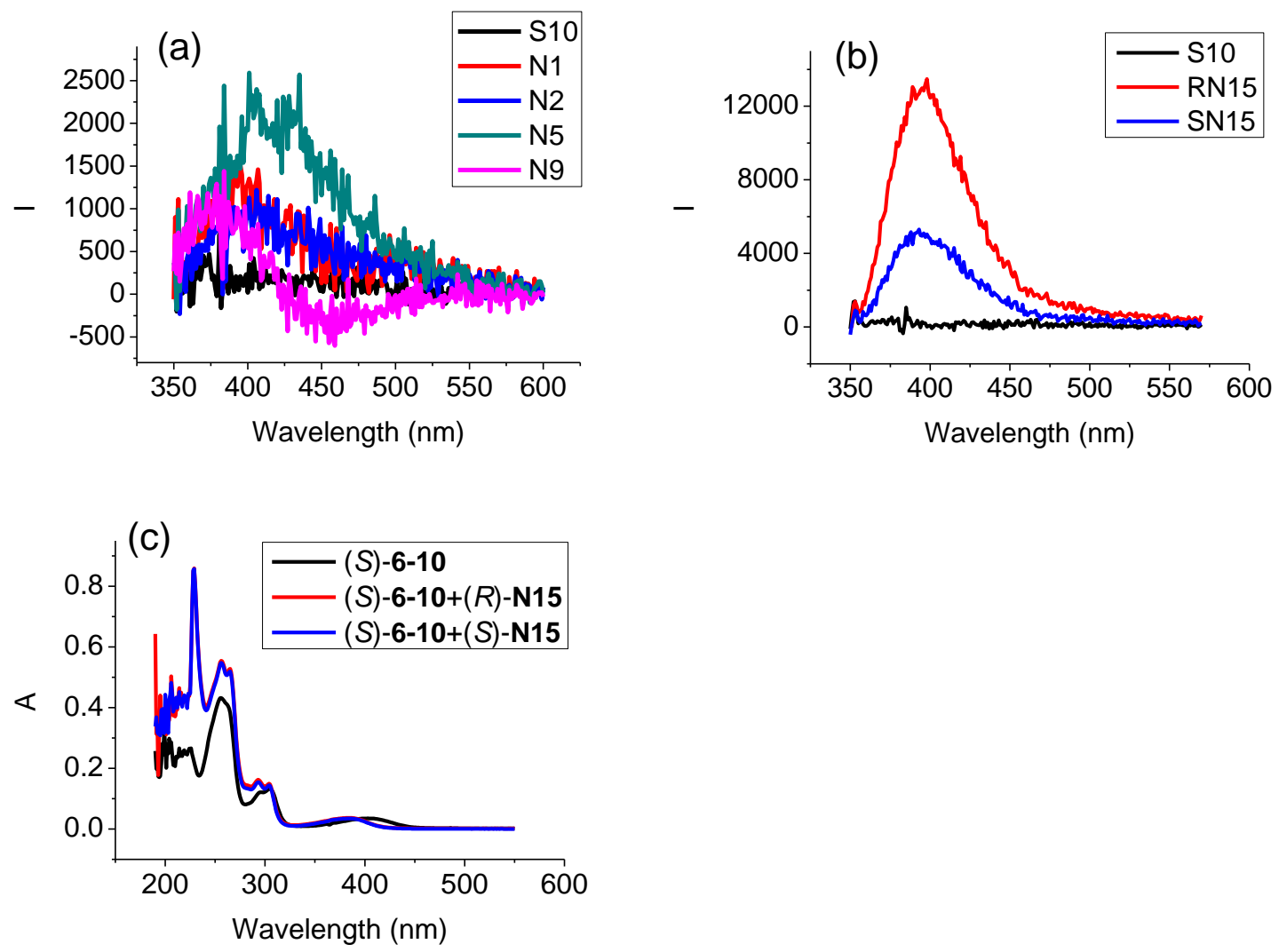

$(S)-6-12$, the BINOL-COCHF 2 analogue, showed similar fluorescence responses as (S)-6-4 toward various amines (Figure 6-17). When its solution $\left(1.0 \times 10^{-5} \mathrm{M}\right.$ in $\left.\mathrm{CH}_{2} \mathrm{Cl}_{2}\right)$ was treated with $(R, R)-\mathbf{N} 15\left(5.0 \times 10^{-3} \mathrm{M}\right)$, a dramatic fluorescent enhancement was observed with dual emissions at $385\left(\lambda_{1}\right)$ and $438\left(\lambda_{2}\right)$ nm with $\mathrm{I}_{1} / \mathrm{I}_{2}=0.64$. When $(S)-6-$ 12 was treated with $(S, S)-\mathbf{N} 15$, a similar large fluorescence enhancement at $\lambda_{1}$ was also observed, but the fluorescence enhancement at $\lambda_{2}$ was much smaller with $I_{1} / I_{2}=1.47$. Therefore, it can serve as an alternative sensor for the enantioselective fluorescent recognition of the chiral diamines.

Figure 6-17. Fluorescence spectra of $(S)-6-12\left(1.0 \times 10^{-5} \mathrm{M}\right.$ in $\left.\mathrm{CH}_{2} \mathrm{Cl}_{2}\right)$ in the presence of N1, N2, N5, N9, $(R, R)-$ or $(S, S)-\mathbf{N 1 5}\left(5.0 \times 10^{-3} \mathrm{M}\right) .\left(\lambda_{\mathrm{exc}}=343 \mathrm{~nm}\right.$, slit $\left.=3.0 / 3.0 \mathrm{~nm}\right)$. 

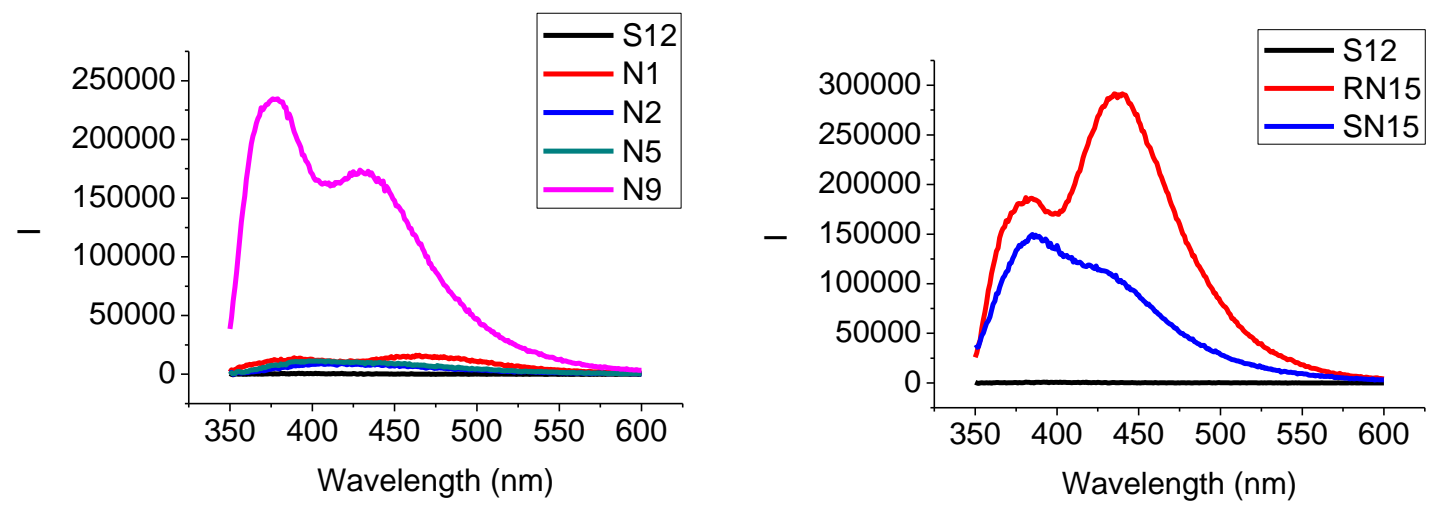

The 6, 6'-analogue (S)-6-16 exhibited significant fluorescence quenching with all of the tested amines, in which chiral diamine $\mathbf{N 1 5}$ quenched over $90 \%$ of its fluorescence with no enantioselectivity (Figure 6-18a,b). Since (S)-6-16 does not have the intramolecular $\mathrm{OH} . . . \mathrm{O}=\mathrm{C}$ hydrogen bonds of $(S)-\mathbf{6 - 4}$, it is strongly emissive. Therefore, the mechanism of fluorescence enhancement for $(S)-\mathbf{6 - 4}$ in the presence of N15 to disrupte the intramolecular $\mathrm{OH} . . \mathrm{O}=\mathrm{C}$ hydrogen bonds cannot operate with $(S)-\mathbf{6 - 1 6}$. The 6,6'-positions of the two trifluoroacetyl groups of (S)-6-16 also make it unlikely to form a cyclic rigid structure with N15. It was reported ${ }^{16}$ that the fluorescence of BINOL can be quenched by amines in various solvents. Our observed fluorescence quneching of $(S)$-616 by the amines appears to be much more efficient than that of BINOL by amines. It was proposed that the fluorescence quenching of BINOL by amines could be attributed to the formation of hydrogen bonded complexes between the BINOL hydroxyl groups and the amine nitrogen as well as the corresponding excited state proton transfer complex. The increased fluorescence quenching of $(S)-\mathbf{6 - 1 6}$ by the amines could be attributed to the increased acidity of the hydroxyl groups of $(S)-\mathbf{6 - 1 6}$ caused by the two electronwithdrawing 6,6'-trifluoroacetyl groups which should increase their interaction with the 
amine bases. Figure 6-18c gives the UV responses of $(S)-6-16$ when treated with R and S-N15. It shows an absorption increase at $228 \mathrm{~nm}$, an decrease at 251, 279 and $323 \mathrm{~nm}$, and an new absorption at $370 \mathrm{~nm}$. No enantioselectivity is observed in the UV absorptions.

Figure 6-18. Fluorescence spectra of $(S)-6-16$ in the presence of N1, N2, N5, N9, N12 (a), and $(R, R)$ - or $(S, S)-\mathbf{N 1 5}(\mathrm{b}) .\left(\lambda_{\mathrm{exc}}=343 \mathrm{~nm}\right.$. slit $=1.0 / 1.0 \mathrm{~nm}$ for N1, N2, N5, N9 and N12. slit $=2.0 / 2.0 \mathrm{~nm}$ for $(R, R)$ - or $(S, S)$-N15). UV spectra of $(S)-6-16$ in the presence of $(R, R)$ - and $(S, S)-\mathrm{N} 15$ (c). (sensor: $1.0 \times 10^{-5} \mathrm{M}$ in $\mathrm{CH}_{2} \mathrm{Cl}_{2}$. amine: $5.0 \times 10^{-3}$ M)
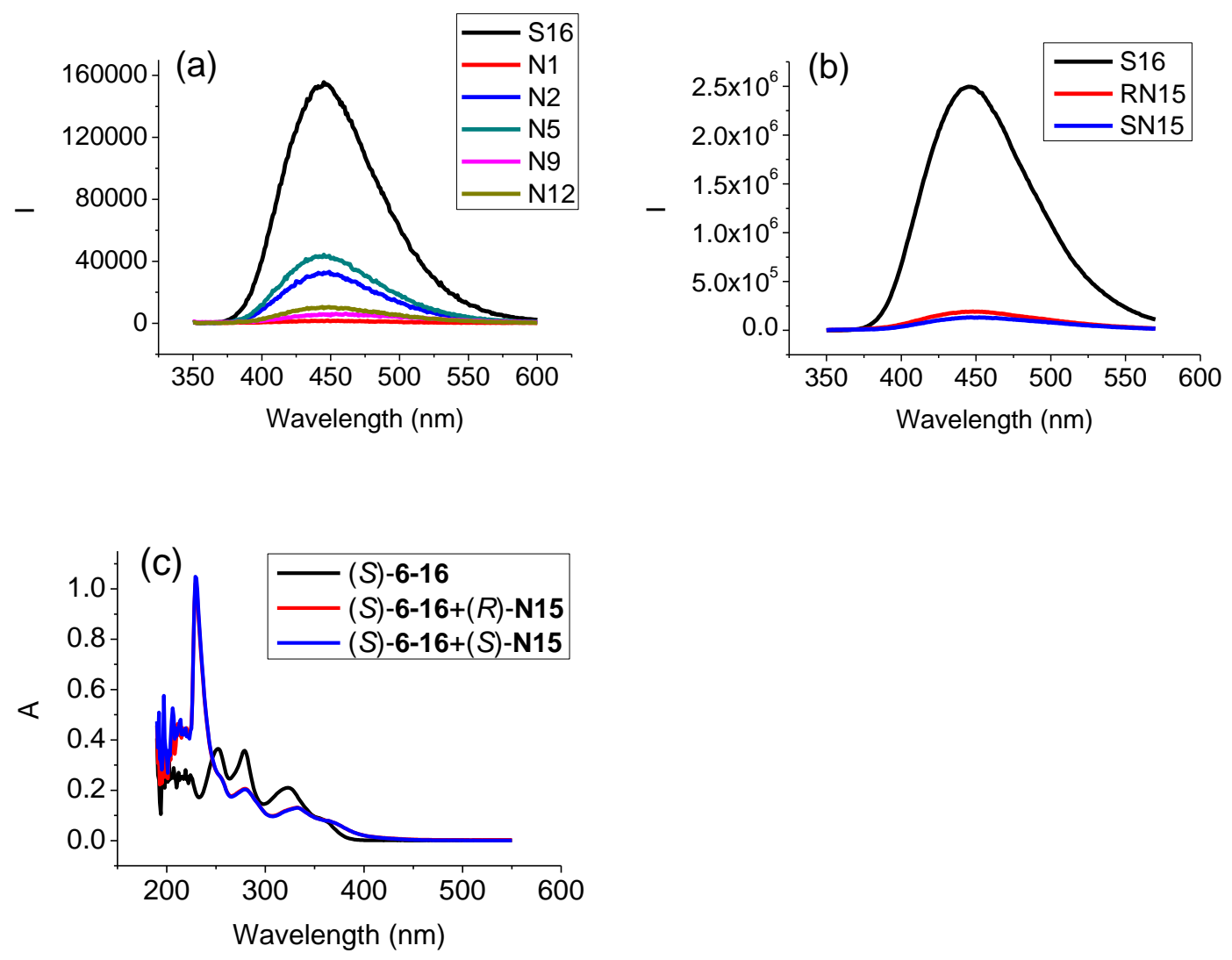
The MOM protected analogue (S)-6-3 was also examined for the interaction with the chiral diamine $(R, R)$ - and $(S, S)-\mathbf{N 1 5}$ in $\mathrm{CH}_{2} \mathrm{Cl}_{2}$ (Figure 6-19). Without the intramolecular $\mathrm{OH}^{\cdots} \mathrm{O}=\mathrm{C}$ hydrogen bonds of $(S)-\mathbf{6 - 4},(S)-\mathbf{6 - 3}$ is also strongly emissive. Both enantiomers of $\mathbf{N 1 5}$ caused a small ( $10 \%)$ fluorescence quenching of $(S)$-6-3 with no enantioselectivity. This demonstrates that the hydroxyl groups of (S)-6-4 are essential for its highly sensitive fluorescence enhancement and excellent enantioselectivity. The UV spetrum of (S)-6-3 showed little change in the presence of R or SN15 except an increase at $1=230 \mathrm{~nm}$ (Figure 6-19b).

Figure 6-19. (a) Fluorescence spectra of $(S)-6-3$ in the presence of $(R, R)$ - and $(S, S)$ N15. $\left(\lambda_{\text {exc }}=343 \mathrm{~nm}\right.$, slit $\left.=2.0 / 2.0 \mathrm{~nm}\right)$. (b) UV spectra of $(S)-6-3$ in the presence of $(R$, $R$ )- and $(S, S)$-N15. (sensor: $1.0 \times 10^{-5} \mathrm{M}^{\text {in }} \mathrm{CH}_{2} \mathrm{Cl}_{2}$. amine: $5.0 \times 10^{-3} \mathrm{M}$ )
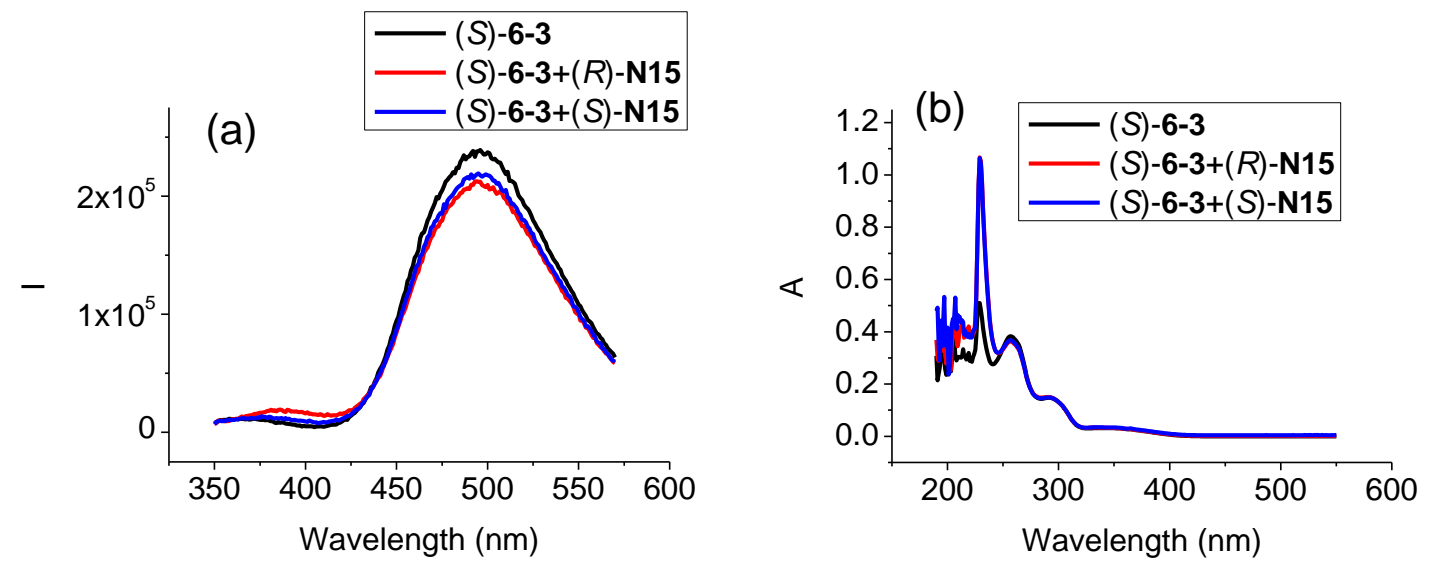

When the excitation wavelength was set to $290 \mathrm{~nm},(S)-6-3$ showed dual emissions at 359 and $494 \mathrm{~nm}$, in which the emission at $359 \mathrm{~nm}$ is much weaker than that at $494 \mathrm{~nm}$ (Figure 6-20). (S)-6-16 also showed a shoulder peak at $359 \mathrm{~nm}$ after treatment with N15. We attribute the short wavelength emission to the locally excited state of binaphthol and 
the long wavelength emission to internal charge shift state.

Figure 6-20. Fluorescence spectra of $(S)-6-3$ (a) and $(S)-6-16$ (b) in the presence of $(R$,

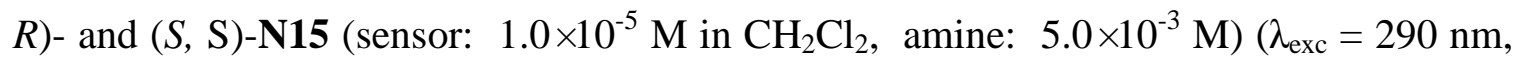
slit $=2.0 / 2.0 \mathrm{~nm})$.
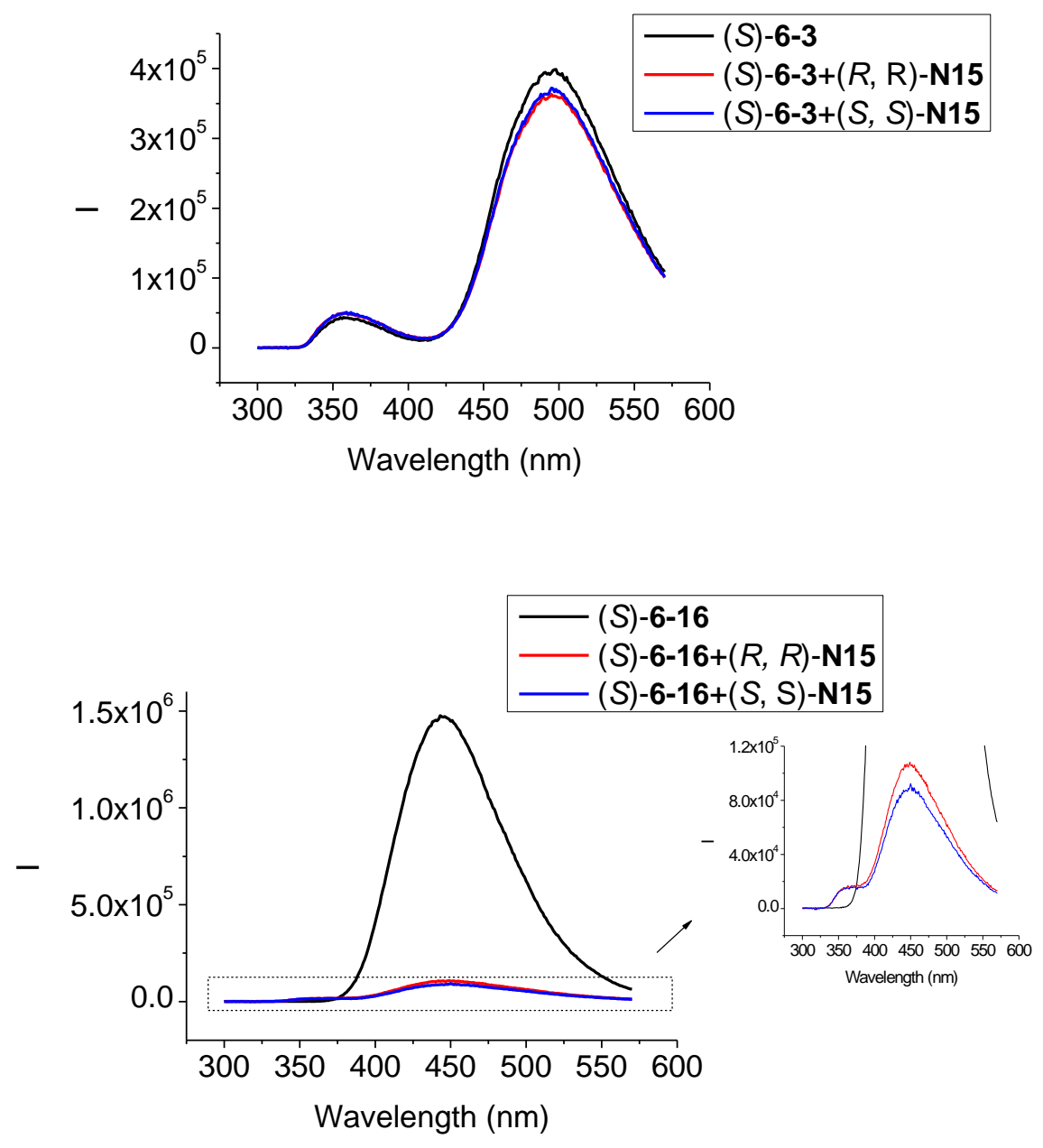

\subsection{Interaction of (S)-6-4 with Tetrabutylammonium Salts of Amino Acids.}

Interactions of $(S)-6-4\left(1 \times 10^{-5} \mathrm{M}\right)$ with various amino acids (as tetrabutylammonium salts, $\left.2.0 \times 10^{-4} \mathrm{M}\right)$ in $\mathrm{CH}_{2} \mathrm{Cl}_{2}$ were also studied. Figure 6-21 gives the fluorescence 
spectra of $(S)-6-4$ in the presence of the tetrabutylammonium salts of D- and L-amino acids. All of the tested amino acids turned on the fluorescence of (S)-6-4. The maximum emission of (S)-6-4 located at $450 \mathrm{~nm}$ when treated with Ser, Ala, Val, Leu, Tyr and at $460 \mathrm{~nm}$ with Asp. Moreover, it showed enantioselectivity toward different enantiomers of amino acids with higher fluorescence intensity for the D-enantiomer. The ratios of $\mathrm{I}_{\mathrm{D}} / \mathrm{I}_{\mathrm{L}}$ for tested amino acids were summarized in table 6-2.

Table 6-2. The ratio of $I_{D} / I_{L}$ of tested amino acid salts. ( $I_{D}$ : the fluorescence intensity maximum of $(S)-6-4$ when treated with D-amino acid salts. $\mathrm{I}_{\mathrm{L}}$ : the fluorescence intensity at maximum of (S)-6-4 when treated with L-amino acid salts.)

\begin{tabular}{|c|c|c|c|c|c|c|}
\hline & Ser & Ala & Val & Leu & Asp & Tyr \\
\hline $\mathrm{I}_{\mathrm{D}} / \mathrm{I}_{\mathrm{L}}$ & 2.0 & 1.9 & 2.8 & 1.6 & 3.2 & 1.5 \\
\hline
\end{tabular}

Figure 6-21. Fluorescence spectra of $(S)-6-4\left(1.0 \times 10^{-5} \mathrm{M}\right)$ in the presence of the tetrabutylammonium salts of D- and L-amino acids $\left(2.0 \times 10^{-4} \mathrm{M}\right) .\left(\lambda_{\mathrm{exc}}=343 \mathrm{~nm}\right.$, slit $=$ $2.0 / 2.0 \mathrm{~nm})$.
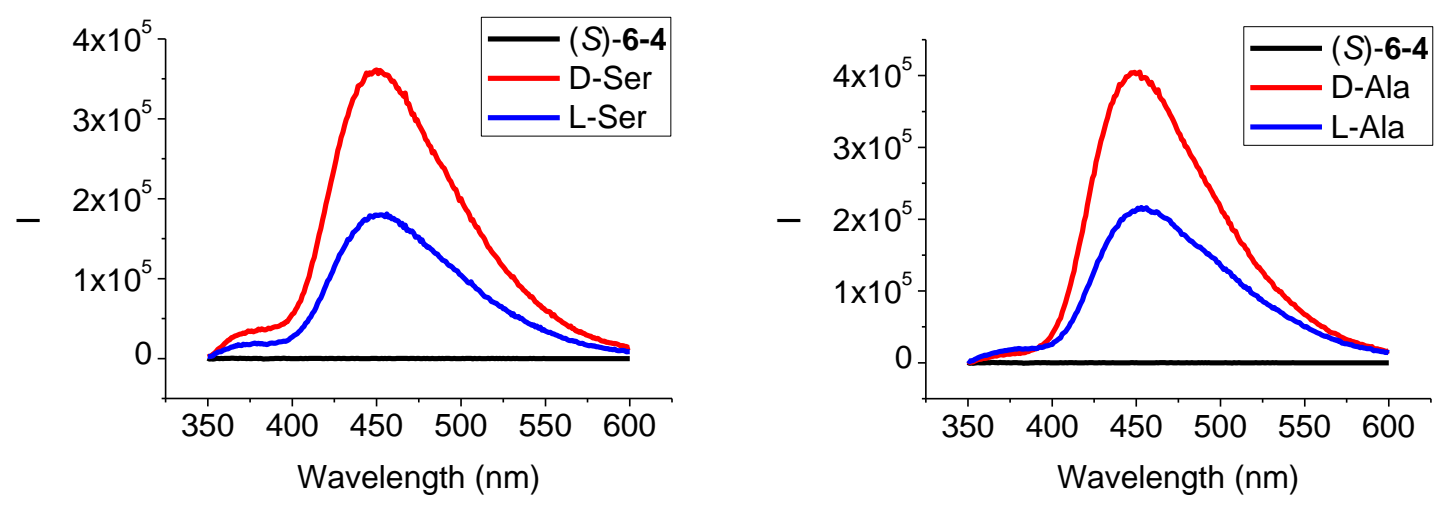

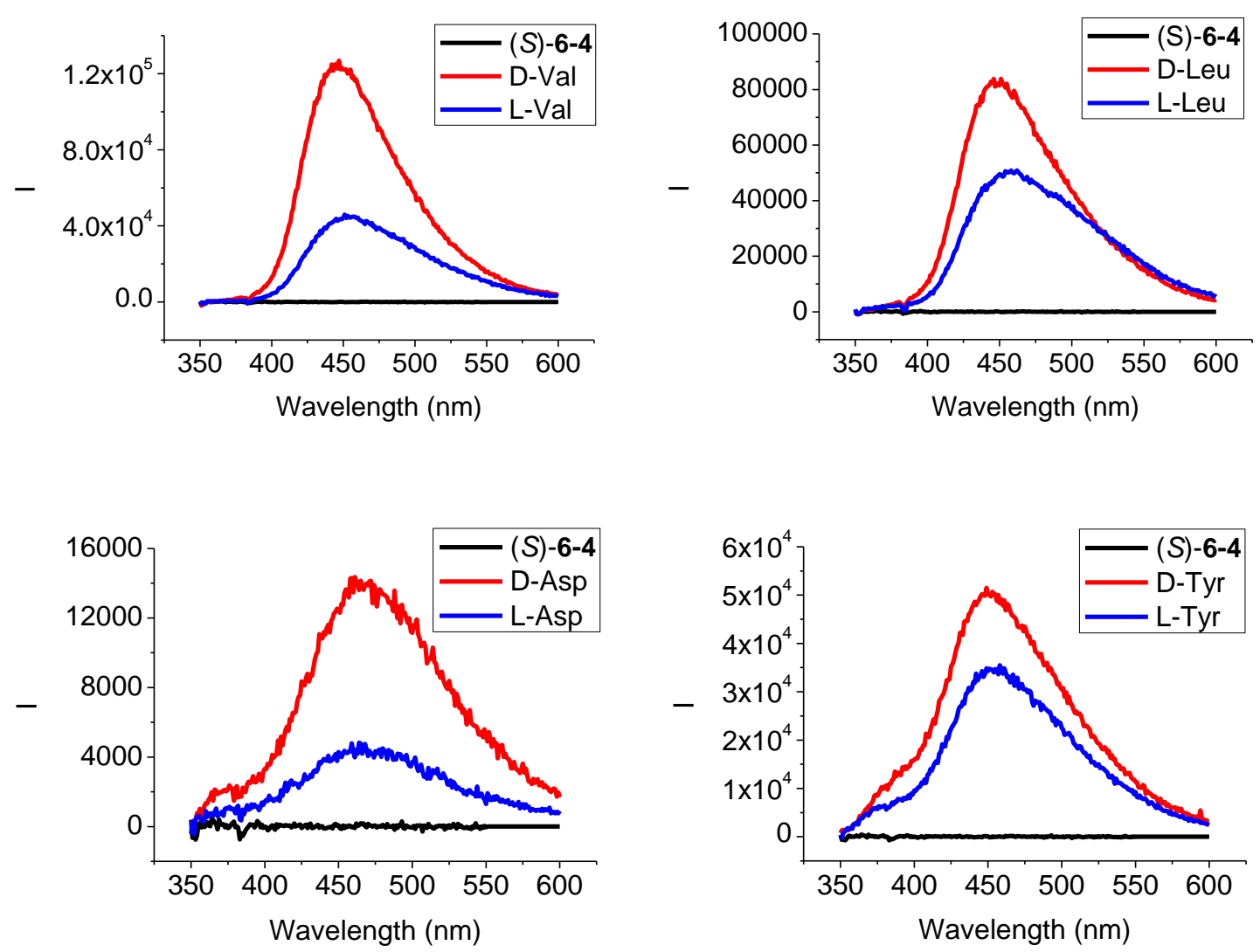

\subsection{Interaction of $(S)-6-4$ with Chiral Amino Alcohols.}

Interactions of $(S)-6-4\left(1 \times 10^{-5} \mathrm{M}\right)$ with chiral amino alcohols AA1, AA2 and AA3 in $\mathrm{CH}_{2} \mathrm{Cl}_{2}$ were also studied. As shown in Figure 6-23, treatment with $(S)$-AA1 turned on the fluorescence of $(S)-\mathbf{6 - 4}$ with maximum emission at $375 \mathrm{~nm}$ while $(R)$-AA1 only caused very little change to the $(S)-6-4$ solution. The fluorescence intensity ratio $I_{S} / I_{R}\left(I_{S}\right.$ : the fluorescence intensity of $(S)-\mathbf{6 - 4}$ at $375 \mathrm{~nm}$ when treated with $(S)$-AA. $\mathrm{I}_{\mathrm{R}}$ : the fluorescence intensity of $(S)-\mathbf{6 - 4}$ at $375 \mathrm{~nm}$ when treated with $(R)$-AA. ) reached 13.4 when the concentration of AA1 was $2.0 \mathrm{mM}$. Both enantiomers of AA2 turned on the fluorescence of $(S)-\mathbf{6 - 4}$ and showed maximum emission at $375 \mathrm{~nm}$. Similar to AA1, $(S)$ AA2 enhanced the fluorescence of $(S)$-6-4 more than $(R)$-AA2 did, with $\mathrm{I}_{\mathrm{S}} / \mathrm{I}_{\mathrm{R}}=3.3$ at 
concentration 2.0 mM. When $\mathbf{A A 3}$ was used, the ratio $I_{S} / I_{R}$ reached 5.1 at concentration $5.0 \mathrm{mM}$. It was noted that treatment of $(R)$-AA2 2 showed dual emissions at $375 \mathrm{~nm}$ and $425 \mathrm{~nm}$. With addition of increasing concentration of $(R)-\mathbf{A A 2}$, the ratio of fluorescence intensities at these two wavelengths, $\lambda_{375} / \lambda_{425}$, was decreasing.

Time-dependent fluorescence was also monitored for the mixture of $(S)-\mathbf{6 - 4}$ with $(S)$ AA1 and the fluorescence kept increasing even after overnight. Therefore, the reaction of (S)-6-4 with amino alcohols was much slower than that with diamines. The spectra shown in Figure 6-23 were taken two hours after preparation. When amino alcohols with higher concentration were used, consistent fluorescent results could not be obtained, which make it difficult for this sensor to be used in quantitative measurement.

Figure 6-22. Structures of tested chiral amino alcohols.

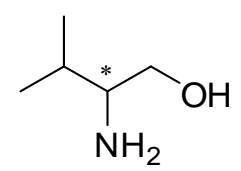

AA1

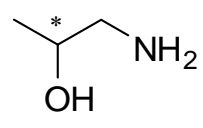

AA2

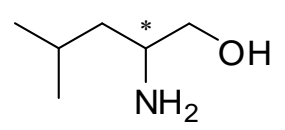

AA3

Figure 6-23. Fluorescence spectra of $(S)-6-4\left(1.0 \times 10^{-5} \mathrm{M}\right.$, in $\left.\mathrm{CH}_{2} \mathrm{Cl}_{2}\right)$ in the presence of chiral amino alcohols $\left(2.0 \times 10^{-4} \mathrm{M}\right)$ and the maximum emission change with increasing concentrations. $\left(\lambda_{\mathrm{exc}}=343 \mathrm{~nm}\right.$, slit $\left.=2.0 / 2.0 \mathrm{~nm}\right)$. 

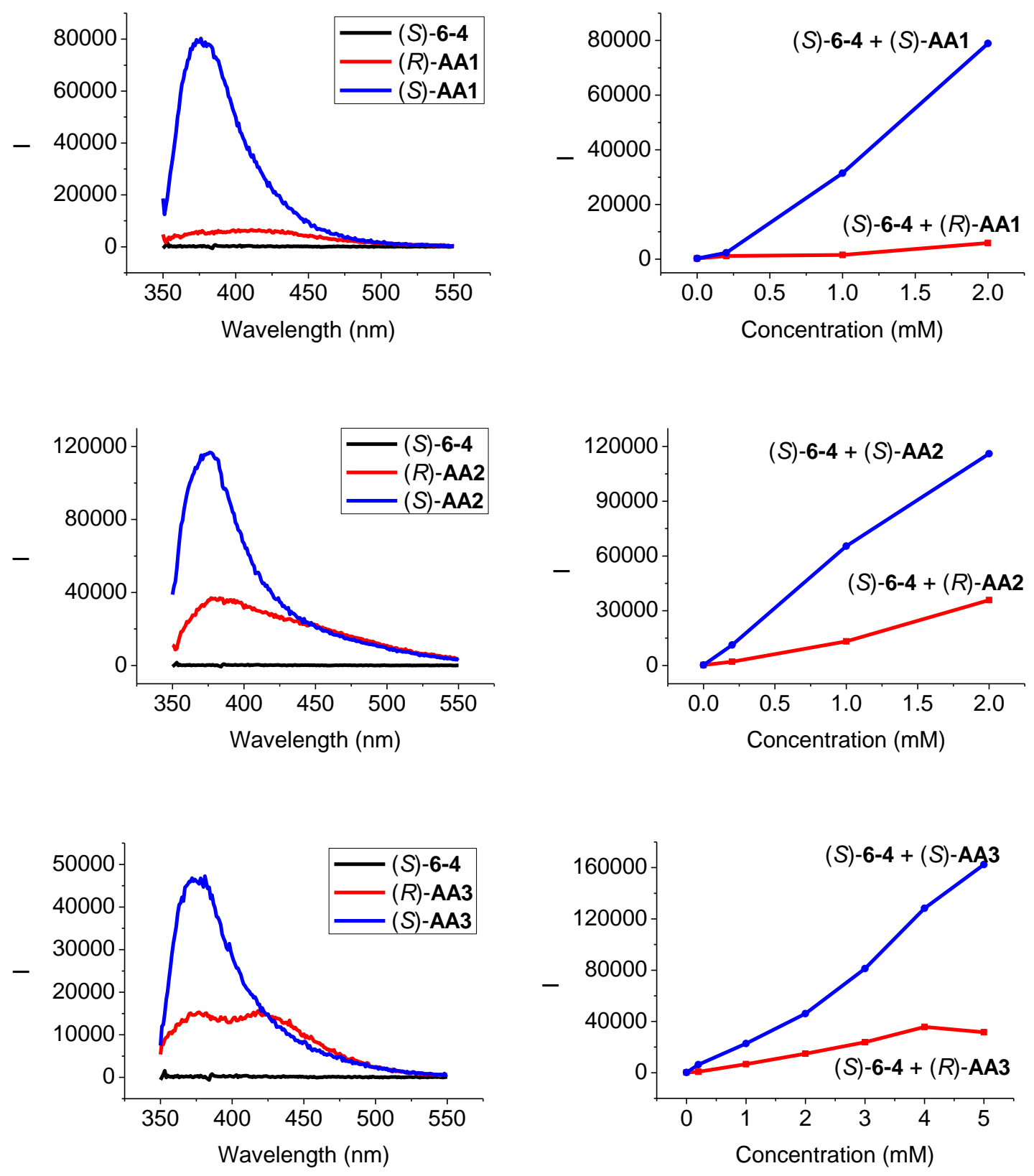

\section{Conclusion}

We have synthesized and studied a series of BINOL-dicarbonyl compounds for fluorescent recognition. This study reveals that the intramolecular hydrogen bonds between the BINOL hydroxyl groups and the carbonyl units of these compounds can turn 
off the fluorescence of the BINOL unit. Binding with nucleophilic substrates transforms the original hydrogen binding and turns on the fluorescence. Formation of more rigid structures with the substrates further enhances the fluorescence. $(S)-\mathbf{6 - 4}$ was proved to be a general enantioselective fluorescent sensor for a variety of substrates with two nucleophilic groups, such as diamines, amino carboxylates and amino alcohols. Some analogs of (S)-6-4, such as compounds (R)-6-10 and $(S)-\mathbf{6 - 1 2}$, also exhibit enantioselective fluorescent responses in the interaction with chiral diamines. Therefore, the BINOL-dicarbonyl compounds represent a promising class of enantioselective fluorescent sensors for functional chiral amines.

\section{Experimental Section}

\subsection{General Data:}

All reactions were carried out under nitrogen unless otherwise noted. All chemicals were purchased from Sigma Aldrich Chemical Co. or Alfa Aesar. THF was distilled over sodium and benzophenone under nitrogen atmosphere. Methylene chloride and diethyl ether were dried by passing through activated alumina columns under nitrogen. Solvents were stored over $4 \AA$ molecular sieves.

Melting points were uncorrected and obtained on a Mel-Temp II capillary melting point apparatus. Optical rotations were measured on a Jasco P-2000 digital polarimeter. NMR spectra were recorded on a Varian-300 MHz or Bruker-600 MHz spectrometer.

Chemical shifts for ${ }^{1} \mathrm{H}$ NMR spectra were reported in parts per million relative to a singlet at $7.26 \mathrm{ppm}$ for deuterated chloroform. Chemical shifts for ${ }^{13} \mathrm{C}$ NMR were 
reported relative to the centerline of a triplet at $77.16 \mathrm{ppm}$ for deuterated chloroform. The ${ }^{19} \mathrm{~F}$ NMR spectra were reported in units of part per million (ppm) relative to trifluoroacetic acid $(\delta-76.55 \mathrm{ppm})$ as an external reference. High resolution mass spectra were obtained from the University of Illinois at Urbana-Champaign (UIUC) Mass Spectrometry Facility. UV-Vis spectra were recorded on a Hewlett-Packard 8452A diode-array spectrophotometer. Steady-state fluorescence emission spectra were recorded on a Horiba FluoroMax-4 spectrofluorometer.

\subsection{Preparation of Samples for Fluorescence Measurement.}

Materials: Sensors were purified by column chromatography followed by recrystallization and then stored in a refrigerator. All of the tested amines were purchased and redistilled. All of the solvents were either HPLC or spectroscopic grade. The stock solutions of the sensors and amines were freshly prepared for each measurement. For the fluorescence study, a sensor solution was mixed with the amine solution at room temperature in a $5 \mathrm{~mL}$ volumetric flask and diluted to the desired concentration. The resulting solution was allowed to stand at room temperature for $0.5 \mathrm{~h}$ before the fluorescence measurement and all the fluorescence spectra were taken within 2 hours.

\subsection{Synthesis and Characterization of Compounds}

Synthesis and Characterization of (S)-6-2. ${ }^{17}$ Under nitrogen, $(S)-1,1$ '-binaphthyl-2,2'diol, $(S)-6-1$ (17.5 mmol, $5.0 \mathrm{~g})$, was dissolved in THF $(200 \mathrm{~mL})$. The solution was cooled to $0{ }^{\circ} \mathrm{C}$, and $\mathrm{NaH}(43.75 \mathrm{mmol}, 60 \%$ in mineral oil, $1.75 \mathrm{~g})$ was added in small portions. The reaction mixture was stirred for $15 \mathrm{~min}$ and then chloromethyl methyl ether (43.75 mmol, $3.3 \mathrm{~mL}$ ) was added slowly. The reaction mixture was allowed to warm to 
room temperature and stirred for $1 \mathrm{~h}$. Water was added slowly to quench the reaction. The organic layer was separated, and the aqueous layer was extracted with ethyl acetate (three $30 \mathrm{~mL}$ aliquots). The combined organic extracts were washed with brine, and dried over $\mathrm{Na}_{2} \mathrm{SO}_{4}$. After evaporation of the solvent, the residue was purified by column chromatography on silica gel eluted with hexane/ethyl acetate (15/1) to afford compound (S)-6-2 as a white solid in 95\% yield (16.6 mmol, 6.22g). $\left.{ }^{1} \mathrm{H} \mathrm{NMR} \mathrm{(300} \mathrm{MHz}, \mathrm{CDCl}_{3}\right) \delta$ $3.15(\mathrm{~s}, 6 \mathrm{H}), 4.98(\mathrm{~d}, \mathrm{~J}=6.9 \mathrm{~Hz}, 2 \mathrm{H}), 5.09(\mathrm{~d}, \mathrm{~J}=6.6 \mathrm{~Hz}, 2 \mathrm{H}), 7.14-7.25(\mathrm{~m}, 4 \mathrm{H}), 7.32-$ $7.37(\mathrm{~m}, 2 \mathrm{H}), 7.58(\mathrm{~d}, \mathrm{~J}=9.3 \mathrm{~Hz}, 2 \mathrm{H}), 7.88(\mathrm{~d}, \mathrm{~J}=8.1 \mathrm{~Hz}, 2 \mathrm{H}), 7.96(\mathrm{~d}, \mathrm{~J}=9.0 \mathrm{~Hz}, 2 \mathrm{H})$.

Synthesis and Characterization of (S)-6-3. Under nitrogen, 2,2'-bis-methoxymethyl1,1'-binaphthyl, $(S)$-6-2 (3.0 mmol, $1.12 \mathrm{~g})$, was dissolved in diethyl ether (36 mL). The solution was cooled to $0^{\circ} \mathrm{C}$, and $\mathrm{n}-\mathrm{BuLi}(12.0 \mathrm{mmol}, 2.5 \mathrm{M}$ in hexane, $4.8 \mathrm{~mL})$ was added dropwise. The reaction mixture was stirred for $2 \mathrm{~h}$ at room temperature and cooled to $0{ }^{0} \mathrm{C}$, and then ethyl trifluoroacetate $(13.5 \mathrm{mmol}, 1.6 \mathrm{~mL})$ was added slowly. The reaction mixture was allowed to warm to room temperature and stirred for $1 \mathrm{~h}$ to afford a cream-like mixture. A saturated aqueous $\mathrm{NH}_{4} \mathrm{Cl}$ solution was added to quench the reaction. The organic layer was separated, and the aqueous layer was extracted with ethyl acetate (three $20 \mathrm{~mL}$ aliquots). The combined organic extracts were washed with brine, and dried over $\mathrm{Na}_{2} \mathrm{SO}_{4}$. After evaporation of the solvent, the residue was purified by column chromatography on silica gel eluted with hexane/methylene chloride (1/3) to afford compound $(S)-2$ as a yellow oil in $62 \%$ yield (1.86 mmol, $1.05 \mathrm{~g}) .{ }^{1} \mathrm{H}$ NMR (300 $\left.\mathrm{MHz}, \mathrm{CDCl}_{3}\right) \delta 2.77(\mathrm{~s}, 6 \mathrm{H}), 4.73(\mathrm{~d}, \mathrm{~J}=6.3 \mathrm{~Hz}, 2 \mathrm{H}), 4.77(\mathrm{~d}, \mathrm{~J}=6.3 \mathrm{~Hz}, 2 \mathrm{H}), 7.25(\mathrm{~d}, \mathrm{~J}=$ 8.7 Hz, 2H), 7.44-7.50 (m, 2H), 7.53-7.58 (m, 2H), 8.05 (d, J = 8.1 Hz, 2H), $8.43(\mathrm{~s}, 2 \mathrm{H})$. 
${ }^{19} \mathrm{~F}$ NMR $\left(282 \mathrm{MHz}, \mathrm{CDCl}_{3}\right) \delta-73.62 .{ }^{13} \mathrm{C} \mathrm{NMR}\left(75 \mathrm{MHz}, \mathrm{CDCl}_{3}\right) \delta 56.6,100.7,116.5$ $(q, J=290 \mathrm{~Hz}), 126.4,126.8,126.9,127.0,129.6,129.8,130.1,132.7,136.2,151.6$, $182.4\left(\mathrm{q}, \mathrm{J}=35.6 \mathrm{~Hz}\right.$ ). HRMS Calcd for $\mathrm{C}_{28} \mathrm{H}_{20} \mathrm{O}_{6} \mathrm{~F}_{6} \mathrm{Na}\left(\mathrm{MNa}^{+}\right)$: 589.1062, Found: 589.1053. $[\alpha]_{\mathrm{D}}=-18.056\left(\mathrm{c}=0.590, \mathrm{CHCl}_{3}\right)$.

Synthesis and Characterization of (S)-6-4. After compound (S)-6-3 (0.25 mmol, 134.2 mg) was dissolved in a minimum amount of $\mathrm{CH}_{2} \mathrm{Cl}_{2}$, trifluroacetic acid $(1.0 \mathrm{~mL})$ was added slowly, and the mixture was stirred at room temperature for $10 \mathrm{~min}$. A saturated aqueous $\mathrm{NaHCO}_{3}$ solution was added to quench the reaction. The organic layer was separated, and the aqueous layer was extracted with $\mathrm{CH}_{2} \mathrm{Cl}_{2}(3 \times 20 \mathrm{~mL})$. The combined organic extracts were washed with brine, and dried over $\mathrm{Na}_{2} \mathrm{SO}_{4}$. After evaporation of the solvent, the residue was purified by column chromatography on silica gel eluted with hexane/methylene chloride (2/1) to afford compound $(S)-\mathbf{6 - 4}$ as an orange solid in $84 \%$ yield (0.21 mmol, $100 \mathrm{mg}) .{ }^{1} \mathrm{H} \mathrm{NMR}\left(300 \mathrm{MHz}, \mathrm{CDCl}_{3}\right) \delta 7.16(\mathrm{~d}, \mathrm{~J}=7.5 \mathrm{~Hz}, 2 \mathrm{H})$, 7.41-7.51 (m, 4H), $8.02(\mathrm{~d}, \mathrm{~J}=7.5 \mathrm{~Hz}, 2 \mathrm{H}), 8.70(\mathrm{~s}, 2 \mathrm{H}), 10.51(\mathrm{~s}, 2 \mathrm{H}) .{ }^{19} \mathrm{~F}$ NMR (282 $\left.\mathrm{MHz}, \mathrm{CDCl}_{3}\right) \delta-70.06 .{ }^{13} \mathrm{C} \mathrm{NMR}\left(150 \mathrm{MHz}, \mathrm{CDCl}_{3}\right) \delta 115.2,116.7(\mathrm{q}, \mathrm{J}=289.5 \mathrm{~Hz})$, $117.8,124.7,125.3,127.2,131.2,132.2,136.1(q, J=3.75 \mathrm{~Hz}), 138.5,155.0,185.1(q, \mathrm{~J}$ $=36.0 \mathrm{~Hz})$. HRMS Calcd for $\mathrm{C}_{24} \mathrm{H}_{13} \mathrm{O}_{4} \mathrm{~F}_{6}\left(\mathrm{MH}^{+}\right): 479.0718$, Found: 479.0719. m.p. 231 ${ }^{0} \mathrm{C} .[\alpha]_{\mathrm{D}}=-167.50\left(\mathrm{c}=0.355, \mathrm{CHCl}_{3}\right)$.

Synthesis and Characterization of $(\boldsymbol{R})-\mathbf{6 - 6} .^{18}$ Under nitrogen, $(R)-5,5^{\prime}, 6,6^{\prime}, 7,7^{\prime}, 8,8^{\prime}-$ octahydro-1,1'-binaphthyl-2,2'-diol ( $\left.\mathrm{H}_{8} \mathrm{BINOL}\right),(R)-\mathbf{6 - 5}$ (25 mmol, $\left.7.4 \mathrm{~g}\right)$, was dissolved in THF (300 mL). The solution was cooled to $0{ }^{\circ} \mathrm{C}$, and $\mathrm{NaH}(62.8 \mathrm{mmol}, 60 \%$ in mineral oil, $2.52 \mathrm{~g}$ ) was added in small portions. The reaction mixture was stirred for 30 
min and then chloromethyl methyl ether $(62.8 \mathrm{mmol}, 4.8 \mathrm{~mL})$ was added slowly. The reaction mixture was allowed to warm to room temperature and stirred for overnight. Water was added slowly to quench the reaction. The organic layer was separated, and the aqueous layer was extracted with ethyl acetate (three $30 \mathrm{~mL}$ aliquots). The combined organic extracts were washed with brine, and dried over $\mathrm{Na}_{2} \mathrm{SO}_{4}$. After evaporation of the solvent, the residue was purified by column chromatography on silica gel eluted with hexane/ethyl acetate (15/1) to afford compound (R)-6-6 as a white solid in $90 \%$ yield (22.5 mmol, 8.6 g). ${ }^{1} \mathrm{H}$ NMR (300 MHz, $\left.\mathrm{CDCl}_{3}\right) \delta 1.65-1.76(\mathrm{~m}, 8 \mathrm{H}), 2.07-2.17(\mathrm{~m}, 2 \mathrm{H})$, 2.27-2.37 (m, 2H), $2.78(\mathrm{t}, \mathrm{J}=6.0 \mathrm{~Hz}, 4 \mathrm{H}), 3.30(\mathrm{~s}, 6 \mathrm{H}), 4.97(\mathrm{~d}, \mathrm{~J}=6.6 \mathrm{~Hz}, 2 \mathrm{H}), 5.03(\mathrm{~d}$, $\mathrm{J}=6.6 \mathrm{~Hz}, 2 \mathrm{H}), 6.99(\mathrm{~d}, \mathrm{~J}=8.4 \mathrm{~Hz}, 2 \mathrm{H}), 7.05(\mathrm{~d}, \mathrm{~J}=8.4 \mathrm{~Hz}, 2 \mathrm{H})$.

Synthesis and Characterization of (R)-6-7. Under nitrogen, (R)-2,2'bis(methoxymethoxy)-5,5',6,6',7,7',8,8'-octahydro-1,1'-binaphthyl, $(R)$-6-6 (4.4 mmol, 1.7 $\mathrm{g})$, was dissolved in diethyl ether $(50 \mathrm{~mL})$. The solution was cooled to $0{ }^{\circ} \mathrm{C}$, and $\mathrm{n}$-BuLi (17.8 mmol, 2.5 $\mathrm{M}$ in hexane, $7.1 \mathrm{~mL}$ ) was added dropwise. The reaction mixture was stirred for $4 \mathrm{~h}$ at room temperature and cooled to $0{ }^{0} \mathrm{C}$, and then ethyl trifluoroacetate (20.0 mmol, $2.4 \mathrm{~mL}$ ) was added slowly. The reaction mixture was allowed to warm to room temperature and stirred for $2.5 \mathrm{~h}$ to afford a cream-like mixture. A saturated aqueous $\mathrm{NH}_{4} \mathrm{Cl}$ solution was added to quench the reaction. The organic layer was separated, and the aqueous layer was extracted with ethyl acetate (three $20 \mathrm{~mL}$ aliquots). The combined organic extracts were washed with brine, and dried over $\mathrm{Na}_{2} \mathrm{SO}_{4}$. After evaporation of the solvent, the residue was purified by column chromatography on silica gel eluted with hexane/methylene chloride (1/3) to afford compound $(R)-6-7$ as a yellow 
oil in $52 \%$ yield $(2.3 \mathrm{mmol}, 1.31 \mathrm{~g}) .{ }^{1} \mathrm{H} \mathrm{NMR}\left(300 \mathrm{MHz}, \mathrm{CDCl}_{3}\right) \delta 1.65-1.82(\mathrm{~m}, 8 \mathrm{H})$, 2.12-2.22 (m, 2H), 2.47-2.57 (m, 2H), 2.83-2.87 (m, 10H), $4.79(\mathrm{~d}, \mathrm{~J}=6.6 \mathrm{~Hz}, 2 \mathrm{H}), 4.83$ $(\mathrm{d}, \mathrm{J}=6.6 \mathrm{~Hz}, 2 \mathrm{H}), 7.49(\mathrm{~s}, 2 \mathrm{H}) .{ }^{13} \mathrm{C} \mathrm{NMR}\left(75 \mathrm{MHz}, \mathrm{CDCl}_{3}\right) \delta 22.5,28.5,29.6,56.2$, $100.8,116.4(q, J=290 \mathrm{~Hz}), 123.2,130.47,130.51,132.2,133.9,145.4,153.5,181.4(q, J$ $=34.5 \mathrm{~Hz})$. HRMS Calcd for $\mathrm{C}_{28} \mathrm{H}_{28} \mathrm{O}_{6} \mathrm{~F}_{6} \mathrm{Na}\left(\mathrm{MNa}^{+}\right)$: 597.1688, Found: 197.1678. [ $\left.\alpha\right]_{\mathrm{D}}$ $=43.34\left(\mathrm{c}=0.39, \mathrm{CHCl}_{3}\right)$.

Synthesis and Characterization of $(\boldsymbol{R})$-6-8. After compound $(R)-6-7(237.8 \mathrm{mg}, 0.41$ mmol) was dissolved in a minimum amount of $\mathrm{CH}_{2} \mathrm{Cl}_{2}$, trifluroacetic acid (1.0 mL) was added slowly, and the mixture was stirred at room temperature for $10 \mathrm{~min}$. A saturated aqueous $\mathrm{NaHCO}_{3}$ solution was added to quench the reaction. The organic layer was separated, and the aqueous layer was extracted with $\mathrm{CH}_{2} \mathrm{Cl}_{2}(3 \times 20 \mathrm{~mL})$. The combined organic extracts were washed with brine, and dried over $\mathrm{Na}_{2} \mathrm{SO}_{4}$. After evaporation of the solvent, the residue was purified by column chromatography on silica gel eluted with hexane/methylene chloride (2/1) to afford compound $(R)-\mathbf{6 - 8}$ as a yellow solid in $86 \%$ yield (0.35 mmol, $172 \mathrm{mg}) .{ }^{1} \mathrm{H}$ NMR $\left(300 \mathrm{MHz}, \mathrm{CDCl}_{3}\right) \delta 1.71-1.88(\mathrm{~m}, 8 \mathrm{H}), 2.25-2.35$ $(\mathrm{m}, 2 \mathrm{H}), 2.50-2.60(\mathrm{~m}, 2 \mathrm{H}), 2.88(\mathrm{~m}, 4 \mathrm{H}), 7.67(\mathrm{~s}, 2 \mathrm{H}), 11.1(\mathrm{~s}, 2 \mathrm{H}) .{ }^{13} \mathrm{C} \mathrm{NMR}(75 \mathrm{MHz}$, $\left.\mathrm{CDCl}_{3}\right) \delta 22.4,22.6,28.3,29.4,112.2,116.8(\mathrm{q}, \mathrm{J}=288 \mathrm{~Hz}), 124.4,129.9,130.6(\mathrm{q}, \mathrm{J}=$ 3.0 Hz), 150.0, 159.4, $184.1(\mathrm{q}, \mathrm{J}=34.5 \mathrm{~Hz})$. HRMS Calcd for $\mathrm{C}_{24} \mathrm{H}_{21} \mathrm{O}_{4} \mathrm{~F}_{6}\left(\mathrm{MH}^{+}\right)$: 487.1344, Found: 487.1338. m.p. $209{ }^{0} \mathrm{C} .[\alpha]_{\mathrm{D}}=-38.5\left(\mathrm{c}=0.36, \mathrm{CHCl}_{3}\right)$.

Synthesis and Characterization of $(\boldsymbol{S})-6-9 .{ }^{6}$ Under nitrogen, 2,2'-bis-methoxymethyl1,1'-binaphthyl, $(S)$-6-2 (3.0 mmol, $1.12 \mathrm{~g})$, was dissolved in diethyl ether (33 mL). The solution was cooled to $0{ }^{\circ} \mathrm{C}$, and $\mathrm{n}$-BuLi (10.5 mmol, $2.5 \mathrm{M}$ in hexane, $4.2 \mathrm{~mL}$ ) was 
added dropwise. The reaction mixture was stirred for $3 \mathrm{~h}$ at room temperature and cooled to $0{ }^{0} \mathrm{C}$, and then anhydrous DMF $(12.0 \mathrm{mmol}, 1.3 \mathrm{~mL})$ was added slowly. The reaction mixture was allowed to warm to room temperature and stirred for $2 \mathrm{~h}$ to afford a creamlike mixture. A saturated aqueous $\mathrm{NH}_{4} \mathrm{Cl}$ solution was added to quench the reaction. The organic layer was separated, and the aqueous layer was extracted with ethyl acetate (three $20 \mathrm{~mL}$ aliquots). The combined organic extracts were washed with brine, and dried over $\mathrm{Na}_{2} \mathrm{SO}_{4}$. After evaporation of the solvent, the residue was purified by column chromatography on silica gel eluted with hexane/ethyl acetate (4/1) to afford compound (S)-6-9 as a yellow oil in 73\% yield $(2.2 \mathrm{mmol}, 943 \mathrm{mg}) .{ }^{1} \mathrm{H} \mathrm{NMR}\left(300 \mathrm{MHz}, \mathrm{CDCl}_{3}\right) \delta$ $2.88(\mathrm{~s}, 6 \mathrm{H}), 4.76(\mathrm{~d}, \mathrm{~J}=1.2 \mathrm{~Hz}, 2 \mathrm{H}), 4.78(\mathrm{~d}, \mathrm{~J}=1.5 \mathrm{~Hz}, 2 \mathrm{H}), 7.27(\mathrm{~d}, \mathrm{~J}=8.4 \mathrm{~Hz}, 2 \mathrm{H})$, $7.42(\mathrm{t}, \mathrm{J}=6.9 \mathrm{~Hz}, 2 \mathrm{H}), 7.53(\mathrm{~d}, \mathrm{~J}=6.9 \mathrm{~Hz}, 2 \mathrm{H}), 8.10(\mathrm{~d}, \mathrm{~J}=8.1 \mathrm{~Hz}, 2 \mathrm{H}), 8.67(\mathrm{~s}, 2 \mathrm{H})$, 10.59 (s, 2H).

Synthesis and Characterization of $(S)-6-10{ }^{6}$ After compound $(S)-6-9(1.1 \mathrm{mmol}$, $471 \mathrm{mg}$ ) was dissolved in a minimum amount of $\mathrm{CH}_{2} \mathrm{Cl}_{2}$, trifluroacetic acid (1.0 mL) was added slowly, and the mixture was stirred at room temperature for $1 \mathrm{~h}$. A saturated aqueous $\mathrm{Na}_{2} \mathrm{CO}_{3}$ solution was added to quench the reaction. The organic layer was separated, and the aqueous layer was extracted with $\mathrm{CH}_{2} \mathrm{Cl}_{2}(3 \times 20 \mathrm{~mL})$. The combined organic extracts were washed with brine, and dried over $\mathrm{Na}_{2} \mathrm{SO}_{4}$. After evaporation of the solvent, the residue was purified by recrystallization with $\mathrm{CH}_{2} \mathrm{Cl}_{2}$ /ethanol to afford compound $(S)-\mathbf{6 - 1 0}$ as a yellow solid in $84 \%$ yield $(0.92 \mathrm{mmol}, 316 \mathrm{mg}) .{ }^{1} \mathrm{H}$ NMR (300 $\left.\mathrm{MHz}, \mathrm{CDCl}_{3}\right) \delta$ 7.19-7.22 (m, 2H), 7.38-7.43 (m, 4H), 7.98-8.02 (m, 2H), $8.35(\mathrm{~s}, 2 \mathrm{H})$, 10.19 (s, 2H), 10.59 (s, 2H). 
Synthesis and Characterization of (S)-6-11. Under nitrogen, 2,2'-bis-methoxymethyl1,1'-binaphthyl, $(S)$-6-2 (3.0 mmol, $1.12 \mathrm{~g})$, was dissolved in diethyl ether (36 mL). The solution was cooled to $0^{\circ} \mathrm{C}$, and $\mathrm{n}-\mathrm{BuLi}$ (10.5 mmol, $2.5 \mathrm{M}$ in hexane, $4.2 \mathrm{~mL}$ ) was added dropwise. The reaction mixture was stirred for $3 \mathrm{~h}$ at room temperature and cooled to $0{ }^{0} \mathrm{C}$, and then ethyl difluoroacetate $(12.0 \mathrm{mmol}, 1.3 \mathrm{~mL})$ was added slowly. The reaction mixture was allowed to warm to room temperature and stirred for $2 \mathrm{~h}$ to afford a cream-like mixture. A saturated aqueous $\mathrm{NH}_{4} \mathrm{Cl}$ solution was added to quench the reaction. The organic layer was separated, and the aqueous layer was extracted with ethyl acetate (three $20 \mathrm{~mL}$ aliquots). The combined organic extracts were washed with brine, and dried over $\mathrm{Na}_{2} \mathrm{SO}_{4}$. After evaporation of the solvent, the residue was purified by column chromatography on silica gel eluted with hexane/methylene chloride (1/2) to afford compound $(S)-\mathbf{6 - 1 1}$ as a yellow oil in $41 \%$ yield $(1.23 \mathrm{mmol}, 652 \mathrm{mg}) .{ }^{1} \mathrm{H}$ NMR $\left(300 \mathrm{MHz}, \mathrm{CDCl}_{3}\right) \delta 2.90(\mathrm{~s}, 6 \mathrm{H}), 4.60(\mathrm{~d}, \mathrm{~J}=5.7 \mathrm{~Hz}, 2 \mathrm{H}), 4.66(\mathrm{~d}, \mathrm{~J}=5.7 \mathrm{~Hz}, 2 \mathrm{H}), 6.69$ $(\mathrm{t}, \mathrm{J}=54 \mathrm{~Hz}, 2 \mathrm{H}), 7.20(\mathrm{~d}, \mathrm{~J}=8.4 \mathrm{~Hz}, 2 \mathrm{H}), 7.44(\mathrm{~m}, 2 \mathrm{H}), 7.54(\mathrm{~m}, 2 \mathrm{H}), 8.05(\mathrm{~d}, \mathrm{~J}=8.1$ $\mathrm{Hz}, 2 \mathrm{H}), 8.44(\mathrm{~s}, 2 \mathrm{H}) .{ }^{19} \mathrm{~F}$ NMR $\left(282 \mathrm{MHz}, \mathrm{CDCl}_{3}\right) \delta-127.93(\mathrm{dd}, \mathrm{J} 1=321 \mathrm{~Hz}, \mathrm{~J} 2=54$ $\mathrm{Hz}, 2 \mathrm{~F}),-126.57(\mathrm{dd}, \mathrm{J} 1=321 \mathrm{~Hz}, 54 \mathrm{~Hz}, 2 \mathrm{~F}) .{ }^{13} \mathrm{C} \mathrm{NMR}\left(75 \mathrm{MHz}, \mathrm{CDCl}_{3}\right) \delta 57.0,100.2$, $110.0(\mathrm{t}, \mathrm{J}=249 \mathrm{~Hz}), 125.4,125.9,126.6,129.4,129.7,129.9,130.0,133.1,136.0,151.0$, $190.6(\mathrm{t}, \mathrm{J}=25.5 \mathrm{~Hz})$. HRMS Calcd for $\mathrm{C}_{28} \mathrm{H}_{22} \mathrm{O}_{6} \mathrm{~F}_{4} \mathrm{Na}\left(\mathrm{MNa}^{+}\right)$: 553.1250, Found: 553.1259. $[\alpha]_{\mathrm{D}}=31.9\left(\mathrm{c}=0.19, \mathrm{CHCl}_{3}\right)$.

Synthesis and Characterization of (S)-6-12. After compound $(S)$-6-11 (0.94 mmol, 500 mg) was dissolved in a minimum amount of $\mathrm{CH}_{2} \mathrm{Cl}_{2}$, trifluroacetic acid $(1.0 \mathrm{~mL})$ was added slowly, and the mixture was stirred at room temperature for $1 \mathrm{~h}$. A saturated 
aqueous $\mathrm{NaHCO}_{3}$ solution was added to quench the reaction. The organic layer was separated, and the aqueous layer was extracted with $\mathrm{CH}_{2} \mathrm{Cl}_{2}(3 \times 20 \mathrm{~mL})$. The combined organic extracts were washed with brine, and dried over $\mathrm{Na}_{2} \mathrm{SO}_{4}$. After evaporation of the solvent, the residue was purified by column chromatography on silica gel eluted with hexane/methylene chloride (1/1) to afford compound $(S)$-6-12 as a light yellow solid in $62 \%$ yield $(0.58 \mathrm{mmol}, 258 \mathrm{mg}) .{ }^{1} \mathrm{H} \mathrm{NMR}\left(600 \mathrm{MHz}, \mathrm{CDCl}_{3}\right) \delta 6.53(\mathrm{t}, \mathrm{J}=53.4 \mathrm{~Hz}, 2 \mathrm{H})$, $7.17(\mathrm{~d}, \mathrm{~J}=7.8 \mathrm{~Hz}, 2 \mathrm{H}), 7.40-7.46(\mathrm{~m}, 4 \mathrm{H}), 8.00(\mathrm{~d}, \mathrm{~J}=7.8 \mathrm{~Hz}, 2 \mathrm{H}), 8.76(\mathrm{~s}, 2 \mathrm{H}), 10.75$ $(\mathrm{s}, 2 \mathrm{H}) .{ }^{19} \mathrm{~F}$ NMR $\left.\left(282 \mathrm{MHz}, \mathrm{CDCl}_{3}\right)\right) \delta-121.31(\mathrm{dd}, \mathrm{J} 1=351 \mathrm{~Hz}, \mathrm{~J} 2=57 \mathrm{~Hz}, 2 \mathrm{~F}),-$ $119.82(\mathrm{dd}, \mathrm{J} 1=351 \mathrm{~Hz}, 57 \mathrm{~Hz}, 2 \mathrm{~F}) .{ }^{13} \mathrm{C} \mathrm{NMR}\left(150 \mathrm{MHz}, \mathrm{CDCl}_{3}\right) \delta 111.3(\mathrm{t}, \mathrm{J}=252$ Hz), 116.6, 117.6, 124.7, 124.9, 127.2, 130.8, 131.6, $135.4(\mathrm{t}, \mathrm{J}=4.5 \mathrm{~Hz}), 138.1,154.8$, $192.2(\mathrm{t}, \mathrm{J}=25.5 \mathrm{~Hz})$. HRMS Calcd for $\mathrm{C}_{24} \mathrm{H}_{15} \mathrm{O}_{4} \mathrm{~F}_{4}\left(\mathrm{MH}^{+}\right)$: 443.0906, Found: 443.0907. m.p. $271{ }^{0} \mathrm{C} .[\alpha]_{\mathrm{D}}=-153.5\left(\mathrm{c}=0.21, \mathrm{CHCl}_{3}\right)$.

Synthesis and Characterization of (S)-6-13. ${ }^{7}(S)-6-1(14.7 \mathrm{mmol}, 4.2 \mathrm{~g})$ was dissolved in $\mathrm{CH}_{2} \mathrm{Cl}_{2}(150 \mathrm{~mL})$ and the solution was cooled to $-78{ }^{0} \mathrm{C} . \mathrm{Br}_{2}(37.8 \mathrm{mmol}, 2 \mathrm{~mL})$ was added dropwise. After the reaction mixture was stirred for $2.5 \mathrm{~h}$, it was warmed up to room temperature and stirred for additional $30 \mathrm{~min} .10 \% \mathrm{NaHSO}_{3}$ solution $(100 \mathrm{~mL})$ was added to quench the reaction. The organic layer was separated, and the aqueous layer was extracted with $\mathrm{CH}_{2} \mathrm{Cl}_{2}(20 \mathrm{~mL} \times 3)$. The combined organic extracts were washed with brine, and dried over $\mathrm{Na}_{2} \mathrm{SO}_{4}$. After evaporation of the solvent, the residue was purified by column chromatography on silica gel eluted with hexane/ethyl acetate (4/1) to afford compound $(S)-\mathbf{6 - 1 3}$ as a white solid in $87 \%$ yield $(12.8 \mathrm{mmol}, 5.68 \mathrm{~g}) .{ }^{1} \mathrm{H}$ NMR $\left(300 \mathrm{MHz}, \mathrm{CDCl}_{3}\right) \delta 5.01(\mathrm{~s}, 2 \mathrm{H}), 6.96(\mathrm{~d}, \mathrm{~J}=9.0 \mathrm{~Hz}, 2 \mathrm{H}), 7.34-7.41(\mathrm{~m}, 4 \mathrm{H}), 7.89$ 
$(\mathrm{d}, \mathrm{J}=9.0 \mathrm{~Hz}, 2 \mathrm{H}), 8.05(\mathrm{~s}, 2 \mathrm{H})$.

Synthesis and Characterization of (S)-6-14. ${ }^{7}$ Under nitrogen, $(S)-6-13$ (8.0 mmol, 3.55 g) was dissolved in THF $(150 \mathrm{~mL})$. The solution was cooled to $0^{\circ} \mathrm{C}$, and $\mathrm{NaH}(20 \mathrm{mmol}$, $60 \%$ in mineral oil, $0.8 \mathrm{~g}$ ) was added in small portions. The reaction mixture was stirred for $30 \mathrm{~min}$ and then chloromethyl methyl ether $(20 \mathrm{mmol}, 1.5 \mathrm{~mL})$ was added slowly. The reaction mixture was allowed to warm to room temperature and stirred for $1 \mathrm{~h}$. Water was added slowly to quench the reaction. The organic layer was separated, and the aqueous layer was extracted with ethyl acetate (three $30 \mathrm{~mL}$ aliquots). The combined organic extracts were washed with brine, and dried over $\mathrm{Na}_{2} \mathrm{SO}_{4}$. After evaporation of the solvent, the residue was purified by column chromatography on silica gel eluted with hexane/ethyl acetate (10/1) to afford compound $(S)-\mathbf{6 - 1 4}$ as a white solid in $95 \%$ yield (7.6 mmol, $4.04 \mathrm{~g}) .{ }^{1} \mathrm{H}$ NMR $\left(300 \mathrm{MHz}, \mathrm{CDCl}_{3}\right) \delta 3.15(\mathrm{~s}, 6 \mathrm{H}), 4.98(\mathrm{~d}, \mathrm{~J}=6.9 \mathrm{~Hz}, 2 \mathrm{H})$, $5.09(\mathrm{~d}, \mathrm{~J}=6.9 \mathrm{~Hz}, 2 \mathrm{H}), 6.98(\mathrm{~d}, \mathrm{~J}=9.0 \mathrm{~Hz}, 2 \mathrm{H}), 7.29(\mathrm{dd}, \mathrm{J} 1=9.0 \mathrm{~Hz}, \mathrm{~J} 2=2.1 \mathrm{~Hz}, 2 \mathrm{H})$, $7.60(\mathrm{~d}, \mathrm{~J}=9.0 \mathrm{~Hz}, 2 \mathrm{H}), 7.86(\mathrm{~d}, \mathrm{~J}=9.0 \mathrm{~Hz}, 2 \mathrm{H}), 8.03(\mathrm{~d}, \mathrm{~J}=2.1 \mathrm{~Hz}, 2 \mathrm{H})$.

Synthesis and Characterization of (S)-6-15. Under nitrogen, $(S)-6-14$ (1.0 mmol, 532 $\mathrm{mg}$ ) was dissolved in THF $(15 \mathrm{~mL})$. The solution was cooled to $-78{ }^{0} \mathrm{C}$, and n-BuLi (4.4 mmol, $2.5 \mathrm{M}$ in hexane, $1.8 \mathrm{~mL}$ ) was added dropwise. The reaction mixture was stirred for an additional $3 \mathrm{~h}$ at the same temperature, and then ethyl trifluoroacetate $(6.6 \mathrm{mmol}$, $0.8 \mathrm{~mL}$ ) was added slowly. The reaction mixture was stirred for $30 \mathrm{~min}$ at $-78{ }^{0} \mathrm{C}$ and for 30 min at rt. A saturated aqueous $\mathrm{NH}_{4} \mathrm{Cl}$ solution was added to quench the reaction. The organic layer was separated, and the aqueous layer was extracted with ethyl acetate (20 $\mathrm{mL} \times 3$ ). The combined organic extracts were washed with brine, and dried over $\mathrm{Na}_{2} \mathrm{SO}_{4}$. 
After evaporation of the solvent, the residue was purified by column chromatography on silica gel eluted with hexane/methylene chloride (1/2) to afford compound $(S)-\mathbf{6 - 1 5}$ as a light yellow oil in $87 \%$ yield $(0.87 \mathrm{mmol}, 493 \mathrm{mg}) .{ }^{1} \mathrm{H} \mathrm{NMR}\left(300 \mathrm{MHz}, \mathrm{CDCl}_{3}\right) \delta 3.24$ $(\mathrm{s}, 6 \mathrm{H}), 5.12(\mathrm{~d}, \mathrm{~J}=7.2 \mathrm{~Hz}, 2 \mathrm{H}), 5.21(\mathrm{~d}, \mathrm{~J}=7.2 \mathrm{~Hz}, 2 \mathrm{H}), 7.27(\mathrm{~d}, \mathrm{~J}=9.0 \mathrm{~Hz}, 2 \mathrm{H}), 7.77$ $(\mathrm{d}, \mathrm{J}=9.0 \mathrm{~Hz}, 2 \mathrm{H}), 7.88(\mathrm{~d}, \mathrm{~J}=9.0 \mathrm{~Hz}, 2 \mathrm{H}), 8.20(\mathrm{~d}, \mathrm{~J}=9.0 \mathrm{~Hz}, 2 \mathrm{H}), 8.72(\mathrm{~s}, 2 \mathrm{H}) .{ }^{19} \mathrm{~F}$ NMR (282 MHz, $\left.\mathrm{CDCl}_{3}\right) \delta-71.63 .{ }^{13} \mathrm{C} \mathrm{NMR}\left(75 \mathrm{MHz}, \mathrm{CDCl}_{3}\right) \delta 56.2,94.5,117.0(\mathrm{t}, \mathrm{J}=$ $290 \mathrm{~Hz}), 117.6,119.8,125.1,125.6,126.1,128.4,132.7,133.7,137.4,156.2,180.2(\mathrm{t}, \mathrm{J}=$ 34.5 Hz). HRMS Calcd for $\mathrm{C}_{28} \mathrm{H}_{20} \mathrm{O}_{6} \mathrm{~F}_{6} \mathrm{Na}\left(\mathrm{MNa}^{+}\right)$: 589.1062, Found: 589.1063. [ $\left.\alpha\right]_{\mathrm{D}}=$ $36.35\left(\mathrm{c}=0.305, \mathrm{CHCl}_{3}\right)$.

Synthesis and Characterization of (S)-6-16. After compound (S)-6-15 (320 mg, 0.56 mmol) was dissolved in a minimum amount of $\mathrm{CH}_{2} \mathrm{Cl}_{2}$, trifluroacetic acid (1 mL) was added slowly, and the mixture was stirred at room temperature for $2 \mathrm{~h}$. A saturated aqueous $\mathrm{Na}_{2} \mathrm{CO}_{3}$ solution was added to quench the reaction. The organic layer was separated, and the aqueous layer was extracted with $\mathrm{CH}_{2} \mathrm{Cl}_{2}(20 \mathrm{~mL}$ x 3$)$. The combined organic extracts were washed with brine, and dried over $\mathrm{Na}_{2} \mathrm{SO}_{4}$. After evaporation of the solvent, the residue was purified by column chromatography on silica gel eluted with hexane/methylene chloride (2/1) to afford compound $(S)$-6-16 as a light yellow solid in $89 \%$ yield (0.50 mmol, $238 \mathrm{mg}) .{ }^{1} \mathrm{H}$ NMR $\left(300 \mathrm{MHz}, \mathrm{CDCl}_{3}\right) \delta 5.33(\mathrm{~s}, 2 \mathrm{H}), 7.19(\mathrm{~d}, \mathrm{~J}=$ $8.7 \mathrm{~Hz}, 2 \mathrm{H}), 7.53(\mathrm{~d}, \mathrm{~J}=9.0 \mathrm{~Hz}, 2 \mathrm{H}), 7.92(\mathrm{~d}, \mathrm{~J}=9.0 \mathrm{~Hz}, 2 \mathrm{H}), 8.21(\mathrm{~d}, \mathrm{~J}=9.0 \mathrm{~Hz}, 2 \mathrm{H})$, 8.69 (s, 2H). ${ }^{19} \mathrm{~F}$ NMR (282 MHz, $\left.\mathrm{CDCl}_{3}\right) \delta-71.78 .{ }^{13} \mathrm{C}$ NMR (150 MHz, DMSO) $\delta$ $115.0,116.8(\mathrm{t}, \mathrm{J}=291 \mathrm{~Hz}), 120.2,123.3,124.4,125.3,126.7,132.5,133.6,137.7,179.1$ $(\mathrm{t}, \mathrm{J}=33 \mathrm{~Hz}) . \quad[\alpha]_{\mathrm{D}}=333.6\left(\mathrm{c}=0.16, \mathrm{CHCl}_{3}\right)$. m.p. $244{ }^{0} \mathrm{C} . \quad \mathrm{HRMS}$ Calcd for 
$\mathrm{C}_{24} \mathrm{H}_{13} \mathrm{O}_{4} \mathrm{~F}_{6}\left(\mathrm{MH}^{+}\right):$479.0718, Found: 479.0714 .

\section{References}

1. Selected references of enantioselective fluorescent sensors: (a) James, T. D.; Sandanayake, K. R. A. S.; Shinkai, S. Nature 1995, 374, 345-347. (b) Pugh, V.; Hu, Q. -S.; Pu, L. Angew. Chem. Int. Ed. 2000, 39, 3638-3641. (c) Reetz, M. T.; Sostmann, S. Tetrahedron 2001, 57, 2515-2520. (d) Korbel, G. A.; Lalic, G.; Shair, M. D. J. Am. Chem. Soc. 2001, 123, 361-362. (e) Jarvo, E. R.; Evans, C. A.; Copeland, G. T.; Miller, S. J. J. Org. Chem. 2001, 66, 5522-5527. (f) Wong, W.-L.; Huang, K.-H.; Teng, P.-F.; Lee, C.-S.; Kwong, H.-L. Chem. Commun. 2004, 384-385.

(g) Zhao, J.-Z.; Fyles, T. M.; James, T. D. Angew. Chem., Int. Ed. 2004, 43, 34613464. (h) Pagliari, S.; Corradini, R.; Galaverna, G.; Sforza, S.; Dossena, A.; Montalti, M.; Prodi, L.; Zaccheroni, N.; Marchelli, R. Chem. Eur. J. 2004, 10, 27492758. (i) Matsushita, H.; Yamamoto, N.; Meijler, M. M.; Wirsching, P.; Lerner, R.A.; Matsushita, M.; Janda, K. D. Mol. Biosyst. 2005, 1. 303-306. (j) Zhu, L.; Anslyn, E. V. J. Am. Chem. Soc. 2004, 126, 3676-3677. (k) Mei, X. F. ; Wolf, C. J. Am. Chem. Soc. 2004, 126, 14736-14737.

2. For reviews on enantioselective fluorescent recognition: (a) Pu, L. Chem. Rev. 2004, 104, 1687-1716. (b) Pu, L. Acc. Chem. Res. 2012, 45, 150-163. (c) A recent review on chiral optical sensors: Leung, D.; Kang, S. O.; Anslyn, E. V. Chem. Soc. Rev. 2012, 41, 448-479.

3. (a) Lin, J.; Hu, Q.-S.; Xu, M. H.; Pu, L. J. Am. Chem. Soc. 2002, 124, 2088-2089.

(b) Xu, M.-H.; Lin, J.; Hu, Q.-S.; Pu, L. J. Am. Chem. Soc. 2002, 124, 14239- 
14246. (c) Li, Z.-B.; Lin, J.; Pu, L. Angew. Chem., Int. Ed. 2005, 44, 1690-1693.

(d) Lin, J.; Rajaram, A. R.; Pu, L. Tetrahedron 2004, 60, 11277-11281. (e) He, X.; Cui, X.; Li, M.; Lin, L.; Liu, X.; Feng, X. Tetrahedron Lett. 2009, 50, 5853-5856.

(f) Liu, H. -L.; Hou, X. -L.; Pu, L. Angew. Chem. Int. Ed. 2009, 48, 382 -385. (g) Chen, X.; Huang, Z.; Chen, S. -Y.; Li, K.; Yu, X. -Q.; Pu, L. J. Am. Chem. Soc. 2010, 132, 7297-7299. (h) Liu, H. -L.; Hou, X. -L; Pu, L. Org. Lett. 2010, 12, 4172-4175. (i) Liu, H. -L.; Peng, Q.; Wu, Y. -D.; Chen, D.; Hou, X. -L.; Sabat, M.; Pu, L. Angew. Chem. Int. Ed. 2010, 49, 602-606. (j) Yu, S.; Pu, L. J. Am. Chem. Soc. 2010, 132, 17698-17700. (k) Yu, S.; DeBerardinis, A. M.; Turlington, M.; Pu, L. J. Org. Chem. 2011, 76, 2814-2819. (1) Yu, S.; Pu, L. Sci. China. Chem. 2013, 56, 301-306.

4. (a) Herman, H. B.; Rechnitz, G. A. Science 1974, 184, 1074-1075. (b) Meyerhoff, M. E.; Pretsch, E.; Welti, D. H.; Simon, W. Anal. Chem. 1987, 59, 144-150. (c) Wang, K.; Seiler, K.; Haug, J. -P.; Lehmann, B.; Hartman, S. W. K.; Simon, W. Anal. Chem. 1991, 63, 970-974. (d) Seiler, K.; Wang, K.; Kuratli, M.; Simon, W. Anal. Chim. Act. 1991, 244, 151-160. (e) Mohr, G. J.; Tirelli, N.; Lohse, C.; SpichigerKeller, U. E. Adv. Mater. 1998, 10, 1353-1357. (f) Mertz, E.; Zimmerman, S. C. J. Am. Chem. Soc. 2003, 125, 3424-3425. (g) Sasaki, S. -i.; Kotegawa, Y.; Tamiaki, H. Tetrahedron Lett. 2006, 47, 4849-4852. (h) Ryu, D.; Park, E.; Kim, D. -S.; Yan, S.; Lee, J. Y.; Chang, B. -Y.; Ahn, K. H. J. Am. Chem. Soc. 2008, 130, 2394-2395. (i) Sambasivan, S.; Kim, D. -s.; Ahn, K. H. Chem. Commun. 2010, 46, 541-543.

5. Yu, S.; Plunkett, W.; Kim, M.; Pu, L. J. Am. Chem. Soc. 2012, 134, 20282-20285. 
6. Cox, P. J.; Wang, W.; Snieckus, V. Tetrahedron Lett. 1992, 33, 2253-2256.

7. Hu, Q. S.; Zheng, X. F.; Pu, L. J. Org. Chem. 1996, 61, 5200.

8. Abou-Eikhair, R. A. I.; Dixon, D. W.; Netzel, T. L. J. Org. Chem. 2009, 74, 4712.

9. (a) Hou, S. Y.; Hetherington, W. M.; Korenowski, G. M.; Eisenthal, K. B. Chem. Phys. Lett. 1979, 68, 282. (b) Ford, D.; Thistlethwaite, P. J.; Woolfe, G. J. Chem. Phys. Lett. 1980, 69, 246.

10. Forster, T. Z. Electrochem. 1950, 54, 531.

11. Wan, P.; Shukla, D. Chem. Rev. 1993, 93, 571.

12. Djoufac-Woumfo, E.; Arnaud, N.; Georges, J. Analyst. 1988, 113, 447.

13. Ofran, M.; Feitelson, J. Chem. Phys. Lett. 1973, 19, 427.

14. Lee, J.; Griffin, R. D.; Robinson, G. W. J. Chem. Phys. 1985, 82, 4920.

15. Law, K-Y.; Shoham, J. J. Phys. Chem. 1994, 98, 3114.

16. Iwanek, W.; Mattay, J. J. Photochem. Photobiol. A. 1992, 67, 209.

17. Yue, Y.; Turlington, M.; Yu, X-Q.; Lin, P. J. Org. Chem. 2009, 74, 8681.

18. DeBerardinis, A. M.; Turlington, M.; Ko, J.; Sole, L.; Lin, P. J. Org. Chem. 2010, 75, 2836. 
Appendix 


\section{Appendix to Chapter 2}
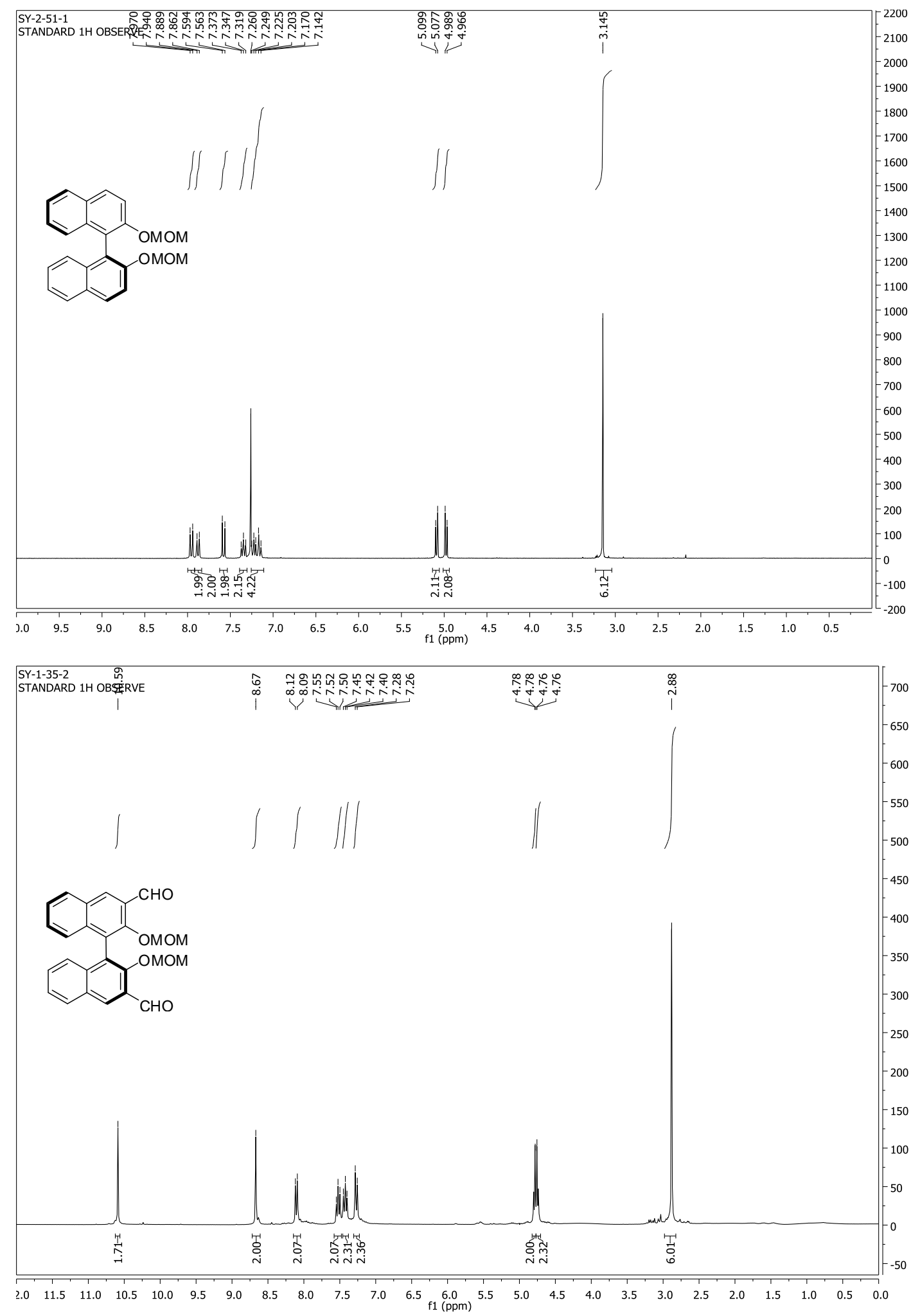

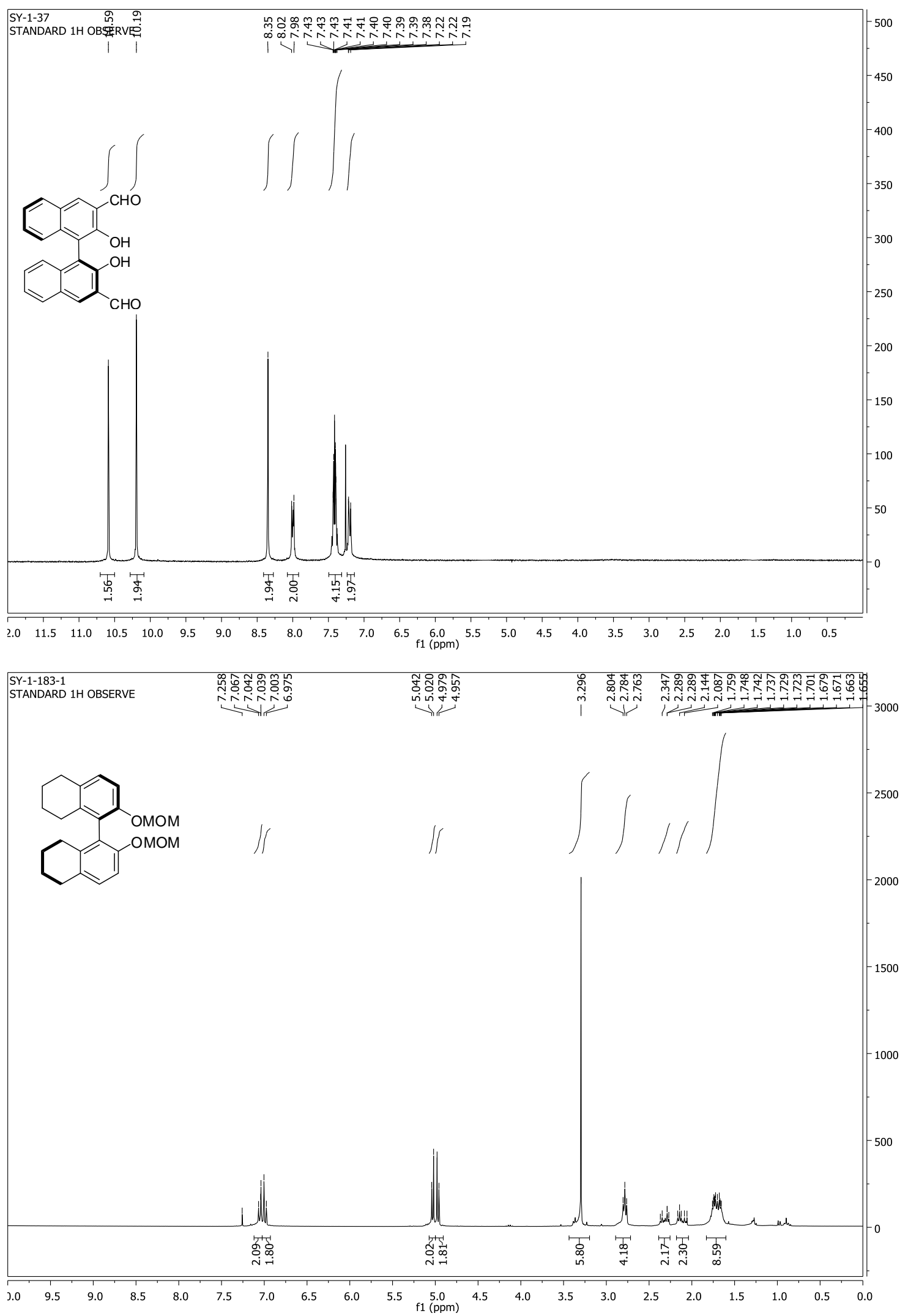

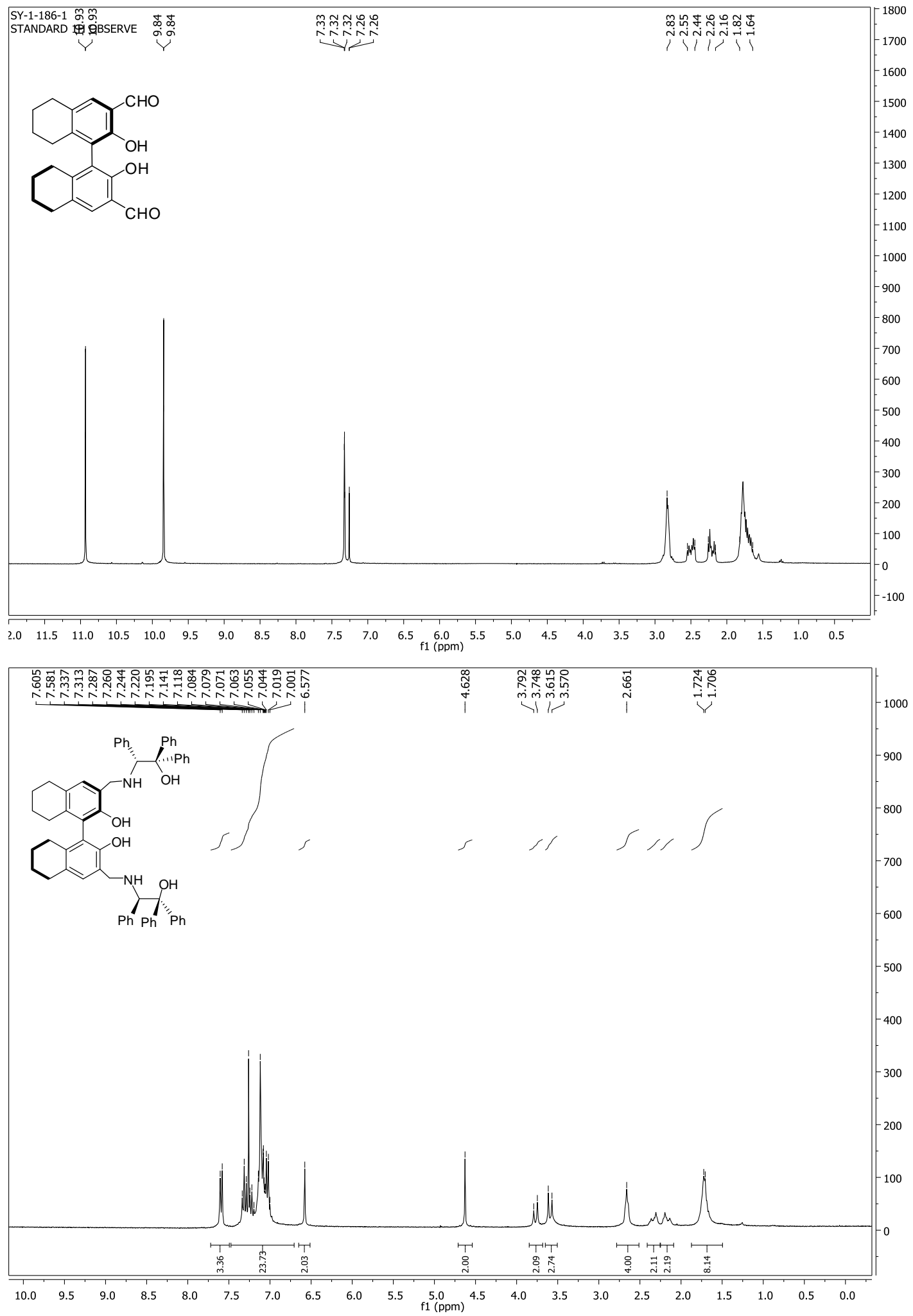


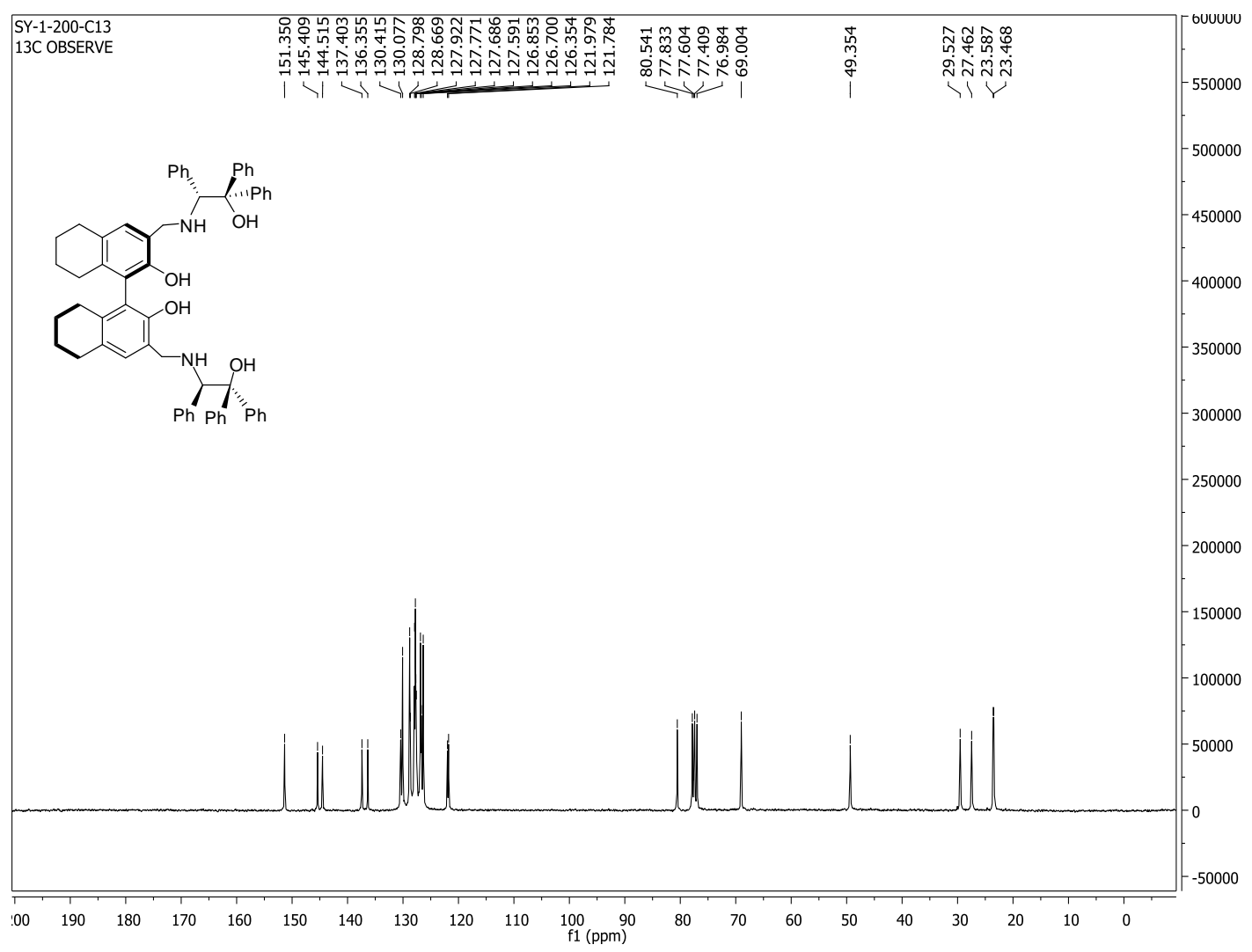




\section{Appendix to Chapter 3}

NMR Experiments for the Interaction of $(S)-3-5$ with $(R)$-MA

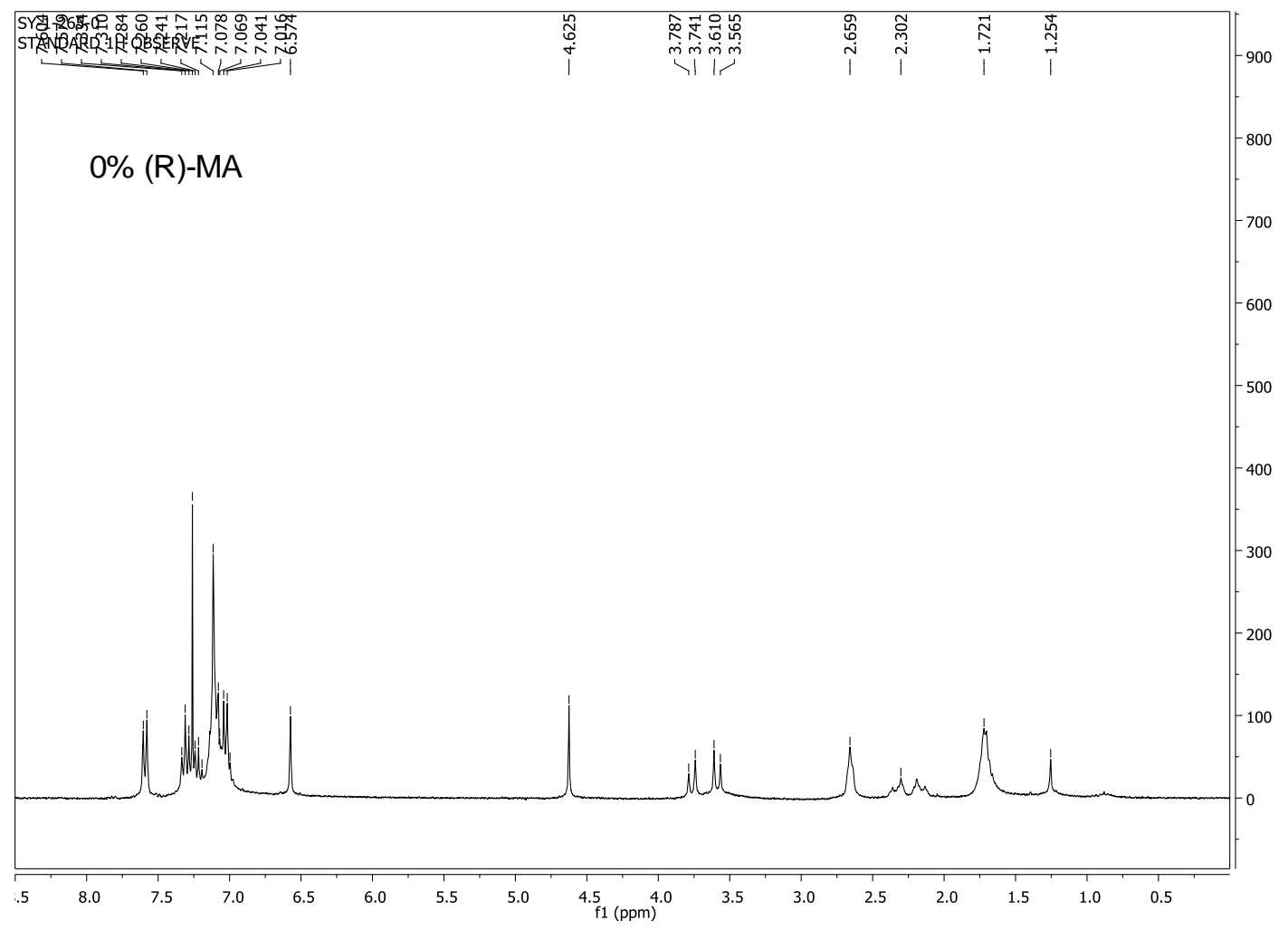



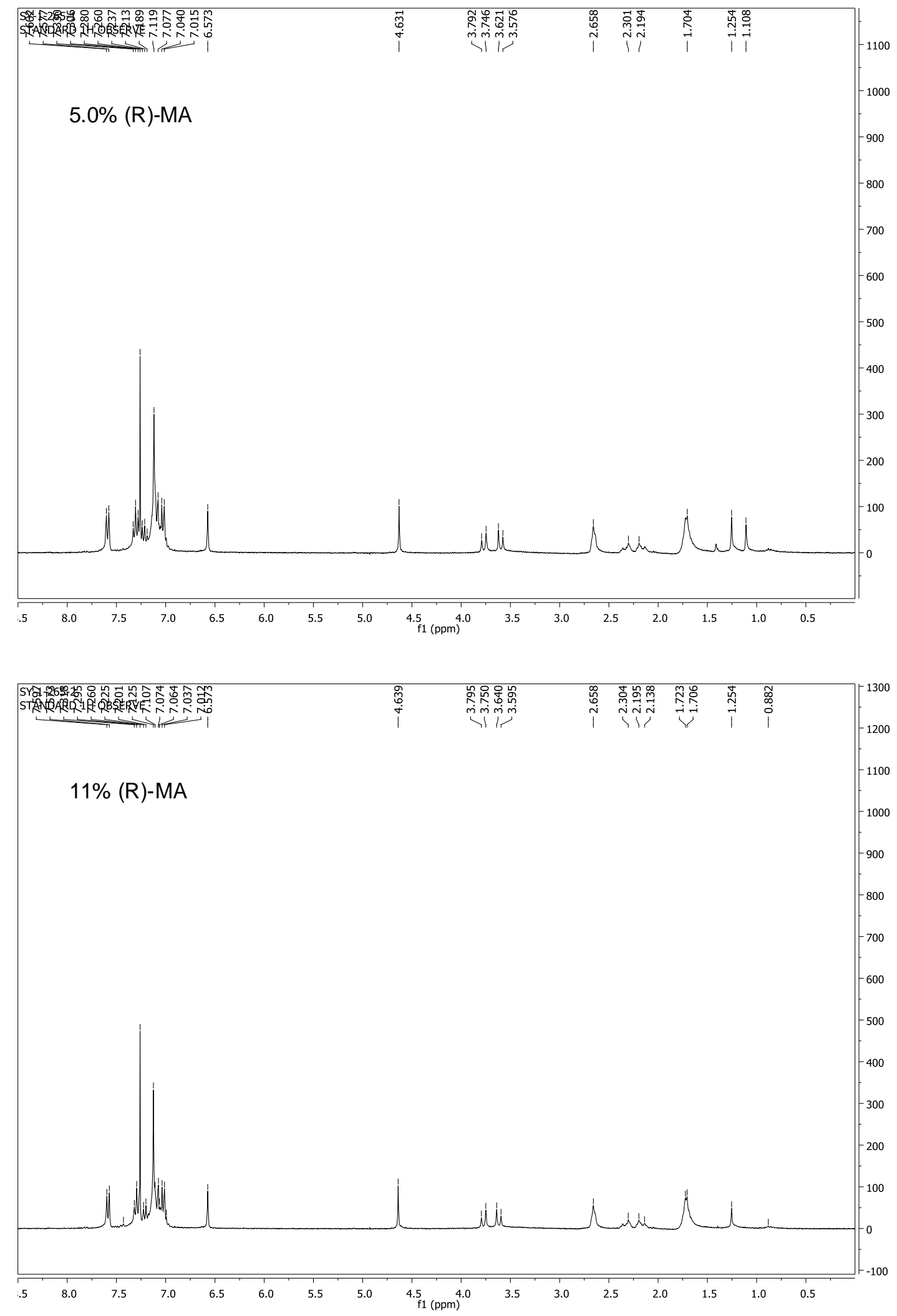

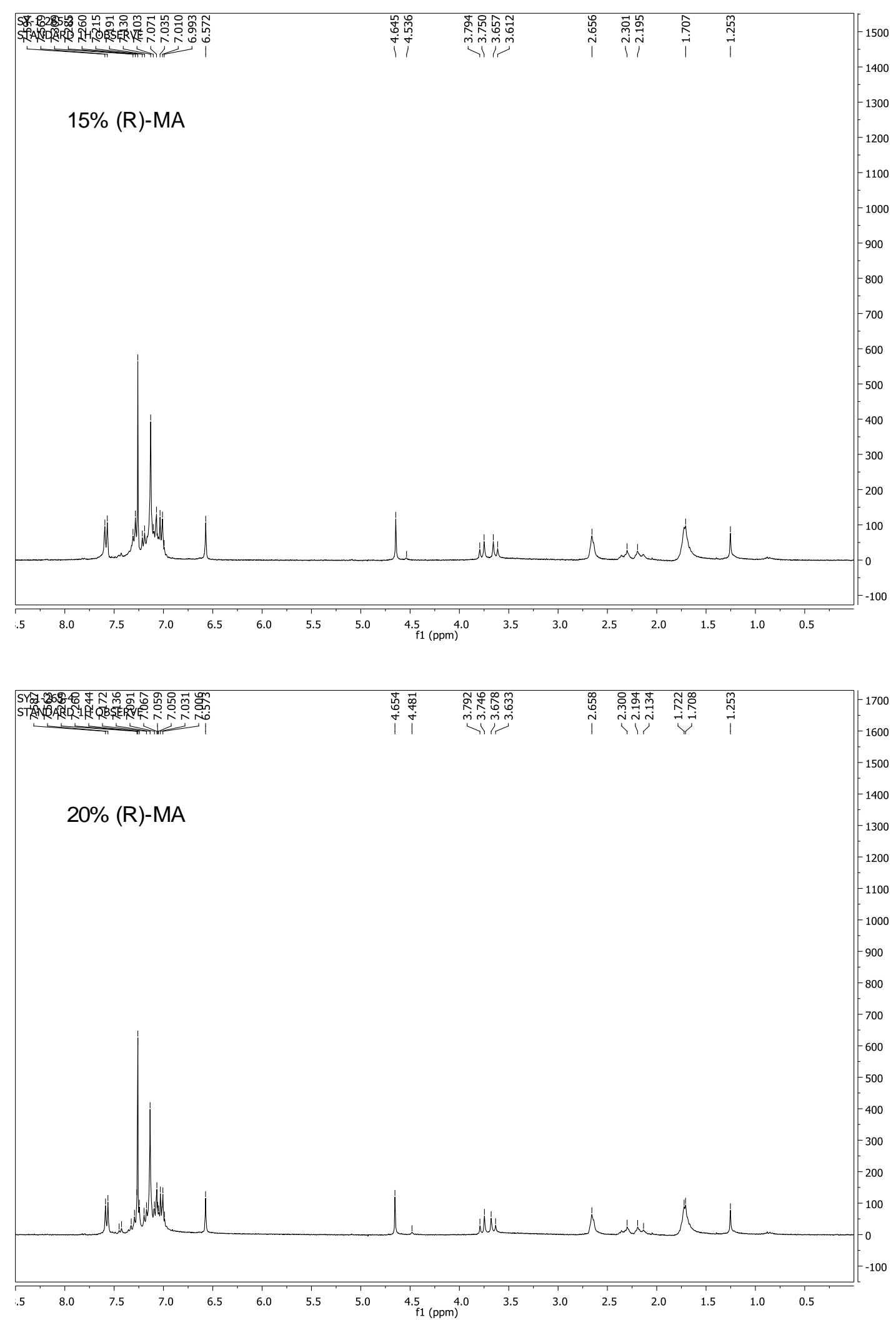

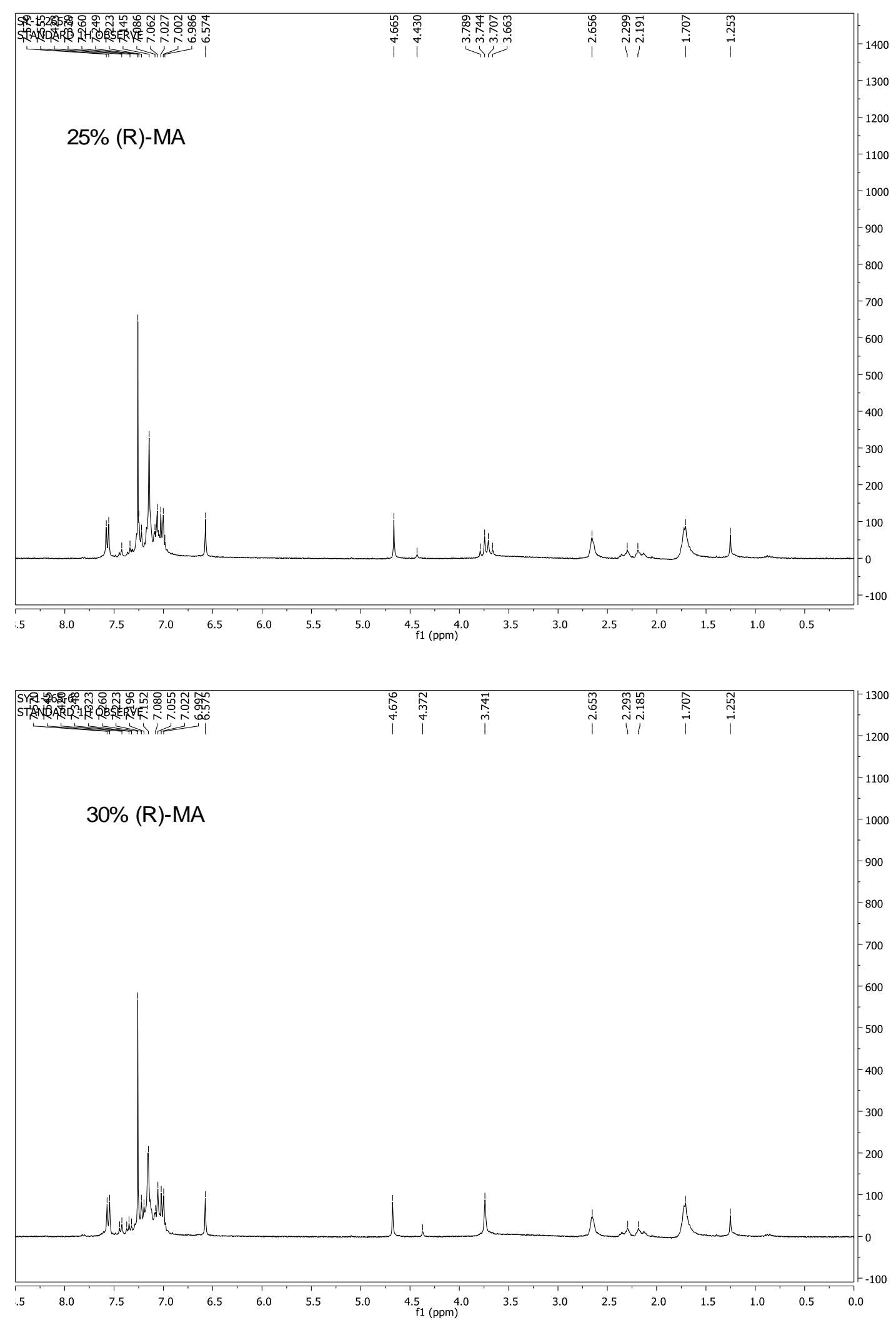

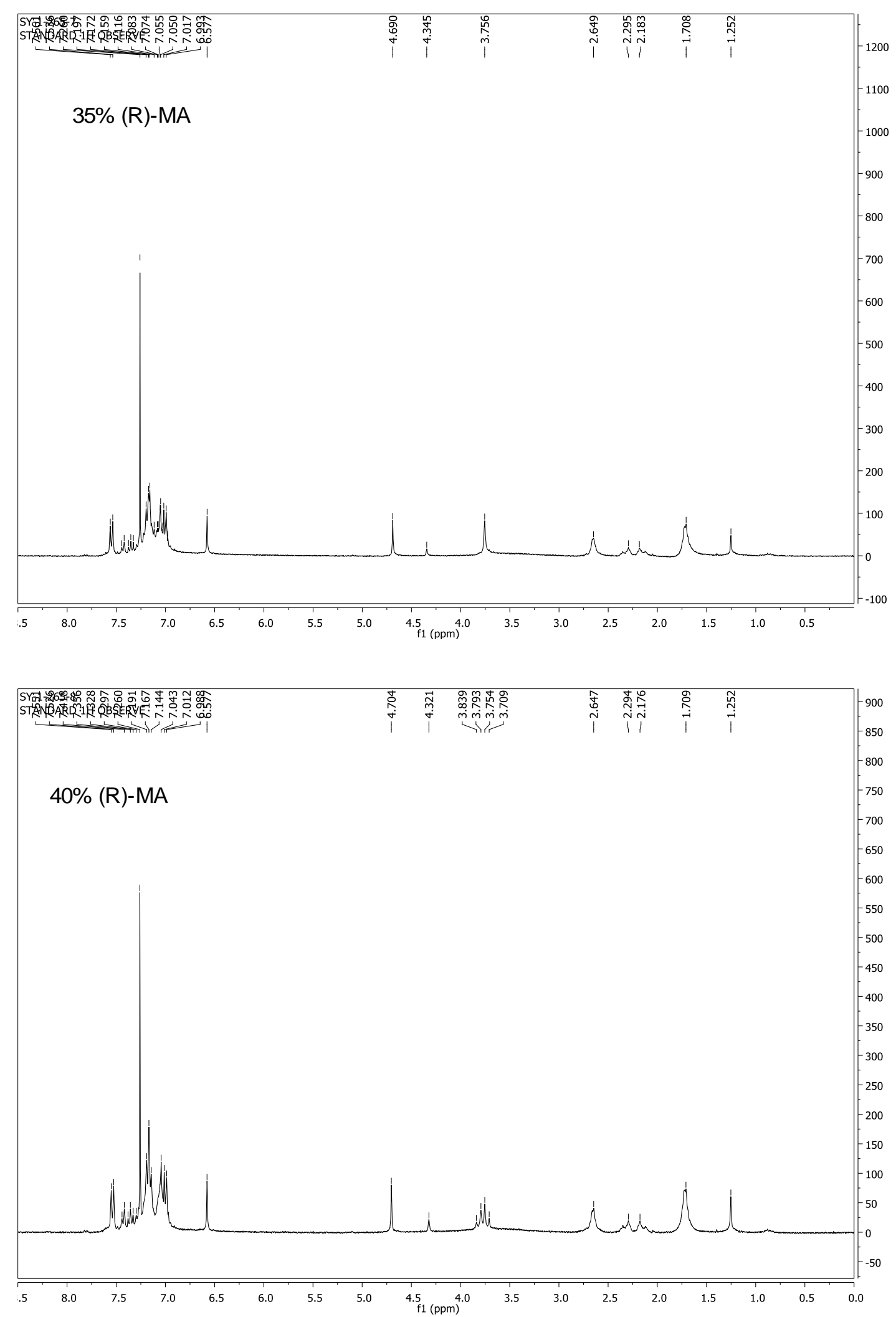

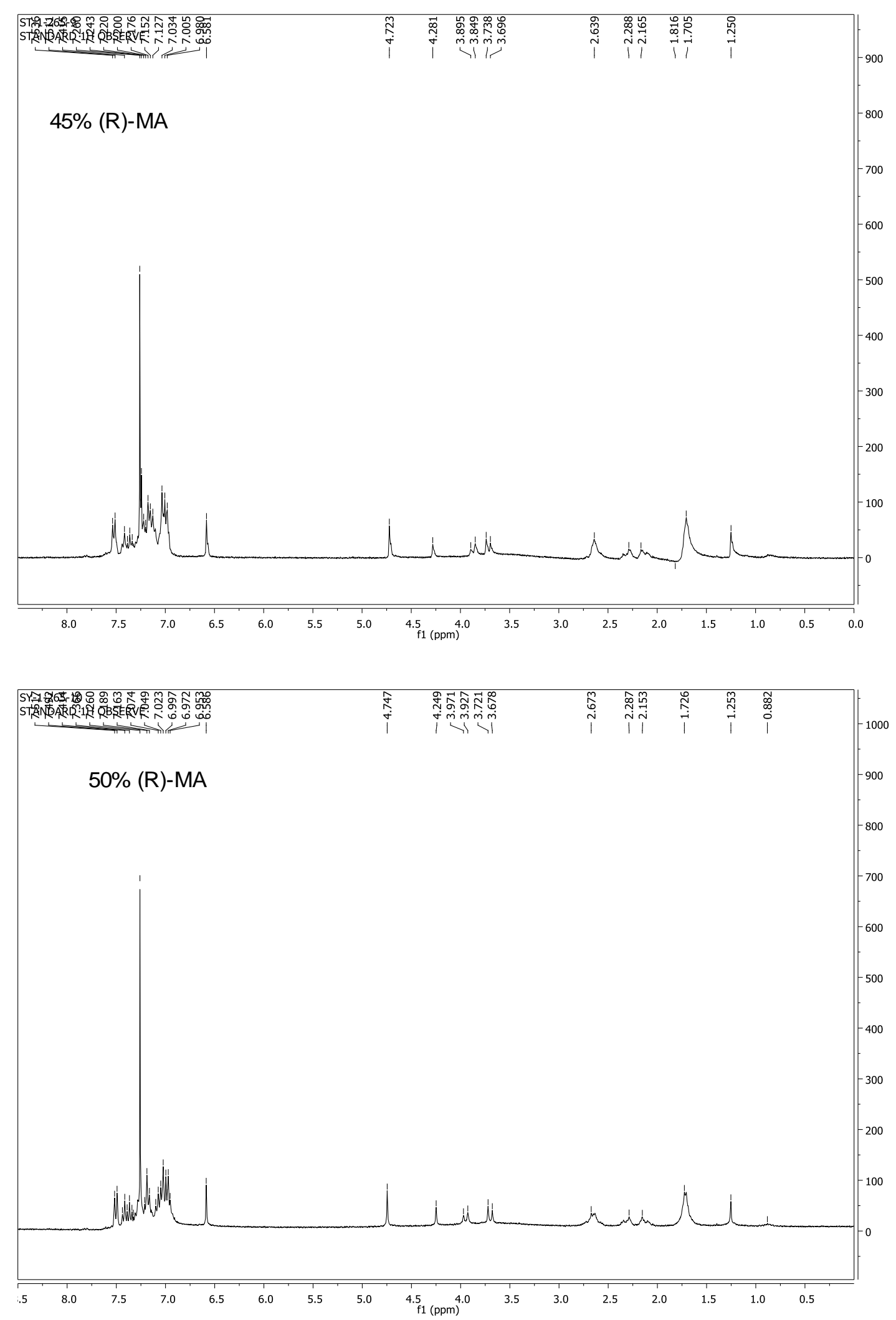

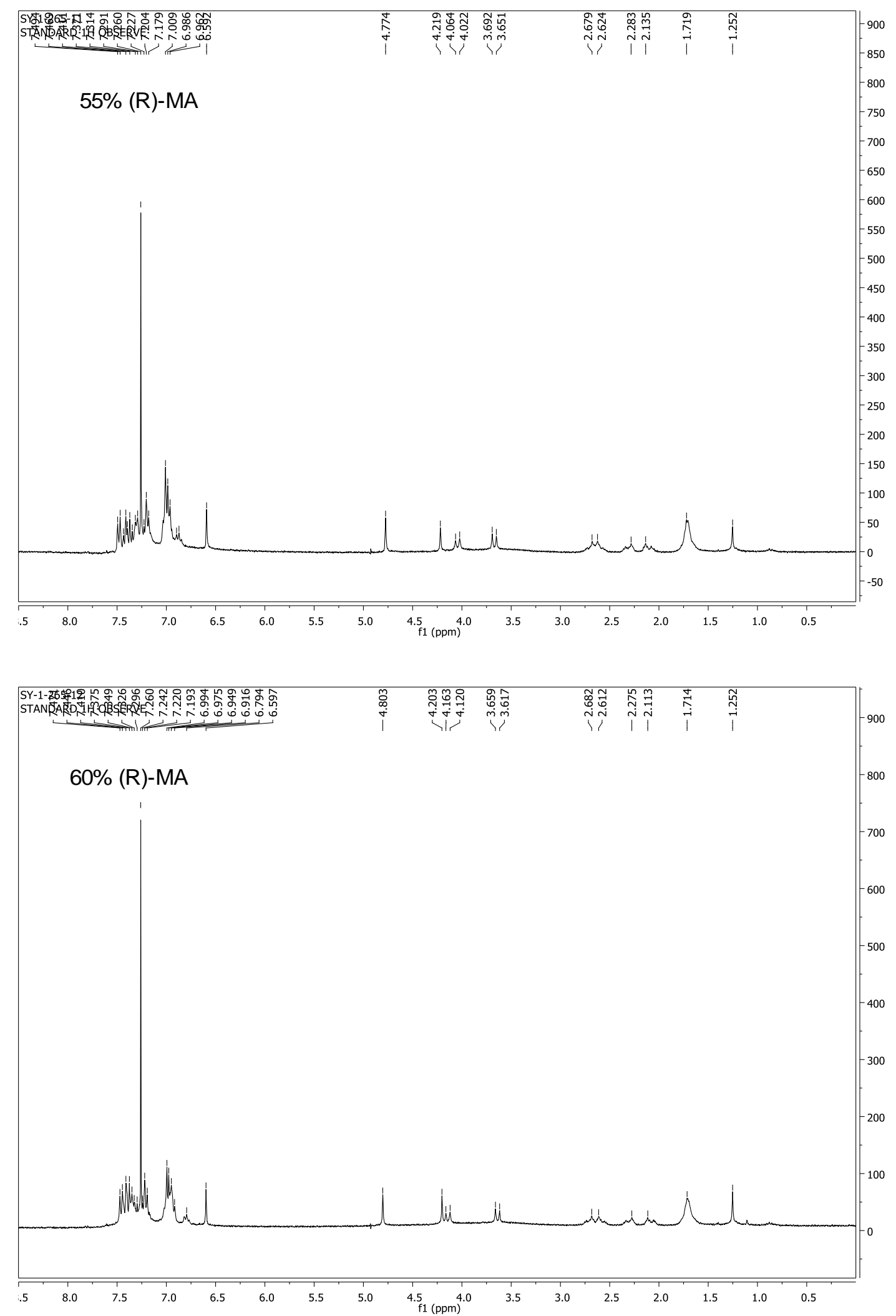

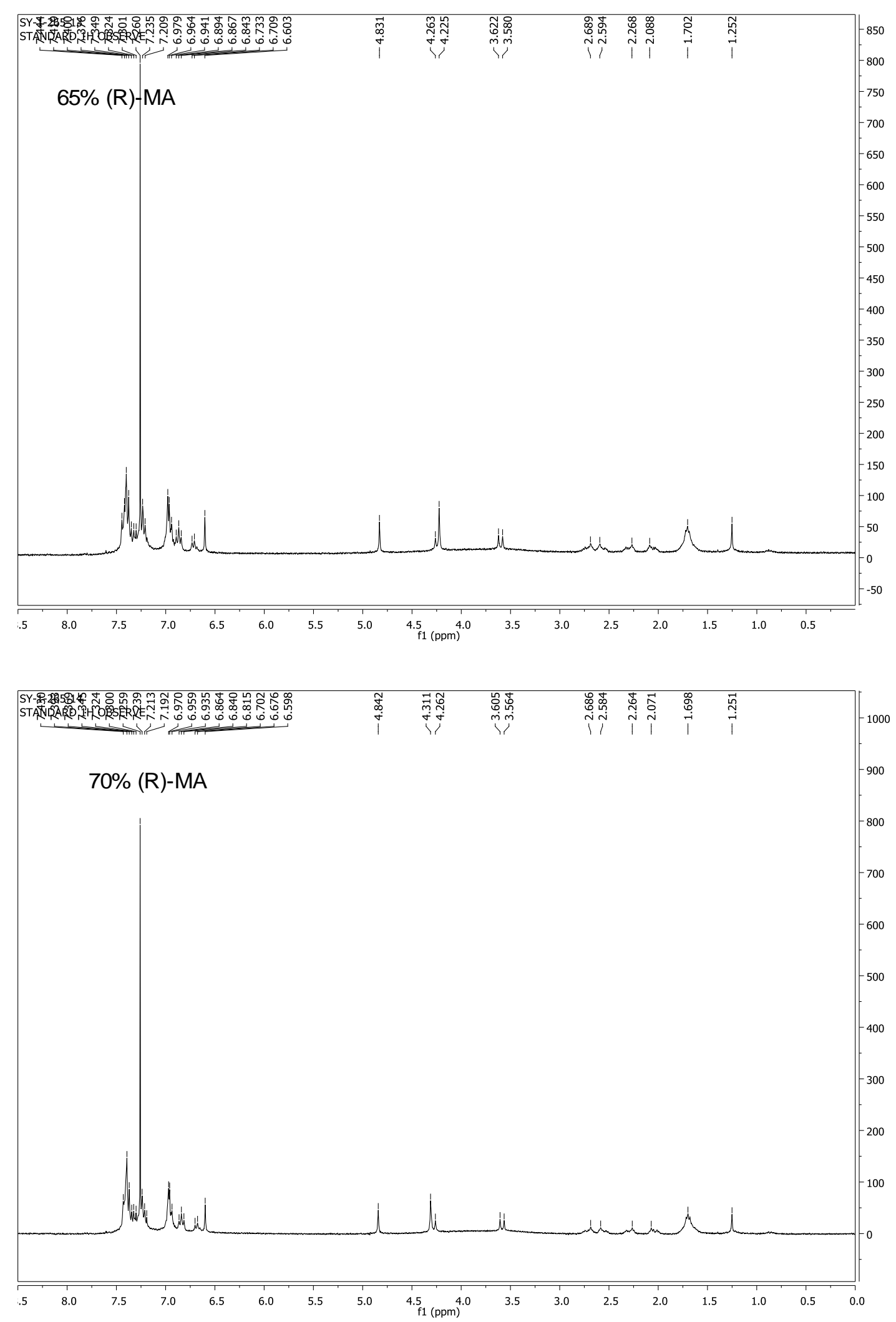

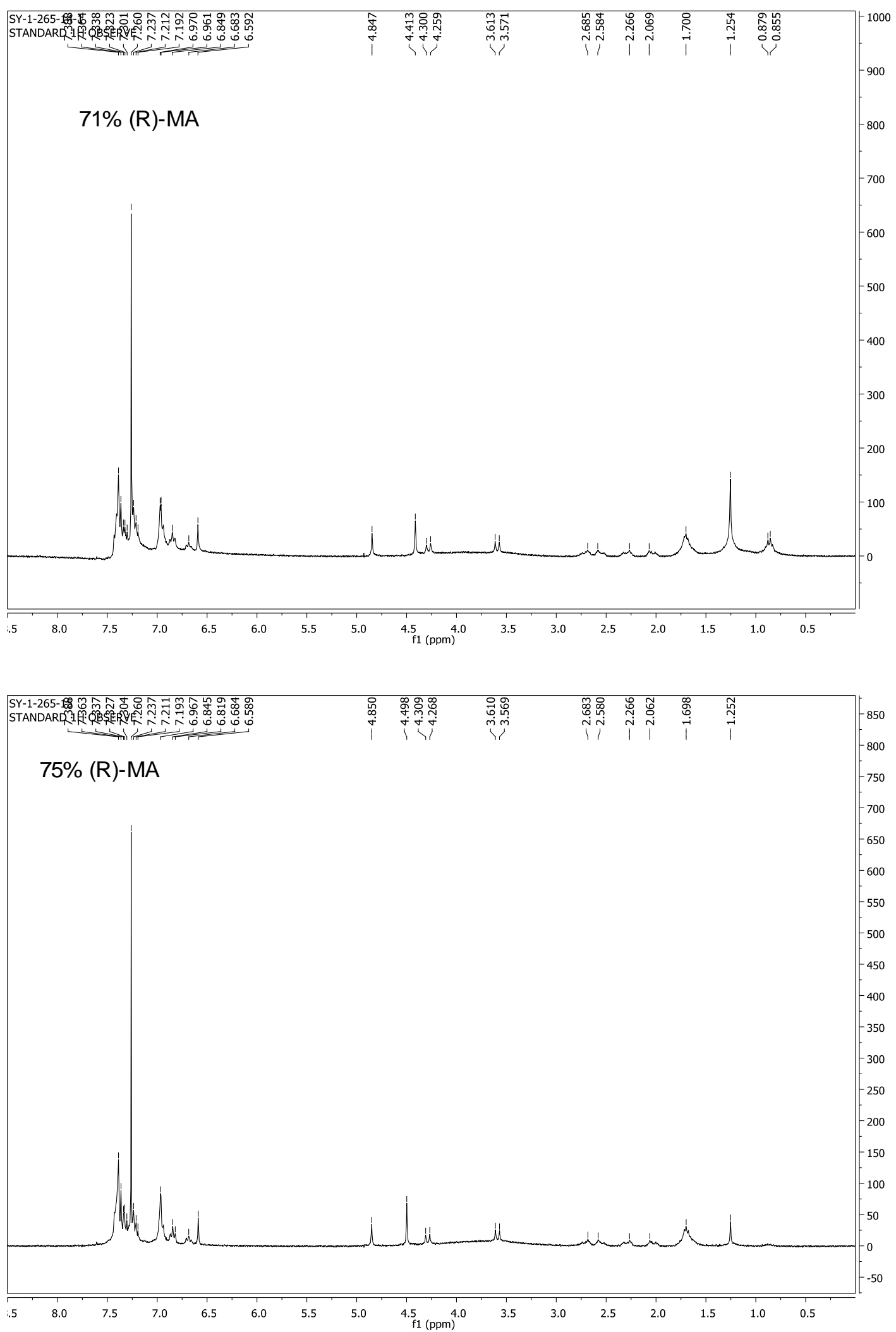

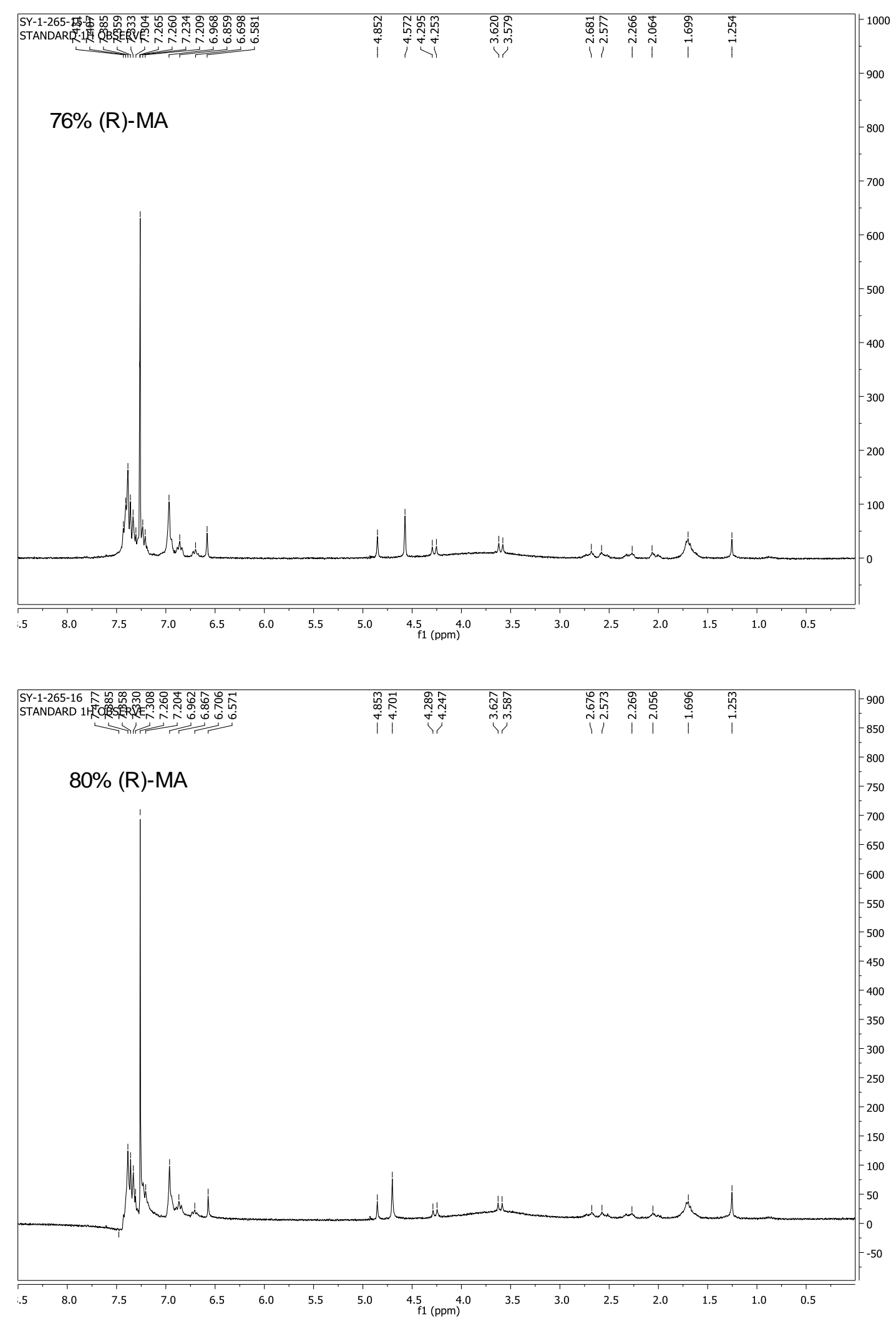

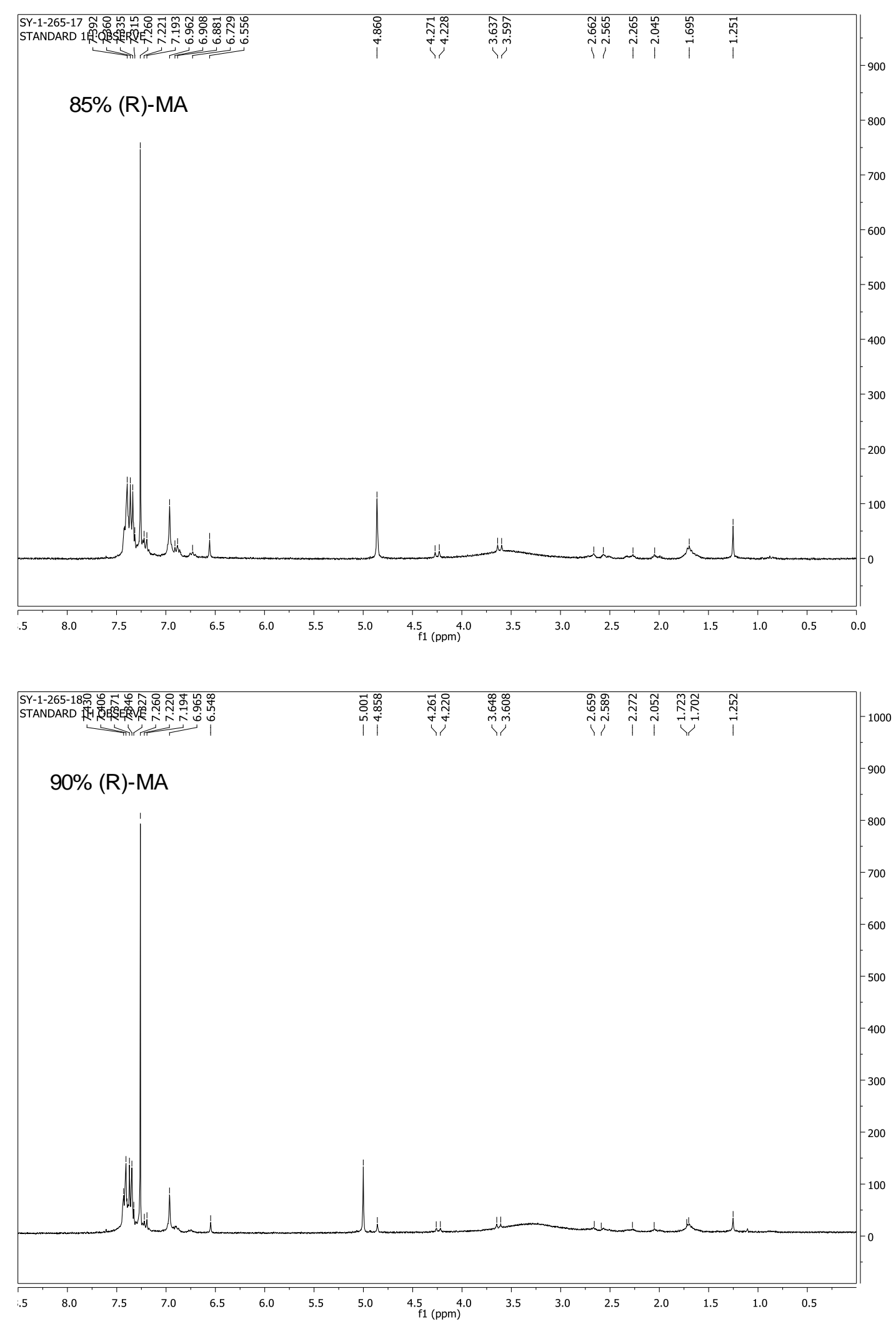

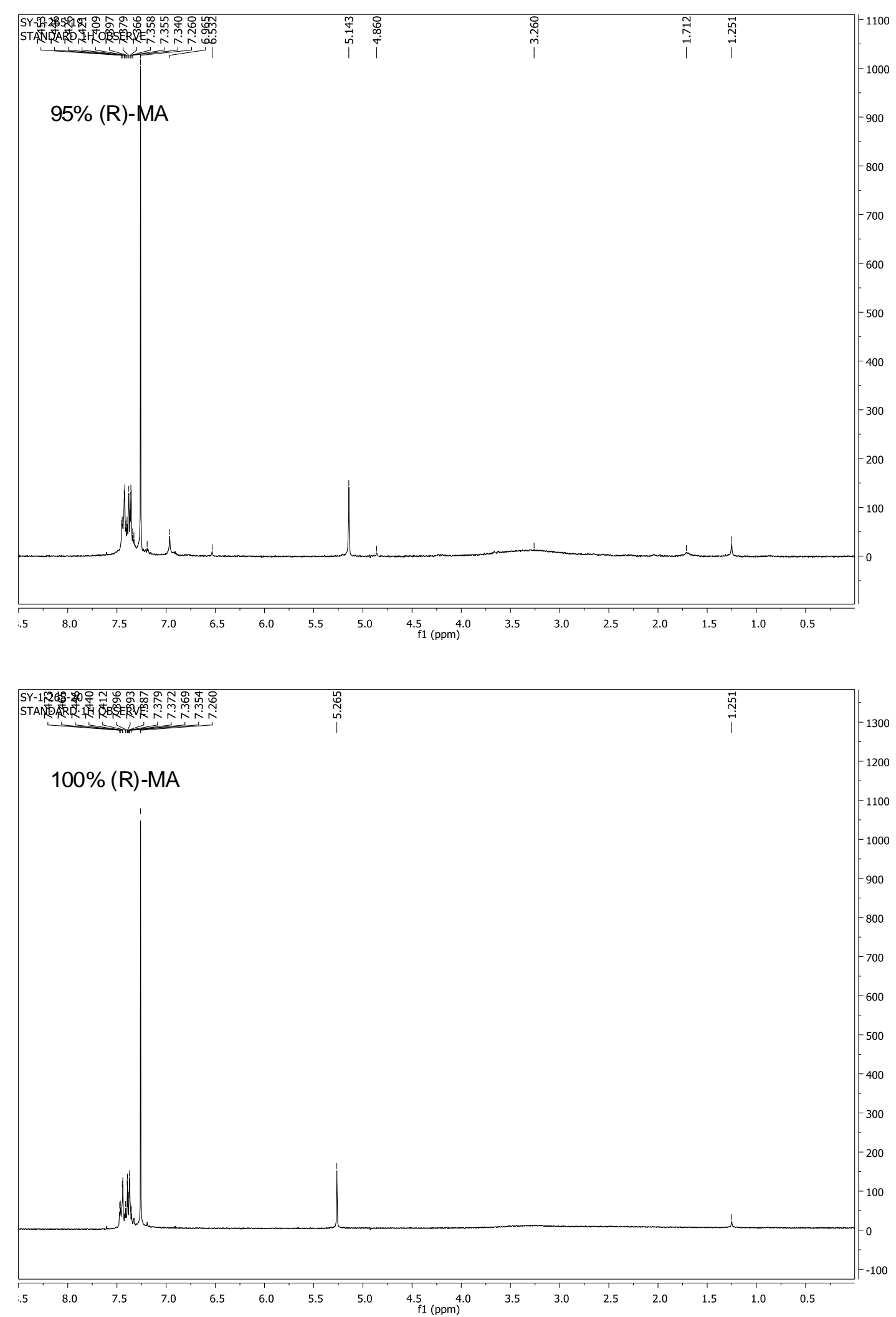


\section{Appendix to Chapter 4}

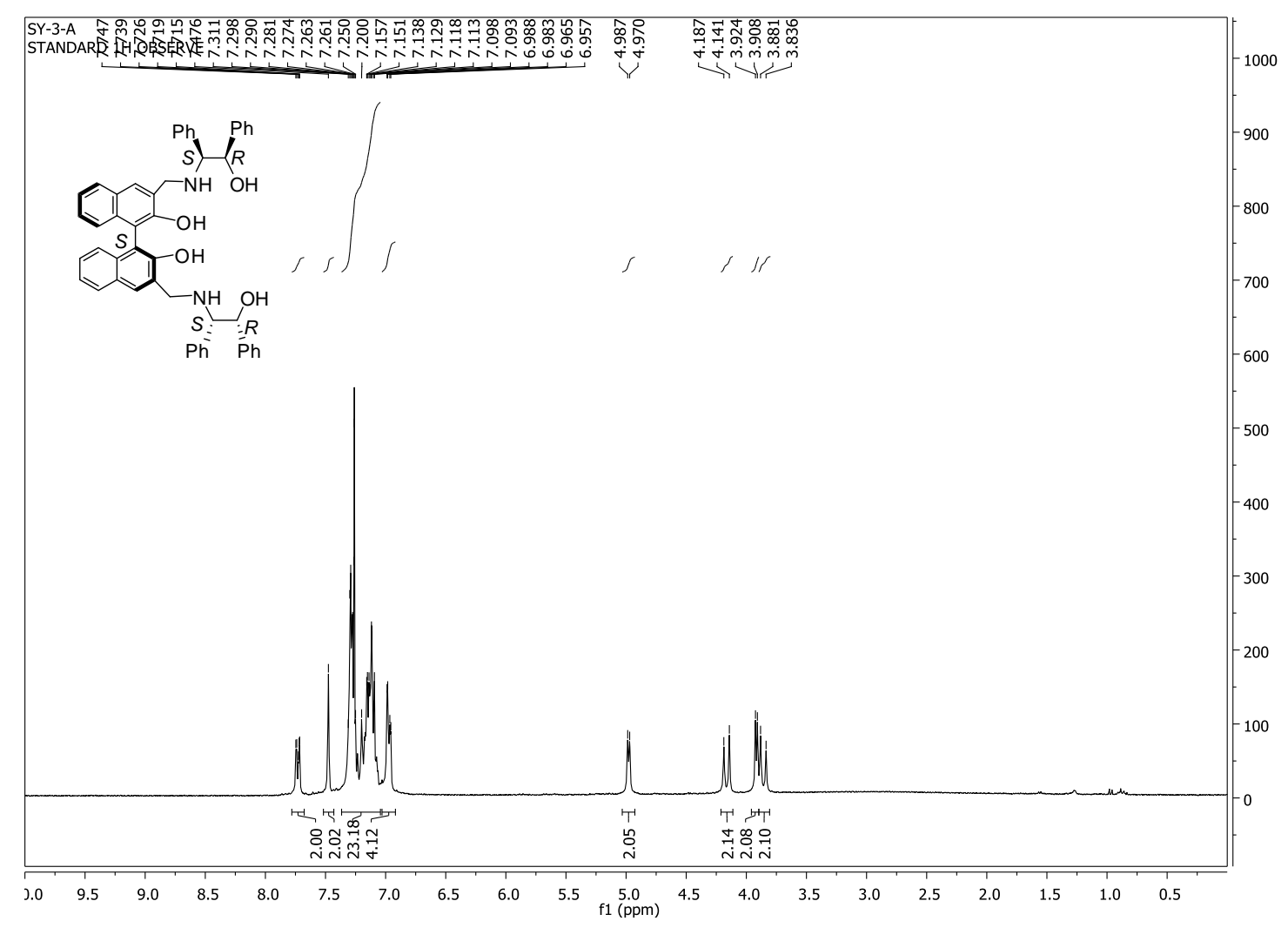



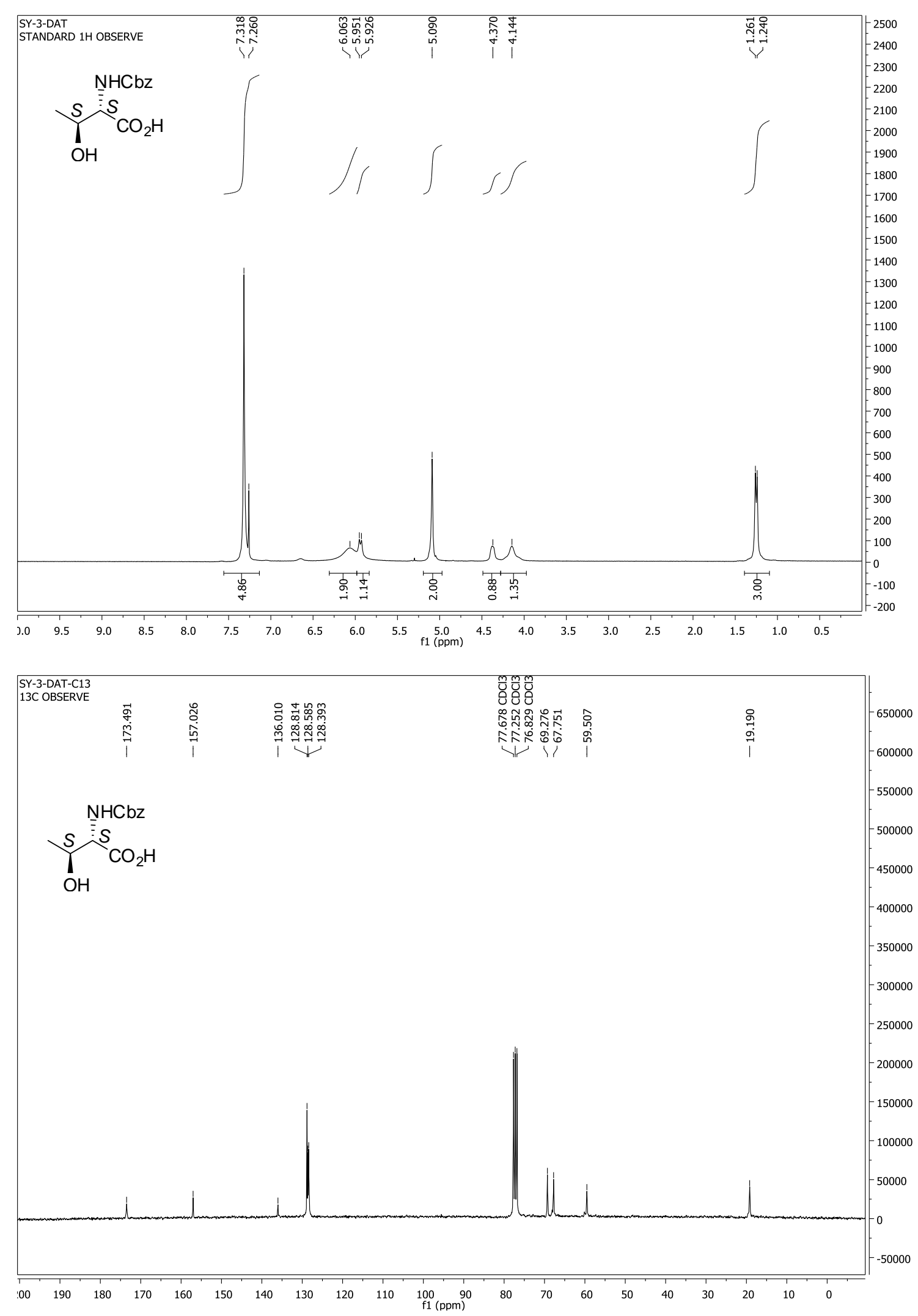
Appendix to Chapter 5

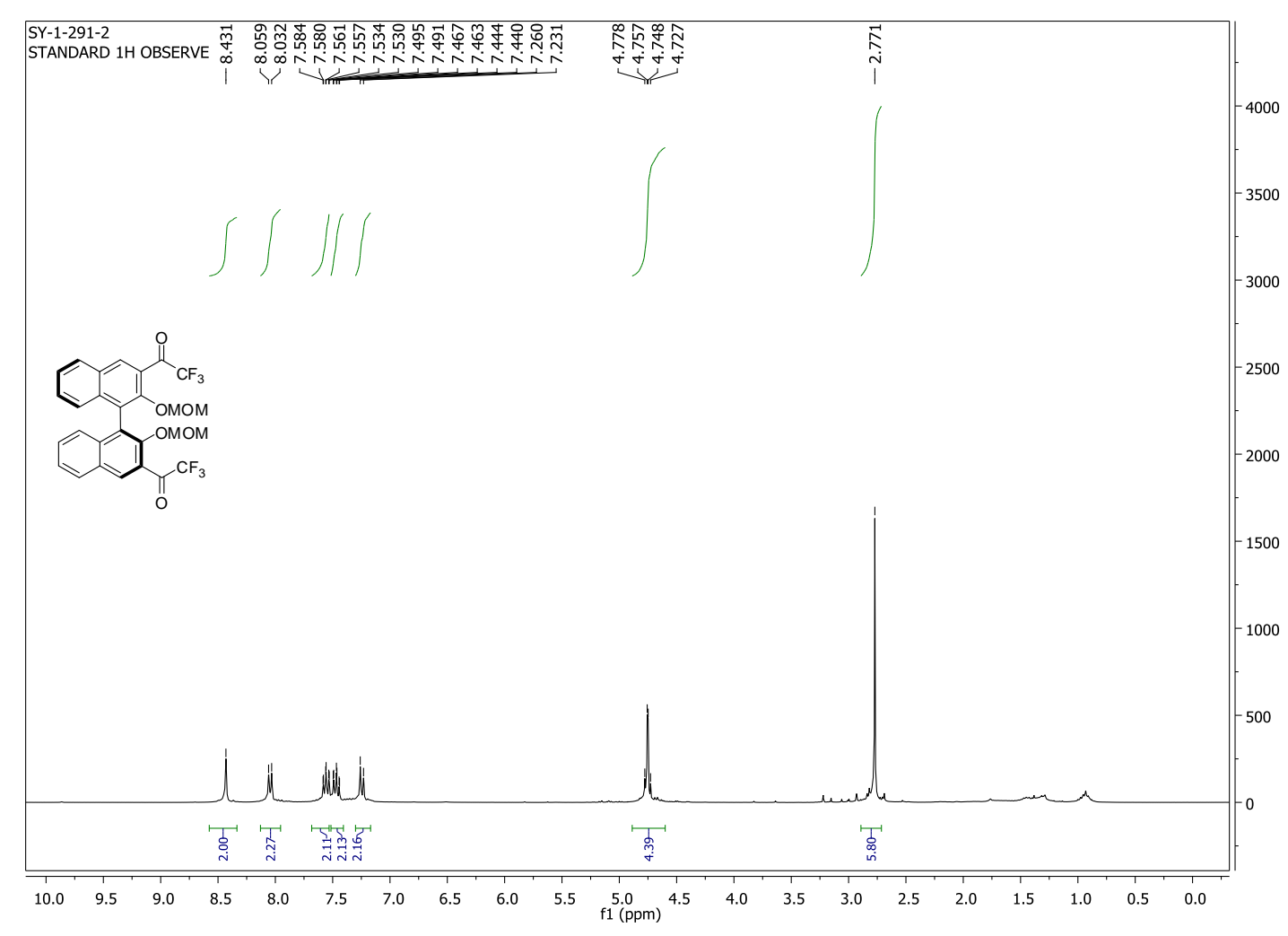




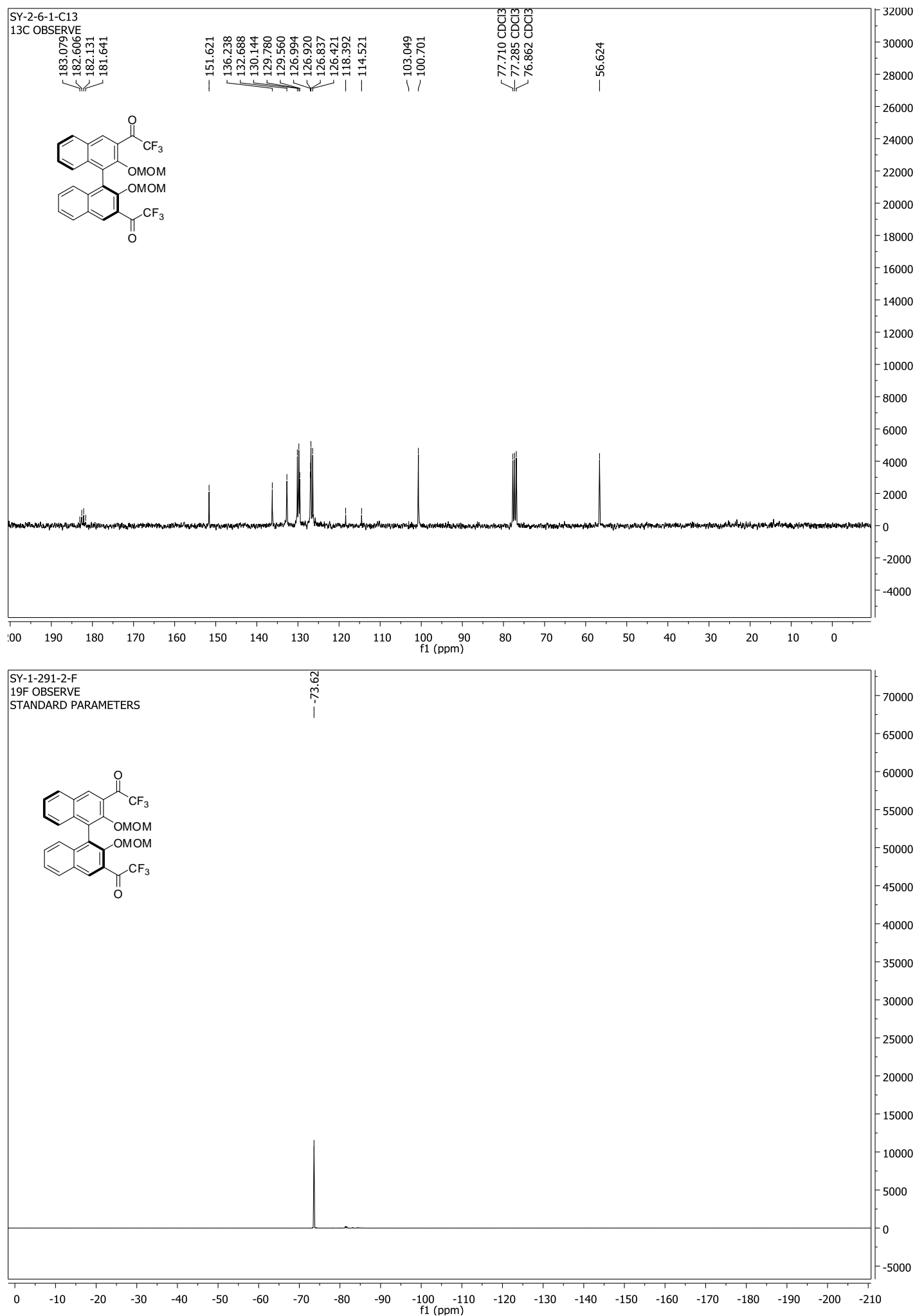



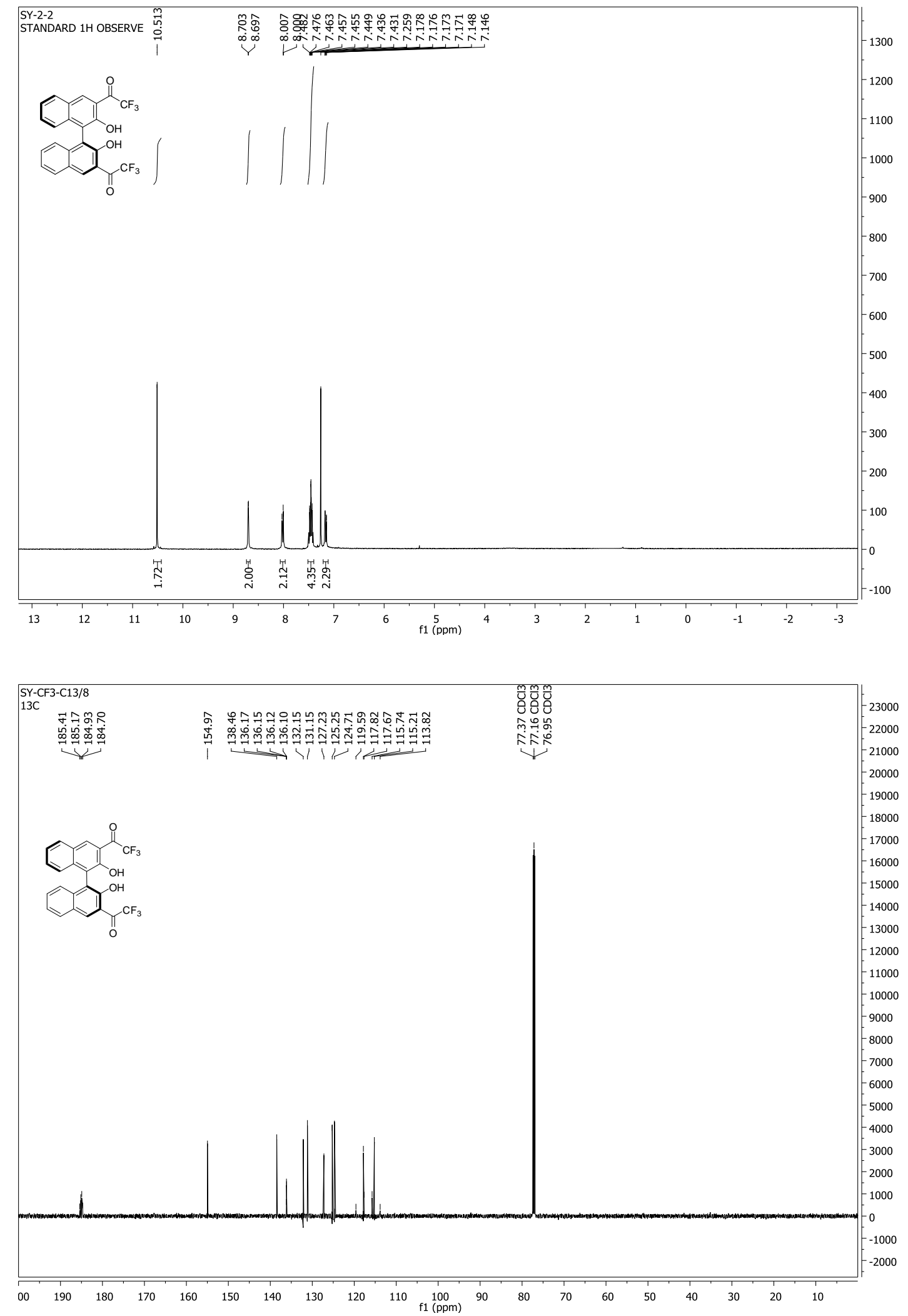

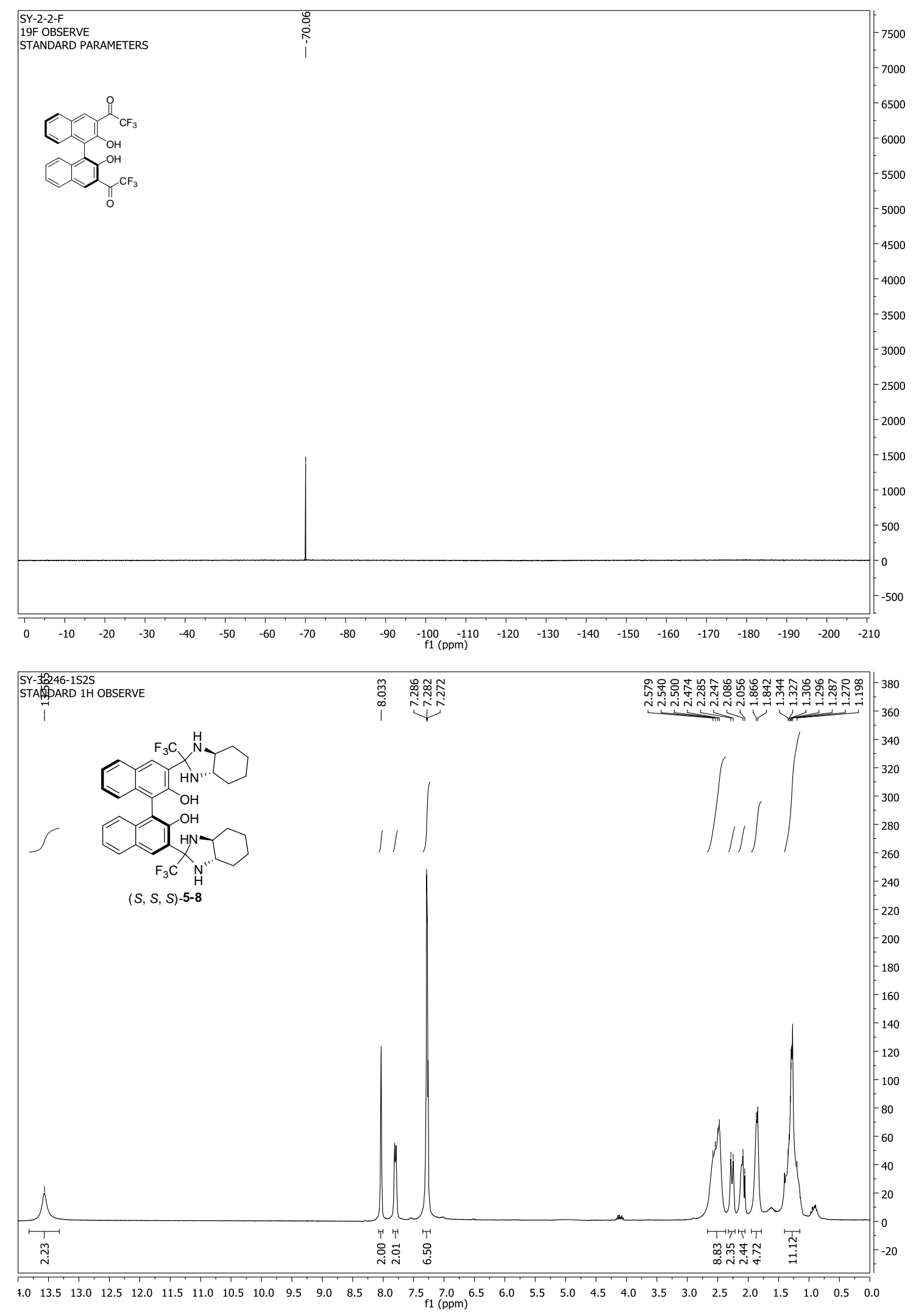

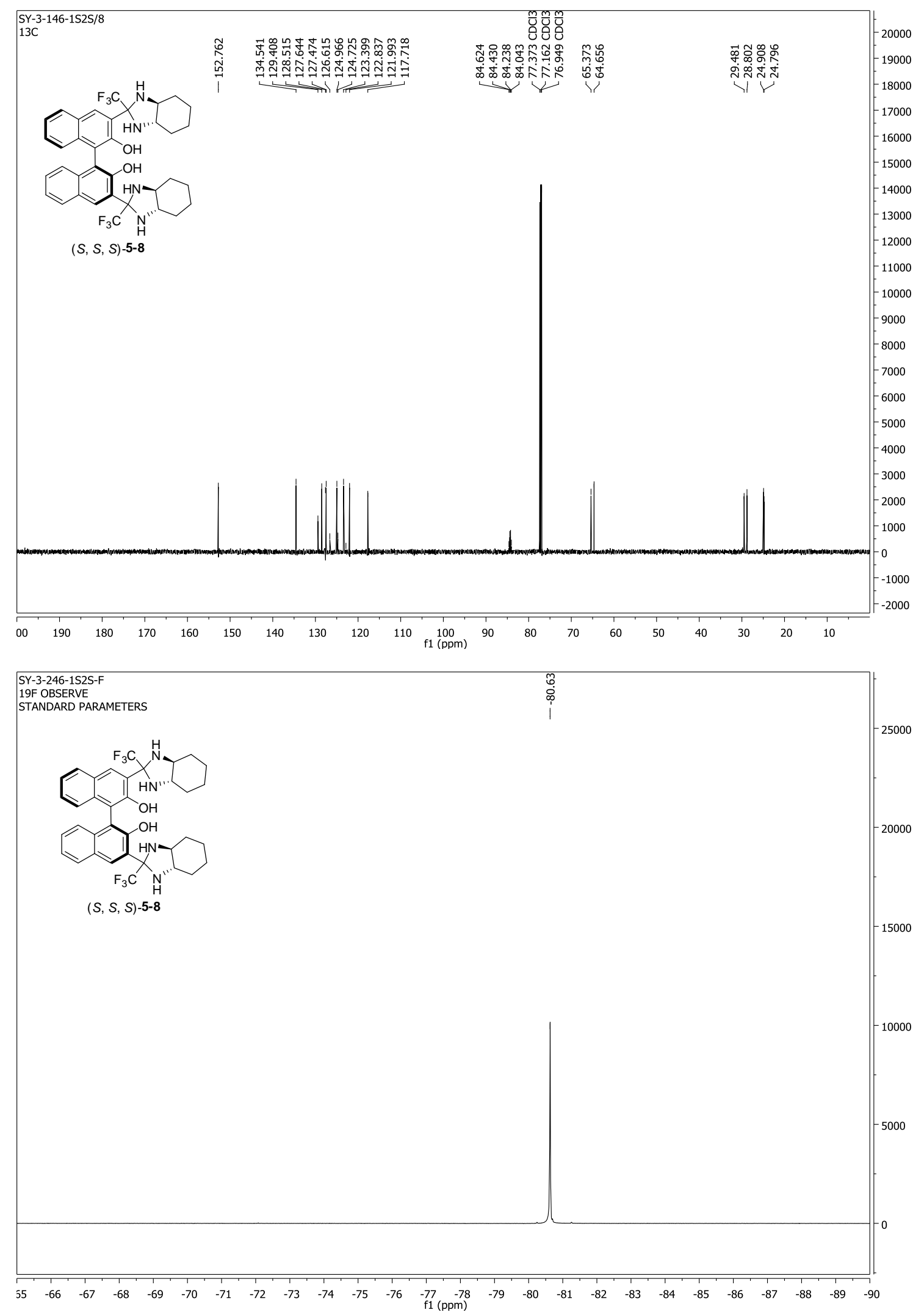

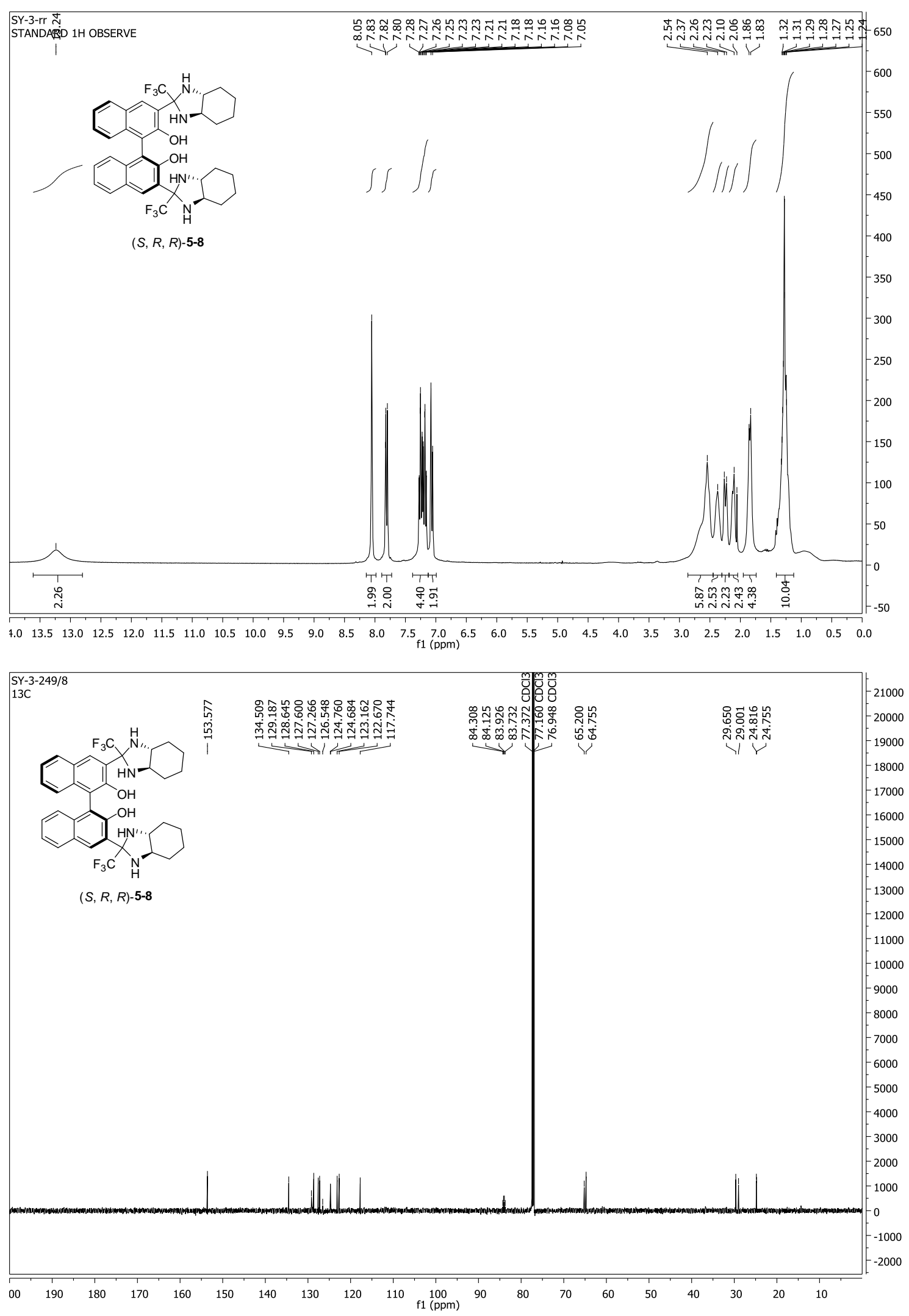


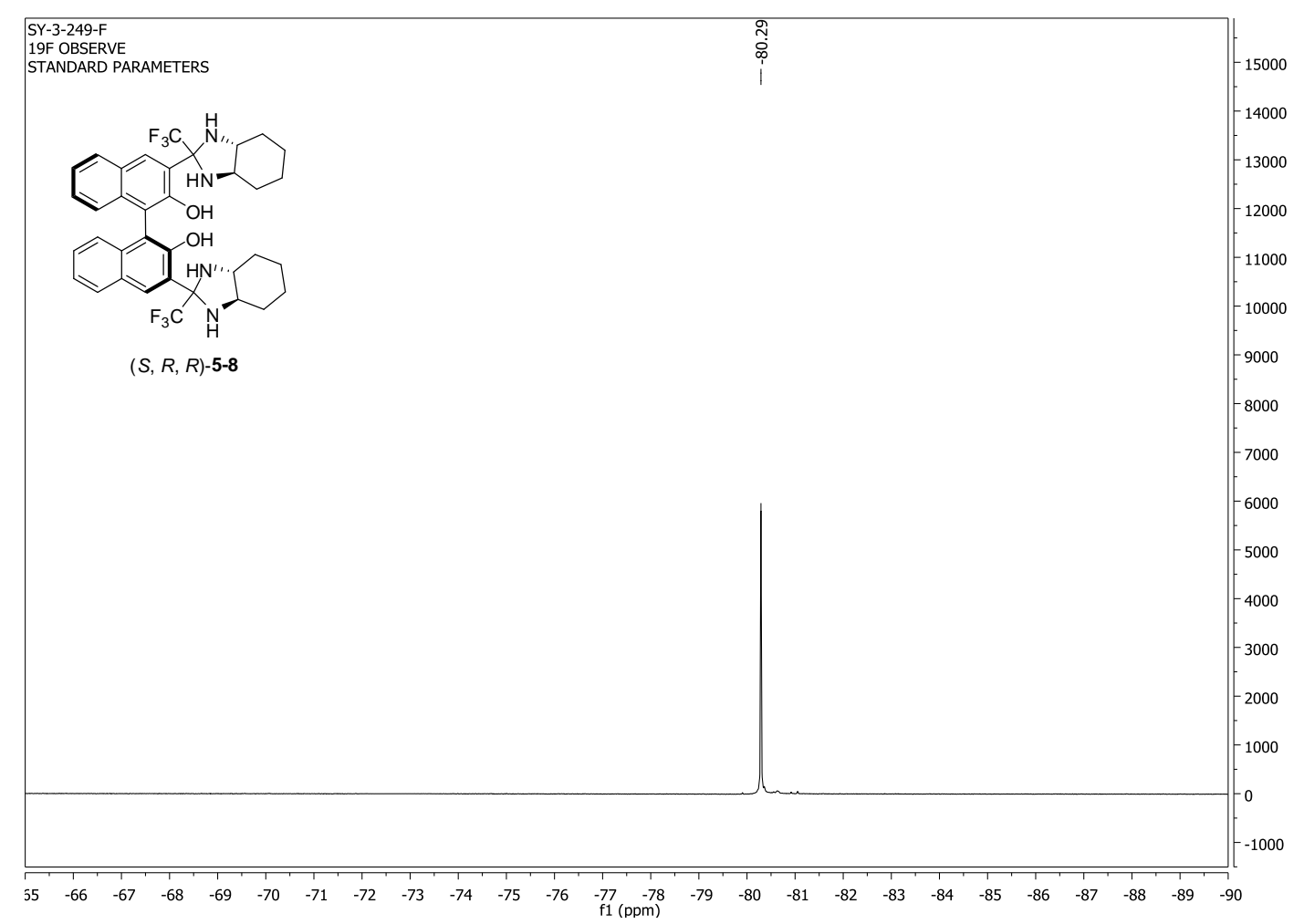

${ }^{19}$ F NMR Titration of $(S)-5-3$ with $(S, S)-5-4$

0 eq of amine

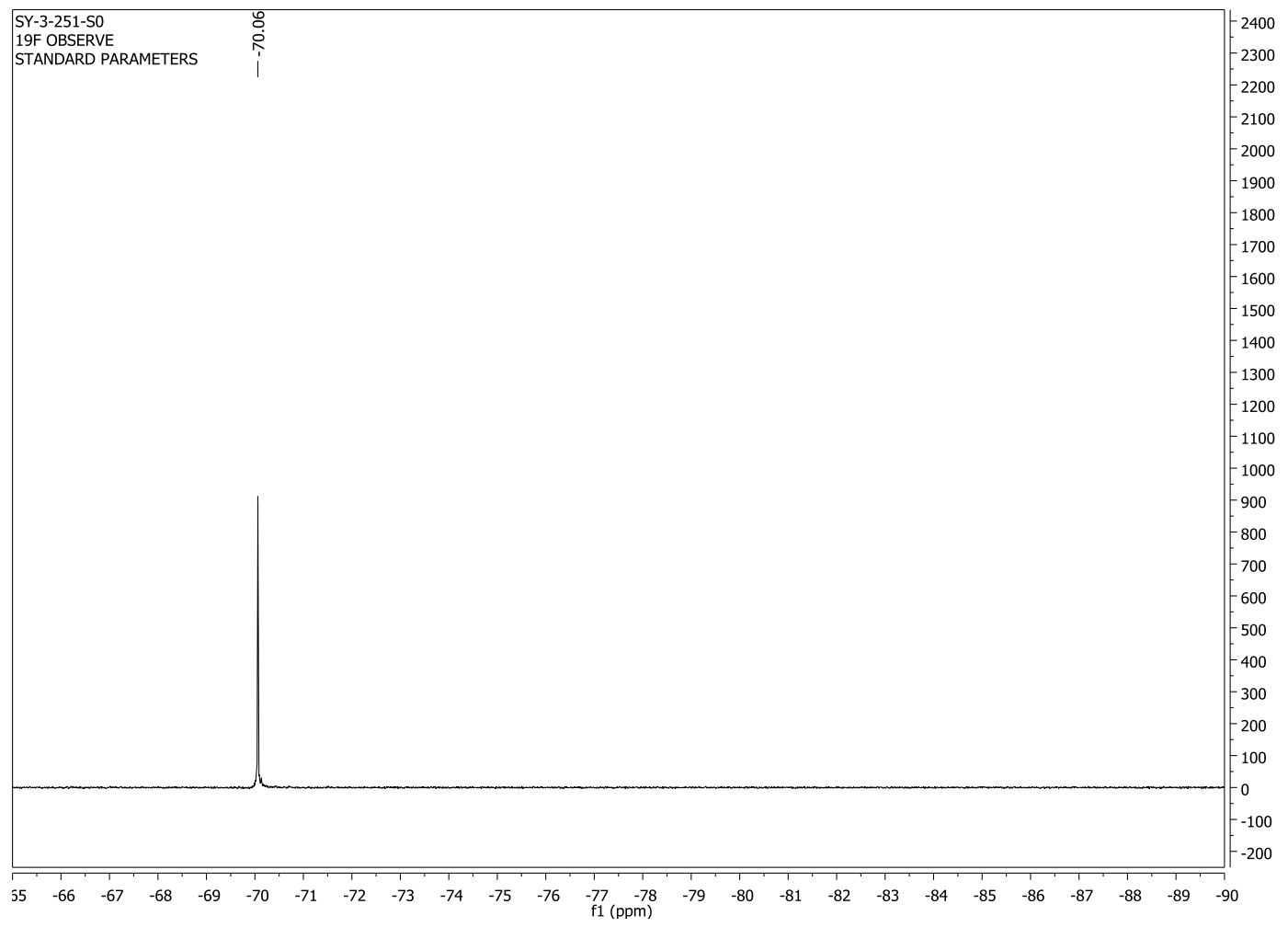




\section{$1.2 \mathrm{eq}$ of amine}

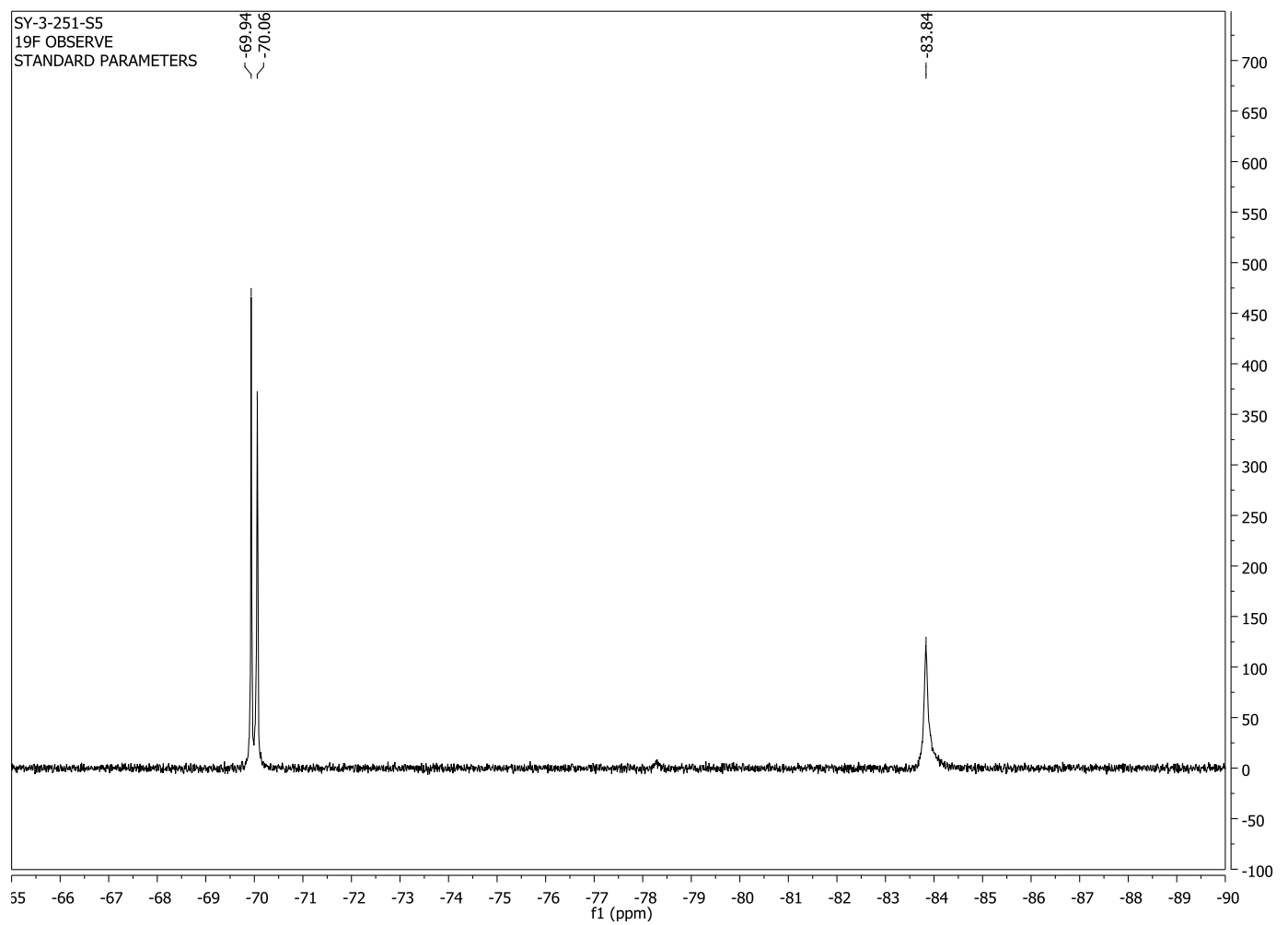

\section{4 eq of amine}

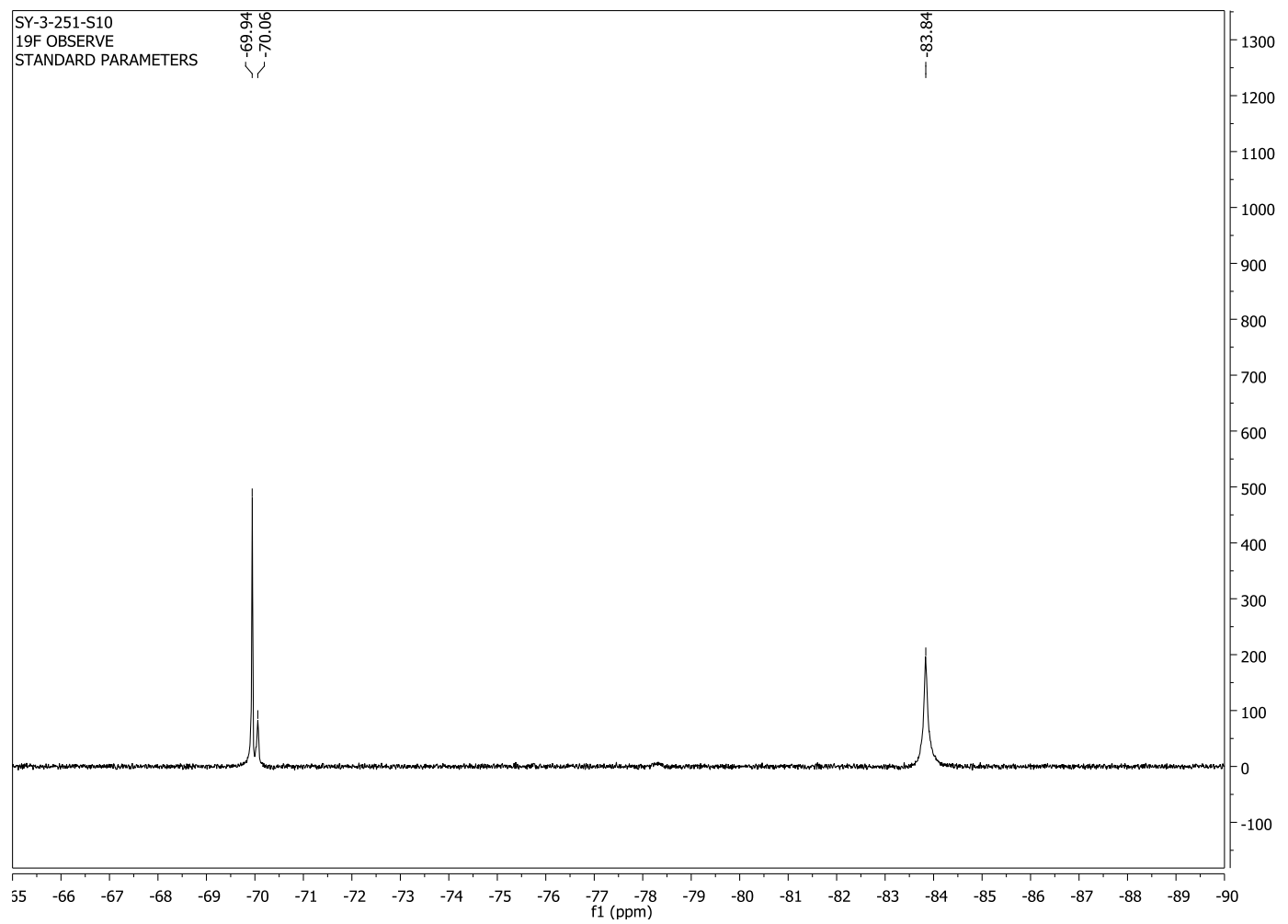




\section{$3.6 \mathrm{eq}$ of amine}

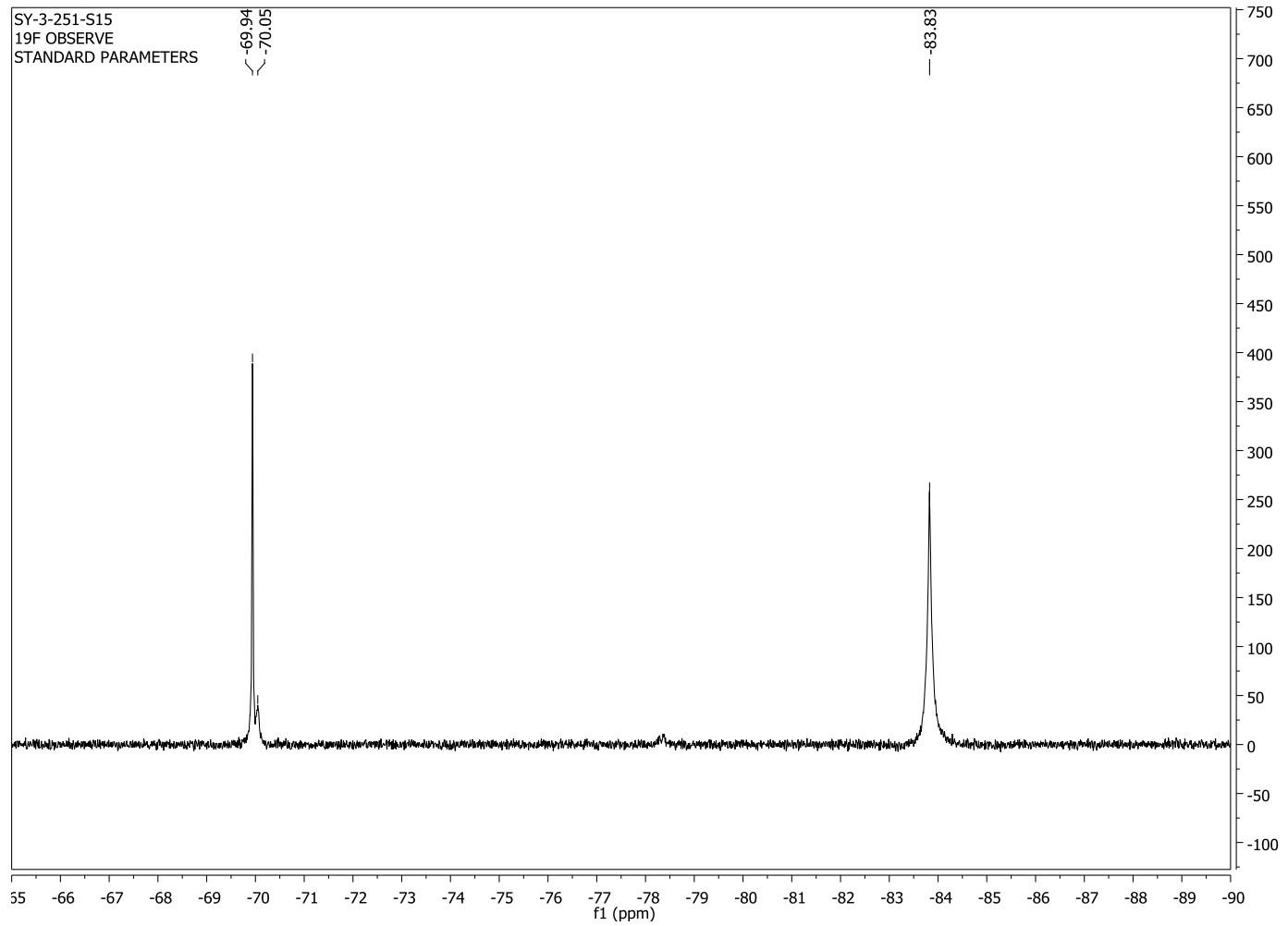

\section{$4.8 \mathrm{eq}$ of amine}

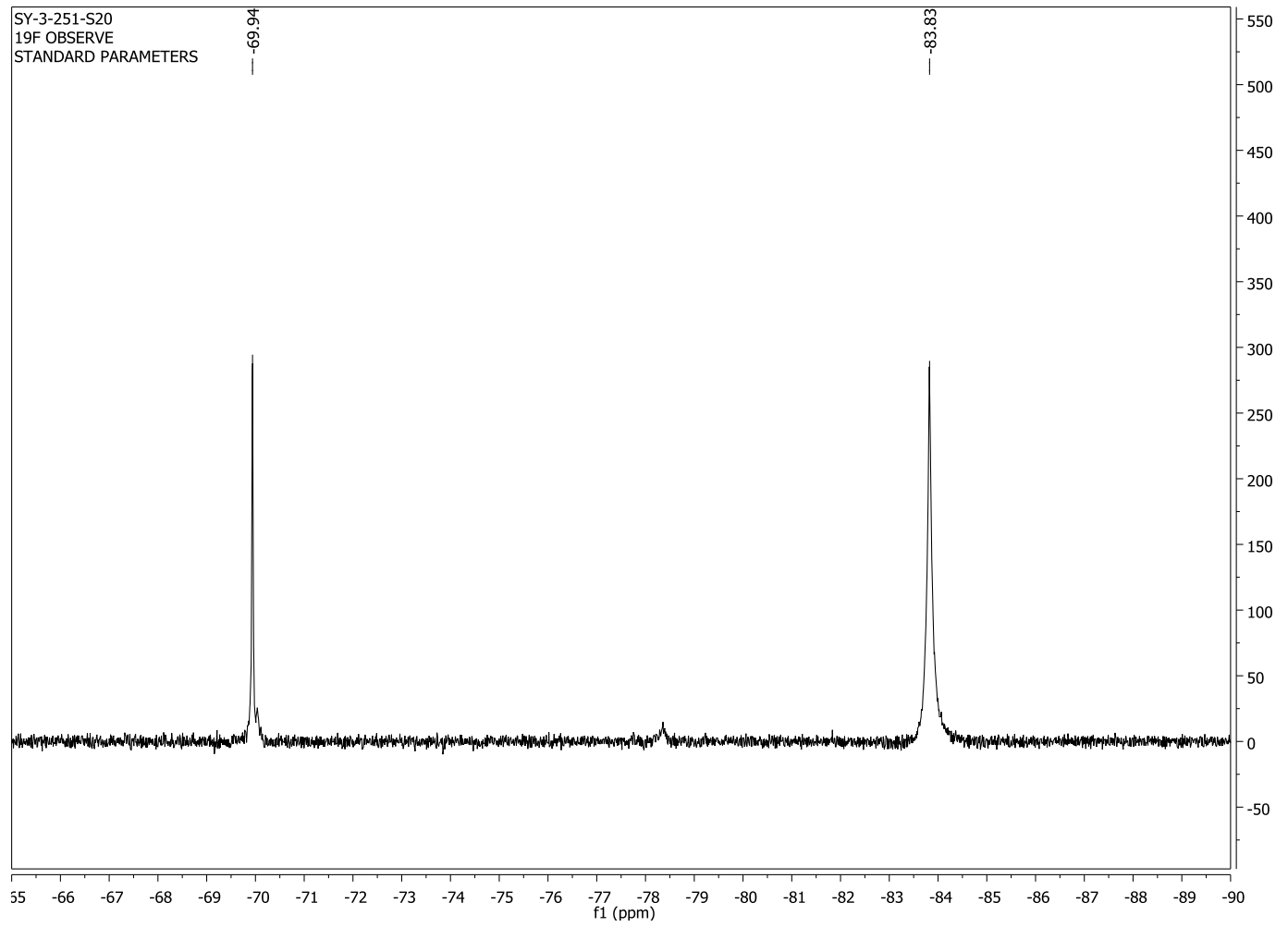




\section{$7.0 \mathrm{eq}$ of amine}

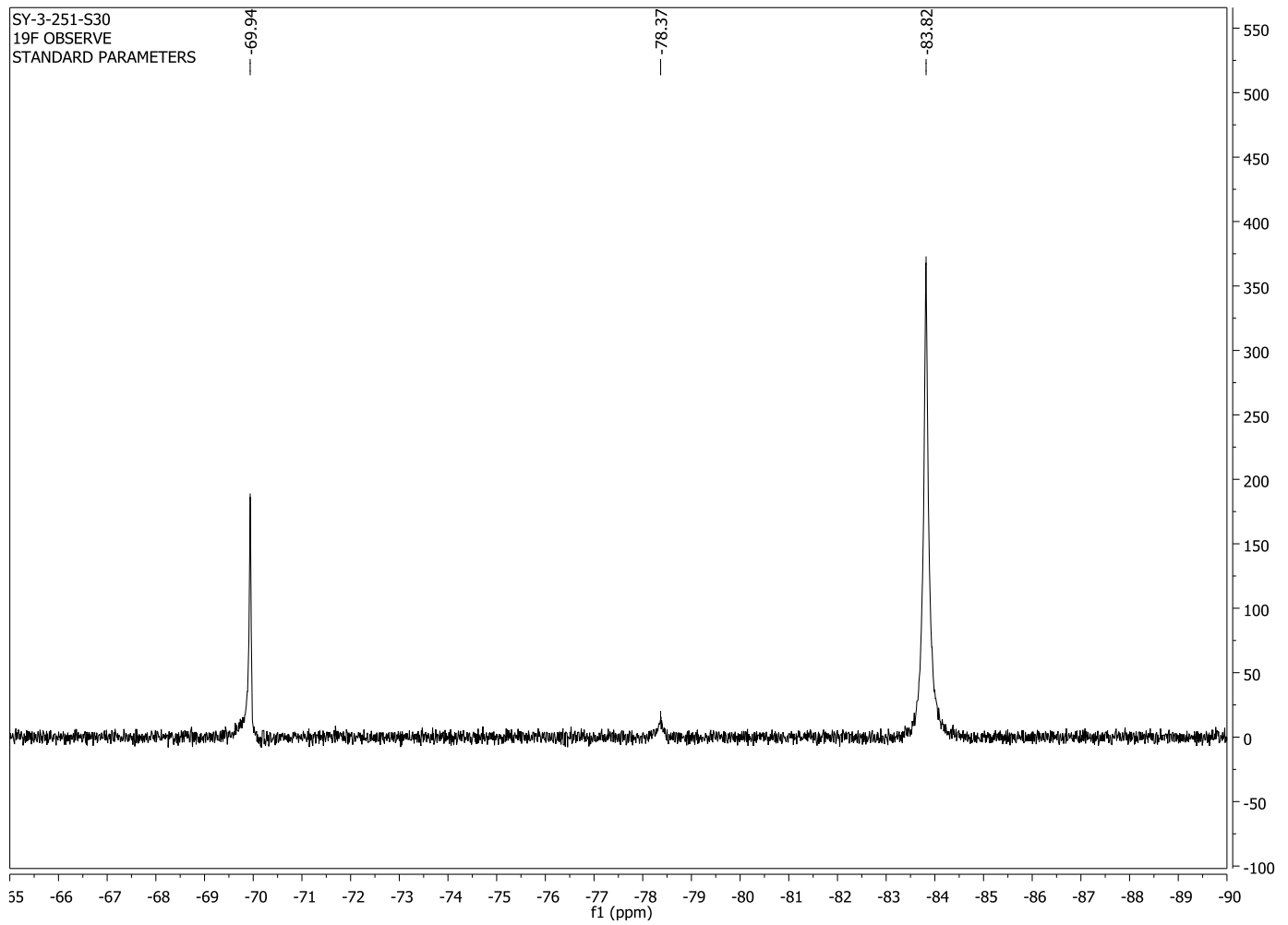

\section{1 eq of amine}

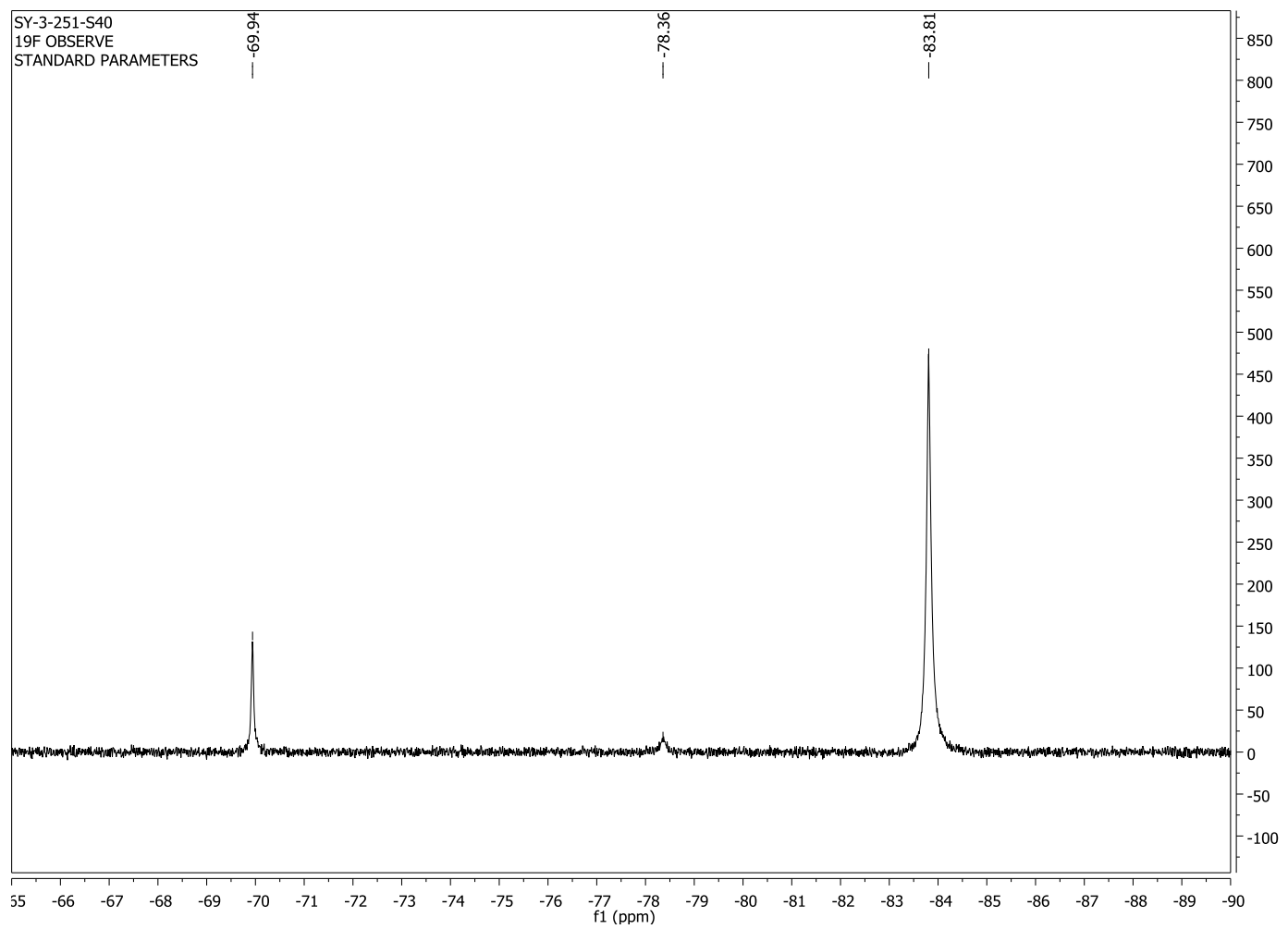




\section{1 eq of amine}

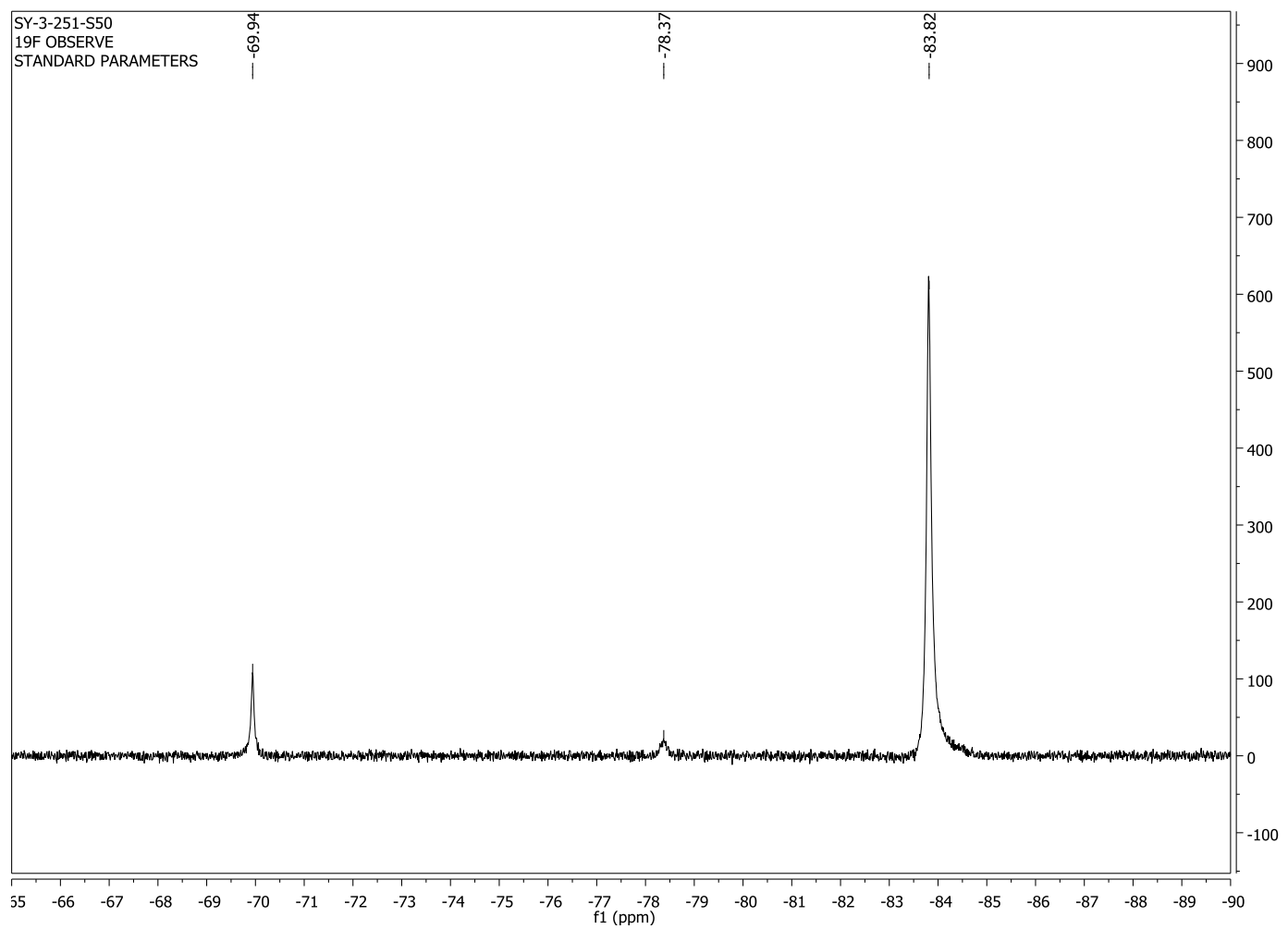

14.9 eq of amine

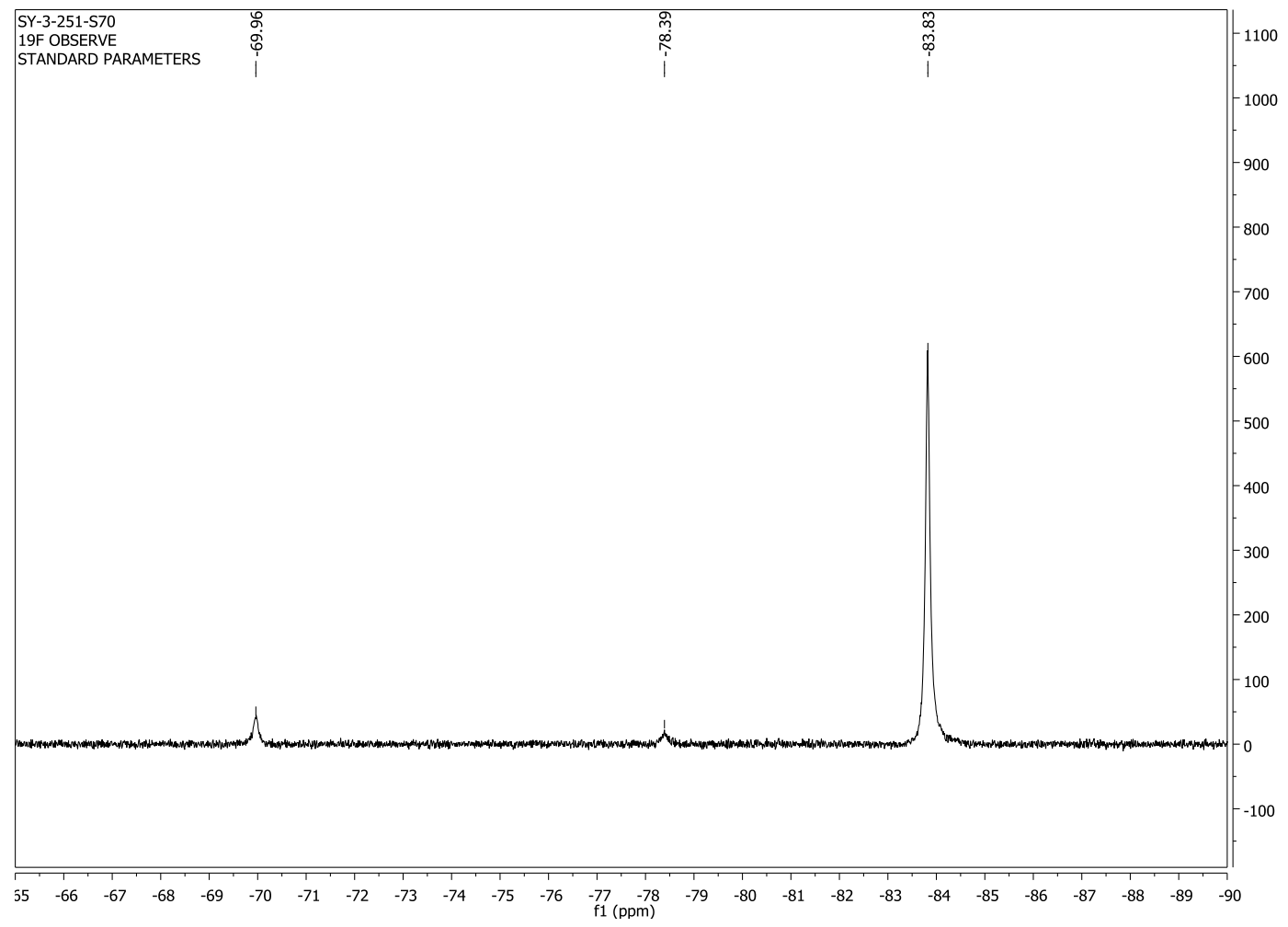




\section{0 eq of amine}

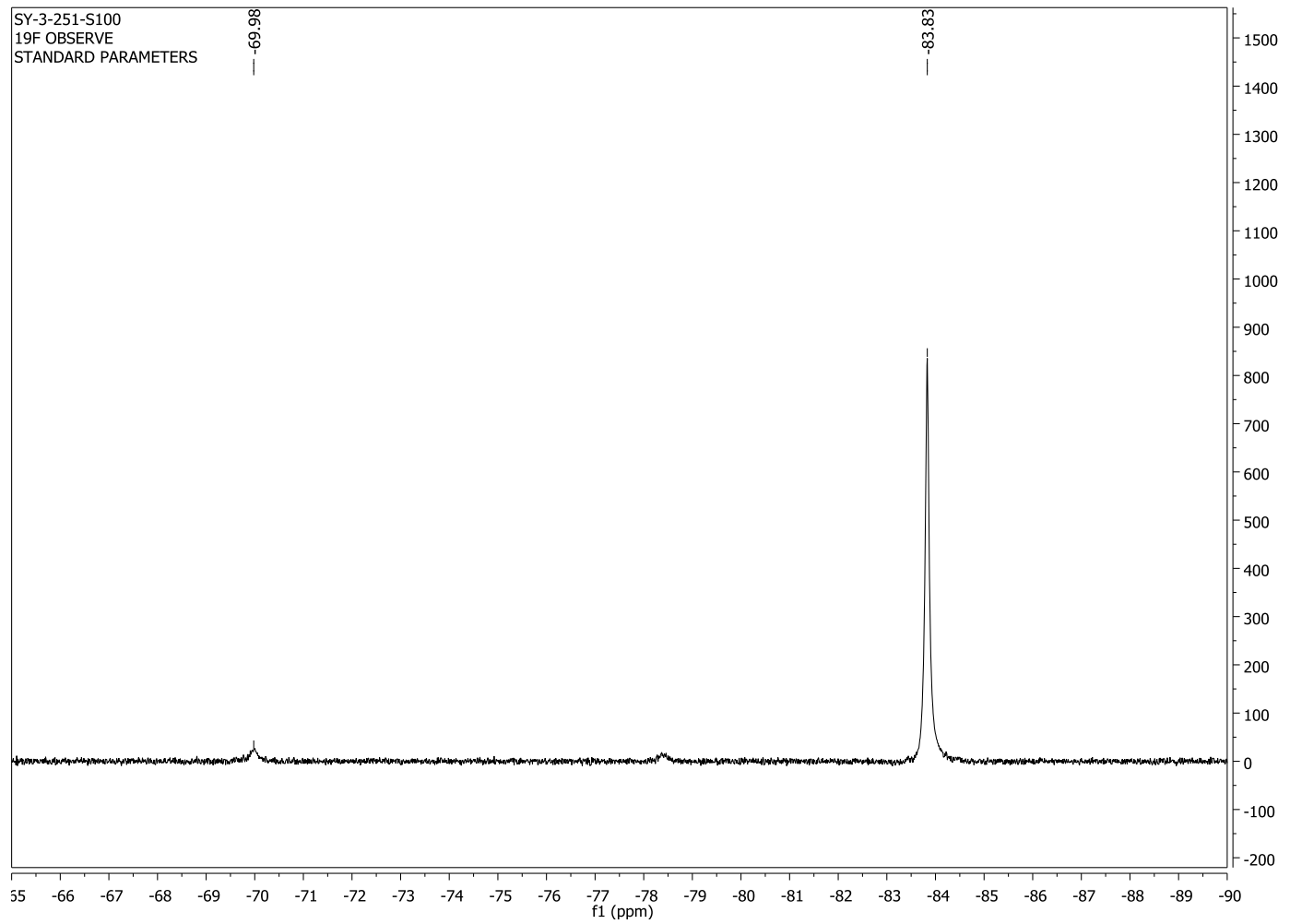

\section{$27.3 \mathrm{eq}$ of amine}

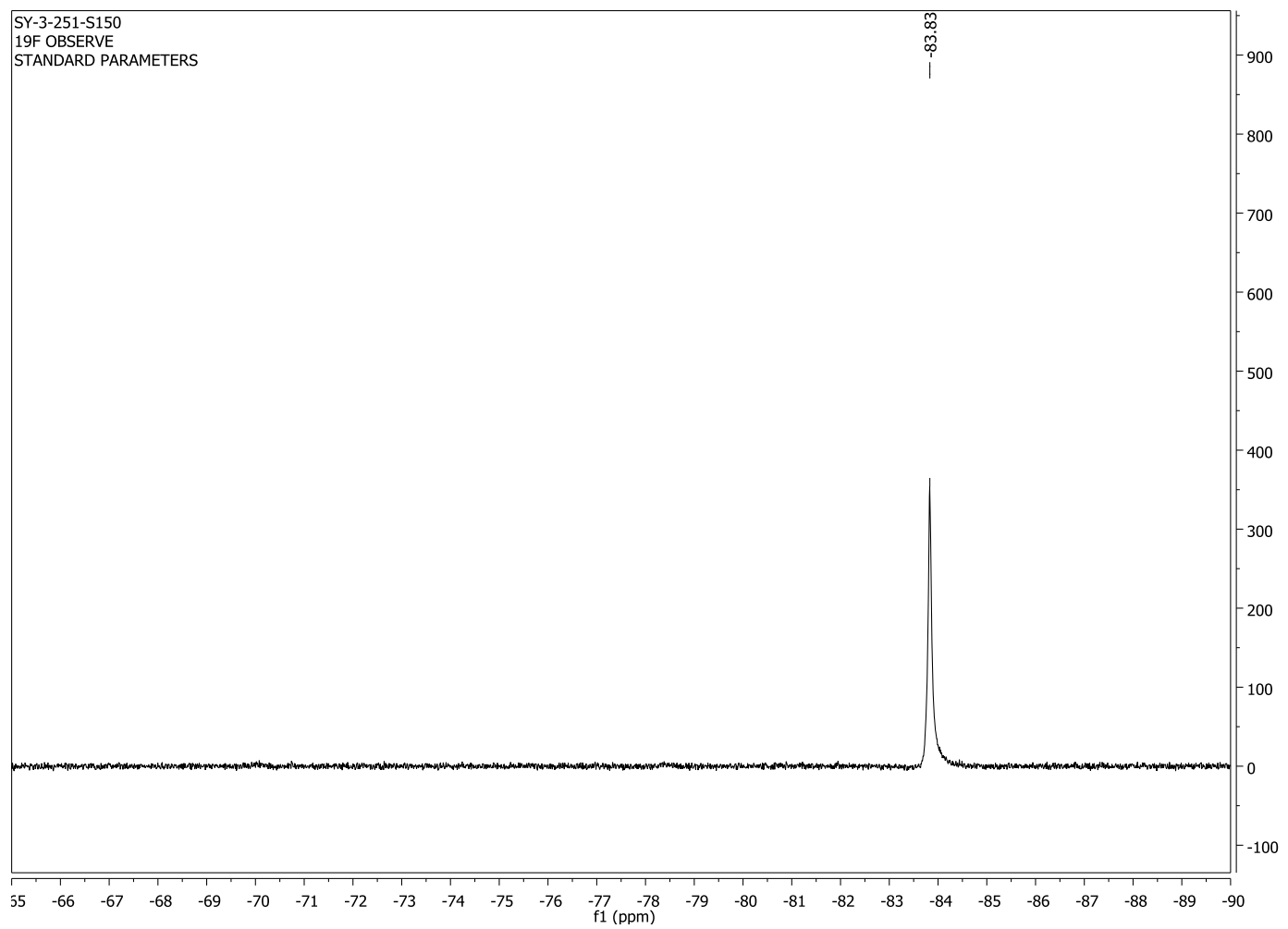


33.3 eq of amine

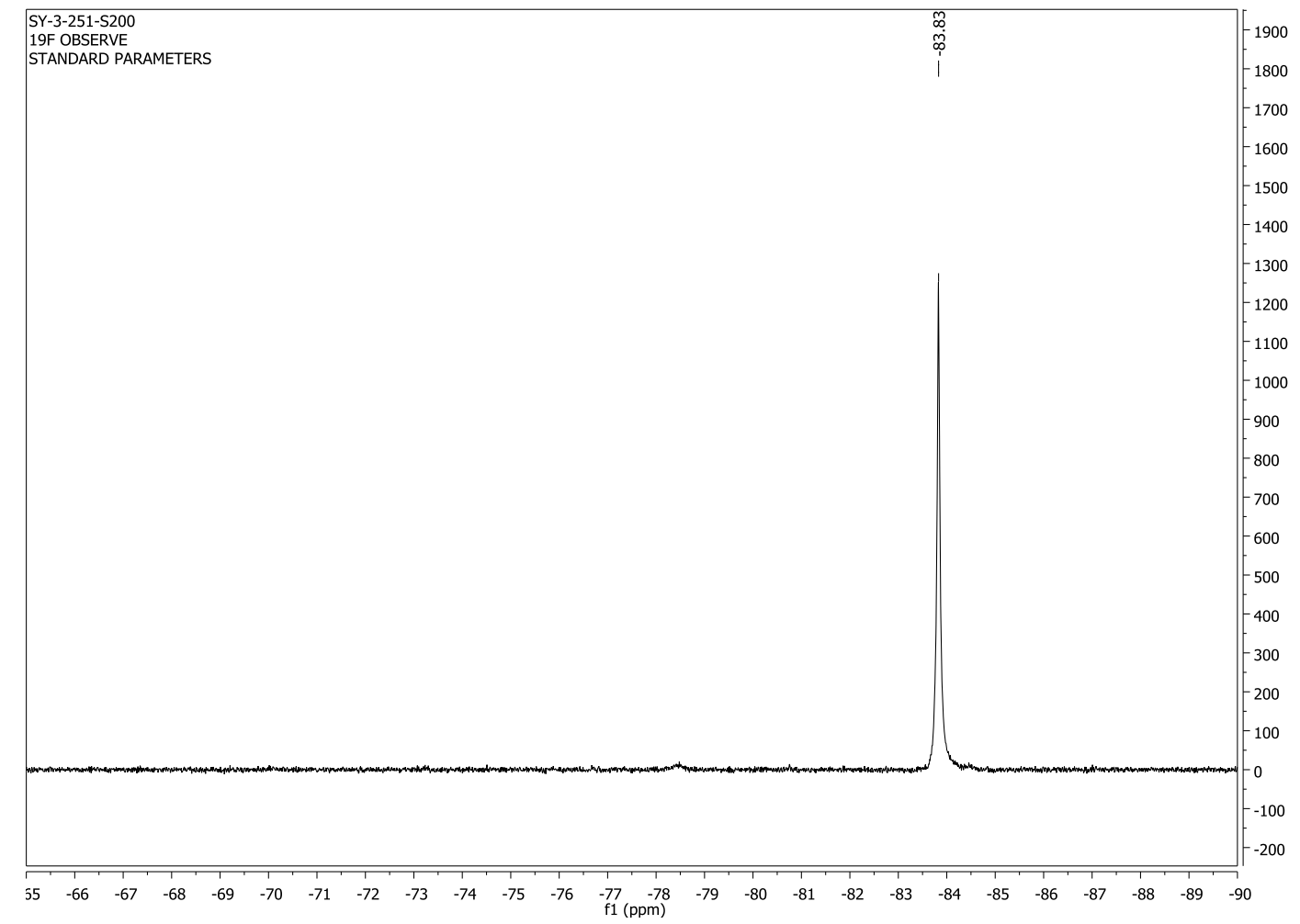

42.9 eq of amine

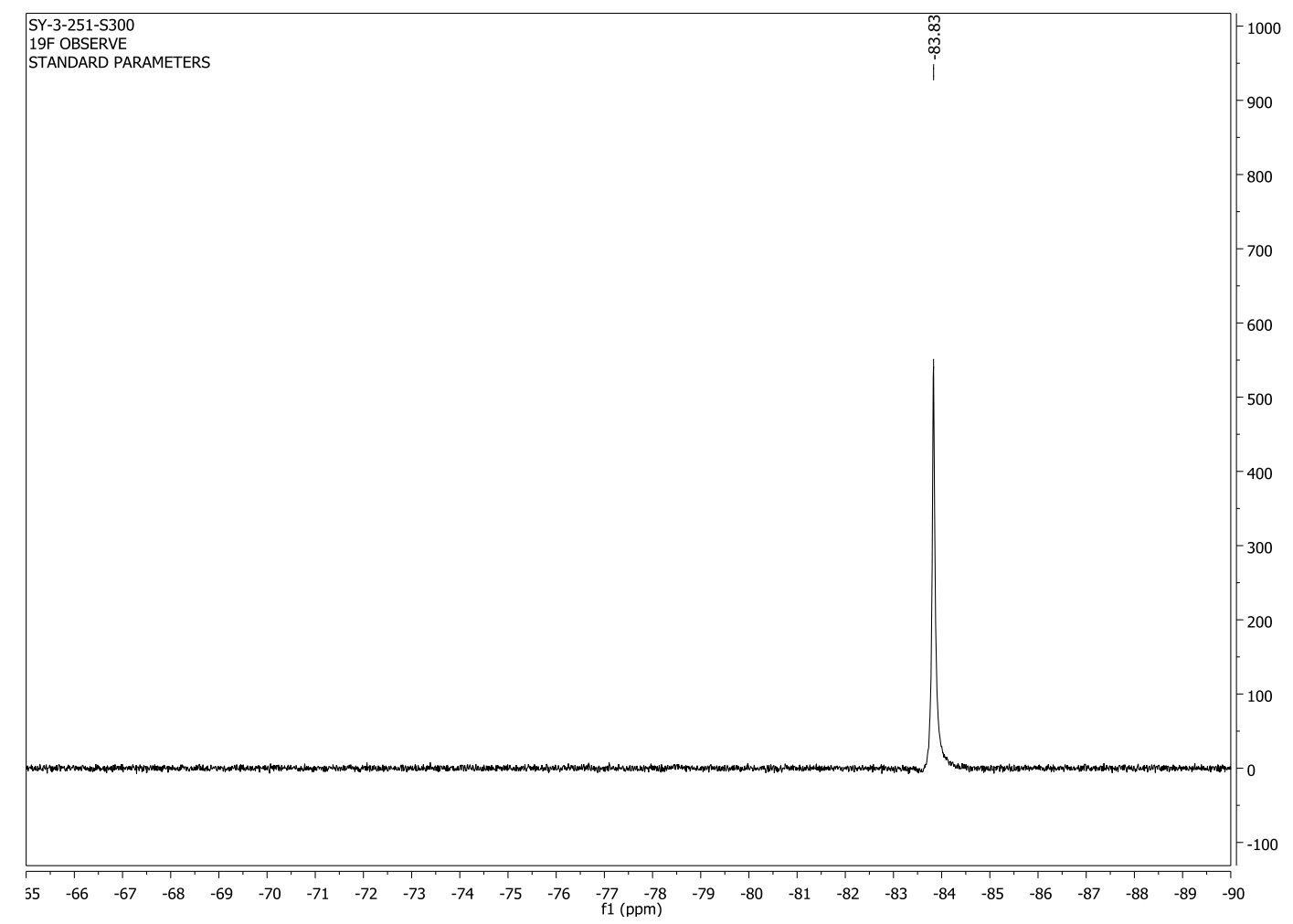




\section{6 eq of amine}

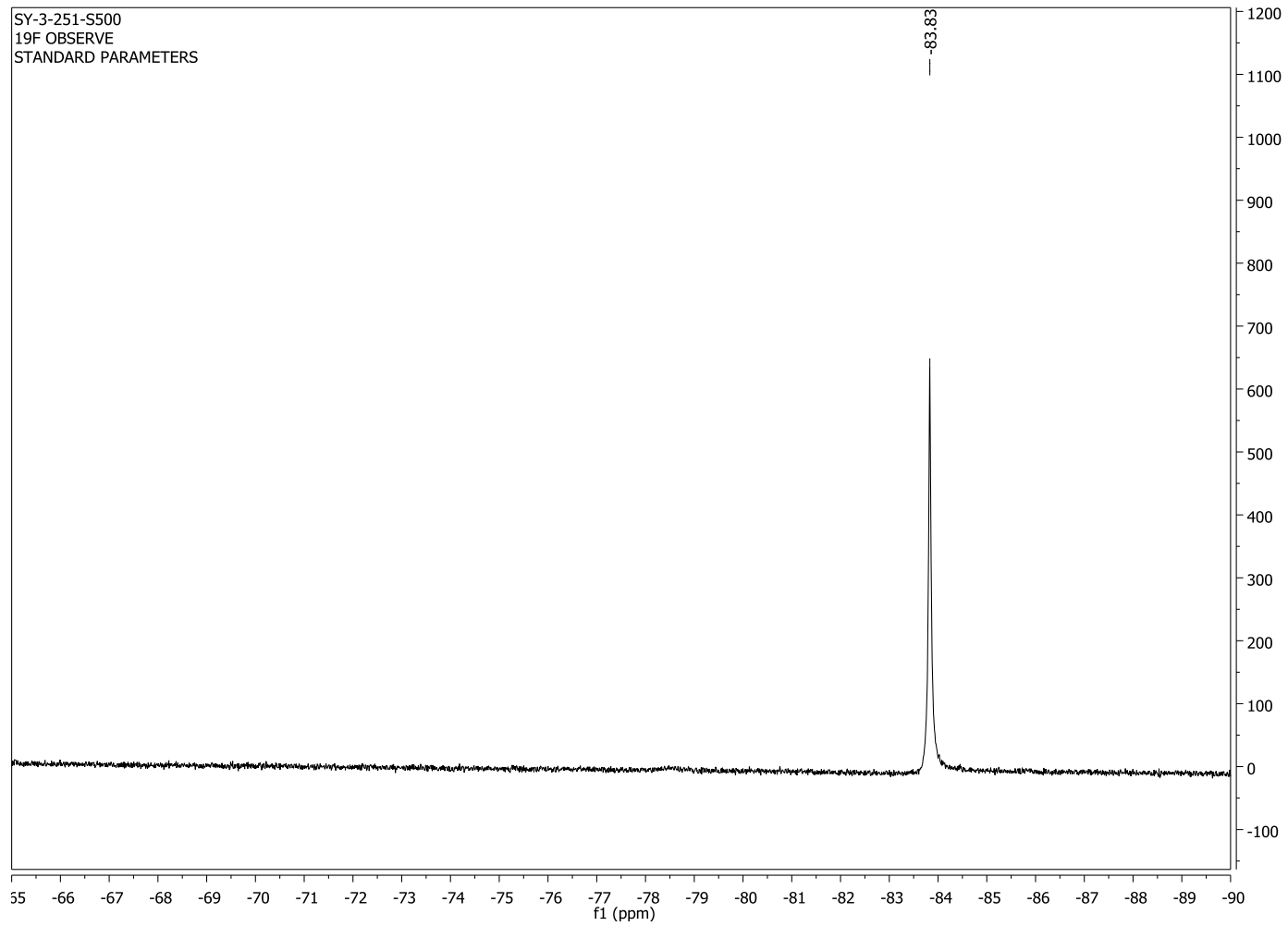

66.7 eq of amine

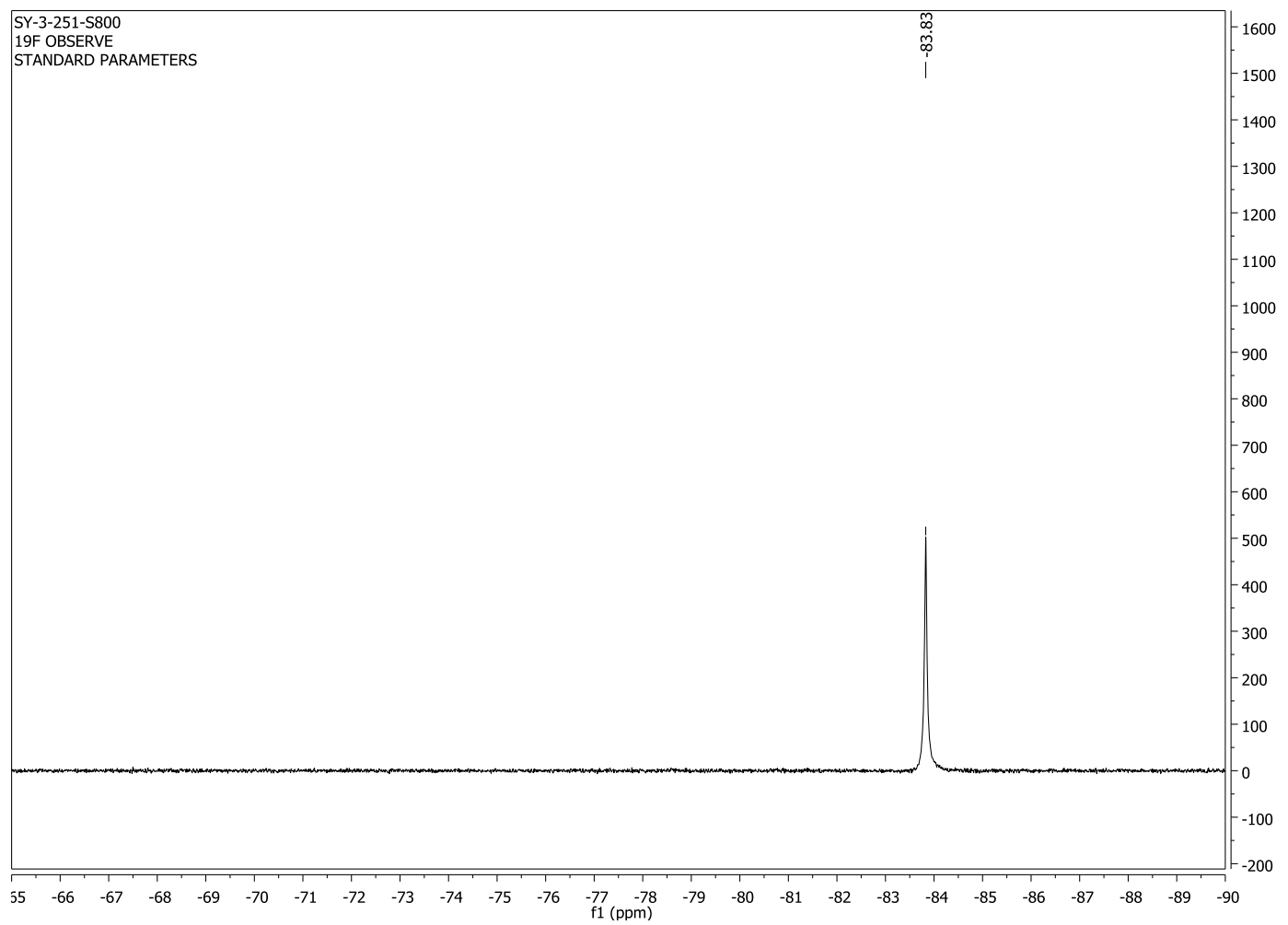


71.0 eq of amine

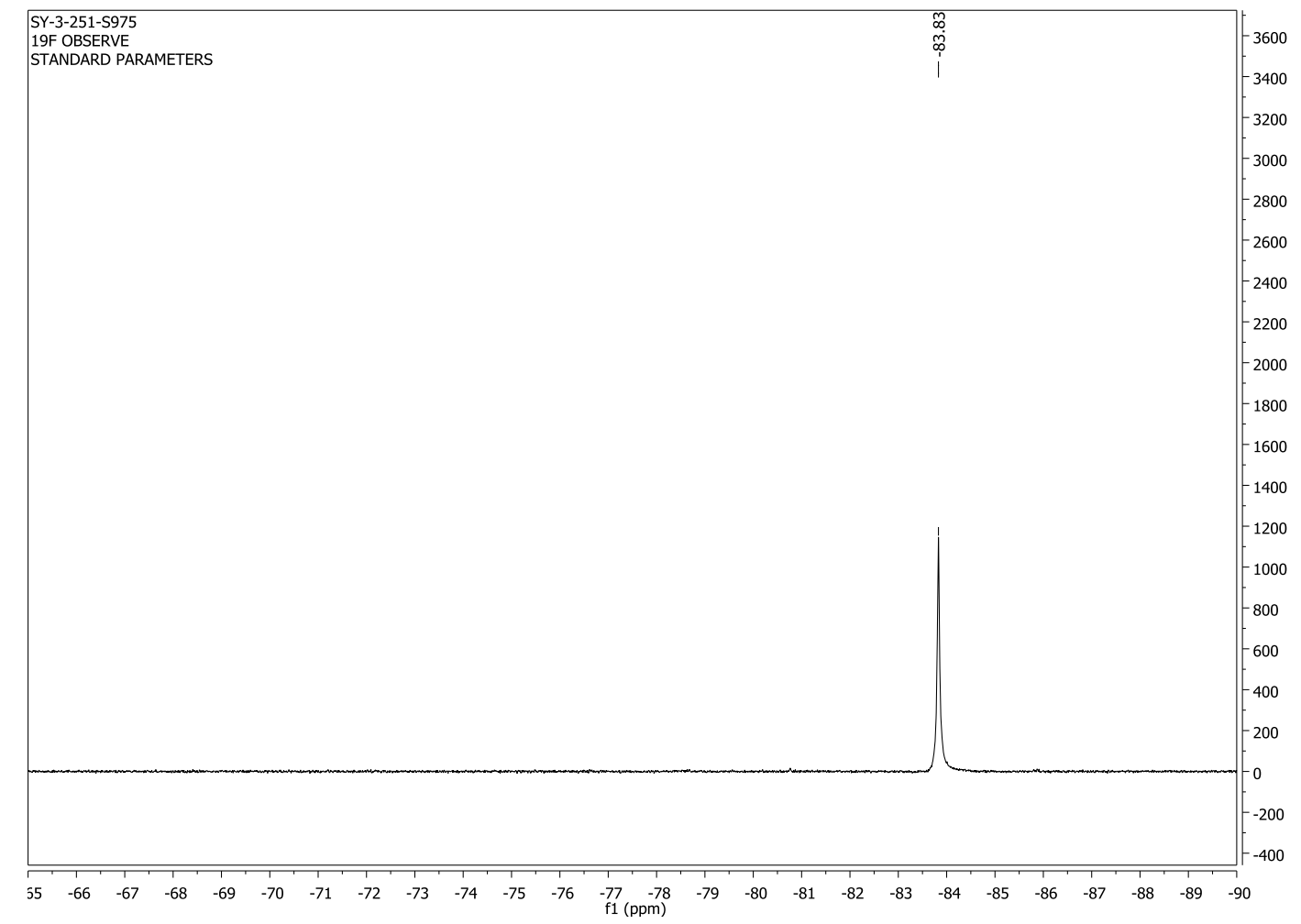

After two days

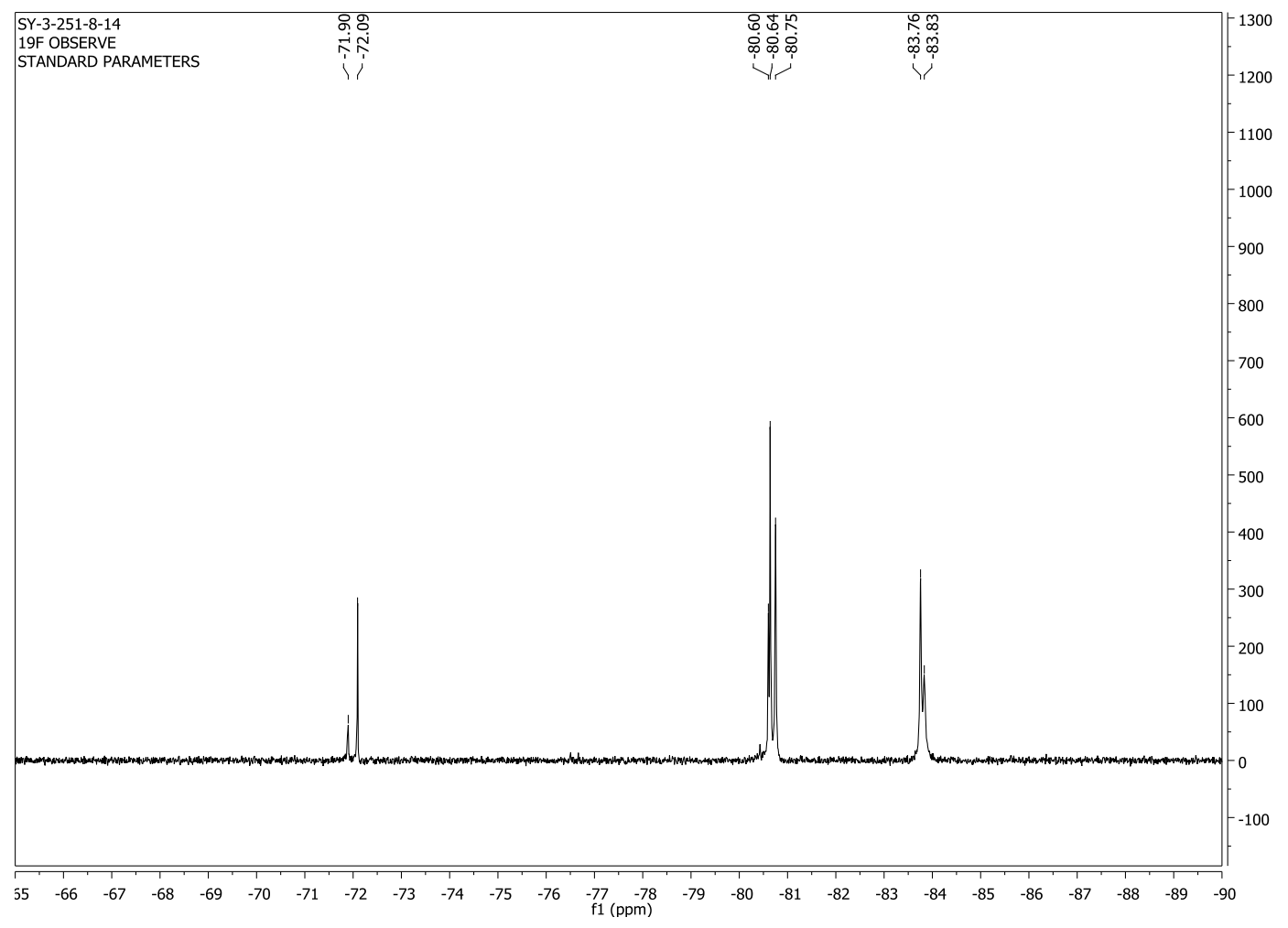


After four days

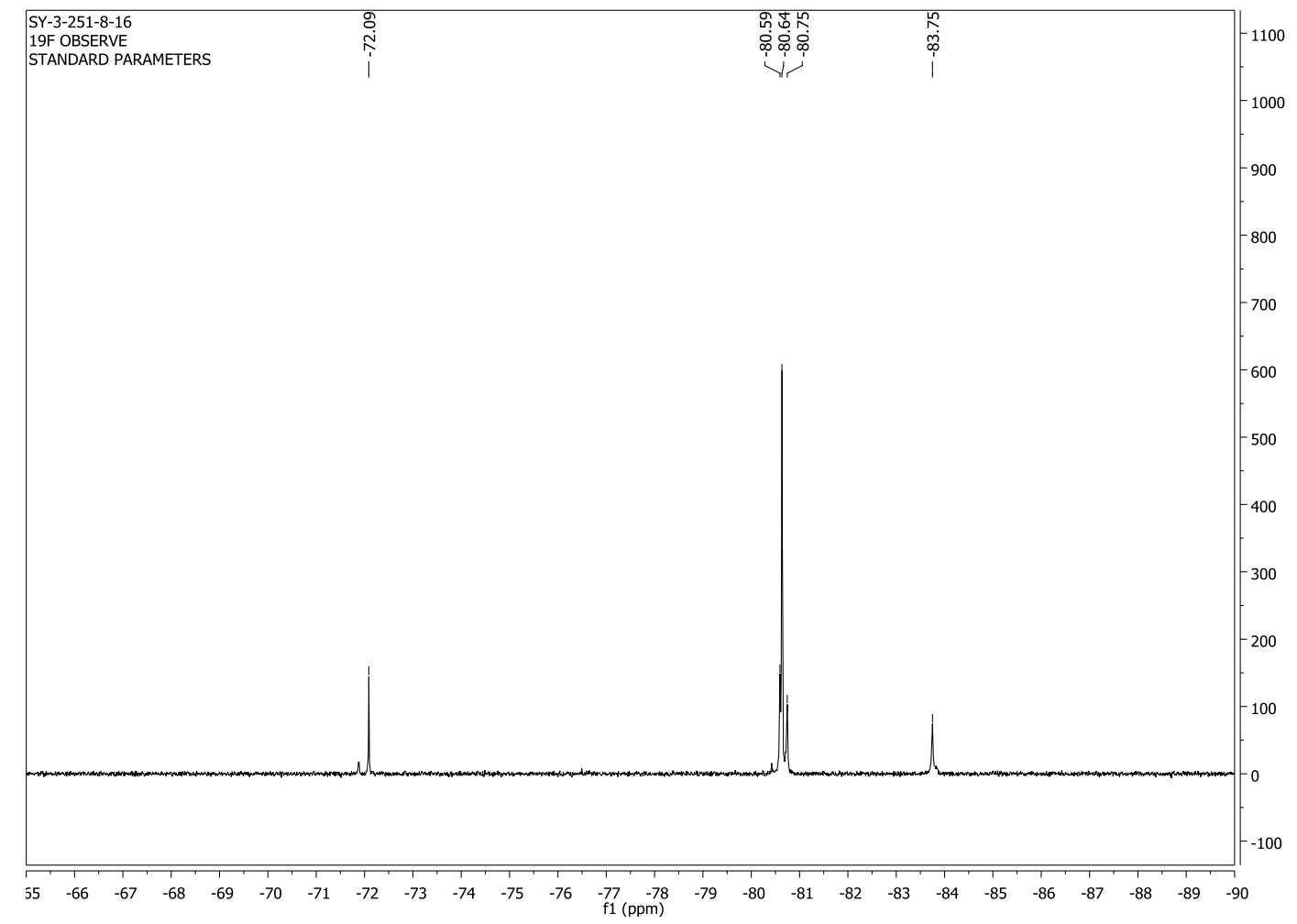

After five days

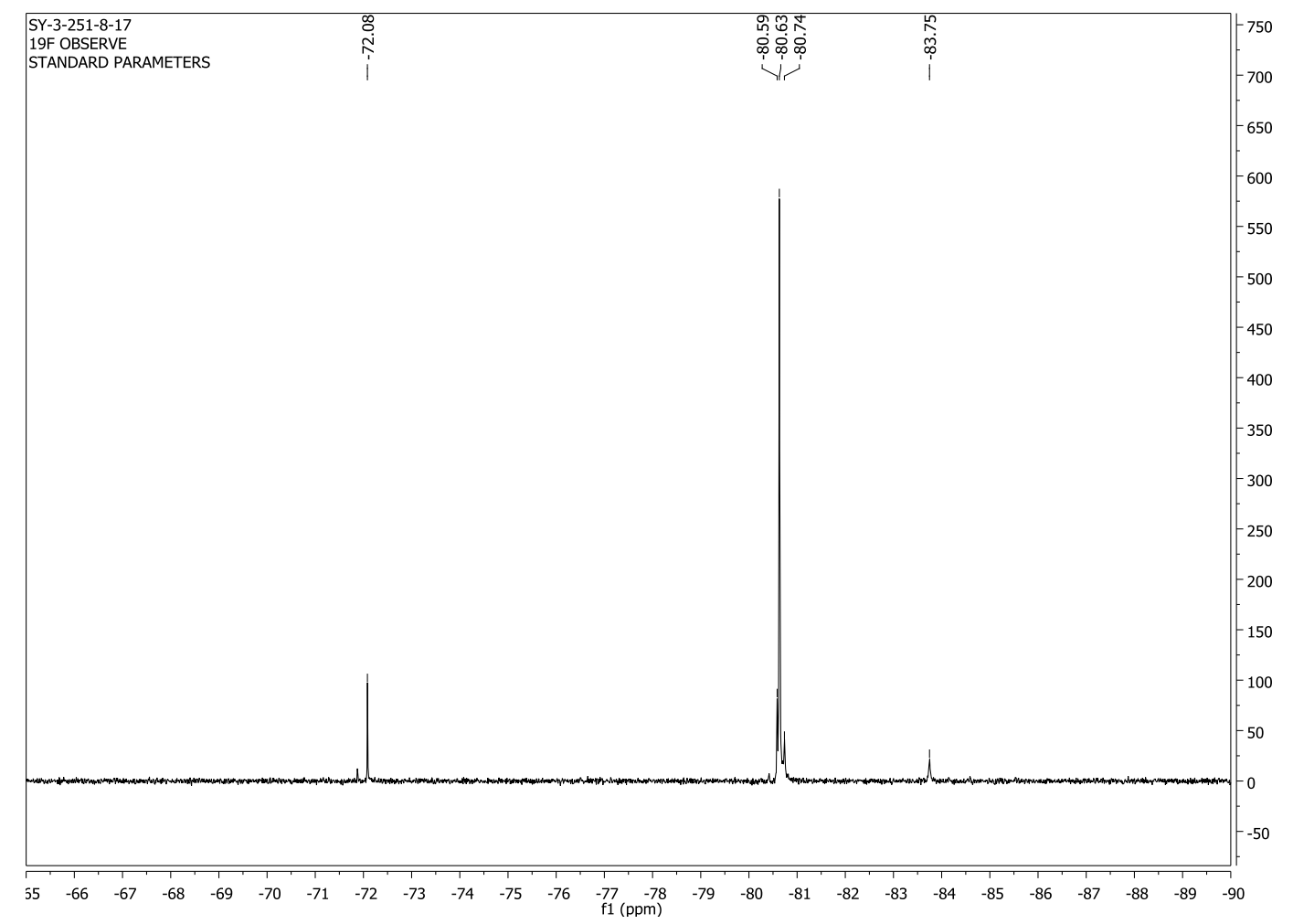


${ }^{19}$ F NMR Titration of $(S)-5-3$ with $(R, R)-5-4$

0 eq of amine

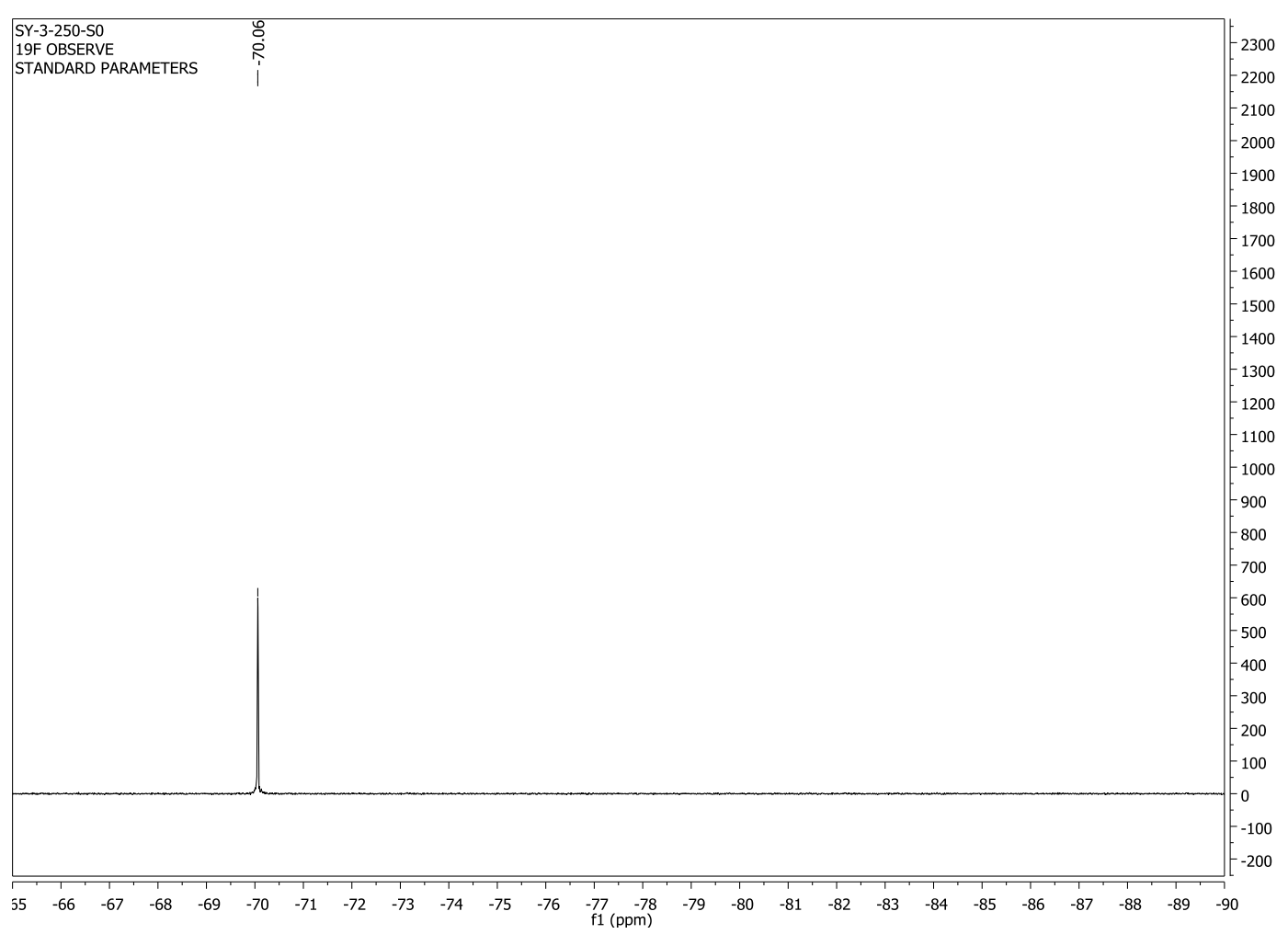




\section{$0.5 \mathrm{eq}$ of amine}

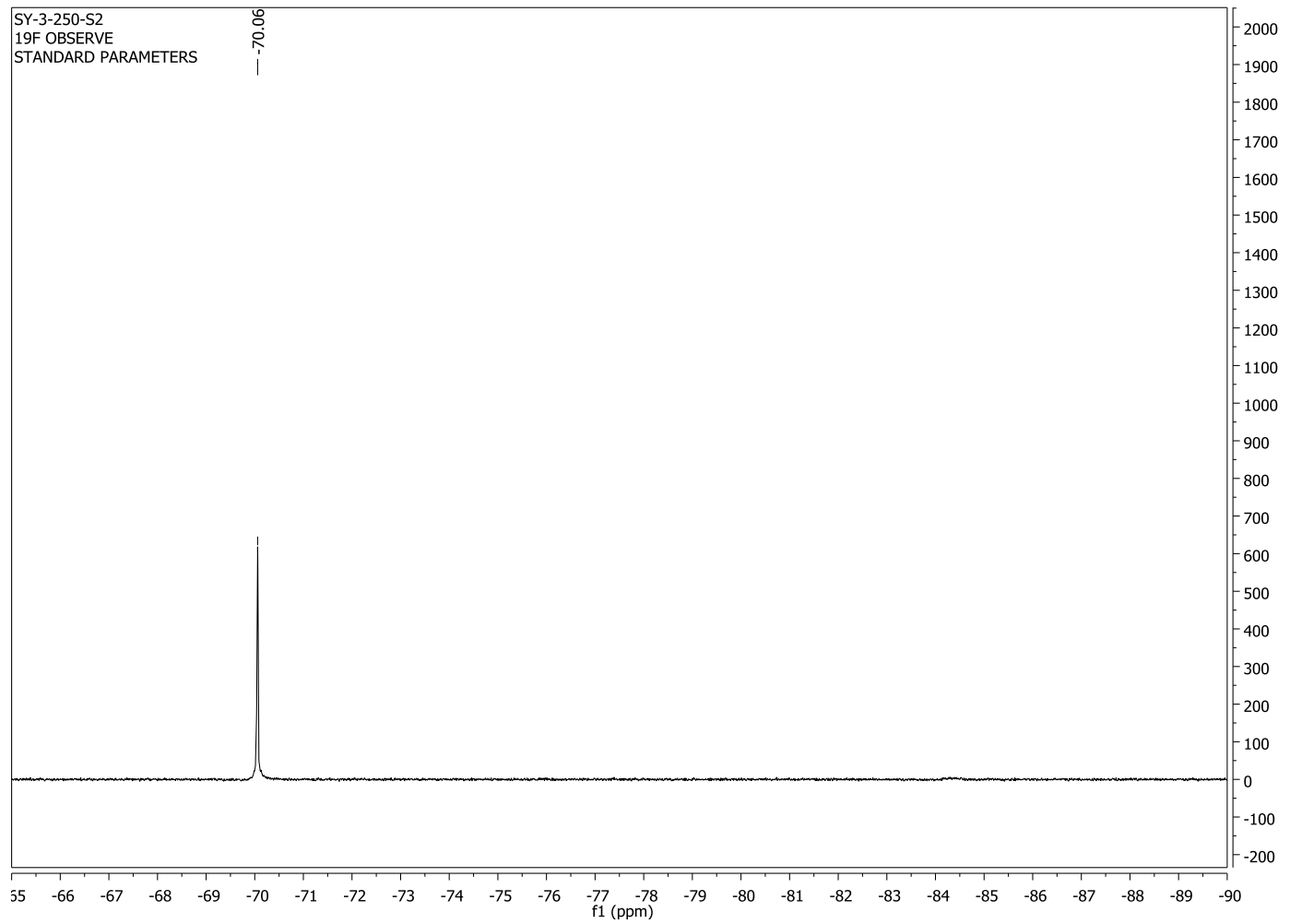

\section{$1.2 \mathrm{eq}$ of amine}

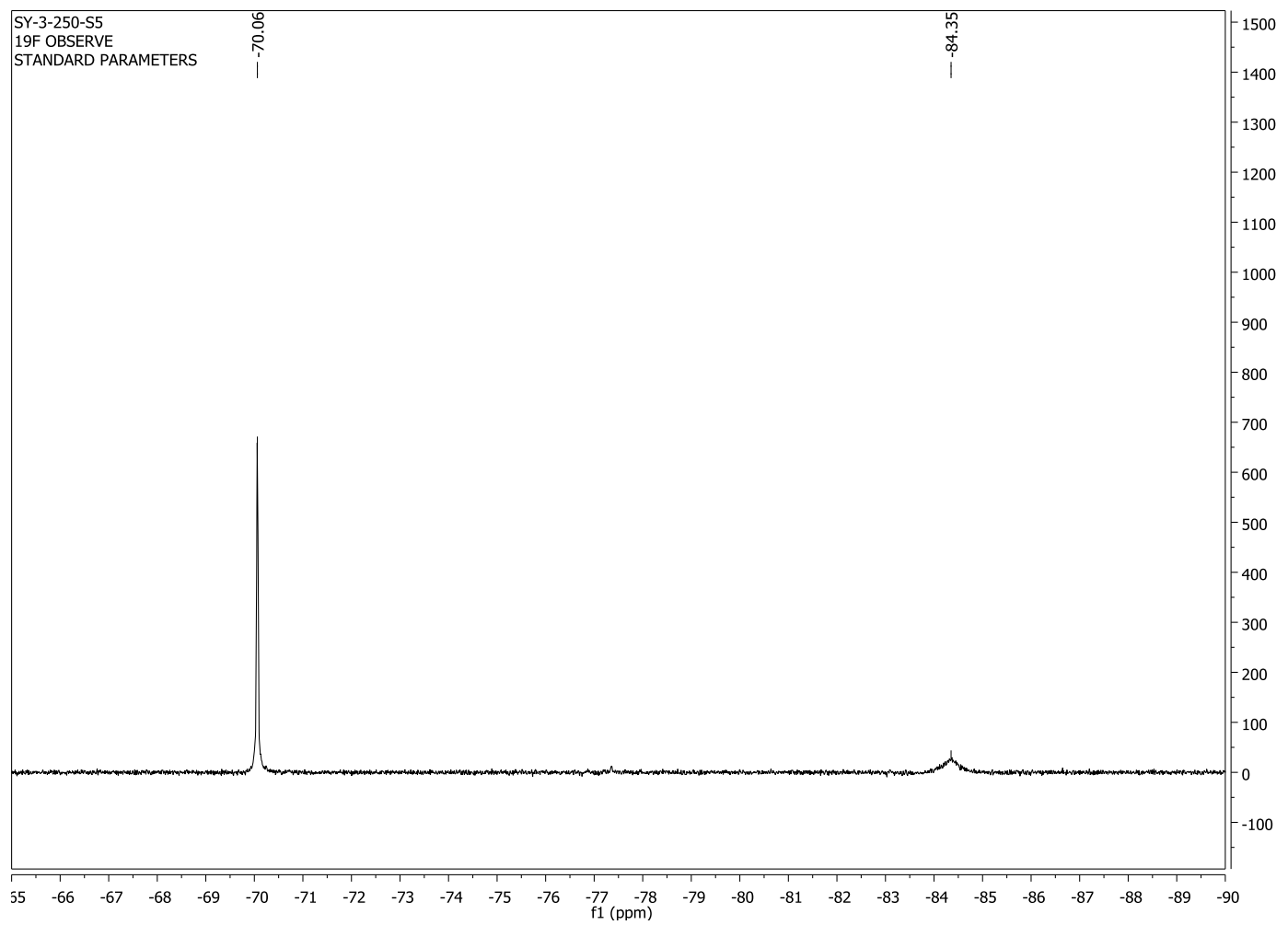




\section{4 eq of amine}

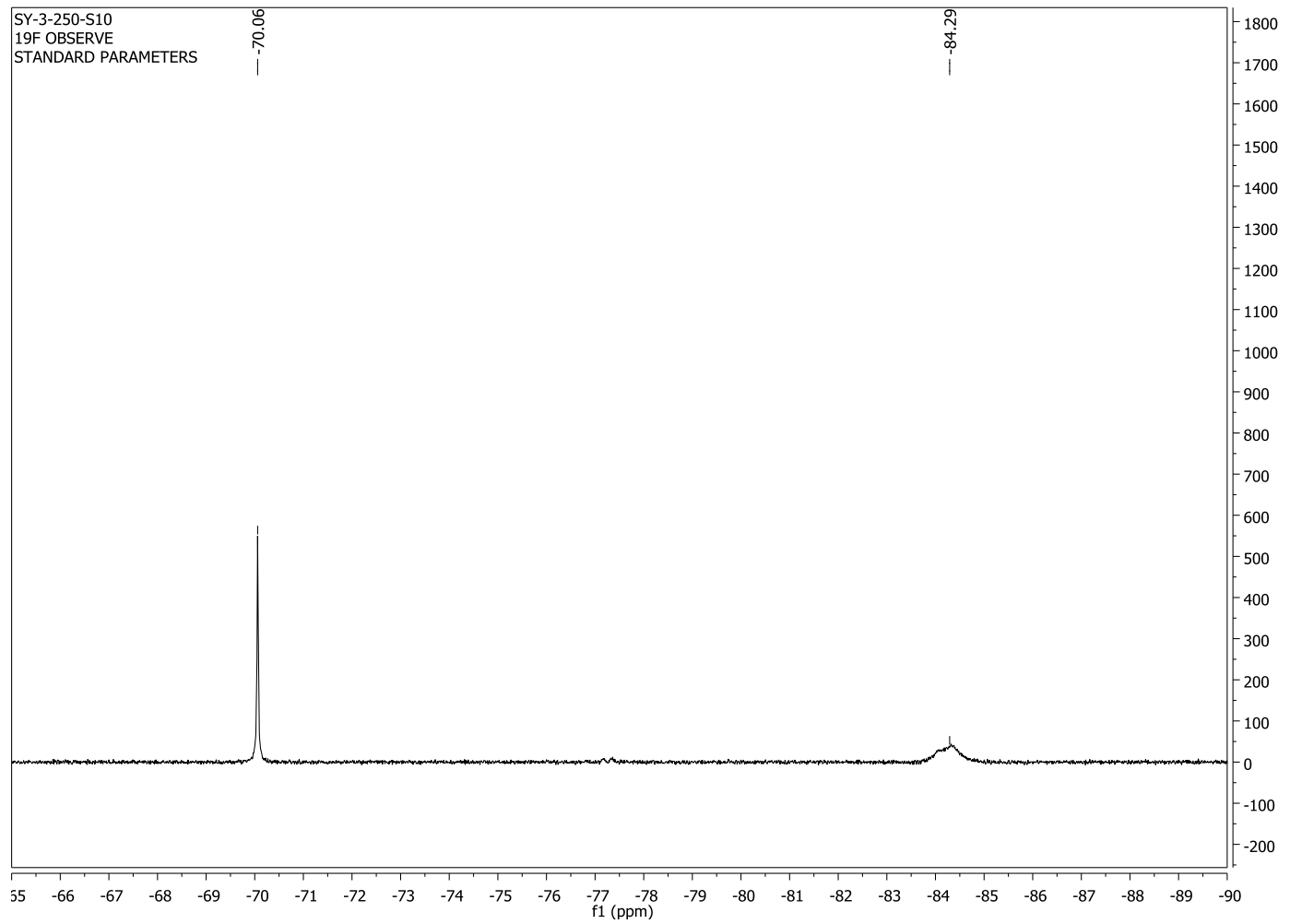

\section{$4.8 \mathrm{eq}$ of amine}

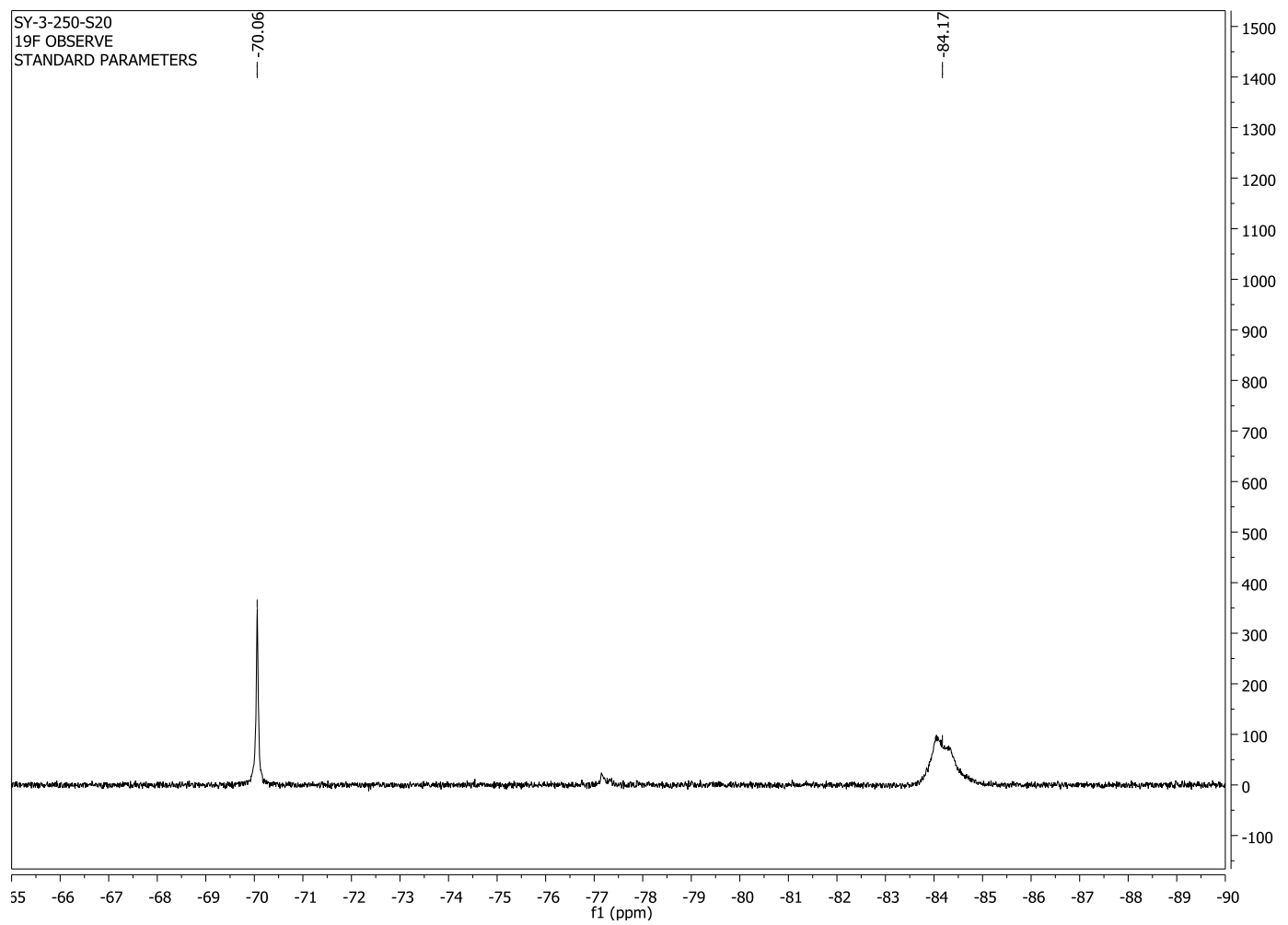




\section{1 eq of amine}

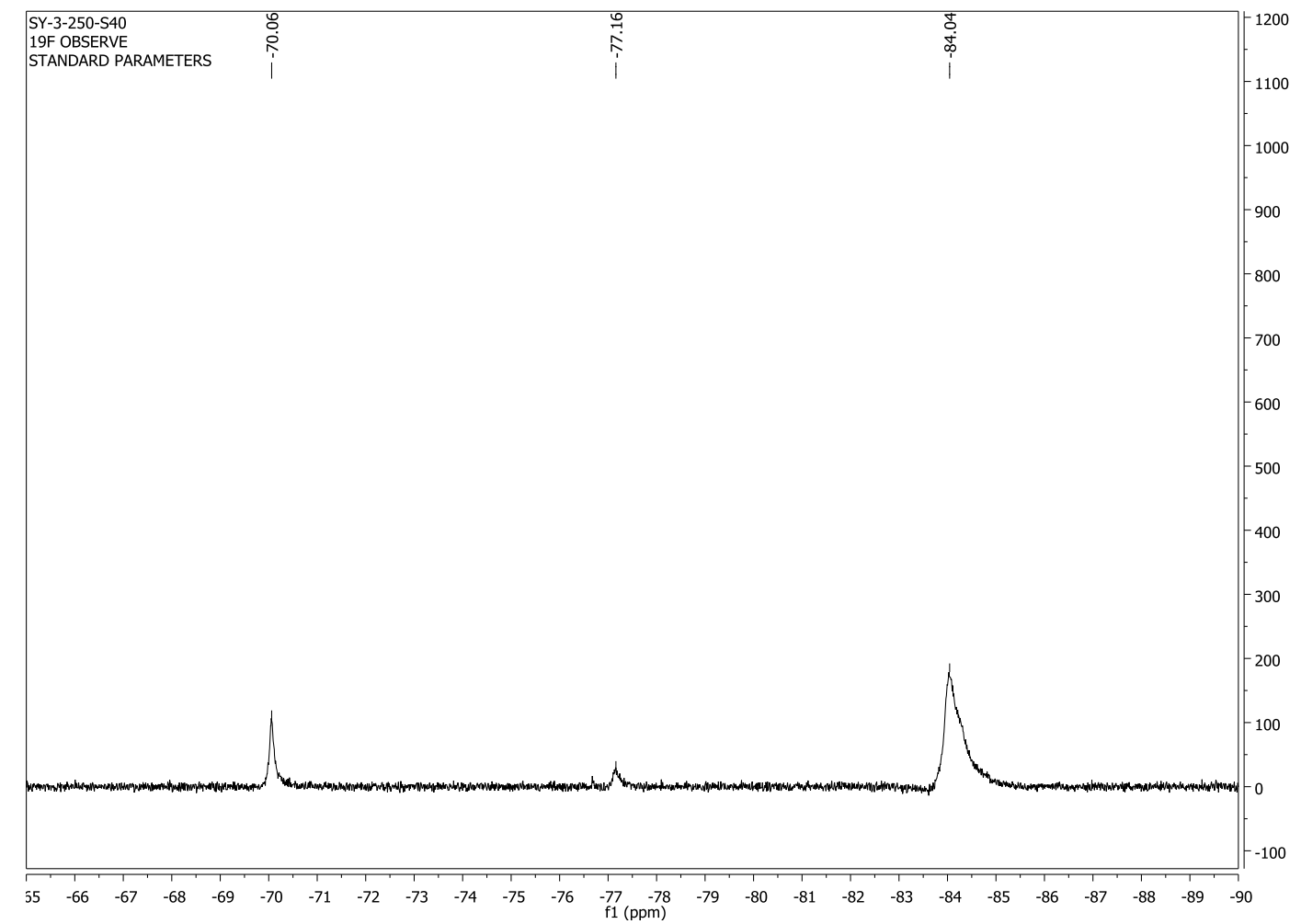

\section{9 eq of amine}

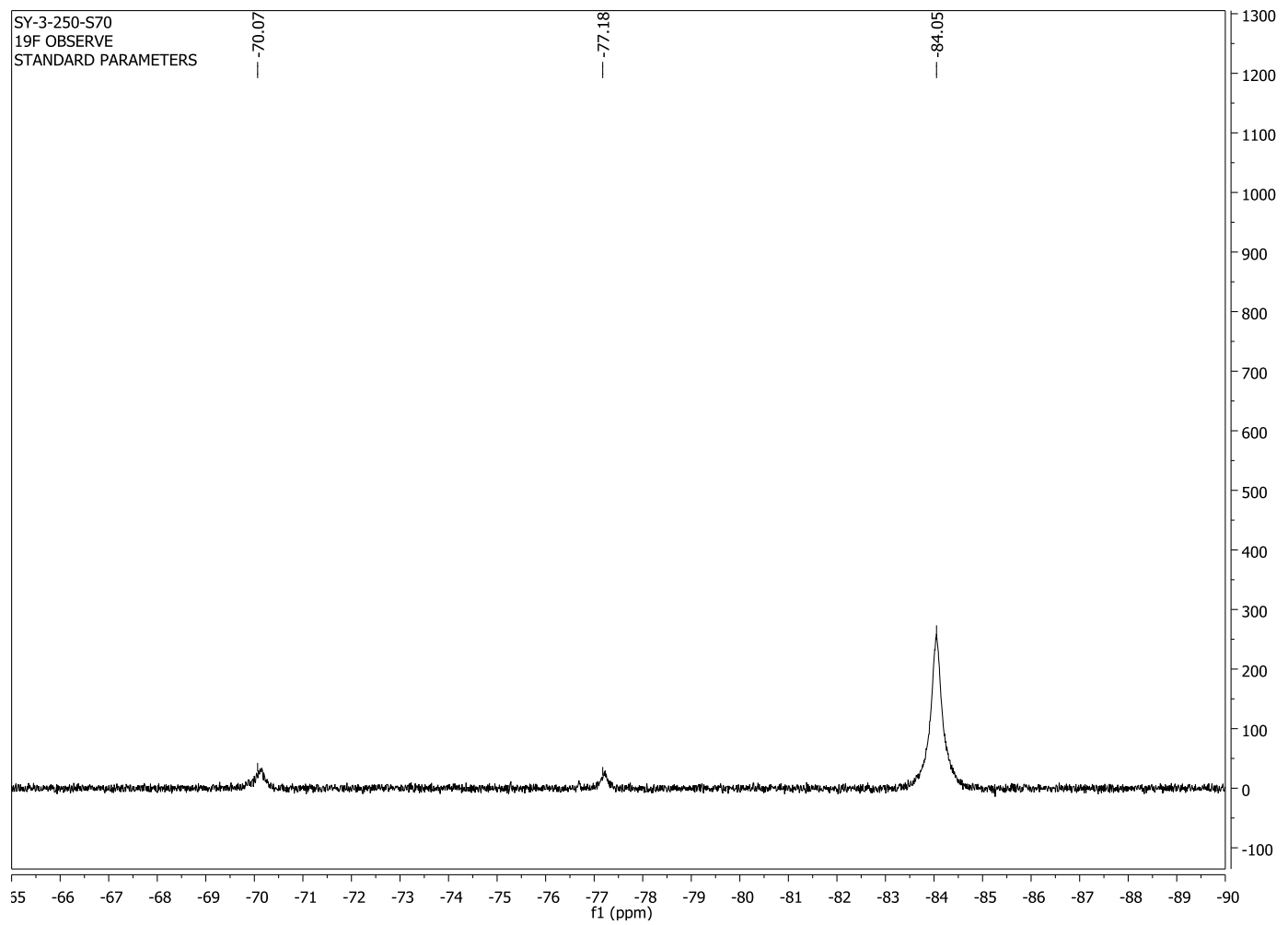




\section{0 eq of amine}

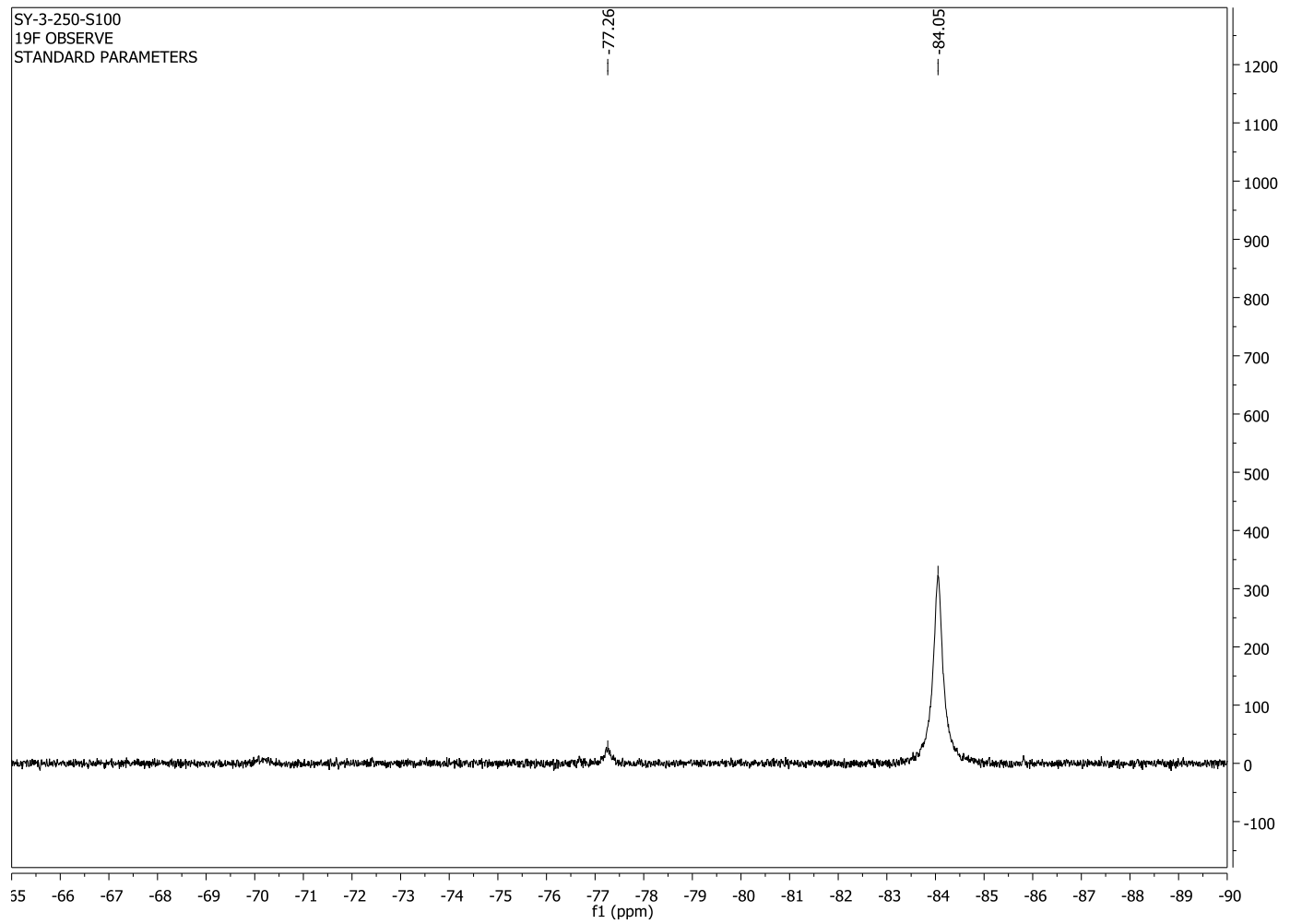

\section{3 eq of amine}

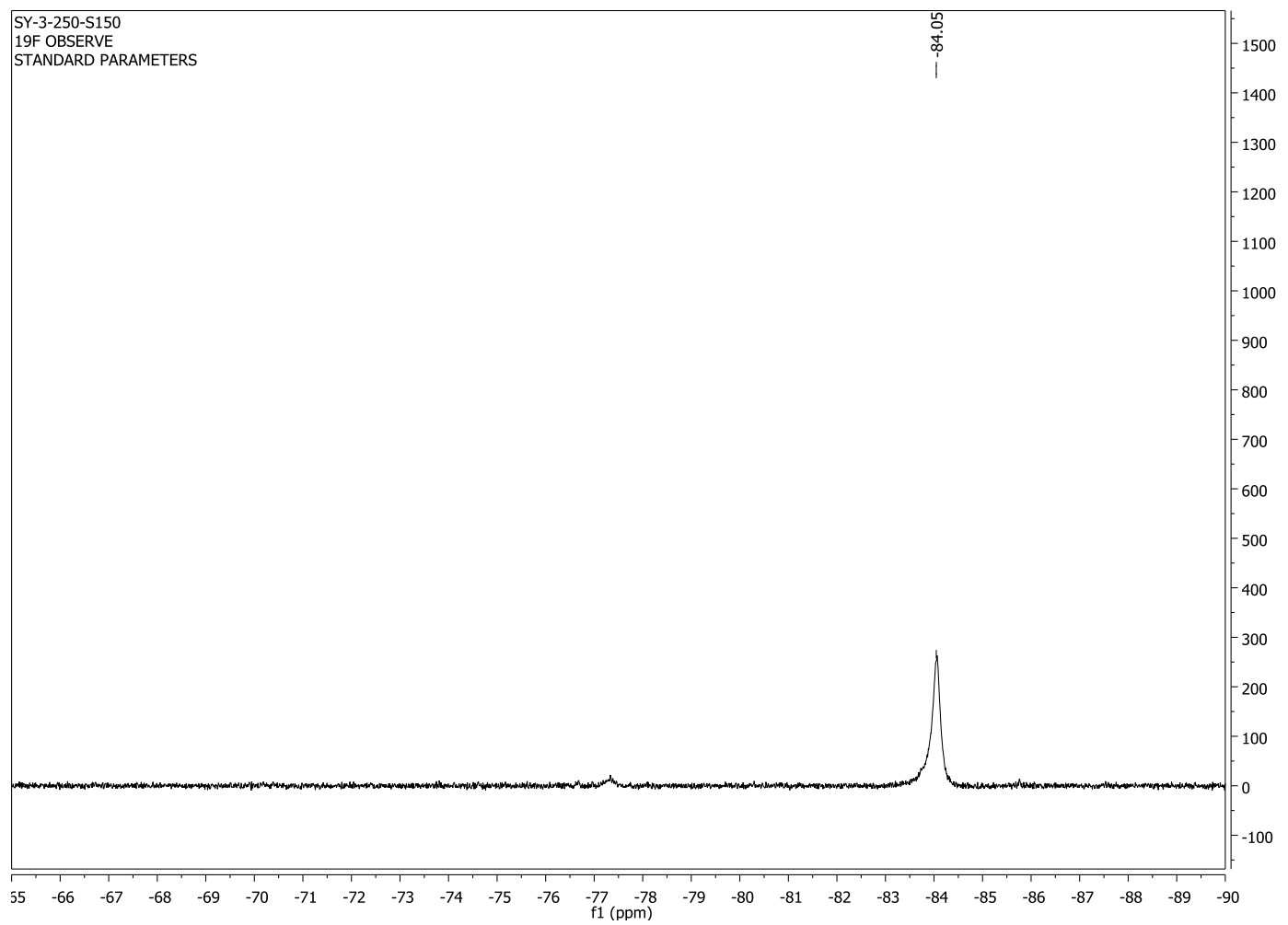




\section{5 eq of amine}

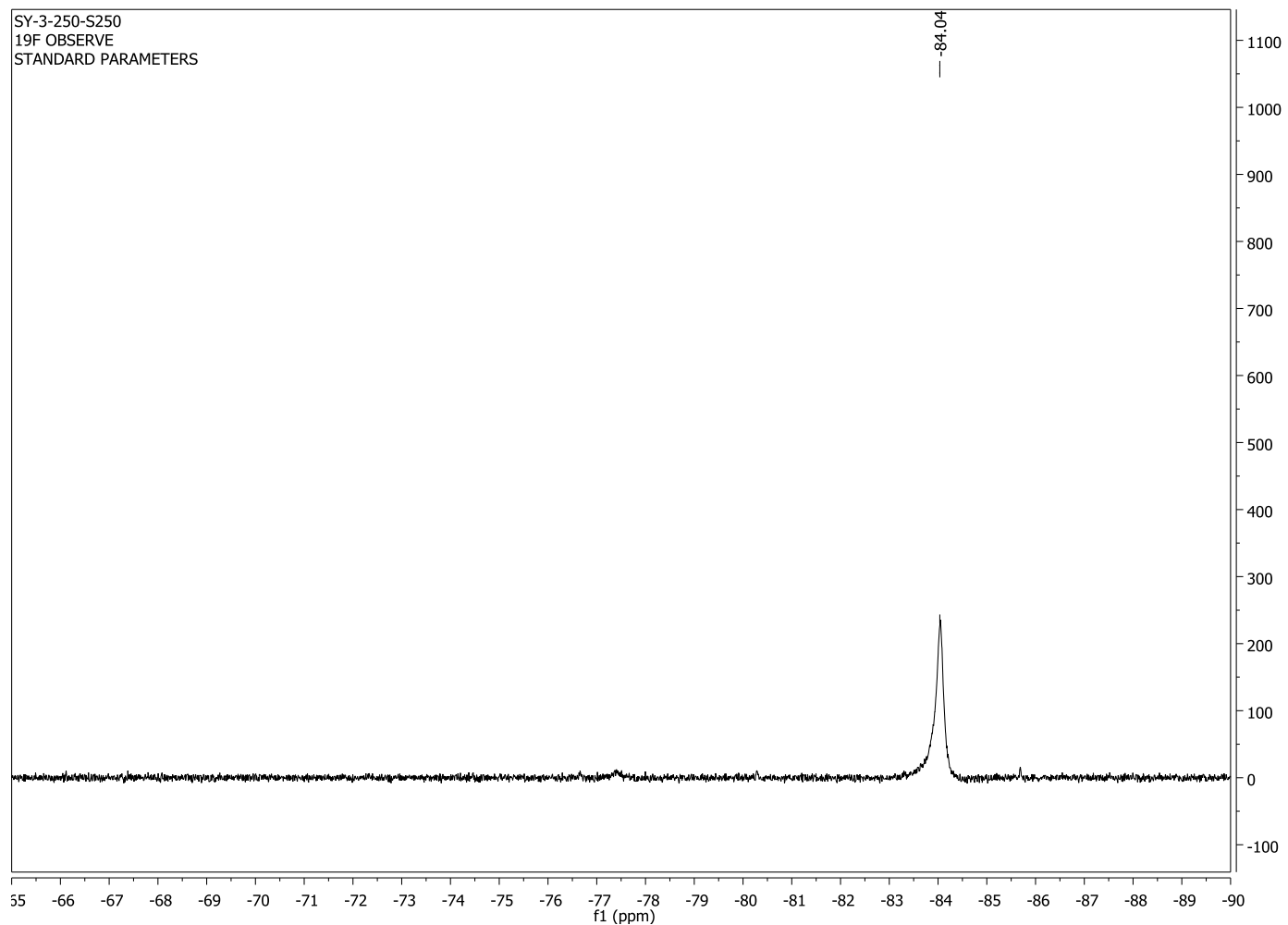

\section{0 eq of amine}

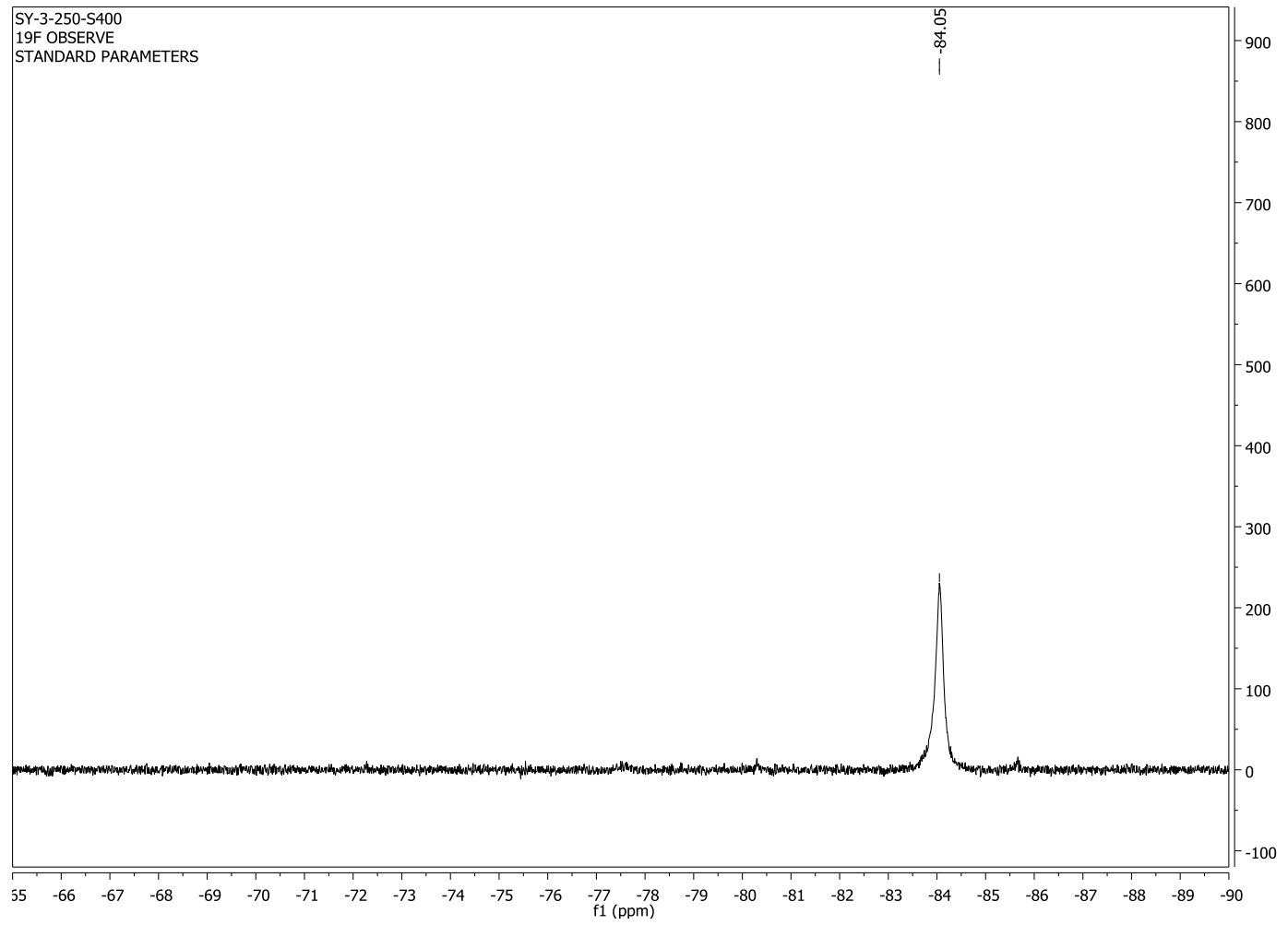


60 eq of amine

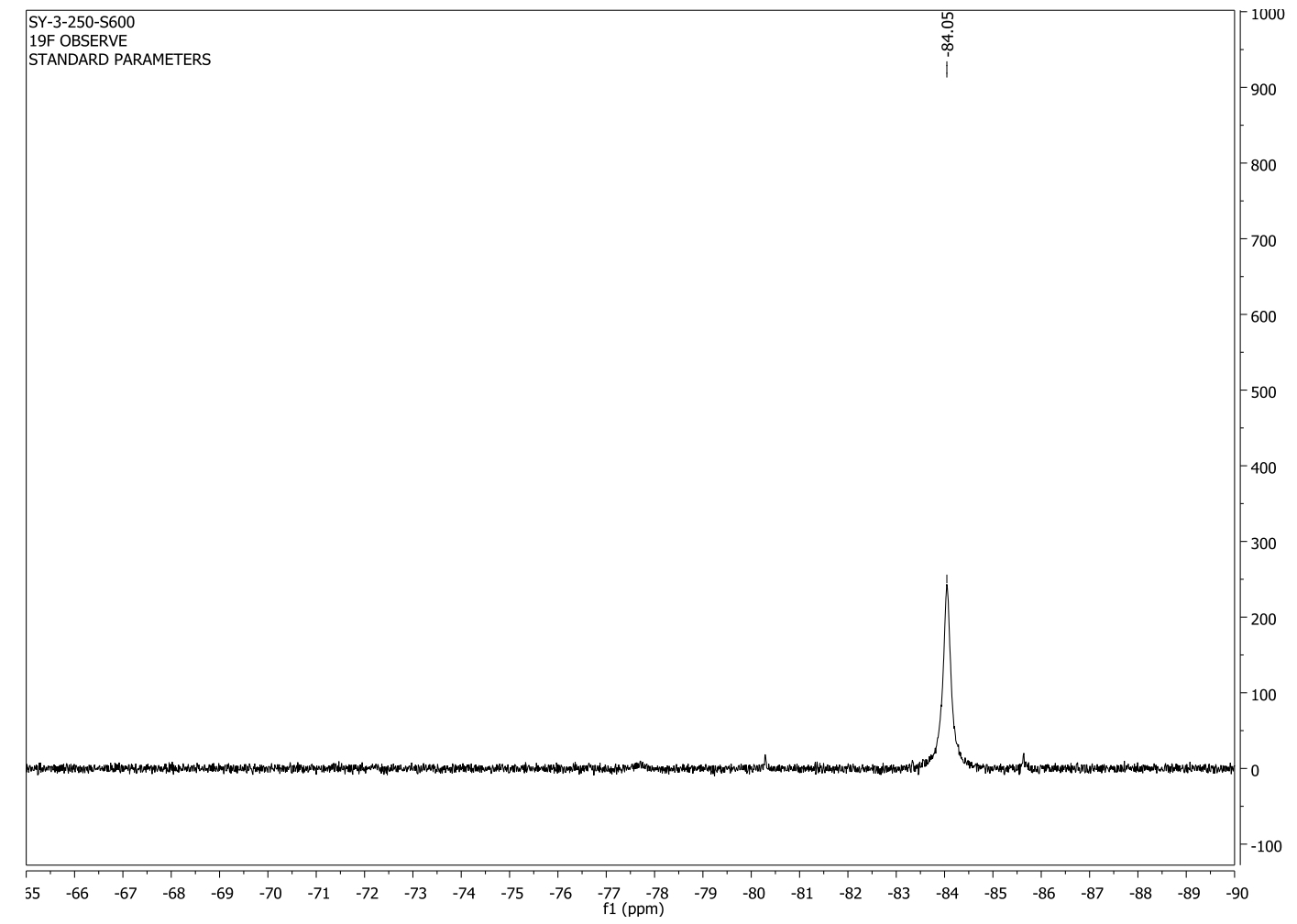

65.5 eq of amine

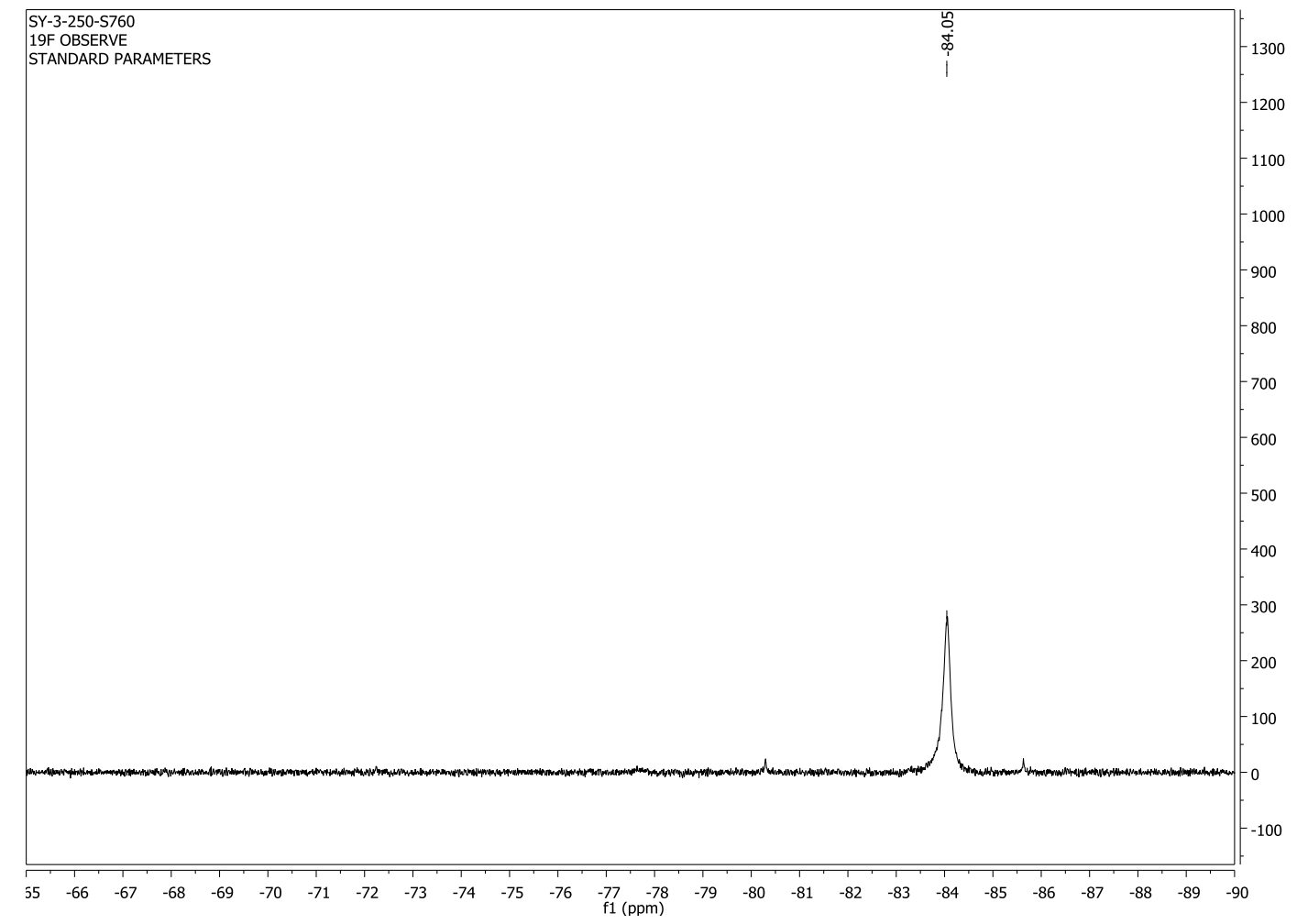


overnight

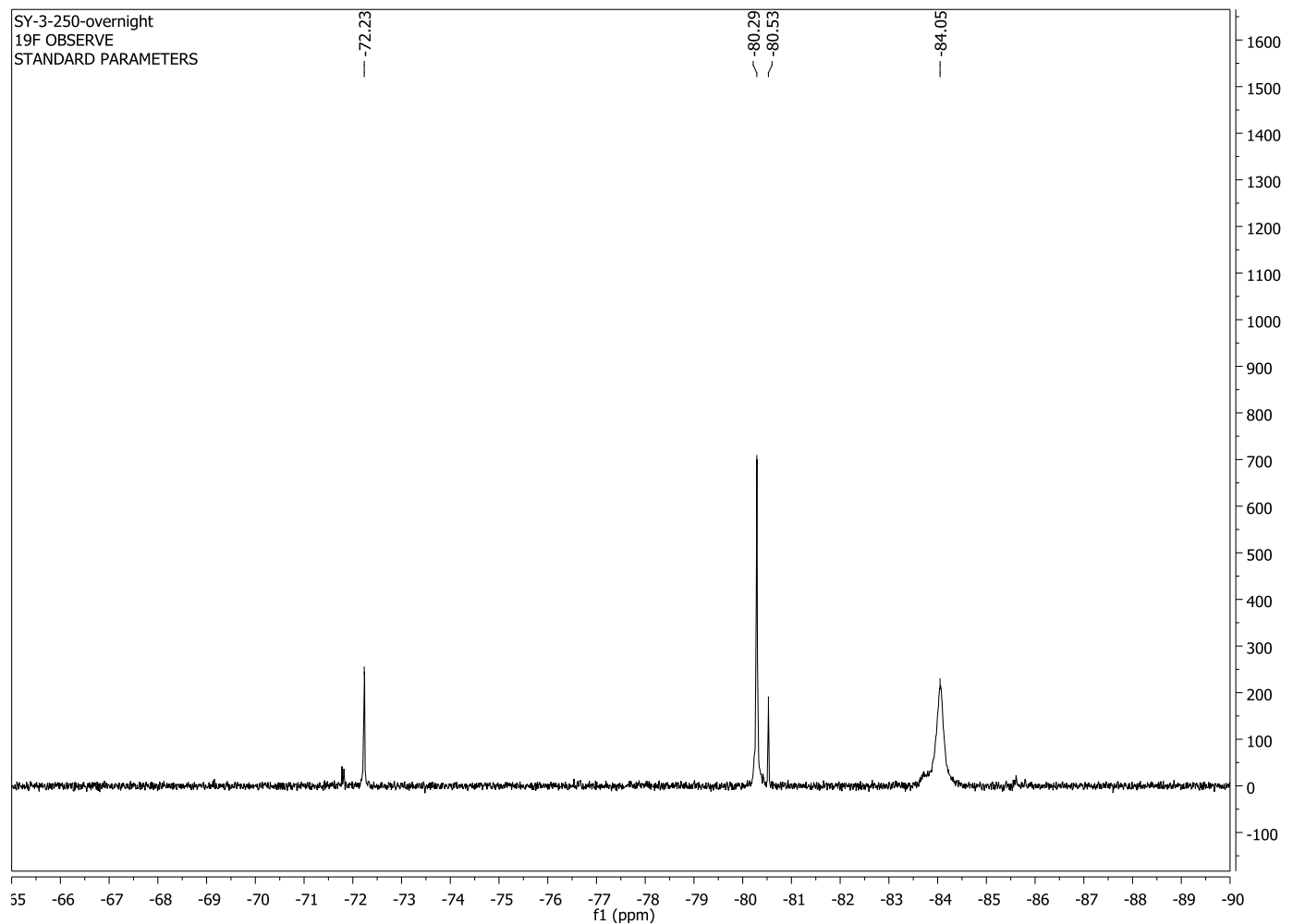

After 3 days

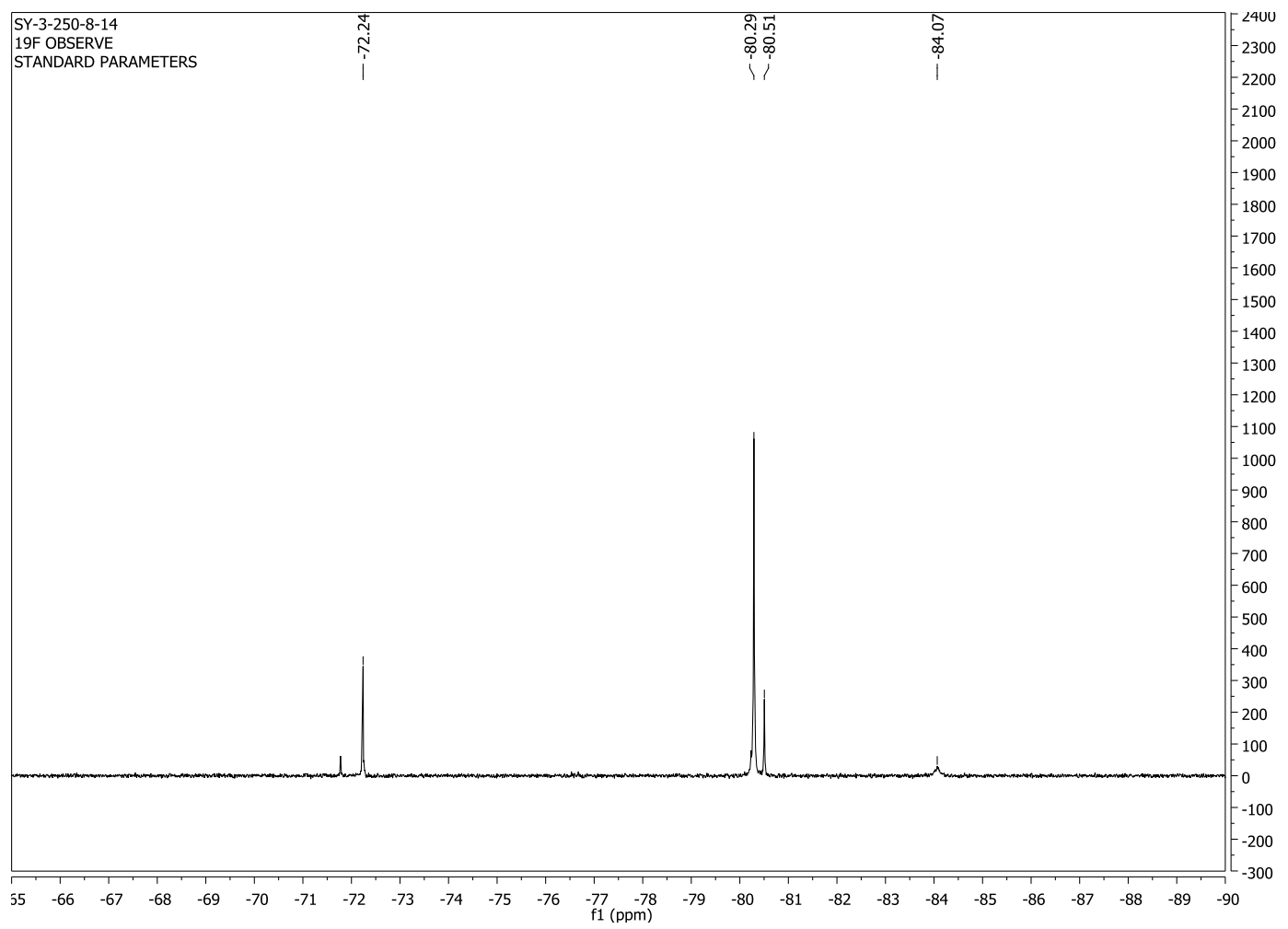


After 5 days

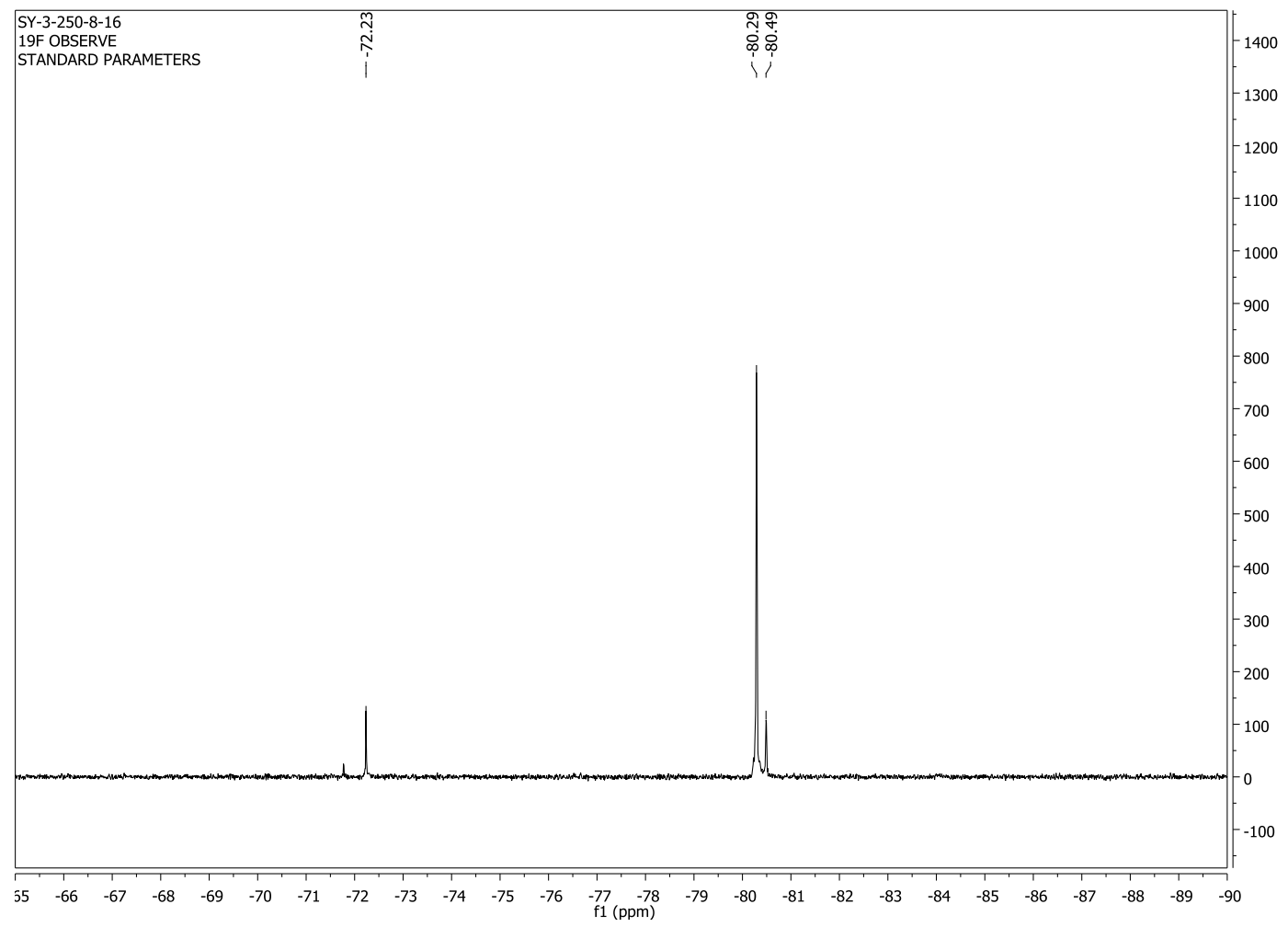

After 6 days

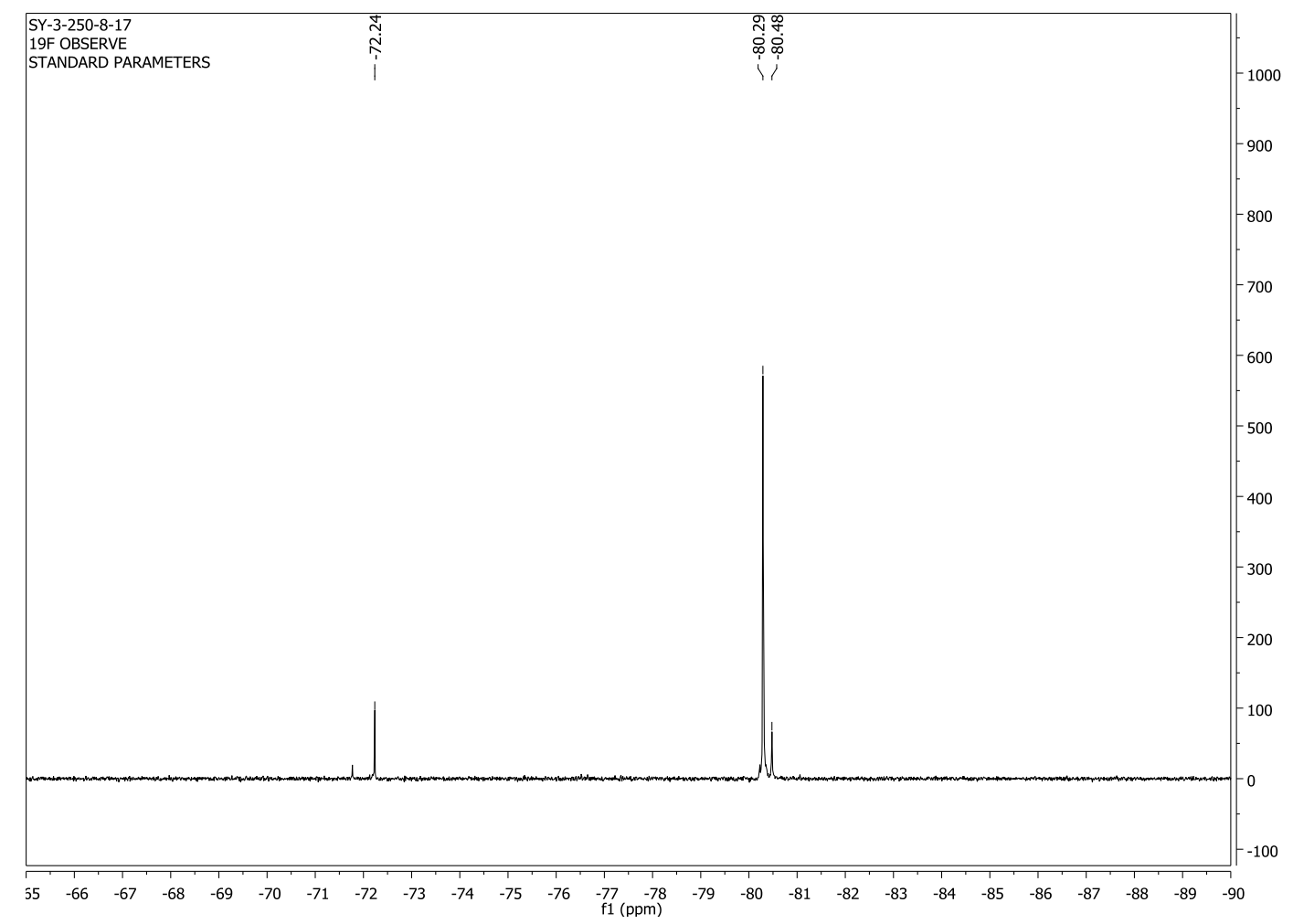




\section{Appendix to Chapter 6}

Figure A6-1. Fluorescence spectra of $(S)-6-4\left(1.0 \times 10^{-5} \mathrm{M}, \mathrm{CH}_{2} \mathrm{Cl}_{2}\right)$ with increasing concentrations of (a) N9 (0-1.6 mM), (b) N12 (0-1.7 mM), (c) (S, S)-N15 (0-5.0 mM), (d) $(R, R)$-N15 (0-5.0 mM), (e) $(S, R)$-N15 (0-5.0 mM), (f) $(S)$-N16 (0-5.0 mM) and $(\mathrm{g})(R)$ N16 $(0-5.0 \mathrm{mM}) .\left(\lambda_{\mathrm{exc}}=343 \mathrm{~nm}\right.$, slit $\left.=2.0 / 2.0 \mathrm{~nm}\right)$.
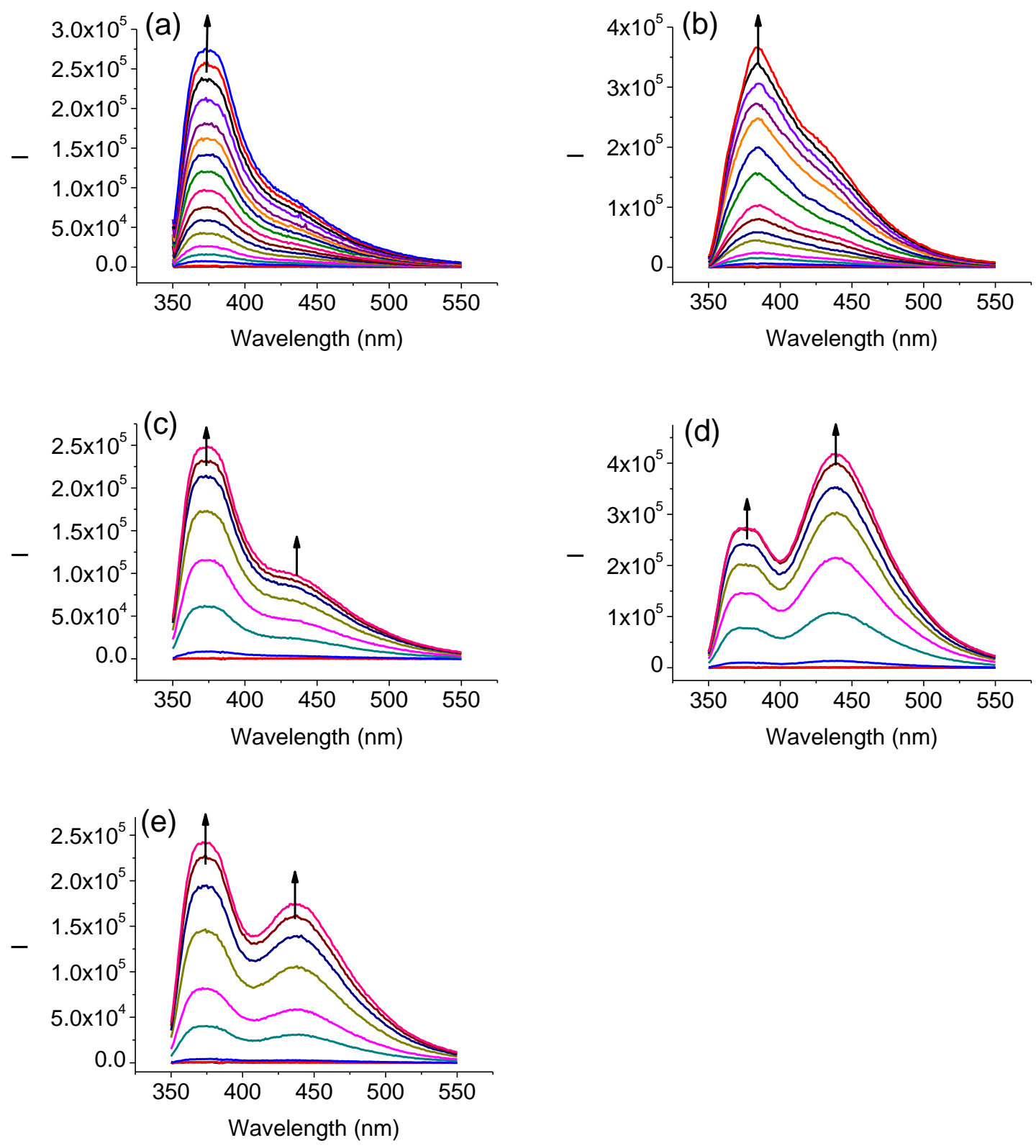

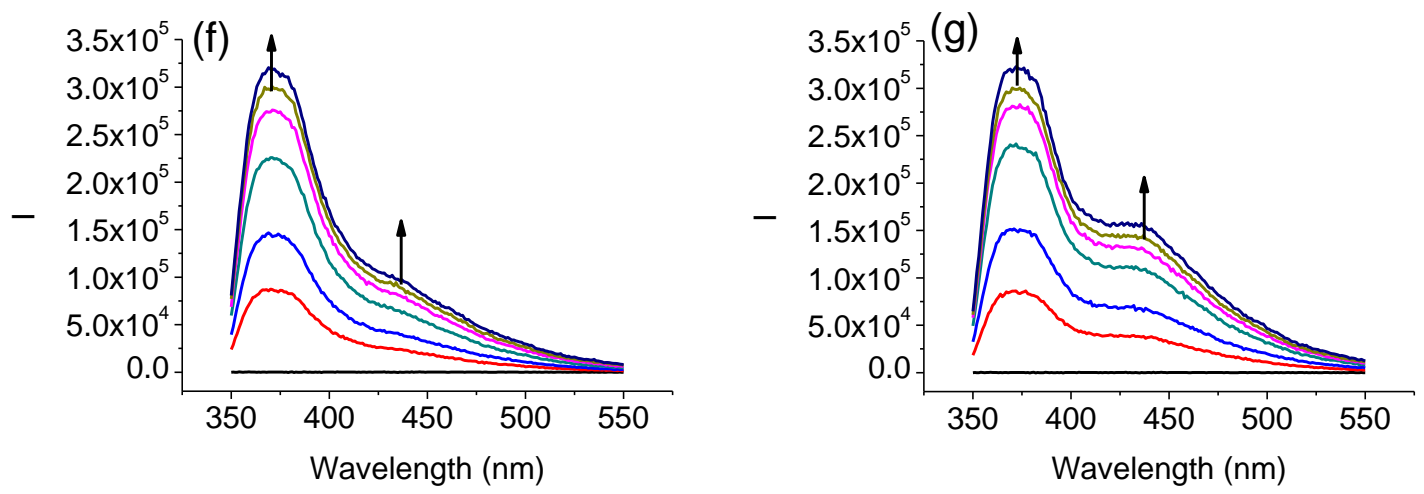

Figure A6-2. UV-Vis absorption spectra of $(S)-6-4\left(1.0 \times 10^{-5} \mathrm{M}, \mathrm{CH}_{2} \mathrm{Cl}_{2}\right)$ with increasing concentrations of (a) N9 (0-8.2 mM), (b) N10 (0-10.3 mM), (c) N12 (0-10.9 mM), (d) $(S$, $S)-\mathbf{N 1 5}(0-5.0 \mathrm{mM}),(\mathrm{e})(R, R)-\mathbf{N 1 5}(0-5.0 \mathrm{mM}),(\mathrm{f})(S, R)-\mathbf{N 1 5}(0-5.0 \mathrm{mM})$.
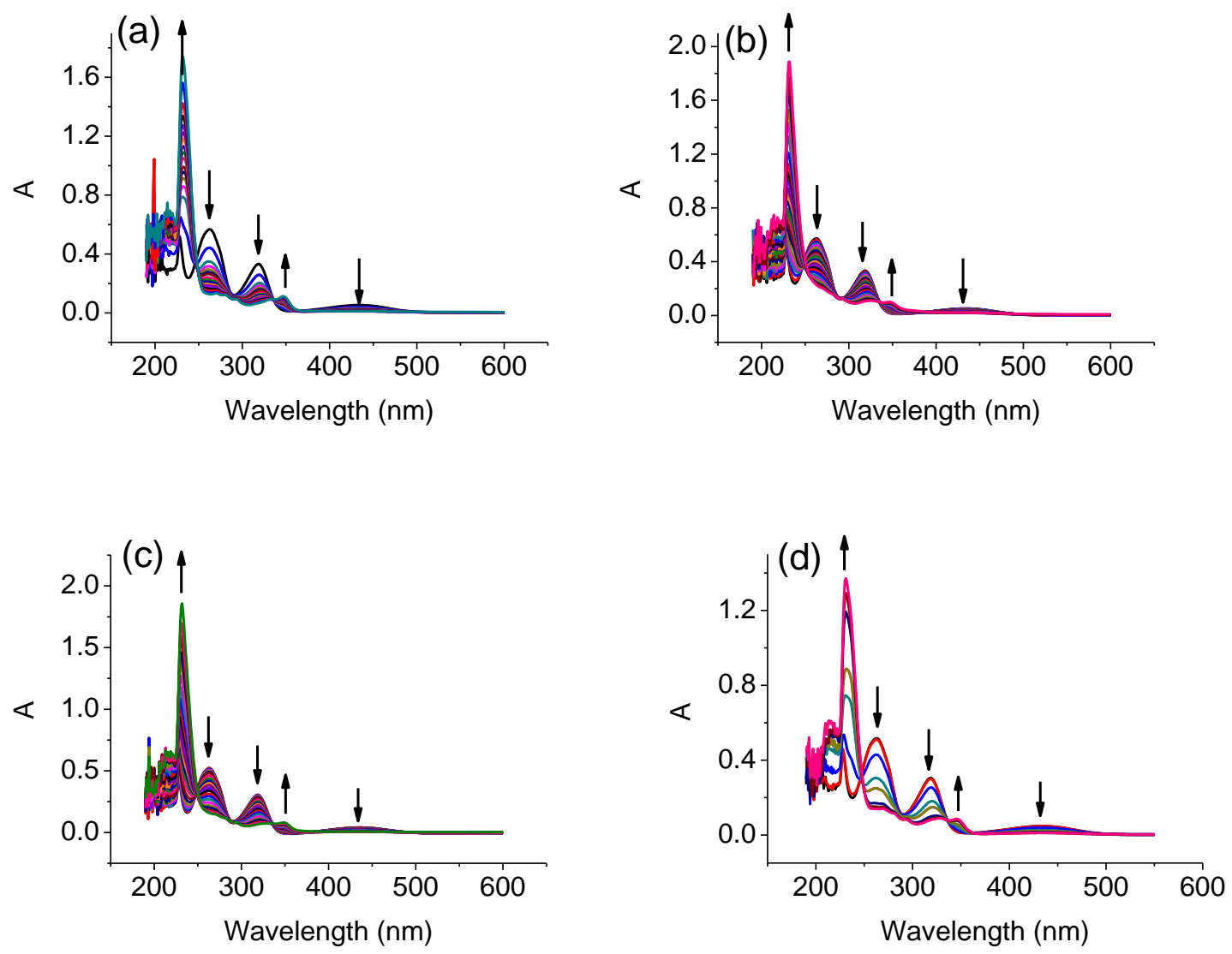

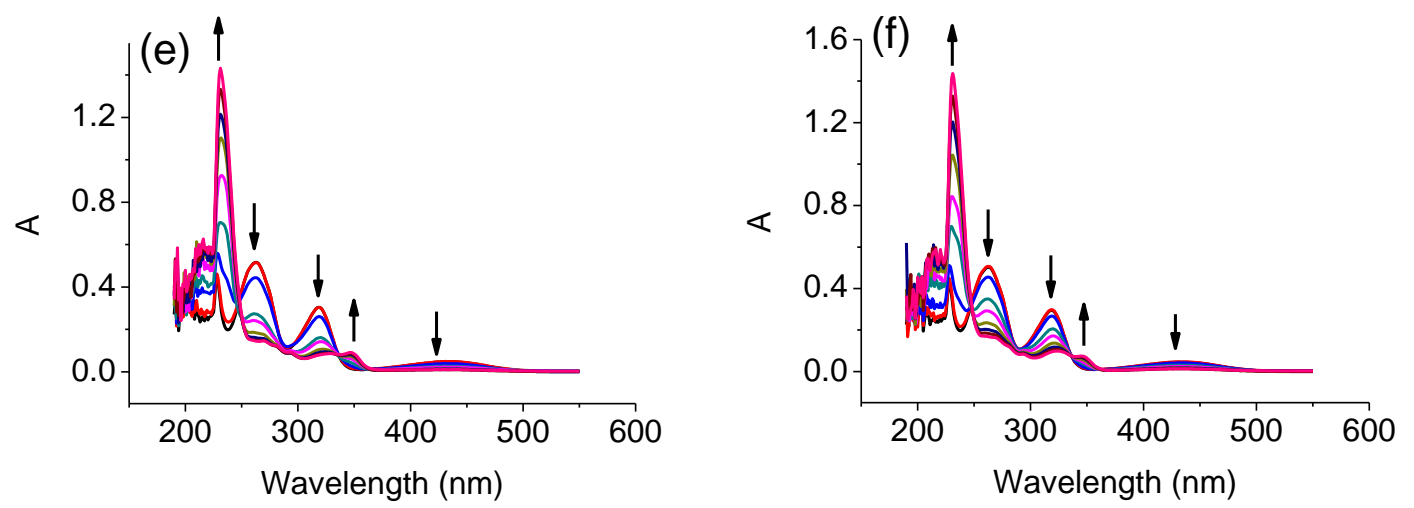

Figure A6-3. Fluorescence spectra of $(S)-6-4\left(1.0 \times 10^{-5} \mathrm{M}, \mathrm{CH}_{2} \mathrm{Cl}_{2}\right)$ with increasing concentrations of AA1, AA2 and AA3. $\left(\lambda_{\mathrm{exc}}=343 \mathrm{~nm}\right.$, slit $\left.=2.0 / 2.0 \mathrm{~nm}\right)$.
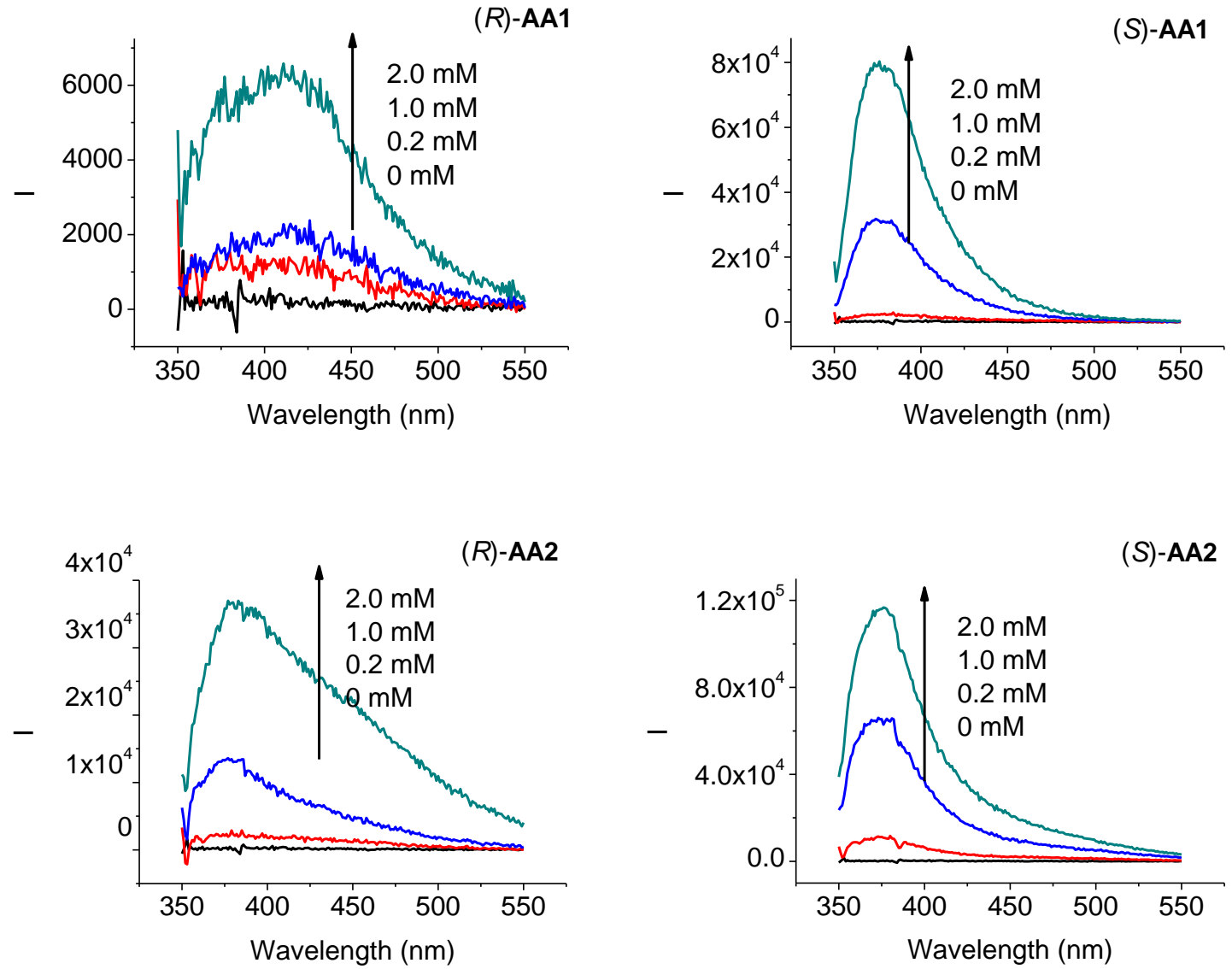

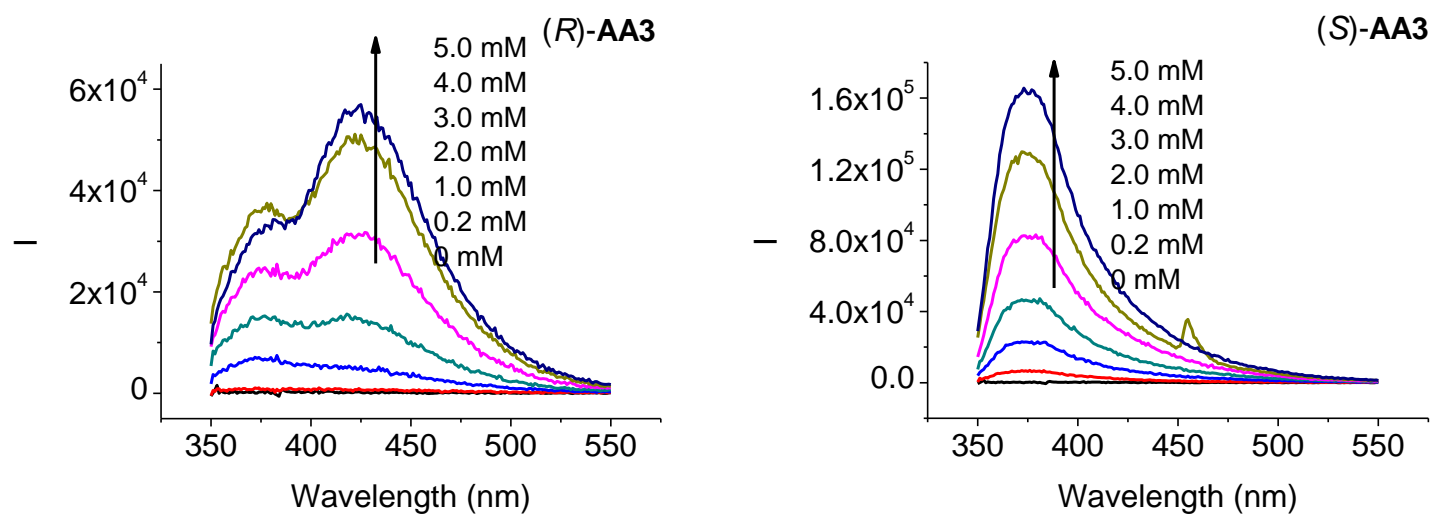

\section{NMR Spectra of Compounds}

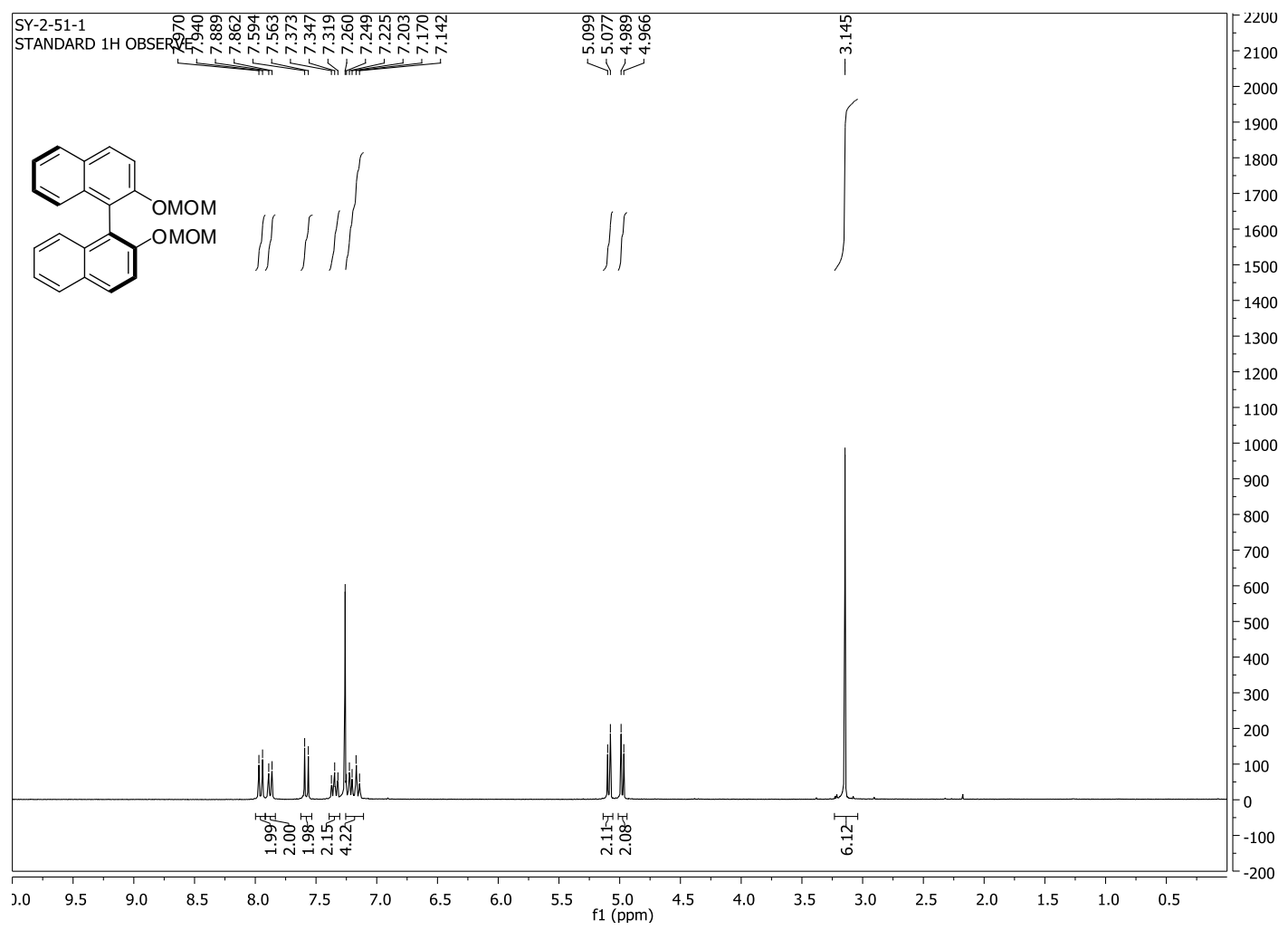



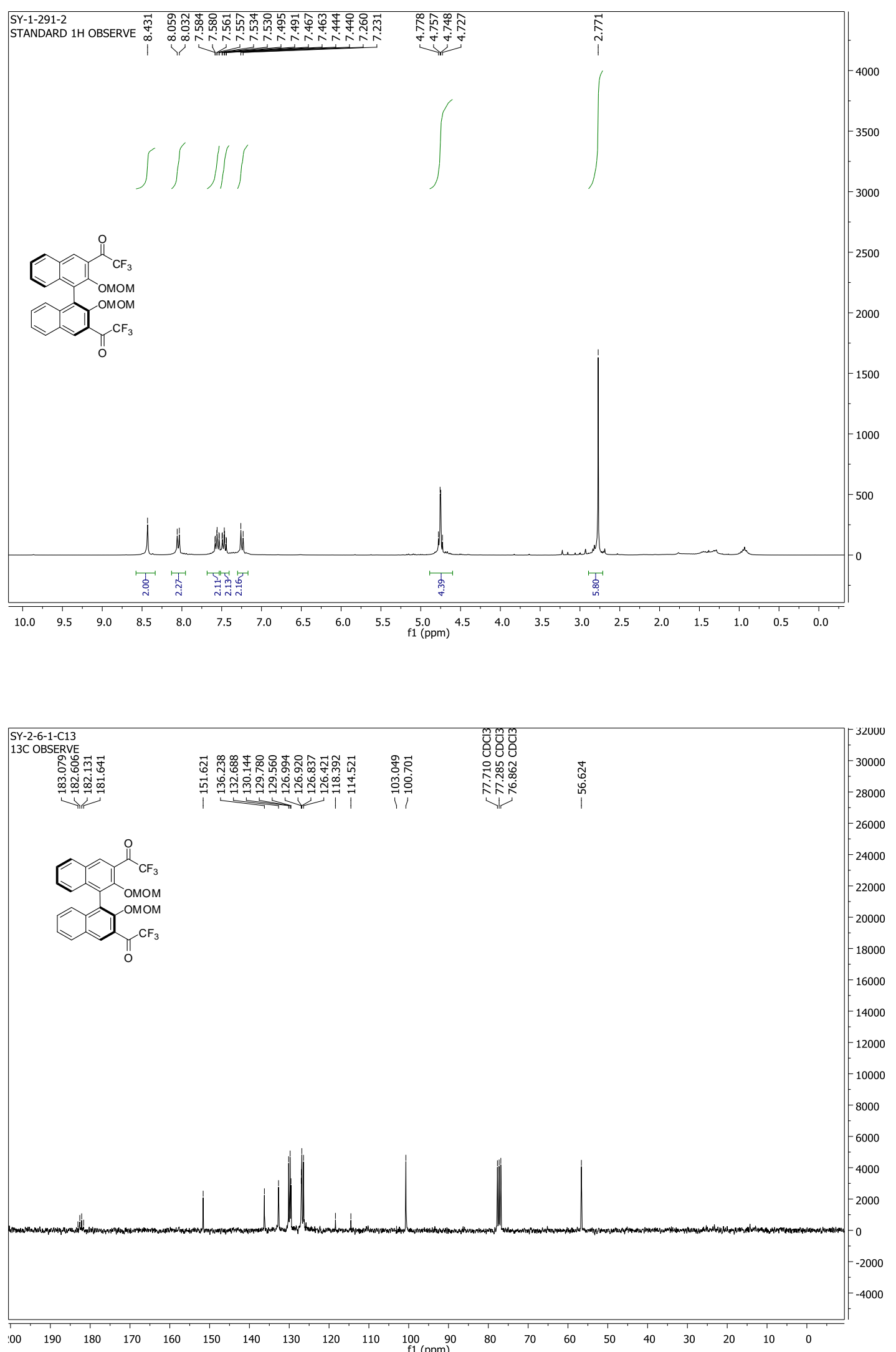

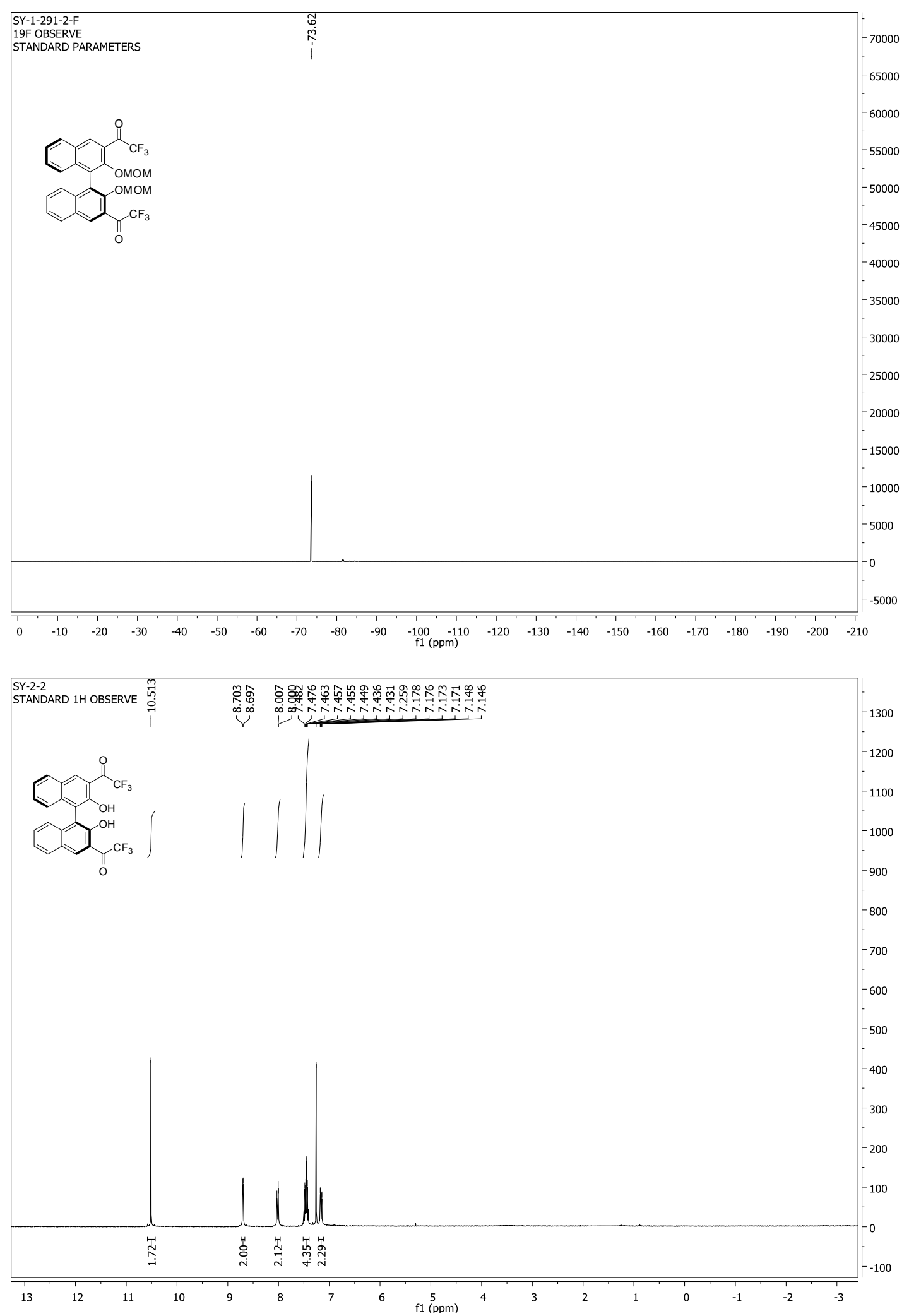


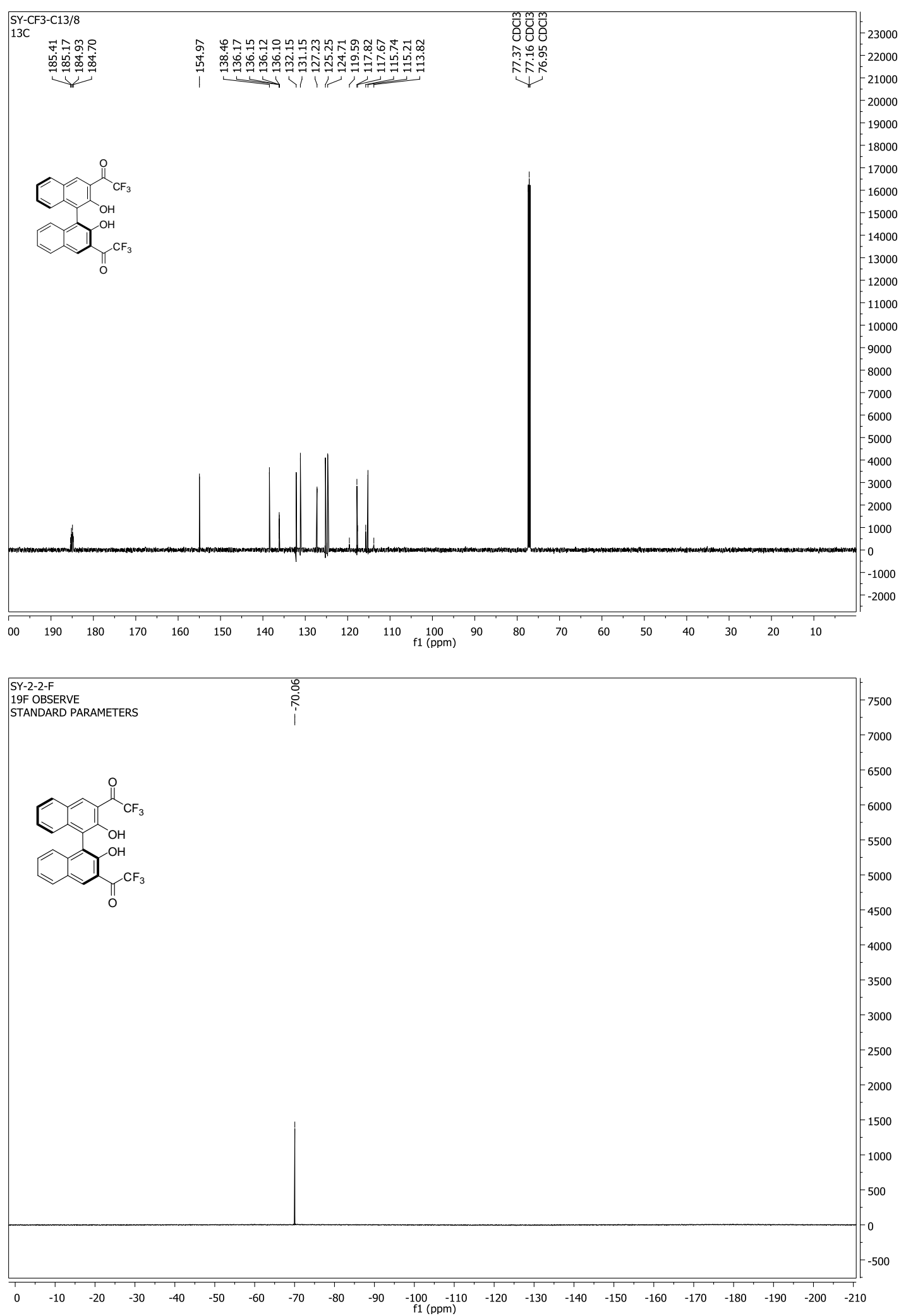



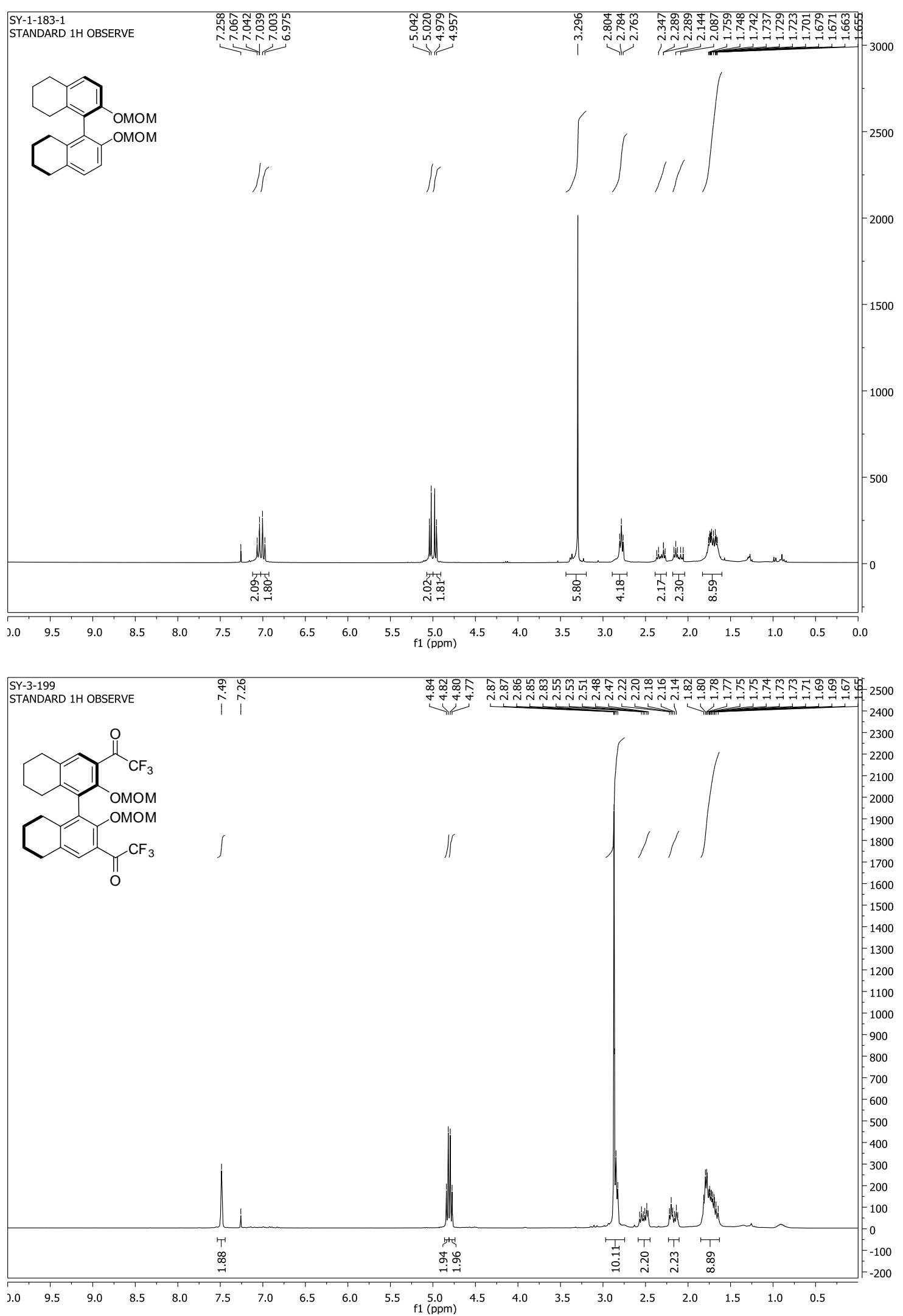

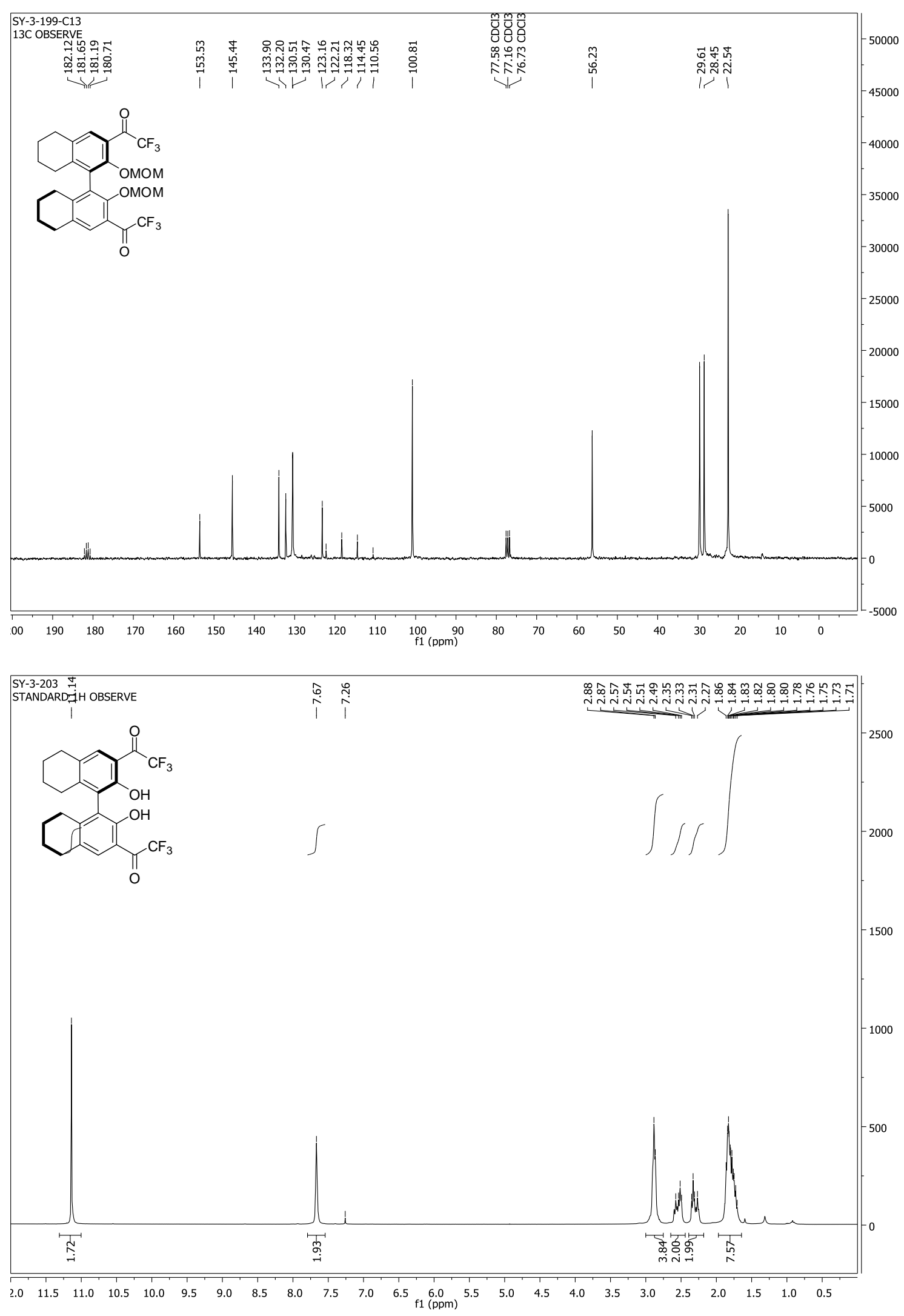

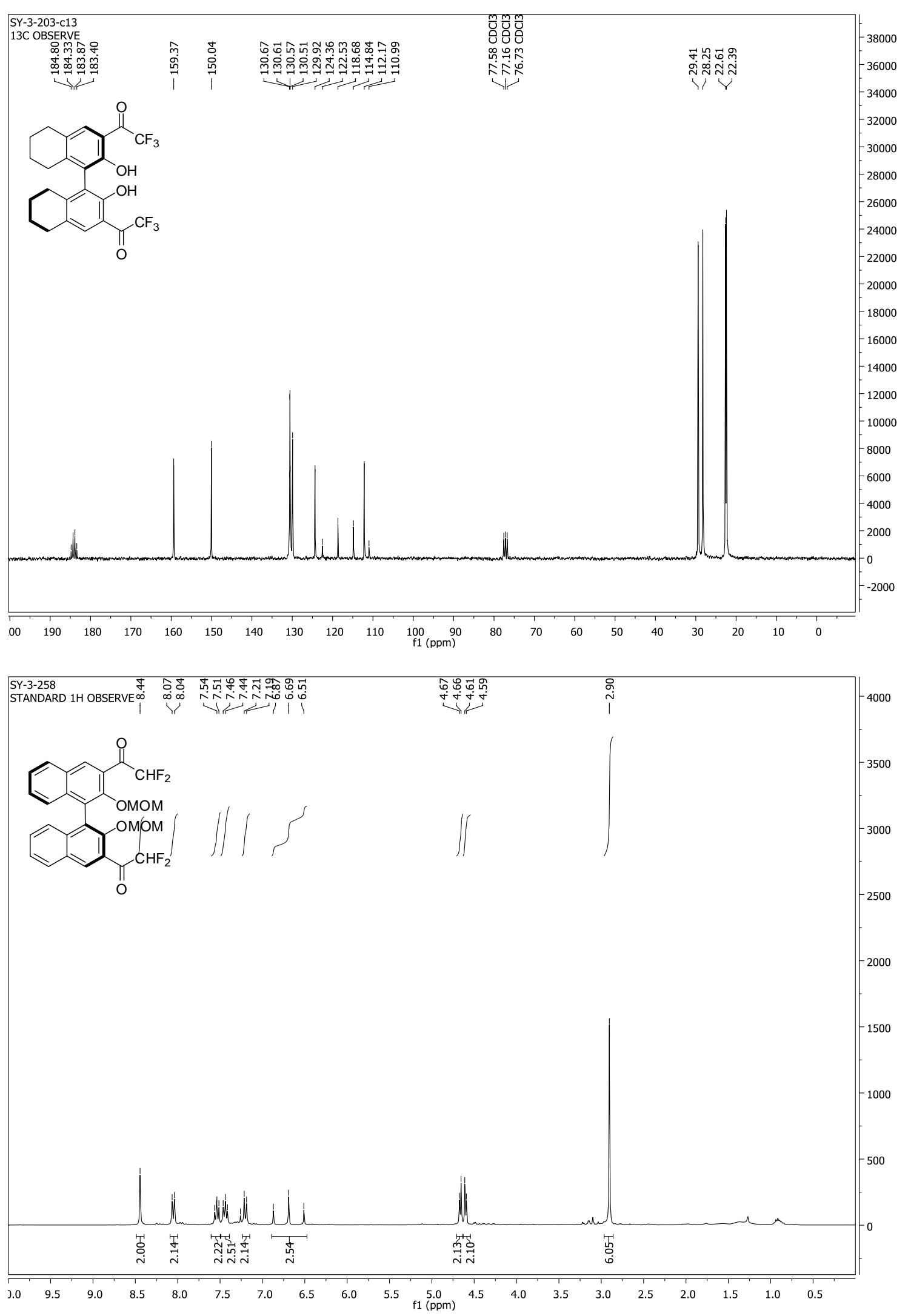


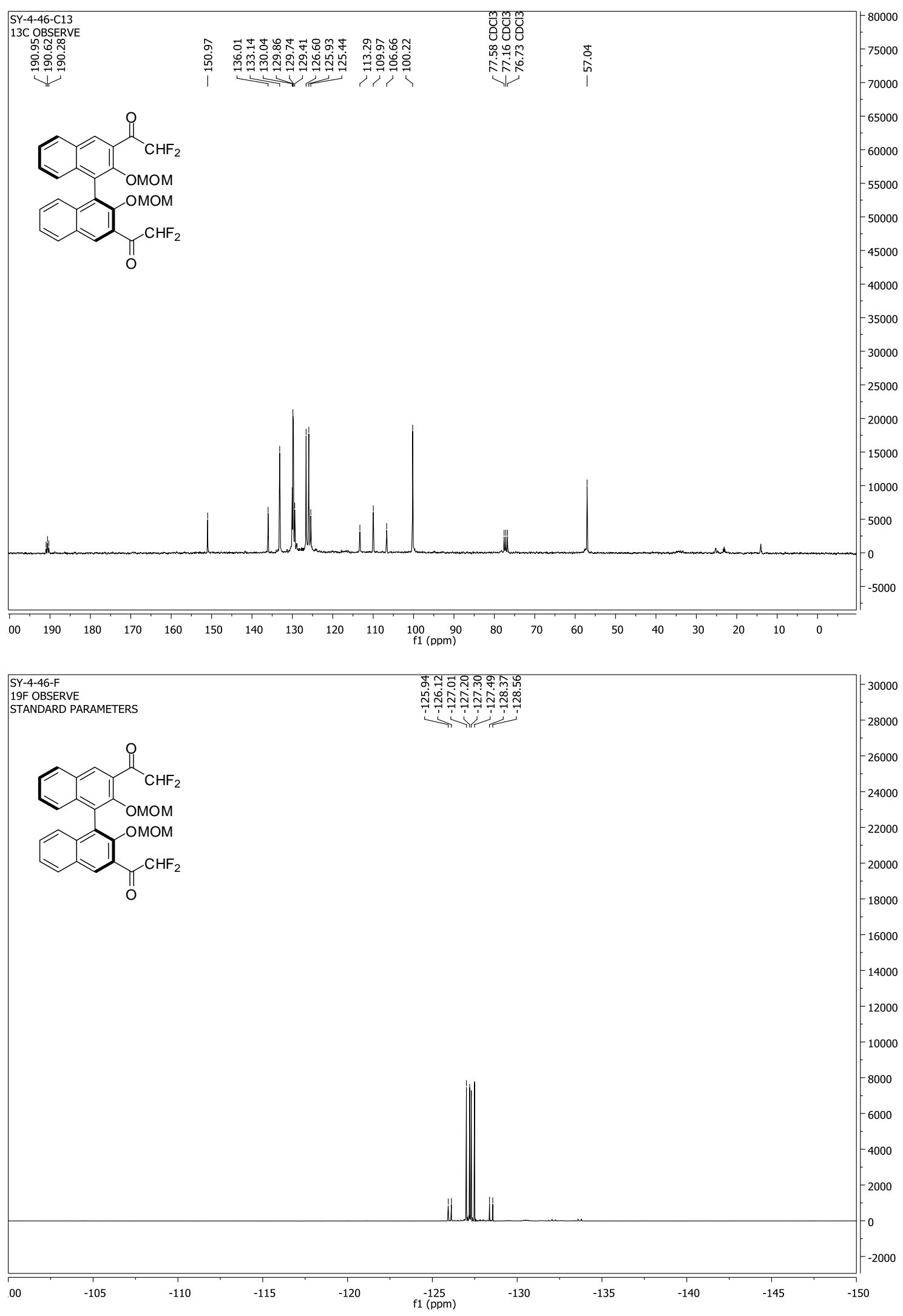



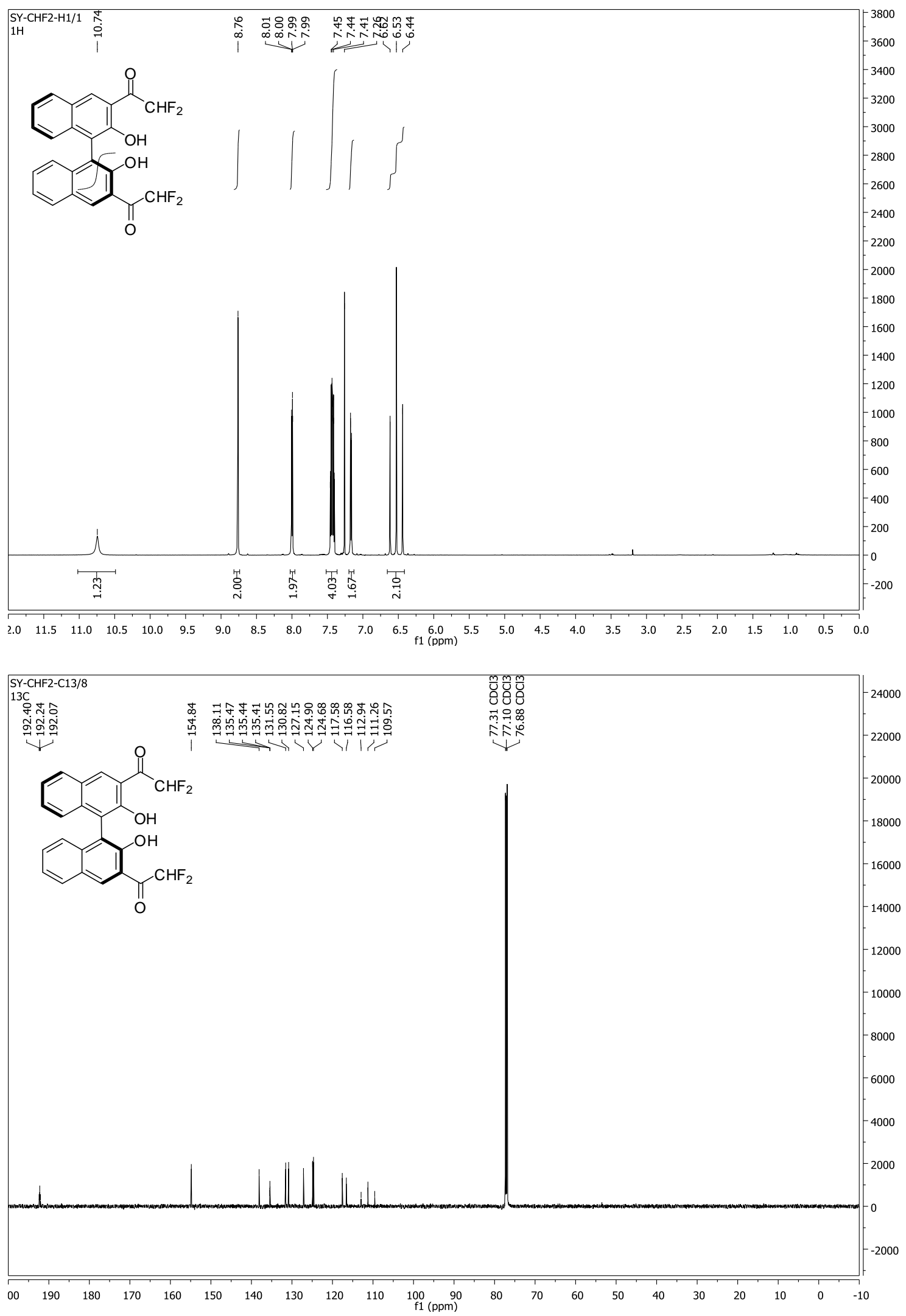

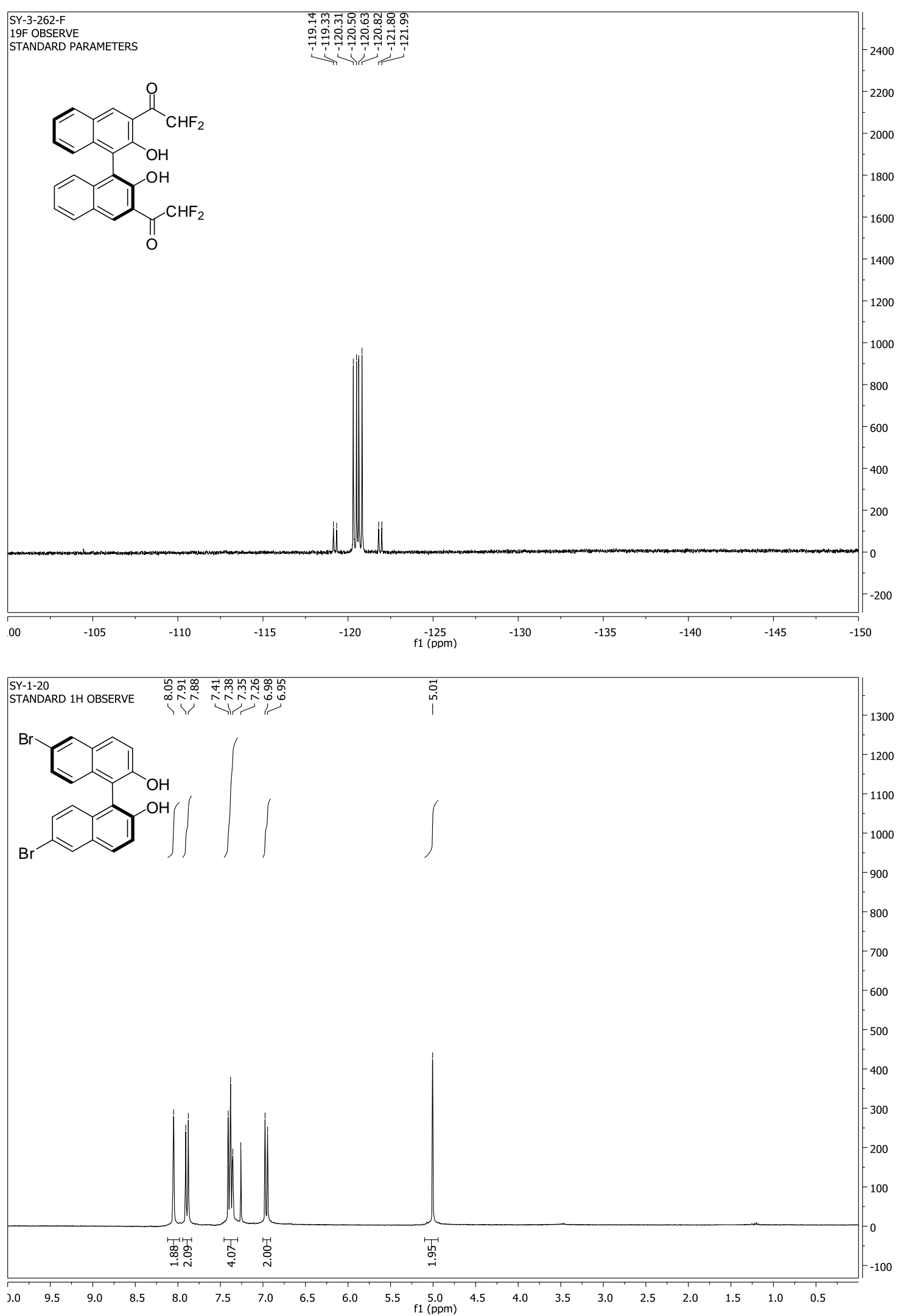

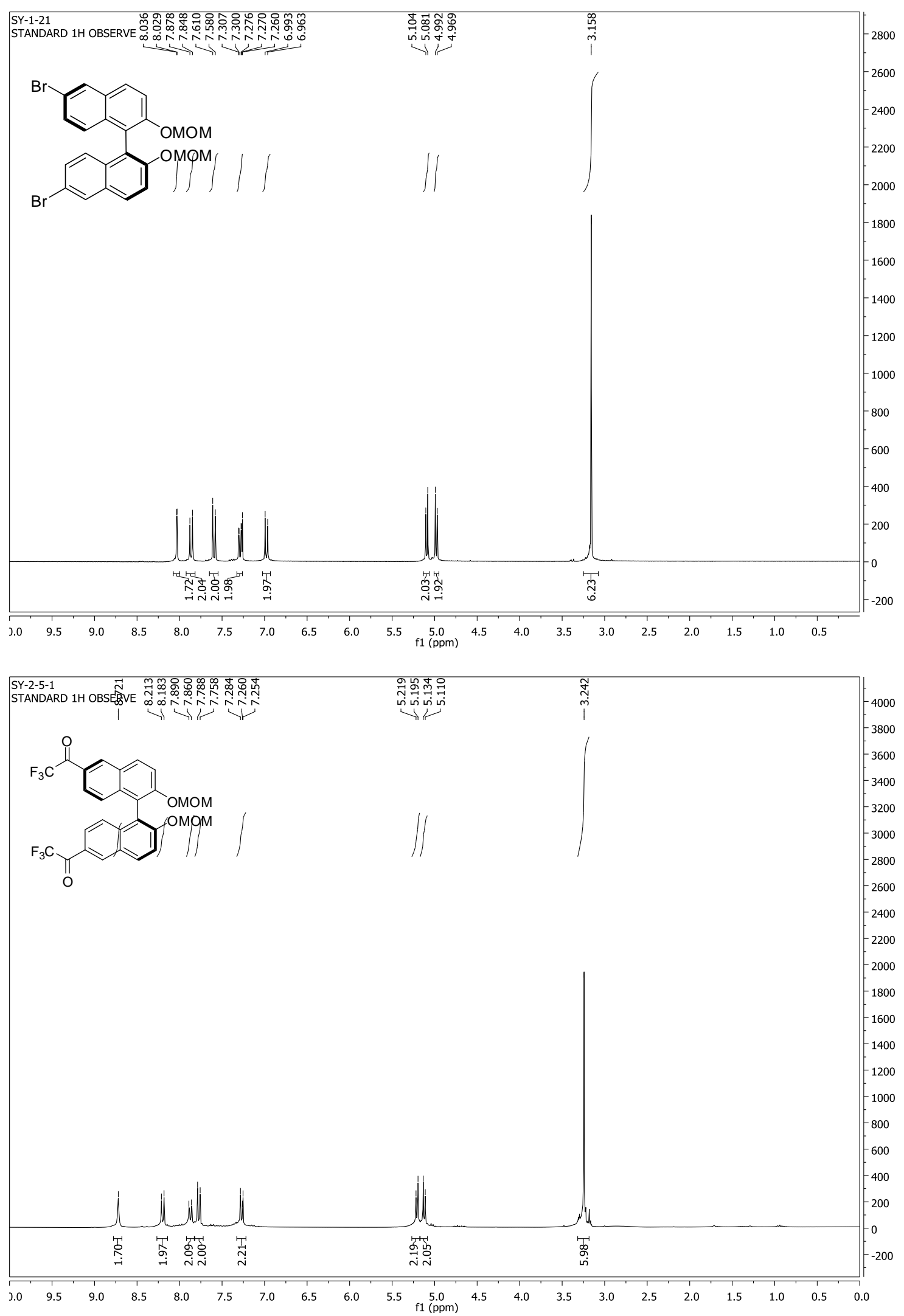


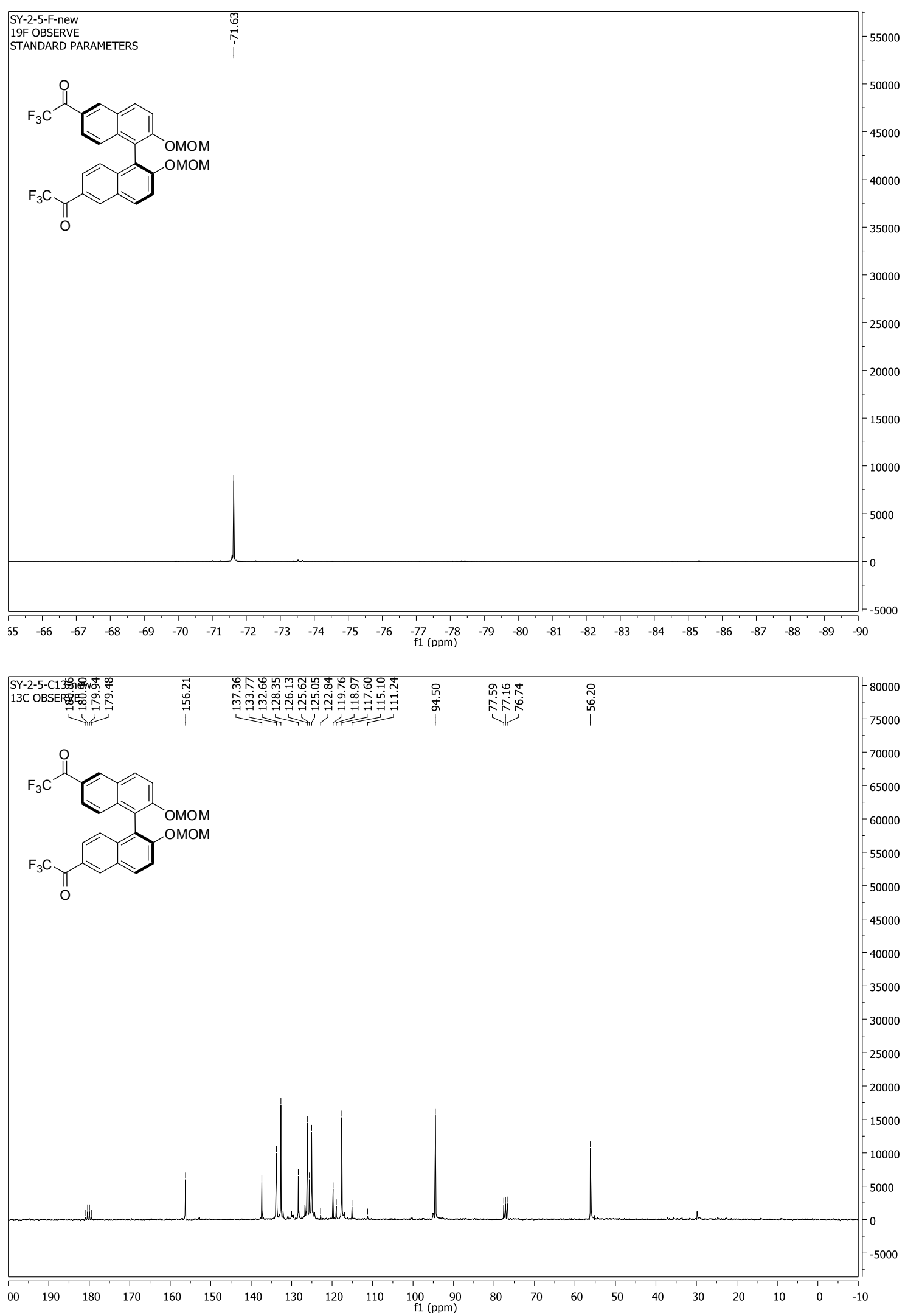



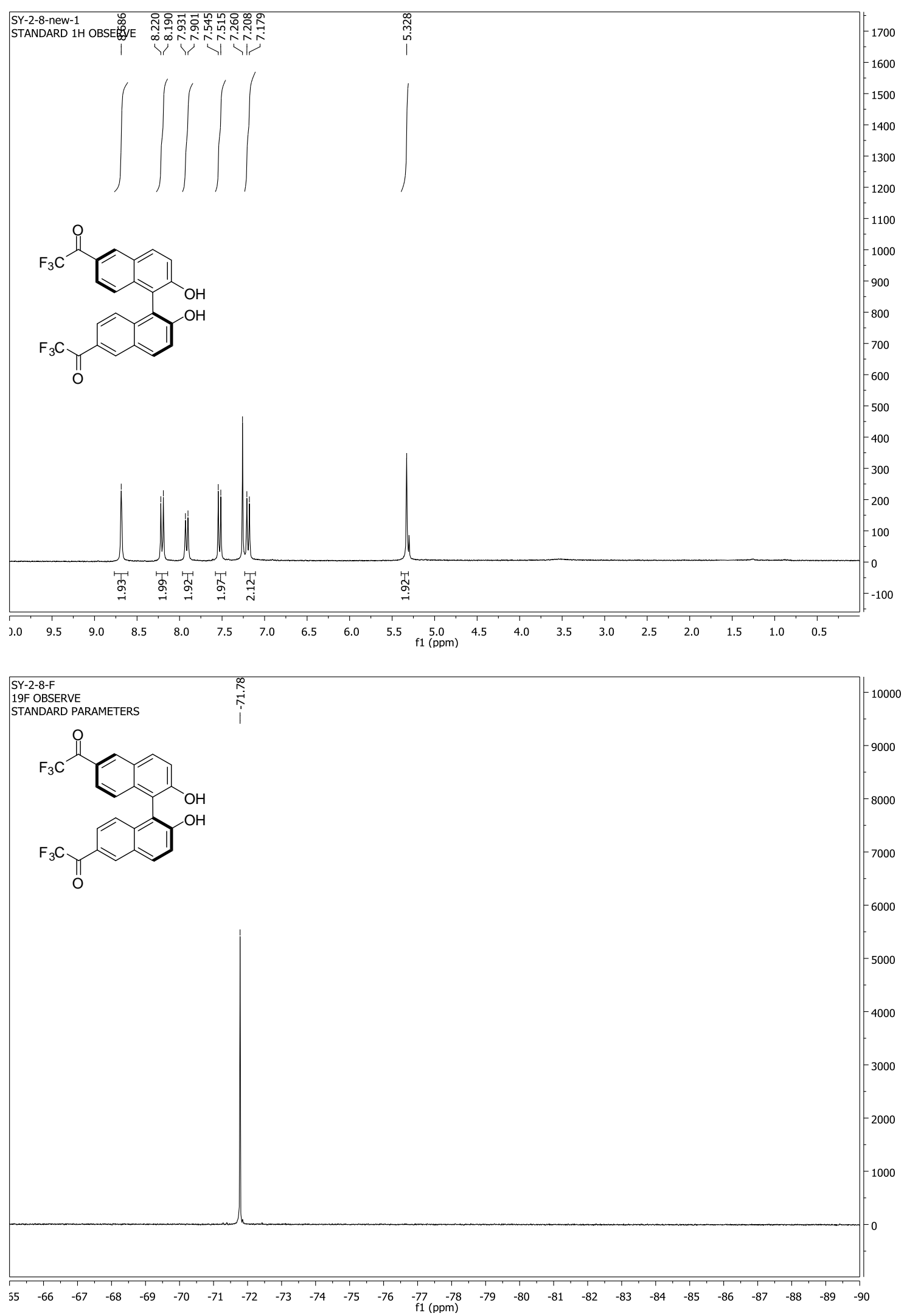


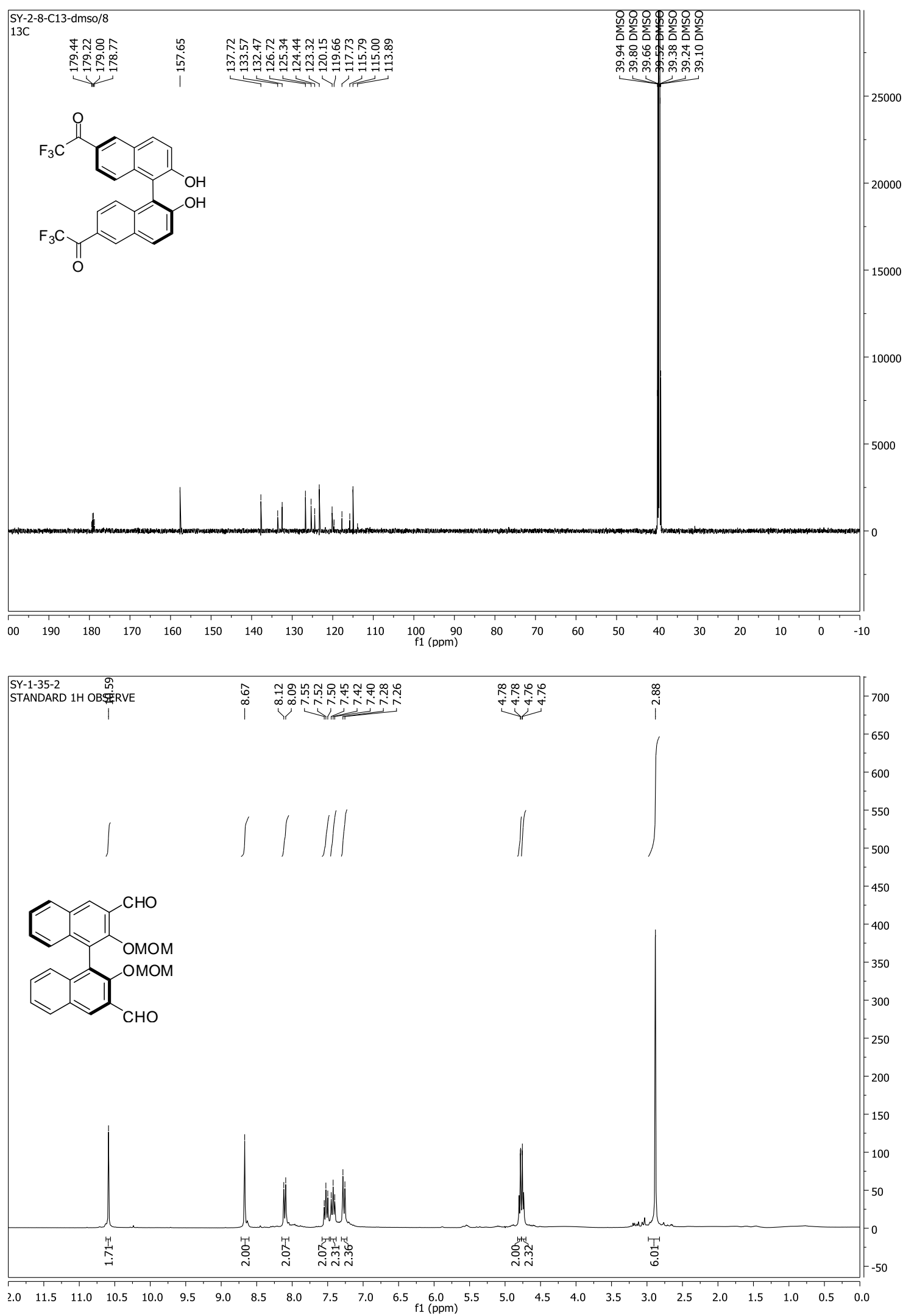




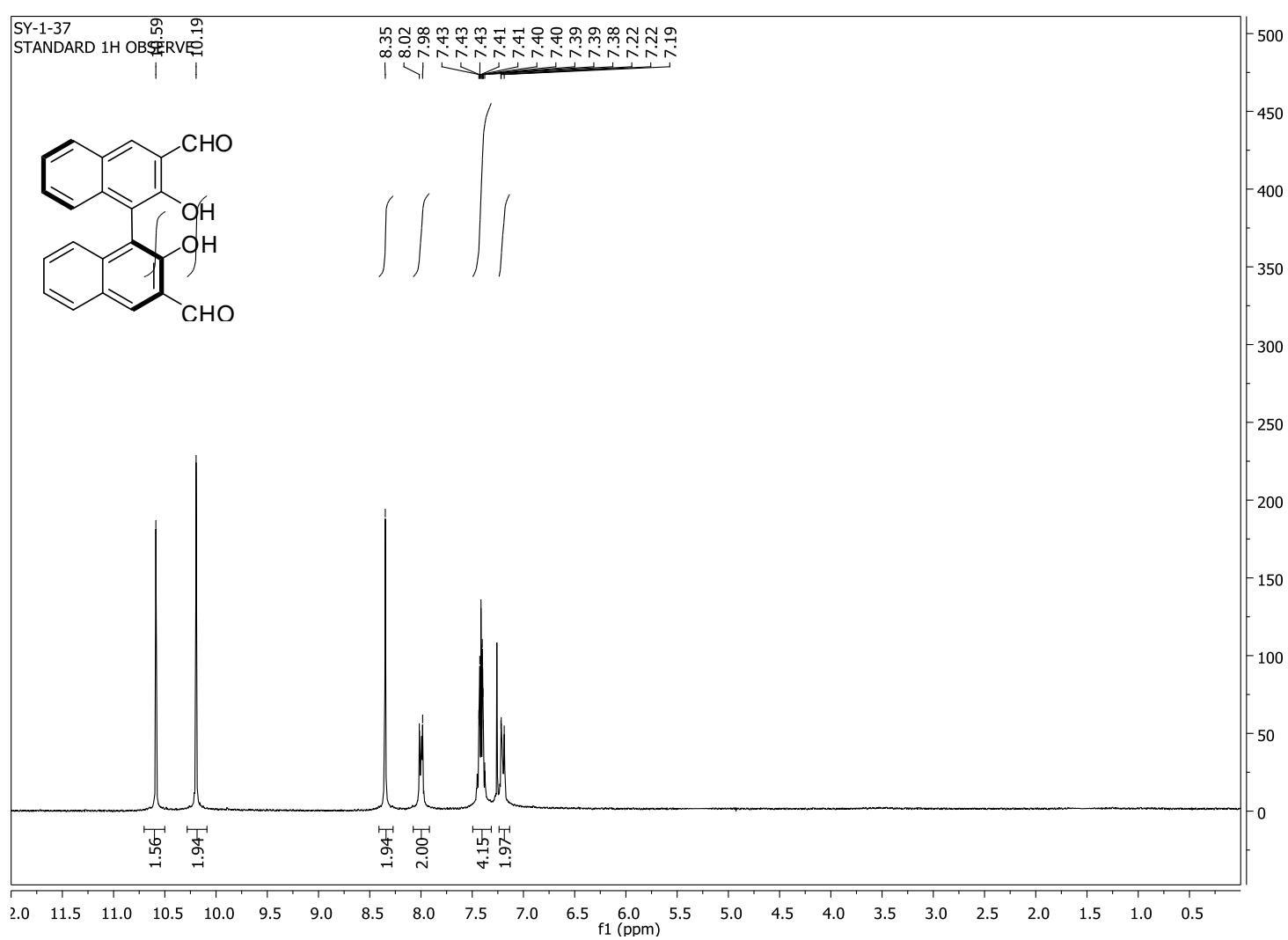




\section{${ }^{19}$ F NMR titration of $(S)-6-4$ with N9}

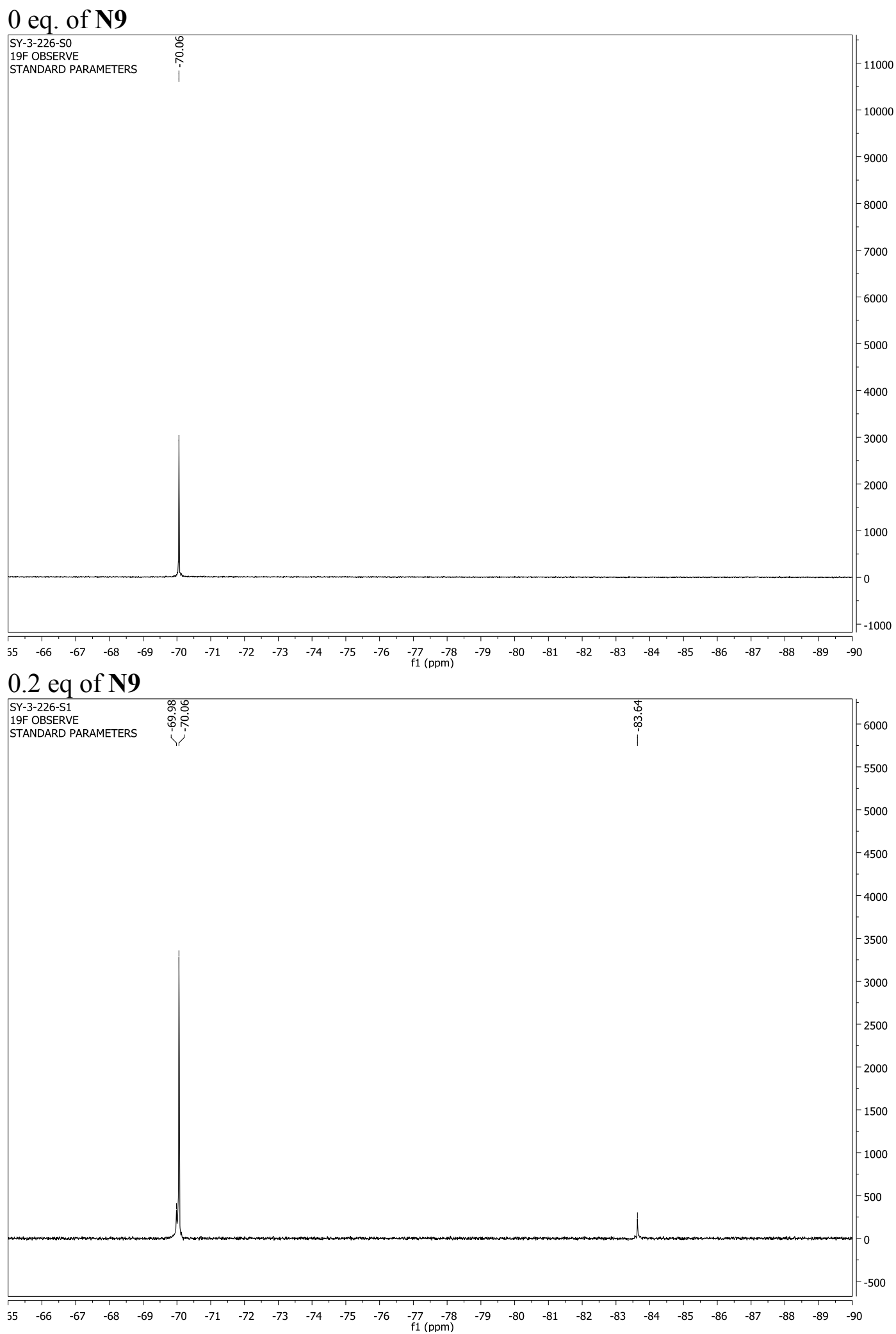




\section{4 eq of $\mathbf{N 9}$}

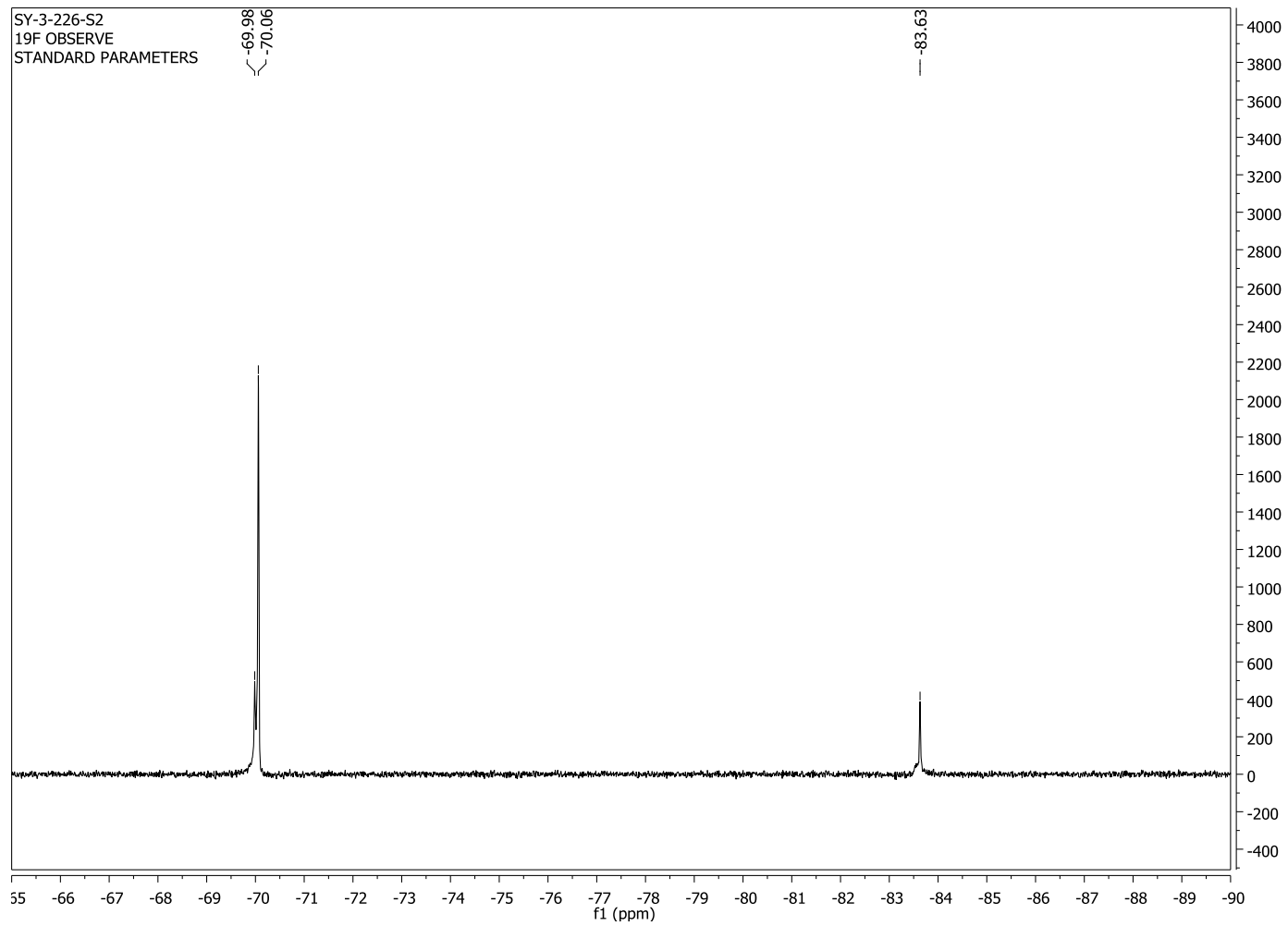

\section{6 eq of $\mathbf{N 9}$}

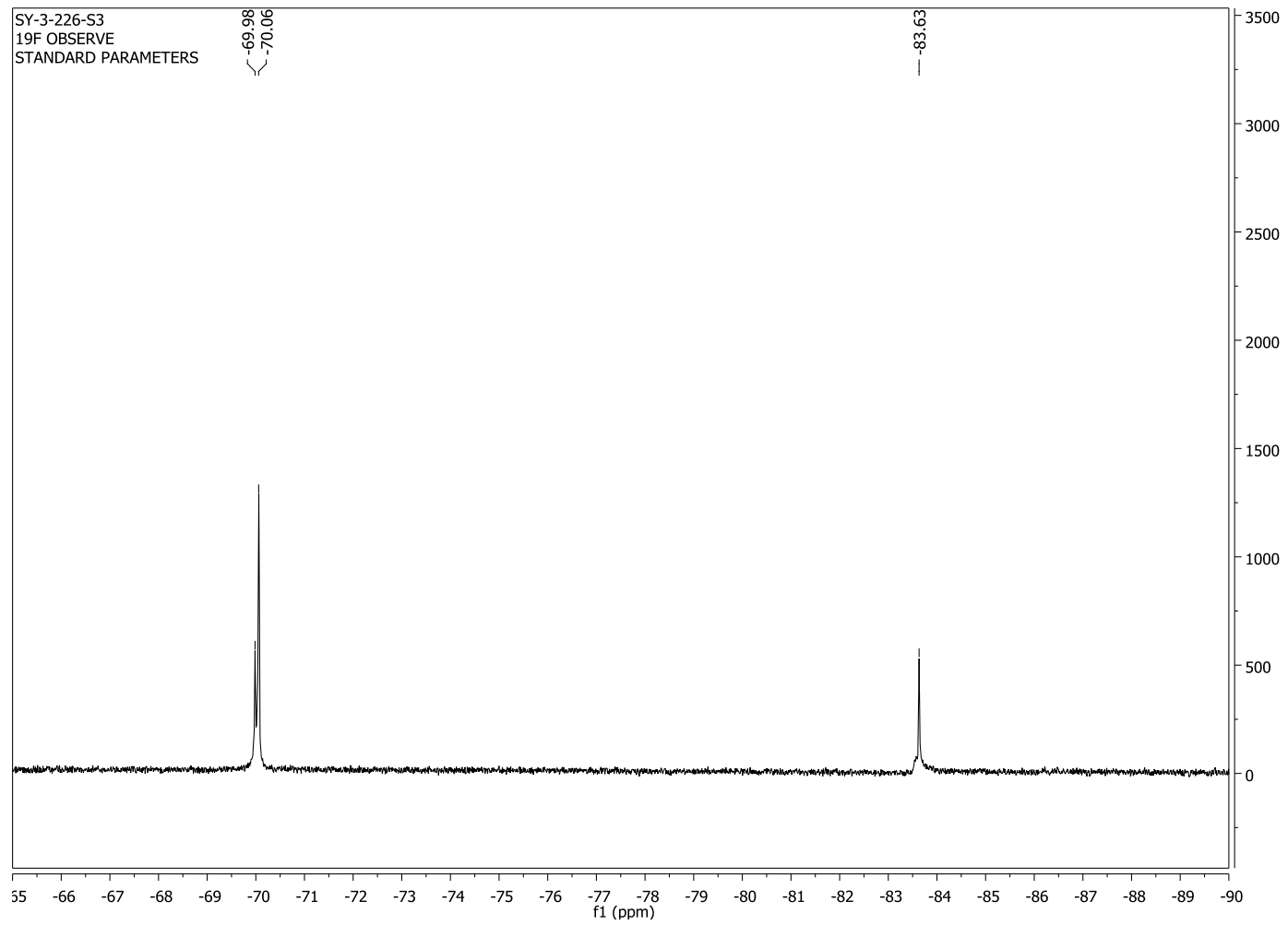




\section{8 eq of $\mathbf{N 9}$}

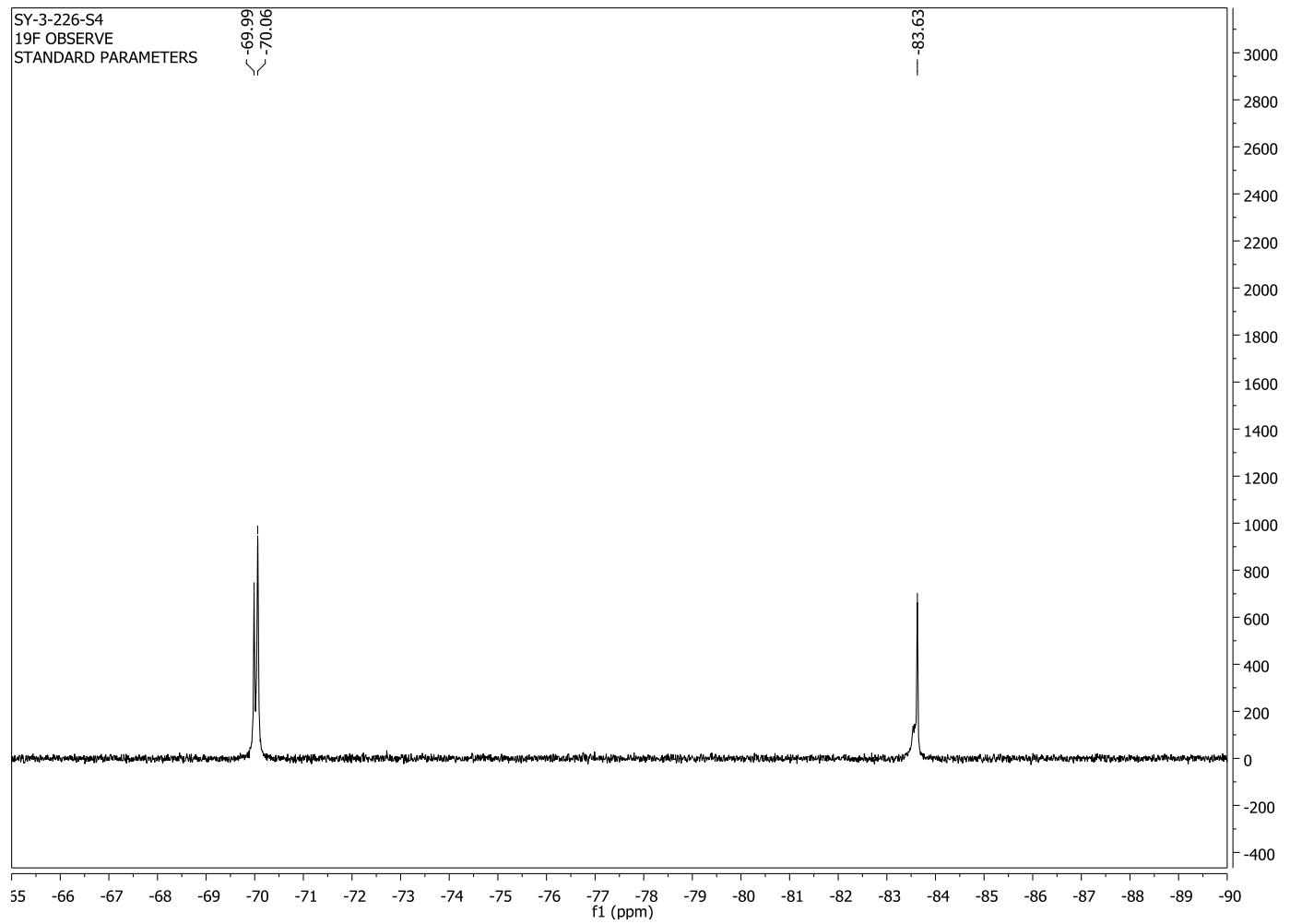

\subsection{5 eq of $\mathbf{N 9}$}

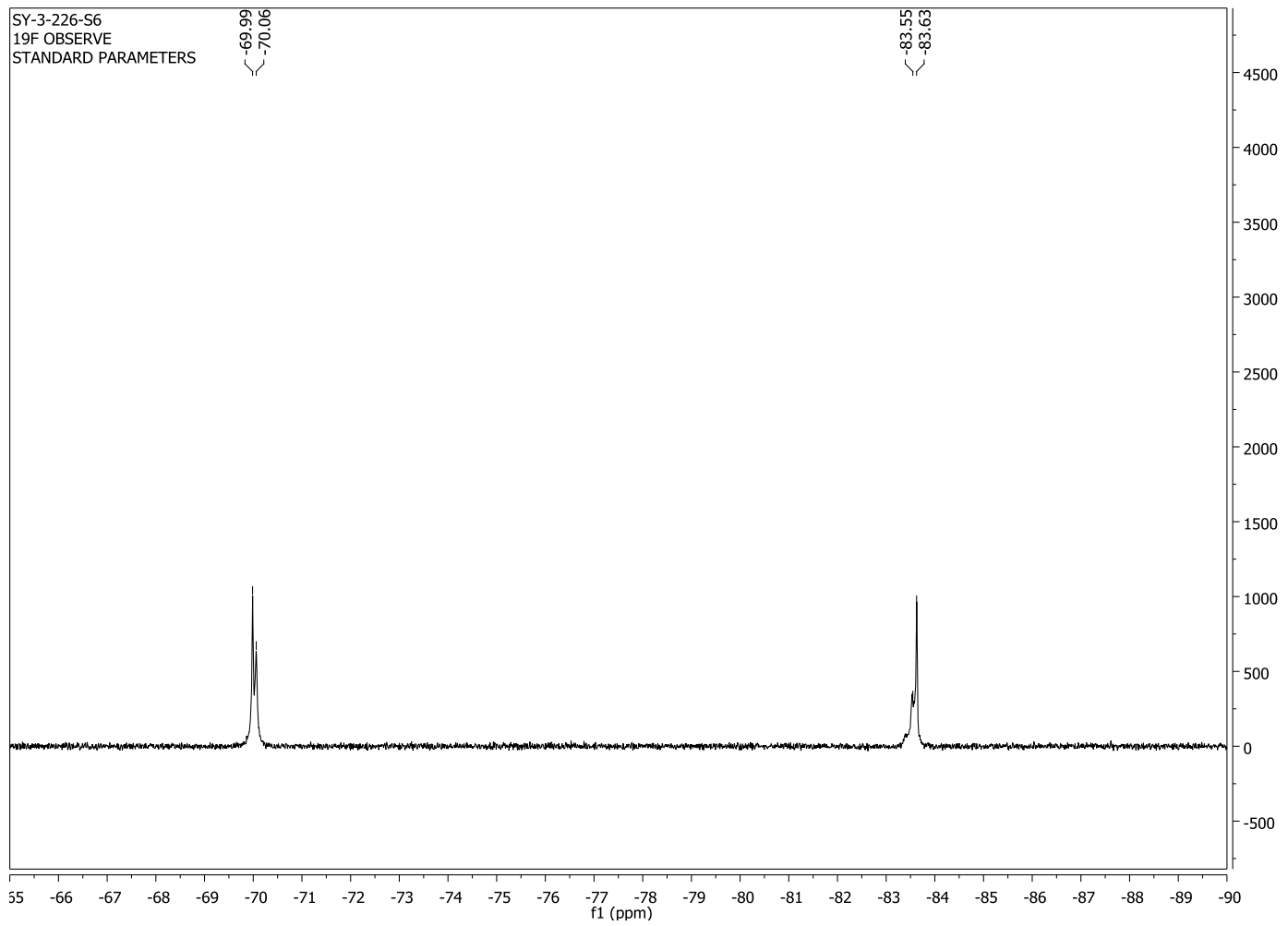




\section{9 eq of $\mathbf{N 9}$}

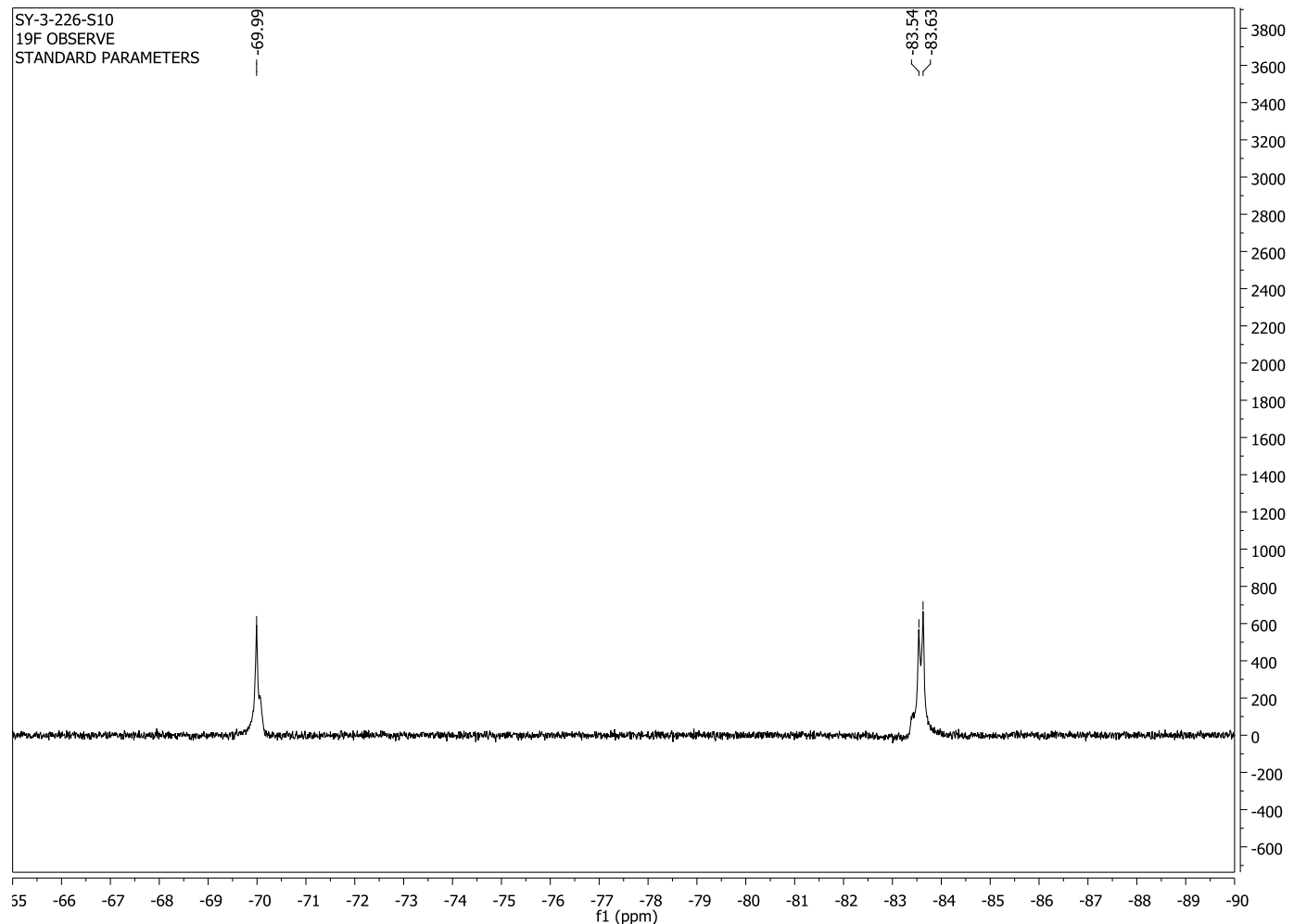

\section{7 eq of N9}

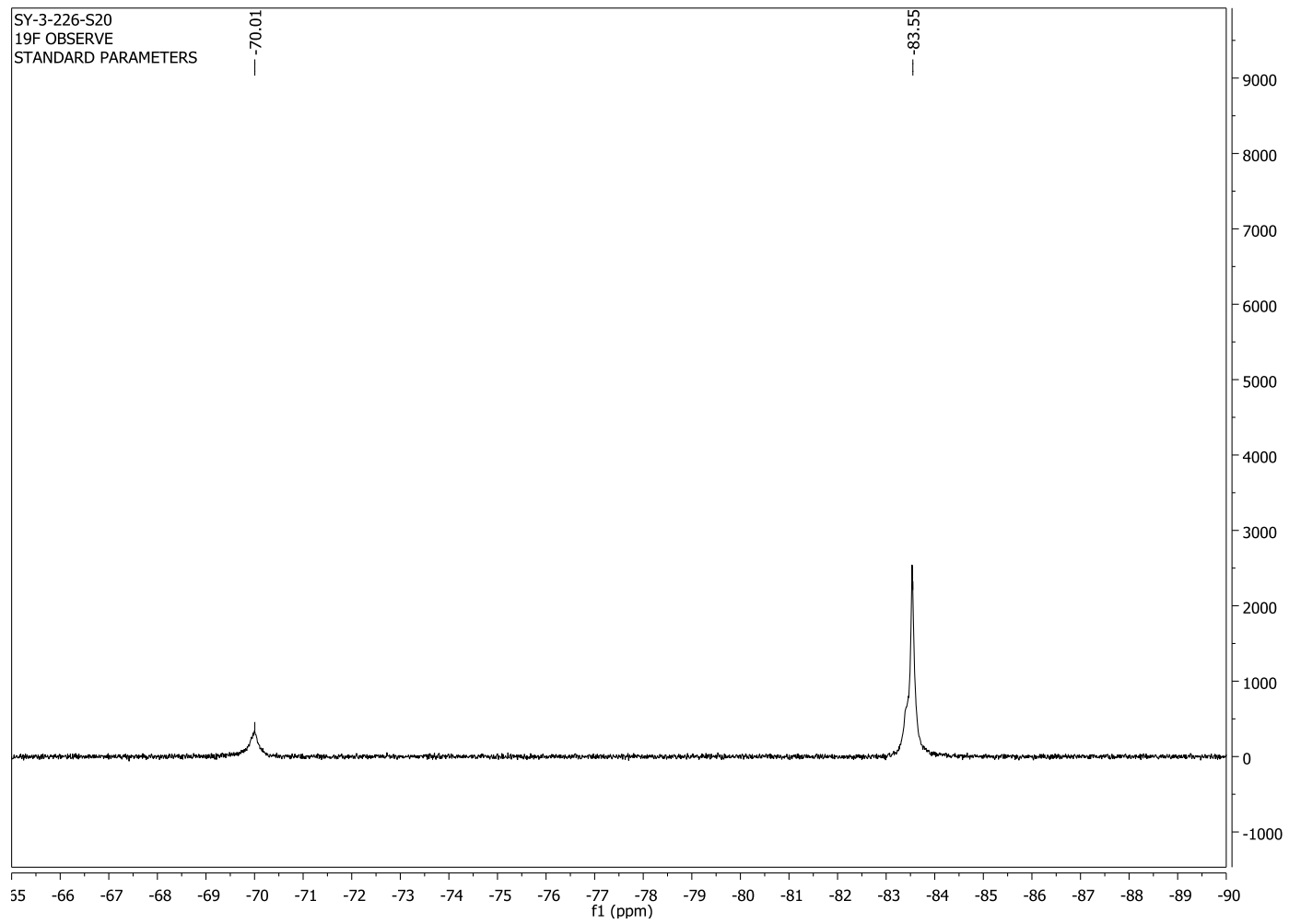




\section{4 eq of N9}

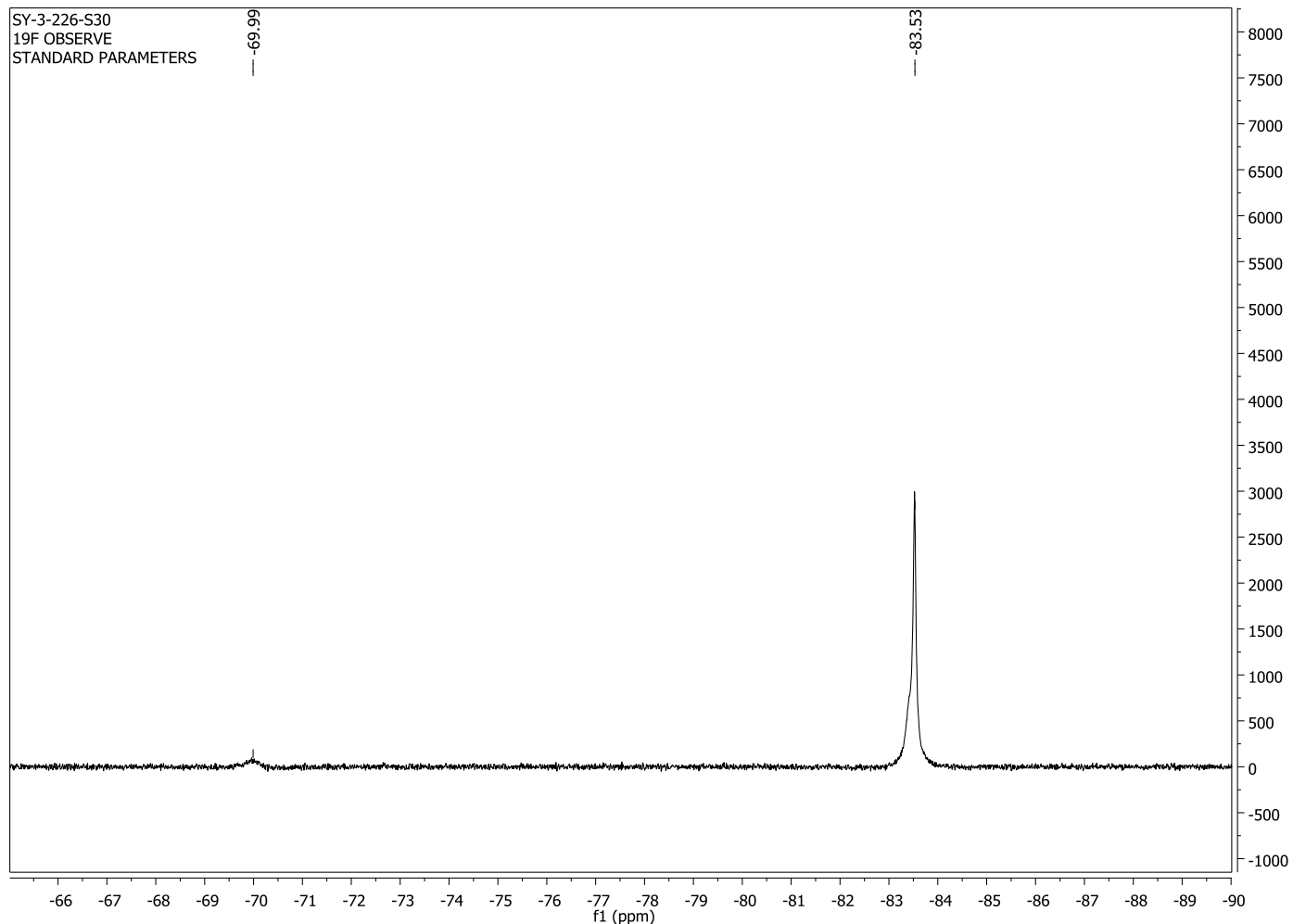

\section{1 eq of $\mathbf{N 9}$}

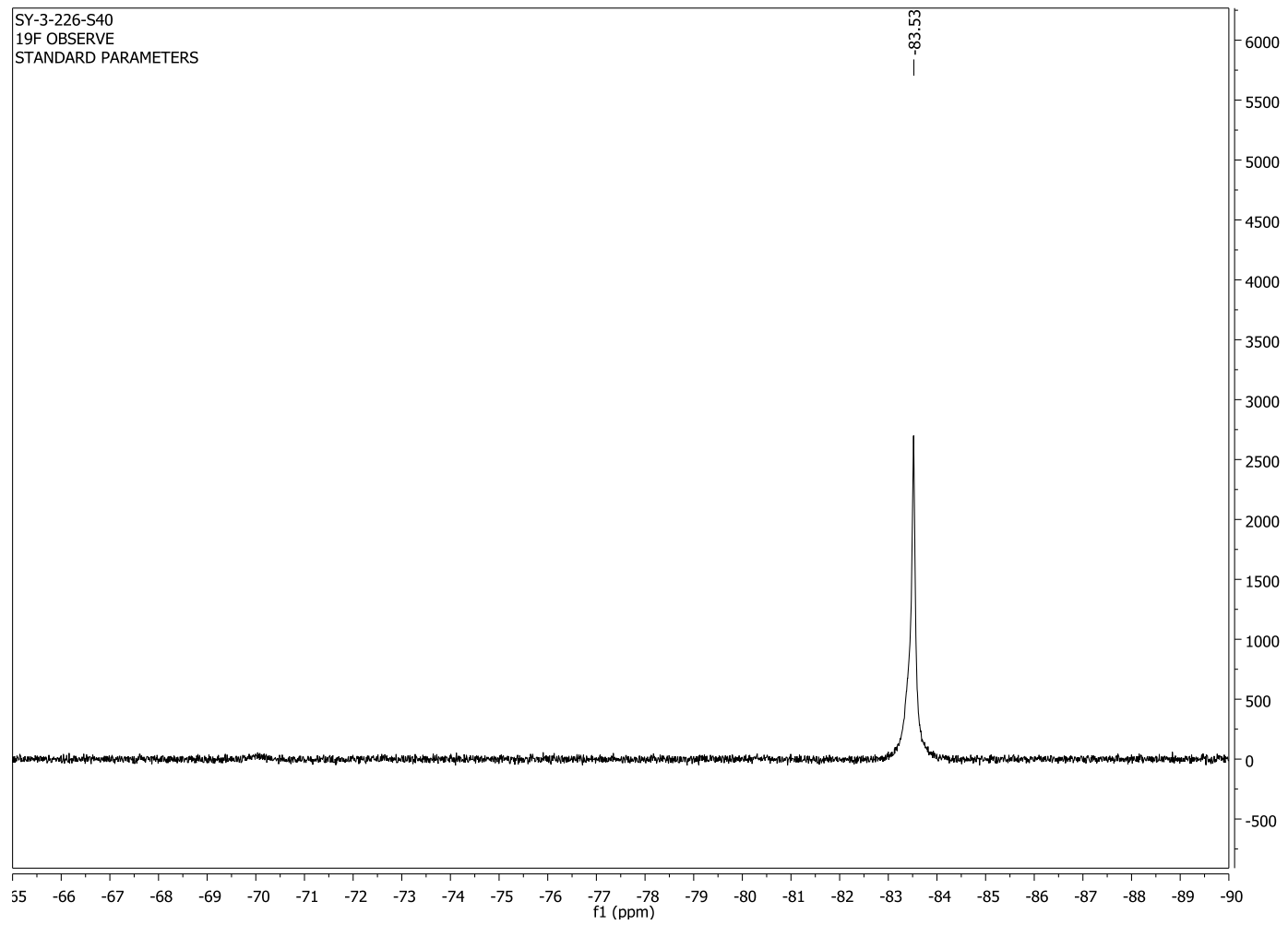




\section{7 eq of N9}

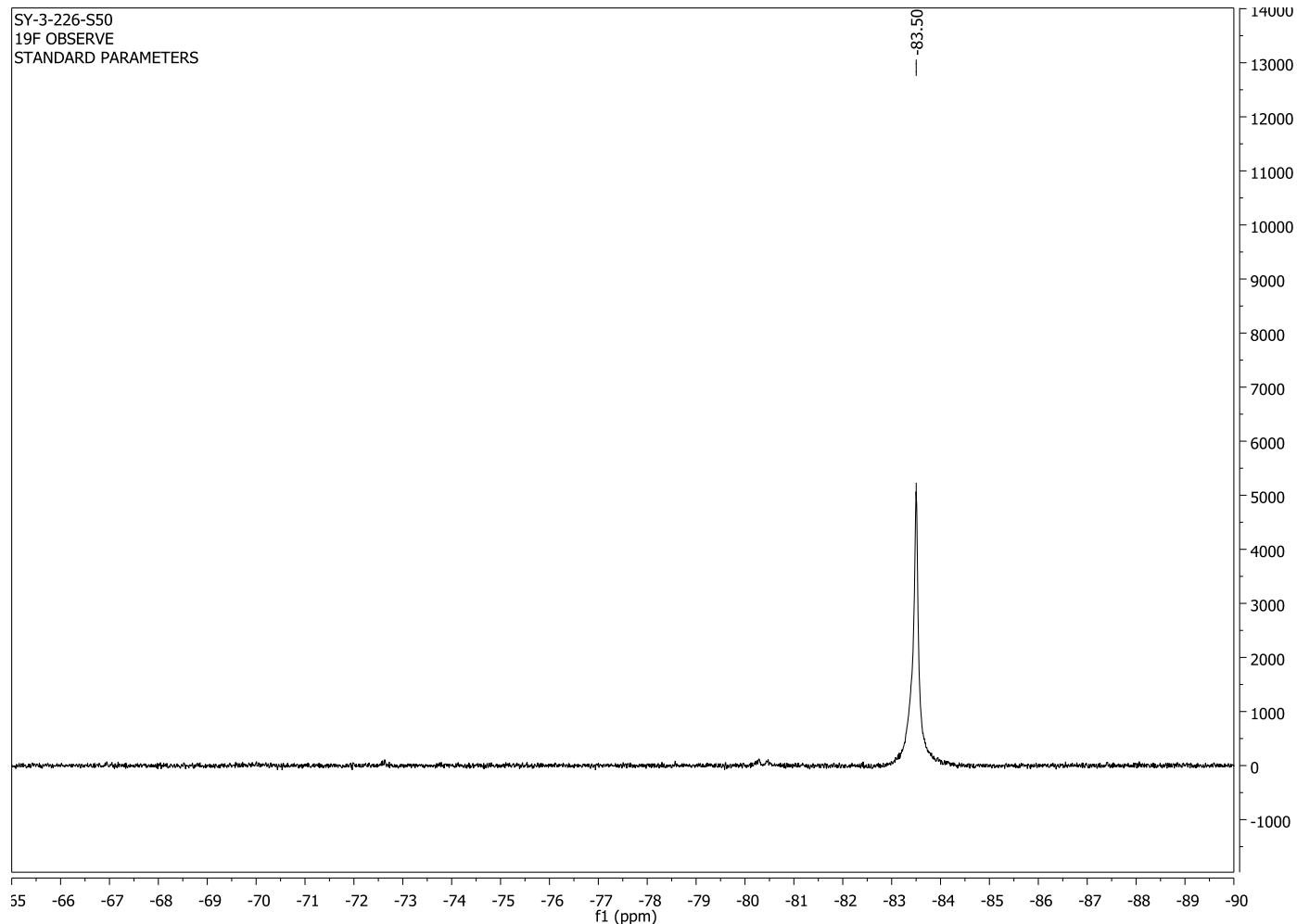

\section{$11.6 \mathrm{eq}$ of $\mathbf{N 9}$}

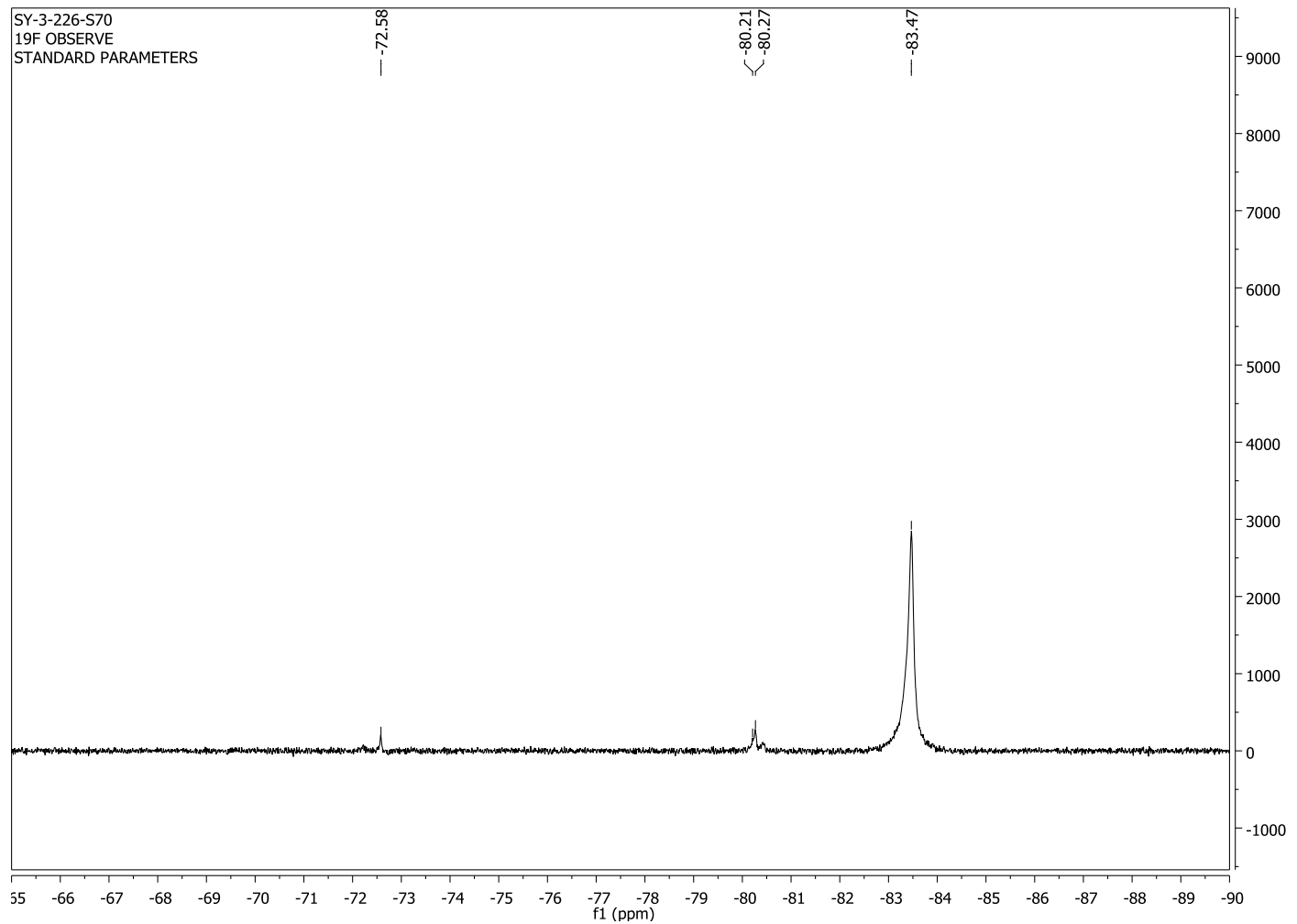




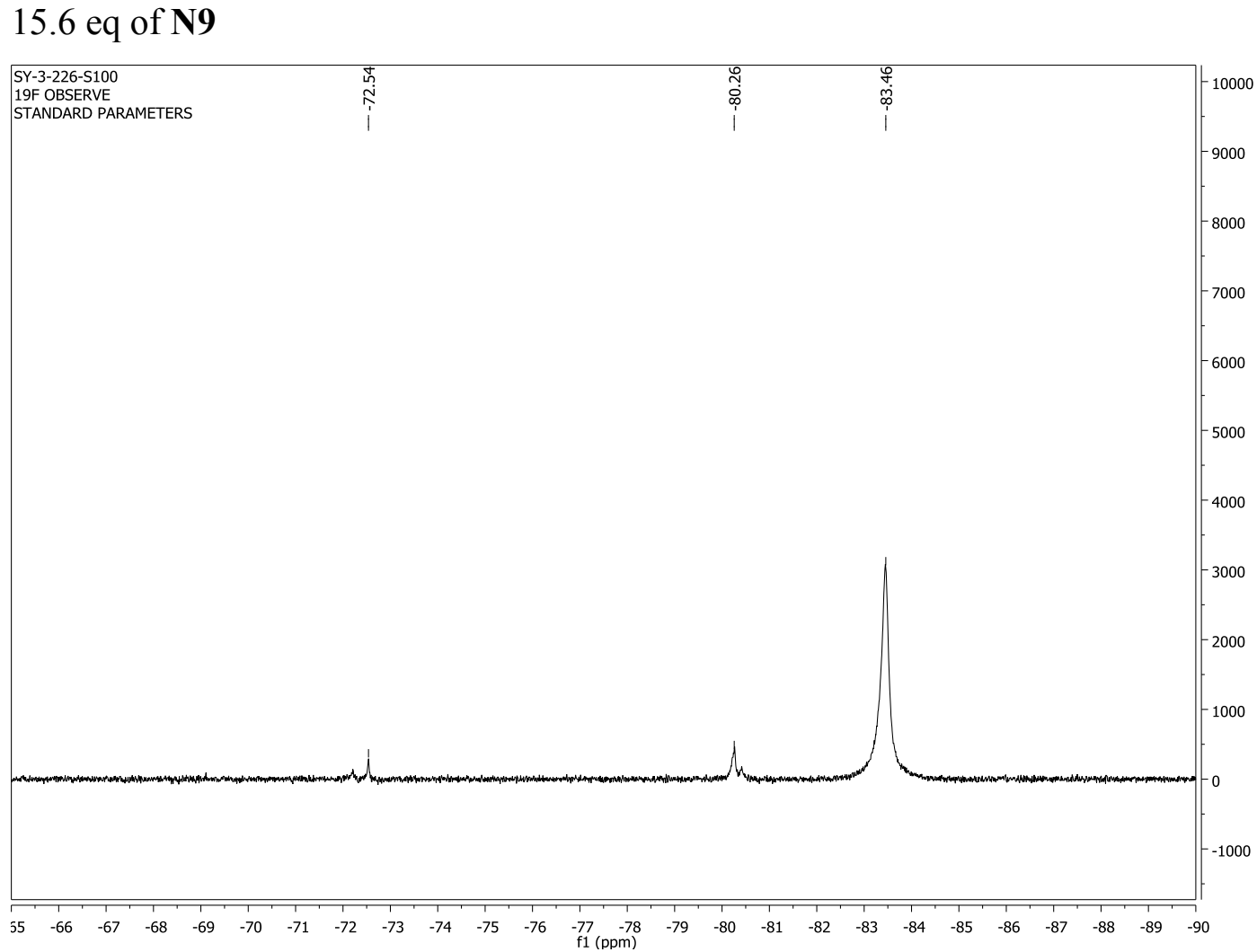

\section{3 eq of $\mathbf{N 9}$}

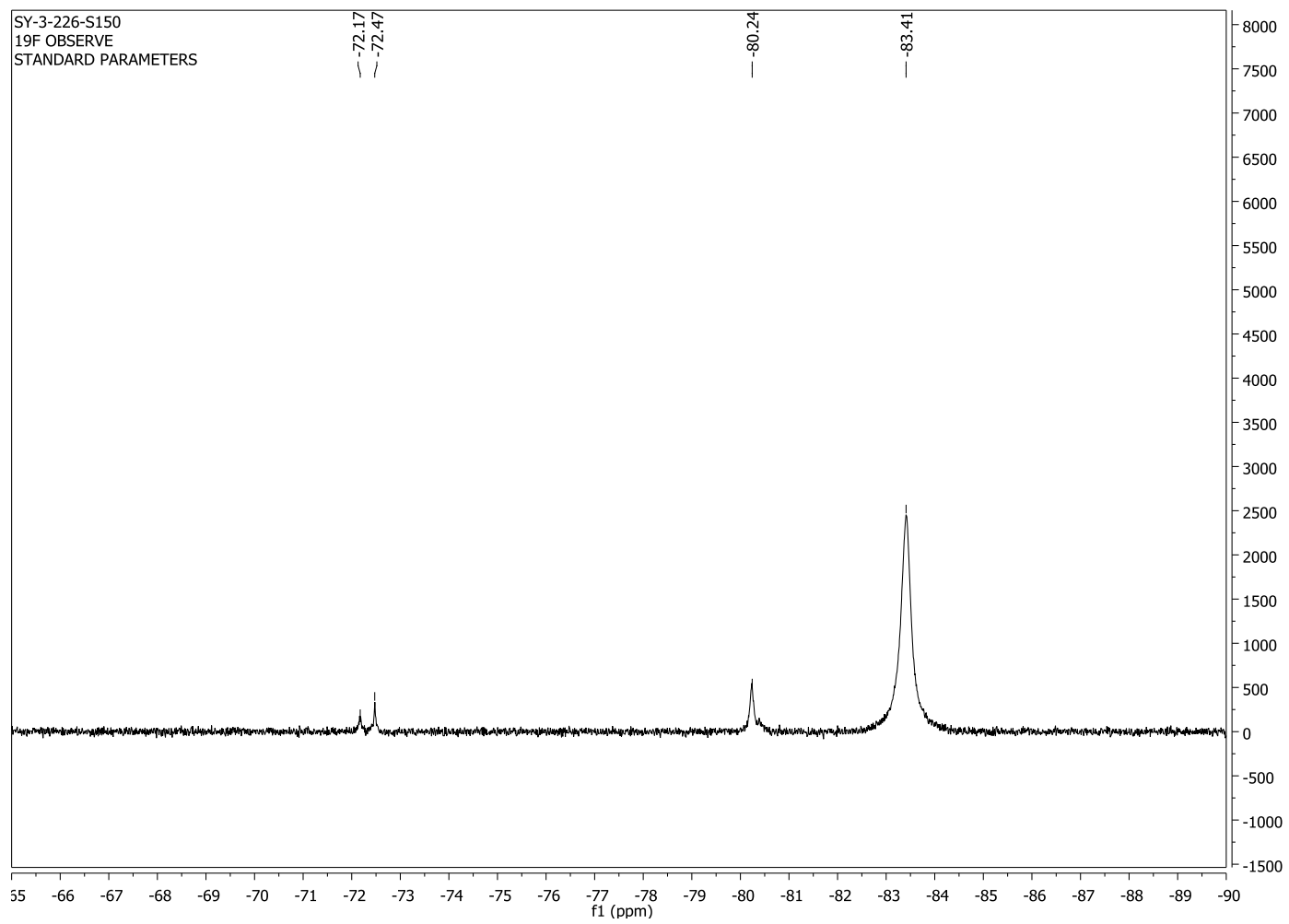




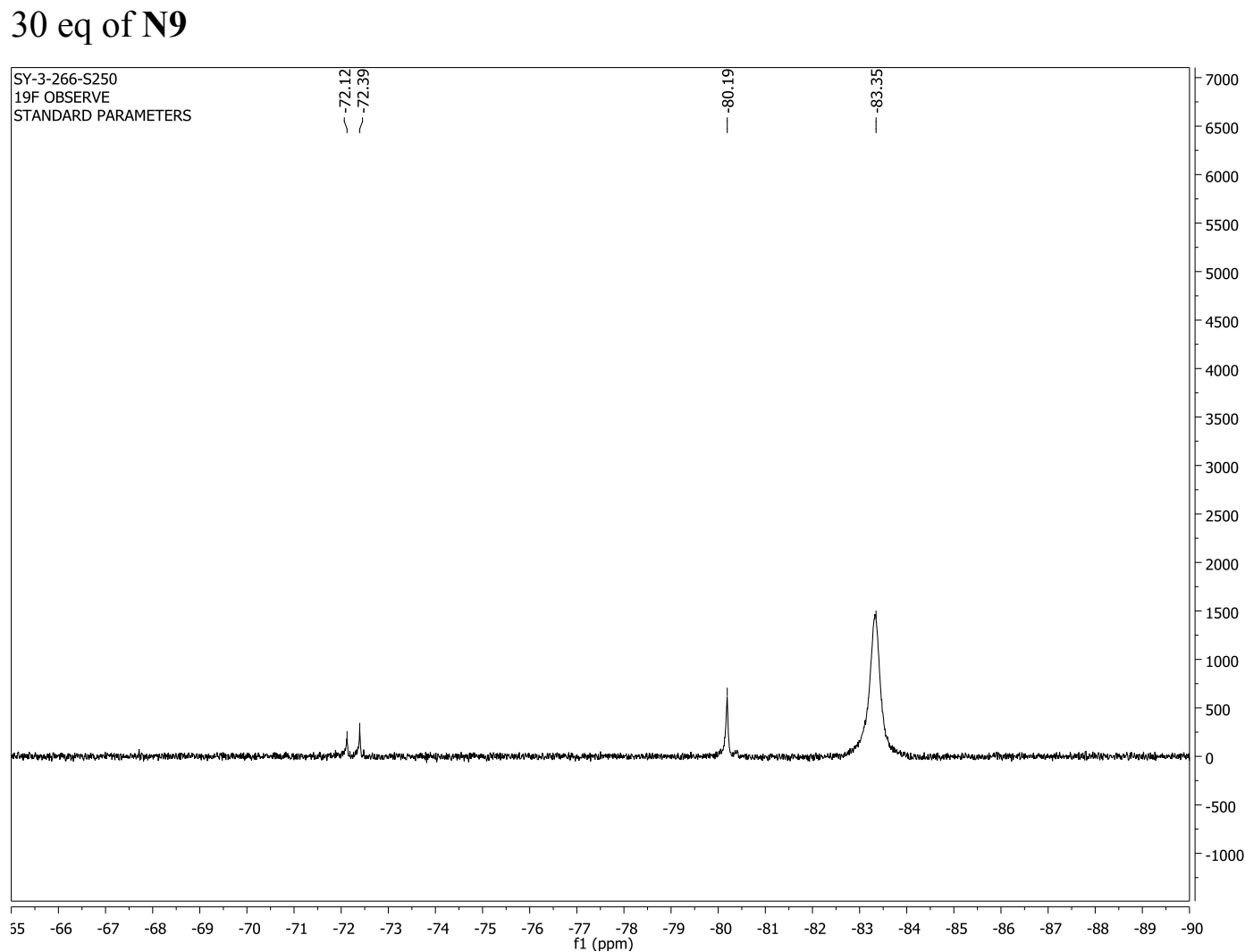

\section{9 eq of N9}

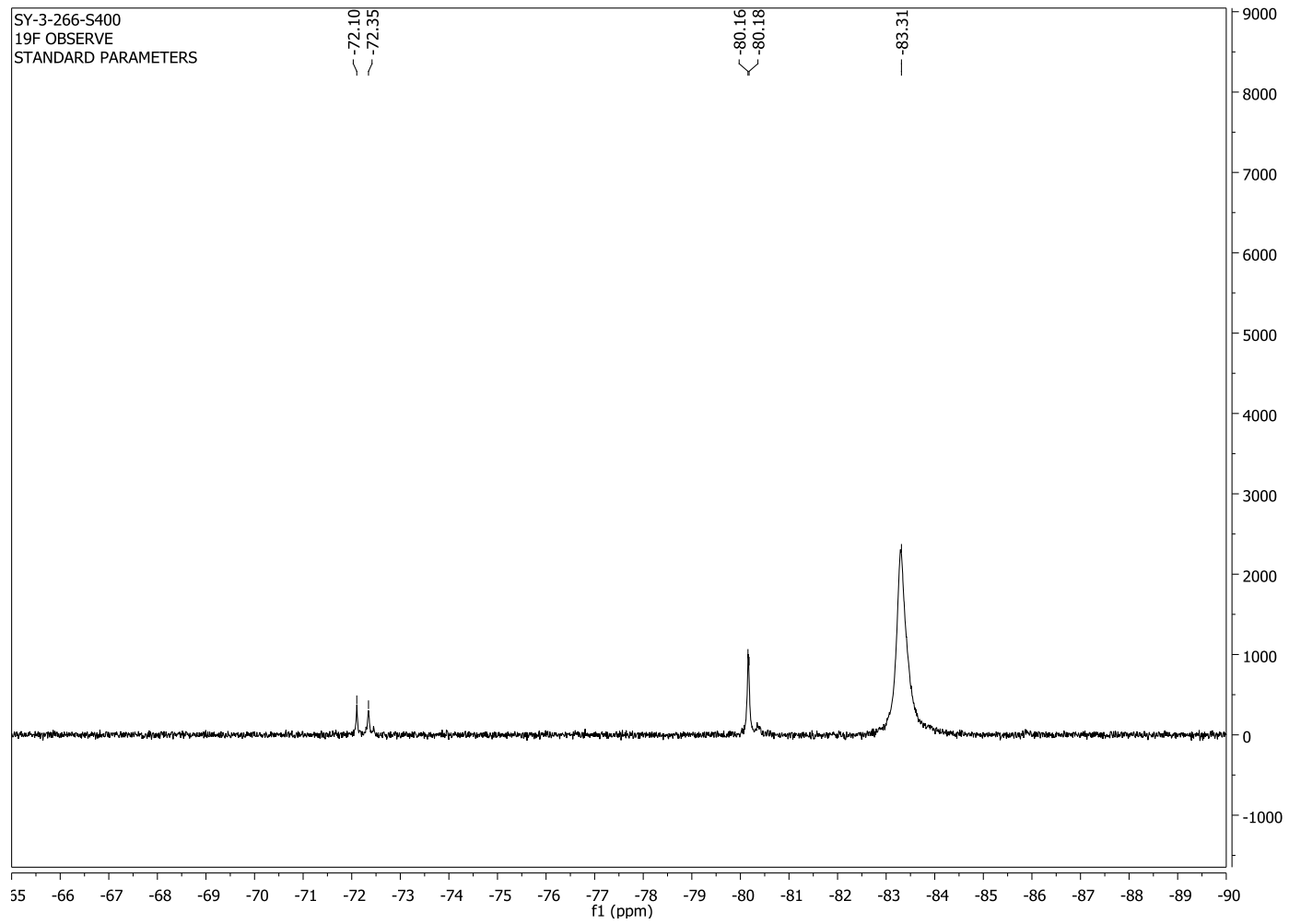




\section{9 eq of $\mathbf{N 9}$}

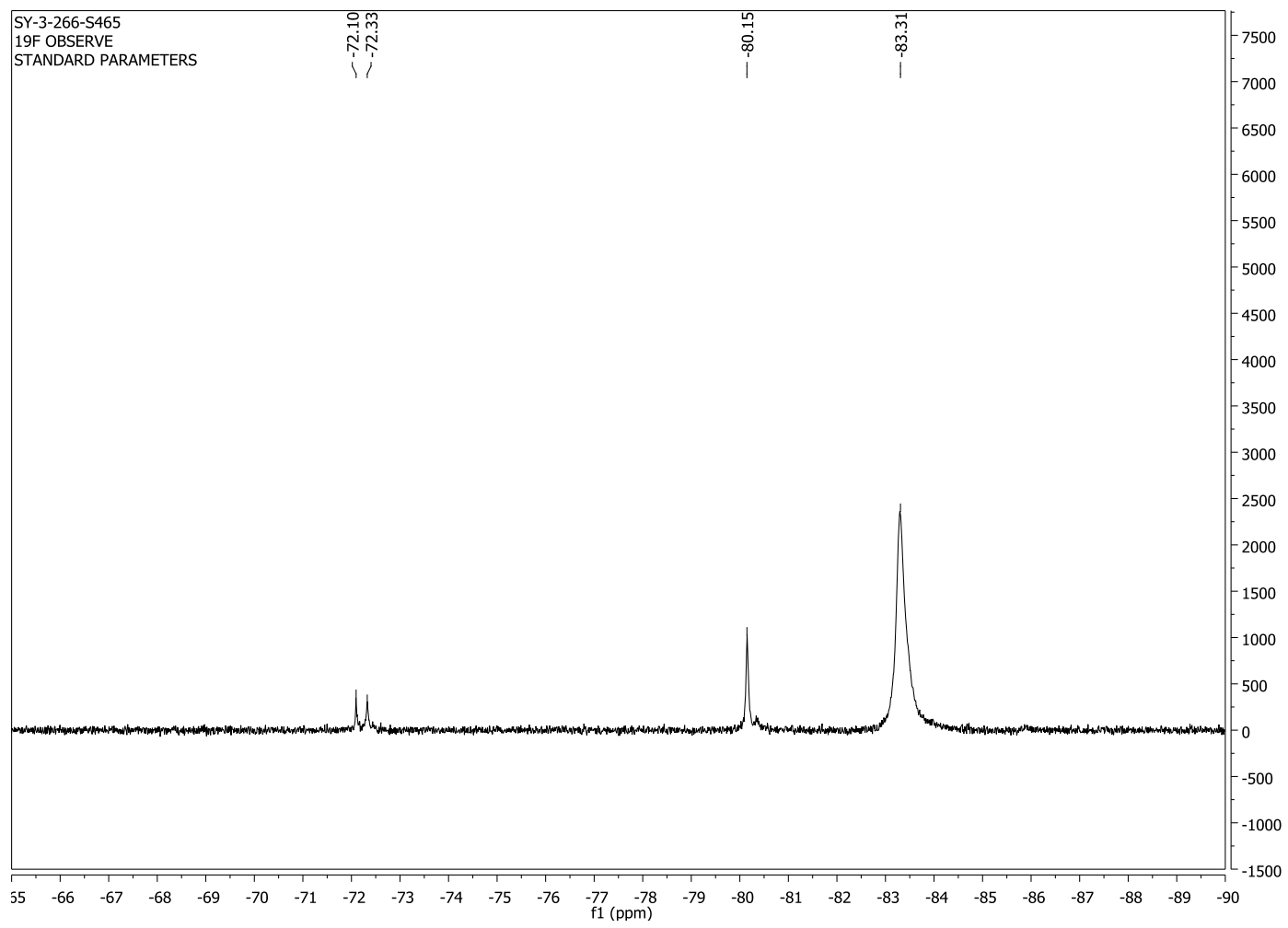

\section{8 eq of $\mathbf{N 9}$}

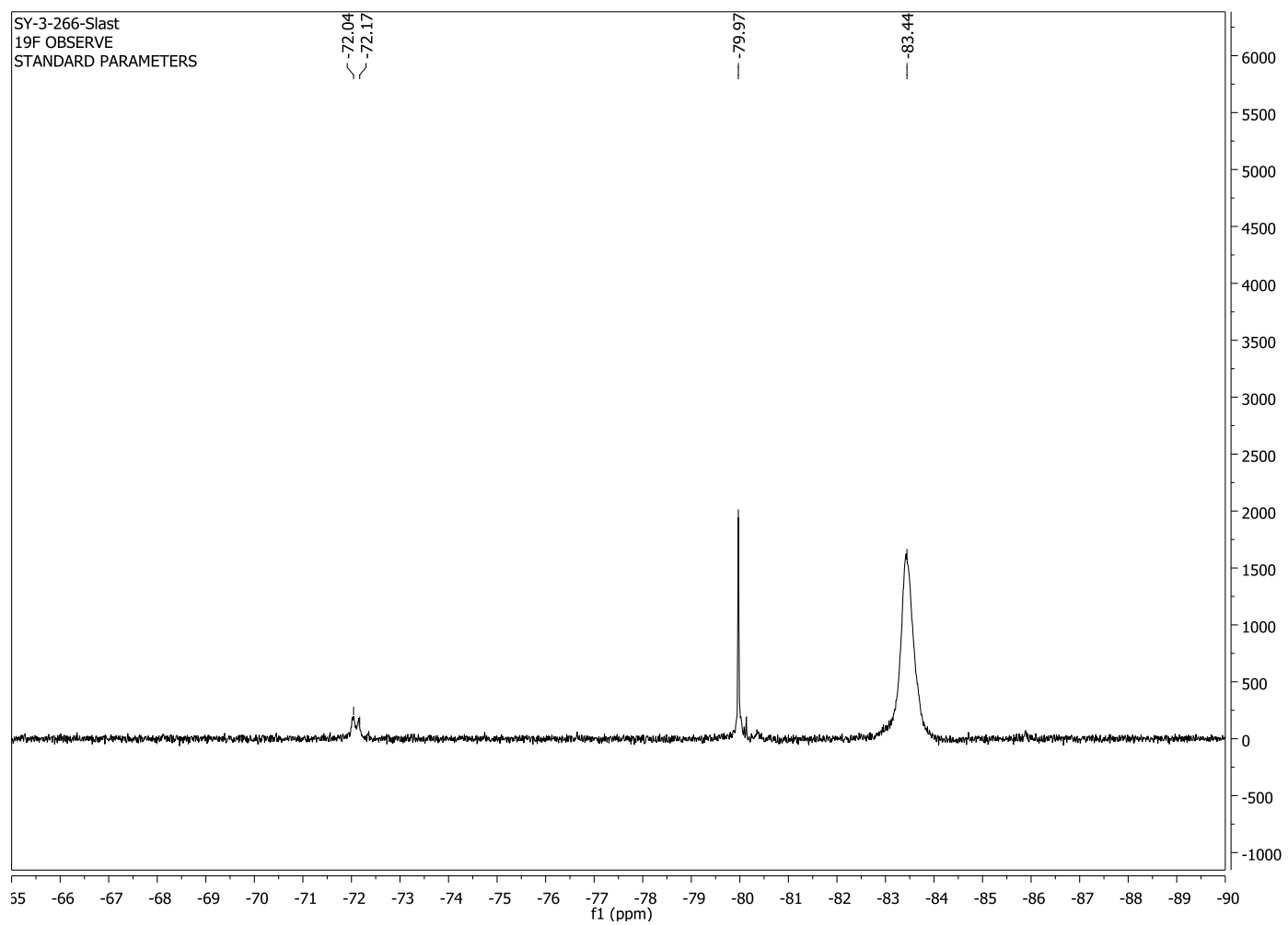




\section{8 eq of $\mathbf{N 9}$ overnight}

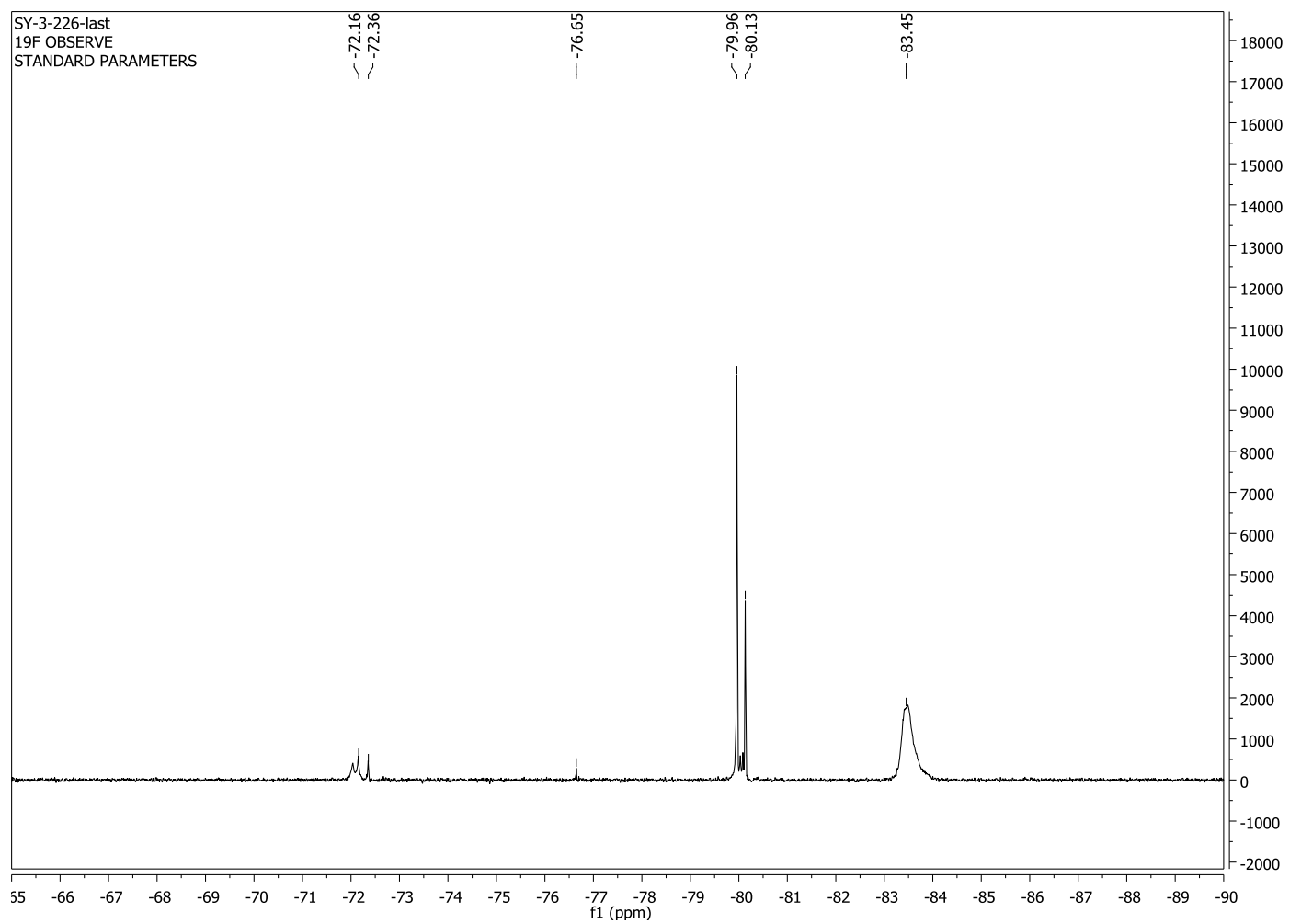


${ }^{19}$ F NMR titration of $(S)-6-4$ with N12

0 eq of N12

SY-3-228-SO
19F OBSERVE

19F OBSERVE

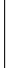




\subsection{7 eq of $\mathbf{N 1 2}$}

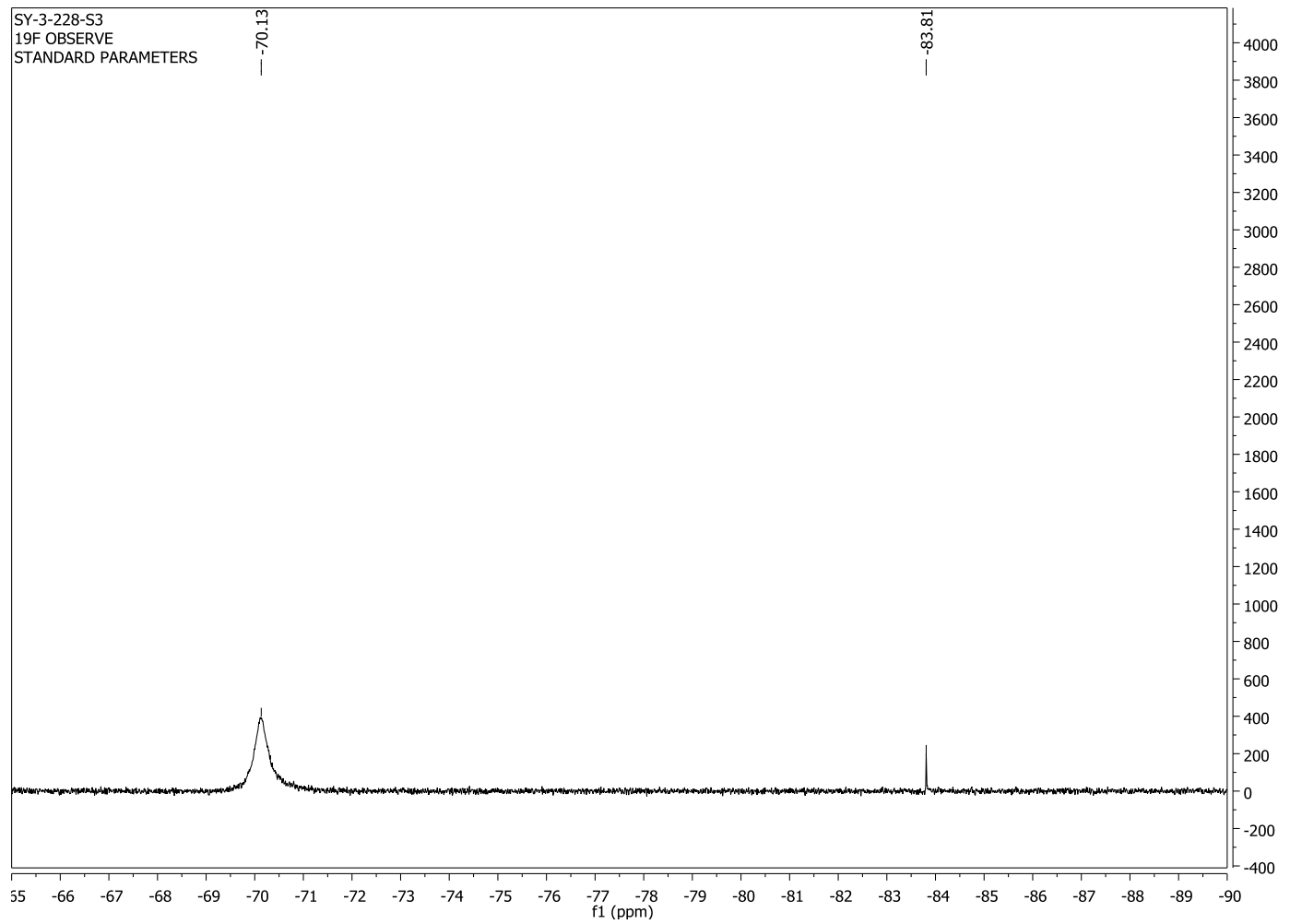

\subsection{4 eq of N12}

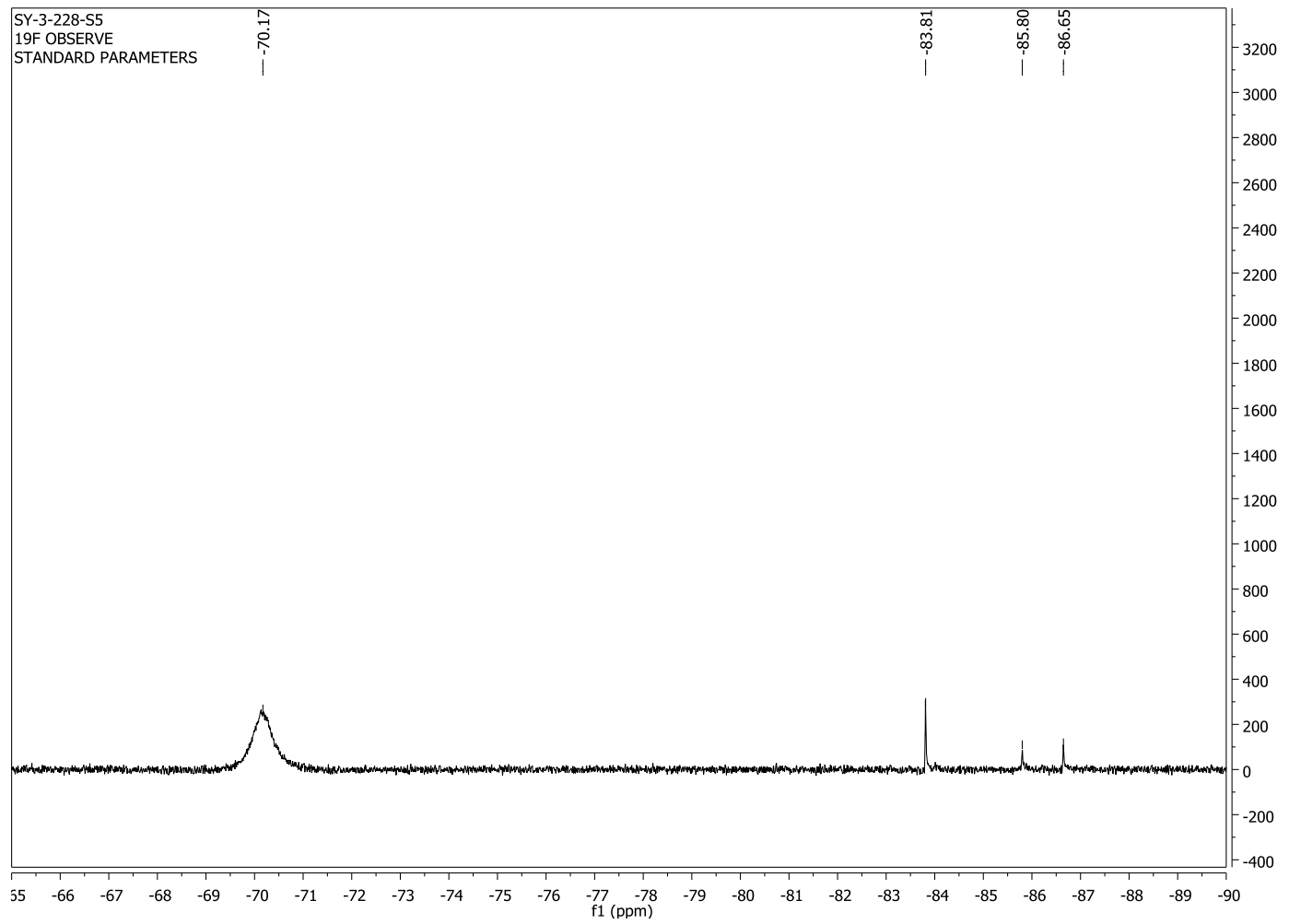




\section{5 eq of $\mathbf{N 1 2}$}

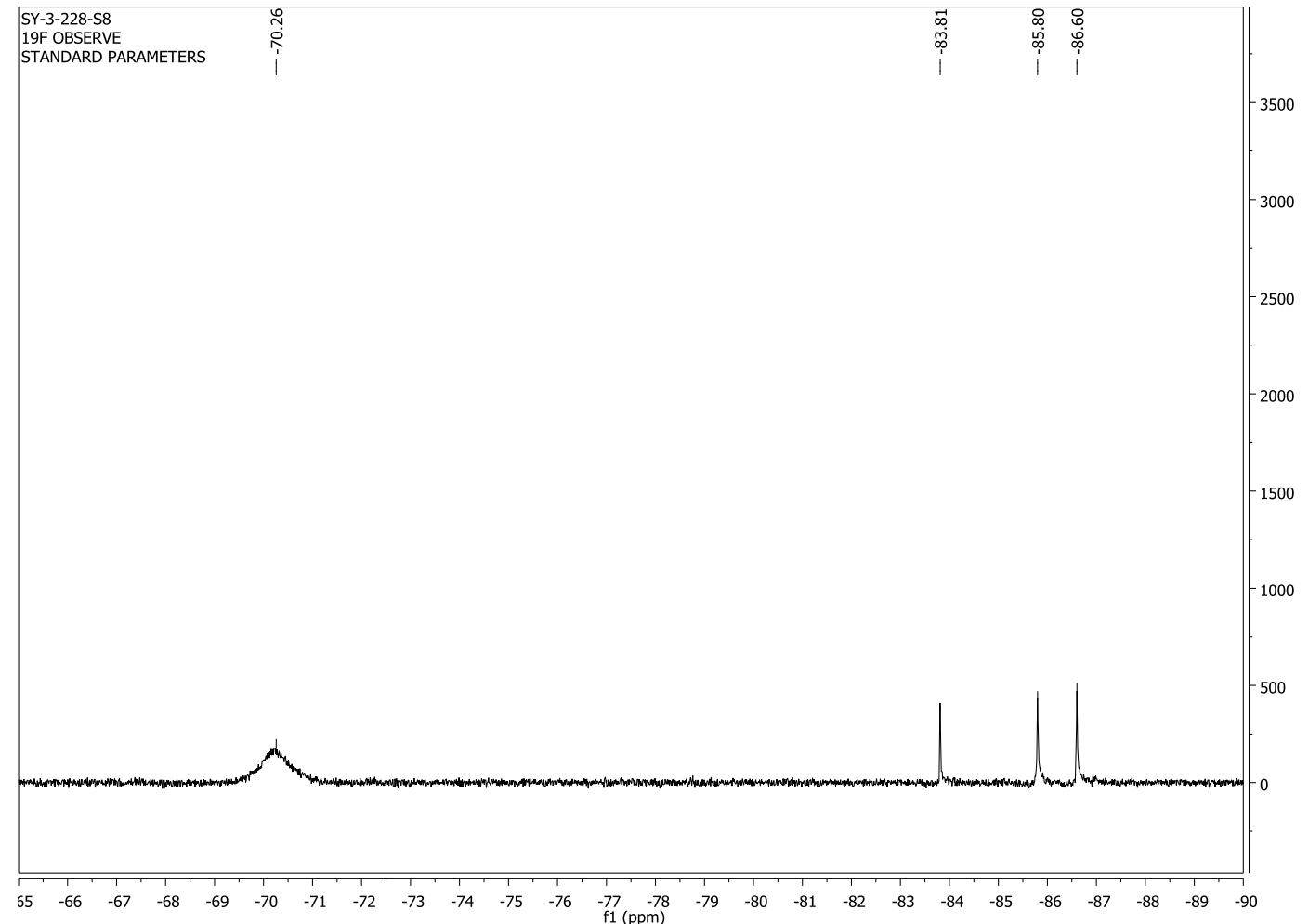

\subsection{6 eq. of $\mathbf{N 1 2}$}

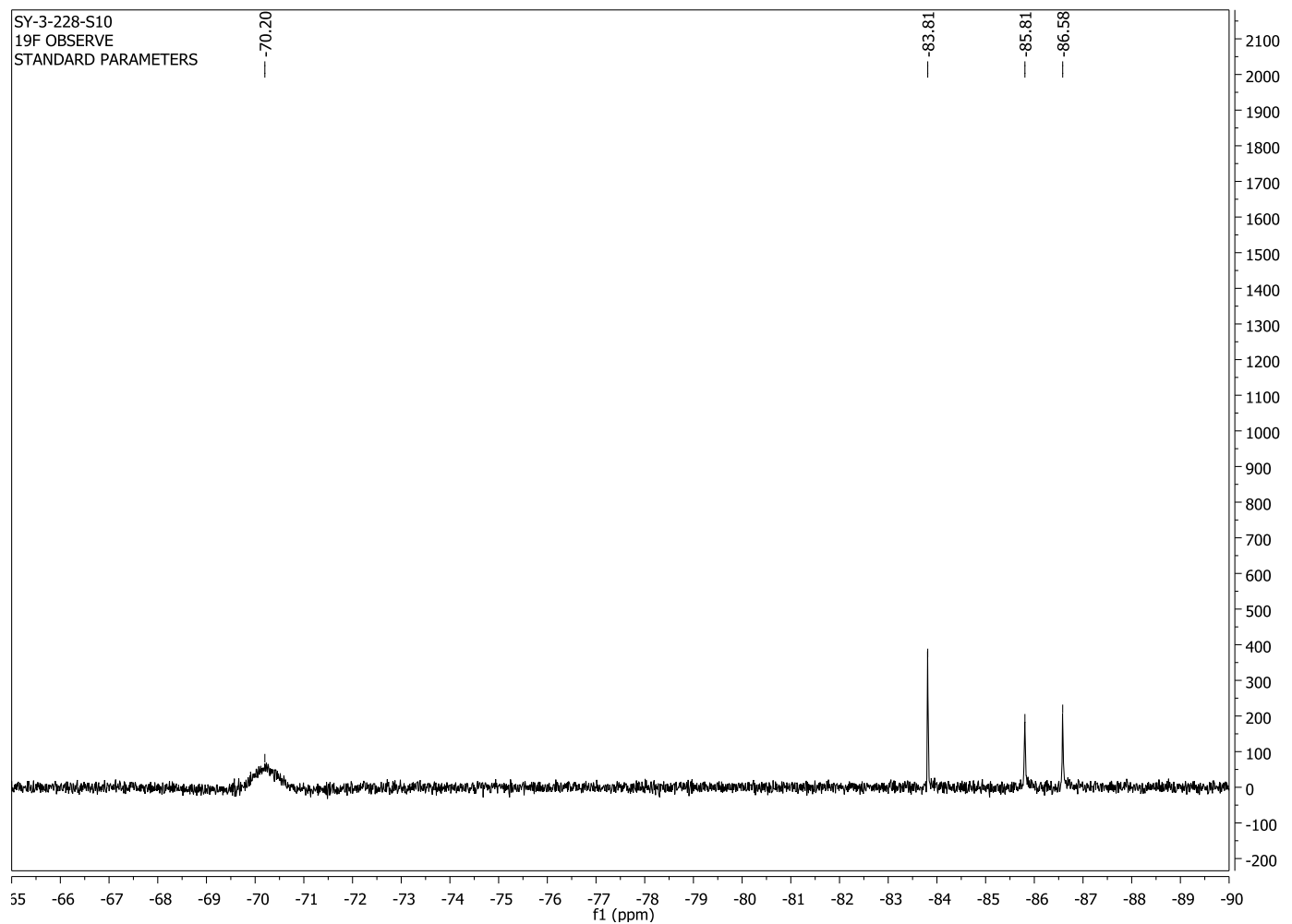




\subsection{5 eq. of N12}

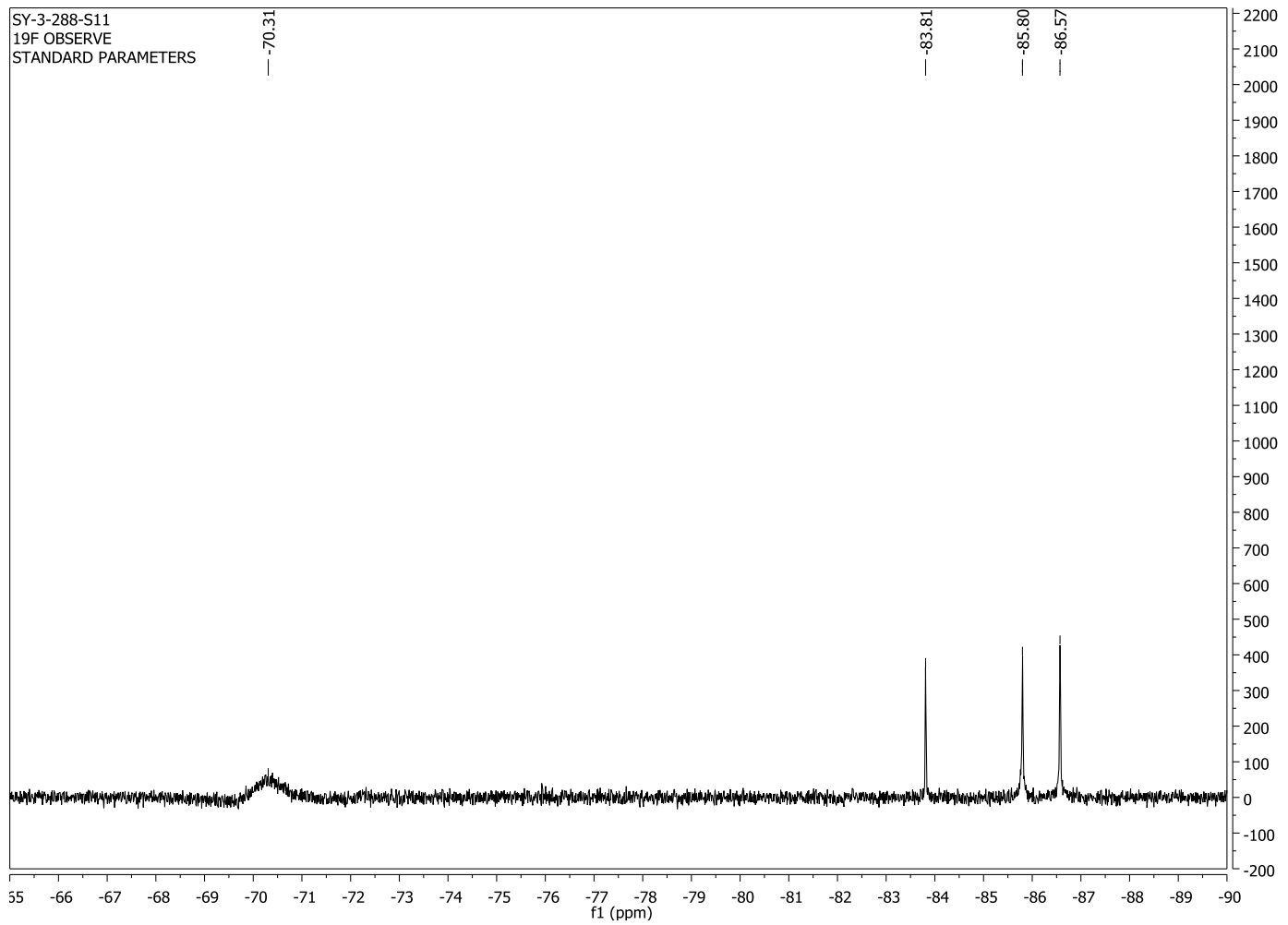

\subsection{6 eq of $\mathbf{N 1 2}$}

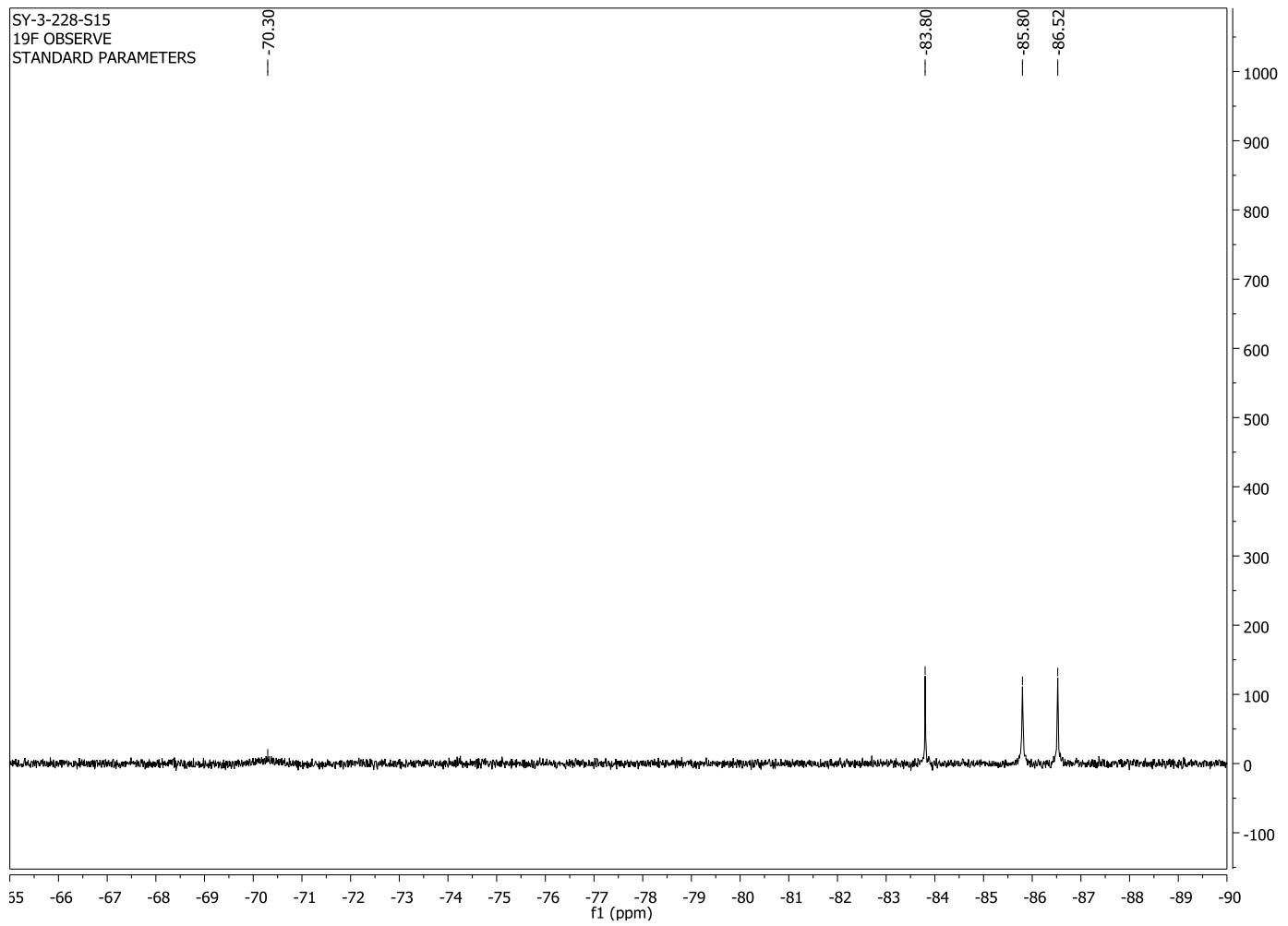




\subsection{3 eq of N12}

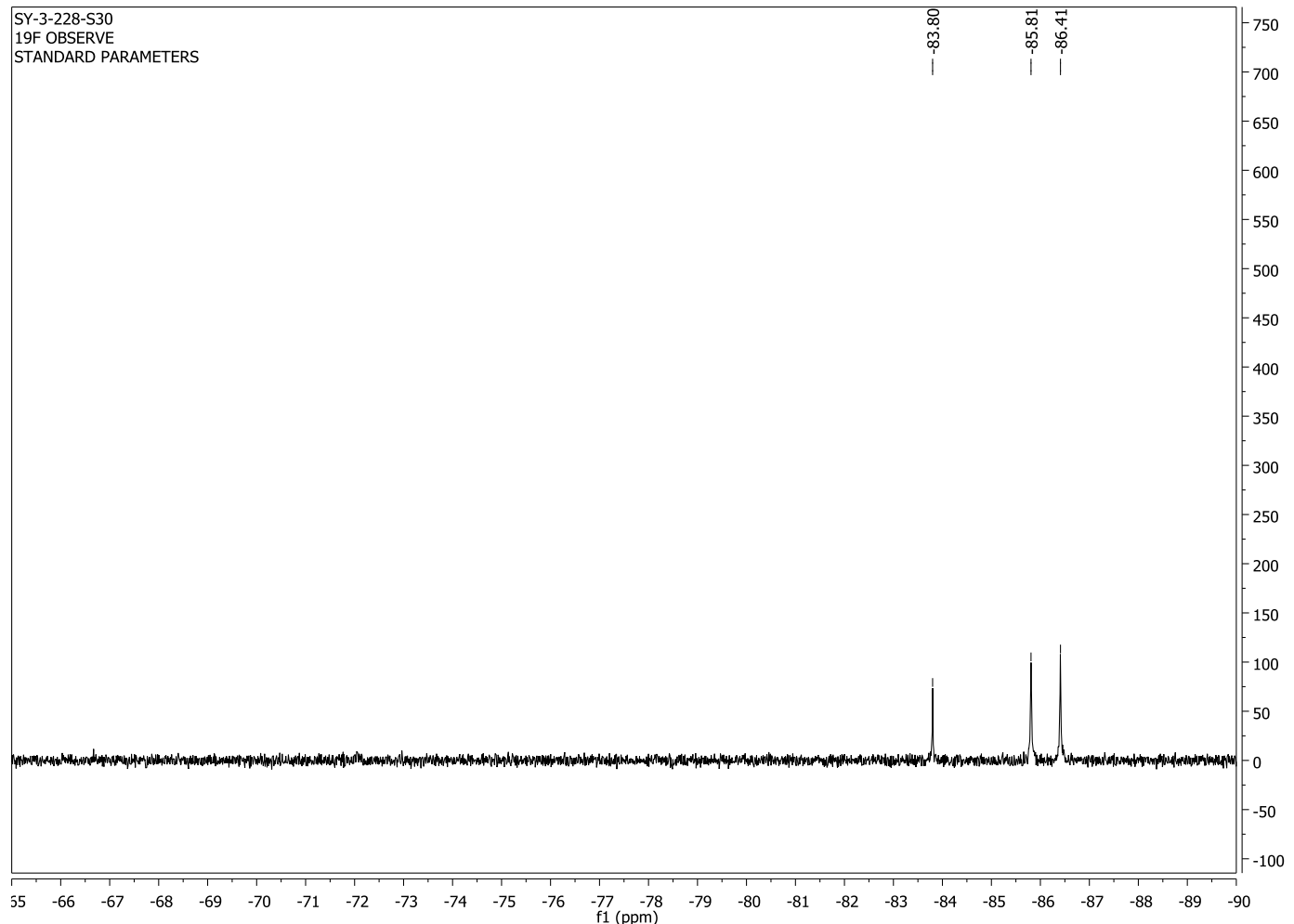

\subsection{9 eq of $\mathbf{N 1 2}$}

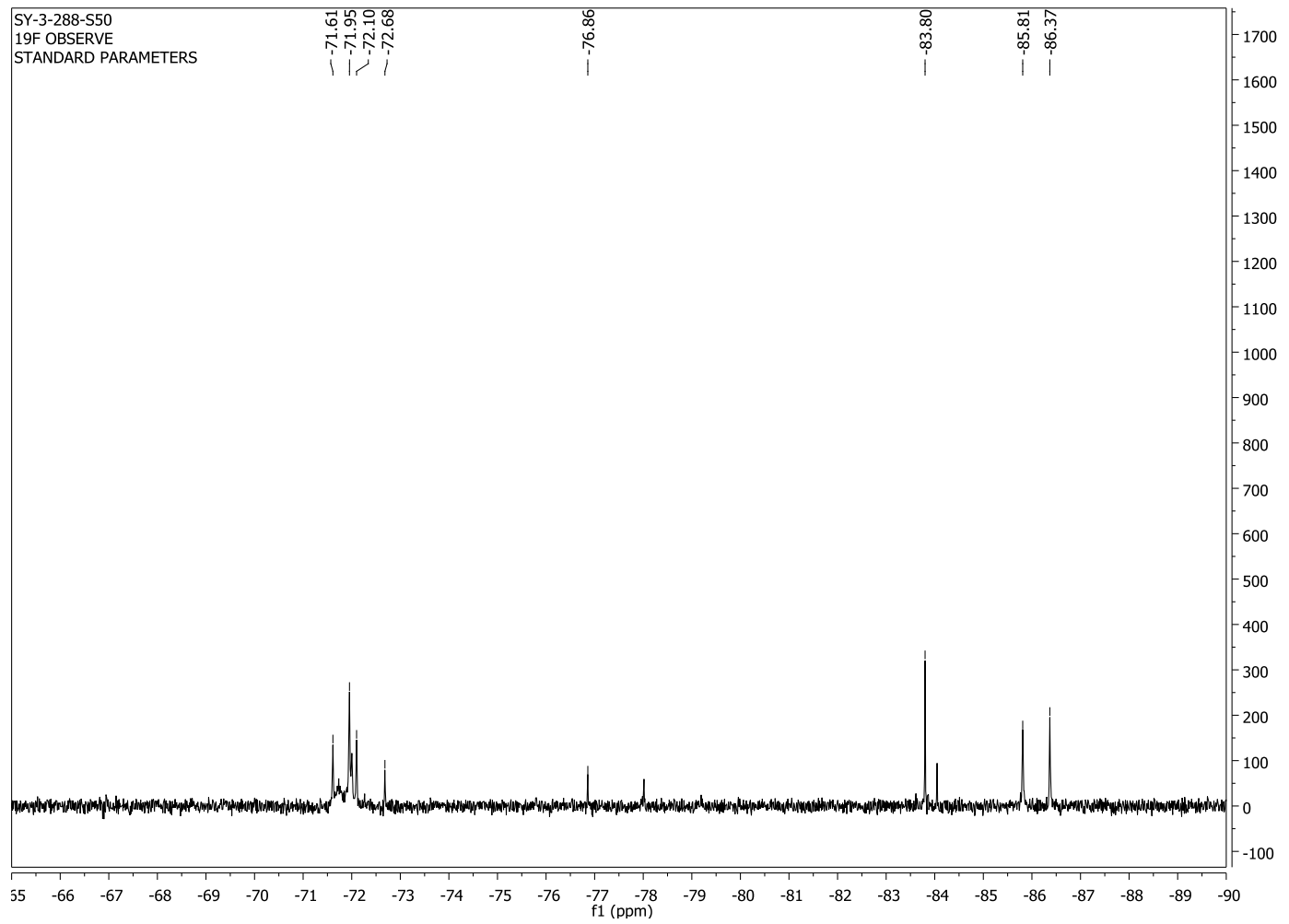


15.3 eq of $\mathbf{N 1 2}$

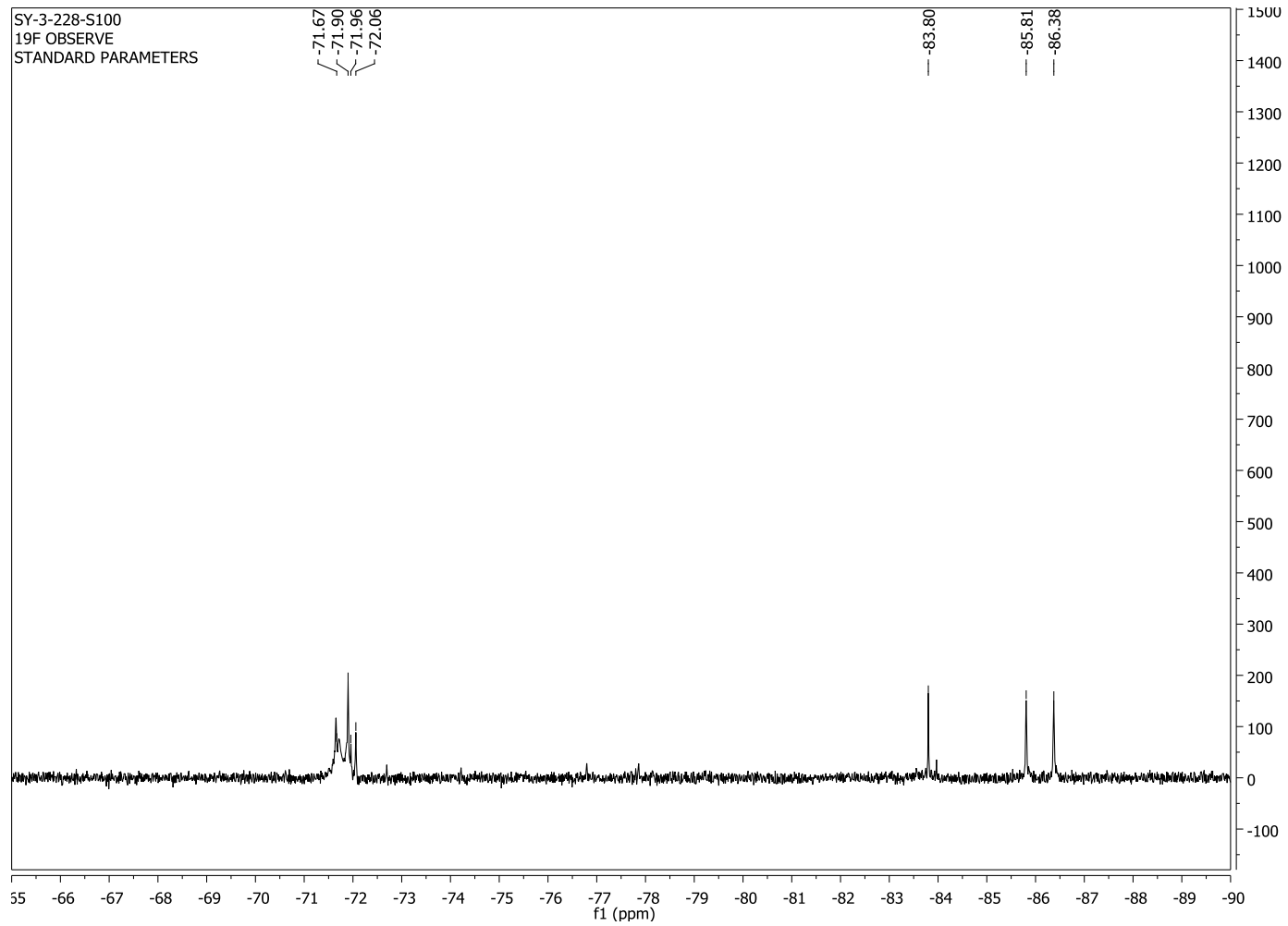

25.5 eq of $\mathbf{N} 12$

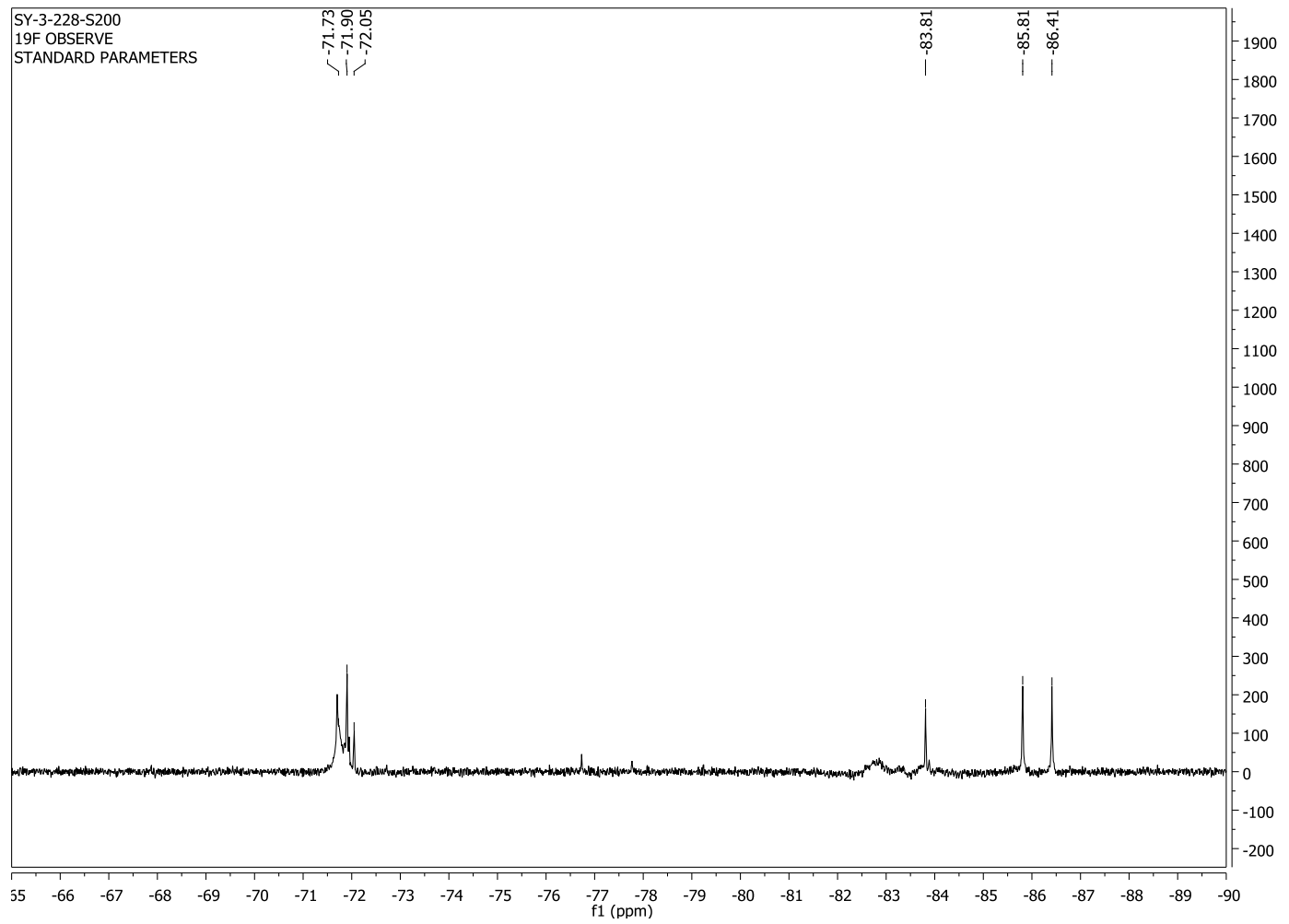


40 eq. of N12

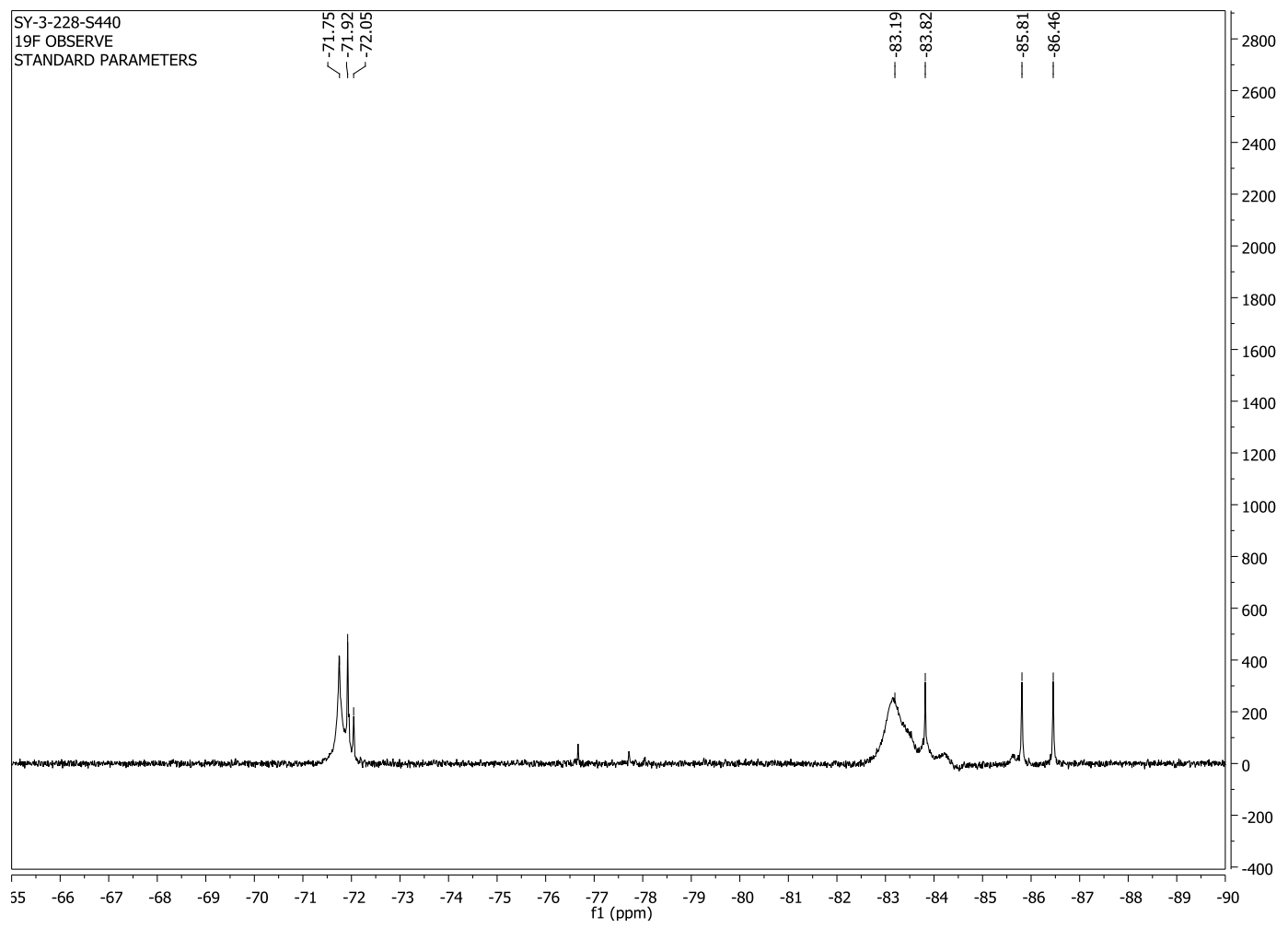


${ }^{19} \mathrm{~F}$ NMR titration of $(S)$-6-4 with $\mathrm{N} 5$ in $\left(\mathrm{CD}_{3}\right)_{2} \mathrm{SO}$

0 eq of $\mathrm{N} 5$

SY-4-77-0

SYF OBSERVE

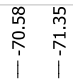

\%ू
लू
1
1

$-2000$

STANDAR PARAMETERS

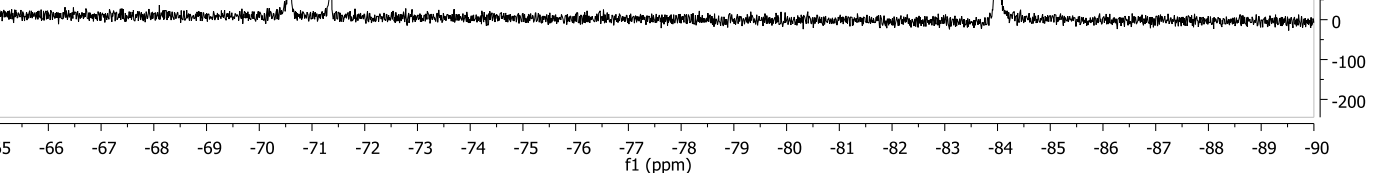

\section{2 eq of $\mathrm{N} 5$}

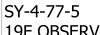

STANDARD PARAMETERS
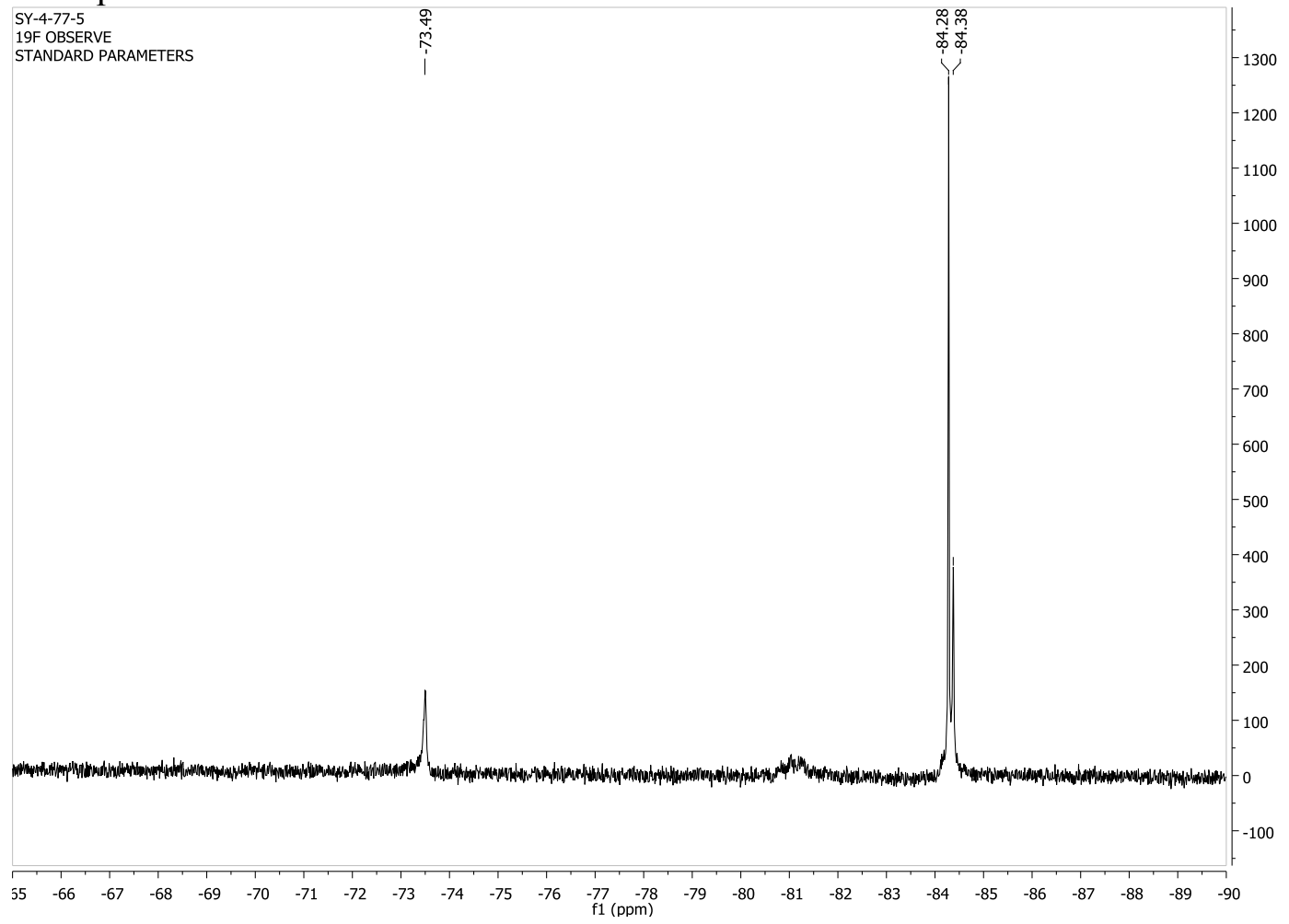


\section{6 eq of N5}

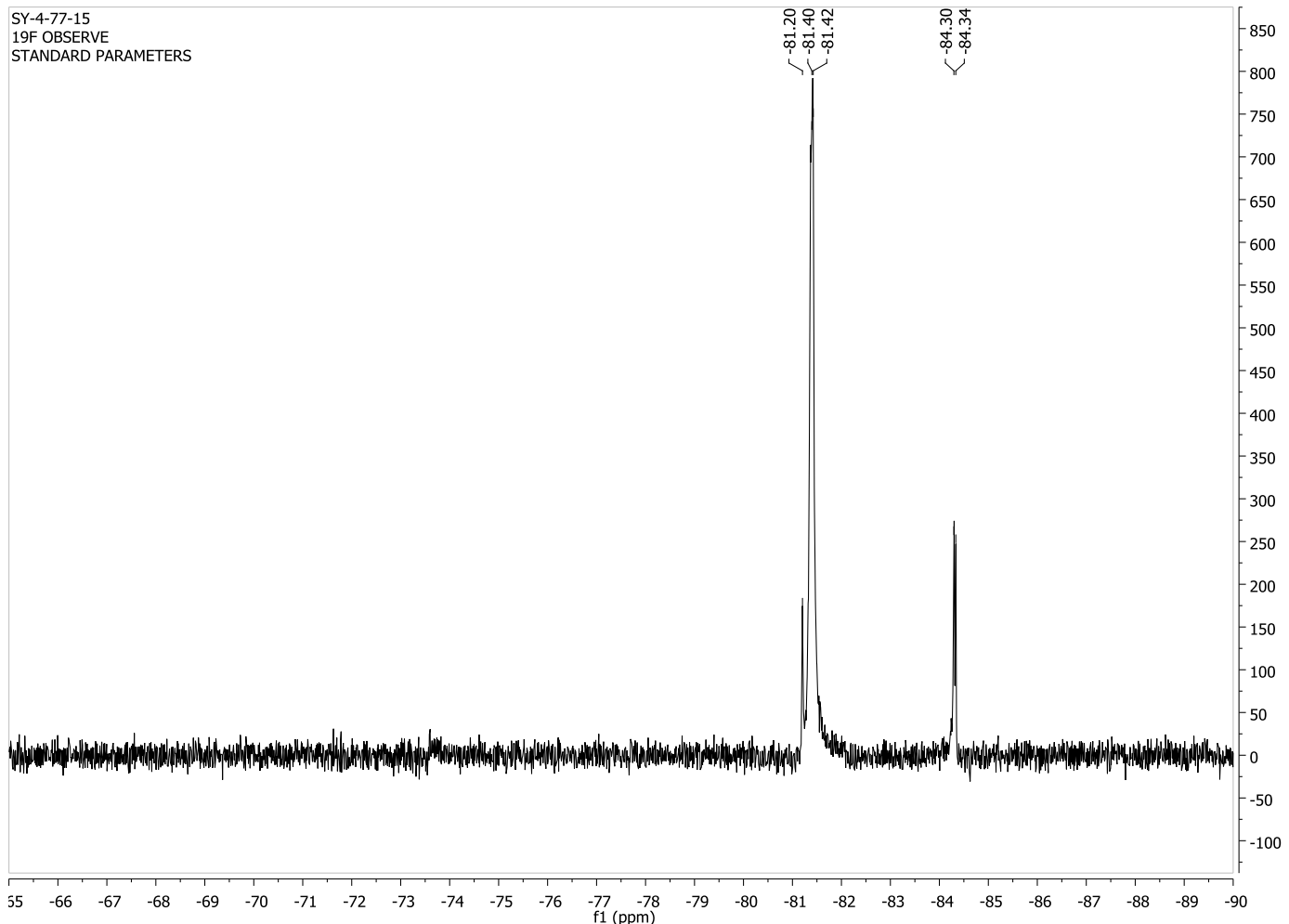

\section{9 eq of N5}

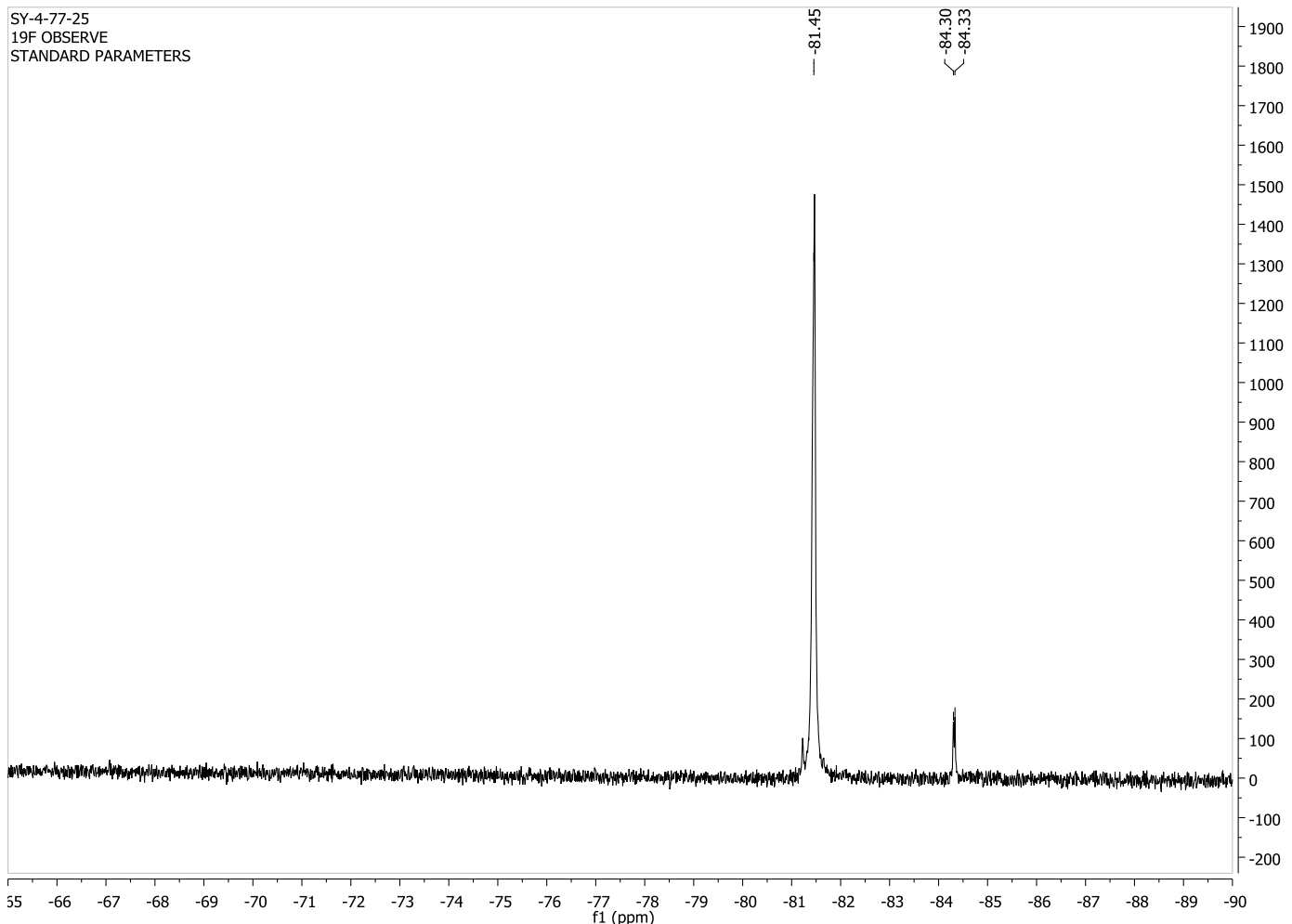




\section{1 eq of $\mathrm{N} 5$}

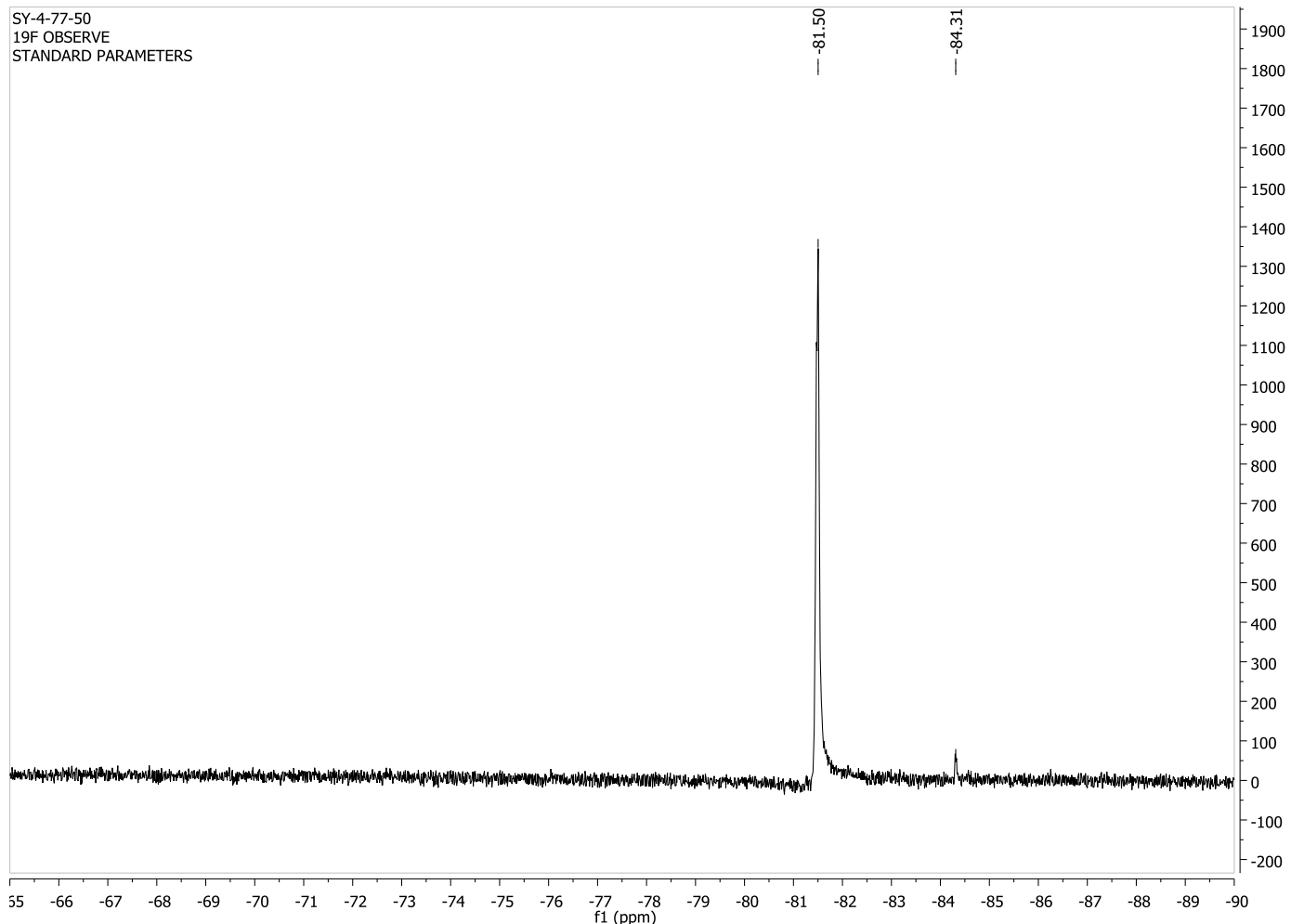

\section{0 eq of N5}

SY-4-77-100
19F OBSERVE

STANDARD PARAMETERS
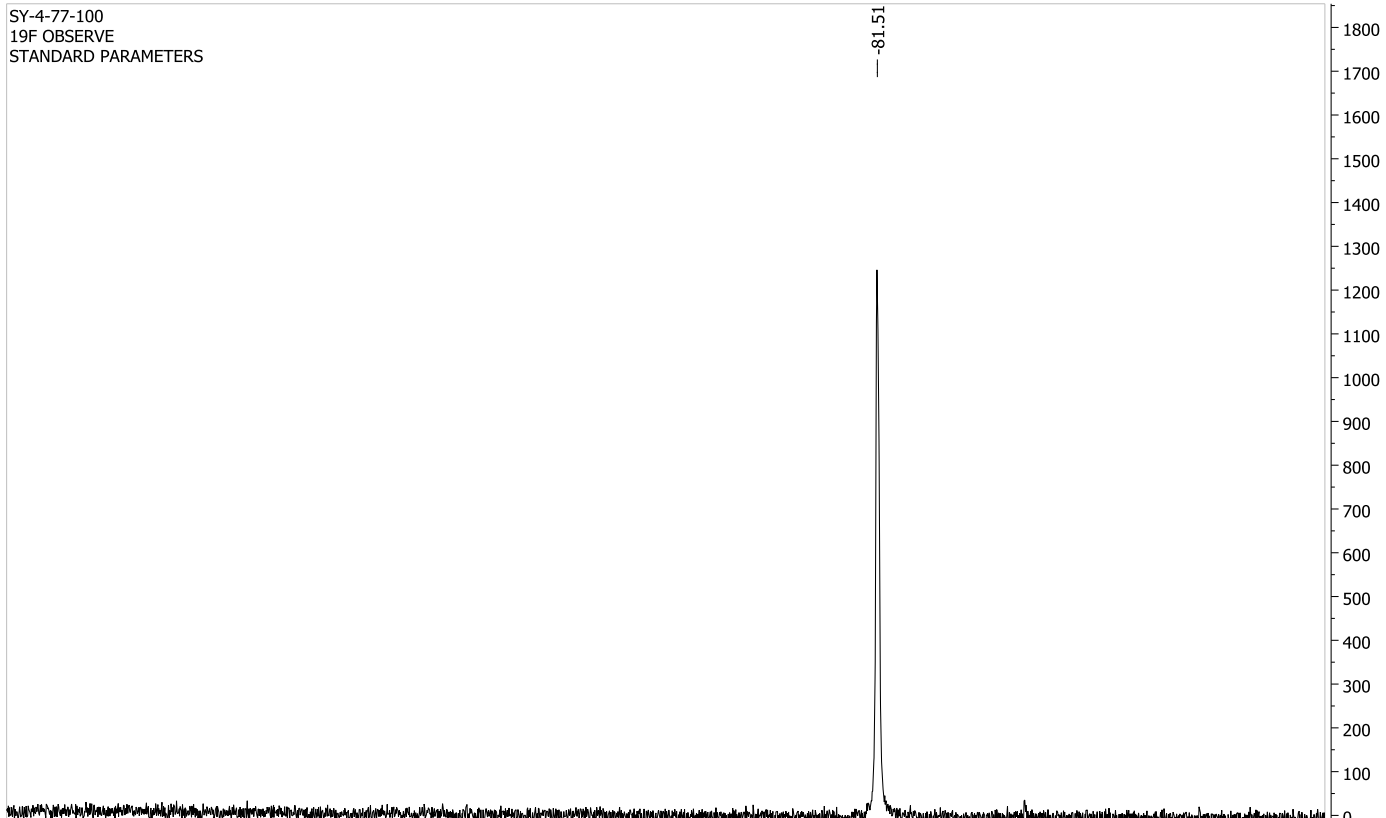

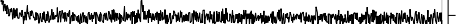

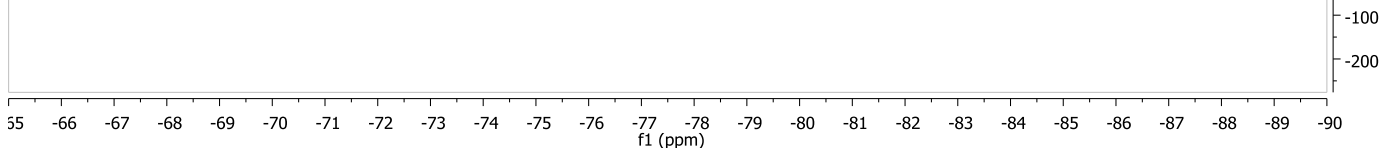


${ }^{1} \mathrm{H}$ NMR spectrum of (S)-6-4 with 5 eq $\mathbf{N 5}$ in $\left(\mathrm{CD}_{3}\right)_{2} \mathrm{SO}$

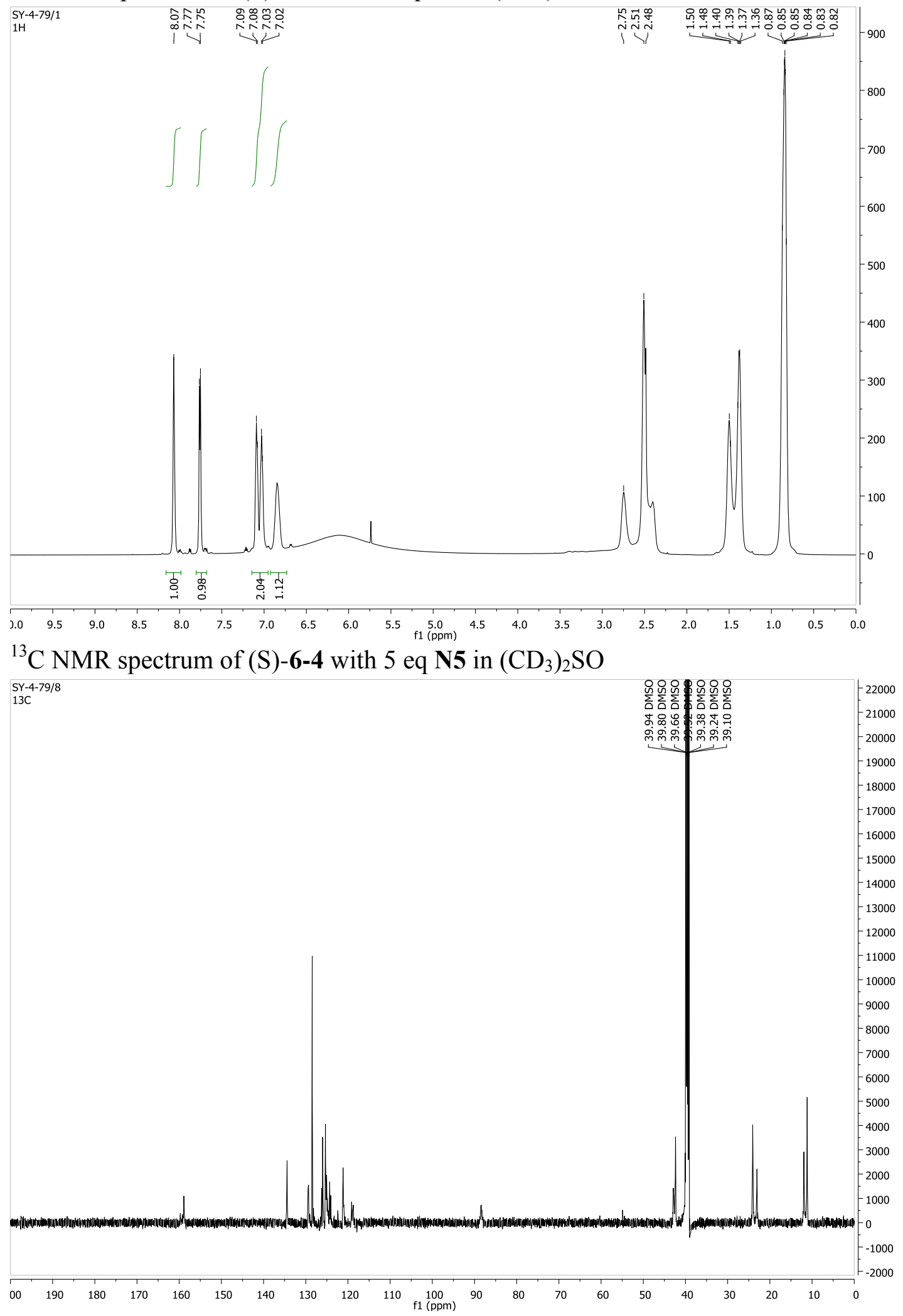




\section{${ }^{19}$ F NMR titration of $(S)-6-16$ with $(S, S)$-N15 in $\mathrm{CDCl}_{3}$}

\section{0 eq of N15 \\ SY-4-74-0}

OBSERVE
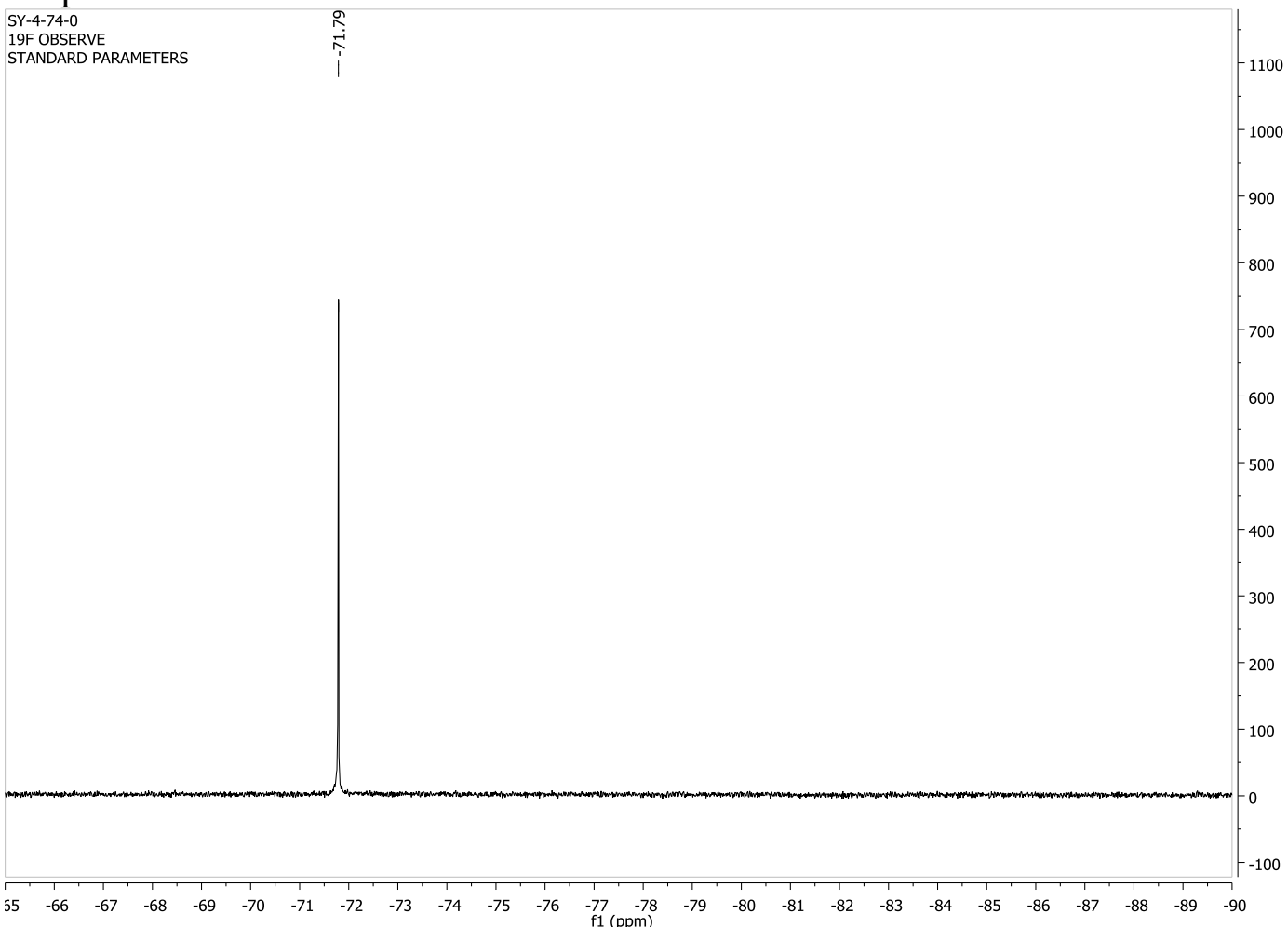

0.2 eq of $\mathrm{N} 15$

SY-4-74-2

STANDARD PARAMETERS
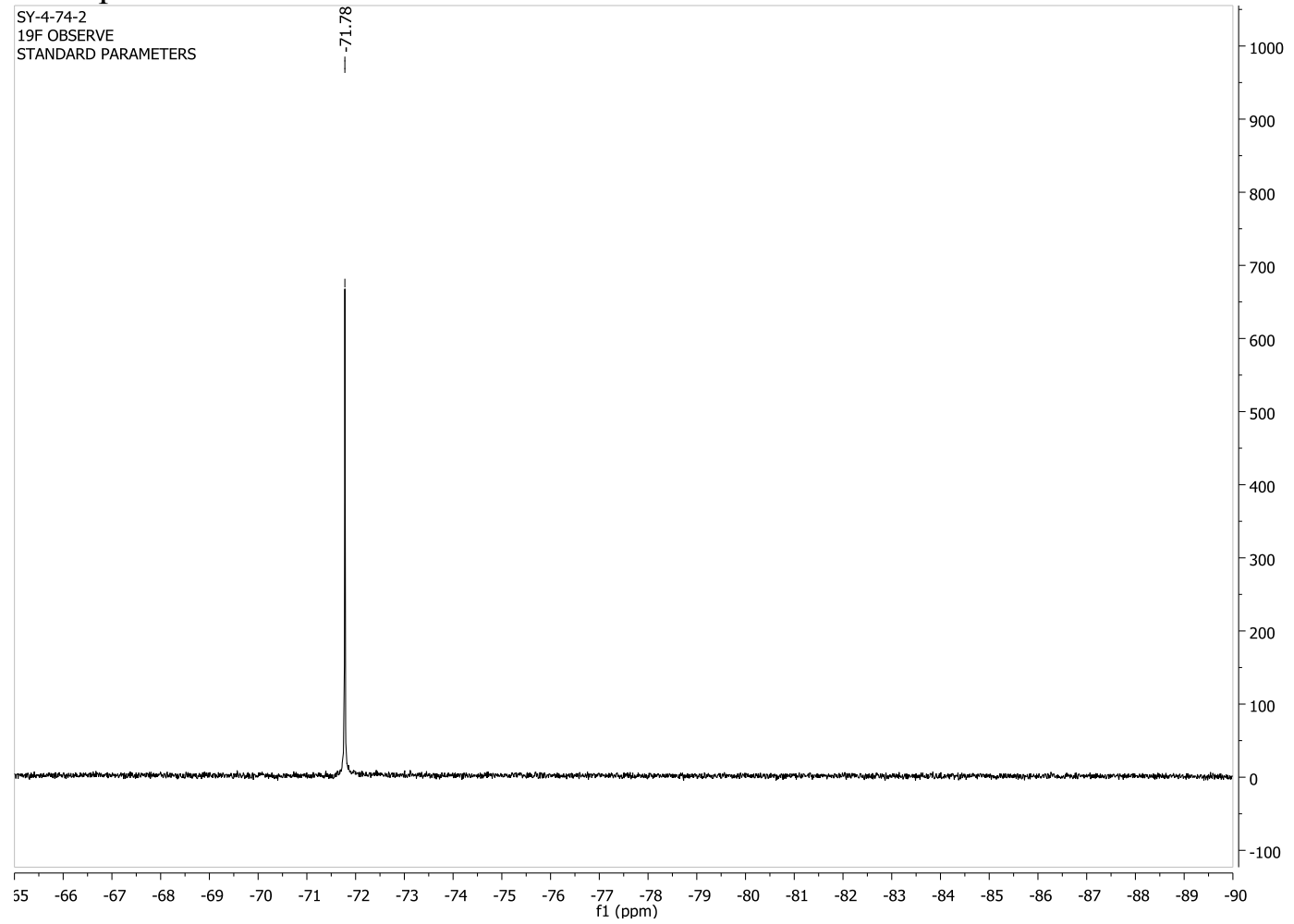


\section{7 eq of N15}

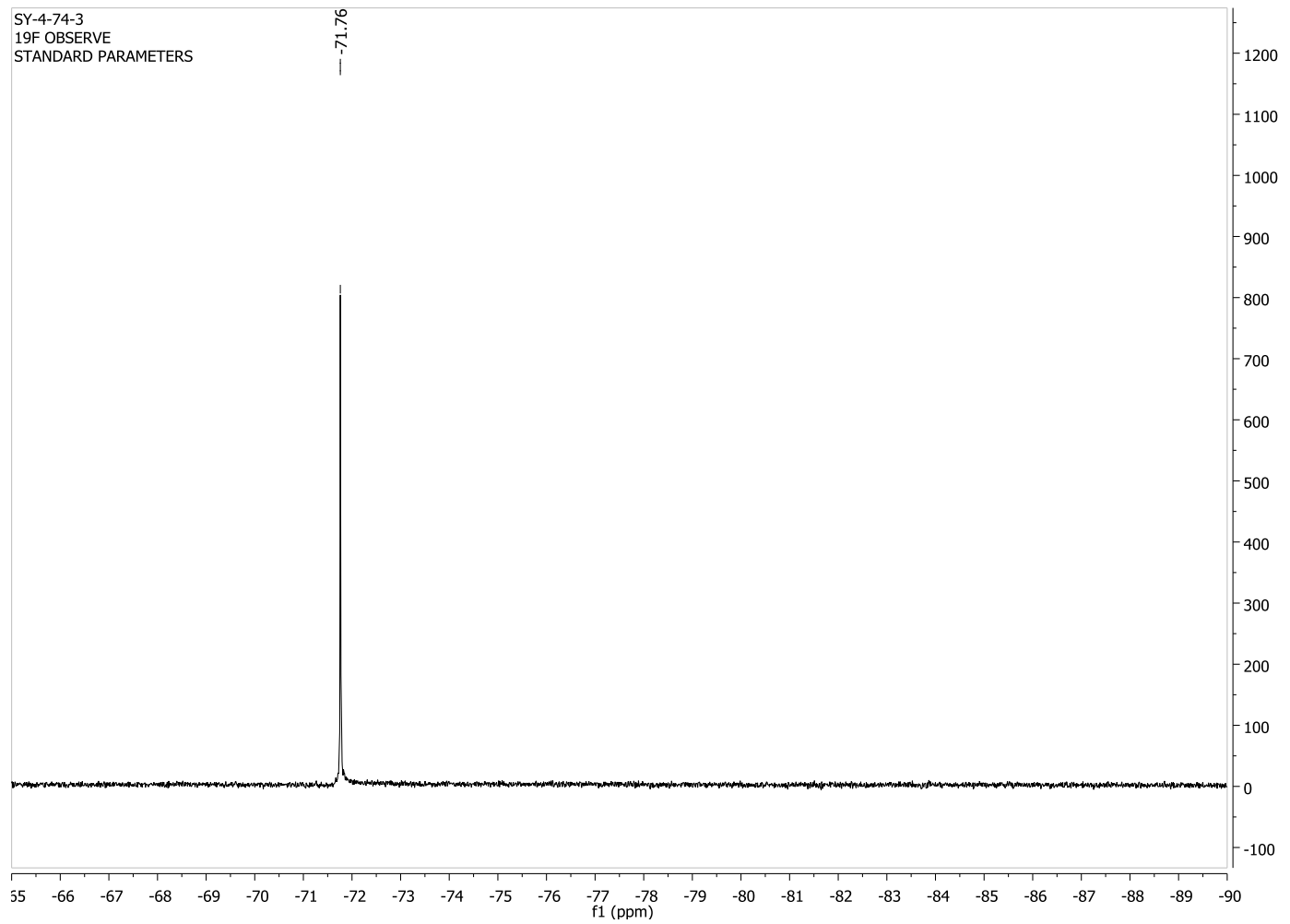

\section{2 eq of N15}

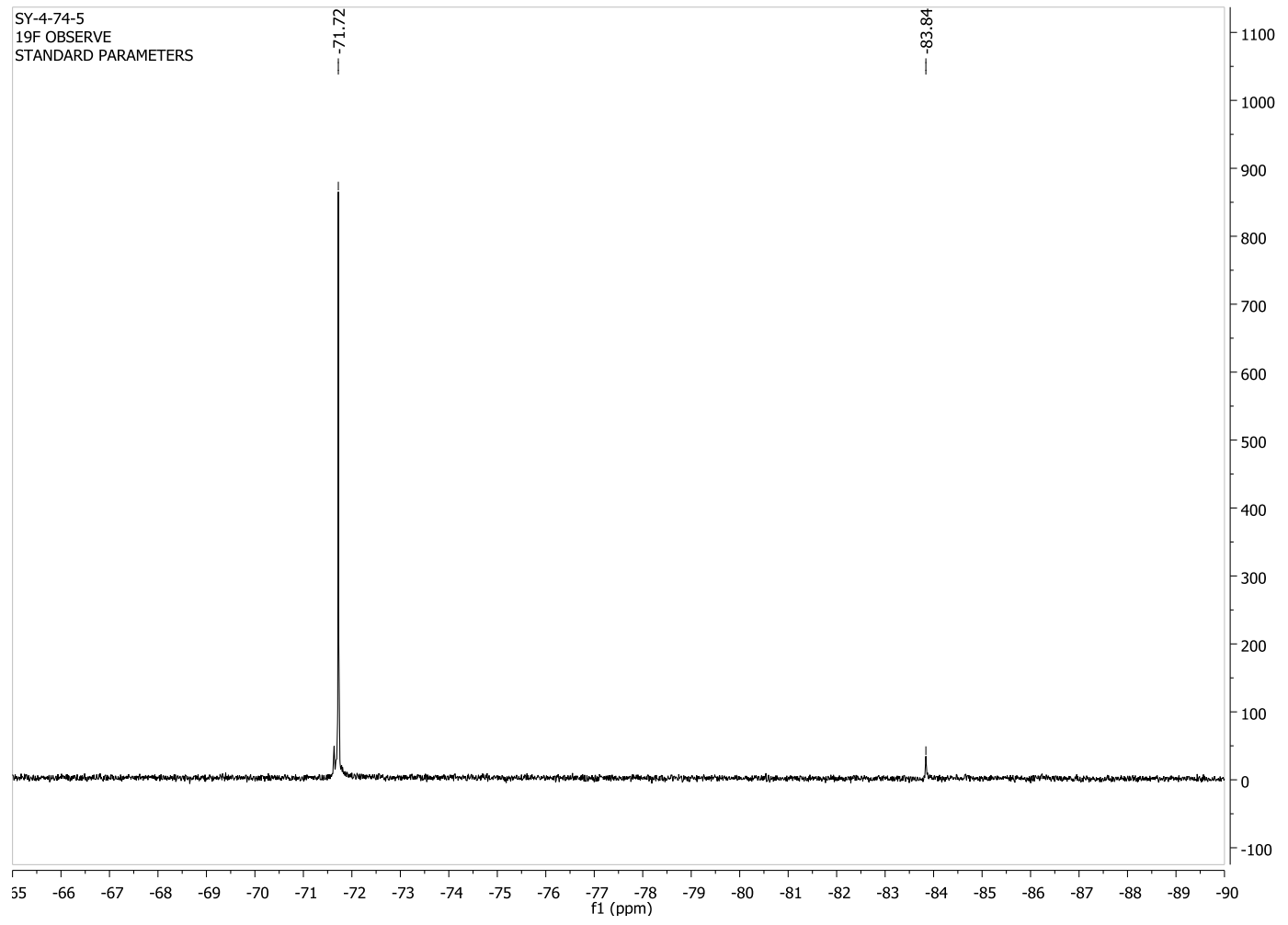




\section{4 eq of N15}

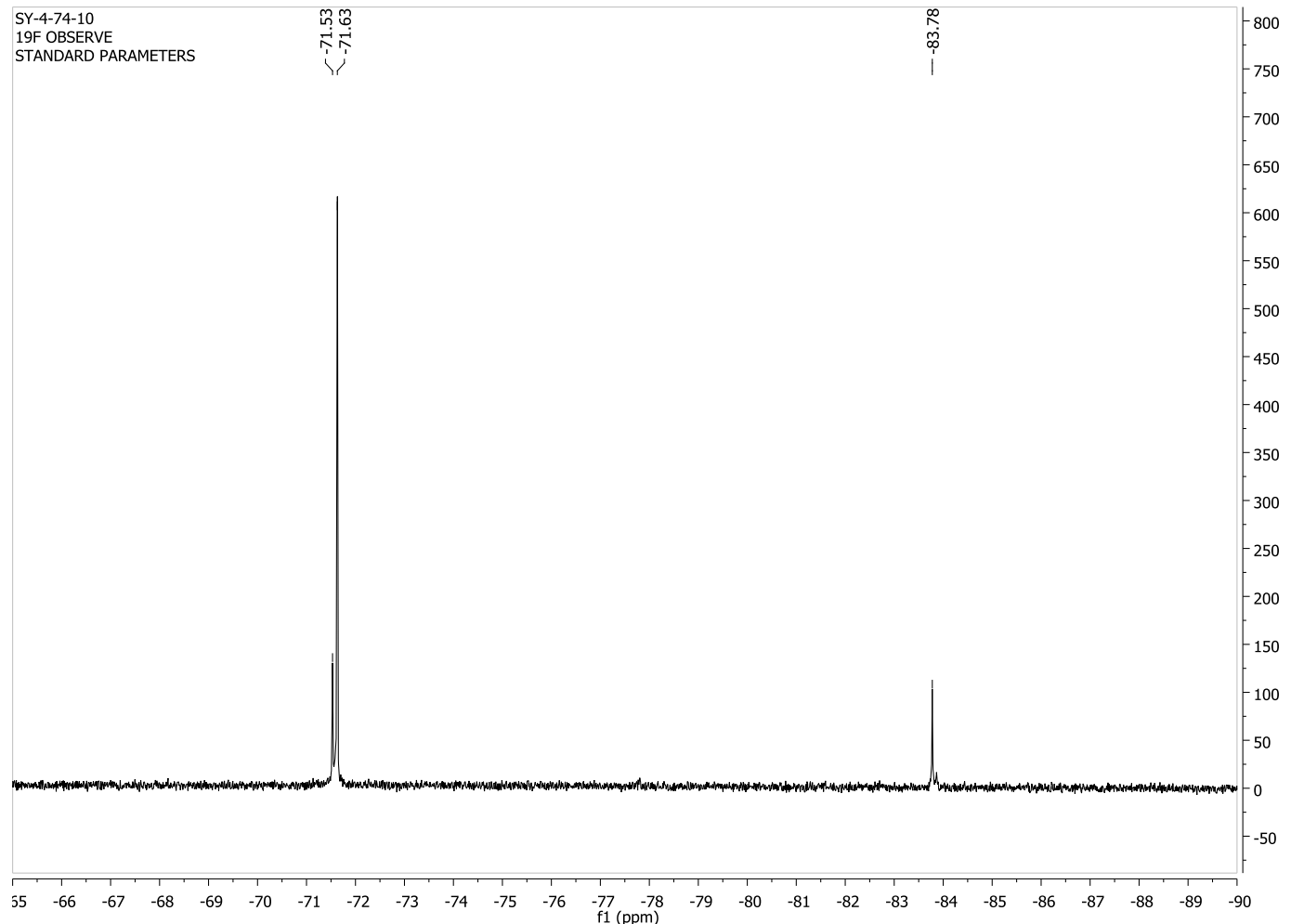

\section{6 eq of N15}

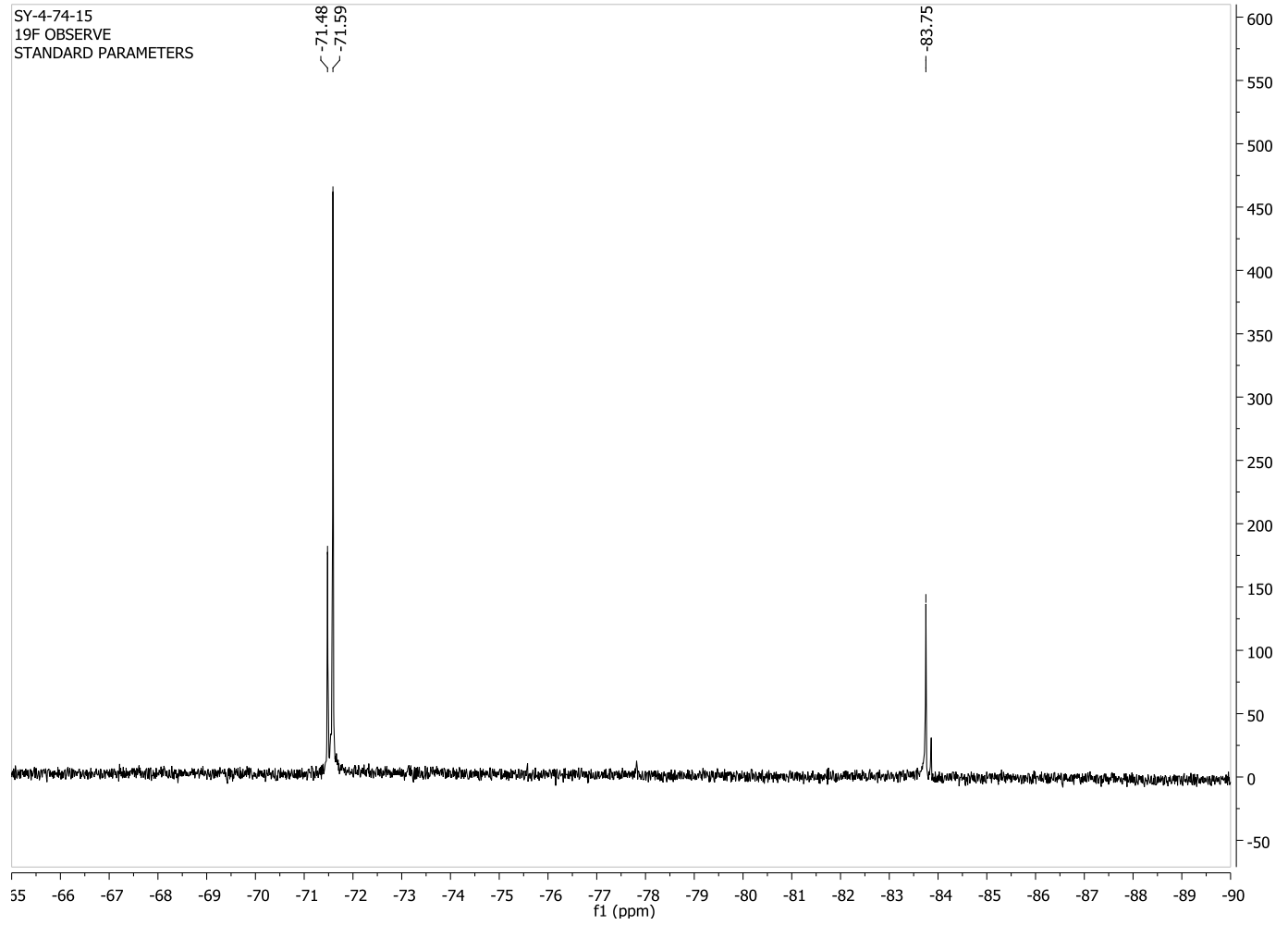




\section{8 eq of N15}

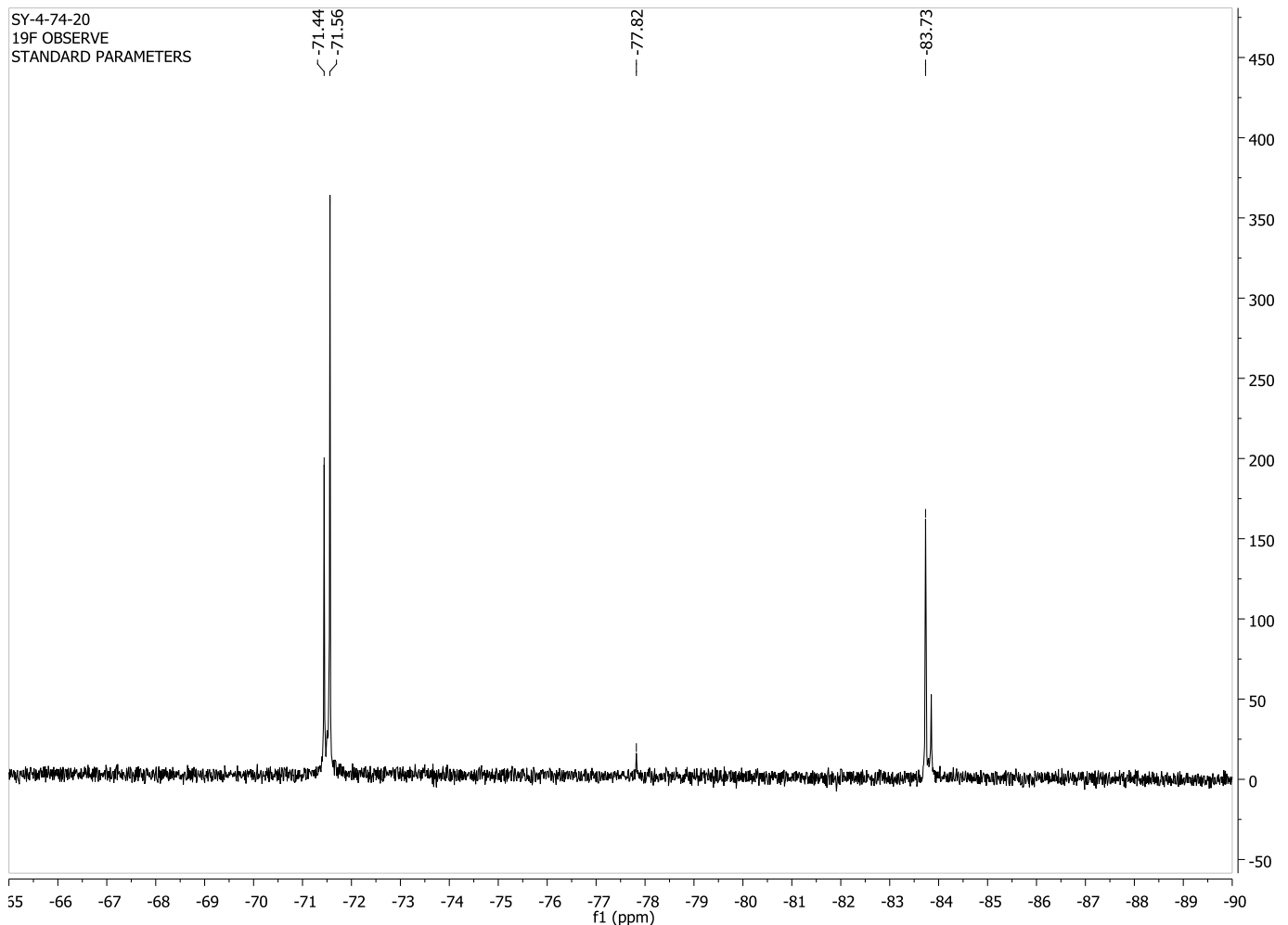

\section{0 eq of N15}

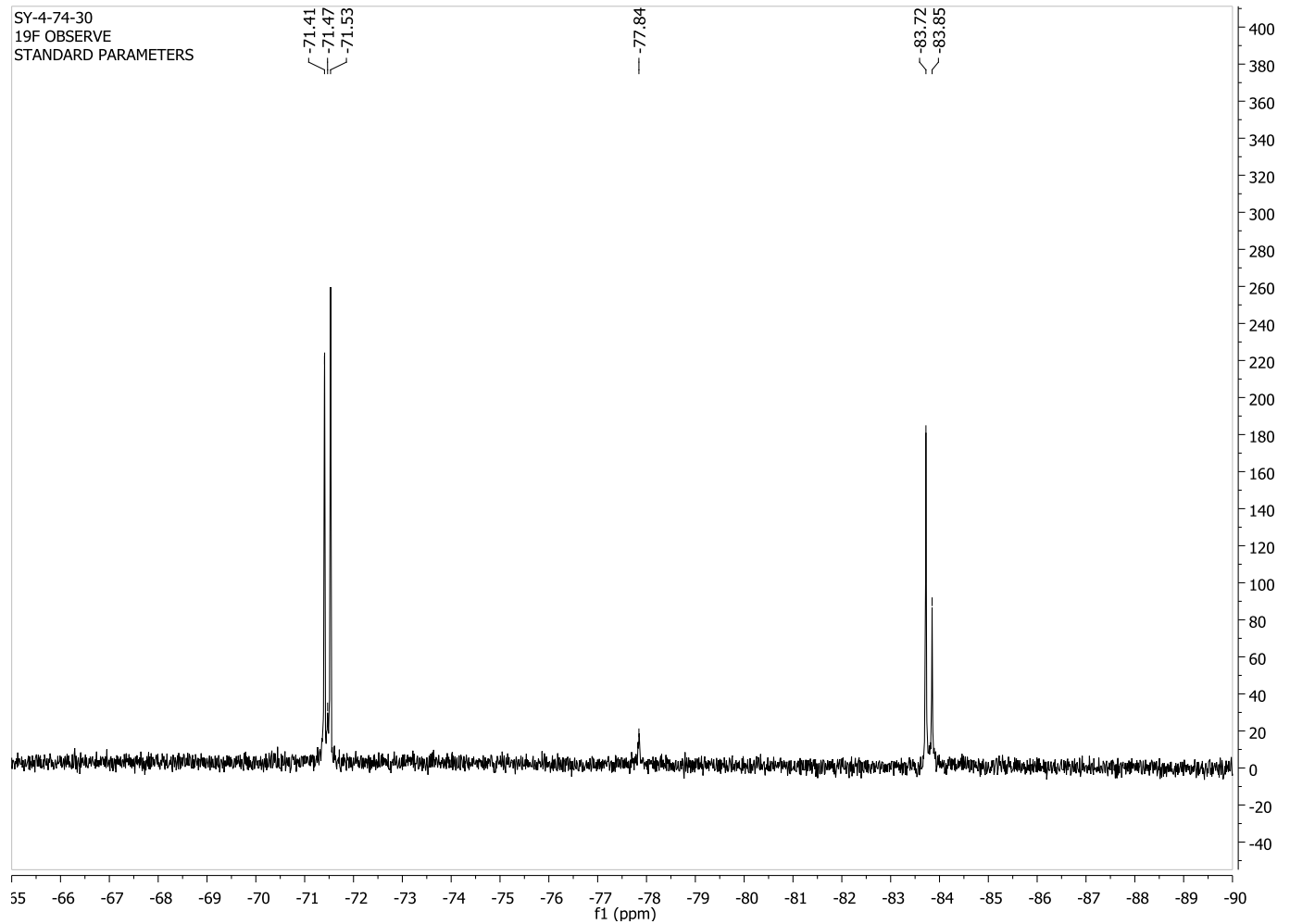




\section{1 eq of N15}

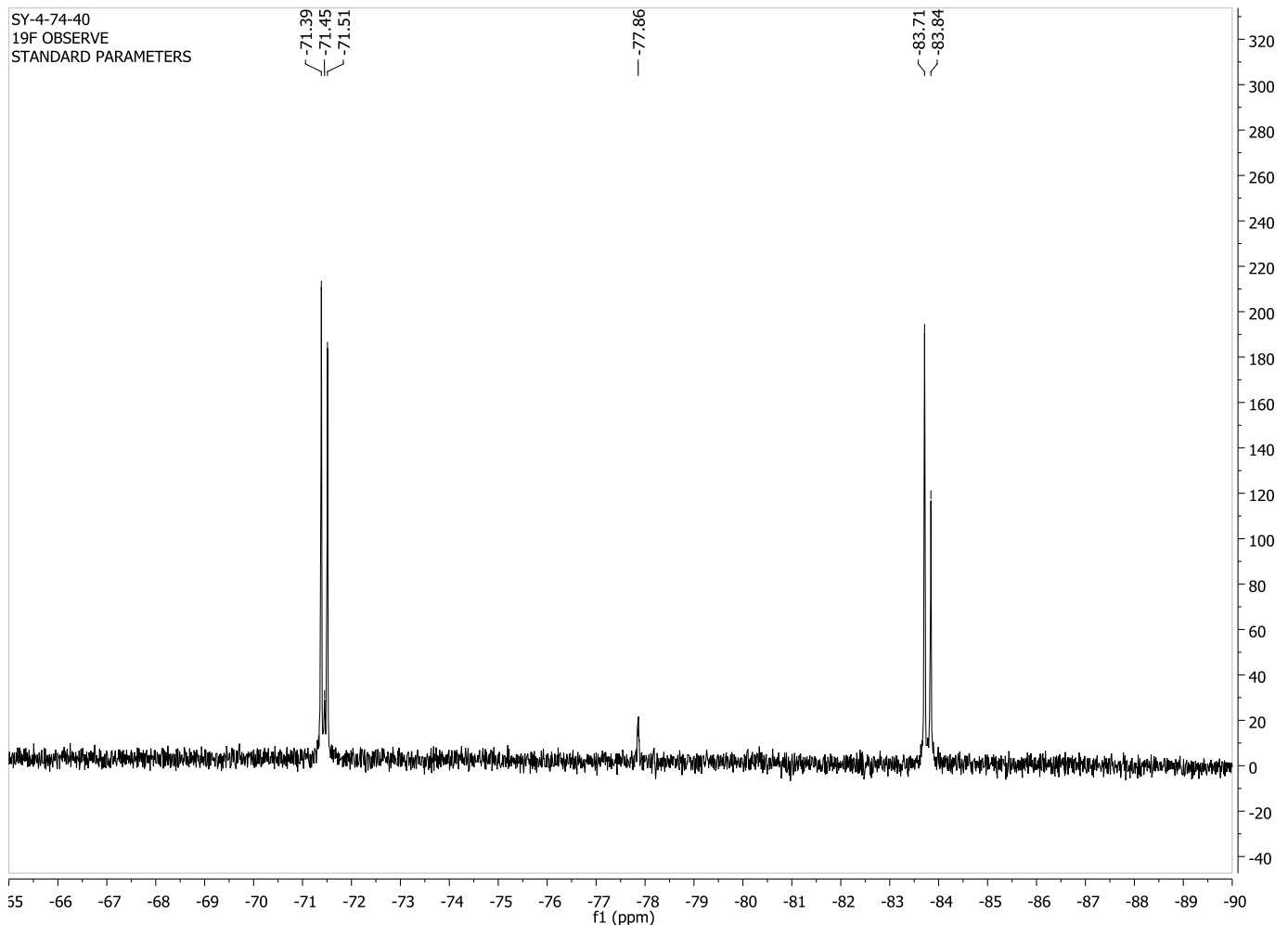

\section{1 eq of N15}

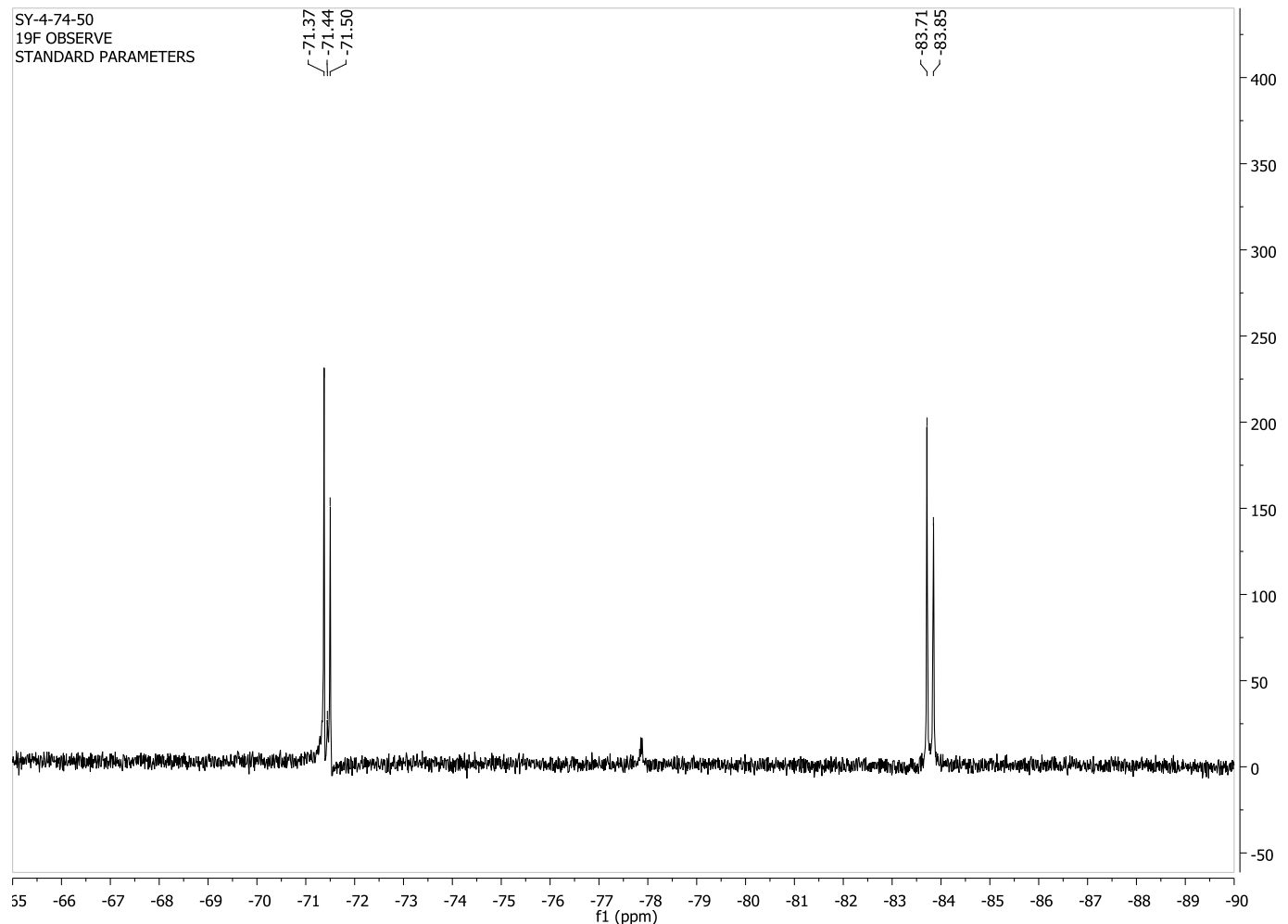


15.8 eq of N15

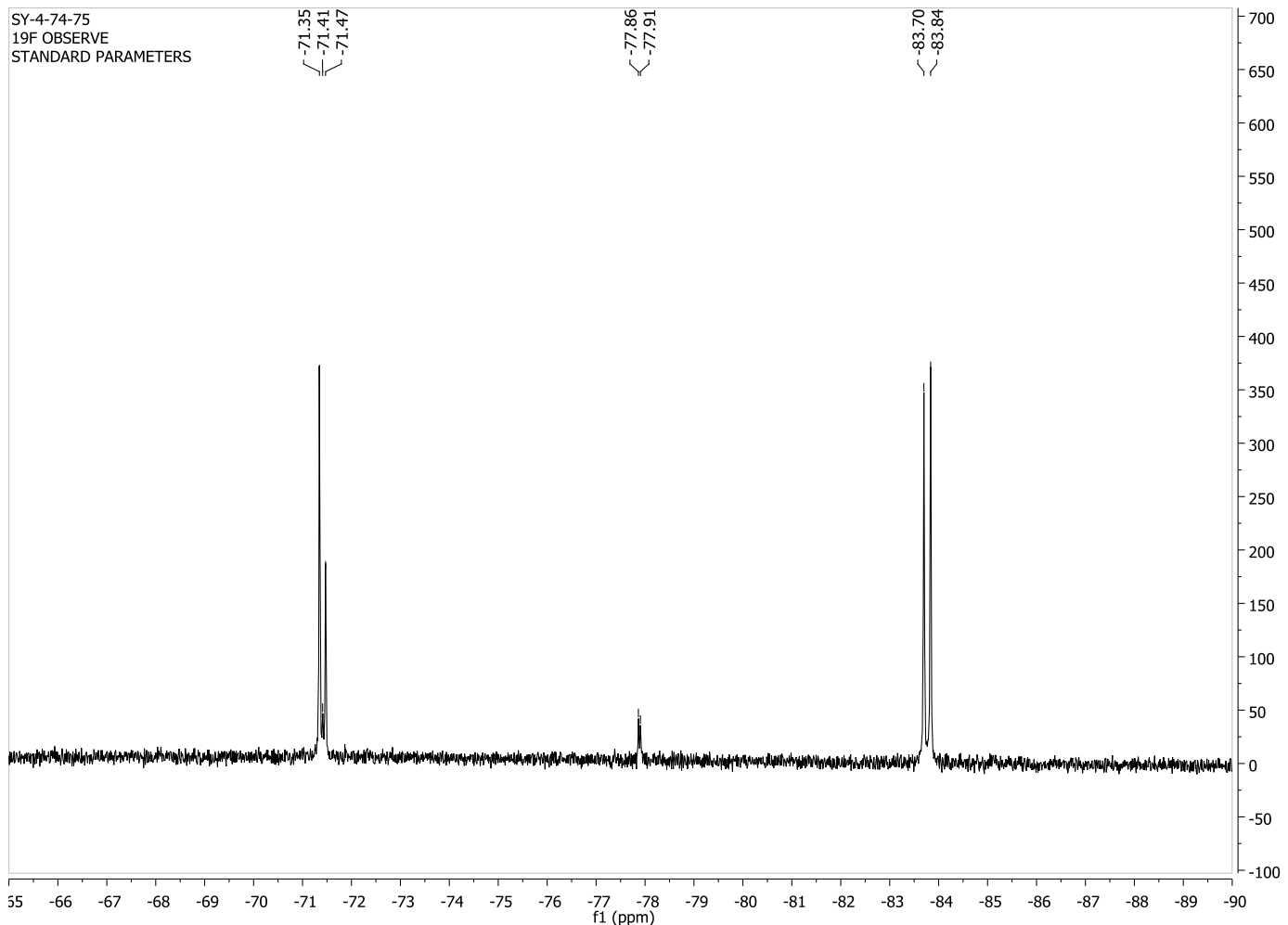

20 eq of N15

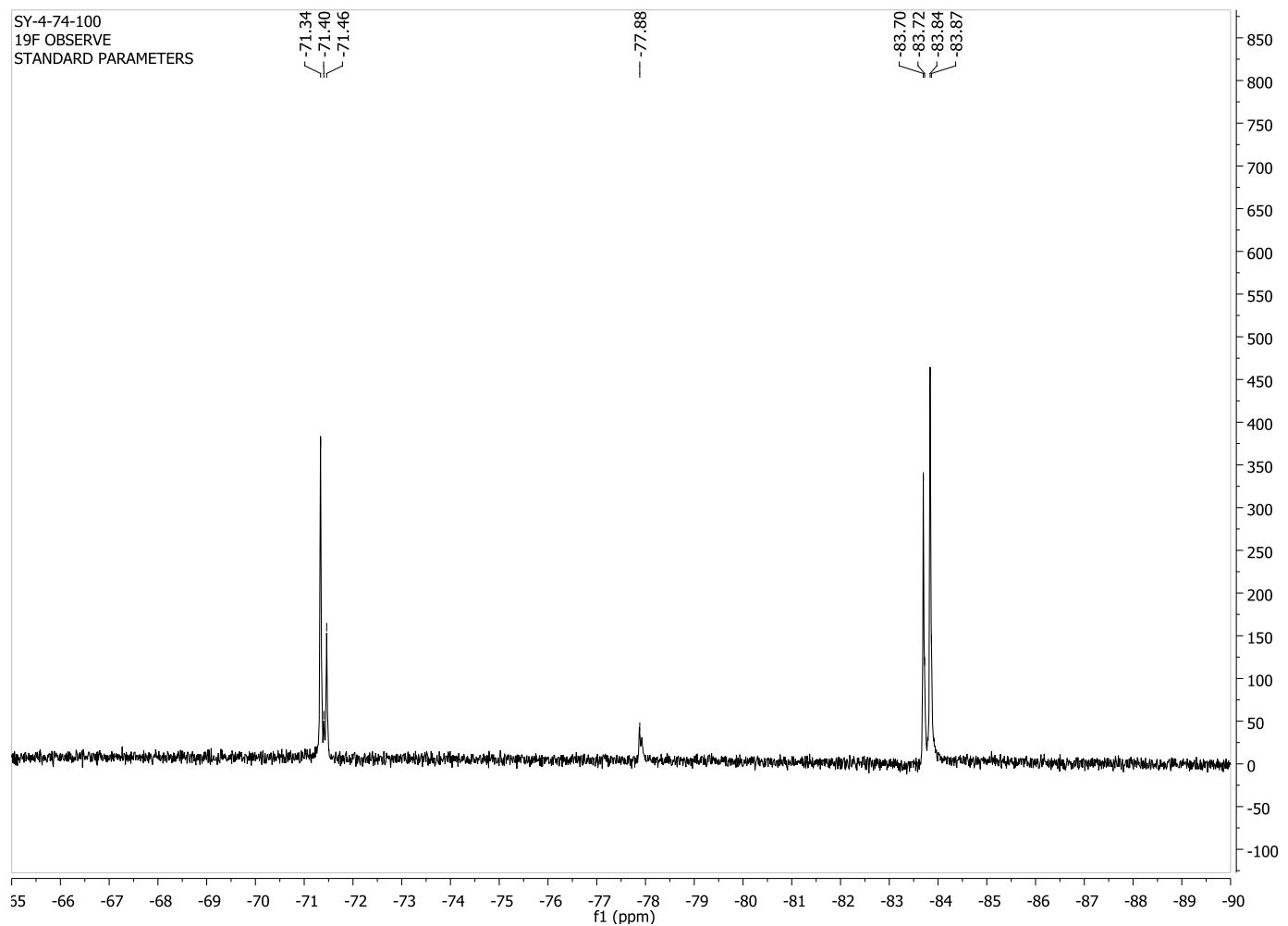




\section{3 eq of N15}

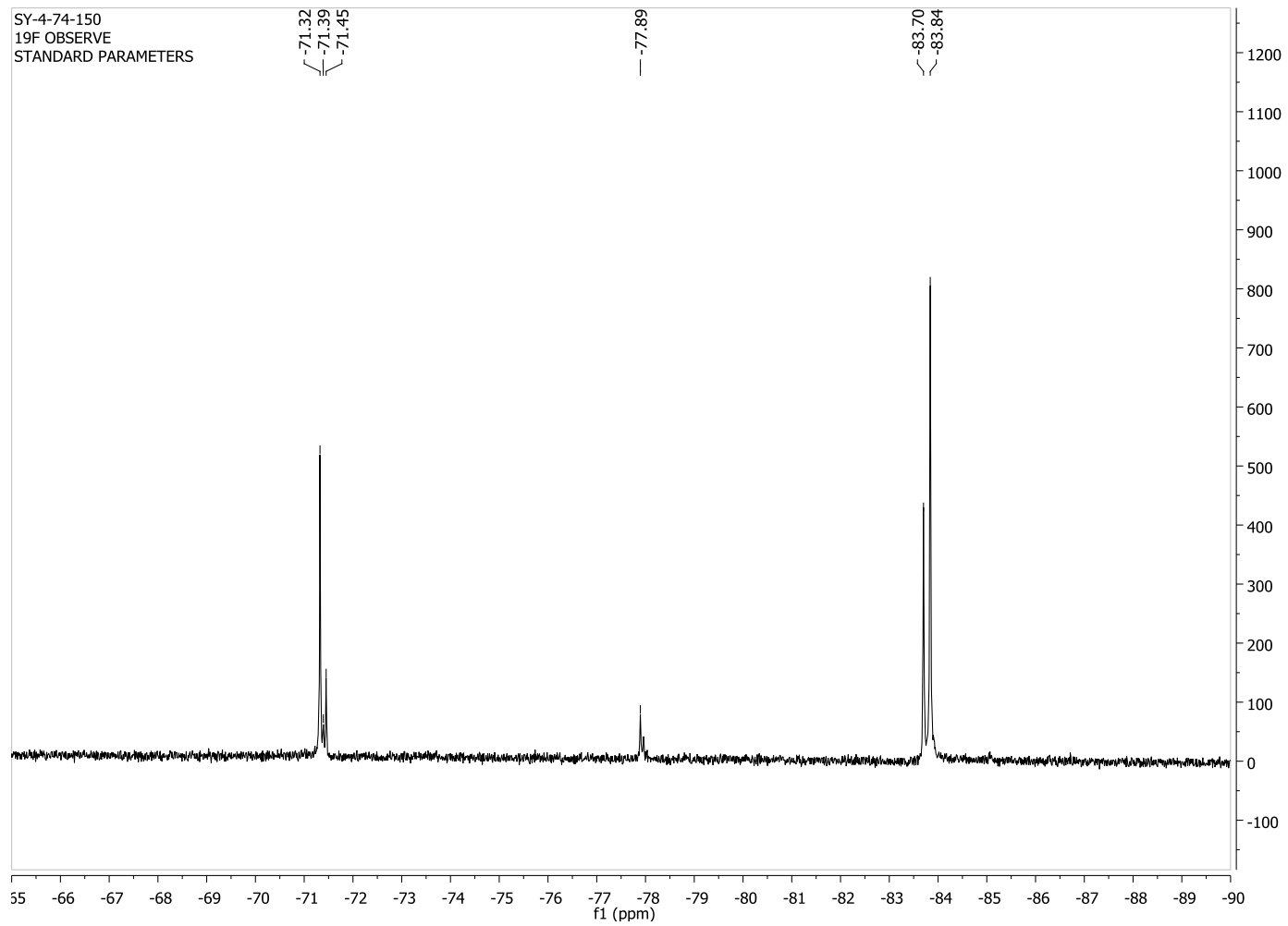

27.3 eq of N15

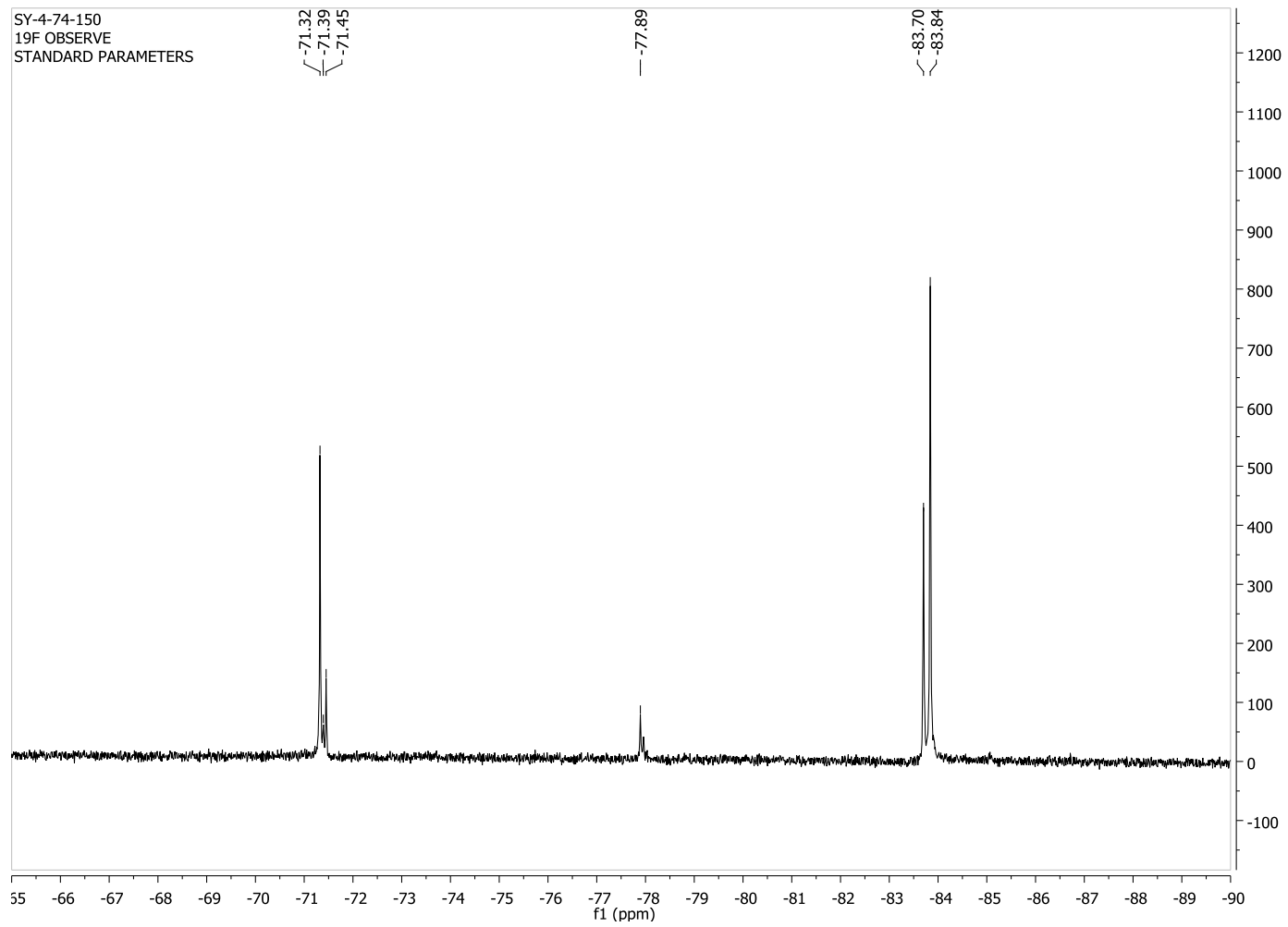


33.3 eq of N15

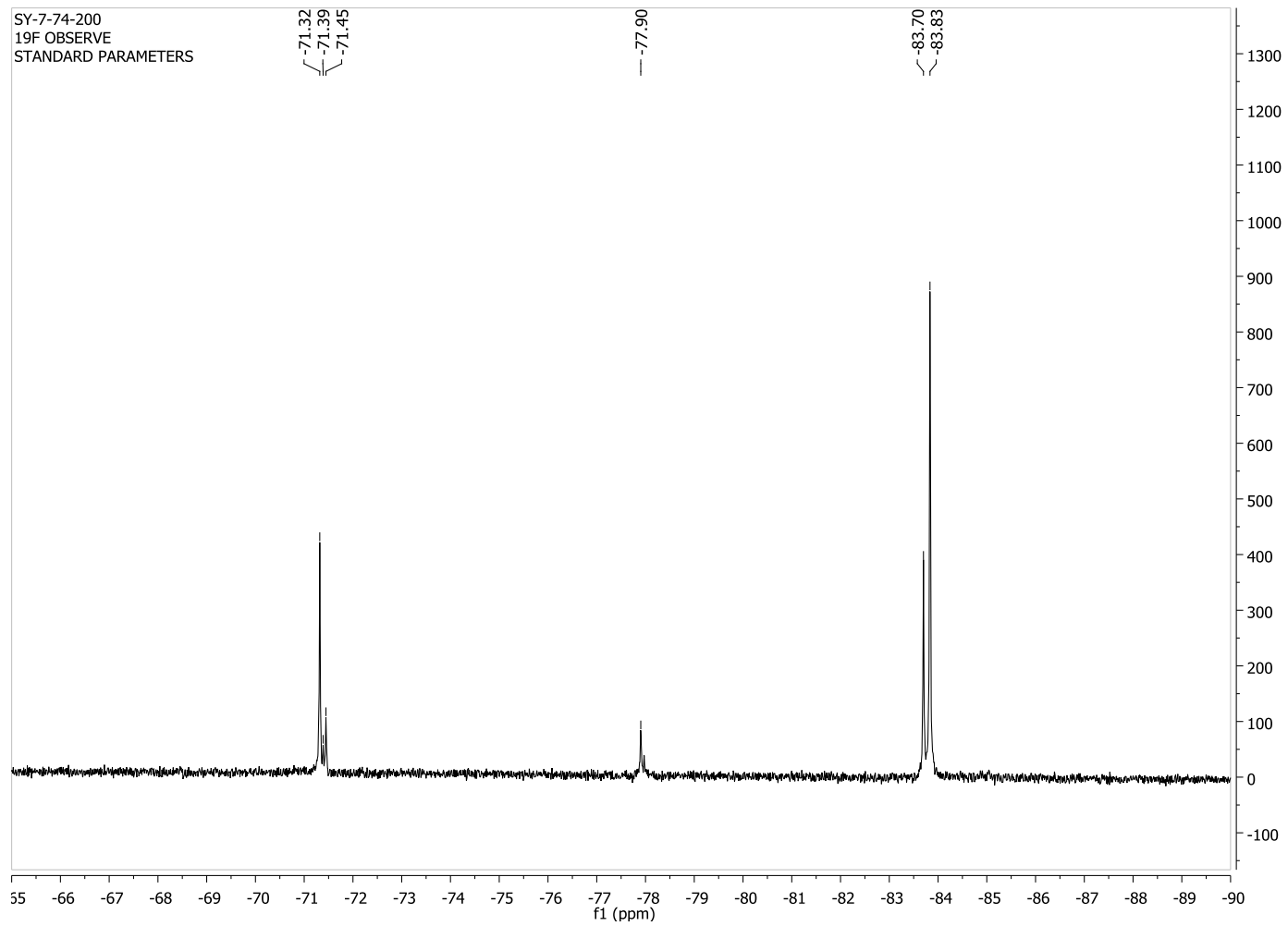

42.9 eq of N15

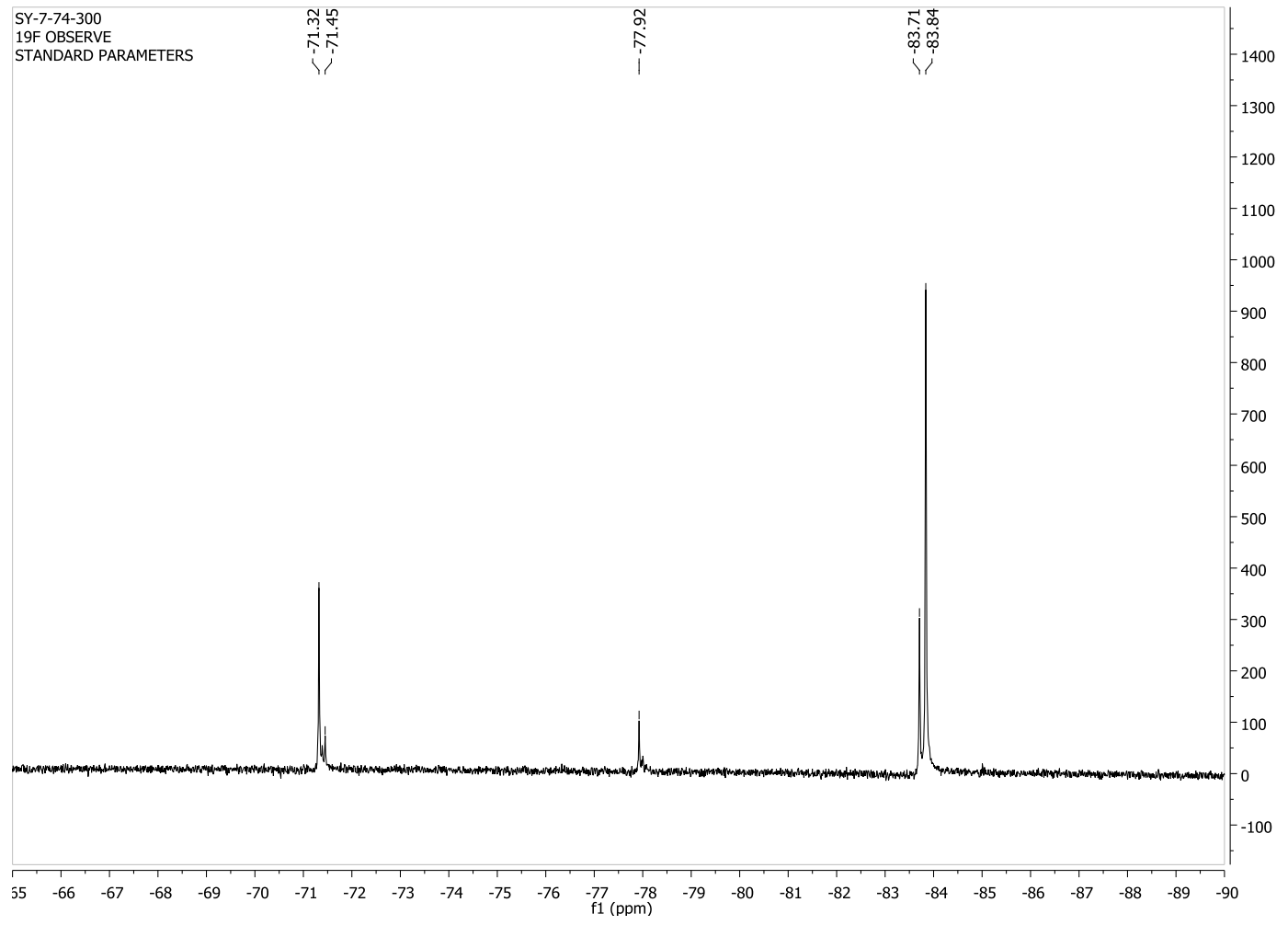




\section{0 eq of N15}

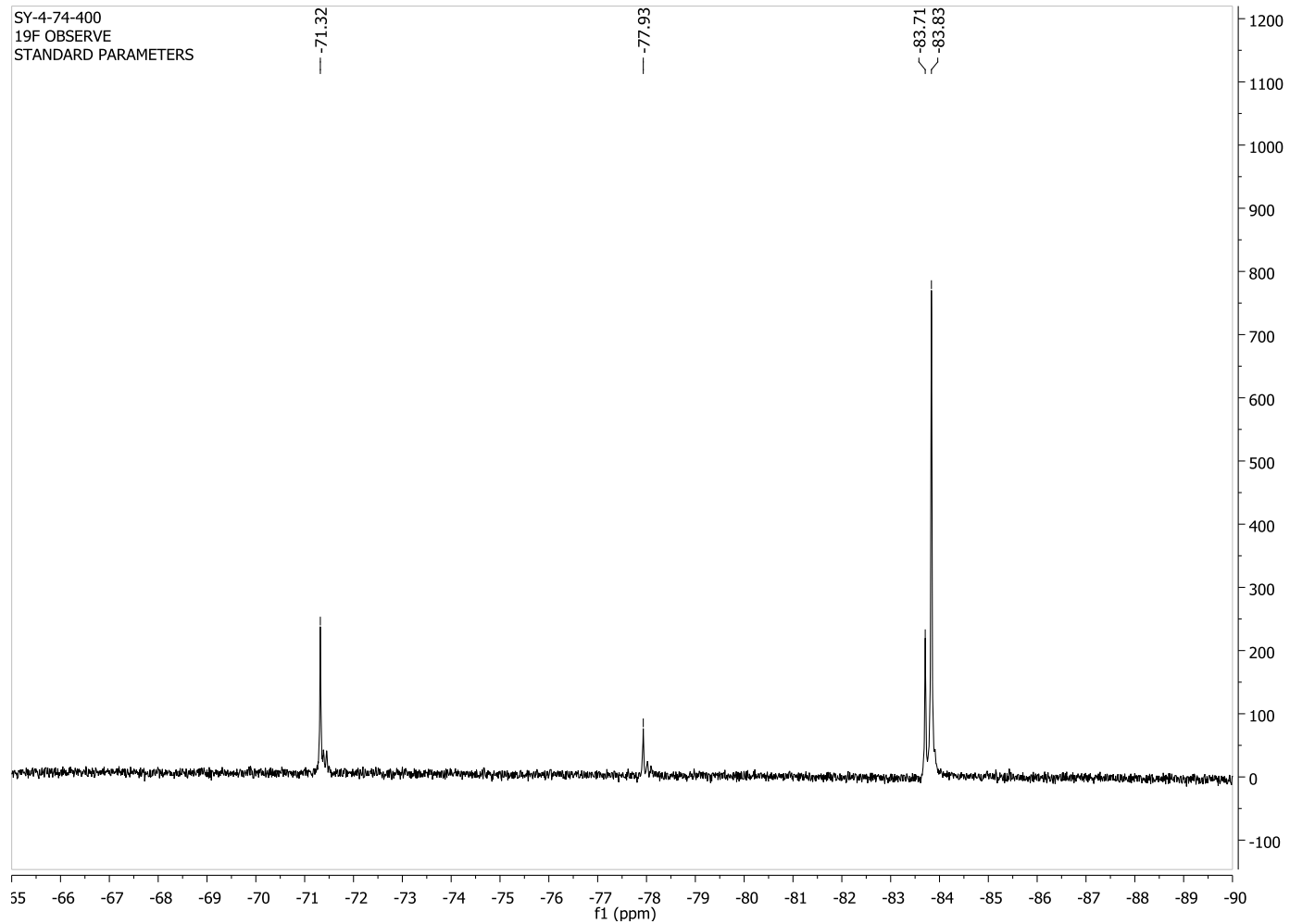

\section{6 eq of N15}

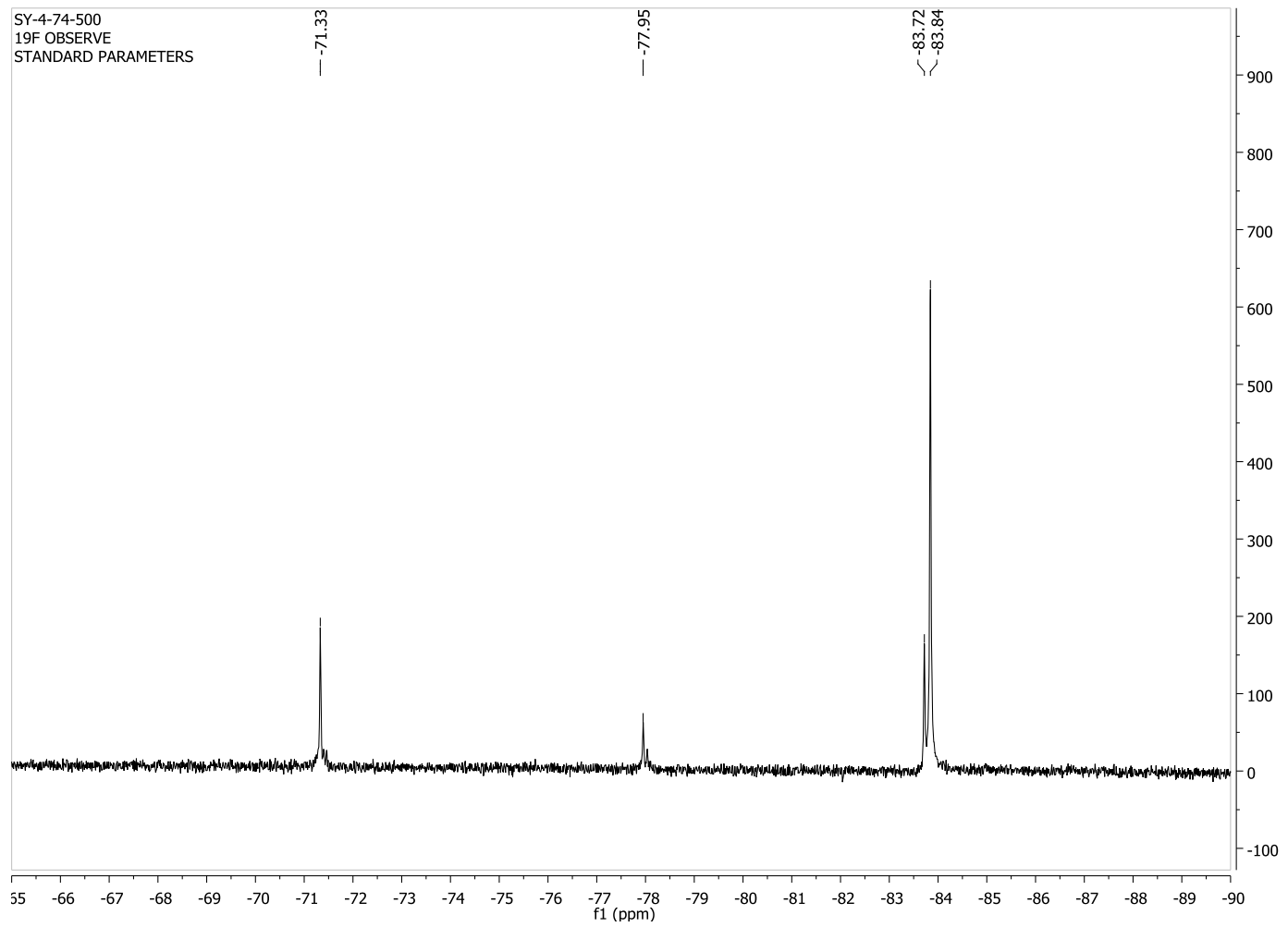




\section{2 eq of N15}

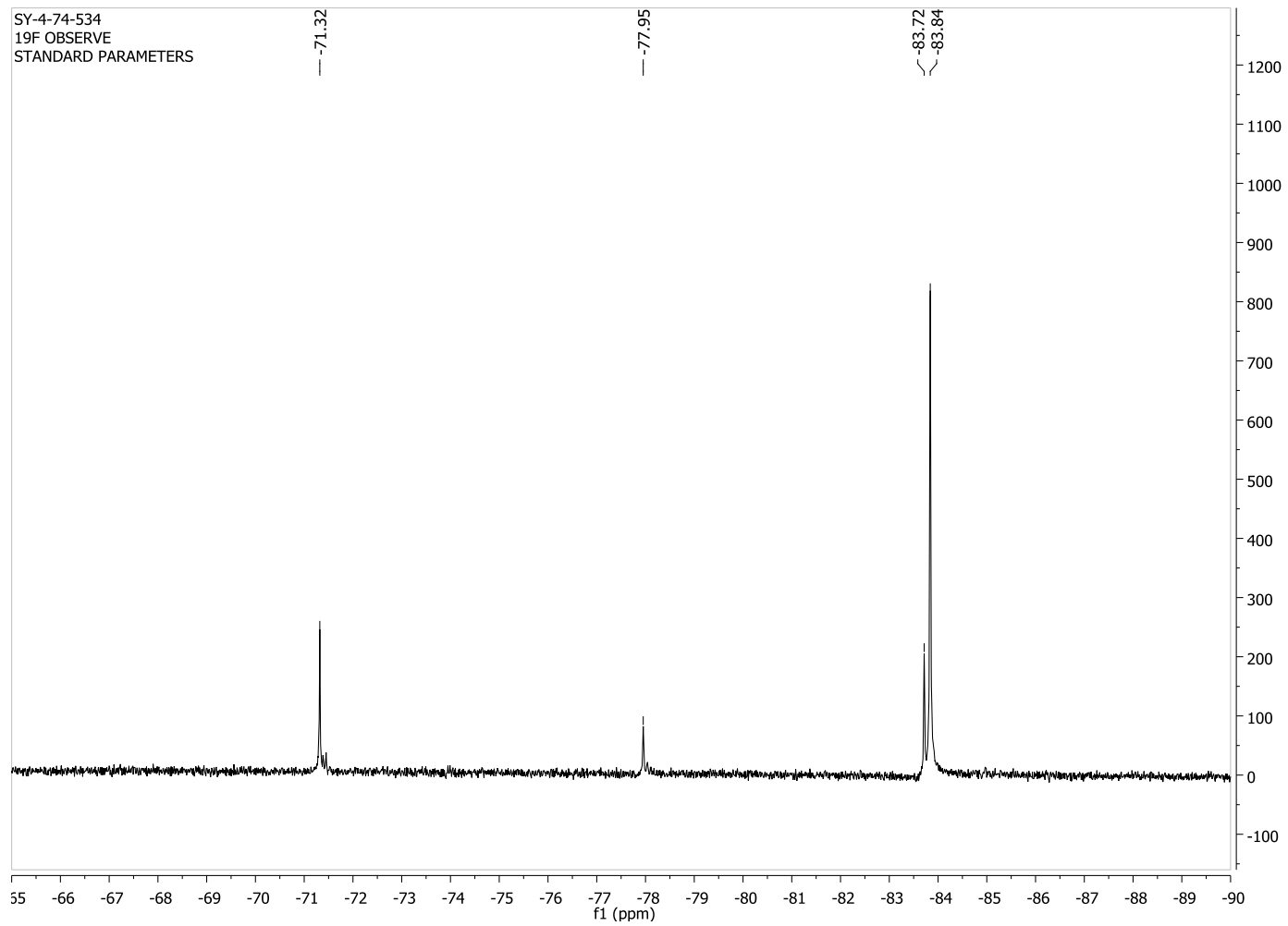


Table A6-1. Crystal data and structure refinement for C28H24F6N4O2.

Empirical formula

Formula weight

Temperature

Wavelength

Crystal system

Space group

Unit cell dimensions

Volume

$\mathrm{Z}$

Density (calculated)

Absorption coefficient

$\mathrm{F}(000)$

Crystal size

Theta range for data collection

Index ranges

Reflections collected

Independent reflections

Completeness to theta $=25.82^{\circ}$

Absorption correction

Max. and min. transmission

Refinement method

Data / restraints / parameters

Goodness-of-fit on $\mathrm{F}^{2}$

Final $\mathrm{R}$ indices [I>2sigma(I)]

$\mathrm{R}$ indices (all data)

Extinction coefficient

Largest diff. peak and hole
C28 H24 F6 N4 O2

562.51

153(2) K

$0.71073 \AA$

Tetragonal

P4(3)

$\mathrm{a}=10.532(3) \AA$

$\mathrm{b}=10.532(3) \AA$

$\mathrm{c}=44.888(12) \AA$

4979(2) $\AA^{3}$

8

$1.501 \mathrm{Mg} / \mathrm{m}^{3}$

$0.127 \mathrm{~mm}^{-1}$

2320

$0.39 \times 0.34 \times 0.32 \mathrm{~mm}^{3}$

3.28 to $25.82^{\circ}$.

$-12<=\mathrm{h}<=12,-12<=\mathrm{k}<=12,-54<=1<=54$

47263

$9416[\mathrm{R}(\mathrm{int})=0.0647]$

$99.1 \%$

Empirical

0.9605 and 0.9522

Full-matrix least-squares on $\mathrm{F}^{2}$

$9416 / 1 / 726$

1.184

$\mathrm{R} 1=0.0889, \mathrm{wR} 2=0.2121$

$\mathrm{R} 1=0.0910, \mathrm{wR} 2=0.2130$

$0.0010(3)$

0.433 and -0.471 e. $\AA^{-3}$ 
Table A6-2. Atomic coordinates ( $\mathrm{x} 10^{4}$ ) and equivalent isotropic displacement parameters $\left(\AA^{2} \times 10^{3}\right)$ for $\mathrm{C} 28 \mathrm{H} 24 \mathrm{~F} 6 \mathrm{~N} 4 \mathrm{O} 2$. U(eq) is defined as one third of the trace of the orthogonalized $\mathrm{Uij}$ tensor.

\begin{tabular}{|c|c|c|c|c|}
\hline & $\mathrm{x}$ & $\mathrm{y}$ & $\mathrm{z}$ & $\mathrm{U}(\mathrm{eq})$ \\
\hline $\mathrm{F}(1)$ & 411(4) & $9671(4)$ & 1111(1) & $42(1)$ \\
\hline $\mathrm{F}(2)$ & $-532(4)$ & $10144(5)$ & $1519(1)$ & $48(1)$ \\
\hline $\mathrm{F}(3)$ & $-147(5)$ & $11596(5)$ & $1195(1)$ & $48(1)$ \\
\hline $\mathrm{F}(4)$ & $-1231(4)$ & $4652(5)$ & $3054(1)$ & $47(1)$ \\
\hline $\mathrm{F}(5)$ & $700(5)$ & $5158(5)$ & $3170(1)$ & $47(1)$ \\
\hline $\mathrm{F}(6)$ & $239(5)$ & $4540(4)$ & $2726(1)$ & $47(1)$ \\
\hline$F(7)$ & $10358(4)$ & $6220(4)$ & $1354(1)$ & $48(1)$ \\
\hline $\mathrm{F}(8)$ & $10475(4)$ & $4731(5)$ & $1678(1)$ & $49(1)$ \\
\hline $\mathrm{F}(9)$ & $9831(4)$ & 4303(4) & $1237(1)$ & $45(1)$ \\
\hline $\mathrm{F}(10)$ & $4840(5)$ & $5527(4)$ & $2886(1)$ & $48(1)$ \\
\hline $\mathrm{F}(11)$ & $3397(5)$ & $5165(5)$ & $3210(1)$ & $50(1)$ \\
\hline $\mathrm{F}(12)$ & $5326(4)$ & $4577(4)$ & $3296(1)$ & $43(1)$ \\
\hline $\mathrm{O}(1)$ & $1405(5)$ & $10113(5)$ & 2104(1) & $34(1)$ \\
\hline $\mathrm{O}(2)$ & $294(5)$ & $7004(5)$ & $2230(1)$ & $34(1)$ \\
\hline $\mathrm{O}(3)$ & 7992(5) & $4694(5)$ & $2178(1)$ & $36(1)$ \\
\hline $\mathrm{O}(4)$ & $4877(5)$ & $3592(6)$ & $2305(1)$ & $38(1)$ \\
\hline $\mathrm{N}(1)$ & $2486(6)$ & $11333(6)$ & $1247(1)$ & $33(1)$ \\
\hline $\mathrm{N}(2)$ & $1438(6)$ & $11829(6)$ & 1691(1) & $33(1)$ \\
\hline $\mathrm{N}(3)$ & $-991(6)$ & $7274(7)$ & $3109(1)$ & $36(2)$ \\
\hline $\mathrm{N}(4)$ & $-1424(6)$ & $6545(5)$ & $2623(1)$ & $28(1)$ \\
\hline $\mathrm{N}(5)$ & $3170(6)$ & $3576(7)$ & $2716(1)$ & $39(2)$ \\
\hline $\mathrm{N}(6)$ & $3660(6)$ & $2504(6)$ & $3159(1)$ & $35(1)$ \\
\hline $\mathrm{N}(7)$ & $8452(5)$ & $6421(6)$ & $1785(1)$ & $28(1)$ \\
\hline $\mathrm{N}(8)$ & $7726(6)$ & $5989(5)$ & $1297(1)$ & $32(1)$ \\
\hline $\mathrm{C}(1)$ & $2460(6)$ & $8180(6)$ & $2035(1)$ & $23(1)$ \\
\hline$C(2)$ & $1965(6)$ & $9299(6)$ & $1915(1)$ & $25(1)$ \\
\hline
\end{tabular}




\begin{tabular}{|c|c|c|c|c|}
\hline $\mathrm{C}(3)$ & $2106(6)$ & $9585(6)$ & $1608(1)$ & $25(1)$ \\
\hline$C(4)$ & $2692(6)$ & $8688(6)$ & $1427(1)$ & $22(1)$ \\
\hline$C(5)$ & $3206(6)$ & $7552(6)$ & $1539(1)$ & $23(1)$ \\
\hline$C(6)$ & $3820(6)$ & $6686(6)$ & $1355(2)$ & $25(1)$ \\
\hline$C(7)$ & $4367(7)$ & $5602(6)$ & $1465(2)$ & $28(1)$ \\
\hline $\mathrm{C}(8)$ & $4294(7)$ & $5370(7)$ & $1775(2)$ & $31(2)$ \\
\hline $\mathrm{C}(9)$ & $3674(7)$ & $6179(6)$ & 1959(2) & $28(1)$ \\
\hline$C(10)$ & $3095(6)$ & $7301(7)$ & 1851(1) & $23(1)$ \\
\hline $\mathrm{C}(11)$ & $1637(6)$ & $10807(6)$ & $1468(1)$ & $26(1)$ \\
\hline$C(12)$ & $2958(8)$ & $12566(7)$ & $1358(2)$ & $38(2)$ \\
\hline $\mathrm{C}(13)$ & $2621(7)$ & $12548(7)$ & $1689(2)$ & $38(2)$ \\
\hline $\mathrm{C}(14)$ & $329(6)$ & $10552(7)$ & $1324(2)$ & $32(2)$ \\
\hline$C(15)$ & $2222(6)$ & $7965(6)$ & $2358(1)$ & $21(1)$ \\
\hline$C(16)$ & $1105(6)$ & $7369(6)$ & $2446(1)$ & $25(1)$ \\
\hline$C(17)$ & $813(6)$ & $7233(6)$ & $2754(1)$ & $20(1)$ \\
\hline $\mathrm{C}(18)$ & $1641(7)$ & $7648(6)$ & 2961(1) & $24(1)$ \\
\hline C(19) & 2795(6) & $8255(6)$ & $2882(1)$ & $22(1)$ \\
\hline$C(20)$ & $3652(6)$ & $8714(7)$ & $3094(2)$ & $27(1)$ \\
\hline $\mathrm{C}(21)$ & $4745(6)$ & $9333(7)$ & $3018(2)$ & $30(2)$ \\
\hline$C(22)$ & $5030(6)$ & $9480(6)$ & $2716(2)$ & $26(1)$ \\
\hline$C(23)$ & $4222(6)$ & $9019(7)$ & 2499(2) & $28(1)$ \\
\hline$C(24)$ & $3093(6)$ & $8403(6)$ & $2573(1)$ & $21(1)$ \\
\hline$C(25)$ & $-447(6)$ & $6589(7)$ & $2854(2)$ & $26(1)$ \\
\hline$C(26)$ & $-2360(7)$ & $7443(8)$ & $3043(2)$ & $38(2)$ \\
\hline$C(27)$ & $-2388(7)$ & $7475(8)$ & $2708(2)$ & $37(2)$ \\
\hline$C(28)$ & $-184(7)$ & $5236(8)$ & $2951(2)$ & $38(2)$ \\
\hline C(29) & $7051(6)$ & $2768(6)$ & $2049(1)$ & 21(1) \\
\hline$C(30)$ & $7629(6)$ & $3894(6)$ & 1961(2) & $23(1)$ \\
\hline $\mathrm{C}(31)$ & $7763(6)$ & $4177(6)$ & $1656(1)$ & $20(1)$ \\
\hline$C(32)$ & $7342(6)$ & $3349(7)$ & $1446(2)$ & $28(1)$ \\
\hline$C(33)$ & $6739(6)$ & $2185(6)$ & $1527(1)$ & 19(1) \\
\hline $\mathrm{C}(34)$ & $6292(6)$ & $1338(6)$ & $1307(2)$ & $25(1)$ \\
\hline$C(35)$ & $5693(6)$ & $258(7)$ & $1390(2)$ & $30(2)$ \\
\hline$C(36)$ & $5549(7)$ & $-36(6)$ & $1692(2)$ & $30(2)$ \\
\hline
\end{tabular}




$\begin{array}{lrrrr}\mathrm{C}(37) & 5974(7) & 752(7) & 1906(2) & 29(2) \\ \mathrm{C}(38) & 6584(6) & 1899(6) & 1833(1) & 21(1) \\ \mathrm{C}(39) & 8420(7) & 5444(6) & 1551(2) & 26(1) \\ \mathrm{C}(40) & 7528(9) & 7386(8) & 1700(2) & 42(2) \\ \mathrm{C}(41) & 7544(9) & 7338(7) & 1359(2) & 39(2) \\ \mathrm{C}(42) & 9750(7) & 5177(7) & 1453(2) & 32(2) \\ \mathrm{C}(43) & 6807(6) & 2548(6) & 2375(1) & 24(1) \\ \mathrm{C}(44) & 5710(6) & 3016(6) & 2491(1) & 23(1) \\ \mathrm{C}(45) & 2893(6) & 2800(1) & 25(1) \\ \mathrm{C}(46) & 2301(6) & 2982(1) & 23(1) \\ \mathrm{C}(47) & 6319(7) & 1802(6) & 2868(1) & 22(1) \\ \mathrm{C}(48) & 6310(6) & 1165(6) & 3053(2) & 26(1) \\ \mathrm{C}(49) & 7436(6) & 613(6) & 2936(2) & 29(2) \\ \mathrm{C}(50) & 8317(6) & 688(7) & 2632(2) & 32(2) \\ \mathrm{C}(51) & 9390(7) & 2449(2) & 26(1) \\ \mathrm{C}(52) & 9622(7) & 2542(6) & 22(1) \\ \mathrm{C}(53) & 8816(7) & 1905(6) & 23(1) & 39(1) \\ \mathrm{C}(54) & 7698(6) & 2382(6) & 2939(2) & 38(2) \\ \mathrm{C}(55) & 4176(6) & 3047(2) & 32(2) \\ \mathrm{C}(56) & 2437(7) & 2048(7) & 2721(2) & \end{array}$


Table A6-3. Bond lengths $[\AA]$ and angles $\left[{ }^{\circ}\right]$ for $\mathrm{C} 28 \mathrm{H} 24 \mathrm{~F} 6 \mathrm{~N} 4 \mathrm{O} 2$.

\begin{tabular}{ll}
\hline $\mathrm{F}(1)-\mathrm{C}(14)$ & $1.334(9)$ \\
$\mathrm{F}(2)-\mathrm{C}(14)$ & $1.333(9)$ \\
$\mathrm{F}(3)-\mathrm{C}(14)$ & $1.340(9)$ \\
$\mathrm{F}(4)-\mathrm{C}(28)$ & $1.344(9)$ \\
$\mathrm{F}(5)-\mathrm{C}(28)$ & $1.357(9)$ \\
$\mathrm{F}(6)-\mathrm{C}(28)$ & $1.324(10)$ \\
$\mathrm{F}(7)-\mathrm{C}(42)$ & $1.347(9)$ \\
$\mathrm{F}(8)-\mathrm{C}(42)$ & $1.350(9)$ \\
$\mathrm{F}(9)-\mathrm{C}(42)$ & $1.339(9)$ \\
$\mathrm{F}(10)-\mathrm{C}(56)$ & $1.319(9)$ \\
$\mathrm{F}(11)-\mathrm{C}(56)$ & $1.352(9)$ \\
$\mathrm{F}(12)-\mathrm{C}(56)$ & $1.341(9)$ \\
$\mathrm{O}(1)-\mathrm{C}(2)$ & $1.342(8)$ \\
$\mathrm{O}(2)-\mathrm{C}(16)$ & $1.351(8)$ \\
$\mathrm{O}(3)-\mathrm{C}(30)$ & $1.342(8)$ \\
$\mathrm{O}(4)-\mathrm{C}(44)$ & $1.356(8)$ \\
$\mathrm{N}(1)-\mathrm{C}(11)$ & $1.446(9)$ \\
$\mathrm{N}(1)-\mathrm{C}(12)$ & $1.477(10)$ \\
$\mathrm{N}(2)-\mathrm{C}(13)$ & $1.458(10)$ \\
$\mathrm{N}(2)-\mathrm{C}(11)$ & $1.483(9)$ \\
$\mathrm{N}(3)-\mathrm{C}(25)$ & $1.470(9)$ \\
$\mathrm{N}(3)-\mathrm{C}(26)$ & $1.483(9)$ \\
$\mathrm{N}(4)-\mathrm{C}(27)$ & $1.462(9)$ \\
$\mathrm{N}(4)-\mathrm{C}(25)$ & $1.463(9)$ \\
$\mathrm{N}(5)-\mathrm{C}(55)$ & $1.474(10)$ \\
$\mathrm{N}(5)-\mathrm{C}(53)$ & $1.472(9)$ \\
$\mathrm{N}(6)-\mathrm{C}(53)$ & $1.457(8)$ \\
$\mathrm{N}(6)-\mathrm{C}(54)$ & $1.465(10)$ \\
$\mathrm{N}(7)-\mathrm{C}(40)$ & $1.459(10)$ \\
$\mathrm{N}(7)-\mathrm{C}(39)$ & $1.472(9)$ \\
$\mathrm{N}(8)-\mathrm{C}(39)$ & $1.472(8)$ \\
$\mathrm{N}(8)-\mathrm{C}(41)$ & $1.461(9)$ \\
& \\
&
\end{tabular}




\begin{tabular}{|c|c|}
\hline $\mathrm{C}(1)-\mathrm{C}(2)$ & $1.396(10)$ \\
\hline$C(1)-C(10)$ & $1.409(9)$ \\
\hline $\mathrm{C}(1)-\mathrm{C}(15)$ & $1.490(8)$ \\
\hline$C(2)-C(3)$ & $1.416(9)$ \\
\hline$C(3)-C(4)$ & $1.390(9)$ \\
\hline $\mathrm{C}(3)-\mathrm{C}(11)$ & $1.516(9)$ \\
\hline$C(4)-C(5)$ & $1.406(9)$ \\
\hline$C(5)-C(6)$ & $1.392(9)$ \\
\hline$C(5)-C(10)$ & $1.426(9)$ \\
\hline$C(6)-C(7)$ & $1.371(9)$ \\
\hline$C(7)-C(8)$ & $1.415(10)$ \\
\hline $\mathrm{C}(8)-\mathrm{C}(9)$ & $1.354(10)$ \\
\hline $\mathrm{C}(9)-\mathrm{C}(10)$ & $1.417(10)$ \\
\hline $\mathrm{C}(11)-\mathrm{C}(14)$ & $1.545(9)$ \\
\hline$C(12)-C(13)$ & $1.527(11)$ \\
\hline$C(15)-C(16)$ & $1.392(9)$ \\
\hline$C(15)-C(24)$ & $1.409(9)$ \\
\hline$C(16)-C(17)$ & $1.419(9)$ \\
\hline $\mathrm{C}(17)-\mathrm{C}(18)$ & $1.347(9)$ \\
\hline$C(17)-C(25)$ & $1.556(9)$ \\
\hline $\mathrm{C}(18)-\mathrm{C}(19)$ & $1.419(9)$ \\
\hline$C(19)-C(20)$ & $1.400(9)$ \\
\hline$C(19)-C(24)$ & $1.429(9)$ \\
\hline$C(20)-C(21)$ & $1.366(9)$ \\
\hline$C(21)-C(22)$ & $1.395(10)$ \\
\hline$C(22)-C(23)$ & $1.384(9)$ \\
\hline$C(23)-C(24)$ & $1.395(9)$ \\
\hline$C(25)-C(28)$ & $1.515(11)$ \\
\hline$C(26)-C(27)$ & $1.505(12)$ \\
\hline $\mathrm{C}(29)-\mathrm{C}(30)$ & $1.391(9)$ \\
\hline $\mathrm{C}(29)-\mathrm{C}(38)$ & $1.422(8)$ \\
\hline $\mathrm{C}(29)-\mathrm{C}(43)$ & $1.503(8)$ \\
\hline$C(30)-C(31)$ & $1.409(9)$ \\
\hline $\mathrm{C}(31)-\mathrm{C}(32)$ & $1.358(10)$ \\
\hline
\end{tabular}




\begin{tabular}{|c|c|}
\hline$C(31)-C(39)$ & $1.575(9)$ \\
\hline $\mathrm{C}(32)-\mathrm{C}(33)$ & $1.428(9)$ \\
\hline$C(33)-C(34)$ & $1.409(9)$ \\
\hline $\mathrm{C}(33)-\mathrm{C}(38)$ & $1.418(9)$ \\
\hline$C(34)-C(35)$ & $1.352(9)$ \\
\hline$C(35)-C(36)$ & $1.399(10)$ \\
\hline $\mathrm{C}(36)-\mathrm{C}(37)$ & $1.347(10)$ \\
\hline $\mathrm{C}(37)-\mathrm{C}(38)$ & $1.407(9)$ \\
\hline $\mathrm{C}(39)-\mathrm{C}(42)$ & $1.495(10)$ \\
\hline$C(40)-C(41)$ & $1.531(11)$ \\
\hline$C(43)-C(44)$ & $1.359(10)$ \\
\hline$C(43)-C(52)$ & $1.410(9)$ \\
\hline$C(44)-C(45)$ & $1.425(9)$ \\
\hline$C(45)-C(46)$ & $1.390(9)$ \\
\hline$C(45)-C(53)$ & $1.540(9)$ \\
\hline$C(46)-C(47)$ & $1.394(9)$ \\
\hline $\mathrm{C}(47)-\mathrm{C}(48)$ & $1.415(9)$ \\
\hline$C(47)-C(52)$ & $1.436(8)$ \\
\hline C(48)-C(49) & $1.374(10)$ \\
\hline$C(49)-C(50)$ & $1.390(10)$ \\
\hline $\mathrm{C}(50)-\mathrm{C}(51)$ & $1.368(10)$ \\
\hline$C(51)-C(52)$ & $1.403(10)$ \\
\hline$C(53)-C(56)$ & $1.532(9)$ \\
\hline$C(54)-C(55)$ & $1.502(12)$ \\
\hline $\mathrm{C}(11)-\mathrm{N}(1)-\mathrm{C}(12)$ & 108.3(6) \\
\hline $\mathrm{C}(13)-\mathrm{N}(2)-\mathrm{C}(11)$ & $104.6(6)$ \\
\hline$C(25)-N(3)-C(26)$ & $106.4(6)$ \\
\hline $\mathrm{C}(27)-\mathrm{N}(4)-\mathrm{C}(25)$ & $106.3(6)$ \\
\hline$C(55)-N(5)-C(53)$ & $103.7(6)$ \\
\hline $\mathrm{C}(53)-\mathrm{N}(6)-\mathrm{C}(54)$ & $107.7(6)$ \\
\hline $\mathrm{C}(40)-\mathrm{N}(7)-\mathrm{C}(39)$ & $106.5(6)$ \\
\hline $\mathrm{C}(39)-\mathrm{N}(8)-\mathrm{C}(41)$ & $107.3(6)$ \\
\hline$C(2)-C(1)-C(10)$ & $120.4(6)$ \\
\hline
\end{tabular}




\begin{tabular}{|c|c|}
\hline $\mathrm{C}(2)-\mathrm{C}(1)-\mathrm{C}(15)$ & $116.1(6)$ \\
\hline$C(10)-C(1)-C(15)$ & $123.4(6)$ \\
\hline $\mathrm{O}(1)-\mathrm{C}(2)-\mathrm{C}(1)$ & $117.4(6)$ \\
\hline $\mathrm{O}(1)-\mathrm{C}(2)-\mathrm{C}(3)$ & $121.7(6)$ \\
\hline $\mathrm{C}(1)-\mathrm{C}(2)-\mathrm{C}(3)$ & $120.9(6)$ \\
\hline$C(4)-C(3)-C(2)$ & $118.1(6)$ \\
\hline$C(4)-C(3)-C(11)$ & $118.6(6)$ \\
\hline$C(2)-C(3)-C(11)$ & $123.3(6)$ \\
\hline$C(5)-C(4)-C(3)$ & $122.7(6)$ \\
\hline$C(4)-C(5)-C(6)$ & $121.6(6)$ \\
\hline$C(4)-C(5)-C(10)$ & $118.4(6)$ \\
\hline$C(6)-C(5)-C(10)$ & $120.0(6)$ \\
\hline$C(7)-C(6)-C(5)$ & $121.6(6)$ \\
\hline$C(6)-C(7)-C(8)$ & $118.5(6)$ \\
\hline$C(9)-C(8)-C(7)$ & $121.1(6)$ \\
\hline$C(8)-C(9)-C(10)$ & $121.5(6)$ \\
\hline $\mathrm{C}(1)-\mathrm{C}(10)-\mathrm{C}(9)$ & $123.4(6)$ \\
\hline$C(1)-C(10)-C(5)$ & $119.4(6)$ \\
\hline$C(9)-C(10)-C(5)$ & $117.2(6)$ \\
\hline $\mathrm{N}(1)-\mathrm{C}(11)-\mathrm{N}(2)$ & $105.8(6)$ \\
\hline $\mathrm{N}(1)-\mathrm{C}(11)-\mathrm{C}(3)$ & $114.1(5)$ \\
\hline $\mathrm{N}(2)-\mathrm{C}(11)-\mathrm{C}(3)$ & $112.5(5)$ \\
\hline $\mathrm{N}(1)-\mathrm{C}(11)-\mathrm{C}(14)$ & $109.3(6)$ \\
\hline $\mathrm{N}(2)-\mathrm{C}(11)-\mathrm{C}(14)$ & $106.4(5)$ \\
\hline$C(3)-C(11)-C(14)$ & $108.5(6)$ \\
\hline $\mathrm{N}(1)-\mathrm{C}(12)-\mathrm{C}(13)$ & $103.8(6)$ \\
\hline $\mathrm{N}(2)-\mathrm{C}(13)-\mathrm{C}(12)$ & $102.2(6)$ \\
\hline $\mathrm{F}(2)-\mathrm{C}(14)-\mathrm{F}(3)$ & $107.1(6)$ \\
\hline $\mathrm{F}(2)-\mathrm{C}(14)-\mathrm{F}(1)$ & $106.9(6)$ \\
\hline $\mathrm{F}(3)-\mathrm{C}(14)-\mathrm{F}(1)$ & $106.6(6)$ \\
\hline $\mathrm{F}(2)-\mathrm{C}(14)-\mathrm{C}(11)$ & $112.7(6)$ \\
\hline $\mathrm{F}(3)-\mathrm{C}(14)-\mathrm{C}(11)$ & $111.8(6)$ \\
\hline $\mathrm{F}(1)-\mathrm{C}(14)-\mathrm{C}(11)$ & $111.3(6)$ \\
\hline$C(16)-C(15)-C(24)$ & $120.2(6)$ \\
\hline
\end{tabular}




\begin{tabular}{|c|c|}
\hline$C(16)-C(15)-C(1)$ & $119.3(6)$ \\
\hline $\mathrm{C}(24)-\mathrm{C}(15)-\mathrm{C}(1)$ & $120.5(6)$ \\
\hline $\mathrm{O}(2)-\mathrm{C}(16)-\mathrm{C}(15)$ & $117.2(6)$ \\
\hline $\mathrm{O}(2)-\mathrm{C}(16)-\mathrm{C}(17)$ & $122.3(6)$ \\
\hline$C(15)-C(16)-C(17)$ & $120.4(6)$ \\
\hline $\mathrm{C}(18)-\mathrm{C}(17)-\mathrm{C}(16)$ & $119.8(6)$ \\
\hline $\mathrm{C}(18)-\mathrm{C}(17)-\mathrm{C}(25)$ & $119.5(6)$ \\
\hline $\mathrm{C}(16)-\mathrm{C}(17)-\mathrm{C}(25)$ & $120.7(6)$ \\
\hline $\mathrm{C}(17)-\mathrm{C}(18)-\mathrm{C}(19)$ & $121.9(6)$ \\
\hline $\mathrm{C}(20)-\mathrm{C}(19)-\mathrm{C}(24)$ & $118.8(6)$ \\
\hline$C(20)-C(19)-C(18)$ & $122.5(6)$ \\
\hline $\mathrm{C}(24)-\mathrm{C}(19)-\mathrm{C}(18)$ & $118.7(6)$ \\
\hline$C(21)-C(20)-C(19)$ & $122.5(6)$ \\
\hline $\mathrm{C}(20)-\mathrm{C}(21)-\mathrm{C}(22)$ & $118.5(6)$ \\
\hline$C(23)-C(22)-C(21)$ & $120.9(6)$ \\
\hline $\mathrm{C}(22)-\mathrm{C}(23)-\mathrm{C}(24)$ & $121.3(6)$ \\
\hline $\mathrm{C}(23)-\mathrm{C}(24)-\mathrm{C}(15)$ & $122.9(6)$ \\
\hline$C(23)-C(24)-C(19)$ & $118.0(6)$ \\
\hline $\mathrm{C}(15)-\mathrm{C}(24)-\mathrm{C}(19)$ & $119.0(6)$ \\
\hline $\mathrm{N}(4)-\mathrm{C}(25)-\mathrm{N}(3)$ & $107.1(6)$ \\
\hline $\mathrm{N}(4)-\mathrm{C}(25)-\mathrm{C}(28)$ & $107.5(5)$ \\
\hline $\mathrm{N}(3)-\mathrm{C}(25)-\mathrm{C}(28)$ & $108.0(6)$ \\
\hline $\mathrm{N}(4)-\mathrm{C}(25)-\mathrm{C}(17)$ & $114.1(5)$ \\
\hline $\mathrm{N}(3)-\mathrm{C}(25)-\mathrm{C}(17)$ & $110.2(5)$ \\
\hline $\mathrm{C}(28)-\mathrm{C}(25)-\mathrm{C}(17)$ & $109.7(6)$ \\
\hline $\mathrm{N}(3)-\mathrm{C}(26)-\mathrm{C}(27)$ & $102.8(6)$ \\
\hline $\mathrm{N}(4)-\mathrm{C}(27)-\mathrm{C}(26)$ & $103.5(6)$ \\
\hline $\mathrm{F}(6)-\mathrm{C}(28)-\mathrm{F}(4)$ & $106.6(6)$ \\
\hline $\mathrm{F}(6)-\mathrm{C}(28)-\mathrm{F}(5)$ & $106.7(6)$ \\
\hline $\mathrm{F}(4)-\mathrm{C}(28)-\mathrm{F}(5)$ & $106.7(7)$ \\
\hline $\mathrm{F}(6)-\mathrm{C}(28)-\mathrm{C}(25)$ & $111.4(7)$ \\
\hline $\mathrm{F}(4)-\mathrm{C}(28)-\mathrm{C}(25)$ & $112.2(6)$ \\
\hline $\mathrm{F}(5)-\mathrm{C}(28)-\mathrm{C}(25)$ & $112.9(6)$ \\
\hline$C(30)-C(29)-C(38)$ & $120.4(6)$ \\
\hline
\end{tabular}




$\begin{array}{ll}\mathrm{C}(30)-\mathrm{C}(29)-\mathrm{C}(43) & 118.9(5) \\ \mathrm{C}(38)-\mathrm{C}(29)-\mathrm{C}(43) & 120.4(6) \\ \mathrm{O}(3)-\mathrm{C}(30)-\mathrm{C}(29) & 117.0(6) \\ \mathrm{O}(3)-\mathrm{C}(30)-\mathrm{C}(31) & 122.9(6) \\ \mathrm{C}(29)-\mathrm{C}(30)-\mathrm{C}(31) & 120.1(6) \\ \mathrm{C}(32)-\mathrm{C}(31)-\mathrm{C}(30) & 120.4(6) \\ \mathrm{C}(32)-\mathrm{C}(31)-\mathrm{C}(39) & 118.7(6) \\ \mathrm{C}(30)-\mathrm{C}(31)-\mathrm{C}(39) & 120.9(5) \\ \mathrm{C}(31)-\mathrm{C}(32)-\mathrm{C}(33) & 121.4(6) \\ \mathrm{C}(34)-\mathrm{C}(33)-\mathrm{C}(32) & 121.0(6) \\ \mathrm{C}(34)-\mathrm{C}(33)-\mathrm{C}(38) & 120.4(6) \\ \mathrm{C}(32)-\mathrm{C}(33)-\mathrm{C}(38) & 118.7(5) \\ \mathrm{C}(35)-\mathrm{C}(34)-\mathrm{C}(33) & 119.7(6) \\ \mathrm{C}(34)-\mathrm{C}(35)-\mathrm{C}(36) & 120.2(6) \\ \mathrm{C}(37)-\mathrm{C}(36)-\mathrm{C}(35) & 121.3(7) \\ \mathrm{C}(36)-\mathrm{C}(37)-\mathrm{C}(38) & 120.9(6) \\ \mathrm{C}(37)-\mathrm{C}(38)-\mathrm{C}(29) & 123.4(6) \\ \mathrm{C}(37)-\mathrm{C}(38)-\mathrm{C}(33) & 117.5(6) \\ \mathrm{C}(29)-\mathrm{C}(38)-\mathrm{C}(33) & 119.1(5) \\ \mathrm{N}(8)-\mathrm{C}(39)-\mathrm{N}(7) & 107.0(5) \\ \mathrm{N}(8)-\mathrm{C}(39)-\mathrm{C}(42) & 108.0(6) \\ \mathrm{N}(7)-\mathrm{C}(39)-\mathrm{C}(42) & 108.7(6) \\ \mathrm{N}(8)-\mathrm{C}(39)-\mathrm{C}(31) & 110.1(5) \\ \mathrm{N}(7)-\mathrm{C}(39)-\mathrm{C}(31) & 112.9(5) \\ \mathrm{C}(42)-\mathrm{C}(39)-\mathrm{C}(31) & 109.9(6) \\ \mathrm{N}(7)-\mathrm{C}(40)-\mathrm{C}(41) & 103.5(6) \\ \mathrm{N}(8)-\mathrm{C}(41)-\mathrm{C}(40) & 103.0(6) \\ \mathrm{F}(9)-\mathrm{C}(42)-\mathrm{F}(8) & 105.4(6) \\ \mathrm{F}(9)-\mathrm{C}(42)-\mathrm{F}(7) & 107.0(6) \\ \mathrm{F}(8)-\mathrm{C}(42)-\mathrm{F}(7) & 105.2(6) \\ \mathrm{F}(9)-\mathrm{C}(42)-\mathrm{C}(39) & 113.7(6) \\ \mathrm{F}(8)-\mathrm{C}(42)-\mathrm{C}(39) & 112.0(6) \\ \mathrm{F}(4)-\mathrm{C}(39) & 112.9(6) \\ & \\ \mathrm{C}(43)-\mathrm{C}(52) & 121.4(6)\end{array}$




$\begin{array}{ll}\mathrm{C}(44)-\mathrm{C}(43)-\mathrm{C}(29) & 117.5(6) \\ \mathrm{C}(52)-\mathrm{C}(43)-\mathrm{C}(29) & 121.1(6) \\ \mathrm{O}(4)-\mathrm{C}(44)-\mathrm{C}(43) & 118.4(6) \\ \mathrm{O}(4)-\mathrm{C}(44)-\mathrm{C}(45) & 120.1(6) \\ \mathrm{C}(43)-\mathrm{C}(44)-\mathrm{C}(45) & 121.5(6) \\ \mathrm{C}(46)-\mathrm{C}(45)-\mathrm{C}(44) & 117.7(6) \\ \mathrm{C}(46)-\mathrm{C}(45)-\mathrm{C}(53) & 119.0(6) \\ \mathrm{C}(44)-\mathrm{C}(45)-\mathrm{C}(53) & 123.2(6) \\ \mathrm{C}(45)-\mathrm{C}(46)-\mathrm{C}(47) & 122.0(6) \\ \mathrm{C}(46)-\mathrm{C}(47)-\mathrm{C}(48) & 121.5(6) \\ \mathrm{C}(46)-\mathrm{C}(47)-\mathrm{C}(52) & 119.5(6) \\ \mathrm{C}(48)-\mathrm{C}(47)-\mathrm{C}(52) & 119.0(6) \\ \mathrm{C}(49)-\mathrm{C}(48)-\mathrm{C}(47) & 121.0(6) \\ \mathrm{C}(48)-\mathrm{C}(49)-\mathrm{C}(50) & 119.7(7) \\ \mathrm{C}(51)-\mathrm{C}(50)-\mathrm{C}(49) & 120.7(7) \\ \mathrm{C}(50)-\mathrm{C}(51)-\mathrm{C}(52) & 121.9(6) \\ \mathrm{C}(51)-\mathrm{C}(52)-\mathrm{C}(43) & 124.5(6) \\ \mathrm{C}(51)-\mathrm{C}(52)-\mathrm{C}(47) & 117.5(6) \\ \mathrm{C}(43)-\mathrm{C}(52)-\mathrm{C}(47) & 117.9(6) \\ \mathrm{N}(6)-\mathrm{C}(53)-\mathrm{N}(5) & 106.2(5) \\ \mathrm{N}(6)-\mathrm{C}(53)-\mathrm{C}(56) & 110.7(6) \\ \mathrm{N}(5)-\mathrm{C}(53)-\mathrm{C}(56) & 107.1(6) \\ \mathrm{N}(6)-\mathrm{C}(53)-\mathrm{C}(45) & 112.3(5) \\ \mathrm{N}(5)-\mathrm{C}(53)-\mathrm{C}(45) & 112.5(5) \\ \mathrm{C}(56)-\mathrm{C}(53)-\mathrm{C}(45) & 107.9(5) \\ \mathrm{N}(6)-\mathrm{C}(54)-\mathrm{C}(55) & 104.3(6) \\ \mathrm{N}(5)-\mathrm{C}(55)-\mathrm{C}(54) & 102.5(6) \\ \mathrm{F}(10)-\mathrm{C}(56)-\mathrm{F}(12) & 108.4(6) \\ \mathrm{F}(10)-\mathrm{C}(56)-\mathrm{F}(11) & 106.4(6) \\ \mathrm{F}(12)-\mathrm{C}(56)-\mathrm{F}(11) & 106.6(6) \\ \mathrm{F}(10)-\mathrm{C}(56)-\mathrm{C}(53) & 112.8(6) \\ \mathrm{F}(12)-\mathrm{C}(56)-\mathrm{C}(53) & 111.0(6) \\ \mathrm{F}(11)-\mathrm{C}(56)-\mathrm{C}(53) & 111.3(6)\end{array}$


Table A6-4. Anisotropic displacement parameters $\left(\AA^{2} \times 10^{3}\right)$ for $\mathrm{C} 28 \mathrm{H} 24 \mathrm{~F} 6 \mathrm{~N} 4 \mathrm{O} 2$. The anisotropic

displacement factor exponent takes the form: $-2 \pi^{2}\left[h^{2} a^{* 2} U^{11}+\ldots+2 h \mathrm{k}^{*} b^{*} U^{12}\right]$

\begin{tabular}{|c|c|c|c|c|c|c|}
\hline & $\mathrm{U}^{11}$ & $\mathrm{U}^{22}$ & $\mathrm{U}^{33}$ & $\mathrm{U}^{23}$ & $\mathrm{U}^{13}$ & $\mathrm{U}^{12}$ \\
\hline $\mathrm{F}(1)$ & $40(3)$ & $46(3)$ & $42(3)$ & $-10(2)$ & $-14(2)$ & $5(2)$ \\
\hline $\mathrm{F}(2)$ & $32(2)$ & $57(3)$ & $55(3)$ & $3(2)$ & $1(2)$ & $-7(2)$ \\
\hline $\mathrm{F}(3)$ & $45(3)$ & $49(3)$ & $48(3)$ & $6(2)$ & $-15(2)$ & $12(2)$ \\
\hline $\mathrm{F}(4)$ & $33(2)$ & $45(3)$ & $64(3)$ & $17(2)$ & $3(2)$ & $-12(2)$ \\
\hline $\mathrm{F}(5)$ & $42(3)$ & $49(3)$ & $49(3)$ & $23(2)$ & $-10(2)$ & $0(2)$ \\
\hline$F(6)$ & $48(3)$ & $33(2)$ & $61(3)$ & $-6(2)$ & $2(2)$ & $1(2)$ \\
\hline $\mathrm{F}(7)$ & $42(3)$ & $36(2)$ & $68(3)$ & $6(2)$ & $16(2)$ & $-14(2)$ \\
\hline $\mathrm{F}(8)$ & $32(2)$ & $45(3)$ & $69(4)$ & $5(2)$ & $-7(2)$ & $7(2)$ \\
\hline $\mathrm{F}(9)$ & $42(3)$ & $43(3)$ & $48(3)$ & $-12(2)$ & $19(2)$ & $-6(2)$ \\
\hline$F(10)$ & $54(3)$ & $35(2)$ & $53(3)$ & $-1(2)$ & $6(2)$ & $1(2)$ \\
\hline $\mathrm{F}(11)$ & $46(3)$ & $47(3)$ & $56(3)$ & $-17(2)$ & $8(2)$ & $19(2)$ \\
\hline$F(12)$ & $45(3)$ & $45(3)$ & $40(3)$ & $-13(2)$ & $-8(2)$ & $6(2)$ \\
\hline $\mathrm{O}(1)$ & $42(3)$ & $38(3)$ & $22(2)$ & $3(2)$ & $8(2)$ & $3(2)$ \\
\hline $\mathrm{O}(2)$ & $35(3)$ & $50(3)$ & $16(2)$ & $-2(2)$ & $-4(2)$ & $-18(2)$ \\
\hline $\mathrm{O}(3)$ & $58(3)$ & $32(3)$ & $17(2)$ & $-1(2)$ & $-2(2)$ & $-19(2)$ \\
\hline $\mathrm{O}(4)$ & $40(3)$ & $52(3)$ & $24(3)$ & $10(2)$ & $-2(2)$ & $8(3)$ \\
\hline $\mathrm{N}(1)$ & $47(4)$ & $33(3)$ & $18(3)$ & $6(2)$ & $4(2)$ & $1(3)$ \\
\hline $\mathrm{N}(2)$ & $48(4)$ & $27(3)$ & $25(3)$ & $-3(2)$ & $5(3)$ & $5(3)$ \\
\hline $\mathrm{N}(3)$ & $27(3)$ & $61(4)$ & $21(3)$ & $-11(3)$ & $5(2)$ & $5(3)$ \\
\hline $\mathrm{N}(4)$ & $34(3)$ & $30(3)$ & $21(3)$ & $-4(2)$ & $-2(2)$ & $-8(3)$ \\
\hline $\mathrm{N}(5)$ & $36(4)$ & $57(4)$ & $23(3)$ & $7(3)$ & $-2(3)$ & $8(3)$ \\
\hline $\mathrm{N}(6)$ & $43(4)$ & $40(4)$ & $21(3)$ & $6(3)$ & $6(3)$ & $-3(3)$ \\
\hline $\mathrm{N}(7)$ & $34(3)$ & $35(3)$ & $15(3)$ & $-1(2)$ & $-2(2)$ & $-4(3)$ \\
\hline $\mathrm{N}(8)$ & $52(4)$ & $24(3)$ & $20(3)$ & $6(2)$ & $-8(3)$ & $-2(3)$ \\
\hline $\mathrm{C}(1)$ & $25(3)$ & $26(3)$ & $19(3)$ & $-3(2)$ & $1(2)$ & $-11(3)$ \\
\hline $\mathrm{C}(2)$ & $31(3)$ & $28(3)$ & $15(3)$ & $-4(2)$ & $5(2)$ & $-9(3)$ \\
\hline
\end{tabular}




\begin{tabular}{|c|c|c|c|c|c|c|}
\hline$C(3)$ & $32(4)$ & $18(3)$ & $24(3)$ & $4(2)$ & $-1(3)$ & $1(3)$ \\
\hline C(4) & $26(3)$ & $20(3)$ & $19(3)$ & $2(2)$ & $0(2)$ & $-3(2)$ \\
\hline$C(5)$ & $26(3)$ & $27(3)$ & $16(3)$ & $-2(2)$ & $5(2)$ & $-7(3)$ \\
\hline$C(6)$ & $27(3)$ & $25(3)$ & $24(3)$ & $-1(3)$ & $3(3)$ & $-1(3)$ \\
\hline $\mathrm{C}(7)$ & $36(4)$ & $18(3)$ & $30(4)$ & $1(3)$ & $0(3)$ & $-1(3)$ \\
\hline $\mathrm{C}(8)$ & $36(4)$ & $25(4)$ & $32(4)$ & $12(3)$ & $8(3)$ & 11(3) \\
\hline $\mathrm{C}(9)$ & $35(4)$ & $28(3)$ & $22(3)$ & $6(3)$ & $3(3)$ & $-9(3)$ \\
\hline$C(10)$ & $15(3)$ & $32(4)$ & $23(3)$ & $2(3)$ & $0(2)$ & $-4(2)$ \\
\hline $\mathrm{C}(11)$ & $25(3)$ & $31(4)$ & $21(3)$ & $-3(3)$ & $-4(2)$ & $7(3)$ \\
\hline $\mathrm{C}(12)$ & $33(4)$ & $37(4)$ & $44(5)$ & $10(3)$ & $-7(3)$ & $-7(3)$ \\
\hline $\mathrm{C}(13)$ & $37(4)$ & $33(4)$ & $44(5)$ & $0(3)$ & $-6(3)$ & $0(3)$ \\
\hline$C(14)$ & $22(3)$ & $40(4)$ & $34(4)$ & $-1(3)$ & $-4(3)$ & $4(3)$ \\
\hline$C(15)$ & $26(3)$ & $12(3)$ & $25(3)$ & $-1(2)$ & $-2(2)$ & $3(2)$ \\
\hline$C(16)$ & $28(3)$ & $28(3)$ & $18(3)$ & $1(3)$ & $-1(3)$ & $2(3)$ \\
\hline$C(17)$ & $23(3)$ & $18(3)$ & $20(3)$ & $-1(2)$ & $2(2)$ & $0(2)$ \\
\hline$C(18)$ & $35(4)$ & $26(3)$ & $13(3)$ & $2(2)$ & $2(3)$ & $0(3)$ \\
\hline C(19) & $19(3)$ & $24(3)$ & $22(3)$ & $0(3)$ & $-2(2)$ & $1(2)$ \\
\hline$C(20)$ & $25(3)$ & $35(4)$ & $20(3)$ & $3(3)$ & $0(3)$ & $-3(3)$ \\
\hline$C(21)$ & $19(3)$ & $36(4)$ & $34(4)$ & $-13(3)$ & $-6(3)$ & $-6(3)$ \\
\hline$C(22)$ & $20(3)$ & $23(3)$ & $36(4)$ & $4(3)$ & $-6(3)$ & $-11(3)$ \\
\hline$C(23)$ & $25(3)$ & $39(4)$ & $20(3)$ & $0(3)$ & $1(3)$ & $-5(3)$ \\
\hline$C(24)$ & $23(3)$ & $17(3)$ & $24(3)$ & $5(2)$ & $2(2)$ & $3(2)$ \\
\hline$C(25)$ & $28(3)$ & $35(4)$ & $17(3)$ & $0(3)$ & $3(3)$ & $-10(3)$ \\
\hline$C(26)$ & $30(4)$ & $37(4)$ & $48(5)$ & $-7(4)$ & $7(3)$ & $8(3)$ \\
\hline$C(27)$ & $23(4)$ & $38(4)$ & $49(5)$ & $-11(4)$ & $-9(3)$ & $11(3)$ \\
\hline$C(28)$ & $26(4)$ & $49(5)$ & $37(4)$ & $3(4)$ & $-6(3)$ & $-3(3)$ \\
\hline$C(29)$ & $27(3)$ & $26(3)$ & $11(3)$ & $-2(2)$ & $-2(2)$ & $-1(3)$ \\
\hline$C(30)$ & $20(3)$ & $26(3)$ & $22(3)$ & $0(3)$ & $1(2)$ & $1(2)$ \\
\hline $\mathrm{C}(31)$ & $17(3)$ & $25(3)$ & $19(3)$ & $4(2)$ & $-1(2)$ & $0(2)$ \\
\hline$C(32)$ & $30(4)$ & $32(4)$ & $21(3)$ & $7(3)$ & $4(3)$ & $3(3)$ \\
\hline $\mathrm{C}(33)$ & $20(3)$ & $16(3)$ & $20(3)$ & $-1(2)$ & $-1(2)$ & $-1(2)$ \\
\hline$C(34)$ & $32(3)$ & $23(3)$ & $21(3)$ & $2(3)$ & $1(3)$ & $-3(3)$ \\
\hline$C(35)$ & $24(3)$ & $32(4)$ & $33(4)$ & $-2(3)$ & $-6(3)$ & $-6(3)$ \\
\hline$C(36)$ & $34(4)$ & $22(3)$ & $33(4)$ & $-2(3)$ & $-9(3)$ & $-6(3)$ \\
\hline
\end{tabular}




$\begin{array}{lllllll}\mathrm{C}(37) & 32(4) & 35(4) & 20(3) & 4(3) & -1(3) & -11(3) \\ \mathrm{C}(38) & 27(3) & 15(3) & 22(3) & 2(2) & 2(3) & -8(2) \\ \mathrm{C}(39) & 33(4) & 26(3) & 18(3) & 4(3) & -2(3) & -7(3) \\ \mathrm{C}(40) & 55(5) & 39(4) & 33(4) & -3(3) & -1(4) & 12(4) \\ \mathrm{C}(41) & 64(5) & 24(4) & 29(4) & 6(3) & -3(4) & -3(3) \\ \mathrm{C}(42) & 21(3) & 33(4) & 41(4) & -2(3) & 2(3) & 1(3) \\ \mathrm{C}(43) & 30(3) & 27(3) & 17(3) & -2(2) & 0(3) & -14(3) \\ \mathrm{C}(44) & 27(3) & 18(3) & 24(3) & 2(2) & 0(3) & -2(3) \\ \mathrm{C}(45) & 36(4) & 27(3) & 13(3) & -4(2) & 2(3) & 0(3) \\ \mathrm{C}(46) & 29(3) & 27(3) & 14(3) & 3(2) & 0(2) & 0(3) \\ \mathrm{C}(47) & 22(3) & 25(3) & 18(3) & 3(2) & 3(2) & 0(2) \\ \mathrm{C}(48) & 24(3) & 28(3) & 24(3) & 7(3) & -4(3) & 0(3) \\ \mathrm{C}(49) & 32(4) & 27(4) & 29(4) & 6(3) & -3(3) & -8(3) \\ \mathrm{C}(50) & 28(4) & 39(4) & 30(4) & 0(3) & 9(3) & 3(3) \\ \mathrm{C}(51) & 35(4) & 23(3) & 22(3) & -2(3) & 1(3) & -14(3) \\ \mathrm{C}(52) & 25(3) & 23(3) & 18(3) & -2(2) & -1(2) & -6(3) \\ \mathrm{C}(53) & 19(3) & 22(3) & 29(3) & 0(3) & 0(3) & -1(2) \\ \mathrm{C}(54) & 34(4) & 23(4) & 60(5) & -8(3) & 17(4) & -4(3) \\ \mathrm{C}(55) & 36(4) & 33(4) & 46(5) & -10(3) & -7(3) & -7(3) \\ \mathrm{C}(56) & 39(4) & 28(4) & 29(4) & -4(3) & 3(3) & -1(3)\end{array}$


Table A6-5. Hydrogen coordinates ( $\mathrm{x} 10^{4}$ ) and isotropic displacement parameters $\left(\AA^{2} \times 10^{3}\right)$

for C28H24F6N4O2.

\begin{tabular}{|c|c|c|c|c|}
\hline & $\mathrm{X}$ & $\mathrm{y}$ & $\mathrm{z}$ & $\mathrm{U}(\mathrm{eq})$ \\
\hline $\mathrm{H}(1 \mathrm{O})$ & 1136 & 10745 & 2009 & 51 \\
\hline $\mathrm{H}(2 \mathrm{O})$ & -362 & 6691 & 2307 & 50 \\
\hline $\mathrm{H}(3 \mathrm{O})$ & 8435 & 5278 & 2104 & 54 \\
\hline $\mathrm{H}(4 \mathrm{O})$ & 4134 & 3375 & 2349 & 58 \\
\hline $\mathrm{H}(1 \mathrm{~N})$ & 2688 & 10978 & 1076 & 39 \\
\hline $\mathrm{H}(2 \mathrm{~N})$ & 757 & 11969 & 1800 & 40 \\
\hline $\mathrm{H}(3 \mathrm{~N})$ & -584 & 7532 & 3270 & 43 \\
\hline $\mathrm{H}(4 \mathrm{~N})$ & -1431 & 6055 & 2464 & 34 \\
\hline $\mathrm{H}(5 \mathrm{~N})$ & 3026 & 4253 & 2607 & 47 \\
\hline $\mathrm{H}(6 \mathrm{~N})$ & 4019 & 2288 & 3329 & 42 \\
\hline $\mathrm{H}(7 \mathrm{~N})$ & 8934 & 6423 & 1945 & 34 \\
\hline $\mathrm{H}(8 \mathrm{~N})$ & 7469 & 5578 & 1137 & 38 \\
\hline $\mathrm{H}(4)$ & 2748 & 8850 & 1220 & 26 \\
\hline $\mathrm{H}(6)$ & 3861 & 6849 & 1147 & 30 \\
\hline $\mathrm{H}(7)$ & 4785 & 5021 & 1337 & 34 \\
\hline $\mathrm{H}(8)$ & 4686 & 4634 & 1856 & 37 \\
\hline $\mathrm{H}(9)$ & 3626 & 5989 & 2166 & 34 \\
\hline $\mathrm{H}(12 \mathrm{~A})$ & 2532 & 13280 & 1255 & 45 \\
\hline $\mathrm{H}(12 \mathrm{~B})$ & 3887 & 12641 & 1329 & 45 \\
\hline $\mathrm{H}(13 \mathrm{~A})$ & 3286 & 12117 & 1807 & 45 \\
\hline $\mathrm{H}(13 \mathrm{~B})$ & 2494 & 13418 & 1767 & 45 \\
\hline $\mathrm{H}(18)$ & 1446 & 7531 & 3165 & 29 \\
\hline $\mathrm{H}(20)$ & 3467 & 8590 & 3299 & 32 \\
\hline $\mathrm{H}(21)$ & 5298 & 9656 & 3167 & 35 \\
\hline $\mathrm{H}(22)$ & 5790 & 9902 & 2660 & 32 \\
\hline
\end{tabular}




\begin{tabular}{|c|c|c|c|}
\hline $\mathrm{H}(23)$ & 4441 & 9124 & 2295 \\
\hline $\mathrm{H}(26 \mathrm{~A})$ & -2685 & 8246 & 3129 \\
\hline $\mathrm{H}(26 \mathrm{~B})$ & -2867 & 6726 & 3122 \\
\hline $\mathrm{H}(27 \mathrm{~A})$ & -3235 & 7230 & 2632 \\
\hline $\mathrm{H}(27 \mathrm{~B})$ & -2171 & 8331 & 2633 \\
\hline $\mathrm{H}(32)$ & 7452 & 3548 & 1241 \\
\hline $\mathrm{H}(34)$ & 6411 & 1525 & 1102 \\
\hline $\mathrm{H}(35)$ & 5369 & -302 & 1243 \\
\hline $\mathrm{H}(36)$ & 5143 & -806 & 1747 \\
\hline $\mathrm{H}(37)$ & 5861 & 530 & 2110 \\
\hline $\mathrm{H}(40 \mathrm{~A})$ & 7785 & 8235 & 1773 \\
\hline $\mathrm{H}(40 \mathrm{~B})$ & 6675 & 7181 & 1778 \\
\hline $\mathrm{H}(41 \mathrm{~A})$ & 6733 & 7648 & 1274 \\
\hline $\mathrm{H}(41 \mathrm{~B})$ & 8251 & 7851 & 1277 \\
\hline $\mathrm{H}(46)$ & 6146 & 2236 & 3189 \\
\hline $\mathrm{H}(48)$ & 8165 & 1118 & 3262 \\
\hline H(49) & 9971 & 183 & 3063 \\
\hline $\mathrm{H}(50)$ & 10347 & 280 & 2550 \\
\hline $\mathrm{H}(51)$ & 9021 & 1417 & 2243 \\
\hline $\mathrm{H}(54 \mathrm{~A})$ & 1724 & 2481 & 3149 \\
\hline $\mathrm{H}(54 \mathrm{~B})$ & 2352 & 1120 & 3077 \\
\hline $\mathrm{H}(55 \mathrm{~A})$ & 2901 & 1707 & 2604 \\
\hline $\mathrm{H}(55 \mathrm{~B})$ & 1589 & 2483 & 2642 \\
\hline
\end{tabular}


Table A6-6. Crystal data and structure refinement for C31H29F6N4O2Cl3.

Empirical formula

Formula weight

Temperature

Wavelength

Crystal system

Space group

Unit cell dimensions

Volume

$\mathrm{Z}$

Density (calculated)

Absorption coefficient

$\mathrm{F}(000)$

Crystal size

Theta range for data collection

Index ranges

Reflections collected

Independent reflections

Completeness to theta $=25.00^{\circ}$

Absorption correction

Max. and min. transmission

Refinement method

Data / restraints / parameters

Goodness-of-fit on $\mathrm{F}^{2}$

Final $\mathrm{R}$ indices [I $>2 \operatorname{sigma}(\mathrm{I})]$

$\mathrm{R}$ indices (all data)

Absolute structure parameter

Largest diff. peak and hole
C 31 H29 Cl3 F6 N4 O2

709.93

153(2) K

$0.71073 \AA$

Orthorhombic

P2(1)2(1)2(1)

$\mathrm{a}=11.5447(4) \AA$

$\mathrm{b}=14.4270(5) \AA$

$\mathrm{c}=19.4717(7) \AA$

3243.1(2) $\AA^{3}$

4

$1.454 \mathrm{Mg} / \mathrm{m}^{3}$

$0.353 \mathrm{~mm}^{-1}$

1456

$0.44 \times 0.28 \times 0.26 \mathrm{~mm}^{3}$

3.49 to $28.81^{\circ}$.

$-15<=\mathrm{h}<=15,-19<=\mathrm{k}<=19,-26<=\mathrm{l}<=26$

54959

$8462[\mathrm{R}(\mathrm{int})=0.0449]$

$99.6 \%$

Empirical

0.9138 and 0.8602

Full-matrix least-squares on $\mathrm{F}^{2}$

8462 / 0 / 423

0.928

$\mathrm{R} 1=0.0536, \mathrm{wR} 2=0.1278$

$\mathrm{R} 1=0.0648, \mathrm{wR} 2=0.1349$

$0.03(10)$

0.549 and -0.649 e. $\AA^{-3}$ 
Table A6-7. Atomic coordinates ( $\mathrm{x} \mathrm{10^{4 }}$ ) and equivalent isotropic displacement parameters $\left(\AA^{2} \times 10^{3}\right)$

for $\mathrm{C} 31 \mathrm{H} 29 \mathrm{~F} 6 \mathrm{~N} 4 \mathrm{O} 2 \mathrm{Cl} 3$. U(eq) is defined as one third of the trace of the orthogonalized Uij tensor.

\begin{tabular}{|c|c|c|c|c|}
\hline & $\mathrm{X}$ & $\mathrm{y}$ & $\mathrm{z}$ & $\mathrm{U}(\mathrm{eq})$ \\
\hline $\mathrm{Cl}(1)$ & $457(1)$ & $7043(2)$ & $7424(1)$ & $113(1)$ \\
\hline $\mathrm{Cl}(2)$ & $2327(1)$ & $6659(2)$ & $6476(1)$ & $134(1)$ \\
\hline $\mathrm{Cl}(3)$ & $1428(2)$ & $5270(2)$ & $7358(1)$ & $138(1)$ \\
\hline $\mathrm{F}(1)$ & $-2255(2)$ & 7333(1) & 9094(1) & $33(1)$ \\
\hline $\mathrm{F}(2)$ & $-2379(1)$ & $8809(1)$ & $8975(1)$ & $31(1)$ \\
\hline $\mathrm{F}(3)$ & $-814(1)$ & $8105(1)$ & $8696(1)$ & $35(1)$ \\
\hline $\mathrm{F}(4)$ & $2845(2)$ & $12196(1)$ & $8066(1)$ & $35(1)$ \\
\hline $\mathrm{F}(5)$ & $4703(2)$ & $12241(1)$ & $8003(1)$ & $40(1)$ \\
\hline $\mathrm{F}(6)$ & $3671(2)$ & $12276(1)$ & $7084(1)$ & $39(1)$ \\
\hline $\mathrm{O}(1)$ & $1231(2)$ & $8476(1)$ & $9505(1)$ & $22(1)$ \\
\hline $\mathrm{O}(2)$ & $1544(2)$ & 10521(1) & $8338(1)$ & $24(1)$ \\
\hline $\mathrm{N}(1)$ & $-2230(2)$ & $8259(2)$ & $10335(1)$ & $21(1)$ \\
\hline $\mathrm{N}(2)$ & $-406(2)$ & $7473(1)$ & $10024(1)$ & $20(1)$ \\
\hline $\mathrm{N}(3)$ & $2853(2)$ & $10516(2)$ & $7242(1)$ & $22(1)$ \\
\hline $\mathrm{N}(4)$ & 4921(2) & $10623(2)$ & $7350(1)$ & $30(1)$ \\
\hline $\mathrm{C}(1)$ & $1179(2)$ & $10087(2)$ & $9673(1)$ & $15(1)$ \\
\hline$C(2)$ & $631(2)$ & $9237(2)$ & $9704(1)$ & $15(1)$ \\
\hline$C(3)$ & $-552(2)$ & $9170(2)$ & 9931(1) & $15(1)$ \\
\hline$C(4)$ & $-1114(2)$ & $9959(2)$ & 10131(1) & $17(1)$ \\
\hline$C(5)$ & $-576(2)$ & $10834(2)$ & $10120(1)$ & $17(1)$ \\
\hline$C(6)$ & $-1161(2)$ & $11644(2)$ & $10338(1)$ & $24(1)$ \\
\hline$C(7)$ & $-639(2)$ & $12490(2)$ & 10311(2) & $32(1)$ \\
\hline$C(8)$ & $513(3)$ & $12558(2)$ & $10071(2)$ & $34(1)$ \\
\hline $\mathrm{C}(9)$ & $1107(2)$ & $11792(2)$ & $9856(2)$ & $26(1)$ \\
\hline$C(10)$ & $584(2)$ & $10900(2)$ & $9875(1)$ & $16(1)$ \\
\hline
\end{tabular}




$\begin{array}{lrrrr}\mathrm{C}(11) & 2380(2) & 10142(2) & 9392(1) & 14(1) \\ \mathrm{C}(12) & 3368(2) & 9977(2) & 9808(1) & 14(1) \\ \mathrm{C}(13) & 3286(2) & 9753(2) & 10512(1) & 19(1) \\ \mathrm{C}(14) & 4254(2) & 9612(2) & 10905(1) & 24(1) \\ \mathrm{C}(15) & 5372(2) & 9674(2) & 10610(1) & 22(1) \\ \mathrm{C}(16) & 5485(2) & 9878(2) & 9929(1) & 20(1) \\ \mathrm{C}(17) & 4495(2) & 10040(2) & 9512(1) & 14(1) \\ \mathrm{C}(18) & 4595(2) & 10282(2) & 8814(1) & 17(1) \\ \mathrm{C}(19) & 3653(2) & 10463(2) & 8411(1) & 17(1) \\ \mathrm{C}(20) & 2516(2) & 10373(2) & 8711(1) & 16(1) \\ \mathrm{C}(21) & -1208(2) & 8250(2) & 9896(1) & 18(1) \\ \mathrm{C}(22) & -1946(2) & 8174(2) & 11072(1) & 24(1) \\ \mathrm{C}(23) & -1162(2) & 7349(2) & 11216(1) & 26(1) \\ \mathrm{C}(24) & -100(2) & 7414(2) & 10755(1) & 24(1) \\ \mathrm{C}(25) & -1662(2) & 8132(2) & 9156(1) & 23(1) \\ \mathrm{C}(26) & 3814(2) & 10832(2) & 7676(1) & 21(1) \\ \mathrm{C}(27) & 2954(3) & 9512(2) & 7087(2) & 32(1) \\ \mathrm{C}(28) & 4103(3) & 9299(3) & 6742(2) & 42(1) \\ \mathrm{C}(29) & 9633(3) & 7197(2) & 39(1) \\ \mathrm{C}(30) & 5082(3) & 11891(2) & 7706(1) & 28(1) \\ \mathrm{C}(31) & 3746(2) & 6248(4) & 6866(2) & 81(2)\end{array}$


Table A6-8. Bond lengths $[\AA]$ and angles $\left[{ }^{\circ}\right]$ for $\mathrm{C} 31 \mathrm{H} 29 \mathrm{~F} 6 \mathrm{~N} 4 \mathrm{O} 2 \mathrm{Cl} 3$.

\begin{tabular}{ll}
\hline $\mathrm{Cl}(1)-\mathrm{C}(31)$ & $1.732(5)$ \\
$\mathrm{Cl}(2)-\mathrm{C}(31)$ & $1.741(5)$ \\
$\mathrm{Cl}(3)-\mathrm{C}(31)$ & $1.754(7)$ \\
$\mathrm{F}(1)-\mathrm{C}(25)$ & $1.346(3)$ \\
$\mathrm{F}(2)-\mathrm{C}(25)$ & $1.328(3)$ \\
$\mathrm{F}(3)-\mathrm{C}(25)$ & $1.327(3)$ \\
$\mathrm{F}(4)-\mathrm{C}(30)$ & $1.329(3)$ \\
$\mathrm{F}(5)-\mathrm{C}(30)$ & $1.345(3)$ \\
$\mathrm{F}(6)-\mathrm{C}(30)$ & $1.336(3)$ \\
$\mathrm{O}(1)-\mathrm{C}(2)$ & $1.355(3)$ \\
$\mathrm{O}(2)-\mathrm{C}(20)$ & $1.355(3)$ \\
$\mathrm{N}(1)-\mathrm{C}(21)$ & $1.458(3)$ \\
$\mathrm{N}(1)-\mathrm{C}(22)$ & $1.477(3)$ \\
$\mathrm{N}(2)-\mathrm{C}(24)$ & $1.470(3)$ \\
$\mathrm{N}(2)-\mathrm{C}(21)$ & $1.474(3)$ \\
$\mathrm{N}(3)-\mathrm{C}(26)$ & $1.468(3)$ \\
$\mathrm{N}(3)-\mathrm{C}(27)$ & $1.483(4)$ \\
$\mathrm{N}(4)-\mathrm{C}(26)$ & $1.459(3)$ \\
$\mathrm{N}(4)-\mathrm{C}(29)$ & $1.470(4)$ \\
$\mathrm{C}(1)-\mathrm{C}(2)$ & $1.380(3)$ \\
$\mathrm{C}(1)-\mathrm{C}(10)$ & $1.416(3)$ \\
$\mathrm{C}(1)-\mathrm{C}(11)$ & $1.493(3)$ \\
$\mathrm{C}(2)-\mathrm{C}(3)$ & $1.439(3)$ \\
$\mathrm{C}(3)-\mathrm{C}(4)$ & $1.367(3)$ \\
$\mathrm{C}(3)-\mathrm{C}(21)$ & $1.531(3)$ \\
$\mathrm{C}(4)-\mathrm{C}(5)$ & $1.408(3)$ \\
$\mathrm{C}(5)-\mathrm{C}(6)$ & $1.414(3)$ \\
$\mathrm{C}(5)-\mathrm{C}(10)$ & $1.425(3)$ \\
$\mathrm{C}(6)-\mathrm{C}(7)$ & $1.363(4)$ \\
$\mathrm{C}(7)-\mathrm{C}(8)$ & $1.413(4)$ \\
$\mathrm{C}(8)-\mathrm{C}(9)$ & $1.366(4)$ \\
$\mathrm{C}(9)-\mathrm{C}(10)$ & $1.422(3)$ \\
&
\end{tabular}




\begin{tabular}{|c|c|}
\hline $\mathrm{C}(11)-\mathrm{C}(20)$ & $1.376(3)$ \\
\hline $\mathrm{C}(11)-\mathrm{C}(12)$ & $1.419(3)$ \\
\hline$C(12)-C(13)$ & $1.411(3)$ \\
\hline$C(12)-C(17)$ & $1.426(3)$ \\
\hline$C(13)-C(14)$ & $1.370(3)$ \\
\hline$C(14)-C(15)$ & $1.416(4)$ \\
\hline$C(15)-C(16)$ & $1.365(4)$ \\
\hline$C(16)-C(17)$ & $1.421(3)$ \\
\hline $\mathrm{C}(17)-\mathrm{C}(18)$ & $1.409(3)$ \\
\hline $\mathrm{C}(18)-\mathrm{C}(19)$ & $1.366(3)$ \\
\hline$C(19)-C(20)$ & $1.442(3)$ \\
\hline$C(19)-C(26)$ & $1.538(3)$ \\
\hline$C(21)-C(25)$ & $1.542(3)$ \\
\hline $\mathrm{C}(22)-\mathrm{C}(23)$ & $1.521(4)$ \\
\hline$C(23)-C(24)$ & $1.522(4)$ \\
\hline$C(26)-C(30)$ & $1.531(4)$ \\
\hline $\mathrm{C}(27)-\mathrm{C}(28)$ & $1.519(4)$ \\
\hline $\mathrm{C}(28)-\mathrm{C}(29)$ & $1.515(5)$ \\
\hline $\mathrm{C}(21)-\mathrm{N}(1)-\mathrm{C}(22)$ & $112.91(18)$ \\
\hline $\mathrm{C}(24)-\mathrm{N}(2)-\mathrm{C}(21)$ & $111.04(19)$ \\
\hline $\mathrm{C}(26)-\mathrm{N}(3)-\mathrm{C}(27)$ & $111.2(2)$ \\
\hline$C(26)-N(4)-C(29)$ & $113.6(2)$ \\
\hline$C(2)-C(1)-C(10)$ & $120.14(19)$ \\
\hline$C(2)-C(1)-C(11)$ & $119.2(2)$ \\
\hline$C(10)-C(1)-C(11)$ & $120.5(2)$ \\
\hline $\mathrm{O}(1)-\mathrm{C}(2)-\mathrm{C}(1)$ & $118.27(19)$ \\
\hline $\mathrm{O}(1)-\mathrm{C}(2)-\mathrm{C}(3)$ & $121.2(2)$ \\
\hline $\mathrm{C}(1)-\mathrm{C}(2)-\mathrm{C}(3)$ & $120.5(2)$ \\
\hline $\mathrm{C}(4)-\mathrm{C}(3)-\mathrm{C}(2)$ & $118.9(2)$ \\
\hline$C(4)-C(3)-C(21)$ & $119.98(19)$ \\
\hline$C(2)-C(3)-C(21)$ & $120.96(19)$ \\
\hline$C(3)-C(4)-C(5)$ & $122.2(2)$ \\
\hline$C(4)-C(5)-C(6)$ & $121.7(2)$ \\
\hline
\end{tabular}




$\begin{array}{ll}\mathrm{C}(4)-\mathrm{C}(5)-\mathrm{C}(10) & 118.7(2) \\ \mathrm{C}(6)-\mathrm{C}(5)-\mathrm{C}(10) & 119.7(2) \\ \mathrm{C}(7)-\mathrm{C}(6)-\mathrm{C}(5) & 121.1(2) \\ \mathrm{C}(6)-\mathrm{C}(7)-\mathrm{C}(8) & 119.4(2) \\ \mathrm{C}(9)-\mathrm{C}(8)-\mathrm{C}(7) & 121.2(2) \\ \mathrm{C}(8)-\mathrm{C}(9)-\mathrm{C}(10) & 120.7(2) \\ \mathrm{C}(1)-\mathrm{C}(10)-\mathrm{C}(5) & 119.6(2) \\ \mathrm{C}(1)-\mathrm{C}(10)-\mathrm{C}(9) & 122.5(2) \\ \mathrm{C}(5)-\mathrm{C}(10)-\mathrm{C}(9) & 117.9(2) \\ \mathrm{C}(20)-\mathrm{C}(11)-\mathrm{C}(12) & 119.89(19) \\ \mathrm{C}(20)-\mathrm{C}(11)-\mathrm{C}(1) & 118.21(19) \\ \mathrm{C}(12)-\mathrm{C}(11)-\mathrm{C}(1) & 121.89(19) \\ \mathrm{C}(13)-\mathrm{C}(12)-\mathrm{C}(11) & 122.6(2) \\ \mathrm{C}(13)-\mathrm{C}(12)-\mathrm{C}(17) & 117.87(19) \\ \mathrm{C}(11)-\mathrm{C}(12)-\mathrm{C}(17) & 119.52(19) \\ \mathrm{C}(14)-\mathrm{C}(13)-\mathrm{C}(12) & 121.5(2) \\ \mathrm{C}(13)-\mathrm{C}(14)-\mathrm{C}(15) & 120.5(2) \\ \mathrm{C}(16)-\mathrm{C}(15)-\mathrm{C}(14) & 119.6(2) \\ \mathrm{C}(15)-\mathrm{C}(16)-\mathrm{C}(17) & 120.9(2) \\ \mathrm{C}(18)-\mathrm{C}(17)-\mathrm{C}(16) & 121.7(2) \\ \mathrm{C}(18)-\mathrm{C}(17)-\mathrm{C}(12) & 118.7(2) \\ \mathrm{C}(16)-\mathrm{C}(17)-\mathrm{C}(12) & 119.6(2) \\ \mathrm{C}(19)-\mathrm{C}(18)-\mathrm{C}(17) & 122.4(2) \\ \mathrm{C}(18)-\mathrm{C}(19)-\mathrm{C}(20) & 118.37(19) \\ \mathrm{C}(18)-\mathrm{C}(19)-\mathrm{C}(26) & 120.2(2) \\ \mathrm{C}(20)-\mathrm{C}(19)-\mathrm{C}(26) & 121.2(2) \\ \mathrm{O}(2)-\mathrm{C}(20)-\mathrm{C}(11) & 117.38(19) \\ \mathrm{O}(2)-\mathrm{C}(20)-\mathrm{C}(19) & 121.53(19) \\ \mathrm{C}(11)-\mathrm{C}(20)-\mathrm{C}(19) & 121.1(2) \\ \mathrm{N}(2)-\mathrm{C}(21)-\mathrm{N}(1) & 114.6(2) \\ \mathrm{N}(2)-\mathrm{C}(21)-\mathrm{C}(25) & 106.71(19) \\ \mathrm{N}(1)-\mathrm{C}(21)-\mathrm{C}(25) & 105.88(18) \\ \mathrm{N}(2)-\mathrm{C}(21)-\mathrm{C}(3) & 109.95(18) \\ \mathrm{N}(1)-\mathrm{C}(21)-\mathrm{C}(3) & 111.51(19) \\ & \end{array}$




$\begin{array}{ll}\mathrm{C}(25)-\mathrm{C}(21)-\mathrm{C}(3) & 107.78(19) \\ \mathrm{N}(1)-\mathrm{C}(22)-\mathrm{C}(23) & 112.1(2) \\ \mathrm{C}(22)-\mathrm{C}(23)-\mathrm{C}(24) & 108.9(2) \\ \mathrm{N}(2)-\mathrm{C}(24)-\mathrm{C}(23) & 112.4(2) \\ \mathrm{F}(3)-\mathrm{C}(25)-\mathrm{F}(2) & 107.5(2) \\ \mathrm{F}(3)-\mathrm{C}(25)-\mathrm{F}(1) & 106.9(2) \\ \mathrm{F}(2)-\mathrm{C}(25)-\mathrm{F}(1) & 106.78(19) \\ \mathrm{F}(3)-\mathrm{C}(25)-\mathrm{C}(21) & 112.50(19) \\ \mathrm{F}(2)-\mathrm{C}(25)-\mathrm{C}(21) & 112.3(2) \\ \mathrm{F}(1)-\mathrm{C}(25)-\mathrm{C}(21) & 110.6(2) \\ \mathrm{N}(4)-\mathrm{C}(26)-\mathrm{N}(3) & 110.2(2) \\ \mathrm{N}(4)-\mathrm{C}(26)-\mathrm{C}(30) & 105.5(2) \\ \mathrm{N}(3)-\mathrm{C}(26)-\mathrm{C}(30) & 107.1(2) \\ \mathrm{N}(4)-\mathrm{C}(26)-\mathrm{C}(19) & 116.1(2) \\ \mathrm{N}(3)-\mathrm{C}(26)-\mathrm{C}(19) & 109.71(19) \\ \mathrm{C}(30)-\mathrm{C}(26)-\mathrm{C}(19) & 107.7(2) \\ \mathrm{N}(3)-\mathrm{C}(27)-\mathrm{C}(28) & 110.8(3) \\ \mathrm{C}(29)-\mathrm{C}(28)-\mathrm{C}(27) & 109.2(2) \\ \mathrm{N}(4)-\mathrm{C}(29)-\mathrm{C}(28) & 109.4(2) \\ \mathrm{F}(4)-\mathrm{C}(30)-\mathrm{F}(6) & 106.9(2) \\ \mathrm{F}(4)-\mathrm{C}(30)-\mathrm{F}(5) & 107.0(2) \\ \mathrm{F}(6)-\mathrm{C}(30)-\mathrm{F}(5) & 106.7(2) \\ \mathrm{F}(4)-\mathrm{C}(30)-\mathrm{C}(26) & 113.0(2) \\ \mathrm{F}(6)-\mathrm{C}(30)-\mathrm{C}(26) & 112.6(2) \\ \mathrm{F}(5)-\mathrm{C}(30)-\mathrm{C}(26) & 110.4(2) \\ \mathrm{Cl}(1)-\mathrm{C}(31)-\mathrm{Cl}(2) & 112.9(3) \\ \mathrm{Cl}(1)-\mathrm{C}(31)-\mathrm{Cl}(3) & 106.7(3) \\ \mathrm{Cl}(2)-\mathrm{C}(31)-\mathrm{Cl}(3) & 108.5(3) \\ & \end{array}$


Table A6-9. Anisotropic displacement parameters $\left(\AA^{2} \times 10^{3}\right)$ for $\mathrm{C} 31 \mathrm{H} 29 \mathrm{~F} 6 \mathrm{~N} 4 \mathrm{O} 2 \mathrm{Cl} 3$. The anisotropic displacement factor exponent takes the form: $-2 \pi^{2}\left[h^{2} a^{* 2} U^{11}+\ldots+2 h \mathrm{k}^{*} b^{*} U^{12}\right]$

\begin{tabular}{|c|c|c|c|c|c|c|}
\hline & $\mathrm{U}^{11}$ & $\mathrm{U}^{22}$ & $\mathrm{U}^{33}$ & $\mathrm{U}^{23}$ & $\mathrm{U}^{13}$ & $\mathrm{U}^{12}$ \\
\hline $\mathrm{Cl}(1)$ & 71(1) & $147(2)$ & $120(1)$ & $-67(1)$ & $-5(1)$ & $18(1)$ \\
\hline $\mathrm{Cl}(2)$ & $65(1)$ & $243(3)$ & $95(1)$ & $-28(1)$ & $15(1)$ & $-33(1)$ \\
\hline $\mathrm{Cl}(3)$ & $161(2)$ & $139(2)$ & $115(2)$ & $-30(1)$ & $-24(1)$ & $22(2)$ \\
\hline $\mathrm{F}(1)$ & $31(1)$ & $29(1)$ & $39(1)$ & $-5(1)$ & $-14(1)$ & $-8(1)$ \\
\hline $\mathrm{F}(2)$ & $29(1)$ & $32(1)$ & $32(1)$ & $3(1)$ & $-8(1)$ & $5(1)$ \\
\hline $\mathrm{F}(3)$ & $28(1)$ & $50(1)$ & $26(1)$ & $-8(1)$ & $3(1)$ & $0(1)$ \\
\hline $\mathrm{F}(4)$ & $44(1)$ & $27(1)$ & $35(1)$ & $2(1)$ & $9(1)$ & $5(1)$ \\
\hline $\mathrm{F}(5)$ & $44(1)$ & $36(1)$ & $41(1)$ & $8(1)$ & $-8(1)$ & $-16(1)$ \\
\hline $\mathrm{F}(6)$ & $48(1)$ & $40(1)$ & $28(1)$ & $17(1)$ & $2(1)$ & $-3(1)$ \\
\hline $\mathrm{O}(1)$ & $13(1)$ & $15(1)$ & $37(1)$ & $-2(1)$ & $6(1)$ & $2(1)$ \\
\hline $\mathrm{O}(2)$ & $14(1)$ & $40(1)$ & $18(1)$ & $5(1)$ & $-2(1)$ & $2(1)$ \\
\hline $\mathrm{N}(1)$ & $8(1)$ & $26(1)$ & $27(1)$ & $1(1)$ & $0(1)$ & $-3(1)$ \\
\hline $\mathrm{N}(2)$ & $16(1)$ & $15(1)$ & $30(1)$ & $-2(1)$ & $0(1)$ & $1(1)$ \\
\hline $\mathrm{N}(3)$ & 21(1) & $29(1)$ & $18(1)$ & $2(1)$ & $-2(1)$ & $4(1)$ \\
\hline $\mathrm{N}(4)$ & $22(1)$ & $46(2)$ & $24(1)$ & $8(1)$ & $10(1)$ & $4(1)$ \\
\hline $\mathrm{C}(1)$ & $10(1)$ & $19(1)$ & $16(1)$ & $2(1)$ & $2(1)$ & $0(1)$ \\
\hline$C(2)$ & $10(1)$ & $18(1)$ & 19(1) & $0(1)$ & $1(1)$ & $2(1)$ \\
\hline$C(3)$ & $10(1)$ & $16(1)$ & $20(1)$ & $1(1)$ & $-1(1)$ & $0(1)$ \\
\hline $\mathrm{C}(4)$ & $8(1)$ & $20(1)$ & $22(1)$ & $0(1)$ & $1(1)$ & $0(1)$ \\
\hline$C(5)$ & $12(1)$ & $17(1)$ & 21(1) & $-1(1)$ & $0(1)$ & $1(1)$ \\
\hline$C(6)$ & $16(1)$ & $22(1)$ & $35(1)$ & $-6(1)$ & $2(1)$ & $4(1)$ \\
\hline$C(7)$ & $26(1)$ & $20(1)$ & $51(2)$ & $-8(1)$ & $3(1)$ & $5(1)$ \\
\hline$C(8)$ & $27(1)$ & $14(1)$ & $59(2)$ & $-4(1)$ & $7(1)$ & $-3(1)$ \\
\hline C(9) & $18(1)$ & 19(1) & $40(1)$ & $0(1)$ & $7(1)$ & $-1(1)$ \\
\hline$C(10)$ & $14(1)$ & $16(1)$ & 19(1) & $0(1)$ & $0(1)$ & $1(1)$ \\
\hline $\mathrm{C}(11)$ & 11(1) & $14(1)$ & $17(1)$ & $0(1)$ & $3(1)$ & $0(1)$ \\
\hline
\end{tabular}




$\begin{array}{lllllll}\mathrm{C}(12) & 11(1) & 13(1) & 17(1) & -1(1) & 0(1) & 0(1) \\ \mathrm{C}(13) & 15(1) & 24(1) & 19(1) & 2(1) & 2(1) & 1(1) \\ \mathrm{C}(14) & 25(1) & 28(1) & 18(1) & 2(1) & 0(1) & 4(1) \\ \mathrm{C}(15) & 15(1) & 28(1) & 24(1) & 0(1) & -7(1) & 3(1) \\ \mathrm{C}(16) & 12(1) & 22(1) & 25(1) & -2(1) & -3(1) & 1(1) \\ \mathrm{C}(17) & 11(1) & 14(1) & 18(1) & -1(1) & 1(1) & 0(1) \\ \mathrm{C}(18) & 12(1) & 19(1) & 21(1) & 0(1) & 4(1) & -1(1) \\ \mathrm{C}(19) & 15(1) & 19(1) & 15(1) & 1(1) & 3(1) & 0(1) \\ \mathrm{C}(20) & 10(1) & 18(1) & 18(1) & 1(1) & 1(1) & -1(1) \\ \mathrm{C}(21) & 11(1) & 16(1) & 26(1) & -1(1) & -1(1) & 0(1) \\ \mathrm{C}(22) & 18(1) & 27(1) & 27(1) & 0(1) & 3(1) & -2(1) \\ \mathrm{C}(23) & 26(1) & 25(1) & 28(1) & 5(1) & -3(1) & -6(1) \\ \mathrm{C}(24) & 18(1) & 20(1) & 34(1) & 3(1) & -7(1) & -2(1) \\ \mathrm{C}(25) & 16(1) & 25(1) & 28(1) & -2(1) & -4(1) & -1(1) \\ \mathrm{C}(26) & 17(1) & 30(1) & 16(1) & 4(1) & 3(1) & 1(1) \\ \mathrm{C}(27) & 39(2) & 31(1) & 26(1) & -3(1) & -10(1) & 6(1) \\ \mathrm{C}(28) & 47(2) & 52(2) & 25(1) & -10(1) & -2(1) & 22(2) \\ \mathrm{C}(29) & 33(2) & 56(2) & 27(1) & -5(1) & 2(1) & 21(1) \\ \mathrm{C}(30) & 29(1) & 33(1) & 24(1) & 9(1) & 2(1) & -4(1) \\ \mathrm{C}(31) & 43(2) & 144(5) & 55(2) & -39(3) & -1(2) & -10(3)\end{array}$


Table A6-10. Hydrogen coordinates ( $\mathrm{x} \mathrm{104}$ ) and isotropic displacement parameters $\left(\AA^{2} \times 10^{3}\right)$ for $\mathrm{C} 31 \mathrm{H} 29 \mathrm{~F} 6 \mathrm{~N} 4 \mathrm{O} 2 \mathrm{Cl} 3$.

\begin{tabular}{|c|c|c|c|c|}
\hline & $\mathrm{x}$ & $\mathrm{y}$ & $\mathrm{z}$ & $\mathrm{U}(\mathrm{eq})$ \\
\hline $\mathrm{H}(1)$ & -2942 & 8310 & 10178 & 25 \\
\hline $\mathrm{H}(2)$ & -145 & 7095 & 9705 & 24 \\
\hline $\mathrm{H}(3)$ & 2290 & 10874 & 7092 & 27 \\
\hline $\mathrm{H}(4 \mathrm{~A})$ & 5442 & 11048 & 7253 & 37 \\
\hline $\mathrm{H}(4)$ & -1895 & 9915 & 10283 & 21 \\
\hline $\mathrm{H}(6)$ & -1932 & 11596 & 10506 & 29 \\
\hline $\mathrm{H}(7)$ & -1046 & 13030 & 10452 & 38 \\
\hline $\mathrm{H}(8)$ & 881 & 13147 & 10060 & 40 \\
\hline $\mathrm{H}(9)$ & 1878 & 11857 & 9691 & 31 \\
\hline $\mathrm{H}(13)$ & 2543 & 9700 & 10718 & 23 \\
\hline $\mathrm{H}(14)$ & 4175 & 9471 & 11379 & 28 \\
\hline $\mathrm{H}(15)$ & 6041 & 9574 & 10885 & 27 \\
\hline $\mathrm{H}(16)$ & 6235 & 9912 & 9730 & 24 \\
\hline $\mathrm{H}(18)$ & 5345 & 10322 & 8616 & 21 \\
\hline $\mathrm{H}(22 \mathrm{~A})$ & -2672 & 8105 & 11338 & 29 \\
\hline $\mathrm{H}(22 \mathrm{~B})$ & -1557 & 8748 & 11228 & 29 \\
\hline $\mathrm{H}(23 \mathrm{~A})$ & -1583 & 6764 & 11123 & 32 \\
\hline $\mathrm{H}(23 \mathrm{~B})$ & -923 & 7351 & 11704 & 32 \\
\hline $\mathrm{H}(24 \mathrm{~A})$ & 356 & 7968 & 10885 & 29 \\
\hline $\mathrm{H}(24 \mathrm{~B})$ & 394 & 6861 & 10830 & 29 \\
\hline $\mathrm{H}(27 \mathrm{~A})$ & 2310 & 9322 & 6782 & 39 \\
\hline $\mathrm{H}(27 \mathrm{~B})$ & 2891 & 9153 & 7519 & 39 \\
\hline $\mathrm{H}(28 \mathrm{~A})$ & 4175 & 8624 & 6664 & 50 \\
\hline $\mathrm{H}(28 \mathrm{~B})$ & 4142 & 9615 & 6291 & 50 \\
\hline $\mathrm{H}(29 \mathrm{~A})$ & 5090 & 9273 & 7630 & 46 \\
\hline H(29B) & 5833 & 9538 & 6961 & 46 \\
\hline $\mathrm{H}(31)$ & 494 & 6072 & 6506 & 97 \\
\hline $\mathrm{H}(1 \mathrm{O} 1)$ & $850(30)$ & $8050(30)$ & $9642(18)$ & $36(10)$ \\
\hline $\mathrm{H}(1 \mathrm{O} 2)$ & $1700(30)$ & $10620(20)$ & 7962(19) & $31(9)$ \\
\hline
\end{tabular}


Table A6-11. Crystal data and structure refinement for C38H38Cl6F6N4O2.

Empirical formula

Formula weight

Temperature

Wavelength

Crystal system

Space group

Unit cell dimensions

Volume

$\mathrm{Z}$

Density (calculated)

Absorption coefficient

$\mathrm{F}(000)$

Crystal size

Theta range for data collection

Index ranges

Reflections collected

Independent reflections

Completeness to theta $=31.46^{\circ}$

Absorption correction

Max. and min. transmission

Refinement method

Data / restraints / parameters

Goodness-of-fit on $\mathrm{F}^{2}$

Final $\mathrm{R}$ indices [I>2sigma(I)]

$\mathrm{R}$ indices (all data)

Absolute structure parameter

Largest diff. peak and hole

\section{C38 H38 Cl6 F6 N4 O2}

909.42

153(2) K

$0.71073 \AA$

Monoclinic

$\mathrm{C} 2$

$\mathrm{a}=18.621(6) \AA$

$\mathrm{b}=9.489(3) \AA$ $\beta=96.732(8)^{\circ}$.

$\mathrm{c}=11.431(3) \AA$

2005.8(10) $\AA^{3}$

2

$1.506 \mathrm{Mg} / \mathrm{m}^{3}$

$0.497 \mathrm{~mm}^{-1}$

932

$0.44 \times 0.34 \times 0.16 \mathrm{~mm}^{3}$

3.94 to $31.46^{\circ}$.

$-26<=\mathrm{h}<=26,-13<=\mathrm{k}<=13,-16<=1<=16$

16129

$6176[\mathrm{R}($ int $)=0.0638]$

$97.5 \%$

Empirical

0.9248 and 0.8111

Full-matrix least-squares on $\mathrm{F}^{2}$

6176 / 1 / 257

1.059

$\mathrm{R} 1=0.0884, \mathrm{wR} 2=0.1977$

$\mathrm{R} 1=0.1001, \mathrm{wR} 2=0.2050$

$0.06(12)$

0.539 and -0.631 e. $\AA^{-3}$ 
Table A6-12. Atomic coordinates ( x 104) and equivalent isotropic displacement parameters $\left(\AA^{2} \times 10^{3}\right)$ for $\mathrm{C} 38 \mathrm{H} 38 \mathrm{Cl} 6 \mathrm{~F} 6 \mathrm{~N} 4 \mathrm{O} 2$. U(eq) is defined as one third of the trace of the orthogonalized $\mathrm{Uij}^{\mathrm{ij}}$ tensor.

\begin{tabular}{|c|c|c|c|c|}
\hline & $\mathrm{x}$ & $\mathrm{y}$ & $\mathrm{z}$ & $\mathrm{U}(\mathrm{eq})$ \\
\hline $\mathrm{Cl}(1)$ & $8769(1)$ & $1822(2)$ & $3162(1)$ & $56(1)$ \\
\hline $\mathrm{Cl}(2)$ & $9360(1)$ & $577(2)$ & $1179(2)$ & $57(1)$ \\
\hline $\mathrm{Cl}(3)$ & $7860(1)$ & $1347(2)$ & $946(1)$ & $48(1)$ \\
\hline $\mathrm{F}(1)$ & $7808(2)$ & $4545(5)$ & $6823(4)$ & $49(1)$ \\
\hline $\mathrm{F}(2)$ & $8437(2)$ & $4612(5)$ & $5361(3)$ & $55(1)$ \\
\hline $\mathrm{F}(3)$ & $7683(2)$ & 2931(5) & $5479(3)$ & $54(1)$ \\
\hline $\mathrm{O}(1)$ & $8917(2)$ & $3379(4)$ & $9422(3)$ & $30(1)$ \\
\hline $\mathrm{N}(1)$ & $9079(2)$ & 1908(6) & $6020(3)$ & $33(1)$ \\
\hline $\mathrm{N}(2)$ & $8373(2)$ & 1941(4) & $7626(3)$ & $24(1)$ \\
\hline $\mathrm{C}(1)$ & $9955(2)$ & $4777(4)$ & $9334(3)$ & $18(1)$ \\
\hline$C(2)$ & 9392(2) & $3985(4)$ & $8764(4)$ & $19(1)$ \\
\hline$C(3)$ & $9323(2)$ & $3793(5)$ & $7508(3)$ & $18(1)$ \\
\hline$C(4)$ & $9812(2)$ & $4490(5)$ & $6879(4)$ & $23(1)$ \\
\hline$C(5)$ & $10356(2)$ & $5378(5)$ & $7430(4)$ & $20(1)$ \\
\hline$C(6)$ & 10811(2) & $6184(6)$ & $6772(4)$ & $26(1)$ \\
\hline$C(7)$ & $11331(2)$ & $7074(5)$ & $7317(4)$ & $27(1)$ \\
\hline $\mathrm{C}(8)$ & $11419(2)$ & $7168(6)$ & $8558(5)$ & $30(1)$ \\
\hline $\mathrm{C}(9)$ & $10998(2)$ & $6418(5)$ & $9229(4)$ & $25(1)$ \\
\hline$C(10)$ & $10434(2)$ & $5520(5)$ & $8684(4)$ & $20(1)$ \\
\hline $\mathrm{C}(11)$ & $8755(2)$ & $2860(6)$ & $6836(4)$ & $24(1)$ \\
\hline $\mathrm{C}(12)$ & $8801(2)$ & $483(7)$ & $6207(4)$ & $33(1)$ \\
\hline$C(13)$ & $8684(2)$ & $518(6)$ & $7496(4)$ & $26(1)$ \\
\hline$C(14)$ & $8263(3)$ & $-769(6)$ & $7835(5)$ & $33(1)$ \\
\hline$C(15)$ & $8705(3)$ & $-2080(7)$ & $7571(5)$ & $40(1)$ \\
\hline$C(16)$ & $8888(3)$ & $-2123(7)$ & $6306(5)$ & $40(1)$ \\
\hline$C(17)$ & $9258(3)$ & $-757(7)$ & $5946(5)$ & $38(1)$ \\
\hline$C(18)$ & $8171(3)$ & $3762(7)$ & $6114(5)$ & $36(1)$ \\
\hline C(19) & $8734(3)$ & $1792(7)$ & $1629(4)$ & $33(1)$ \\
\hline
\end{tabular}


Table A6-13. Bond lengths $[\AA]$ and angles $\left[{ }^{\circ}\right]$ for $\mathrm{C} 38 \mathrm{H} 38 \mathrm{Cl} 6 \mathrm{~F} 6 \mathrm{~N} 4 \mathrm{O} 2$.

\begin{tabular}{ll}
\hline $\mathrm{Cl}(1)-\mathrm{C}(19)$ & $1.746(5)$ \\
$\mathrm{Cl}(2)-\mathrm{C}(19)$ & $1.760(6)$ \\
$\mathrm{Cl}(3)-\mathrm{C}(19)$ & $1.772(5)$ \\
$\mathrm{F}(1)-\mathrm{C}(18)$ & $1.338(7)$ \\
$\mathrm{F}(2)-\mathrm{C}(18)$ & $1.318(6)$ \\
$\mathrm{F}(3)-\mathrm{C}(18)$ & $1.349(6)$ \\
$\mathrm{O}(1)-\mathrm{C}(2)$ & $1.355(5)$ \\
$\mathrm{N}(1)-\mathrm{C}(12)$ & $1.472(8)$ \\
$\mathrm{N}(1)-\mathrm{C}(11)$ & $1.477(6)$ \\
$\mathrm{N}(2)-\mathrm{C}(13)$ & $1.484(6)$ \\
$\mathrm{N}(2)-\mathrm{C}(11)$ & $1.495(5)$ \\
$\mathrm{C}(1)-\mathrm{C}(2)$ & $1.388(6)$ \\
$\mathrm{C}(1)-\mathrm{C}(10)$ & $1.415(5)$ \\
$\mathrm{C}(1)-\mathrm{C}(1) \# 1$ & $1.512(8)$ \\
$\mathrm{C}(2)-\mathrm{C}(3)$ & $1.438(5)$ \\
$\mathrm{C}(3)-\mathrm{C}(4)$ & $1.391(5)$ \\
$\mathrm{C}(3)-\mathrm{C}(11)$ & $1.517(6)$ \\
$\mathrm{C}(4)-\mathrm{C}(5)$ & $1.408(6)$ \\
$\mathrm{C}(5)-\mathrm{C}(6)$ & $1.421(6)$ \\
$\mathrm{C}(5)-\mathrm{C}(10)$ & $1.430(5)$ \\
$\mathrm{C}(6)-\mathrm{C}(7)$ & $1.377(6)$ \\
$\mathrm{C}(7)-\mathrm{C}(8)$ & $1.412(7)$ \\
$\mathrm{C}(8)-\mathrm{C}(9)$ & $1.361(6)$ \\
$\mathrm{C}(9)-\mathrm{C}(10)$ & $1.436(6)$ \\
$\mathrm{C}(11)-\mathrm{C}(18)$ & $1.545(7)$ \\
$\mathrm{C}(12)-\mathrm{C}(17)$ & $1.503(8)$ \\
$\mathrm{C}(12)-\mathrm{C}(13)$ & $1.515(7)$ \\
$\mathrm{C}(13)-\mathrm{C}(14)$ & $1.526(7)$ \\
$\mathrm{C}(14)-\mathrm{C}(15)$ & $1.541(8)$ \\
$\mathrm{C}(15)-\mathrm{C}(16)$ & $1.524(8)$ \\
$\mathrm{C}(16)-\mathrm{C}(17)$ & $1.546(9)$ \\
& \\
&
\end{tabular}




$\begin{array}{ll}\mathrm{C}(12)-\mathrm{N}(1)-\mathrm{C}(11) & 107.0(3) \\ \mathrm{C}(13)-\mathrm{N}(2)-\mathrm{C}(11) & 104.5(3) \\ \mathrm{C}(2)-\mathrm{C}(1)-\mathrm{C}(10) & 120.7(4) \\ \mathrm{C}(2)-\mathrm{C}(1)-\mathrm{C}(1) \# 1 & 117.5(4) \\ \mathrm{C}(10)-\mathrm{C}(1)-\mathrm{C}(1) \# 1 & 121.8(4) \\ \mathrm{O}(1)-\mathrm{C}(2)-\mathrm{C}(1) & 118.3(4) \\ \mathrm{O}(1)-\mathrm{C}(2)-\mathrm{C}(3) & 120.9(4) \\ \mathrm{C}(1)-\mathrm{C}(2)-\mathrm{C}(3) & 120.8(4) \\ \mathrm{C}(4)-\mathrm{C}(3)-\mathrm{C}(2) & 117.9(4) \\ \mathrm{C}(4)-\mathrm{C}(3)-\mathrm{C}(11) & 118.4(4) \\ \mathrm{C}(2)-\mathrm{C}(3)-\mathrm{C}(11) & 123.7(3) \\ \mathrm{C}(3)-\mathrm{C}(4)-\mathrm{C}(5) & 122.2(4) \\ \mathrm{C}(4)-\mathrm{C}(5)-\mathrm{C}(6) & 121.9(4) \\ \mathrm{C}(4)-\mathrm{C}(5)-\mathrm{C}(10) & 119.2(4) \\ \mathrm{C}(6)-\mathrm{C}(5)-\mathrm{C}(10) & 118.8(4) \\ \mathrm{C}(7)-\mathrm{C}(6)-\mathrm{C}(5) & 121.5(4) \\ \mathrm{C}(6)-\mathrm{C}(7)-\mathrm{C}(8) & 119.1(4) \\ \mathrm{C}(9)-\mathrm{C}(8)-\mathrm{C}(7) & 121.8(4) \\ \mathrm{C}(8)-\mathrm{C}(9)-\mathrm{C}(10) & 120.4(4) \\ \mathrm{C}(1)-\mathrm{C}(10)-\mathrm{C}(9) & 122.8(4) \\ \mathrm{C}(1)-\mathrm{C}(10)-\mathrm{C}(5) & 118.9(4) \\ \mathrm{C}(9)-\mathrm{C}(10)-\mathrm{C}(5) & 118.3(4) \\ \mathrm{N}(1)-\mathrm{C}(11)-\mathrm{N}(2) & 106.5(4) \\ \mathrm{N}(1)-\mathrm{C}(11)-\mathrm{C}(3) & 111.4(3) \\ \mathrm{N}(2)-\mathrm{C}(11)-\mathrm{C}(3) & 112.8(3) \\ \mathrm{N}(1)-\mathrm{C}(11)-\mathrm{C}(18) & 108.4(4) \\ \mathrm{N}(2)-\mathrm{C}(11)-\mathrm{C}(18) & 106.9(3) \\ \mathrm{C}(3)-\mathrm{C}(11)-\mathrm{C}(18) & 110.7(4) \\ \mathrm{N}(1)-\mathrm{C}(12)-\mathrm{C}(17) & 118.2(4) \\ \mathrm{N}(1)-\mathrm{C}(12)-\mathrm{C}(13) & 102.4(4) \\ \mathrm{C}(17)-\mathrm{C}(12)-\mathrm{C}(13) & 111.0(5) \\ \mathrm{N}(2)-\mathrm{C}(13)-\mathrm{C}(14) & 118.9(4) \\ \mathrm{N}(2)-\mathrm{C}(13)-\mathrm{C}(12) & 102.7(4) \\ \mathrm{C}(14)-\mathrm{C}(13)-\mathrm{C}(12) & 111.5(4)\end{array}$




$\begin{array}{ll}\mathrm{C}(13)-\mathrm{C}(14)-\mathrm{C}(15) & 107.0(4) \\ \mathrm{C}(16)-\mathrm{C}(15)-\mathrm{C}(14) & 113.0(5) \\ \mathrm{C}(17)-\mathrm{C}(16)-\mathrm{C}(15) & 112.7(5) \\ \mathrm{C}(12)-\mathrm{C}(17)-\mathrm{C}(16) & 108.8(4) \\ \mathrm{F}(2)-\mathrm{C}(18)-\mathrm{F}(1) & 108.1(6) \\ \mathrm{F}(2)-\mathrm{C}(18)-\mathrm{F}(3) & 106.7(4) \\ \mathrm{F}(1)-\mathrm{C}(18)-\mathrm{F}(3) & 107.2(4) \\ \mathrm{F}(2)-\mathrm{C}(18)-\mathrm{C}(11) & 113.1(4) \\ \mathrm{F}(1)-\mathrm{C}(18)-\mathrm{C}(11) & 111.0(4) \\ \mathrm{F}(3)-\mathrm{C}(18)-\mathrm{C}(11) & 110.6(5) \\ \mathrm{Cl}(1)-\mathrm{C}(19)-\mathrm{Cl}(2) & 110.8(3) \\ \mathrm{Cl}(1)-\mathrm{C}(19)-\mathrm{Cl}(3) & 111.7(3) \\ \mathrm{Cl}(2)-\mathrm{C}(19)-\mathrm{Cl}(3) & 108.7(3)\end{array}$

Symmetry transformations used to generate equivalent atoms:

$\# 1-\mathrm{x}+2, \mathrm{y},-\mathrm{z}+2$ 
Table A6-14. Anisotropic displacement parameters $\left(\AA^{2} \times 10^{3}\right)$ for C38H38Cl6F6N4O2. The anisotropic

displacement factor exponent takes the form: $-2 \pi^{2}\left[h^{2} a^{* 2} U^{11}+\ldots+2 h k a^{*} b^{*} U^{12}\right]$

\begin{tabular}{|c|c|c|c|c|c|c|}
\hline & $\mathrm{U}^{11}$ & $\mathrm{U}^{22}$ & $\mathrm{U}^{33}$ & $\mathrm{U}^{23}$ & $\mathrm{U}^{13}$ & $\mathrm{U}^{12}$ \\
\hline $\mathrm{Cl}(1)$ & $65(1)$ & $70(1)$ & $33(1)$ & $8(1)$ & $9(1)$ & $-25(1)$ \\
\hline $\mathrm{Cl}(2)$ & $29(1)$ & $62(1)$ & $81(1)$ & $3(1)$ & $9(1)$ & 11(1) \\
\hline $\mathrm{Cl}(3)$ & $23(1)$ & $74(1)$ & $48(1)$ & $5(1)$ & $6(1)$ & $4(1)$ \\
\hline $\mathrm{F}(1)$ & $30(2)$ & $57(3)$ & $61(2)$ & $3(2)$ & $8(2)$ & $5(2)$ \\
\hline$F(2)$ & $34(2)$ & $82(3)$ & $46(2)$ & $29(2)$ & $-5(1)$ & $-19(2)$ \\
\hline $\mathrm{F}(3)$ & $28(2)$ & $87(3)$ & $44(2)$ & $13(2)$ & $-8(1)$ & $-18(2)$ \\
\hline $\mathrm{O}(1)$ & $32(2)$ & $33(2)$ & $26(2)$ & $-3(1)$ & $12(1)$ & $-15(2)$ \\
\hline $\mathrm{N}(1)$ & $25(2)$ & $52(3)$ & $24(2)$ & $-5(2)$ & $11(1)$ & $-8(2)$ \\
\hline $\mathrm{N}(2)$ & $21(2)$ & $25(2)$ & $27(2)$ & $-2(2)$ & $10(1)$ & $-3(1)$ \\
\hline $\mathrm{C}(1)$ & $17(2)$ & $17(2)$ & $19(2)$ & $2(1)$ & $4(1)$ & $0(1)$ \\
\hline $\mathrm{C}(2)$ & $21(2)$ & $16(2)$ & $22(2)$ & $0(1)$ & $8(1)$ & $2(1)$ \\
\hline $\mathrm{C}(3)$ & $16(2)$ & $19(2)$ & $21(2)$ & $2(1)$ & $6(1)$ & $1(1)$ \\
\hline $\mathrm{C}(4)$ & $17(2)$ & $32(2)$ & $22(2)$ & $-2(2)$ & $8(1)$ & $-4(2)$ \\
\hline $\mathrm{C}(5)$ & $21(2)$ & $18(2)$ & $22(2)$ & $2(1)$ & $8(1)$ & $-2(1)$ \\
\hline$C(6)$ & $22(2)$ & $31(2)$ & $27(2)$ & $1(2)$ & $10(2)$ & $-2(2)$ \\
\hline $\mathrm{C}(7)$ & $24(2)$ & $22(2)$ & $36(2)$ & $-2(2)$ & $10(2)$ & $-8(2)$ \\
\hline $\mathrm{C}(8)$ & $21(2)$ & $30(3)$ & $39(2)$ & $0(2)$ & $3(2)$ & $-5(2)$ \\
\hline $\mathrm{C}(9)$ & $22(2)$ & $25(2)$ & $27(2)$ & $-2(2)$ & $3(1)$ & $-1(2)$ \\
\hline$C(10)$ & $18(2)$ & $18(2)$ & $23(2)$ & $-2(2)$ & $6(1)$ & $4(1)$ \\
\hline $\mathrm{C}(11)$ & $19(2)$ & $35(2)$ & $19(2)$ & $4(2)$ & $7(1)$ & $-4(2)$ \\
\hline$C(12)$ & $20(2)$ & $48(3)$ & $33(2)$ & $-6(2)$ & $4(2)$ & $0(2)$ \\
\hline$C(13)$ & $22(2)$ & $27(2)$ & $30(2)$ & $-5(2)$ & $5(2)$ & $3(2)$ \\
\hline$C(14)$ & $37(2)$ & $25(3)$ & $39(3)$ & $0(2)$ & $12(2)$ & $-5(2)$ \\
\hline$C(15)$ & $44(3)$ & $27(3)$ & $50(3)$ & $-3(2)$ & $4(2)$ & $0(2)$ \\
\hline$C(16)$ & $37(3)$ & $41(3)$ & $41(3)$ & $-7(3)$ & $0(2)$ & $10(2)$ \\
\hline$C(17)$ & $27(2)$ & $52(4)$ & $35(2)$ & $-12(2)$ & $6(2)$ & $2(2)$ \\
\hline$C(18)$ & $23(2)$ & $51(4)$ & $31(2)$ & $7(2)$ & $-2(2)$ & $-10(2)$ \\
\hline$C(19)$ & $29(2)$ & $39(3)$ & $32(2)$ & $3(2)$ & $6(2)$ & $-9(2)$ \\
\hline
\end{tabular}


Table A6-15. Hydrogen coordinates ( $\mathrm{x} \mathrm{104)}$ and isotropic displacement parameters $\left(\AA^{2} \times 10^{3}\right)$

for $\mathrm{C} 38 \mathrm{H} 38 \mathrm{Cl} 6 \mathrm{~F} 6 \mathrm{~N} 4 \mathrm{O} 2$.

\begin{tabular}{|c|c|c|c|c|}
\hline & $\mathrm{x}$ & $\mathrm{y}$ & $\mathrm{z}$ & $\mathrm{U}(\mathrm{eq})$ \\
\hline $\mathrm{H}(1)$ & 9381 & 2147 & 5517 & 40 \\
\hline $\mathrm{H}(2)$ & 8038 & 2188 & 8070 & 29 \\
\hline $\mathrm{H}(4)$ & 9777 & 4364 & 6050 & 28 \\
\hline $\mathrm{H}(6)$ & 10756 & 6108 & 5938 & 31 \\
\hline $\mathrm{H}(7)$ & 11627 & 7616 & 6864 & 32 \\
\hline $\mathrm{H}(8)$ & 11782 & 7772 & 8935 & 36 \\
\hline $\mathrm{H}(9)$ & 11077 & 6488 & 10063 & 30 \\
\hline $\mathrm{H}(12)$ & 8318 & 387 & 5728 & 40 \\
\hline $\mathrm{H}(13)$ & 9170 & 495 & 7972 & 32 \\
\hline $\mathrm{H}(14 \mathrm{~A})$ & 7779 & -796 & 7370 & 40 \\
\hline $\mathrm{H}(14 \mathrm{~B})$ & 8198 & -734 & 8682 & 40 \\
\hline $\mathrm{H}(15 \mathrm{~A})$ & 9160 & -2093 & 8114 & 48 \\
\hline $\mathrm{H}(15 \mathrm{~B})$ & 8427 & -2936 & 7724 & 48 \\
\hline $\mathrm{H}(16 \mathrm{~A})$ & 9212 & -2932 & 6216 & 48 \\
\hline $\mathrm{H}(16 \mathrm{~B})$ & 8438 & -2273 & 5768 & 48 \\
\hline $\mathrm{H}(17 \mathrm{~A})$ & 9745 & -672 & 6392 & 45 \\
\hline $\mathrm{H}(17 \mathrm{~B})$ & 9313 & -783 & 5095 & 45 \\
\hline H(19) & 8860 & 2752 & 1355 & 39 \\
\hline $\mathrm{H}(1 \mathrm{O})$ & $8670(40)$ & $3080(90)$ & $8970(70)$ & $50(20)$ \\
\hline
\end{tabular}




\section{Appendix I. Derivatives of Binol-Pyridine Compounds}

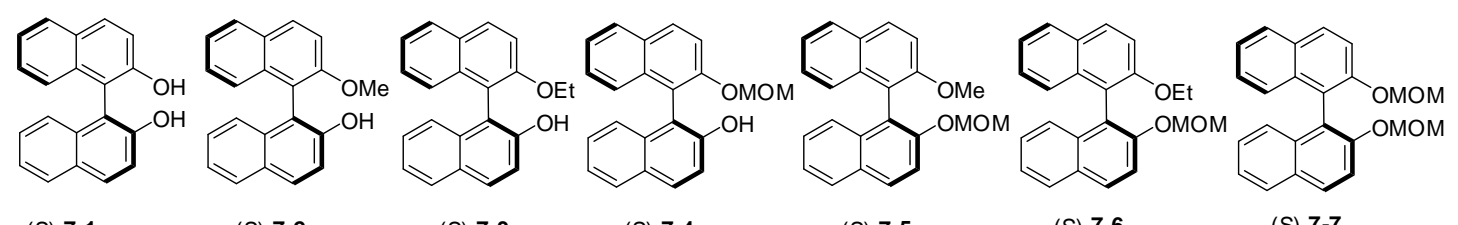

(S) -7-1

(S) -7-2

(S) -7-3

(S) -7-4

(S) -7-5

(S) -7-6

(S) -7-7

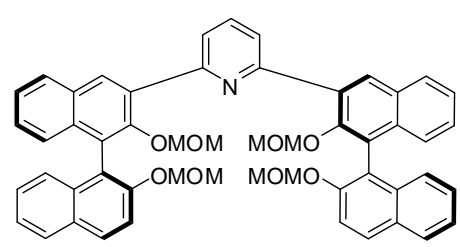

(S)-7-8

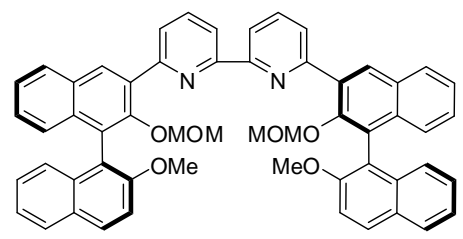

(S) -7-10

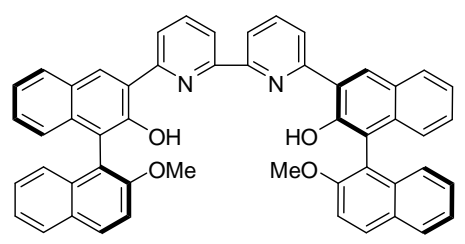

(S) -7-13

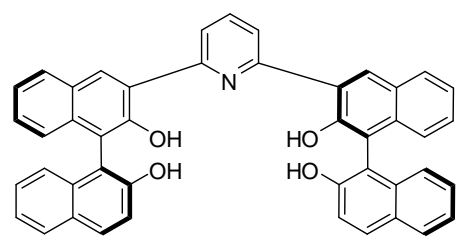

(S) -7-9

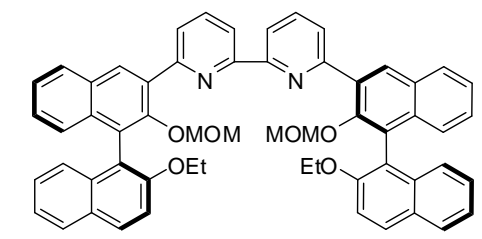

(S)-7-11

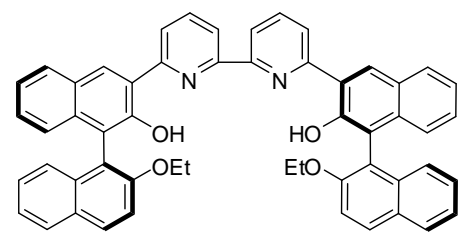

(S)-7-14

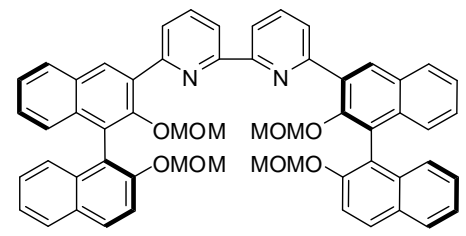

(S) -7-12

(S) -7-15

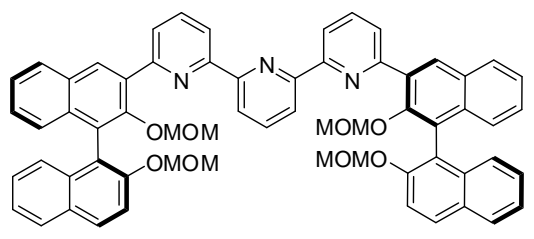

(S) -7-20

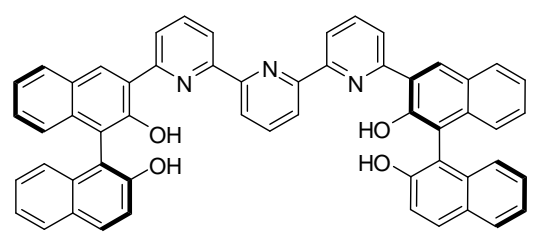

(S)-7-21 


\section{Preparation and Characterization of Compounds}
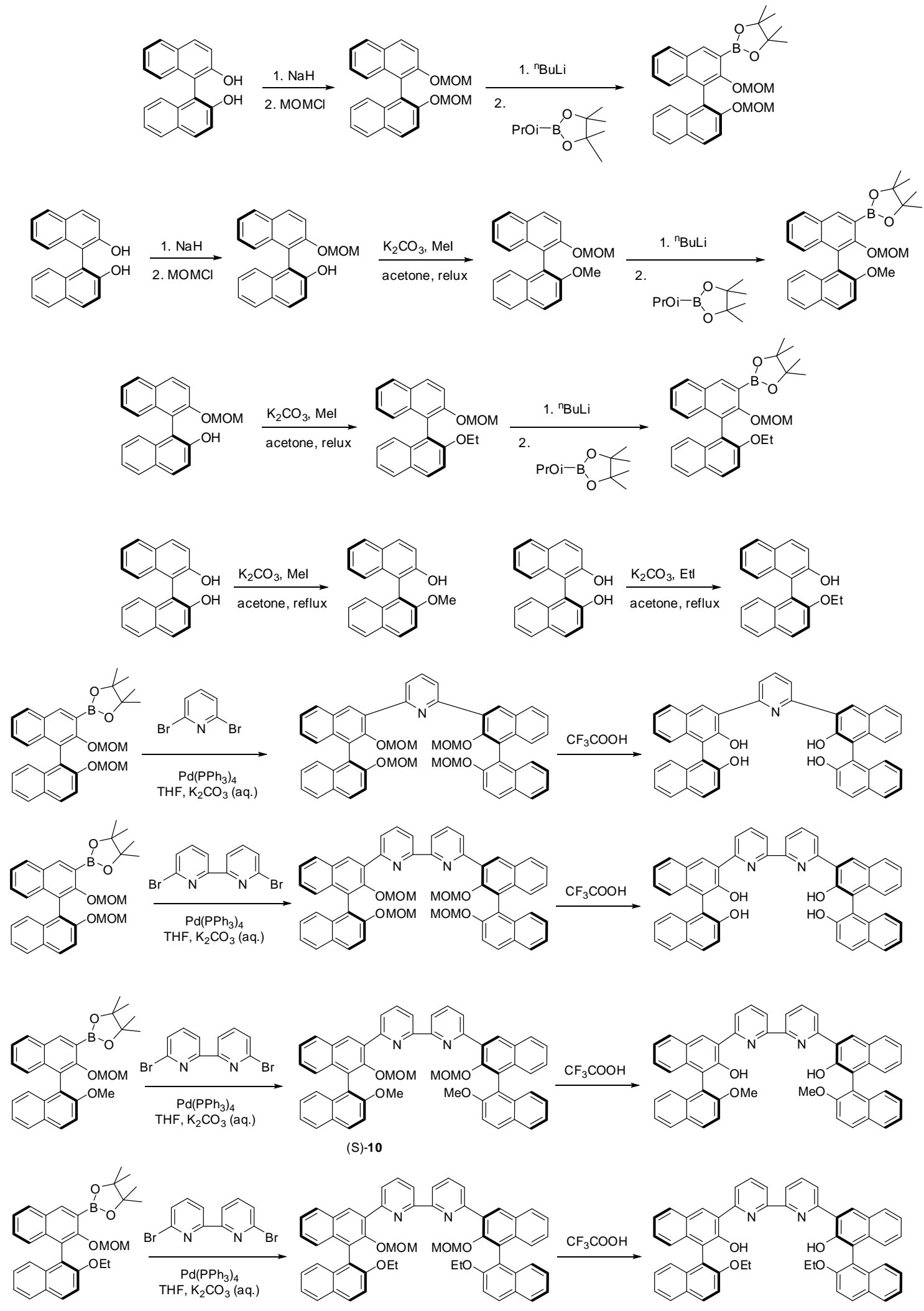

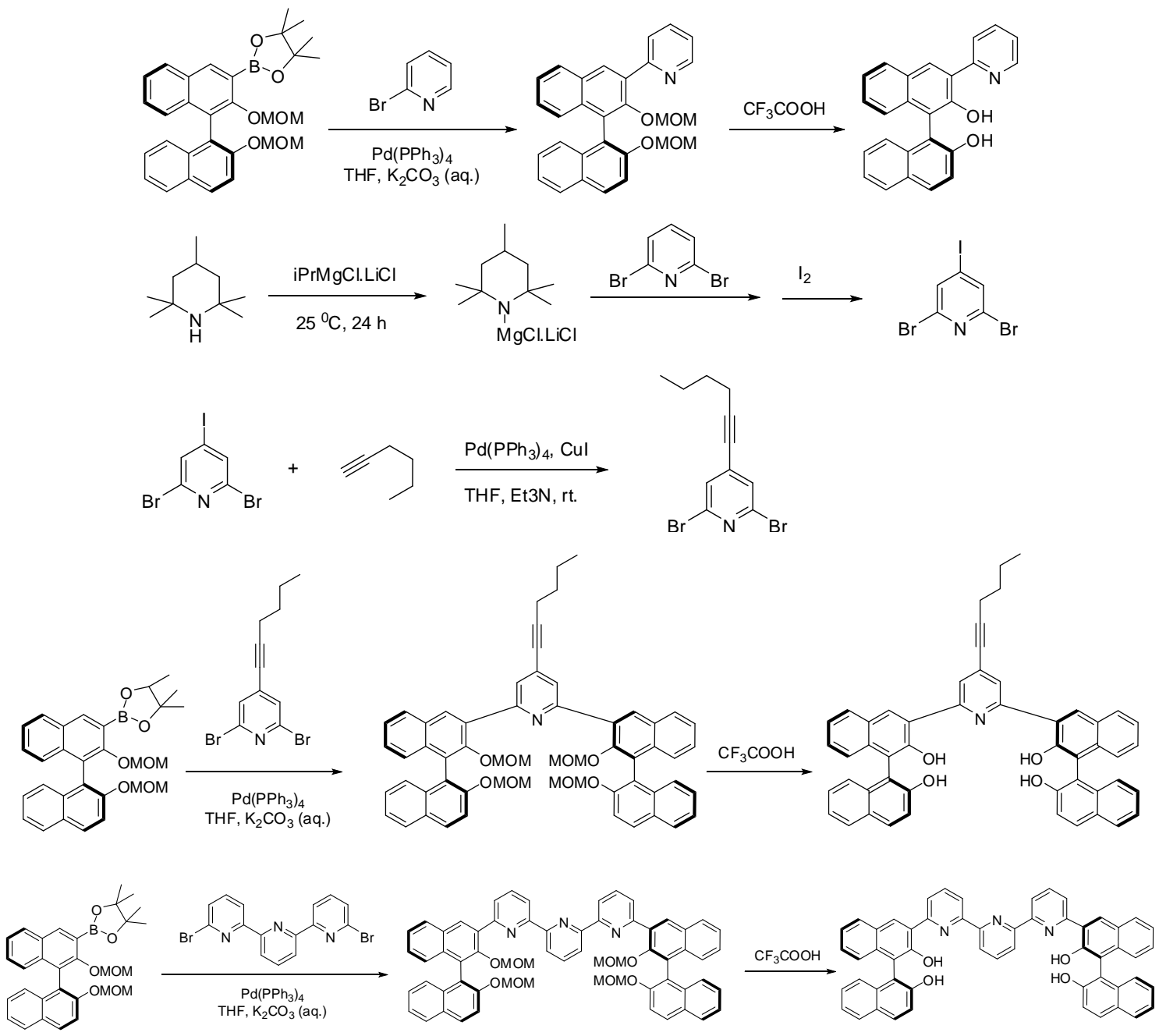

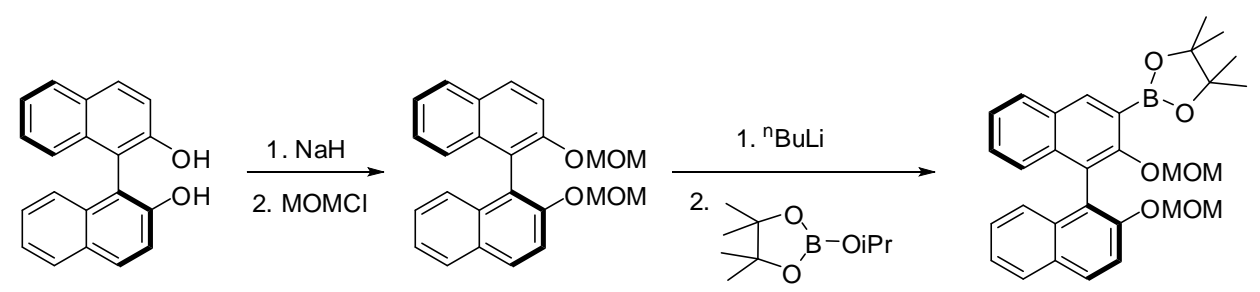

Preparation of (S)-2,2'-bis(methoxymethoxy)-1,1'-binaphthy. Under nitrogen, in a $500 \mathrm{~mL}$ flame-dried flask, (S)-BINOL (5.0 g, $17.5 \mathrm{mmol}$ ) was dissolved in $200 \mathrm{~mL}$ THF and cooled to $0^{\circ} \mathrm{C}$. $\mathrm{NaH}(60 \%$ in mineral oil, $1.75 \mathrm{~g}, 43.75 \mathrm{mmol})$ was added to the solution in 3 portions. After the mixture was stirred for $15 \mathrm{~min}, \mathrm{MOMCl}(3.3 \mathrm{~mL}, 43.75$ mmol) was added and the reaction mixture was allowed to warm to room temperature. After the mixture was stirred for $1 \mathrm{~h}$, the reaction was quenched with water $(20 \mathrm{~mL})$. The organic layer was separated and the aqueous layer was extracted with ethyl acetated $(2 \mathrm{x}$ $30 \mathrm{~mL})$. The combined organic layer was washed with brine $(30 \mathrm{~mL})$ and dried over anhydrous $\mathrm{Na}_{2} \mathrm{SO}_{4}$. After evaporation of the solvent, the residue was purified by column chromatography on silica gel eluted with hexanes/ethyl acetate (10/1) to afford desired product as a white solid in $92 \%$ yield. ${ }^{1} \mathrm{H}$ NMR $\left(300 \mathrm{MHz}, \mathrm{CDCl}_{3}\right) \delta 3.15(\mathrm{~s}, 6 \mathrm{H}), 4.98(\mathrm{~d}$, $2 \mathrm{H}, \mathrm{J}=6.9 \mathrm{~Hz}), 5.09(\mathrm{~d}, 2 \mathrm{H}, \mathrm{J}=6.9 \mathrm{~Hz}), 7.14-7.25(\mathrm{~m}, 4 \mathrm{H}), 7.32-7.37(\mathrm{~m}, 2 \mathrm{H}), 7.58(\mathrm{~d}$, $2 \mathrm{H}, \mathrm{J}=9.3 \mathrm{~Hz}), 7.88(\mathrm{~d}, 2 \mathrm{H}, \mathrm{J}=8.1 \mathrm{~Hz}), 7.96(\mathrm{~d}, 2 \mathrm{H}, \mathrm{J}=9.0 \mathrm{~Hz})$.

\section{Preparation of (S)-2-(2,2'-bis(methoxymethoxy)-1,1'-binaphthyl-3-yl)-4,4,5,5-}

tetramethyl-1,3,2-dioxaborolane. Under nitrogen (S)-2,2'-bis(methoxymethoxy)-1,1'binaphthy $(3.74 \mathrm{~g}, 10 \mathrm{mmol})$ was dissolved in $110 \mathrm{~mL}$ ether and cooled to $0^{\circ} \mathrm{C} . \mathrm{nBuLi}$ (2.5 M, $5.2 \mathrm{~mL}, 13 \mathrm{mmol}$ ) was added dropwise and the reaction mixture was allow to 
warm to room temperature over $3 \mathrm{~h}$. Then the reaction mixture was cooled to The reaction mixture was cooled to $-78{ }^{\circ} \mathrm{C}$ and 2-isopropoxy-4,4,5,5-tetramethyl-1,3,2dioxaborolane $(3 \mathrm{~mL}, 15.0 \mathrm{mmol})$ was added. The mixture was allowed to warm to room temperature and stirred for overnight. The generated salts were removed by filtration through a Buchner funnel and rinsed with $\mathrm{CH}_{2} \mathrm{Cl}_{2}$. The combined organic layer was concentrated and purified by flash chromatography on silica gel eluted with hexanes/ethyl acetate (10/1) to afford desired product as a white solid in $42 \%$ yield. ${ }^{1} \mathrm{H}$ NMR (300 MHz, $\left.\mathrm{CDCl}_{3}\right) \delta 1.39(\mathrm{~s}, 2 \mathrm{H}), 2.39(\mathrm{~s}, 2 \mathrm{H}), 3.11(\mathrm{~s}, 2 \mathrm{H}), 4.84(\mathrm{~d}, \mathrm{~J}=6.0 \mathrm{~Hz}$, $1 \mathrm{H}), 4.89(\mathrm{~d}, \mathrm{~J}=6.0 \mathrm{~Hz}, 1 \mathrm{H}), 5.08(\mathrm{~d}, \mathrm{~J}=6.6 \mathrm{~Hz}, 1 \mathrm{H}), 5.16(\mathrm{~d}, \mathrm{~J}=6.9 \mathrm{~Hz}, 1 \mathrm{H}), 6.94(\mathrm{~d}, \mathrm{~J}$ $=8.4 \mathrm{~Hz}, 1 \mathrm{H}), 7.01(\mathrm{~s}, \mathrm{~J}=8.4 \mathrm{~Hz}, 1 \mathrm{H}), 7.25(\mathrm{t}, \mathrm{J}=7.2 \mathrm{~Hz}, 1 \mathrm{H}), 7.31-7.41(\mathrm{~m}, 2 \mathrm{H}), 7.43$ $(\mathrm{t}, \mathrm{J}=7.2 \mathrm{~Hz}, 1 \mathrm{H}), 7.63(\mathrm{~d}, \mathrm{~J}=9.0 \mathrm{~Hz}, 1 \mathrm{H}), 7.94(\mathrm{~d}, \mathrm{~J}=8.1 \mathrm{~Hz}, 1 \mathrm{H}), 8.04-8.07(\mathrm{~m}, 2 \mathrm{H})$, $8.49(\mathrm{~s}, 1 \mathrm{H})$

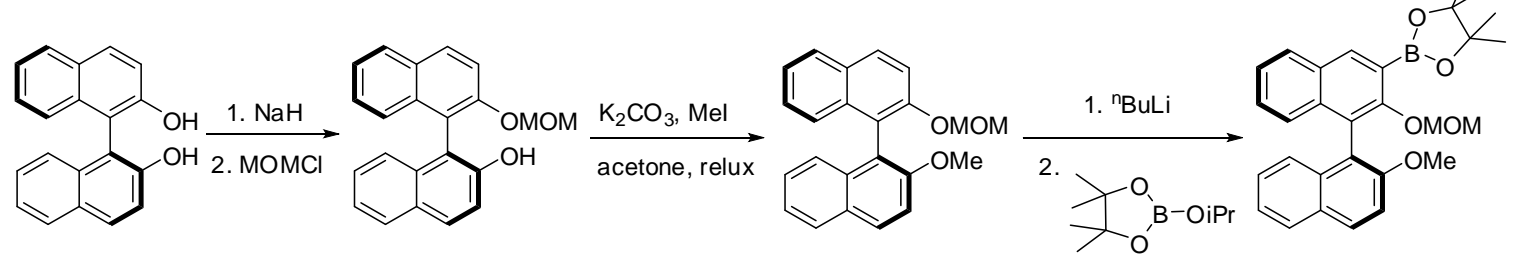

Preparation of (S)-2'-(methoxymethoxy)-1,1'-binaphthyl-2-ol. Under nitrogen, in a $500 \mathrm{~mL}$ flame-dried flask, (S)-BINOL (5.0 g, $17.5 \mathrm{mmol})$ was dissolved in $200 \mathrm{~mL}$ THF and cooled to $0{ }^{\circ} \mathrm{C} . \mathrm{NaH}(60 \%$ in mineral oil, $0.77 \mathrm{~g}, 19.2 \mathrm{mmol})$ was added to the solution in 3 portions. After the mixture was stirred for $15 \mathrm{~min}$, $\mathrm{MOMCl}(1.5 \mathrm{~mL}, 19.2$ mmol) was added and the reaction mixture was allowed to warm to room temperature. 
After the mixture was stirred for $1 \mathrm{~h}$, the reaction was quenched with water $(20 \mathrm{~mL})$. The organic layer was separated and the aqueous layer was extracted with ethyl acetated $(2 \mathrm{x}$ $30 \mathrm{~mL})$. The combined organic layer was washed with brine $(30 \mathrm{~mL})$ and dried over anhydrous $\mathrm{Na}_{2} \mathrm{SO}_{4}$. After evaporation of the solvent, the residue was purified by column chromatography on silica gel eluted with hexanes/ethyl acetate (15/1) to afford desired product as a white solid in $71 \%$ yield. ${ }^{1} \mathrm{H} \mathrm{NMR}\left(300 \mathrm{MHz}, \mathrm{CDCl}_{3}\right) \delta 3.19(\mathrm{~s}, 3 \mathrm{H}), 4.95(\mathrm{~s}$, 1H), $5.06(\mathrm{~d}, \mathrm{~J}=6.9 \mathrm{~Hz}, 1 \mathrm{H}), 5.11(\mathrm{~d}, \mathrm{~J}=6.9 \mathrm{~Hz}, 1 \mathrm{H}), 7.08(\mathrm{~d}, \mathrm{~J}=7.8 \mathrm{~Hz}, 1 \mathrm{H}), 7.18-7.43$ $(\mathrm{m}, 6 \mathrm{H}), 7.60(\mathrm{~d}, \mathrm{~J}=9.0 \mathrm{~Hz}, 1 \mathrm{H}), 7.86(\mathrm{~d}, \mathrm{~J}=8.1 \mathrm{~Hz}, 1 \mathrm{H}), 7.91(\mathrm{~d}, \mathrm{~J}=8.7 \mathrm{~Hz}, 2 \mathrm{H}), 8.03$ $(\mathrm{d}, \mathrm{J}=9.0 \mathrm{~Hz}, 1 \mathrm{H})$.

Preparation of (S)-2-methoxy-2'-(methoxymethoxy)-1,1'-binaphthyl. To a $100 \mathrm{~mL}$ flask, (S)-2'-(methoxymethoxy)-1,1'-binaphthyl-2-ol (1.65g, $5 \mathrm{mmol}), \mathrm{K}_{2} \mathrm{CO}_{3}(0.864 \mathrm{~g}$, $6.26 \mathrm{mmol}$ ) and $50 \mathrm{~mL}$ acetone were added. After the reaction mixture was stirred under reflux for $1 \mathrm{~h}$, MeI $(0.47 \mathrm{~mL}, 7.5 \mathrm{mmol})$ was added and the resulting mixture was stirred under relux overnight. When it cooled down, the reaction mixture was poured in to 100 $\mathrm{mL}$ water and extracted with $\mathrm{CH}_{2} \mathrm{Cl}_{2}(3 \times 30 \mathrm{~mL})$. The combined organic layer was washed with brine $(30 \mathrm{~mL})$ and dried over anhydrous $\mathrm{Na}_{2} \mathrm{SO}_{4}$. After evaporation of the solvent, the residue was purified by column chromatography on silica gel eluted with hexanes/ethyl acetate (15/1) to afford desired product as a white solid in $81 \%$ yield. ${ }^{1} \mathrm{H}$ NMR (300 MHz, $\left.\mathrm{CDCl}_{3}\right) \delta 3.17(\mathrm{~s}, 3 \mathrm{H}), 3.77(\mathrm{~s}, 3 \mathrm{H}), 4.99(\mathrm{~d}, \mathrm{~J}=6.6 \mathrm{~Hz}, 1 \mathrm{H}), 5.07$ (d, J $=6.6 \mathrm{~Hz}, 1 \mathrm{H}), 7.13(\mathrm{~d}, \mathrm{~J}=8.4 \mathrm{~Hz}, 2 \mathrm{H}), 7.20-7.38(\mathrm{~m}, 4 \mathrm{H}), 7.46(\mathrm{~d}, \mathrm{~J}=9.0 \mathrm{~Hz}, 1 \mathrm{H}), 7.58$ 
$(\mathrm{d}, \mathrm{J}=9.0 \mathrm{~Hz}, 1 \mathrm{H}), 7.87(\mathrm{~d}, \mathrm{~J}=8.1 \mathrm{~Hz}, 1 \mathrm{H}), 7.88(\mathrm{~d}, \mathrm{~J}=8.1 \mathrm{~Hz}, 1 \mathrm{H}), 7.97(\mathrm{t}, \mathrm{J}=9.6 \mathrm{~Hz}$, $2 \mathrm{H})$.

Preparation of (S)-2-(2'-methoxy-2-(methoxymethoxy)-1,1'-binaphthyl-3-yl)4,4,5,5-tetra-methyl-1,3,2-dioxaborolane. Under nitrogen (S)-2-methoxy-2'(methoxymethoxy)-1,1'-binaphthyl (1.20 g, $3.5 \mathrm{mmol})$ was dissolved in $40 \mathrm{~mL}$ ether and cooled to $0^{\circ} \mathrm{C}$. $\mathrm{nBuLi}(2.5 \mathrm{M}, 1.67 \mathrm{~mL}, 4.2 \mathrm{mmol})$ was added dropwise and the reaction mixture was allow to warm to room temperature over $3 \mathrm{~h}$. Then the reaction mixture was cooled to The reaction mixture was cooled to $-78{ }^{\circ} \mathrm{C}$ and 2-isopropoxy-4,4,5,5-tetramethyl-1,3,2-dioxaborolane (1.1 mL, $5.2 \mathrm{mmol})$ was added. The mixture was allowed to warm to room temperature and stirred for overnight. The generated salts were removed by filtration through a Buchner funnel and rinsed with $\mathrm{CH}_{2} \mathrm{Cl}_{2}$. The combined organic layer was concentrated and purified by flash chromatography on silica gel eluted with hexanes/ethyl acetate (10/1) to afford desired product as a white solid in $78 \%$ yield. ${ }^{1} \mathrm{H}$ NMR $\left(300 \mathrm{MHz}, \mathrm{CDCl}_{3}\right) \delta 1.39(\mathrm{~s}, 12 \mathrm{H}), 2.42$ (s, 3H), 3.77 (s, 3H), 4.82 (d, J = 6.0 Hz, 1H), 4.88 (d, J = 5.7 Hz, 1H), 7.13-7.44 (m, 7H), $7.83(\mathrm{~d}, \mathrm{~J}=8.1 \mathrm{~Hz}, 1 \mathrm{H}), 7.94(\mathrm{t}, \mathrm{J}=9.0 \mathrm{~Hz}, 2 \mathrm{H}), 8.49(\mathrm{~s}, 1 \mathrm{H}) .{ }^{13} \mathrm{C} \mathrm{NMR}\left(75 \mathrm{MHz}, \mathrm{CDCl}_{3}\right)$ $\delta 25.1,55.9,56.1,56.8,84.0,100.2,113.7,120.0,123.7,124.9,126.0,126.1,126.4$, 126.6, 127.6, 127.8, 128.8, 129.1, 129.6, 130.6, 134.6, 136.0, 139.5, 155.4, 156.9. HRMS Calcd for $\mathrm{C}_{29} \mathrm{H}_{31} \mathrm{BO}_{5} \mathrm{Na}\left(\mathrm{MNa}^{+}\right)$: 493.2162. Found: 493.2162. m.p. 73-74 ${ }^{0} \mathrm{C} . \quad[\alpha]_{\mathrm{D}}=$ $-55.441\left(\mathrm{c}=0.43, \mathrm{CHCl}_{3}\right)$. 

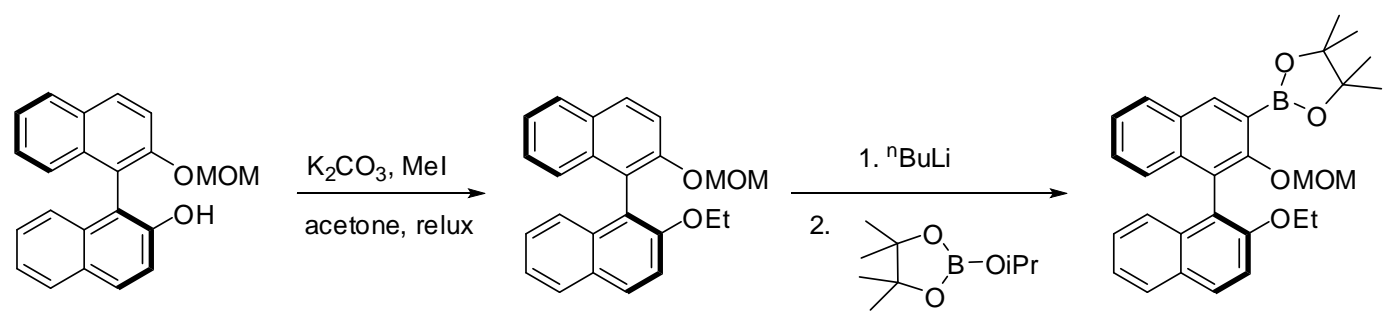

Preparation of (S)-2-ethoxy-2'-(methoxymethoxy)-1,1'-binaphthyl. To a $100 \mathrm{~mL}$ flask, (S)-2'-(methoxymethoxy)-1,1'-binaphthyl-2-ol (1.65g, $5 \mathrm{mmol}), \mathrm{K}_{2} \mathrm{CO}_{3}(1.04 \mathrm{~g}, 7.5$ $\mathrm{mmol}$ ) and $50 \mathrm{~mL}$ acetone were added. After the reaction mixture was stirred under reflux for $1 \mathrm{~h}$, EtI $(0.8 \mathrm{~mL}, 10 \mathrm{mmol})$ was added and the resulting mixture was stirred under relux overnight. When it cooled down, the reaction mixture was poured in to $100 \mathrm{~mL}$ water and extracted with $\mathrm{CH}_{2} \mathrm{Cl}_{2}(3 \times 30 \mathrm{~mL})$. The combined organic layer was washed with brine $(30 \mathrm{~mL})$ and dried over anhydrous $\mathrm{Na}_{2} \mathrm{SO}_{4}$. After evaporation of the solvent, the residue was purified by column chromatography on silica gel eluted with hexanes/ethyl acetate (15/1) to afford desired product as a white solid in $85 \%$ yield. ${ }^{1} \mathrm{H}$ NMR (300 MHz, $\left.\mathrm{CDCl}_{3}\right) \delta 1.17(\mathrm{t}, \mathrm{J}=6.9 \mathrm{~Hz}, 3 \mathrm{H}), 3.29(\mathrm{~s}, 3 \mathrm{H}), 4.15(\mathrm{~m}, 2 \mathrm{H}), 5.10(\mathrm{~d}, \mathrm{~J}$ $=6.6 \mathrm{~Hz}, 1 \mathrm{H}), 5.20(\mathrm{~d}, \mathrm{~J}=6.6 \mathrm{~Hz}, 1 \mathrm{H}), 7.31-7.48(\mathrm{~m}, 6 \mathrm{H}), 7.53(\mathrm{~d}, \mathrm{~J}=9.0 \mathrm{~Hz}, 1 \mathrm{H}), 7.73$ $(\mathrm{d}, \mathrm{J}=9.0 \mathrm{~Hz}, 1 \mathrm{H}), 7.98(\mathrm{~d}, \mathrm{~J}=8.1 \mathrm{~Hz}, 2 \mathrm{H}), 8.05(\mathrm{~d}, \mathrm{~J}=9.0 \mathrm{~Hz}, 2 \mathrm{H}) .{ }^{13} \mathrm{C} \mathrm{NMR}(75 \mathrm{MHz}$, $\left.\mathrm{CDCl}_{3}\right) \delta 15.4,56.1,65.4,95.6,116.0,117.7,120.7,121.2,124.0,124.2,124.3,125.8$ 126.0, 126.5, 126.7, 128.3, 129.6, 129.7, 130.3, 134.5, 134.6, 153.1, 154.7. HRMS Calcd for $\mathrm{C}_{24} \mathrm{H}_{22} \mathrm{O}_{3} \mathrm{Na}\left(\mathrm{MNa}^{+}\right)$: 381.1467. Found: 381.1473. m.p. $62{ }^{0} \mathrm{C} .[\alpha]_{\mathrm{D}}=-76.367(\mathrm{c}=$ $\left.0.29, \mathrm{CHCl}_{3}\right)$.

Preparation of (S)-2-(2'-ethoxy-2-(methoxymethoxy)-1,1'-binaphthyl-3-yl)- 
4,4,5,5-tetra-methyl-1,3,2-dioxaborolane. Under nitrogen (S)-2-ethoxy-2'(methoxymethoxy)-1,1'-binaphthyl (1.20 g, $3.5 \mathrm{mmol})$ was dissolved in $30 \mathrm{~mL}$ ether and cooled to $0^{\circ} \mathrm{C}$. nBuLi $(2.5 \mathrm{M}, 1.27 \mathrm{~mL}, 3.2 \mathrm{mmol})$ was added dropwise and the reaction mixture was allow to warm to room temperature over $3 \mathrm{~h}$. Then the reaction mixture was cooled to The reaction mixture was cooled to $-78{ }^{\circ} \mathrm{C}$ and 2-isopropoxy-4,4,5,5-tetramethyl-1,3,2-dioxaborolane $(0.81 \mathrm{~mL}, 4.0 \mathrm{mmol})$ was added. The mixture was allowed to warm to room temperature and stirred for overnight. The generated salts were removed by filtration through a Buchner funnel and rinsed with $\mathrm{CH}_{2} \mathrm{Cl}_{2}$. The combined organic layer was concentrated and purified by flash chromatography on silica gel eluted with hexanes/ethyl acetate (10/1) to afford desired product as a white solid in $82 \%$ yield. ${ }^{1} \mathrm{H}$ NMR $\left(300 \mathrm{MHz}, \mathrm{CDCl}_{3}\right) \delta 1.03(\mathrm{td}, \mathrm{J} 1=6.9$ $\mathrm{Hz}, \mathrm{J} 2=3.6 \mathrm{~Hz}, 3 \mathrm{H}), 1.41(\mathrm{~d}, \mathrm{~J}=3.6,12 \mathrm{H}), 2.45(\mathrm{~d}, \mathrm{~J}=4.2 \mathrm{~Hz}, 3 \mathrm{H}), 4.15(\mathrm{~m}, 2 \mathrm{H}), 4.86$ $(\mathrm{dd}, \mathrm{J} 1=5.4 \mathrm{~Hz}, \mathrm{~J} 2=3.9 \mathrm{~Hz}, 1 \mathrm{H}), 5.20(\mathrm{dd}, \mathrm{J} 1=6.0 \mathrm{~Hz}, \mathrm{~J} 2=3.9 \mathrm{~Hz}, 1 \mathrm{H}), 7.16-7.42(\mathrm{~m}$, 7H), $7.82(\mathrm{dd}, \mathrm{J} 1=8.1 \mathrm{~Hz}, \mathrm{~J} 2=3.3 \mathrm{~Hz}, 1 \mathrm{H}), 7.93(\mathrm{dd}, \mathrm{J} 1=8.7 \mathrm{~Hz}, \mathrm{~J} 2=3.6 \mathrm{~Hz}, 2 \mathrm{H})$, $8.48(\mathrm{~d}, \mathrm{~J}=3.9 \mathrm{~Hz}, 2 \mathrm{H}) .{ }^{13} \mathrm{C} \mathrm{NMR}\left(75 \mathrm{MHz}, \mathrm{CDCl}_{3}\right) \delta 15.2,25.1,25.2,56.1,65.2,84.0$, $100.2,115.3,120.8,123.7,124.8,125.8,126.1,126.2,126.5,127.4,127.7,128.7,129.2$, 129.5, 130.6, 134.6, 136.0, 139.2, 154.8, 156.9. HRMS Calcd for $\mathrm{C}_{30} \mathrm{H}_{33} \mathrm{BO}_{5} \mathrm{Na}\left(\mathrm{MNa}^{+}\right)$: 507.2319. Found: 507.2318. m.p. $64{ }^{0} \mathrm{C} .[\alpha]_{\mathrm{D}}=-77.764\left(\mathrm{c}=0.34, \mathrm{CHCl}_{3}\right)$. 


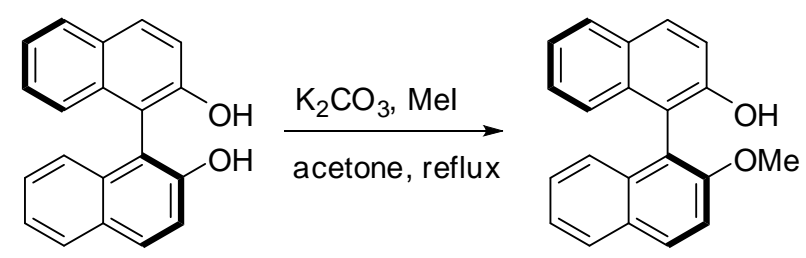

Preparation of (S)-2'-methoxy-1,1'-binaphthyl-2-ol. To a $100 \mathrm{~mL}$ flask, (S)-BINOL (1.44 g, $5.0 \mathrm{mmol}), \mathrm{K}_{2} \mathrm{CO}_{3}(0.76 \mathrm{~g}, 5.5 \mathrm{mmol})$ and $50 \mathrm{~mL}$ acetone were added. After the reaction mixture was stirred under reflux for $2 \mathrm{~h}$, MeI $(0.374 \mathrm{~mL}, 6.0 \mathrm{mmol})$ was added and the resulting mixture was stirred under reflux overnight. When it cooled down, the reaction mixture was poured in to $100 \mathrm{~mL}$ water and extracted with $\mathrm{CH}_{2} \mathrm{Cl}_{2}(3 \times 30 \mathrm{~mL})$. The combined organic layer was washed with brine $(30 \mathrm{~mL})$ and dried over anhydrous $\mathrm{Na}_{2} \mathrm{SO}_{4}$. After evaporation of the solvent, the residue was purified by column chromatography on silica gel eluted with hexanes/ $\mathrm{CH}_{2} \mathrm{Cl}_{2}$ (2/1) to afford desired product as a white solid in $79 \%$ yield. ${ }^{1} \mathrm{H}$ NMR $\left(300 \mathrm{MHz}, \mathrm{CDCl}_{3}\right) \delta 3.81(\mathrm{~s}, 3 \mathrm{H}), 4.91(\mathrm{~s}, 1 \mathrm{H})$, $7.05(\mathrm{~d}, \mathrm{~J}=9.0 \mathrm{~Hz}, 1 \mathrm{H}), 7.16-7.41(\mathrm{~m}, 6 \mathrm{H}), 7.49(\mathrm{~d}, 1 \mathrm{H}, 9.3 \mathrm{~Hz}), 7.85-7.92,(\mathrm{~m}, 3 \mathrm{H})$, $7.86(\mathrm{~d}, \mathrm{~J}=9.0 \mathrm{~Hz}, 1 \mathrm{H})$.

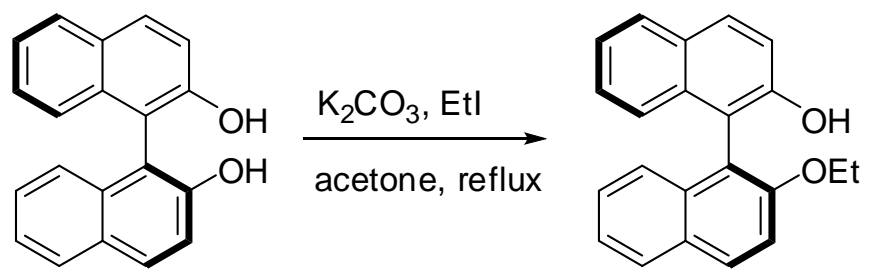

Preparation of (S)-2'-ethoxy-1,1'-binaphthyl-2-ol. To a $100 \mathrm{~mL}$ flask, (S)-BINOL (1.44 g, $5.0 \mathrm{mmol}), \mathrm{K}_{2} \mathrm{CO}_{3}(1.04 \mathrm{~g}, 7.5 \mathrm{mmol})$ and $50 \mathrm{~mL}$ acetone were added. After the reaction mixture was stirred under reflux for $2 \mathrm{~h}$, EtI $(0.60 \mathrm{~mL}, 7.5 \mathrm{mmol})$ was added and the resulting mixture was stirred under reflux overnight. When it cooled down, the 
reaction mixture was poured in to $100 \mathrm{~mL}$ water and extracted with $\mathrm{CH}_{2} \mathrm{Cl}_{2}(3 \times 30 \mathrm{~mL})$. The combined organic layer was washed with brine $(30 \mathrm{~mL})$ and dried over anhydrous $\mathrm{Na}_{2} \mathrm{SO}_{4}$. After evaporation of the solvent, the residue was purified by column chromatography on silica gel eluted with hexanes/ethyl acetate (15/1) to afford desired product as a white solid in $83 \%$ yield. ${ }^{1} \mathrm{H}$ NMR $\left(300 \mathrm{MHz}, \mathrm{CDCl}_{3}\right) \delta 1.11(\mathrm{t}, \mathrm{J}=6.9 \mathrm{~Hz}$, 3H), 4.01-4.16 (m, 2H), $4.97(\mathrm{~s}, 1 \mathrm{H}), 7.07(\mathrm{~d}, \mathrm{~J}=8.4 \mathrm{~Hz}, 1 \mathrm{H}), 7.17-7.40(\mathrm{~m}, 6 \mathrm{H}), 7.46(\mathrm{~d}$, $\mathrm{J}=9.0 \mathrm{~Hz}, 1 \mathrm{H}), 7.85-7.92(\mathrm{~m}, 3 \mathrm{H}), 8.02(\mathrm{~d}, \mathrm{~J}=9.0 \mathrm{~Hz}, 1 \mathrm{H})$.

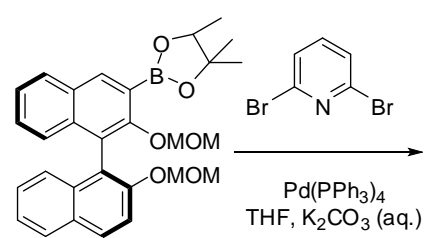

Preparation of
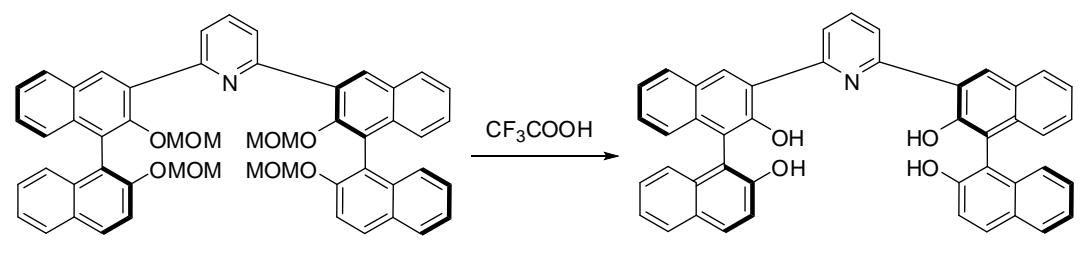

2,6-bis((S)-2,2'-bis(methoxymethoxy)-1,1'-binaphthyl-3-

yl)pyridine. Under nitrogen, to a $100 \mathrm{~mL}$ flame-dried Schlenk flask was loaded (S)-2-(2,2'-bis(methoxymethoxy)-1,1'-binaphthyl-3-yl)-4,4,5,5-tetramethyl-1,3,2-dioxabo rolane (1.0 g, $2.0 \mathrm{mmol})$, 2,6-dibromopyridine (189.5 mg, $0.8 \mathrm{mmol}), \mathrm{Pd}\left(\mathrm{PPh}_{3}\right)_{4}(92 \mathrm{mg}$, $0.08 \mathrm{mmol})$. Degassed THF $(10 \mathrm{~mL})$ and $2 \mathrm{M} \mathrm{K}_{2} \mathrm{CO}_{3}(10 \mathrm{~mL})$ were transferred into the flask via cannula. The reaction mixture was stirred under reflux for overnight. After it cooled to room temperature, the organic layer was separated and the aqueous layer was extracted with $\mathrm{CH}_{2} \mathrm{Cl}_{2}(3 \times 30 \mathrm{~mL})$. The combined organic layer was washed with brine (30 mL) and dried over anhydrous $\mathrm{Na}_{2} \mathrm{SO}_{4}$. After evaporation of the solvent, the residue was purified by column chromatography on silica gel eluted with hexanes/ethyl acetate 
(6/1) to afford desired product as a white solid in $88 \%$ yield. ${ }^{1} \mathrm{H}$ NMR $\left(300 \mathrm{MHz}, \mathrm{CDCl}_{3}\right)$ $\delta 2.51(\mathrm{~s}, 6 \mathrm{H}), 3.29(\mathrm{~s}, 6 \mathrm{H}), 4.59(\mathrm{~d}, \mathrm{~J}=6.0 \mathrm{~Hz}, 2 \mathrm{H}), 4.61(\mathrm{~d}, \mathrm{~J}=6.0 \mathrm{~Hz}, 2 \mathrm{H}), 5.14(\mathrm{~d}, \mathrm{~J}=$ $6.9 \mathrm{~Hz}, 2 \mathrm{H}), 5.23(\mathrm{~d}, \mathrm{~J}=6.9 \mathrm{~Hz}, 2 \mathrm{H}), 7.32-7.50(\mathrm{~m}, 12 \mathrm{H}), 7.69(\mathrm{~d}, \mathrm{~J}=9.0 \mathrm{~Hz}, 2 \mathrm{H})$, 7.85-7.94 (m, 3H), 8.01-8.05 (m, 4H), $8.11(\mathrm{~d}, \mathrm{~J}=8.1 \mathrm{~Hz}, 2 \mathrm{H}), 8.66(\mathrm{~s}, 2 \mathrm{H}) .{ }^{13} \mathrm{C}$ NMR $\left(75 \mathrm{MHz}, \mathrm{CDCl}_{3}\right) \delta 56.3,56.5,95.3,99.5,116.9,121.3,124.1,124.5,125.6,126.1$, $126.85,126.95,127.0,128.2,129.0,130.0,130.1,131.5,131.8,134.4,134.5,134.8$, 136.2, 151.1, 153.3, 157.0. HRMS Calcd for $\mathrm{C}_{53} \mathrm{H}_{46} \mathrm{NO}_{8}\left(\mathrm{MH}^{+}\right)$: 824.3223. Found: 824.3228. m.p. $110{ }^{0} \mathrm{C} .[\alpha]_{\mathrm{D}}=-98.860\left(\mathrm{c}=0.275, \mathrm{CHCl}_{3}\right)$.

\section{Preparation of (1S,1'S)-3,3'-(pyridine-2,6-diyl)di-1,1'-bibenzobenzene-2,2'-diol.}

Under nitrogen, 2,6-bis((S)-2,2'-bis(methoxymethoxy)-1,1'-binaphthyl-3-yl)pyridine (576.8 $\mathrm{mg}, 0.7 \mathrm{mmol}$ ) was dissolved in $10 \mathrm{ml} \mathrm{CH}_{2} \mathrm{Cl}_{2}$ and trifluoroacetic acid $(1.0 \mathrm{~mL}$ ) was added. After the reaction mixture was stirred at room temperature of $2 \mathrm{~h}$, saturated $\mathrm{NaHCO}_{3}$ (aq.) was added to adjust the $\mathrm{pH}$ to 8 . Then the mixture was extracted with $\mathrm{CH}_{2} \mathrm{Cl}_{2}(3 \times 30 \mathrm{~mL})$ washed with brine $(30 \mathrm{~mL})$ and dried over anhydrous $\mathrm{Na}_{2} \mathrm{SO}_{4}$. After evaporation of the solvent, the residue was purified by column chromatography on silica gel eluted with hexanes/ethyl acetate (2/1) to afford desired product as a light yellow solid in $97 \%$ yield. ${ }^{1} \mathrm{H}$ NMR $\left(300 \mathrm{MHz}, \mathrm{CDCl}_{3}\right) \delta 7.11-7.37(\mathrm{~m}, 14 \mathrm{H}), 7.86(\mathrm{~d}, \mathrm{~J}=8.1 \mathrm{~Hz}$, 2H), $7.92(\mathrm{~d}, \mathrm{~J}=9.0 \mathrm{~Hz}, 4 \mathrm{H}), 8.00-8.08(\mathrm{~m}, 3 \mathrm{H}), 8.46(\mathrm{~s}, 2 \mathrm{H}) .{ }^{13} \mathrm{C} \mathrm{NMR}(300 \mathrm{MHz}$, $\left.\mathrm{CDCl}_{3}\right) \delta 113.2,113.6,118.0,121.8,123.9,124.4,124.6,124.8,127.3,128.5,128.7$ 129.3, 129.6, 130.7, 131.0, 133.7, 134.7, 138.5, 152.4, 152.9, 155.4. HRMS Calcd for 
$\mathrm{C}_{45} \mathrm{H}_{30} \mathrm{NO}_{4}\left(\mathrm{MH}^{+}\right)$: 648.2175. Found: 648.2175. m.p. $225{ }^{0} \mathrm{C} .[\alpha]_{\mathrm{D}}=-194.4(\mathrm{c}=0.355$, $\left.\mathrm{CHCl}_{3}\right)$.

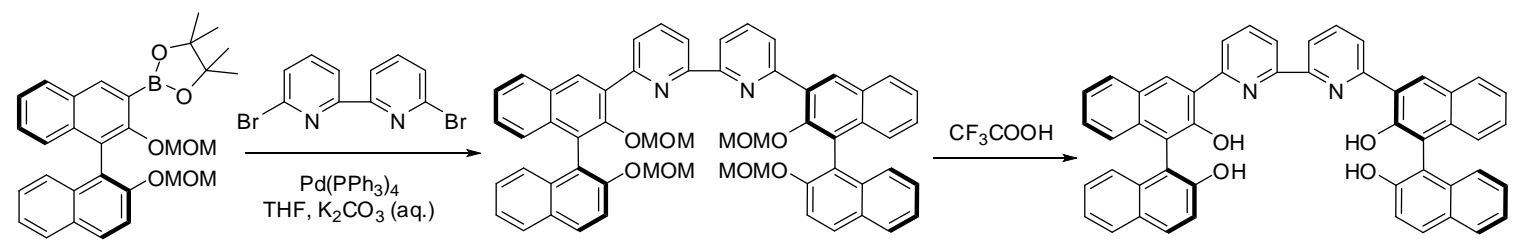

Preparation of 6,6'-bis((S)-2,2'-bis(methoxymethoxy)-1,1'-binaphthyl-3-yl)-2,2'-

bipyridine. Under nitrogen, to a $100 \mathrm{~mL}$ flame-dried Schlenk flask was loaded (S)-2-(2,2'-bis(methoxymethoxy)-1,1'-binaphthyl-3-yl)-4,4,5,5-tetramethyl-1,3,2-dioxabo rolane (1.0 g, $2.0 \mathrm{mmol}), 6,6$ '-dibromo-2,2'-bipyridine (250 mg, $0.8 \mathrm{mmol}), \mathrm{Pd}\left(\mathrm{PPh}_{3}\right)_{4}(92$ mg, $0.08 \mathrm{mmol})$. Degassed THF $(10 \mathrm{~mL})$ and $2 \mathrm{M} \mathrm{K}_{2} \mathrm{CO}_{3}(10 \mathrm{~mL})$ were transferred into the flask via cannula. The reaction mixture was stirred under reflux for overnight. After it cooled to room temperature, the organic layer was separated and the aqueous layer was extracted with $\mathrm{CH}_{2} \mathrm{Cl}_{2}(3 \times 30 \mathrm{~mL})$. The combined organic layer was washed with brine $(30 \mathrm{~mL})$ and dried over anhydrous $\mathrm{Na}_{2} \mathrm{SO}_{4}$. After evaporation of the solvent, the residue was purified by column chromatography on silica gel eluted with hexanes/ethyl acetate (6/1) to afford desired product as a white solid in $90 \%$ yield. ${ }^{1} \mathrm{H}$ NMR $\left(300 \mathrm{MHz}, \mathrm{CDCl}_{3}\right)$ $\delta 2.41(\mathrm{~s}, 3 \mathrm{H}), 3.26(\mathrm{~s}, 3 \mathrm{H}), 4.49(\mathrm{dd}, \mathrm{J} 1=5.4 \mathrm{~Hz}, \mathrm{~J} 2=1.2 \mathrm{~Hz}, 2 \mathrm{H}), 4.52(\mathrm{dd}, \mathrm{J} 1=5.4 \mathrm{~Hz}$, $\mathrm{J} 2=1.2 \mathrm{~Hz}, 2 \mathrm{H}), 5.10(\mathrm{dd}, \mathrm{J} 1=6.9 \mathrm{~Hz}, \mathrm{~J} 2=0.9 \mathrm{~Hz}, 2 \mathrm{H}), 5.19(\mathrm{dd}, \mathrm{J} 1=6.9 \mathrm{~Hz}, \mathrm{~J} 2=0.9$ $\mathrm{Hz}, 2 \mathrm{H}), 7.24-7.48(\mathrm{~m}, 12 \mathrm{H}), 7.63(\mathrm{dd}, \mathrm{J} 1=9.0 \mathrm{~Hz}, \mathrm{~J} 2=0.9 \mathrm{~Hz}, 2 \mathrm{H}), 7.87-8.06(\mathrm{~m}, 10 \mathrm{H})$, $8.54(\mathrm{~s}, 2 \mathrm{H}), 8.68(\mathrm{~d}, \mathrm{~J}=7.8 \mathrm{~Hz}, 2 \mathrm{H}) .{ }^{13} \mathrm{C} \mathrm{NMR}\left(300 \mathrm{MHz}, \mathrm{CDCl}_{3}\right) \delta 56.2,56.4,95.2$, 
$99.5,116.8,119.9,121.2,124.4,125.5,125.6,126.0,126.1,126.8,126.9,128.1,128.9$, 129.9, 130.0, 131.3, 131.7, 134.3, 134.4, 134.6, 137.2, 151.2, 153.2, 156.2, 156.5. HRMS Calcd for $\mathrm{C}_{58} \mathrm{H}_{49} \mathrm{~N}_{2} \mathrm{O}_{8}\left(\mathrm{MH}^{+}\right)$: 901.3489. Found: 901.3484. m.p. 113-115 ${ }^{0} \mathrm{C} .[\alpha]_{\mathrm{D}}=$ $-108.80\left(\mathrm{c}=0.365, \mathrm{CHCl}_{3}\right)$.

\section{Preparation of (1S,1'S)-3,3'-(2,2'-bipyridine-6,6'-diyl)di-1,1'-bibenzobenzene-}

2,2'-diol. Under nitrogen, 6,6'-bis((S)-2,2'-bis(methoxymethoxy)-1,1'-binaphthyl-3-yl)2,2'-bipyridine (630.7 $\mathrm{mg}, 0.7 \mathrm{mmol}$ ) was dissolved in $10 \mathrm{ml} \mathrm{CH}_{2} \mathrm{Cl}_{2}$ and trifluoroacetic acid $(0.5 \mathrm{~mL})$ was added. After the reaction mixture was stirred at room temperature for overnight, the solvent was evaporated and saturated $\mathrm{NaHCO}_{3}$ (aq.) was added. Then the mixture was filtered and the residue was washed with water, methanol and $\mathrm{CH}_{2} \mathrm{Cl}_{2}$ to afford desired product as a yellow solid in $95 \%$ yield. ${ }^{1} \mathrm{H} \mathrm{NMR}\left(300 \mathrm{MHz},(\mathrm{CD})_{2} \mathrm{SO}\right) \delta$ $6.95(\mathrm{~d}, \mathrm{~J}=7.8 \mathrm{~Hz}, 2 \mathrm{H}), 7.02(\mathrm{~d}, \mathrm{~J}=8.4 \mathrm{~Hz}, 2 \mathrm{H}), 7.15-7.39(\mathrm{~m}, 10 \mathrm{H}), 7.90(\mathrm{t}, \mathrm{J}=7.8 \mathrm{~Hz}$, 4H), $8.02(\mathrm{t}, \mathrm{J}=8.4 \mathrm{~Hz}, 4 \mathrm{H}), 8.29(\mathrm{t}, \mathrm{J}=8.1 \mathrm{~Hz}, 2 \mathrm{H}), 8.67(\mathrm{~d}, \mathrm{~J}=8.4 \mathrm{~Hz}, 2 \mathrm{H}), 8.93(\mathrm{~s}$, 2H), $9.31(\mathrm{~s}, 2 \mathrm{H}) .{ }^{13} \mathrm{C} \mathrm{NMR}\left(75 \mathrm{MHz},(\mathrm{CD})_{2} \mathrm{SO}\right) \delta 115.9,118.4,119.3,120.3,121.6$, $122.8,123.1,123.8,124.8,125.0,126.8,128.1,128.2,128.6,128.8,128.9,129.5,129.7$, 134.7, 135.6, 141.2, 151.9, 153.7, 154.6, 158.0. HRMS Calcd for $\mathrm{C}_{50} \mathrm{H}_{33} \mathrm{~N}_{2} \mathrm{O}_{4}\left(\mathrm{MH}^{+}\right)$: 725.2440. Found: 725.2437. m.p. higher than $400{ }^{0} \mathrm{C} .[\alpha]_{\mathrm{D}}=-301.13(\mathrm{c}=0.355, \mathrm{DMSO})$.
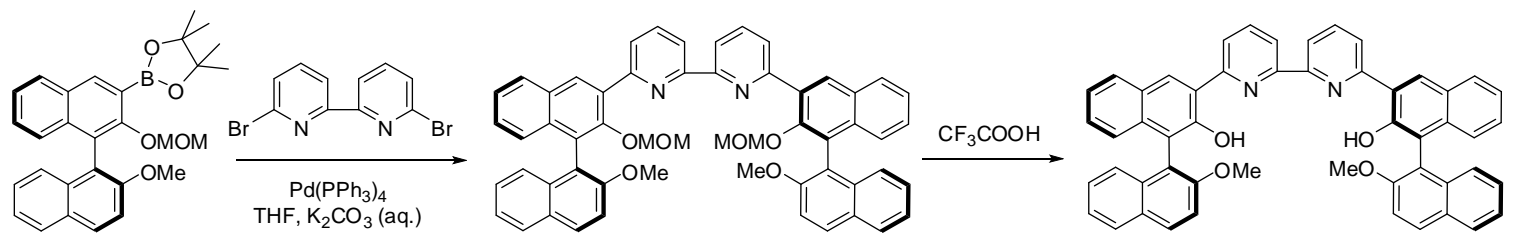


\section{Preparation of 6,6'-bis((S)-2'-methoxy-2-(methoxymethoxy)-1,1'-binaphthyl-3-}

yl)-2,2'-bipyridine. Under nitrogen, to a $50 \mathrm{~mL}$ flame-dried Schlenk flask was loaded (S)-2-(2'-methoxy-2-(methoxymethoxy)-1,1'-binaphthyl-3-yl)-4,4,5,5-tetramethyl-1,3,2-d ioxaborolane (706 mg, $1.5 \mathrm{mmol})$, 6,6'-dibromo-2,2'-bipyridine (188 mg, $0.6 \mathrm{mmol})$, $\mathrm{Pd}\left(\mathrm{PPh}_{3}\right)_{4}(69 \mathrm{mg}, 0.06 \mathrm{mmol})$. Degassed THF $(8 \mathrm{~mL})$ and $2 \mathrm{M} \mathrm{K}_{2} \mathrm{CO}_{3}(8 \mathrm{~mL})$ were transferred into the flask via cannula. The reaction mixture was stirred under reflux for overnight. After it cooled to room temperature, the organic layer was separated and the aqueous layer was extracted with $\mathrm{CH}_{2} \mathrm{Cl}_{2}(3 \times 30 \mathrm{~mL})$. The combined organic layer was washed with brine $(30 \mathrm{~mL})$ and dried over anhydrous $\mathrm{Na}_{2} \mathrm{SO}_{4}$. After evaporation of the solvent, the residue was recrystallized with hexanes/ethyl acetate (1/1) to afford desired product as a white solid in $71 \%$ yield. ${ }^{1} \mathrm{H}$ NMR $\left(300 \mathrm{MHz}, \mathrm{CDCl}_{3}\right) \delta 2.44(\mathrm{~s}, 6 \mathrm{H}), 3.85(\mathrm{~s}$, $6 \mathrm{H}), 4.47(\mathrm{~d}, \mathrm{~J}=5.4 \mathrm{~Hz}, 2 \mathrm{H}), 4.54(\mathrm{~d}, \mathrm{~J}=5.4 \mathrm{~Hz}, 2 \mathrm{H}), 7.21-7.38(\mathrm{~m}, 10 \mathrm{H}), 7.43-7.50(\mathrm{~m}$, 4H), 7.87-8.01 (m, 10H), $8.52(\mathrm{~s}, 2 \mathrm{H}), 8.68(\mathrm{~d}, \mathrm{~J}=7.8 \mathrm{~Hz}, 2 \mathrm{H}) .{ }^{13} \mathrm{C}$ NMR $(75 \mathrm{MHz}$, $\left.\mathrm{CDCl}_{3}\right) \delta 56.5,56.9,99.5,113.9,119.9,123.9,125.4,125.7,126.0,126.8,126.9,128.1$ $128.9,129.3,130.1,131.3,131.6,134.3,134.4,134.7,137.1,151.2,155.4,156.3,156.4$. HRMS Calcd for $\mathrm{C}_{56} \mathrm{H}_{45} \mathrm{~N}_{2} \mathrm{O}_{6}\left(\mathrm{MH}^{+}\right)$: 841.4278. Found: 841.3282. m.p. $242{ }^{0} \mathrm{C} .[\alpha]_{D}=$ $-106.99\left(\mathrm{c}=0.175, \mathrm{CHCl}_{3}\right)$.

Preparation of (1S,1'S)-3,3'-(2,2'-bipyridine-6,6'-diyl)bis(2'-methoxy-1,1'bibenzobenzen-2-ol). Under nitrogen, 6,6'-bis((S)-2'-methoxy-2-(methoxymethoxy)-1,1'binaphthyl-3-yl)-2,2'-bipyridine (251.7 $\mathrm{mg}, 0.3 \mathrm{mmol}$ ) was dissolved in $10 \mathrm{ml} \mathrm{CH}_{2} \mathrm{Cl}_{2}$ 
and trifluoroacetic acid $(0.1 \mathrm{~mL})$ was added. After the reaction mixture was stirred at room temperature for overnight, saturated $\mathrm{NaHCO} 3$ (aq.) was added to adjust the $\mathrm{pH}$ to 8 . Then the mixture was extracted with $\mathrm{CH}_{2} \mathrm{Cl}_{2}(3 \times 30 \mathrm{~mL})$ washed with brine $(30 \mathrm{~mL})$ and dried over anhydrous $\mathrm{Na}_{2} \mathrm{SO}_{4}$. After evaporation of the solvent, the residue was recrystallized with $\mathrm{CH}_{2} \mathrm{Cl}_{2}$ / hexanes to afford desired product as a light yellow solid in $99 \%$ yield. ${ }^{1} \mathrm{H}$ NMR $\left(300 \mathrm{MHz},\left(\mathrm{CD}_{3}\right)_{2} \mathrm{SO}\right) \delta 3.71(\mathrm{~s}, 6 \mathrm{H}), 6.85(\mathrm{~d}, \mathrm{~J}=8.1 \mathrm{~Hz}, 2 \mathrm{H}), 7.05(\mathrm{~d}, \mathrm{~J}$ $=8.4 \mathrm{~Hz}, 2 \mathrm{H}), 7.04-7.33(\mathrm{~m}, 8 \mathrm{H}), 7.63(\mathrm{~d}, \mathrm{~J}=9.3 \mathrm{~Hz}, 2 \mathrm{H}), 7.95-8.02(\mathrm{~m}, 4 \mathrm{H}), 8.10(\mathrm{~d}, \mathrm{~J}$ $=9.0 \mathrm{~Hz}, 2 \mathrm{H}), 8.26(\mathrm{t}, \mathrm{J}=8.1 \mathrm{~Hz}, 2 \mathrm{H}), 8.64(\mathrm{~d}, \mathrm{~J}=8.1 \mathrm{~Hz}, 2 \mathrm{H}), 8.92(\mathrm{~s}, 2 \mathrm{H}) . \mathrm{HRMS}$ Calcd for $\mathrm{C}_{52} \mathrm{H}_{37} \mathrm{~N}_{2} \mathrm{O}_{4}\left(\mathrm{MH}^{+}\right)$: 753.2753. Found: 753.2758. m.p. $354{ }^{0} \mathrm{C} .[\alpha]_{\mathrm{D}}=-286.63(\mathrm{c}$ $\left.=0.10, \mathrm{CHCl}_{3}\right)$.

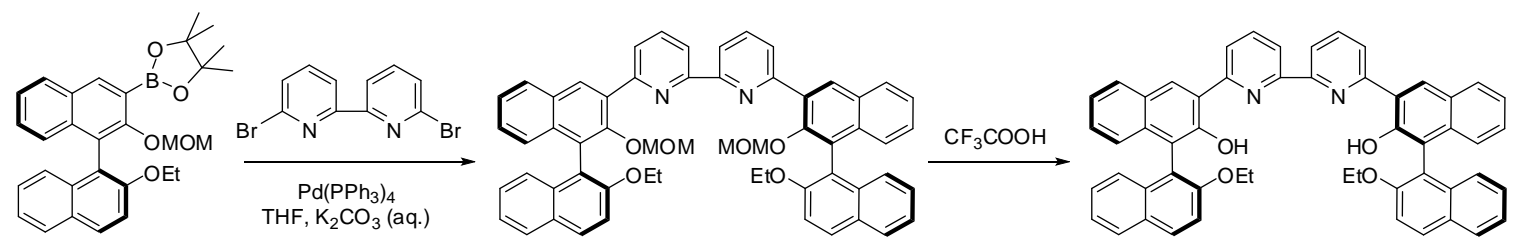

Preparation of 6,6'-bis((S)-2'-ethoxy-2-(methoxymethoxy)-1,1'-binaphthyl-3-yl)-

2,2'-bipyridine. Under nitrogen, to a $50 \mathrm{~mL}$ flame-dried Schlenk flask was loaded (S)-2-(2'-ethoxy-2-(methoxymethoxy)-1,1'-binaphthyl-3-yl)-4,4,5,5-tetramethyl-1,3,2-dio xaborolane (727mg, $1.5 \mathrm{mmol}$ ), 6,6'-dibromo-2,2'-bipyridine (188 mg, $0.6 \mathrm{mmol}$ ), $\mathrm{Pd}\left(\mathrm{PPh}_{3}\right)_{4}(69 \mathrm{mg}, 0.06 \mathrm{mmol})$. Degassed THF $(8 \mathrm{~mL})$ and $2 \mathrm{M} \mathrm{K}_{2} \mathrm{CO}_{3}(8 \mathrm{~mL})$ were transferred into the flask via cannula. The reaction mixture was stirred under reflux for overnight. After it cooled to room temperature, the organic layer was separated and the 
aqueous layer was extracted with $\mathrm{CH}_{2} \mathrm{Cl}_{2}(3 \times 30 \mathrm{~mL})$. The combined organic layer was washed with brine $(30 \mathrm{~mL})$ and dried over anhydrous $\mathrm{Na}_{2} \mathrm{SO}_{4}$. After evaporation of the solvent, the residue was purified by column chromatography on silica gel eluted with hexanes/ethyl acetate (6/1) to afford desired product as a white solid in $89 \%$ yield. ${ }^{1} \mathrm{H}$ NMR (300 MHz, CDCl $) \delta 1.15(\mathrm{t}, \mathrm{J}=6.9 \mathrm{~Hz}, 6 \mathrm{H}), 2.44(\mathrm{~s}, 6 \mathrm{H}), 4.06-4.23(\mathrm{~m}, 4 \mathrm{H}), 4.46$ $(\mathrm{d}, \mathrm{J}=5.4 \mathrm{~Hz}, 2 \mathrm{H}), 4.54(\mathrm{~d}, \mathrm{~J}=5.4 \mathrm{~Hz}, 2 \mathrm{H}), 7.26-7.48(\mathrm{~m}, 14 \mathrm{H}), 7.86-8.06(\mathrm{~m}, 10 \mathrm{H})$, $8.55(\mathrm{~s}, 2 \mathrm{H}), 8.69(\mathrm{~d}, \mathrm{~J}=7.8 \mathrm{~Hz}, 2 \mathrm{H}) .{ }^{13} \mathrm{C} \mathrm{NMR}\left(75 \mathrm{MHz}, \mathrm{CDCl}_{3}\right) \delta 15.4,56.5,65.3$, $99.5,115.4,120.0,120.4,123.9,125.3,125.78,125.83,126.1,126.8,126.9,128.1,128.9$, 129.3, 129.9, 131.3, 131.4, 134.3, 134.4, 134.6, 137.1, 151.2, 154.8, 156.3, 156.5. HRMS Calcd for $\mathrm{C}_{58} \mathrm{H}_{49} \mathrm{~N}_{2} \mathrm{O}_{6}\left(\mathrm{MH}^{+}\right)$: 869.3591. Found: 869.3591. m.p. $130{ }^{0} \mathrm{C}$. $[\alpha]_{\mathrm{D}}=-112.22(\mathrm{c}$ $\left.=1.05, \mathrm{CHCl}_{3}\right)$.

Preparation of $\quad\left(1 \mathrm{~S}, 1^{\prime} ' S\right)-3,3 '-(2,2 '$ 'bipyridine-6,6'-diyl)bis(2'-ethoxy-1,1'bibenzobenzen-2-ol). Under nitrogen, 6,6'-bis((S)-2'-ethoxy-2-(methoxymethoxy)-1,1'binaphthyl-3-yl)-2,2'-bipyridine (286 mg, $0.33 \mathrm{mmol}$ ) was dissolved in $10 \mathrm{ml} \mathrm{CH}_{2} \mathrm{Cl}_{2}$ and trifluoroacetic acid $(0.1 \mathrm{~mL})$ was added. After the reaction mixture was stirred at room temperature for overnight, saturated $\mathrm{NaHCO}_{3}$ (aq.) was added to adjust the $\mathrm{pH}$ to 8 . Then the mixture was extracted with $\mathrm{CH}_{2} \mathrm{Cl}_{2}(3 \times 30 \mathrm{~mL})$ washed with brine $(30 \mathrm{~mL})$ and dried over anhydrous $\mathrm{Na}_{2} \mathrm{SO}_{4}$. After evaporation of the solvent, the residue the residue was purified by column chromatography on silica gel eluted with $\mathrm{CH}_{2} \mathrm{Cl}_{2}$ to afford desired product as a light yellow solid in $99 \%$ yield. ${ }^{1} \mathrm{H}$ NMR $\left(300 \mathrm{MHz},(\mathrm{CD})_{2} \mathrm{SO}\right) \delta 0.95(\mathrm{t}, \mathrm{J}=$ 
$6.9 \mathrm{~Hz}, 6 \mathrm{H}), 4.07(\mathrm{q}, \mathrm{J}=6.9 \mathrm{~Hz}, 4 \mathrm{H}), 6.90(\mathrm{~d}, \mathrm{~J}=8.4 \mathrm{~Hz}, 2 \mathrm{H}), 7.08(\mathrm{~d}, \mathrm{~J}=8.4 \mathrm{~Hz}, 2 \mathrm{H})$, 7.20-7.35 (m, 8H), $7.62(\mathrm{~d}, \mathrm{~J}=8.7 \mathrm{~Hz}, 2 \mathrm{H}), 7.95-8.09(\mathrm{~m}, 8 \mathrm{H}), 8.28(\mathrm{t}, \mathrm{J}=8.4 \mathrm{~Hz}, 2 \mathrm{H})$, $8.66(\mathrm{~d}, \mathrm{~J}=7.8 \mathrm{~Hz}, 2 \mathrm{H}), 8.92(\mathrm{~s}, 2 \mathrm{H}) .{ }^{13} \mathrm{C} \mathrm{NMR}\left(75 \mathrm{MHz},(\mathrm{CD})_{2} \mathrm{SO}\right) \delta 15.5,65.0,116.6$ $118.3,120.1,120.5,121.5,122.8,123.8,124.1,124.6,125.4,127.0,128.0,128.2,128.7$ 129.0, 129.6, 129.7, 129.9, 134.2, 135.4, 141.2, 152.1, 154.3, 154.8, 158.0. HRMS Calcd for $\mathrm{C}_{54} \mathrm{H}_{41} \mathrm{~N}_{2} \mathrm{O}_{4}\left(\mathrm{MH}^{+}\right)$: 781.3066. Found: 781.3059. m.p. $210{ }^{0} \mathrm{C} .[\alpha]_{\mathrm{D}}=-298.15(\mathrm{c}=$ $\left.0.285, \mathrm{CHCl}_{3}\right)$.<smiles>COc1ccc2ccccc2c1-c1cc2ccccc2cc1B1OC(C)(C)C(C)(C)O1</smiles>

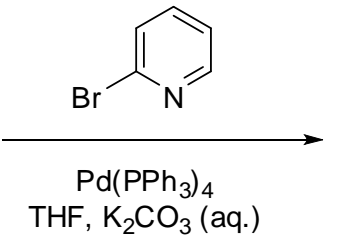<smiles>COc1ccc2ccccc2c1-c1c(-c2ccccn2)cc2ccccc2c1OC</smiles><smiles>O=C(O)C(F)(F)F</smiles>

Preparation of (S)-2-(2,2'-bis(methoxymethoxy)-1,1'-binaphthyl-3-yl)pyridine.

Under nitrogen, to a $50 \mathrm{~mL}$ flame-dried Schlenk flask was loaded (S)-2-(2,2'-bis(methoxymethoxy)-1,1'-binaphthyl-3-yl)-4,4,5,5-tetramethyl-1,3,2-dioxabo rolane (438 mg, $0.875 \mathrm{mmol}), 2$-bromopyridine $(67 \mu \mathrm{L}, 0.7 \mathrm{mmol}), \mathrm{Pd}\left(\mathrm{PPh}_{3}\right)_{4}(40 \mathrm{mg}$, $0.035 \mathrm{mmol})$. Degassed THF $(5 \mathrm{~mL})$ and $2 \mathrm{M} \mathrm{K}_{2} \mathrm{CO}_{3}(5 \mathrm{~mL})$ were transferred into the flask via cannula. The reaction mixture was stirred under reflux for overnight. After it cooled to room temperature, the organic layer was separated and the aqueous layer was extracted with $\mathrm{CH}_{2} \mathrm{Cl}_{2}(3 \times 30 \mathrm{~mL})$. The combined organic layer was washed with brine (30 mL) and dried over anhydrous $\mathrm{Na}_{2} \mathrm{SO}_{4}$. After evaporation of the solvent, the residue 
was purified by column chromatography on silica gel eluted with hexanes/ethyl acetate (6/1) to afford desired product as a white solid in $95 \%$ yield. ${ }^{1} \mathrm{H}$ NMR $\left(300 \mathrm{MHz}, \mathrm{CDCl}_{3}\right)$ $\delta 2.45(\mathrm{~s}, 3 \mathrm{H}), 3.24(\mathrm{~s}, 3 \mathrm{H}), 4.47(\mathrm{~d}, \mathrm{~J}=5.4 \mathrm{~Hz}, 1 \mathrm{H}), 4.49(\mathrm{~d}, \mathrm{~J}=5.7 \mathrm{~Hz}, 1 \mathrm{H}), 5.11(\mathrm{~d}, \mathrm{~J}=$ $6.9 \mathrm{~Hz}, 1 \mathrm{H}), 5.20(\mathrm{~d}, \mathrm{~J}=6.9 \mathrm{~Hz}, 1 \mathrm{H}), 7.26-7.47(\mathrm{~m}, 7 \mathrm{H}), 7.65(\mathrm{~d}, \mathrm{~J}=9.3 \mathrm{~Hz}, 1 \mathrm{H}), 7.76$ $(\mathrm{td}, \mathrm{J} 1=7.8 \mathrm{~Hz}, \mathrm{~J} 2=1.5 \mathrm{~Hz}, 1 \mathrm{H}), 7.90(\mathrm{~d}, \mathrm{~J}=7.8 \mathrm{~Hz}, 2 \mathrm{H}), 7.98-8.03(\mathrm{~m}, 3 \mathrm{H}), 8.41(\mathrm{~s}$, $1 \mathrm{H}), 8.83(\mathrm{~d}, \mathrm{~J}=4.8 \mathrm{~Hz}, 1 \mathrm{H}) .{ }^{13} \mathrm{C} \mathrm{NMR}\left(75 \mathrm{MHz}, \mathrm{CDCl}_{3}\right) \delta 56.2,56.4,95.3,99.4,116.9$, $121.3,122.4,124.5,125.6,125.7,126.0,126.1,126.8,126.9,127.0,128.1,128.9,130.0$, 130.1, 131.3, 131.5, 134.3, 134.4, 134.5, 136.3, 150.0, 150.9, 153.2, 157.0. HRMS Calcd for $\mathrm{C}_{29} \mathrm{H}_{26} \mathrm{NO}_{4}\left(\mathrm{MH}^{+}\right)$: 452.1862. Found: 452.1873. m.p. $136{ }^{\circ} \mathrm{C}$. $[\alpha]_{\mathrm{D}}=-102.11(\mathrm{c}=0.36$, $\left.\mathrm{CHCl}_{3}\right)$.

Preparation of (S)-3-(pyridin-2-yl)-1,1'-binaphthyl-2,2'-diol. Under nitrogen, (S)-2-(2,2'-bis(methoxymethoxy)-1,1'-binaphthyl-3-yl)pyridine (149 mg, $0.33 \mathrm{mmol}$ ) was dissolved in $10 \mathrm{ml} \mathrm{CH}_{2} \mathrm{Cl}_{2}$ and trifluoroacetic acid $(0.15 \mathrm{~mL})$ was added. After the reaction mixture was stirred at room temperature for overnight, saturated $\mathrm{NaHCO} 3$ (aq.) was added to adjust the $\mathrm{pH}$ to 8 . Then the mixture was extracted with $\mathrm{CH}_{2} \mathrm{Cl}_{2}(3 \times 30 \mathrm{~mL})$ washed with brine $(30 \mathrm{~mL})$ and dried over anhydrous $\mathrm{Na}_{2} \mathrm{SO}_{4}$. After evaporation of the solvent, the residue the residue was purified by column chromatography on silica gel eluted with hexanes/ethyl acetate (4/1) to afford desired product as a yellow solid in $90 \%$ yield. ${ }^{1} \mathrm{H}$ NMR (300 MHz, $\left.\mathrm{CDCl}_{3}\right) \delta$ 7.19-7.37 (m, 7H), $7.45(\mathrm{~d}, \mathrm{~J}=8.7 \mathrm{~Hz}, 1 \mathrm{H}), 7.82(\mathrm{td}$, $\mathrm{J} 1=8.4 \mathrm{~Hz}, \mathrm{~J} 2=1.8 \mathrm{~Hz}, 1 \mathrm{H}), 7.90-7.97(\mathrm{~m}, 3 \mathrm{H}), 8.15(\mathrm{~d}, \mathrm{~J}=8.1 \mathrm{~Hz}, 1 \mathrm{H}), 8.39(\mathrm{~d}, \mathrm{~J}=$ 
$5.1 \mathrm{~Hz}, 1 \mathrm{H}), 8.52(\mathrm{~s}, 1 \mathrm{H}) .{ }^{13} \mathrm{C} \mathrm{NMR}\left(75 \mathrm{MHz}, \mathrm{CDCl}_{3}\right) \delta 114.8,115.7,118.1,120.5,121.6$, $122.8,123.6,124.1,124.8,125.3,126.8,128.0,128.3,128.5,128.6,129.3,129.7,130.1$, 134.1, 135.5, 138.6, 146.1, 151.8, 156.1, 157.5. HRMS Calcd for $\mathrm{C}_{25} \mathrm{H}_{18} \mathrm{NO}_{2}\left(\mathrm{MH}^{+}\right)$: 364.1338. Found: 364.1342 . m.p. $177{ }^{0} \mathrm{C} .[\alpha]_{\mathrm{D}}=-75.720\left(\mathrm{c}=0.215, \mathrm{CHCl}_{3}\right)$.

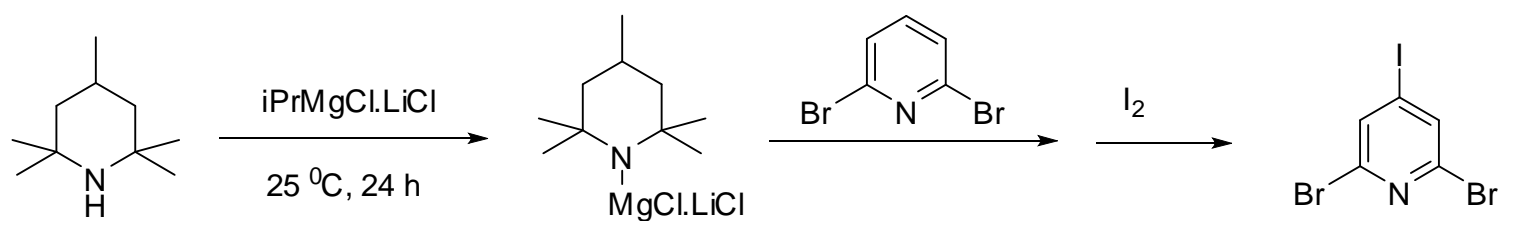

Preparation of 2,6-dibromo-4-iodopyridine. Under nitrogen to a flame-dried flask, $i \mathrm{PrMgCl} \cdot \mathrm{LiCl}(1.25 \mathrm{M}, 21.4 \mathrm{~mL}, 26.7 \mathrm{mmol})$ was added. THF $(26 \mathrm{~mL})$ solution of 2,2,4,6,6-pentamethypiperdine $(3.67 \mathrm{~g}, 26.0 \mathrm{mmol}$ ) was added dropwise. The mixture was stirred at room temperature for $24 \mathrm{~h}$. Then the mixture was added to the $10 \mathrm{~mL}$ THF solution of 2,6-dibromopyridine $(3.8 \mathrm{~g}, 16.0 \mathrm{mmol})$ via annula at $-30^{\circ} \mathrm{C}$. After $1 \mathrm{~h}$ stirring, $\mathrm{I}_{2}(5.1 \mathrm{~g}, 20.1 \mathrm{mmol})$ in THF $(10 \mathrm{~mL})$ was added to quench the reaction. The reaction mixture was washed with saturated $\mathrm{NaHSO}_{3}$ (aq. $10 \mathrm{~mL}$ ) and extracted with ethyl acetate $(3 \times 30 \mathrm{~mL})$. The combined organic layer was washed with brine $(30 \mathrm{~mL})$ and dried over anhydrous $\mathrm{Na}_{2} \mathrm{SO}_{4}$. After evaporation of the solvent, the residue the residue was purified by column chromatography on silica gel eluted with hexanes/ethyl acetate (8/1) to afford desired product as a white solid in $82 \%$ yield. ${ }^{1} \mathrm{H}$ NMR $\left(300 \mathrm{MHz}, \mathrm{CDCl}_{3}\right) \delta 7.84(\mathrm{~s}, 2 \mathrm{H})$.
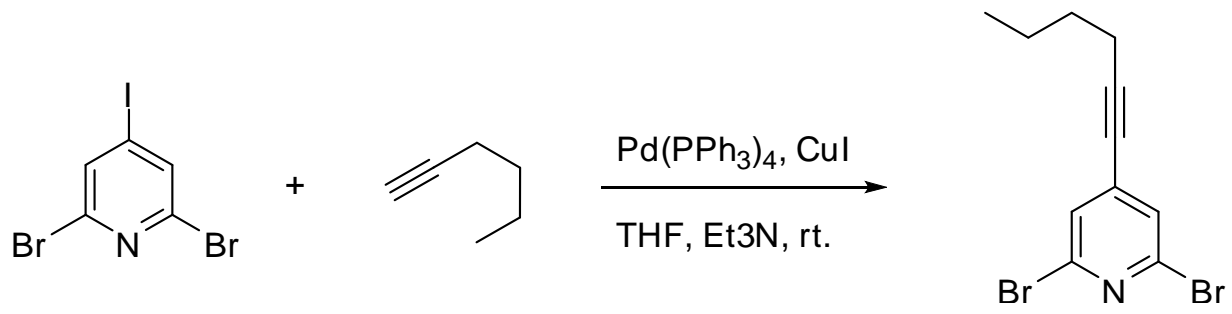
Preparation of 2,6-dibromo-4-(hex-1-ynyl)pyridine. Under nitrogen, to a $50 \mathrm{~mL}$ flame-dried Schlenk flask was loaded 2,6-dibromo-4-iodopyridine (363 mg, 2 mmol), $\mathrm{Pd}\left(\mathrm{PPh}_{3}\right)_{4}(231 \mathrm{mg}, 0.02 \mathrm{mmol}), \mathrm{CuI}(38.2 \mathrm{mg}, 0.2 \mathrm{mmol})$. Degassed THF (64 mL) and $\mathrm{Et}_{3} \mathrm{~N}(16 \mathrm{~mL})$ were transferred into the flask via cannula. 1-Hexyne $(290 \mu \mathrm{L}, 2.4 \mathrm{mmol})$ was added with syringe. The reaction mixture was stirred at room temperature for $24 \mathrm{~h}$. The organic layer was separated and the aqueous layer was extracted with ethyl acetate (3 x $30 \mathrm{~mL})$. The combined organic layer was washed with brine $(30 \mathrm{~mL})$ and dried over anhydrous $\mathrm{Na}_{2} \mathrm{SO}_{4}$. After evaporation of the solvent, the residue was purified by column chromatography on silica gel eluted with hexanes/ethyl acetate (50/1) to afford desired product as colorless liquid in $72 \%$ yield. ${ }^{1} \mathrm{H}$ NMR $\left(300 \mathrm{MHz}, \mathrm{CDCl}_{3}\right) \delta 0.95(\mathrm{t}, \mathrm{J}=7.2$ $\mathrm{Hz}, 3 \mathrm{H}), 1.39-1.51(\mathrm{~m}, 2 \mathrm{H}), 1.52-1.63(\mathrm{~m}, 2 \mathrm{H}), 2.42(\mathrm{t}, \mathrm{J}=7.2 \mathrm{~Hz}, 2 \mathrm{H}), 7.39(\mathrm{~s}, 2 \mathrm{H}) .{ }^{13} \mathrm{C}$ NMR (75 MHz, $\left.\mathrm{CDCl}_{3}\right) \delta 13.8,19.5,22.3,76.4,99.9,129.1,137.0,140.8$. HRMS Calcd for $\mathrm{C}_{11} \mathrm{H}_{12} \mathrm{NBr}_{2}\left(\mathrm{MH}^{+}\right)$: 315.9336 . Found: 315.9344 .

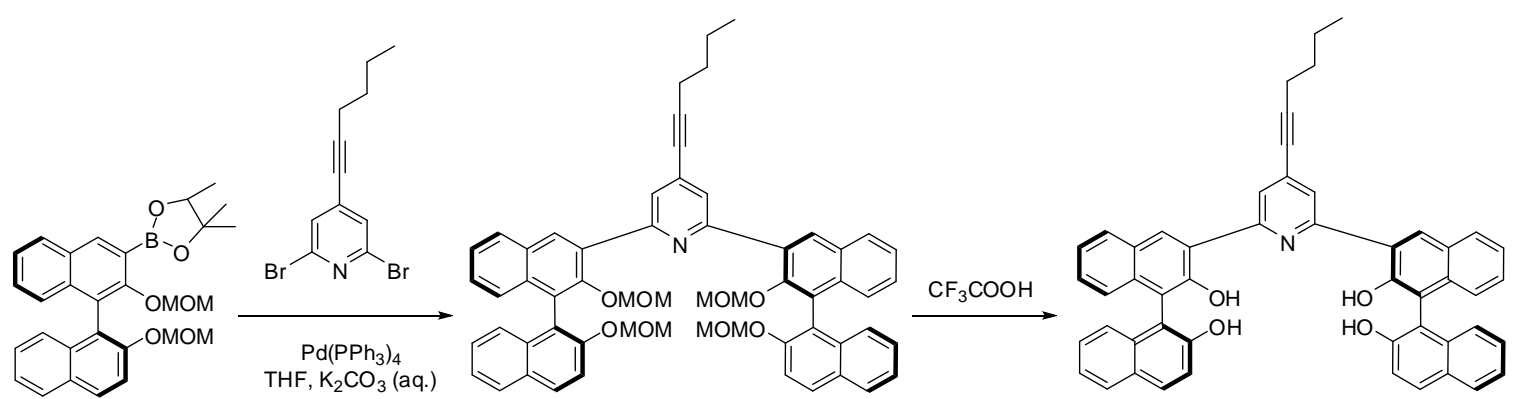

Preparation of 2,6-bis((S)-2,2'-bis(methoxymethoxy)-1,1'-binaphthyl-3-yl)-4-

(hex-1-ynyl)pyridine. Under nitrogen, to a $50 \mathrm{~mL}$ flame-dried Schlenk flask was loaded (S)-2-(2,2'-bis(methoxymethoxy)-1,1'-binaphthyl-3-yl)-4,4,5,5-tetramethyl-1,3,2-dioxabo 
rolane (438 mg, $0.875 \mathrm{mmol}$ ), 2,6-dibromo-4-(hex-1-ynyl)pyridine (110.7 mg, 0.35 mmol), $\mathrm{Pd}\left(\mathrm{PPh}_{3}\right)_{4}(40 \mathrm{mg}, 0.035 \mathrm{mmol})$. Degassed THF $(7 \mathrm{~mL})$ and $2 \mathrm{M} \mathrm{K}_{2} \mathrm{CO}_{3}(7 \mathrm{~mL})$ were transferred into the flask via cannula. The reaction mixture was stirred under reflux for overnight. After it cooled to room temperature, the organic layer was separated and the aqueous layer was extracted with $\mathrm{CH}_{2} \mathrm{Cl}_{2}(3 \times 30 \mathrm{~mL})$. The combined organic layer was washed with brine $(30 \mathrm{~mL})$ and dried over anhydrous $\mathrm{Na}_{2} \mathrm{SO}_{4}$. After evaporation of the solvent, the residue was purified by column chromatography on silica gel eluted with hexanes/ethyl acetate (6/1) to afford desired product as a white solid in $86 \%$ yield. ${ }^{1} \mathrm{H}$ NMR $\left(300 \mathrm{MHz}, \mathrm{CDCl}_{3}\right) \delta 1.02(\mathrm{t}, \mathrm{J}=7.2 \mathrm{~Hz}, 3 \mathrm{H}), 1,49-1.59(\mathrm{~m}, 2 \mathrm{H}), 1.64-1.73(\mathrm{~m}, 2 \mathrm{H})$, $2.51(\mathrm{t}, \mathrm{J}=6.9 \mathrm{~Hz}, 2 \mathrm{H}), 2.60(\mathrm{~s}, 6 \mathrm{H}), 3.27(\mathrm{~s}, 6 \mathrm{H}), 4.65(\mathrm{~d}, \mathrm{~J}=5.1 \mathrm{~Hz}, 2 \mathrm{H}), 4.70(\mathrm{~d}, \mathrm{~J}=$ $5.1 \mathrm{~Hz}, 2 \mathrm{H}), 5.13(\mathrm{~d}, \mathrm{~J}=6.6 \mathrm{~Hz}, 2 \mathrm{H}), 5.25(\mathrm{~d}, \mathrm{~J}=6.6 \mathrm{~Hz}, 2 \mathrm{H}), 7.34-7.49(\mathrm{~m}, 12 \mathrm{H}), 7.69$ $(\mathrm{d}, \mathrm{J}=8.7 \mathrm{~Hz}, 2 \mathrm{H}), 7.93(\mathrm{~d}, \mathrm{~J}=8.4 \mathrm{~Hz}, 2 \mathrm{H}), 8.01-8.12(\mathrm{~m}, 6 \mathrm{H}), 8.66(\mathrm{~s}, 2 \mathrm{H}) .{ }^{13} \mathrm{C} \mathrm{NMR}$ $\left(75 \mathrm{MHz}, \mathrm{CDCl}_{3}\right) \delta 14.0,19.6,22.4,30.8,56.3,56.6,77.7,79.4,95.3,95.8,99.8,117.0$ $121.4,124.5,125.6,126.1,126.9,127.0,127.1,128.2,129.0,130.0,130.1,131.4,131.8$ 132.6, 134.4, 151.4, 153.3, 157.0. HRMS Calcd for $\mathrm{C}_{59} \mathrm{H}_{54} \mathrm{NO}_{8}\left(\mathrm{MH}^{+}\right)$: 904.3849. Found: 904.3839. m.p. $120{ }^{0} \mathrm{C} .[\alpha]_{\mathrm{D}}=-135.14\left(\mathrm{c}=0.325, \mathrm{CHCl}_{3}\right)$.

Preparation of $\quad\left(1 S, 1^{\prime \prime} S\right)-3,3 '-(4-(h e x-1-y n y l) p y r i d i n e-2,6-d i y l) d i-1,1^{\prime}-$ bibenzobenzene-2,2'-diol. Under nitrogen, 2,6-bis((S)-2,2'-bis(methoxymethoxy)1,1'-binaphthyl-3-yl)-4-(hex-1-ynyl)pyridine (136 mg, $0.15 \mathrm{mmol})$ was dissolved in 10 $\mathrm{ml} \mathrm{CH}_{2} \mathrm{Cl}_{2}$ and trifluoroacetic acid $(0.15 \mathrm{~mL})$ was added. After the reaction mixture was 
stirred at room temperature for overnight, saturated $\mathrm{NaHCO} 3$ (aq.) was added to adjust the $\mathrm{pH}$ to 8 . Then the mixture was extracted with $\mathrm{CH}_{2} \mathrm{Cl}_{2}(3 \times 30 \mathrm{~mL})$ washed with brine (30 mL) and dried over anhydrous $\mathrm{Na}_{2} \mathrm{SO}_{4}$. After evaporation of the solvent, the residue the residue was purified by column chromatography on silica gel eluted with hexanes/ethyl acetate (4/1) to afford desired product as an orange yellow solid in $91 \%$ yield. ${ }^{1} \mathrm{H}$ NMR $\left(300 \mathrm{MHz}, \mathrm{CDCl}_{3}\right) \delta 0.99(\mathrm{t}, \mathrm{J}=7.2 \mathrm{~Hz}, 3 \mathrm{H}), 1.46-1.58(\mathrm{~m}, 2 \mathrm{H})$, 1.61-1.70 (m, 2H), $2.49(\mathrm{t}, \mathrm{J}=7.2 \mathrm{~Hz}, 2 \mathrm{H}), 7.12(\mathrm{t}, \mathrm{J}=8.4 \mathrm{~Hz}, 4 \mathrm{H}), 7.20-7.39(\mathrm{~m}, 10 \mathrm{H})$, $7.84(\mathrm{~d}, \mathrm{~J}=7.8 \mathrm{~Hz}, 2 \mathrm{H}), 7.90(\mathrm{~d}, \mathrm{~J}=8.7 \mathrm{~Hz}, 4 \mathrm{H}), 8.01(\mathrm{~s}, 2 \mathrm{H}), 8.41(\mathrm{~s}, 2 \mathrm{H}),{ }^{13} \mathrm{C} \mathrm{NMR}$ $\left(150 \mathrm{MHz}, \mathrm{CDCl}_{3}\right) \delta 13.8,19.5,22.3,30.6,78.8,113.0,114.0,118.1,123.8,123.9,124.4$, 124.6, 124.7, 127.1, 128.4, 128.5, 128.6, 129.2, 129.5, 130.6, 130.8, 133.6, 134.8, 152.4. HRMS Calcd for $\mathrm{C}_{51} \mathrm{H}_{38} \mathrm{NO}_{4}\left(\mathrm{MH}^{+}\right)$: 728.2801 . Found: 728.2791. m.p. $176{ }^{0} \mathrm{C} .[\alpha]_{\mathrm{D}}=$ $-176.89\left(\mathrm{c}=0.205, \mathrm{CHCl}_{3}\right)$.

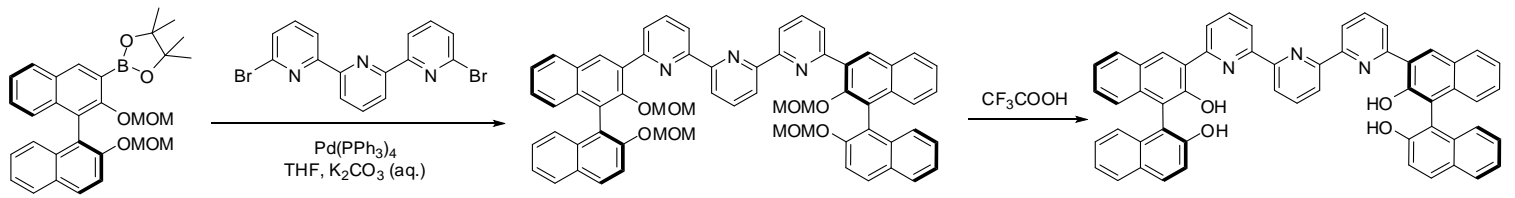

Preparation of 6,6'-bis((S)-2,2'-bis(methoxymethoxy)-1,1'-binaphthyl-3-yl)2,2':6',2'-terpyridine. Under nitrogen, to a $50 \mathrm{~mL}$ flame-dried Schlenk flask was loaded (S)-2-(2,2'-bis(methoxymethoxy)-1,1'-binaphthyl-3-yl)-4,4,5,5-tetramethyl-1,3,2-dioxabo rolane (750.6 mg, $1.5 \mathrm{mmol}), 6,6^{\prime \prime}$-dibromo-2,2':6',2"'-terpyridine (234.6 mg, $0.6 \mathrm{mmol}$ ), $\mathrm{Pd}\left(\mathrm{PPh}_{3}\right)_{4}(57.8 \mathrm{mg}, 0.05 \mathrm{mmol})$. Degassed THF $(10 \mathrm{~mL})$ and $2 \mathrm{M} \mathrm{K}_{2} \mathrm{CO}_{3}(10 \mathrm{~mL})$ were transferred into the flask via cannula. The reaction mixture was stirred under reflux for 
overnight. After it cooled to room temperature, the organic layer was separated and the aqueous layer was extracted with $\mathrm{CH}_{2} \mathrm{Cl}_{2}(3 \times 30 \mathrm{~mL})$. The combined organic layer was washed with brine $(30 \mathrm{~mL})$ and dried over anhydrous $\mathrm{Na}_{2} \mathrm{SO}_{4}$. After evaporation of the solvent, the residue was purified by column chromatography on silica gel eluted with hexanes/ethyl acetate (3/1) to afford desired product as a white solid in $92 \%$ yield. ${ }^{1} \mathrm{H}$ NMR (600 MHz, $\left.\mathrm{CDCl}_{3}\right) \delta 2.42(\mathrm{~s}, 6 \mathrm{H}), 3.27(\mathrm{~s}, 6 \mathrm{H}), 4.50(\mathrm{~d}, \mathrm{~J}=5.4 \mathrm{~Hz}, 2 \mathrm{H}), 4.52(\mathrm{~d}, \mathrm{~J}$ $=5.4 \mathrm{~Hz}, 2 \mathrm{H}), 5.19(\mathrm{~d}, \mathrm{~J}=7.2 \mathrm{~Hz}, 2 \mathrm{H}), 5.20(\mathrm{~d}, \mathrm{~J}=6.6 \mathrm{~Hz}, 2 \mathrm{H}), 7.27-7.35(\mathrm{~m}, 8 \mathrm{H})$, 7.37-7.40 (m, 2H), 7.45-7.47 (m, 2H), $7.64(\mathrm{~d}, \mathrm{~J}=9.0 \mathrm{~Hz}, 2 \mathrm{H}), 7.89(\mathrm{~d}, \mathrm{~J}=8.4 \mathrm{~Hz}, 2 \mathrm{H})$, 7.94-8.00 (m, 4H), 8.03-8.06 (m, 5H), 8.55 (s, 2H), 8.93 (dd, J1 = 7.8Hz, J2 = $1.2 \mathrm{~Hz}$, $2 \mathrm{H}), 8.77(\mathrm{~d}, \mathrm{~J}=7.8 \mathrm{~Hz}, 2 \mathrm{H}) .{ }^{13} \mathrm{C} \mathrm{NMR}\left(150 \mathrm{MHz}, \mathrm{CDCl}_{3}\right) \delta 56.2,56.3,95.1,99.4,116.7$, $119.6,121.1,121.5,124.3,125.4,125.6,125.9,126.0,126.69,126.73,126.8,128.0$ $128.8,129.8,129.9,131.6,134.2,134.3,134.5,137.0,138.0,151.0,153.1,155.6,156.1$, 156.3. HRMS Calcd for $\mathrm{C}_{63} \mathrm{H}_{52} \mathrm{~N}_{3} \mathrm{O}_{8}\left(\mathrm{MH}^{+}\right)$: 978.3754 . Found: 978.3763 . m.p. $146{ }^{0} \mathrm{C}$. $[\alpha]_{\mathrm{D}}=-92.583\left(\mathrm{c}=0.28, \mathrm{CHCl}_{3}\right)$.

Preparation of $\quad\left(1 \mathrm{~S}, 1^{\prime \prime} \mathrm{S}\right)-3,3^{\prime}-\left(2,2^{\prime}: 6^{\prime}, 2^{\prime \prime}\right.$-terpyridine-6,6"-diyl)di-1,1'bibenzobenzene-2,2'-diol. Under nitrogen, 6,6'-bis((S)-2,2'-bis(methoxymethoxy)-1,1'binaphthyl-3-yl)-2,2':6',2"-terpyridine (244 mg, $0.25 \mathrm{mmol}$ ) was dissolved in $10 \mathrm{ml}$ $\mathrm{CH}_{2} \mathrm{Cl}_{2}$ and trifluoroacetic acid $(0.3 \mathrm{~mL})$ was added. After the reaction mixture was stirred at room temperature for overnight, saturated $\mathrm{NaHCO} 3$ (aq.) was added to adjust the $\mathrm{pH}$ to 8 . Then the mixture was extracted with $\mathrm{CH}_{2} \mathrm{Cl}_{2}(3 \times 30 \mathrm{~mL})$ washed with brine 
$\left(30 \mathrm{~mL}\right.$ ) and dried over anhydrous $\mathrm{Na}_{2} \mathrm{SO}_{4}$. After evaporation of the solvent, the residue the residue was purified by column chromatography on silica gel eluted with $\mathrm{CH}_{2} \mathrm{Cl}_{2} /$ methanol (50/1) to afford desired product as a yellow solid in $95 \%$ yield. ${ }^{1} \mathrm{H}$ NMR (600 MHz, $\left.\left(\mathrm{CD}_{3}\right)_{2} \mathrm{SO}\right) \delta 9.99(\mathrm{~d}, \mathrm{~J}=8.4 \mathrm{~Hz}, 2 \mathrm{H}), 7.09(\mathrm{~d}, \mathrm{~J}=8.4 \mathrm{~Hz}, 2 \mathrm{H}), 7.18(\mathrm{td}$, $\mathrm{J} 1=7.8 \mathrm{~Hz}, \mathrm{~J} 2=1.8 \mathrm{~Hz}, 2 \mathrm{H}), 7.23-7.31(\mathrm{~m}, 6 \mathrm{H}), 7.38(\mathrm{~d}, \mathrm{~J}=9.0 \mathrm{~Hz}, 2 \mathrm{H}), 7.89-7.93(\mathrm{~m}$, $6 \mathrm{H}), 8.00(\mathrm{~d}, \mathrm{~J}=9.6 \mathrm{~Hz}, 2 \mathrm{H}), 8.02-8.05(\mathrm{~m}, 3 \mathrm{H}), 8.46(\mathrm{~d}, \mathrm{~J}=7.8 \mathrm{~Hz}, 2 \mathrm{H}), 8.56(\mathrm{~d}, \mathrm{~J}=8.4$ $\mathrm{Hz}, 2 \mathrm{H}), 8.89$ (s, 2H), 9.29 (s, 2H), 14.62 (s, 2H). $\left.{ }^{13} \mathrm{C} \mathrm{NMR} \mathrm{(150} \mathrm{MHz,}\left(\mathrm{CD}_{3}\right)_{2} \mathrm{SO}\right) \delta$ $115.5,117.7,118.6,120.3,120.8,120.9,121.4,122.4,124.1,124.5,126.0,127.4,127.8$ 127.9, 128.2, 128.7, 129.0, 134.1, 134.9, 139.7, 151.6, 153.0, 153.1, 154.4, 156.8. HRMS Calcd for $\mathrm{C}_{55} \mathrm{H}_{36} \mathrm{~N}_{3} \mathrm{O}_{4}\left(\mathrm{MH}^{+}\right)$: 802.2706. Found: 802.2714. m.p. $248{ }^{0} \mathrm{C} .[\alpha]_{\mathrm{D}}=-217.83(\mathrm{c}$ $\left.=0.165, \mathrm{CHCl}_{3}\right)$.

\section{Measurement of the Fluorescence Quautum Yield}

The fluorescence quantum yield of compounds was estimated by using a 2-aminopyridine solution in $0.1 \mathrm{~N} \mathrm{H}_{2} \mathrm{SO}_{4}\left(\phi_{\mathrm{F}}=0.60\right)$ as the standard. The fluorescence spectra were recorded on a Horiba FluoroMax-4 spectrofluorometer. Excited wavelength was $290 \mathrm{~nm}$. The excitation slit was set at $1.5 \mathrm{~nm}$ and the emission slit at $1.5 \mathrm{~nm}$. The scan speed was set at $1.0 \mathrm{~nm} / \mathrm{s}$. After subtracting the solvent background signal and noise signals, the integration of the area under the fluorescence signal of the sensor was calculated from $300 \mathrm{~nm}$ to $550 \mathrm{~nm}$. The UV spectra were recorded on a Hewlett-Packard 8452A diode-array spectrophotometer. 
Following equation is applied to calculate the quantum yield:

$$
\phi_{\mathrm{F}}=\phi_{\mathrm{F}, \mathrm{ref}}\left(\frac{A_{\mathrm{ref}}}{A}\right)\left(\frac{n_{\mathrm{D}}}{n_{\mathrm{D}, \mathrm{ref}}}\right)^{2}\left(\frac{a}{a_{\mathrm{ref}}}\right)
$$

Wherein $\phi_{\mathrm{F}, \text { ref }}, A_{\text {ref }}, n_{\mathrm{D} \text {,ref }}$ and $a_{\text {ref }}$ are the reference quantum yield, the reference absorbance, the refraction index of the solvent of the reference, and the integration of the area under the fluorescence signal of the reference, respectively. $n_{\mathrm{D}}$ of THF: 1.4242 . $n_{\mathrm{D}, \mathrm{ref}}$ of $0.1 \mathrm{~N}$ sulfuric acid: 1.3330 .

Table AI-1. Measurement of fluorescence quantum yield of $(S)-7-1-(S)-7-21$.

\begin{tabular}{|c|c|c|c|}
\hline Sensor & $A$ & $a$ & $\phi_{\mathrm{F}}$ \\
\hline 2-aminopyridine & 0.086993 & 29644375.0000000 & 0.60 \\
\hline$(S)-7-1$ & 0.066947 & 2814137.0000000 & 0.082107 \\
\hline$(S)-7-2$ & 0.063777 & 1893047.0000000 & 0.057978 \\
\hline$(S)-7-4$ & 0.078642 & 3467837.0000000 & 0.086133 \\
\hline$(S)-7-5$ & 0.085512 & 17226787.0000000 & 0.3935 \\
\hline$(S)-7-6$ & 0.079736 & 16590987.0000000 & 0.406429 \\
\hline$(S)-7-7$ & 0.101477 & 17318687.0000000 & 0.333361 \\
\hline & & & $1.25497 \mathrm{E} 8$ \\
\hline$(S-$ aminopyridine & 0.097859 & 33507480.0000000 & 0.161739 \\
\hline$(S)-7-8$ & 0.107704 & 65505.6039700 & 0.000367 \\
\hline$(S)-7-9$ & 0.092876 & 31025980.0000000 & 0.162311 \\
\hline$(S)-7-10$ & 0.099376 & 29981180.0000000 & 0.166424 \\
\hline$(S)-7-11$ & 0.093656 & 34428480.0000000 & 0.172904 \\
\hline$(S)-7-12$ & 0.103518 & 17410.0044100 & $8.7 \mathrm{E}-05$ \\
\hline$(S)-7-13$ & 0.104049 & 6552.0634100 & $3.88 \mathrm{E}-05$ \\
\hline$(S)-7-14$ & 0.087769 & 13024.2522100 & $6.55 \mathrm{E}-05$ \\
\hline$(S)-7-15$ & 0.103434 & 9058540.0000000 & 0.061416 \\
\hline$(S)-7-3$ & 0.07668 & & \\
\hline
\end{tabular}




\begin{tabular}{|c|c|c|c|}
\hline & & & \\
\hline 2-aminopyridine & 0.094995 & 127810657.0000000 & 0.60 \\
\hline$(\mathrm{S})-\mathbf{7 - 1 6}$ & 0.097006 & 25793500.0000000 & 0.131543 \\
\hline$(\mathrm{S})-\mathbf{7 - 1 7}$ & 0.086143 & 243608.0000000 & 0.001399 \\
\hline$(\mathrm{S})-\mathbf{7 - 1 8}$ & 0.10428 & 72424800.0000000 & 0.343594 \\
\hline$(\mathrm{S})-\mathbf{7 - 1 9}$ & 0.092089 & 572068.0000000 & 0.003073 \\
\hline$(\mathrm{S})-\mathbf{7 - 2 0}$ & 0.085861 & 29730900.0000000 & 0.171306 \\
\hline$(\mathrm{S})-\mathbf{7 - 2 1}$ & 0.10577 & 60393.0000000 & 0.000282 \\
\hline
\end{tabular}

\section{Measurement of Lifetime}

Table AI-2. Fluorescence lifetime of $(S)$-7-1 - $(S)$-7-21.

\begin{tabular}{|c|c|c|}
\hline$(S)-7-1$ & $0.279 \mathrm{~ns}$ & \\
\hline$(S)-7-2$ & $0.255 \mathrm{~ns}$ & \\
\hline$(S)-7-3$ & $0.292 \mathrm{~ns}$ & \\
\hline$(S)-7-4$ & $0.256 \mathrm{~ns}$ & \\
\hline$(S)-7-5$ & $0.247 \mathrm{~ns}(97.42 \%)$ & $5.6 \mathrm{~ns}(2.58 \%)$ \\
\hline$(S)-7-6$ & $0.244 \mathrm{~ns}(97.26 \%)$ & $5.5 \mathrm{~ns}(2.74 \%)$ \\
\hline$(S)-7-7$ & $0.248 \mathrm{~ns}$ & \\
\hline$(S)-7-8$ & $0.266 \mathrm{~ns}(92.24 \%)$ & $4.09 \mathrm{~ns}(7.76 \%)$ \\
\hline$(S)-7-9$ & $0.256 \mathrm{~ns}$ & \\
\hline$(S)-\mathbf{7 - 1 0}$ & $0.264 \mathrm{~ns}(91.14 \%)$ & $4.01 \mathrm{~ns}(8.86 \%)$ \\
\hline$(S)-7-11$ & $0.272 \mathrm{~ns}(90.17 \%)$ & $3.86 \mathrm{~ns}(9.83 \%)$ \\
\hline$(S)-7-12$ & $0.271 \mathrm{~ns}(92.10 \%)$ & $4.24 \mathrm{~ns}(7.90 \%)$ \\
\hline$(S)-7-13$ & $0.251 \mathrm{~ns}$ & \\
\hline$(S)-7-14$ & $0.250 \mathrm{~ns}$ & \\
\hline$(S)-7-15$ & $0.252 \mathrm{~ns}$ & \\
\hline (S)-7-16 & $0.258 \mathrm{~ns}$ & \\
\hline (S)-7-17 & $0.232 \mathrm{~ns}$ & \\
\hline (S)-7-18 & $0.23 \mathrm{~ns}(94.43 \%)$ & $3.63 \mathrm{~ns}(5.57 \%)$ \\
\hline (S)-7-19 & $0.219 \mathrm{~ns}$ & \\
\hline (S)-7-20 & $0.223 \mathrm{~ns}(94.86 \%)$ & $4.15 \mathrm{~s}(5.14 \%)$ \\
\hline
\end{tabular}




\section{UV-Vis Absorption of Compounds}

Figure AI-1. UV-Vis absorption spectra of compounds (1.0x $10^{-5} \mathrm{M}$ in THF).
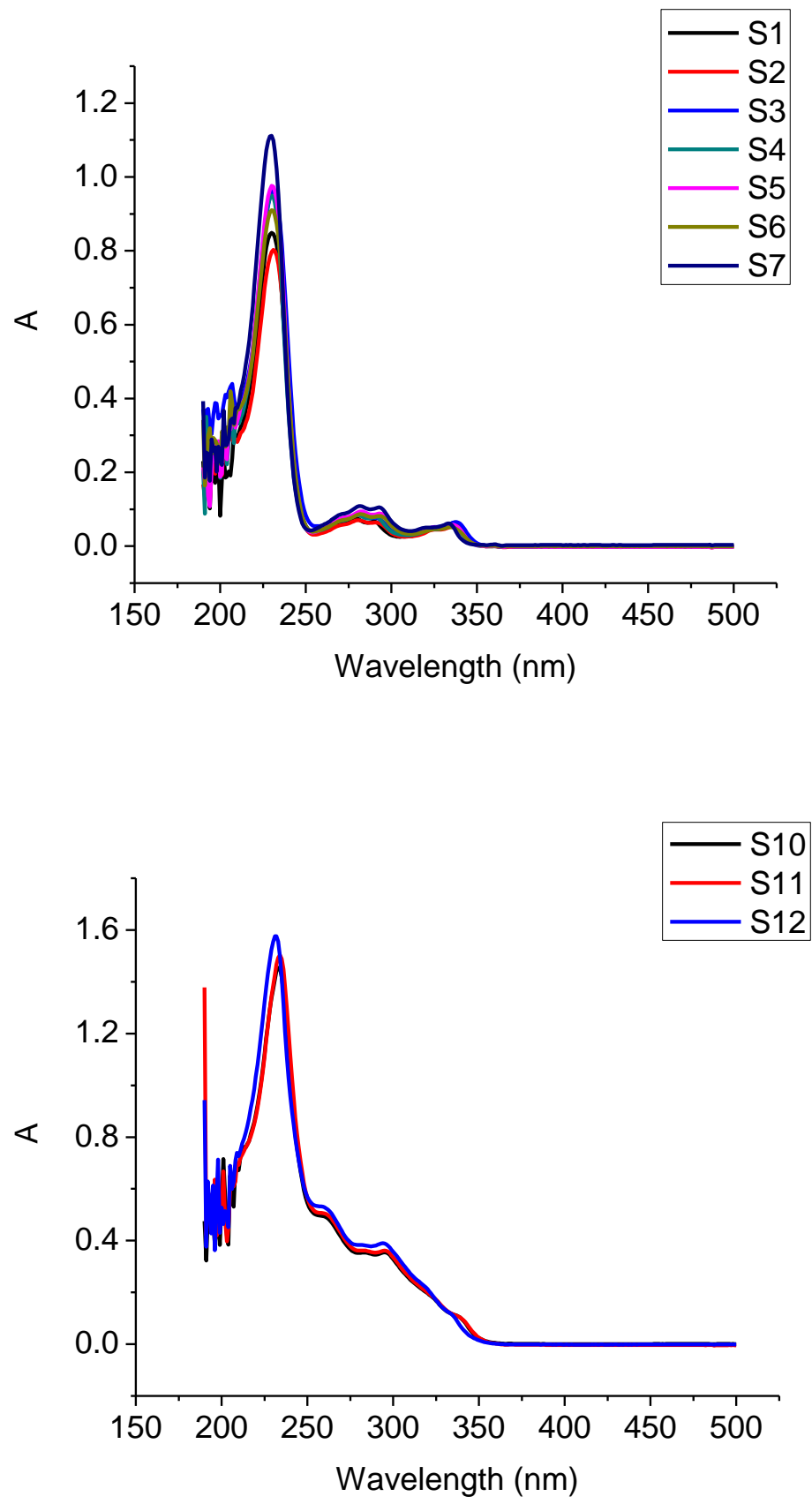

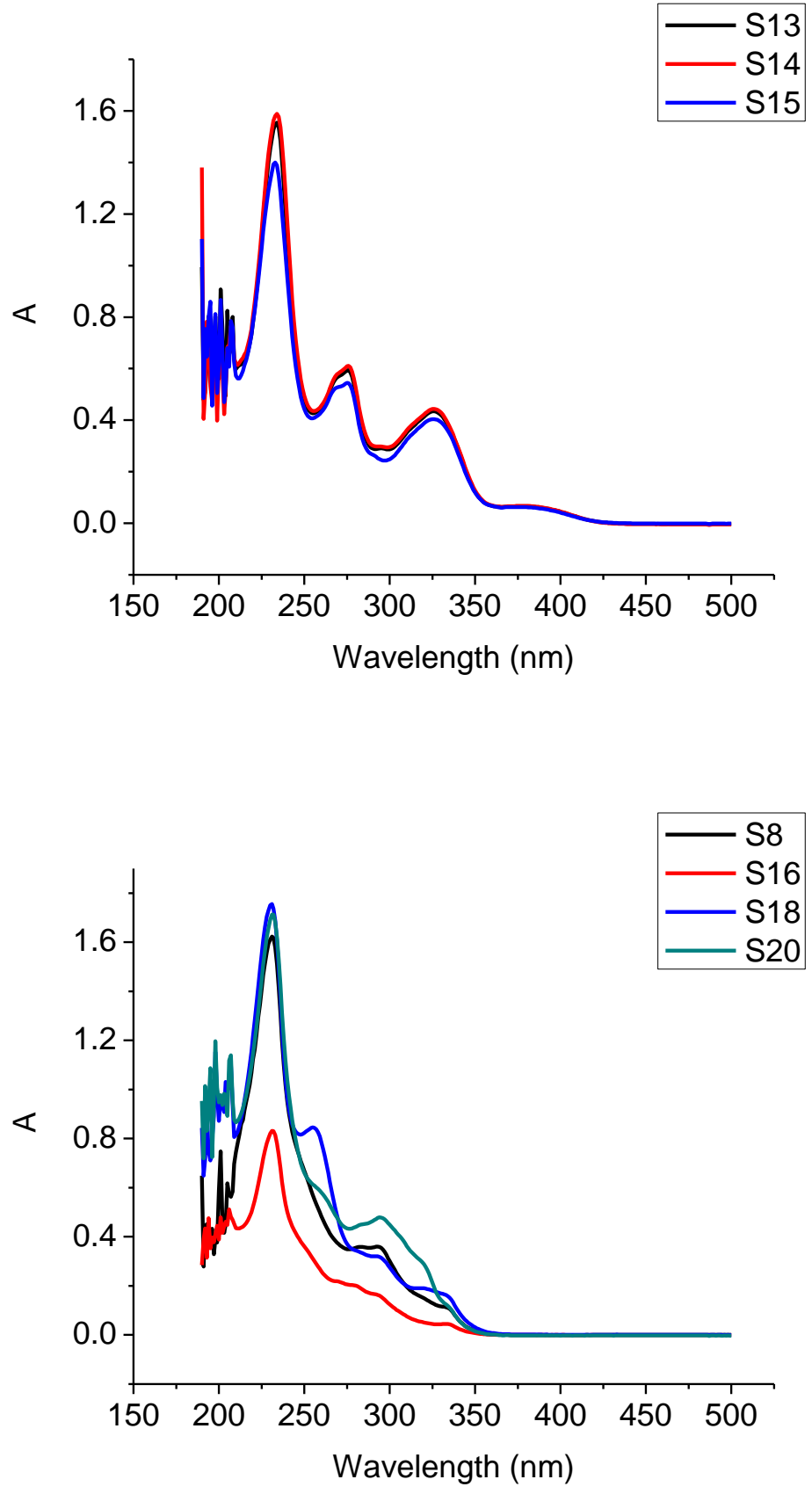


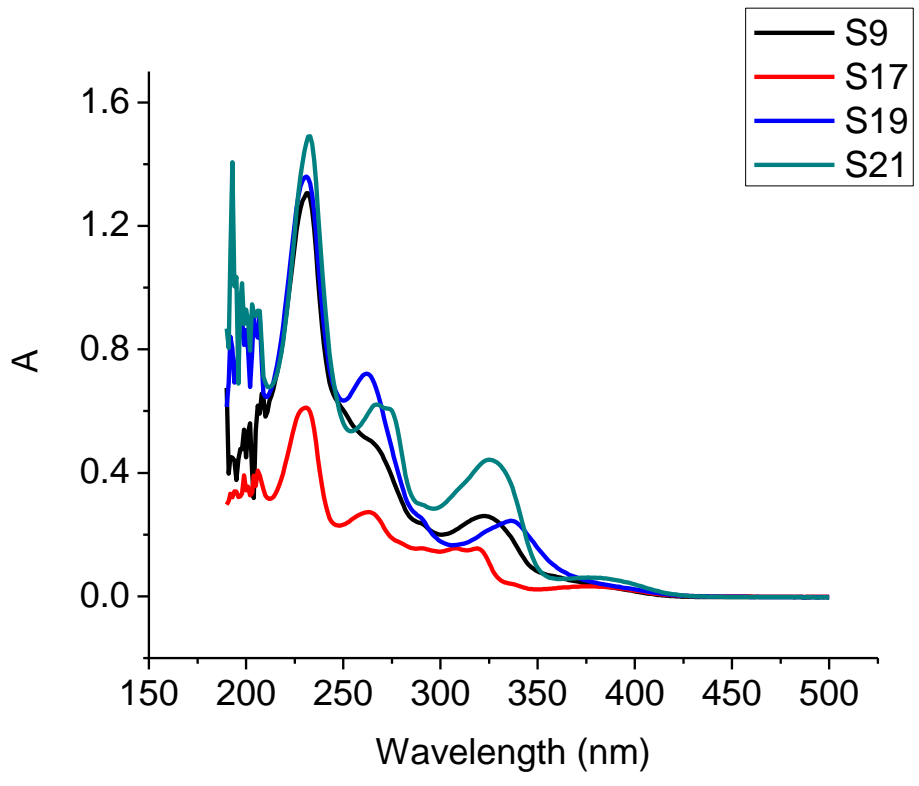

Table AI-3. Maximum absorption peaks and extinction coffecient of (S)-7-1 - (S)-7-21 $\left(1.0 \times 10^{-5} \mathrm{M}\right.$ in THF).

\begin{tabular}{|l|l|}
\hline & \multicolumn{1}{|c|}{$\lambda_{\max }(\varepsilon)$} \\
\hline$(S)-7-1$ & $230\left(8.5 \times 10^{4}\right), 279\left(7.6 \times 10^{3}\right), 290\left(6.7 \times 10^{3}\right), 325\left(4.7 \times 10^{3}\right), 337\left(6.5 \times 10^{3}\right) \mathrm{nm}$ \\
\hline$(S)-7-2$ & $231\left(8.0 \times 10^{4}\right), 281\left(7.1 \times 10^{3}\right), 291\left(6.4 \times 10^{3}\right), 326\left(4.5 \times 10^{3}\right), 338\left(5.9 \times 10^{3}\right) \mathrm{nm}$ \\
\hline$(S)-7-3$ & $231\left(9.6 \times 10^{4}\right), 280\left(8.6 \times 10^{3}\right), 291\left(7.7 \times 10^{3}\right), 326\left(5.2 \times 10^{3}\right), 338\left(6.5 \times 10^{3}\right) \mathrm{nm}$ \\
\hline$(S)-7-4$ & $230\left(9.5 \times 10^{4}\right), 280\left(8.5 \times 10^{3}\right), 291\left(7.9 \times 10^{3}\right), 326\left(4.6 \times 10^{3}\right), 336\left(5.7 \times 10^{3}\right) \mathrm{nm}$ \\
\hline$(S)-7-5$ & $230\left(9.8 \times 10^{4}\right), 282\left(9.4 \times 10^{3}\right), 293\left(8.8 \times 10^{3}\right), 325\left(5.1 \times 10^{3}\right), 335\left(5.9 \times 10^{3}\right) \mathrm{nm}$ \\
\hline$(S)-7-6$ & $230\left(9.1 \times 10^{4}\right), 282\left(8.7 \times 10^{3}\right), 293\left(8.2 \times 10^{3}\right), 325\left(4.6 \times 10^{3}\right), 334\left(5.2 \times 10^{3}\right) \mathrm{nm}$ \\
\hline$(S)-7-7$ & $230\left(1.1 \times 10^{5}\right), 282\left(1.1 \times 10^{4}\right), 293\left(1.1 \times 10^{4}\right), 323\left(5.1 \times 10^{3}\right), 333\left(6.1 \times 10^{3}\right) \mathrm{nm}$ \\
\hline$(S)-7-8$ & $231\left(1.6 \times 10^{5}\right), 293\left(3.6 \times 10^{4}\right), 334\left(1.1 \times 10^{4}\right) \mathrm{nm}$ \\
\hline$(S)-7-9$ & $231\left(1.3 \times 10^{5}\right), 268\left(4.8 \times 10^{4}\right), 323\left(2.6 \times 10^{4}\right) \mathrm{nm}$ \\
\hline$(S)-7-10$ & $233\left(1.5 \times 10^{5}\right), 261\left(4.9 \times 10^{4}\right), 297\left(3.5 \times 10^{4}\right) \mathrm{nm}$ \\
\hline$(S)-7-11$ & $234\left(1.5 \times 10^{5}\right), 262\left(5.0 \times 10^{4}\right), 297\left(3.6 \times 10^{4}\right) \mathrm{nm}$ \\
\hline$(S)-7-12$ & $231\left(1.6 \times 10^{5}\right), 261\left(5.2 \times 10^{4}\right), 295\left(3.9 \times 10^{4}\right) \mathrm{nm}$ \\
\hline$(S)-7-13$ & $234\left(1.6 \times 10^{5}\right), 276\left(5.9 \times 10^{4}\right), 326\left(4.3 \times 10^{4}\right), 386\left(6.3 \times 10^{3}\right) \mathrm{nm}$ \\
\hline$(S)-7-14$ & $234\left(1.6 \times 10^{5}\right), 276\left(6.1 \times 10^{4}\right), 326\left(4.4 \times 10^{4}\right), 386\left(6.4 \times 10^{3}\right) \mathrm{nm}$ \\
\hline$(S)-7-15$ & $233\left(1.4 \times 10^{5}\right), 275\left(5.4 \times 10^{4}\right), 326\left(4.0 \times 10^{4}\right), 386\left(6.0 \times 10^{3}\right) \mathrm{nm}$ \\
\hline
\end{tabular}




\begin{tabular}{|c|c|}
\hline$(\mathrm{S})-7-16$ & $231\left(8.3 \times 10^{4}\right), 278\left(2.0 \times 10^{4}\right), 294\left(1.6 \times 10^{4}\right), 333\left(4.5 \times 10^{3}\right) \mathrm{nm}$ \\
\hline$(\mathrm{S})-7-17$ & $230\left(6.1 \times 10^{4}\right), 263\left(2.7 \times 10^{4}\right), 308\left(1.6 \times 10^{4}\right), 319\left(1.5 \times 10^{4}\right), 376\left(3.2 \times 10^{3}\right) \mathrm{nm}$ \\
\hline$(\mathrm{S})-7-18$ & $231\left(1.8 \times 10^{5}\right), 255\left(8.5 \times 10^{4}\right), 293\left(3.2 \times 10^{4}\right), 332\left(1.7 \times 10^{4}\right) \mathrm{nm}$ \\
\hline$(\mathrm{S})-7-19$ & $231\left(1.4 \times 10^{5}\right), 262\left(7.2 \times 10^{4}\right), 336\left(2.4 \times 10^{4}\right) \mathrm{nm}$ \\
\hline$(\mathrm{S})-7-20$ & $231\left(1.7 \times 10^{5}\right), 295\left(4.8 \times 10^{4}\right) \mathrm{nm}$ \\
\hline$(S)-7-21$ & $233\left(1.5 \times 10^{5}\right), 267\left(6.2 \times 10^{4}\right), 325\left(4.4 \times 10^{4}\right), 386\left(5.7 \times 10^{3}\right) \mathrm{nm}$ \\
\hline
\end{tabular}

\section{Fluorescent Properties of Compounds}

Figure AI-2. Fluorescence spectra of (S)-7-1 - (S)-7-21 (1.0x10 $0^{-5} \mathrm{M}$ in THF). $\left(\lambda_{\text {exc }}=290\right.$ nm. slit $=1.5 / 1.5 \mathrm{~nm})$.
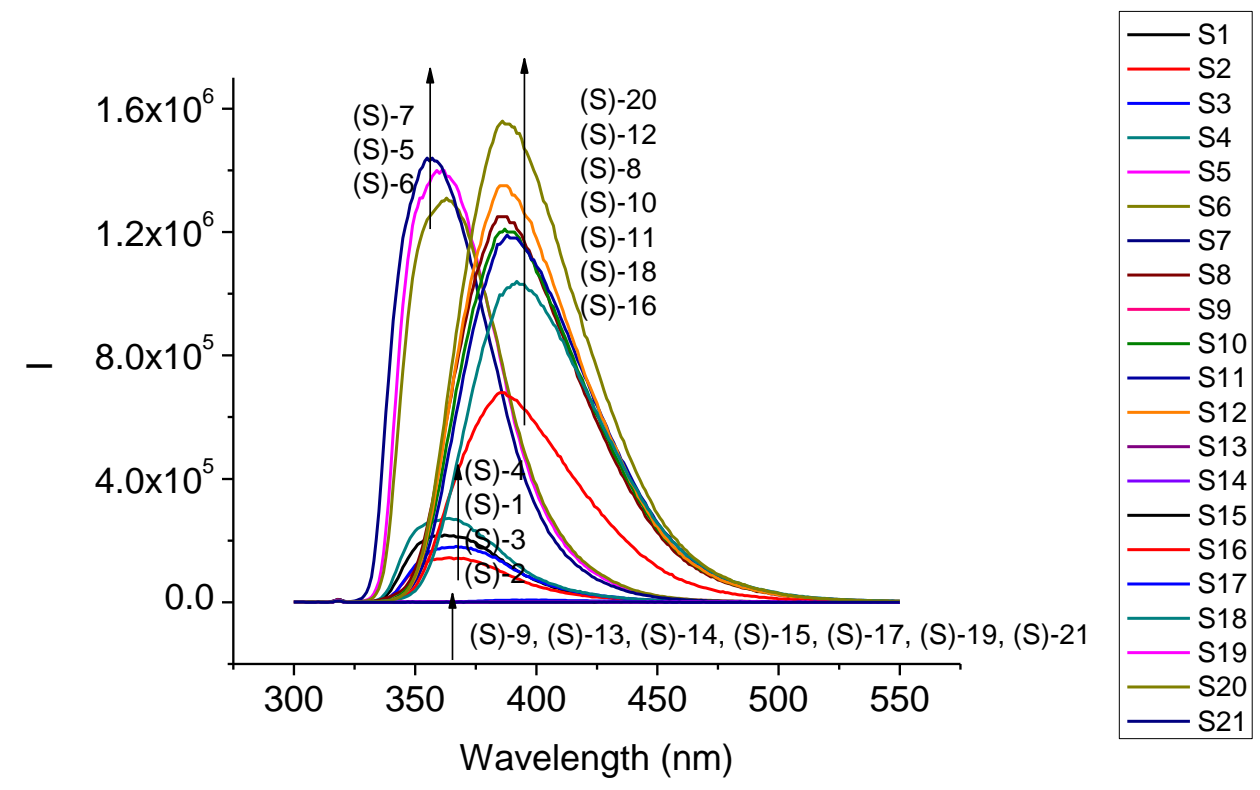

Figure AI-3. Fluorescence spectra of (S)-7-1 - (S)-7-21 (1.0x10 ${ }^{-5} \mathrm{M}$ in THF). $\left(\lambda_{\text {exc }}=335\right.$ nm. slit $=1.5 / 1.5 \mathrm{~nm})$. 

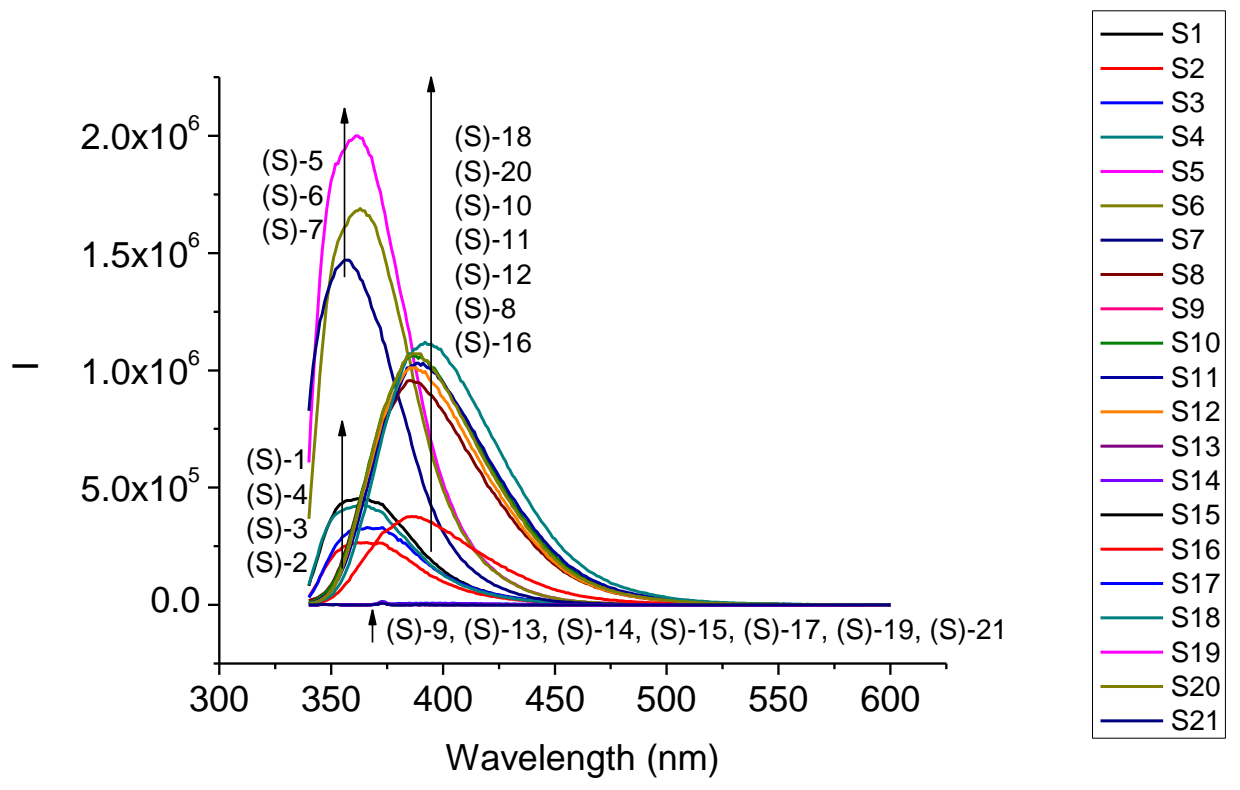

\section{Interaction of (S)-7-1 with Pyridine and Bipyridine.}

$335 \mathrm{~nm}$ was used as the excitation wavelength because both pyridine and bipyridine have absorption at $290 \mathrm{~nm}$. Pyridine has no absorption at all at $335 \mathrm{~nm}$ even when the concentration is as high as $7 \mathrm{M}$. Bipyridine started to show absorption at $335 \mathrm{~nm}$ when the concentration is higher than $0.01 \mathrm{M}$. When treated with pyridine, (S)-7-1 showed fluorescence quenching when the pyridine concentration was higher than $0.01 \mathrm{M}$. Bipyridine caused no change to the fluorescence of (S)-7-1 when its concentration was lower than 0.01 M.

Figure AI-4. UV absorption of pyridine (3.5 M, 7M in THF) and bipyridine (0.01, 0.05, $0.1,0.2,0.4,0.8 \mathrm{M})$ 

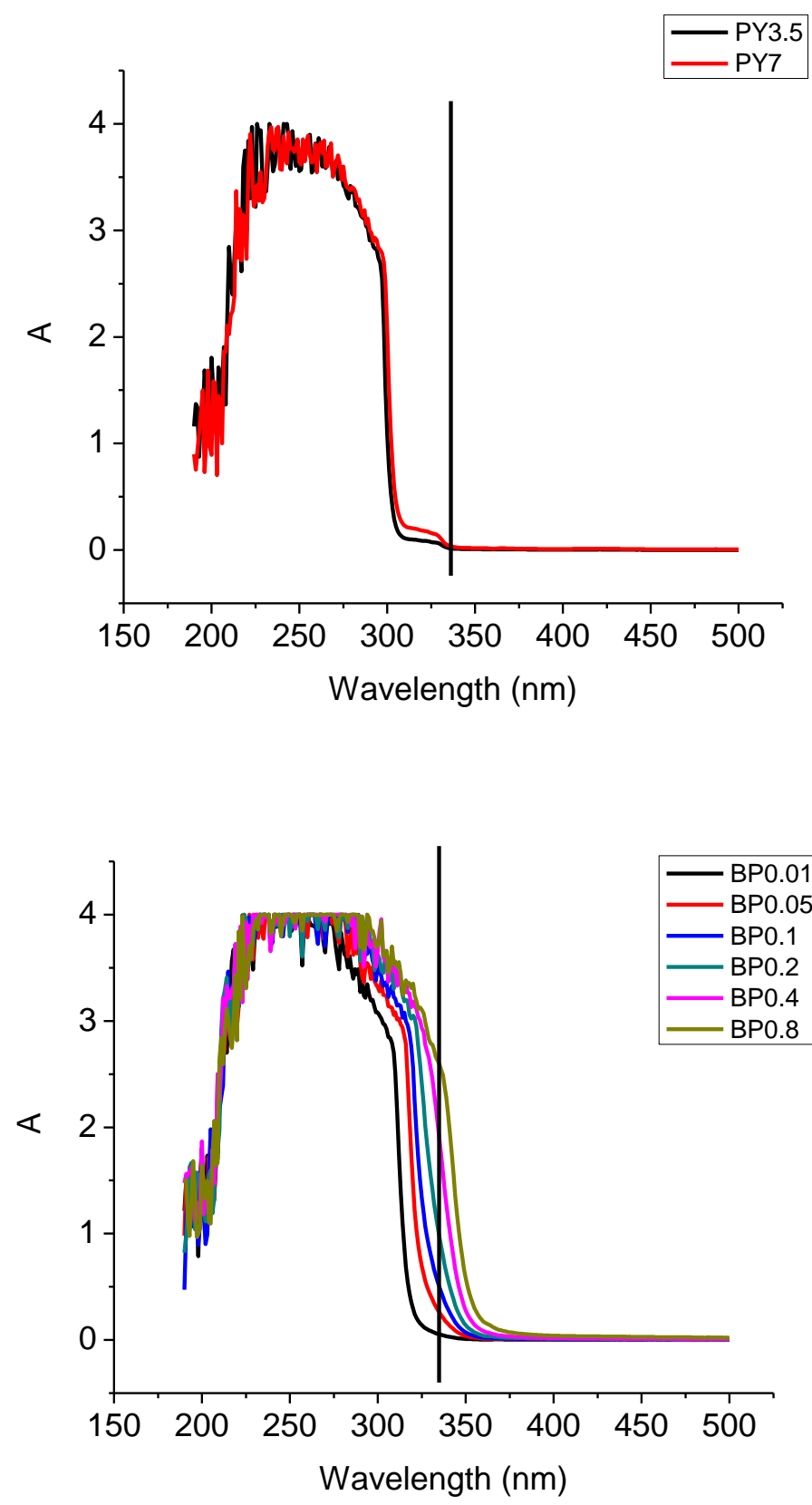

Figure AI-5. Fluorescence spectra of (S)-7-1(2.0x10 $10^{-5} \mathrm{M}$ in THF) in the presence of pyridine at various concentrations $(2 \mathrm{E}-5,2 \mathrm{E}-4,0.001,0.002,0.005,0.01,0.02,0.05,0.1$, $0.2,0.3,0.4,0.5,0.6,0.7,0.8,0.9,1,2,3,4,5,6,7 \mathrm{M}) .\left(\lambda_{\mathrm{exc}}=335 \mathrm{~nm} . \mathrm{slit}=1.5 / 1.5 \mathrm{~nm}\right)$ 

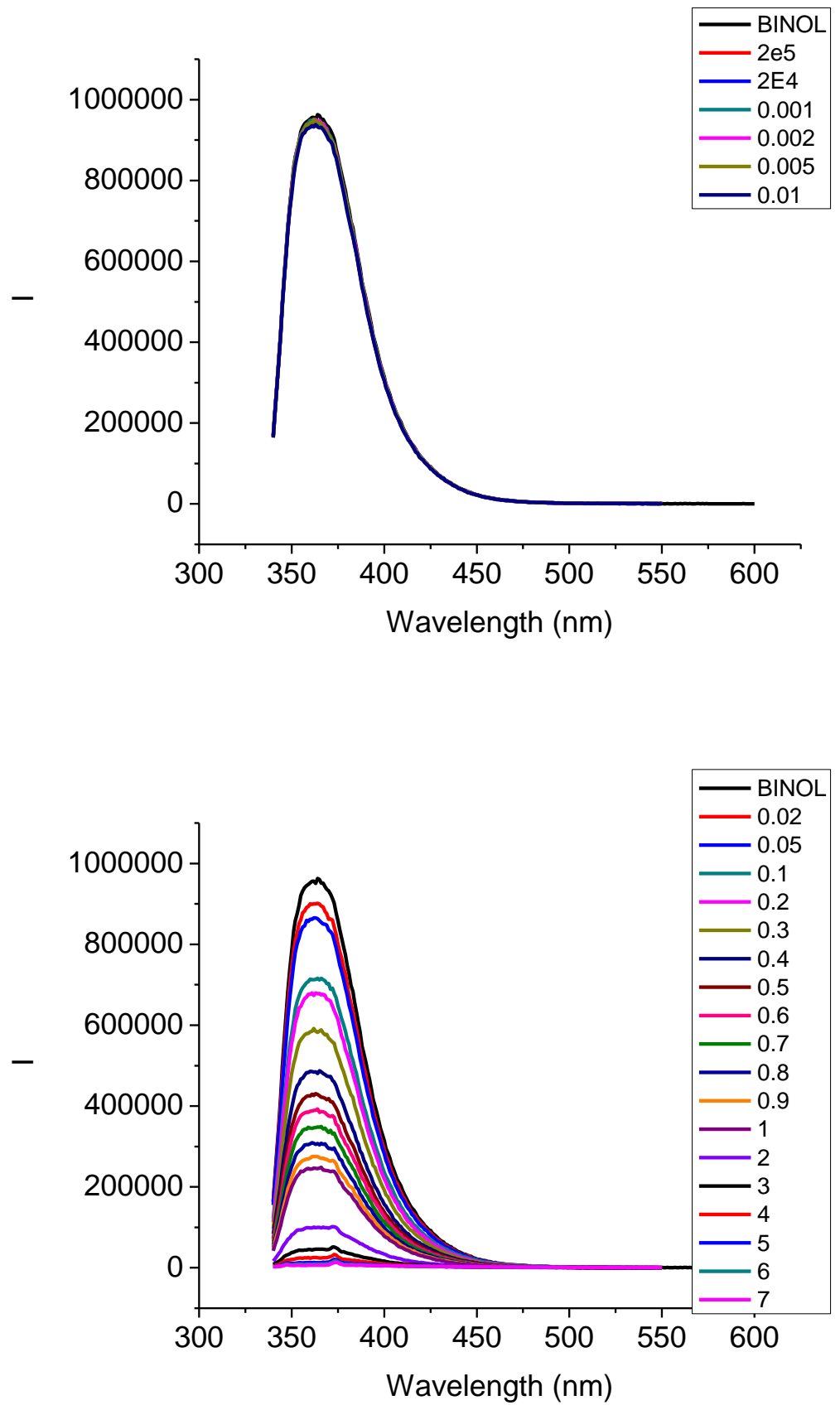

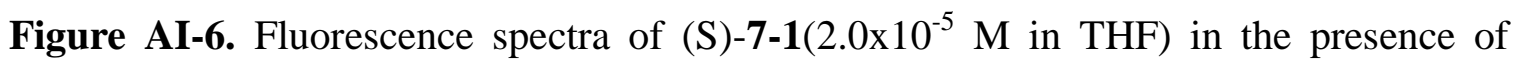
bipyridine at various concentrations $(2 \mathrm{E}-5,2 \mathrm{E}-4,0.001,0.002,0.005,0.01,0.02,0.05$, $0.1,0.2,0.3,0.4,0.5,0.6,0.7,0.8 \mathrm{M}) .\left(\lambda_{\mathrm{exc}}=335 \mathrm{~nm}\right.$. slit $\left.=1.5 / 1.5 \mathrm{~nm}\right)$ 

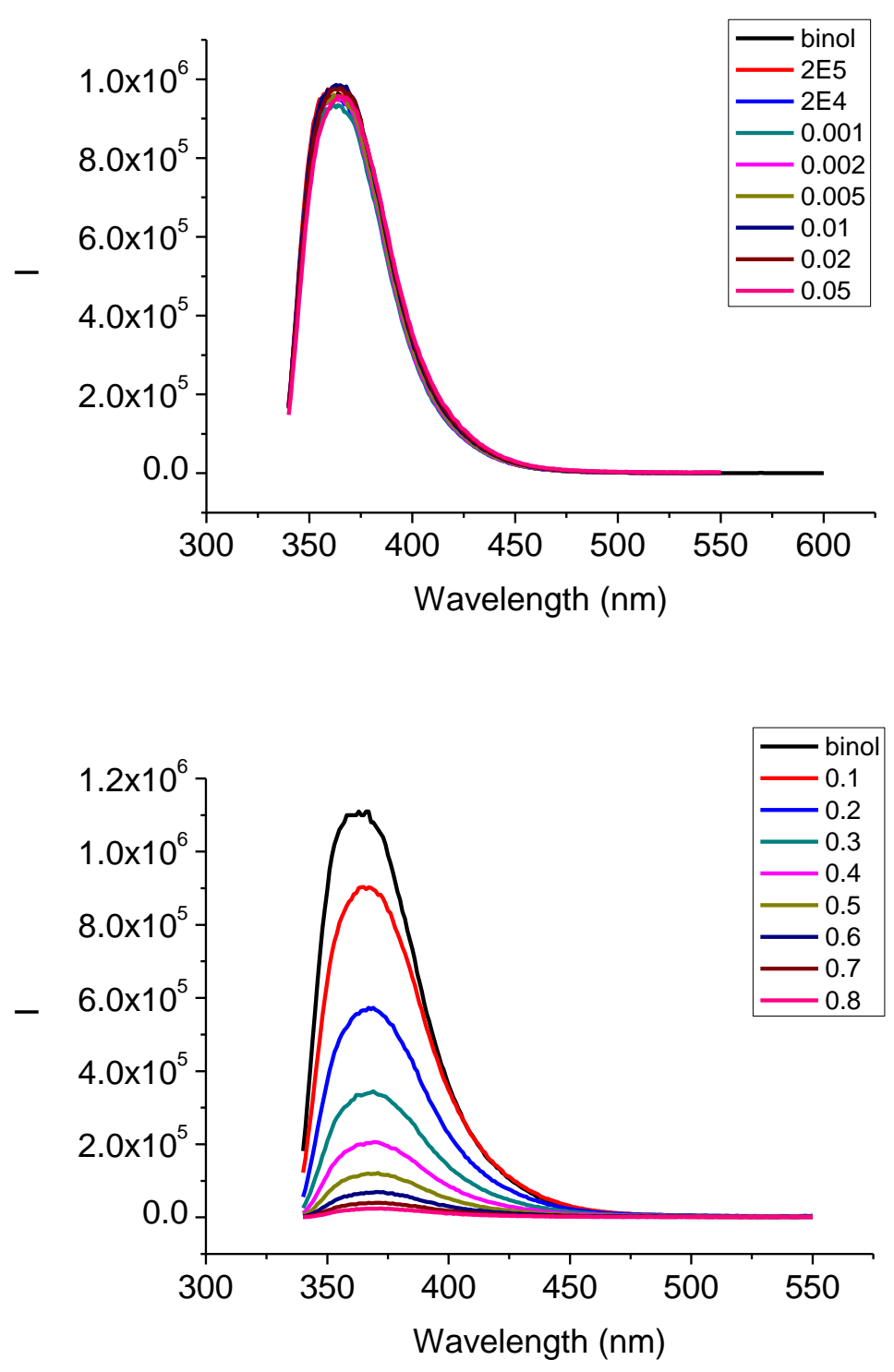

\section{Preparation of $\mathrm{Cu}$ complex}

Copper complex of BINOL-pyridine and BINOL-bipyridine compounds (S)-7-10 (S)-7-15 were prepared by mixing $(\mathrm{S})-\mathbf{7 - 1 0}-(\mathrm{S})-\mathbf{7 - 1 5}(0.5 \mathrm{mmol})$ and $\mathrm{CuCl}_{2} \cdot 2 \mathrm{H}_{2} \mathrm{O}(0.6$ $\mathrm{mmol})$ in methanol $(10 \mathrm{~mL})$ at room temperature for about $0.5 \mathrm{~h}$. The reaction was monitored by TLC. After the reaction was complete, the solution was concentrated and 
water $(2 \mathrm{~mL})$ was added to generate precipitate. The solid was collected through filtration, washed with water $(3 \times 1 \mathrm{~mL})$ and methanol (several drops) and then dried under vacuum.

Copper complex of BINOl-terpyridine compounds (S)-7-20 and (S)-7-21 were prepared at $70{ }^{\circ} \mathrm{C}$.

\section{8. pH effect}

Figure AI-7. Fluorescence spectra of (S)-7-9 $\left(1 \times 10^{-5} \mathrm{M}\right.$ in $5 \mathrm{~mL}$ THF) with addition of (a) $3 \mathrm{M} \mathrm{HCl}$ or (b) $3 \mathrm{M} \mathrm{NaOH}(1,5,10,20,50 \mu \mathrm{L}) .\left(\lambda_{\mathrm{exc}}=325 \mathrm{~nm}\right.$. slit $\left.=5.0 / 5.0 \mathrm{~nm}\right)$

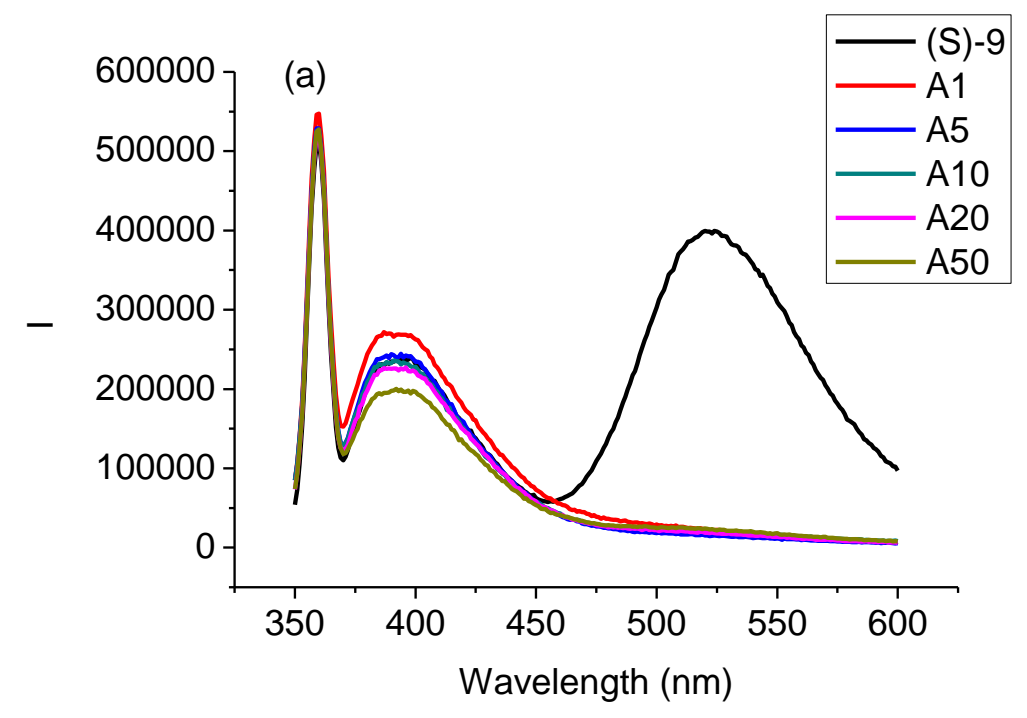




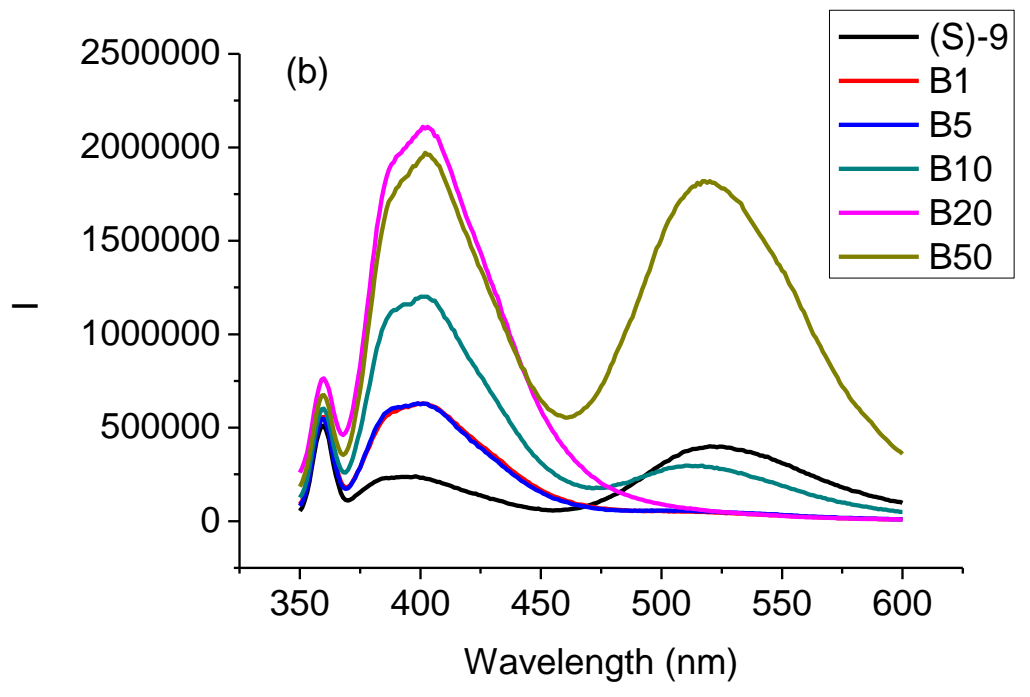

Figure AI-8. Fluorescence spectra of (S)-7-9 $\left(1 \times 10^{-5} \mathrm{M}\right.$ in $5 \mathrm{~mL}$ THF/ $\mathrm{H}_{2} \mathrm{O}$ 5:1) with addition of $3 \mathrm{M} \mathrm{NaOH}(1,5,10,20 \mu \mathrm{L}) .\left(\lambda_{\mathrm{exc}}=325 \mathrm{~nm} . \mathrm{slit}=3.0 / 3.0 \mathrm{~nm}\right)$

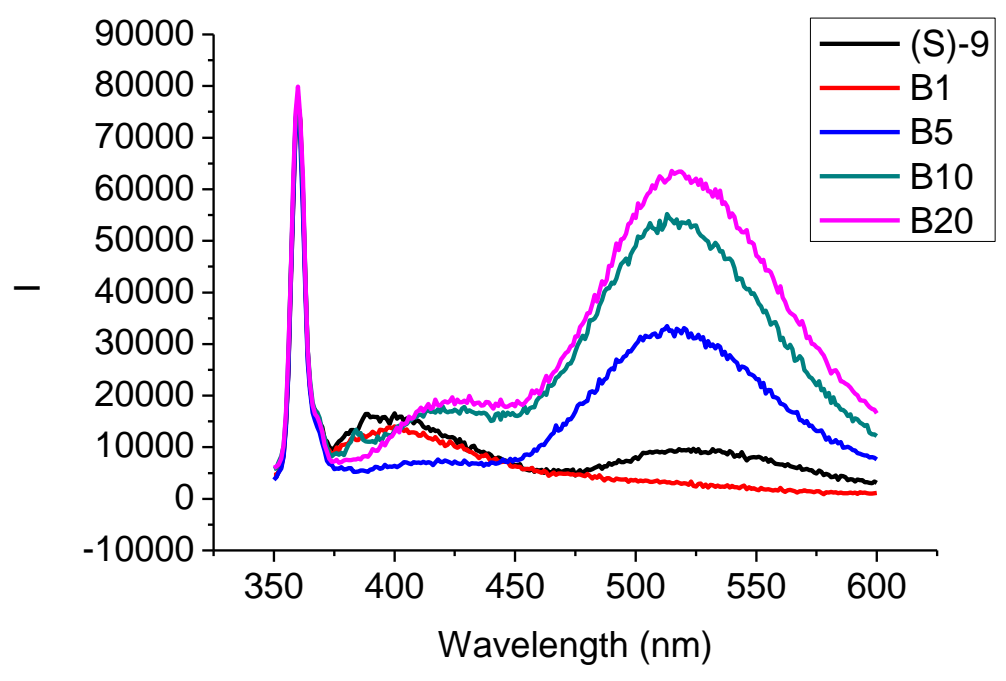

Figure AI-9. Fluorescence spectra of (S)-7-15 $\left(1 \times 10^{-5} \mathrm{M}\right.$ in $5 \mathrm{~mL}$ DMF) with addition of (a) $3 \mathrm{M} \mathrm{HCl}$ or (b) $3 \mathrm{M} \mathrm{NaOH}(1,5,10,20,50 \mu \mathrm{L}) .\left(\lambda_{\text {exc }}=325 \mathrm{~nm}\right.$. slit $\left.=5 \cdot 0 / 5 \cdot 0 \mathrm{~nm}\right)$ 

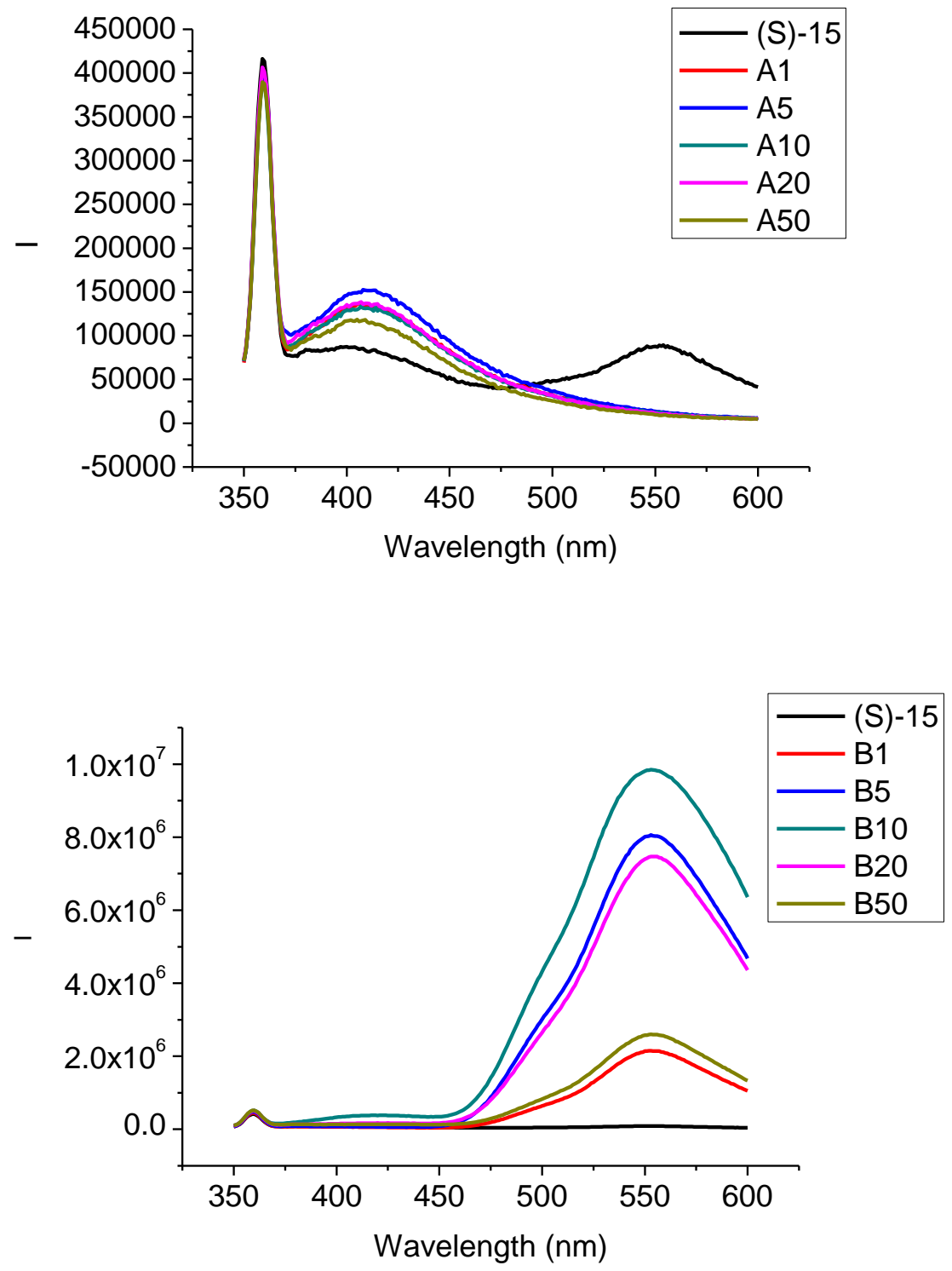

Figure AI-10. Fluorescence spectra of (S)-7-15 $\left(1 \times 10^{-5} \mathrm{M}\right.$ in $5 \mathrm{~mL}$ THF/ $\left.\mathrm{H}_{2} \mathrm{O} 5: 1\right)$ with addition of $3 \mathrm{M} \mathrm{NaOH}(1,5,10,20 \mu \mathrm{L}) \cdot\left(\lambda_{\mathrm{exc}}=325 \mathrm{~nm} . \mathrm{slit}=3.0 / 3.0 \mathrm{~nm}\right)$ 


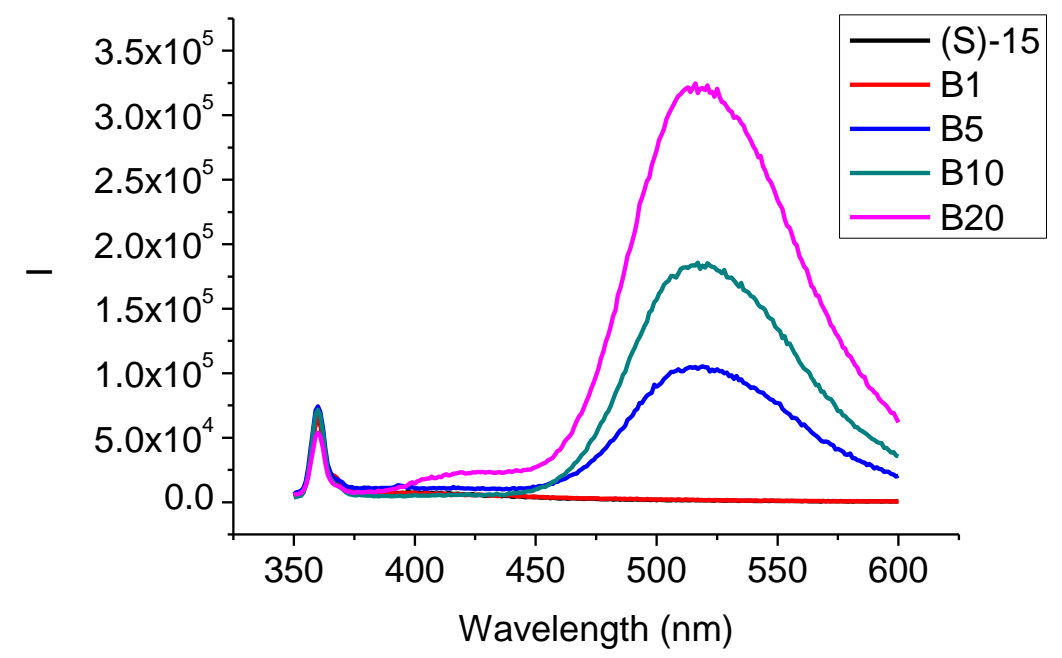

Figure AI-11. Fluorescence spectra of (S)-7-13 $\left(1 \times 10^{-5} \mathrm{M}\right.$ in $5 \mathrm{~mL}$ THF) with addition of (a) $3 \mathrm{M} \mathrm{HCl}$ or (b) $3 \mathrm{M} \mathrm{NaOH}(1,5,10,20,50 \mu \mathrm{L}) \cdot\left(\lambda_{\text {exc }}=325 \mathrm{~nm}\right.$. slit $\left.=5 \cdot 0 / 5 \cdot 0 \mathrm{~nm}\right)$

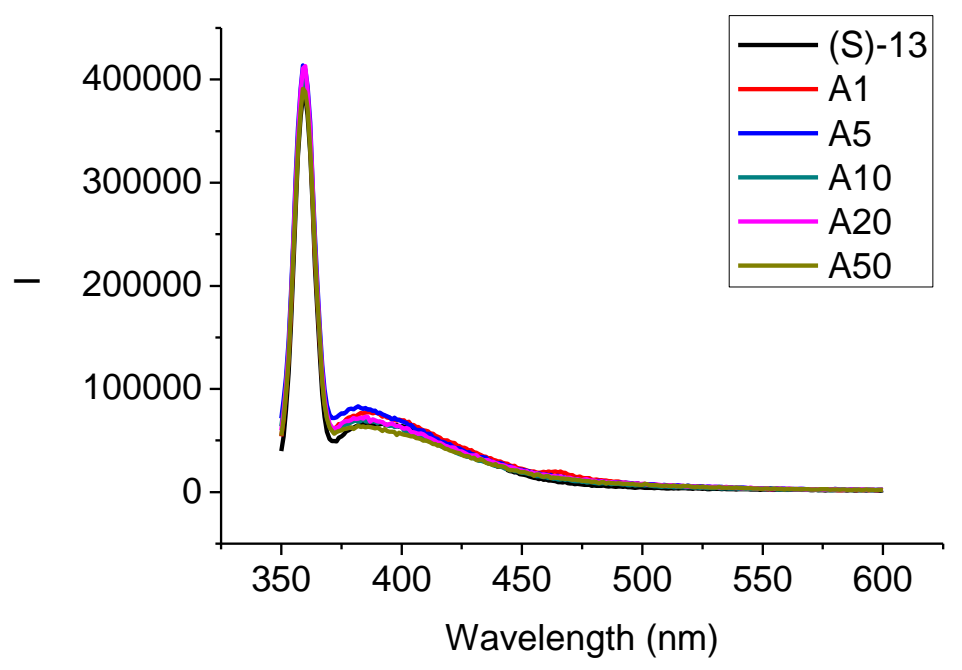




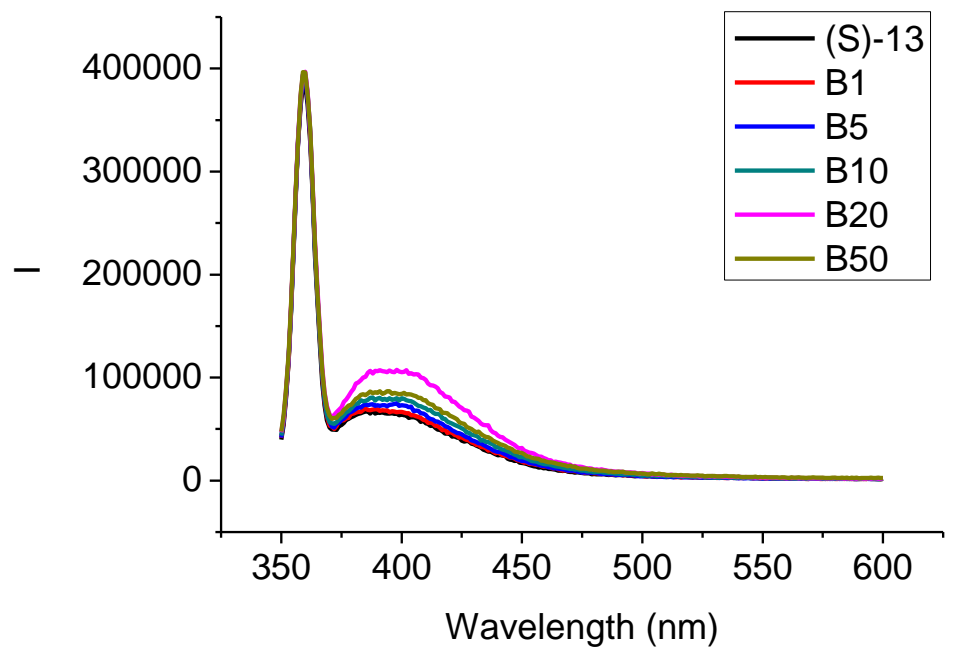

Figure AI-12. Fluorescence spectra of (S)-7-14 $\left(1 \times 10^{-5} \mathrm{M}\right.$ in $5 \mathrm{~mL}$ THF) with addition of (a) $3 \mathrm{M} \mathrm{HCl}$ or (b) $3 \mathrm{M} \mathrm{NaOH}(1,5,10,20,50 \mu \mathrm{L}) \cdot\left(\lambda_{\mathrm{exc}}=325 \mathrm{~nm}\right.$. slit $\left.=5 \cdot 0 / 5.0 \mathrm{~nm}\right)$

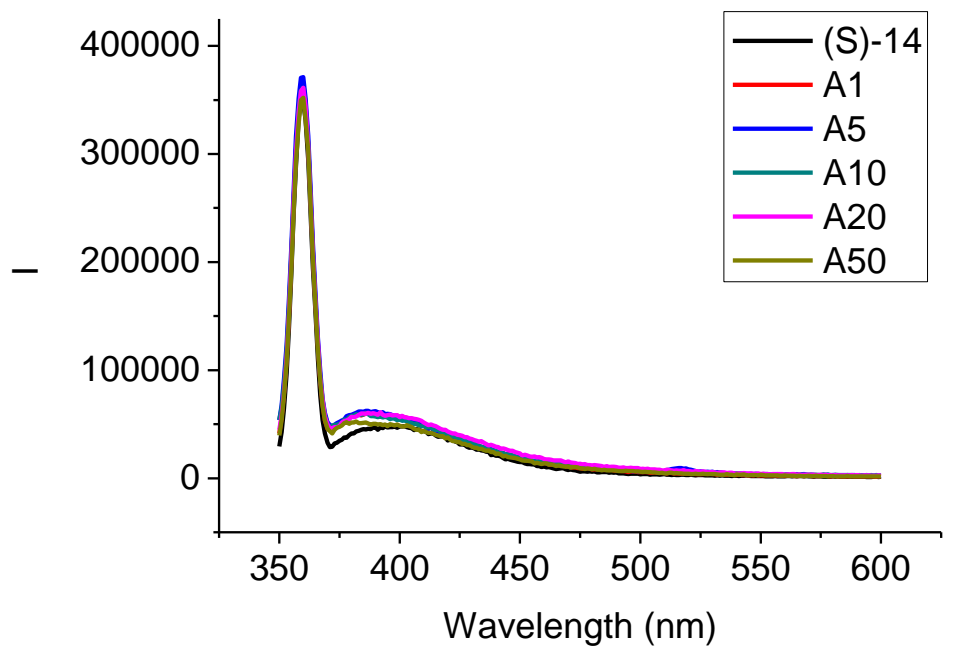




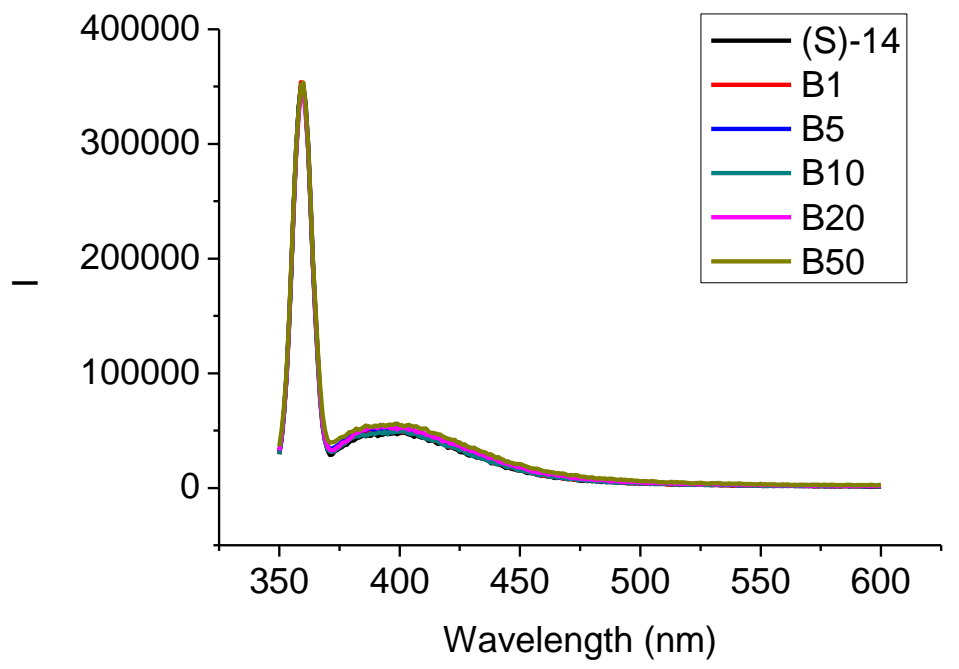

Figure AI-13. Fluorescence spectra of (S)-7-15. $\mathrm{CuCl}_{2}\left(1 \times 10^{-5} \mathrm{M}\right.$ in $\left.5 \mathrm{~mL} \mathrm{DMF}\right)$ at different pH. $\left(\lambda_{\mathrm{exc}}=320 \mathrm{~nm}\right.$. slit $\left.=5.0 / 5.0 \mathrm{~nm}\right) \mathrm{SY}-2-207$

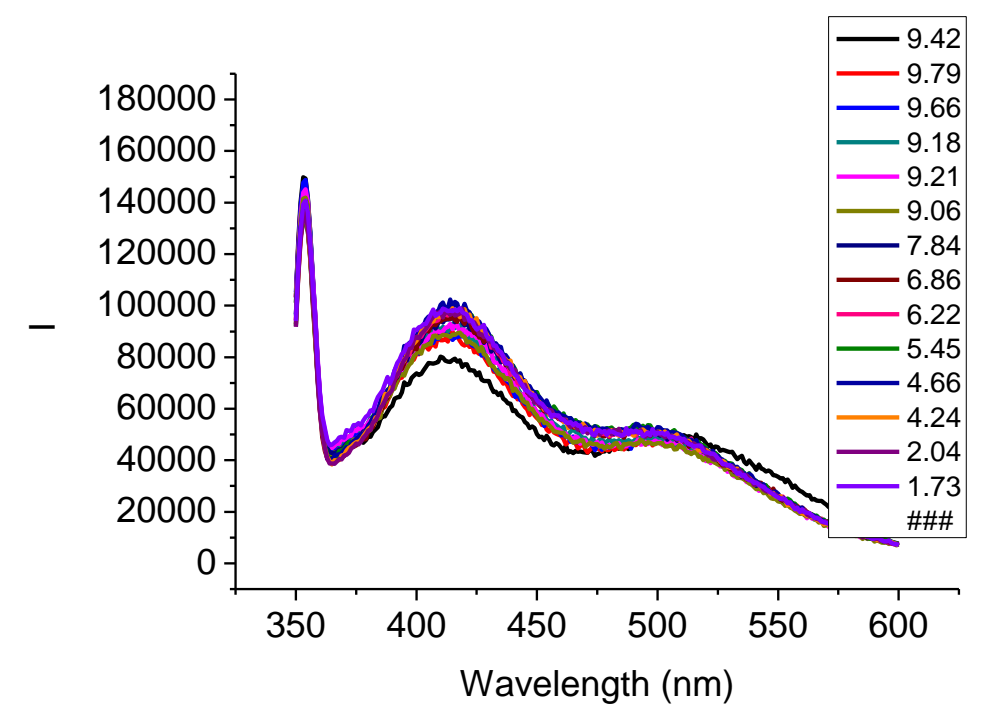




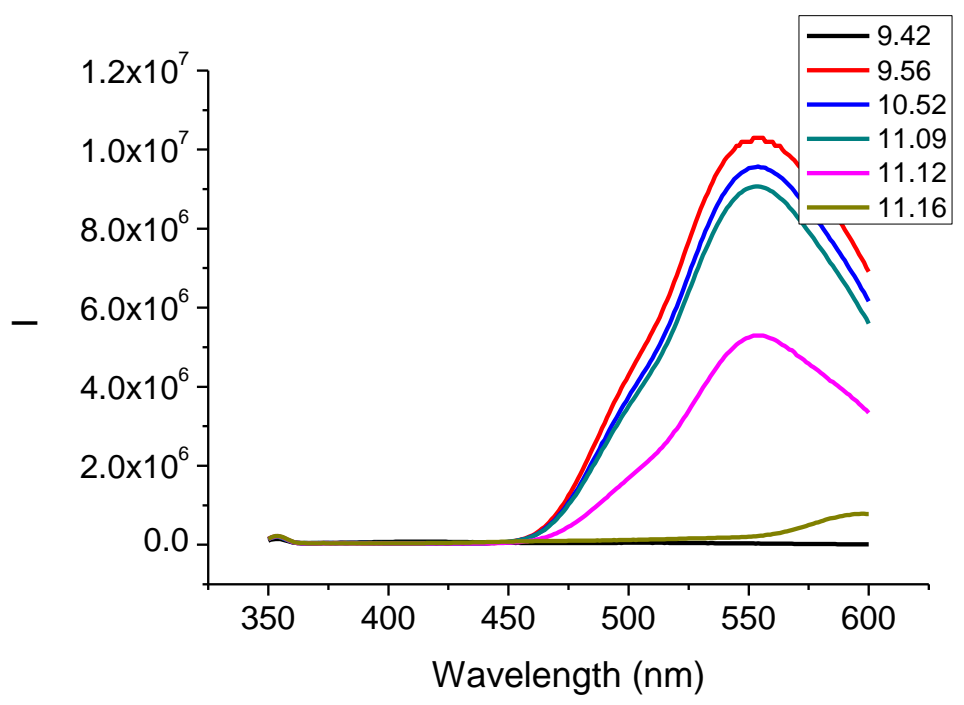

9. Interaction of $\mathrm{Cu}$ complexes of (S)-7-9, (S)-7-13, (S)-7-14, (S)-7-15 with substrates

Figure AI-14. UV-Vis absorption spectra of (S)-7-9 and its copper complex $\left(2 \times 10^{-5} \mathrm{M}\right.$ in THF).

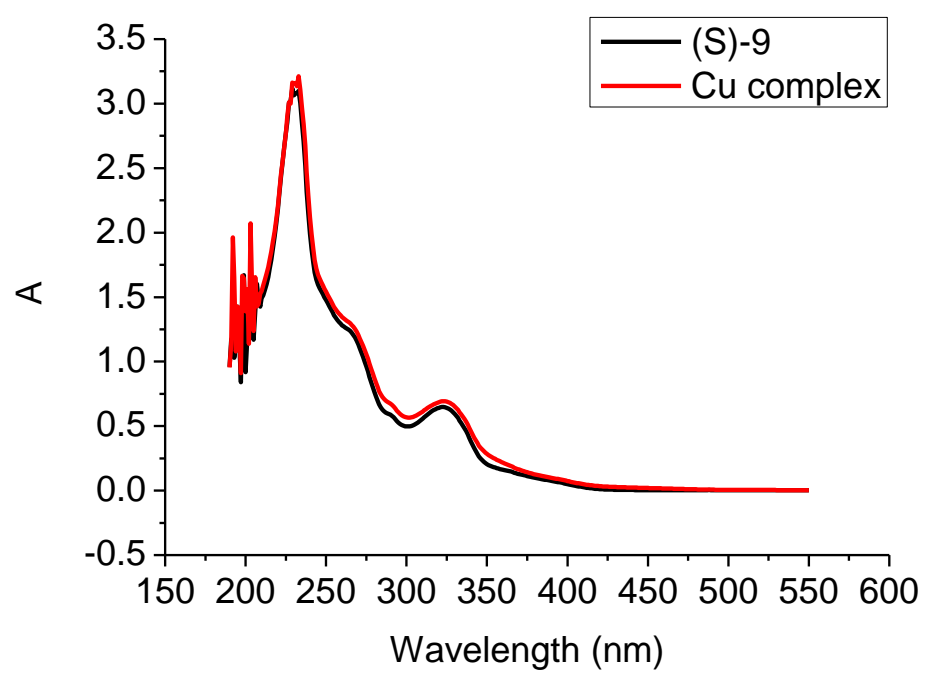

Figure AI-15. Fluorescence spectra of (S)-7-9, its copper complex $\left(2 \times 10^{-5} \mathrm{M}\right.$ in 
THF/water 4.2/0.8) and its copper complex in the presence of mandelic acid, Leu, Ser and $\operatorname{Arg}\left(1 \times 10^{-4} \mathrm{M}\right) .\left(\lambda_{\text {exc }}=323 \mathrm{~nm}\right.$. slit $\left.=7.0 / 7.0 \mathrm{~nm}\right)$

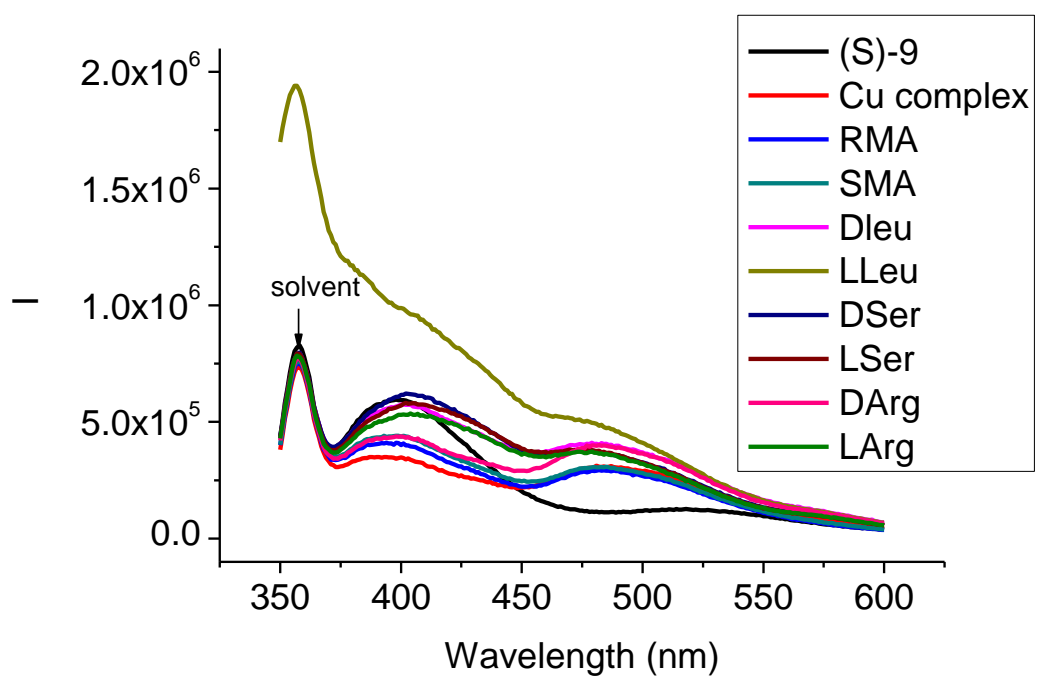

Figure AI-16. UV-Vis absorption spectra of (S)-7-13 and its copper complex $\left(1 \times 10^{-5} \mathrm{M}\right.$ in $\left.\mathrm{THF} / \mathrm{H}_{2} \mathrm{O} 5: 1\right)$.

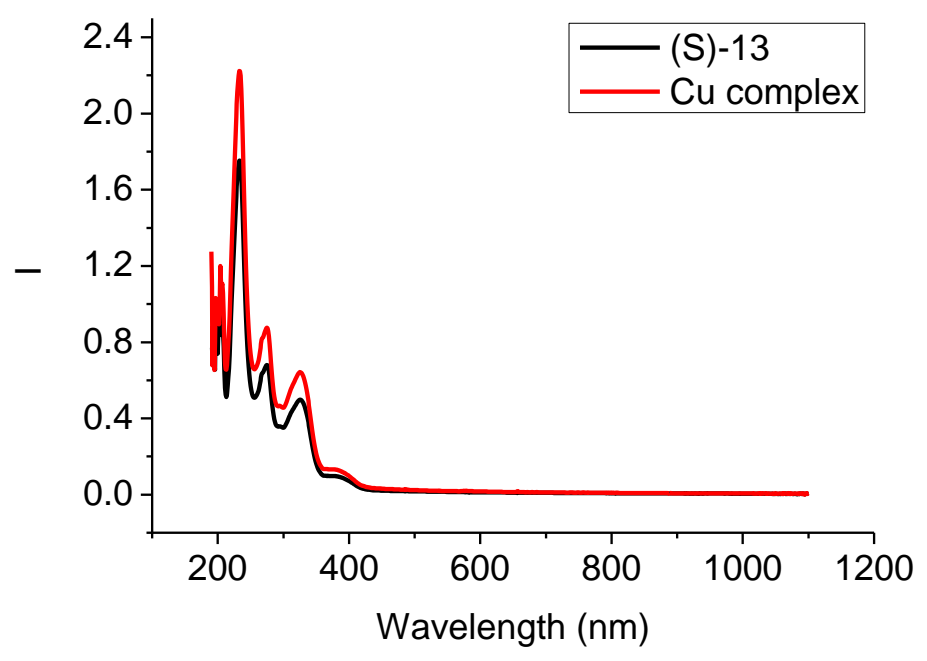

Figure AI-17. Fluorescence spectra of (S)-7-13, (S)-7-13. $\mathrm{CuCl}_{2}$ and $(\mathrm{S})-\mathbf{7 - 1 3} \cdot \mathrm{CuCl}_{2}$ 
$\left(1 \times 10^{-5} \mathrm{M}\right.$ in $\left.\mathrm{THF} / \mathrm{H}_{2} \mathrm{O} 5: 1\right)$ in the presence of D-Ser, L-Ser and L-His $\left(1 \times 10^{-5} \mathrm{M}, 5 \times 10^{-5}\right.$ $\left.\mathrm{M}, 1 \times 10^{-4} \mathrm{M}\right) .\left(\lambda_{\mathrm{exc}}=325 \mathrm{~nm}\right.$. slit $\left.=3 \cdot 0 / 3.0 \mathrm{~nm}\right)$

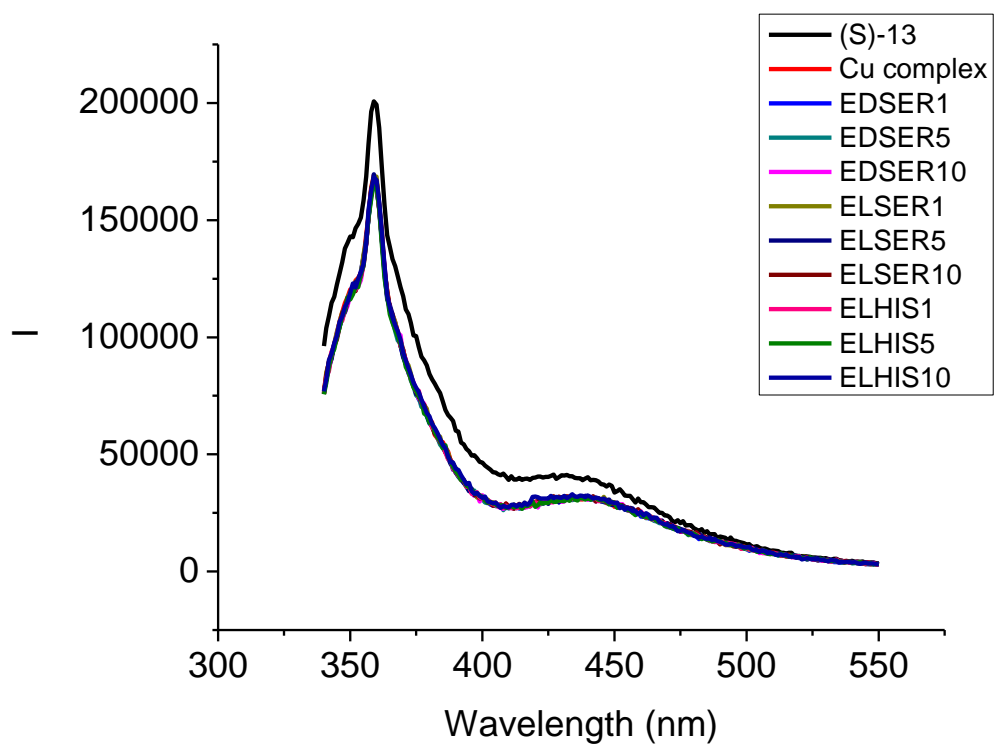

Figure AI-18. UV-Vis absorption spectra of (S)-7-14 and its copper complex $\left(1 \times 10^{-5} \mathrm{M}\right.$ in $\mathrm{THF} / \mathrm{H}_{2} \mathrm{O}$ 5:1).

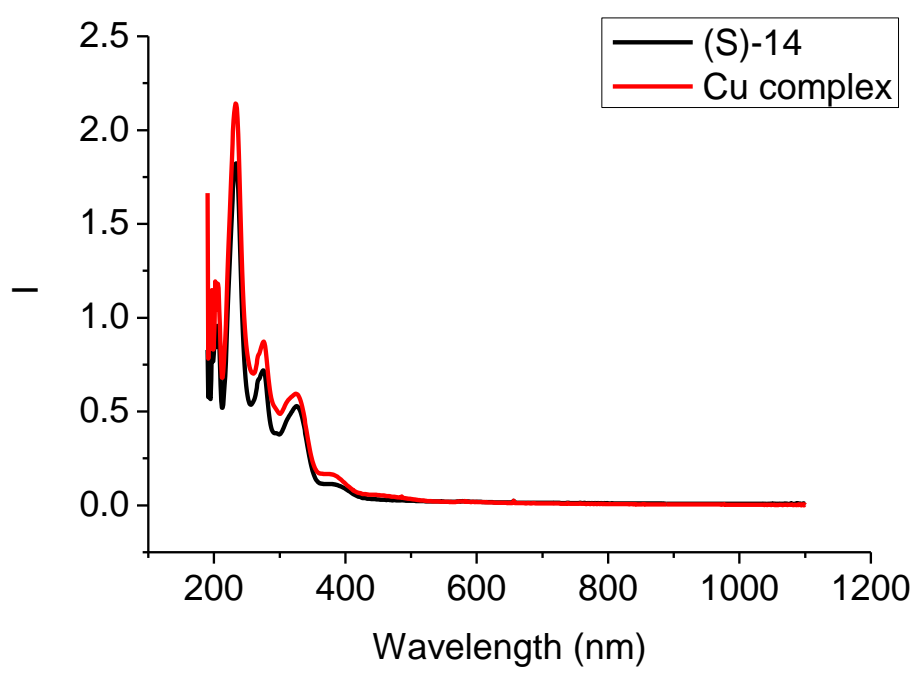


Figure AI-19. Fluorescence spectra of (S)-7-14, (S)-7-14. $\mathrm{CuCl}_{2}$ and (S)-7-14. $\mathrm{CuCl}_{2}$ $\left(1 \times 10^{-5} \mathrm{M}\right.$ in $\left.\mathrm{THF} / \mathrm{H}_{2} \mathrm{O} 5: 1\right)$ in the presence of D-Ser, L-Ser and L-His $\left(1 \times 10^{-5} \mathrm{M}, 5 \times 10^{-5}\right.$ $\left.\mathrm{M}, 1 \times 10^{-4} \mathrm{M}\right) .\left(\lambda_{\mathrm{exc}}=325 \mathrm{~nm}\right.$. slit $\left.=3 \cdot 0 / 3.0 \mathrm{~nm}\right)$

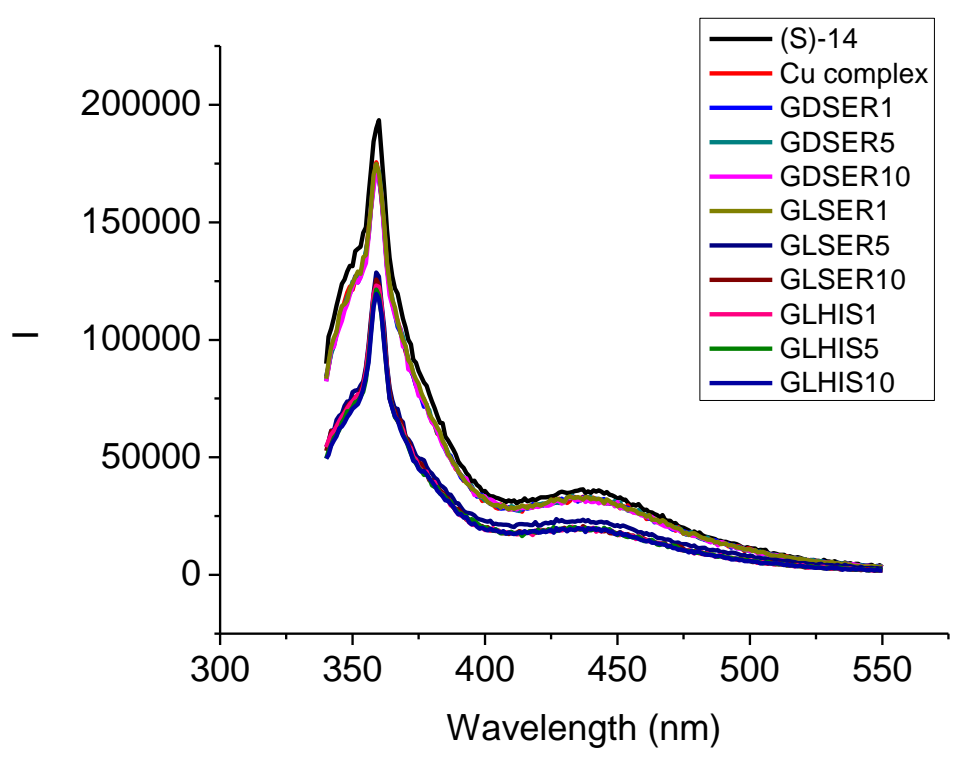

Figure AI-20. UV-Vis absorption spectra of (S)-7-15 and its copper complex $\left(2 \times 10^{-5} \mathrm{M}\right.$ in DMF).

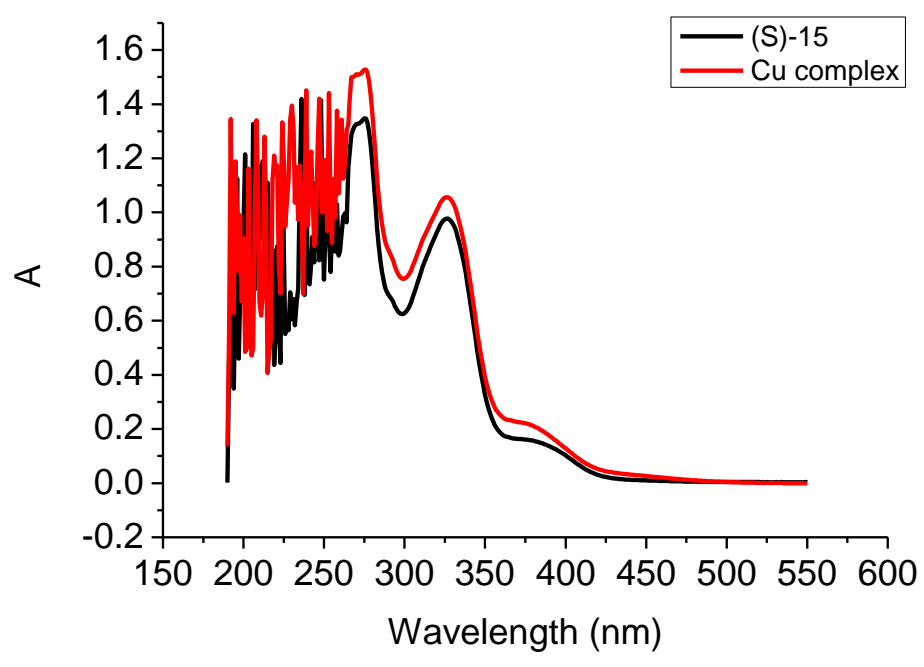


Figure AI-21. Fluorescence spectra of (S)-7-15, its copper complex $\left(2 \times 10^{-5} \mathrm{M}\right.$ in DMF/water 4.2/0.8) and its copper complex in the presence of mandelic acid, Leu, Ser and $\operatorname{Arg}\left(1 \times 10^{-4} \mathrm{M}\right) .\left(\lambda_{\text {exc }}=326 \mathrm{~nm}\right.$. slit $\left.=7.0 / 7.0 \mathrm{~nm}\right)$

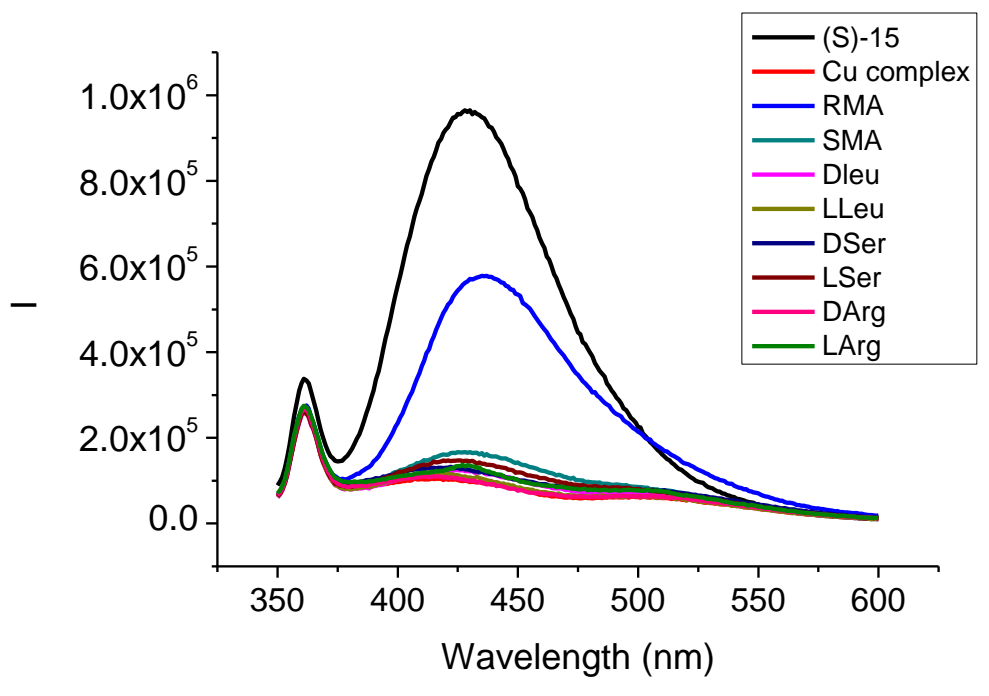

Figure AI-22. Fluorescence spectra of (S)-7-15. $\mathrm{CuCl}_{2}\left(2 \times 10^{-5} \mathrm{M}\right.$ in DMF) in the presence of phenylalaninol $\left(2 \times 10^{-4} \mathrm{M}, 4 \times 10^{-4} \mathrm{M}, 6 \times 10^{-4} \mathrm{M}\right) .\left(\lambda_{\mathrm{exc}}=326 \mathrm{~nm} . \mathrm{slit}=6 / 6 \mathrm{~nm}\right)$

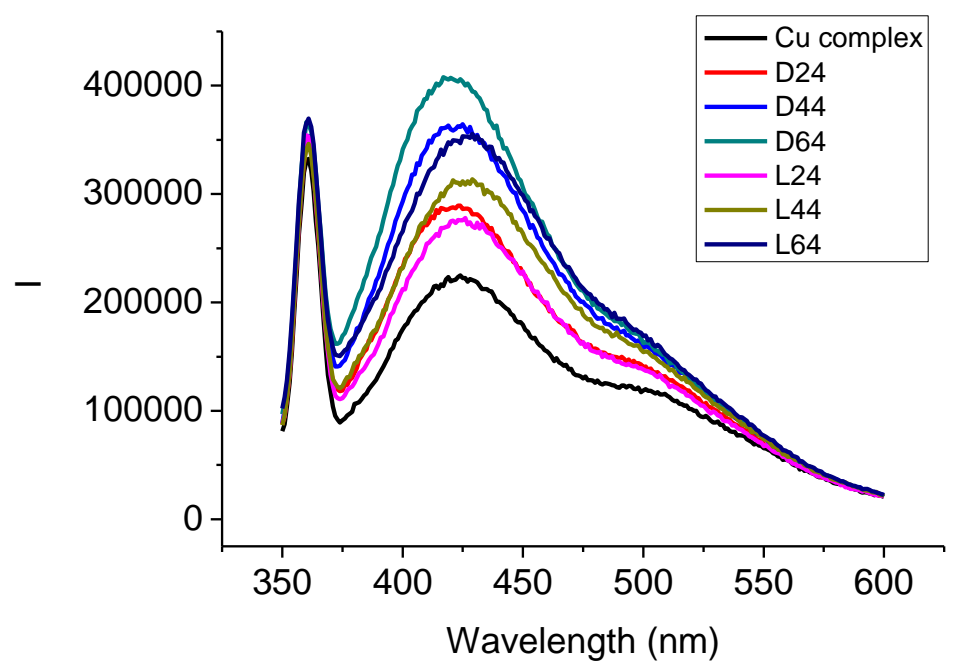


Figure AI-23. Fluorescence spectra of $(\mathrm{S})-\mathbf{7 - 1 5} \cdot \mathrm{CuCl}_{2}\left(2 \times 10^{-5} \mathrm{M}\right.$ in DMSO $)$ in the presence of phenylalaninol $\left(2 \times 10^{-4} \mathrm{M}, 1 \times 10^{-3} \mathrm{M}, 2 \times 10^{-3} \mathrm{M}\right) .\left(\lambda_{\mathrm{exc}}=326 \mathrm{~nm} . \mathrm{slit}=5.0 / 5.0\right.$ $\mathrm{nm})$

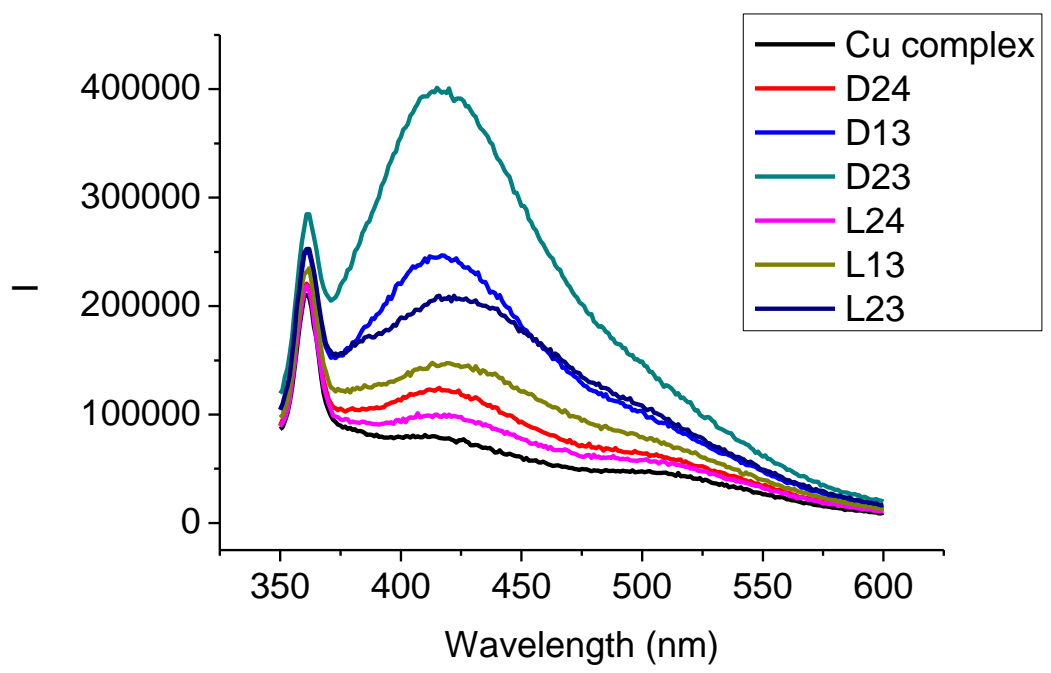

Figure AI-24. Fluorescence spectra of (S)-7-15. $\mathrm{CuCl}_{2}\left(2 \times 10^{-5} \mathrm{M}\right.$ in DMSO/ $\mathrm{H}_{2} \mathrm{O}$ 4.1/0.9) in the presence of Leu $\left(2 \times 10^{-4} \mathrm{M}, 1 \times 10^{-3} \mathrm{M}, 2 \times 10^{-3} \mathrm{M}\right) .\left(\lambda_{\mathrm{exc}}=326 \mathrm{~nm}\right.$. slit $\left.=5.0 / 5.0 \mathrm{~nm}\right)$

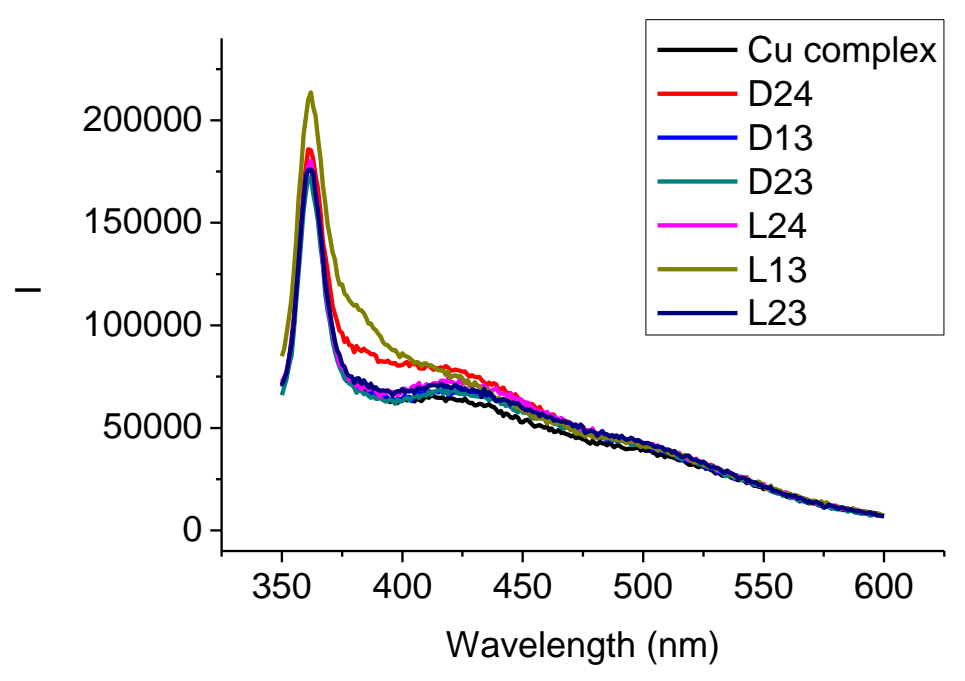


Figure AI-25. Fluorescence spectra of (S)-7-15. $\mathrm{CuCl}_{2}\left(2 \times 10^{-5} \mathrm{M}\right.$ in DMSO/ $\mathrm{H}_{2} \mathrm{O}$ 4.1/0.9) in the presence of Ser $\left(2 \times 10^{-4} \mathrm{M}, 1 \times 10^{-3} \mathrm{M}, 2 \times 10^{-3} \mathrm{M}\right) .\left(\lambda_{\mathrm{exc}}=326 \mathrm{~nm}\right.$. slit $\left.=5.0 / 5.0 \mathrm{~nm}\right)$

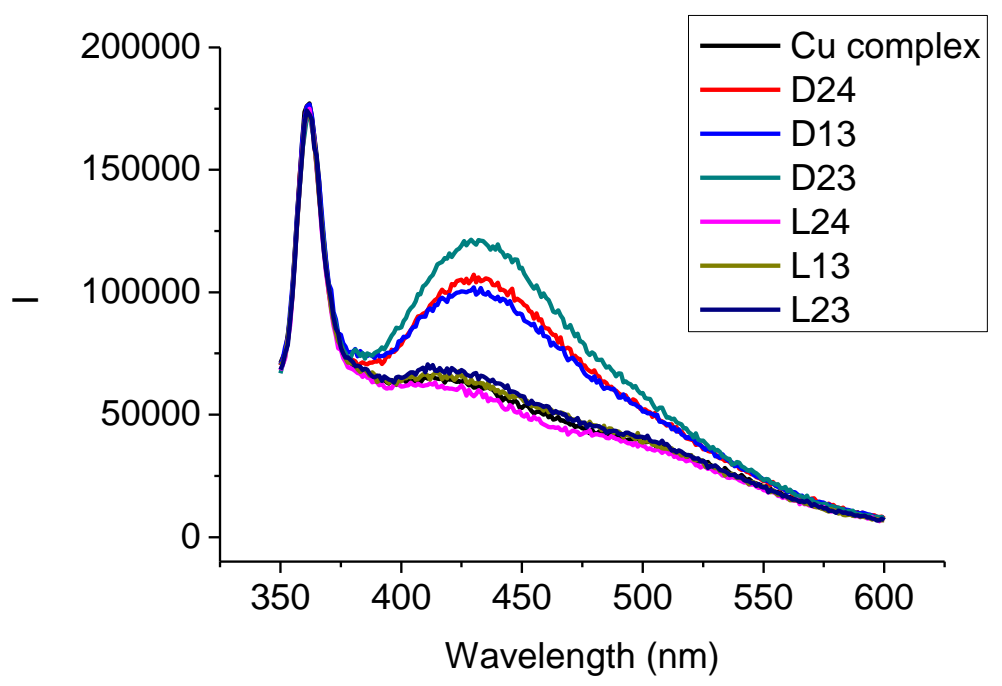

10. Interaction of $\mathrm{Cu}$ complexes of (S)-7-10, (S)-7-11, (S)-7-12, (S)-7-20 with substrates

Figure AI-26. UV-Vis absorption spectra of (S)-7-10 and its copper complex $\left(1 \times 10^{-5} \mathrm{M}\right.$ in $\left.\mathrm{THF} / \mathrm{H}_{2} \mathrm{O} 5: 1\right)$. 


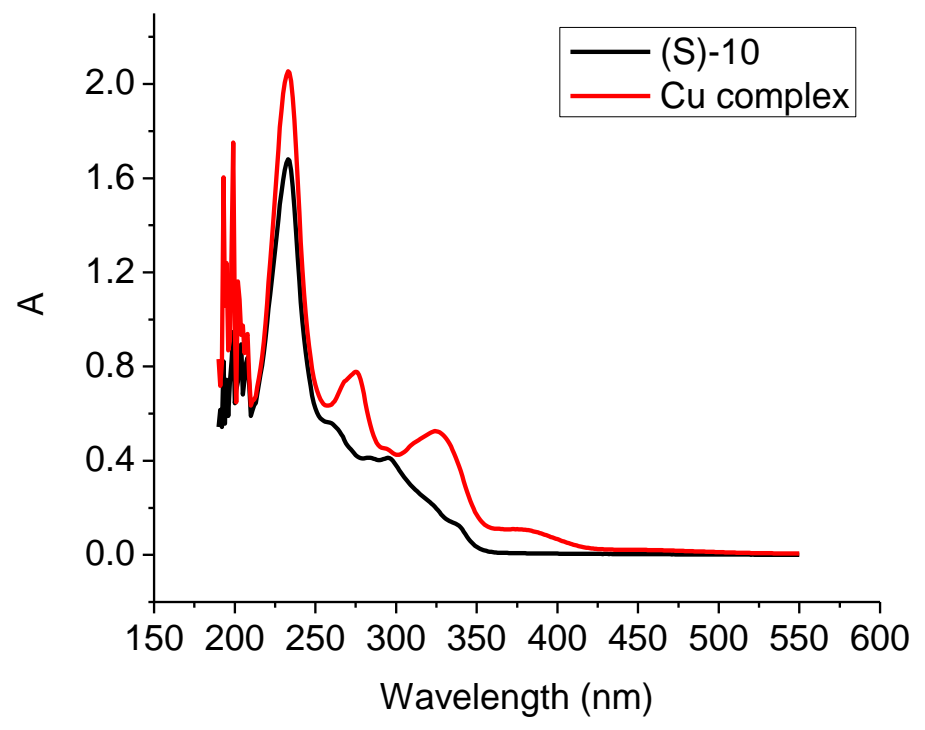

Figure AI-27. Fluorescence spectra of (S)-7-10 and its copper complex $\left(1 \times 10^{-5} \mathrm{M}\right.$ in $\left.\mathrm{THF} / \mathrm{H}_{2} \mathrm{O} 5: 1\right) .\left(\lambda_{\mathrm{exc}}=325 \mathrm{~nm}\right.$. slit $\left.=1.5 / 1.5 \mathrm{~nm}\right)$

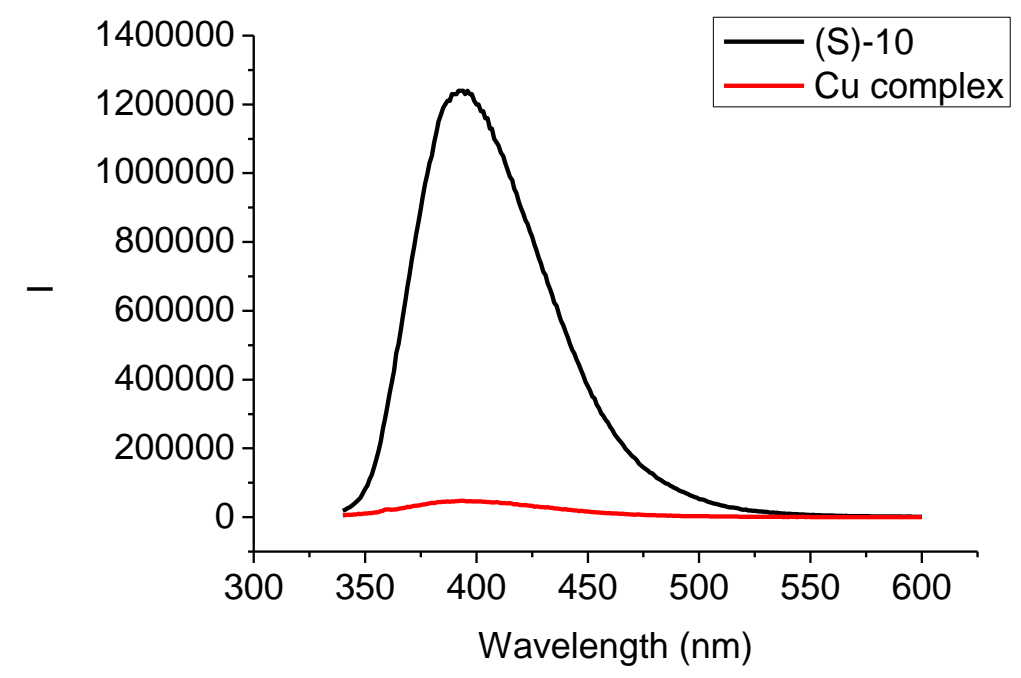

Figure AI-28. Fluorescence spectra of $(\mathrm{S})-\mathbf{7 - 1 0} \cdot \mathrm{CuCl}_{2}\left(1 \times 10^{-5} \mathrm{M}\right.$ in $\mathrm{THF} / \mathrm{H}_{2} \mathrm{O}$ 5:1) in the presence of $\operatorname{Ser}\left(1 \times 10^{-5} \mathrm{M}, 5 \times 10^{-5} \mathrm{M}, 1 \times 10^{-4} \mathrm{M}\right) .\left(\lambda_{\mathrm{exc}}=325 \mathrm{~nm} . \mathrm{slit}=1.5 / 1.5 \mathrm{~nm}\right)$ 


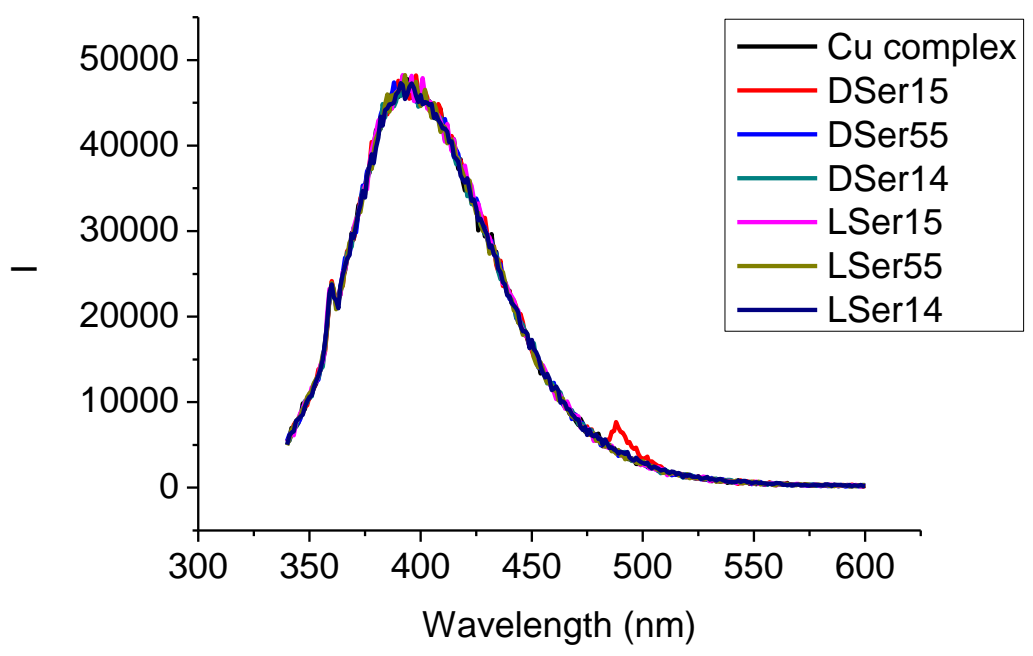

Figure AI-29. Fluorescence spectra of (S)-7-10. $\mathrm{CuCl}_{2}\left(1 \times 10^{-5} \mathrm{M}\right.$ in $\mathrm{THF} / \mathrm{H}_{2} \mathrm{O}$ 5:1) in the presence of mandelic acid $\left(1 \times 10^{-4} \mathrm{M}, 1 \times 10^{-3} \mathrm{M}, 1 \times 10^{-2} \mathrm{M}\right) .\left(\lambda_{\mathrm{exc}}=325 \mathrm{~nm} . \mathrm{slit}=1.5 / 1.5\right.$ nm)

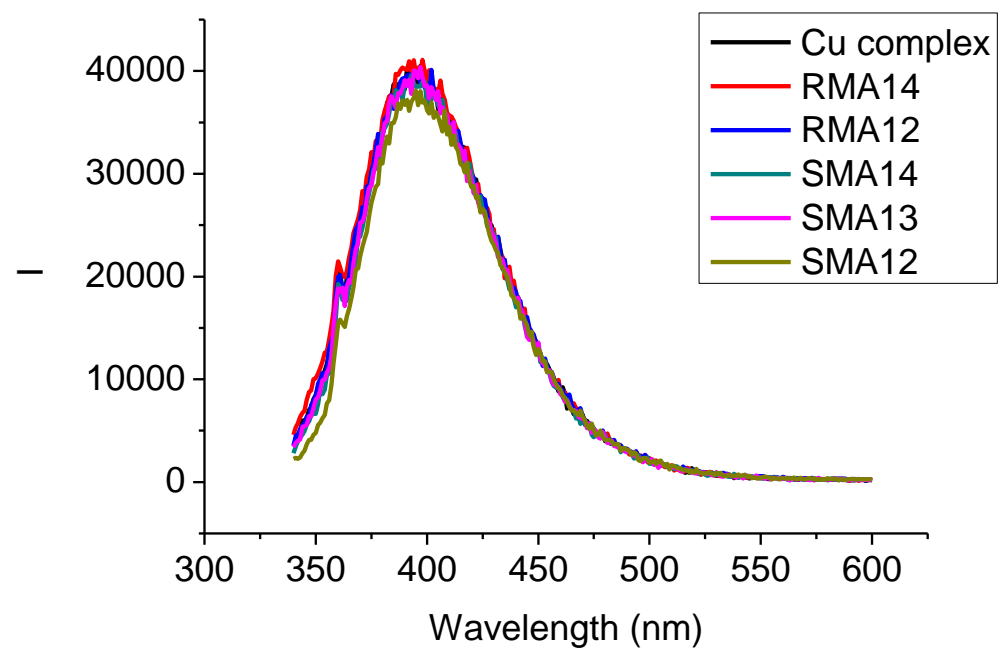

Figure AI-30. Fluorescence spectra of (S)-7-10. $\mathrm{CuCl}_{2}\left(1 \times 10^{-5} \mathrm{M}\right.$ in $\left.\mathrm{THF} / \mathrm{H}_{2} \mathrm{O} 5: 1\right)$ in the presence of phenylalaninol $\left(1 \times 10^{-4} \mathrm{M}, 1 \times 10^{-3} \mathrm{M}, 1 \times 10^{-2} \mathrm{M}\right) .\left(\lambda_{\mathrm{exc}}=325 \mathrm{~nm} . \mathrm{slit}=1.5 / 1.5\right.$ $\mathrm{nm)}$ 


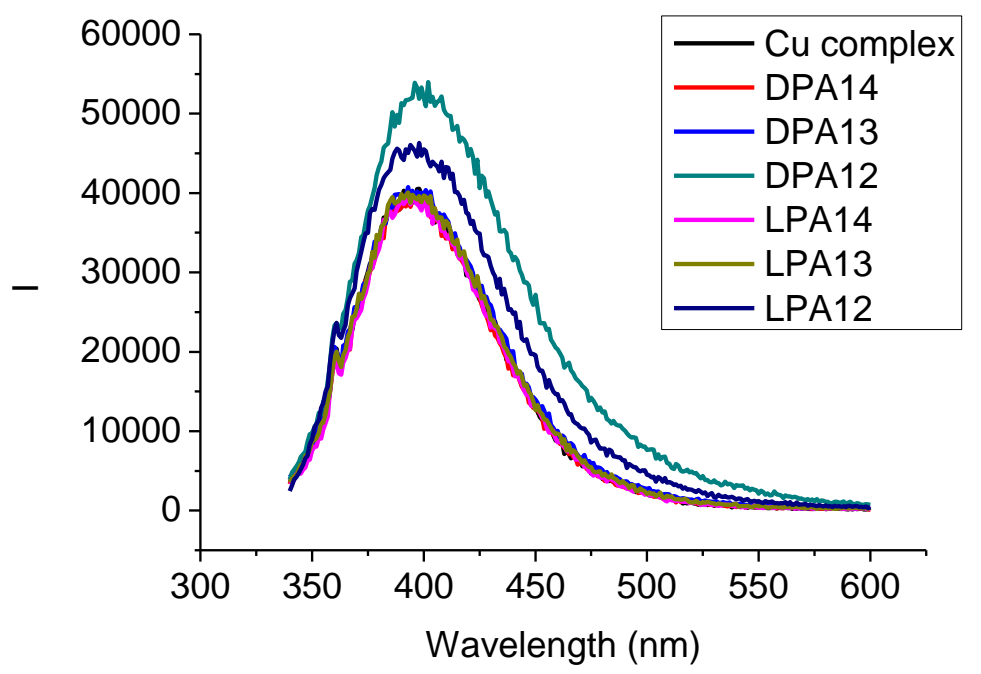

Figure AI-31. Fluorescence spectra of $(\mathrm{S})-\mathbf{7 - 1 0} \cdot \mathrm{CuCl}_{2}\left(1 \times 10^{-5} \mathrm{M}\right.$ in $\left.\mathrm{THF} / \mathrm{H}_{2} \mathrm{O} 5: 1\right)$ in the presence of Alanine $\left(1 \times 10^{-4} \mathrm{M}, 1 \times 10^{-3} \mathrm{M}, 1 \times 10^{-2} \mathrm{M}\right) .\left(\lambda_{\mathrm{exc}}=325 \mathrm{~nm} . \mathrm{slit}=1.5 / 1.5 \mathrm{~nm}\right)$

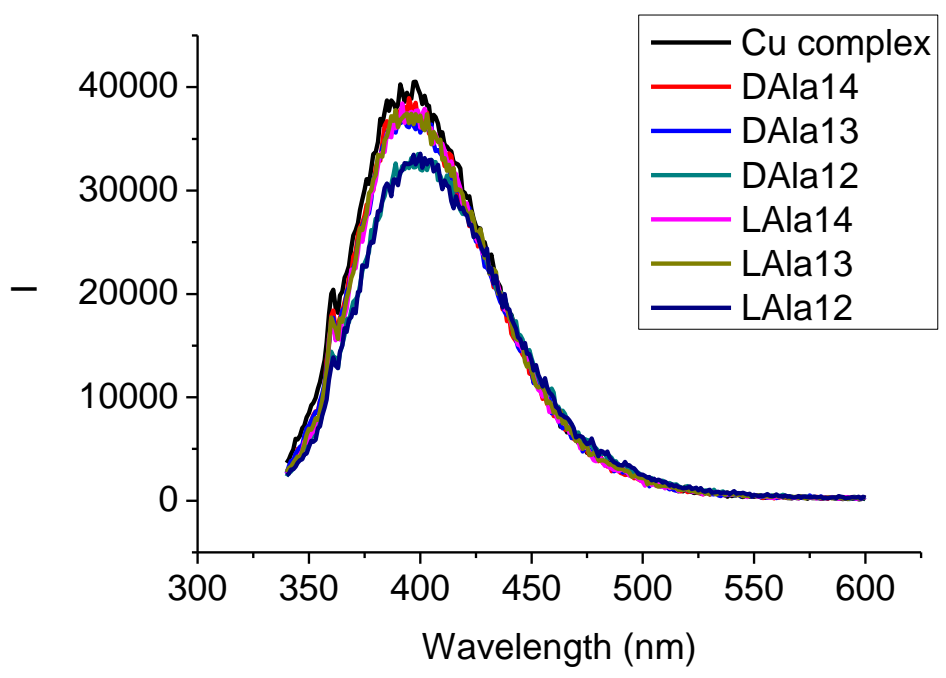

Figure AI-32. Fluorescence spectra of $(\mathrm{S})-\mathbf{7 - 1 0} \cdot \mathrm{CuCl}_{2}\left(1 \times 10^{-5} \mathrm{M}\right.$ in $\mathrm{THF}: \mathrm{CH} 3 \mathrm{OH}$ : water $=1: 10.8: 8.5)$ in the presence of mandelic acid $\left(1 \times 10^{-4} \mathrm{M}, 1 \times 10^{-3} \mathrm{M}, 1 \times 10^{-2} \mathrm{M}\right)$. $\left(\lambda_{\text {exc }}=325 \mathrm{~nm} . \mathrm{slit}=1.5 / 1.5 \mathrm{~nm}\right)$ 


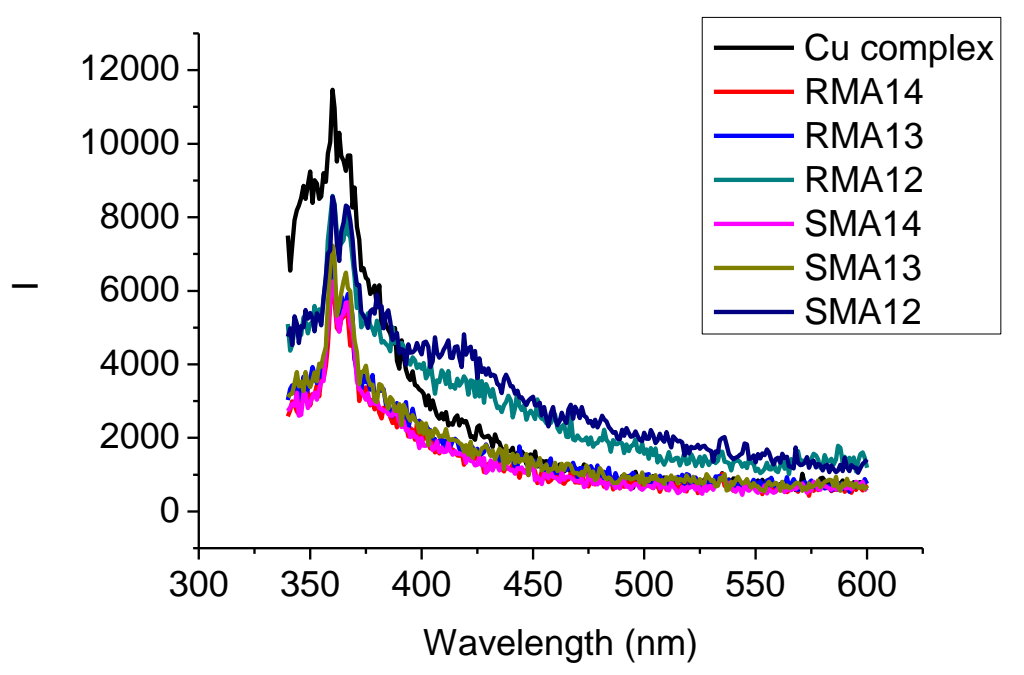

Figure AI-33. Fluorescence spectra of $(\mathrm{S})-\mathbf{7 - 1 0} \cdot \mathrm{CuCl}_{2}\left(1 \times 10^{-5} \mathrm{M}\right.$ in $\mathrm{THF}: \mathrm{CH} 3 \mathrm{OH}$ : water $=1: 10.8: 8.5)$ in the presence of phenylalaninol $\left(1 \times 10^{-4} \mathrm{M}, 1 \times 10^{-3} \mathrm{M}, 1 \times 10^{-2} \mathrm{M}\right)$. $\left(\lambda_{\text {exc }}=325 \mathrm{~nm}\right.$. slit $\left.=1.5 / 1.5 \mathrm{~nm}\right)$

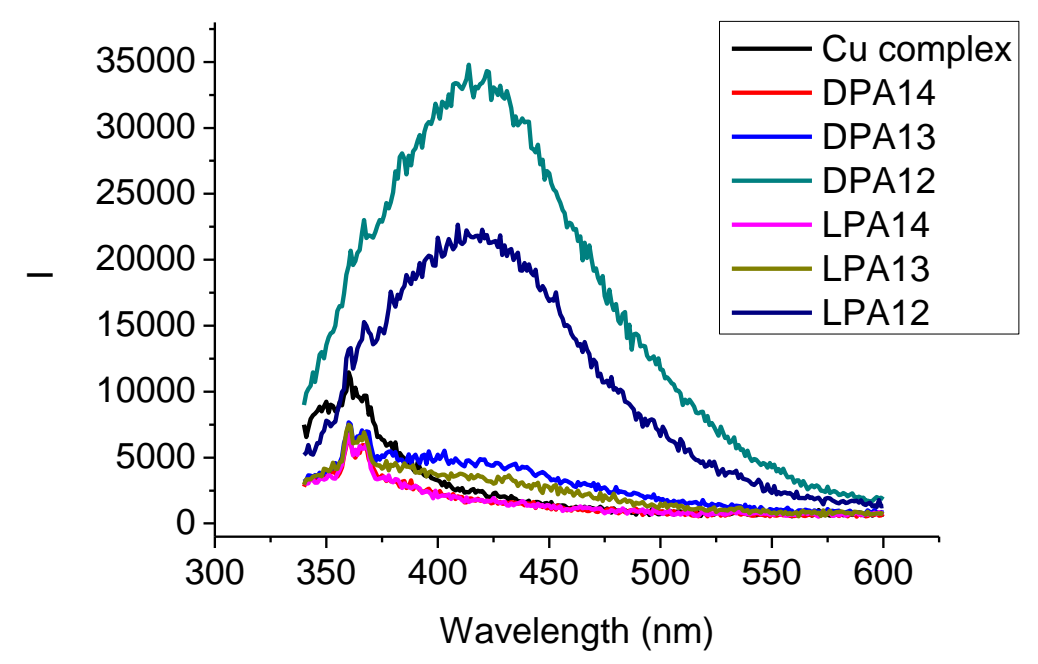

Figure AI-34. Fluorescence spectra of (S)-7-10 $\cdot \mathrm{CuCl}_{2}\left(1 \times 10^{-5} \mathrm{M}\right.$ in THF : $\mathrm{CH} 3 \mathrm{OH}$ : water $=1: 10.8: 8.5)$ in the presence of Alanine $\left(1 \times 10^{-4} \mathrm{M}, 1 \times 10^{-3} \mathrm{M}, 1 \times 10^{-2} \mathrm{M}\right) .\left(\lambda_{\mathrm{exc}}=\right.$ $325 \mathrm{~nm}$. slit $=1.5 / 1.5 \mathrm{~nm})$ 


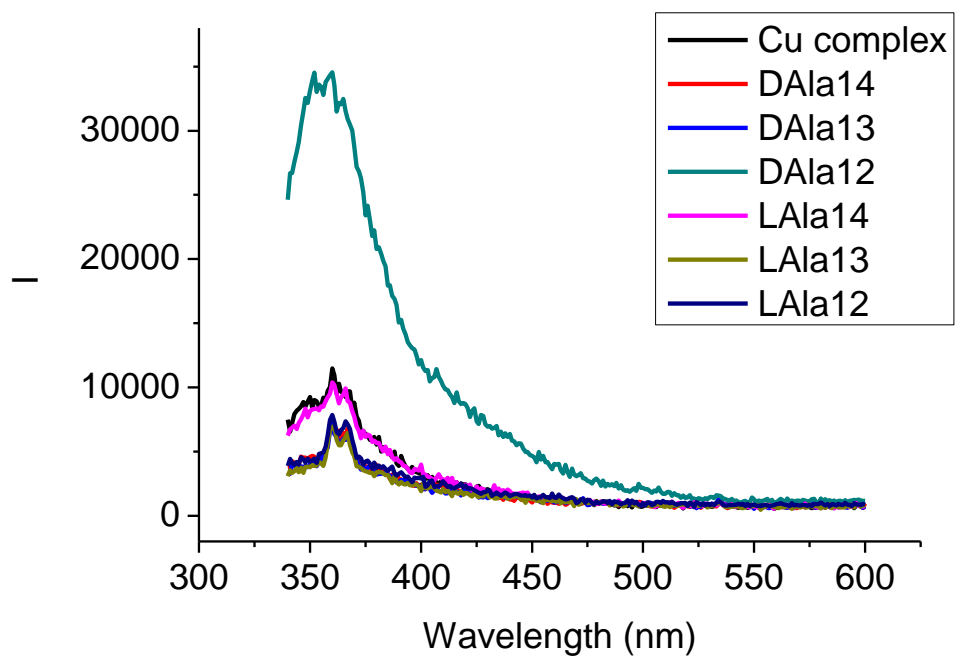

Figure AI-35. UV-Vis absorption spectra of (S)-7-11 and its copper complex $\left(1 \times 10^{-5} \mathrm{M}\right.$ in $\mathrm{THF} / \mathrm{H}_{2} \mathrm{O}$ 5:1).

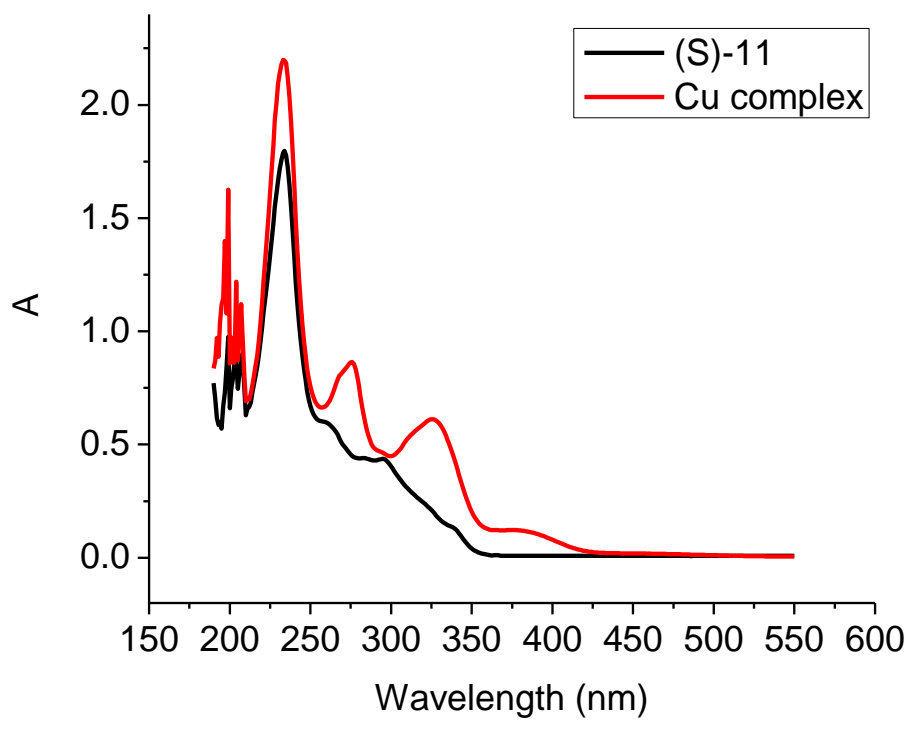

Figure AI-36. Fluorescence spectra of (S)-7-11 and its copper complex $\left(1 \times 10^{-5} \mathrm{M}\right.$ in $\left.\mathrm{THF} / \mathrm{H}_{2} \mathrm{O} 5: 1\right) .\left(\lambda_{\mathrm{exc}}=325 \mathrm{~nm} . \mathrm{slit}=1.5 / 1.5 \mathrm{~nm}\right)$ 


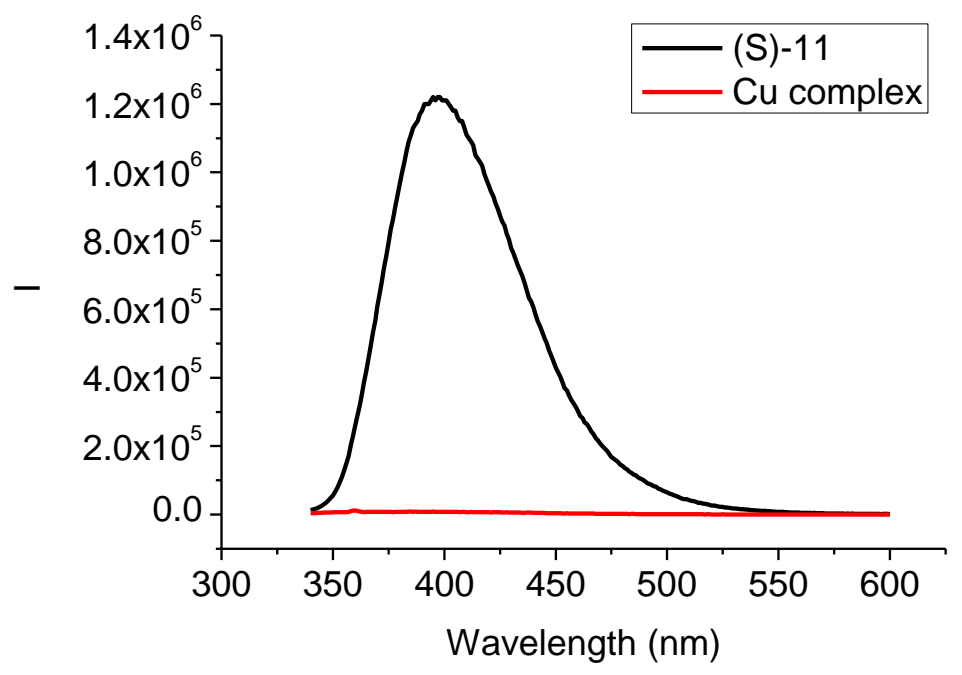

Figure AI-37. Fluorescence spectra of (S)-7-11 $\cdot \mathrm{CuCl}_{2}\left(1 \times 10^{-5} \mathrm{M}\right.$ in $\mathrm{THF} / \mathrm{H}_{2} \mathrm{O}$ 5:1) in the presence of $\operatorname{Ser}\left(1 \times 10^{-5} \mathrm{M}, 5 \times 10^{-5} \mathrm{M}, 1 \times 10^{-4} \mathrm{M}\right) .\left(\lambda_{\text {exc }}=325 \mathrm{~nm} . \mathrm{slit}=1.5 / 1.5 \mathrm{~nm}\right)$

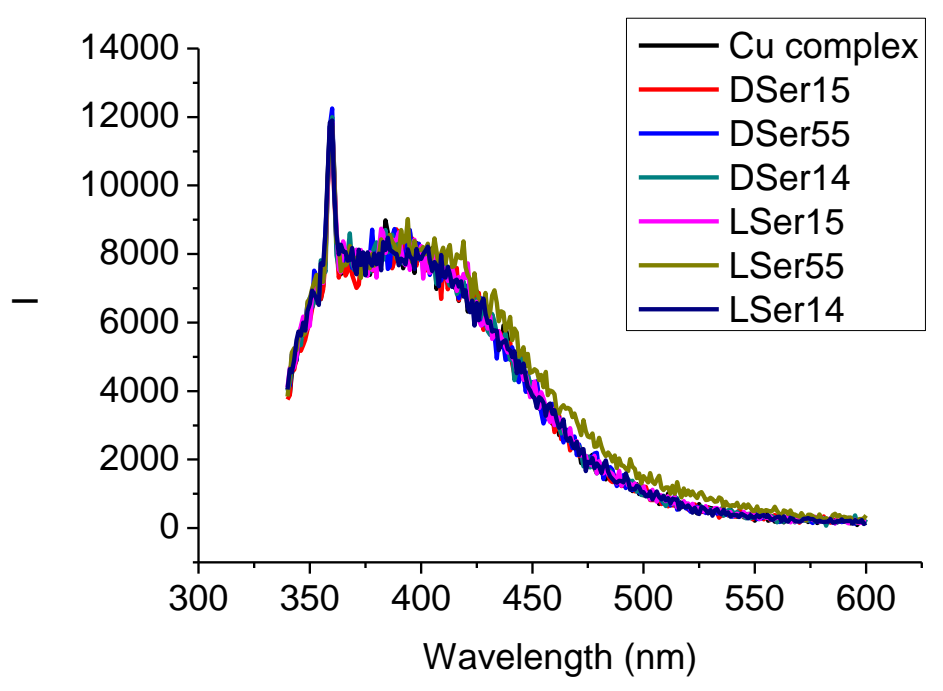

Figure AI-38. Fluorescence spectra of (S)-7-11. $\mathrm{CuCl}_{2}\left(1 \times 10^{-5} \mathrm{M}\right.$ in $\mathrm{THF} / \mathrm{H}_{2} \mathrm{O}$ 5:1) in the presence of mandelic acid $\left(1 \times 10^{-4} \mathrm{M}, 1 \times 10^{-3} \mathrm{M}, 1 \times 10^{-2} \mathrm{M}\right) .\left(\lambda_{\mathrm{exc}}=325 \mathrm{~nm} . \mathrm{slit}=1.5 / 1.5\right.$ $\mathrm{nm}$ ) 


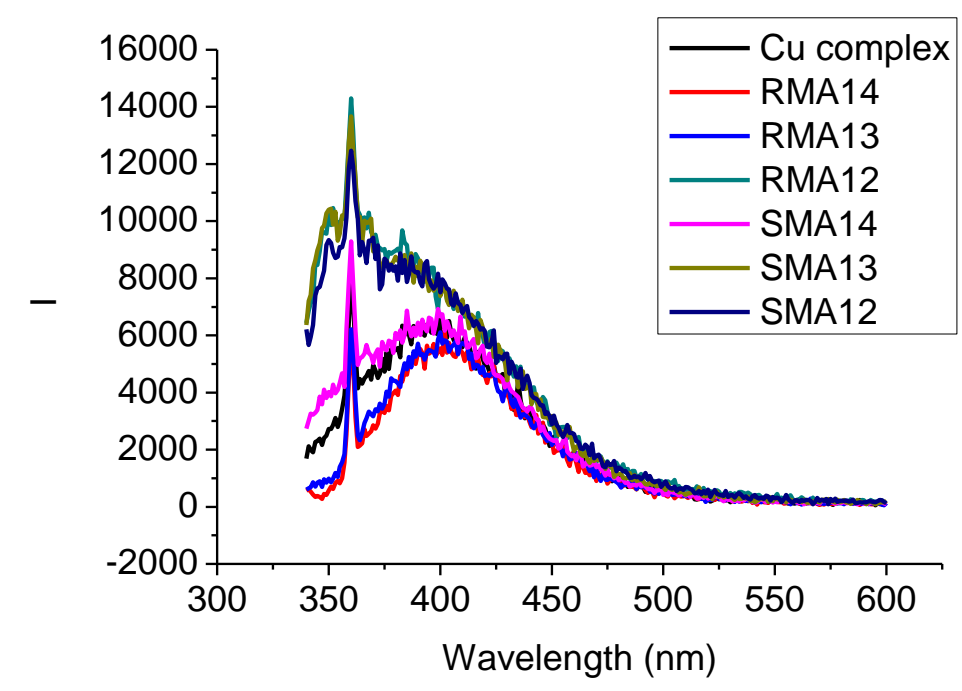

Figure AI-39. Fluorescence spectra of $(\mathrm{S})-\mathbf{7 - 1 1} \cdot \mathrm{CuCl}_{2}\left(1 \times 10^{-5} \mathrm{M}\right.$ in $\mathrm{THF} / \mathrm{H}_{2} \mathrm{O}$ 5:1) in the presence of phenylalaninol $\left(1 \times 10^{-4} \mathrm{M}, 1 \times 10^{-3} \mathrm{M}, 1 \times 10^{-2} \mathrm{M}\right) .\left(\lambda_{\mathrm{exc}}=325 \mathrm{~nm}\right.$. slit $=1.5 / 1.5$ $\mathrm{nm})$

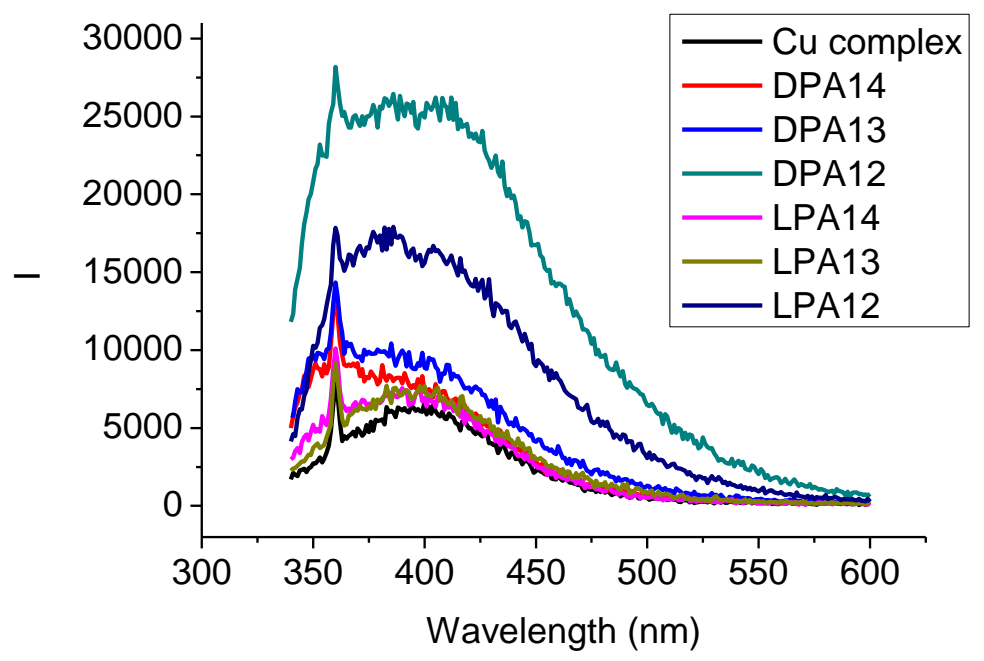

Figure AI-40. Fluorescence spectra of (S)-7-11. $\mathrm{CuCl}_{2}\left(1 \times 10^{-5} \mathrm{M}\right.$ in $\mathrm{THF} / \mathrm{H}_{2} \mathrm{O}$ 5:1) in the presence of Alanine $\left(1 \times 10^{-4} \mathrm{M}, 1 \times 10^{-3} \mathrm{M}, 1 \times 10^{-2} \mathrm{M}\right) .\left(\lambda_{\mathrm{exc}}=325 \mathrm{~nm} . \mathrm{slit}=1.5 / 1.5 \mathrm{~nm}\right)$ 


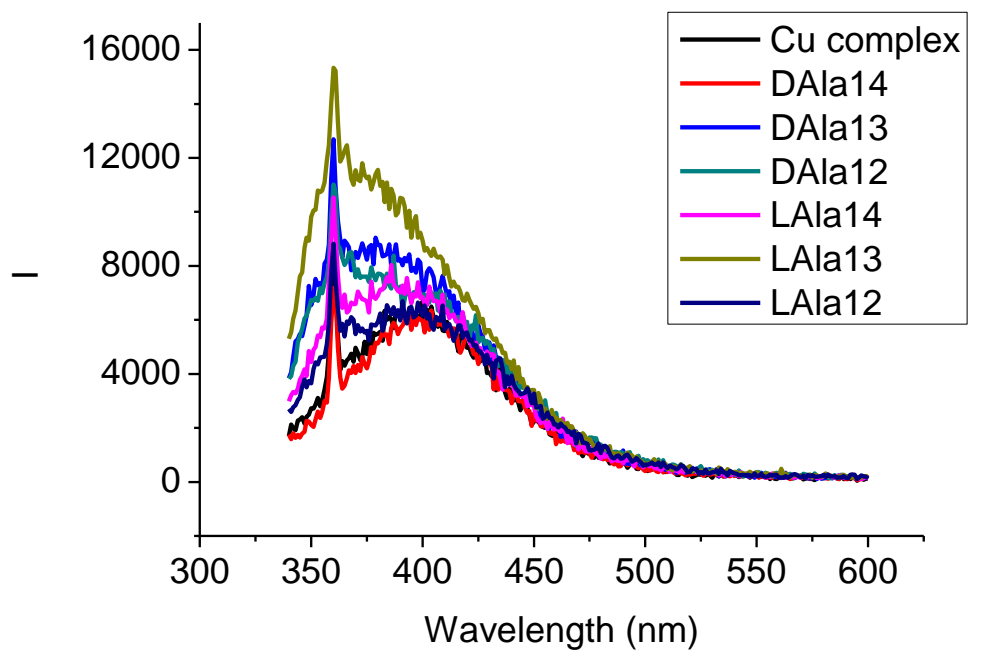

Figure AI-41. Fluorescence spectra of (S)-7-11. $\mathrm{CuCl}_{2}\left(1 \times 10^{-5} \mathrm{M}\right.$ in THF : $\mathrm{CH} 3 \mathrm{OH}$ : water $=1: 16.6: 12.6)$ in the presence of mandelic acid $\left(1 \times 10^{-4} \mathrm{M}, 1 \times 10^{-3} \mathrm{M}, 1 \times 10^{-2} \mathrm{M}\right)$. $\left(\lambda_{\text {exc }}=325 \mathrm{~nm}\right.$. slit $\left.=1.5 / 1.5 \mathrm{~nm}\right)$

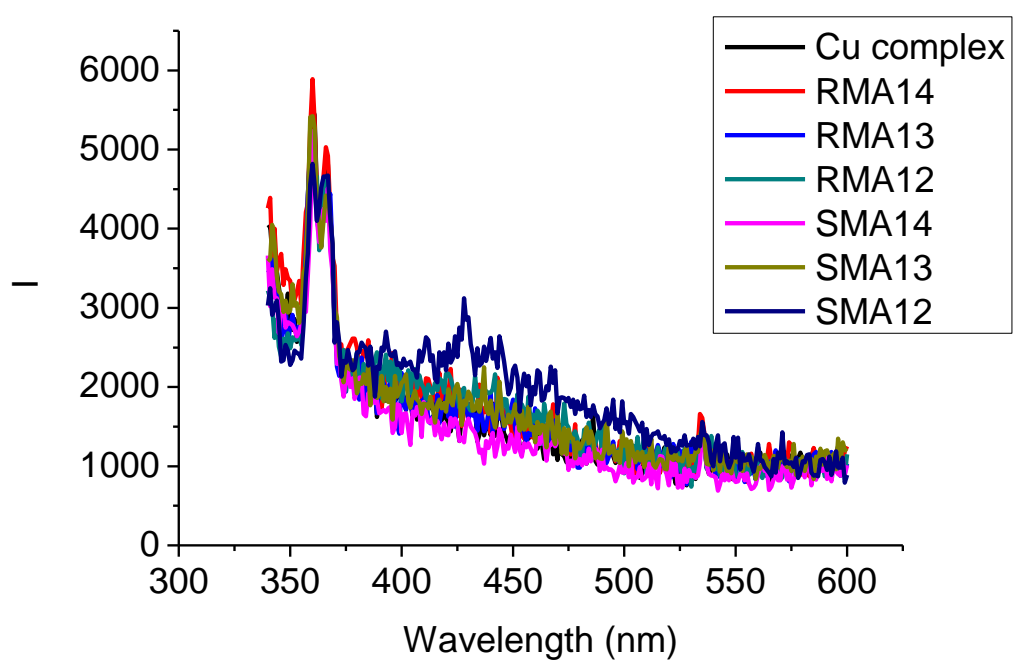

Figure AI-42. Fluorescence spectra of $(\mathrm{S})-7-11 \cdot \mathrm{CuCl}_{2}\left(1 \times 10^{-5} \mathrm{M}\right.$ in THF : $\mathrm{CH} 3 \mathrm{OH}$ : water $=1: 16.6: 12.6)$ in the presence of phenylalaninol $\left(1 \times 10^{-4} \mathrm{M}, 1 \times 10^{-3} \mathrm{M}, 1 \times 10^{-2} \mathrm{M}\right)$. $\left(\lambda_{\text {exc }}=325 \mathrm{~nm}\right.$. slit $\left.=1.5 / 1.5 \mathrm{~nm}\right)$ 


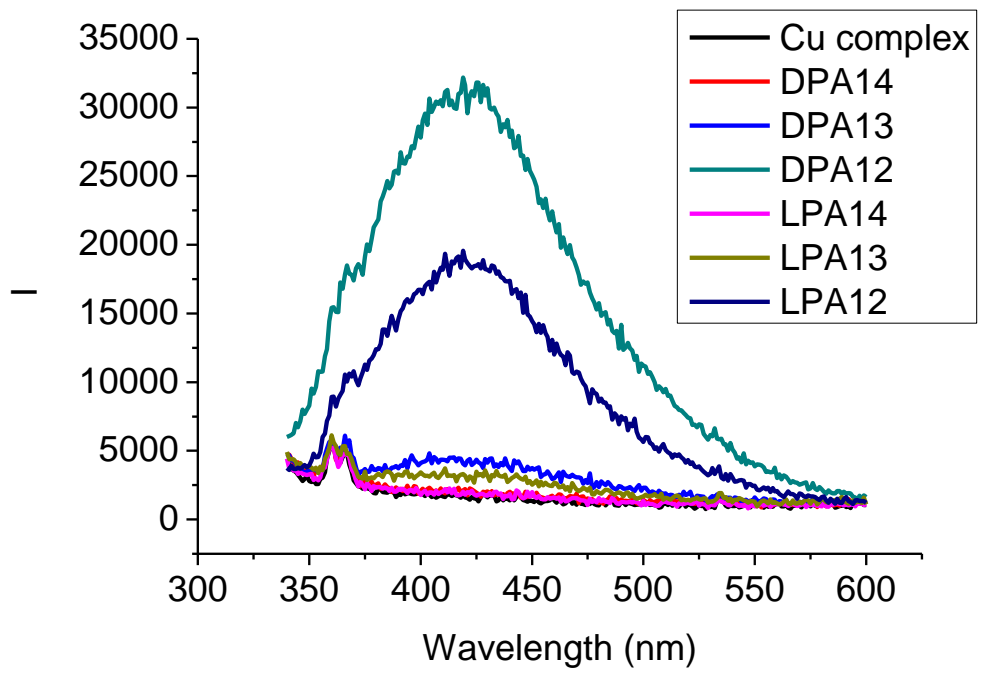

Figure AI-43. Fluorescence spectra of $(\mathrm{S})-\mathbf{7 - 1 1} \cdot \mathrm{CuCl}_{2}\left(1 \times 10^{-5} \mathrm{M}\right.$ in THF : $\mathrm{CH}_{3} \mathrm{OH}$ : water $=1: 16.6: 12.6)$ in the presence of Alanine $\left(1 \times 10^{-4} \mathrm{M}, 1 \times 10^{-3} \mathrm{M}, 1 \times 10^{-2} \mathrm{M}\right) .\left(\lambda_{\text {exc }}\right.$ $=325 \mathrm{~nm} . \mathrm{slit}=1.5 / 1.5 \mathrm{~nm})$

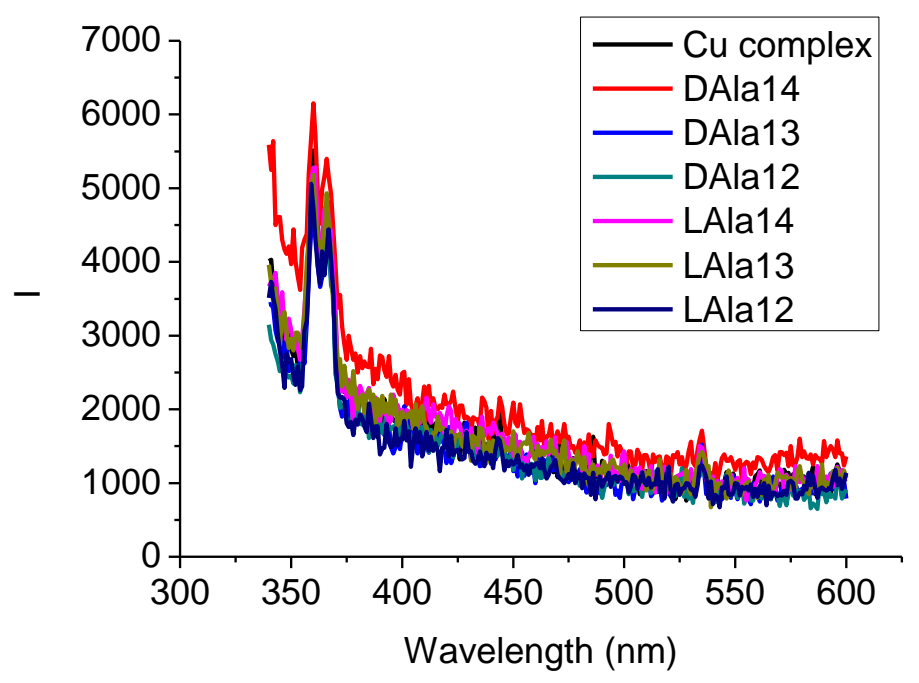

Figure AI-44. UV-Vis absorption spectra of (S)-7-12 and its copper complex $\left(1 \times 10^{-5} \mathrm{M}\right.$ in $\mathrm{THF} / \mathrm{H}_{2} \mathrm{O} 5: 1$ ). 


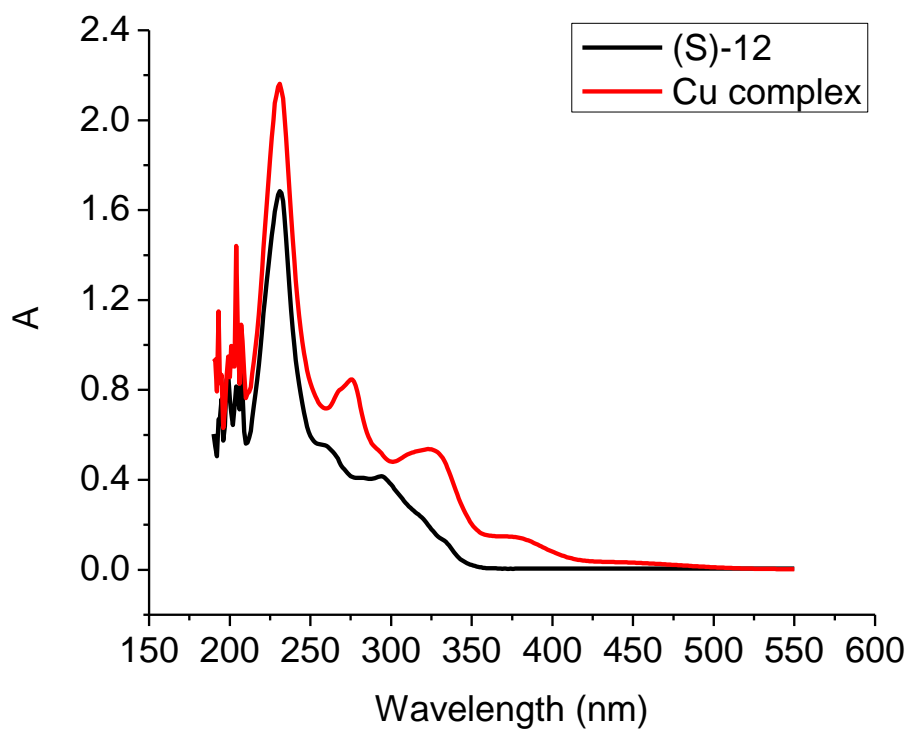

Figure I-45. Fluorescence spectra of (S)-7-12 and its copper complex $\left(1 \times 10^{-5} \mathrm{M}\right.$ in $\left.\mathrm{THF} / \mathrm{H}_{2} \mathrm{O} 5: 1\right) .\left(\lambda_{\mathrm{exc}}=325 \mathrm{~nm}\right.$. slit $\left.=1.5 / 1.5 \mathrm{~nm}\right)$

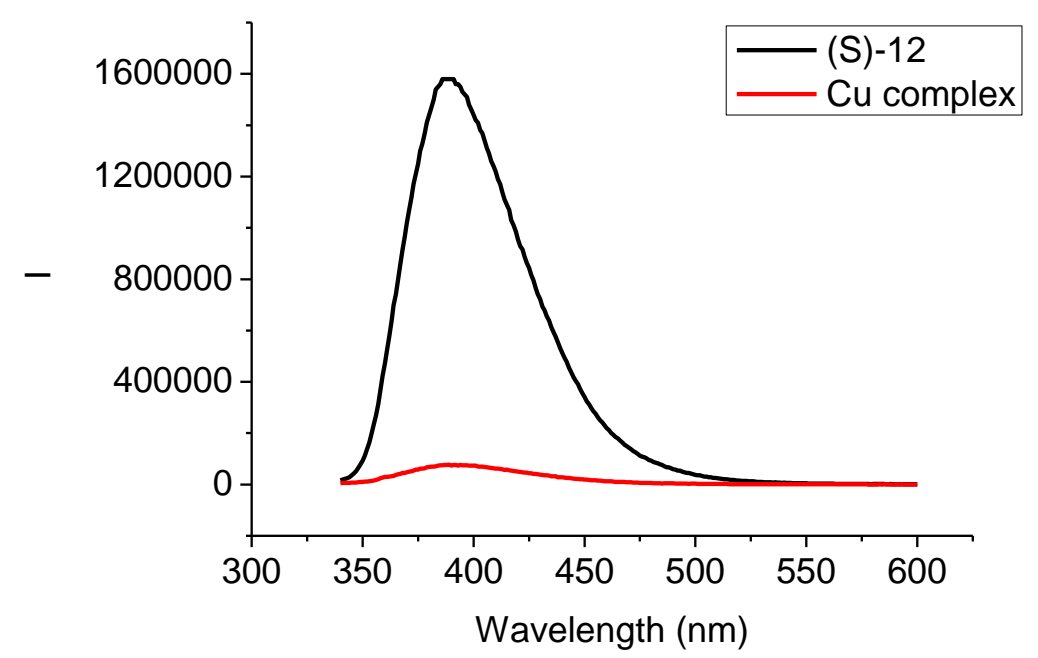

Figure AI-46. Fluorescence spectra of (S)-7-12. $\mathrm{CuCl}_{2}\left(1 \times 10^{-5} \mathrm{M}\right.$ in $\left.\mathrm{THF} / \mathrm{H}_{2} \mathrm{O} 5: 1\right)$ in the presence of $\operatorname{Ser}\left(1 \times 10^{-5} \mathrm{M}, 5 \times 10^{-5} \mathrm{M}, 1 \times 10^{-4} \mathrm{M}\right) .\left(\lambda_{\mathrm{exc}}=325 \mathrm{~nm}\right.$. slit $\left.=1.5 / 1.5 \mathrm{~nm}\right)$ 


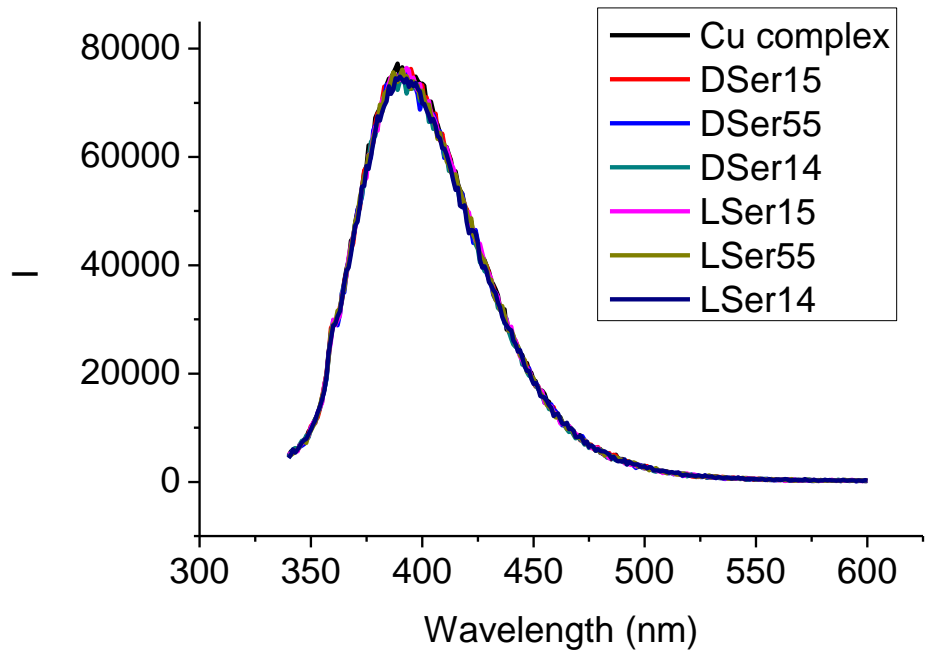

Figure AI-47. Fluorescence spectra of (S)-7-12. $\mathrm{CuCl}_{2}\left(1 \times 10^{-5} \mathrm{M}\right.$ in $\mathrm{THF} / \mathrm{H}_{2} \mathrm{O}$ 5:1) in the presence of mandelic acid $\left(1 \times 10^{-4} \mathrm{M}, 1 \times 10^{-3} \mathrm{M}, 1 \times 10^{-2} \mathrm{M}\right) .\left(\lambda_{\mathrm{exc}}=325 \mathrm{~nm} . \mathrm{slit}=1.5 / 1.5\right.$ nm)

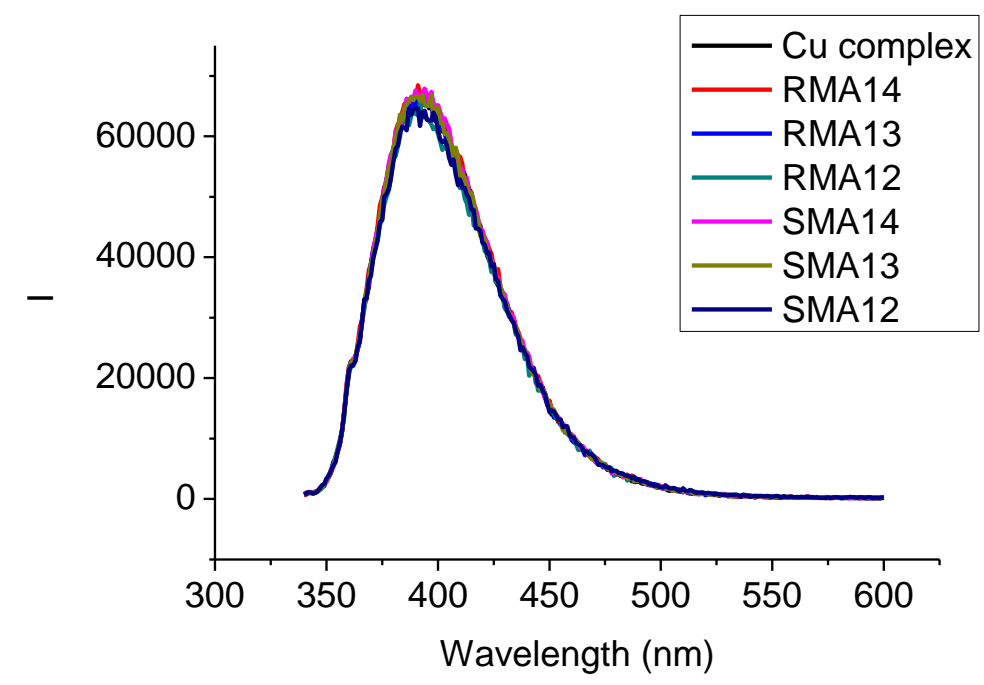

Figure AI-48. Fluorescence spectra of (S)-7-12. $\mathrm{CuCl}_{2}\left(1 \times 10^{-5} \mathrm{M}\right.$ in $\mathrm{THF} / \mathrm{H}_{2} \mathrm{O}$ 5:1) in the presence of phenylalaninol $\left(1 \times 10^{-4} \mathrm{M}, 1 \times 10^{-3} \mathrm{M}, 1 \times 10^{-2} \mathrm{M}\right) .\left(\lambda_{\text {exc }}=325 \mathrm{~nm}\right.$. slit $=1.5 / 1.5$ 
nm)

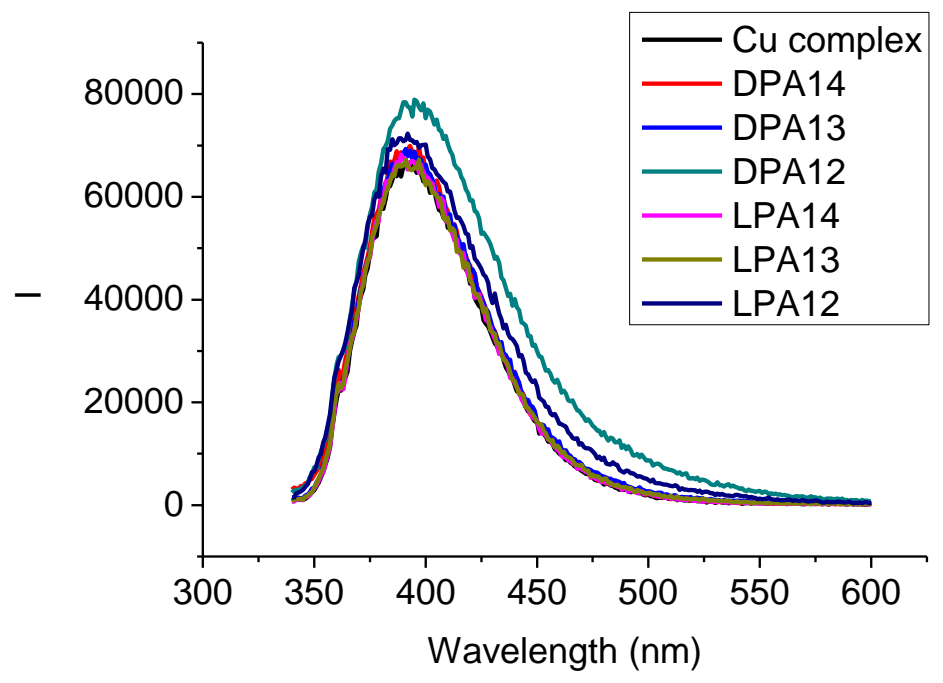

Figure AI-49. Fluorescence spectra of (S)-7-12. $\mathrm{CuCl}_{2}\left(1 \times 10^{-5} \mathrm{M}\right.$ in $\mathrm{THF} / \mathrm{H}_{2} \mathrm{O}$ 5:1) in the presence of Alanine $\left(1 \times 10^{-4} \mathrm{M}, 1 \times 10^{-3} \mathrm{M}, 1 \times 10^{-2} \mathrm{M}\right) .\left(\lambda_{\mathrm{exc}}=325 \mathrm{~nm} . \mathrm{slit}=1.5 / 1.5 \mathrm{~nm}\right)$

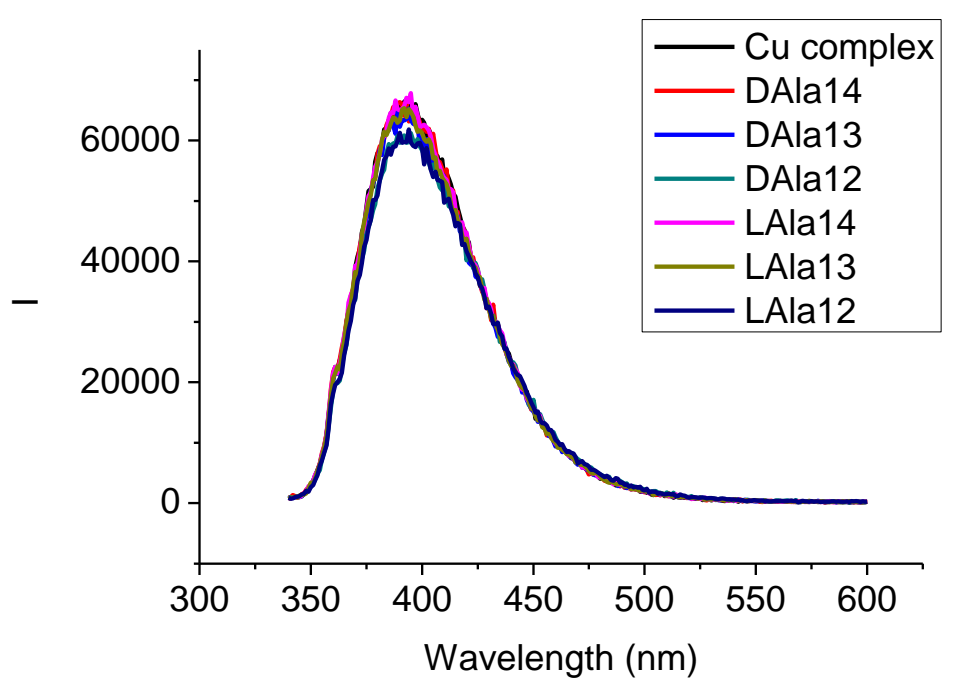

Figure AI-50. Fluorescence spectra of $(\mathrm{S})-\mathbf{7 - 1 2} \cdot \mathrm{CuCl}_{2}\left(1 \times 10^{-5} \mathrm{M}\right.$ in $\mathrm{CH}_{3} \mathrm{OH}$ : water = $1.2: 1)$ in the presence of mandelic acid $\left(1 \times 10^{-4} \mathrm{M}, 1 \times 10^{-3} \mathrm{M}, 1 \times 10^{-2} \mathrm{M}\right) .\left(\lambda_{\text {exc }}=325 \mathrm{~nm}\right.$. 
slit $=1.5 / 1.5 \mathrm{~nm})$

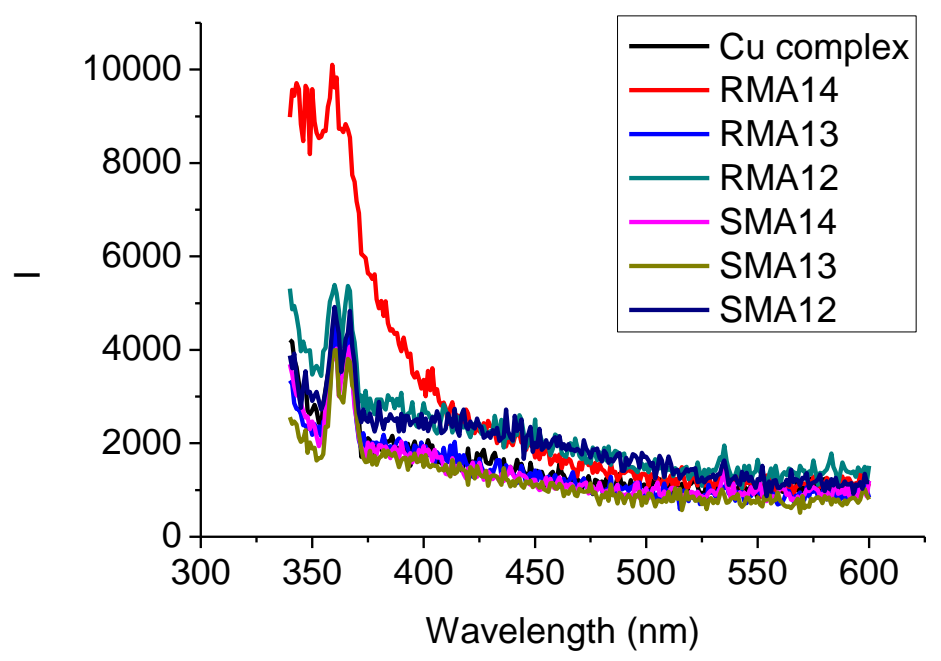

Figure AI-51. Fluorescence spectra of (S)-7-12. $\mathrm{CuCl}_{2}\left(1 \times 10^{-5} \mathrm{M}\right.$ in $\mathrm{CH}_{3} \mathrm{OH}$ : water = $1.2: 1)$ in the presence of phenylalaninol $\left(1 \times 10^{-4} \mathrm{M}, 1 \times 10^{-3} \mathrm{M}, 1 \times 10^{-2} \mathrm{M}\right) .\left(\lambda_{\mathrm{exc}}=325 \mathrm{~nm}\right.$. slit $=1.5 / 1.5 \mathrm{~nm})$

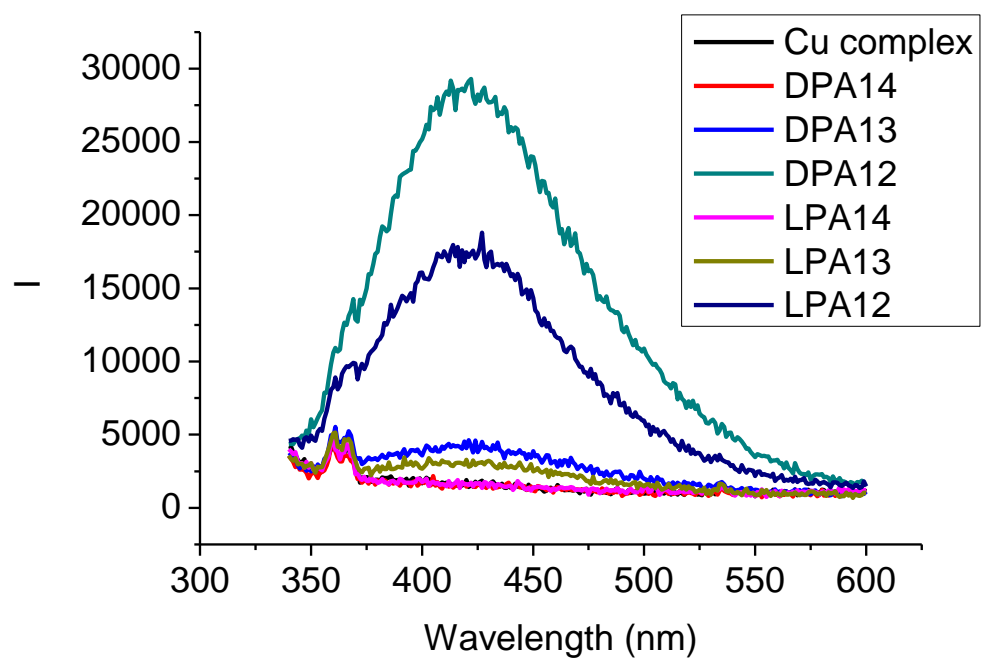

Figure AI-52. Fluorescence spectra of $(\mathrm{S})-\mathbf{7 - 1 2} \cdot \mathrm{CuCl}_{2}\left(1 \times 10^{-5} \mathrm{M}\right.$ in $\mathrm{CH}_{3} \mathrm{OH}$ : water = $1.2: 1)$ in the presence of alanine $\left(1 \times 10^{-4} \mathrm{M}, 1 \times 10^{-3} \mathrm{M}, 1 \times 10^{-2} \mathrm{M}\right) .\left(\lambda_{\mathrm{exc}}=325 \mathrm{~nm}\right.$. slit 
$=1.5 / 1.5 \mathrm{~nm})$

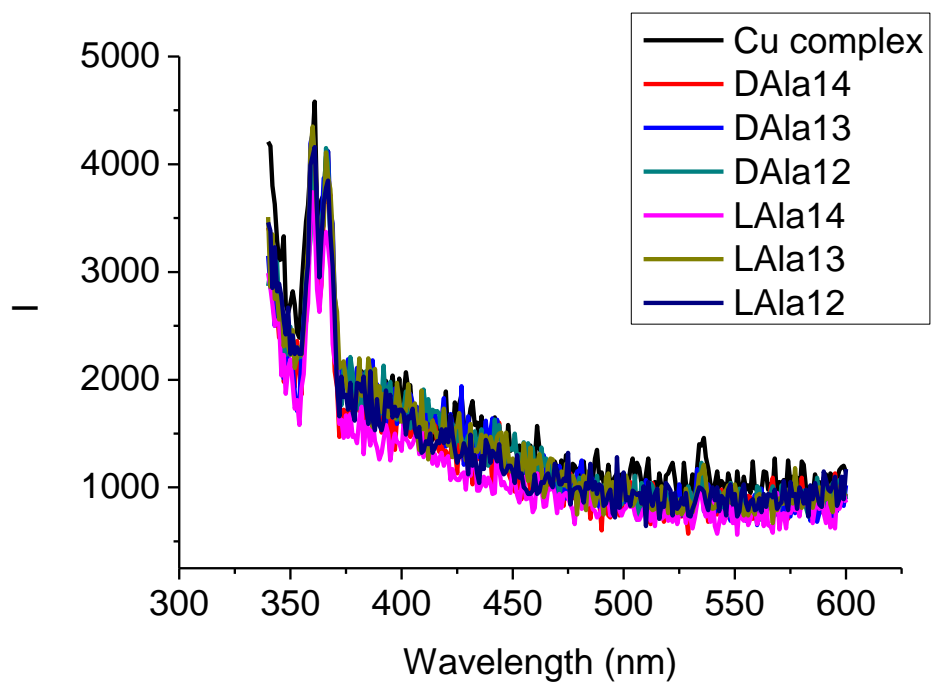

Figure AI-53. UV-Vis absorption spectra of (S)-7-20 and its copper complex $\left(1 \times 10^{-5} \mathrm{M}\right.$ in $\left.\mathrm{THF} / \mathrm{H}_{2} \mathrm{O} 5: 1\right)$.

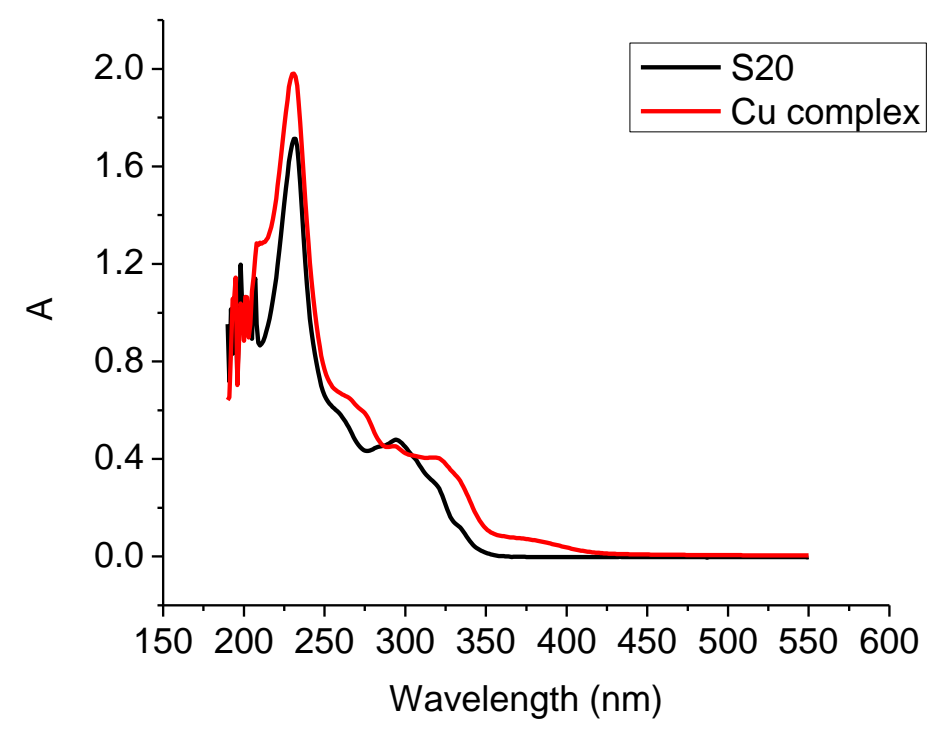

Figure AI-54. Fluorescence spectra of (S)-7-20 and its copper complex $\left(1 \times 10^{-5} \mathrm{M}\right.$ in THF). $\left(\lambda_{\text {exc }}=290 \mathrm{~nm}\right.$. slit $\left.=1.5 / 1.5 \mathrm{~nm}\right)$ 


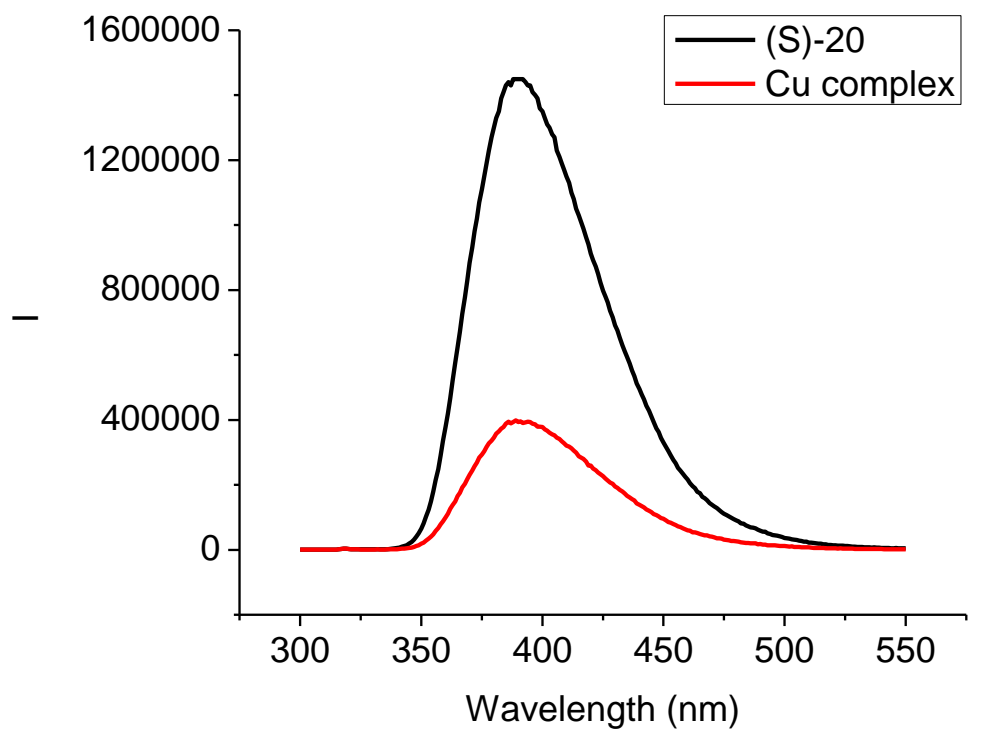

Figure AI-55. Fluorescence spectra of $(\mathrm{S})-\mathbf{7 - 2 0} \cdot \mathrm{CuCl}_{2}\left(1 \times 10^{-5} \mathrm{M}\right.$ in $\left.\mathrm{THF} / \mathrm{H}_{2} \mathrm{O} 5: 1\right)$ in the absence and presence of D- or L-Ala, Ser and $\operatorname{Arg}\left(1 \times 10^{-5} \mathrm{M}, 5 \times 10^{-5} \mathrm{M}, 1 \times 10^{-4} \mathrm{M}\right) .\left(\lambda_{\text {exc }}\right.$ $=290 \mathrm{~nm}$. slit $=1.5 / 1.5 \mathrm{~nm})$

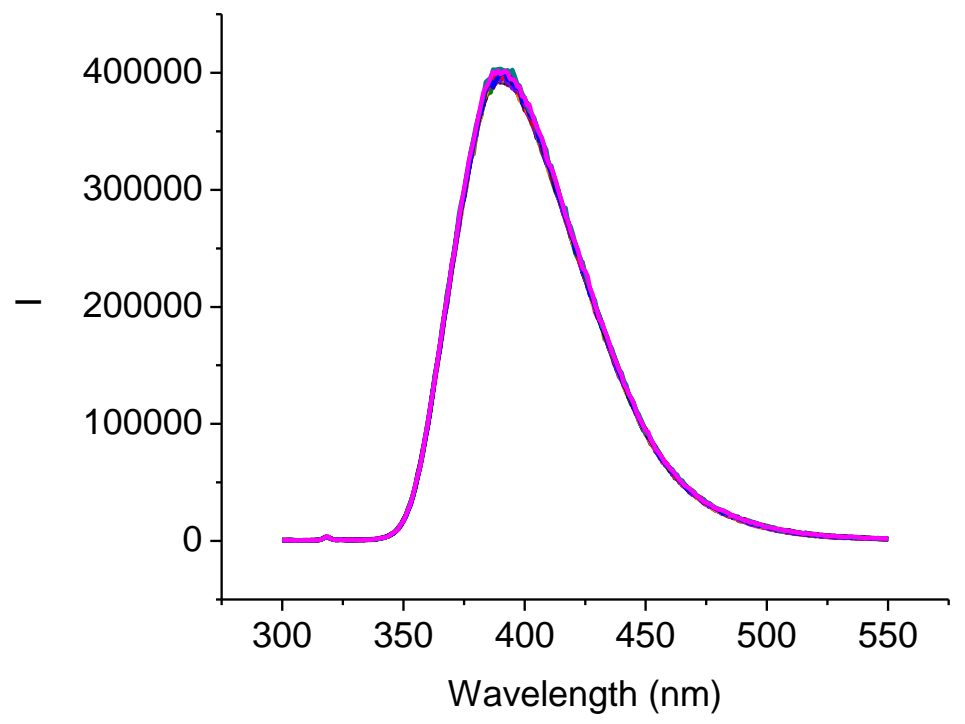

Figure AI-56. Fluorescence spectra of (S)-7-20 $\cdot \mathrm{CuCl}_{2}\left(1 \times 10^{-5} \mathrm{M}\right.$ in DCM/hexane 2:3) in 
the absence and presence of $(\mathrm{R})$ - or $(\mathrm{S})$-leucinol and 2-amino-3-phenylpropanol $\left(4.5 \times 10^{-3}\right.$ M). $\left(\lambda_{\mathrm{exc}}=290 \mathrm{~nm} . \mathrm{slit}=1.5 / 1.5 \mathrm{~nm}\right)$

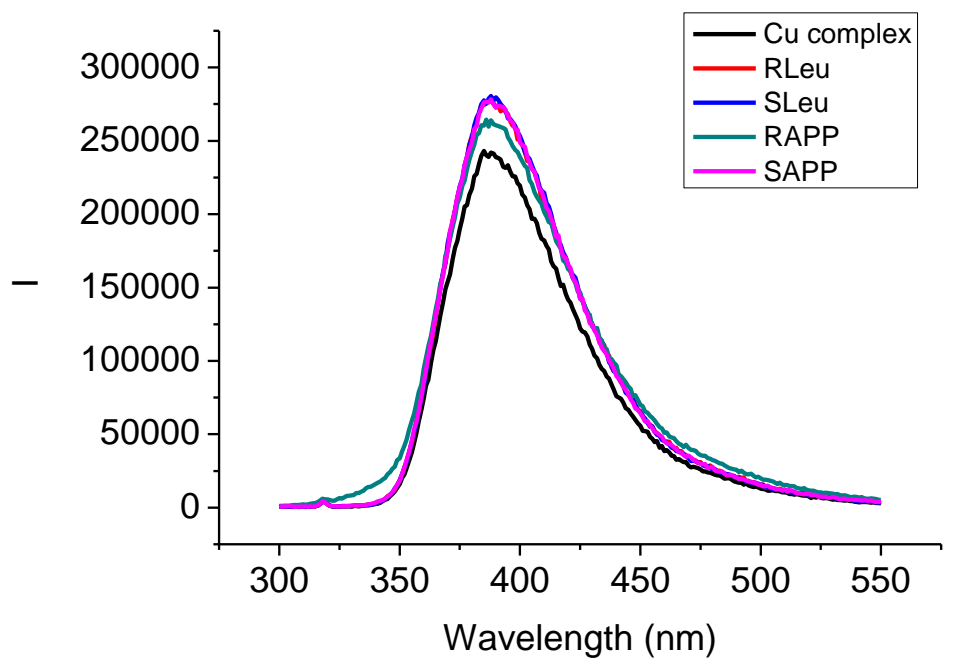

Figure AI-57. Fluorescence spectra of $(\mathrm{S})-\mathbf{7 - 2 0} \cdot \mathrm{CuCl}_{2}\left(5 \times 10^{-7} \mathrm{M}\right.$ in DCM/hexane 2:3) in the absence and presence of $(\mathrm{R})$ - or $(\mathrm{S})$-leucinol and 2-amino-3-phenylpropanol $\left(5 \times 10^{-4}\right.$ M). $\left(\lambda_{\text {exc }}=290 \mathrm{~nm}\right.$. slit $\left.=1.5 / 1.5 \mathrm{~nm}\right)$

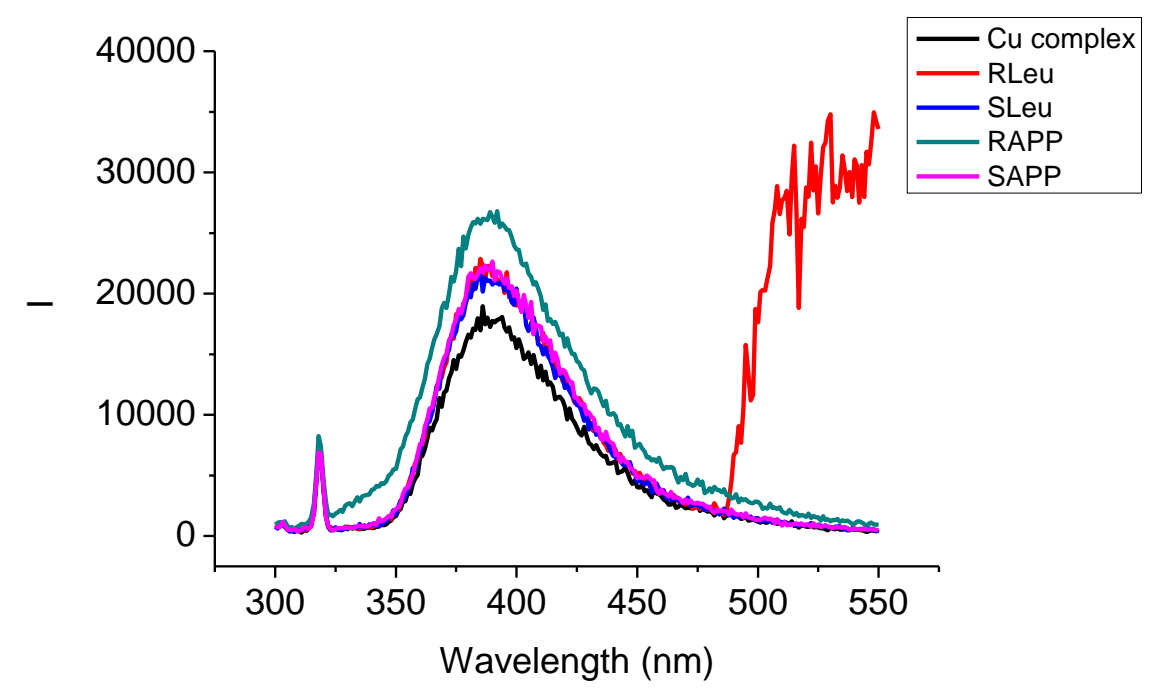


Figure AI-58. Fluorescence spectra of (S)-7-20 and its copper complex $\left(1 \times 10^{-5} \mathrm{M}\right.$ in THF, $\mathrm{THF} / \mathrm{H}_{2} \mathrm{O}$ and methanol $) .\left(\lambda_{\mathrm{exc}}=290 \mathrm{~nm} . \mathrm{slit}=1.5 / 1.5 \mathrm{~nm}\right)$

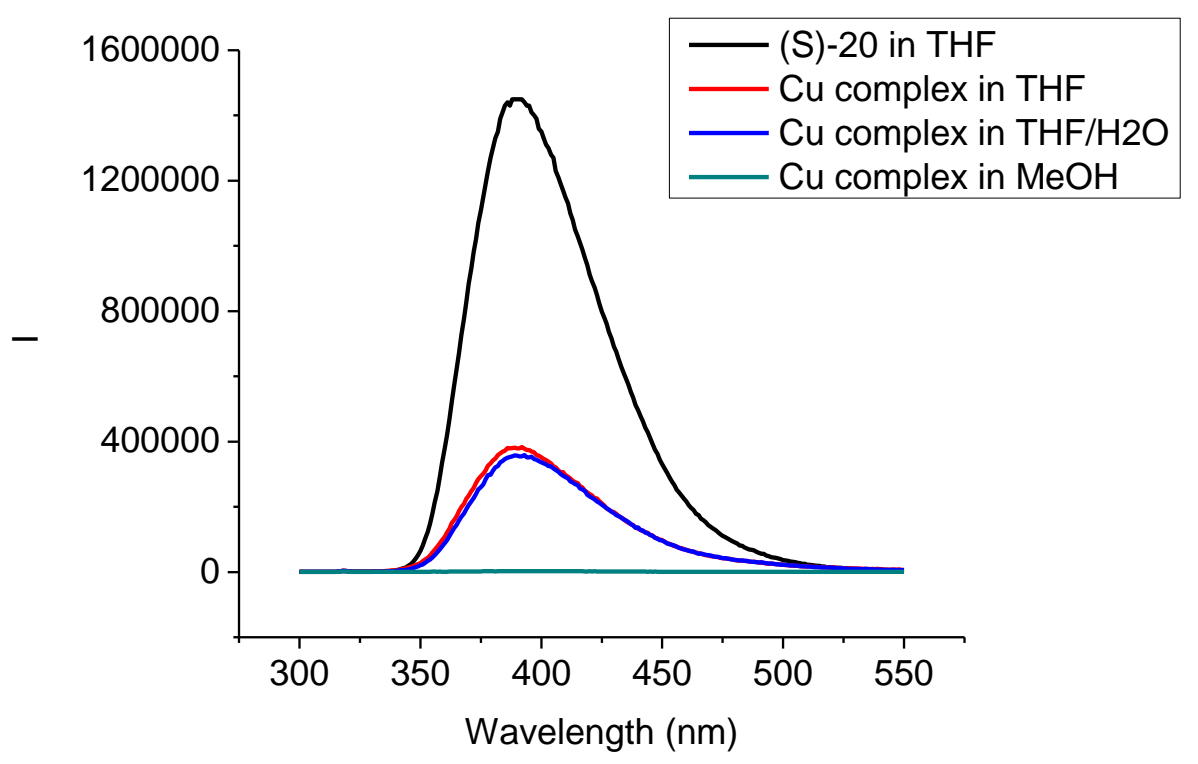

Figure AI-59. Fluorescence spectra of (S)-7-20. $\mathrm{CuCl}_{2}\left(1 \times 10^{-5} \mathrm{M}\right.$ in THF) in the absence and presence of (R)- or (S)-mandelic acid $\left[1 \times 10^{-5} \mathrm{M}\right.$ in THF $\left(0.1 \%\right.$ methanol), $1 \times 10^{-4} \mathrm{M}$ $(1 \%$ methanol $), 1 \times 10^{-3} \mathrm{M}(10 \%$ methanol $\left.)\right] .\left(\lambda_{\mathrm{exc}}=290 \mathrm{~nm} . \mathrm{slit}=1.5 / 1.5 \mathrm{~nm}\right)$ 


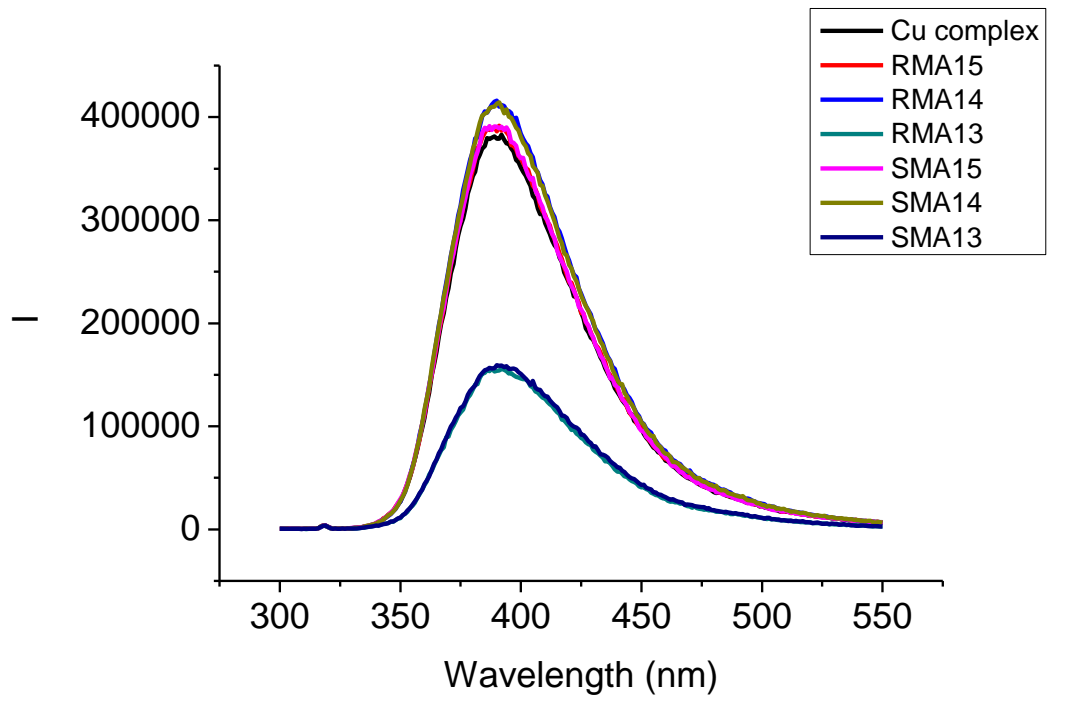

Figure AI-60. Fluorescence spectra of $(\mathrm{S})-\mathbf{7 - 2 0} \cdot \mathrm{CuCl}_{2}\left(1 \times 10^{-5} \mathrm{M}\right.$ in $\left.\mathrm{MeOH}\right)$ in the absence and presence of (R)- or (S)-mandelic acid $\left[1 \times 10^{-5} \mathrm{M}, 1 \times 10^{-4} \mathrm{M}, 1 \times 10^{-3} \mathrm{M}\right] .\left(\lambda_{\text {exc }}\right.$ $=290 \mathrm{~nm}$. slit $=1.5 / 1.5 \mathrm{~nm})$

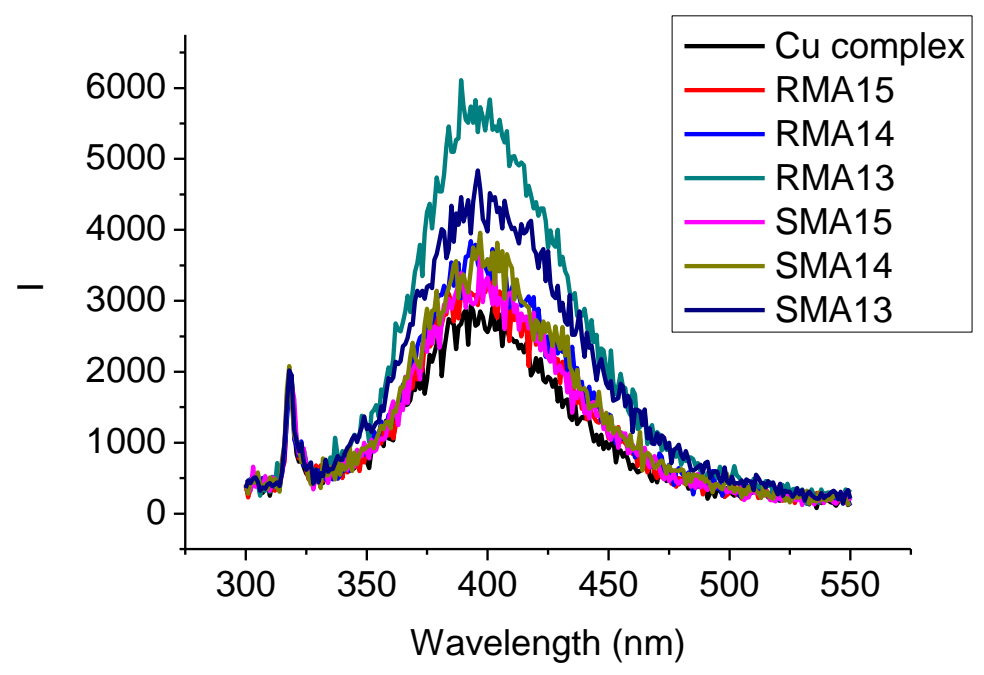

Figure AI-61. Fluorescence spectra of (S)-7-20. $\mathrm{CuCl}_{2}\left(1 \times 10^{-5} \mathrm{M}\right.$ in THF) in the absence and presence of L-Histidine $\left[1 \times 10^{-5} \mathrm{M}\right.$ in THF $\left(0.1 \% \mathrm{H}_{2} \mathrm{O}\right), 1 \times 10^{-4} \mathrm{M}$ in THF $\left(1 \% \mathrm{H}_{2} \mathrm{O}\right)$, 
$1 \times 10^{-3} \mathrm{M}$ in $\left.\operatorname{THF}\left(10 \% \mathrm{H}_{2} \mathrm{O}\right)\right] .\left(\lambda_{\mathrm{exc}}=290 \mathrm{~nm} . \mathrm{slit}=1.5 / 1.5 \mathrm{~nm}\right)$

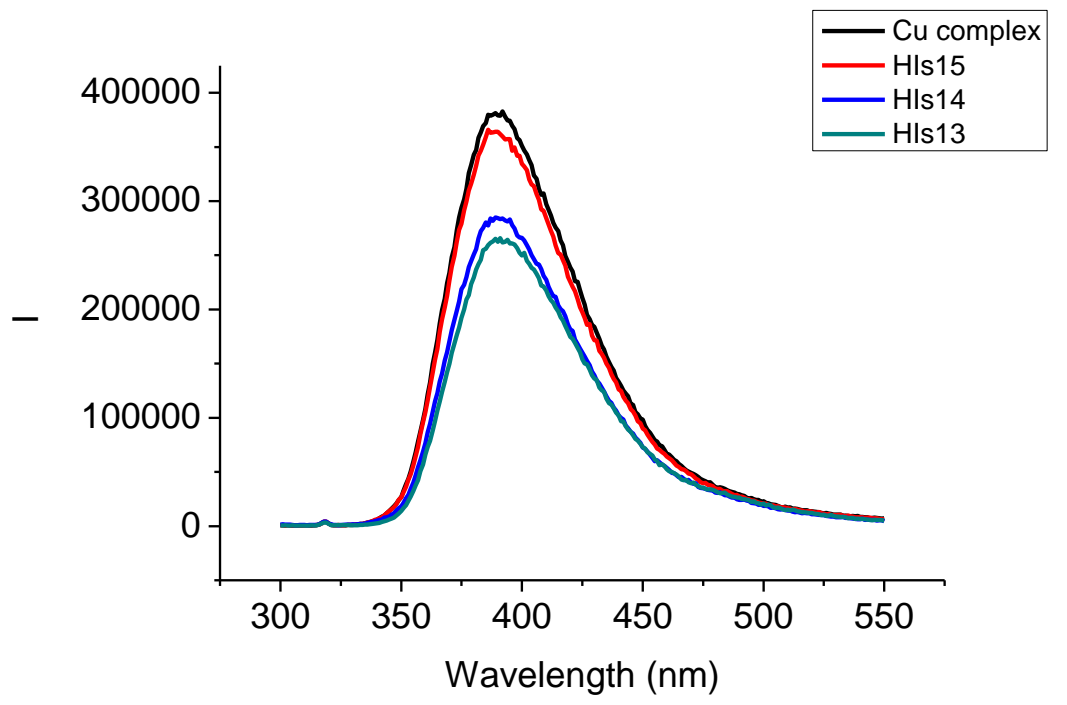

Figure AI-62. Fluorescence spectra of $(\mathrm{S})-\mathbf{7 - 2 0} \cdot \mathrm{CuCl}_{2}\left(1 \times 10^{-5} \mathrm{M}\right.$ in $\left.\mathrm{MeOH}\right)$ in the absence and presence of L-Histidine $\left[1 \times 10^{-5} \mathrm{M}\right.$ in $\mathrm{MeOH}\left(0.1 \% \mathrm{H}_{2} \mathrm{O}\right), 1 \times 10^{-4} \mathrm{M}$ in $\mathrm{MeOH}\left(1 \% \mathrm{H}_{2} \mathrm{O}\right), 1 \times 10^{-3} \mathrm{M}$ in $\left.\mathrm{MeOH}\left(10 \% \mathrm{H}_{2} \mathrm{O}\right)\right] .\left(\lambda_{\mathrm{exc}}=290 \mathrm{~nm}\right.$. slit $\left.=1.5 / 1.5 \mathrm{~nm}\right)$

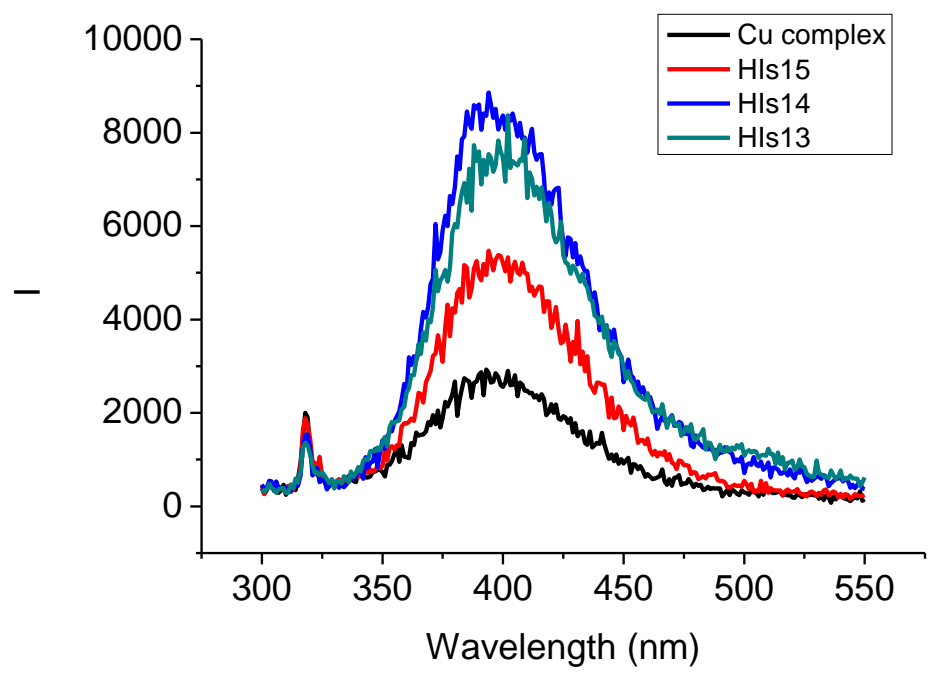

11. Interaction of (S)-7-9, (S)-7-13, (S)-7-14, (S)-7-15 and (S)-7-21 with substrates 
Figure AI-63. Fluorescence spectra of (S)-7-9 $\left(1 \times 10^{-5} \mathrm{M}\right.$ in $\left.\mathrm{THF} / \mathrm{H}_{2} \mathrm{O} 5: 1\right)$ in the absence and presence of D- or L-Alanine $\left(1 \times 10^{-4} \mathrm{M}, 1 \times 10^{-3} \mathrm{M}, 1 \times 10^{-2} \mathrm{M}\right) .\left(\lambda_{\text {exc }}=325 \mathrm{~nm}\right.$. slit $=3.0 / 3.0 \mathrm{~nm})$

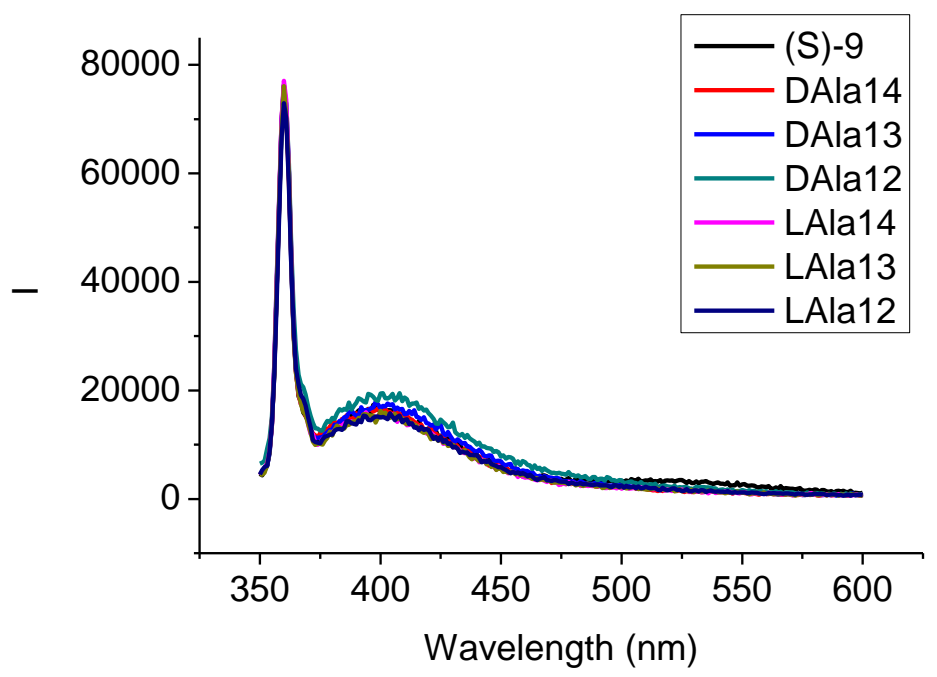

Figure AI-64. Fluorescence spectra of (S)-7-9 $\left(1 \times 10^{-5} \mathrm{M}\right.$ in $\left.\mathrm{THF} / \mathrm{H}_{2} \mathrm{O} 5: 1\right)$ in the absence and presence of D- or L-Serine $\left(1 \times 10^{-4} \mathrm{M}, 1 \times 10^{-3} \mathrm{M}, 1 \times 10^{-2} \mathrm{M}\right) .\left(\lambda_{\mathrm{exc}}=325 \mathrm{~nm}\right.$. slit $=3.0 / 3.0 \mathrm{~nm})$ 


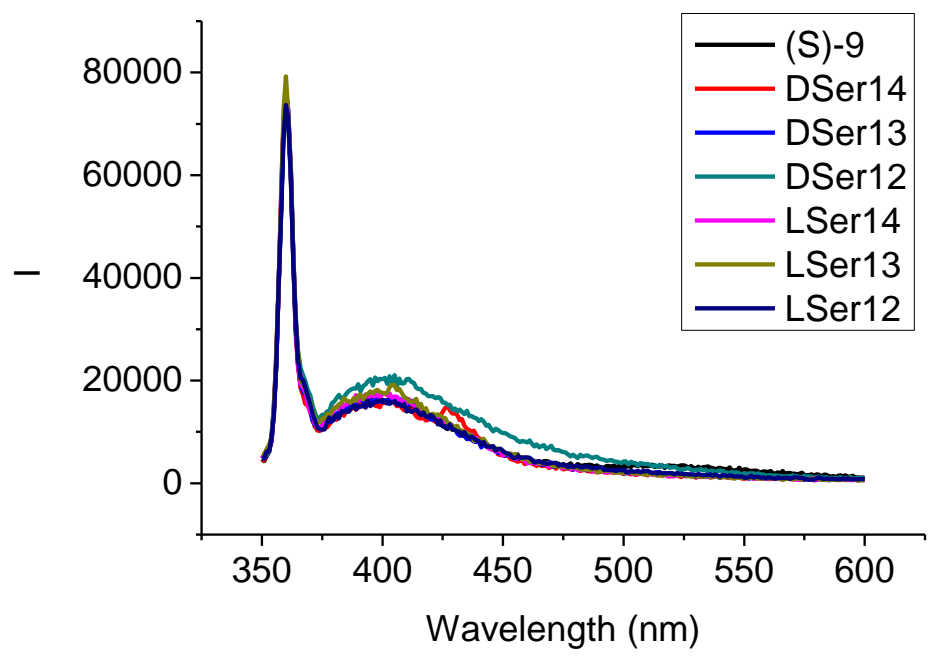

Figure AI-65. Fluorescence spectra of $(\mathrm{S})-\mathbf{7 - 9}\left(1 \times 10^{-5} \mathrm{M}\right.$ in $\left.\mathrm{THF} / \mathrm{H}_{2} \mathrm{O} 5: 1\right)$ in the absence and presence of D- or L-Arginine $\left(1 \times 10^{-4} \mathrm{M}, 1 \times 10^{-3} \mathrm{M}, 1 \times 10^{-2} \mathrm{M}\right) .\left(\lambda_{\mathrm{exc}}=325 \mathrm{~nm}\right.$. slit $=3.0 / 3.0 \mathrm{~nm})$

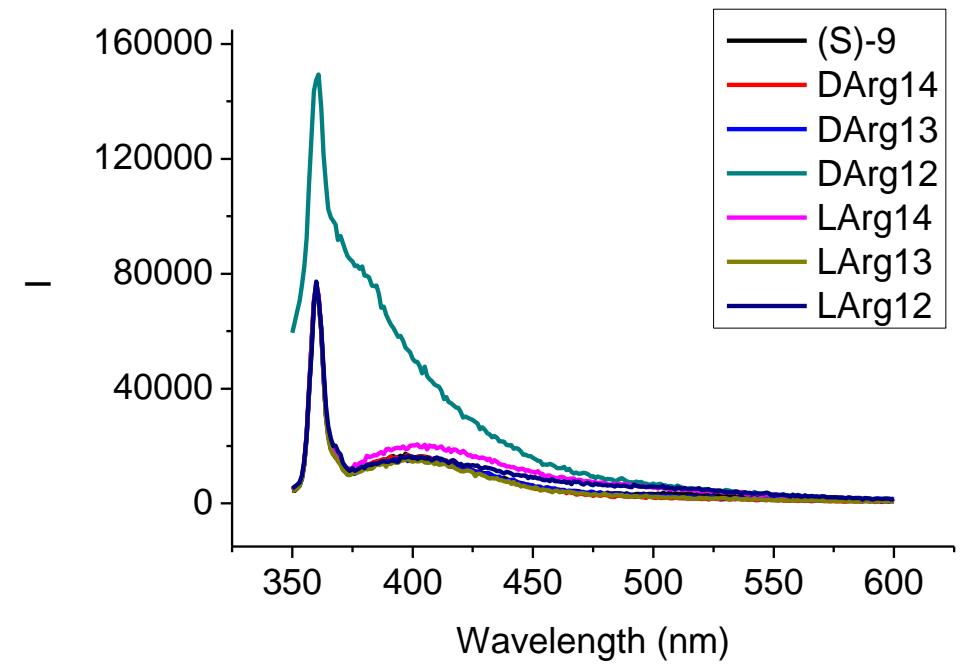

Figure AI-66. Fluorescence spectra of (S)-7-13 $\left(1 \times 10^{-5} \mathrm{M}\right.$ in $\mathrm{THF} / \mathrm{H}_{2} \mathrm{O}$ 5:1) in the absence and presence of D- or L-Alanine $\left(1 \times 10^{-4} \mathrm{M}, 1 \times 10^{-3} \mathrm{M}, 1 \times 10^{-2} \mathrm{M}\right) .\left(\lambda_{\text {exc }}=325 \mathrm{~nm}\right.$. 
slit $=3.0 / 3.0 \mathrm{~nm})$

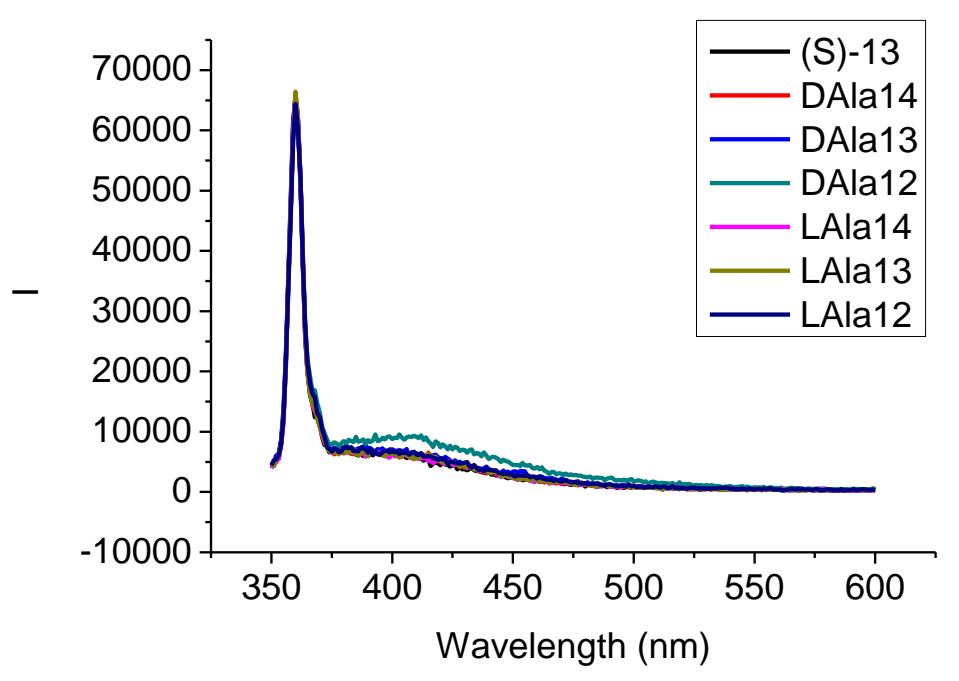

Figure AI-67. Fluorescence spectra of (S)-7-13 $\left(1 \times 10^{-5} \mathrm{M}\right.$ in $\mathrm{THF} / \mathrm{H}_{2} \mathrm{O}$ 5:1) in the absence and presence of D- or L-Serine $\left(1 \times 10^{-4} \mathrm{M}, 1 \times 10^{-3} \mathrm{M}, 1 \times 10^{-2} \mathrm{M}\right) .\left(\lambda_{\mathrm{exc}}=325 \mathrm{~nm}\right.$. slit $=3.0 / 3.0 \mathrm{~nm})$

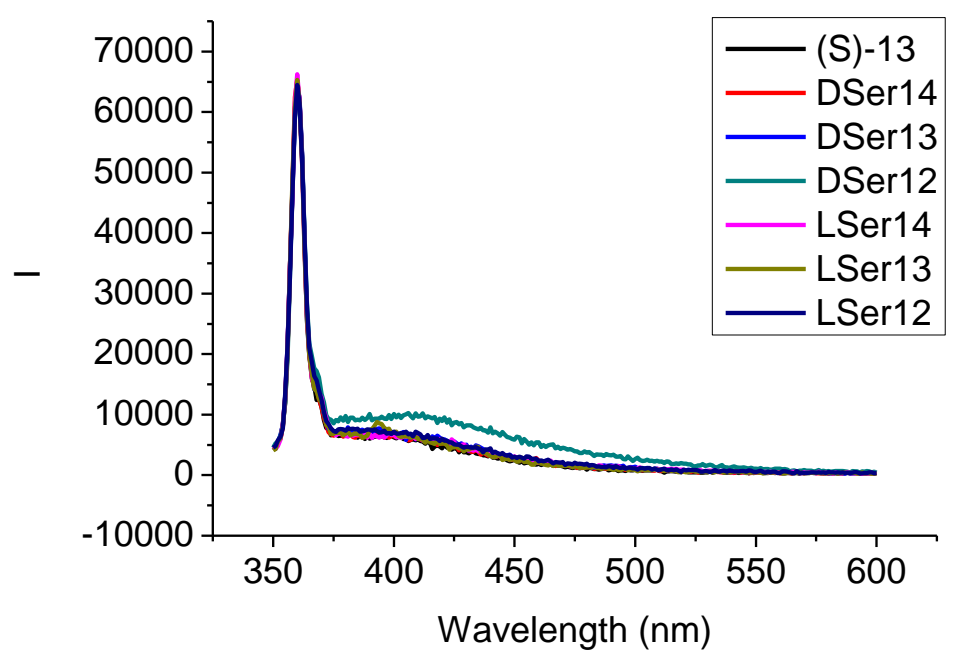

Figure AI-68. Fluorescence spectra of $(\mathrm{S})-\mathbf{7 - 1 3}\left(1 \times 10^{-5} \mathrm{M}\right.$ in $\left.\mathrm{THF} / \mathrm{H}_{2} \mathrm{O} 5: 1\right)$ in the absence and presence of D- or L-Arginine $\left(1 \times 10^{-4} \mathrm{M}, 1 \times 10^{-3} \mathrm{M}, 1 \times 10^{-2} \mathrm{M}\right) .\left(\lambda_{\text {exc }}=325\right.$ 
nm. slit $=3 \cdot 0 / 3.0 \mathrm{~nm}$ )

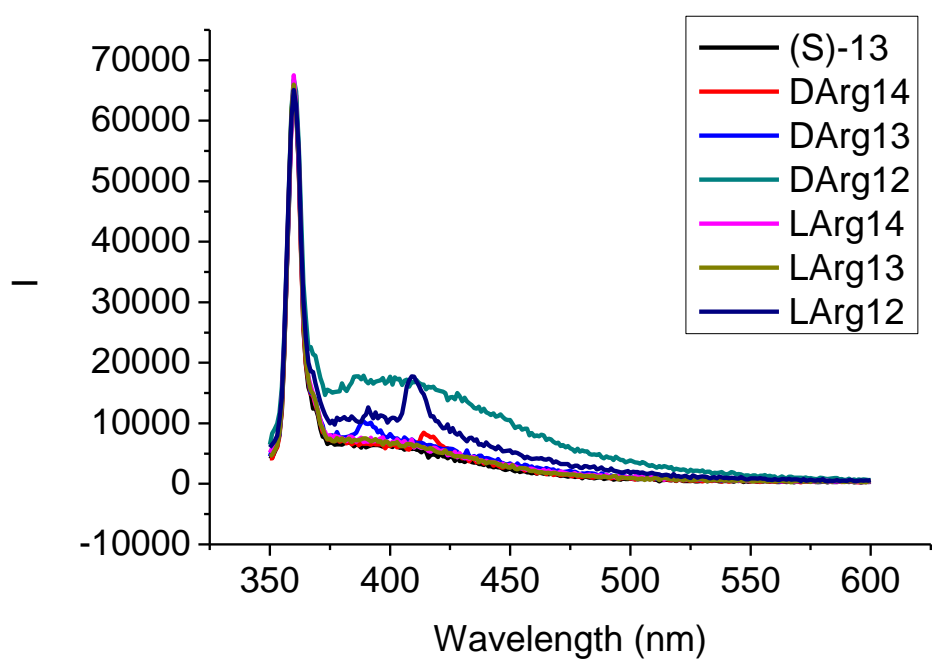

Figure AI-69. Fluorescence spectra of $(\mathrm{S})-\mathbf{7 - 1 4}\left(1 \times 10^{-5} \mathrm{M}\right.$ in $\mathrm{THF} / \mathrm{H}_{2} \mathrm{O}$ 5:1) in the absence and presence of D- or L-Alanine $\left(1 \times 10^{-4} \mathrm{M}, 1 \times 10^{-3} \mathrm{M}, 1 \times 10^{-2} \mathrm{M}\right) .\left(\lambda_{\mathrm{exc}}=325 \mathrm{~nm}\right.$. slit $=3.0 / 3.0 \mathrm{~nm}$ )

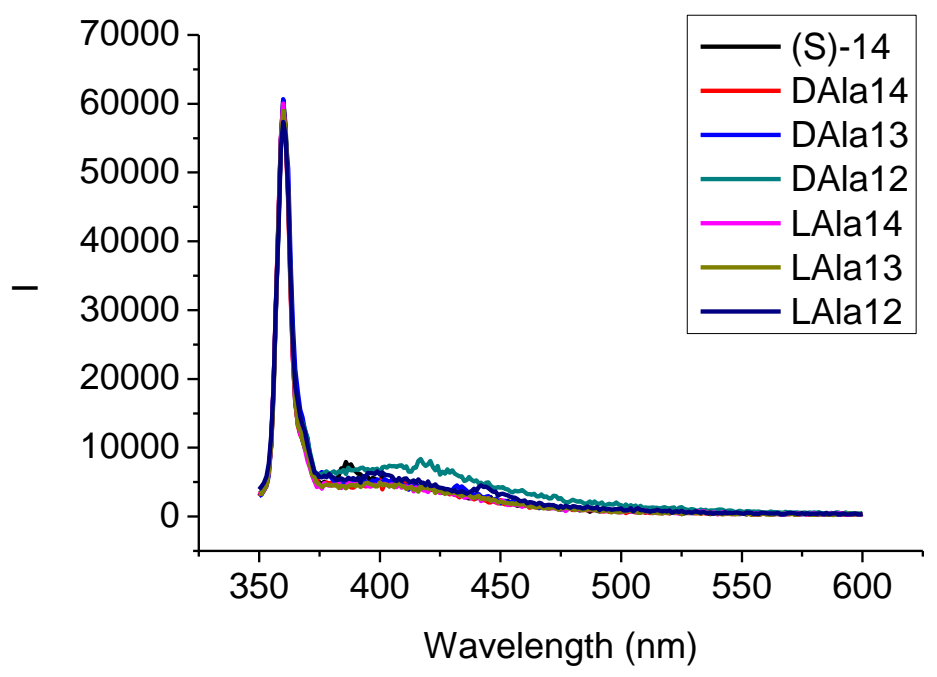

Figure AI-70. Fluorescence spectra of $(\mathrm{S})-\mathbf{7 - 1 4}\left(1 \times 10^{-5} \mathrm{M}\right.$ in $\mathrm{THF} / \mathrm{H}_{2} \mathrm{O}$ 5:1) in the 
absence and presence of D- or L-Serine $\left(1 \times 10^{-4} \mathrm{M}, 1 \times 10^{-3} \mathrm{M}, 1 \times 10^{-2} \mathrm{M}\right) .\left(\lambda_{\mathrm{exc}}=325 \mathrm{~nm}\right.$. slit $=3.0 / 3.0 \mathrm{~nm})$

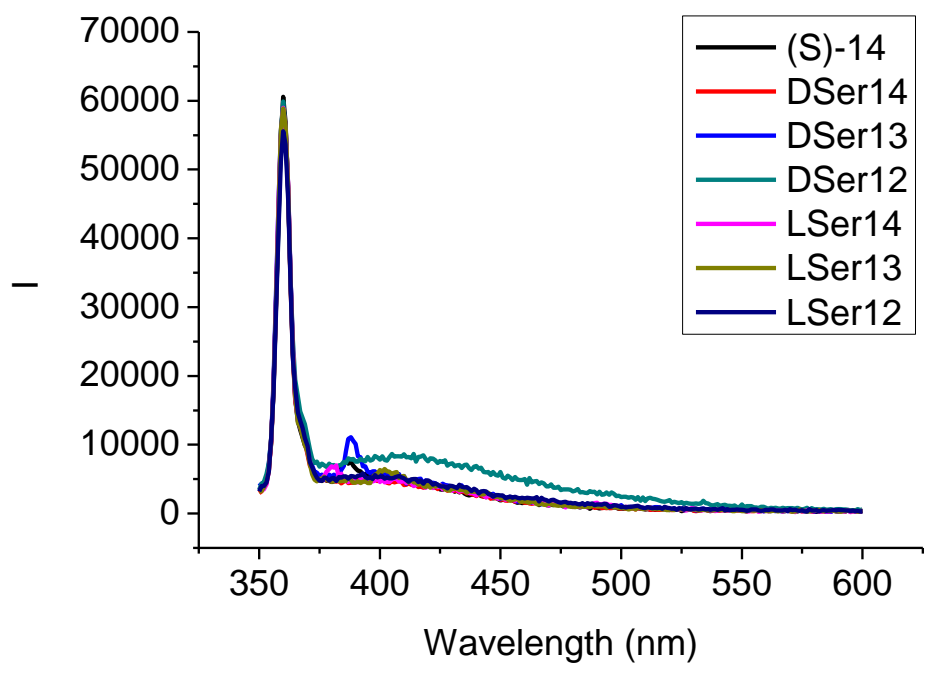

Figure AI-71. Fluorescence spectra of (S)-7-14 $\left(1 \times 10^{-5} \mathrm{M}\right.$ in $\left.\mathrm{THF} / \mathrm{H}_{2} \mathrm{O} 5: 1\right)$ in the absence and presence of D- or L-Arginine $\left(1 \times 10^{-4} \mathrm{M}, 1 \times 10^{-3} \mathrm{M}, 1 \times 10^{-2} \mathrm{M}\right) \cdot\left(\lambda_{\text {exc }}=325\right.$ nm. slit $=3.0 / 3.0 \mathrm{~nm})$

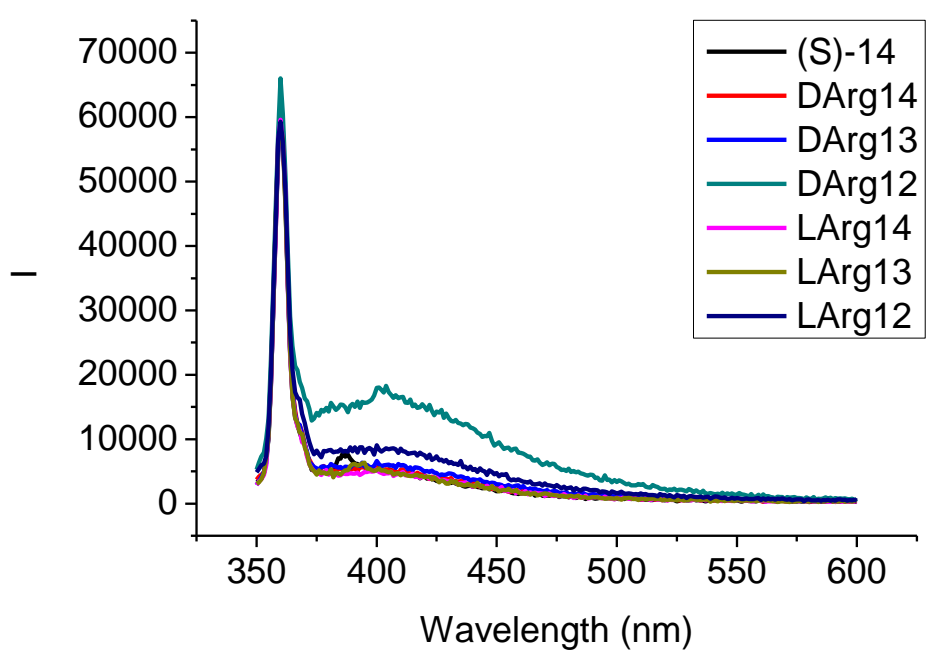


Figure AI-72. Fluorescence spectra of $(\mathrm{S})-\mathbf{7 - 1 5}\left(1 \times 10^{-5} \mathrm{M}\right.$ in $\left.\mathrm{THF} / \mathrm{H}_{2} \mathrm{O} 5: 1\right)$ in the absence and presence of D- or L-Alanine $\left(1 \times 10^{-4} \mathrm{M}, 1 \times 10^{-3} \mathrm{M}, 1 \times 10^{-2} \mathrm{M}\right) .\left(\lambda_{\mathrm{exc}}=325 \mathrm{~nm}\right.$. slit $=3.0 / 3.0 \mathrm{~nm})$

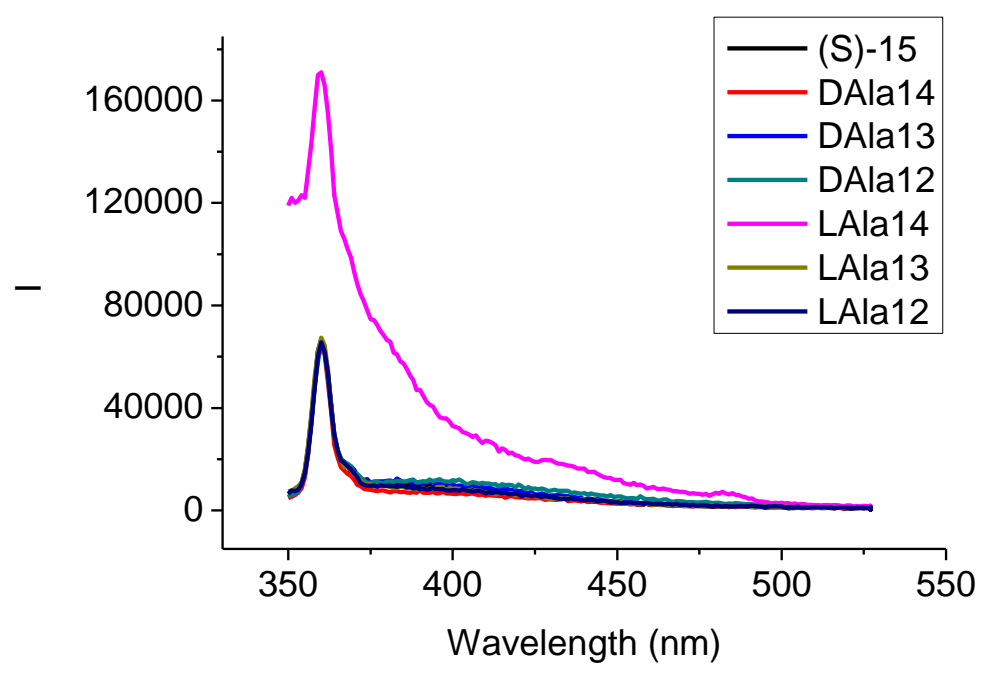

Figure AI-73. Fluorescence spectra of $(\mathrm{S})-\mathbf{7 - 1 5}\left(1 \times 10^{-5} \mathrm{M}\right.$ in $\left.\mathrm{THF} / \mathrm{H}_{2} \mathrm{O} 5: 1\right)$ in the absence and presence of D- or L-Serine $\left(1 \times 10^{-4} \mathrm{M}, 1 \times 10^{-3} \mathrm{M}, 1 \times 10^{-2} \mathrm{M}\right) .\left(\lambda_{\mathrm{exc}}=325 \mathrm{~nm}\right.$. slit $=3.0 / 3.0 \mathrm{~nm})$

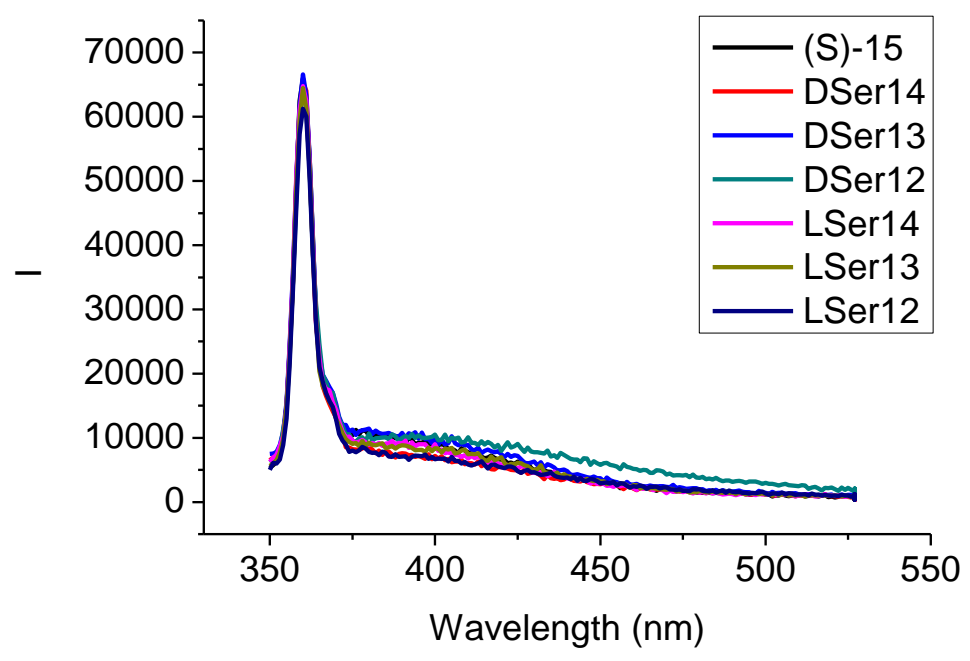


Figure AI-74. Fluorescence spectra of $(\mathrm{S})-7-15\left(1 \times 10^{-5} \mathrm{M}\right.$ in $\left.\mathrm{THF} / \mathrm{H}_{2} \mathrm{O} 5: 1\right)$ in the absence and presence of D- or L-Arginine $\left(1 \times 10^{-4} \mathrm{M}, 1 \times 10^{-3} \mathrm{M}, 1 \times 10^{-2} \mathrm{M}\right) .\left(\lambda_{\text {exc }}=325\right.$ nm. slit $=3.0 / 3.0 \mathrm{~nm})$

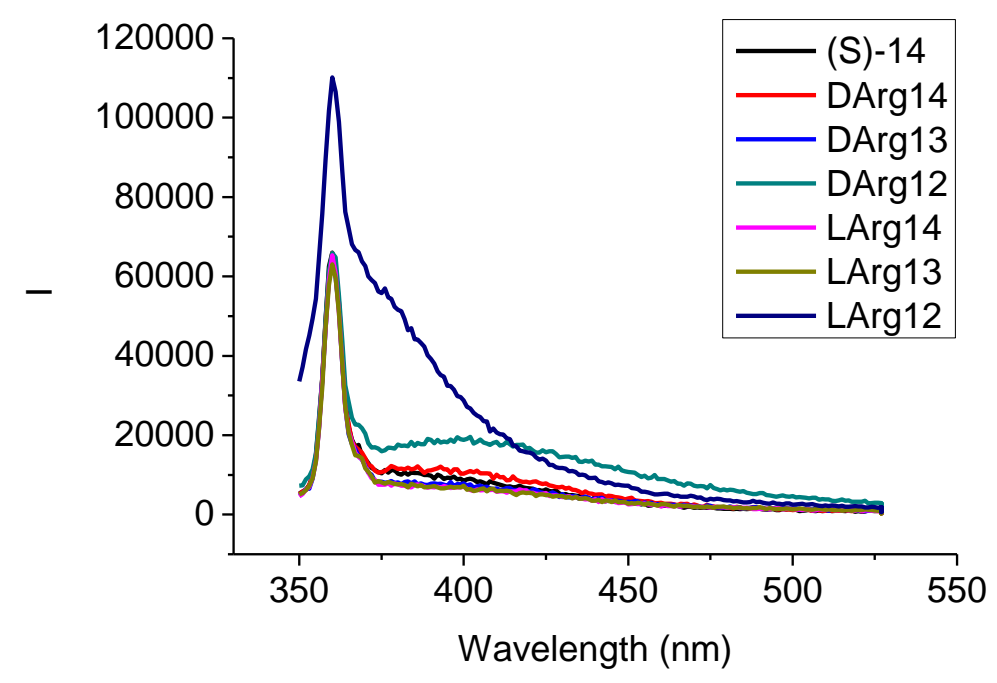

Figure AI-75. Fluorescence spectra of $(\mathrm{S})-\mathbf{7 - 2 1}\left(1 \times 10^{-5} \mathrm{M}\right.$ in $\left.\mathrm{THF} / \mathrm{H}_{2} \mathrm{O} 5: 1\right)$ in the absence and presence of D- or L-Alanine $\left(1 \times 10^{-4} \mathrm{M}, 1 \times 10^{-3} \mathrm{M}, 1 \times 10^{-2} \mathrm{M}\right) .\left(\lambda_{\text {exc }}=325 \mathrm{~nm}\right.$. slit $=3.0 / 3.0 \mathrm{~nm})$ 


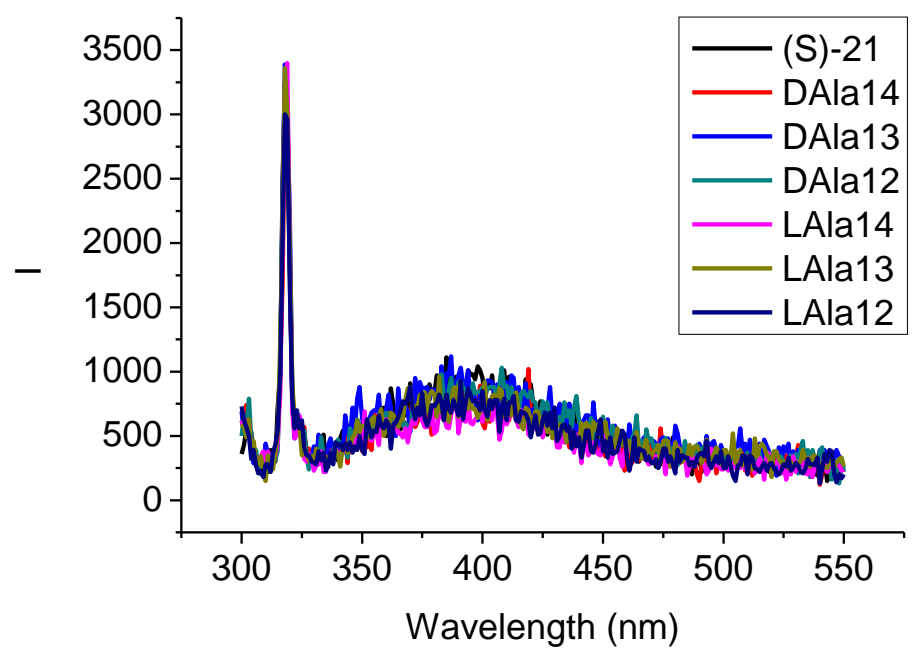

Figure AI-76. Fluorescence spectra of $(\mathrm{S})-\mathbf{7 - 2 1}\left(1 \times 10^{-5} \mathrm{M}\right.$ in $\left.\mathrm{THF} / \mathrm{H}_{2} \mathrm{O} 5: 1\right)$ in the absence and presence of D- or L-Serine $\left(1 \times 10^{-4} \mathrm{M}, 1 \times 10^{-3} \mathrm{M}, 1 \times 10^{-2} \mathrm{M}\right) .\left(\lambda_{\mathrm{exc}}=325 \mathrm{~nm}\right.$. slit $=3.0 / 3.0 \mathrm{~nm})$

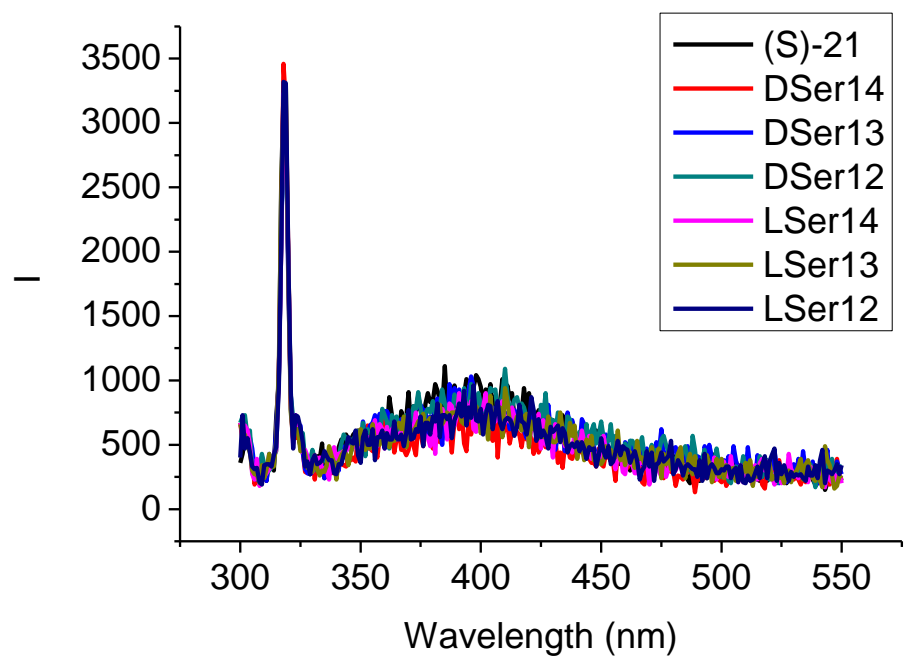

Figure AI-77. Fluorescence spectra of (S)-7-21 $\left(1 \times 10^{-5} \mathrm{M}\right.$ in $\left.\mathrm{THF} / \mathrm{H}_{2} \mathrm{O} 5: 1\right)$ in the absence and presence of D- or L-Arginine $\left(1 \times 10^{-4} \mathrm{M}, 1 \times 10^{-3} \mathrm{M}, 1 \times 10^{-2} \mathrm{M}\right) \cdot\left(\lambda_{\text {exc }}=325\right.$ nm. slit $=3.0 / 3.0 \mathrm{~nm}$ ) 


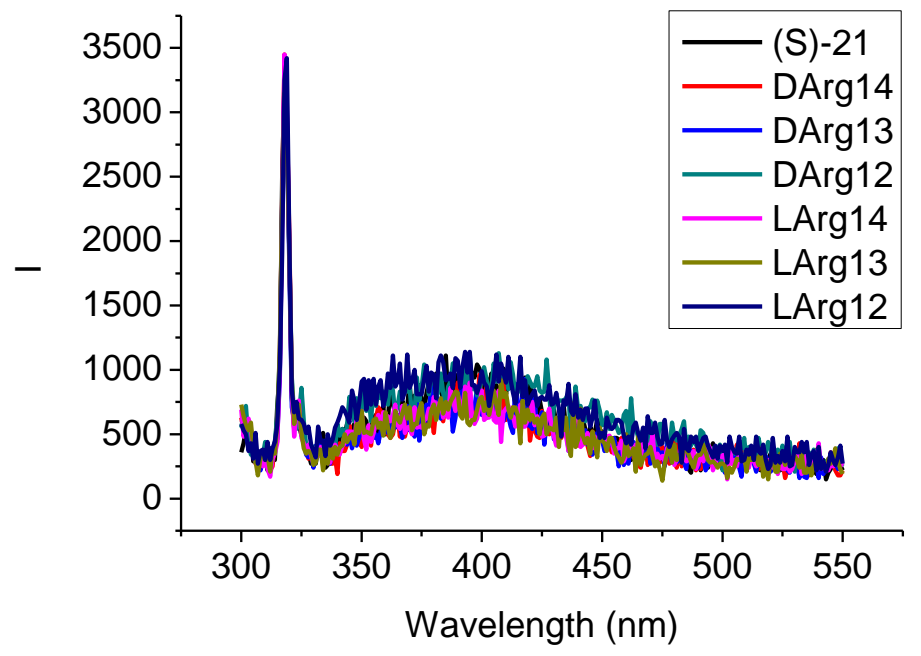

12. NMR spectra

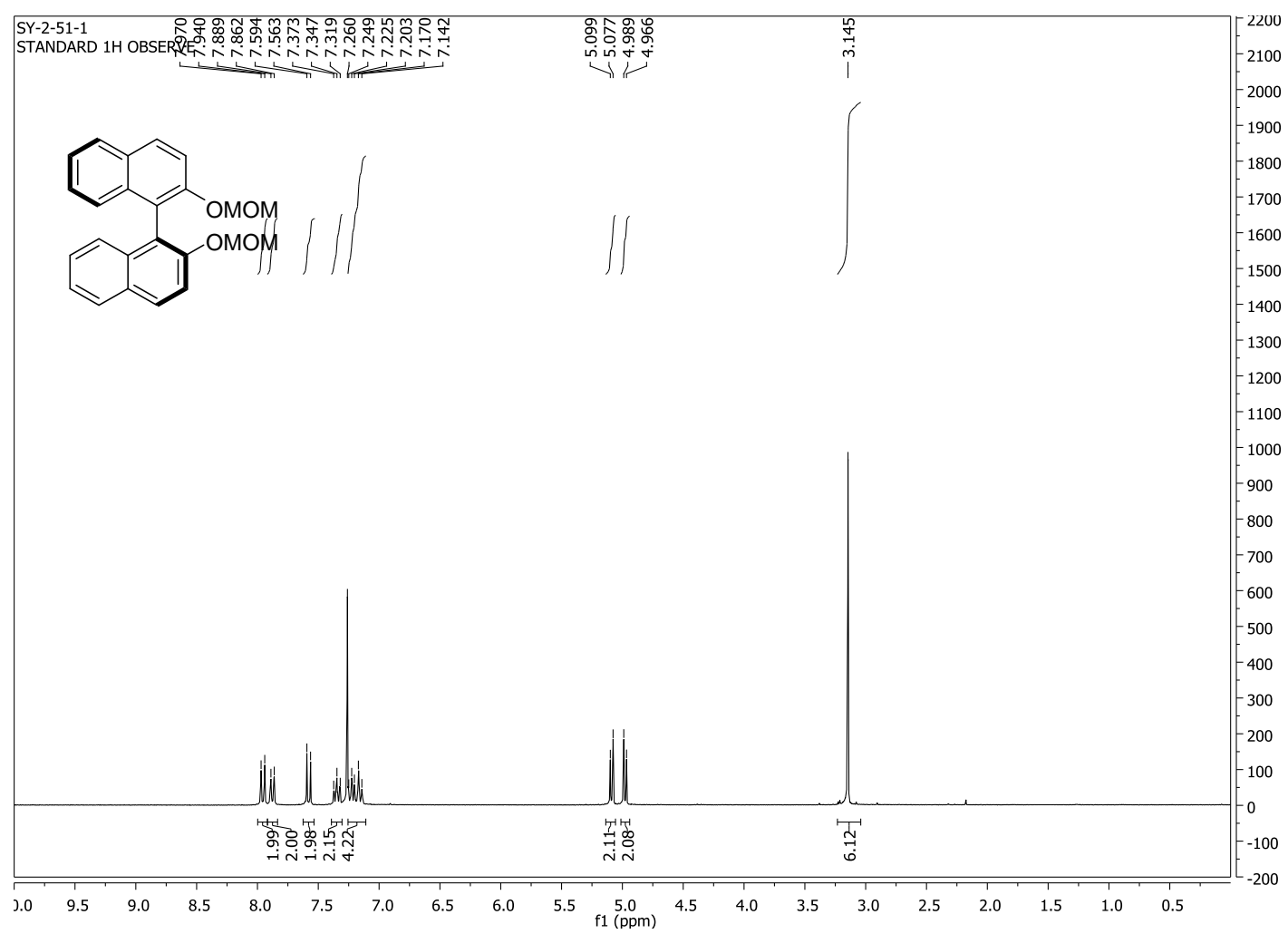




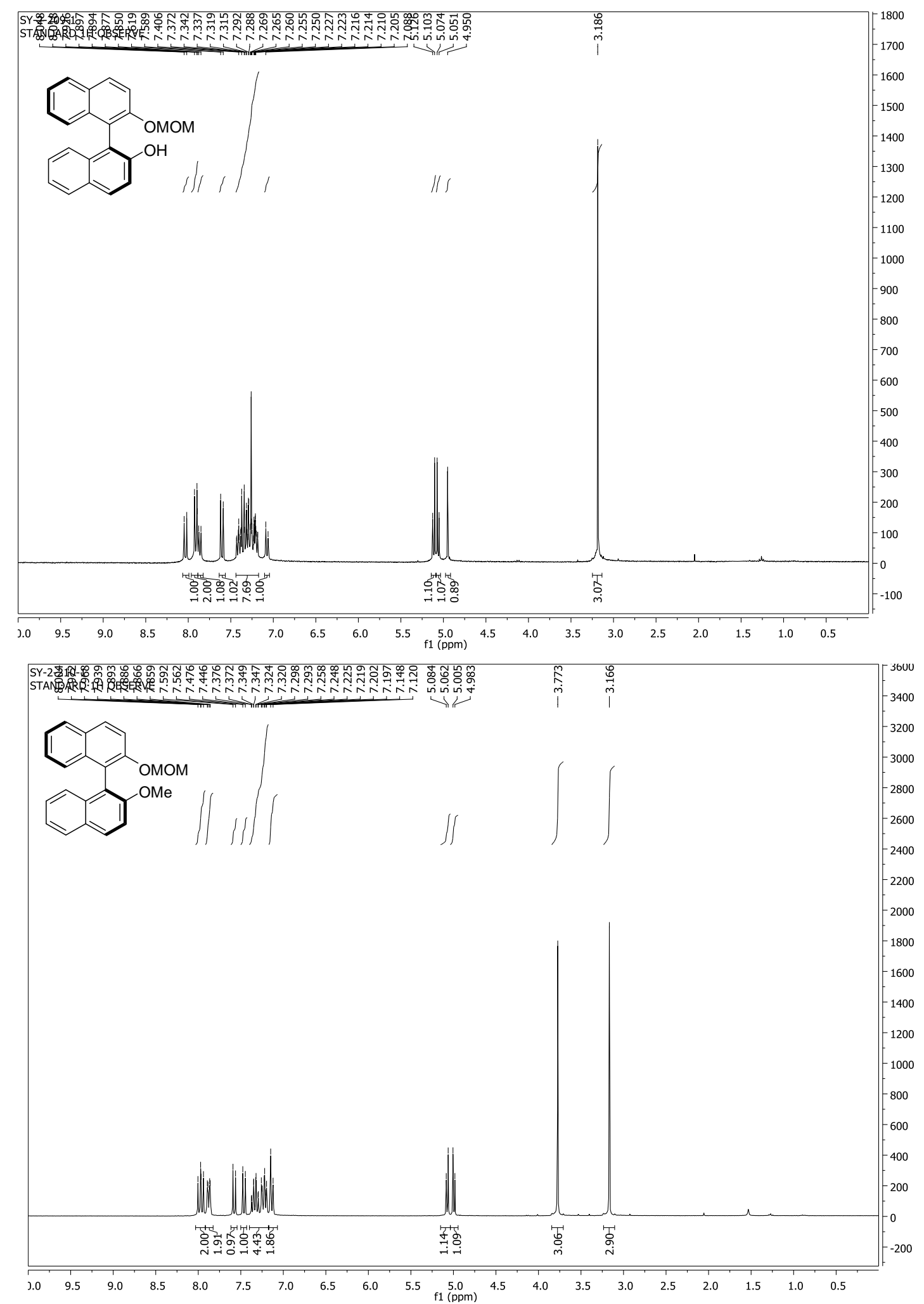




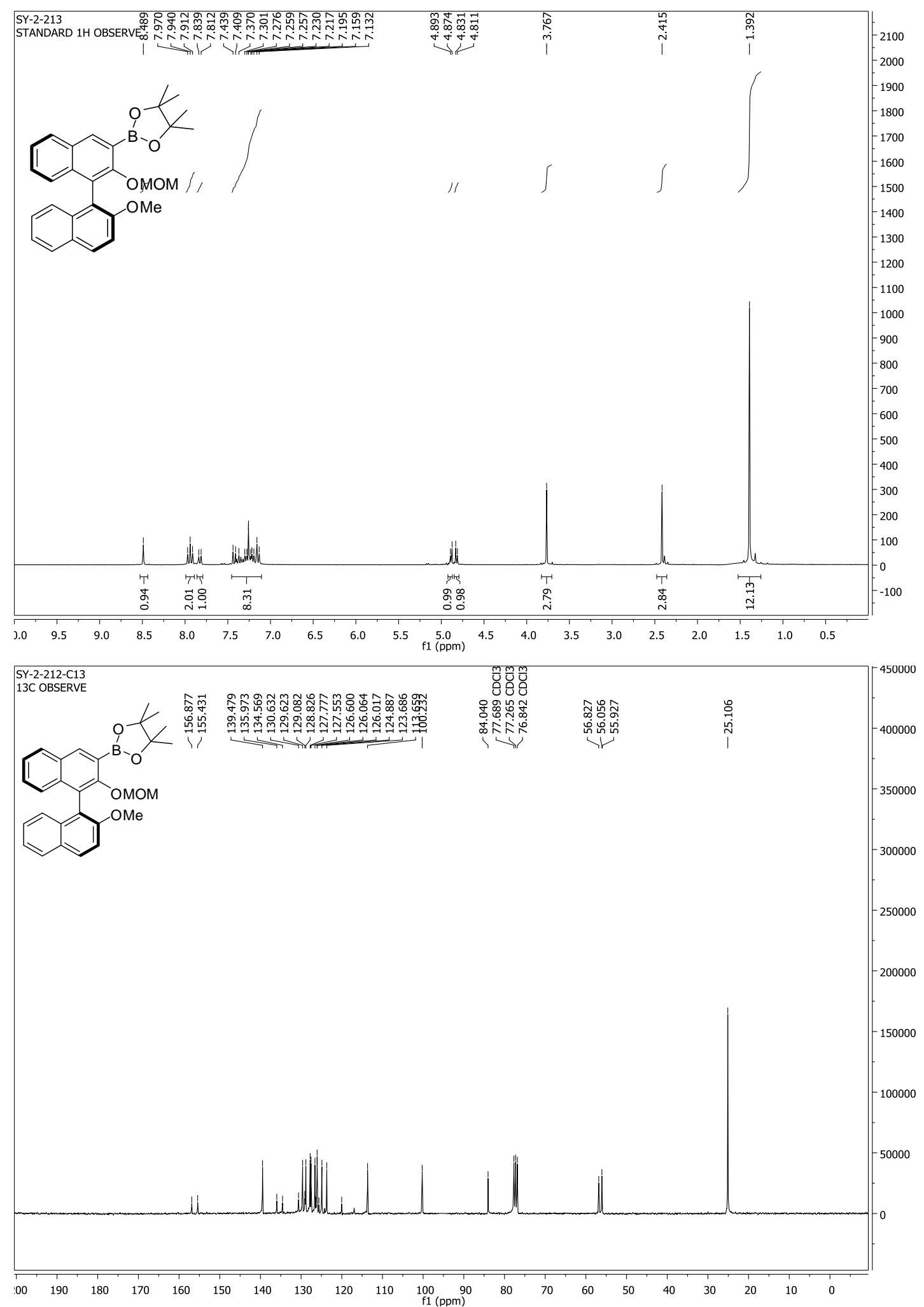




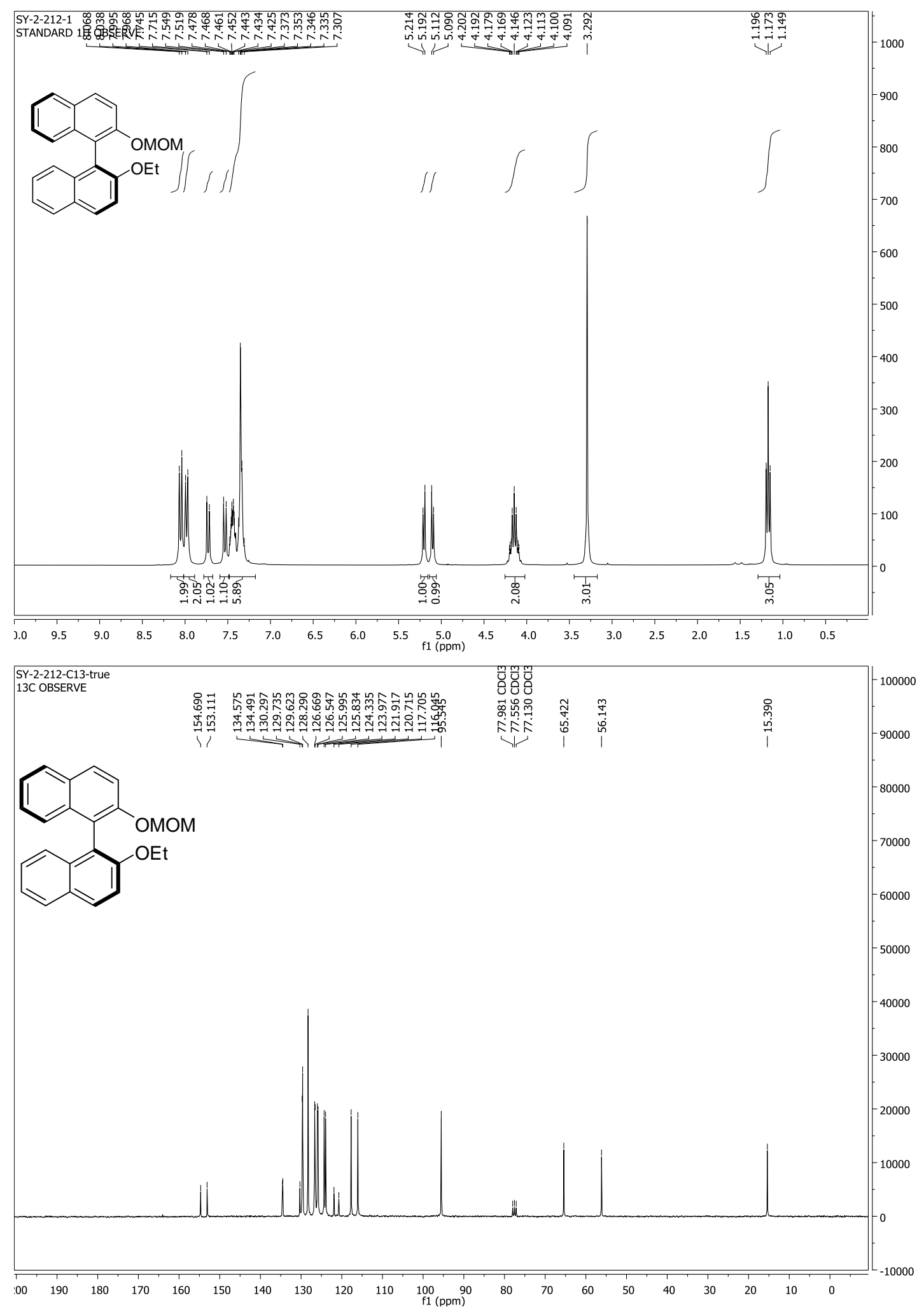



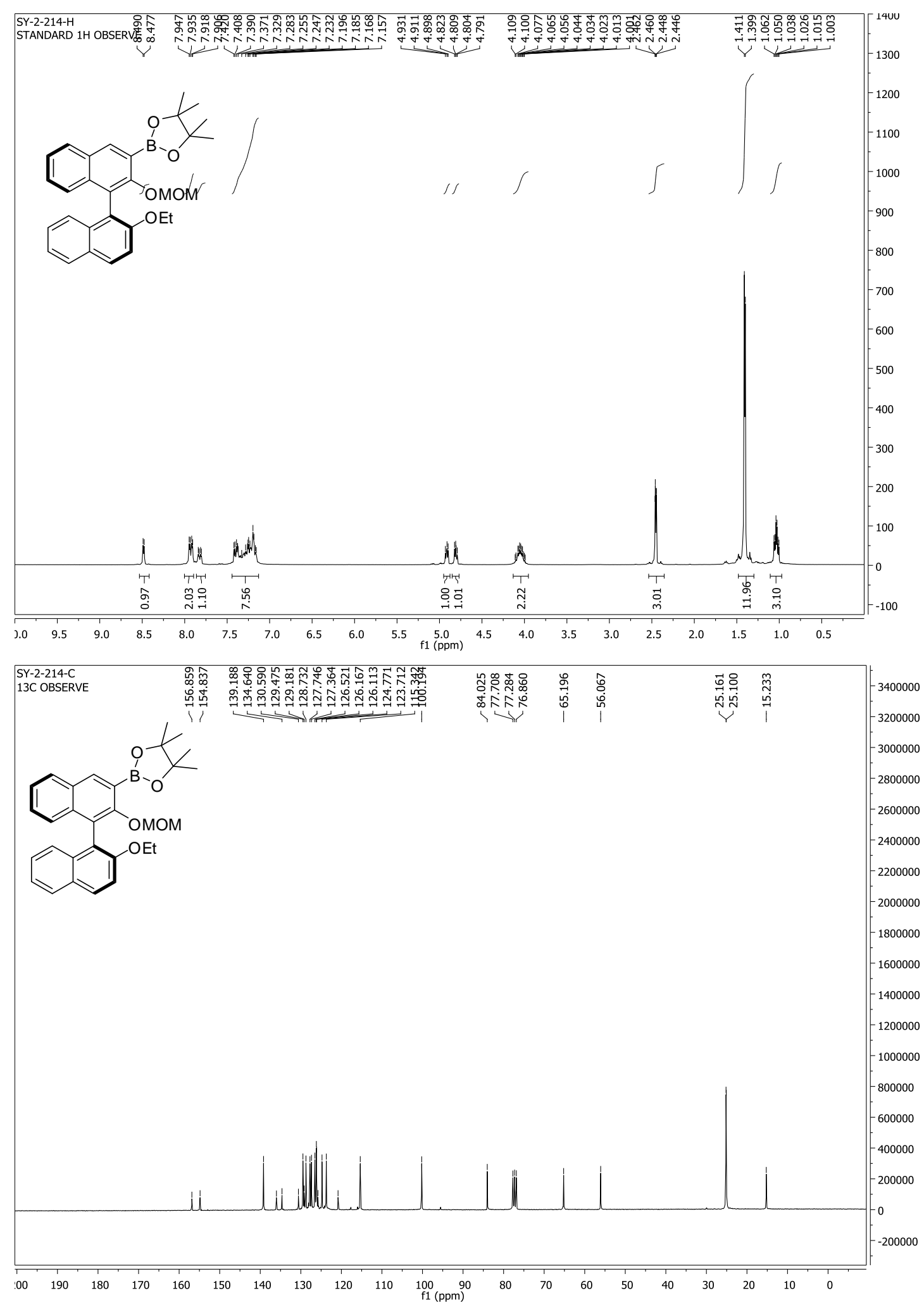

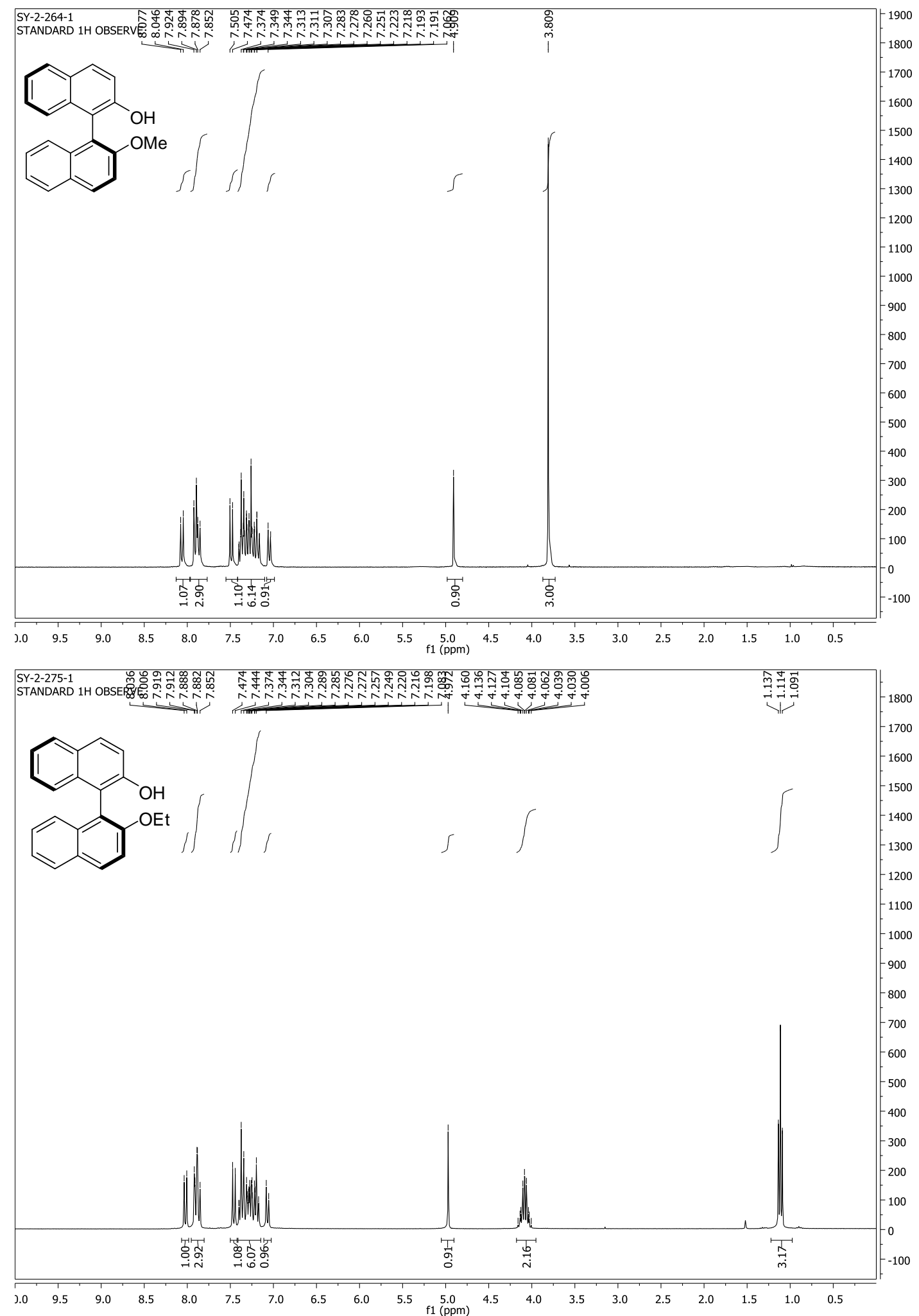


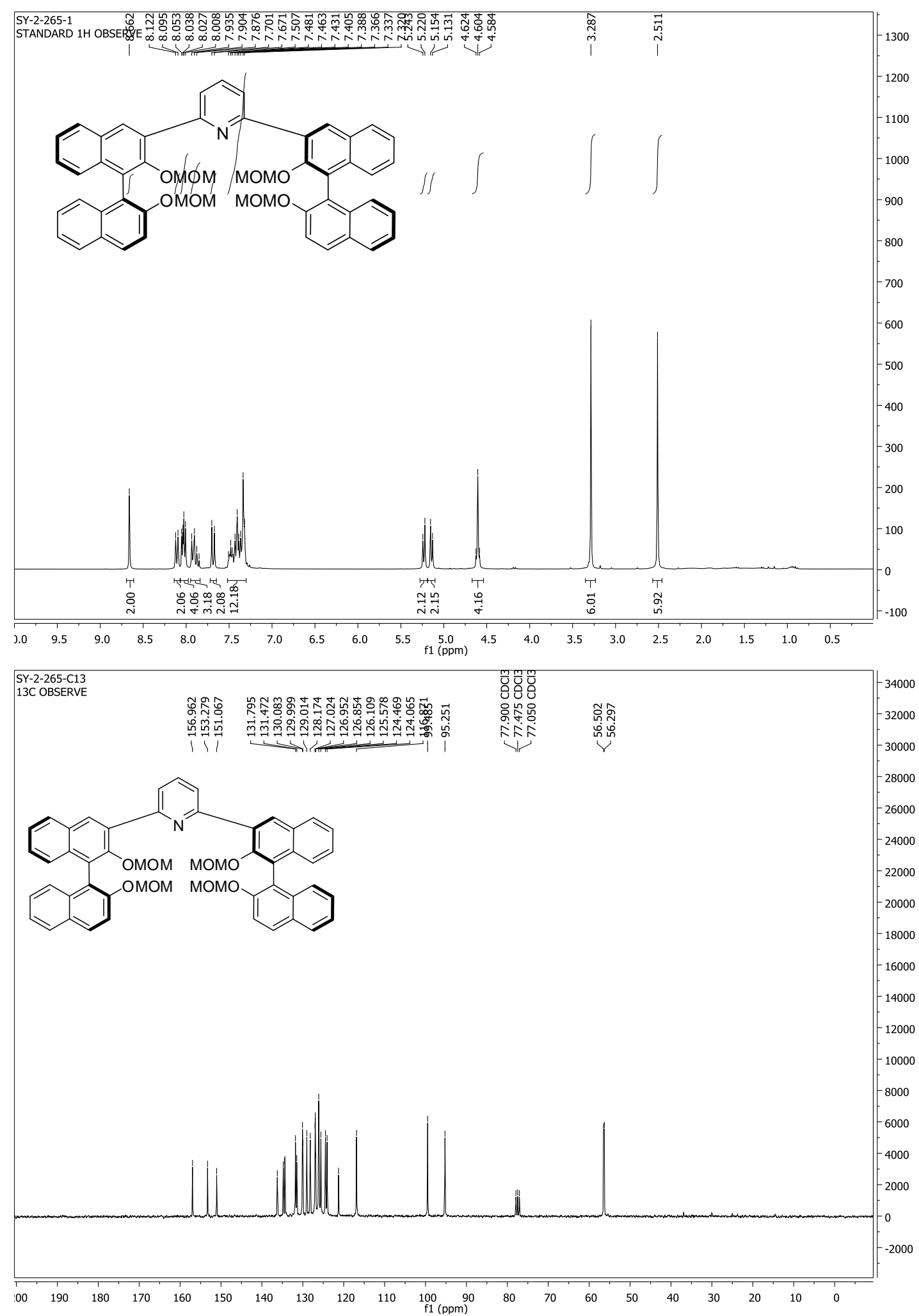



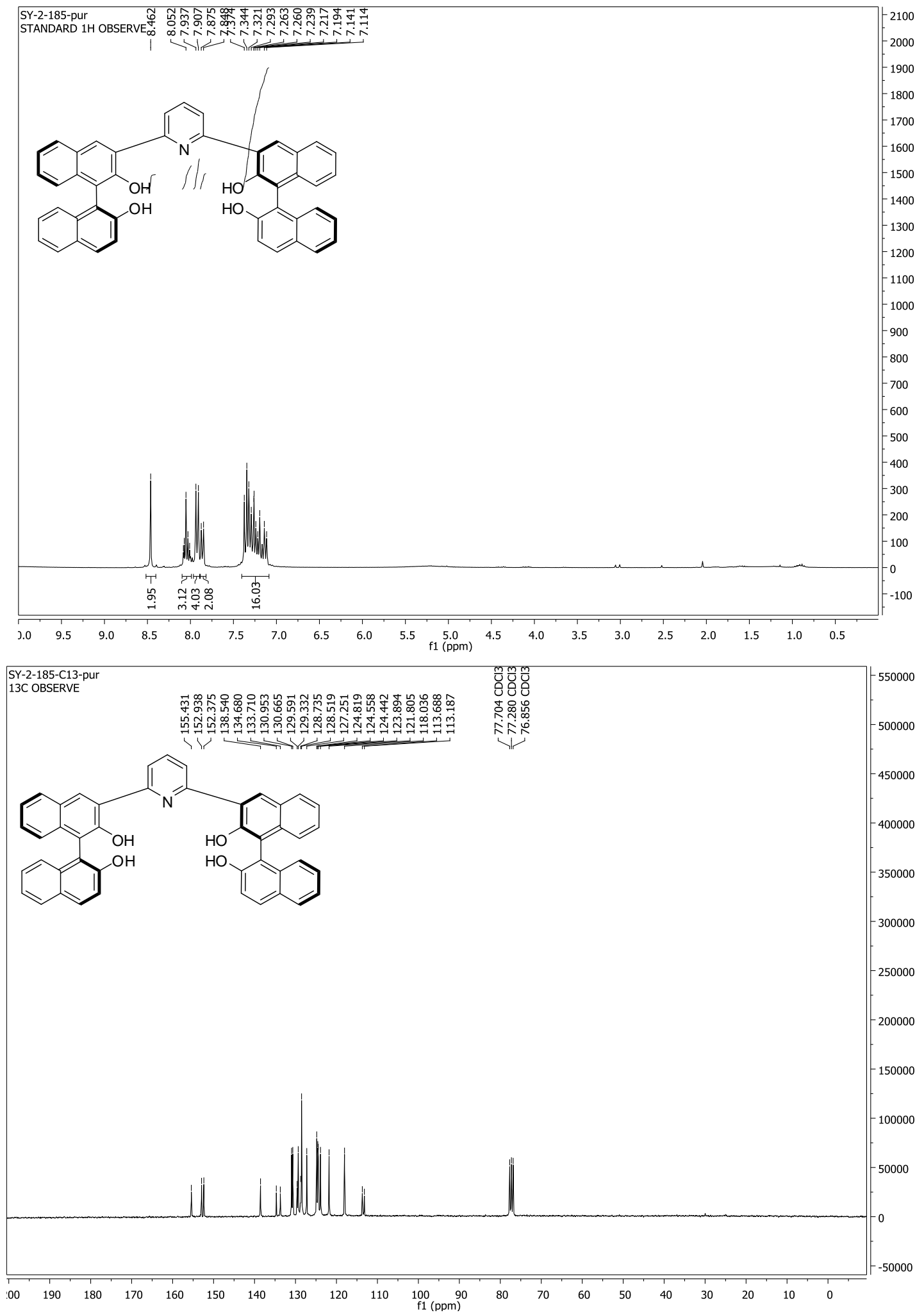


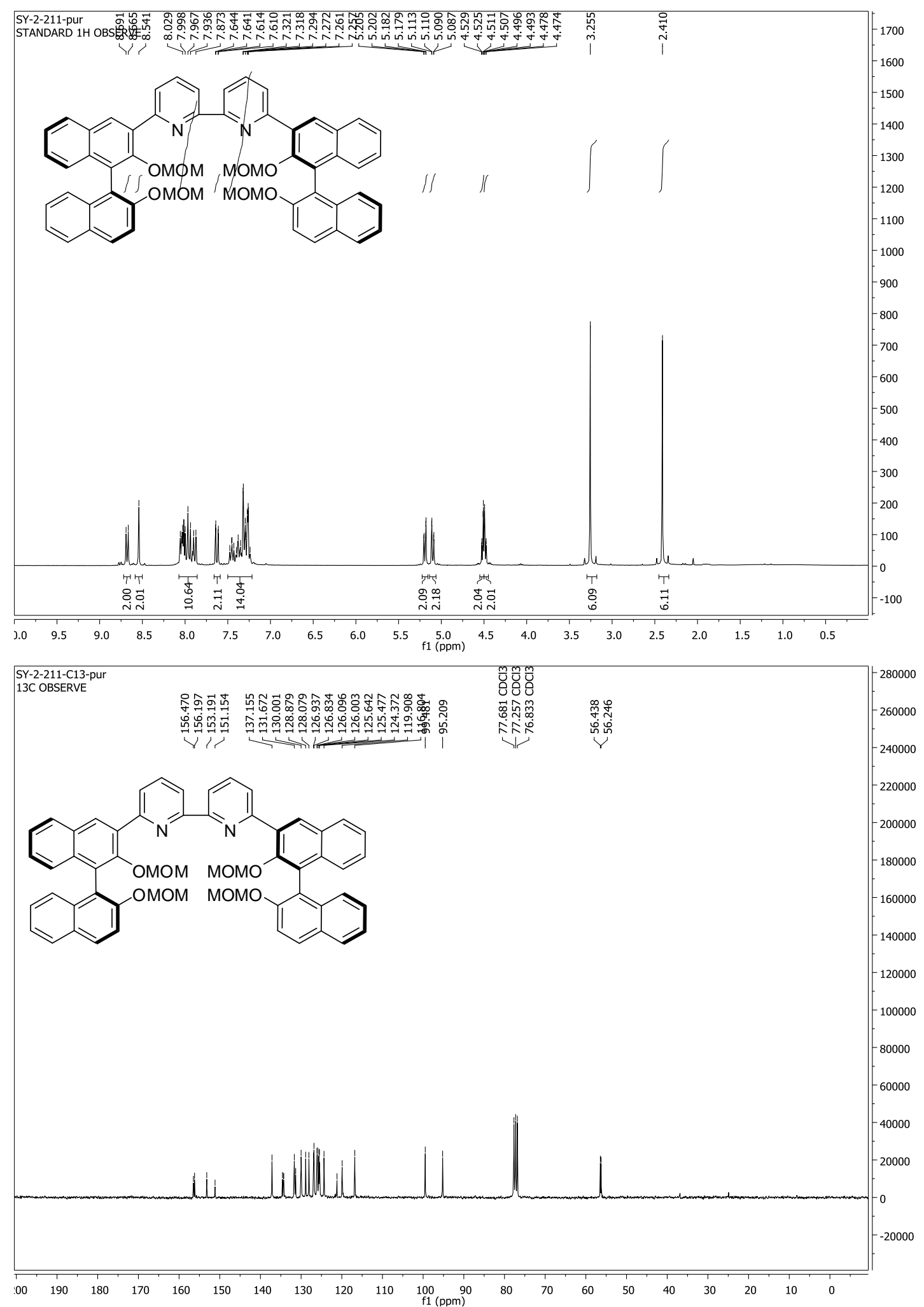



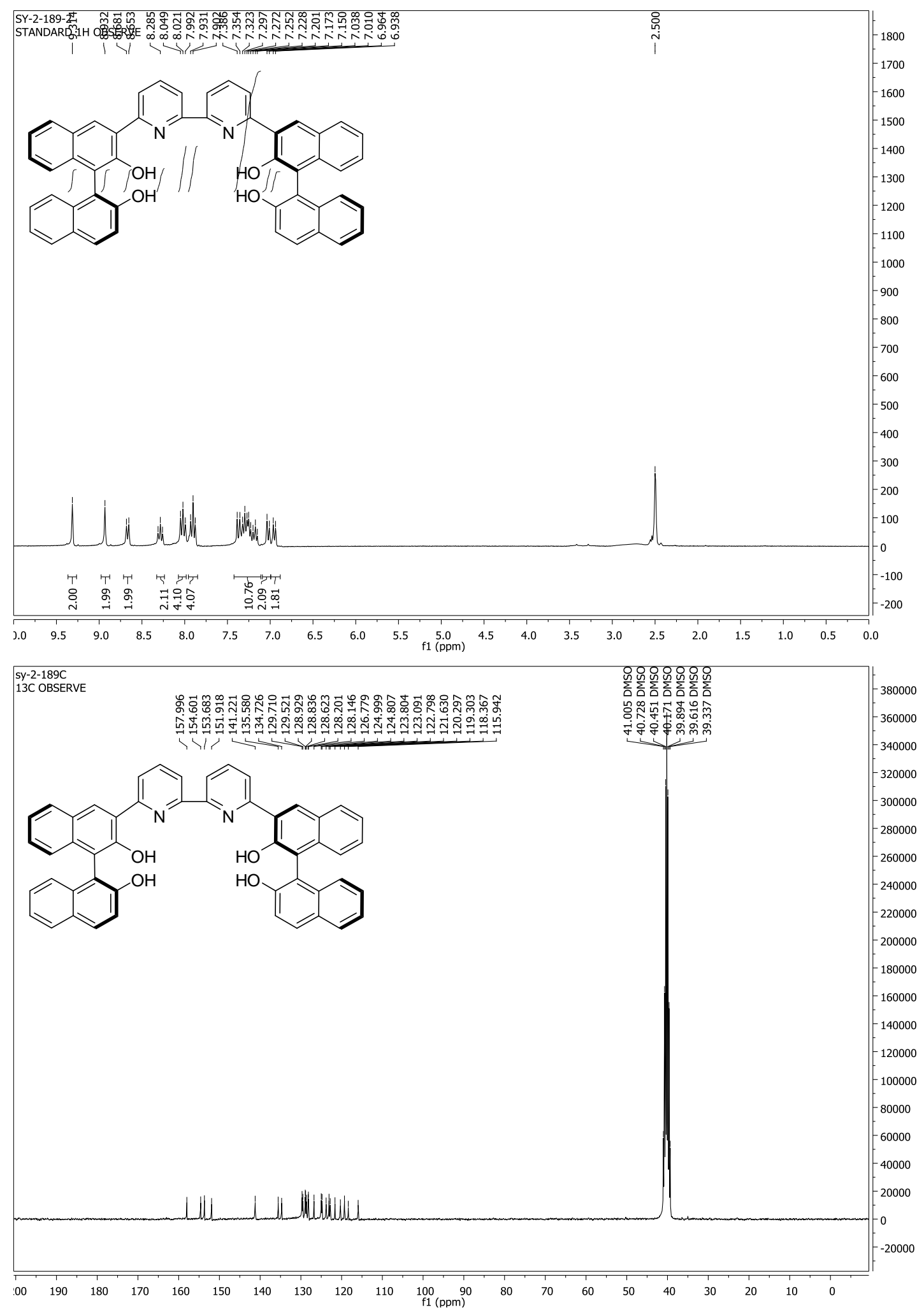


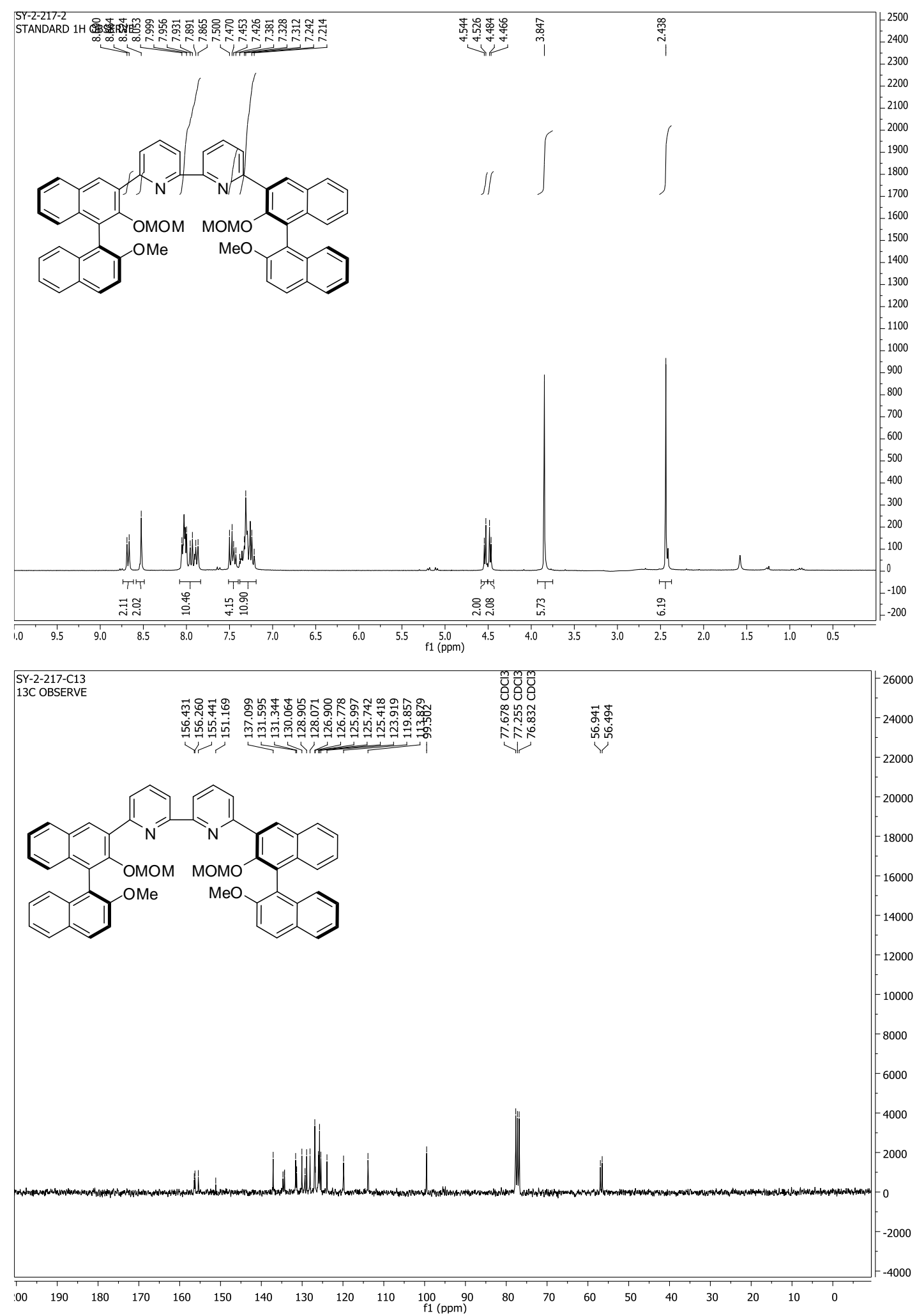




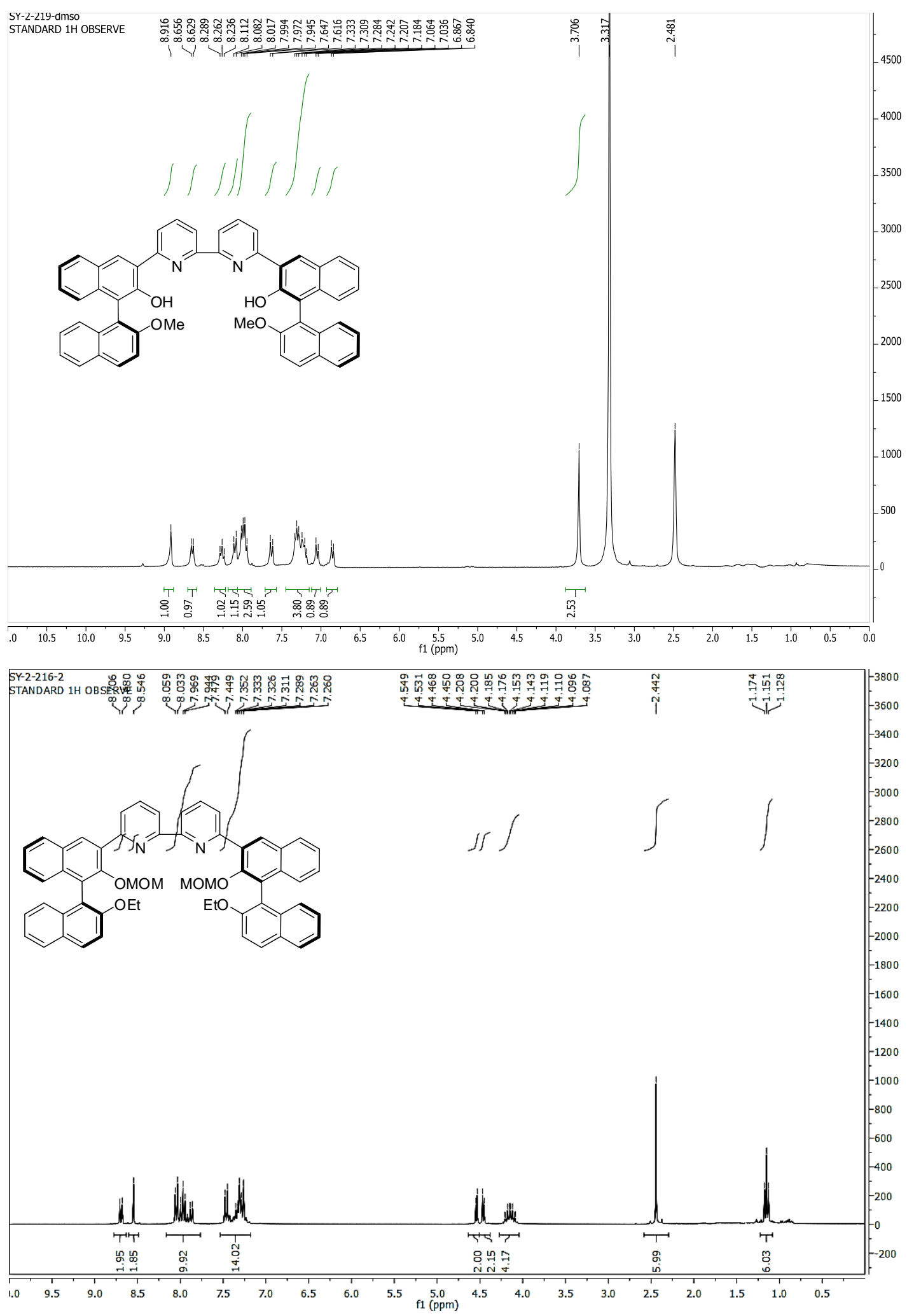




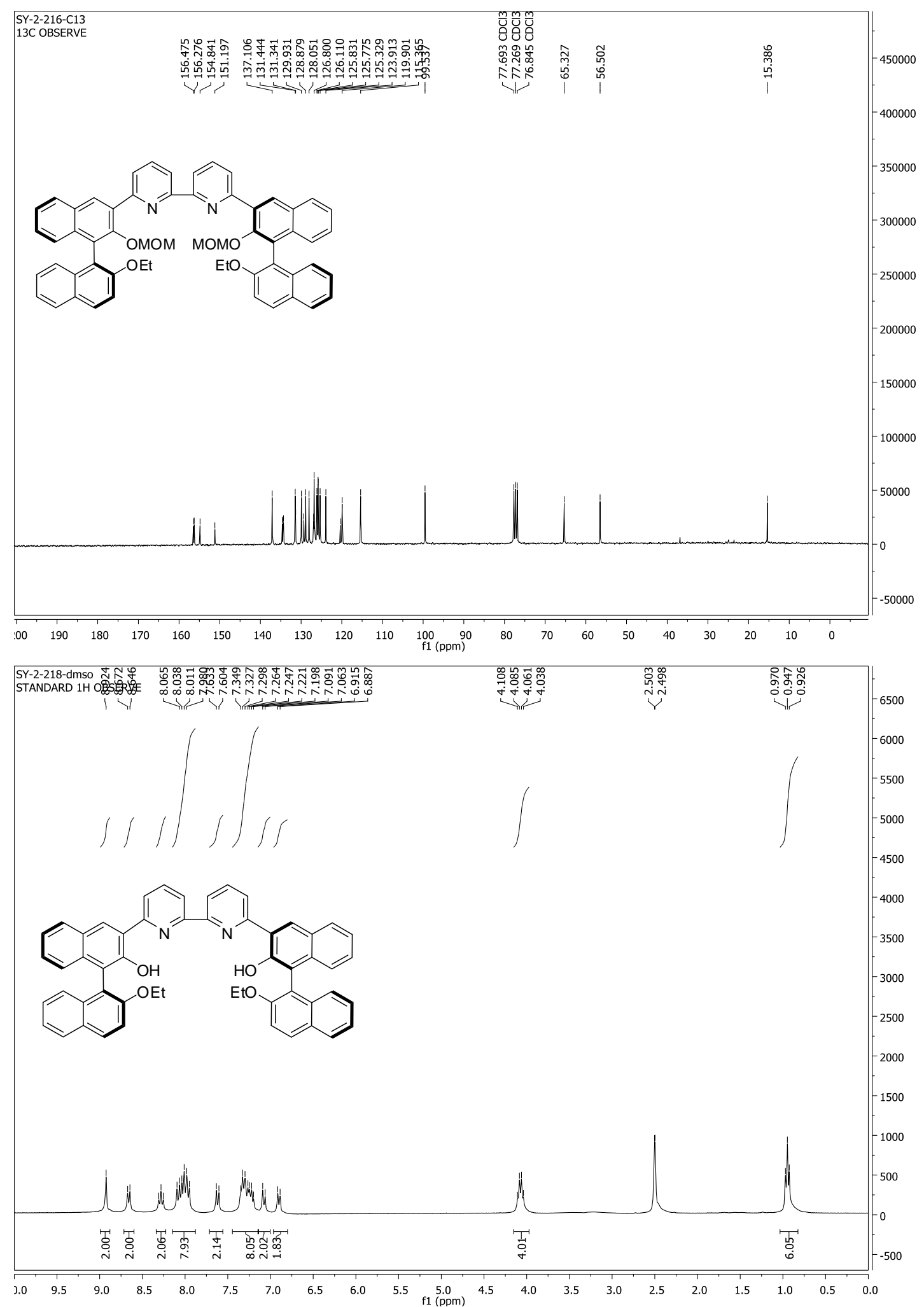



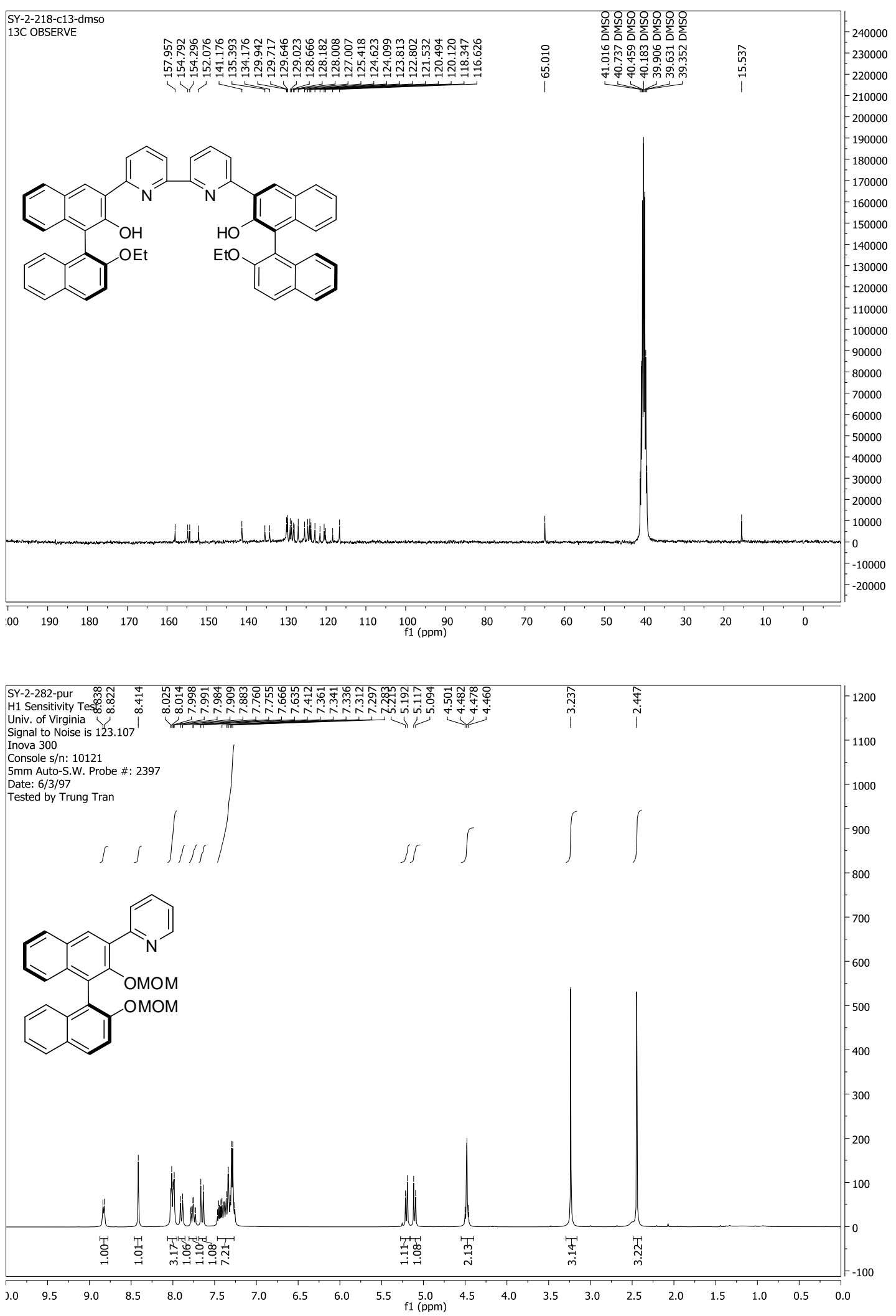

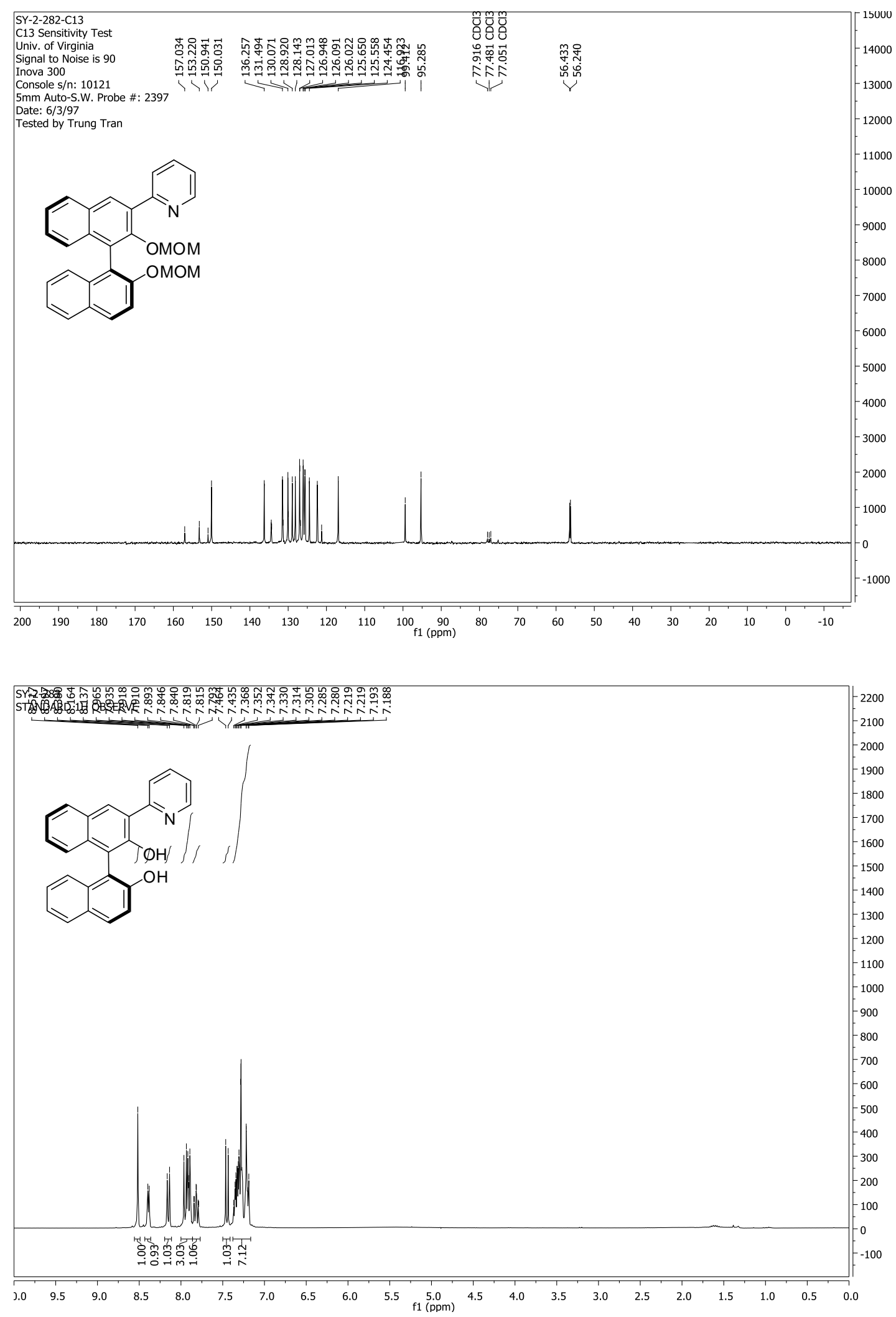

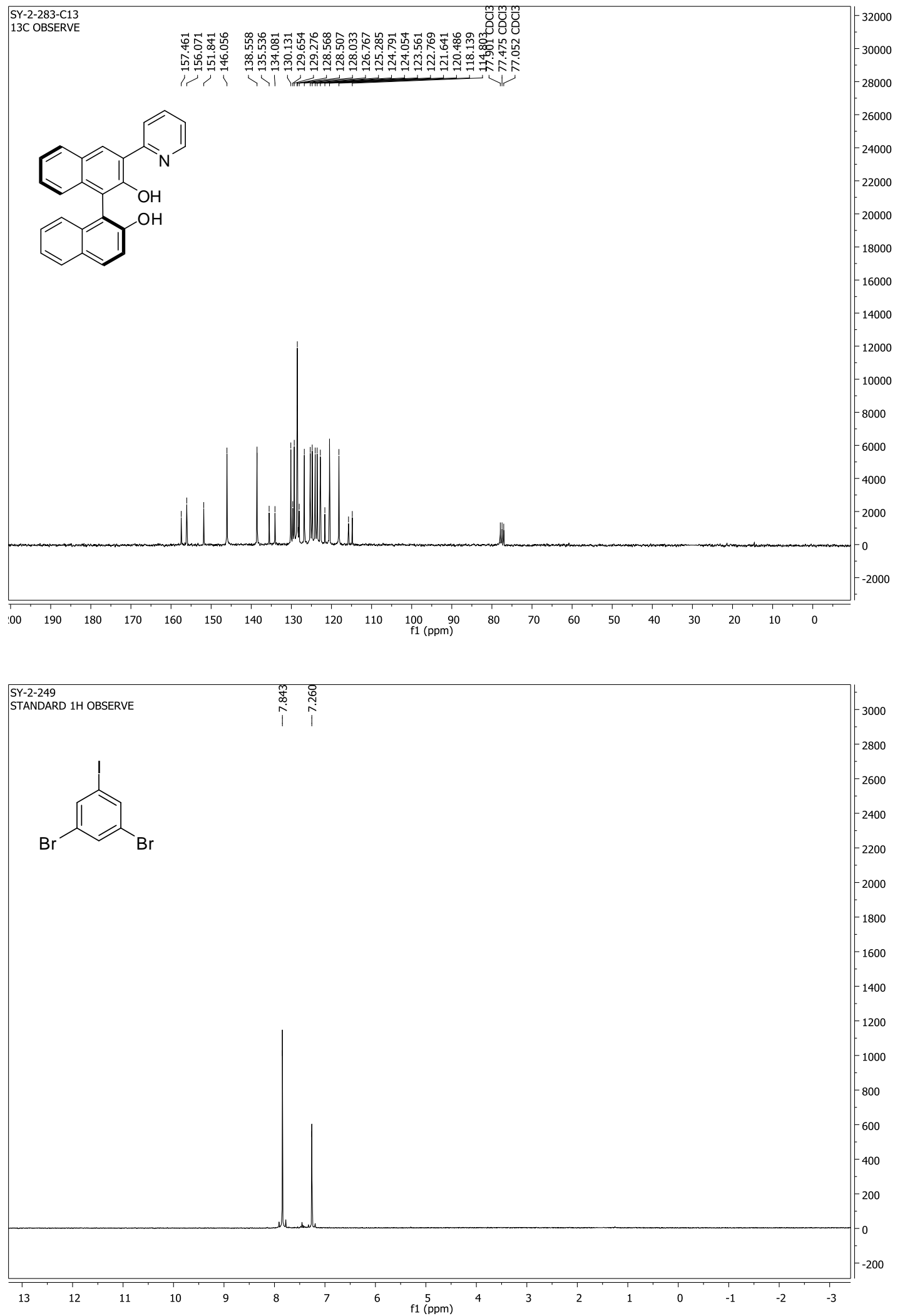


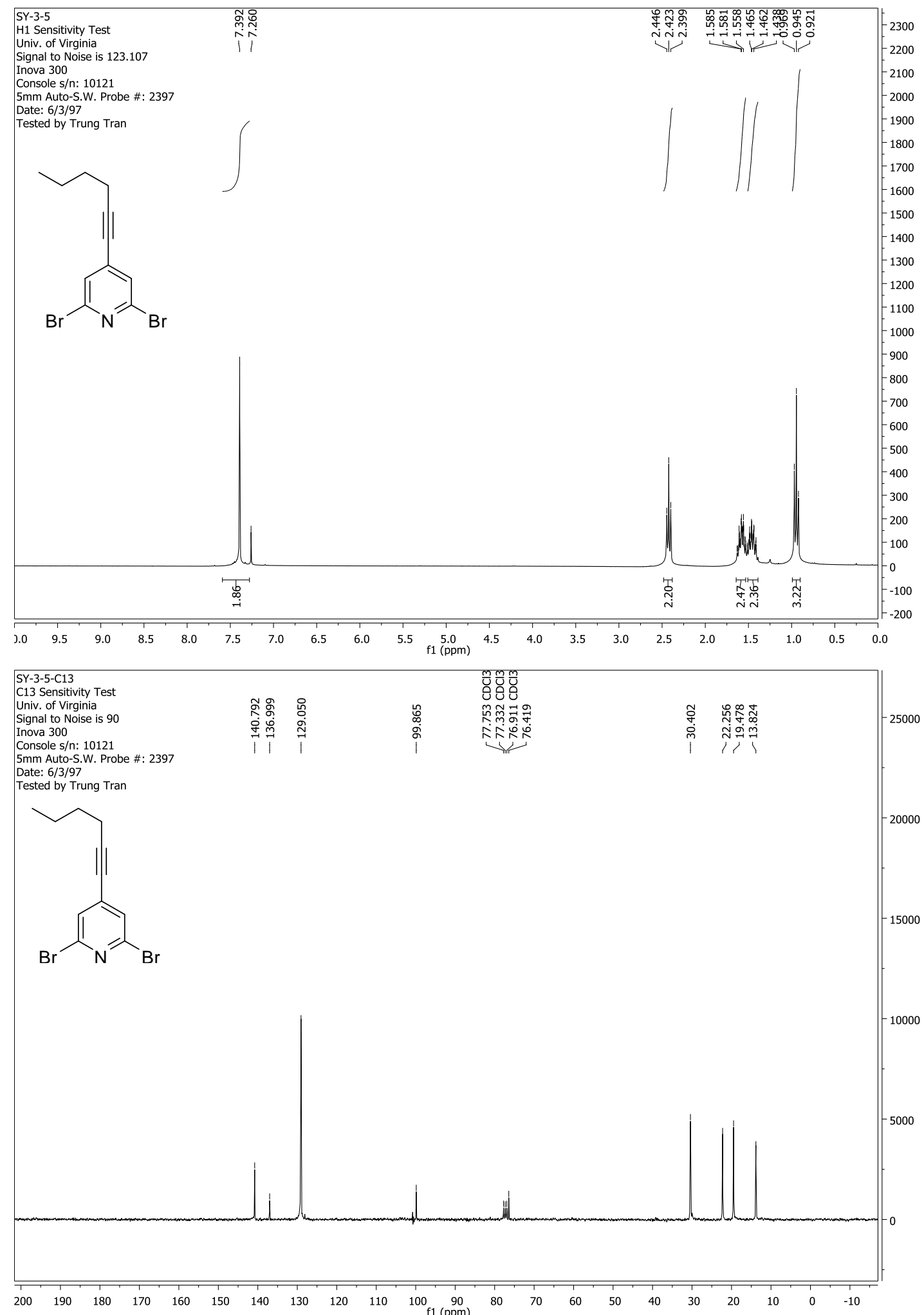



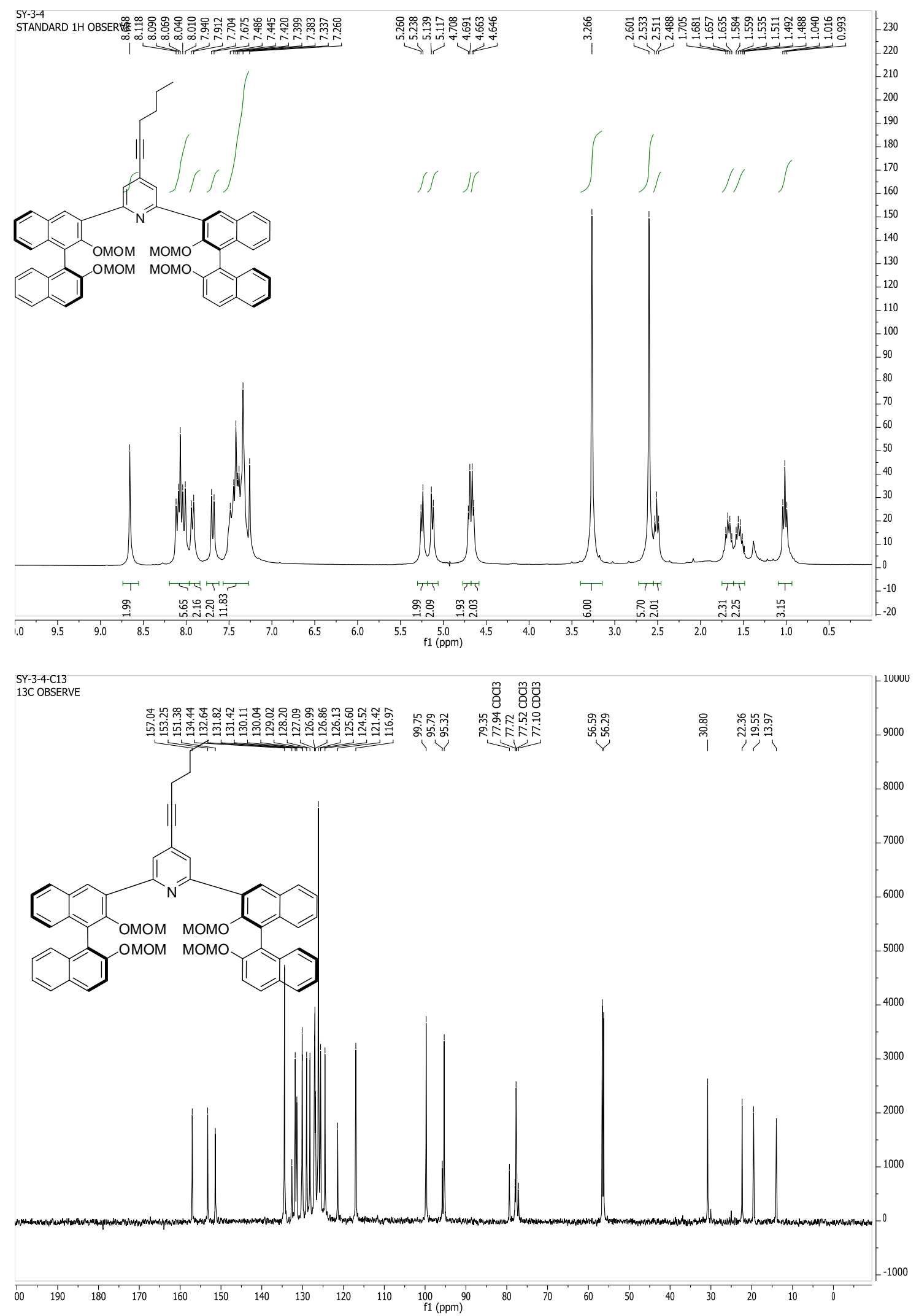

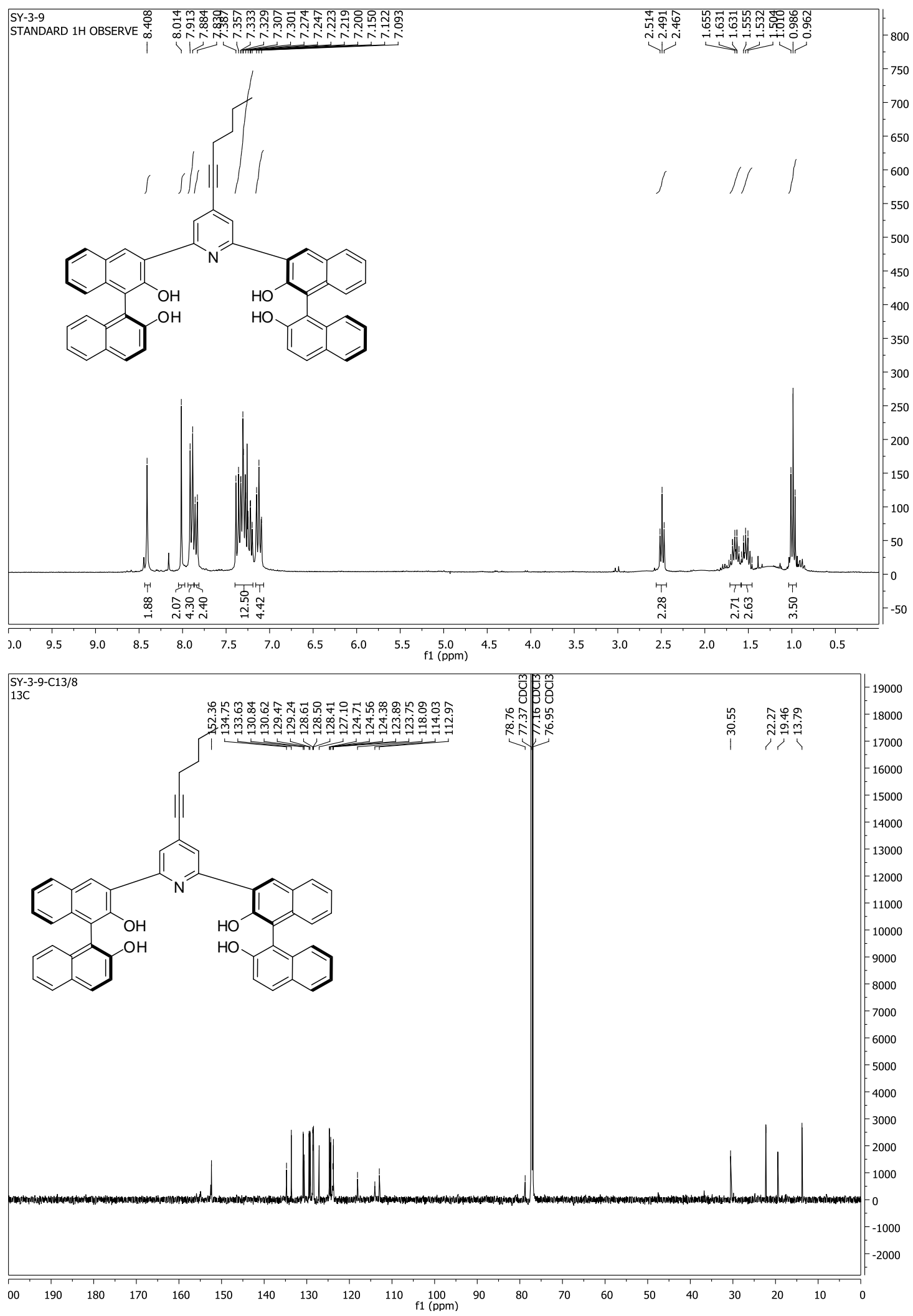

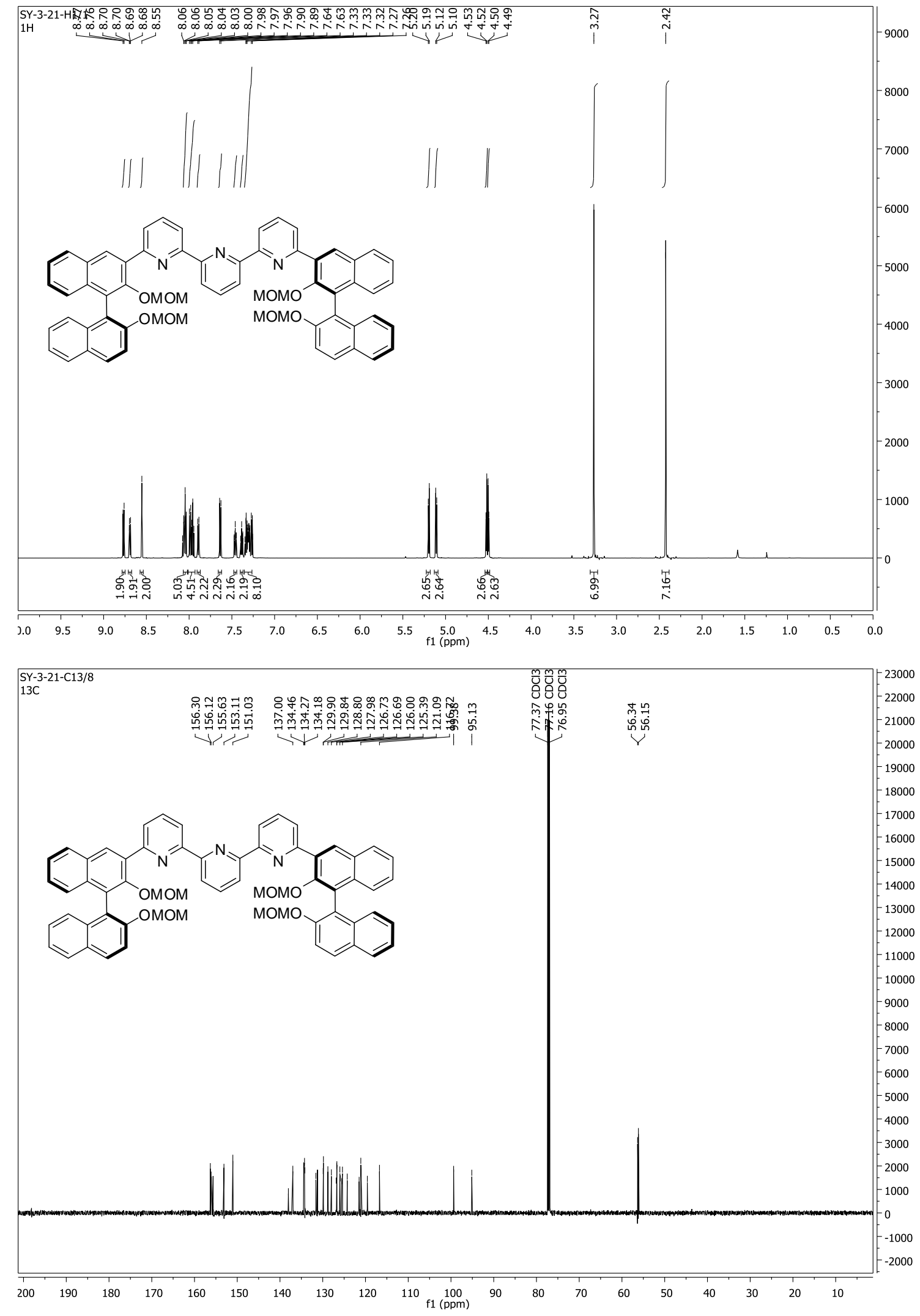

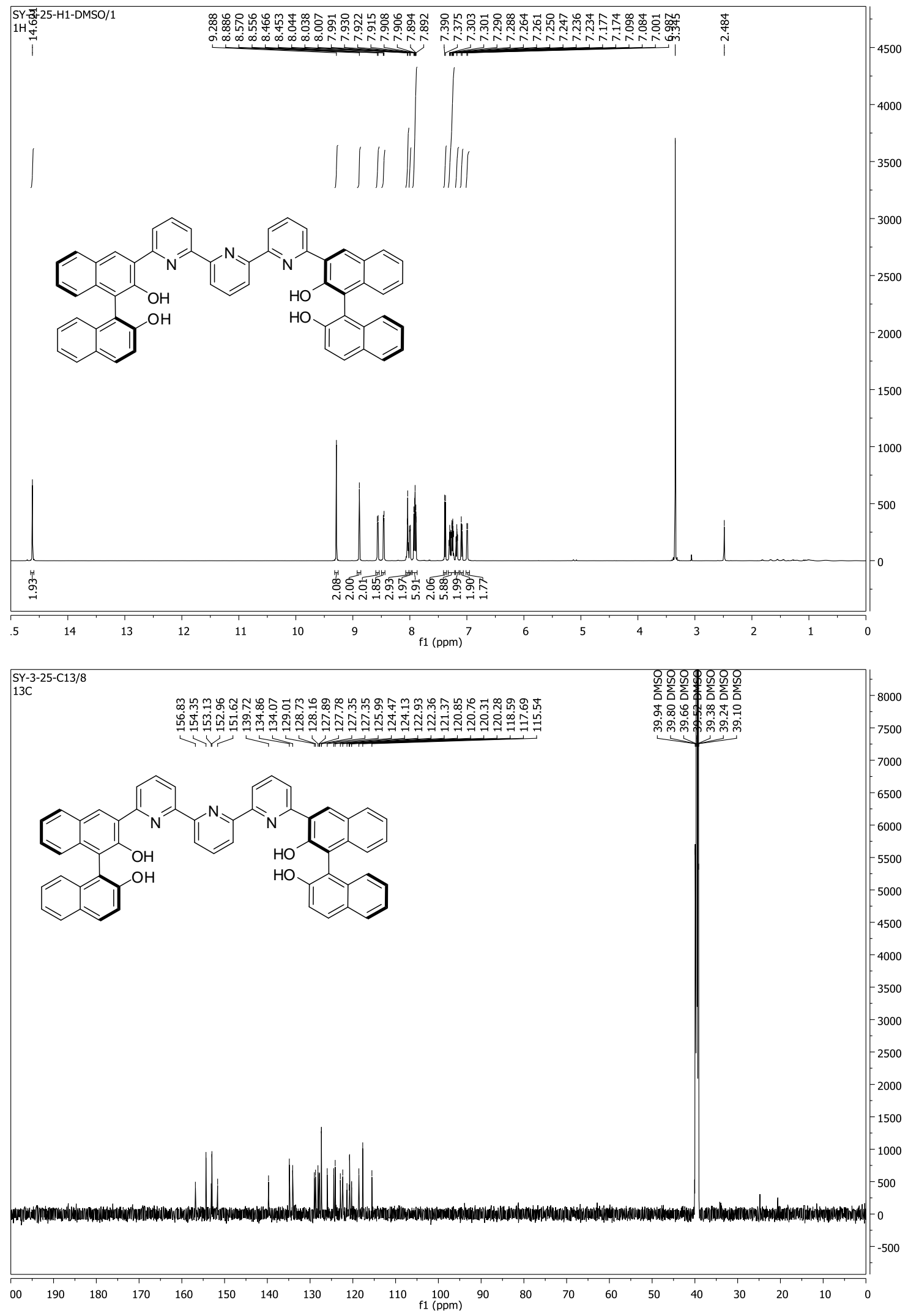


\section{Appendix II. Indolizine-Terpyridine Compound}

\section{Preparation and Characterization of Compounds}
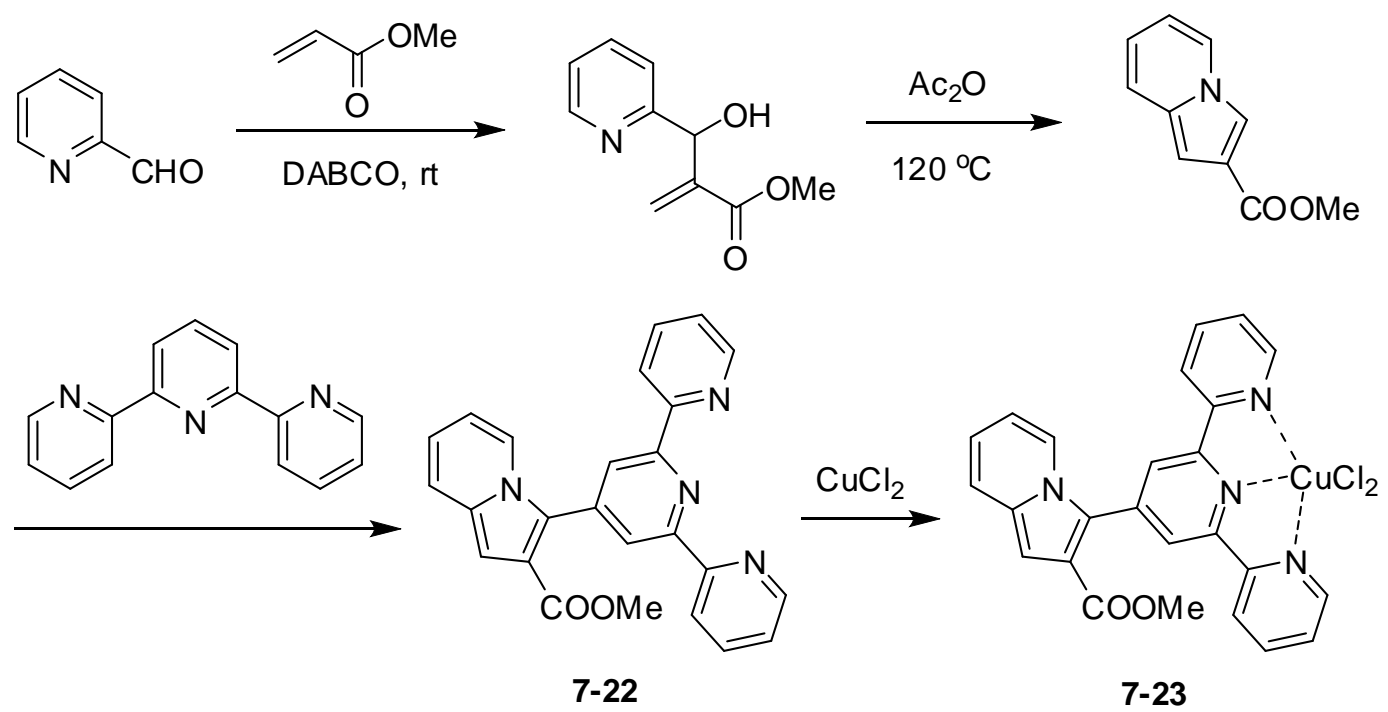

Preparation of methyl 2-(hydroxy(pyridin-2-yl)methyl)acrylate. A solution of methyl acrylate $(2.50 \mathrm{~g}, 0.029 \mathrm{~mol}), \mathrm{DABCO}(0.15 \mathrm{~g}, 1.34 \mathrm{mmol})$ and pyridine-2carbalerhyde $(2.95 \mathrm{~g}, 0.028 \mathrm{mmol})$ in $\mathrm{CHCl}_{3}(2 \mathrm{~mL})$ was allowed to stand at room temperature for $3 \mathrm{~d}$. The solvent was evaporated and the crude product purified by flash chromatography on silica gel eluted with EtOAc to afford a colorless oil (5.08 g) in 94\% yield. ${ }^{1} \mathrm{H}$ NMR $\left(300 \mathrm{MHz}, \mathrm{CDCl}_{3}\right) \delta 3.68(\mathrm{~s}, 3 \mathrm{H}), 4.83(\mathrm{~s}, \mathrm{br}, 1 \mathrm{H}), 5.61(\mathrm{~d}, \mathrm{~J}=5.4 \mathrm{~Hz}$, 1H), $5.90(\mathrm{~s}, 1 \mathrm{H}), 6.33(\mathrm{~s}, 1 \mathrm{H}), 7.15(\mathrm{t}, \mathrm{J}=6.0 \mathrm{~Hz}, 1 \mathrm{H}), 7.36(\mathrm{~d}, \mathrm{~J}=7.2 \mathrm{~Hz}, 1 \mathrm{H}), 7.55-$ $7.60(\mathrm{~m}, 1 \mathrm{H}), 8.48(\mathrm{~d}, \mathrm{~J}=4.8 \mathrm{~Hz}, 1 \mathrm{H})$.

Preparation of methyl indolizine-2-carboxylate. The hydroxyl precursor methyl 2(hydroxy(pyridin-2-yl)methyl)acrylate (1g, $5.2 \mathrm{mmol})$ was heated in $\mathrm{Ac}_{2} \mathrm{O}(5 \mathrm{~mL})$ at 110 
${ }^{\circ} \mathrm{C}$ for $2 \mathrm{~h}$. The cooled mixture was poured in to aq. NaHCO3-ice and stirred for $0.5 \mathrm{H}$. The mixture was then extracted with $\mathrm{Et}_{2} \mathrm{O}(3 \times 30 \mathrm{~mL})$, washed with brine. The solvent was evaporated and the residue was purified by flash chromatography on silica eluted with hexane-EtOAc (3:1) to afford the desired product as yellowish crystal in $50 \%$ yield. ${ }^{1} \mathrm{H}$ NMR $\left(300 \mathrm{MHz}, \mathrm{CDCl}_{3}\right) \delta 3.88(\mathrm{~s}, 3 \mathrm{H}), 6.52(\mathrm{t}, \mathrm{J}=6.6 \mathrm{~Hz}, 1 \mathrm{H}), 6.67(\mathrm{t}, \mathrm{J}=7.8 \mathrm{~Hz}$ 1H), $6.81(\mathrm{~s}, 1 \mathrm{H}), 7.35(\mathrm{~d}, \mathrm{~J}=9.3 \mathrm{~Hz}), 7.79(\mathrm{~s}, 1 \mathrm{H}), 7.85(\mathrm{~d}, \mathrm{~J}=7.2 \mathrm{~Hz}, 1 \mathrm{H})$.

\section{Preparation of methyl 3-([2,2':6',2''-terpyridin $\left.]-4^{\prime}-y l\right)$ indolizine-2-carboxylate.}

Under nitrogen, to a $10 \mathrm{~mL}$ flame-dried Schlenk flask was loaded methyl indolizine-2carboxylate (43.8 mg, $0.25 \mathrm{mmol}), 4^{\prime}$ '-chloro-2,2':6',2"'-terpyridine (133.9 mg, 0.5 $\mathrm{mmol}), \mathrm{Pd}(\mathrm{OAc})_{2}(2.8 \mathrm{mg}, 0.0125 \mathrm{mmol})$, tricyclohexylphosphine $(0.025 \mathrm{mmol}, 7 \mathrm{mg})$, $\mathrm{Cs}_{2} \mathrm{CO}_{3}(0.75 \mathrm{mmol}, 244 \mathrm{mg})$, toluene $(0.5 \mathrm{~mL})$. The reaction mixture was stirred under reflux for overnight. After it cooled to room temperature, $10 \mathrm{~mL}$ water was added and the mixture was extracted with EtOAc $(3 \times 20 \mathrm{~mL})$. The combined organic layer was washed with brine $(20 \mathrm{~mL})$ and dried over anhydrous $\mathrm{Na}_{2} \mathrm{SO}_{4}$. After evaporation of the solvent, the residue was purified by column chromatography on silica gel eluted with hexanes/ethyl acetate (2/1) to afford desired product as a yellow solid in 56\% yield (60 mg). ${ }^{1} \mathrm{H}$ NMR (300 MHz, $\left.\mathrm{CDCl}_{3}\right) \delta 3.73(\mathrm{~s}, 3 \mathrm{H}), 6.51(\mathrm{t}, \mathrm{J}=6.3 \mathrm{~Hz}, 1 \mathrm{H}), 6.73(\mathrm{t}, \mathrm{J}=7.8$ $\mathrm{Hz}, 1 \mathrm{H}), 7.01(\mathrm{~s}, 1 \mathrm{H}), 7.31(\mathrm{t}, \mathrm{J}=6.3 \mathrm{~Hz}, 2 \mathrm{H}), 7.42(\mathrm{~d}, \mathrm{~J}=9.0 \mathrm{~Hz}, 1 \mathrm{H}), 7.84-7.94(\mathrm{~m}$, $3 \mathrm{H}), 8.62-8.70(\mathrm{~m}, 6 \mathrm{H}) .{ }^{13} \mathrm{C} \mathrm{NMR}\left(75 \mathrm{MHz}, \mathrm{CDCl}_{3}\right) \delta 51.7,102.5,113.1,118.4,119.1$, 120.6, 121.5, 123.1, 124.2, 132.9, 137.1, 141.1, 149.4, 156.1, 156.2, 165.4. HRMS Calcd 
for $\mathrm{C}_{25} \mathrm{H}_{19} \mathrm{~N}_{4} \mathrm{O}_{2}\left(\mathrm{MH}^{+}\right)$: 407.1508. Found: 407.1506. m.p. $225{ }^{0} \mathrm{C}$.

Preparation of compound 7-23. 7-22 $(53.2 \mathrm{mg}, 0.13 \mathrm{mmol})$ and $\mathrm{CuCl}_{2} \cdot 2 \mathrm{H}_{2} \mathrm{O}$ (26.6 $\mathrm{mg}, 0.16 \mathrm{mmol})$ in methanol $(10 \mathrm{~mL})$ at room temperature for about $0.5 \mathrm{~h}$. The reaction was monitored by TLC. After the reaction was complete, the solution was concentrated and water $(2 \mathrm{~mL})$ was added to generate precipitate. The solid was collected through filtration, washed with water $(1 \mathrm{~mL})$ and methanol (several drops) and then dried under vacuum.

\section{Fluorescent study}

Figure AII-1. Fluorescence spectra of 7-22 and 7-23 $\left[1 \times 10^{-5} \mathrm{M}\right.$ in $25 \mathrm{mM}$ HEPES (pH 7.46)]. $\left(\lambda_{\mathrm{exc}}=360 \mathrm{~nm}, \mathrm{slit}=2.0 / 2.0 \mathrm{~nm}\right)$

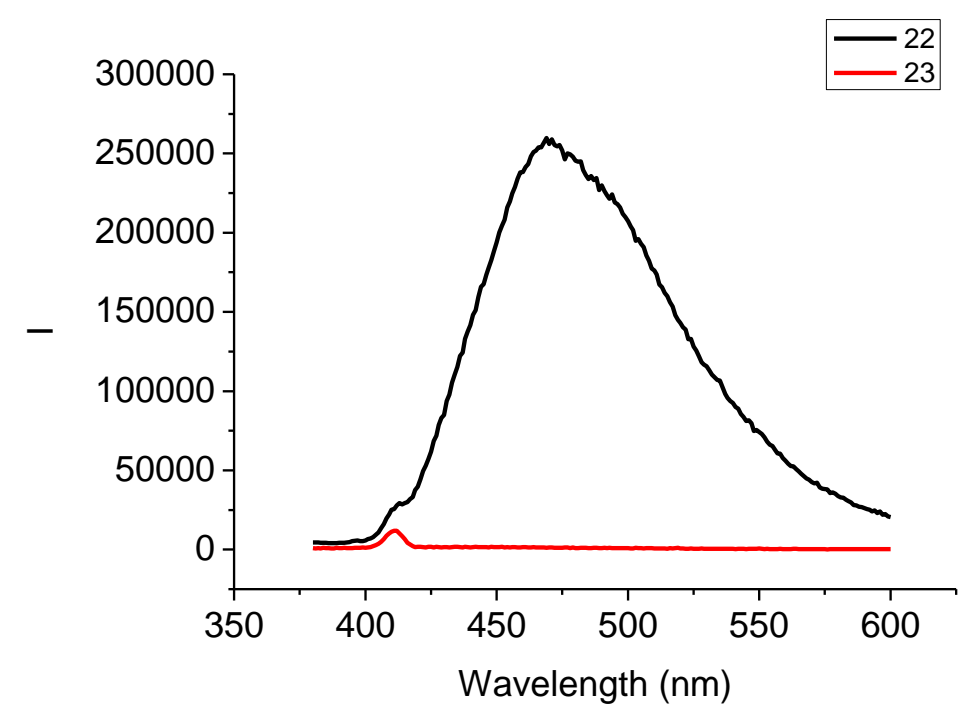

Figure AII-2. Fluorescence spectra of 7-23 $\left[1 \times 10^{-5} \mathrm{M}\right.$ in $25 \mathrm{mM}$ HEPES $\left.(\mathrm{pH} 7.46)\right]$ in the absence and presence of L-Histidine $(1,10,50,100,250,500 \mathrm{eq}) \cdot\left(\lambda_{\mathrm{exc}}=360 \mathrm{~nm}\right.$, slit 
$=3.0 / 3.0 \mathrm{~nm}$ )

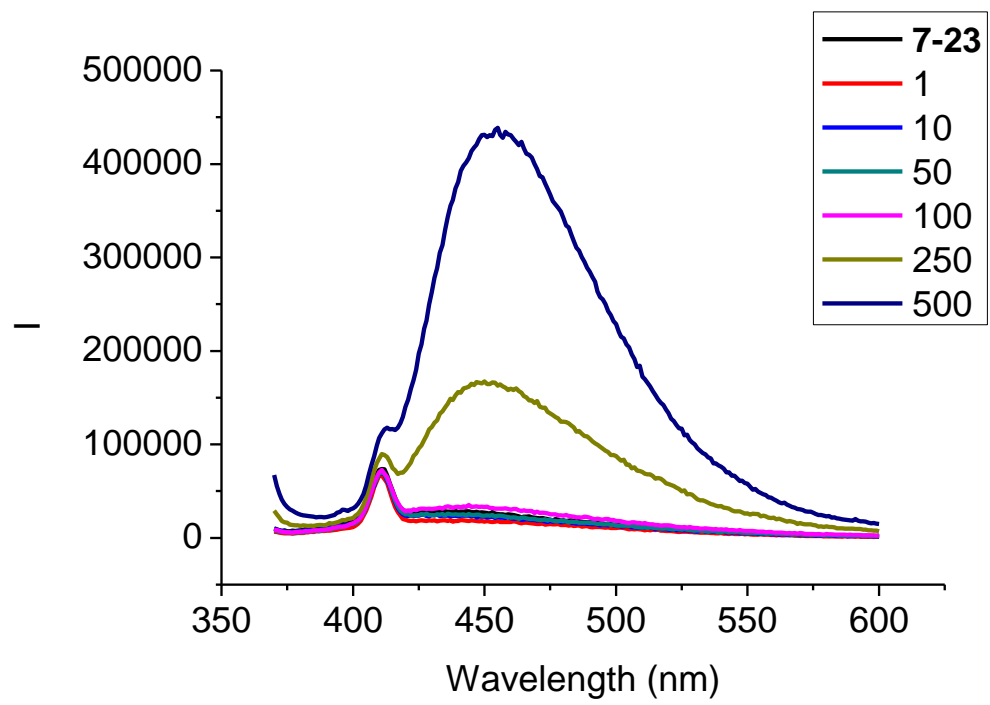

Figure AII-3. Fluorescence spectra of 7-23 $\left[1 \times 10^{-5} \mathrm{M}\right.$ in $25 \mathrm{mM}$ HEPES $\left.(\mathrm{pH} 7.46)\right]$ in the absence and presence of L-Cysteine $(1,10,50,100,250,500 \mathrm{eq}) .\left(\lambda_{\mathrm{exc}}=360 \mathrm{~nm}\right.$, slit $=3.0 / 3.0 \mathrm{~nm})$

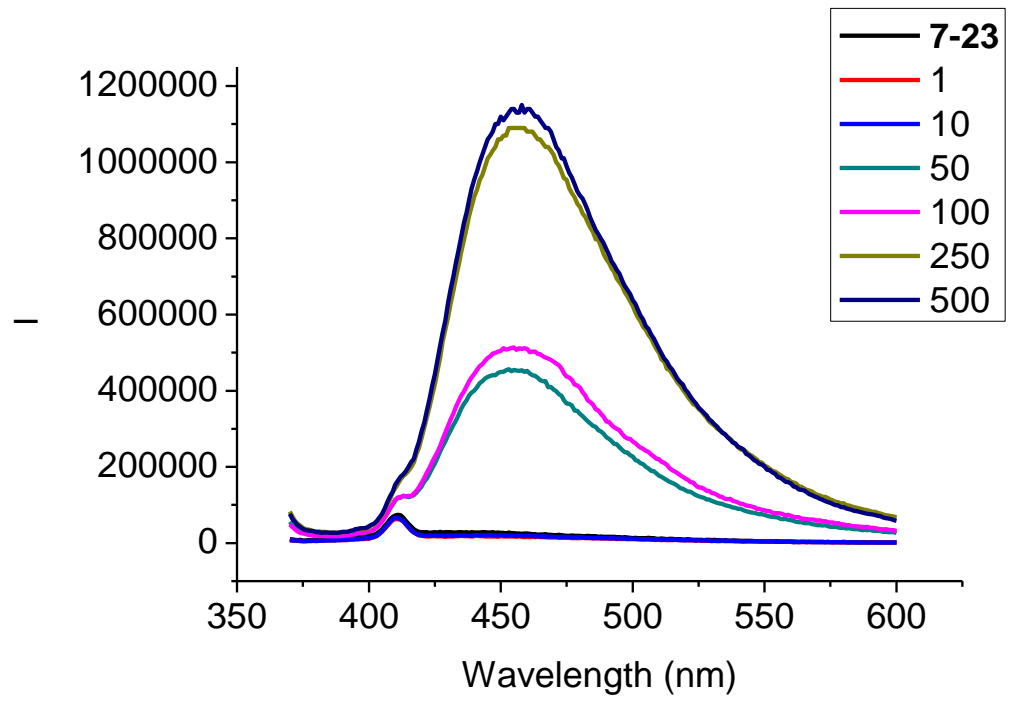

Figure AII-4. Fluorescence spectra of 7-23 $\left[1 \times 10^{-5} \mathrm{M}\right.$ in degassed $25 \mathrm{mM}$ HEPES $(\mathrm{pH}$ 
7.46)] in the absence and presence of L-Cysteine $\left(1,2,3,4,5,6,7,8,9,10\right.$ eq). ( $\lambda_{\text {exc }}=$ $360 \mathrm{~nm}$, slit $=3.0 / 3.0 \mathrm{~nm})$

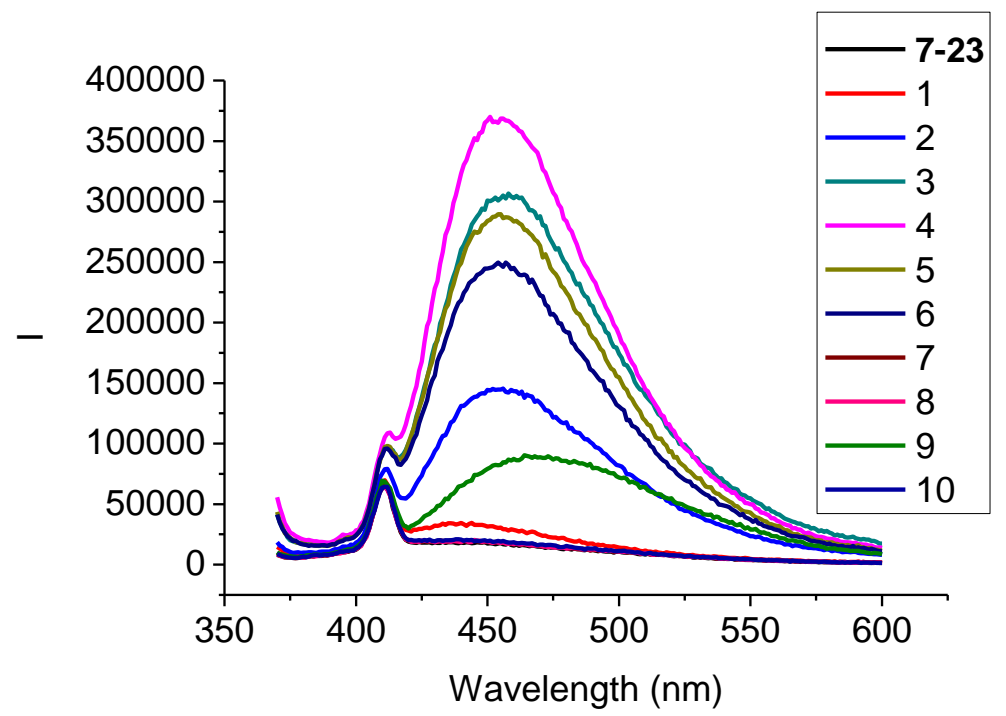

Figure AII-2. Fluorescence intensities of 7-22 $\left(1 \times 10^{-5} \mathrm{M}\right.$ in methanol) at $500 \mathrm{~nm}$ in the absence and presence of 1 eq of $\mathrm{NaCl}, \mathrm{KCl}, \mathrm{Cu}\left(\mathrm{NO}_{3}\right)_{2} \cdot 2.5 \mathrm{H}_{2} \mathrm{O}, \mathrm{Zn}\left(\mathrm{NO}_{3}\right)_{2} \cdot 6 \mathrm{H}_{2} \mathrm{O}$, $\mathrm{BaCl}_{2} \cdot 2 \mathrm{H}_{2} \mathrm{O}, \mathrm{CaCl}_{2}, \mathrm{MnCl}_{2} \cdot 4 \mathrm{H}_{2} \mathrm{O}, \mathrm{SnCl}_{4} \cdot 5 \mathrm{H}_{2} \mathrm{O}, \mathrm{Al}\left(\mathrm{NO}_{3}\right)_{3}, \mathrm{AgNO}_{3}, \mathrm{NiCl}_{2}, \mathrm{TbCl}_{3} \cdot 6 \mathrm{H}_{2} \mathrm{O}$, $\mathrm{CoCl}_{2}, \mathrm{CeCl}_{3} \cdot 7 \mathrm{H}_{2} \mathrm{O}, \mathrm{La}\left(\mathrm{NO}_{3}\right)_{3} \cdot 5 \mathrm{H}_{2} \mathrm{O}, \mathrm{FeCl}_{3} \cdot 6 \mathrm{H}_{2} \mathrm{O} .\left(\lambda_{\text {exc }}=360 \mathrm{~nm}\right.$, slit $\left.=3.0 / 3.0 \mathrm{~nm}\right)$ 


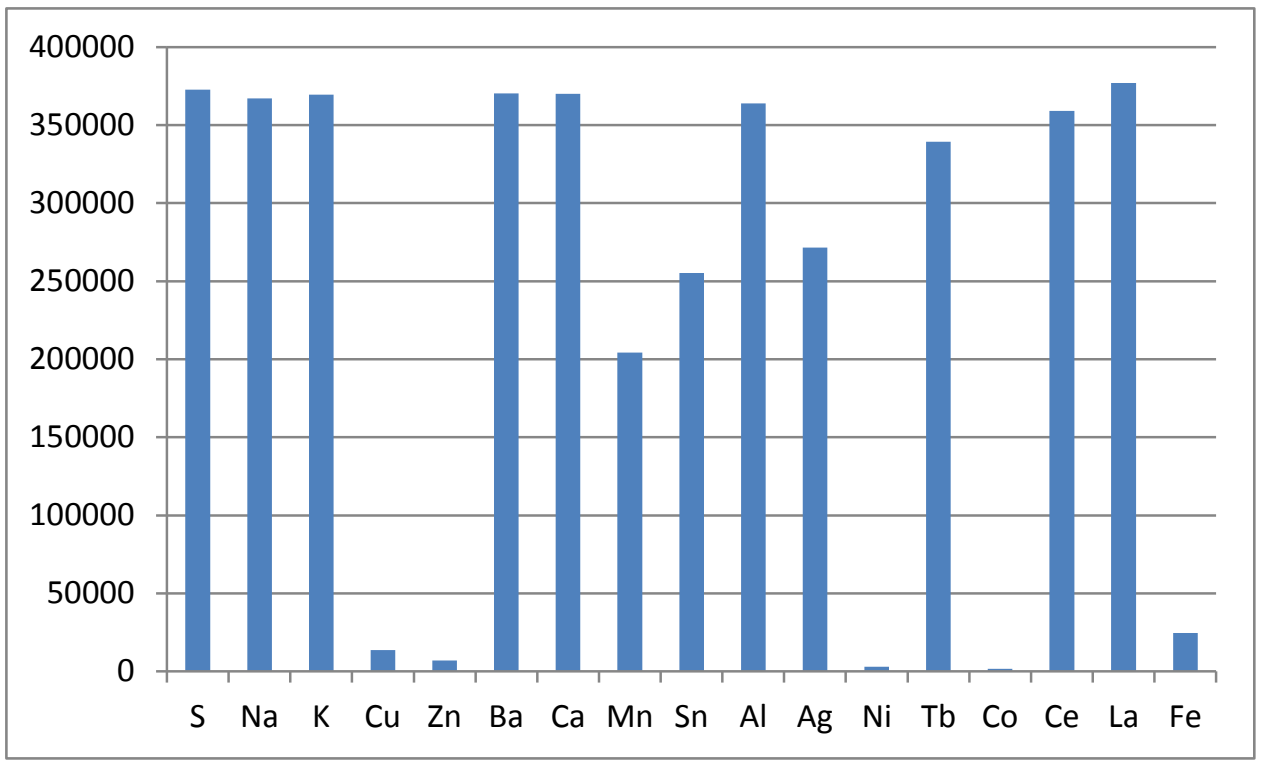

When 7-23 $\left[1 \times 10^{-5} \mathrm{M}\right.$ in $25 \mathrm{mM}$ HEPES (pH 7.46)] was treated with 10 eq of various amino acids, including Cysteine, Valine, Proline, Histidine, Lysine, Alanine, Serine, Arginine, Glutamic acid, Aspartic acid, Glysine, Leucine, no change in fluorescence was observed half an hour after preparation. Cysteine showed fluorescence enhancement right after preparation but the fluorescence diminished quickly. 


\section{NMR spectra}

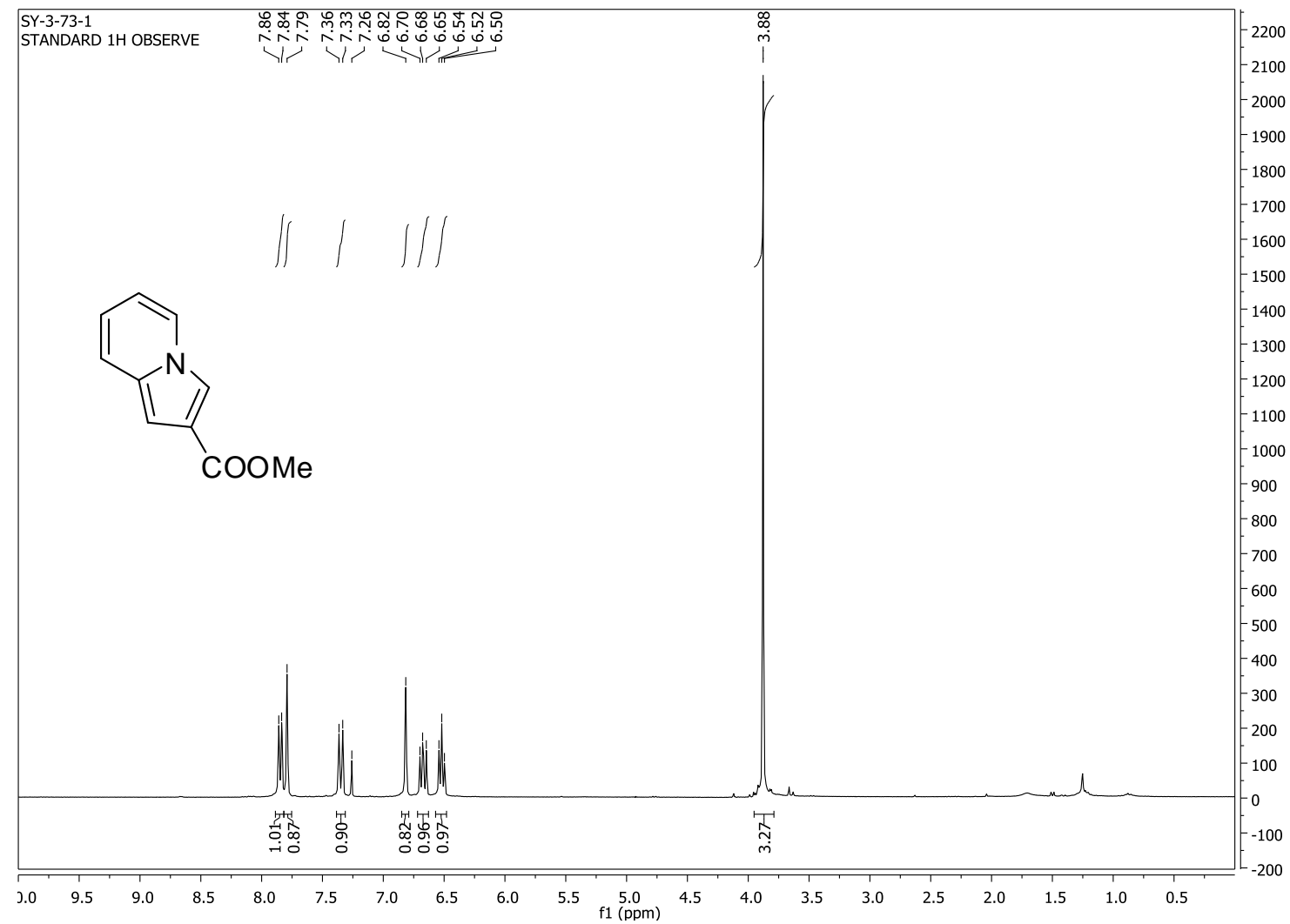



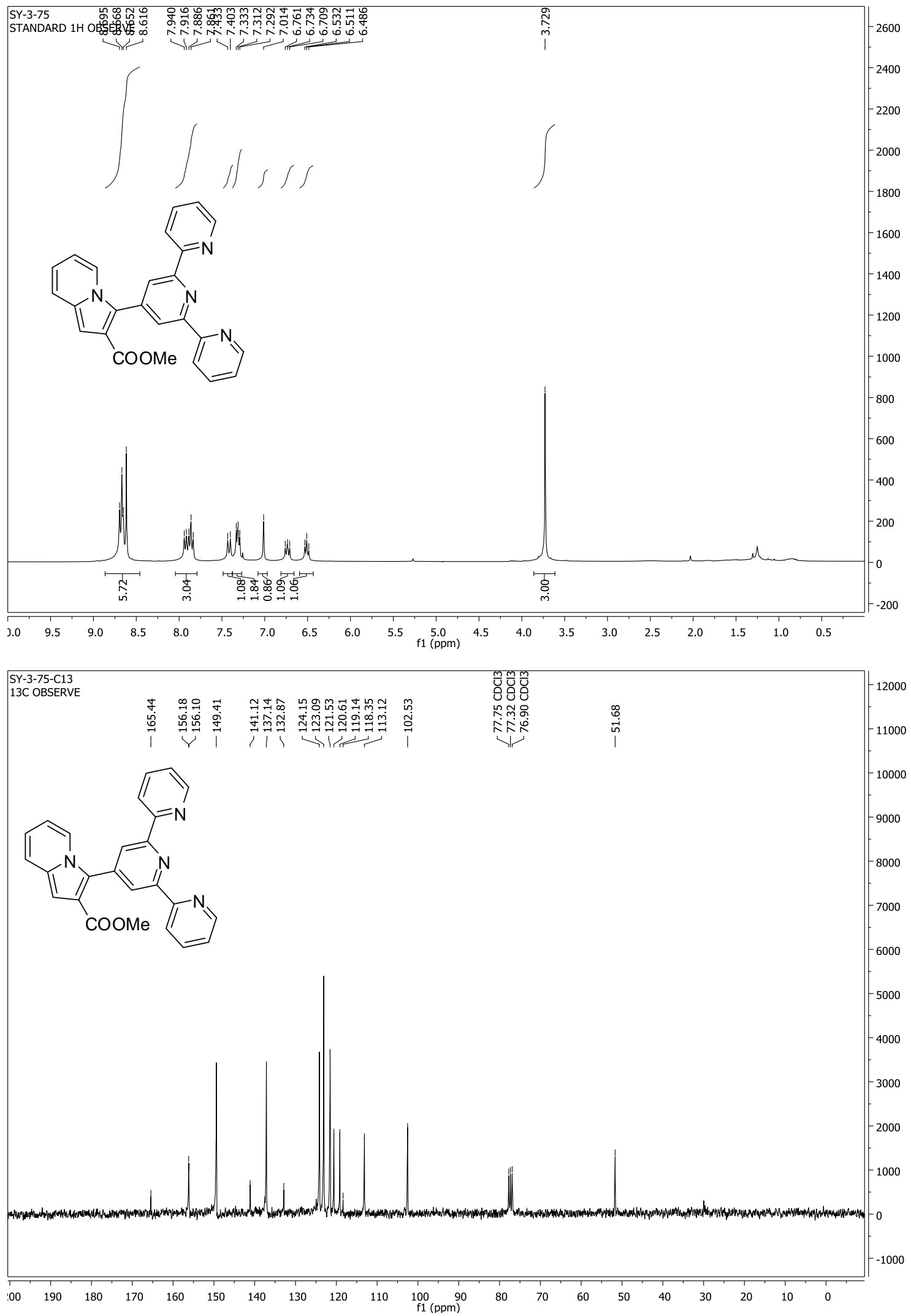


\section{Appendix III. Study of Aggregation-Induced Emission (AIE)}

\section{in Fluorescence Sensing}

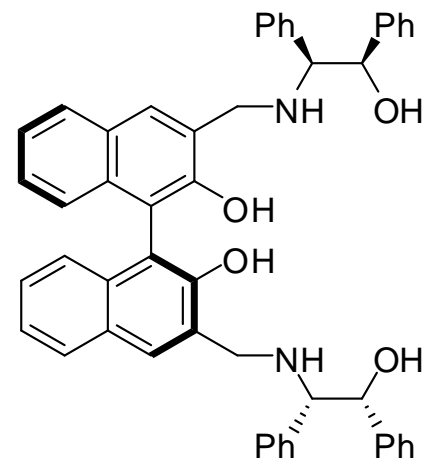

(S)-7-24

\section{Study of the fluorescent properties}

Figure AIII-1. Fluorescence spectra of $(\mathrm{S})-\mathbf{7 - 2 4}\left(2 \times 10^{-4} \mathrm{M}\right)$ in $\mathrm{THF} / \mathrm{H}_{2} \mathrm{O}(5 / 0,4 / 1,3 / 2$, $2 / 3,1 / 4,0.1 / 4.9) .\left(\lambda_{\mathrm{exc}}=341 \mathrm{~nm}\right.$, slit $\left.=2.0 / 2.0 \mathrm{~nm}\right)$

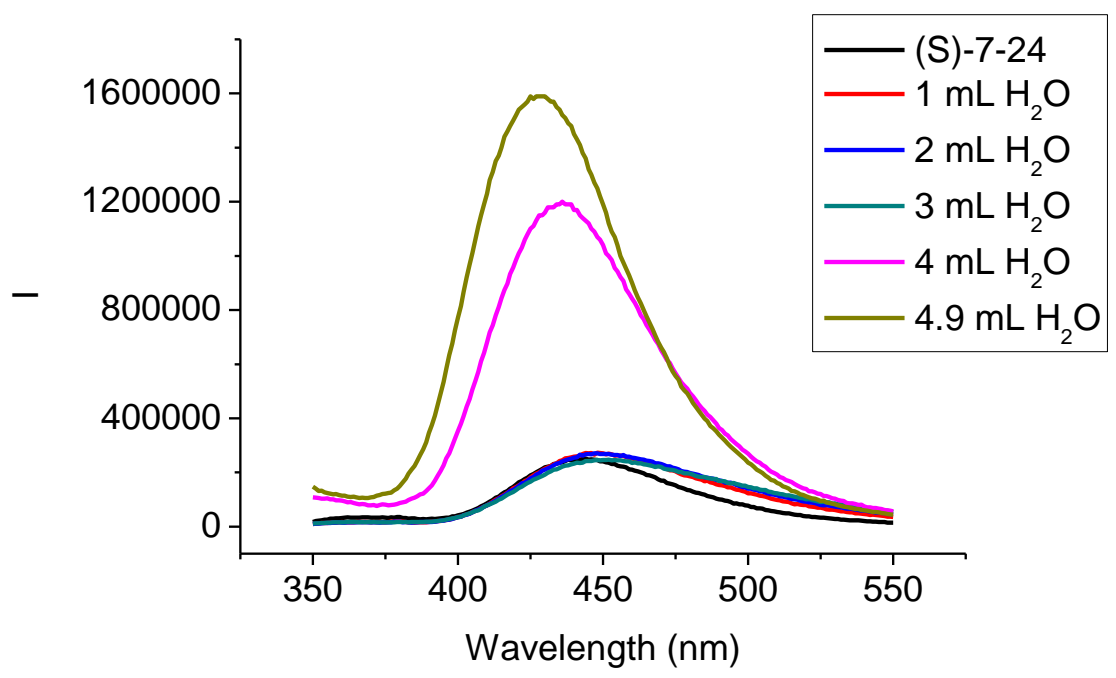

Figure AIII-2. Fluorescence spectra of (S)-7-24 $\left(2 \times 10^{-4} \mathrm{M}\right.$ in $\mathrm{THF} / \mathrm{H}_{2} \mathrm{O}$ 0.1/4.9) in the presence of 1 eq $\mathrm{CuCl}_{2} \cdot 2 \mathrm{H}_{2} \mathrm{O}, \mathrm{Zn}\left(\mathrm{NO}_{3}\right)_{2} \cdot 6 \mathrm{H}_{2} \mathrm{O}, \mathrm{FeCl}_{3} \cdot 6 \mathrm{H}_{2} \mathrm{O} \cdot\left(\lambda_{\text {exc }}=341 \mathrm{~nm}\right.$, slit $=2.0 / 2.0$ $\mathrm{nm})$ 


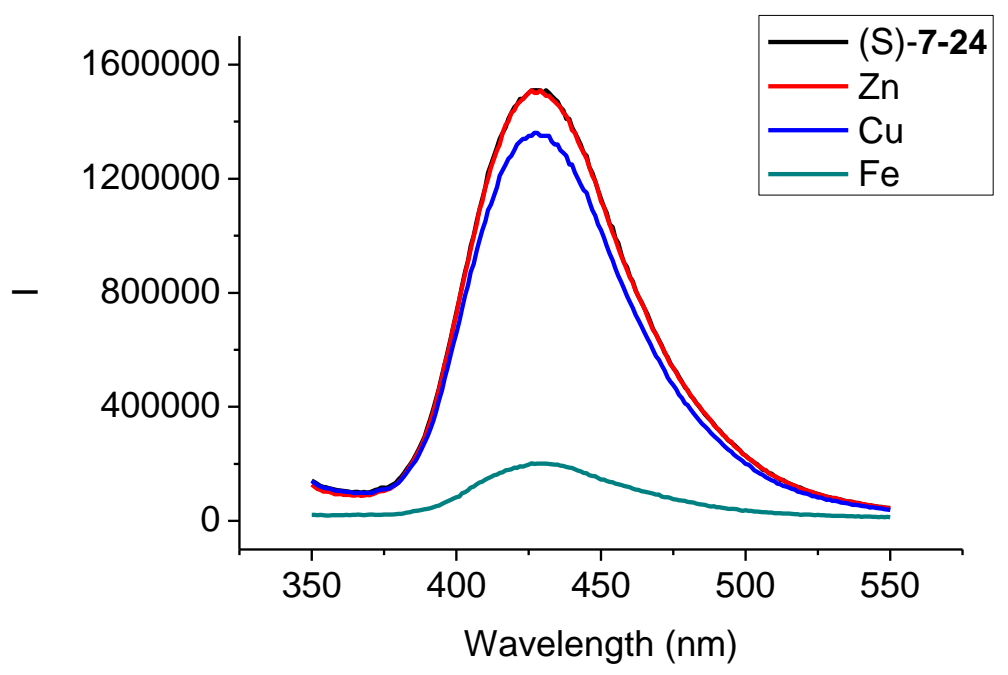

Figure AIII-3. Fluorescence spectra of (S)-7-24 $\left(1 \times 10^{-4} \mathrm{M}\right.$ in THF/ $\left.\mathrm{H}_{2} \mathrm{O} \quad 0.05 / 4.95\right)$, (S)7-24 +1 eq $\mathrm{FeCl}_{3} \cdot 6 \mathrm{H}_{2} \mathrm{O}$ and $(\mathrm{S})-\mathbf{7 - 2 4}+1$ eq $\mathrm{FeCl}_{3} \cdot 6 \mathrm{H}_{2} \mathrm{O}+\mathrm{Gly}(1,10,100$ eq $) \cdot\left(\lambda_{\text {exc }}=\right.$ $341 \mathrm{~nm}, \mathrm{slit}=2.0 / 2.0 \mathrm{~nm})$

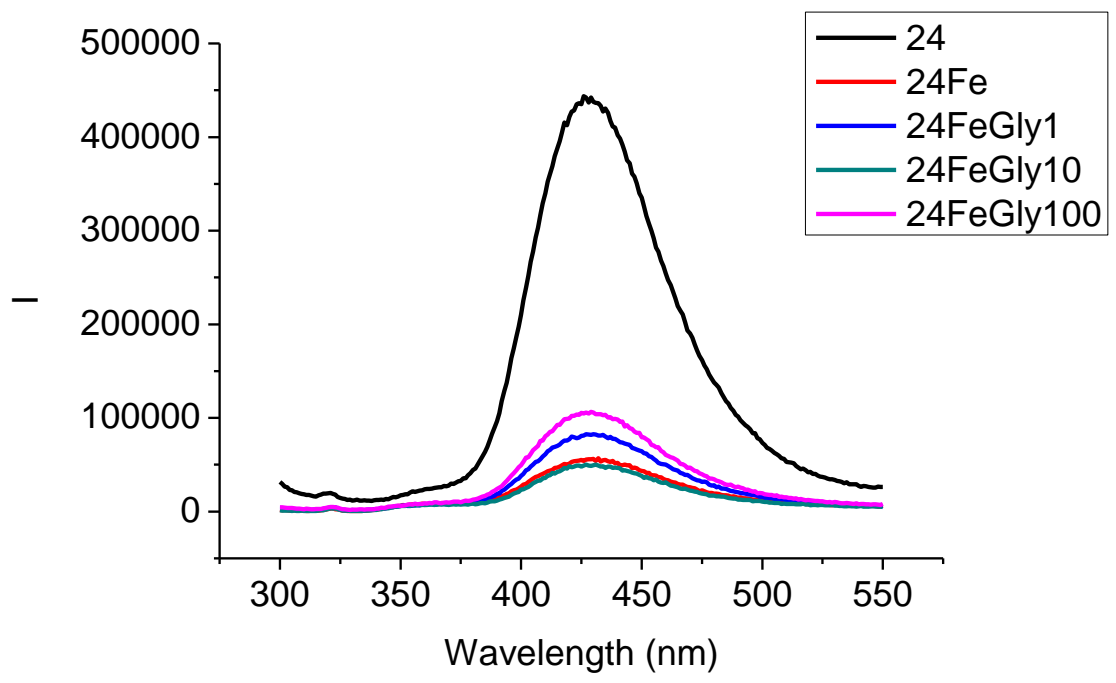

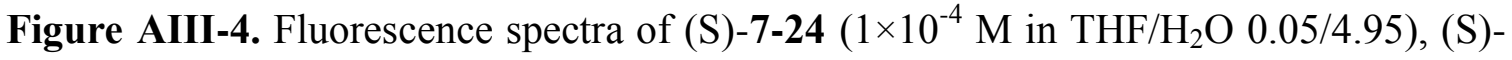
7-24 +1 eq $\mathrm{FeCl}_{3} \cdot 6 \mathrm{H}_{2} \mathrm{O}$ and $(\mathrm{S})-\mathbf{7 - 2 4}+1$ eq $\mathrm{FeCl}_{3} \cdot 6 \mathrm{H}_{2} \mathrm{O}+1$ eq Gly $+\mathrm{NaOH}(1,10,50$ eq $) \cdot\left(\lambda_{\text {exc }}=341 \mathrm{~nm}\right.$, slit $\left.=2.0 / 2.0 \mathrm{~nm}\right)$ 


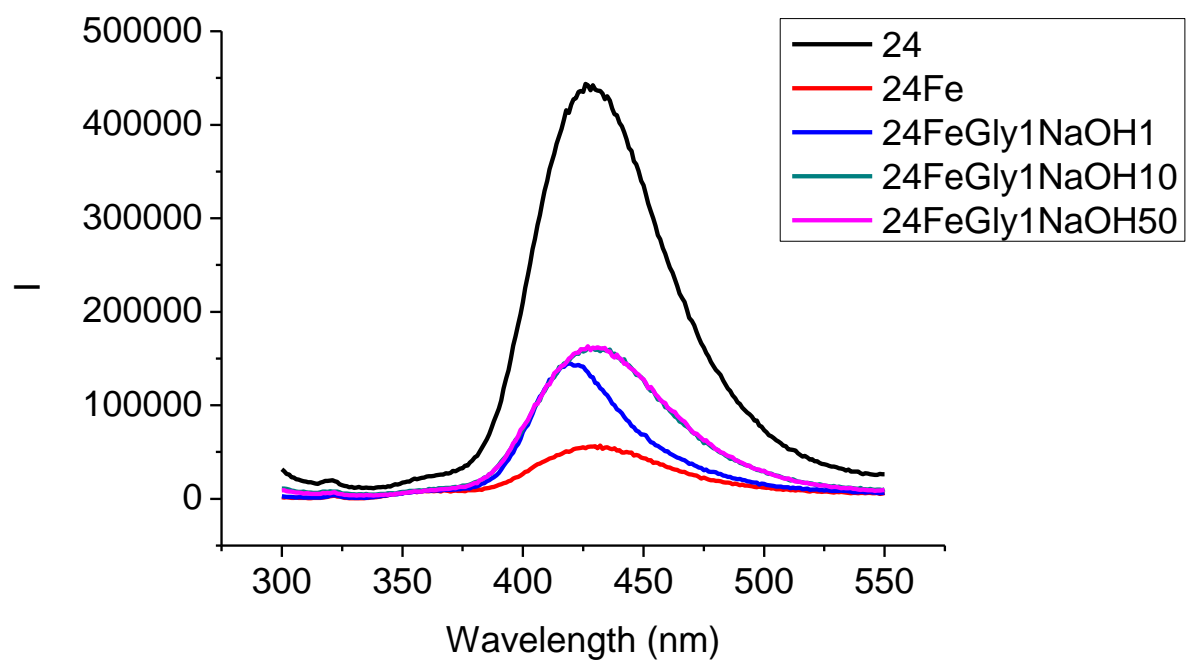

Figure AIII-3. Fluorescence spectra of (S)-7-24 $\left(1 \times 10^{-4} \mathrm{M}\right.$ in $\left.\mathrm{THF} / \mathrm{H}_{2} \mathrm{O} 0.05 / 4.95\right)$, (S)7-24 +1 eq $\mathrm{FeCl}_{3} \cdot 6 \mathrm{H}_{2} \mathrm{O}$ and $(\mathrm{S})-\mathbf{7 - 2 4}+1$ eq $\mathrm{FeCl}_{3} \cdot 6 \mathrm{H}_{2} \mathrm{O}+10$ eq Gly $+\mathrm{NaOH}(10,50$, $100 \mathrm{eq}) \cdot\left(\lambda_{\mathrm{exc}}=341 \mathrm{~nm}, \mathrm{slit}=2.0 / 2.0 \mathrm{~nm}\right)$

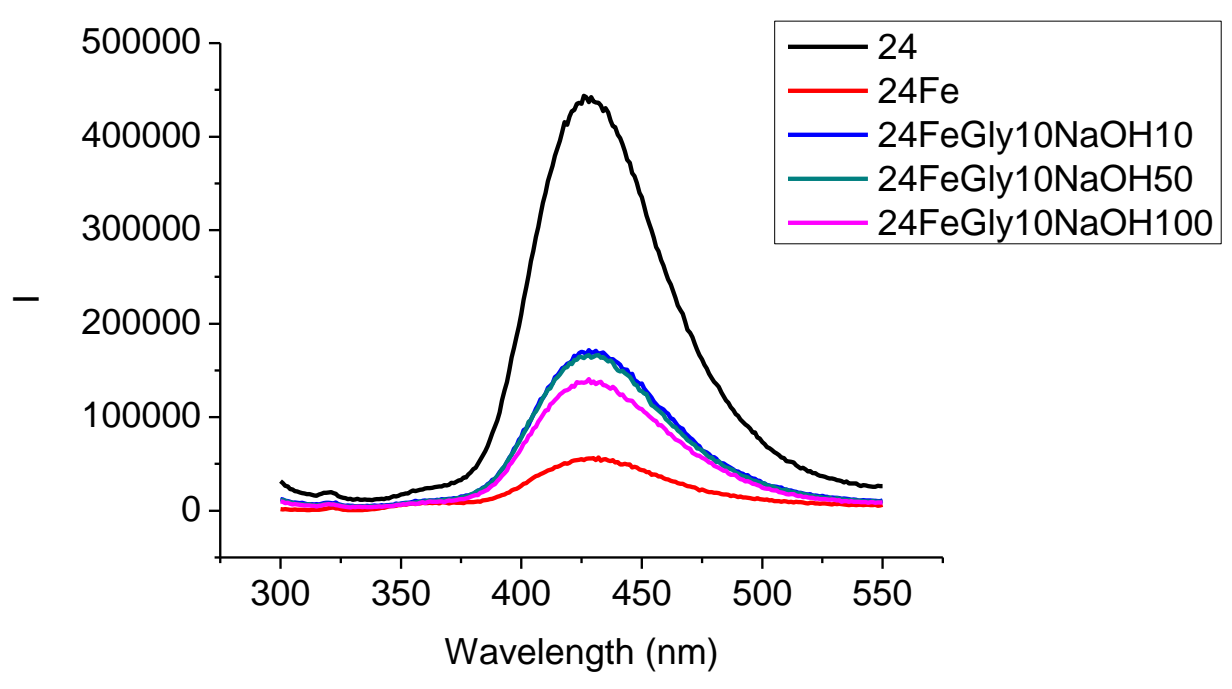




\section{Appendix IV. Near-Infrared (NIR) Dyes}

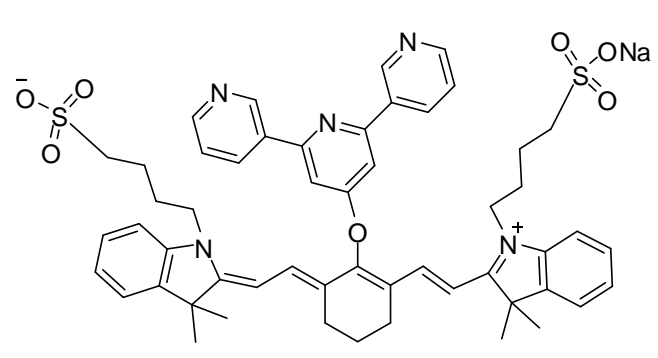

7-25

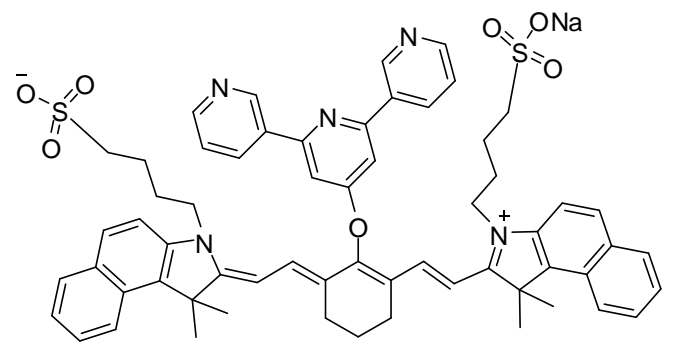

7-26

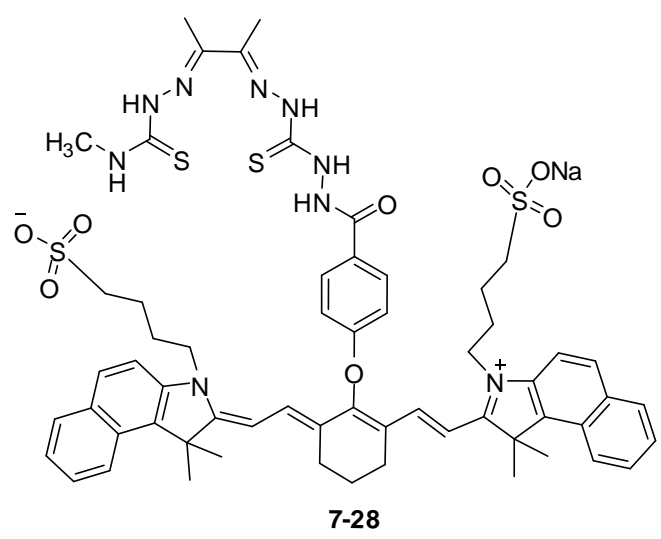

\section{Synthesis and characterization of compounds}

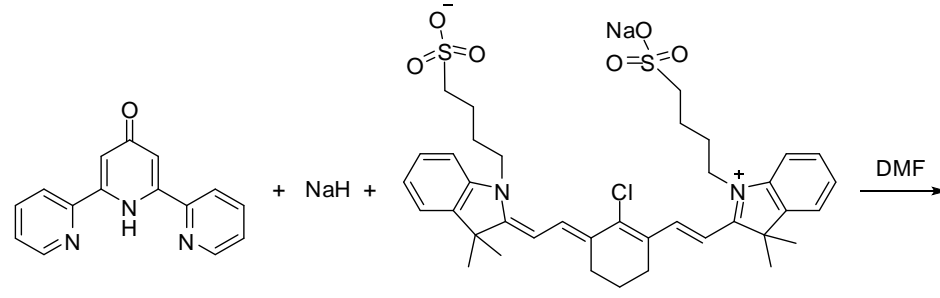

IR-783

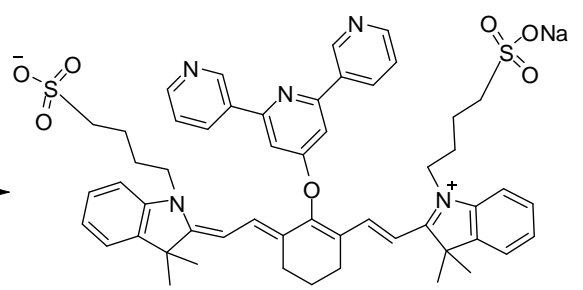

7-25

Preparation and characterization of compound 7-25. Under nitrogen, to a DMF (1.5 mL) solution of 6-bis(2-pyridyl)-4(1H)-pyridone $(24.9 \mathrm{mg}, 0.1 \mathrm{mmol})$ was added $\mathrm{NaH}(60 \%$ oil dispension, $4 \mathrm{mg}, 0.1 \mathrm{mmol})$ at $0{ }^{\circ} \mathrm{C}$. After $30 \mathrm{~min}$, DMF solution $(1 \mathrm{~mL})$ of IR-783 (37.5 mg, $0.05 \mathrm{mmol})$ was added to the reaction mixture. After $18 \mathrm{~h}$, the reaction was quenched with dry ice and the mixture as purified by preparative HPLC to afford 7-25 (25 mg) in 52\% yield. ${ }^{1} \mathrm{H}$ NMR (300 MHz, $\left.\left(\mathrm{CD}_{3}\right)_{2} \mathrm{SO}\right) \delta 1.26(\mathrm{~s}, 12 \mathrm{H}), 1.73$ 
(m, 8H), $2.00(\mathrm{t}, \mathrm{J}=6.3 \mathrm{~Hz}, 2 \mathrm{H}), 2.59(\mathrm{t}, 4 \mathrm{H}), 2.82(\mathrm{t}, \mathrm{J}=5.1 \mathrm{~Hz}, 4 \mathrm{H}), 4.14(\mathrm{t}, \mathrm{J}=7.5 \mathrm{~Hz}$, 4H), $6.32(\mathrm{~d}, \mathrm{~J}=14.4 \mathrm{~Hz}, 2 \mathrm{H}), 7.14(\mathrm{t}, \mathrm{J}=7.2 \mathrm{~Hz}, 2 \mathrm{H}), 7.27-7.43(\mathrm{~m}, 6 \mathrm{H}), 7.62(\mathrm{t}, \mathrm{J}=6.0$ $\mathrm{Hz}, 2 \mathrm{H}), 7.77(\mathrm{~d}, \mathrm{~J}=14.4 \mathrm{~Hz}, 2 \mathrm{H}), 8.11(\mathrm{t}, \mathrm{J}=8.1 \mathrm{~Hz}, 2 \mathrm{H}), 8.23(\mathrm{~s}, 2 \mathrm{H}), 8.72(\mathrm{~d}, \mathrm{~J}=7.2$ $\mathrm{Hz}, 2 \mathrm{H}), 8.82(\mathrm{~d}, \mathrm{~J}=4.2 \mathrm{~Hz}, 2 \mathrm{H})$.

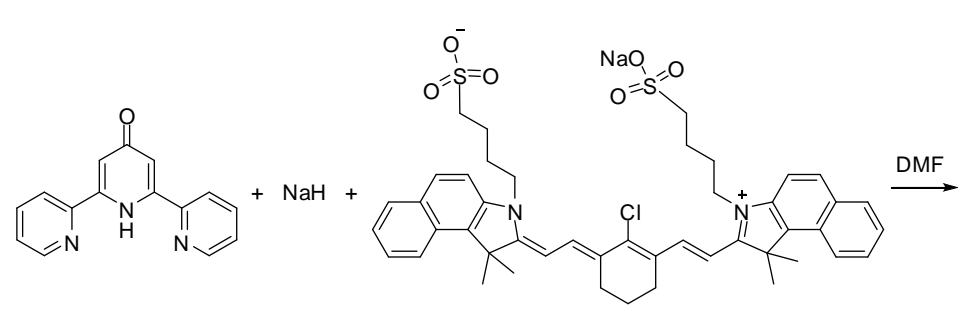

IR-820

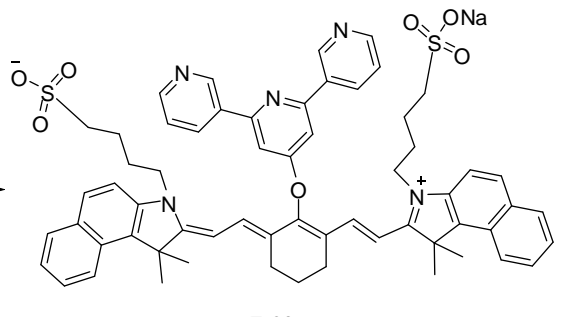

7-26

Preparation and characterization of compound 7-26. Under nitrogen, to a DMF (1.5 mL) solution of 6-bis(2-pyridyl)-4(1H)-pyridone $(24.9 \mathrm{mg}, 0.1 \mathrm{mmol})$ was added $\mathrm{NaH}(60 \%$ oil dispension, $4 \mathrm{mg}, 0.1 \mathrm{mmol})$ at $0{ }^{\circ} \mathrm{C}$. After $30 \mathrm{~min}$, DMF solution $(1 \mathrm{~mL})$ of IR-820 (42.5 mg, $0.05 \mathrm{mmol})$ was added to the reaction mixture. After $18 \mathrm{~h}$, the reaction was quenched with dry ice and the mixture as purified by preparative HPLC to afford 7-26 (15 mg) in 28\% yield. ${ }^{1} \mathrm{H}$ NMR (300 MHz, $\left.\left(\mathrm{CD}_{3}\right)_{2} \mathrm{SO}\right) \delta 1.54(\mathrm{~s}, 12 \mathrm{H}), 1.75$ (m, 8H), $2.01(\mathrm{t}, 2 \mathrm{H}), 2.52(\mathrm{t}, \mathrm{J}=6.9 \mathrm{~Hz}, 4 \mathrm{H}), 2.84(\mathrm{t}, 4 \mathrm{H}), 4.25(\mathrm{t}, 4 \mathrm{H}), 6.33(\mathrm{~d}, \mathrm{~J}=14.1$ $\mathrm{Hz}, 2 \mathrm{H}), 7.38-7.50(\mathrm{~m}, 4 \mathrm{H}), 7.64-7.72(\mathrm{~m}, 4 \mathrm{H}), 7.85-8.00(\mathrm{~m}, 8 \mathrm{H}), 8.15(\mathrm{t}, \mathrm{J}=7.8 \mathrm{~Hz}$ 2H), $8.32(\mathrm{~s}, 2 \mathrm{H}), 8.76(\mathrm{~d}, \mathrm{~J}=7.8 \mathrm{~Hz}, 2 \mathrm{H}), 8.87$ (d, J = 4.2 Hz, 2H).

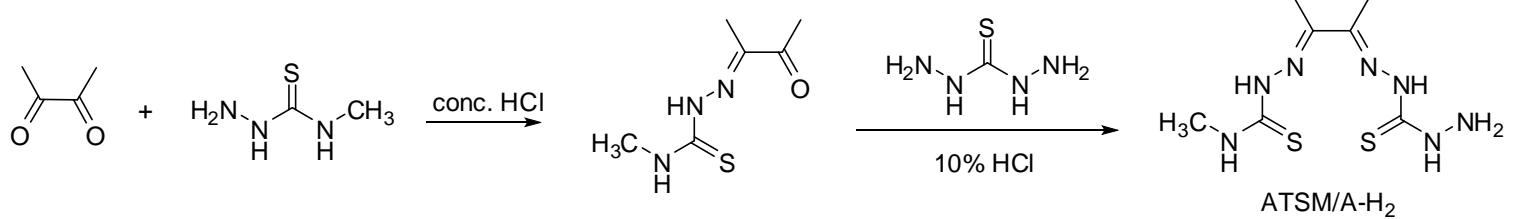

\section{Preparation of (E)-N-methyl-2-(3-oxobutan-2-ylidene)hydrazinecarbothioamide.} 2,3-Butanedione $(2.08 \mathrm{~mL}, 24 \mathrm{mmol})$ and conc. $\mathrm{HCl}(1 \mathrm{~mL})$ were added to 4-methyl-3thiosemicarbazide $(2.1 \mathrm{~g}, 20 \mathrm{mmol})$ in water $(40 \mathrm{~mL})$. The mixture was cooled in an ice 
bath and stirred for $30 \mathrm{~min}$. A bulky wihte solid settle out of solution and was filtered, washed with cold water and dried under vacuum. The product was isolated as a white solid in $65 \%$ yield.

Preparation and characterization of ATSM/A-H2. Thiocarbohydrazide $(530.8 \mathrm{mg}$, $5 \mathrm{mmol})$ was added to ethanol $(30 \mathrm{~mL})$ and the suspension stirred at $50 \mathrm{oC}$. A total of 1 eq of (E)-N-methyl-2-(3-oxobutan-2-ylidene)hydrazinecarbothioamide was added in portions over $2 \mathrm{~h}$. After the final addition, 5 drops of $10 \% \mathrm{HCl}$ was added and the reaction heated under reflux for $5 \mathrm{~h}$. The mixture was allowed to cool to room temperature, and then the precipitate was collected by filtration, washed with ethanol $(2 \times 30 \mathrm{~mL})$ and diethyl ether $(5 \times 30 \mathrm{~mL})$, and then dried in vacuo to give the product in $76 \%$ yield. ${ }^{1} \mathrm{H}$ NMR $\left(300 \mathrm{MHz},\left(\mathrm{CD}_{3}\right)_{2} \mathrm{SO}\right) \delta 2.16(\mathrm{~s}, 6 \mathrm{H}), 3.00(\mathrm{~d}, \mathrm{~J}=4.5 \mathrm{~Hz}, 3 \mathrm{H}), 4.94$ (s, 2H), 8.34 (q, J = 3.9 Hz, 1H), 9.67 (s, 1H), $10.18(\mathrm{~s}, 1 \mathrm{H}), 10.20(\mathrm{~s}, 1 \mathrm{H})$.
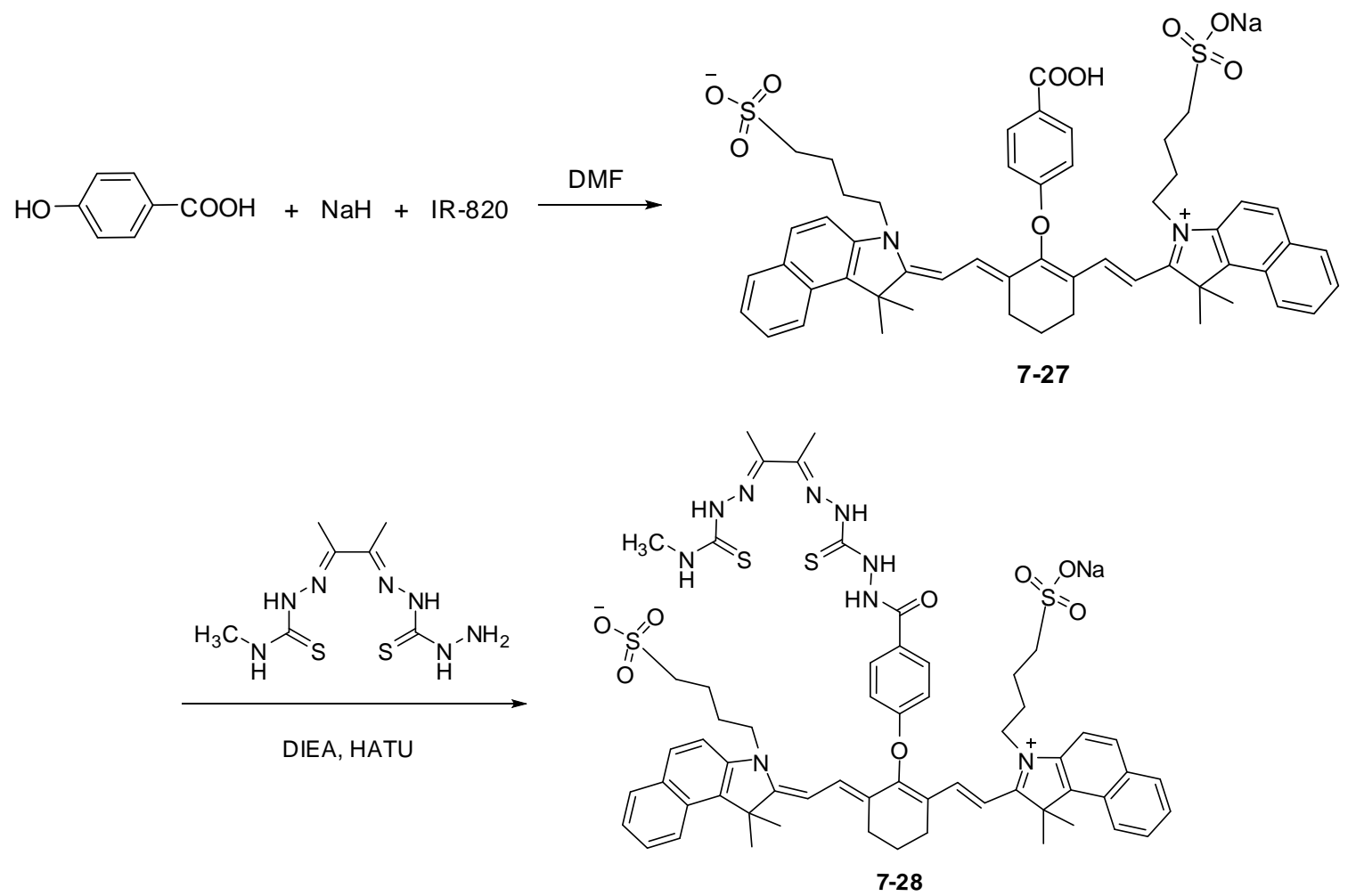
Preparation and characterization of compound 7-27. Under nitrogen, to an anhydrous DMF (1.5 mL) solution of 4-hydroxybenzoic acid $(13.8 \mathrm{mg}, 0.1 \mathrm{mmol})$ was added $\mathrm{NaH}(60 \%$ oil dispension, $8 \mathrm{mg}, 0.2 \mathrm{mmol})$ at $0{ }^{\circ} \mathrm{C}$. After $30 \mathrm{~min}$, DMF solution (1 $\mathrm{mL})$ of IR-820 (42.5 $\mathrm{mg}, 0.05 \mathrm{mmol})$ was added to the reaction mixture. After $18 \mathrm{~h}$, the reaction was quenched with dry ice and the mixture as purified by preparative HPLC to afford 7-27 (17 mg) in 36\% yield. ${ }^{1} \mathrm{H}$ NMR $\left(300 \mathrm{MHz},\left(\mathrm{CD}_{3}\right)_{2} \mathrm{SO}\right) \delta 1.55(\mathrm{~s}, 12 \mathrm{H}), 1.73-$ $1.97(\mathrm{~m}, 10 \mathrm{H}), 2.53(\mathrm{t}, \mathrm{J}=6.0 \mathrm{~Hz}, 4 \mathrm{H}), 2.77(\mathrm{t}, 4 \mathrm{H}), 4.25(\mathrm{t}, 4 \mathrm{H}), 6.28(\mathrm{~d}, \mathrm{~J}=14.7 \mathrm{~Hz}$ 2H), $7.33(\mathrm{t}, \mathrm{J}=6.9 \mathrm{~Hz}, 2 \mathrm{H}), 7.45(\mathrm{t}, \mathrm{J}=6.0 \mathrm{~Hz}, 2 \mathrm{H}), 7.58(\mathrm{t}, \mathrm{J}=7.8 \mathrm{~Hz}, 2 \mathrm{H}), 7.72(\mathrm{~d}, \mathrm{~J}=$ $9.0 \mathrm{~Hz}, 2 \mathrm{H}), 7.86(\mathrm{~d}, \mathrm{~J}=14.4 \mathrm{~Hz}, 2 \mathrm{H}), 8.00-8.13(\mathrm{~m}, 8 \mathrm{H})$.

Preparation and characterization of compound 7-28. ATSM/A- $\mathrm{H}_{2}(7 \mathrm{mg}, 0.027$ mmol) was suspended in the minimum amount of DMF. Compound 7-27 (17 mg, 0.018 mmol), diisopropylethylamine $(3.5 \mathrm{mg}, 0.027 \mathrm{mmol})$ and HATU (10.3 $\mathrm{mg}, 0.027 \mathrm{mmol})$ were added and the mixture was stirred at room temperature for overnight. After reaction diethylether $(50 \mathrm{~mL})$ was added and the corresponding precipitate was collected through filtration. The residue was purified by preparative HPLC to afford 7-28 (7.6 mg) in 35\% yield. ${ }^{1} \mathrm{H}$ NMR $\left(300 \mathrm{MHz},\left(\mathrm{CD}_{3}\right)_{2} \mathrm{SO}\right) \delta 1.57(\mathrm{~s}, 12 \mathrm{H}), 1.71-1.81(\mathrm{~m}, 8 \mathrm{H}), 1.99(\mathrm{~s}, 2 \mathrm{H})$, $2.17(\mathrm{~s}, 3 \mathrm{H}), 2.22(\mathrm{~s}, 3 \mathrm{H}), 2.52(\mathrm{t}, \mathrm{J}=6.0 \mathrm{~Hz}, 4 \mathrm{H}), 2.78(\mathrm{t}, 4 \mathrm{H}), 3.00(\mathrm{~d}, \mathrm{~J}=4.2 \mathrm{~Hz}, 3 \mathrm{H})$, $4.26(\mathrm{t}, 4 \mathrm{H}), 6.29(\mathrm{~d}, \mathrm{~J}=14.4 \mathrm{~Hz}, 2 \mathrm{H}), 7.38(\mathrm{~d}, \mathrm{~J}=8.7 \mathrm{~Hz}, 2 \mathrm{H}), 7.45(\mathrm{t}, \mathrm{J}=7.8 \mathrm{~Hz}, 2 \mathrm{H})$, $7.58(\mathrm{t}, \mathrm{J}=7.5 \mathrm{~Hz}, 2 \mathrm{H}), 7.73(\mathrm{~d}, \mathrm{~J}=9.0 \mathrm{~Hz}, 2 \mathrm{H}), 7.90$ (d, J = 14.4 Hz, 2H), 7.99-8.14 (m, $8 \mathrm{H}), 8.38(\mathrm{~d}, \mathrm{~J}=4.2 \mathrm{~Hz}, 1 \mathrm{H}), 10.11(\mathrm{~s}, 1 \mathrm{H}), 10.20(\mathrm{~s}, 1 \mathrm{H}), 10.56(\mathrm{~s}, 1 \mathrm{H}), 10.61(\mathrm{~s}, 1 \mathrm{H})$

\section{Fluorescent study}

Figure AIV-1. Excitation spectrum of $(\mathrm{S})-7-25\left(1 \times 10^{-5} \mathrm{M}\right.$ in $\left.\mathrm{H}_{2} \mathrm{O}\right) .\left(\lambda_{\mathrm{emi}}=795 \mathrm{~nm}\right.$, slit 
$=5.0 / 5.0 \mathrm{~nm})$

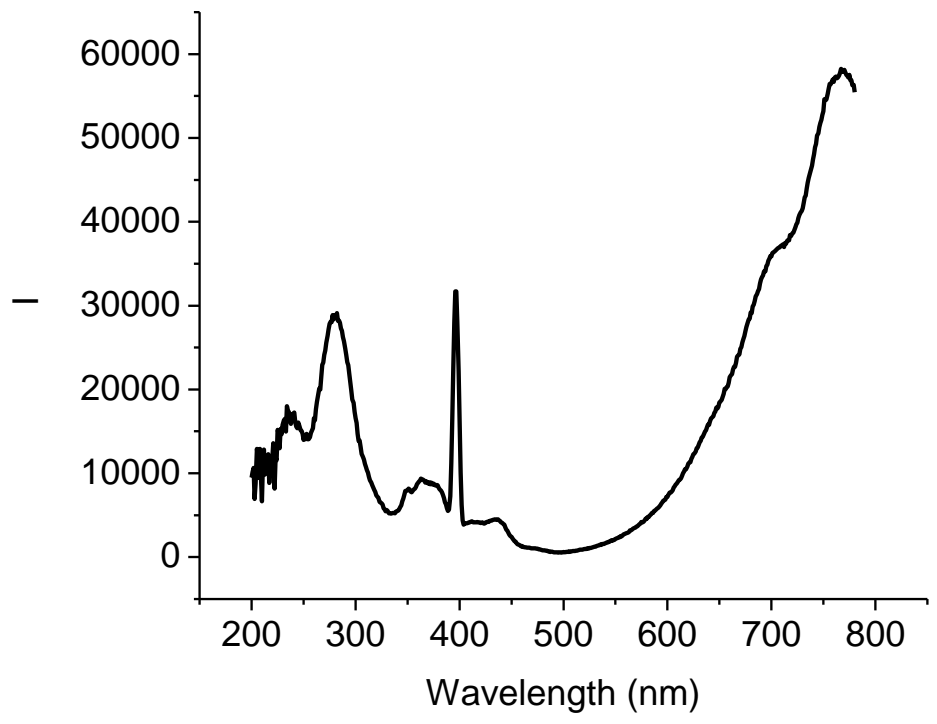

Figure AIV-2. Fluorescence spectra of $(\mathrm{S})-7-25\left(1 \times 10^{-5} \mathrm{M}\right.$ in $\left.\mathrm{H}_{2} \mathrm{O}\right)$ in the absence and presence of 1 eq $\mathrm{CuCl}_{2} \cdot 2 \mathrm{H}_{2} \mathrm{O} .\left(\lambda_{\mathrm{exc}}=280 \mathrm{~nm}\right.$, slit $\left.=5.0 / 5.0 \mathrm{~nm}\right)$

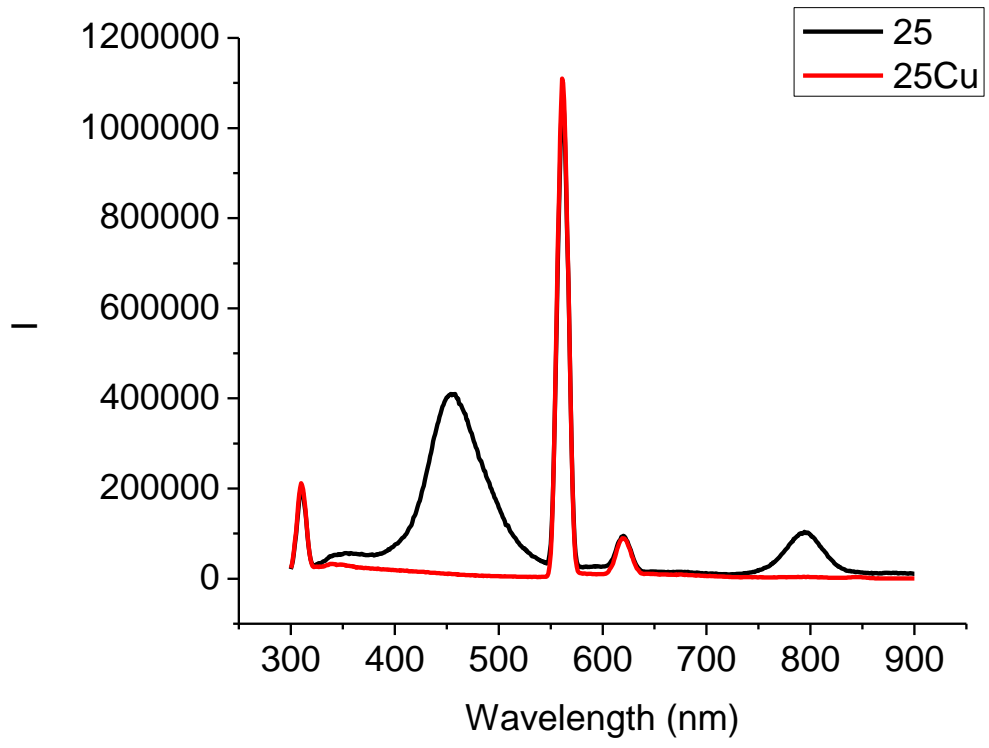

Figure AIV-3. Fluorescence spectra of $(\mathrm{S})-7-25\left(1 \times 10^{-5} \mathrm{M}\right.$ in $\left.\mathrm{H}_{2} \mathrm{O}\right)$ in the absence and 
presence of 1 eq $\mathrm{CuCl}_{2} \cdot 2 \mathrm{H}_{2} \mathrm{O} .\left(\lambda_{\text {exc }}=765 \mathrm{~nm}\right.$, slit $\left.=5.0 / 5.0 \mathrm{~nm}\right)$

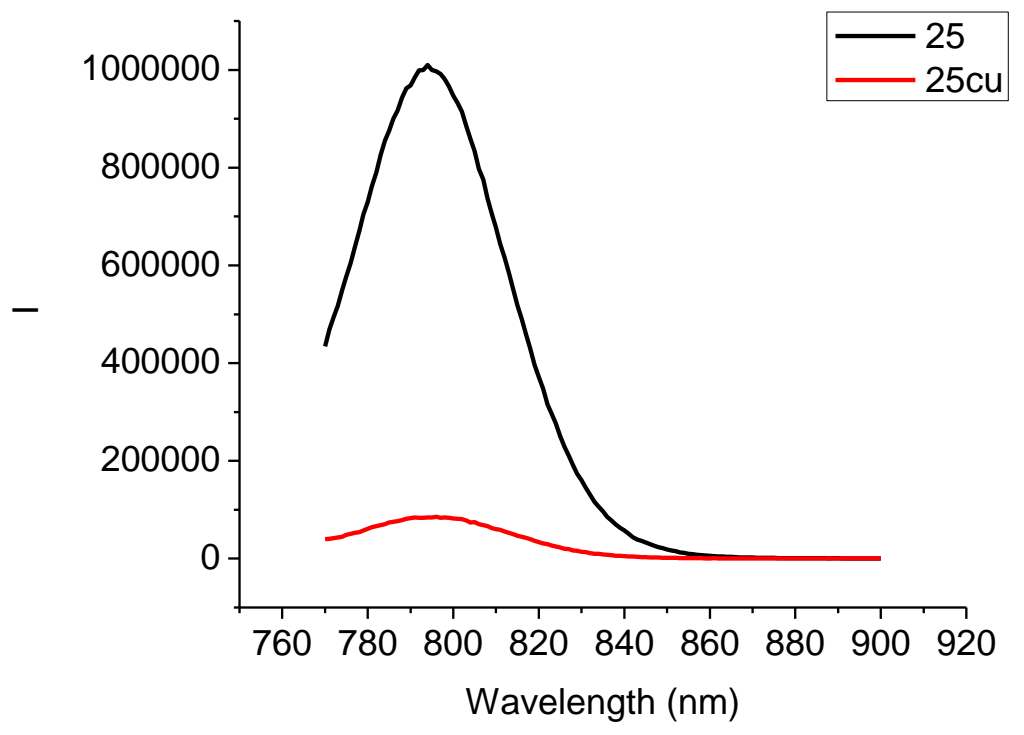

Figure AIV-4. Fluorescence spectra of $(\mathrm{S})-7-25\left(1 \times 10^{-5} \mathrm{M}\right.$ in $\left.\mathrm{H}_{2} \mathrm{O}\right)$ in the absence and presence of 1 eq $\mathrm{CuCl}_{2} \cdot 2 \mathrm{H}_{2} \mathrm{O} .\left(\lambda_{\text {exc }}=700 \mathrm{~nm}\right.$, slit $\left.=5.0 / 5.0 \mathrm{~nm}\right)$

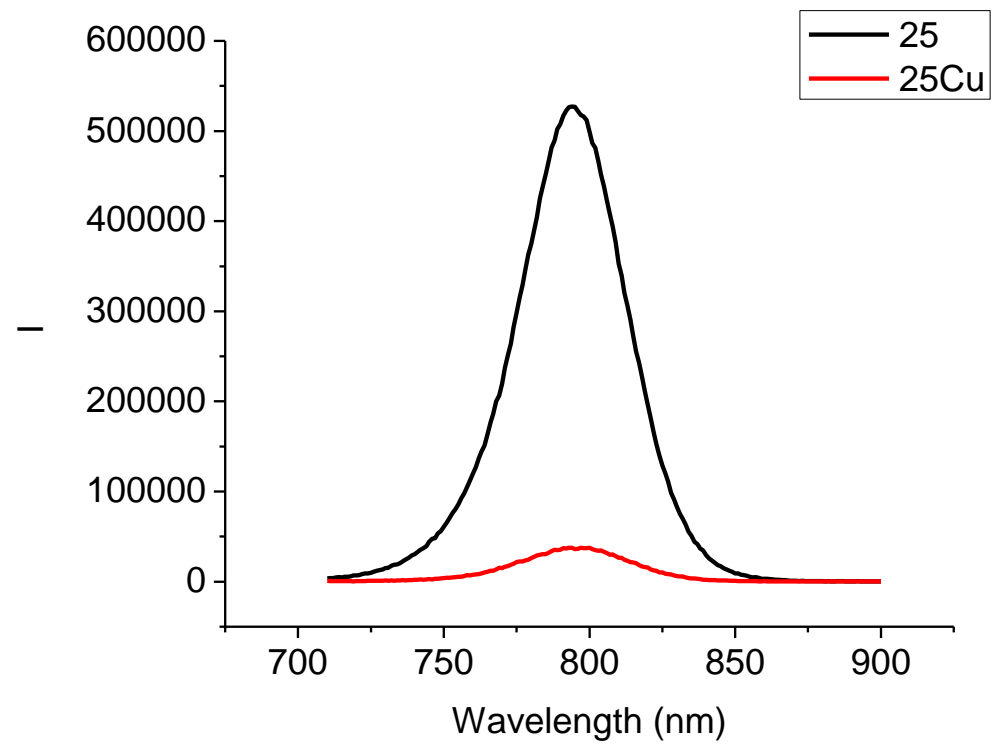

Figure AIV-5. Fluorescence titratoin of (S)-7-25 +1 eq $\mathrm{CuCl}_{2}\left(1 \times 10^{-5} \mathrm{M}\right.$ in $50 \mathrm{mM}$ 
HEPES) with L-Cysteine $\left(1,20,40,60,80,100,150,200\right.$ eq). ( $\lambda_{\text {exc }}=700 \mathrm{~nm}$, slit $=5.0 / 5.0 \mathrm{~nm})$

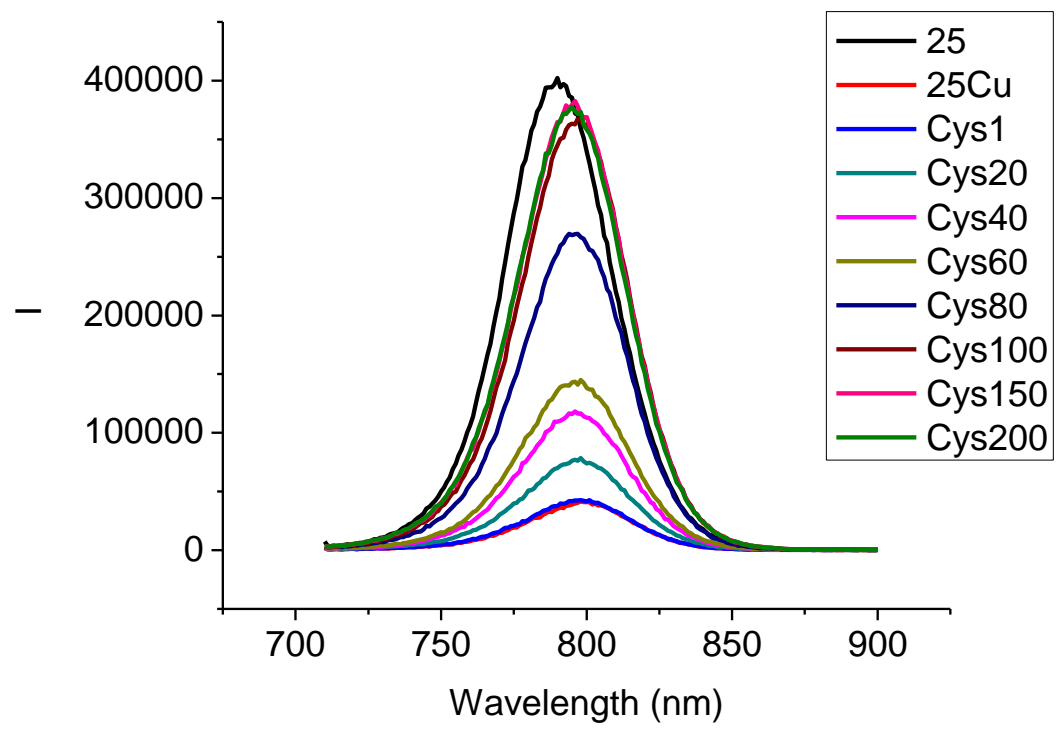

Figure AIV-6. Fluorescence titratoin of (S)-7-25 +1 eq $\mathrm{CuCl}_{2}\left(1 \times 10^{-5} \mathrm{M}\right.$ in $50 \mathrm{mM}$ HEPES) with L-Histidine (10, 40, 80, 100, 150, 200, 250, 300, 350, 400, 450, 500 eq). $\left(\lambda_{\text {exc }}=700 \mathrm{~nm}\right.$, slit $\left.=5.0 / 5.0 \mathrm{~nm}\right)$

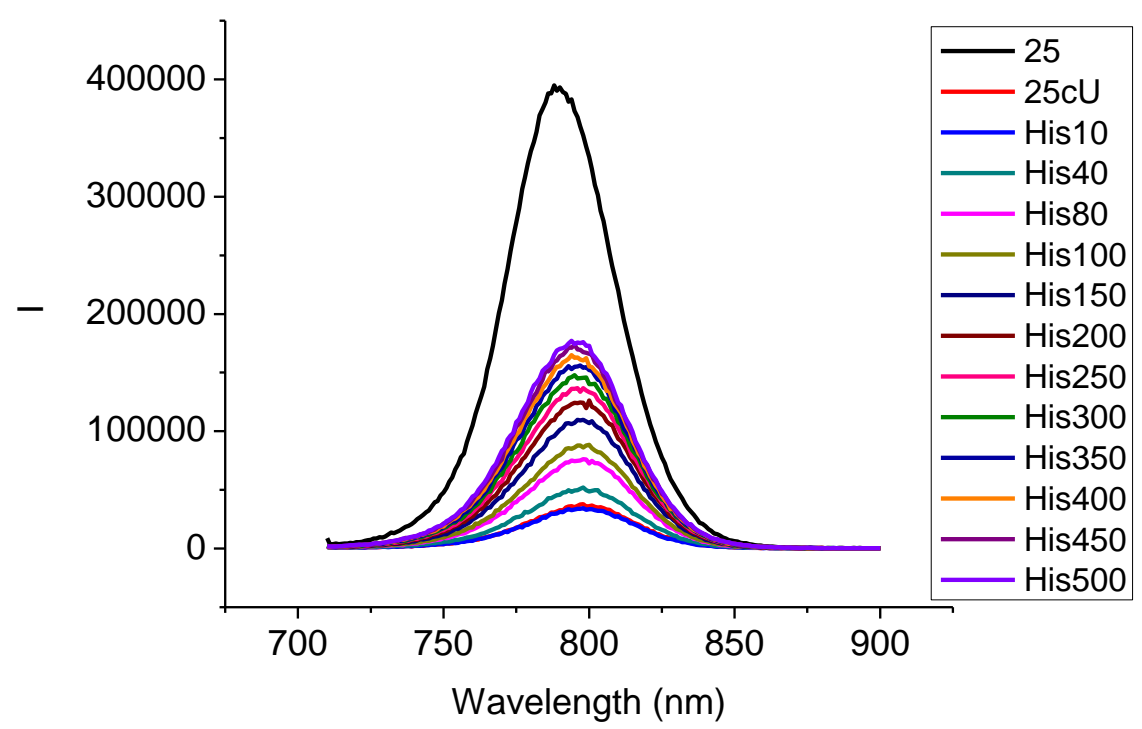


3. NMR spectra

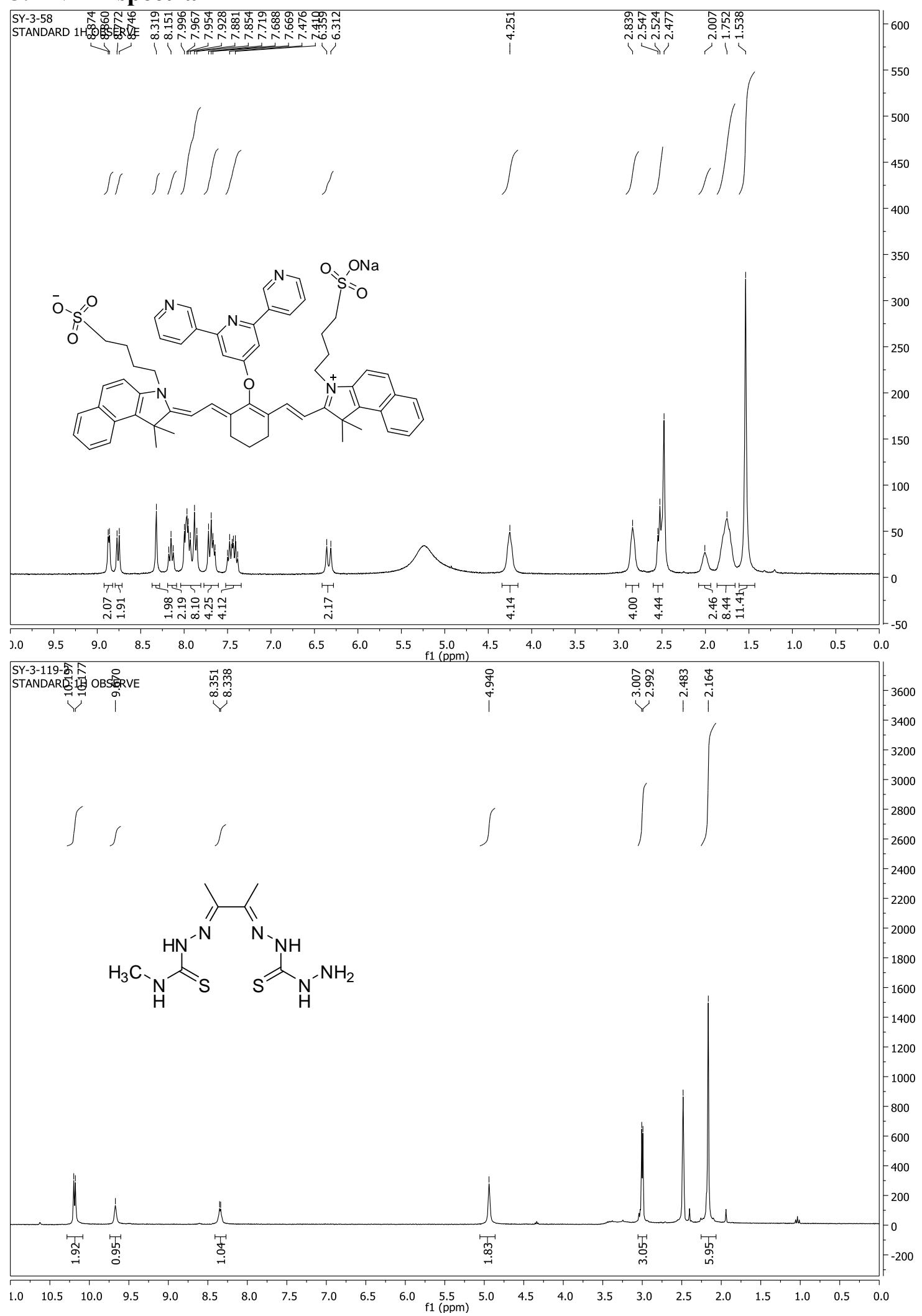



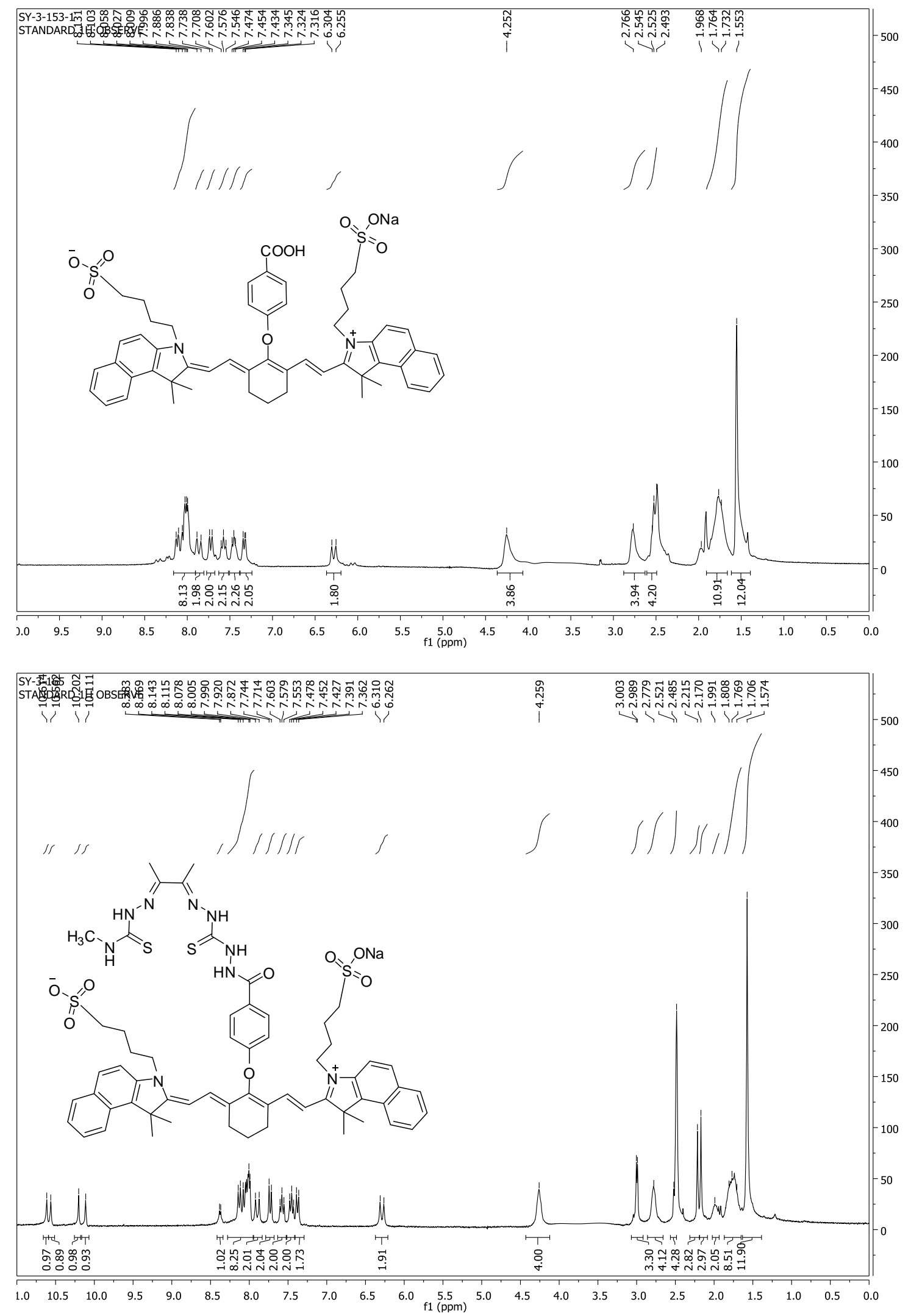


\section{Appendix V. Derivatives of Binol-Amino Alcohol Sensors}

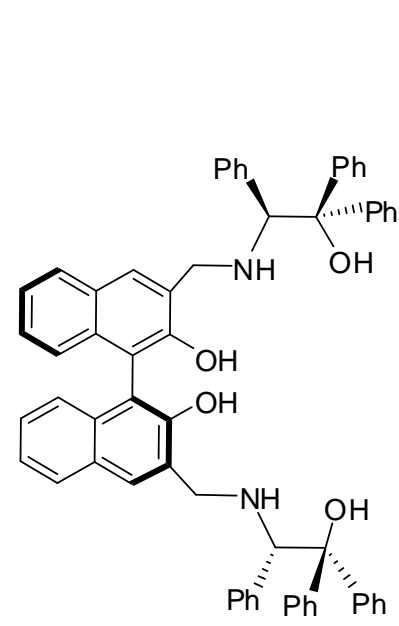

$(S)-7-35$

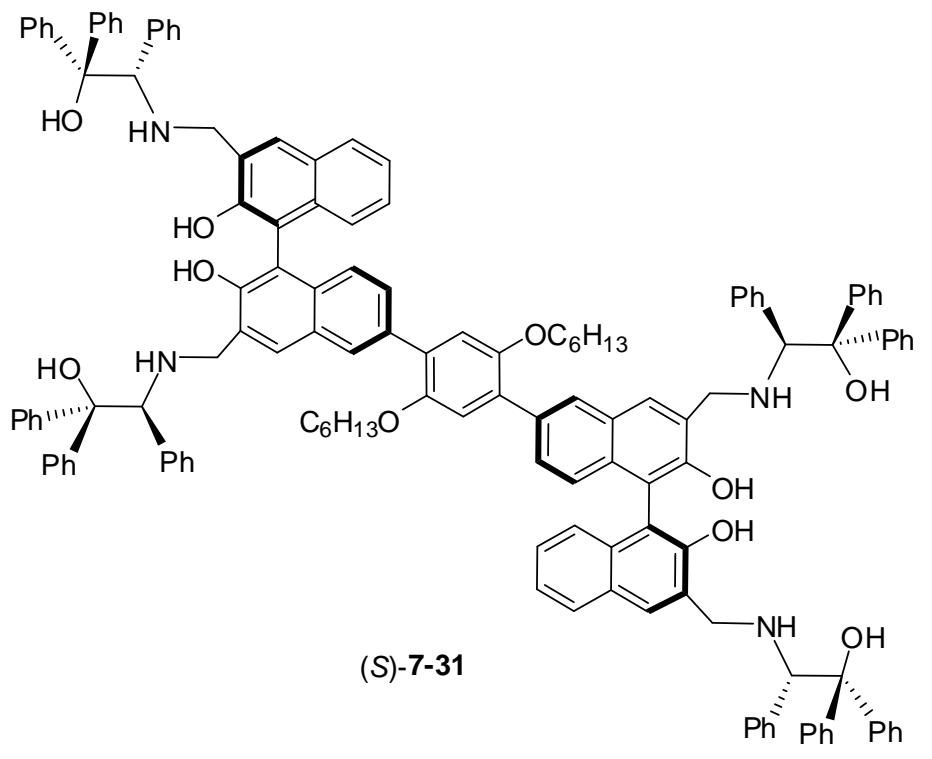

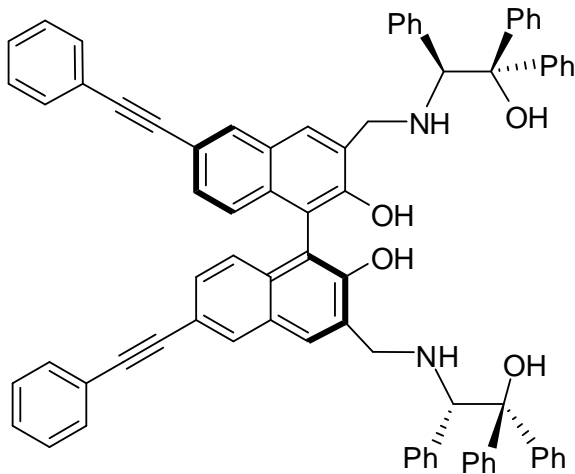

(S)-7-32

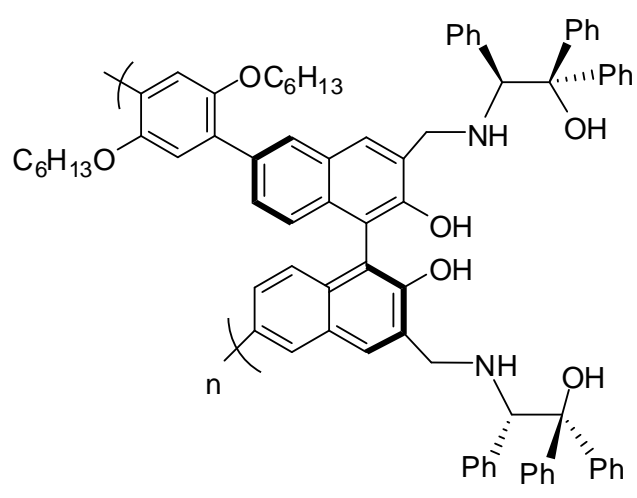

(S) $-7-33 \quad M n=3330$

\section{Preparation and Characterization of Compounds}<smiles>O=Cc1cc2ccccc2c(-c2c(C=O)cc3ccccc3c2O)c1O</smiles><smiles>O=Cc1cc2ccccc2c(-c2c(O)c(C=O)cc3cc(Br)ccc23)c1O</smiles>
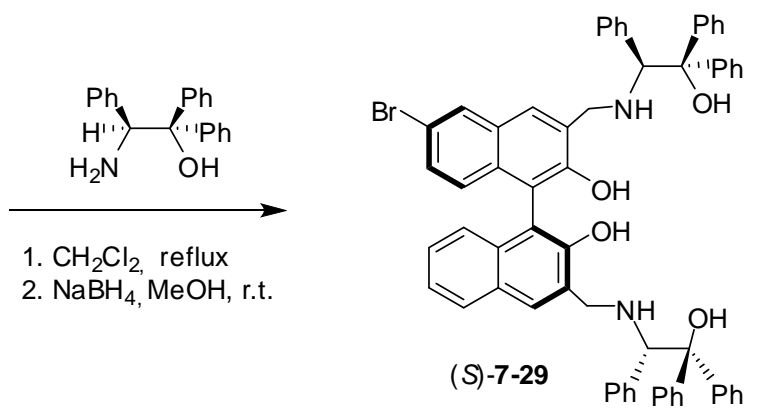


\section{Preparation and characterization of (S)-6-bromo-2,2'-dihydroxy-1,1'-} binaphthyl-3,3'-dicarbaldehyde. Under nitrogen, (S)-3, 3'-diformylBINOL (240 mg, $0.70 \mathrm{mmol})$ was dissolved in methylene chloride $(20 \mathrm{~mL})$. To the solution was added dropwise $0.13 \mathrm{~mL}(2.5 \mathrm{mmol})$ of bromine. The resulting solution was stirred overnight at room temperature. The remaining bromine was quenched by the addition of $\mathrm{Na}_{2} \mathrm{SO}_{3}$ aq. Then the aqueous layer was extracted with methylene chloride $(2 \times 10 \mathrm{~mL})$. The combined methylene chloride layer was washed with brine $(10 \mathrm{~mL})$ and dried over anhydrous $\mathrm{Na}_{2} \mathrm{SO}_{4}$. After evaporation of the solvent, the residue was purified by column chromatography on silica gel eluted with dichloromethane/hexanes (3/2) to afford desired product as a yellow solid in $51 \%$ yield. ${ }^{1} \mathrm{H}$ NMR $\left(\left(\mathrm{CD}_{3}\right)_{2} \mathrm{SO}, 300 \mathrm{MHz}\right) \delta 6.94(\mathrm{~d}, \mathrm{~J}=9.0$ $\mathrm{Hz}, 1 \mathrm{H}), 6.98-7.01(\mathrm{~m}, 1 \mathrm{H}), 7.42-7.45(\mathrm{~m}, 2 \mathrm{H}), 7.53(\mathrm{dt}, \mathrm{J} 1=9.0 \mathrm{~Hz}, \mathrm{~J} 2=2.1 \mathrm{~Hz}, 1 \mathrm{H})$, 8.14-8.17 (m, 1H), $8.45(\mathrm{~s}, 1 \mathrm{H}), 8.63(\mathrm{~s}, 1 \mathrm{H}), 8.67(\mathrm{~s}, 1 \mathrm{H}), 10.18(\mathrm{~d}, \mathrm{~J}=1.5 \mathrm{~Hz}, 1 \mathrm{H})$, $10.21(\mathrm{~d}, \mathrm{~J}=1.5 \mathrm{~Hz}, 1 \mathrm{H}), 10.35(\mathrm{~d}, \mathrm{~J}=1.8 \mathrm{~Hz}, 1 \mathrm{H}), 10.37(\mathrm{~d}, \mathrm{~J}=1.8 \mathrm{~Hz}, 1 \mathrm{H}) .{ }^{13} \mathrm{C} \mathrm{NMR}$ $\left(\left(\mathrm{CD}_{3}\right)_{2} \mathrm{SO}, 75 \mathrm{MHz}\right) \delta 115.9,117.1,117.7,123.9,124.77,124.83,125.0,127.3,128.1$, $129.3,131.1,132.5,133.5,135.5,135.6,136.0,137.4,137.5,154.2,154.6,196.2,197.0$. HRMS Calcd for $\mathrm{C}_{22} \mathrm{H}_{14} \mathrm{BrO}_{4}\left(\mathrm{MH}^{+}\right)$: 421.0075, Found: 421.0075. HRMS Calcd for $\mathrm{C}_{22} \mathrm{H}_{13} \mathrm{BrO}_{4} \mathrm{Na}\left(\mathrm{MNa}^{+}\right)$: 442.9895, Found: 442.9898. $[\alpha]_{\mathrm{D}}=-190.17(\mathrm{c}=0.31, \mathrm{DMSO})$.

Preparation and characterization of compound (S)-7-29. (1) Under nitrogen, (S)6-bromo-2,2'-dihydroxy-1,1'-binaphthyl-3,3'-dicarbaldehyde (152 mg, $0.36 \mathrm{mmol})$ was dissolved in $\mathrm{CH}_{2} \mathrm{Cl}_{2}(15 \mathrm{~mL})$ and combined with (S)-2-amino-1,1,2-triphenylenthanol (211 $\mathrm{mg}, 0.72 \mathrm{mmol}$ ). The reaction mixture was heated at reflux for overnight, and monitored by using ${ }^{1} \mathrm{H}$ NMR spectroscopy. When the reaction was complete, the solution was cooled to room temperature and dried over anhydrous $\mathrm{Na}_{2} \mathrm{SO}_{4}$. After filtration, the 
filtrate was concentration under vacuum. The residue was passed through a silica gel column eluted with hexanes/ethyl acetate (8/1) to give the corresponding Schiff base. (2) The Schiff base was dissolved in methanol $(15 \mathrm{~mL})$ and cooled down to $0{ }^{\circ} \mathrm{C} . \mathrm{NaBH}_{4}(55$ $\mathrm{mg}, 1.44 \mathrm{mmol}$ ) was added in small portions. The reaction temperature was maintained at $0{ }^{\circ} \mathrm{C}$ until the solution became colorless and transparent. Then it was allowed to proceed at room temperature for additional $30 \mathrm{~min}$. Methanol was removed, and the residue was dissolved in ethyl acetate $(25 \mathrm{~mL})$ and washed with water $(10 \mathrm{~mL})$. The aqueous layer after separation was extracted with ethyl acetate $(3 \times 10 \mathrm{~mL})$. The combined ethyl acetate layer was washed with brine $(10 \mathrm{~mL})$ and dried over anhydrous $\mathrm{Na}_{2} \mathrm{SO}_{4}$. After evaporation of the solvent, the residue was purified by flash column chromatography on silica gel eluted with hexanes/ethyl acetate (3/1) to afford $(S)-7-29$ as a white solid in $43 \%$ yield. ${ }^{1} \mathrm{H}$ NMR $\left(\mathrm{CDCl}_{3}, 300 \mathrm{MHz}\right) \delta 3.31(\mathrm{~s}, 1 \mathrm{H}), 3.53(\mathrm{~s}, 1 \mathrm{H}), 3.73(\mathrm{~d}, \mathrm{~J}=14.1 \mathrm{~Hz}$, 1H), $3.81(\mathrm{~d}, \mathrm{~J}=14.1 \mathrm{~Hz}, 1 \mathrm{H}), 4.08(\mathrm{~d}, \mathrm{~J}=6.3 \mathrm{~Hz}, 1 \mathrm{H}), 4.12(\mathrm{~d}, \mathrm{~J}=7.2 \mathrm{~Hz}, 1 \mathrm{H}), 4.57(\mathrm{~s}$, 1H), $4.63(\mathrm{~s}, 1 \mathrm{H}), 6.88-7.51(\mathrm{~m}, 37 \mathrm{H}), 7.76-7.79(\mathrm{~m}, 1 \mathrm{H}), 7.89(\mathrm{~d}, \mathrm{~J}=1.8 \mathrm{~Hz}, 1 \mathrm{H})$.
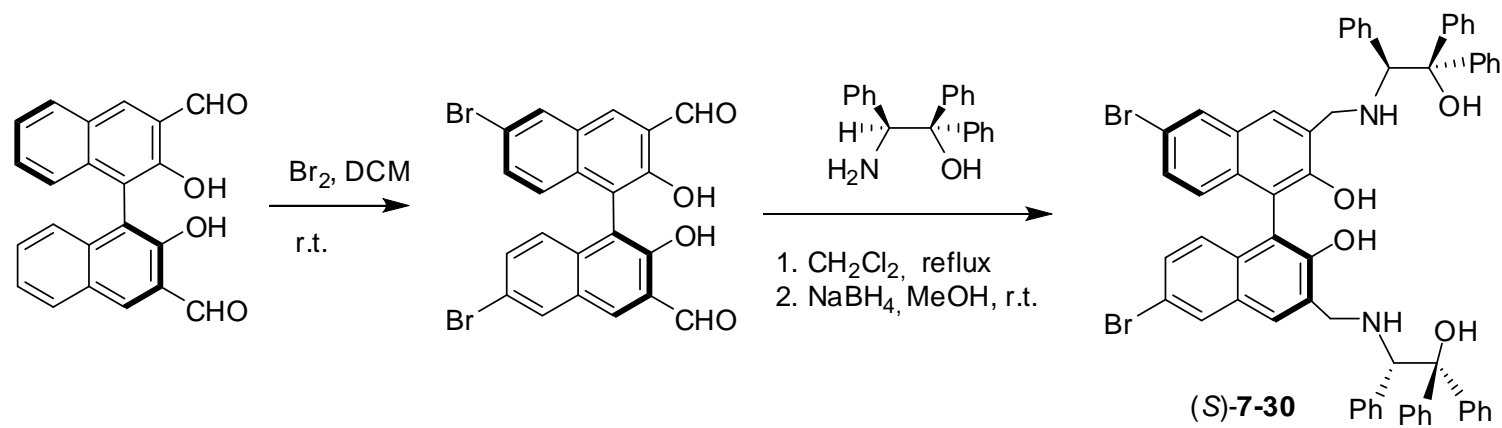

Preparation and characterization of (S)-6,6'-dibromo-2,2'-dihydroxy- 1,1'binaphthyl-3,3'-dicarbaldehyde. Under nitrogen, (S)-3, 3'-diformylBINOL (120 mg, $0.35 \mathrm{mmol}$ ) was dissolved in methylene chloride $(7 \mathrm{~mL})$. To the solution was added dropwise $0.09 \mathrm{~mL}(1.75 \mathrm{mmol})$ of bromine. The resulting solution was stirred overnight 
at room temperature. The remaining bromine was quenched by the addition of $\mathrm{Na}_{2} \mathrm{SO}_{3}$ aq. Then the aqueous layer was extracted with methylene chloride $(2 \times 10 \mathrm{~mL})$. The combined methylene chloride layer was washed with brine $(10 \mathrm{~mL})$ and dried over anhydrous $\mathrm{Na}_{2} \mathrm{SO}_{4}$. After evaporation of the solvent, the residue was purified by column chromatography on silica gel eluted with dichloromethane/hexanes (3/2) to afford $(S)-7-3$ as a yellow solid in $71 \%$ yield. ${ }^{1} \mathrm{H}$ NMR $\left(\left(\mathrm{CD}_{3}\right)_{2} \mathrm{SO}, 300 \mathrm{MHz}\right) \delta 6.90(\mathrm{~d}, J=9.0 \mathrm{~Hz}$, 2H), $7.52(\mathrm{dd}, \mathrm{J}=2.1,9.0 \mathrm{~Hz}, 2 \mathrm{H}), 8.43(\mathrm{~s}, 2 \mathrm{H}), 8.43(\mathrm{~s}, 2 \mathrm{H}), 10.18(\mathrm{~S}, 2 \mathrm{H}), 10.35$ (s, $2 \mathrm{H}) .{ }^{13} \mathrm{C} \mathrm{NMR}\left(\left(\mathrm{CD}_{3}\right)_{2} \mathrm{SO}, 75 \mathrm{MHz}\right) \delta 116.4,117.7,124.9,127.1,129.3,132.5,133.6$, 135.6, 135.9, 154.7, 196.1. $[\alpha]_{\mathrm{D}}=-143.4(\mathrm{c}=0.30, \mathrm{DMSO})$.

Preparation and characterization of compound (S)-7-30. (1) Under nitrogen, (S)6,6'-dibromo-2,2'-dihydroxy- 1,1'-binaphthyl-3,3'-dicarbaldehyde (298 mg, $0.60 \mathrm{mmol}$ ) was dissolved in $\mathrm{CH}_{2} \mathrm{Cl}_{2}(200 \mathrm{~mL})$ and combined with (S)-2-amino-1,1,2triphenylenthanol (345 $\mathrm{mg}, 1.20 \mathrm{mmol})$. The reaction mixture was heated at reflux for overnight, and monitored by using ${ }^{1} \mathrm{H}$ NMR spectroscopy. When the reaction was complete, the solution was cooled to room temperature and dried over anhydrous $\mathrm{Na}_{2} \mathrm{SO}_{4}$. After filtration, the filtrate was concentration under vacuum. The residue was passed through a silica gel column eluted with hexanes/ethyl acetate $(8 / 1)$ to give the corresponding Schiff base. (2) The Schiff base was dissolved in anhydrous ethanol (60 $\mathrm{mL})$ and cooled down to $0{ }^{\circ} \mathrm{C}$. $\mathrm{NaBH}_{4}(69 \mathrm{mg}, 1.80 \mathrm{mmol})$ was added in small portions. The reaction temperature was maintained at $0{ }^{\circ} \mathrm{C}$ until the solution became colorless and transparent. Then it was allowed to proceed at room temperature for additional $30 \mathrm{~min}$. Ethanol was removed, and the residue was dissolved in ethyl acetate $(50 \mathrm{~mL})$ and washed with water $(15 \mathrm{~mL})$. The aqueous layer after separation was extracted with ethyl acetate 
$(3 \times 30 \mathrm{~mL})$. The combined ethyl acetate layer was washed with brine $(15 \mathrm{~mL})$ and dried over anhydrous $\mathrm{Na}_{2} \mathrm{SO}_{4}$. After evaporation of the solvent, the residue was purified by flash column chromatography on silica gel eluted with hexanes/ethyl acetate (3/1) to afford $(S)-7-5$ as a white silid in 72\% yield. ${ }^{1} \mathrm{H}$ NMR $\left(\mathrm{CDCl}_{3}, 300 \mathrm{MHz}\right) \delta 3.30(\mathrm{~s}, 2 \mathrm{H})$, $3.79(\mathrm{~d}, \mathrm{~J}=13.8 \mathrm{~Hz}), 4.08(\mathrm{~d}, \mathrm{~J}=13.8 \mathrm{~Hz}), 4.60(\mathrm{~s}, 2 \mathrm{H}), 6.99-7.36(\mathrm{~m}, 34 \mathrm{H}), 7.48$ (dd, J1 $=8.1 \mathrm{~Hz}, \mathrm{~J} 2=1.5 \mathrm{~Hz}, 4 \mathrm{H}), 7.90(\mathrm{~d}, \mathrm{~J}=2.1 \mathrm{~Hz})$. HRMS Calcd for $\mathrm{C}_{62} \mathrm{H}_{51} \mathrm{~N}_{2} \mathrm{O}_{4}\left(\mathrm{MH}^{+}\right)$: 1045.2216, Found: 1045.2211. $[\alpha]_{\mathrm{D}}=-42.3\left(\mathrm{c}=0.51, \mathrm{CHCl}_{3}\right)$.

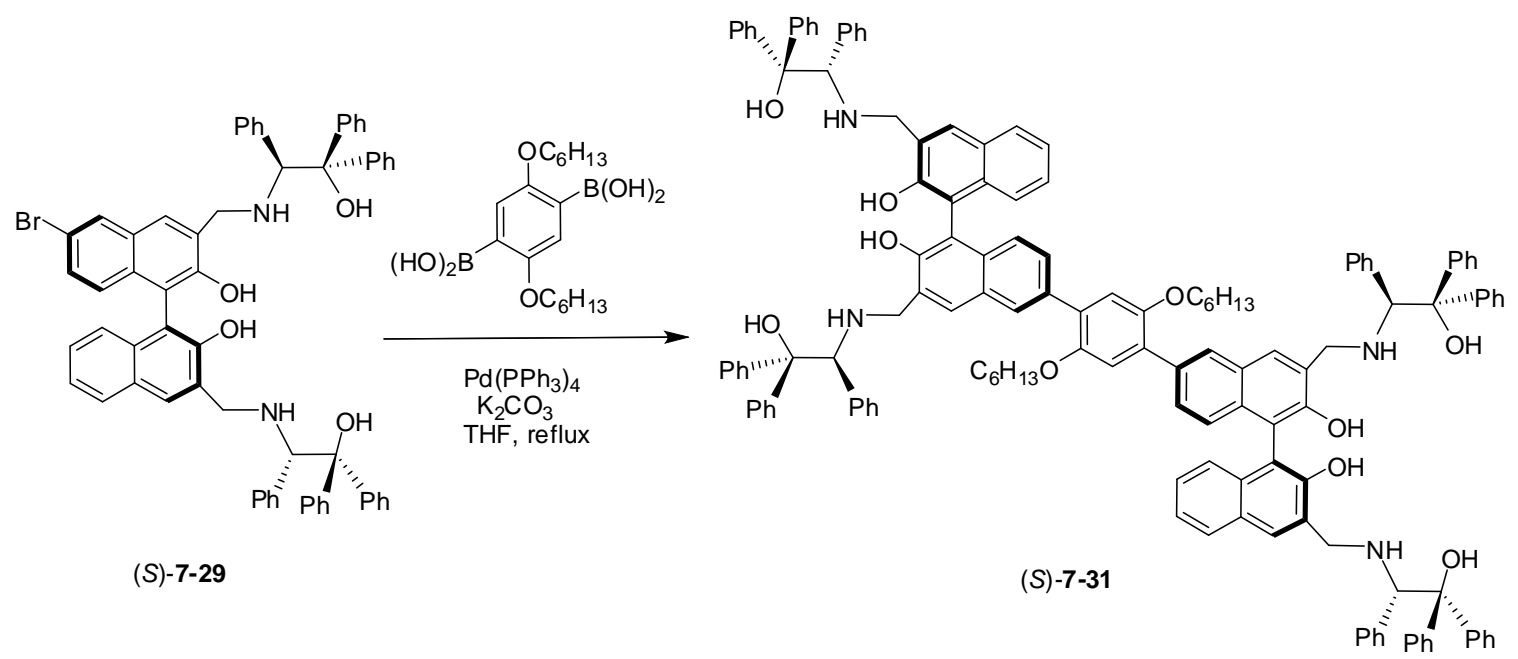

Preparation and characterization of compound (S)-7-31. Under nitrogen, a mixture of $(S)$-7-29 (281 mg, $0.29 \mathrm{mmol})$, 2,5-bis(hexyloxy)-1,4-phenylenediboronic acid (53 mg, $0.15 \mathrm{mmol})$, and $\mathrm{Pd}\left(\mathrm{PPh}_{3}\right)_{4}(17 \mathrm{mg}, 0.015 \mathrm{mmol}, 5 \mathrm{~mol} \%)$ in THF(4.5 mL) and $2 \mathrm{M} \mathrm{K}_{2} \mathrm{CO}_{3}(4.5 \mathrm{~mL})$ was heated at reflux for overnight. The organic layer was separated and then diluted with $\mathrm{CH}_{2} \mathrm{Cl}_{2}(20 \mathrm{~mL})$. The solution was washed with $1 \mathrm{~N} \mathrm{HCl}(10 \mathrm{~mL})$ and brine $(10 \mathrm{~mL})$ and dried over anhydrous $\mathrm{Na}_{2} \mathrm{SO}_{4}$. After evaporation of the solvent, the residue was purified by flash column chromatography on silica gel eluted with $\mathrm{CH}_{2} \mathrm{Cl}_{2}$ /methanol (100/1) to afford (S)-7-31 as a light yellow solid in $60 \%$ yield. ${ }^{1} \mathrm{H}$ 
$\operatorname{NMR}\left(\mathrm{CDCl}_{3}, 300 \mathrm{MHz}\right) \delta 0.77(\mathrm{t}, \mathrm{J}=6.0 \mathrm{~Hz}, 6 \mathrm{H}), 1.16-1.31(\mathrm{~m}, 12 \mathrm{H}), 1.62(\mathrm{t}, 6.6 \mathrm{~Hz}$, 2H), 3.68-3.78 (m, 8H), $3.90(\mathrm{t}, \mathrm{J}=6.0 \mathrm{~Hz}, 4 \mathrm{H}), 4.09-4.16(\mathrm{~m}, 4 \mathrm{H}), 4.65(\mathrm{~s}, 4 \mathrm{H}), 6.95-$ $7.83(\mathrm{~m}, 78 \mathrm{H}), 8.07(\mathrm{~s}, 2 \mathrm{H}) .{ }^{13} \mathrm{C} \mathrm{NMR}\left(\mathrm{CDCl}_{3}, 75 \mathrm{MHz}\right) \delta 14.2,22.8,25.9,29.5,31.6$, $49.2,67.7,67.8,69.8,80.6,114.5,114.7,116.7,123.9,124.5,125.1,126.0,126.5,126.9$, $127.4,127.7,128.0,128.3,128.7,128.9,130.0,130.1,130.2,130.7,133.0,133.97$, 134.03, 137.05, 144.5, 145.0, 150.7, 152.8, 152.9. HRMS Calcd for $\mathrm{C}_{142} \mathrm{H}_{132} \mathrm{~N}_{4} \mathrm{O}_{10} / 2$ $\left([\mathrm{M}+2 \mathrm{H}]^{2+}\right): 1026.4972$, Found: 1026.4972.

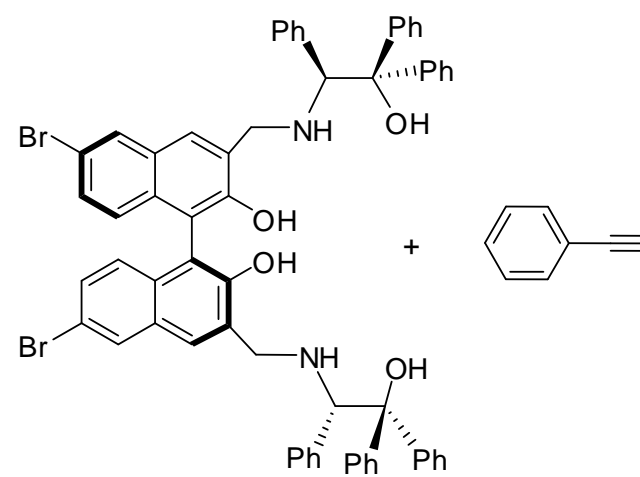

(S)-7-30

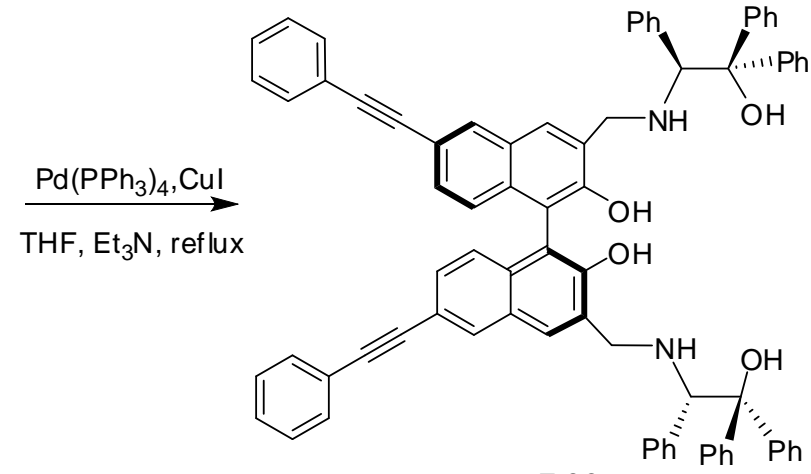

$(S)-7-32$

Preparation and characterization of compound (S)-7-32. Under nitrogen, to a 25 $\mathrm{mL}$ flame-dried Schlenk flask was loaded (S)-7-30 (105 mg, $0.10 \mathrm{mmol})$, ethynylbenzene (44 $\mu \mathrm{L}, 0.40 \mathrm{mmol})$, triethylamine $(1.5 \mathrm{~mL})$, and THF $(1.5 \mathrm{~mL})$. The resulting solution was degassed for $30 \mathrm{~min}$ and then was combined with $\mathrm{Pd}\left(\mathrm{PPh}_{3}\right)_{4}(12 \mathrm{mg}, 0.010 \mathrm{mmol})$ and $\mathrm{CuI}(2 \mathrm{mg}, 0.010 \mathrm{mmol})$ in the dry box. After this reaction mixture was stirred at reflux for $24 \mathrm{~h}$, it was filtered to remove the insoluble triethylamine hydrobromide salt. The salt was rinsed with diethyl ether until the filtrate was clear. After evaporation of the solvent, the residue was purified by flash column chromatography on silica gel eluted with hexane/ethyl acetate (3/1) to afford (S)-7-32 as a white solid in 51\% yield. ${ }^{1} \mathrm{H}$ NMR $\left(\mathrm{CDCl}_{3}, 300 \mathrm{MHz}\right) \delta 3.49(\mathrm{~s}, 2 \mathrm{H}), 3.77(\mathrm{~d}, \mathrm{~J}=13.8 \mathrm{~Hz}, 2 \mathrm{H}), 4.08(\mathrm{~d}, \mathrm{~J}=13.8 \mathrm{~Hz}, 2 \mathrm{H})$, 
$4.63(\mathrm{~s}, 2 \mathrm{H}), 6.99-7.60(\mathrm{~m}, 44 \mathrm{H}), 8.01(\mathrm{~s}, 2 \mathrm{H}) .{ }^{13} \mathrm{C} \mathrm{NMR}\left(\mathrm{CDCl}_{3}, 75 \mathrm{MHz}\right) \delta 49.5,67.9$, $80.7,89.6,90.2,115.2,118.4,123.6,125.1,126.0,126.4,126.7,127.1,127.5,127.8$ $128.2,128.4,128.5,128.6,128.8,129.4,129.5,130.0,131.9,133.5,139.9,144.5,144.8$, 154.0. HRMS Calcd for $\mathrm{C}_{78} \mathrm{H}_{61} \mathrm{~N}_{2} \mathrm{O}_{4}\left(\mathrm{MH}^{+}\right)$: 1089.4631 , Found: 1089.4611 .

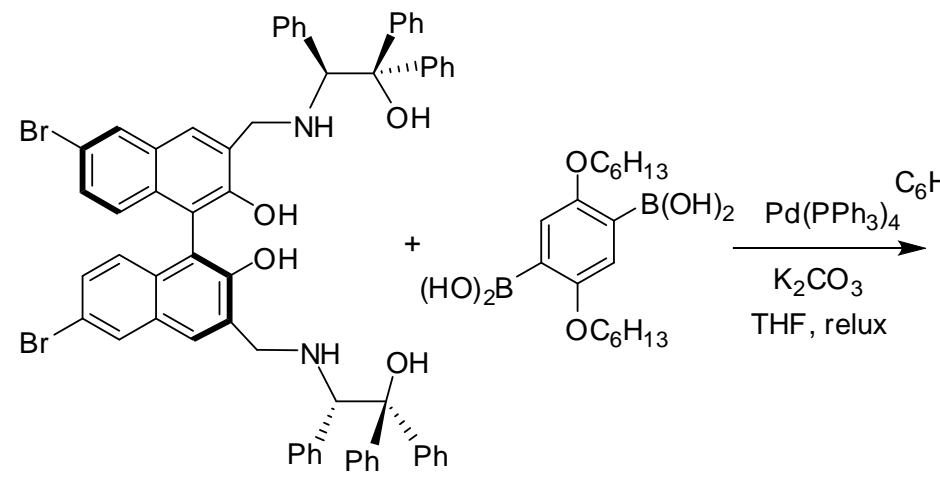

(S)-7-30

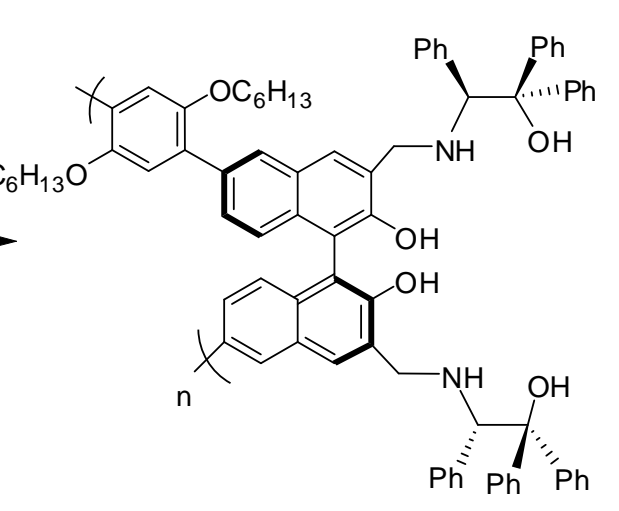

(S) $-7-33 \quad M n=3330$

(S) $-7-34 \quad M n=4496$

Preparation and characterization of compound (S)-7-33. Under nitrogen, a mixture of $(S)$-7-30 (105 mg, $0.10 \mathrm{mmol})$, 2,5-bis(hexyloxy)-1,4-phenylenediboronic acid (37 mg, $0.10 \mathrm{mmol})$, and $\mathrm{Pd}\left(\mathrm{PPh}_{3}\right)_{4}(10 \mathrm{mg}, 0.010 \mathrm{mmol}, 10 \mathrm{~mol} \%)$ in $\mathrm{THF}(2.0 \mathrm{~mL})$ and $2 \mathrm{M} \mathrm{K}_{2} \mathrm{CO}_{3}(2.0 \mathrm{~mL})$ was heated at reflux for $48 \mathrm{~h}$. The organic layer was separated and then diluted with $\mathrm{CH}_{2} \mathrm{Cl}_{2}(20 \mathrm{~mL})$. The solution was washed with $1 \mathrm{~N} \mathrm{HCl}(10 \mathrm{~mL})$ and brine $(10 \mathrm{~mL})$. After separation, the solvent of the organic layer was removed to give a yellowish solid. The solid was redissoved in $\mathrm{CH}_{2} \mathrm{Cl}_{2}$ and precipitated out twice with $\mathrm{MeOH}$. The solid, $(S)$-7-33, was isolated by centrifugation and was dried under vacuum at room temperature for $24 \mathrm{~h}$. The yield was $67 \%$. ${ }^{1} \mathrm{H} \mathrm{NMR}\left(\mathrm{CDCl}_{3}, 300 \mathrm{MHz}\right) \delta 0.71(\mathrm{t}$, $\mathrm{J}=6.0 \mathrm{~Hz}, 6 \mathrm{H}), 1.23-1.33(\mathrm{~m}, 12 \mathrm{H}), 1.53-1.63(\mathrm{~m}, 4 \mathrm{H}), 3.75-3.94(\mathrm{~m}, 8 \mathrm{H}), 4.14(\mathrm{~d}, \mathrm{~J}=$ $12.9 \mathrm{~Hz}, 2 \mathrm{H}), 4.64(\mathrm{~s}, 2 \mathrm{H}), 6.84-7.78(\mathrm{~m}, 38 \mathrm{H}), 8.04(\mathrm{~s}, 2 \mathrm{H}) . \mathrm{M}_{\mathrm{n}}=3330 . \mathrm{M}_{\mathrm{w}} / \mathrm{M}_{\mathrm{n}}=1.40$.

Preparation and characterization of compound (S)-7-34. Under nitrogen, a 
mixture of $(S)$-7-30 (105 mg, $0.10 \mathrm{mmol}), 2,5$-bis(hexyloxy)-1,4-phenylenediboronic acid (37 mg, $0.10 \mathrm{mmol})$, and $\mathrm{Pd}\left(\mathrm{PPh}_{3}\right)_{4}(10 \mathrm{mg}, 0.010 \mathrm{mmol}, 10 \mathrm{~mol} \%)$ in $\mathrm{THF}(2.0 \mathrm{~mL})$ and $2 \mathrm{M} \mathrm{K}_{2} \mathrm{CO}_{3}(2.0 \mathrm{~mL})$ was heated at reflux for $60 \mathrm{~h}$. The organic layer was separated and then diluted with $\mathrm{CH}_{2} \mathrm{Cl}_{2}(20 \mathrm{~mL})$. The solution was washed with $1 \mathrm{~N} \mathrm{HCl}(10 \mathrm{~mL})$ and brine $(10 \mathrm{~mL})$. After separation, the solvent of the organic layer was removed to give a yellowish solid. The solid was redissoved in $\mathrm{CH}_{2} \mathrm{Cl}_{2}$ and precipitated out twice with $\mathrm{MeOH}$. The solid, $(S)$-7-34, was isolated by centrifugation and was dried under vacuum at room temperature for $24 \mathrm{~h}$. The yield was $85 \% .{ }^{1} \mathrm{H}$ NMR $\left(\mathrm{CDCl}_{3}, 300 \mathrm{MHz}\right) \delta 0.70(\mathrm{t}$, $\mathrm{J}=6.0 \mathrm{~Hz}, 6 \mathrm{H}), 1.11-1.31(\mathrm{~m}, 12 \mathrm{H}), 1.58(\mathrm{t}, \mathrm{J}=6.0 \mathrm{~Hz}, 4 \mathrm{H}), 3.74-3.94(\mathrm{~m}, 8 \mathrm{H}), 4.14(\mathrm{~d}$, $\mathrm{J}=10.8 \mathrm{~Hz}, 2 \mathrm{H}), 4.64(\mathrm{~s}, 2 \mathrm{H}), 6.84-7.76(\mathrm{~m}, 38 \mathrm{H}), 8.04(\mathrm{~s}, 2 \mathrm{H}) . \mathrm{M}_{\mathrm{n}}=4496 . \mathrm{M}_{\mathrm{w}} / \mathrm{M}_{\mathrm{n}}=$ 1.43.

\section{Fluorescent study}

Figure AV-1. Fluorescence spectra of (S)-7-31, (S)-7-32, (S)-7-33 and (S)-7-35 $\left(5 \times 10^{-6}\right.$ $\mathrm{M}$ in benzene). $\left(\lambda_{\mathrm{exc}}=334 \mathrm{~nm}\right.$ for $(\mathrm{S})-\mathbf{7 - 3 1},(\mathrm{S})-\mathbf{7 - 3 3}$ and $(\mathrm{S})-\mathbf{7 - 3 5}, \lambda_{\mathrm{exc}}=314 \mathrm{~nm}$ for $(\mathrm{S})$ 7-32, slit $=2.0 / 2.0 \mathrm{~nm})$ 


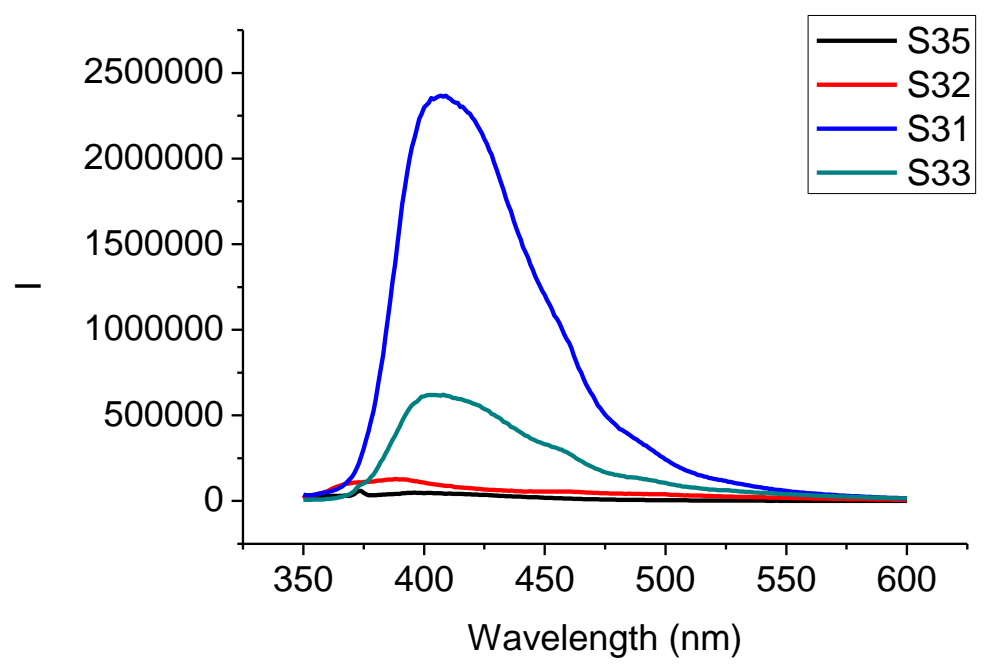

Figure AV-2. Fluorescence spectra of (S)-7-35, (S)-7-31, (S)-7-32 $\left(6 \times 10^{-5} \mathrm{M}\right)$ with/without mandelic acid $\left(3.0 \times 10^{-3} \mathrm{M}\right.$ in benzene $\left.(0.4 \% \mathrm{DME})\right) .\left(\lambda_{\mathrm{exc}}=334 \mathrm{~nm}\right.$ for $(\mathrm{S})-$ 7-31 and $(\mathrm{S})-\mathbf{7 - 3 5}, \lambda_{\mathrm{exc}}=314 \mathrm{~nm}$ for $(\mathrm{S})-\mathbf{7 - 3 2}$, slit $\left.=2.5 / 2.5 \mathrm{~nm}\right)$

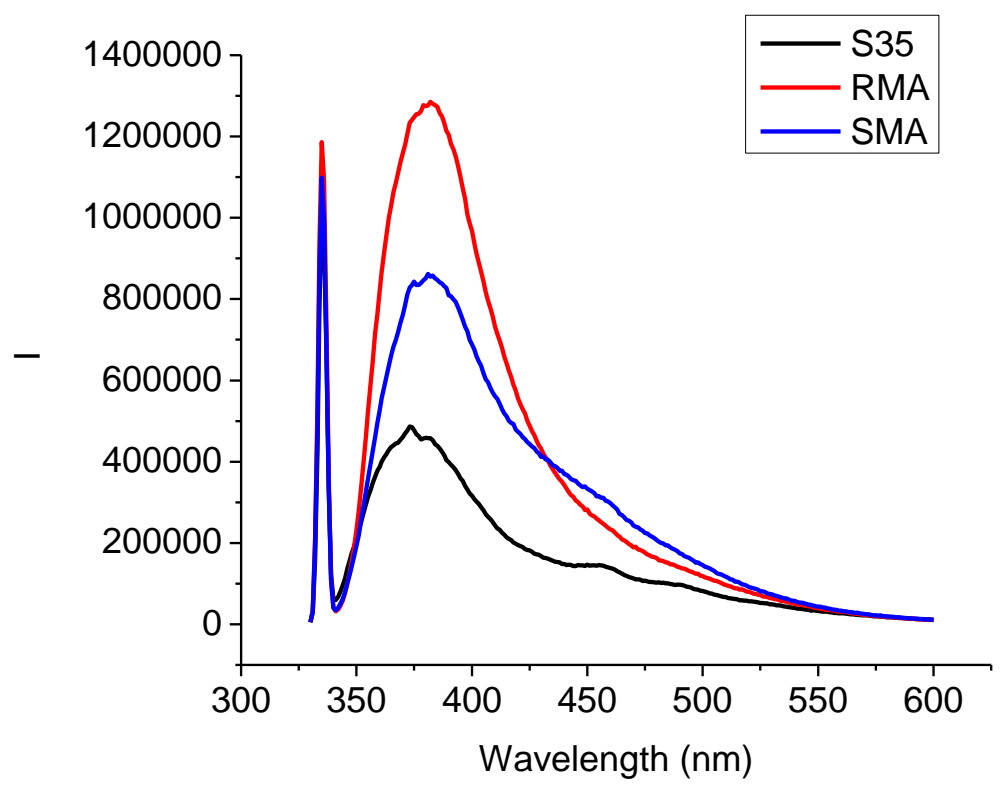



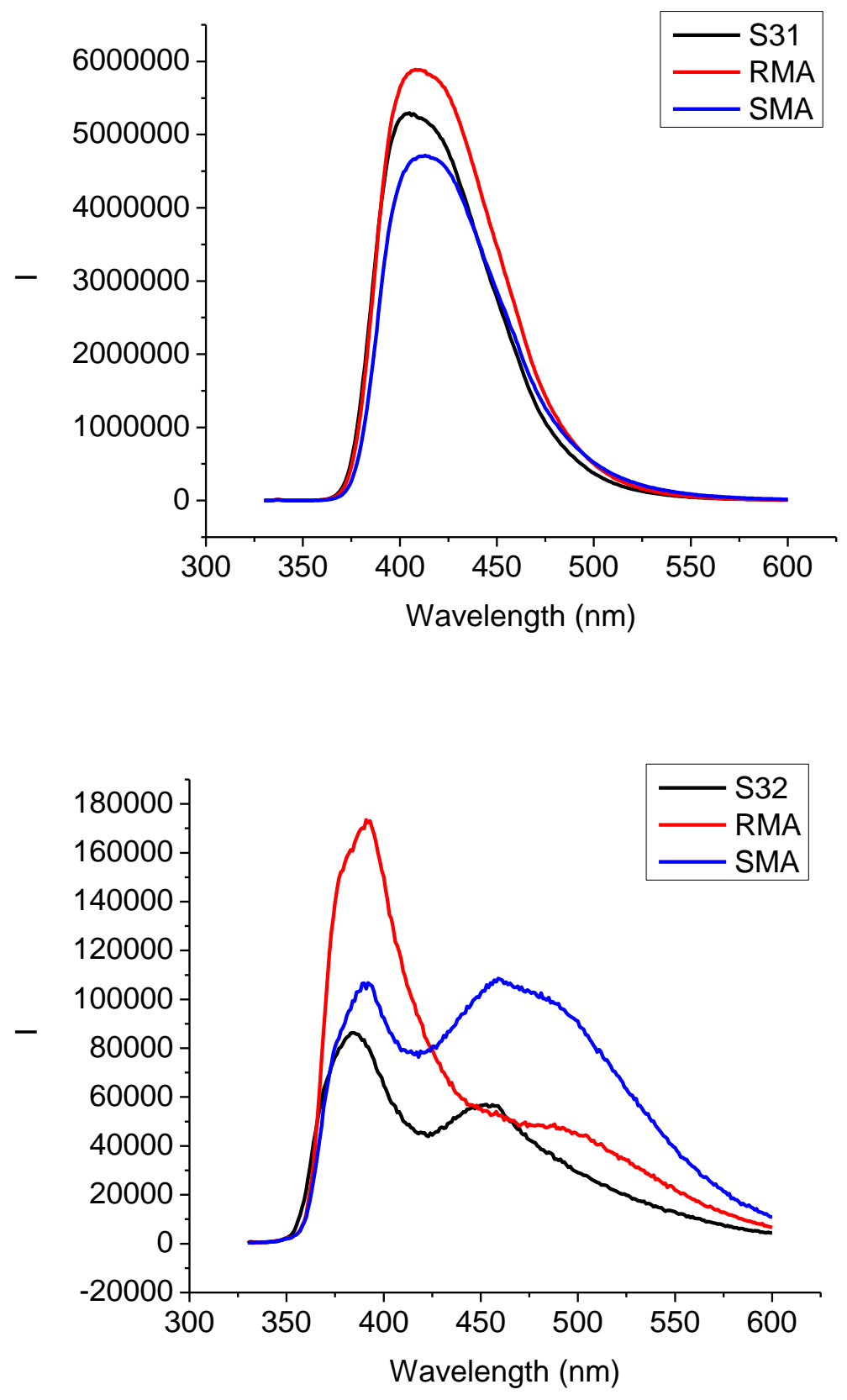

Figure AV-3. Fluorescence spectra of (S)-7-35, (S)-7-31, (S)-7-32 $\left(6 \times 10^{-5} \mathrm{M}\right)$ with/without phenyllactic acid $\left(3.0 \times 10^{-3} \mathrm{M}\right.$ in benzene $\left.(0.4 \% \mathrm{DME})\right) .\left(\lambda_{\mathrm{exc}}=334 \mathrm{~nm}\right.$ for $(\mathrm{S})-7-31$ and $(\mathrm{S})-7-35, \lambda_{\mathrm{exc}}=314 \mathrm{~nm}$ for $(\mathrm{S})-\mathbf{7 - 3 2}$, slit $\left.=2.5 / 2.5 \mathrm{~nm}\right)$ 

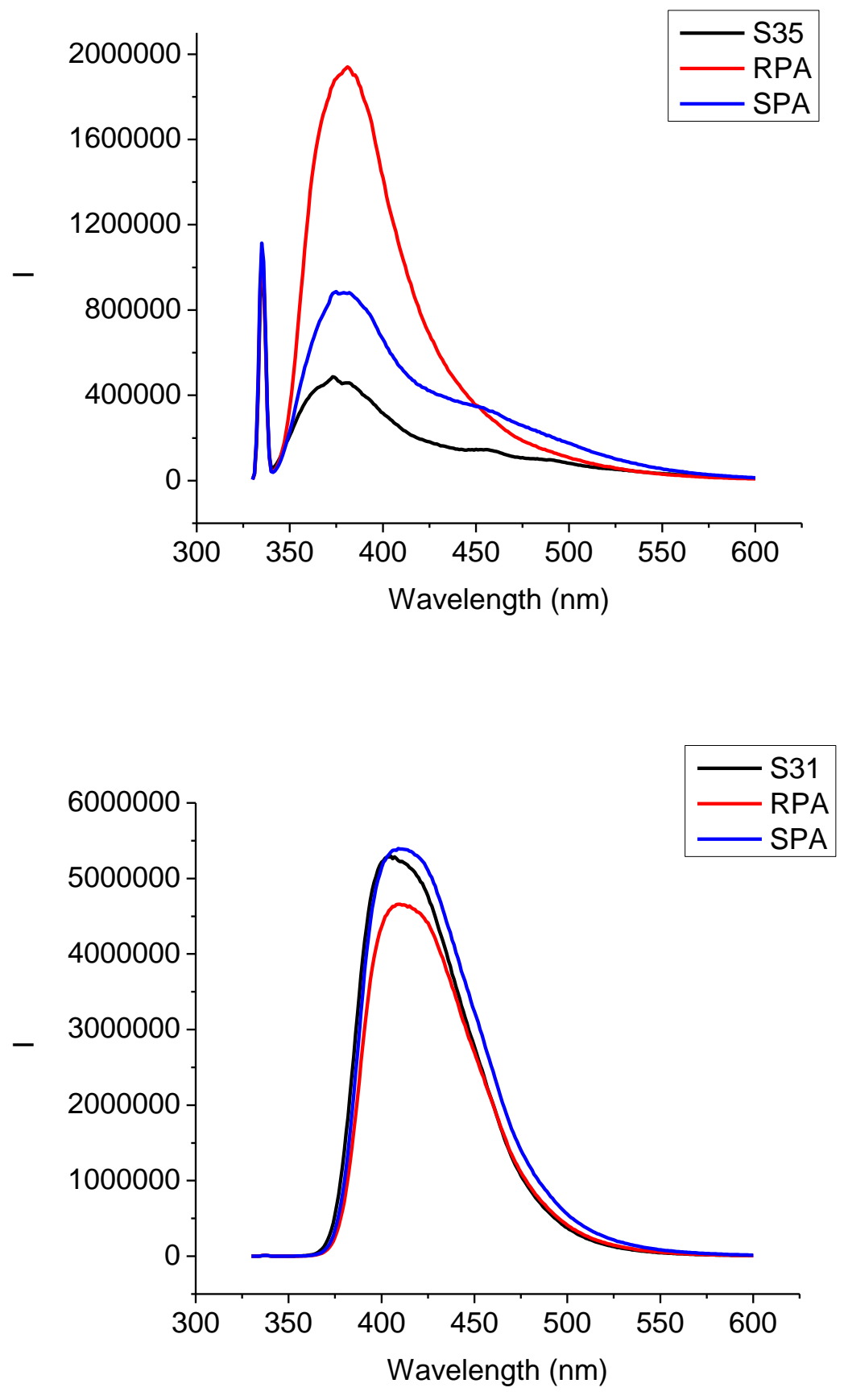


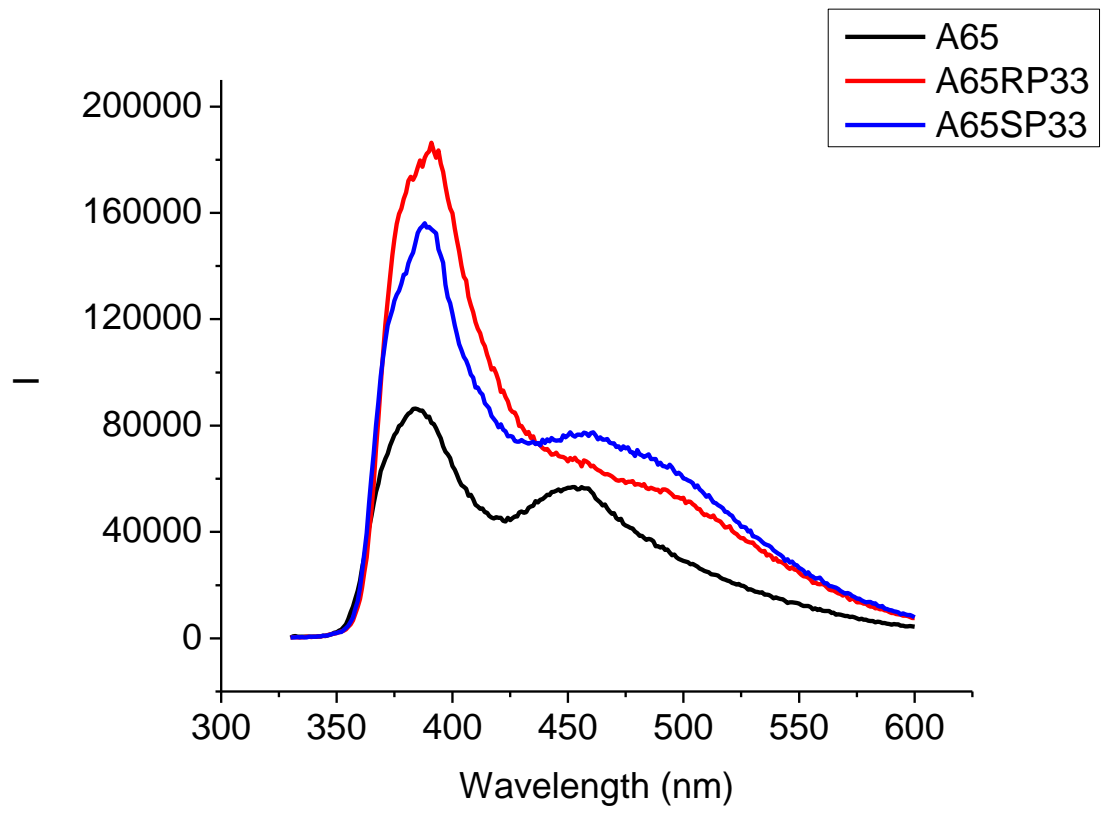

Figure AV-4. Fluorescence spectra of (S)-7-31, (S)-7-32, (S)-7-33 and (S)-7-34 $\left(5 \times 10^{-8}\right.$ M) with/without mandelic $\left(1.0 \times 10^{-3} \mathrm{M}\right.$ in $\left.\mathrm{CH}_{2} \mathrm{Cl}_{2}\right)$. $\left(\lambda_{\text {exc }}=336 \mathrm{~nm}\right.$ for $(\mathrm{S})-\mathbf{7 - 3 1},(\mathrm{S})-\mathbf{7 - 3 3}$ and (S)-7-34, $\lambda_{\text {exc }}=313 \mathrm{~nm}$ for $(\mathrm{S})-\mathbf{7 - 3 2}$, slit $\left.=4.0 / 4.0 \mathrm{~nm}\right)$

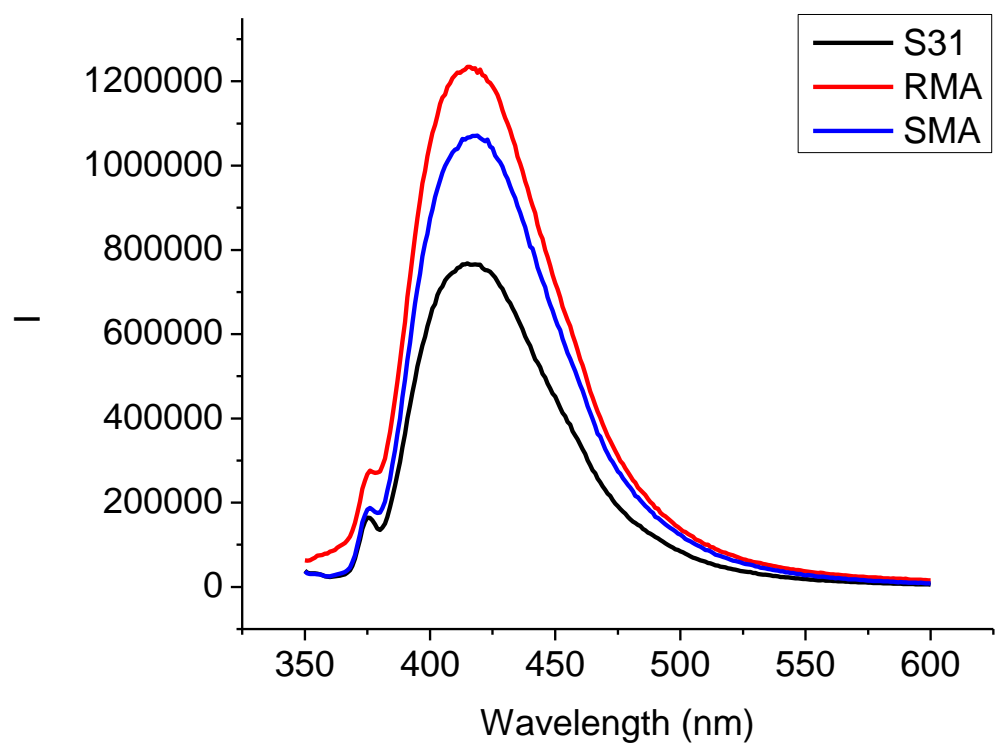



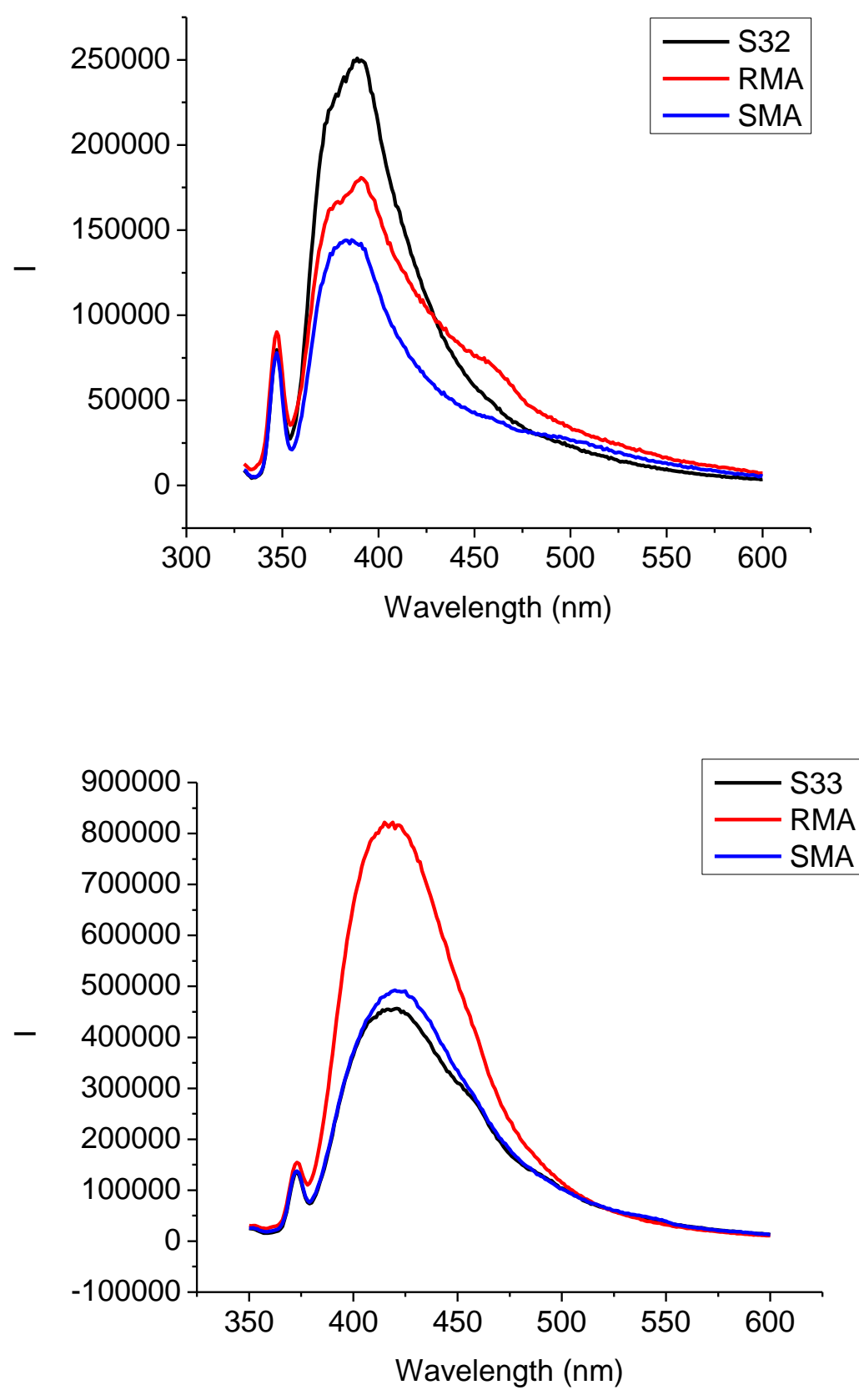


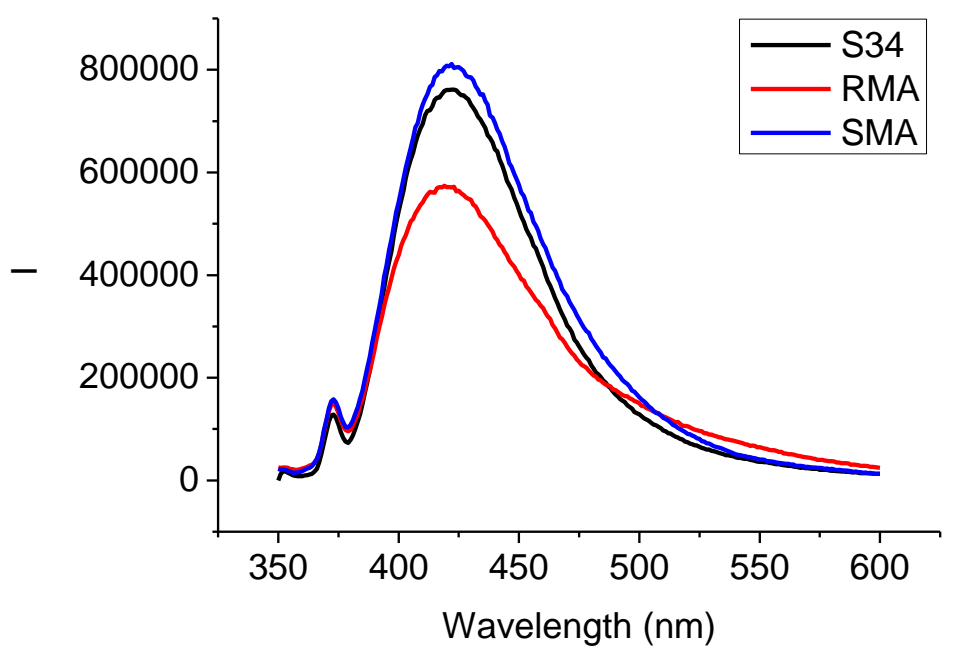

\section{NMR spectra}

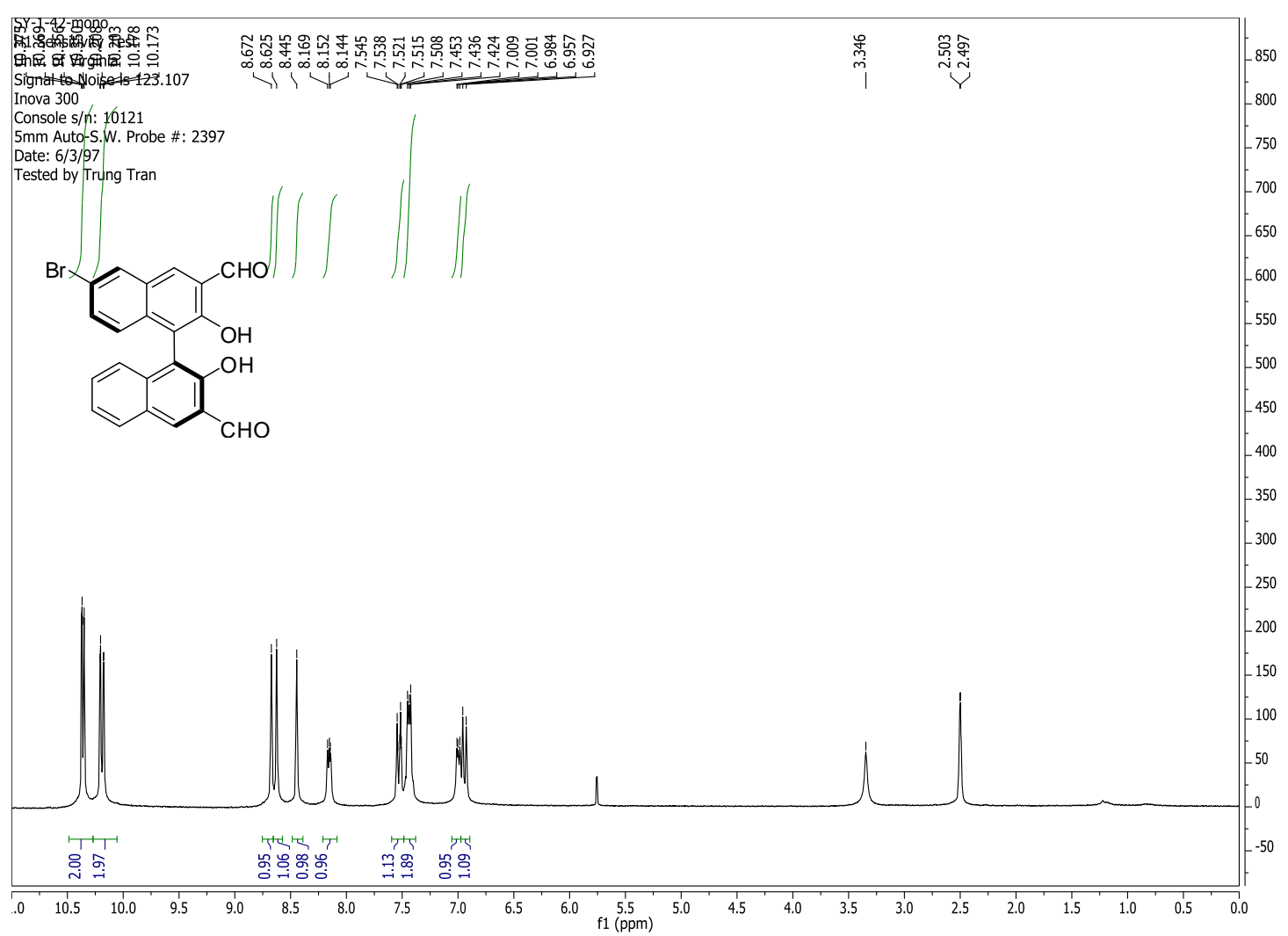



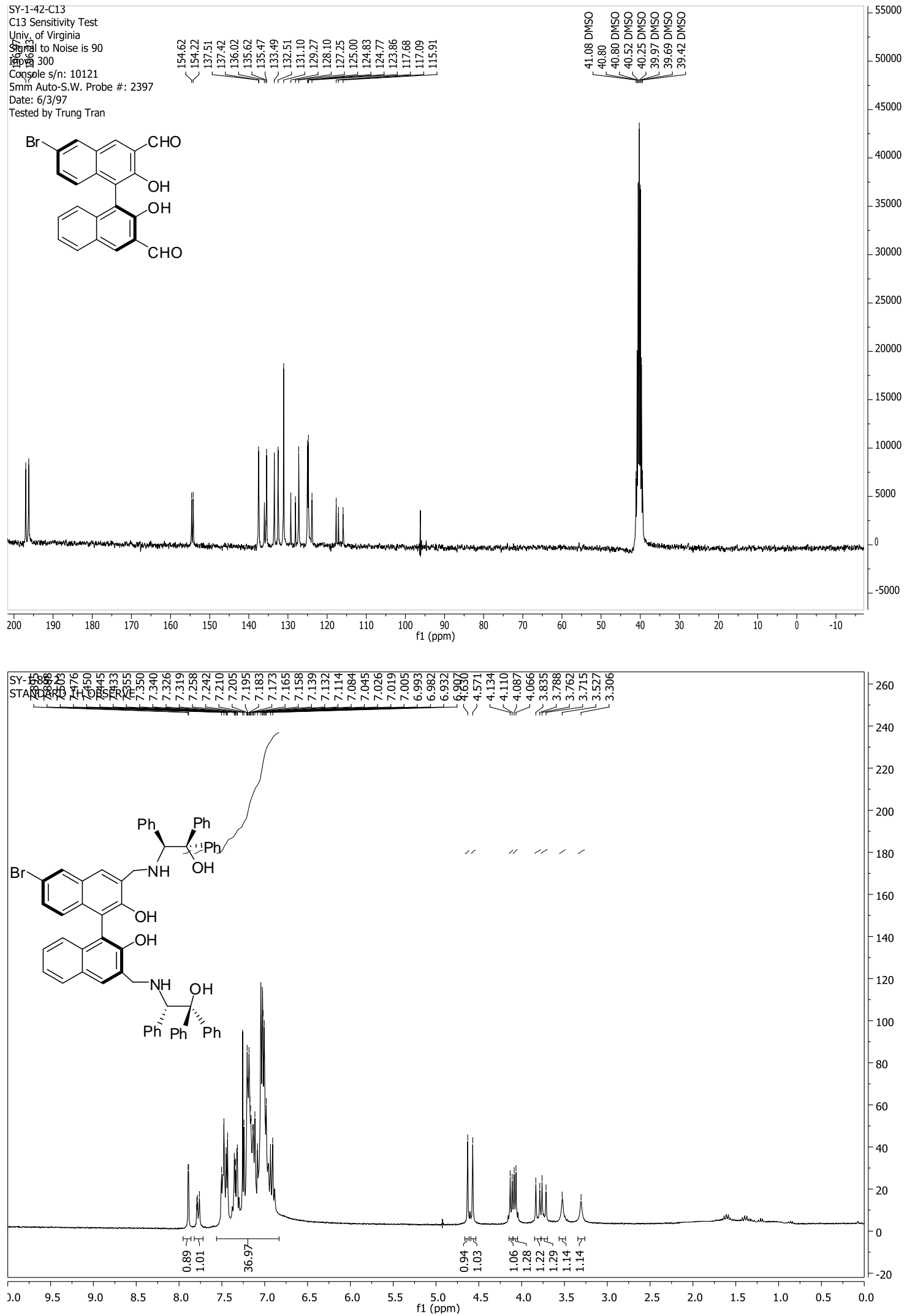

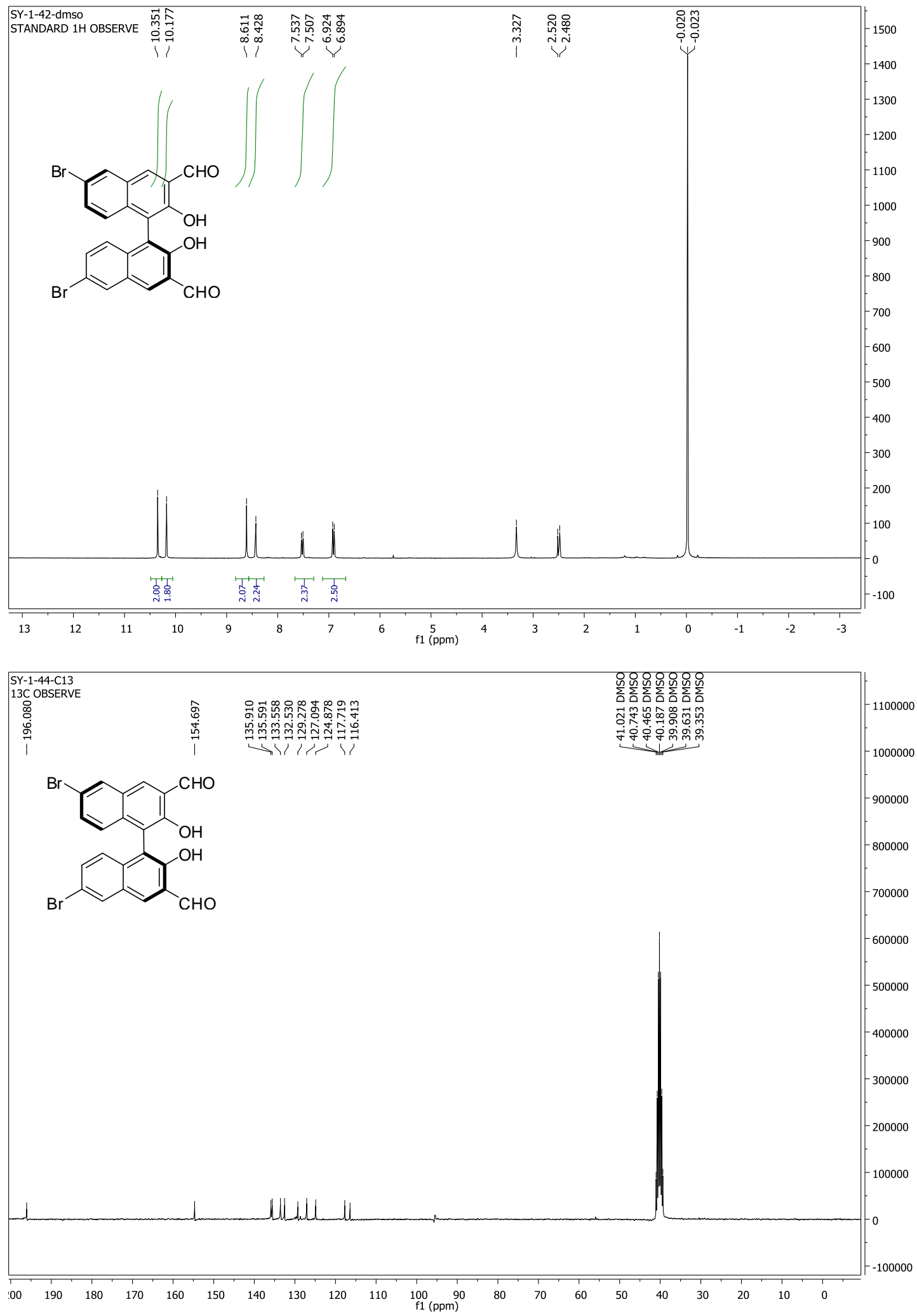

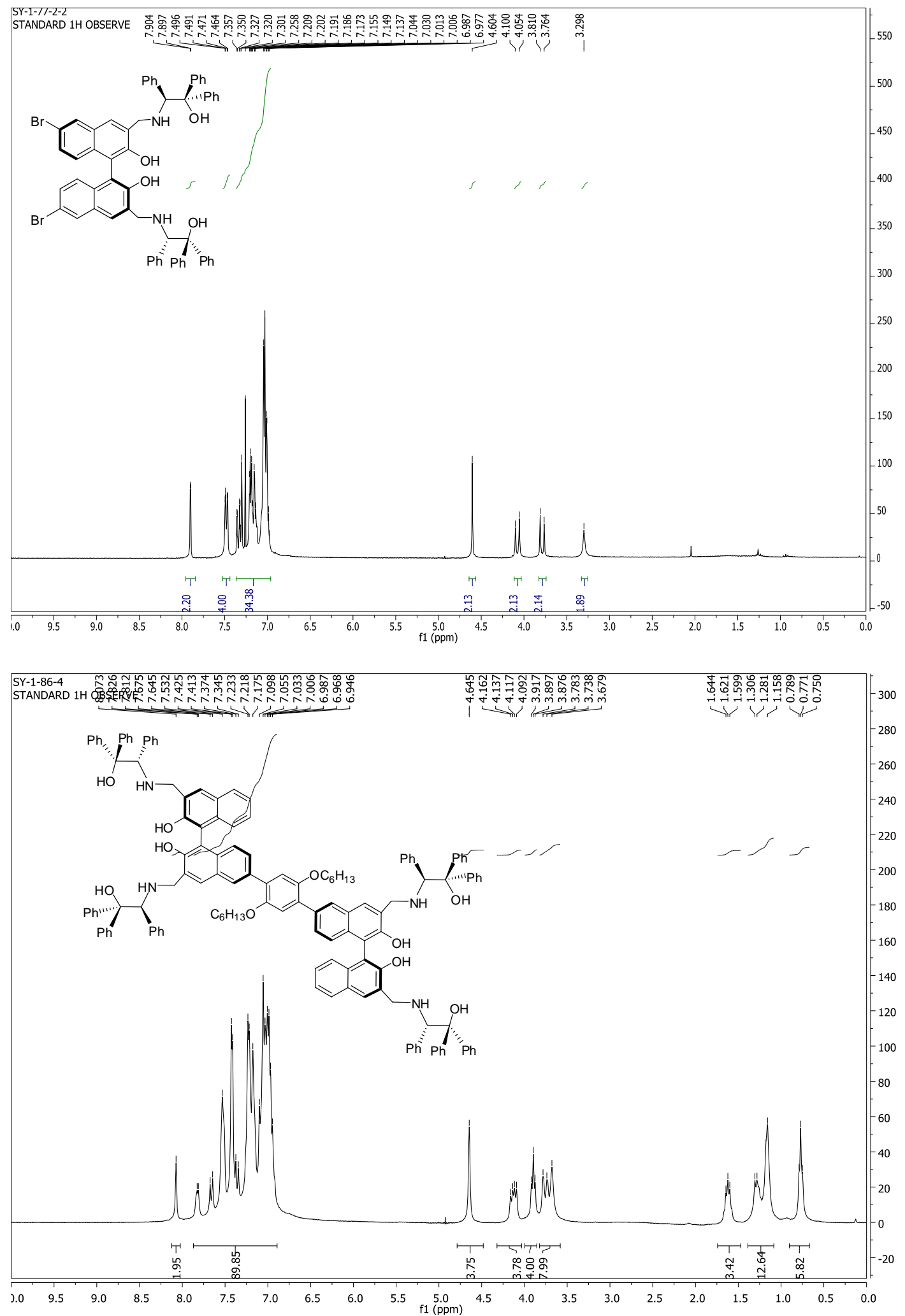

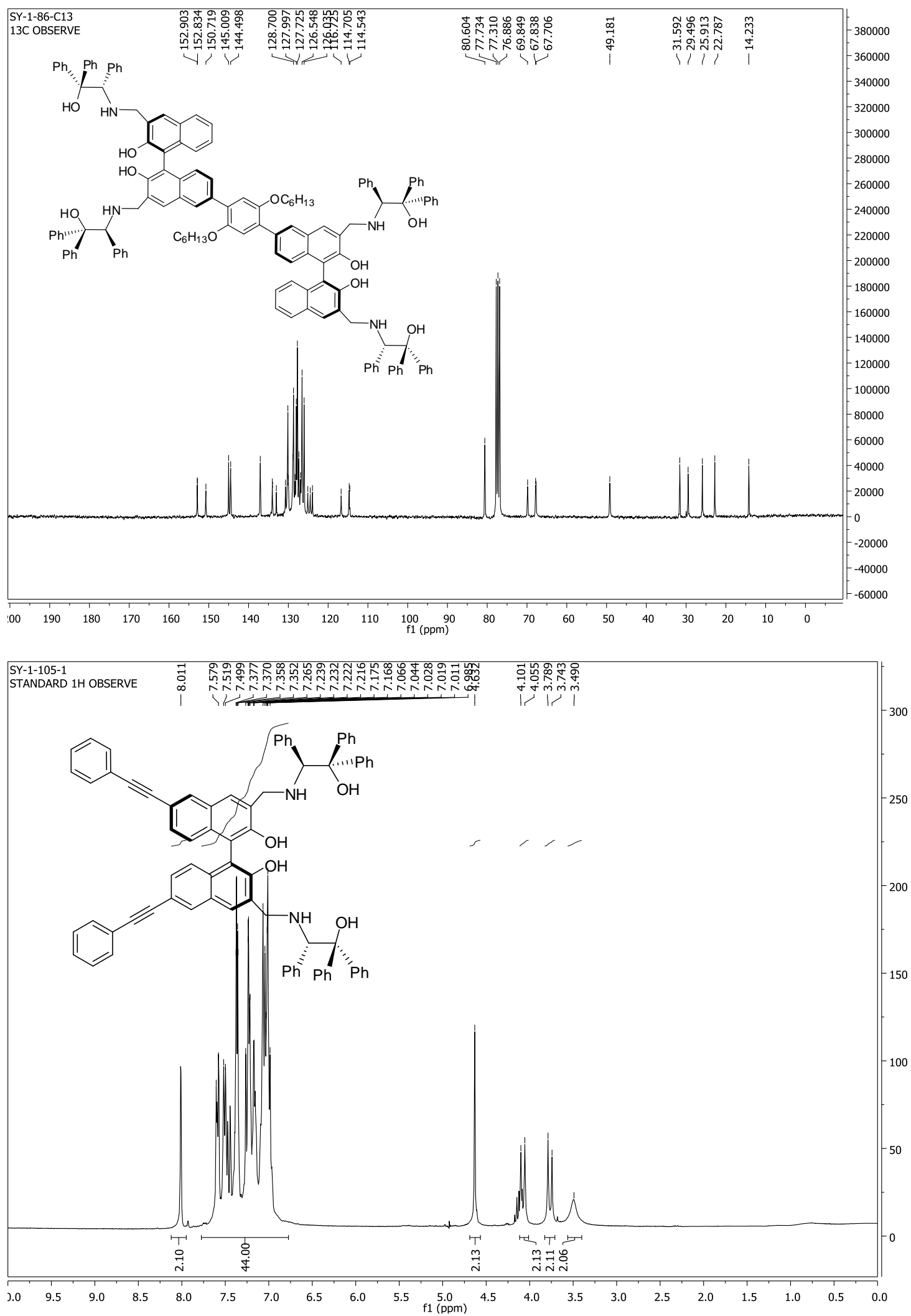

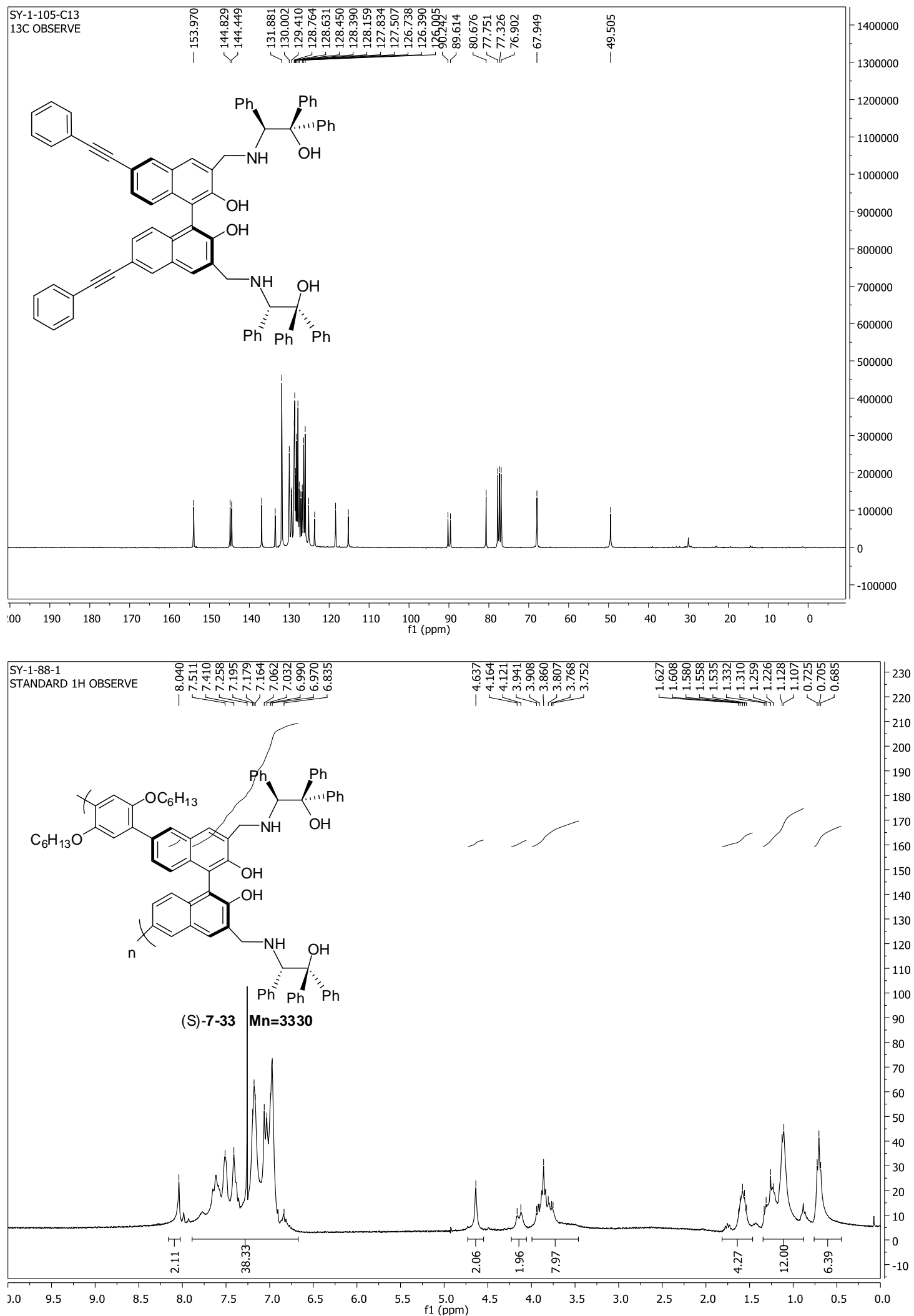


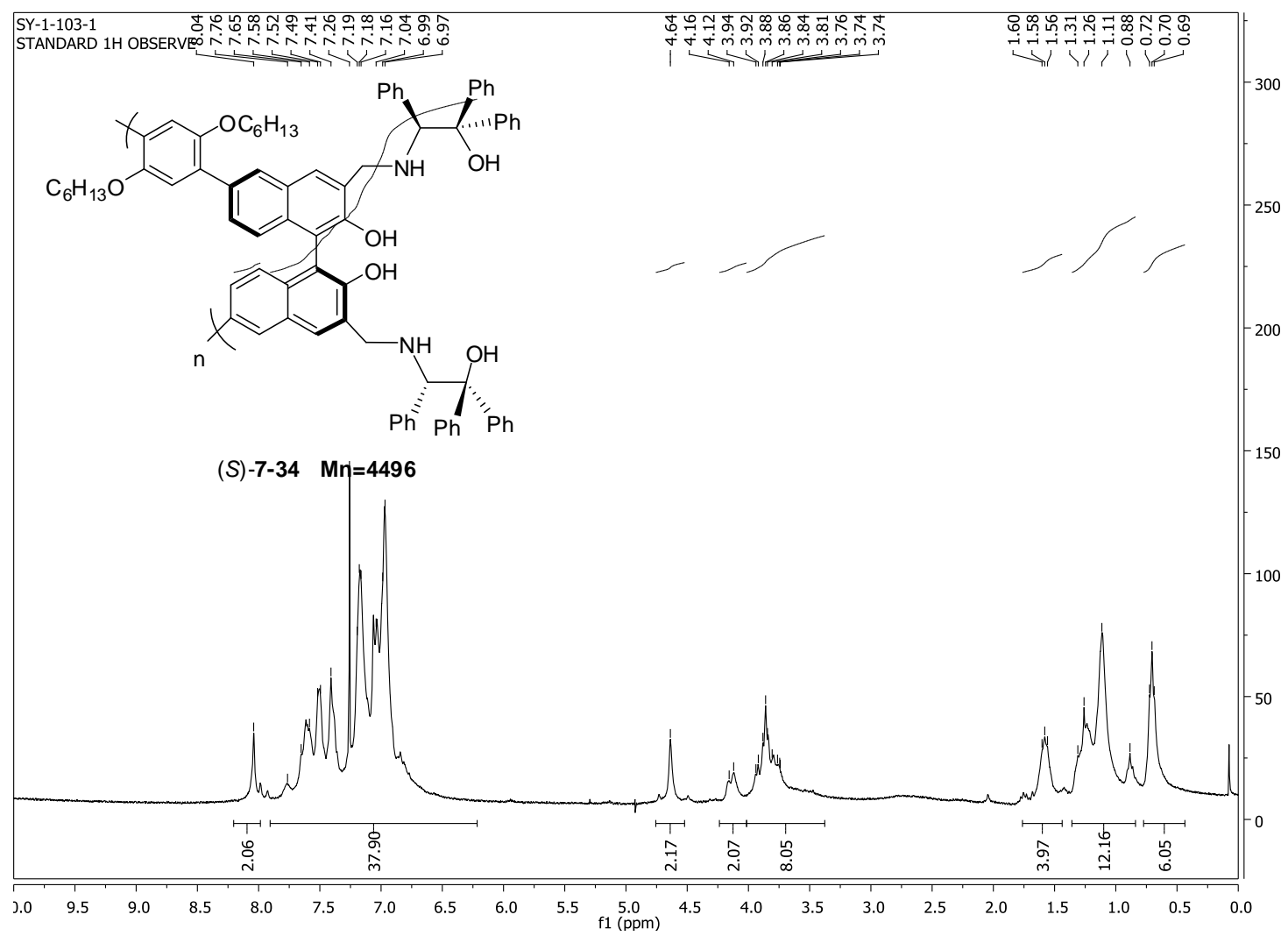

